\title{
Studies Toward Selenium- $\pi$-Acid Catalyzed Oxidative Functionalizations of Olefinic and Acetylenic Multiple Bonds
}

\author{
Dissertation \\ zur Erlangung des mathematisch-naturwissenschaftlichen Doktorgrades \\ "Doctor rerum naturalium" \\ der Georg-August-Universität Göttingen \\ GEORG-AUGUST-UNIVERSITÄT
GÖTTINGEN \\ im Promotionsprogramm \\ Catalysis for Sustainable Synthesis

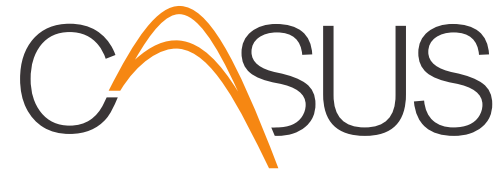 \\ der Georg-August University School of Science (GAUSS)
}

vorgelegt von

Katharina Rode

aus Hamburg

Göttingen, 2020 



\section{Betreuungsausschuss}

Prof. Dr. Alexander Breder, Institut für Organische Chemie, Universität Regensburg

Prof. Dr. Lutz Ackermann, Institut für Organische und Biomolekulare Chemie

Prof. Dr. Daniel B. Werz, Institut für Organische Chemie, TU Braunschweig

\section{Mitglieder der Prüfungskommission}

Referent: Prof. Dr. Alexander Breder, Institut für Organische Chemie, Universität Regensburg Koreferent: Prof. Dr. Lutz Ackermann, Institut für Organische und Biomolekulare Chemie

\section{Weitere Mitglieder der Prüfungskommission:}

Dr. Holm Frauendorf, Institut für Organische und Biomolekulare Chemie

Prof. Dr. Konrad Koszinowski, Institut für Organische und Biomolekulare Chemie

Prof. Dr. Inke Siewert, Institut für Anorganische Chemie

Prof. Dr. Dietmar Stalke, Institut für Anorganische Chemie

Tag der mündlichen Prüfung: 19.08.2020 

"Ja, aber Pippi", sagte Thomas.

"Du kannst ja wohl nicht Klavier spielen!"

"Wie soll ich das wissen, wenn ich es noch nie versucht hab?", fragte Pippi.

Kapitän Langstrumpf stand eine Weile still. "Mach es wie du willst", sagte er schließlich. "Das hast du immer getan."

Pippi nickte zustimmend. "Ja, das habe ich immer getan", sagte sie ruhig.

Pippi Langstrumpf /Pippi geht an Bord

Astrid Lindgren 



\section{Contents}

\section{Introduction}

1.1 Chalcogens in oxidative alkene functionalization reactions

1.1.1 Activation modes in chalcogen-mediated reactions

1.1.2 Endogenous and exogenous nucleophiles

1.2 Examples for selenium-catalyzed oxidative functionalizations of alkenes

1.2.1 Formation of $\mathrm{C}-\mathrm{N}$ bonds

1.2.2 Formation of $\mathrm{C}-\mathrm{O}$ bonds

1.2.3 Formation of $\mathrm{C}-\mathrm{X}$ bonds

2 Objectives

3 Results and Discussion

3.1 Intra- and intermolecular etherification via photo-aerobic selenium- $\pi$-acid catalysis

3.1.1 Intramolecular etherification - Preliminary investigations and optimization

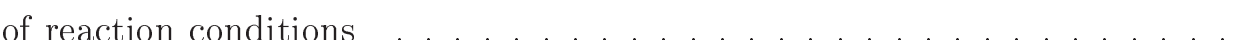

3.1.2 Intramolecular etherification - Synthesis and cyclization of unsaturated alcohols . . . . . . . . . . . . . . . . . .

3.1.3 Intermolecular etherification - Preliminary investigations and optimization of reaction conditions . . . . . . . . . . . . . . . .

3.1.4 Intermolecular etherification - Synthesis and etherification of alkenes . . . 39

3.2 Synthesis and lactonization of unsaturated acids via photo-aerobic selenium- $\pi$-acid catalysis . . . . . . . . . . . . . . . . . . .

3.2.1 Synthesis of unsaturated acids . . . . . . . . . . . . . . . . .

3.2.2 Lactonization of unsaturated acids . . . . . . . . . . . . . 48

3.3 Photo-aerobic selenium- $\pi$-acid catalysed phosphatation of alkenes . . . . . . . 52

3.4 Investigations toward the selenium-catalyzed amination of olefins . . . . . . . . 58

3.4 .1 Intermolecular light-driven amination . . . . . . . . . . . . . 58

3.4 .2 Intramolecular light-driven amination . . . . . . . . . . . . 62

3.4.3 Synthesis and cyclization of ortho-vinyl homobenzylamides . . . . . . . 68

3.5 Selenium- $\pi$-acid catalyzed synthesis of allenylamides . . . . . . . . . . 74

3.5.1 Preliminary investigations and optimization of reaction conditions . . . 74

3.5.2 Synthesis of alkynes and allenylamides . . . . . . . . . . . . . . 80

3.5.3 Mechanistic investigations . . . . . . . . . . . . . . . . . 84

3.6 Synthesis and application of chiral diselenide catalysts . . . . . . . . . . 89

3.6.1 Examination of selenation methods . . . . . . . . . . . . . . . . 89

3.6.2 Synthesis of di(binaphthyl) diselenides . . . . . . . . . . . . . . . . . . . . . . . . . . . . .

3.6.3 Synthesis of arylated binaphthyl diselenides . . . . . . . . . . . . . 95 
3.6.4 Application of diselenide catalysts ................ 98

4 Conclusion and Outlook 100

5 Experimental Section $\quad 102$

5.1 General Methods . . . . . . . . . . . . . . . . . . . . . . . . 102

$5.1 .1 \quad$ Preparative Methods . . . . . . . . . . . . . . . . . . . . 102

5.1 .2 Chromatographic Methods . . . . . . . . . . . . . . . . . . . 102

$5.1 .3 \quad$ Instrumental Analysis . . . . . . . . . . . . . . . . . . . 102

5.2 Synthesis of diaryl diselenides and photosensitizer 95 . . . . . . . . . . 103

5.3 Intramolecular etherification via photo-aerobic selenium- $\pi$-acid catalysis . . . . . 105

5.3.1 Synthesis of unsaturated alcohols 126 and $139 \ldots \ldots \ldots$. . . . . . . 105

5.3.2 Synthesis of tetrahydrofurans and tetrahydropyrans 127 and 144 . . . . 117

5.4 Intermolecular etherification via photo-aerobic selenium- $\pi$-acid catalysis . . . . 123

5.4 .1 Synthesis of alkenes 148 . . . . . . . . . . . . . . . . 123

5.4 .2 Synthesis of allylic ethers $149 \ldots \ldots \ldots \ldots$. . . . . . . . . . 125

5.5 Synthesis and lactonization of unsaturated acids via photo-aerobic selenium- $\pi$-acid catalysis . . . . . . . . . . . . . . . . . . 131

5.5.1 Synthesis of unsaturated carboxylic acids 34, 137 and $167 \ldots \ldots \ldots 131$

5.5.2 Synthesis of lactones $\mathbf{7 7}, \mathbf{1 7 0}$ and $\mathbf{1 7 1} \ldots \ldots \ldots \ldots$. . . . . . . . 137

5.6 Synthesis and phosphatation of alkenes . . . . . . . . . . . . . . 142

5.6 .1 Synthesis of alkenes $174 \ldots \ldots \ldots$. . . . . . . . . . . . . 142

5.6.2 Synthesis of phosphates $173 \ldots \ldots \ldots \ldots \ldots$

5.7 Light-driven intermolecular amination . . . . . . . . . . . . . . . 149

5.8 Synthesis of tetrahydroisoquinolines . . . . . . . . . . . . . 152

5.8.1 Synthesis of ortho-vinyl homobenzylamides 207 . . . . . . . . . . . . 152

5.8 .2 Synthesis of tetrahydroisoquinolines $209 \ldots \ldots \ldots \ldots$

5.9 Synthesis of allenylamides . . . . . . . . . . . . . . . . 167

5.9 .1 Synthesis of alkynes $242 \ldots \ldots \ldots \ldots \ldots$

5.9 .2 Synthesis of imidoallenes 253 . . . . . . . . . . . . . . 170

5.10 Syntheses towards binaphthyl diselenides and catalysis . . . . . . . . . . . 174

6 References 182

List of Abbreviations 192

\begin{tabular}{ll} 
List of Figures & 196 \\
\hline
\end{tabular}

$\begin{array}{ll}\text { List of Schemes } & 196\end{array}$

$\begin{array}{ll}\text { List of Tables } & 197\end{array}$ 
Acknowledgements

Spectra

203 



\section{Introduction}

Simple olefins are important starting materials for organic syntheses. Due to their easy availability from petrochemical industrial processes, they are abundant and inexpensive. Therefore, the functionalization of alkenes, especially oxidative functionalizations, which are a step-

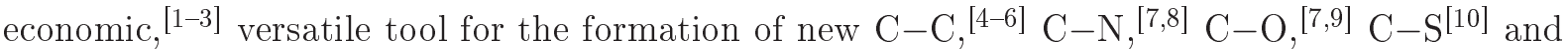
$\mathrm{C}-\mathrm{Hal}{ }^{[11,12]}$ bonds, has been the subject of extensive research in the last decades. The larger part of the methods developed thus far involve the use of transition metal catalysts such as palladium $[4,7,13,14]$ or copper complexes. [9] Although a large variety of different methods for the functionalization of terminal alkenes has been developed, the functionalization of internal alkenes remains challenging. ${ }^{[13]}$ This is due to many transition metal catalysts being prone to isomerization of the double bond to the terminal position, which often leads to mixtures of products. [13] During the last decades, the use of organocatalytic alternatives for the oxidative functionalization of terminal and internal alkenes has been explored. Especially the use of electrophilic, hypervalent iodine(III) reagents turned out to be a versatile and mild method for the formation of C-O, $\underline{\underline{15}}-\underline{18}]$ $\mathrm{C}-\mathrm{N} \underline{[19}-22]$ and $\mathrm{C}-\mathrm{Hal} \underline{23}-\underline{25]}$ bonds. In most cases, vicinal difunctionalizations of alkenes $\mathbf{1}$ took place by forming iodofunctionalized intermediate $\mathbf{3}$ followed by a substitution of the iodo-moiety with a second nucleophile, yielding the syn-product 4 (Scheme 1.1). $.26,27]$

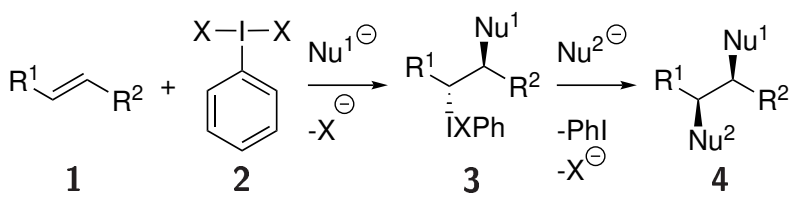

Scheme 1.1: Iodine(III)-mediated oxidative vicinal functionalization of alkenes.

Furthermore, an allylic amination of $\alpha$-methyl styrene derivatives was accomplished by MUÑIZ et al. by eliminating the iodine moiety from intermediate $\mathbf{3}$ instead of substituting it. [28] The elimination was preferred due to the iodo-moiety being attached to a tertiary carbon. The iodine(III)-mediated functionalizations were further improved by the development of catalytic variants of the previous reactions. $27,29-31]$ Because in the elimination step an iodide(I) species is formed, a suitable oxidant is needed to facilitate the reformation of the catalytically active iodine(III) species. The group of FUCHIGAMI was able to perform the reoxidation electrochemically, [29] while the groups of KITA and OCHIAI employed $m$ CPBA as the terminal oxidant. $[30,31]$ Another important achievement was the development of stereo- and enantioselective variants. FUJITA and colleagues were able to perform intramolecular oxylactonizations of ortho-alkenylbenzoates with $e e^{\prime}$ 's up to $98 \%, \underline{[32,33]}$ and WIRTH and colleagues were able to achieve the cyclization of bisnucleophilic urea derivatives to isourea derivatives with up to $99 \%$ ee by using chiral nonracemic hypervalent iodine(III) species at low reaction temperatures. ${ }^{34]}$ The application of a chiral iodine(III) catalyst was first realized by MUÑIZ and colleagues. ${ }^{[35]}$ They were able to 
accomplish the catalytic intermolecular diacetoxylation of styrenes using peracetic acid as the terminal oxidant with up to $94 \%$ ee.

A method that is conceptually cognate to the iodine(III)-mediated reactions is the application of electrophilic sulfur and selenium compounds in the oxidative functionalization of alkenes. After the development of stoichiometric methods for the oxidative chalcogenofunctionalization of alkenes, the identification of suitable oxidants facilitated the investigation of several catalytic methods. $\underline{[36,37]}$ As chalcogen-catalyzed reactions usually afford allylic or vinylic products, they beneficially complement the available repertoire of transition-metal and iodine(III)-catalyzed oxidative functionalizations of alkenes.

\subsection{Chalcogens in oxidative alkene functionalization reactions}

The first functionalization reactions of alkenes with chalcogens were addition reactions of electrophilic sulfenyl chlorides. In 1925, LECHER and colleagues described the addition of phenyl-, $p$-tolyl- and 2-nitrobenzenesulfenyl chloride to ethene which yielded chlorosulfenylated adducts 6 Equation 1.1. [38] This reactivity was further examined by KHARASCH et al., who added 2,4dinitrophenylsulfenyl chloride to cyclohexene Equation 1.2, and by Turner and Connor, who added 2-nitro-4-chlorobenzenesulfenyl chloride to several alkenes. $\underline{[39,40]}$
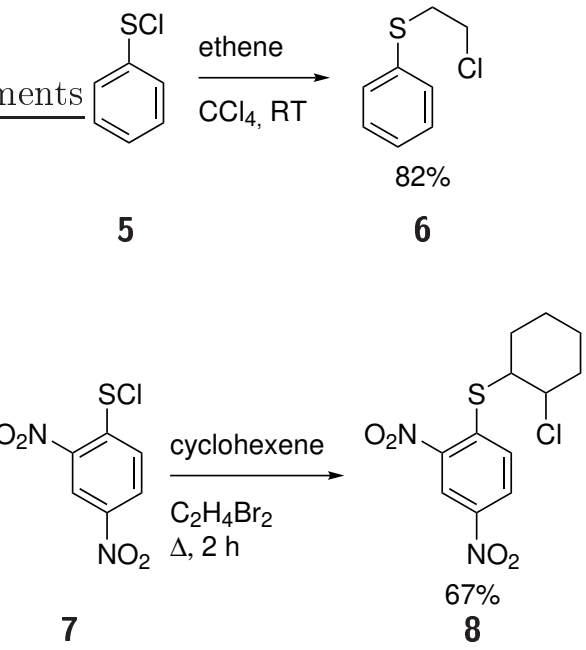

Shortly after these findings, JENNY und HöLzLE expanded this methodology to selenium elec-

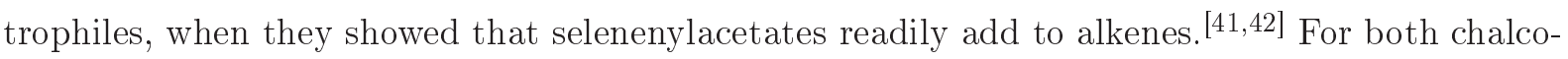
gens, the addition reaction was found to be stereospecific (trans-addition) and following MARKOVNIKOV's rule. $[\underline{40,43-45]}$ Based on this, KHARASCH and Buess proposed a thiiranium ion as an intermediate in the addition of sulfenyl chlorides to nonsymmetric alkenes. $\underline{43]}$ Such an ion could be formed either by homolytic cleavage of the $\mathrm{S}-\mathrm{Cl}$ bond of a sulfenyl chloride followed by a radical reaction, or by a polarization of the $\mathrm{S}-\mathrm{Cl}$ bond which enables an electrophilic attack of the sulfur (Scheme 1.2). 


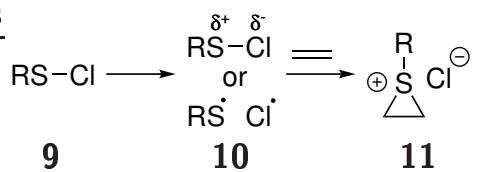

Scheme 1.2: Formation of thiiranium ion 11 via an ionic or a radical pathway.

Evidence for the existence of thiiranium ions was provided in the 1960s, when PETTIT and HelmKamP isolated several thiiranium compounds (e.g., Figure 1.1, 12). 47,48$]$ Similarly, Garatt and SCHMID isolated and characterized a series of seleniranium ions as well as episelenurane 14, which is one of only few examples of this compound class ever reported Figure 1.1, 13 and 14). $[49,50]$

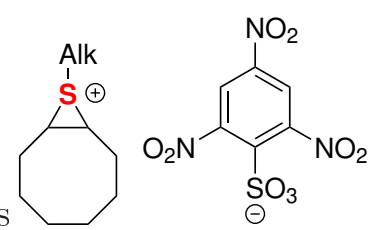

12

Pettit, Helmkamp

1963 and 1964

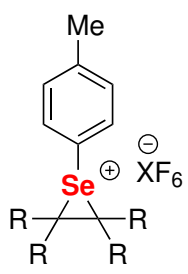

13
Garratt, Schmid

1974 and 1975

Figure 1.1: First synthesized thiiranium and seleniranium ions.

When the chalcogeniranium ions were subjected to nucleophiles, they gave way to the same products that were obtained in the functionalization of the respective alkenes with the chalcogen compounds. [50,51] This supports the assumption that chalcogeniranium ions are intermediates in the functionalization reaction of alkenes.

An important reactivity of the chalcogenofunctionalized products is the possibility to eliminate the chalcogen moieties under oxidative conditions, which results in the formation of alkenes. This was first found by KingsBury and CRAm in 1960, when they showed that sulfoxides 15 eliminate to alkenes 16 in good yields when they are heated (Equation 1.3). [52] Jones and colleagues observed a similar behavior in the case of selenoxides (Equation 1.4. .53 . However, a striking difference between sulfur and selenium is that for the elimination reaction of sulfur, high temperatures are needed, whereas selenium compounds readily eliminate at room temperature or even at $0{ }^{\circ} \mathrm{C} . \underline{[53]}$

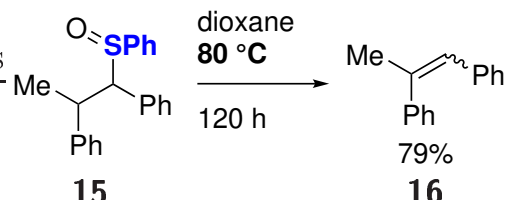




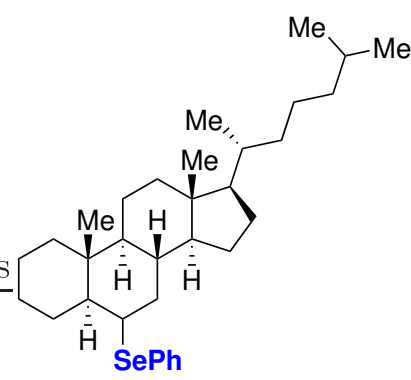

17

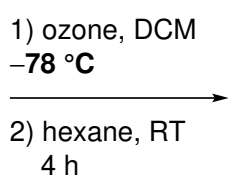

$4 \mathrm{~h}$

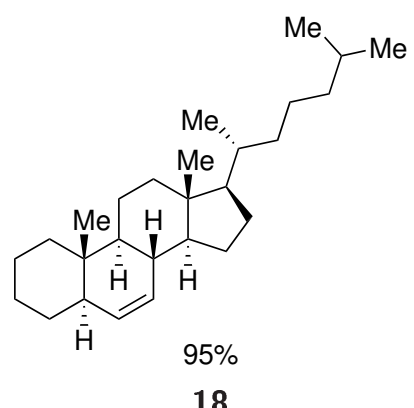

18

Sharpless and LAUER exploited this reactivity when they converted epoxides $\mathbf{1 9}$ into allyl alcohols 21 (Scheme 1.3). They opened the epoxide with a phenylselenyl anion to form hydroxy selenide 20. The subsequent oxidation of the selenide by $\mathrm{H}_{2} \mathrm{O}_{2}$ afforded the corresponding allylic alcohols $\mathbf{2 1}$ in yields ranging from 40-98\%.

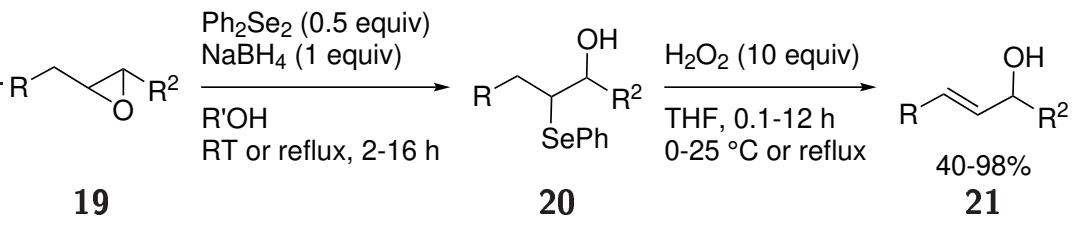

Scheme 1.3: Allylic alcohol synthesis by SHARPLESS and LAUER.

A few years later, SHARPLESS advanced this methodology further, when he and HoRI developed the first selenium-catalyzed chlorination of olefins with 3 mol\% diphenyl diselenide, 1.1 equiv $N$-chlorosuccinimide (NCS, 22) as the oxidant and chloride source and $10 \mathrm{~mol} \%$ pyridine (Equation $1 . 5 \longdiv { . 1 1 1 }$

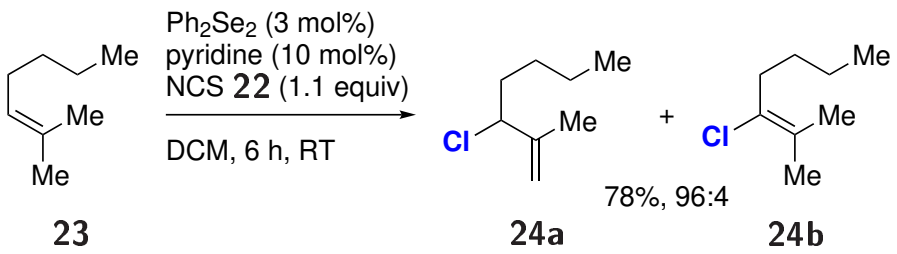

They hypothesized that diphenyl diselenide (25) and NCS (22) react with each other to form $\mathrm{PhSeCl}$ (27), which adds to the double bond of alkene 23 (Scheme 1.4). The reaction of selenide 28 with NCS eventually results in product formation by the elimination of $\mathrm{PhSeCl}$ along with succinimide. Following this first catalytic reaction, different methods for the selenium-catalyzed oxidative functionalization of alkenes were developed.

\subsubsection{Activation modes in chalcogen-mediated reactions}

The activation modes in chalcogen-mediated reactions are based on two distinct roles of the chalcogen atom. In the first activation mode, the chalcogen atom acts as a LEWIS base and 


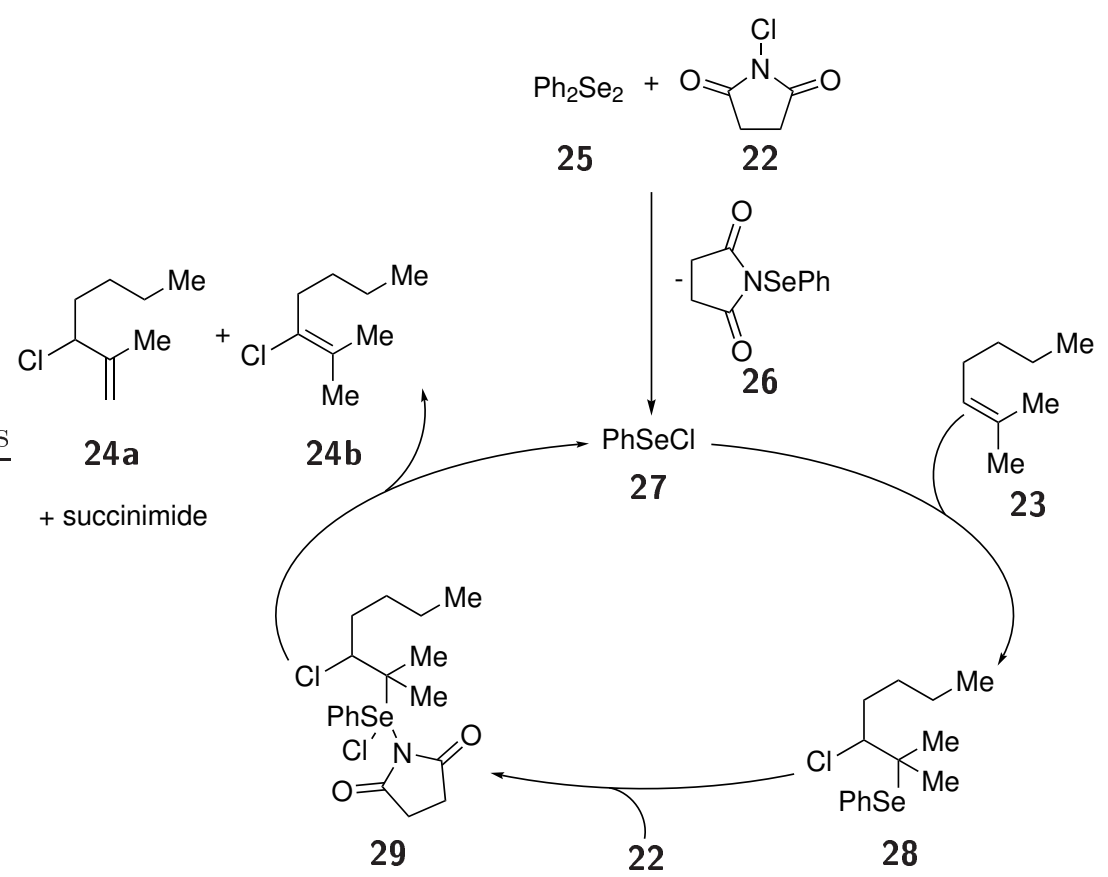

Scheme 1.4: Proposed mechanism of the catalytic chlorination by HORI and SHARPLESS.

activates an electrophile $\mathbf{3 0}$, which can react in its activated form $\mathbf{3 2}$ with an unactivated alkene (Scheme 1.5). .54

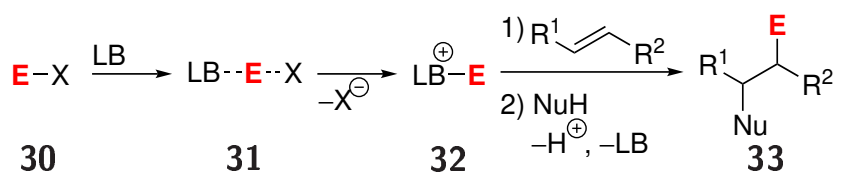

Scheme 1.5: LEwIS base activation of an electrophile and subsequent alkene functionalization.

Important examples for this kind of activation are the chlorination of alkenes with NCS by SHARPLESS and HorI (Scheme 1.4 and cognate halogenations, 1 11.12] as well as halocyclization reactions. 5 [5-59] One of the first examples of a selenium-catalyzed bromolactonization was de-

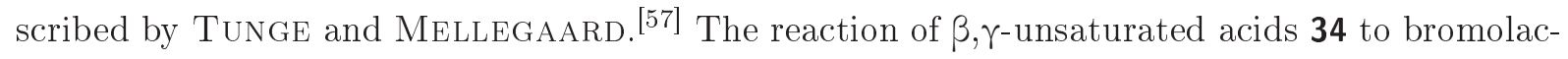
tones 35 was realized by the LEWIS base activation of $N$-bromosuccinimide (NBS) by diphenyl diselenide (Equation 1.6). The authors first suspected a reaction of diphenyl diselenide and NBS to $\mathrm{PhSeBr}$ and phenylselenyl succinimide, reminiscent of the reaction with NCS in the chlorination reaction by SHARPLESS and Hori (cf. Scheme 1.4). As both $\mathrm{PhSeBr}$ and phenylselenyl succinimide did not reproduce the results with diphenyl diselenide, they hypothesized that the Se-Se bond remains intact and proposed the formation of bromonium ion $\mathbf{3 6}$ and the subsequent attack of the carboxylate moiety. 


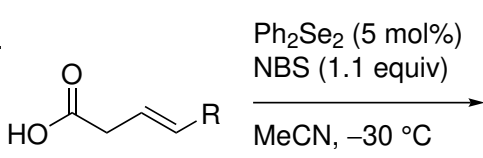

34<smiles>O=C1CC(Br)C(Br)O1</smiles>

35

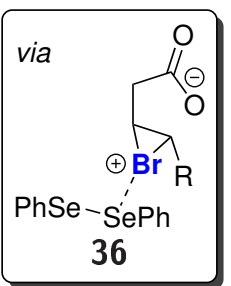

In the other activation mode of chxalcogen-mediated reactions, the electrophilic chalcogen atom activates the double bond of an olefin for a nucleophilic attack by acting as a LEWIS acid. In the transition state of this activation, the antibonding $\sigma^{*}$-orbital of the $\mathrm{Ch}-\mathrm{X}$ bond receives electron density from the binding $\pi$-orbital of the double bond (donation) and the non-bonding $\mathrm{n}$ orbitals of the chalcogen donate electrons into the antibonding $\pi^{*}$-orbital of the double bond (back-donation) (Figure 1.2 a). $\underline{[37,60]}$ This interaction is somewhat reminiscent of the binding situation found in certain transition metal-olefin complexes, which has been described by the DEWAR-CHATT-DUNCANSON model. In these systems, the donating interaction is between a $\pi$-orbital of the double bond and a $\sigma^{*}$-orbital of a metal-ligand bond or an empty d orbital of the metal. The back-donation into the $\pi^{*}$-orbital of the double bond results from a filled $\mathrm{d}$ orbital of the metal (Figure 1.2p).
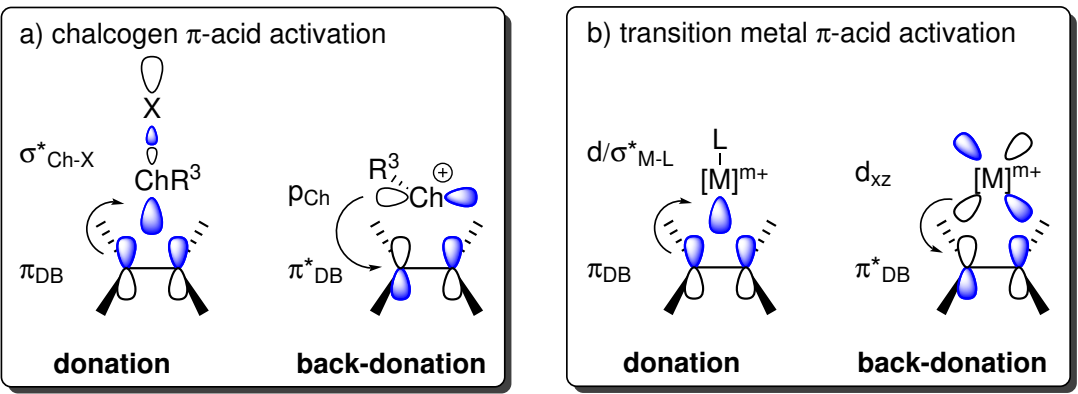

Figure 1.2: Proposed orbital interactions in the $\pi$-acid activation of olefins by a) chalcogenium ions and b) transition metals.

The overall mechanism of the $\pi$-acid activation of an alkene $\mathbf{3 7}$ with a chalcogen atom and the following functionalization with a nucleophile is depicted in Scheme 1.6. During the electrophilic attack of the chalcogen LEWIS acid $\mathbf{3 8}$ on the $\pi$-bond, chalcogeniranium ion $\mathbf{3 9}$ is formed, which is activated for a nucleophilic attack. Subsequent oxidation and elimination of the chalcogen species affords functionalized product 40.62]

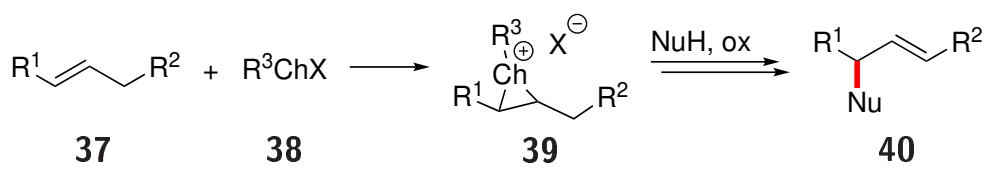

Scheme 1.6: Oxidative functionalization of alkene 37 via $\pi$-acid activation. 


\subsubsection{Endogenous and exogenous nucleophiles}

In the course of their close examination of the mechanism of the PhSeCl-mediated selenolactonization reaction, DENMARK and EDWARDS were able to provide NMR evidence for the formation of seleniranium ion $\mathbf{4 1}$ as an intermediate of the reaction. $\underline{62]}$ Furthermore, they observed the reversible formation of chlorinated side products $\mathbf{4 2}$ and $\mathbf{4 3}$ from this intermediate by attack of the nucleophilic chloride counter ion (Scheme 1.7). By conducting NMR experiments at low temperatures, they were able to identify adduct $\mathbf{4 2}$ as the kinetic adduct. When instead of the free acid the methyl ester was used and the reaction mixture was warmed to room temperature, lactone formation could not occur and they observed the preferred formation of adduct 43, and thus identified this species as the thermodynamic adduct.

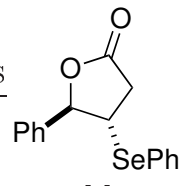

44
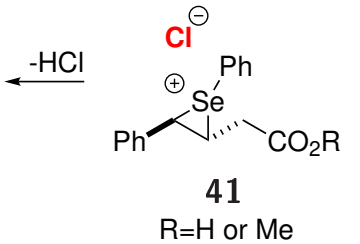<smiles>O=C(O)CC([SeH])C(Cl)c1ccccc1</smiles>

42<smiles>O=C(O)CC(Cl)C(c1ccccc1)c1ccccc1</smiles>

43

kinetic

thermodynamic

Scheme 1.7: Formation of selenolactone 44 and adducts 42 and 43 from seleniranium ion 41.

The observation that the attack of the counterion can in certain cases be faster than the attack of the intramolecular nucleophile illustrates a challenge in the selenium- $\pi$-acid catalyzed functionalization of alkenes. In some cases, nucleophiles originating from the oxidant (endogenous nucleophiles) outcompete intramolecular or intermolecular external nucleophiles (exogenous nucleophiles), leading to the formation of an undesired side product. Therefore, the choice of the nucleophile and the oxidant can be crucial for the course of the reaction.

\subsection{Examples for selenium-catalyzed oxidative functionalizations of alkenes}

Following the catalytic chlorination by SHARPLESS and HORI, various other selenium-catalyzed oxidative functionalizations of alkenes were developed, which promote the formation of $\mathrm{C}-\mathrm{N}$, $\mathrm{C}-\mathrm{O}$ and $\mathrm{C}-\mathrm{X}$ bonds and differ in the oxidants and nucleophiles used.

\subsubsection{Formation of $\mathrm{C}-\mathrm{N}$ bonds}

The first allylic and vinylic amination of alkenes via selenium- $\pi$-catalysis was reported in 2013 by BREDER et al. ${ }^{[63]}$ Remarkably, they were able to use $N$-fluorobenzenesulfonimide (NFSI) as the oxidant and nitrogen source, whereas other $N$-halogenated oxidants usually serve as a halogen source (e.g. allylic chlorinations with NCS by SHARPLESs et al. and Tunge et al.). [11,12] Allyl imides $\mathbf{4 6}$ were obtained from alkenes $\mathbf{4 5}$, bearing an electron-withdrawing group (EWG) in the 
allylic position, in yields between 49 and $84 \%$ using 5 mol\% diphenyldiselenide (25) and 1 equiv NFSI at room temperature (Scheme 1.8, top). The imidation of cyclic alkenes $\mathbf{4 7}$ was realized under slightly changed conditions and afforded vinylic imides $\mathbf{4 8}$ in yields between 37 and $95 \%$ (Scheme 1.8, bottom).

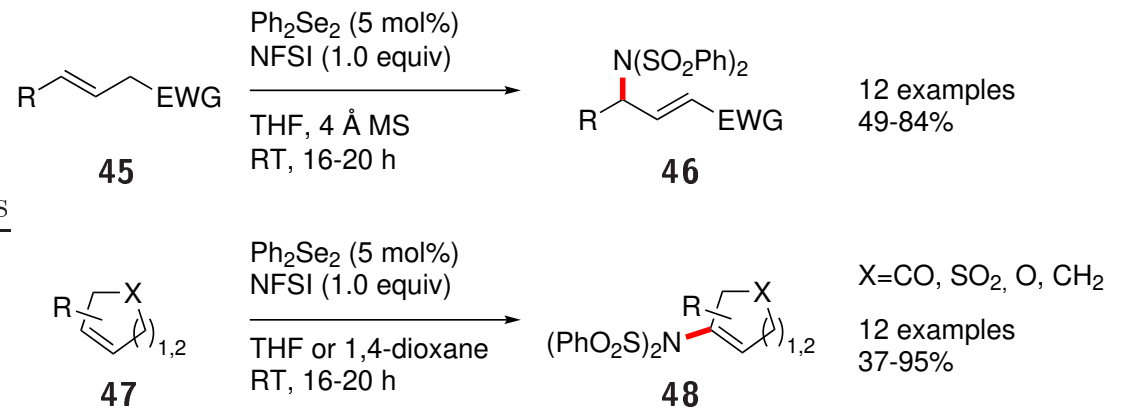

Scheme 1.8: Selenium- $\pi$-acid catalyzed allylic and vinylic imidation by BREDER et al. $\underline{[63]}^{[6}$

The use of NFSI as the nitrogen source and oxidant in selenium-catalyzed amination reactions turned out to be a groundbreaking discovery and led to several following reactions using $\mathrm{N}$ fluorinated oxidants. In 2015, ZHAO et al. were able to use NFSI in the amination of allylic alcohols $\mathbf{4 9}$ to obtain 3-amino-allylalcohols $\mathbf{5 0}$ in good yields between 63 and 95\% (Equation 1.7). [64] The high selectivity for the formation of the anti-MARKOVNIKOV product was rationalized by the authors to originate from a stabilizing interaction of the hydroxy group with a cationic seleniranium intermediate.
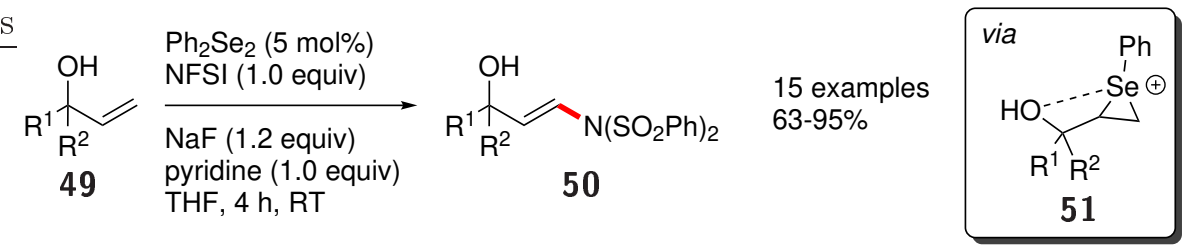

In the same year, the groups of both BREDER and ZHAO independently discovered that the intramolecular $\mathrm{C}-\mathrm{H}$ amination of simple alkenes, which was previously dominated by metalcatalyzed methods, could also be facilitated by the use of diphenyl diselenide and NFSI as the oxidant. $[65,66]$ Both groups were able to catalyze the formation of indoles from ortho-vinylated anilines under conditions that differ in solvent, temperature and catalyst loading (Scheme 1.9). While ZHAO et al. conducted the reaction in 1,4-dioxane at $30{ }^{\circ} \mathrm{C}$ with $10 \mathrm{~mol} \%$ of diphenyl diselenide, BREDER et al. were able to decrease the loading to $2.5 \mathrm{~mol} \%$ in most cases, but needed to perform the reaction in toluene at $100{ }^{\circ} \mathrm{C}$. Futhermore, they showed that with their method azaindoles could also be obtained in good yields between 57 and $81 \%$.

During their attempt to expand this methodology to non-aromatic amines, ZHAO et al. tested several oxidants for the formation of tetrahydrofurans, tetrahydropyrans and cyclic tosyl amides. [67] They found out that $N$-fluoropyridinium triflate (57), first disclosed by DENMARK and colleagues 


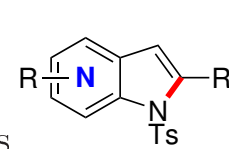

53

Breder et al.

25 examples $42-99 \%$
$\mathrm{Ph}_{2} \mathrm{Se}_{2}(10 \mathrm{~mol} \%)$

$\stackrel{\mathrm{NFSI}(1.05 \text { equiv) }}{\longrightarrow} \mathrm{R}$

1,4-dioxane

$30^{\circ} \mathrm{C}, 18 \mathrm{~h}$

52

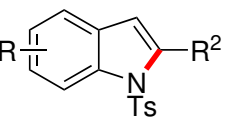

54

Zhao et al.

22 examples

$31-96 \%$

Scheme 1.9: Indole formation under different conditions by BREDER et al. and ZHAO et al. $.65,66]$

in their work on selenium-catalyzed dichlorinations, $\underline{[68]}$ was a potent oxidant for this reaction. The formation of cyclic tosyl amides $\mathbf{5 6}$ from tosyl amides $\mathbf{5 5}$ in yields between 54 and $93 \%$ was performed by treating them with $10 \mathrm{~mol} \%$ of diphenyl diselenide, 1.2 equiv of $\mathbf{5 7}$ and 1.0 equiv $\mathrm{NaF}$ (Equation 1.8).

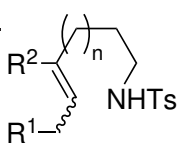

55

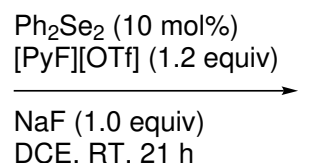

DCE, RT, $21 \mathrm{~h}$

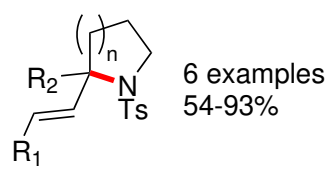

56

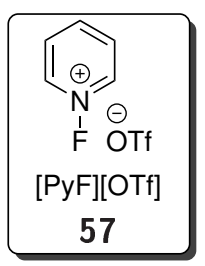

This new $N$-fluorinated oxidant could also be used in the pyridination reaction of 1,3-dienes and alkenes. ${ }^{[69]}$ Interestingly, the reaction of dienes $\mathbf{5 8}$ with 20 mol\% of dibenzyl diselenide and 1.2 equiv of $[\mathrm{PyF}][\mathrm{OTf}] \mathbf{5 7}$ selectively afforded products $\mathbf{5 9}$ with the pyridinium moiety at C-2 in yields between 40 and $89 \%$ Equation 1.9. In previous studies that rely on transition metal catalysts, the functionalization usually occurs with anti-MARKOVNIKOV selectivity. $\underline{[0}-\underline{73}]$

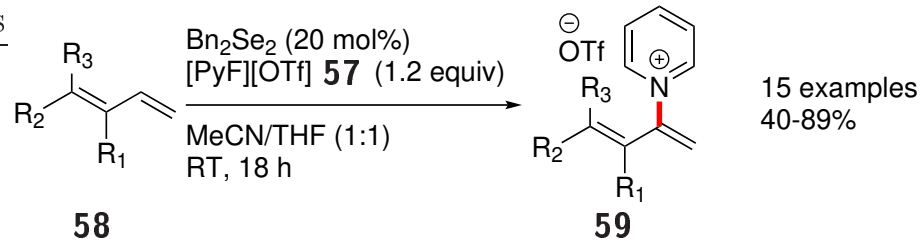

The selectivity holds true for styrene derivatives as well and products $\mathbf{6 1}$ were obtained under otherwise unaltered conditions in good yields between 62-96\% Equation 1.10). However, when a non-styrenic alkene was used, the selectivity changed to $\mathrm{C}-1$ pyridination.

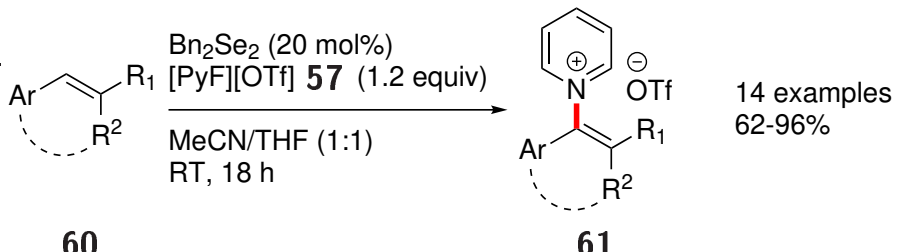


During their experiments for the pyridination of dienes with different $N$-fluoropyridinium salts as oxidants and nitrogen source Equation 1.9, ZHAO et al. noticed that bulky $N$-fluorinated pyridinium salts like $\mathrm{N}$-fluoro-2,4,6-trimethylpyridinium tetrafluoroborate (65) did not lead to product formation and attributed this to them being unsuitable nucleophiles due to the steric shielding of the nitrogen lone pair. As a consequence, they tried to use pyridines $\mathbf{6 3}$ as exogenous nucleophiles together with pyridinium salt $\mathbf{6 5}$ (1.2 equiv) as the oxidant and Selectfluor (0.5 equiv) as the co-oxidant for a faster deselenenylation. Under these conditions, they were able to obtain pyridinium salts 64 in good yields Equation 1.11). Remarkably, this transformation is one of only few examples for a selenium-catalyzed reaction with an exogenous nucleophile.

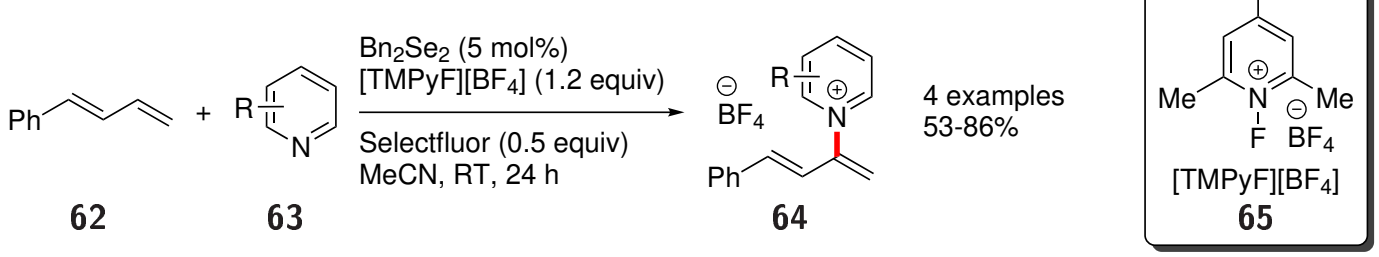

Although the employment of pyridine derivatives $\mathbf{6 3}$ as exogenous nitrogen nucleophiles is an exciting finding, it is important to develop further methods that can use different nitrogen sources in the selenium-catalyzed allylic and vinylic functionalization of alkenes. In the design of the established methods, the choice of oxidant played a key role. Therefore it is important to further examine different oxidants, especially concerning competing reactions between exogenous and endogenous nucleophiles.

\subsubsection{Formation of $\mathrm{C}-\mathrm{O}$ bonds}

One of the first selenium-catalyzed allylic oxyfunctionalizations of alkenes was realized as early as in 1992 by IWAOKA and TOMODA. They were able to insert a methoxy- or acetoxy moiety into simple alkenes like 4-octene (66) by using $10 \mathrm{~mol} \%$ of diselenide catalyst $\mathbf{6 9}$ together with the same amount of copper(II) nitrate and 1.0 equiv sodium peroxodisulfate as the oxidant in methanol or acetic acid as the solvent Equation 1.12. ${ }^{[74]}$ In previous reactions, the selenium compound could not be used catalytically. This was attributed to the formation of selenenic acid during oxidative elimination using oxidants such as $\mathrm{O}_{3}, \mathrm{NaIO}_{4}$ or peroxides, which could not be reintroduced into the catalytic cycle. As a possible reason, the subsequent disproportionation reaction was suggested. To avoid this, the amine backbone of diselenide $\mathbf{6 9}$ served to stabilize the respective selenenic acid after its elimination from the functionalized alkene. The allylic ethers or esters were obtained in low to moderate yields between 16 and $51 \%$ and the scope remained limited. 

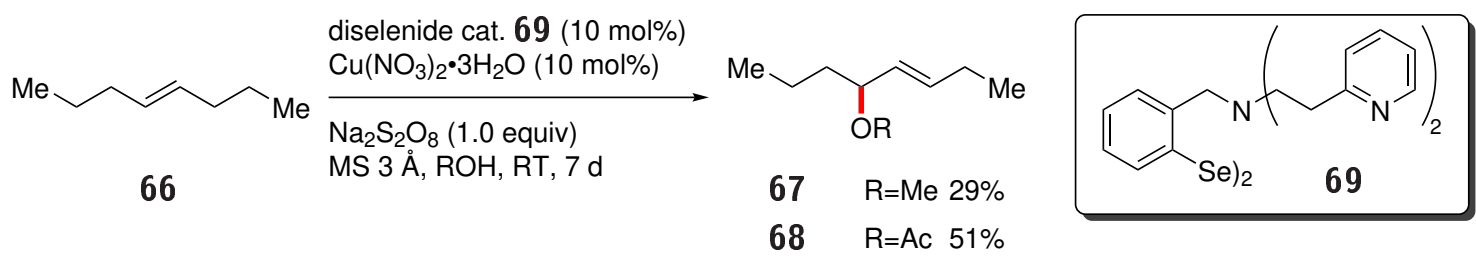

Shortly after, TIECCO and colleagues reported a procedure for the alkoxy and hydroxy functionalization of alkenes using simple diphenyl diselenide (25) as the catalyst and an excess of ammonium peroxodisulfate as the oxidant Equation 1.13. .75$]$ They obtained allylic alcohols and ethers 70 in $23-90 \%$ yield in short reaction times of 2-7 hours. In contrast to IwAOKA and TOMODA, they used substrates that contained electron-withdrawing groups and heated the reaction mixture to $60{ }^{\circ} \mathrm{C}$.

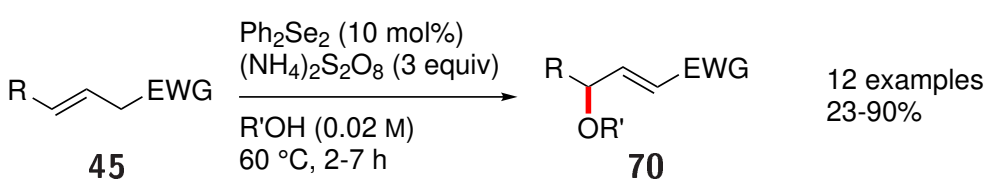

Having identified ammonium peroxodisulfate as a suitable oxidant for selenylation-deselenylation reactions, the group of TIECCO applied this strategy to several other functionalization reactions. Under slightly modified conditions, they were able to catalyze the formation of butenolides 72 from $\beta, \gamma$-unsaturated acids 71 in high yields [Scheme 1.10a). ${ }^{[76]}$ Furthermore, the formation of dihydrofuranes $\mathbf{7 4}$ was catalyzed in excellent yields (Scheme 1.10 $\mathrm{b}$ ). $\underline{\text { [77] }}$ The reaction of $\beta, \gamma$-unsaturated ketones $\mathbf{7 5}$ to furanes $\mathbf{7 6}$ was also realized, but the diselenide had to be used stoichiometrically to prevent the decomposition by the starting material by the oxidant (Scheme 1.10 $).[78]$

a)

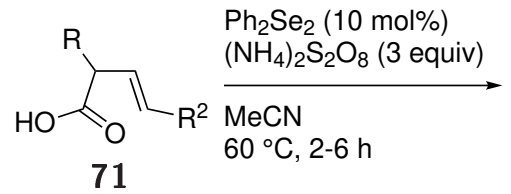

b)

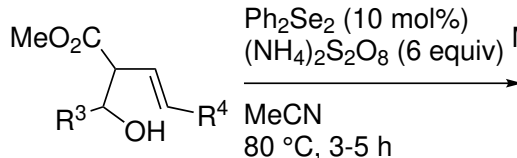

73

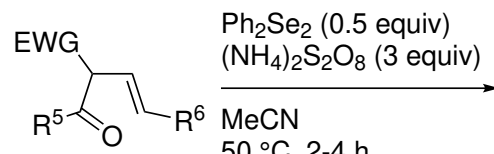

75<smiles>[R]C1=CC([R])OC1=O</smiles>

8 examples $21-97 \%$

10 examples $89-96 \%$

10 examples $45-91 \%$

Scheme 1.10: Subsequent oxygenation reactions by TIECCO et al. 
Although ammonium peroxodisulfate has the advantages that no endogenous nucleophile can arise from it and only non-toxic sulfate is formed as a side product, the reactions need elevated temperatures and in some cases, e.g., the formation of furans (Scheme 1.10), the oxidant is potent enough to oxidize the substrate. $\stackrel{78]}{ }$

As an alternative to peroxodisulfate, hypervalent iodine compounds have been found to work equally well as oxidants in phenylselenylation reactions with diphenyl diselenide. $\underline{\text { [79-81] }}$ Their application in catalytic selenation-deselenylation reactions was first disclosed by the group of WIRTH in 2007. The lactonization of $\beta, \gamma$-unsaturated acids 34 with 5 mol\% of diphenyl diselenide and 1.05 equiv of [bis(trifluoroacetoxy)iodo]benzene (PIFA) as the oxidant afforded lactones $\mathbf{7 7}$ in moderate to excellent yields between 49 and 96\% [Equation 1.14. ${ }^{[82]}$ Compared to the previous works, lower catalyst and oxidant loadings could be used and the reaction worked at room temperature. Electronically neutral acids worked well $(\mathrm{R}=n$-propyl, $n$-butyl or $n$-decyl, yield $>65 \%$ ), however, as only simple alkyl and aryl moieties are present in the scope, the functional group tolerance of the method could not be evaluated.

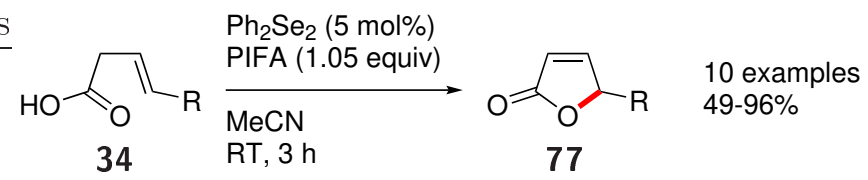

The conditions for the formation of butenolides $\mathbf{7 7}$ were also applied to other lactonization reactions. When styrylbenzoic acids $78\left(\mathrm{R}^{1}=\mathrm{Ar}, \mathrm{R}^{2}=\mathrm{H}\right)$ were subjected to PIFA and a catalytic amount of diphenyl diselenide, isocoumarins $\mathbf{7 9}$ were obtained. However, in order to ensure complete conversion of the selenofunctionalized intermediate into the product, the catalyst loading had to be increased to $10 \mathrm{~mol} \%$ and the reaction time to $16 \mathrm{~h}$ (Scheme 1.11, top). cyclization of aryl-substituted $\beta, \gamma$-unsaturated acids $78\left(\mathrm{R}^{1}, \mathrm{R}^{2}=\mathrm{Ar}\right)$ was examined, it was realized that the reaction time could be reduced to 30 min when ultrasound was used (Scheme 1.11, bottom).

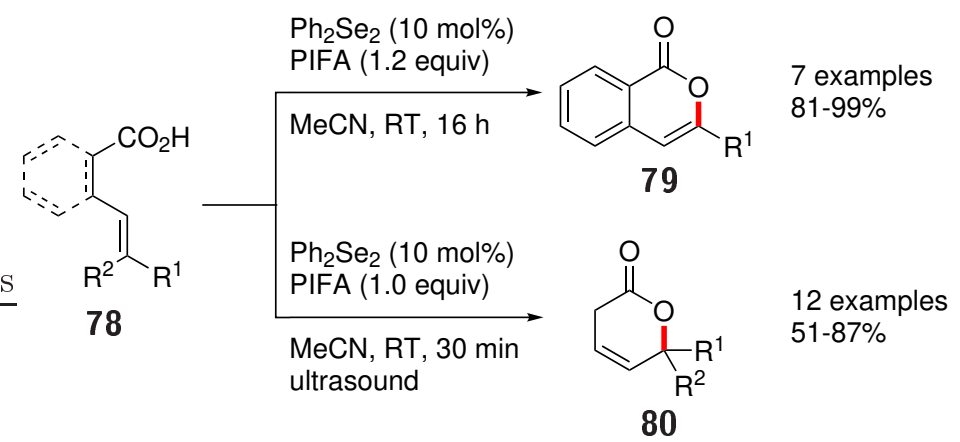

Scheme 1.11: Formation of isocoumarins 79 and dihydropyranones 80 by WIRTH et al.

Hypervalent iodine reagents are a valuable addition to the oxidants available for seleniumcatalyzed functionalization reactions, as only equimolar amounts are needed (compared to the 
high excess that is needed when peroxodisulfates are used). Furthermore, they usually need low reaction temperatures. Unfortunately, only functionalizations with acid nucleophiles were examined.

The possibility of using electrochemistry for the formation of phenylselenium cations from diphenyl diselenide for oxyselenylation reactions as well as the oxidation of selenides with the following elimination of the selenium moiety came up in the early 1980s. $\underline{\text { [85-87] }}$ In contrast to the conventional methods, where the oxidations are two-electron processes, electrochemical oxidations take place as one-electron oxidations. TORI and colleagues were the first to disclose such an electrochemical oxyselenylation/elimination sequence using only a catalytic amount of diphenyl diselenide Equation 1.15. [88] With water or methanol as the nucleophiles, allylic alcohols and methyl ethers $\mathbf{8 2}$ were obtained from alkenes $\mathbf{8 1}$ in good yields between 69 and $91 \%$.

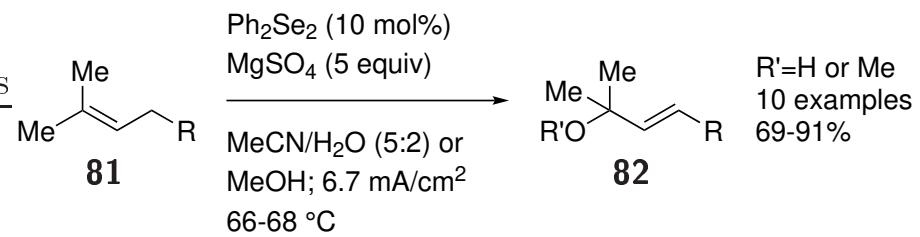

Although a promising alternative to reactions with conventional oxidants, selenium-catalyzed electrochemical functionalizations of alkenes remain sparse. A later example by the group of WIRTH depicts potential difficulties that arise with the method. They examined the allylic methoxylation of alkene 83 using 10 mol\% of diphenyl diselenide along with 1.0 equiv of $\mathrm{Et}_{4} \mathrm{NBr}$, which served both as the electrolyte and a redox catalyst. The reactive selenium species $\mathrm{PhSeBr}$ was presumably formed by the reaction of diphenyl diselenide and bromine, which originated from the oxidation of bromide ions. WIRTH and colleagues observed that the ratio of the formed products depended strongly on the applied current [Equation 1.16). .89$]$ When only $0.5 \mathrm{~mA}$ was used, the mixture contained $64 \%$ of the desired product $\mathbf{8 5}$, but also $19 \%$ of the selenofunctionalized intermediate $\mathbf{8 4}$ and $17 \%$ of additional side products. The product formation could be increased when the current was raised to $3 \mathrm{~mA}$ ( $83 \%$ of $\mathbf{8 5}, 5 \%$ of $\mathbf{8 4}$ and $12 \%$ side products), but with higher currents, overfunctionalized product $\mathbf{8 6}$ began to form (36\% at $10 \mathrm{~mA}$, together with $46 \%$ of desired product $\mathbf{8 5}$ and $18 \%$ side products). The desired product $\mathbf{8 5}$ was isolated from the reaction under a $3 \mathrm{~mA}$ current, with a $50 \%$ yield. Further experiments with water, ethanol, isopropanol and acetic acid led to moderate yields between 48 and $62 \%$.

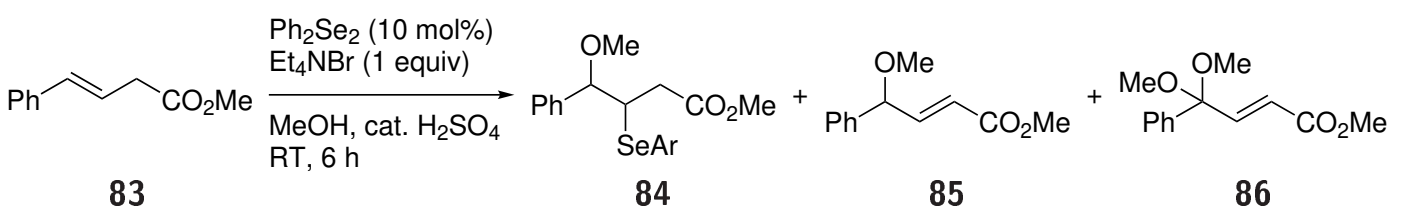


As demonstrated by these last reports, electrochemistry can be used in the functionalization of alkenes with exogenous nucleophiles under mild conditions. The formation of side products can be controlled by tuning the applied current. Nevertheless, the yields in WIRTH's experiments were only moderate and the development of more efficient conditions is desirable.

While in previous studies $N$-fluorinated oxidants were successfully applied in amination reactions, in which these reagents were also the source for endogenous nitrogen nucleophiles, BREDER et al. succeeded in using NFSI to promote a selenium-catalyzed allylic oxyfunctionalization. The synthesis of isobenzufuranones $\mathbf{8 8}$ from benzoic acid derivatives $\mathbf{8 7}$ was achieved with 10 mol\% diphenyl diselenide and 1.0 equiv NFSI in yields of up to $81 \%$ [Equation 1.17). [90] Interestingly, the substrates did not undergo the expected 6-exo-trig cyclization, but formed the corresponding 5 -membered ring.

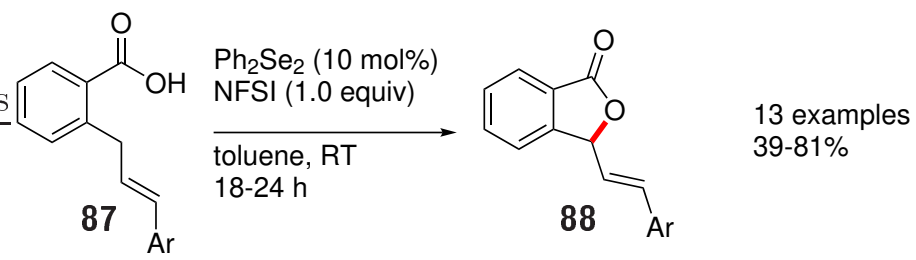

The mechanism for the product formation was therefore examined more closely. Through their following experiments, the authors were able to rule out different hypothesized scenarios and developed the following mechanistic rationale for the formation of isobenzofuranones $\mathbf{8 8}$ (Scheme 1.12). According to this hypothesis, the reaction starts with an attack of an electrophilic selenium-species on the double bond, leading to the formation of seleniranium ion 89. Instead of the direct nucleophilic attack of the carboxy group (red arrow), the opening of the seleniranium ion occurs via the elimination of a benzylic hydrogen atom, leading to the formation of styrene derivative 90. After a subsequent oxidation of the selenium moiety, an $\mathrm{S}_{\mathrm{N}} 2^{\prime}$ reaction by attack of the carboxy group on the double bond leads to elimination of the selenium species and formation of product $\mathbf{8 8}$.<smiles>O=C(O)c1ccccc1C/C=C/[Mg]</smiles>

87

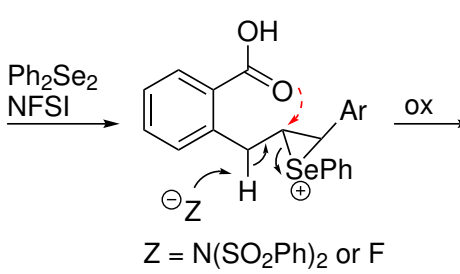

89<smiles>O=C(O)/C=C(\C(=O)O)c1ccccc1/C=C/C(=O)O</smiles>

90<smiles>Cc1ccc2c(c1)C(/C=C/[Al])OC2=O</smiles>

88

Scheme 1.12: Suggested mechanism for the formation of isobenzofuranones 88 .

In the course of their investigation of the cyclization of tosylamides 55, ZHAO et al. were also able to perform the corresponding oxyfunctionalizations with $N$-fluoropyridinium triflate as the oxidant, resulting in the formation of tetrahydrofuranes and -pyranes $\mathbf{9 2}$ from unsaturated 
alcohols 91 [Equation 1.18. [67] The reaction tolerated a lower catalyst loading than the amination reaction (5 instead of 10 mol\% diphenyl diselenide) and proceeded in good yields of $67-96 \%$.

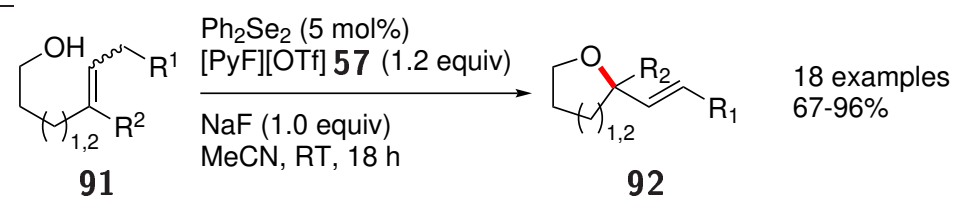

In the presented reactions, the intramolecular nucleophiles outcompete the endogenous nucleophiles originating from the oxidant. However, this competition still poses a problem for intermolecular functionalization reactions. Therefore, the identification of a different oxidant, which does not release strong endogenous nucleophiles, is necessary.

In this context, BREDER et al. disclosed a new approach that was based on a photoredox catalyst that permits the use of ambient air as the terminal oxidant and therefore enables the use of exogenous nucleophiles. [91] Their idea was based on findings by PANDEY and RAGAIns, who were able to facilitate the oxidative cleavage of diphenyl diselenide as well as the cleavage of selenides under photoredox conditions. $[92-96]$ While PANDEY and colleagues used 1,4-dicyanonaphthalene (DCN), which acts as a one-electron acceptor in its excited state, RAGAINs et al. initiated the homolytic cleavage of diphenyl diselenide by irradiation with blue light $(\lambda=455 \mathrm{~nm})$.

BREDER and colleagues speculated that a selenylation-deselenylation reaction of alkenes could be performed if a photosensitizer with a suitable redox potential (reduction potential of the excited state is higher than the oxidation potential of diphenyl diselenide, $E_{0 x}=1.35 \mathrm{~V}$ vs. SCE) ${ }^{[97]}$ was used as a one-electron-shuttle together with air as the terminal oxidant. ${ }^{\text {[91] }}$ They identified 2,4,6tris(4-methoxyphenyl)pyrylium tetrafluoroborate $(\mathbf{9 5}, p$-MeO-TPT) as a suitable photocatalyst and achieved the intermolecular esterification of alkenes $\mathbf{9 3}$ with different carboxylic acids in good yields using 10 mol\% of diphenyl diselenide and 5 mol\% of photosensitizer 95 under an atmosphere of air and irradiation with blue light Equation 1.19.

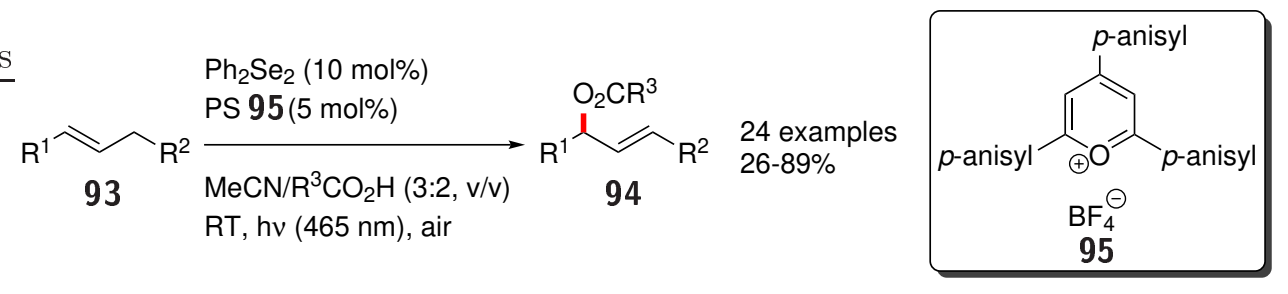

The oxidation steps involving photosensitizer $\mathbf{9 5}$ were envisioned to proceed via its excitated state after irradiation with blue light (intermediate 95*, Scheme 1.13). In this state, a single electron transfer (SET) from either diphenyl diselenide or the selenofunctionalized intermediate $\mathbf{9 9}$ can occur and radical $\mathbf{9 6}$ is formed, which is reconverted to its ground state $\mathbf{9 5}$ by an oxidation by oxygen. The superoxide, which is formed by the reduction of oxygen, undergoes further reactions to finally give water as the terminal reduction product of oxygen reduction. 


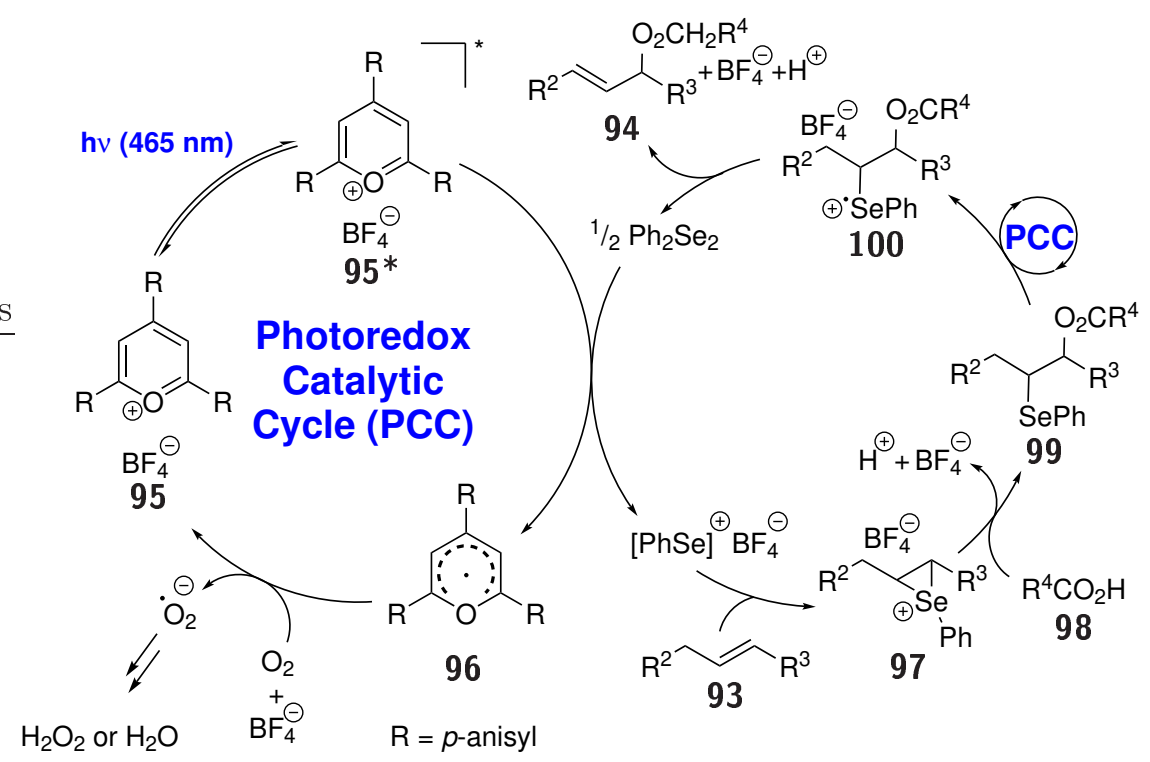

Scheme 1.13: Envisioned catalytic cycle for the intermolecular esterification.

These last results show a new possibility for the incorporation of exogenous nucleophiles into alkenes via selenium-catalysis. It is an important task to further examine the scope of this reaction regarding different nucleophiles and types of bond formation.

\subsubsection{Formation of $\mathrm{C}-\mathrm{X}$ bonds}

As discussed, the first selenium-catalyzed oxidative functionalizations of alkenes was the allylic chlorination of alkenes, where NCS (22) was activated by the reaction with diphenyl diselenide (cf. Equation 1.5. [11] This method was further studied by Tunge and MellegaARD who directly used $\mathrm{PhSeCl}$ (27) (10 mol\%) and NCS for the allylic chlorination of allylic acids, esters, arenes and nitriles $\mathbf{4 5}$ in yields of $62-89 \%$ Equation 1.20 . 12 ]

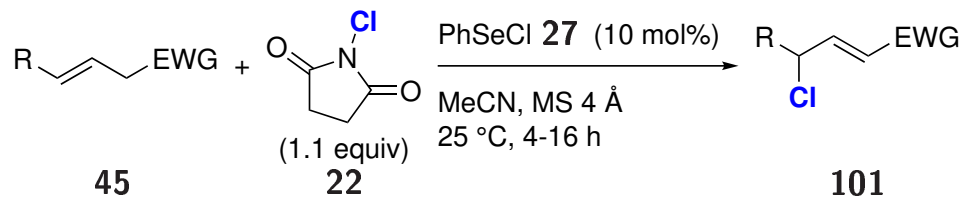

A seminal work concerning selenium-catalyzed dichlorinations of alkenes was published in 2015 by Denmark et al. ${ }^{[68]}$ This work was remarkable in several points, as on the one hand, it was the first selenium-catalyzed difunctionalization reaction, and on the other hand the reaction was synstereospecific, while in the traditional dichlorination methods using $\mathrm{Cl}_{2}$ an anti-dichlorination takes place. ${ }^{[98]}$ The syn-dichloro motif is especially common in chlorosulfolipids, a class of polychlorinated marine natural products. ${ }^{[99-102]}$ The selectivity for a syn-dichlorination was achieved by the judicious choice of the oxidant and chloride as the nucleophile. The authors reasoned that the oxidant had to fulfill several criteria: it must not oxidize the substrate or chloride ions, it 
must not release endogenous nucleophiles that outcompete chloride, and it must not transform the selenium moiety to a species that is capable of a fast syn-elimination, which would lead to the allylic product. They identified $N$-fluoropyridinium tetrafluoroborate as a suitable oxidant and obtained syn-dichlorides 103 in up to $91 \%$ yield and up to $>99: 1 \mathrm{dr}$ using 5 mol\% diphenyl diselenide, 3.0 equiv $\mathrm{BnEt}_{3} \mathrm{NCl}$ as the chloride source, 1.3 equiv $[\mathrm{PyF}]\left[\mathrm{BF}_{4}\right]$ as the oxidant, 2.0 equiv $\mathrm{Me}_{3} \mathrm{SiCl}$ as a fluoride scavenger and 1.0 equiv 2,6-lutidine $N$-oxide as a LEWIS base additive Equation 1.21). In the proposed mechanism, the syn-selectivity was rationalized by the intermediate formation of chloroselenenylated product 104 and a subsequent $\mathrm{S}_{\mathrm{N}} 2$-reaction at the selenium moiety by a chloride ion.
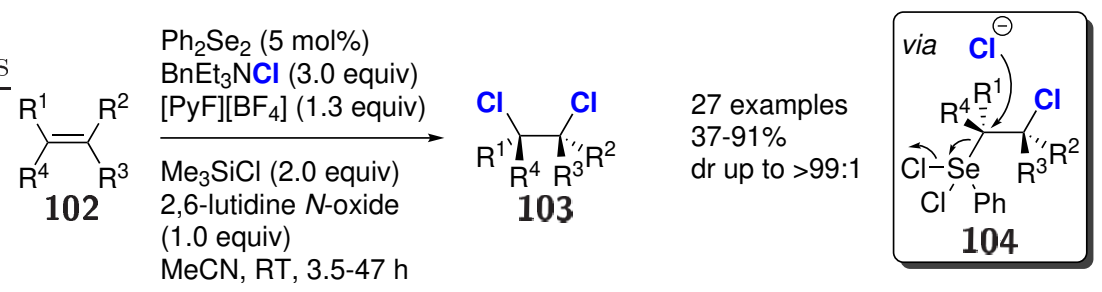

Until recently, $\mathrm{N}$-fluorinated compounds could only be used as oxidant and/or nitrogen source and not for fluorination reactions, as the endogenous nitrogen nucleophiles react faster than the fluorides. Zнао et al. observed that bulky $N$-fluorinated pyridinium salts like $N$-fluoro-2,4,6trimethylpyridinium tetrafluoroborate $(\mathbf{6 5})$ were weak nucleophiles and used them to promote a pyridination reaction with exogenous pyridine nucleophiles (Equation 1.11, Subsection 1.2.1). [69] They realized that they could also apply this idea to fluorination reactions and were successful in promoting the allylic fluorination of electron-poor alkenes 106 using 2.0 equiv of [TMPyF][OTf] (105) as the oxidant and fluoride source along with $10 \mathrm{~mol} \%$ of dibenzyl diselenide as the catalyst (Equation 1.22. 103$]$ Additionally, 0.5 equiv of TEMPO were used as an additive to prevent the decomposition of the diselenide catalyst. Under these conditions, fluorides $\mathbf{1 0 7}$ were obtained in good yields between 59 and $97 \%$.

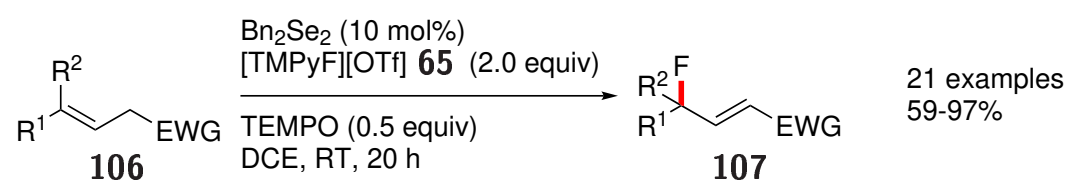

\subsubsection{Asymmetric selenium-catalysis}

Ever since the first successful selenium-catalyzed reactions were found, the endeavor to develop asymmetric reactions has been ongoing. As the reactions with electrophilic selenium reagents were found to proceed via a seleniranium ion that is opened in a trans-fashion, the efforts were directed towards the synthesis of chiral selenium species that allow for the stereoselective formation of these intermediates (see Subsection 1.1.1). $.62,104]$ In this context, the configurational stability of chalcogeniranium ions is an important aspect and was therefore examined more closely. Com- 
putational studies by the groups of RADOM and BORODKIN suggest that it is possible to transfer the chalcogenium ion from a chalcogeniranium ion to another olefin. $105-107]$ WIRTH et al. and DENMARK et al. were able to support this hypothesis by NMR studies and additionally showed that the formation of chalcogeniranium ions is reversible. [62,108,109] The olefin-to-olefin transfer of chalcogenium ions is a mechanism that leads to racemization of enantioenriched chalcogeniranium ions and therefore prevents the formation of enantioenriched chalcogenofunctionalization products $\underline{\text { Scheme 1.14 }}$, path a)..$\underline{37,110]}$ Another pathway that leads to racemization of enantioenriched chalcogeniranium ions is the attack of a nucleophile on the chalcogenium moiety and the following dissociation of the chalcogeniranium ion, leading to the loss of the stereoinformation (Scheme 1.14, path b). $\underline{.37,110]}$

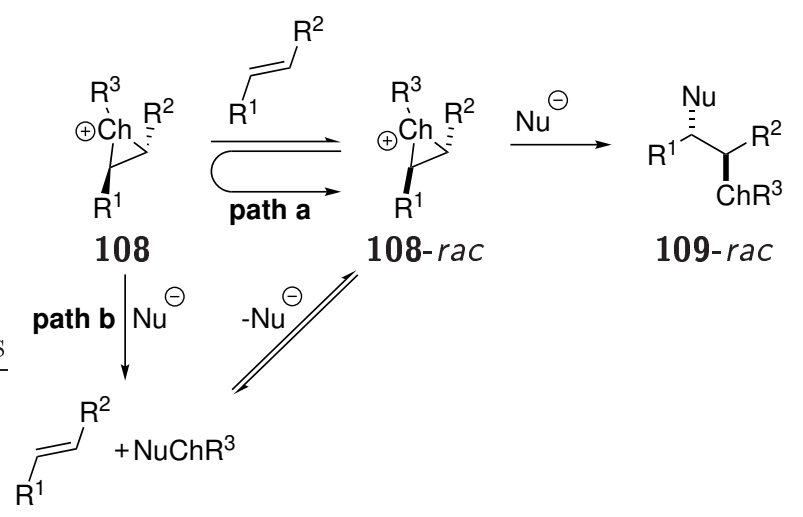

Scheme 1.14: Erosion of enantioenriched chalcogeniranium ion 108 via a) olefin-to-olefin transfer and b) nucleophilic attack on the chalcogenium. $\underline{[37.110]}$

In the course of asymmetric selenium-catalyzed reactions, several strategies for the stabilization of seleniranium ions and the consequential synthesis of enantioenriched selenofunctionalized products were developed. $37,104,110,111]$ A frequently applied method is the use of LEWIS basic side chains in vicinity to the selenium atom. The interaction of a lone pair from these side chains with the LUMO of the selenium cation is supposed to block the free coordination site and prevent the attack of another alkene on the selenium ion (Figure 1.3a). A similar approach is the use of sterically demanding groups, which would prevent attack on the selenium atom by alkenes as well as nucleophiles (Figure 1.3p). For the third strategy, electron-withdrawing groups are attached to the selenium-moiety, polarizing the endocyclic $\mathrm{Se}-\mathrm{C}$ bonds (Figure 1.3 ). On the one hand, this polarization leads to a higher electrophilicity of the selenium atom and thus a stronger binding to the carbon centers. On the other hand, it increases the probability of a nucleophilic attack at these carbon centers instead of the selenium atom.

The application of chiral selenium compounds with the mentioned features in the synthesis of enantioenriched selenofunctionalized products was successful and products with high diastereomeric excess were obtained. $\stackrel{[104]}{[}$ For example, the selenolactonization of acid $\mathbf{1 1 3}$ with diferrocenyldiselenide $\mathbf{1 1 5}$ afforded selenolactone $\mathbf{1 1 4}$ in $84 \%$ yield with a diastereomeric excess of $>95 \%$ Equation 1.23. .112 
a)<smiles>[R]C1C[Te]12Cc1ccccc1C[Te]2</smiles>

110 b)

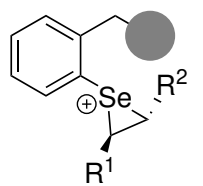

111 c)

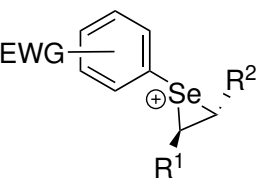

112

Figure 1.3: Strategies for the stabilization of enantioenriched seleniranium ions: a) LEWIS basic side chains, b) steric bulk, c) electron-withdrawing substituents at the selenium moiety.

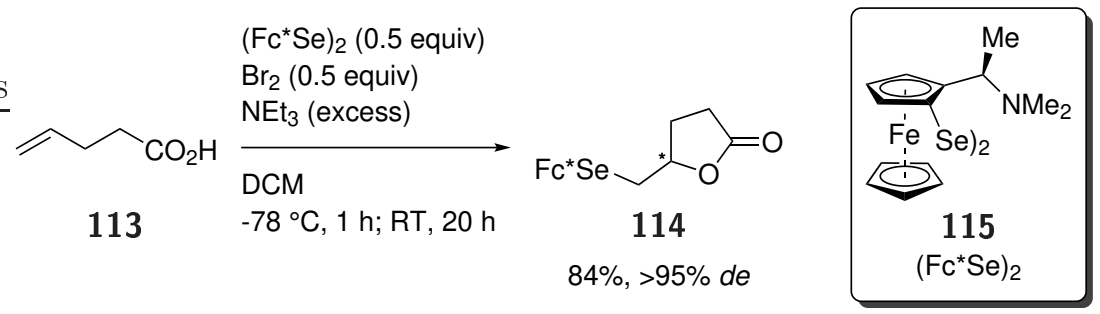

Unfortunately, these findings could not be translated into a catalytic processes. The efforts by TOMOda, [113] FukUZawa, [114] WiRTh, [82,89] TieCCO 115,116$]$ and their respective groups using diselenides 115, 116, 117 and 118 (Figure 1.4) in the catalytic oxyfunctionalization of alkenes led to low enantiomeric excesses (20-66\% ee), an exception being ee's of 75 and $82 \%$ that were achieved in methoxylation reactions by WIRTH ${ }^{[117]}$ and TIECCO $\stackrel{[18]}{ }$ when they used catalyst 117 with $\mathrm{R}^{2}=\mathrm{OMe}$.<smiles>O=S(=O)(O)c1ccccc1CN1C2COC(c3ccccc3)OC2[C@@H]2OC(c3ccccc3)OC[C@H]21</smiles>

116

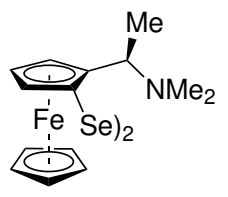

115<smiles>[R7]c1cccc(C([R])[X])c1[R6]</smiles>

$\mathrm{R}=\mathrm{OH}, \mathrm{NMe}_{2}$, SMe, SEt

$\mathrm{R}^{2}=\mathrm{H}$ or $\mathrm{OMe}$

117<smiles>CC1C2CCCC(C1(C)C)C2(C)C</smiles>

118

Tomoda 1994

\section{Wirth 1998, 2006, 2007 Tiecco 1998/1999 \\ Tiecco 2002}

Figure 1.4: Chiral selenium catalysts synthesized by Tomoda, FukuzaWA, Wirth, and Tiecco.

The difference in selectivity between the selenofunctionalization reactions and the selenylationdesenylation sequences can be explained by the temperatures that were applied during the reactions. While the selenylation reactions could be conducted at low temperatures $\left(-78{ }^{\circ} \mathrm{C}\right)$, the catalytic reactions were conducted at room temperature because they need higher temperatures for the elimination of the selenium species from the selenylated intermediates $\left(0{ }^{\circ} \mathrm{C}\right.$ or higher; cf. Subsection 1.1). 53] As Denmark and colleagues showed in their NMR studies, the transfer of selenium ions between olefins already proceeds at $-70{ }^{\circ} \mathrm{C} .{ }^{109]}$ It is therefore safe to assume 
that this transfer is even faster at room temperature, degrading the enantiomeric excess of the formed seleniranium ions.

Despite these challenges, MARUOKA et al. accomplished the enantioselective lactonization of unsaturated acids 34 in high yields (60-99\%) and excellent selectivities (75-97\%) using 10 mol\% of chiral selenide 119, 1.1 equiv NFSI and a base (Equation 1.24 at room temperature.119] Their catalyst features a sterically demanding side chain (OTBS) and a rigid structure based on indanol.
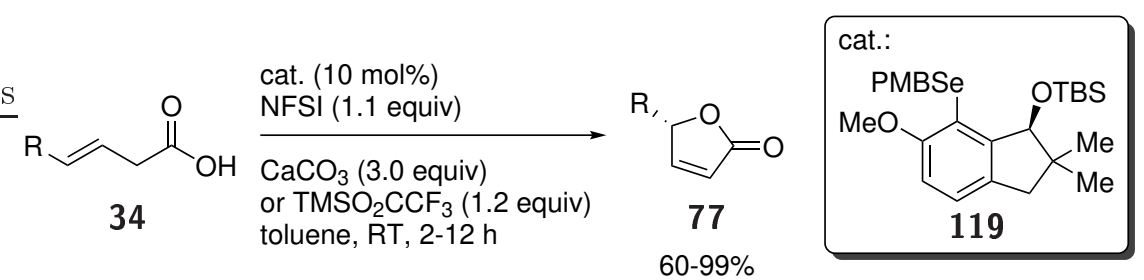

Although MARUOKA's work is groundbreaking, it is still of interest to find further reliable ways for the stabilization of enantioenriched seleniranium ions in order to find general methods for asymmetric selenium-catalyzed functionalization reactions. 


\section{Objectives}

The work during this thesis was focused on the development of new selenium-catalyzed functionalizations. As described in the previous section (Subsection 1.2), the choice of the right oxidant for these transformations is crucial. Conventional oxidants, especially $N$-halogenated oxidants,

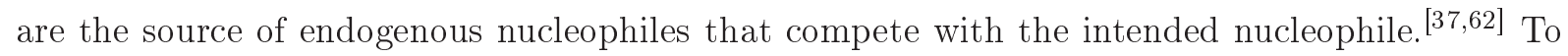
be able to use weak exogenous nucleophiles in these reactions, an alternative to conventional oxidants is necessary. In this context, the recently developed dual selenium/photoredox catalysis by BREDER et al. is a promising method for the functionalization of alkenes with exogenous nucleophiles. $\left.{ }^{[1]}\right]$

In the first experiments with dual selenium/photoredox catalysis, acids were used as nucleophiles. Therefore the question occurred whether the reaction conditions would also promote reactions with weak and challenging nucleophiles. As suitable candidates, a number of different nucleophiles come to mind. Alcohols have been used in selenium-catalyzed oxidative functionalizations, but the reactions often needed special substrates or had selectivity issues (cf. Subsection 1.2.2. $\cdot 67,74,75,88,89]$ In this context, it was to be investigated if selenium/photoredox catalysis allows for simple substrates and high selectivity. Other interesting nucleophiles are amines, which in conventional transformations are often limited to endogenous nucleophiles originating from the $N$-halogenated oxidant (an exception being the pyridination by ZHAO et al.). [69] Selenium/photoredox catalysis could allow for new types of nitrogen nucleophiles that are not derived from oxidants. For the above-mentioned nucleophiles, intramolecular as well as intermolecular transformations were to be investigated in this work (Equation 2.1). Furthermore, it was to be determined if instead of alkenes, alkynes could be used as substrates for the described transformations.
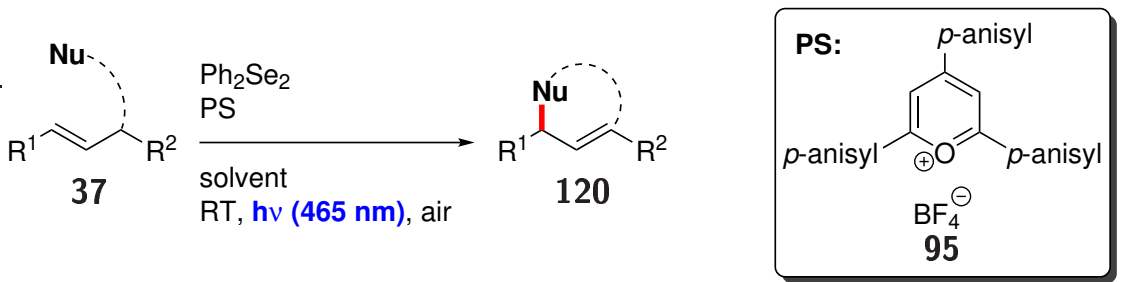

In addition to the development of new selenium-catalyzed functionalizations, the possibility to conduct these reactions in an asymmetric fashion should be examined. For this purpose, asymmetric diselenides should be synthesized and used as catalysts. As already explained in the previous section (Subsection 1.2.4, methods for asymmetric selenium-catalyzed oxidative functionalizations are sparse. Most of the attempts made focus on the use of diselenides bearing LEWIS basic side chains for the stabilization of the seleniranium intermediate in order to prevent its racemization. Another option to prevent racemization of the seleniranium ion by olefin-toolefin transfer is the application of diselenides with attached bulky groups, which would block the free coordination site from the attack of a second alkene. Additionally, a rigid structure was 
expected to keep the steric bulk in place. A suitable structure motif that could fulfill these requirements is the binaphthyl moiety. As binaphthyl-derived catalysts have been applied as chiral brønsted acids in MANNICH-type reactions or transfer hydrogenations,, $120-123]$ the question arose if binaphthyl-derived diselenides could be selective catalysts in selenium-catalyzed transformations. Therefore, two possible binaphthyl-based catalyst structures were envisioned and were to be synthesized during this work (Figure 2.1).<smiles>[Se]c1c(-c2cc[R]cc2)cc2ccccc2c1-c1c(-c2ccccc2)cc2ccccc2c1[Se]</smiles>

121

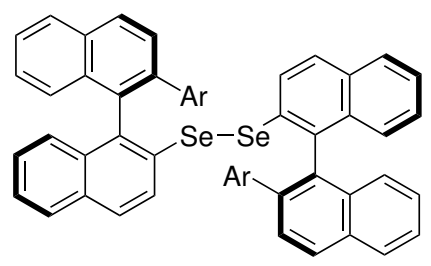

122

Figure 2.1: Binaphthyl derived chiral diselenides. 


\section{Results and Discussion}

\subsection{Intra- and intermolecular etherification via photo-aerobic selenium- $\pi$-acid catalysis}

\subsubsection{Intramolecular etherification - Preliminary investigations and optimization of reaction conditions}

During the total synthesis of $(+)$-Greek tobacco lactone (124), the groups of STARK, Breder and Christmann investigated the selenium-catalyzed cyclization of alcohol $\mathbf{1 2 3}$ as an alternative to the reaction with palladium trifluoroacetate. ${ }^{[124]}$ While in the palladium-mediated reaction the desired product 124 was obtained in only $41 \%$ isolated yield and $15 \%$ of tetrahydrooxepin side product 125 was formed (Scheme 3.1, top), the selenium- $\pi$-acid-catalyzed transformation led to the clean formation of lactone $\mathbf{1 2 4}$ in $60 \%$ yield when NFSI was used as the oxidant Scheme 3.1, middle). Moreover, under the conditions of selenium- $\pi$-acid/photoredox catalysis, the yield was even higher $(83 \%)$ and the dr of 84:16 was comparable to that of the product of the palladiumcatalyzed reaction (Scheme 3.1, bottom).

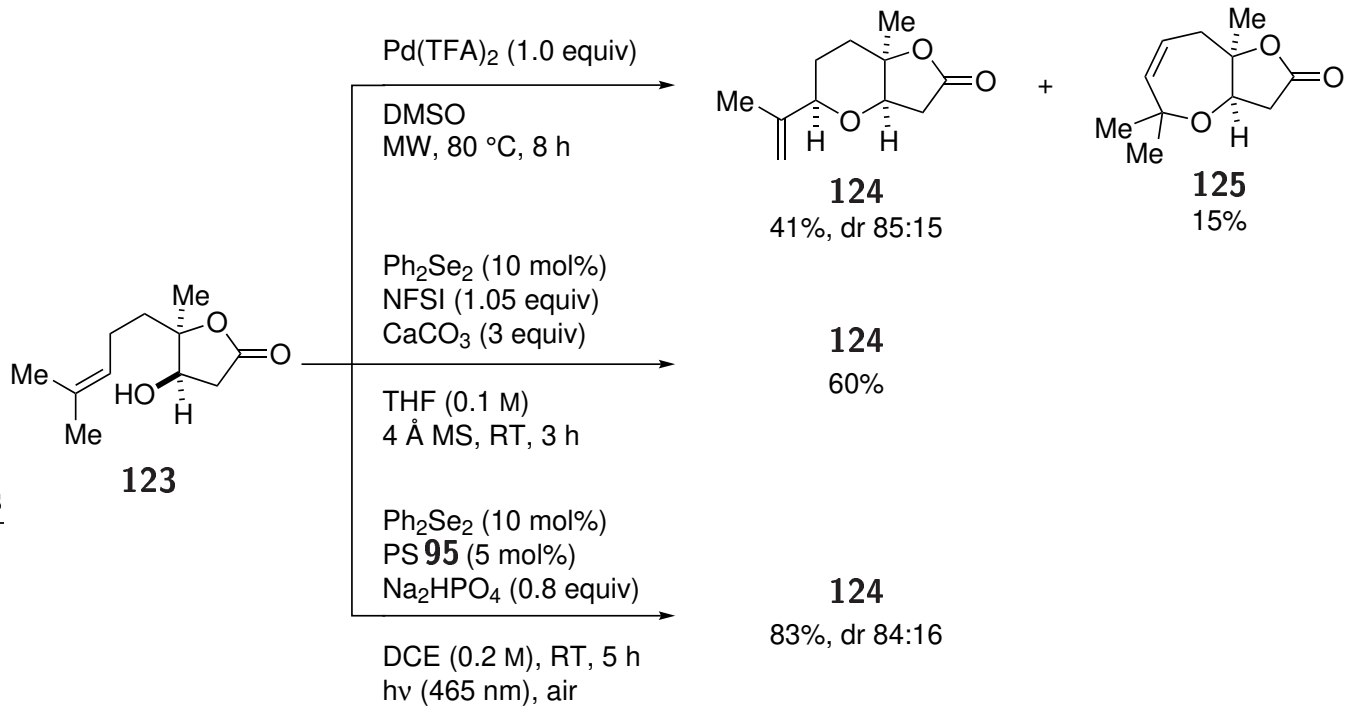

Scheme 3.1: Palladium-mediated (top), selenium/NFSI-mediated (middle) and selenium-photoredoxmediated (bottom) formation of $(+)$-Greek tobacco lactone $(\mathbf{1 2 4})$.

These impressive findings raised the question if the selenium- $\pi$-acid/photoredox-catalyzed intramolecular etherification, which worked well for a rigid system such as alcohol 123, was also possible for more flexible substrates. To find an answer to this question, $(Z)$-dec-4-en-1-ol (126a) was subjected to the conditions that were used in the synthesis of $(+)$-Greek tobacco lactone (124). Gratifyingly, when the alcohol was treated with diphenyl diselenide (10 mol\%), $p$-MeOTPT (5 mol\%), $\mathrm{Na}_{2} \mathrm{HPO}_{4}$ (0.8 equiv) and irradiation at $\lambda=465 \mathrm{~nm}$ under air in DCE $(0.2 \mathrm{M})$, $27 \%$ of a product was observed in ${ }^{1} \mathrm{H}$ NMR Equation 3.1), and identified as (E)-2-(hex-1-en- 
1-yl)tetrahydrofuran (127a) via ${ }^{1} \mathrm{H}$ NMR spectroscopy by the characteristic shift and splitting of the signals of the three carbinol protons. While the signal of the proton at C-1 was observed as a quartet at $4.22 \mathrm{ppm}$, the two protons at C-2 appeared as a doublet of doublets of doublets at $3.89 \mathrm{ppm}$ and a triplet of doublets at $3.75 \mathrm{ppm}$. The formation of product 127 a was also confirmed by comparison with literature data. $\frac{125]}{1 n t r i g u e d ~ b y ~ t h i s ~ r e s u l t, ~ i t ~ w a s ~ e x a m-~}$ ined whether the reaction would run more efficiently in $\mathrm{MeCN}$, which had been the best solvent in the intermolecular selenium- $\pi$-acid/photoredox-catalyzed esterification. [91] Unfortunately, the reaction in MeCN but under otherwise unchanged conditions led to the same yield as in DCE Equation 3.1).

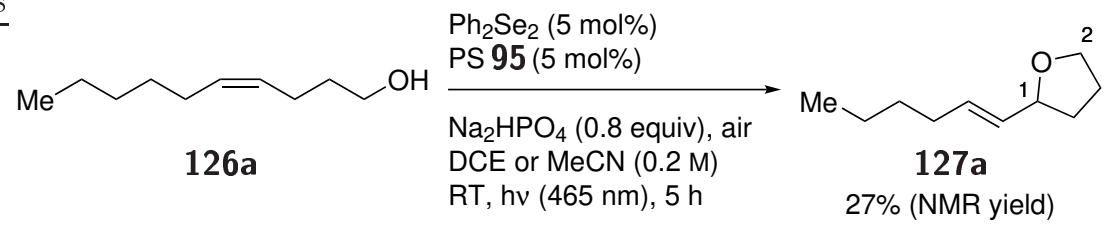

Following these initial results, it was hypothesized that a different combination of diselenide and photosensitizer could lead to improved yields due to a better match of the reduction potential of the excited state of the photosensitizer with the oxidation potential of the diselenide on the one hand, and the one of the selenofunctionalization intermediate on the other hand. For further investigations, diselenides $129\left(E_{o x}=1.22 \mathrm{~V} \text { vs. SCE }\right)^{[126]}$ and $130\left(E_{o x}=1.53 \mathrm{~V}\right.$ vs. SCE $) \underline{[126]}$ were synthesized according to a procedure by DENMARK and obtained in yields of 50 and $44 \%$ Equation 3.2).

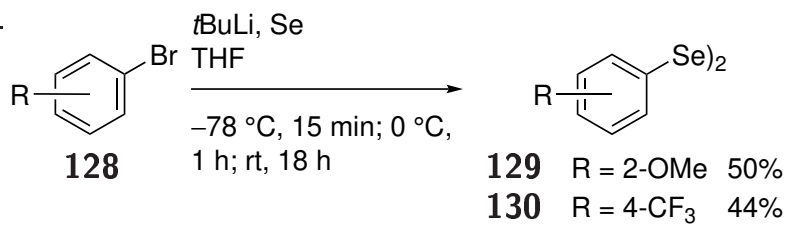

In order to identify potent combinations of the synthesized diselenides with different photosensitizers, their interactions were examined in fluorescence quenching experiments by Dr. S. Ortgies. ${ }^{[127]}$ The STERN-Volmer constants were determined for the combination of diselenides 25, 129 and 130 with photosensitizers $p$-MeO-TPT (95), 10-(3,5-dimethoxyphenyl)-9-mesityl-1,3,6,8tetramethoxyacridinium tetrafluoroborate (131, DMTA) and $\mathrm{Ru}(\mathrm{bpz})_{3} \mathrm{PF}_{6}$ (132) (Table 3.1). The Stern-Volmer constant for the combination of diphenyl diselenide $(\mathrm{R}=\mathrm{H})$ and $p$-MeOTPT, which was used in the starting experiment for the intramolecular etherification, was determined to be $K_{S V}=(116 \pm 2.52) \mathrm{L} \cdot \mathrm{mol}^{-1}$. The quenching of $p$-MeO-TPT with electron-poor diselenide $130\left(\mathrm{R}=4-\mathrm{CF}_{3}\right)$ was less efficient with a constant of $K_{S V}=(85.1 \pm 4.70) \mathrm{L} \cdot \mathrm{mol}^{-1}$. For DMTA, both diselenides 129 and 130 showed higher quenching than diphenyl diselenide $\left(K_{S V}=(76.9 \pm 4.12) \mathrm{L} \cdot \mathrm{mol}^{-1}\right)$, with STERN-VOLMER constants of $K_{S V}=(103 \pm 5.61) \mathrm{L} \cdot \mathrm{mol}^{-1}$ $(\mathrm{R}=2-\mathrm{OMe})$ and $K_{S V}=(96.3 \pm 6.00) \mathrm{L} \cdot \mathrm{mol}^{-1}\left(\mathrm{R}=4-\mathrm{CF}_{3}\right)$, respectively. Using ruthenium 
complex 132, higher quenching than in the other experiments was observed, with diselenide $\mathbf{1 2 9}$ $(\mathrm{R}=2$-OMe $)$ having the highest observed constant $\left(K_{S V}=(992 \pm 18.8) \mathrm{L} \cdot \mathrm{mol}^{-1}\right)$ and diphenyl diselenide having a constant of $K_{S V}=(140 \pm 29.6) \mathrm{L} \cdot \mathrm{mol}^{-1}$. The high values can be attributed to the significantly longer lifetime of the excited triplet state of photocatalyst $132\left(\tau_{f}=1.04 \mu \mathrm{s}\right){ }^{[128]}$ compared to the excited states of photocatalysts 95 and $\left.131\left(\tau_{f}=4-12 \mathrm{~ns}\right) .129 .130\right]$ Due to the quenching of the different photosensitizers with electron-poor diselenide $\mathbf{1 3 0}$ being lower than that of $p$-MeO-TPT with diphenyl diselenide, $\mathbf{1 3 0}$ was ruled out as a potential catalyst. Electron-rich diselenide 129 quenched photosensitizers 131 and 132 more efficiently than diphenyl diselenide in the respective combinations, indicating stronger interactions. Therefore, it was applied in further test reactions for the intramolecular etherification.

Table 3.1: Stern-Volmer constants for different diselenide/photosensitizer combinations, determined by Dr. S. Ortgies. $\frac{[127]}{\underline{10}}$

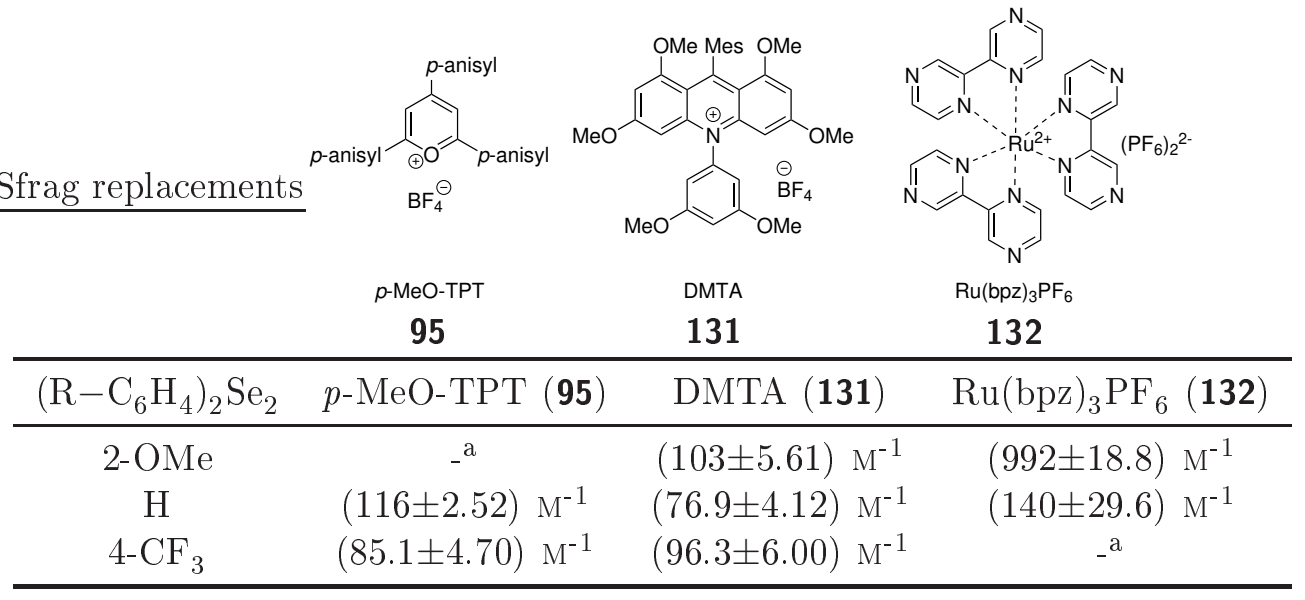

${ }^{\mathrm{a}}$ Values not determined. $\mathrm{bpz}=2,2^{\prime}$-bipyrazine

In the following experiments, the cycloetherification of alcohol 126a with (2-anisyl) ${ }_{2} \mathrm{Se}_{2}(\mathbf{1 2 9})$ and photosensitizers 95, 131 and 132 was examined. For both the diselenide and the photosensitizers, an amount of $5 \mathrm{~mol} \%$ was used. With the new diselenide catalyst, the yield of the transformation with $p$-MeO-TPT increased to $31 \%$ in DCE and 45\% in MeCN (Table 3.2, entries 1 and 2). With the other photosensitizers, product 127a was observed in lower yields. With DMTA, it was formed in $27 \%$ in DCE and $6 \%$ in MeCN (Table 3.2, entries 3 and 4). With $\mathrm{Ru}(\mathrm{bpz})_{3} \mathrm{PF}_{6}$, the product was formed in $24 \%$ in MeCN and no reaction occurred in DCE (Table 3.2, entries 5 and 6). In both solvents, the quenching of the fluorescence was observed. This suggests the decomposition of the photosensitizer, possibly by a selenium species.

As it was assumed that the product decomposed when exposed to the photoconditions for too long, the reaction time was shortened ( $5 \mathrm{~h}$ instead of $16 \mathrm{~h}$ ) and the product development monitored during the reaction by 1,1,2,2-tetrachloroethane (TCE) as an internal NMR standard in MeCN- $d_{3}$. These changes of the reaction conditions increased the yield with $p$-MeO-TPT to $69 \%$ (Table 3.3, entry 1), whereas with DMTA, tetrahydrofuran 127a was formed in 29\%, a similar 
Table 3.2: Comparison of photosensitizers in different solvents in the intramolecular etherification.

\begin{tabular}{|c|c|c|c|}
\hline \multirow{2}{*}{$\begin{array}{c}\mathbf{1 2 6 a} \\
\text { entry }\end{array}$} & $\begin{array}{l}\begin{array}{c}(2 \text {-anisyl) } \\
\text { photoser }\end{array} \\
\mathrm{Na}_{2} \mathrm{HPO}_{4} \\
\text { solvent }( \\
\mathrm{hv}(465 \mathrm{n}\end{array}$ & $\begin{array}{l}\mathrm{Se}_{2}(5 \mathrm{~mol} \% \\
\text { sitizer (5 mo } \\
\text { (0.8 equiv), } \\
.2 \mathrm{M}), \mathrm{RT}, \\
\text { n), } 16 \mathrm{~h}\end{array}$ & $\stackrel{\mathrm{o})}{\longrightarrow} \mathrm{Me} \underbrace{}_{12}$ \\
\hline & photosensitizer & solvent & NMR yield $^{\mathrm{a}}$ \\
\hline 1 & $p$-MeO-TPT & DCE & $31 \%$ \\
\hline 2 & $p$-MeO-TPT & $\mathrm{MeCN}$ & $45 \%$ \\
\hline 3 & DMTA & $\mathrm{DCE}$ & $27 \%$ \\
\hline 4 & DMTA & $\mathrm{MeCN}$ & $6 \%$ \\
\hline 5 & $\mathrm{Ru}(\mathrm{bpz})_{3} \mathrm{PF}_{6}$ & DCE & - \\
\hline 6 & $\mathrm{Ru}(\mathrm{bpz})_{3} \mathrm{PF}_{6}$ & $\mathrm{MeCN}$ & $24 \%$ \\
\hline
\end{tabular}

yield as after $16 \mathrm{~h}$ reaction time (Table 3.3 , entry 2$)$. The yield with $\mathrm{Ru}(\mathrm{bpz})_{3} \mathrm{PF}_{6}$ decreased to $9 \%$ (Table 3.3, entry 3).

Table 3.3: Comparison of photosensitizers in the intramolecular etherification.

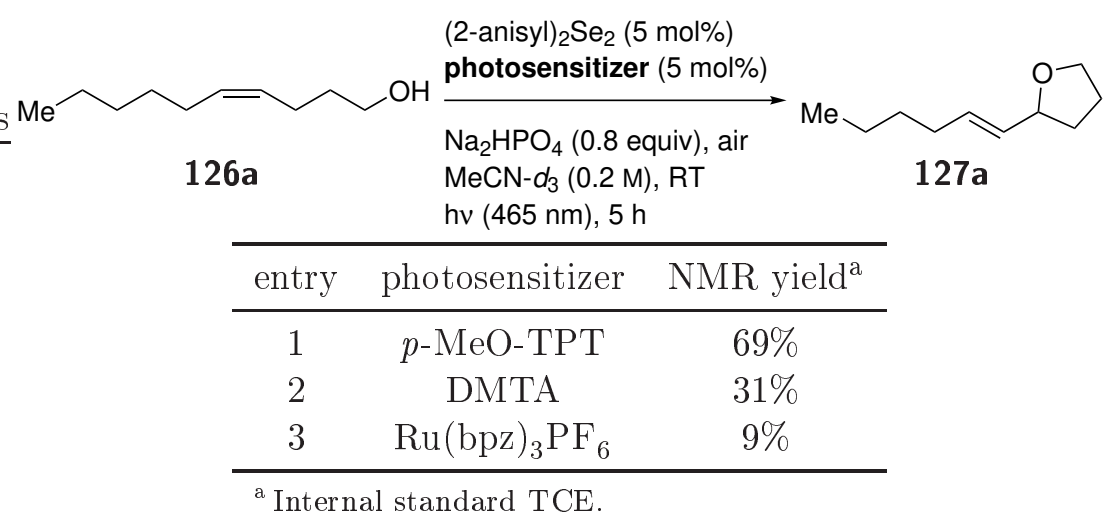

As the combination of pyrylium derivative 95 and (2-anisyl) ${ }_{2} \mathrm{Se}_{2}$ showed the best results in MeCN (69\% yield, Table 3.4, entry 1), these conditions were used to optimize the catalyst loadings. In an initial experiment, the influence of the amount of the photosensitizer on the yield was investigated. In fact, decreasing the photosensitizer loading to $3 \mathrm{~mol} \%$ decreased the yield only slightly to $65 \%$ after $5 \mathrm{~h}$ reaction time (Table 3.4 entry 2). In combination with a higher loading of diselenide $\mathbf{1 2 9}$ (10 mol\%), the formation of tetrahydrofuran 127a with a yield of $68 \%$ was observed after $7 \mathrm{~h}$ (Table 3.4, entry 3). Because the difference between the yields was very small, it was decided to go with the lower catalyst loadings of 5 mol\% diselenide and $3 \mathrm{~mol} \%$ photosensitizer for further reactions. During all preceding experiments, air was used as the oxidant. Alternatively, using pure oxygen could increase the reaction rate and yield, but it could also lead to the formation of oxidized side products. In the experiments on the selenium- $\pi$-acid/photoredox-catalyzed esterification by Dr. S. Ortgies, the formation of an allylic 
hydroperoxide was observed as the probable consequence of the SCHENCK-ene reaction of the alkene with ${ }^{1} \mathrm{O}_{2} \cdot \frac{[127,131,132]}{1 n}$ the following investigations, the addition of molecular sieves was found to reduce the hydroperoxide formation. ${ }^{[127]}$ Therefore, to examine the influence of oxygen on the intramolecular etherification, $4 \AA$ molecular sieves and oxygen instead of air were used with $5 \mathrm{~mol} \%$ (2-anisyl) ${ }_{2} \mathrm{Se}_{2}$ and $3 \mathrm{~mol} \%$-MeO-TPT. The reaction resulted in a yield of $67 \%$, which is comparable to the yield obtained with air (Table 3.4, entry 4). Air was chosen in further experiments to minimize the formation of side products by the ScHENCK-ene reaction. To make sure that both catalysts and air were neccessary for the reaction, two control experiments leaving out one of the catalysts and one experiment under an argon atmosphere were conducted. No product development was observed in either case (Table 3.4 entries 5-7). As irradiation of the reaction mixture could lead to elevated temperatures in the reaction flask, the influence of higher or lower reaction temperatures was examined during reactions using a double-walled flask with a cryostat. Increasing the temperature to $60{ }^{\circ} \mathrm{C}$ led to a decreased yield of $57 \%$ (Table 3.4, entry 8). Decreasing the temperature to $0{ }^{\circ} \mathrm{C}$ led to the shutdown of the reaction and no product formation was observed (Table 3.4, entry 9).

Table 3.4: Optimization of catalyst loading and control experiments in the intramolecular etherification.

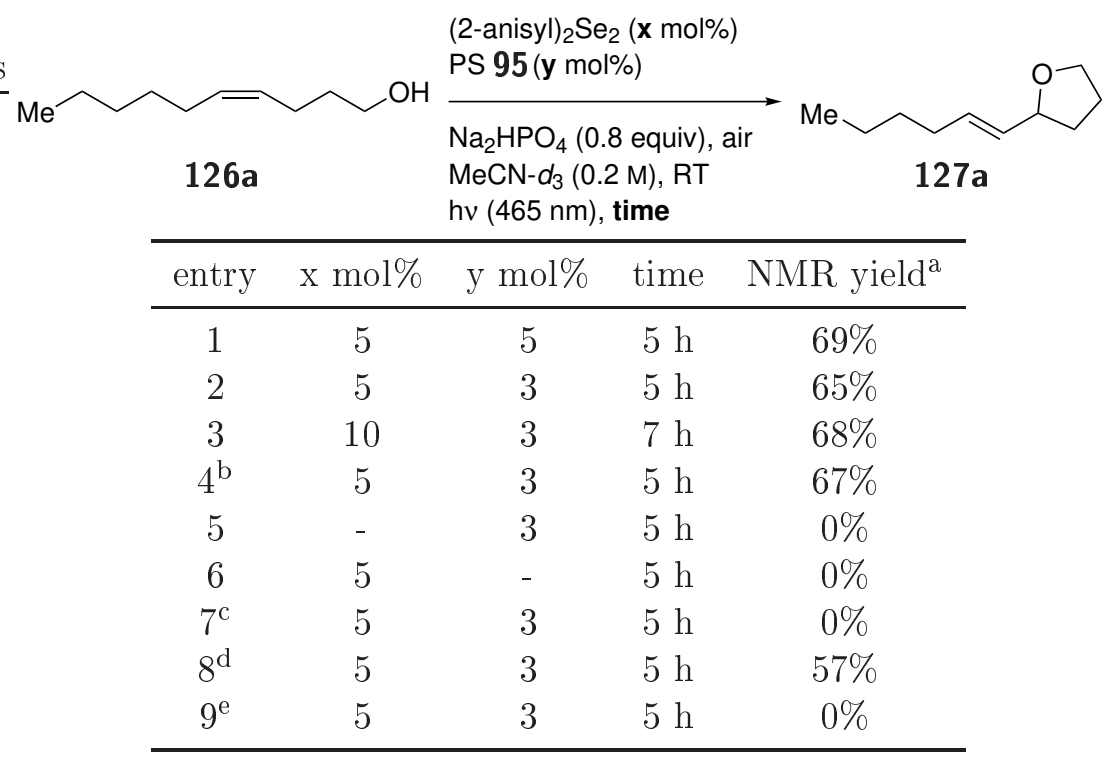

\footnotetext{
${ }^{\mathrm{a}}$ Standard: TCE. ${ }^{\mathrm{b}}$ Reaction with oxygen and molecular sieves instead of air. ${ }^{c}$ Reaction under an argon atmosphere using degassed solvents. ${ }^{\mathrm{d}}$ Reaction at $60{ }^{\circ} \mathrm{C}$.

${ }^{\mathrm{e}}$ Reaction at $0{ }^{\circ} \mathrm{C}$.
}

As the last step of the optimization of the reaction conditions, the effect of different bases on the yield was investigated with TMB as an external NMR standard. The use of different phosphate bases led to good yields of 47 and $53 \%$ with sodium phosphates $\mathrm{Na}_{2} \mathrm{HPO}_{4}$ and $\mathrm{NaH}_{2} \mathrm{PO}_{4}$, respectively, and to significantly lower yields of $0-14 \%$ with potassium phosphates (Table 3.5, entries 1-5). This observation is probably due to the deactivation of the photosensitizer, as in these last reactions, complete quenching of fluorescence was observed. While with KF no reac- 
tion occurred and fluorescence quenching was observed, using $\mathrm{CaF}_{2}$ afforded the product in $51 \%$ yield (Table 3.5, entries 6 and 7). When carbonate bases were used, the yields were between 14 and $50 \%$, with $\mathrm{Li}_{2} \mathrm{CO}_{3}$ giving the best result (Table 3.5, entries 8-10). Acetates were no effective bases for the reaction. Only with NaOAc the reaction occurred and led to only $9 \%$ vield (Table 3.5, entries 11-13). In order to check if the base was important for the reaction, an experiment without any added base was conducted. The product was observed in $33 \%$ yield, indicating a possible inhibition of the reaction by some bases as in the case of potassium phosphates, sodium carbonates and acetates (Table 3.5, entry 14). As some bases proved to be beneficial for the reaction, the influence of the loading was examined. With 0.5 equiv of $\mathrm{Na}_{2} \mathrm{HPO}_{4}$, the product was obtained in $47 \%$ yield, whereas an increase of the loading to 1 or 1.5 equiv resulted in a lower yield of $41 \%$, respectively (Table 3.5, entries 15-17).

Table 3.5: Optimization of bases used in the intramolecular etherification.

\begin{tabular}{|c|c|c|c|}
\hline $126 a$ & $\begin{array}{l}\begin{array}{l}(2-a n i \\
\text { PS } 95\end{array} \\
\begin{array}{l}\text { base, } \\
\text { hv }(46\end{array}\end{array}$ & 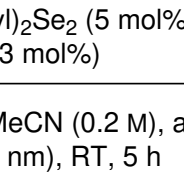 & $127 a$ \\
\hline entry & base & amount & NMR yield ${ }^{\mathrm{a}}$ \\
\hline 1 & $\mathrm{Na}_{2} \mathrm{HPO}_{4}$ & 0.8 equiv & $47 \%$ \\
\hline 2 & $\mathrm{NaH}_{2} \mathrm{PO}_{4}$ & 0.8 equiv & $53 \%$ \\
\hline 3 & $\mathrm{KH}_{2} \mathrm{PO}_{4}$ & 0.8 equiv & $14 \%$ \\
\hline 4 & $\mathrm{~K}_{2} \mathrm{HPO}_{4}$ & 0.8 equiv & $12 \%$ \\
\hline 5 & $\mathrm{~K}_{3} \mathrm{PO}_{4}$ & 0.8 equiv & - \\
\hline 6 & $\mathrm{KF}$ & 0.8 equiv & - \\
\hline 7 & $\mathrm{CaF}_{2}$ & 0.8 equiv & $51 \%$ \\
\hline 8 & $\mathrm{NaHCO}_{3}$ & 0.8 equiv & $28 \%$ \\
\hline 9 & $\mathrm{Na}_{2} \mathrm{CO}_{3}$ & 0.8 equiv & $14 \%$ \\
\hline 10 & $\mathrm{Li}_{2} \mathrm{CO}_{3}$ & 0.8 equiv & $50 \%$ \\
\hline 11 & $\mathrm{LiOAc} \cdot 2 \mathrm{H}_{2} \mathrm{O}$ & 0.8 equiv & - \\
\hline 12 & $\mathrm{NaOAc}$ & 0.8 equiv & $9 \%$ \\
\hline 13 & KOAc & 0.8 equiv & - \\
\hline 14 & - & 0.8 equiv & $33 \%$ \\
\hline 15 & $\mathrm{Na}_{2} \mathrm{HPO}_{4}$ & 0.5 equiv & $47 \%$ \\
\hline 16 & $\mathrm{Na}_{2} \mathrm{HPO}_{4}$ & 1.0 equiv & $41 \%$ \\
\hline 17 & $\mathrm{Na}_{2} \mathrm{HPO}_{4}$ & 1.5 equiv & $41 \%$ \\
\hline
\end{tabular}

\subsubsection{Intramolecular etherification - Synthesis and cyclization of unsaturated alcohols}

For the evaluation of the scope and limitations of the selenium- $\pi$-acid/photoredox-catalyzed intramolecular etherification, a series of unsaturated alcohols was synthesized. In order to investigate the formation of cyclic ethers with different ring sizes, it was intended to synthesize 
4-alken-1-ol derivatives as well as 5-alken-1-ol derivatives. The first path that allowed for the synthesis of both kinds of alcohols was the synthesis and reduction of unsaturated acids $\mathbf{1 3 6}$ and 137. The WitTig reaction of 1.0 equiv of aldehyde $\mathbf{1 3 3}$ with 2.0 equiv of either 3 -carboxypropyl triphenylphosphonium bromide (138a) or 4-carboxybutyl triphenylphosphonium bromide (138b) and 4.0 equiv potassium tert-butoxide afforded alkenoic acids $\mathbf{1 3 6} \mathbf{a}-\mathbf{d}$ and $\mathbf{1 3 7 a - j}$ in high yields between 54 and $99 \%$ Table 3.6 products $136(\mathrm{n}=0)$ with yields in gray synthesized by Dr. M. Palomba). 133,134$]$ Acid $\mathbf{1 3 6 f}$ was obtained in only $20 \%$, presumably due to the formation of dechlorinated side products. The isolation of acid 136e was supposedly impaired by the volatility of the product, which was obtained in $38 \%$. In the reaction with 3,3,3-trifluoropropanal, no transformation into acid $\mathbf{1 3 7 \mathbf { k }}$ was observed. The product formation of unsaturated acids $\mathbf{1 3 6}$ and 137 was confirmed in the ${ }^{1} \mathrm{H}$ NMR spectrum, where the singlet of the aldehyde proton at around 9-10 ppm disappeared and a new multiplet signal indicative of the protons corresponding to the double bond between 5 and $6 \mathrm{ppm}$ appeared.

Table 3.6: WitTig reaction of aldehydes 133 to carboxylic acids $\mathbf{1 3 6}$ and $\mathbf{1 3 7}$. Products $\mathbf{1 3 6}(\mathrm{n}=0)$ with yields in gray synthesized by Dr. M. Palomba.
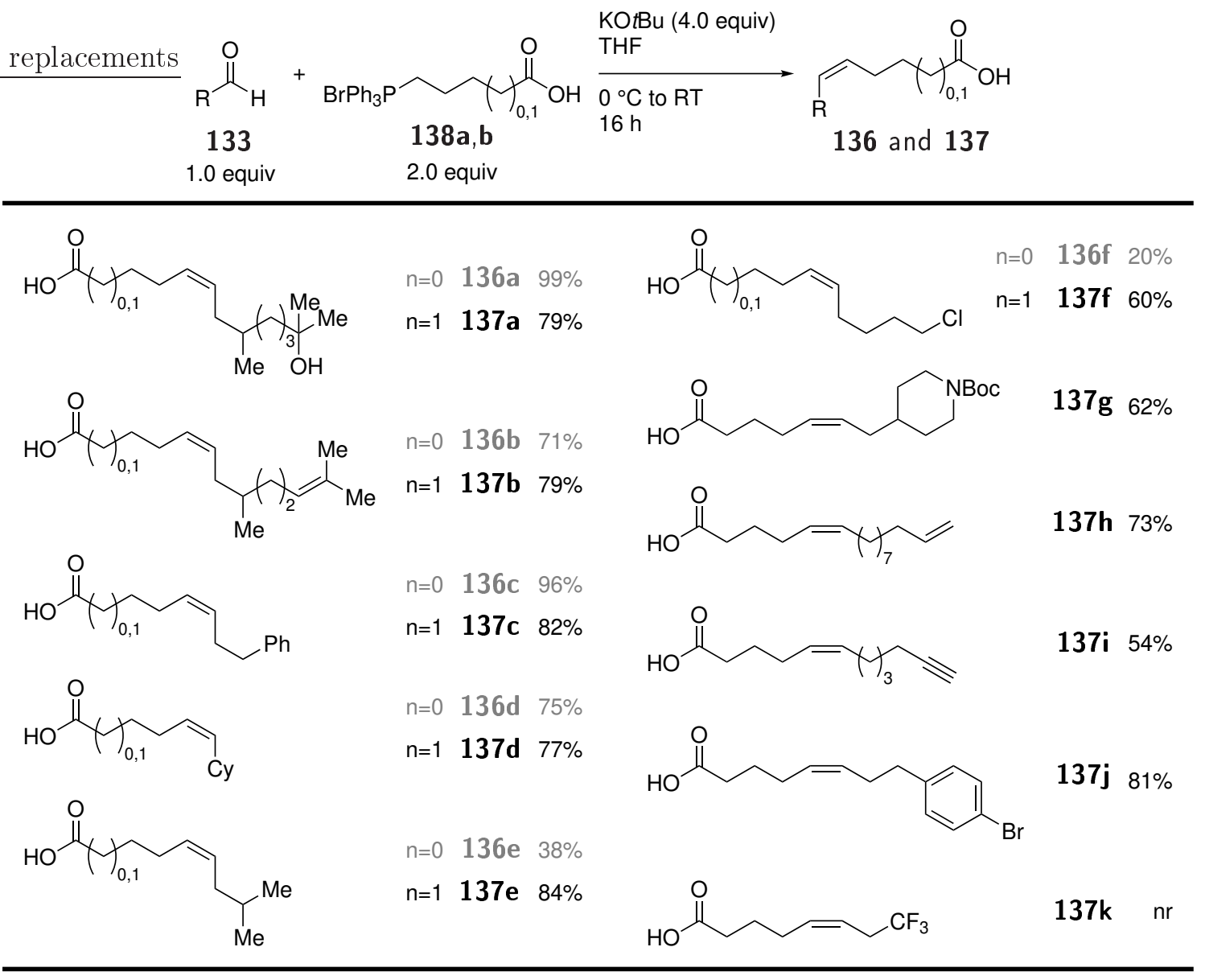
The following reduction of alkenoic acids $\mathbf{1 3 6}$ and $\mathbf{1 3 7}$ was achieved by the reaction with 1.5 equiv $\mathrm{LiAlH}_{4}$ in THF. Alcohols $\mathbf{1 2 6}$ and $\mathbf{1 3 9}$ were synthesized in overall good yields between 36 and $99 \%$ (Table 3.7, products 126 ( $\mathrm{n}=0$ ) with yields in gray synthesized by Dr. M. Palomba). ${ }^{\text {133.134] The }}$ lowest yield was obtained for chloride 139f, which suffered from partial dehalogenation during the reaction. The successful formation of alcohols $\mathbf{1 2 6}$ and $\mathbf{1 3 9}$ was observed in ${ }^{1} \mathrm{H}$ NMR, where the triplet of the newly formed methylene moiety next to the hydroxyl group was observed at around $3.6 \mathrm{ppm}$.

Table 3.7: Reduction of carboxylic acids 136 and 137 to alcohols 126 and 139. Products $126(n=0)$ with yields in gray synthesized by Dr. M. Palomba. $\stackrel{133,134]}{[}$

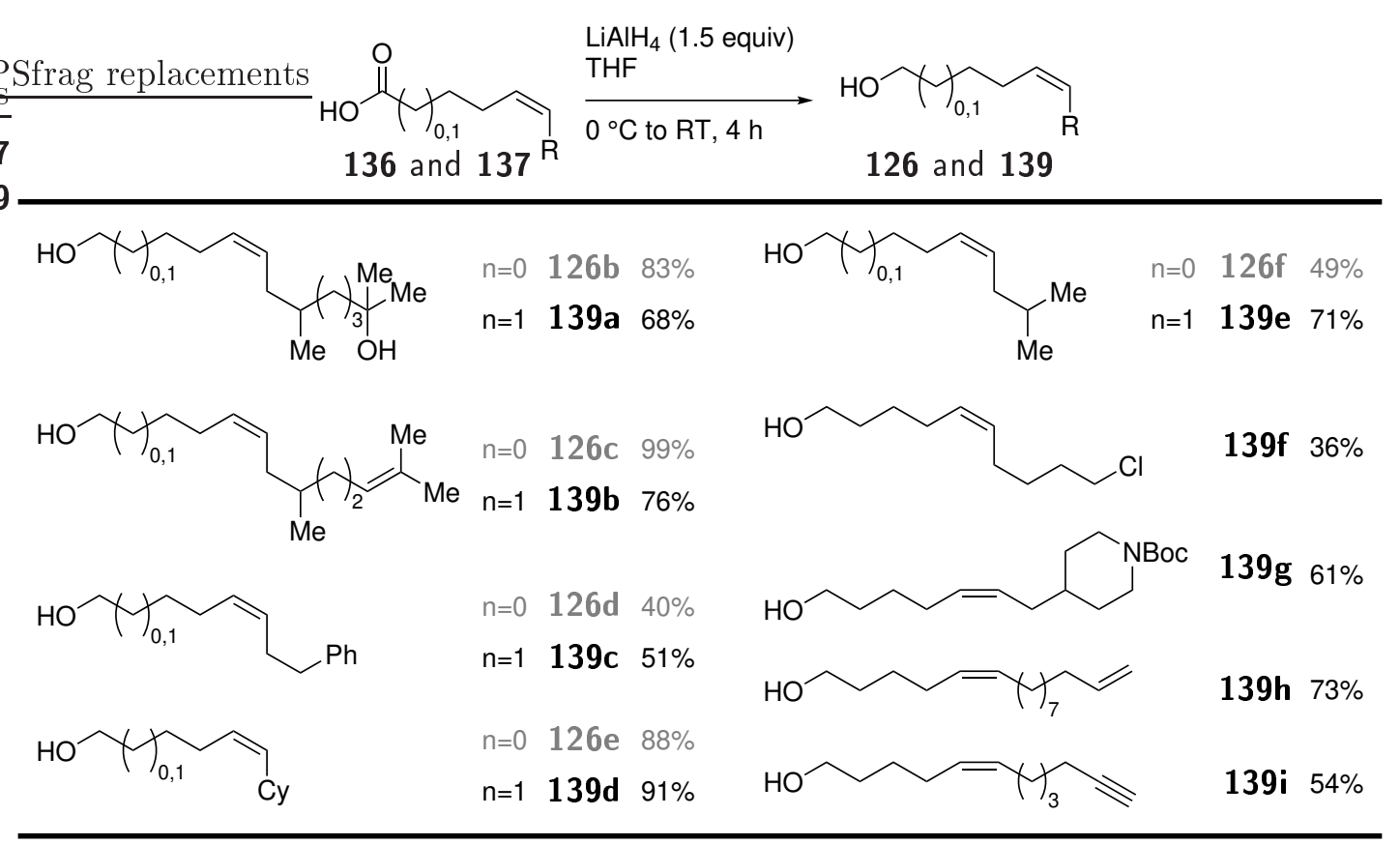

When the reduction of alkenoic acid $\mathbf{1 3 7} \mathbf{j}$ was attempted with $\mathrm{LiAlH}_{4}$, the desired alcohol 139j was obtained in $69 \%$ as an inseparable mixture with the debrominated alcohol 139c Equation 3.3. top). Therefore, other reduction methods were tested. In the reaction with $\mathrm{NaBH}_{4}$ (2.0 equiv) and boron trifluoride diethyl etherate (2.0 equiv), the double bond was also reduced along with the acid moieties (Equation 3.3. middle). The formation of the methyl ester followed by treatment with DIBAL-H (2.5 equiv) afforded an impure fraction of the desired product $139 \mathrm{j}$ in $22 \%$ yield, but most starting material underwent a transesterification to isobutyl ester 141 Equation 3.3. bottom). 


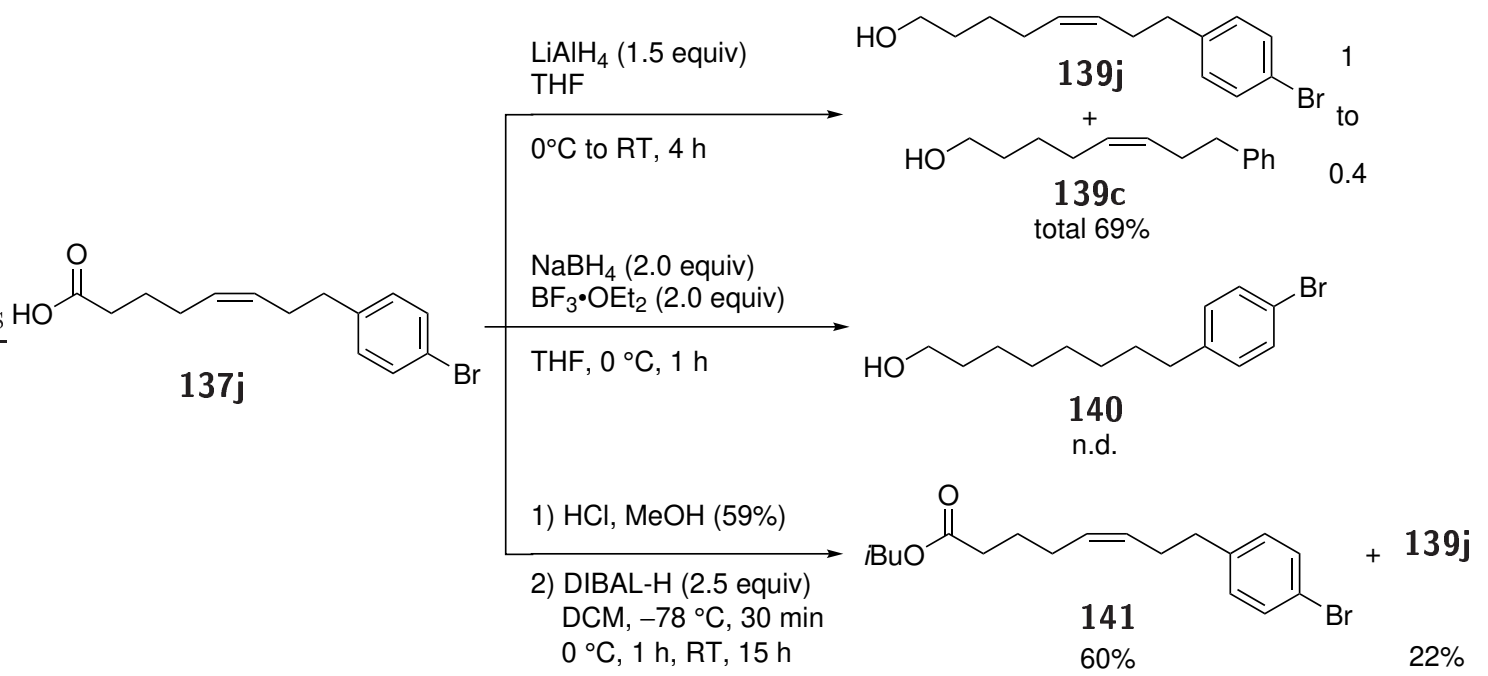

In order to avoid problems with halogenated substances and to be able to access substrates with functional groups that would not tolerate the conditions of the WITTIG reaction and the reduction (e.g. aldehydes, nitriles), another synthetic path to unsaturated alcohols $\mathbf{1 2 6}$ and $\mathbf{1 3 9}$ was needed. An easy and step-economic alternative to the previous route was provided by the cross-metathesis of terminal alkenes 142 with alkenol 143. Terminal alkenes 142 were reacted with 4-pentene-1-ol (143) (3.0 equiv) using the 2nd generation GRUBBS catalyst ( $5 \mathrm{~mol} \%$ ) to give alcohols $\mathbf{1 2 6} \mathbf{g}-\mathbf{I}$ as $E / Z$ mixtures in $22-74 \%$ yield Table 3.8 , grayed alcohols were synthesized by Dr. M. Palomba). ${ }^{[133,134]}$ Possible side products in this reaction are the products of the homocoupling of the two starting materials. In order to assure easy separation of the desired product from the side products by column chromatography, an excess of the alcohol was used, so that the homocoupling of alkene $\mathbf{1 4 2}$ was avoided. The diol that results from the homocoupling of alcohol $\mathbf{1 4 3}$ is very polar and therefore easier to separate. The discrimination between the starting material and the products is also easy in ${ }^{1} \mathrm{H}$ NMR. The olefinic protons of terminal alkene 4-pentene-1-ol (143) result in three signals between 4.8 and $6.0 \mathrm{ppm}$ (Figure 3.1, red). The signal of $\mathrm{H}^{\mathrm{a}}$, a doublet of doublets of triplets at $5.84 \mathrm{ppm}$, has coupling constants of 17.0, 10.2 and $6.7 \mathrm{~Hz}$ that correspond to the trans-coupling to $\mathrm{H}^{\mathrm{b}}$, the cis-coupling to $\mathrm{H}^{\mathrm{c}}$ and the coupling to the neighboring $\mathrm{CH}_{2}$ group, respectively. The signal of $\mathrm{H}^{\mathrm{b}}$, a doublet of quartets at $5.05 \mathrm{ppm}$, shows coupling to $\mathrm{H}^{\mathrm{a}}$ with $17.0 \mathrm{~Hz}$ and to $\mathrm{H}^{\mathrm{c}}$ with $1.7 \mathrm{~Hz}$. The coupling constants of the doublet of doublets of triplets at $4.98 \mathrm{ppm}\left(\mathrm{H}^{\mathrm{c}}\right)$ are $10.2 \mathrm{~Hz}$ (coupling to $\left.\mathrm{H}^{\mathrm{a}}\right), 1.7 \mathrm{~Hz}$ (coupling to $\mathrm{H}^{\mathrm{b}}$ ) and $1.3 \mathrm{~Hz}$ (coupling to the $\mathrm{CH}_{2}$ group). The olefinic protons of alcohol $\mathbf{1 2 6} \mathbf{j}$, however, show two signals at 5.50 and $5.36 \mathrm{ppm}$ (Figure 3.1, green) and in the spectrum of diol $\mathbf{1 2 6} \mathbf{m}$, there is only one multiplet at $5.43 \mathrm{ppm}$ for both double bond protons, resulting from the symmetry of the molecule (Figure 3.1, blue). 
Table 3.8: Synthesis of alcohols $\mathbf{1 2 6 g}-\mathbf{l}$. Grayed products were synthesized by Dr. M. Palomba. ${ }^{[133,134]}$
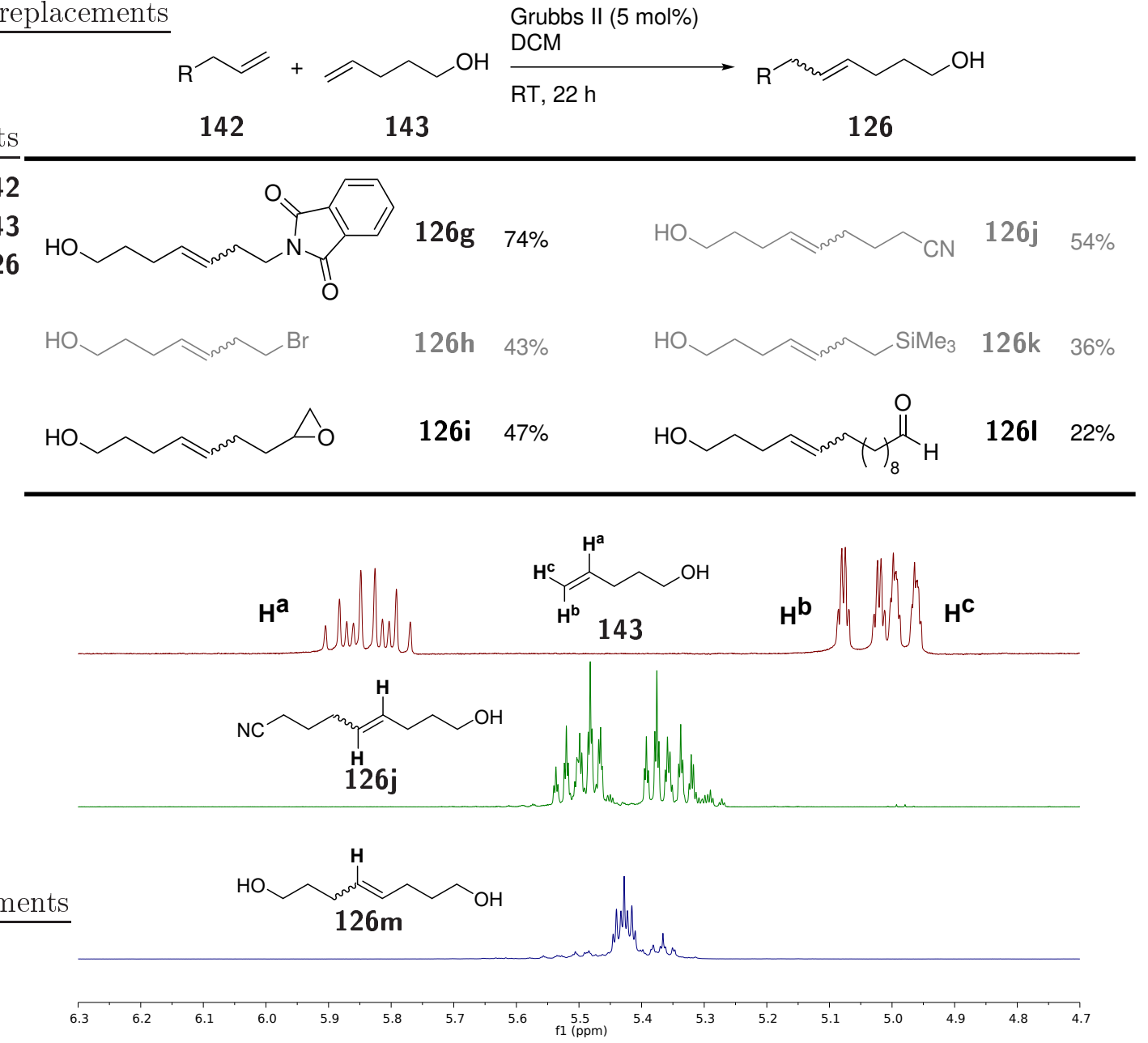

Figure 3.1: Comparison of the ${ }^{1} \mathrm{H}$ NMR spectra of 4-pentene-1-ol (143), alcohol 126j and diol 126m.

The homocoupling of 4-pentene-1-ol (143) was also a useful path to substrates with additional free or functionalized hydroxyl groups. Oct-4-ene-1,8-diol $(\mathbf{1 2 6} \mathbf{m})$ was synthesized in $45 \%$ from alcohol 143 using the 2nd generation GruBbs catalyst (0.5 mol\%) following a procedure by Marshall and Sabatini (Equation 3.4.

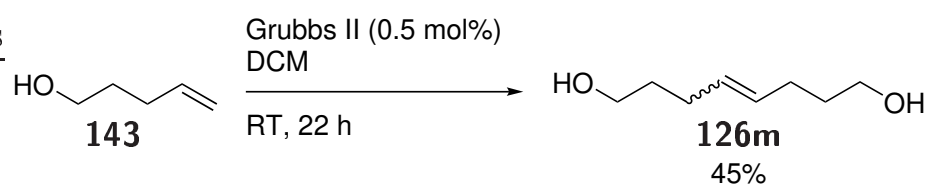

Diol $\mathbf{1 2 6 m}$ was then transformed to monocarbonate $\mathbf{1 2 6} \mathbf{n}$, monopivalic ester $\mathbf{1 2 6 0}$ and monosilylether 126p in yields between 35 and $40 \%$ Equation 3.5. 


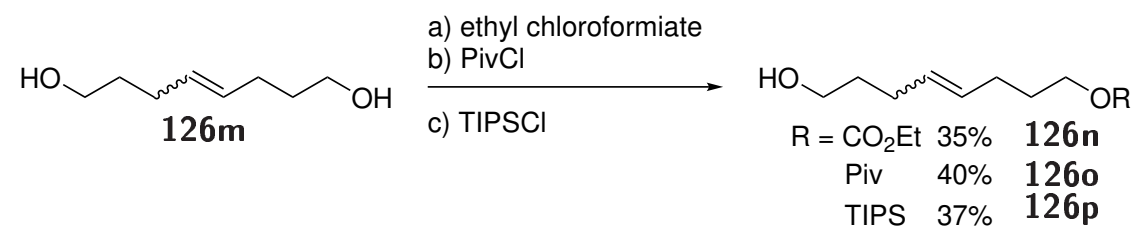

With the substrates in hand, the cycloetherification was attempted using (2-anisyl) ${ }_{2} \mathrm{Se}_{2}$ ( $5 \mathrm{~mol} \%$ ), photosensitizer 95 (3 mol\%) and $\mathrm{NaH}_{2} \mathrm{PO}_{4}$ (0.8 equiv) in MeCN under irradiation at $\lambda=465 \mathrm{~nm}$ and air at room temperature. Tetrahydrofurans $\mathbf{1 2 7} \mathbf{a}-\mathbf{j}, \mathbf{I}-\mathbf{p}$ were formed in yields between 14 and $67 \%$ (Table 3.9, grayed products were synthesized by Dr. M. Palomba).1133,134] Their formation was easily observable by ${ }^{1} \mathrm{H}$ NMR, where the carbinol proton typically resonates at around $4.2 \mathrm{ppm}$ as a quartet with a coupling constant of $7 \mathrm{~Hz}$. The newly formed double bonds have two signals between 5 and $6 \mathrm{ppm}$ and show a coupling of $15 \mathrm{~Hz}$, meaning they are $(E)$-configured. The method tolerated various functional groups very well (aryl, imide, nitrile, free alcohol, carbonate, ester). The formation of ether 127e with a trisubstituted double bond resulted in a moderate yield of $39 \%$. Other substrates proved to be more challenging. Ether 127c was obtained in

Table 3.9: Synthesis of tetrahydrofurans 127. ${ }^{\mathrm{a}}$ Grayed products were synthesized by Dr. M. Palomba.

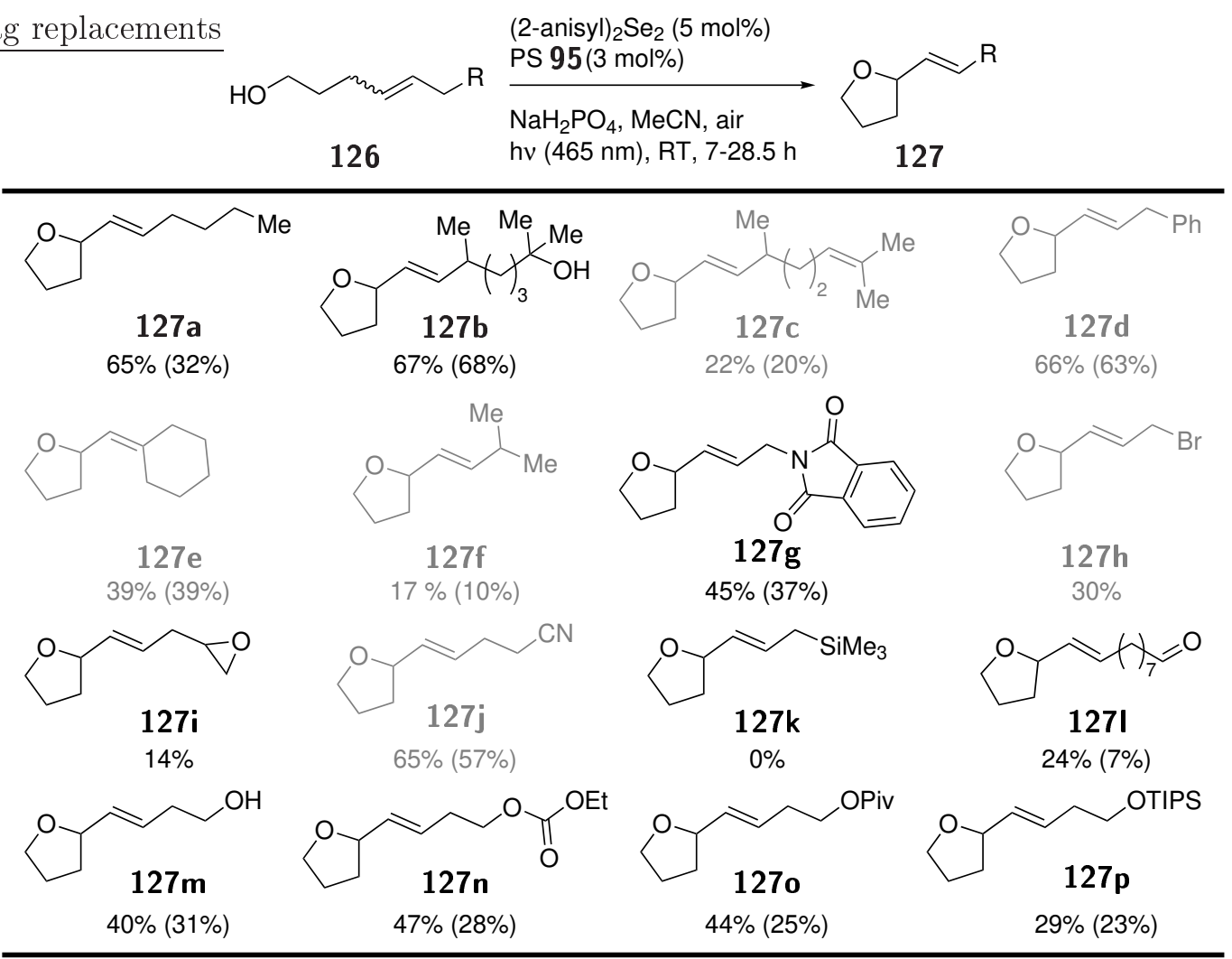

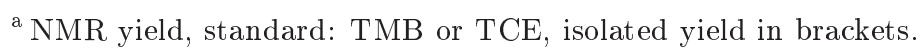


only $22 \%$ yield, presumably due to side reactions with the second double bond. Compound 127f was volatile, which diminished both the NMR and isolated yield. The same was assumed for aldehyde 127l, the formation of which was observed in $24 \%$ yield in NMR, but only $7 \%$ was isolated. Bromide $\mathbf{1 2 6}$ and epoxide 126i seemed to tolerate the reaction conditions and product formation was determined via NMR with yields of 30 and $14 \%$, respectively. However, the products could not be isolated, as the bromide degraded during isolation attempts and the epoxide could only be isolated as a mixture with unidentifiable side products. The NMR yields were based on the signals of the assumed carbinol protons as a quartet at 4.32 and $4.26 \mathrm{ppm}$, respectively (Figure 3.2). In the case of assumed bromide $\mathbf{1 2 7 h}$, there were also two clear signals that were assigned to the double bond, a doublet of triplets of doublets at $5.90 \mathrm{ppm}(J=15.3$, 7.3 and $0.9 \mathrm{~Hz})$ and a doublet of doublets at $5.76 \mathrm{ppm}(J=15.3$ and $6.5 \mathrm{~Hz})$.

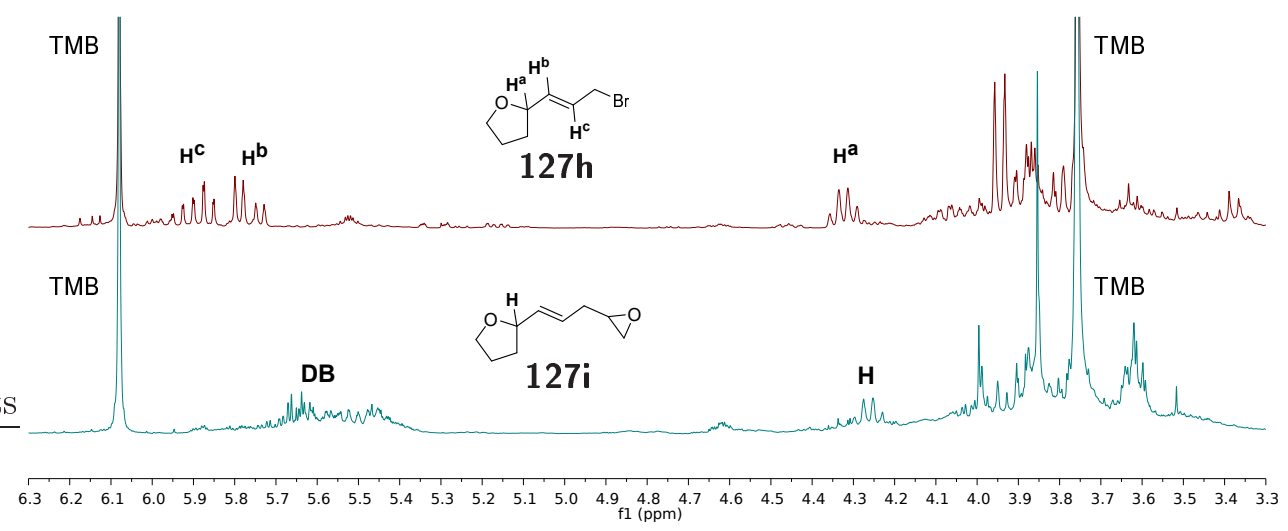

Figure 3.2: Crude ${ }^{1} \mathrm{H}$ NMR spectra of assumed products $\mathbf{1 2 7 h}$ and $\mathbf{1 2 7 i}$.

Substrates containing silyl moieties turned out to be problematic. With silyl ether 126p, the reaction to ether $\mathbf{1 2 7 p}$ proceeded in a low yield of $29 \%$ and the fluorescence was partly quenched. During the attempted cyclization of silane $\mathbf{1 2 6}$, the fluorescence was quenched completely and in the ${ }^{1} \mathrm{H}$ NMR spectrum, no signals for the product $\mathbf{1 2 7}$, but new signals around 0 ppm were observed. As at the same time, the signals of the starting material disappeared, it was assumed that it decomposed under the applied conditions.

The cycloetherification of alcohols $\mathbf{1 3 9}$ under the same conditions proceeded smoothly and delivered tetrahydropyrans $\mathbf{1 4 4}$ in mostly high yields between and 19 and $72 \%$ (Table 3.10). 1134] In contrast to tetrahydrofuran derivatives $\mathbf{1 2 7}$, the carbinol proton is shifted to higher field $(4.0 \mathrm{ppm})$. Compounds with functional groups that were well tolerated in the formation of tetrahydrofurans delivered ethers 144 in high yields as well (compounds 144a,c,d), while an additional internal double bond seemed to also inhibit the reaction (compound 144b). Moreover, ether $\mathbf{1 4 4 e}$ was less volatile than its furan-equivalent and chloride $\mathbf{1 4 4 f}$ and alkene $\mathbf{1 4 4 h}$ were also obtained in very good yields. Alkyne 144i was obtained in moderate yield. Unfortunately, the protected piperidine derivative $\mathbf{1 3 9 g}$ showed no conversion and also showed partial quenching of the fluorescence. 
Table 3.10: Synthesis of tetrahydropyrans $144 .^{\text {a[134] }}$

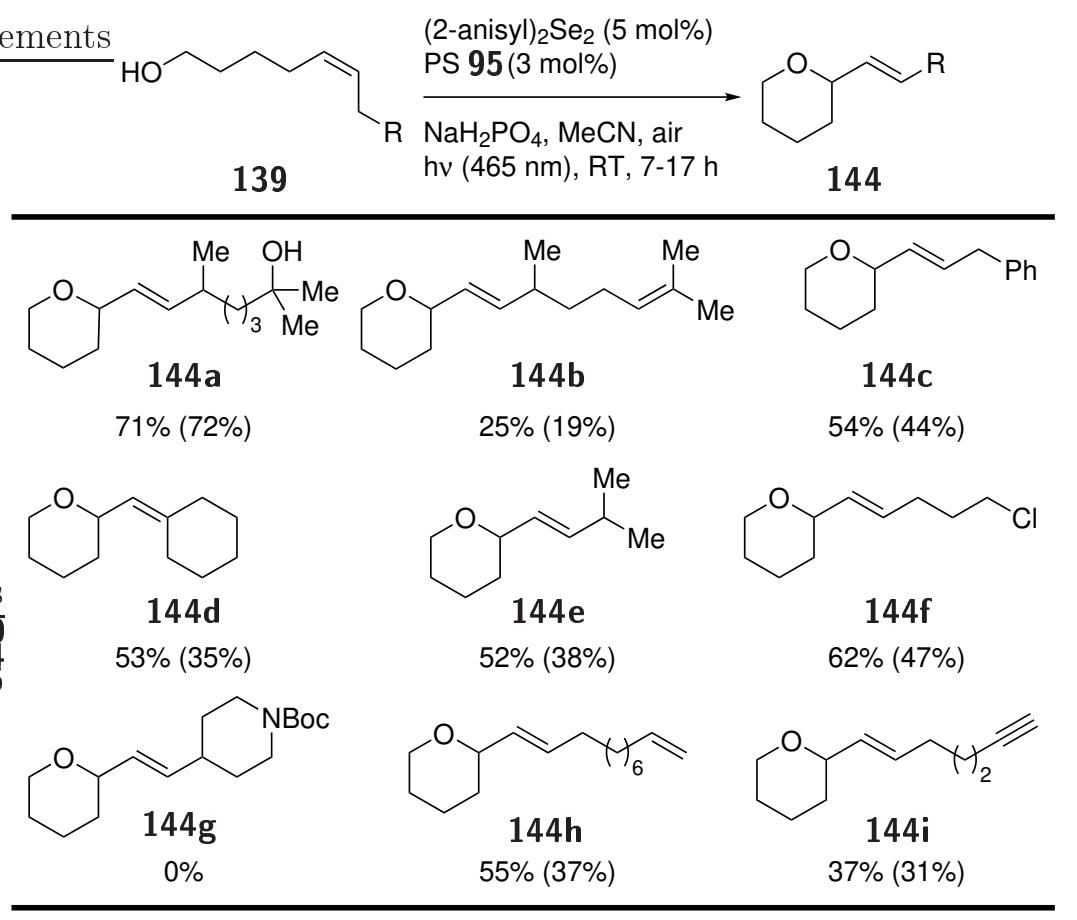

${ }^{\text {a }}$ NMR yield, standard: TMB or TCE, isolated yield in brackets.

In order to evaluate the performance of an aryl bromide, the reaction of the mixture of alcohols 139j and 139c under the established conditions was examined (Equation 3.6). As it was also not possible to separate the formed ethers from each other, only the NMR yield was determined. The differentiation of the signals of the two ethers was simplified by comparison with the previously obtained clean NMR spectrum of compound 144c (Figure 3.3, bottom). As the signals of the double bond protons and the carbinol proton of both compounds overlapped, the NMR yield was determined by integration of the signal of the benzylic protons, a doublet of doublets at 3.37 or $3.31 \mathrm{ppm}$, respectively (Figure 3.3 , top). Bromide 144j was obtained in an excellent yield of $68 \%$, the formation of ether $\mathbf{1 4 4 c}$ was observed in $53 \%$, consistent with the result of pure alcohol 139c (Table 3.10).

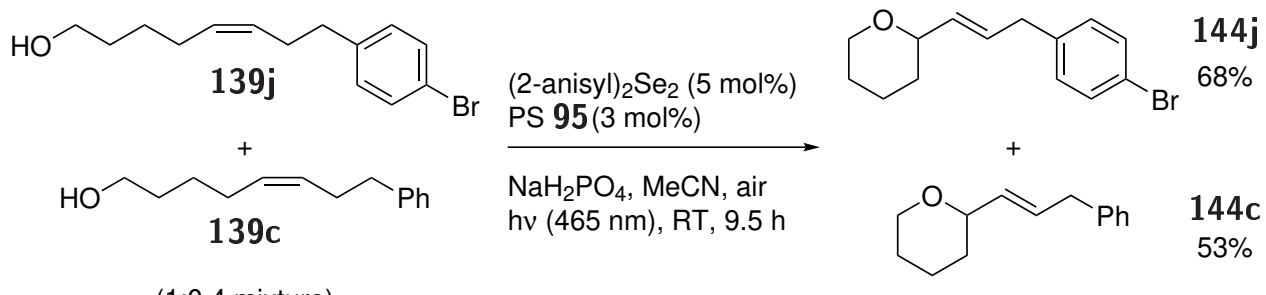

(1:0.4 mixture) 


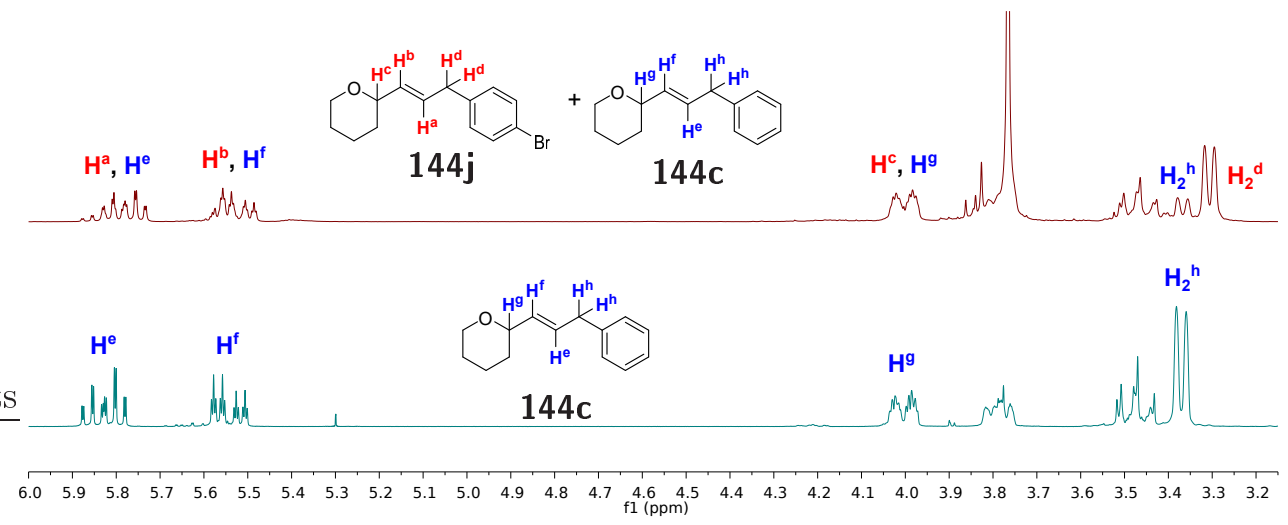

Figure 3.3: Crude ${ }^{1} \mathrm{H}$ NMR spectrum of ethers $144 \mathrm{j}$ and $144 \mathrm{c}$ compared with a clean spectrum of compound 144c.

As the cyclization of primary alcohols proceeded well under the established conditions, the question came up whether secondary alcohols would perform equally well and if one diastereomer would be formed preferentially. For this purpose, secondary alcohols 146a-c were synthesized by Dr. S. Ortgies via the reaction of cis-4-hepten-1-al (145) with aryl magnesium bromide derivatives (1.2 equiv) in 76 to $83 \%$ yield (Equation 3.7.

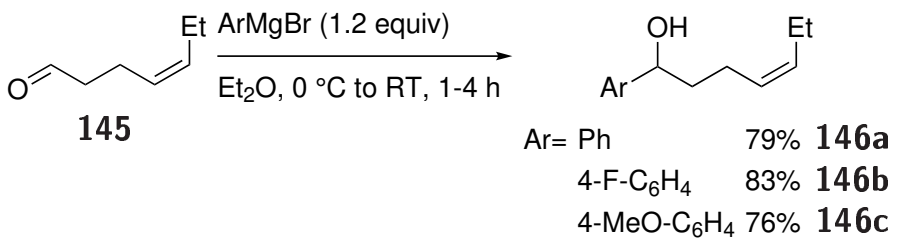

Gratifyingly, the selenium/photoredox catalyzed cyclization of alcohols 146a-c yielded tetrahydrofurans 147a-c in good yields with approx. 1:1 diastereomeric ratios Table 3.11, experiments by Dr. S. Ortgies). $\underline{134,136]}$ Electron-rich product 147c was obtained in the lowest yield (56\%), electron-poor product $\mathbf{1 4 7 \mathbf { b }}$ in the highest (69\%).

Table 3.11: Synthesis of tetrahydrofurans $147 .^{\text {a }}$ All syntheses by Dr. S. Ortgies. 134,136$]$
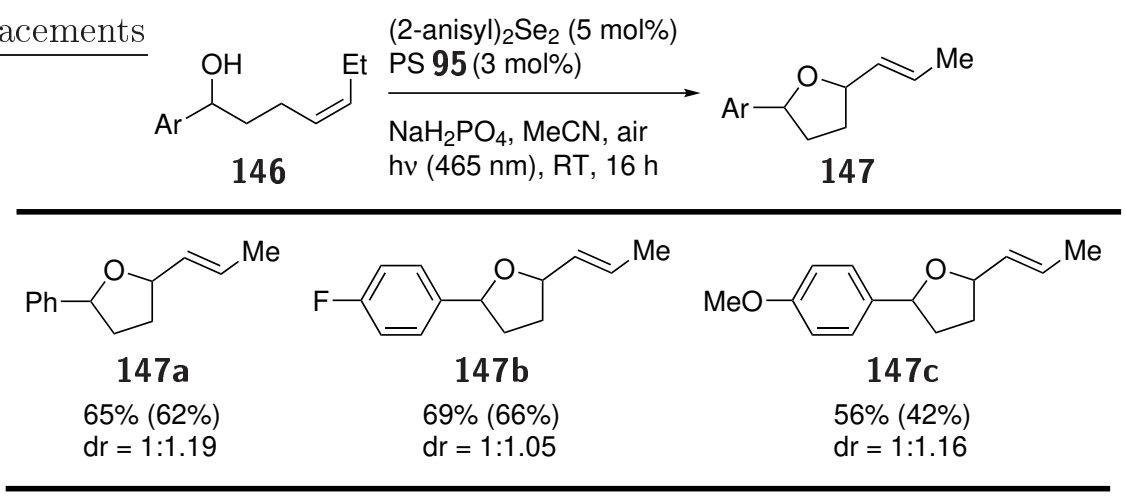

a NMR yield, standard: TCE, isolated yield in brackets. 


\subsubsection{Intermolecular etherification - Preliminary investigations and optimization of reaction conditions}

As the aerobic intramolecular etherification reactions proceeded in good yields, it was hypothesized that it was also possible to accomplish the alkoxylation of alkenes with selenium/photoredox catalysis. This reaction had been previously conducted using selenium catalysts together with high amounts of persulfate oxidants or in electrochemical reactions, which lacked selectivity (see Subsection 1.2.2.. [74,75,88,89] In an initial experiment, R. Rieger examined the reaction of hex-3enoic acid benzyl ester (148a) with diphenyl diselenide (10 mol\%) and p-MeO-TPT (5 mol\%) in methanol under air Equation 3.8). 137$]$ He observed an NMR yield of ether 149a of $17 \%$. The product was identified by the characteristic ${ }^{1} \mathrm{H}$ NMR signals of the double bond, the carbinol and the matching signal of the methoxy group. The signals of the double bond are doublets of doublets at 6.85 and $6.05 \mathrm{ppm}$. This splitting and chemical shift is typical for a double bond that is conjugated to a carbonyl group. ${ }^{[138]}$ The carbinol proton is shifted to higher field, compared to the cyclic ethers. It is a quartet of doublets at $3.67 \mathrm{ppm}$. The methoxy group appears as a singlet at $3.31 \mathrm{ppm}$, which is slightly shifted compared to methanol (3.49 ppm). [139]

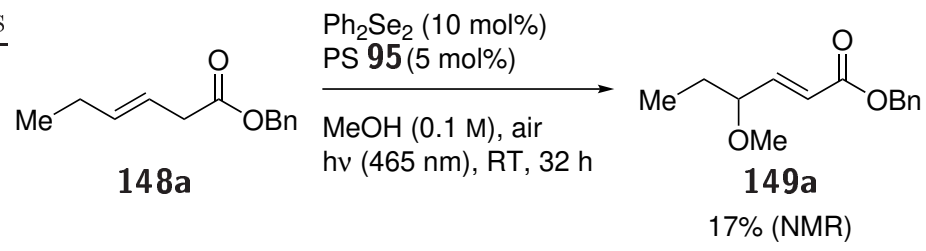

In order to find out if concentration had an impact on the reaction, as a first measure, it was increased to $0.2 \mathrm{M}$, which was the used concentration in the intramolecular reaction. Indeed, ether 149a was observed in $33 \%$ yield Equation 3.9). As (2-anisyl) ${ }_{2} \mathrm{Se}_{2}$ proved to furnish good yields in the intramolecular etherification, the reaction was repeated using this catalyst and the product was obtained in $32 \%$ yield, indicating that the used selenium-catalyst did not have an influence on this reaction Equation 3.9.

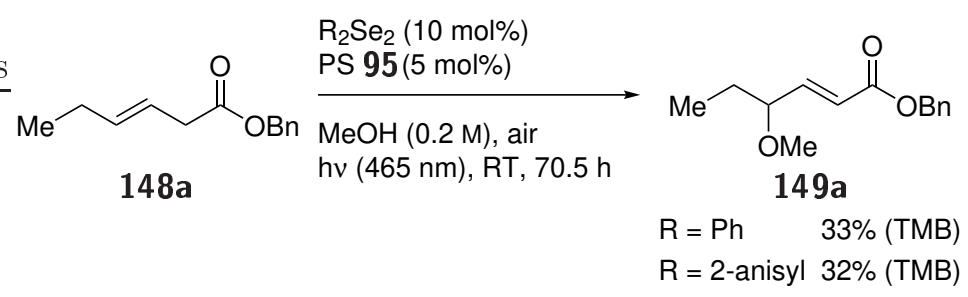

As the reactions by TIECCO et al. needed electron-poor substrates like ester 148a, it was interesting to find out if under selenium/photoredox catalyzed conditions, simple alkenes could be used as substrates instead. Therefore, 5-decene (150) was chosen as a suitable substrate for further investigations. As the resulting methoxy ether would probably be volatile, benzyl alcohol was used as the nucleophile. When the reaction was tested under previous conditions in benzyl alcohol 
as the solvent, it turned out that the solubility of $p$-MeO-TPT in benzyl alcohol was too low to catalyze the reaction and no conversion was observed (Table 3.12, entry 1). For this reason, the next experiments were conducted in $\mathrm{MeCN}-d_{3}$ with 3.0 equiv $\mathrm{BnOH}$ and 2.4 equiv $\mathrm{NaH}_{2} \mathrm{PO}_{4}$. The solvent was used as an internal NMR standard and the integral of its signal compared to one of the doublets of the benzyl group at $4.34 \mathrm{ppm}$. This signal was chosen because the signals of the double bond between 5.3 and $5.7 \mathrm{ppm}$ were expected to overlap with the SCHENCK-ene side product (allyl alcohol or hydroperoxide) and the signal of the carbinol proton, which was expected to be between 3.5 and $4 \mathrm{ppm}$, could not be identified due to several overlapping signals in this region. The reaction turned out to work more efficiently with (2-anisyl) ${ }_{2} \mathrm{Se}_{2}$, where the ratio of product to solvent was 0.22 to 1 , compared to 0.10 to 1 with diphenyl diselenide (Table 3.12, entries 2 and 3).

Table 3.12: Comparison of diselenides in the intermolecular etherification of 5-trans-decen.

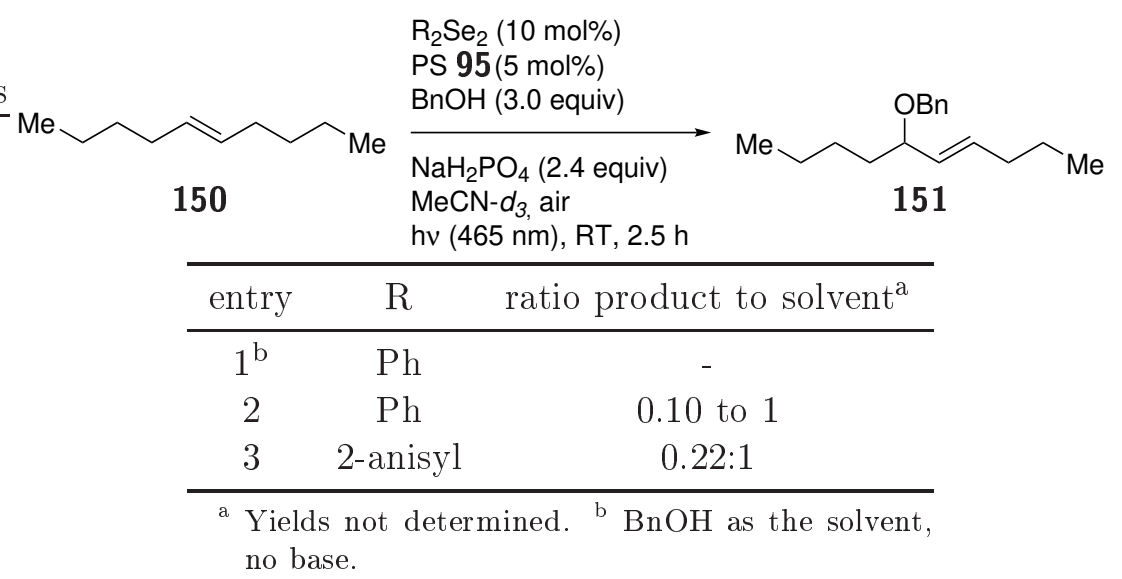

An important observation in the last reactions was the formation of benzaldehyde as a side product. In order to avoid the loss of nucleophile by oxidation, the ratio of alkene and benzyl alcohol was reversed, so that the excess of alkene would lead to preferred attack of the alcohol. Unfortunately, in the reaction of benzyl alcohol with 3 equiv 5-decene (150) with 10 mol\% (2-anisyl $)_{2} \mathrm{Se}_{2}, 5 \mathrm{~mol} \% p$-MeO-TPT and 0.8 equiv $\mathrm{NaH}_{2} \mathrm{PO}_{4}$, only traces of product $\mathbf{1 5 1}$ were formed, but $15 \%$ of the alkene underwent a SCHENK-ene reaction to the allyl alcohol or the allylic hydroperoxide 152 (Table 3.13, entry 1). The formation of the target product 151 could be achieved in $14 \%$ when no base was added to the reaction mixture, but still $15 \%$ of the side product was formed (Table 3.13, entry 2).

Since the functionalization of a simple alkene proved challenging due to overoxidation of either the substrate or the nucleophile, further investigations focused again on alkenes that contained electron-withdrawing groups. However, it was concluded that the reaction proceeded more efficiently with catalyst (2-anisyl $)_{2} \mathrm{Se}_{2}$ and without a base. 
Table 3.13: Influence of base on the intermolecular etherification of 5-trans-decen.

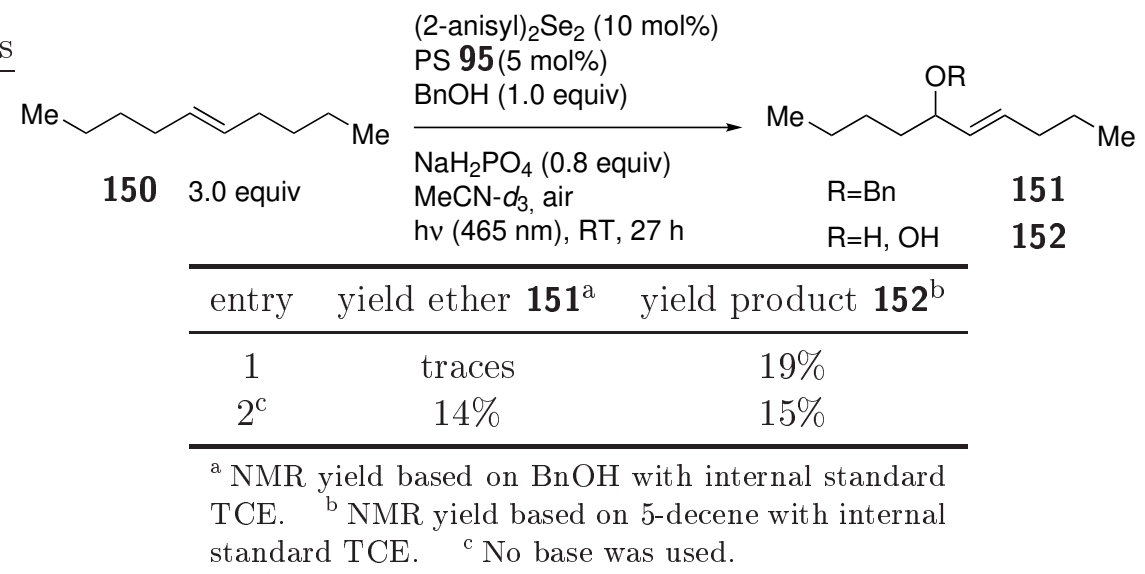

\subsubsection{Intermolecular etherification - Synthesis and etherification of alkenes}

For the evaluation of the scope and limitations of the intermolecular etherification, two additional substrates were synthesized according to literature procedures. Imide 148b was obtained in $32 \%$ in a reaction of 2-oxazolidinone (153) with 3 -hexenoic acid (1.3 equiv), using DCC (1.31 equiv) and DMAP (0.13 equiv) as coupling reagents [Equation 3.10).

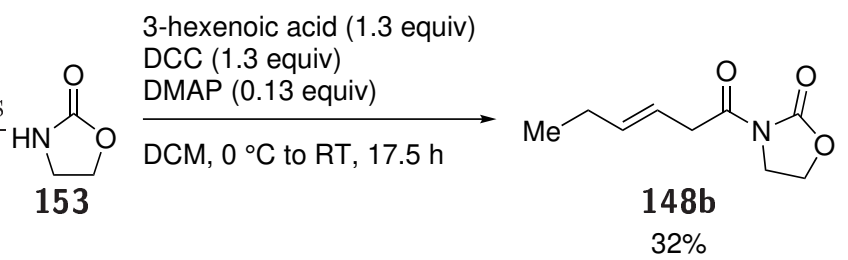

The reaction of $(E)$-crotylbromide (154) with 1.1 equiv $n$ BuLi and 1.1 equiv diethyl phosphite afforded phosphonate 148c in $61 \%$ yield Equation 3.11.140] The success of the reaction was confirmed by ${ }^{31} \mathrm{P}$ NMR by the shift of the phosphorus signal from $7.27 \mathrm{ppm}$ to $28.1 \mathrm{ppm}$ and by the additional coupling of the protons to the phosphorus in ${ }^{1} \mathrm{H}$ NMR (e.g., the coupling of the $\mathrm{CH}_{2}$ group at $\left.2.52 \mathrm{ppm},{ }^{2} J_{H P}=21.4 \mathrm{~Hz}\right)$. 141$]$

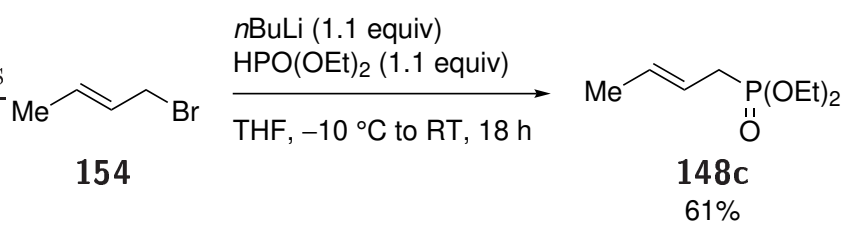

With the substrates in hand, the reactions with alcohols of different carbon chain length were tested using $10 \mathrm{~mol} \%$ of (2-anisyl) ${ }_{2} \mathrm{Se}_{2}$ and $5 \mathrm{~mol} \%$ of $p$-MeO-TPT under irradiation with blue light with the respective alcohol as the solvent. The functionalization of benzyl ester 148a in methanol, ethanol and isopropanol afforded the respective ethers $\mathbf{1 4 9} \mathbf{a}-\mathbf{c}$ in similar yields of 28

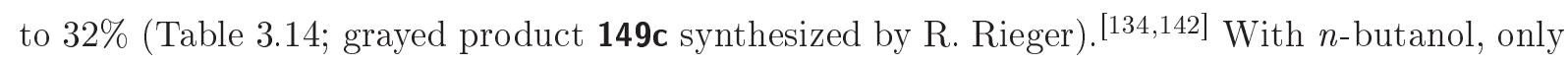


traces of product formation and later decomposition of the substrate was observed. The reactions were run until completion was determined via NMR or TLC, which often took several days. The same observation was made by IwAOKA and TOMODA, whose oxyfunctionalization of 5-decene ran for 7 days (cf. Equation 1.12, Subsection 1.2.2).

Table 3.14: Synthesis of allylic ethers $149 a-d{ }^{a}$ Grayed product $149 c$ was synthesized by $R$. Rieger. $\underline{[134,142]}$
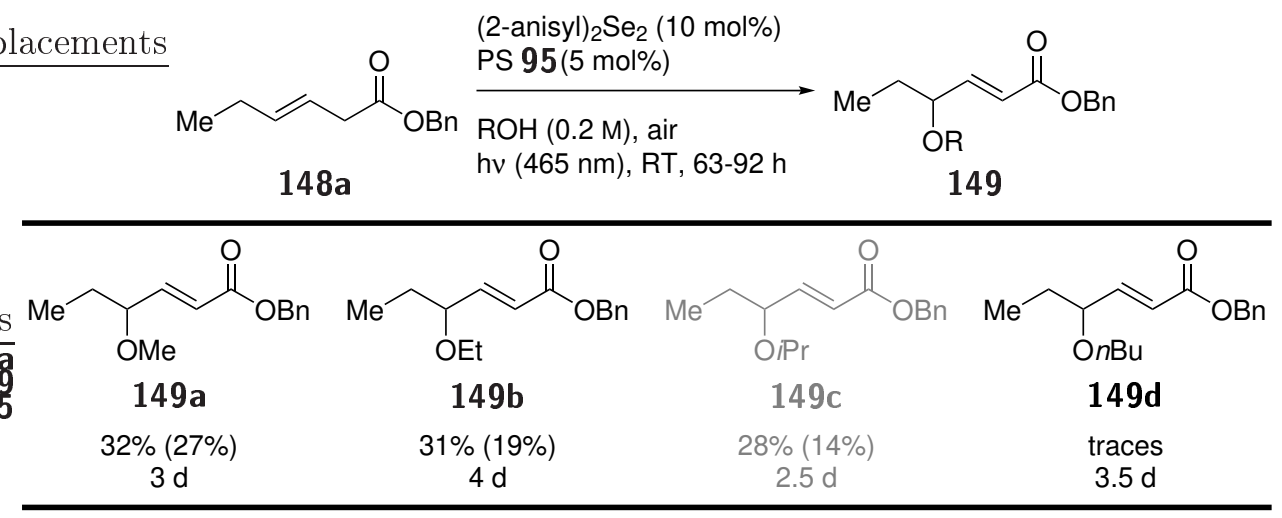

${ }^{\text {a }}$ NMR yield with standard TCE or TMB, isolated yield in brackets.

Unfortunately, imide $\mathbf{1 4 8 b}$ proved to be less reactive under the established conditions than ester 148a. Therefore, the reaction was conducted with diphenyl diselenide (10 mol\%) as the catalyst instead. After 4 days, ether 149e was obtained in 11\% NMR yield (10\% isolated yield) Equation 3.12. Similar to ethers 149a-c, the change of the signals of the double bond protons in NMR from multiplets around $5.6 \mathrm{ppm}$ to the characteristic doublets of doublets at 7.36 and $6.96 \mathrm{ppm}$ along with a singlet at $3.32 \mathrm{ppm}$ with the integral 3 from the methoxy group indicated the product formation.

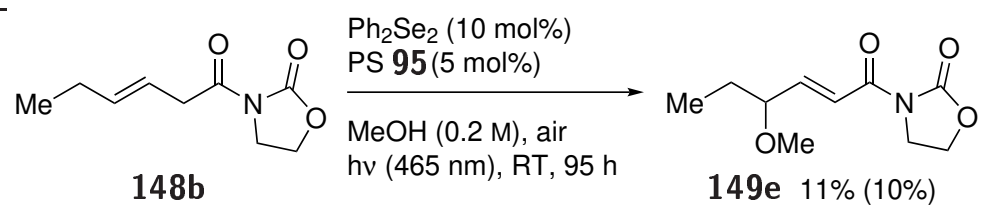

In contrast to imide $\mathbf{1 4 8 b}$, phosphonate $\mathbf{1 4 8 c}$ turned out to be a high-yielding substrate for the intermolecular etherification. The functionalization with simple alcohols methanol, ethanol and isopropanol afforded ethers $\mathbf{1 4 9 f - h}$ in good to excellent NMR yields of 43 to $99 \%$, functionalization with benzyl alcohol derivatives led to yields between 27 and $81 \%$ (products $\mathbf{1 4 9} \mathbf{k}-\mathbf{m}$, Table 3.15). The occasionally high difference between NMR yields and isolated yields (e.g. for products $\mathbf{1 4 9 f}$ and $\mathbf{1 4 9 m}$ ) resulted from difficulties during chromatographic purification due to the high polarity of both products and starting materials. The progress of the reactions was determined via ${ }^{31} \mathrm{P}$ NMR because the signal of the phosphorus atom was shifted to high field from $28.1 \mathrm{ppm}$

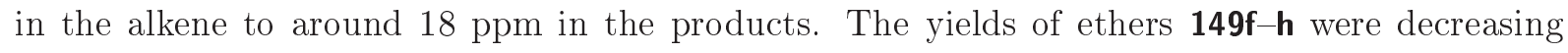
with increasing carbon chain length of the alcohol, which is confirmed by the results with $n$ - 
butanol (13\%) and cyclopentanol (traces), where the reactions stagnated after 5.5 days (149i,j). This trend could be attributed to a lower reactivity of longer-chained alcohols, leading to longer reaction times. At first glance, the results with benzyl ester 148a and methanol, ethanol and isopropanol contradict these assumptions, because they do not show decreasing yields, although the reaction with $n$-butanol afforded only traces of the desired product. While the reactions with ester 148a showed complete conversion (caused by reaction to the product or by oxidative decomposition) after 3-4 days, the reactions with phosphonate 148c needed more than twice this time for full conversion but also afforded higher yields of the products. A possible explanation for these findings is the different susceptibility of the substrates to oxidation which leads to a high amount of the ester being oxidized before the reaction can occur. The phosphonate seems to be less prone to oxidation, being available for the functionalization with isopropanol for as long as 12 days. The reaction times were shorter (up to 5.5 days) with electron-poor benzyl alcohol derivatives, which we attributed to them being more easily deprotonated by side products of the oxidation cycle, which would increase their nucleophilicity. The reaction with 4-chlorobenzyl alcohol had to be conducted in MeCN due to the alcohol being a solid. The lower yield from this reaction, compared to the other benzyl alcohol derivatives, was attributed to the lower amount of alcohol used (1.5 equiv vs. $>10$-fold excess with the alcohol as the solvent). Due to the high

Table 3.15: Synthesis of allylic ethers $149 \mathbf{f}-\mathbf{m} .^{a[134]}$
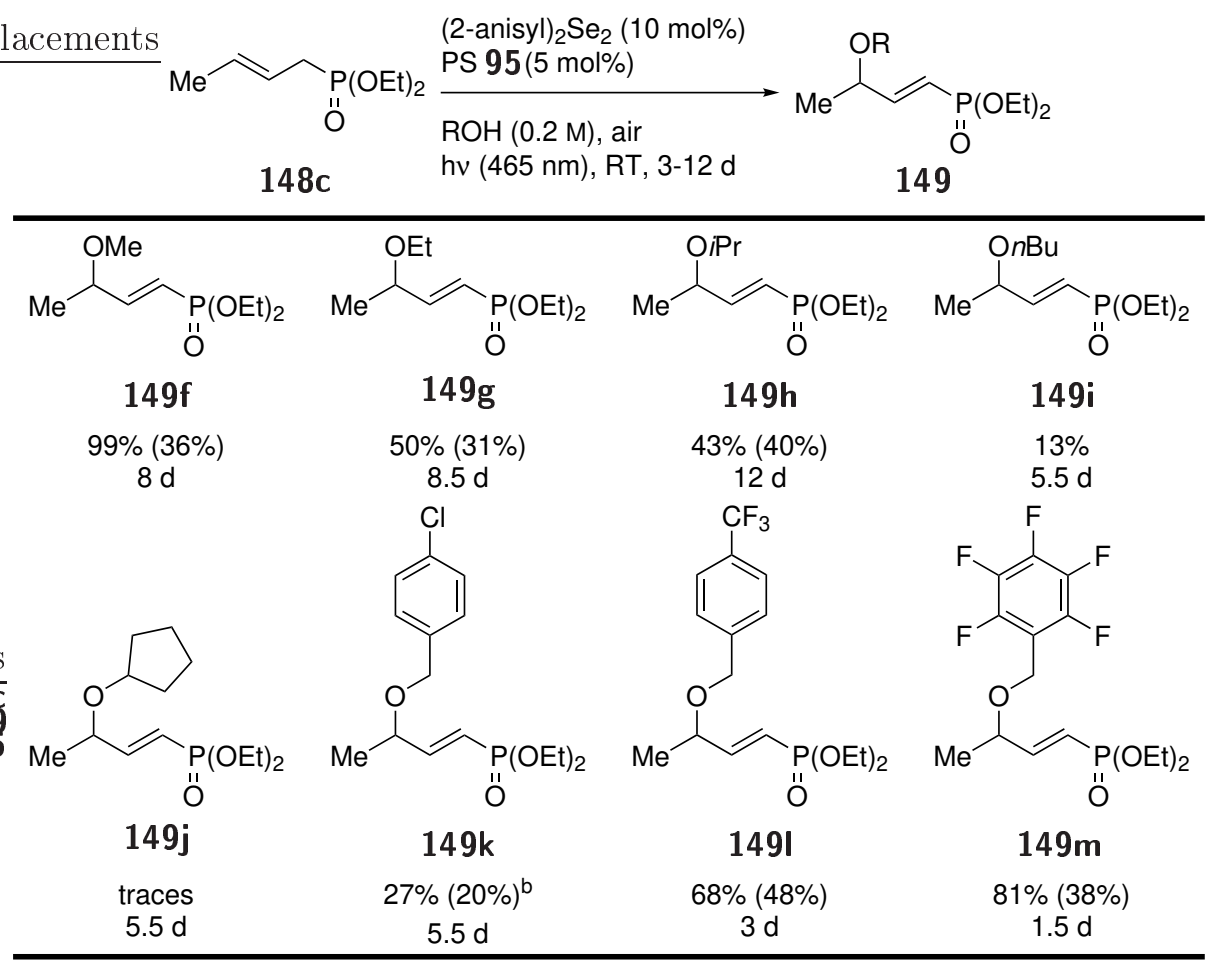

${ }^{a}$ NMR yield with standard TCE, TMB, phthalide or benzaldehyde, isolated yield in brackets. $\quad{ }^{\mathrm{b}}$ Reaction in $\mathrm{MeCN}(0.4 \mathrm{M}), 1.5$ equiv $\mathrm{ROH}$. 
polarity of the products, the isolated yields sometimes differ from the NMR yields because of difficulties in the separation from the alcohols.

Being intrigued by the results with phosphonate 148c, the reactivity of a nitrile was examined with commercially available 3 -pentenenitrile (148d). Under the established conditions, ethers 149n-p were obtained in 18 to $54 \%$ yield (Table 3.16). Interestingly, in contrast to the previous products, not only the $(E)$-configured ethers were obtained, but in all cases, also significant amounts of the $(Z)$-configured product were formed. A possible explanation is the smaller steric bulk of the nitrile moiety compared to the phosphonate, the imide and the ester group. While the ${ }^{1} \mathrm{H}$ NMR shifts of the double bond protons are similar for both isomers (6.3-6.7 ppm and $5.4-5.6 \mathrm{ppm})$, the carbinol proton has a shift of $4.0 \mathrm{ppm}$ in the $(E)$-isomer and a shift of $4.5 \mathrm{ppm}$ in the $(Z)$-isomer. Furthermore, the reactions showed complete conversion after 1 day, which could be attributed to fast oxidation of the substrate. While the use of 4-(trifluoromethyl)benzyl alcohol led to a good yield, probably due to the higher nucleophilicity, the reactions with 2phenylethanol and 4-chlorobenzyl alcohol afforded the products in lower yields. In the latter case, the lower amount of alcohol (1.5 equiv) could be the reason. In the experiment with trifluoroethanol, the desired product was not obtained. Instead, a mixture of the $(E)$ and the (Z)-configured allylic alcohols 155 (ratio 3.5:1) was isolated. This conclusion was based on the appearance of two doublets at 1.95 and $1.37 \mathrm{ppm}(J=\sim 4 \mathrm{~Hz})$, that were attributed to the $\mathrm{O}-\mathrm{H}$ protons, and the appearance of the carbinol proton as a doublet of doublets of doublets of a quartet at $4.48 \mathrm{ppm}(J=8.7,6.7,4.5,2.3 \mathrm{~Hz})$ and a multiplet at $4.81 \mathrm{ppm}$ (Figure 3.4. The matching coupling constant of $4.5 \mathrm{ppm}$ and the roof effect of the doublets in question supported this assumption.

Table 3.16: Synthesis of allylic ethers $149 n-\mathbf{q}^{\text {a[134] }}$
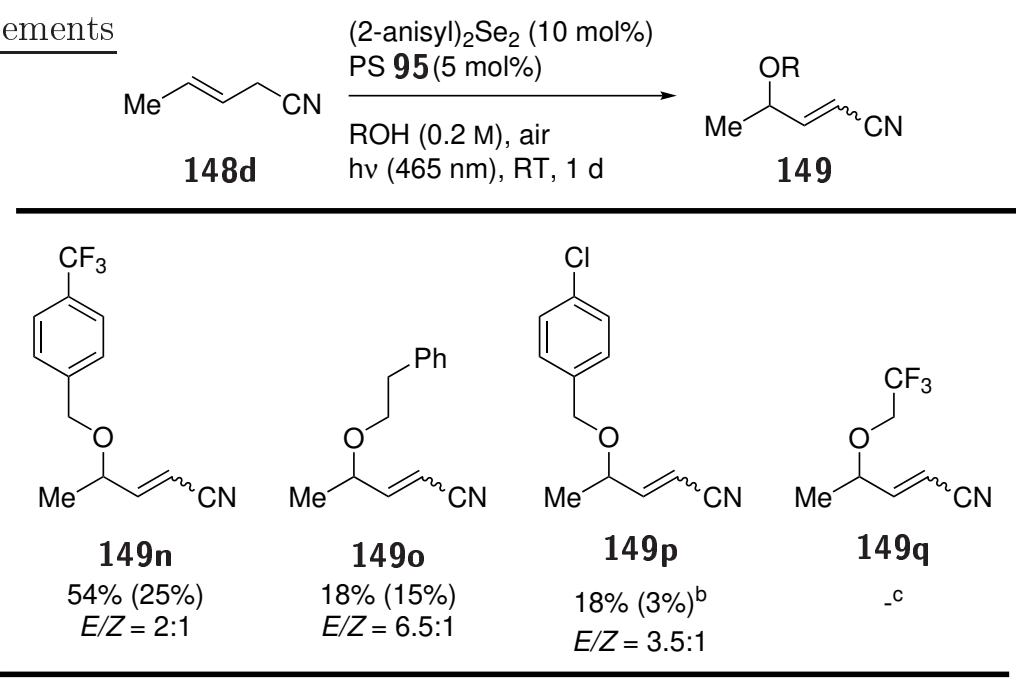

${ }^{a}$ NMR yield with standard TCE or TMB, isolated yield in brackets. ${ }^{\mathrm{b}}$ Reaction in MeCN $(0.4 \mathrm{M}), 1.5$ equiv $\mathrm{ROH} .{ }^{\mathrm{c}}(E / Z)$ mixture $(3.5: 1)$ of the respective allyl alcohol was isolated. 


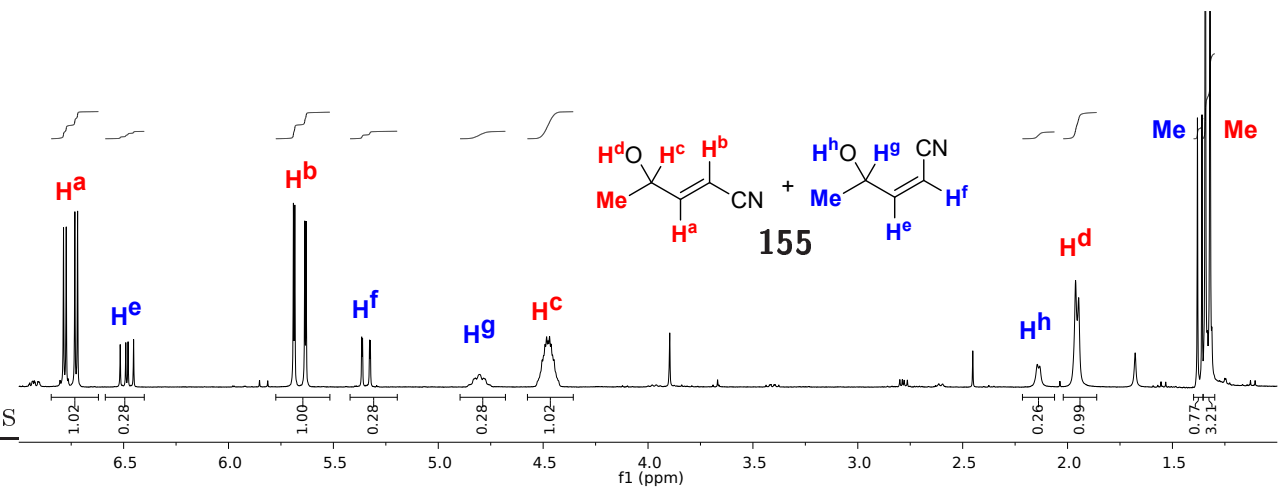

Figure 3.4: ${ }^{1} \mathrm{H}$ NMR spectrum of assumed allyl alcohols 155. 
3.2 Synthesis and lactonization of unsaturated acids via photo-aerobic selenium- $\pi$-acid catalysis

\subsection{Lactonization of unsaturated acids via photo-aerobic selenium- $\pi$-acid catalysis}

After the successful application of the dual selenium- $\pi$-acid/photoredox catalysis in the intermolecular acyloxylation [91] and in the etherification, ${ }^{[134]}$ it was speculated that the lactonization of unsaturated acids could also be accomplished with this method. Due to lactones being an important group of natural products with different biological activities, various synthetic methods for their preparation have been developed, amongst others selenium-catalyzed variants. $[76,82,83,89,90,143]$ Compared to these methods, the selenium/photoredox catalysis would have the advantage of a high atom economy through consequential prevention of waste from the used oxidants, as air would be the terminal oxidant and water the sole co-product. The examination and optimization of the lactonization of 3-hexenoic acid (34a) under selenium/photoredox conditions was performed by R. Rieger. $\stackrel{[137.144]}{ }$ He was able to obtain lactone $\mathbf{7 7 a}$ in $>95 \%$ NMR yield using 5 mol\% diphenyl diselenide and 5 mol\% $p$-MeO-TPT in MeCN (0.1 M) under air and irradiation with blue light $(465 \mathrm{~nm})$. A lower loading of the diselenide or the photosensitizer resulted in lower yields. In toluene or ethereal solvents such as THF or 1,4-dioxane, no product formation was observed, probably due to low solubility of the photosensitizer in these solvents. Using acetone or DCE led to good and very good yields, respectively, but both did not reach the level of product formation observed with MeCN.

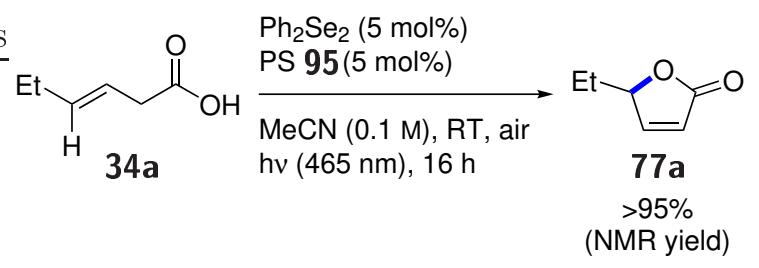

\subsubsection{Synthesis of unsaturated acids}

With the optimized reaction conditions in hand, the synthesis of unsaturated acids was pursued. In addition to the formation of butenolides $\mathbf{7 7}$ from 3-alkenoic acids 34, the formation of 6 - and 7-membered ring lactones was to be examined by using 4-, 5- or 6 -alkenoic acids. The Doebnertype Knoevenagel reaction was identified as an easy way to obtain $\beta, \gamma$-unsaturated acids from aldehydes. In order to examine the applicability of aromatic substrates in the seleniumcatalyzed lactonization, 3-arylpropanal derivatives $\mathbf{1 5 6}$ a and $\mathbf{1 5 6} \mathbf{b}$ were transformed to acids $\mathbf{3 4 \mathbf { b }}$ and 34c in 28 and $43 \%$ yield using 2.2 equiv malonic acid and 0.02 equiv piperidine and acetic acid, respectively (Table 3.17, entries 1 and 2). The success of the reaction was visible via the appearance of two signals for the newly formed double bond in the ${ }^{1} \mathrm{H}$ NMR spectrum, two doublets of triplets of triplets at around 5.6 and $5.75 \mathrm{ppm}$ with couplings of approx. 15, 6.5 and $1.0 \mathrm{~Hz}$. Other characteristic signals are the doublets at around 3.1 and $3.4 \mathrm{ppm}$, which are caused by the methylene groups next to the double bond. With aldehyde 156c, no reaction occurred 
and only the starting material was observed in the ${ }^{1} \mathrm{H}$ NMR spectrum of the crude reaction, a very characteristic signal being the triplet of the aldehyde proton at $9.7 \mathrm{ppm}$.

Table 3.17: Synthesis of carboxylic acids 34b-d.[144]

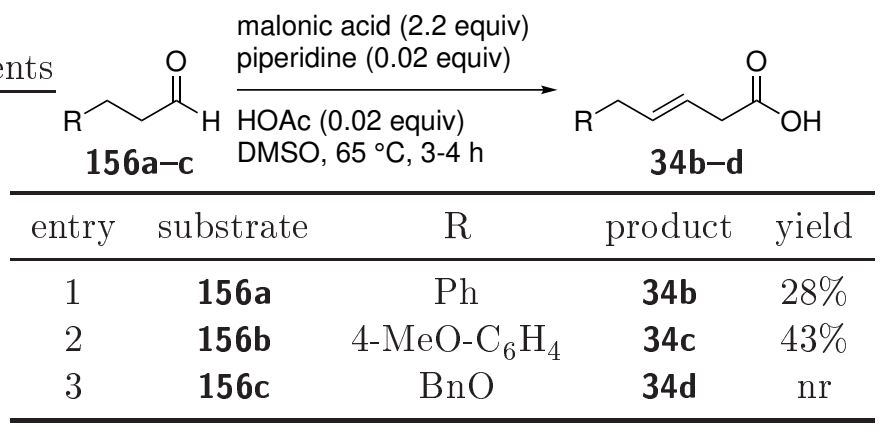

In addition to aromatic substrates, it was interesting to find out if ketones and aldehydes would react under photocatalytic conditions. Therefore, the synthesis of a ketone-containing acid was conducted following a procedure of CASTELLANO et al. ${ }^{[145]}$ In order to obtain 7-oxooctanal (161) for a Knoevenagel reaction, 5-bromopentanol (157) was transformed to $\beta$-keto ester 159 in $40 \%$ yield by coupling it to ethyl acetoacetate (158) using 1.0 equiv NaOEt (Scheme 3.2). The hydrolysis of the ethyl ester with aq. $\mathrm{NaOH}$ (2.5 equiv), followed by the decarboxylation with aq. $\mathrm{H}_{2} \mathrm{SO}_{4}$ (2.0 equiv) gave ketone $\mathbf{1 6 0}$ in $94 \%$ yield. Aldehyde $\mathbf{1 6 1}$ was obtained in $28 \%$ yield after a Dess-MARTiN oxidation using 2.0 equiv Dess-MarTin periodinane. $\underline{146]}$

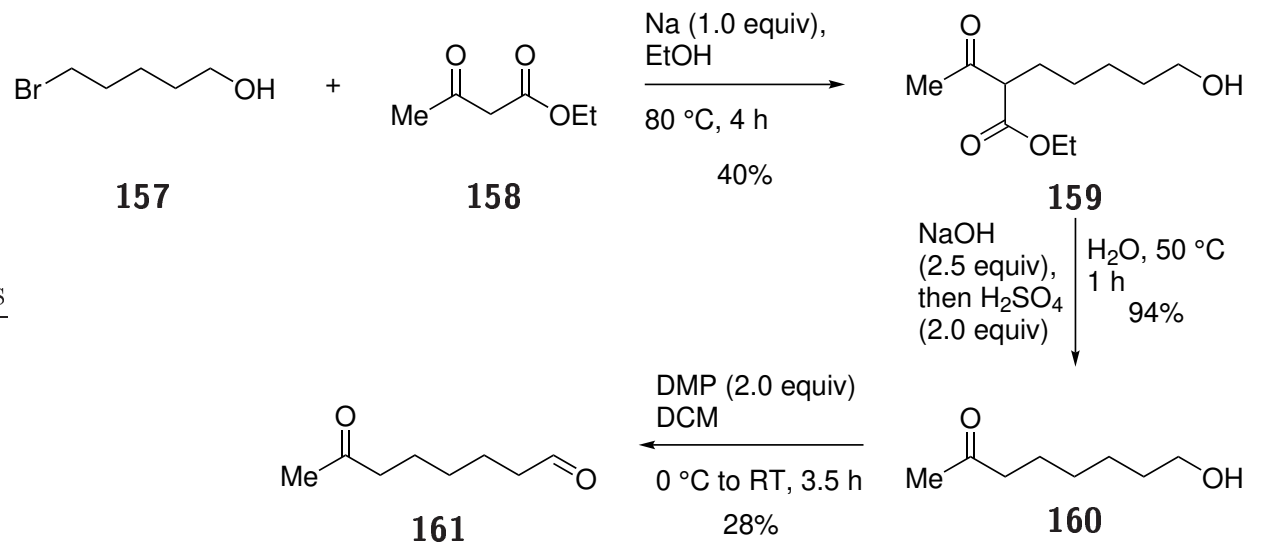

Scheme 3.2: Synthesis of 7-oxooctanal (161). $\underline{\underline{[145}]}$

In order to selectively synthesize the $\beta, \gamma$-unsaturated acid, a procedure of LIST and coworkers was applied to the aldehyde. $\underline{\underline{147]}}$ The KNoEvenAGEL reaction proceeded with 1.0 equiv malonic acid and 0.1 equiv DMAP and provided the $\beta, \gamma$-unsaturated acid 34e in a 1.5:1 mixture with the $\alpha, \beta$-unsaturated acid $\mathbf{1 6 2}$ in $23 \%$ yield (Figure 3.5, top). The ratio of the products was determined in ${ }^{1} \mathrm{H}$ NMR spectroscopy by comparison of the signals of the protons of the double bond. The $\alpha, \beta$-unsaturated acid $\mathbf{1 6 2}$ shows two doublets of triplets at 7.04 and $5.80 \mathrm{ppm}$ with an identical integral (Figure 3.5, bottom). They have coupling constants of 15.6 and $7.0 \mathrm{~Hz}$, and 
15.6 and $1.5 \mathrm{~Hz}$, respectively. The protons at the double bond of the $\beta, \gamma$-unsaturated acid 34e have only one multiplet signal at 5.68-5.40 ppm.

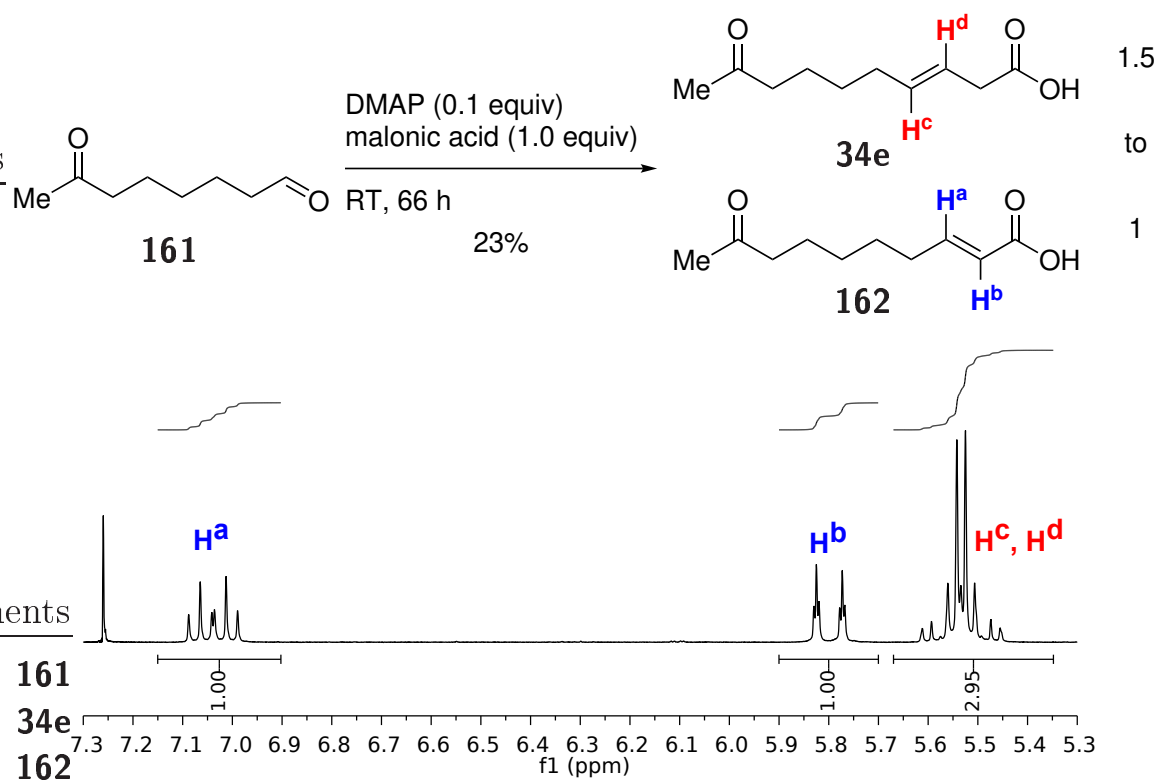

Figure 3.5: Knoevenagel reaction of aldehyde $\mathbf{1 6 1}$ (top), extract from the ${ }^{1} \mathrm{H}$ NMR spectrum of the mixture of 34e and 162 (bottom).

For the synthesis of the aldehyde substrate, the KnOEvEnAGEL reaction was not an option, as it uses an aldehyde as the starting material and a second aldehyde moiety could lead to selectivity problems. In order to avoid the introduction and removal of protecting groups, a step-economic cross-metathesis reaction was used. Acid $\mathbf{3 4 f}$ was obtained in $57 \%$ yield from the reaction of 10-undecenyl aldehyde (163, 3.0 equiv) and 4-butenoic acid (164, 1.0 equiv) using 5 mol\% 2nd generation Hoveyda-Grubbs catalyst (Equation 3.14).

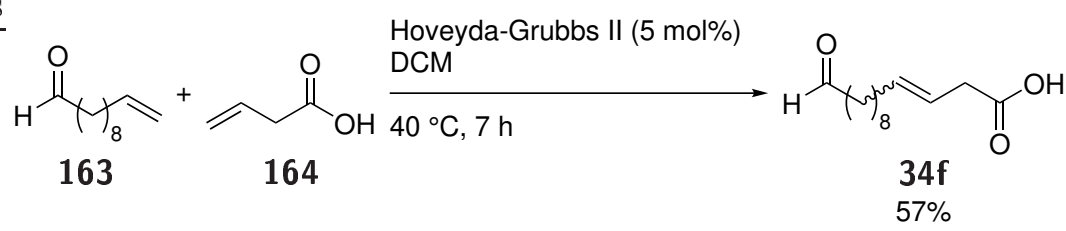

The synthesis of 5-alkenoic acids via a WITTIG reaction was already performed during the synthesis of unsaturated alcohols (see Subsection 3.1.2). Therefore, alkenoic acids 137, 166 and 167 were synthesized according to the established procedure using 2.0 equiv of either (3-carboxypropyl)triphenylphosphoniumbromide, (4-carboxybutyl)triphenylphosphoniumbromide or (5-carboxypentyl)triphenylphosphoniumbromide $(\mathbf{1 3 8 a - c})$ and $\mathrm{KO} t \mathrm{Bu}$ (4.0 equiv) as the base in THF at $0{ }^{\circ} \mathrm{C}$ (Table 3.18, products with yields in gray were synthesized by L. Löffler and Dr. S. Ortgies). $\underline{[144,148,149]}$ The acids were obtained in moderate to excellent yields of 37-92\%. The lowest yield of $12 \%$ was obtained for ether $\mathbf{1 3 7 t}$. No reaction occurred with diphenylacetaldehyde (product 137u). 
Table 3.18: WitTig reaction of aldehydes 133 to carboxylic acids 137, 166 and 167 . Acids with yield in gray synthesized by L. Löffler and Dr. S. Ortgies. $\underline{\underline{144,148,149]}}$

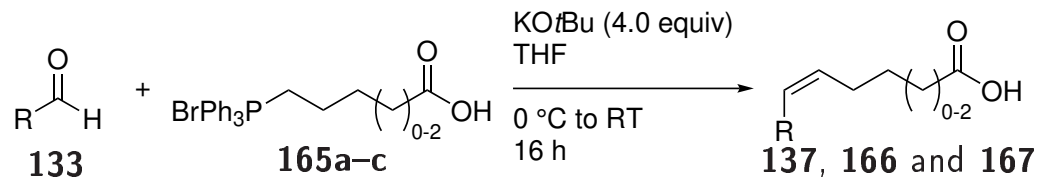

2.0 equiv<smiles>CCC/C=C\CCC(=O)O</smiles><smiles>CC(CC/C=C\CCCC(=O)O)CC(C)(C)O</smiles><smiles>CC(C)=CCCC=CCCCC(=O)O</smiles><smiles>O=C(O)CCC/C=C\CCc1ccccc1</smiles><smiles>C#C/C=C\CCCC(=O)O</smiles><smiles>CC(C)C/C=C\CCCCC(=O)O</smiles><smiles>CC(C)(C)OC1CCC(C/C=C/CCCC(=O)O)CC1</smiles>

166 $37 \%$<smiles>CC(C)/C=C\CCCC(=O)O</smiles>

137a $79 \%$<smiles>O=C(O)CCC/C=C\CC[N+]=[Nb]</smiles>

$137 m \quad 47 \%$

137b<smiles>O=C(O)CCC/C=C\CCc1ccc(P)cc1</smiles>

\begin{tabular}{llr}
$\mathrm{R}=t \mathrm{BBu}$ & $137 \mathrm{n}$ & $92 \%$ \\
$\mathrm{R}=\mathrm{CF}_{3}$ & $137 \mathrm{o}$ & $58 \%$ \\
$\mathrm{R}=\mathrm{F}$ & $137 \mathrm{p}$ & $7 \%$ \\
& \multicolumn{2}{c}{ (2 steps) } \\
$\mathrm{R}=\mathrm{CN}$ & $\mathbf{1 3 7 q}$ & $51 \%$ \\
$\mathrm{R}=\mathrm{OMe}$ & $\mathbf{1 3 7 r}$ & $75 \%$
\end{tabular}

137c $82 \%$<smiles>O=C(O)CCCC=CC1CC1</smiles>

$137 s \quad \begin{array}{r}55 \% \\ Z / E 7: 1\end{array}$

137t $12 \%$

137u $\mathrm{nr}$

$137 \mathrm{~g} 42 \%$

For all of the synthesized acids, the configuration of the double bond could not be determined by the coupling constants of the double bond protons in ${ }^{1} \mathrm{H}$ NMR. Instead, substrate $\mathbf{1 3 7 I}$ was examined in 2D-NMR spectroscopy. All signals were assigned to the protons by COSY. In the NOESY spectrum, a coupling of the $\mathrm{CH}_{2}$ group $\mathbf{c}$ to the $\mathrm{CHMe}_{2}$ group a was observed. This coupling would not be possible if the molecule was in $(E)$-configuration (Figure 3.6). To further support these findings, analytical data of acid $\mathbf{1 6 7}$ were compared to those of petroselinic acid, which is the same molecule with the double bond in $(Z)$-configuration. The data were in 
agreement with each other, meaning acid $\mathbf{1 6 7}$ is in fact petroselinic acid. From this evidence, it was concluded that in all cases, the WITTIG reaction was selective for the $(Z)$-alkene.

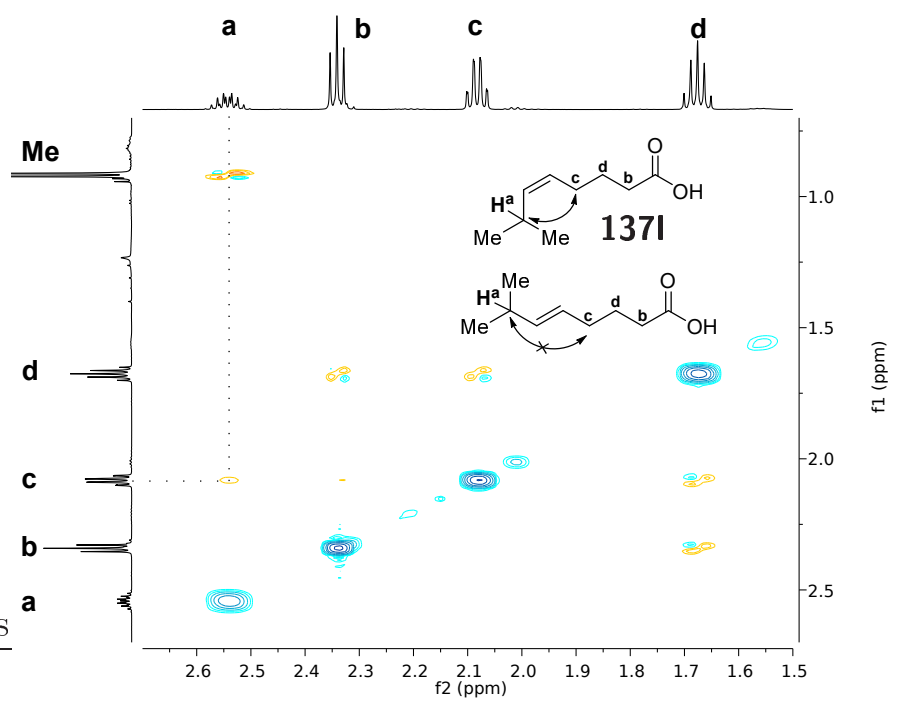

Figure 3.6: NOESY spectrum of unsaturated acid $\mathbf{1 3 7 1 .}$

\subsubsection{Lactonization of unsaturated acids}

The lactonization of 3-alkenoic acids $\mathbf{3 4}$ was performed under the established conditions with 5 mol\% diphenyl diselenide and photosensitizer 95, respectively, in acetonitrile at room temperature under air for $16 \mathrm{~h}$ and under irradiation with blue light. ${ }^{[137]}$ Butenolides $\mathbf{7 7 b} \mathbf{b}-\mathbf{e}$ were obtained

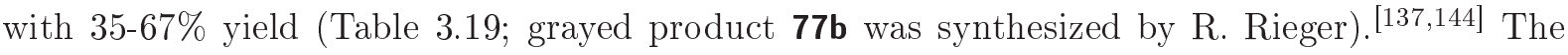
products were identified by their NMR signals. The protons of the double bond are a doublet of doublet of triplets at $6.1 \mathrm{ppm}$ and a doublet of doublets at $7.4 \mathrm{ppm}$ and show only a small coupling to each other $(J=5.7 \mathrm{~Hz})$, which can be justified by the $(Z)$-configuration needed in the ring. The protons of the double bond in the unsaturated acid had signals between 5.4 and $5.9 \mathrm{ppm}$. The changes are also visible in the ${ }^{13} \mathrm{C}$ spectrum, where the former double bond

Table 3.19: Synthesis of lactones $\mathbf{7 7}$. Grayed product $\mathbf{7 7 b}$ synthesized by R. Rieger.1137.144]

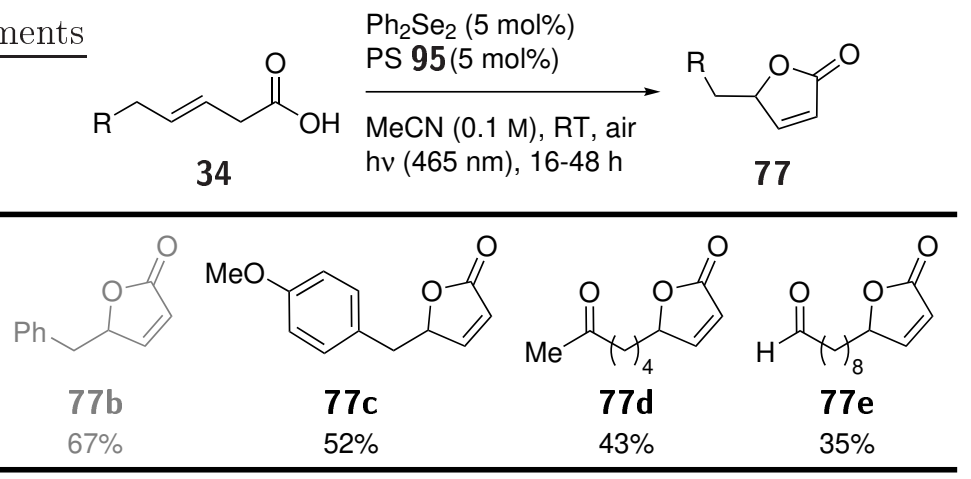


had two signals at 120 and $135 \mathrm{ppm}$ and the signals of the new double bond are at 121 and $156 \mathrm{ppm}$. Additionally, the carbinol proton and carbon show a shift to low field and have signals at around $5 \mathrm{ppm}$ (proton) and $83 \mathrm{ppm}$ (carbon). Lactones 77b and 77c with aromatic groups were obtained in good yields of 52 and $67 \%$, which is similar to the yield that was obtained in the intramolecular etherification (cf. Table 3.9 in Subsection 3.1.2, 127d). Gratifyingly, ketone 34e and aldehyde $\mathbf{3 4 f}$ were also reactive under the photoredox conditions and afforded products 77d and 77e in moderate yields of 35 and $43 \%$. In the intramolecular etherification, the use of an aldehyde led to only low yield (cf. Table 3.9 in Subsection 3.1.2, 127I).

In the lactonization of 4-alkenoic acid 166, there is the possibility of a 5-exo-trig-cyclization or a 6-endo-trig-cyclization. Under the standard conditions, the formation of a product was observed in a good yield of $64 \%$ Equation 3.15). The constitution and configuration of the molecule were deduced from the signals in ${ }^{1} \mathrm{H}$ NMR and the COSY couplings (Table 3.20). The coupling constants of the double bond protons with signals at $5.80 \mathrm{ppm}(\mathrm{dtd}, J=15.3,6.6,1.0 \mathrm{~Hz}$ ) and $5.48 \mathrm{ppm}(\mathrm{ddt}, J=15.3,7.1,1.5 \mathrm{~Hz})$ indicate an $(E)$-configuration. This hints already at the exo-product, since the endo-product needs a $(Z)$-configured double bond because of the ring geometry. Furthermore, in COSY it was visible that the double bond protons $\mathbf{a}$ and $\mathbf{b}$ couple to the carbinol proton $\mathbf{c}$ at $4.89 \mathrm{ppm}$, but also to the $\mathrm{CH}_{2}$ group $\mathbf{f}$. In the 6 -membered ring lactone 169, this would be the $\mathrm{CH}_{2}$ group next to the carbonyl moiety, which would have no further coupling partners. However, protons $\mathbf{f}$ couple to the alkyl chain (h and $\mathbf{i}$ ), revealing that indeed,

Table 3.20: Assignment of ${ }^{1} \mathrm{H}$ NMR signals of lactone 168 and COSY couplings.

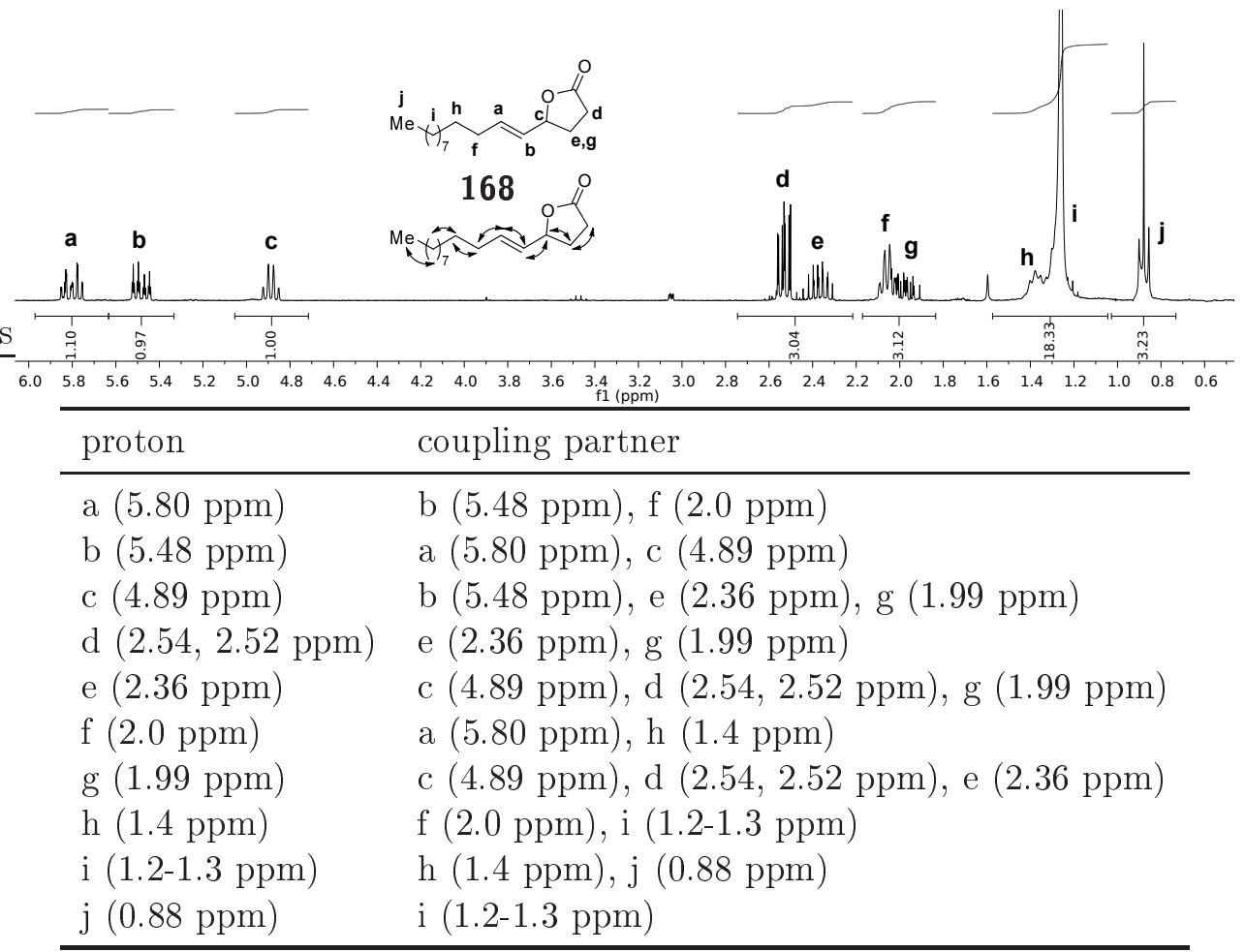


the 5-membered ring product $\mathbf{1 6 8}$ was formed. The signals $\mathbf{d}$, e and $\mathbf{g}$ at 2.54, 2.52, 2.36 and $1.99 \mathrm{ppm}$ with a combined integral of four were assigned to the methylene groups in the lactone ring, as $\mathbf{e}$ and $\mathbf{g}$ both couple to carbinol $\mathbf{c}$. The multiplets $\mathbf{h}$ and $\mathbf{i}$ at $1.49-1.15 \mathrm{ppm}$ belong to the alkyl chain and the triplet at $0.88 \mathrm{ppm}(\mathbf{j})$ to the methyl group.

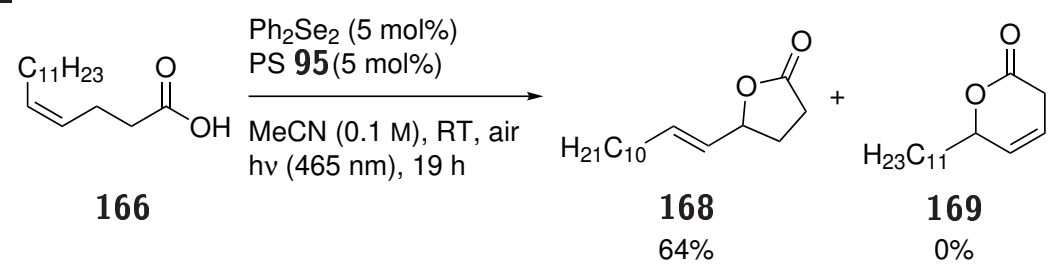

The lactonization of $\delta, \varepsilon$-unsaturated acids 137 under the same conditions led to the formation of six-membered ring lactones with an exocyclic double bond Table 3.21, grayed products were synthesized by L. Löffler, Dr. S. Ortgies and R. Rieger).[137.144.148,149] This was visible from similar aspects of the ${ }^{1} \mathrm{H}$ NMR spectrum as for lactone 168. The protons at the double bond show two characteristic multiplets at around 5.5 and $5.7 \mathrm{ppm}$ that couple with each other with a coupling constant of approx. $15 \mathrm{~Hz}$. The proton in the carbinol position can be found in the spectra as a doublet of doublets of doublets of doublets at around $4.7 \mathrm{ppm}$. It couples with the

Table 3.21: Lactonization of acids 137. Grayed products synthesized by L. Löffler, Dr. S. Ortgies and R. Rieger. $.137,144,148,149]$
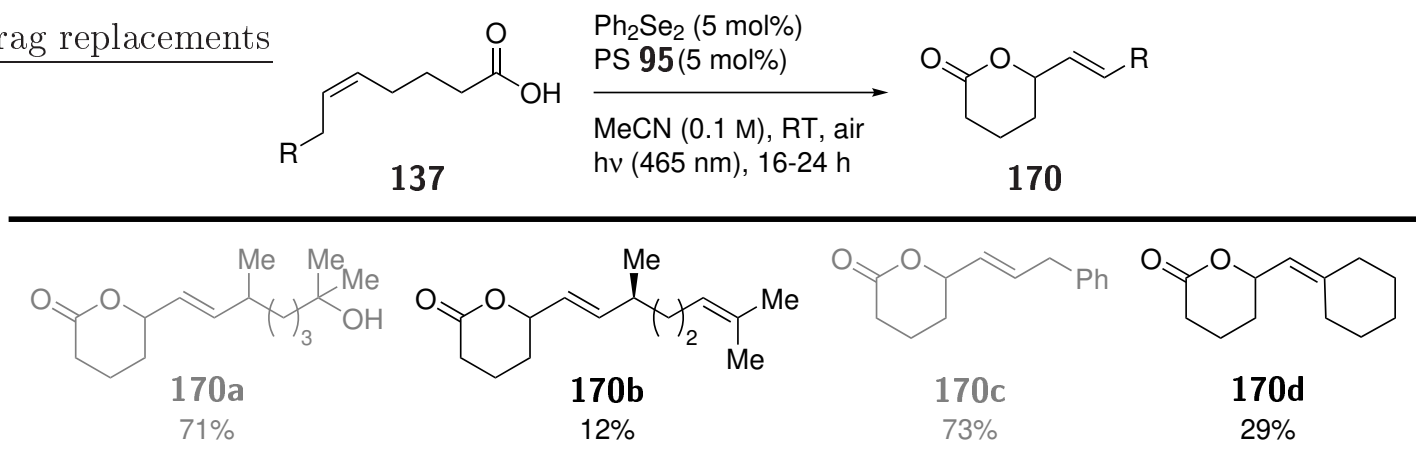<smiles>CC(C)C=CC1CCCC(=O)O1</smiles>

$170 \mathrm{e}$ $69 \%$

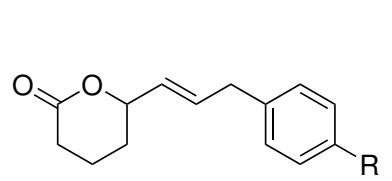<smiles>CC(C)(C)OC1CCC(C=CC2CCC(=O)O2)CC1</smiles>

$170 f$ $78 \%$<smiles>CC(C)=CC1CCCC(=O)O1</smiles>

$170 \mathrm{~g}$ $33 \%$ $R=t B u \quad 170 i \quad 74 \%$ $\mathrm{R}=\mathrm{CF}_{3} \quad \mathbf{1 7 0 j} \quad 73 \%$ $R=F \quad 170 k \quad 83 \%$$$
\mathrm{R}=\mathrm{CN} \quad \mathbf{1 7 0 |} 81 \%
$$$$
\mathrm{R}=\mathrm{OMe} \quad 170 \mathrm{~m} \quad \text {-a }
$$<smiles>O=C1CCCC(/C=C/CNc2ccccc2)O1</smiles>

170h

$57 \%$<smiles>O=C1CCCC(C=C2CC2)O1</smiles>

170n

\footnotetext{
${ }^{a}$ Decomposition of the starting material.
} 
two protons at the double bond and the two protons in the adjacent $\mathrm{CH}_{2}$-group. The signals of the $\mathrm{CH}_{2}$ groups in the lactone ring are multiplets between 1.5 and $2.7 \mathrm{ppm}$. The reactions proceeded with yields between 12 and $81 \%$. The functional group tolerance is comparable to that observed in the formation of tetrahydropyrans (cf. Table 3.10 in Subsection 3.1.2). Lactones 170a and 170e were obtained in good yields of 71 and $69 \%$. The formation of lactone 170b proceeded only with a low yield of $12 \%$, probably due to side reactions of the additional double bond. Similarly, the formation of lactones with trisubstituted double bonds was impaired and products $\mathbf{1 7 0 d}$ and $\mathbf{1 7 0 g}$ were formed in 29 and $33 \%$ yield, while the cyclopropyl-substituted acid 137s did not react at all. Aryl moieties were well tolerated and products $\mathbf{1 7 0 c}, \mathbf{h}-\mathbf{I}$ were obtained in very good yields of 73-81\%, an exception being methoxy-substituted lactone $\mathbf{1 7 0 m}$, which decomposed under the applied conditions. In contrast to the intramolecular etherification, piperidine derivative $\mathbf{1 3 7}$ g reacted in a high yield of $78 \%$ to the respective lactone $\mathbf{1 7 0 f}$.

As the formation of 5- and 6-membered ring lactones proceeded smoothly under selenium/photoredox catalysis, the possible reaction of petroselinic acid (167) in a 7-exo-trig-cyclization was examined. Indeed, the subjection of the acid to the established conditions led to the formation of 7 -membered ring lactone 171, albeit in 5\% yield (Equation 3.16). The configuration of the double bond was determined to be $(E)$ by the coupling constant of $15.4 \mathrm{~Hz}$ of the two protons at 5.74 and $5.53 \mathrm{ppm}$, which suggested again an exocyclic double bond. The signal of the carbinol proton, a doublet of doublets at $4.70 \mathrm{ppm}$, has a similar shift as in the 5- and 6-membered ring lactones.

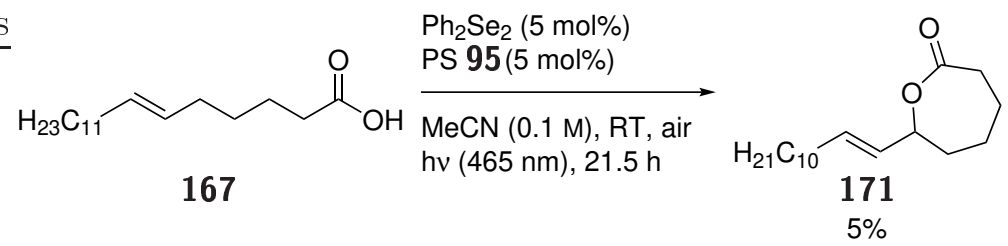




\subsection{Photo-aerobic selenium- $\pi$-acid catalysed phosphatation of alkenes}

After the successful use of acids and alcohols as exogenous nucleophiles in selenium- $\pi$-acidcatalyzed reactions, the question came up if other oxygen nucleophiles could be applied as well. Phosphoric acids came to mind, as their acidity is comparable to that of carboxylic acids, which suggests the feasibility of the reaction. $\frac{[150]}{2}$

Phosphate esters have several biological, synthetical and industrial applications, e.g. they are found in the DNA, as phospholipids in cell membranes and in the ATP-based energy transfer of organisms, they serve as chiral brønsted acid catalysts and substrates in cross-coupling reactions, and they are used in flame retardants and plastisizers. $\underline{[151-155]}$ Previous methods for their synthesis needed prefunctionalized or preactivated reagents, such as alcohols or phosphoric acid chlorides, and the synthesis via selenium- $\pi$-acid-catalysis was unprecedented at the outset of this project. $[156,157]$

Therefore, the direct phosphatation of alkenes with phosphoric acids under photo-aerobic selenium$\pi$-acid catalysis was investigated by our group. C. Depken and R. Rieger were able to optimize conditions for the functionalization of trans-dec-5-ene (150) with dibutyl phosphate (172). The use of the alkene in an excess of 3.0 equiv, 10 mol\% of diphenyl diselenide and $p$-MeO-TPT (95), respectively, $4 \AA \mathrm{MS}$ and 0.8 equiv $\mathrm{Na}_{2} \mathrm{HPO}_{4}$ in DCE under irradiation at $465 \mathrm{~nm}$ and an oxygen atmosphere led to the formation of phosphate 173a in $89 \%$ yield [Equation 3.17). $142,158,159]$ When MeCN was used as the solvent, the phosphate was still obtained in a good yield of $81 \%$, other solvents led to low or moderate yields. With excess of the phosphate or the base, lower yields were achieved. The reaction only proceeded with $\mathrm{Na}_{2} \mathrm{HPO}_{4}, \mathrm{NaHCO}_{3}$ or $\mathrm{KF}$, other carbonate or fluoride bases did not lead to product formation.

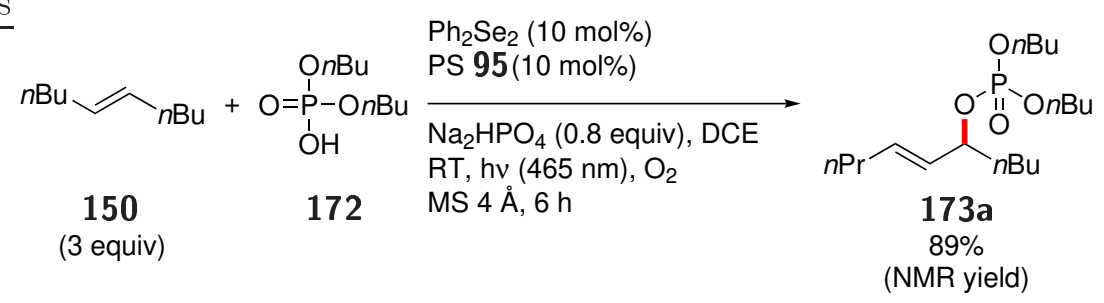

The reaction of trans-dec-5-ene (150) with different phosphates was examined by C. Depken and F. Krätzschmar. $\stackrel{158-160]}{-1 n}$ order to evaluate the reactivity of different alkenes, a series of symmetric olefins was to be synthesized. For this purpose, oct-4-ene-1,8-diol (126m) was functionalized with different groups. First, diesters 174a-e were synthesized in yields between 89 and $>99 \%$ by performing an esterification of diol $\mathbf{1 2 6} \mathbf{m}$ with 2.2 equiv of benzoic acid chlorides and 2.2 equiv DMAP (Table 3.22). Additionally, the two hydroxy groups of diol $\mathbf{1 2 6}$ were transformed into silyl ether or carbonate groups to obtain alkenes $\mathbf{1 7 4 f}$ and $\mathbf{1 7 4 g}$. The reactions with TBSCl (2.2 equiv) or ethyl chloroformiate (2.0 equiv) afforded the products in 93 and $76 \%$ yield, respectively (Equation 3.18). [161] 
Table 3.22: Esterification of diol $126 \mathrm{~m}$.
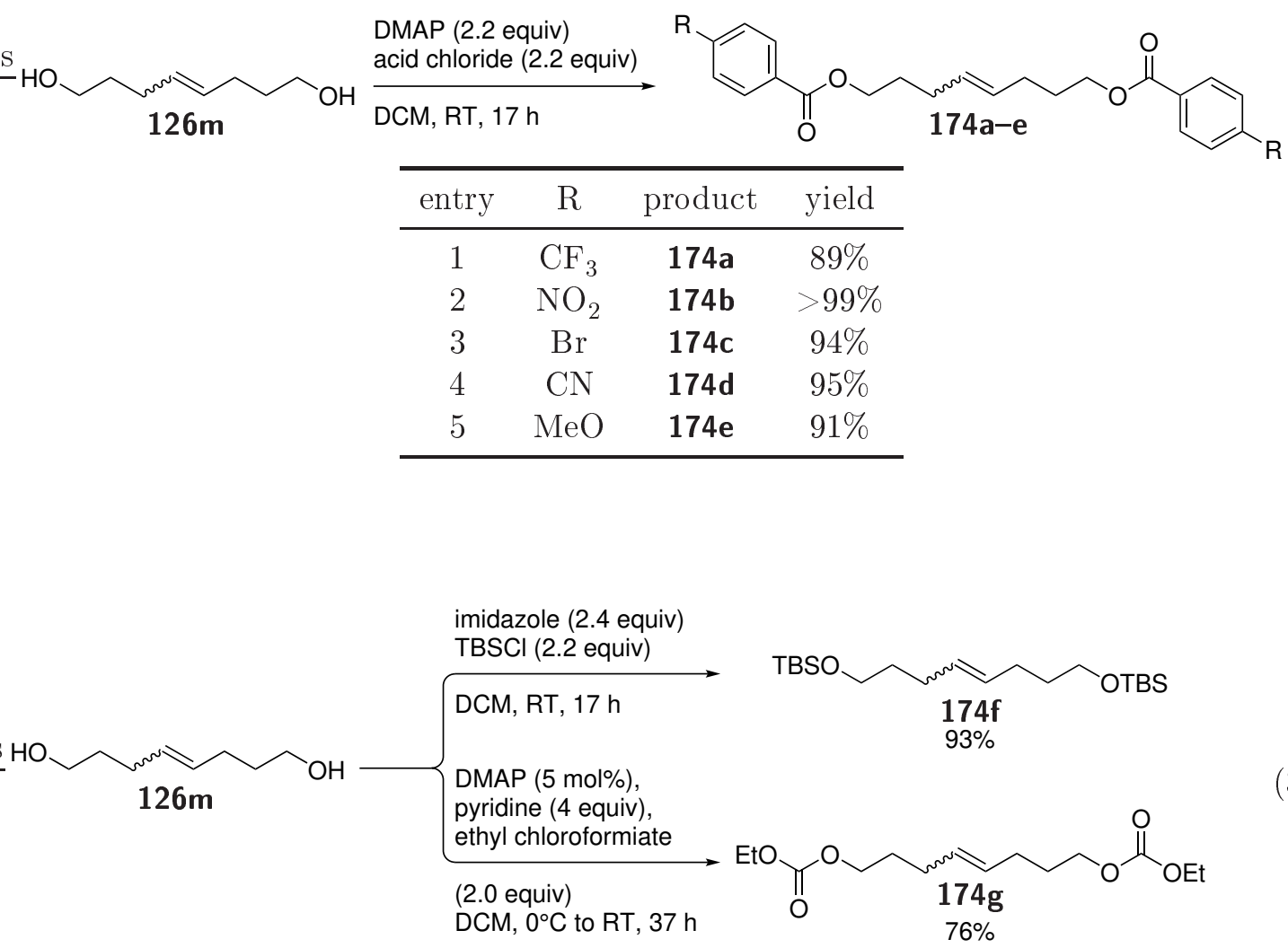

As further substrates, a number of unsymmetric alkenes were to be synthesized. For this purpose, three alcohols and one acid were functionalized in different ways. The pivalic acid esters of 4hexene-1-ol (175) and geraniol (176) were formed with 1.1 equiv DMAP and 1.1 equiv pivalic acid chloride in $75 \%$ and $80 \%$ yield (Equation 3.19 ).

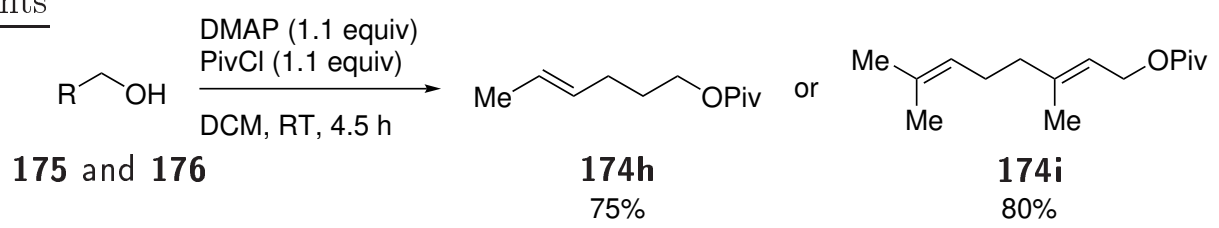

In order to examine the reactivity of an aldehyde in the selenium-catalyzed phosphatation reaction, $(Z)$-dec-4-en-1-ol (126a) was oxidized to aldehyde 174j with 2.0 equiv DMP in $75 \%$ yield Equation 3.20. Finally, the methyl ester of $(Z)-15$-tetracosenoic acid (177) was afforded in a reaction in $\mathrm{MeOH}$ with 5 mol\% PTSA as a catalyst in $94 \%$ yield Equation 3.21.

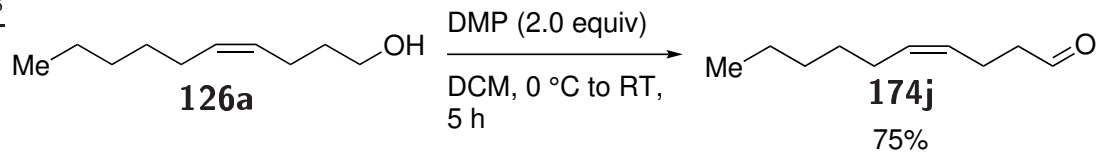




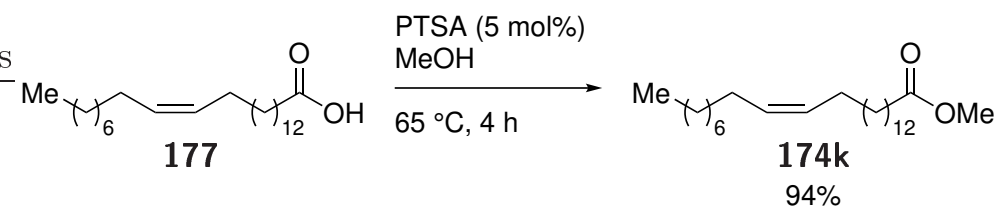

With the aforementioned substrates in hand, the phosphatation with dibutyl phosphate (172) was conducted under the established conditions using alkenes $\mathbf{1 7 4 a -} \mathbf{g}$ in an excess of 3.0 equiv, $10 \mathrm{~mol} \%$ of diphenyl diselenide and $p$-MeO-TPT, respectively, $4 \AA \mathrm{MS}$ and 0.8 equiv $\mathrm{Na}_{2} \mathrm{HPO}_{4}$ in DCE under irradiation at $465 \mathrm{~nm}$ and an oxygen atmosphere (Table 3.23 , reactions for products highlighted in gray were conducted by F. Krätzschmar and R. Rieger). $[142,159,160]$ In the reactions with the benzoic acid esters, only methoxylated product $\mathbf{1 7 3 \mathbf { f }}$ was isolated in $53 \%$, in the reactions of 174a-d, decomposition of the starting material was observed.142,159,160] The allylic phosphate was identified in ${ }^{31} \mathrm{P}$ NMR, where the phosphorus atom was shifted to higher field from $1.3 \mathrm{ppm}$ in dibutyl phosphate to $-1.42 \mathrm{ppm}$ in the product. In the ${ }^{1} \mathrm{H}$ NMR spectrum, the double bond protons were shifted to lower field, compared to alkene $\mathbf{1 7 4 e}$, and appeared as a doublet of doublets of doublets at $5.84 \mathrm{ppm}$ and a doublet of doublets of triplets at $5.63 \mathrm{ppm}$. The signal of the carbinol proton was a quintet at $4.80 \mathrm{ppm}$. The loss of symmetry of the product compared to the starting material was visible in the appearance of two singlets for the methoxy

Table 3.23: Phosphatation reactions of asymmetric alkenes $174 .^{\text {a }}$ Reactions for products in gray conducted by F. Krätzschmar and R. Rieger.
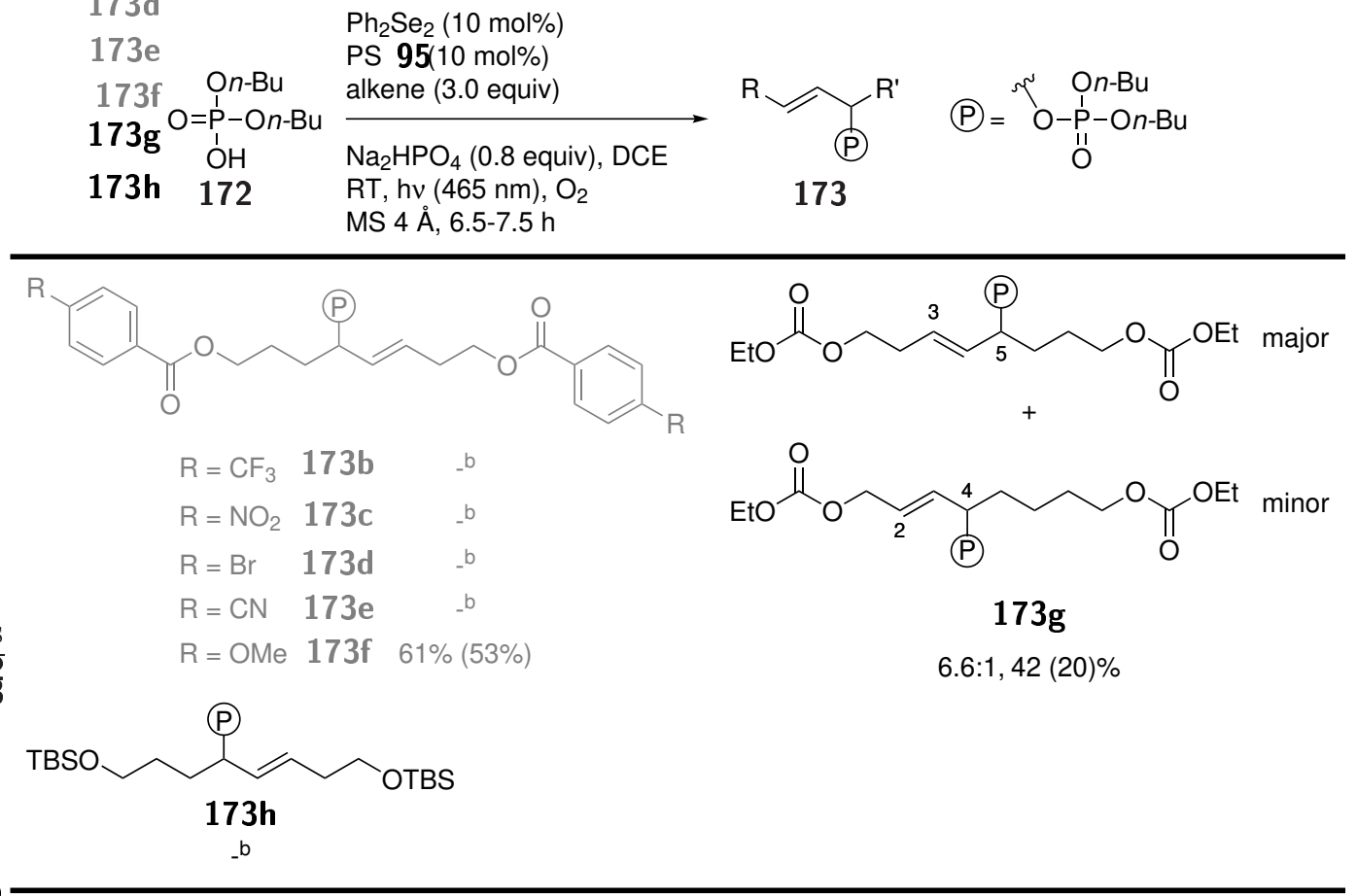

${ }^{\text {a }}$ NMR yields, standard: TMB or $\mathrm{PPh}_{3}$, (isolated yields). $\quad{ }^{\mathrm{b}}$ Decomposition. 
groups at 3.86 and $3.83 \mathrm{ppm}$ and two triplets for the $\mathrm{CH}_{2} \mathrm{O}$ groups next to the ester units at 4.33 and $4.25 \mathrm{ppm}$. In the reaction of disilyl ether $\mathbf{1 7 4 f}$, the crude ${ }^{31} \mathrm{P}$ NMR spectrum showed two signals at -1.49 and $-8.94 \mathrm{ppm}$. As the target products usually had a signal at around $-1.4 \mathrm{ppm}$, the signal at $-1.49 \mathrm{ppm}$ probably belonged to the expected product, the signal at $-8.49 \mathrm{ppm}$ probably belonged to a side product that resulted from an attack of the phophorus at silicon. This assumption was supported by literature data of a TMS phosphite, where the signal in the ${ }^{31} \mathrm{P}$ NMR was at $-7.70 \mathrm{ppm} .{ }^{162]}$ In the ${ }^{1} \mathrm{H}$ NMR spectrum, a complete shift of the signals of the TBS group from 0.90 and $0.05 \mathrm{ppm}$ to 0.84 and $0.00 \mathrm{ppm}$ was observed, indicating a complete consumption of the alkene by a side reaction. Unfortunately, none of the phosphate species was isolated due to problems in the chromatographic purification. A similar problem was observed in the intramolecular etherification when silylated alkene 126k was used and the alcohol decomposed under the used conditions. Other silyl compounds seemed to tolerate the photoconditions better, as the intramolecular etherification of triisopropylsilyl ether 126p afforded the product in 29\%. Following this observation, F. Krätzschmar achieved the phosphatation of a triisopropylsilyl ether in $71 \%$ yield. 159,160$]$ The reaction with carbonate $\mathbf{1 7 4} \mathrm{g}$ led to the formation of an inseparable mixture of two products $173 \mathrm{~g}$ with the ratio of 6.6:1 in $42 \%$ yield, which was isolated in $20 \%$ yield. When the products were examined closely, the major product was identified as the expected product with the double bond in 3-position and the phosphate moiety in 5-position. Surprisingly, the minor product had the double bond in 2 -position and the phosphate moiety in 4-position. The assignment of the structures was done by $1 \mathrm{D}$ and 2D-NMR spectroscopy and started from the signals of the carbinol protons at 4.73 (major product) and $4.83 \mathrm{ppm}$ (minor product), which were assigned because of their chemical shift and by $\mathrm{P}, \mathrm{H}-\mathrm{HMBC}$ couplings (Figure 3.7). The ${ }^{13} \mathrm{C}$ NMR signals of the carbinol carbons were assigned by HSQC to be at $78 \mathrm{ppm}$. Via HMBC, the coupling of the carbinol moiety to the double bond and the sequential coupling to $\mathrm{CH}_{2}$ groups was determined. For the major product, two methylene units were found to be between the double bond and the carbonate group. For the minor product, only one unit with a doublet at $4.62 \mathrm{ppm}$ connected the double bond with the carbonate moiety. The position of the double bond of the side product was explained by the possible impureness of the used alkene. During the synthesis, the double bond could have partly isomerized from the 4-position to the 3-position which would lead to the formation of the minor product.
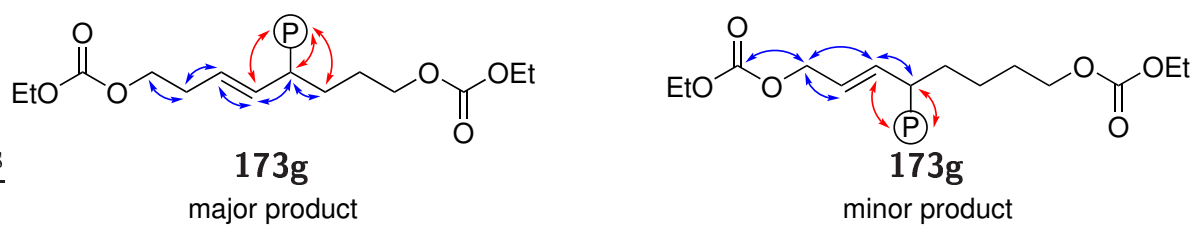

Figure 3.7: Selected H,C-HMBC (blue) and P,H-HMBC (red) couplings of product mixture 173g. 
During the phosphatation reactions of asymmetric alkenes $\mathbf{1 7 4 h}-\mathbf{k}$, product formation was observed only with hexenol derivative $\mathbf{1 7 4 h}$. The inseparable mixture (3.4:1) of allylic phosphates 173i was obtained in $74 \%$ yield. The major product was the terminal alkene, the minor product had an internal double bond. Their ratio was determined via the integrals of the double bond protons in ${ }^{1} \mathrm{H}$ NMR. The signals of the terminal alkene were a doublet of doublets of doublets at $5.82 \mathrm{ppm}(J=17.2,10.4,6.9 \mathrm{~Hz})$, a doublet of triplets at $5.32 \mathrm{ppm}(J=17.2,1.2 \mathrm{~Hz})$ and a doublet of triplets at $5.22 \mathrm{ppm}(J=10.4,1.1 \mathrm{~Hz})$ with couplings to each other and the carbinol proton at $4.77 \mathrm{ppm}$. The internal alkene had two double bond signals at $5.70 \mathrm{ppm}(\mathrm{dt}$, $J=15.5,6.5 \mathrm{~Hz})$ and $5.60 \mathrm{ppm}(\mathrm{ddt}, J=15.5,6.6,1.2 \mathrm{~Hz})$ that coupled with each other and the carbinol proton or the $\mathrm{CH}_{2}$ group next to the double bond. The signal of the carbinol proton was a sextet at $4.88 \mathrm{ppm}$. The high multiplicity could be explained by the coupling to one of the double bond protons, the three protons of the methyl group and the coupling to the phosphorus atom. With geraniol derivative 174i, only the starting material was observed after $8 \mathrm{~h}$. A possible reason could be the trisubstitution of the double bonds, which could hinder the formation of the seleniranium ion. The attempted phosphatation of aldehyde $\mathbf{1 7 4} \mathbf{j}$ did not occur, but the aldehyde was consumed in an unknown side reaction, which was visible by the disappearance of the aldehyde signal at $9.77 \mathrm{ppm}$ in NMR. The functionalization of fatty acid ester $\mathbf{1 7 4 \mathbf { k }}$ was observed in ${ }^{31} \mathrm{P}$ NMR by the appearance of two singlets at -1.44 and $-1.51 \mathrm{ppm}$ which were attributed to two formed isomers. In ${ }^{1} \mathrm{H}$ NMR, a doublet of triplets at $5.61 \mathrm{ppm}(J=14.1$, $6.6 \mathrm{~Hz})$ and a quintet at $4.57 \mathrm{ppm}(J=7.0 \mathrm{~Hz})$ indicated the formation of a product with an allylic functionalization. Unfortunately, only an allylic alcohol was isolated in $16 \%$ yield. For the pure compound, no signal appeared in ${ }^{31} \mathrm{P}$ NMR and in ${ }^{1} \mathrm{H}$ NMR, the signal of the carbinol proton was shifted to higher field and appeared as a quartet at $4.03 \mathrm{ppm}$, which is reminiscent of

Table 3.24: Phosphatation reactions of asymmetric alkenes $174{ }^{\mathrm{a}[159]}$
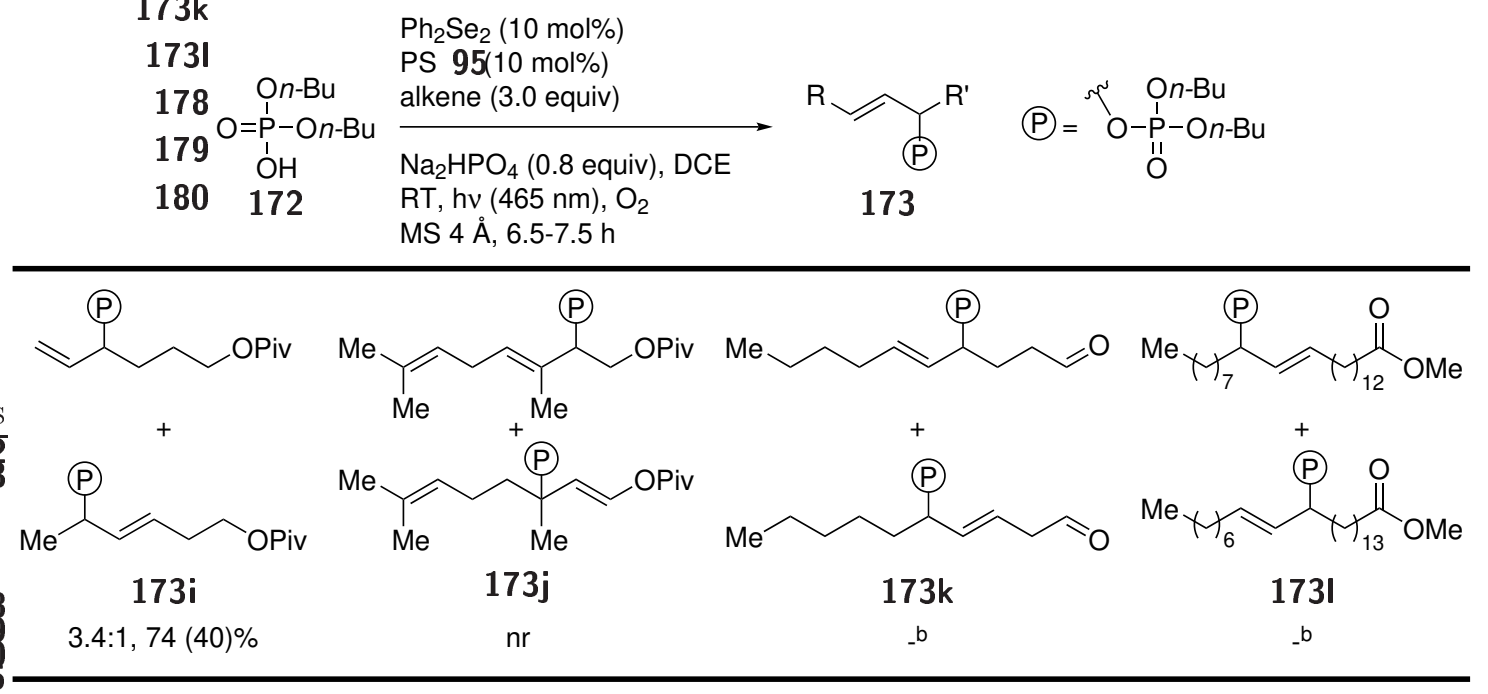

${ }^{\mathrm{a}}$ NMR yield, standard: TMB, (isolated yields). ${ }^{\mathrm{b}}$ Decomposition. 
the shift of the carbinol proton in allylic ethers 149, which was typically between 3.5 and $4.5 \mathrm{ppm}$. The change of the multiplicity could be explained by phosphorus as the coupling partner in the phosphate and therefore one less coupling partner in the alcohol. The signals of the double bond did not suffer from a shift and appeared at 5.62 and $5.44 \mathrm{ppm}$. The formation of the alcohol was further confirmed with ESI mass spectrometry. 


\subsection{Investigations toward the selenium-catalyzed amination of olefins}

\subsubsection{Intermolecular light-driven amination}

As the oxidative functionalization of alkenes with several exogenous oxygen nucleophiles was realized by our group using photo-aerobic selenium- $\pi$-acid catalysis, the application of this method in the functionalization of alkenes with exogenous nitrogen nucleophiles was examined. [91,134.144.159] In previous works, only the use of $N$-halogenated oxidants led to the formation of aminated products. However, the intermolecular nucleophiles in these reactions originated from the oxidant (with one exception; cf. Subsection 1.2.1). $63-67,69]$ Therefore, experiments on the light-driven amination of alkenes were conducted, using cis-cyclooctene (181) as a simple, unfunctionalized substrate. Saccharin (182) and phthalimide (183) were chosen as nucleophiles due to their easy availability and the potential transformation of the corresponding amidation products into amines Figure 3.8). Furthermore, dibenzenesulfonimide (184) was used because the imidation with the corresponding $\mathrm{N}$-fluorinated compound as an oxidant and nucleophile source worked well. $[\underline{63]}$

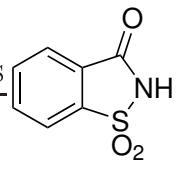

182<smiles>O=C1NC(=O)c2ccccc21</smiles>

183<smiles></smiles>

184

Figure 3.8: Nucleophiles for the amination of olefins.

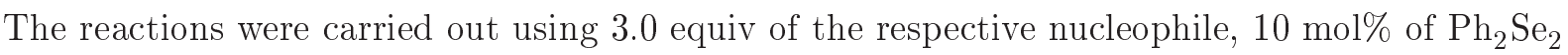
and $5 \mathrm{~mol} \%$ of photocatalyst 95 in acetonitrile- $d_{3}$ (Table 3.25). In order to increase the nucleophilicity of the used amines, 2.5 equiv of pyridine derivatives $\mathbf{1 8 6}$ were used as a base. Under these conditions, only saccharin showed reactivity and the presumed amination product was isolated in $42 \%$ yield with pyridine as the base, in 32 and $15 \%$ yield when 2,6-dimethylpyridine or 2,6-di-tert-butylpyridine were used (Table 3.25, entries 1-3).

Further analysis of the isolated product of the reactions with saccharin showed that not only one, but two products had been formed. In mass spectrometry, both products contained the signals of the mass of the desired product, which led to the conclusion that the products were constitutional isomers. The ${ }^{1} \mathrm{H}$ NMR spectra of both isomers had two signals for the double bond protons at approx. 6.0 and $5.8 \mathrm{ppm}$ and a signal with a shift for a proton in $\alpha$-position to a heteroatom. These $\alpha$-protons and the corresponding carbon atoms of the two products differed strongly in their chemical shift and the signals were at $5.21 \mathrm{ppm}\left({ }^{1} \mathrm{H}\right)$ and $52.2 \mathrm{ppm}\left({ }^{13} \mathrm{C}\right)$ for compound 185a and at $5.64 \mathrm{ppm}\left({ }^{1} \mathrm{H}\right)$ and $81.2 \mathrm{ppm}\left({ }^{13} \mathrm{C}\right)$ for compound 185b Figure 3.9). Therefore, it was concluded that in both products, cyclooctene had been substituted in the allylic position by saccharin, but one of the products was the desired amination product, whereas the other was the result of the nucleophilic attack with the oxygen of the carbonyl moiety. It was assumed that the 
Table 3.25: Amination of cyclooctene (181) with different nucleophiles and bases.

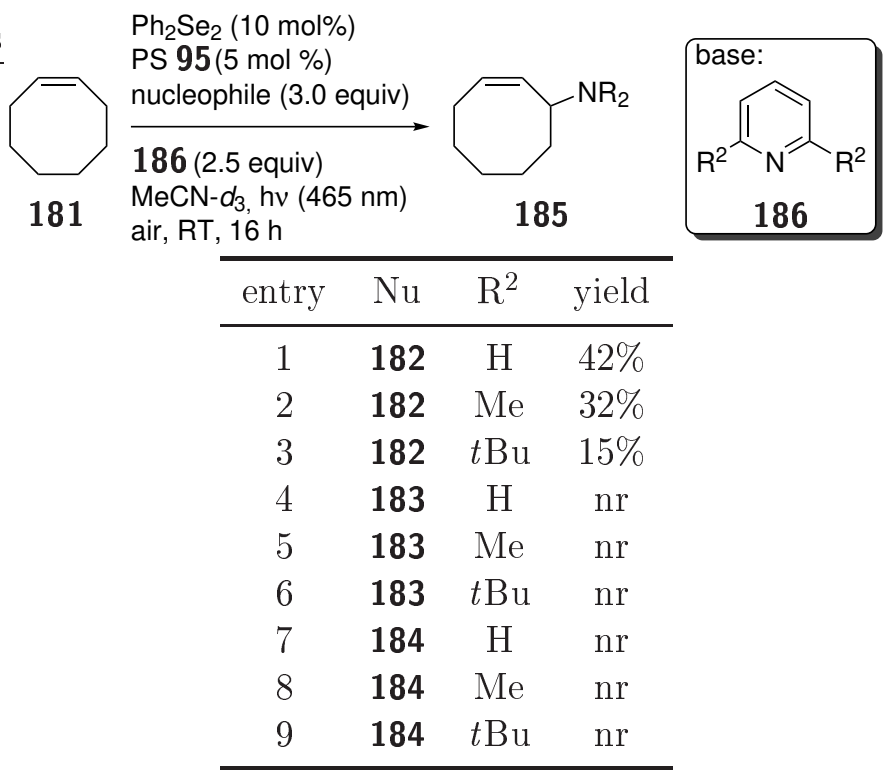

product 185a with the lower shift of the allylic proton was the desired amination product. To further support this hypothesis, an N,H-HMBC experiment was conducted for both products, which would show couplings between the nitrogen atom and protons over three bonds. In the nitrogen-substituted product, couplings are possible to one of the double bond protons and the $\mathrm{CH}_{2}$ group in the homoallylic position. In the oxygen-substituted molecule, the protons are too far away for a coupling. Indeed, the spectrum of alkene 185a showed a coupling between the nitrogen atom and the mentioned methylene group, whereas for alkene $\mathbf{1 8 5} \mathbf{b}$, no couplings were observed. The IR spectrum served as a final evidence. A strong signal at $1726 \mathrm{~cm}^{-1}$ for compound 185a indicated a $\mathrm{C}=\mathrm{O}$ bond, whereas for compound $\mathbf{1 8 5 b}$, only a weak signal at $1728 \mathrm{~cm}^{-1}$ was observed. Instead, a strong signal at $1611 \mathrm{~cm}^{-1}$ indicated an imine bond.

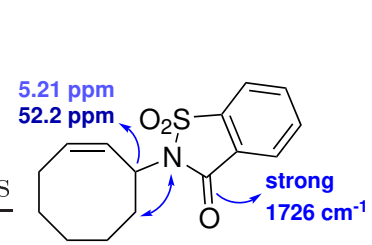

$185 a$

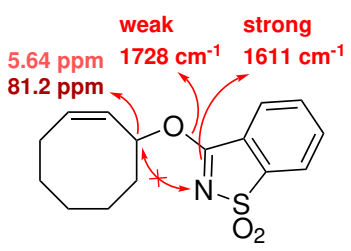

$185 b$

Figure 3.9: Spectroscopic data used for the assignment of the structures of compounds $\mathbf{1 8 5}$ a and $\mathbf{1 8 5 b}$.

As saccharin was the only nucleophile that showed reactivity in the first experiments, the influence of different bases on the yield and selectivity of this reaction was examined. With most carbonate bases, no reaction occurred and the fluorescence of the photocatalyst was quenched (Table 3.26, entries 2-4). Only with $\mathrm{NaHCO}_{3}$ products 185 were formed in $14 \%$ combined yield in a ratio of 1:1 (Table 3.26, entry 1). With fluoride bases $\mathrm{KF}, \mathrm{CsF}$ and $\mathrm{CaF}_{2}$, the products were obtained 
in 5, 19 and 43\% combined yield (Table 3.26, entries 5-7). While with KF and CsF, more of the oxygen-substitution product $\mathbf{1 8 5 b}$ was formed, the ratio was $1: 1$ with $\mathrm{CaF}_{2}$. Except for the reaction with $\mathrm{CaF}_{2}$, the extinction of the fluorescence was observed. This observation was also made in similar experiments and was explained with the probable nucleophilic attack of fluoride ions on the photocatalyst 95.127] With phosphate bases, combined yields between 23 and 59\% were obtained (Table 3.26, entries 8-11). Only with $\mathrm{K}_{3} \mathrm{PO}_{4}$, no product formation was observed and the fluorescence was quenched (Table 3.26, entry 12). The product ratios were all approx. 1:1, the highest yield was obtained with $\mathrm{KH}_{2} \mathrm{PO}_{4}$ as the base.

Table 3.26: Amination of cyclooctene (181) with saccharin and different bases.

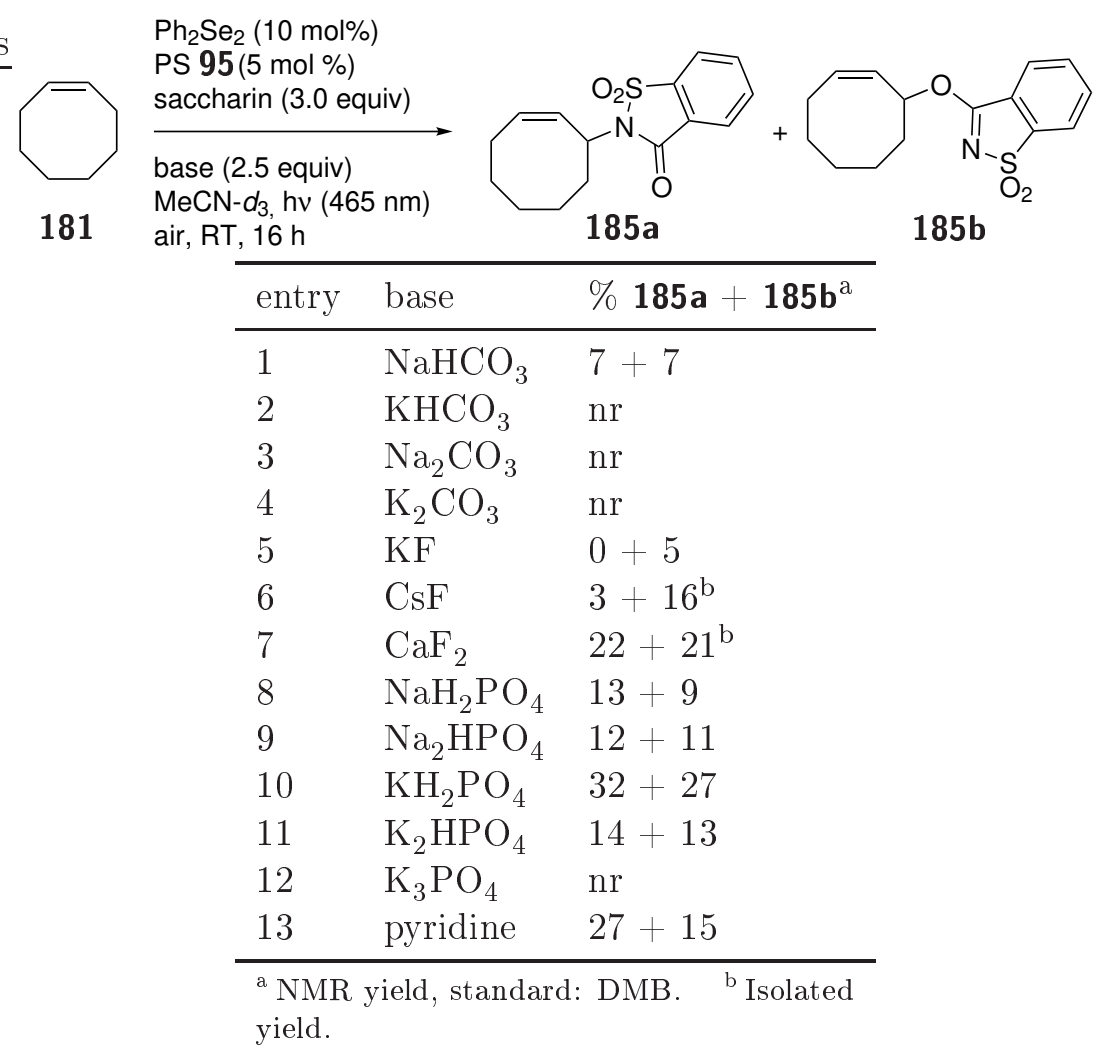

Because the formation of the oxygen-linked side product $\mathbf{1 8 5 b}$ could not be avoided during the reactions with saccharin, more experiments were conducted with other nucleophiles. For this purpose, sulfonamide derivatives were synthesized. Tosylanilide (188) was synthesized according to literature in quantitative yield from aniline using 1.2 equiv $p$-toluenesulfonylchoride and 1.5 equiv $\mathrm{NEt}_{3}$ (Equation 3.22). $\stackrel{[163]}{ }$ In order to increase the nucleophilicity of the nitrogen, sulfonamide 190 with an additional tosyloxy moiety was synthesized from hydroxylamine hydrochloride (189) in $18 \%$ yield using $p$-toluenesulfonylchoride (2.1 equiv) and DMAP (0.1 equiv) Equation 3.23. 

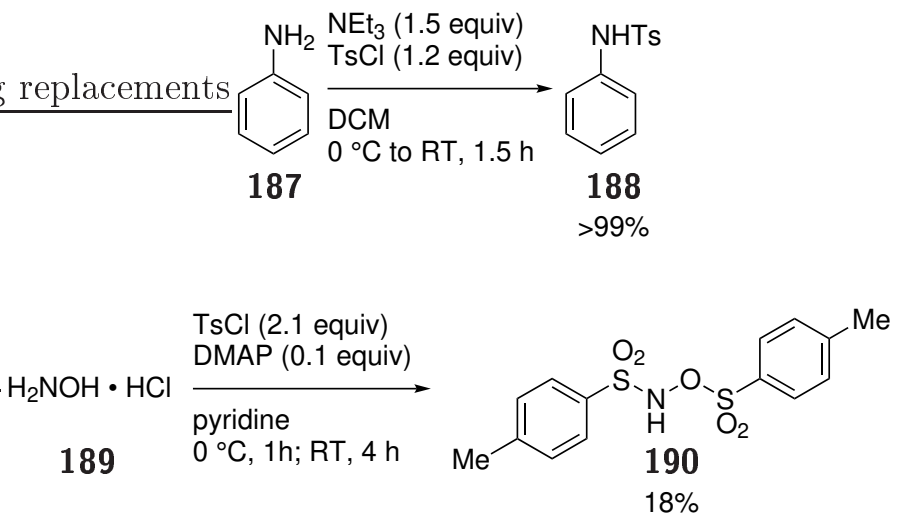

As the use of $\mathrm{KH}_{2} \mathrm{PO}_{4}$ was beneficial in the reaction with saccharin, it was used as the base in the following experiments. Under the altered conditions, the use of phthalimide $\mathbf{1 8 3}$ and electronpoor chloro- and nitro-substituted phthalimide derivatives did not lead to product formation (Table 3.27, entries 1-4). With dibenzenesulfonimide (184), traces of the desired product were obtained and identified by the ${ }^{1} \mathrm{H}$ NMR signals of the double bond and the proton in $\alpha$ position to the nitrogen at $5.98 \mathrm{ppm}(\mathrm{dd}, J=10.6,8.5 \mathrm{~Hz}), 5.62 \mathrm{ppm}(\mathrm{dt}, J=10.2,8.8 \mathrm{~Hz})$ and $5.11 \mathrm{ppm}$ (ddd, $J=12.1,8.5,3.9 \mathrm{~Hz}$ ), respectively (Table 3.27, entry 5). In order to make the reagent more nucleophilic, the sodium salt of dibenzenesulfonimide was formed and used in the reaction without an additional base (Table 3.27, entry 6).165] Unfortunately, the reactivity of the salt was comparable to the amine and the product was obtained in $3 \%$ yield. As a similar

Table 3.27: Attempted amination of cyclooctene (181) with different nucleophiles.

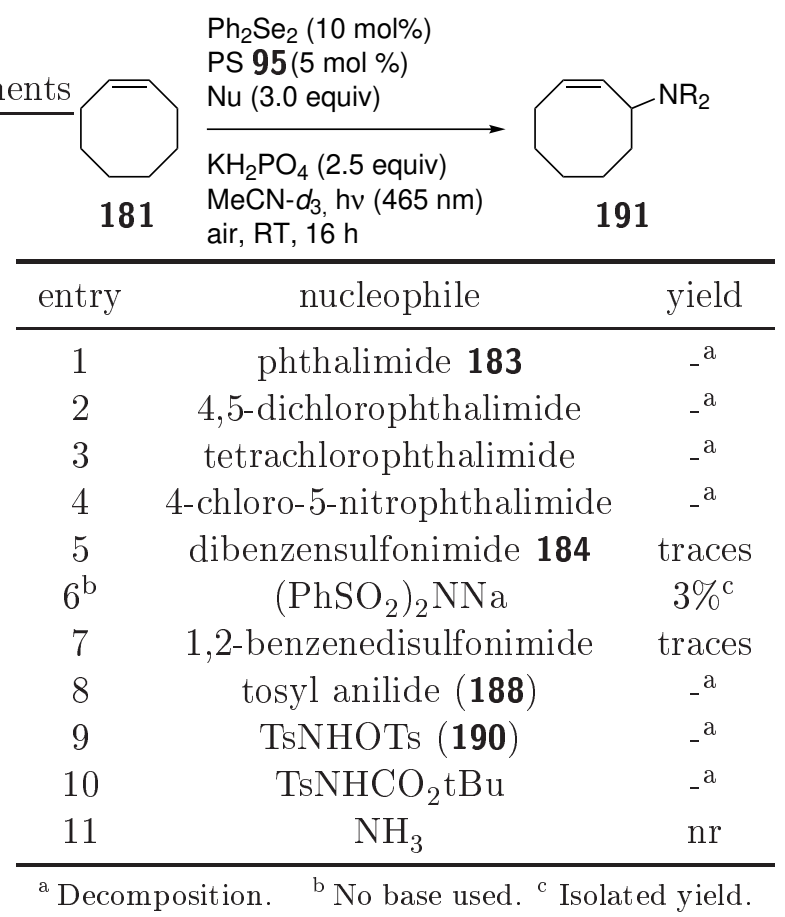


nucleophile, 1,2-benzenedisulfonimide was used and indeed, product formation was observed in mass spectrometry (Table 3.27, entry 7). In ${ }^{1} \mathrm{H}$ NMR, a doublet of doublets of doublets at $5.14 \mathrm{ppm}$ with coupling constants 12.4, 8.3 and $5.2 \mathrm{~Hz}$ (comparable to the signal at $5.21 \mathrm{ppm}$ in the spectrum of amine 185a) and two multiplets at 6.04 and $5.89 \mathrm{ppm}$ (presumably the protons at the double bond) hinted at product formation. Unfortunately, the assumed product could not be isolated and the formation was not quantified. When tosylanilide (188) and sulfonamide 190 were used, only decomposition of the starting material was observed (Table 3.27, entries 8 and 9). Inspired by the type of nucleophile used by WHITE et al., tert-butyl tosylcarbamate was applied in the reaction, but also did not lead to product formation (Table 3.27, entry 10). When simple ammonia was used for the direct formation of an allylic amine, the decomposition of the photocatalyst was observed and no reaction occurred (Table 3.27, entry 11).

In order to further examine the use of ammonia, but avoid the reaction with the photocatalyst, sulfamic acid was used. This reagent was first used by CARREIRA et al. as an ammonia equivalent in the iridium-catalyzed formation of allylic amines from allylic alcohols. ${ }^{[167]}$ When sulfamic acid was used without an added base, only the decomposition of the starting material was observed (Table 3.28, entry 1). The same observation was made with $\mathrm{KH}_{2} \mathrm{PO}_{4}, \mathrm{CaF}_{2}$ and pyridine as base additives (Table 3.28, entries 2-4). In the reaction of CARREIRA, DMF as the solvent was needed to form an adduct with sulfamic acid and liberate ammonia. Unfortunately, when 5.0 equiv of DMF were added to the reaction, in one case along with $\mathrm{KH}_{2} \mathrm{PO}_{4}$ as the base, no reaction was observed (Table 3.28, entries 5 and 6).

Table 3.28: Attempted functionalization of cyclooctene (181) with sulfamic acid.

\begin{tabular}{|c|c|c|}
\hline & $\begin{array}{l}\mathrm{Ph}_{2} \mathrm{Se}_{2}(10 \mathrm{~mol} \%) \\
\mathrm{PS} 95(5 \mathrm{~mol} \%) \\
\text { sulfamic acid (3.0 equiv) } \\
\begin{array}{l}\text { base }(2.5 \text { equiv }) \\
\text { MeCN }-d_{3}, \mathrm{hv}(465 \mathrm{~nm}) \\
\text { air, } \mathrm{RT}, 16 \mathrm{~h}\end{array}\end{array}$ & $\mathrm{NR}_{2}$ \\
\hline entry & base/additive & observation \\
\hline 1 & - & decomposition \\
\hline 2 & $\mathrm{KH}_{2} \mathrm{PO}_{4}$ & decomposition \\
\hline 3 & $\mathrm{CaF}_{2}$ & decomposition \\
\hline 4 & pyridine & decomposition \\
\hline 5 & $\mathrm{KH}_{2} \mathrm{PO}_{4} ; \mathrm{DMF}$ (5.0 equiv) & $\mathrm{nr}$ \\
\hline 6 & DMF (5.0 equiv) & $\mathrm{nr}$ \\
\hline
\end{tabular}

\subsubsection{Intramolecular light-driven amination}

As the intermolecular light-driven amination reaction proved to be challenging due to low reactivity of the used nucleophiles or decomposition of the starting material under photoconditions, the use of photo-aerobic selenium- $\pi$-acid catalysis in the intramolecular amination of alkenes was 
investigated. Our group has already reported the intramolecular amination of alkenes for the formation of (aza-)indoles with NFSI as the terminal oxidant. ${ }^{[65,127]}$ Therefore, tosyl amid 192] was subjected to photoconditions using diphenyl diselenide (10 mol\%) and p-MeO-TPT (10 mol\%) in $\mathrm{MeCN}$ under air and irradiation with blue light (Equation 3.24). Gratifyingly, indole 193 was formed in $17 \%$ yield.

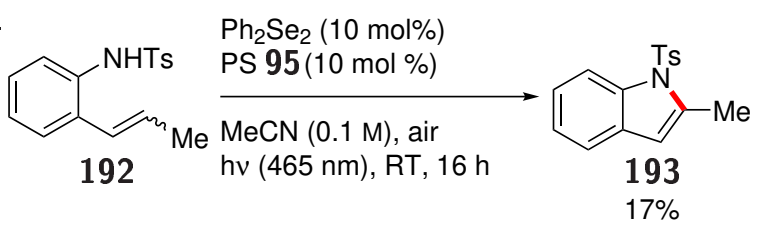

Motivated by these findings, the formation of tetrahydroisoquinolines 194 under photo-aerobic selenium- $\pi$-acid catalysis was examined. Conventional methods for the synthesis of this class of natural products are the reduction of isoquinolines 195, the nucleophilic substitution of a halide by nitrogen (compound 196) and the PICTET-Spengler reaction (Scheme 3.3). [168-171] Furthermore, it was shown by TIETZE et al. that the HECK reaction can be used for the formation of diastereo-enriched tetrahydroisoquinolines from halides 198. 1172.173]

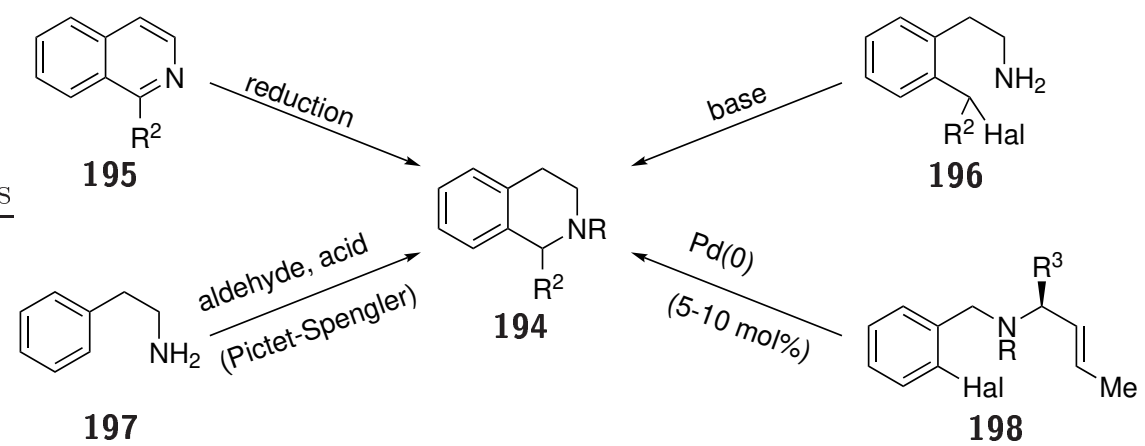

Scheme 3.3: Previous methods for the synthesis of tetrahydroisoquinolines (194).

In order to make the formation of tetrahydroisoquinolines more efficient, catalytic methods for the direct allylic $\mathrm{C}-\mathrm{H}$ amination were developed by the groups of KAWAI, FERINGA, Eustache and Yамамото. The reactions proceeded with bismuth, iridium or palladium catalysts in high yields between 78 and $87 \%$ (Scheme 3.4). 174-177) With bismuth, the reaction proceeded stereospecifically due to the 1,3-chirality transfer in the reaction of alcohol 199. The use of an iridium catalyst and a chiral ligand in the stereoselective reaction of carbonate $\mathbf{2 0 0}$ yielded the desired product in $94 \%$ ee.

As the presented reactions all proceed via an $\mathrm{S}_{\mathrm{N}} 2^{\prime}$ reaction and thus need prefunctionalized starting materials with a leaving group in the allylic position, selenium-catalysis could be a useful alternative to improve the redox economy in these regimes. ${ }^{[178]}$ This suggestion is supported by

\footnotetext{
${ }^{1}$ Tosyl amid 192 was synthesized by Dr. S. Ortgies. ${ }^{[127]}$
} 


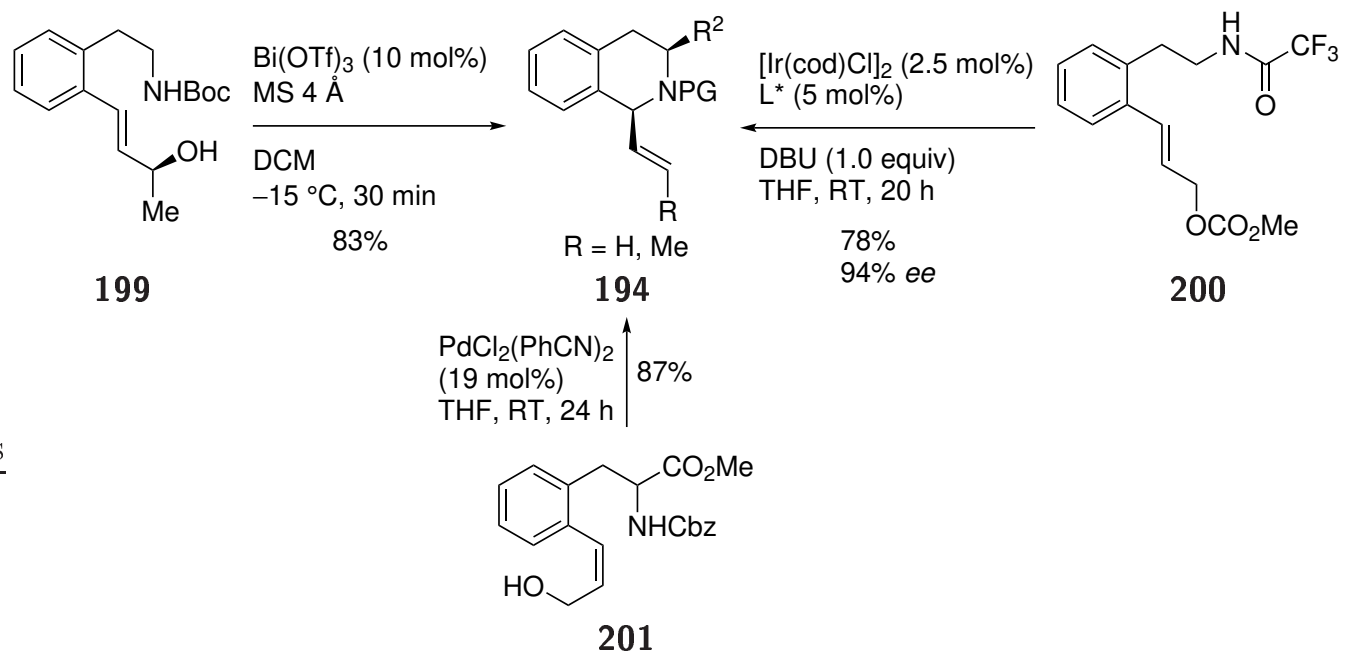

Scheme 3.4: Synthesis of tetrahydroisoquinolines (194) via direct allylic $\mathrm{C}-\mathrm{H}$ amination.

the work of CLIVE and coworkers who have reported the formation of selenated tetrahydroisoquinoline $\mathbf{2 0 3}$ from compound 202 in $87 \%$ yield [Equation 3.25). 179

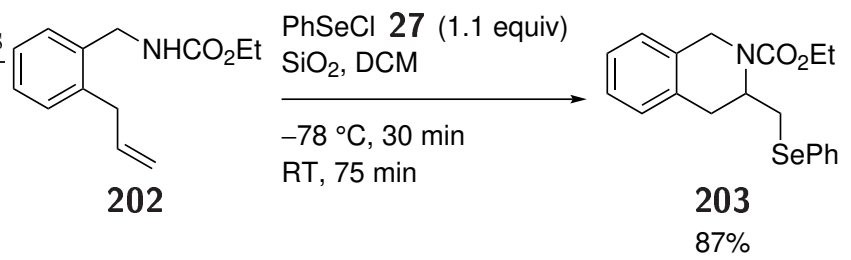

For the experiments on the photo-aerobic selenium- $\pi$-acid catalyzed formation of tetrahydroisoquinolines, tosyl amide 207a was synthesized in three steps starting from bromide 204a (Scheme 3.5). The SuzUKI coupling with 1-pentenylboronic acid (1.1 equiv) afforded nitrile

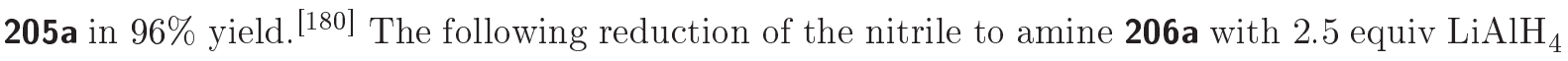
proceeded with quantitative yield and the product was then .transformed into tosyl amide 207a with 1.05 equiv $\mathrm{TsCl}$ and 1.1 equiv $\mathrm{NEt}_{3}$ in $35 \%$ yield, following a procedure by ZHou et al.181]

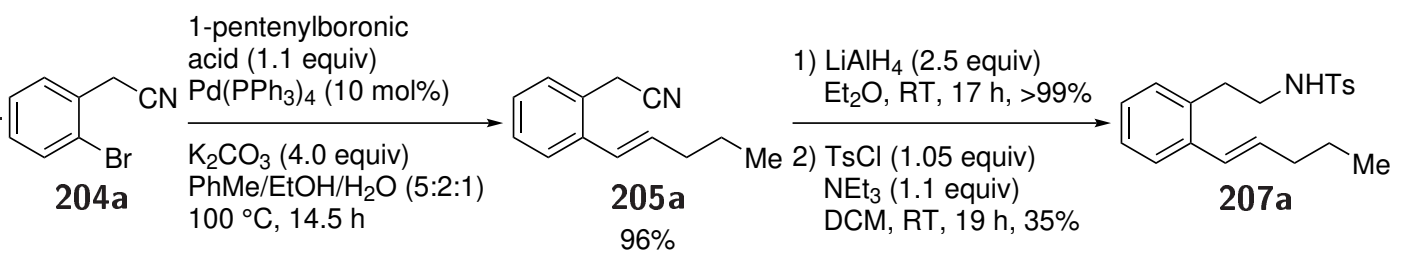

Scheme 3.5: Synthesis of tosyl amide 207a. 180,181$]$

With the starting material in hand, the cyclization was attempted using $10 \mathrm{~mol} \%$ diphenyl diselenide, 5 mol\% $p$-MeO-TPT in MeCN under air and irradiation with blue light Equation 3.26). Gratifyingly, tetrahydroisoquinoline $\mathbf{2 0 8}$ was obtained in 16\% yield. A first hint for the desired 
product formation was given by the HR-ESI mass spectrum and the IR spectrum, where the $\mathrm{N}-\mathrm{H}$ vibration at $3271 \mathrm{~cm}^{-1}$ disappeared. The structure of the product was verified by NMR spectroscopy. In $\mathrm{CDCl}_{3}$, the ${ }^{1} \mathrm{H}$ NMR signals of the double bond and another proton overlapped between 5.37-5.60 ppm and did not allow further analysis of the coupling constants, but the agreement of the data with data of similar structures supported the suggested structure.174] For further analysis, $1 \mathrm{D}$ and 2D NMR spectra were measured in benzene- $d_{6}$, where the signal separated into a multiplet between 5.47 and $5.45 \mathrm{ppm}$ for the vinylic protons and a doublet at $5.75 \mathrm{ppm}$, most likely the signal of proton $\mathbf{d}$ at the stereogenic center Figure 3.10). Starting from the triplet of the methyl group $\mathbf{m}$ at $0.72 \mathrm{ppm}$, the structure of the side chain was determined by the COSY couplings and it was confirmed that proton $\mathbf{d}$ was vicinal to the double bond. More evidence for the formation of tetrahydroisoquinoline $\mathbf{2 0 8}$ was supplied by the signals of the methylene groups adjacent to the nitrogen atom. Their splitting into two signals for each $\mathrm{CH}_{2}$ moiety and their chemical shifts of $3.85,3.13,2.57$ and $2.14 \mathrm{ppm}$ together with high coupling constants of 13 or $17 \mathrm{~Hz}$ for the geminal couplings and up to $11 \mathrm{~Hz}$ for the vicinal couplings suggested the formation of a cyclic structure containing a stereocenter.
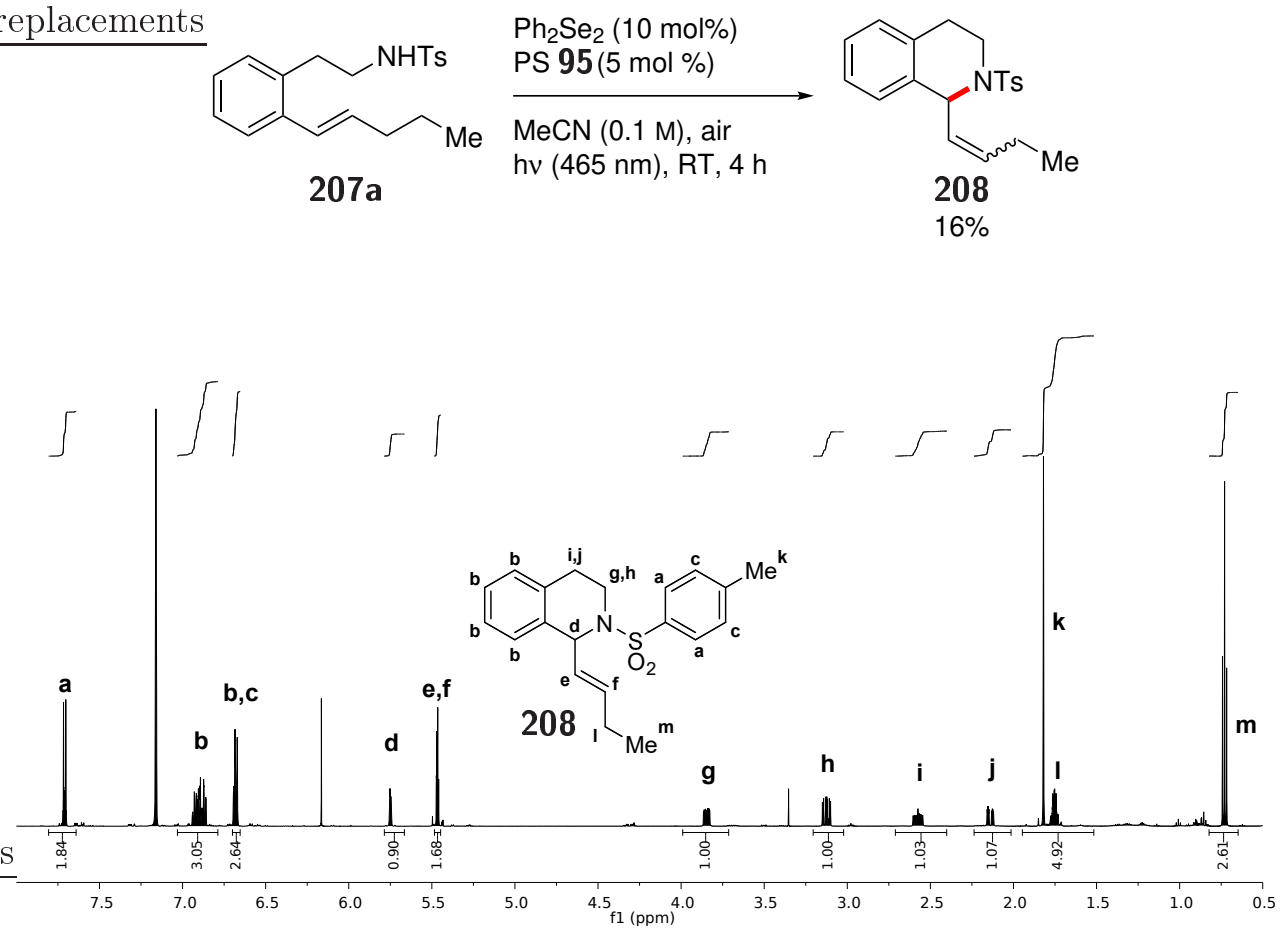

Figure 3.10: Assignment of the ${ }^{1} \mathrm{H}$ NMR signals of tetrahydroisoquinoline 208.

In order to improve the yield of the reaction, the influence of different solvents was examined. When acetone was used instead of acetonitrile, the product was formed in $11 \%$ yield Table 3.29, entry 2). Ethereal solvents, like THF and 1,4-dioxane, led to yields of 11 and 7\%, respectively (Table 3.29, entries 3 and 4). With nitromethane, product 208 was obtained in $9 \%$ vield (Table 3.29 , entry 5). The use of both DCE and toluene resulted in only trace amounts of the 
product Table 3.29, entries 6 and 7). As the yield of the first experiment in MeCN, 16\%, could not be improved, the following experiments were also conducted in this solvent.

Table 3.29: Optimization of used solvents in the intramolecular amination.

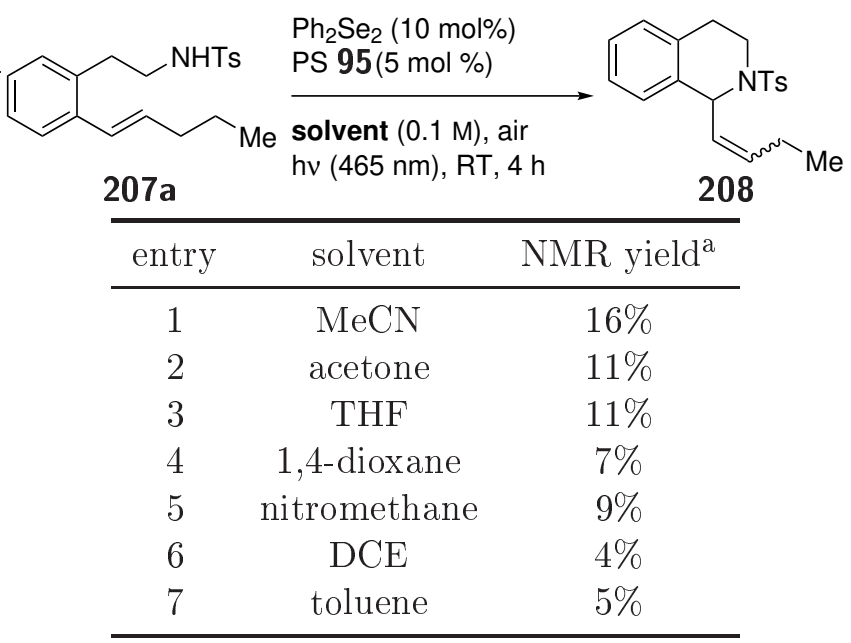

${ }^{a}$ Standard: DMB

Subsequently, it was investigated if the yield of tetrahydroisoquinoline $\mathbf{2 0 8}$ could be improved by adding bases. Due to the experience that some bases, e.g. $\mathrm{K}_{3} \mathrm{PO}_{4}, \mathrm{KF}$ and $\mathrm{CsF}$, led to decomposition of the photocatalyst and stagnation of the reaction, these bases were not considered (cf. Table 3.5 and Table 3.26). Phosphate bases gave the best results: using $\mathrm{NaH}_{2} \mathrm{PO}_{4}$ and $\mathrm{KH}_{2} \mathrm{PO}_{4}$ resulted in $13 \%$ yield, respectively, and with $\mathrm{Na}_{2} \mathrm{HPO}_{4}$, the product was obtained in $10 \%$ yield (Table 3.30, entries 2-4). The use of $\mathrm{K}_{2} \mathrm{HPO}_{4}$ led to decomposition of the photocatalyst and no reaction occurred (Table 3.30, entry 5). With $\mathrm{NaHCO}_{3}$ and $\mathrm{CaF}_{2}$, the product was afforded in 7 and 10\% yield, respectively (Table 3.30, entries 6 and 7). The formation of the product did not occur with NaOAc as the base and decomposition of the photocatalyst was observed Table 3.30. entry 8).

As the addition of the discussed bases did not improve the yield of product 208, the question came up if the use of oxygen instead of air, which was used in the previous experiments, was beneficial. Consequently, two reactions with oxygen were conducted (Equation 3.27). Using only oxygen led to a yield of $9 \%$, additional use of molecular sieves did influence the yield and the product was obtained in $8 \%$.

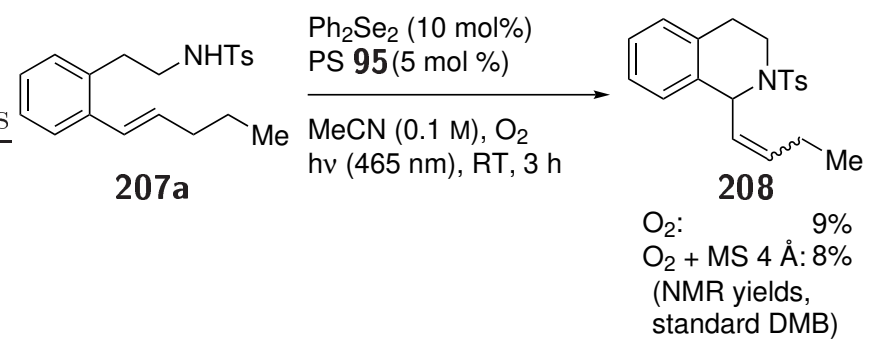


Table 3.30: Optimization of used bases in the intramolecular amination.

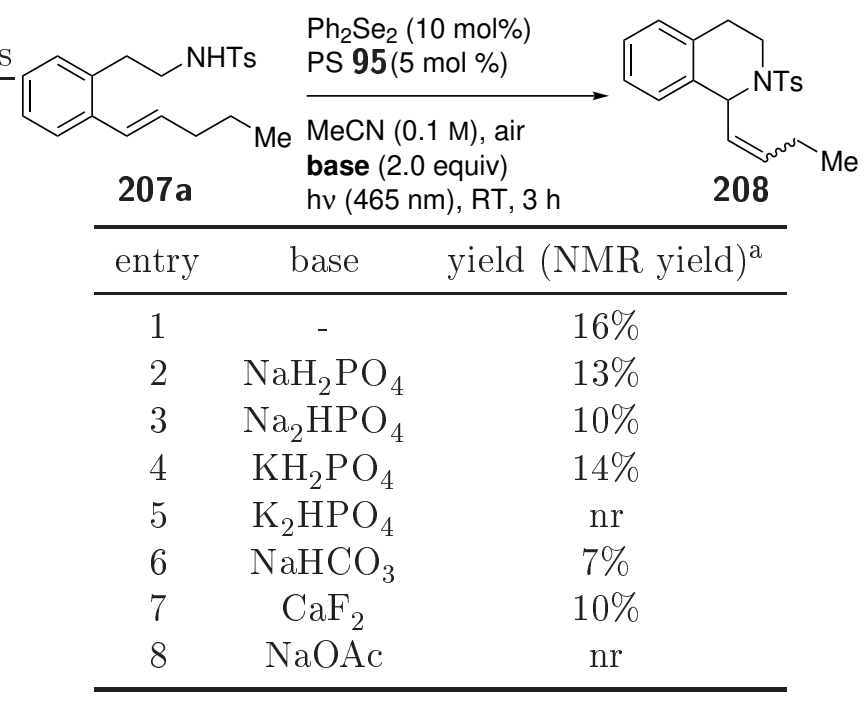

${ }^{a}$ Standard: DMB.

As the yield in the formation of tetrahydroisoquinoline $\mathbf{2 0 8}$ under photoconditions could not be increased with different solvents or bases, but full conversion of the starting material to unidentified side products was observed, it was assumed that it was decomposed under the reaction conditions. Oxidative decomposition could be induced by the used photocatalyst, which has a redox potential of the excited state $E_{\text {red }}^{*}=1.84 \mathrm{~V}$ vs. SCE. 129, 182] As the redox potential of tosyl amides was reported as $E_{o x}=2.38 \mathrm{~V}$ vs. SCE, it was assumed that the observed decomposition was not an oxidation of the amide moiety. ${ }^{[183]}$ However, the oxidation of the styrenic double bond seemed more probable, as NICEWICZ and colleagues reported the oxidation potentials of different styrene derivatives as $E_{o x}=1.2-1.7 \mathrm{~V}$ vs. SCE. [184] To avoid this side reaction, the oxidation potential of the double bond could be increased by introducing electronwithdrawing substituents at the aromatic ring. A second possibility to avoid overoxidation is to use a photocatalyst with a lower reduction potential than $p$-MeO-TPT (95), like acridinium salt $131\left(E_{\text {red }}^{*}=1.65 \mathrm{~V}\right.$ vs. SCE$)$ or ruthenium complex $132\left(E_{\text {red }}^{*}=1.45 \mathrm{~V}\right.$ vs. SCE), which were also tested in the intramolecular etherification reaction. 128,130$]$ Furthermore, changing the protecting group at the nitrogen atom and thereby the $\mathrm{p} K_{\mathrm{a}}$ of the $\mathrm{N}-\mathrm{H}$ unit could influence the turnover of the desired reaction, as was also observed in the selenium-catalyzed formation of indoles. .65$]$ An interesting protecting group could be a carbamate moiety, like it was used in the reaction of CLIVE et al. 1 [179]

In order to evaluate if the formation of tetrahydroisoquinolines 209 was more efficient under non-photochemical conditions, the cyclization of tosyl amides $\mathbf{2 0 7}$ under selenium-catalysis with NFSI as the oxidant was investigated by C. Schlawis. $\frac{[185]}{10}$ He was able to optimize the conditions for the formation of products $\mathbf{2 0 9}$ to the use of 5 mol\% diphenyl diselenide, 1.05 equiv NFSI and $4 \AA$ molecular sieves in toluene at room temperature and obtained the desired products in good yields of up to $69 \%$ Equation 3.28). Surprisingly, the change of the oxidant also led to a change 
in the structure of the product. Instead of the formation of a stereogenic center in the benzylic position, the formation of an isomer with a double bond conjugated to the aromatic system was observed. This was indicated by the ${ }^{1} \mathrm{H}$ NMR signals of the vinylic proton, a triplet at $6.24 \mathrm{ppm}$, and the methylene groups in the ring, which did not split into separate signals for the geminal protons. Their signals appeared as a multiplet at $2.41-2.55 \mathrm{ppm}$, which overlapped with the signal of the $\mathrm{CH}_{2}$ group next to the double bond, and as a broad singlet at $\left.3.83 \mathrm{ppm} .185\right]$

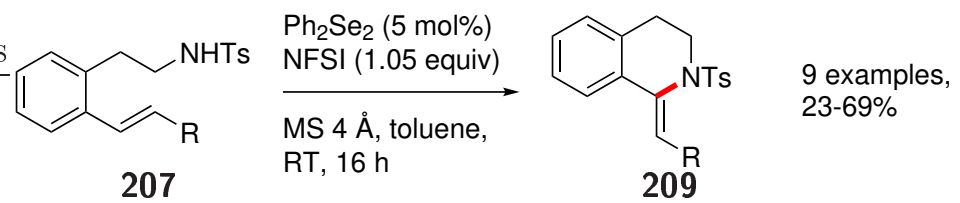

\subsubsection{Synthesis and cyclization of ortho-vinyl homobenzylamides}

The formation of tetrahyroisoquinolines 209 with different substituents in the side chain was examined by C. Schlawis. 185$]$ It was further interesting to evaluate the influence of different substituents on the aromatic ring. Therefore, several ortho-vinyl homobenzylamide derivatives 207 were synthesized using different synthetic strategies. In the first synthetic path, the desired compounds were synthesized in an analogous manner to the synthesis of amide 207a (cf. [Scheme 3.5). Starting from bromophenylacetonitriles 204, the SUZUKI coupling to 1-pentenylboronic acid afforded nitriles 205b-f in good yields between 83 and $88 \%$ (Table 3.31).

Table 3.31: SuZUKI reaction of bromophenylacetonitriles 204 to nitriles 205. 180$]$
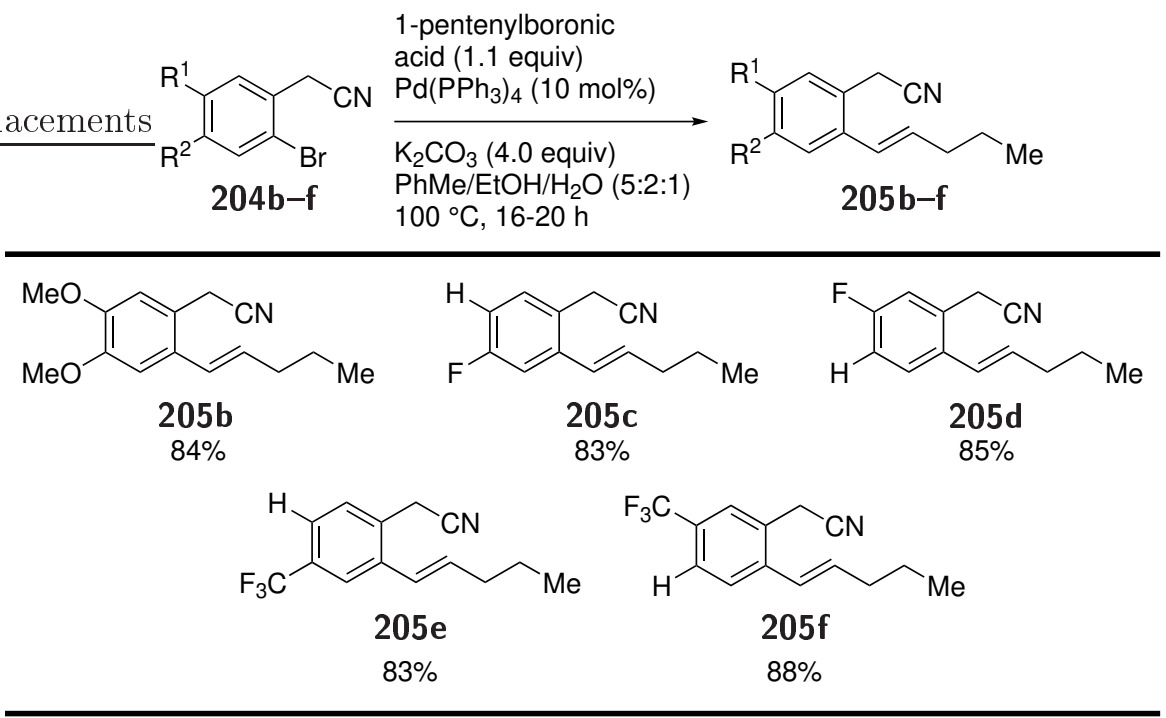

In the next step, the nitrile function was reduced to the amine with lithium aluminum hydride (Table 3.32). The products 206b-f were used without further purification in the following reaction with toluenesulfonic acid chloride to provide tosyl amides 207b-f in overall yields between 5 and $23 \%$. 
Table 3.32: Reduction of nitriles 205 to amines 206 and subsequent reaction to tosyl amides 207. $\stackrel{\text { a } 181]}{[}$

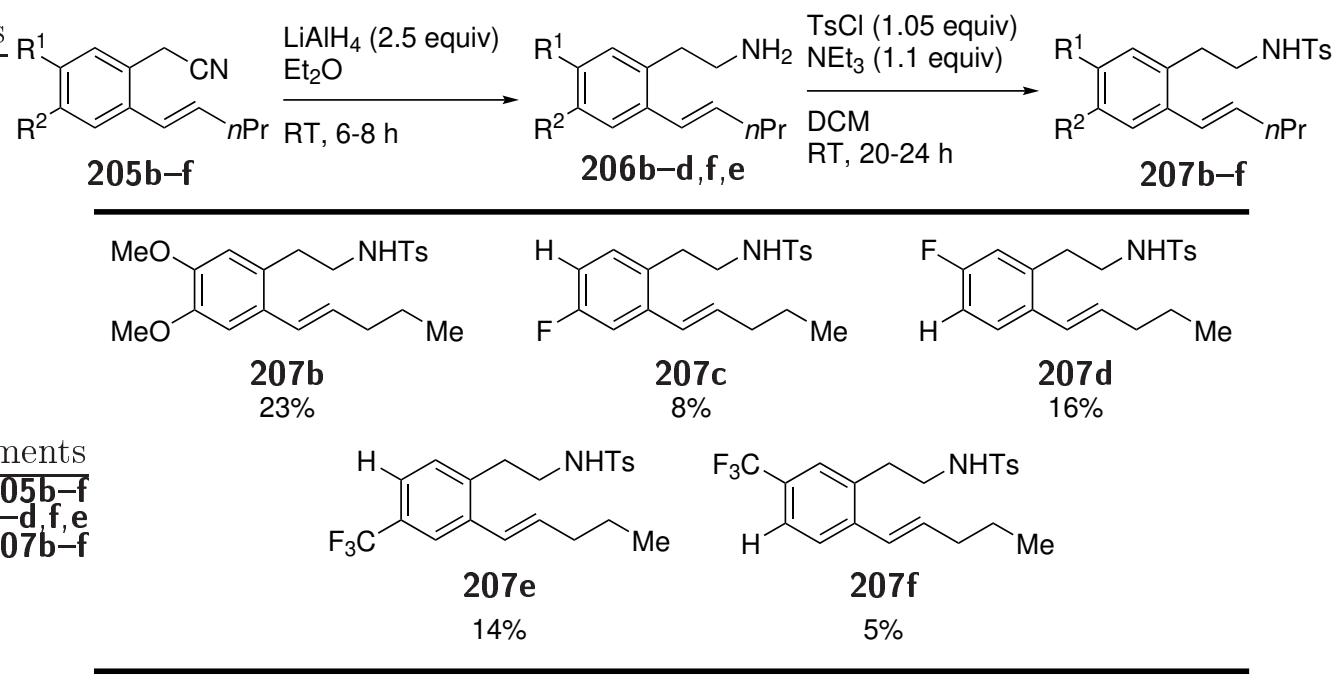

${ }^{a}$ Isolated yield respective to nitriles 205 (two steps).

The synthesis of a mono methoxy substituted and a benzo[ $d][1,3]$ dioxole derived ortho-vinyl homobenzylamide started from 1-bromo-2-(bromomethyl)-4-methoxybenzene (210) and 5-bromo6-(bromomethyl)benzo[ $d][1,3]$ dioxole (211) with a nitromethylation, following a procedure by WATSON and coworkers. $\stackrel{186]}{[1}$ The reaction with nitromethane (7.5 equiv) and sodium tert-butoxide (1.2 equiv) was catalyzed by copper(I)bromide ( 0.2 equiv) with $m$-xylyl-nacnac (214) (0.2 equiv) as the ligand. It afforded the methoxy-subtituted product $\mathbf{2 1 2}$ in $69 \%$ yield and the benzodioxole derivative $\mathbf{2 1 3}$ in $35 \%$ yield Equation 3.29.
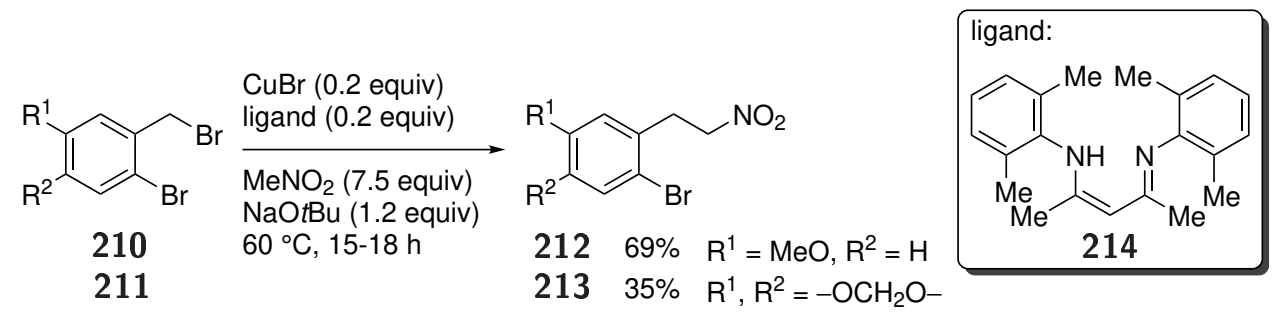

Nitro compounds $\mathbf{2 1 2}$ and $\mathbf{2 1 3}$ were then reduced in a CLEMMENSEN-type reduction following a procedure by GONG and coworkers. .187$]$ The reaction with zinc (20 equiv) in a solvent mixture of methanol/acetic acid/2 $\mathrm{M} \mathrm{HCl}(3: 1: 2, \mathrm{v} / \mathrm{v} / \mathrm{v})$ afforded the desired amines 217 and 218 in 51 and $94 \%$ yield. The amine groups were subsequently converted into tosyl amide groups using the previously applied method (cf. Scheme 3.5 and Table 3.32) to obtain tosyl amides $\mathbf{2 1 5}$ and 216 in 67 and $71 \%$ yield (Scheme 3.6).

In the last step, tosyl amides $\mathbf{2 1 5}$ and $\mathbf{2 1 6}$ were reacted with 1-pentenylboronic acid in a SuzukI coupling using the established conditions (cf. Table 3.31) to afford ortho-vinyl homobenzylamides 207g and 207h in 67 and $47 \%$ yield [Equation 3.30). 


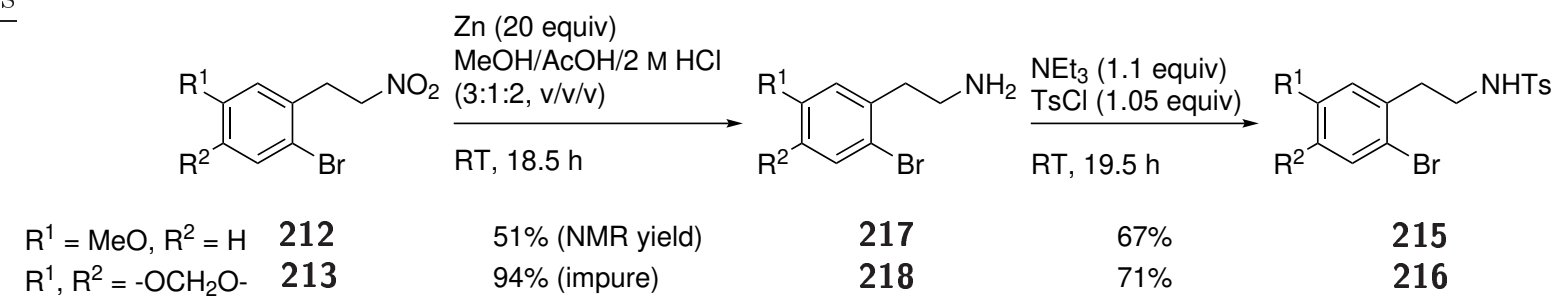

Scheme 3.6: Reduction and following tosyl amide protection of nitro compounds 212 and 213. [181,187]

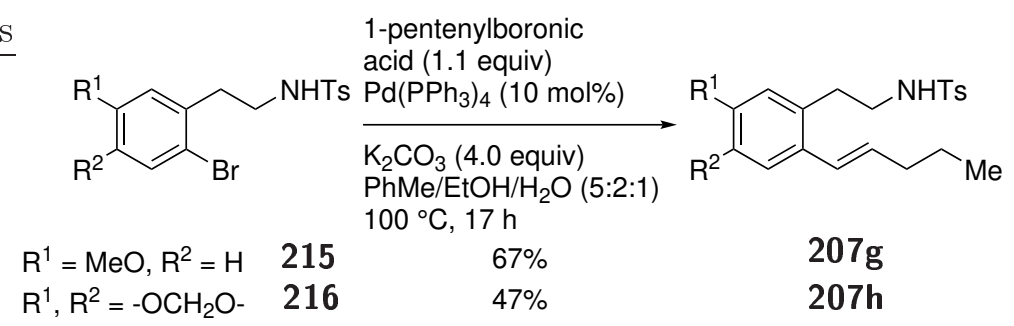

In addition to tosyl amides $\mathbf{2 0 7} \mathbf{b}-\mathbf{h}$, arylated derivatives were to be synthesized. For this purpose, a synthetic path was developed which allowed for the introduction of different aryl moieties in the penultimate step (Scheme 3.7). Consequently, compounds 219 could be obtained from dihalide 220 via two subsequent Suzuki reactions. The introduction of the tosylated side chain could be achieved in the established synthetic path from either nitrile $\mathbf{2 2 1}$ or benzyl bromide 224, which could both be obtained from benzyl alcohol 222.

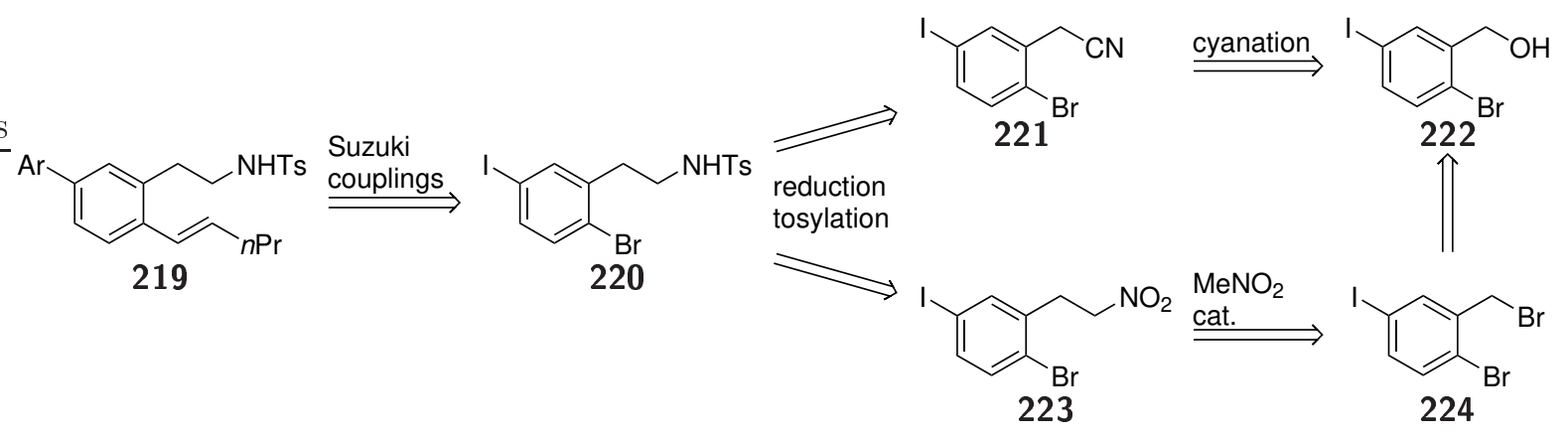

Scheme 3.7: Retrosynthetic considerations for the synthesis of arylated ortho-vinyl homobenzylamides 219.

First, the synthesis via nitrile 221 was attempted using a procedure by Hu and Zuo. 188$]$ The cyanation of 2-bromo-5-iodobenzylalcohol (222) was conducted by transferring it into the mesylalcohol with triethylamine (1.5 equiv) and methanesulfonyl chloride (1.1 equiv) and then substituting the mesyl alcohol with sodium cyanate (1.5 equiv) (Scheme 3.8). Nitrile 221 was obtained in $80 \%$ yield. The reduction of nitrile $\mathbf{2 2 1}$ and the following tosyl amide protection were conducted under the established conditions. ${ }^{[181]}$ Unfortunately, the desired product 220 was ob- 
tained in an inseparable mixture with protodeiodinated side product $\mathbf{2 2 6}$ and other unidentified side products.
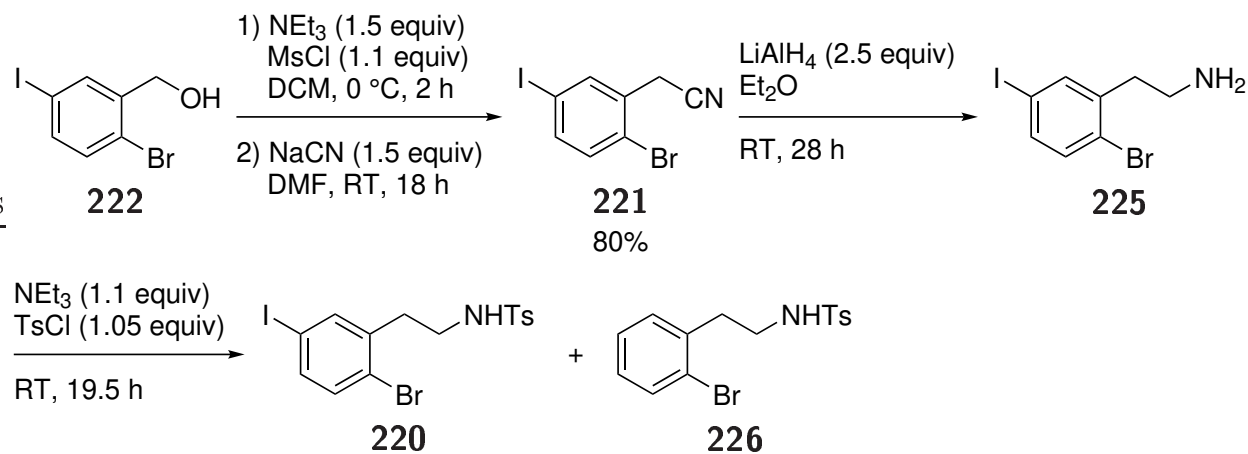

Scheme 3.8: Cyanation and attempted reduction and following tosyl amide protection of benzyl alcohol 221.

In order to avoid this problem, the alternative synthesis via the nitro compound was attempted. The synthesis started with the substitution of the hydroxyl group of 2-bromo-5-iodobenzylalcohol (222) by a bromide. Using $\mathrm{PBr}_{3}$ (0.43 equiv) in THF afforded 1-bromo-2-(bromomethyl)-4iodobenzene (224) in a yield of $63 \%$ (Scheme 3.9). With benzyl bromide 224 in hand, the nitromethylation was conducted according to previous experiments (cf. Equation 3.29) and afforded the nitro compound 223 in $30 \%$ yield. $\stackrel{[186]}{ }$

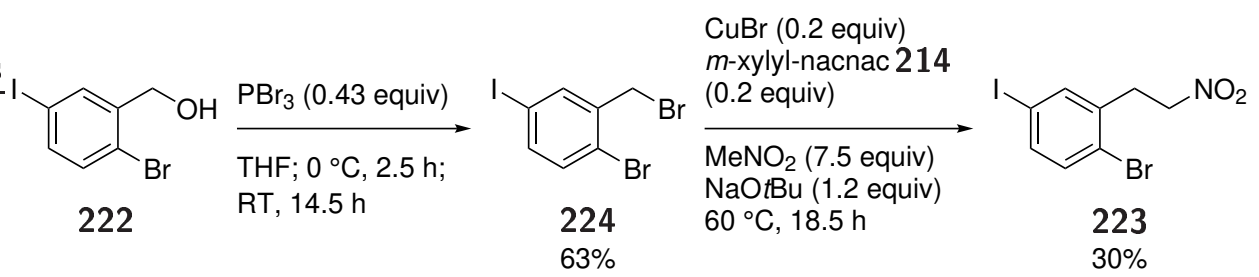

Scheme 3.9: Bromination and following nitromethylation of benzyl alcohol 222.

Unfortunately, the reduction of nitro compound $\mathbf{2 2 3}$ to amine $\mathbf{2 2 7}$ analogous to previous reactions (cf. Scheme 3.6) afforded the protodeiodinated amine $\mathbf{2 2 8}$ in $81 \%$ (Equation 3.31). The formation of this side product was determined from the combined integral of the aromatic region of 4 .

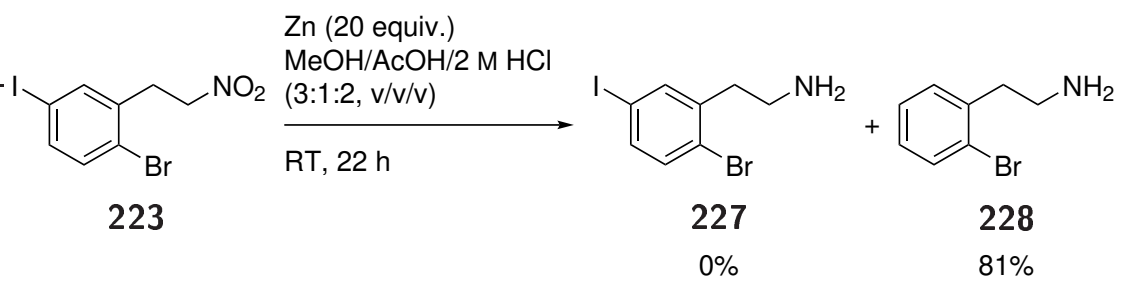

As the hydrogenation of both the nitrile or the nitro function led to cleavage of the iodide, it was attempted to perform the SUZUKI couplings before the reduction reactions. The reaction of nitrile 221 with either 4-(trifluoromethyl)phenylboronic acid or 4-methylphenylboronic acid pincacol 
ester under the established conditions afforded product $\mathbf{2 2 9}$ (trifluoromethyl substituent) in $36 \%$ yield. Product 230 (methyl substituent) could only be obtained in a mixture with an unidentified side product (Equation 3.32). 180$]$ Product 229 was identified by the signals of the benzyl group at $3.92 \mathrm{ppm}$ (singlet, integral 2) and the aromatic protons at 7.80-7.60 ppm (multiplet, integral 6) and $7.42 \mathrm{ppm}$ (doublet of doublets, integral 1). In the spectrum of product $\mathbf{2 3 0}$, there were two singlets at 3.89 (integral 2) and $3.90 \mathrm{ppm}$ (integral 1.2), presumably the benzyl group, and two singlets at 2.40 (integral 3) and $2.42 \mathrm{ppm}$ (integral 1.5), presumably the methyl group. Together with the high integral of 33 of the aromatic region between 7.2 and $7.8 \mathrm{ppm}$, this led to the conclusion that the impureness could be a protodehalogenated side product.

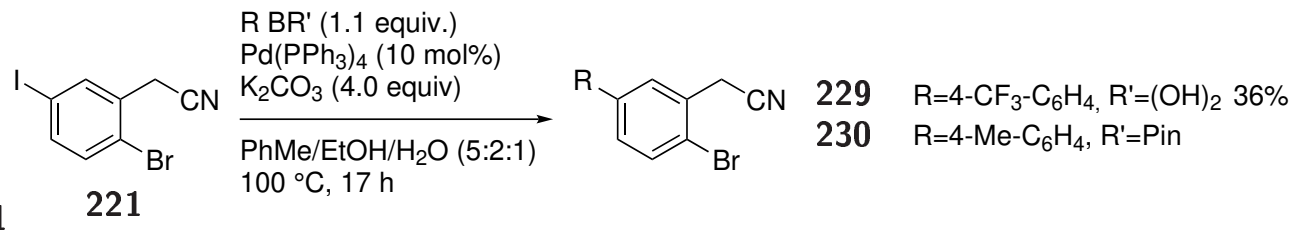

Aryl substituted nitrile $\mathbf{2 2 9}$ was then coupled to 1-pentenylboronic acid in another Suzuki reaction to obtain nitrile 232 in $89 \%$ yield (Scheme 3.10). 180 The following reduction and tosylation of nitrile 232 under the established conditions afforded an inseparable mixture of the desired tosyl amide 233 and an unidentified side product. [181] The formation of the amine was indicated by the appearance of two multiplets at 2.89 and $2.93 \mathrm{ppm}$ for the amine side chain and confirmed via ESI mass spectrometry. The successful reaction of the amine to the amide was indicated by several ${ }^{1} \mathrm{H}$ NMR signals, such as a doublet and a doublet of triplets for the protons at the double bond at 6.61 and $6.09 \mathrm{ppm}$, a multiplet at $4.44 \mathrm{ppm}$ that was assigned to the proton at the nitrogen, and signals of the $n$-propyl moiety $(0.98,1.51$, and $2.22 \mathrm{ppm})$ as well as the ethylene moiety (3.15 and $3.36 \mathrm{ppm})$. The signals in the aromatic region showed an integral that was too high (16 instead of expected 11) and additional signals like a doublet at $1.26 \mathrm{ppm}$ with the integral 3 and a doublet at $2.43 \mathrm{ppm}$ (integral 0.75 ) belonged to an unidentified side product which could not be separated.

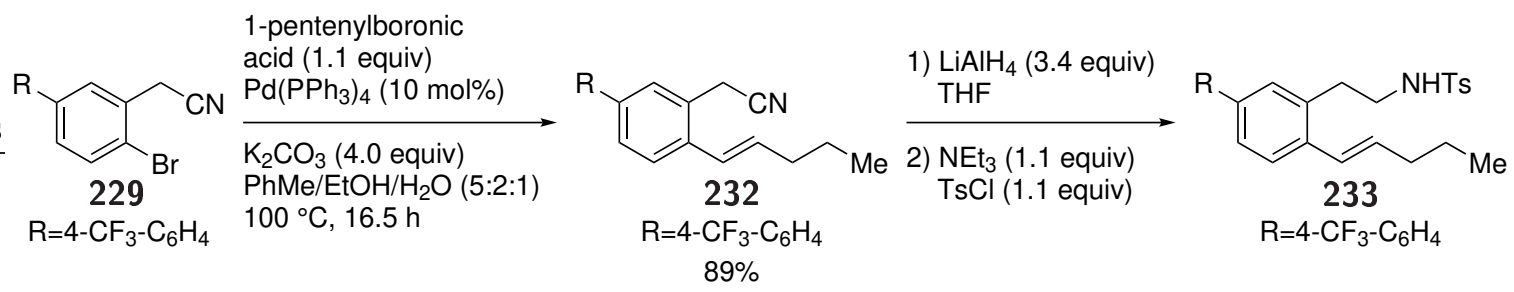

Scheme 3.10: SuZUKI reaction and attempted tosylation of aryl bromide 229.

Since the attempted syntheses of arylated ortho-vinyl homobenzylamides led to the formation of various side products, the catalytic cyclization of synthesized compounds 207 was conducted under the previously established conditions with 5 mol\% diphenyl diselenide, 1.05 equiv NFSI and $4 \AA$ molecular sieves in toluene at room temperature (Table 3.33). ${ }^{1855}$ Tetrahydroisoquinolines 
209c-h were obtained in 21-57\% yield. In the attempted formation of tetrahydroisoquinoline 209b, only the decomposition of the starting material was observed. The products were identified in ${ }^{1} \mathrm{H}$ NMR by the triplet of the vinylic proton between 6.1 and $6.3 \mathrm{ppm}$ and the broad singlet at approx. $3.8 \mathrm{ppm}$ that was assigned to the methylene group $\alpha$ to the nitrogen. The position and nature of the substituents seemed to have a great influence on the yield. Products with electron-withdrawing substituents in meta-position to the double bond were obtained in higher yields (products 209c and 209e) than the analogous products with the substituent in paraposition (products 209d and 209f). Electron-donating moieties led to higher yields than electronwithdrawing groups, also when they were attached in para-position to the double bond (products 209g and 209h).

Table 3.33: Cyclization of tosyl amides 207. ${ }^{\mathrm{a}}$
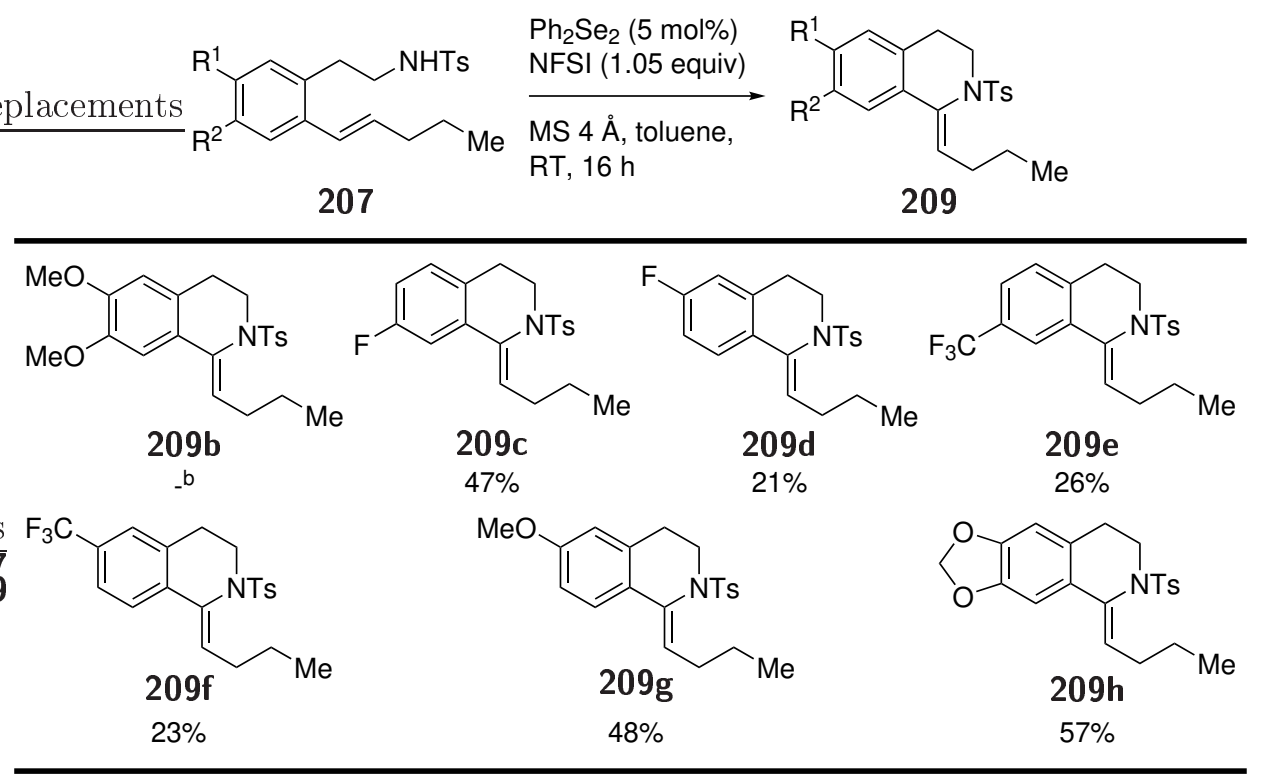

${ }^{\mathrm{a}}$ Isolated yield. $\quad{ }^{\mathrm{b}}$ Decomposition. 


\subsection{Selenium- $\pi$-acid catalyzed synthesis of allenylamides}

\subsubsection{Preliminary investigations and optimization of reaction conditions}

After the examination of reactions with challenging nucleophiles such as alcohols and amines, the question occurred if it was possible to also conduct the reactions with substrates with different $\pi$ bonds; and alkynes attracted our attention. The $\pi$-acid activation of the triple bond by selenium followed by a nucleophilic attack would lead to a vinylic selenofunctionalization product. In the context of a selenium-catalyzed reaction, it is interesting to find out if oxidative elimination of the selenium species is possible and which product is formed. Previously, only few seleniummediated reactions with alkynes as substrates have been reported. Although the formation of a thiirenium ion followed by thiofunctionalization was reported by KHARASCH and AssONY already in $1953,[189]$ the respective selenium-variant was not disclosed until 1974. In the course of his investigations into the addition of phenylselenyl trifluoroacetate to alkenes, REICH also demonstrated its addition to phenyl acetylene (234) (Scheme 3.11). 190]

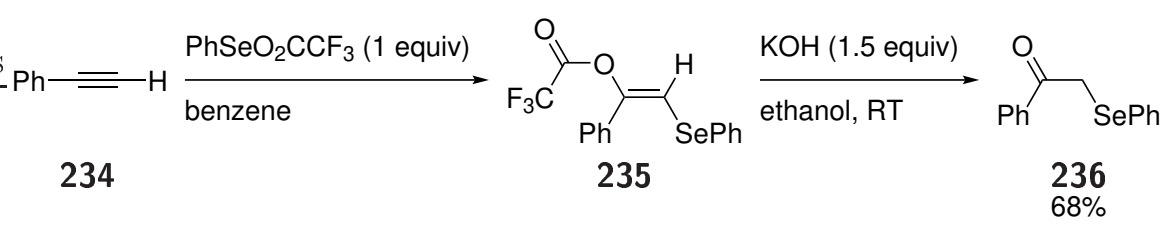

Scheme 3.11: Addition of phenylselenyl trifluoroacetate to phenyl acetylene (234) and the following hydrolysis.

However, REICH did not conduct the oxidative elimination of the selenium species, but hydrolized the acetate, which led to the formation of $\beta$-phenylselenyl ketone 236. The elimination was first examined by BACK and colleagues in 1981.191] They demonstrated the addition of Se-phenyl $p$-tolueneselenosulfonate to terminal alkynes $\mathbf{2 3 7}$ and the following elimination of the selenium moiety by oxidation with $m$ CPBA to acetylenic sulfone 239 in up to $88 \%$ yield (Scheme 3.12). When they subjected alkenes $\mathbf{2 3 8}$ first to basic conditions to induce isomerization of the double bond and then oxidized the resulting vinylic selenides $\mathbf{2 4 0}$, they were able to obtain allenic sulfones $\mathbf{2 4 1}$ in up to $98 \%$ yield instead. $\underline{\underline{192,193]}}$

The formation of functionalized allenes from internal alkynes was realized by the same group under similar conditions to the formation of acetylenic sulfones 239. The addition of Se-phenyl $p$-tolueneselenosulfonate (1.0 equiv) to 5 -decyne (242a) proceeded in $63 \%$ yield and the elimination was initiated by oxidation with $m$ CPBA (1.1 equiv) and heating (Scheme 3.13). ${ }^{194}$ As vinyl selenide $\mathbf{2} \mathbf{4 3}$ has a tetrasubstituted double bond, the elimination of a vinylic hydrogen, like in the case of vinyl selenides 238, is not possible and an allylic hydrogen is eliminated instead, leading to the formation of allene $\mathbf{2 4 4}$ in $96 \%$ yield.

The first procedure for the oxidative functionalization of alkynes that used a substoichiometric amount of a selenium species was the propargylic oxidation of alkynes by the group of ZHAO.195] They were able to form ynones $\mathbf{2 4 6}$ from propargylphosphonates and -esters $\mathbf{2 4 5}$ in yields between 


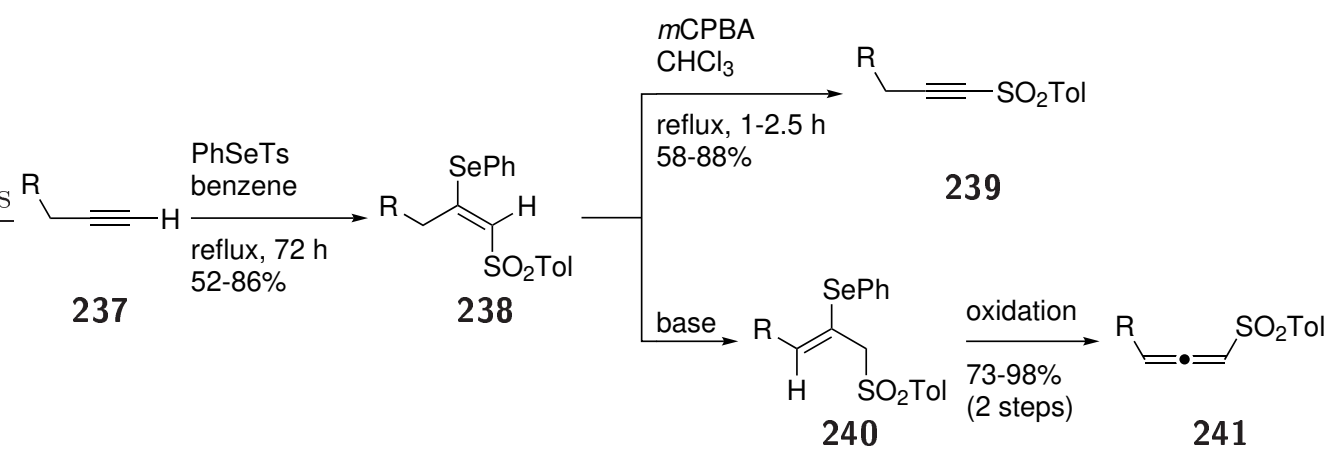

Scheme 3.12: Addition of Se-phenyl $p$-tolueneselenosulfonate to alkynes 237 and the following reactions to alkynes $\mathbf{2 3 9}$ or allenes $\mathbf{2 4 1}$.

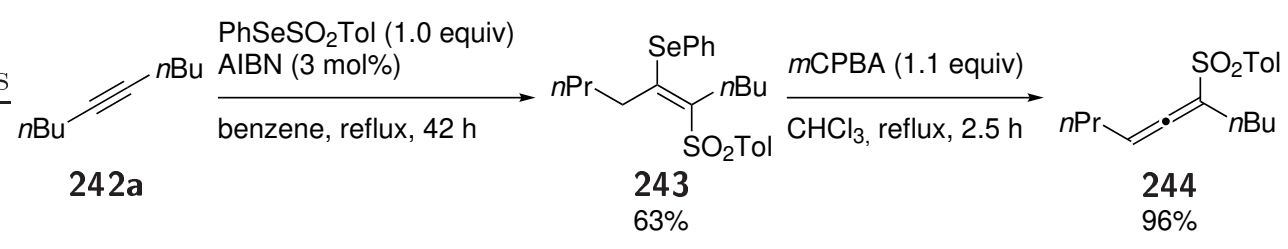

Scheme 3.13: Addition of Se-phenyl $p$-tolueneselenosulfonate to 5-decyne (242a) and the following elimination to allene 244.

41 and $86 \%$ using 10 mol\% of diphenyl diselenide, 2.2 equiv of [PyF][OTf] (57) as the oxidant and 2.0 equiv $\mathrm{H}_{2} \mathrm{O}$ Equation 3.33). Though they were able to use substrates with aromatic and aliphatic alkynes, a drawback of their method is that they need substrates with an oxygencontaining group $\mathrm{R}^{2}$ in the propargylic position.

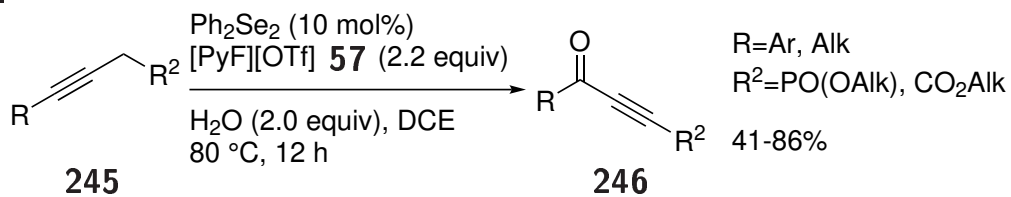

The required nature of the substrates is explained in the proposed mechanism, as the oxygen of the phosphonate or ester group is needed to open selenirenium ion 247 (Scheme 3.14). Oxaphosphole $\mathbf{2 4 8}$ is opened by $\mathrm{H}_{2} \mathrm{O}$ and subsequent isomerization of the double bond yields allyl alcohol 249, which is oxidized to ketone 250. In the last step, oxidation of the selenium moiety leads to elimination and formation of ynone 251.

In order to avoid prefunctionalized substrates and to introduce external nucleophiles, our group performed experiments on the oxidative functionalization of 5-decyne (242a). In the experiments of Dr. S. Ortgies under the conditions of the photo-aerobic selenium- $\pi$-acid catalysis ${ }^{[91]}$ with acetic acid as an exogenous nucleophile, the isolated product could not be separated from leftover

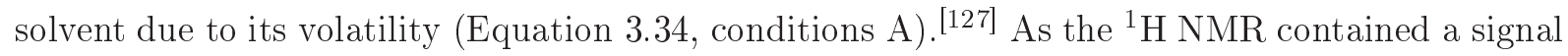
at $4.36 \mathrm{ppm}$, which would fit the vinylic proton, the product was assumed to be allene $\mathbf{2 5 2}$. Unfortunately, the aliphatic signals overlapped with solvent signals and the product could not 


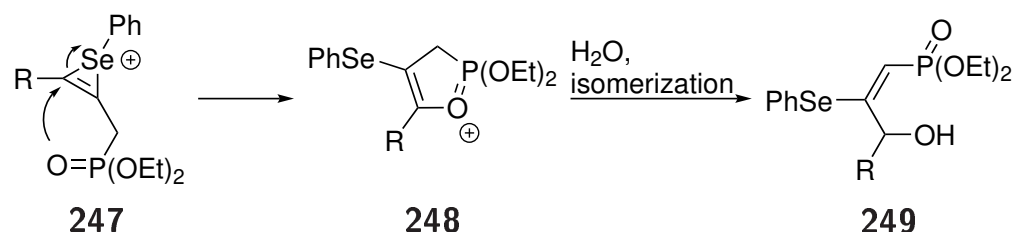

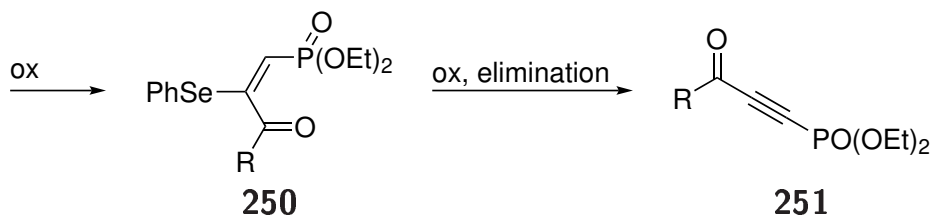

Scheme 3.14: Proposed mechanism of the propargylic oxidation by ZнаO et al.

be definitely identified. ${ }^{[127]}$ Therefore, the reaction was carried out using conditions based on the selenium-catalyzed formation of indoles with NFSI as the oxidant and nucleophile Equation 3.34, conditions B). $\underline{65.127]}$ The reaction with diphenyl diselenide (10 mol\%) and NFSI (1.0 equiv) in toluene at $100{ }^{\circ} \mathrm{C}$ led to the formation of allene 253a in $38 \%$ yield (along with $8 \%$ of the selenofunctionalized intermediate).

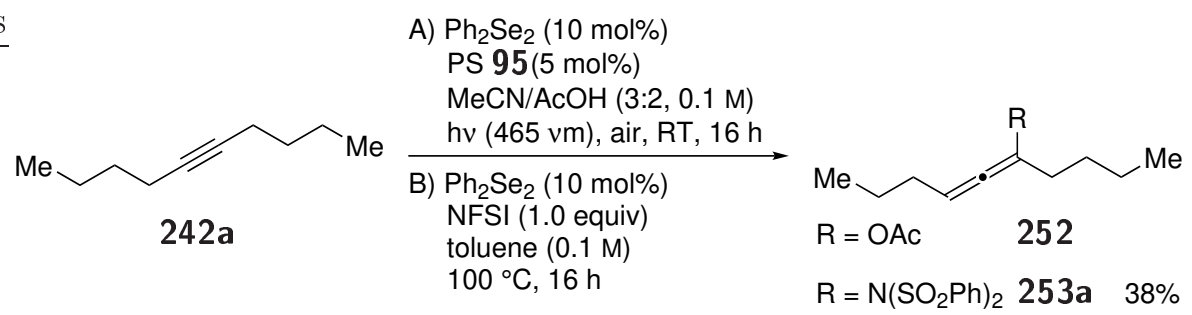

Based on these findings, the conditions for the allene formation were examined further during this work. First, the performance of different diselenide catalysts was compared. In addition to diphenyl diselenide, the performance of (2-anisyl $)_{2} \mathrm{Se}_{2}$, which had been successfully applied in the intramolecular etherification, was evaluated. The reactions were carried out with $10 \mathrm{~mol} \%$ of the respective diselenide and 1.0 equiv NFSI in toluene at $100{ }^{\circ} \mathrm{C}$ for $16 \mathrm{~h}$ (Table 3.34, entries 1 and 2). 196$]$ Product 253a was obtained in $36 \%$ with diphenyl diselenide, confirming the result of the original experiment. With more electron-rich (2-anisyl $)_{2} \mathrm{Se}_{2}$, it was obtained in $45 \%$. Therefore, further experiments were conducted using this catalyst. To assure that the reaction was indeed catalyzed by the diselenide, a control reaction was conducted. When only the alkyne and NFSI were used, no reaction occurred, leading to the conclusion that the diselenide catalyst was needed for the reaction (Table 3.34, entry 3).

Next, the influence of the temperature and different solvents was examined. In order to avoid the formation of side products by residual water in the reaction mixture, molecular sieves was added to the reactions. As lowering the temperature to $50{ }^{\circ} \mathrm{C}$ decreased the vield to $7 \%$ (Table 3.35 , entry 2), the reaction was conducted in different solvents at $100{ }^{\circ} \mathrm{C}$ by R. Rieger. 196$]$ 
Table 3.34: Experiments on the used catalyst in the allenylation. Grayed reaction by R. Rieger. $[196]$

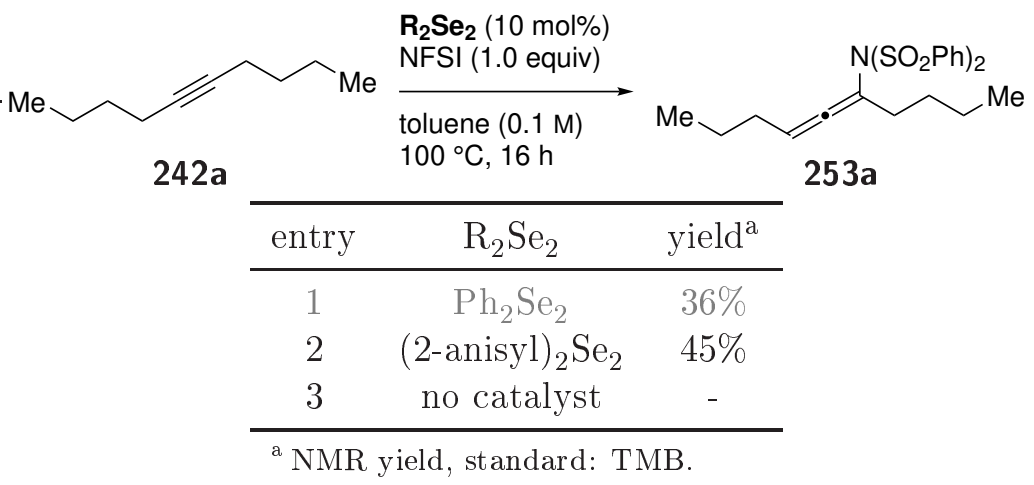

When chlorinated solvents, like 1,1,2,2-tetrachloroethane and 1,2-dichloroethane, were used, allene 253a was obtained in 19 and 9\% yield, respectively (Table 3.35, entries 3 and 4). The use of propionitrile resulted in $17 \%$ yield (Table 3.35 , entry 5). With nitromethane, the product was only obtained in $8 \%$ yield (Table 3.35, entry 6). Ethereal solvents led to yields that were comparable to the one achieved with toluene. With 1,4-dioxane, the product was obtained in $36 \%$ yield, with THF in $40 \%$ yield (Table 3.35, entries 7 and 8 ). This result matches the results of the amination of alkenes by BREDER et al., where ethereal solvents gave the best results. 63$]$

Table 3.35: Experiments on temperature and used solvents in the allenylation. Grayed reactions by R. Rieger. .196$]$

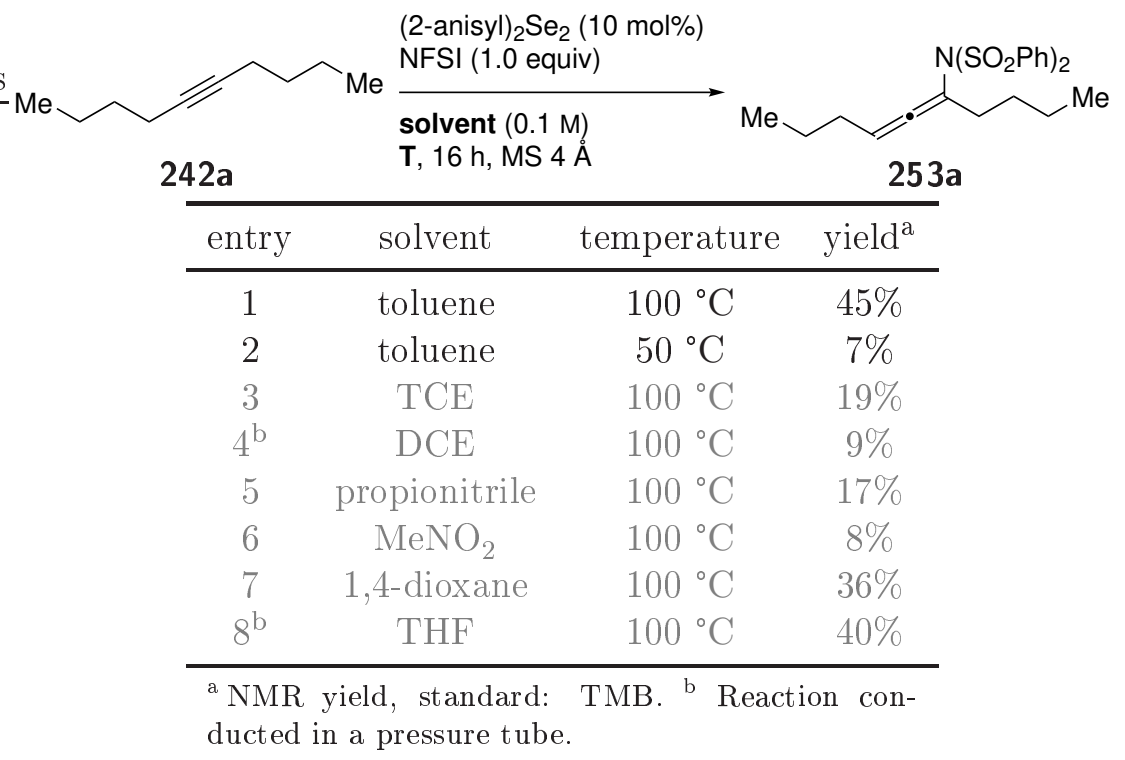

After toluene was identified as the best solvent, it was examined if changing the ratio between starting material and oxidant would increase the yield of the reaction. In the first experiments, 5-decyne (242a) was used in an excess of 1.5, 3.0 and 5.0 equiv and product 253a was obtained in 50,36 and $36 \%$ yield, respectively (Table 3.36, entries 1-3). In the experiments with the higher decyne loading, the decomposition of NFSI was observed. Therefore, the ratio of the reagents 
was reversed to avoid the stagnation of the reaction due to missing nucleophile. With 1.2 equiv NFSI, allene 253a was obtained in 53\% yield, with 3 equiv NFSI it was still obtained in $41 \%$ yield (Table 3.36, entries 4 and 5).

Table 3.36: Optimization of reagent amounts in the allenylation. Grayed reactions by R. Rieger. $\underline{\underline{196]}}$

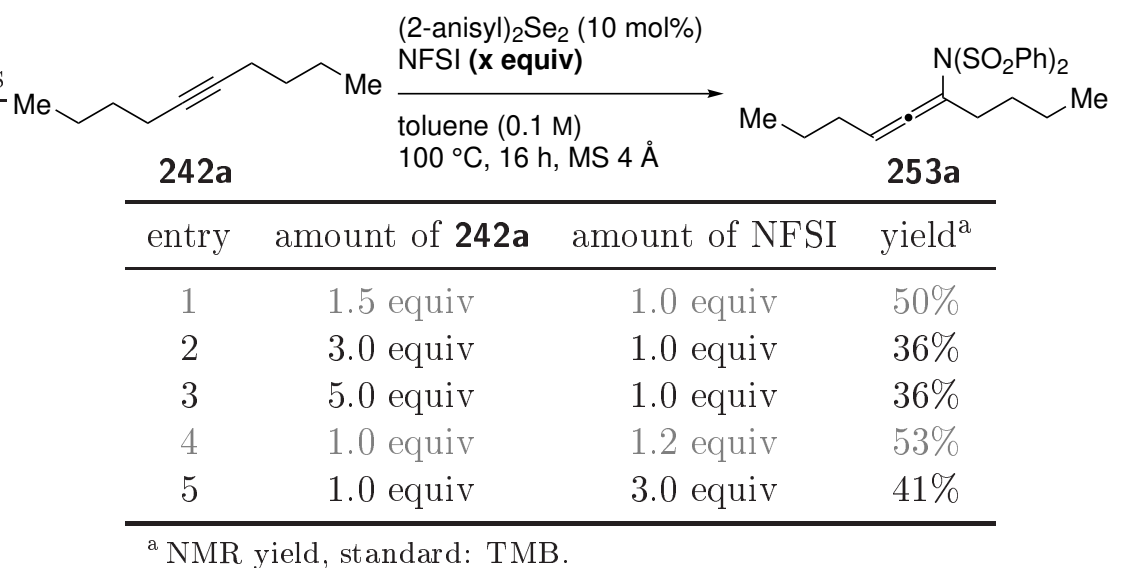

Having determined that a slight excess of NFSI results in higher yields, the effect of different bases on the reaction was investigated. NFSI was added gradually as a solution in toluene to further prevent its decomposition. As complete conversion of the alkyne was observed as soon as the addition of NFSI was complete, the reaction time was shortened to $4 \mathrm{~h}$. The addition of $\mathrm{NaH}_{2} \mathrm{PO}_{4}, \mathrm{Na}_{2} \mathrm{HPO}_{4}$ or $\mathrm{Na}_{3} \mathrm{PO}_{4}$ led to yields of 38,56 or $65 \%$, respectively (Table 3.37, entries 1-3; grayed reaction conducted by R. Rieger). $\stackrel{[196]}{ }$ With potassium phosphates $\mathrm{KH}_{2} \mathrm{PO}_{4}$ and $\mathrm{K}_{2} \mathrm{HPO}_{4}$, allene 253a was also obtained in good yields of 52 and $65 \%$ (Table 3.37, entries 4 and 5). Only $\mathrm{K}_{3} \mathrm{PO}_{4}$ led to a low yield of $11 \%$ (Table 3.37, entry 6 ). The addition of carbonate bases to the reaction had a beneficial effect in most cases. With sodium carbonates $\mathrm{NaHCO}_{3}$ and $\mathrm{Na}_{2} \mathrm{CO}_{3}$, the desired product was obtained in 57 and $64 \%$ yield Table 3.37, entries 7 and 8), lithium carbonate and calcium carbonate afforded the product in the highest yields of 68 and $66 \%$ (Table 3.37, entries 11 and 13). The addition of potassium carbonates $\mathrm{KHCO}_{3}$ and $\mathrm{K}_{2} \mathrm{CO}_{3}$ or caesium carbonate led to moderate yields of 27,29 or $32 \%$ yield Table 3.37, entries 9,10 and 12). With NaOAc and KOAc, the product was obtained in only 9 and $16 \%$ yield Table 3.37 , entries 14 and 15).

In many of the previous reactions, the formation of a side product was observed after the full conversion of the alkyne. A first clue to this side product being the selenofunctionalized compound 254 gave the appearance of additional signals in the aromatic region of the ${ }^{1} \mathrm{H}$ NMR spectrum. In addition to the signals at 6.75-7.08 ppm (integral 2), $7.31 \mathrm{ppm}$ (integral 1) and approx. $7.49 \mathrm{ppm}$ (overlap with signal of desired product; integral approx. 1), a singlet at $3.90 \mathrm{ppm}$ (integral 3, probably the methoxy group) was observed (Figure 3.11, signals marked in red). When the side product was isolated, its identity was further hinted at by the missing signals of the carbon atoms of the allene moiety at 99.0, 106.8 and $206 \mathrm{ppm}$ in ${ }^{13} \mathrm{C}$ NMR. Instead, two signals for the vinylic 
Table 3.37: Optimization of bases used in the allenylation. Grayed reaction by R. Rieger. ${ }^{[196]}$

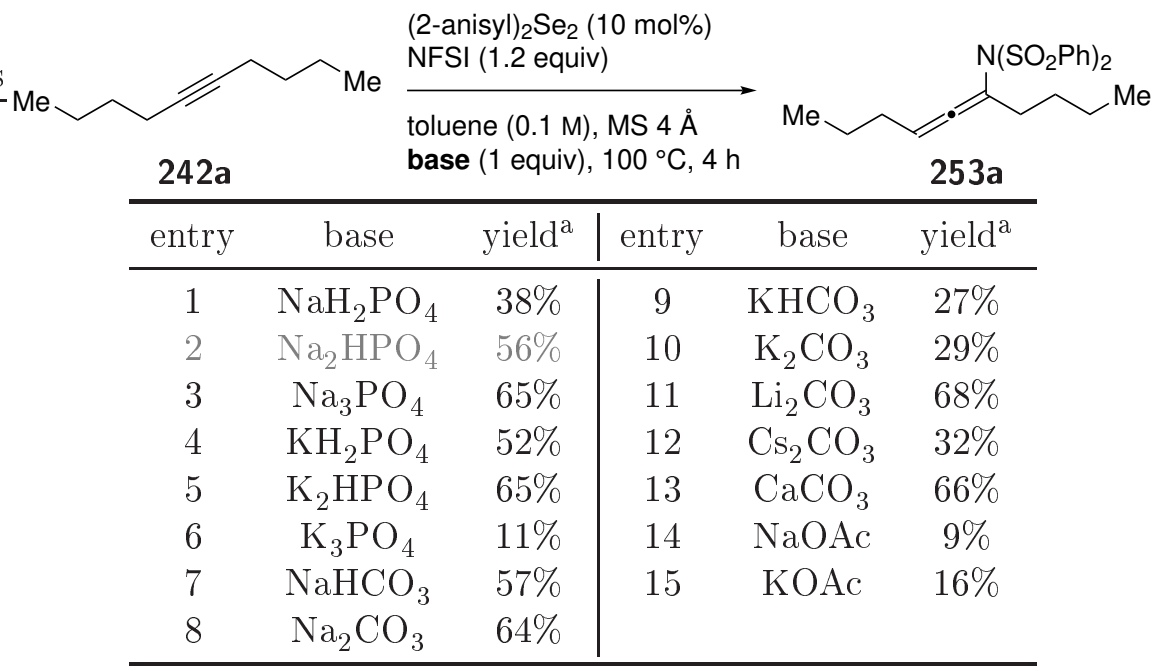

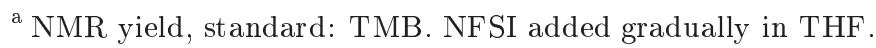

carbon atoms at 133.5 and $146.7 \mathrm{ppm}$ were observed. Additionally, a signal in the ${ }^{77} \mathrm{Se}$ NMR spectrum at $314.1 \mathrm{ppm}$ and mass spectrometric analysis supported the identification of the side product as selenofunctionalized compound 254 .

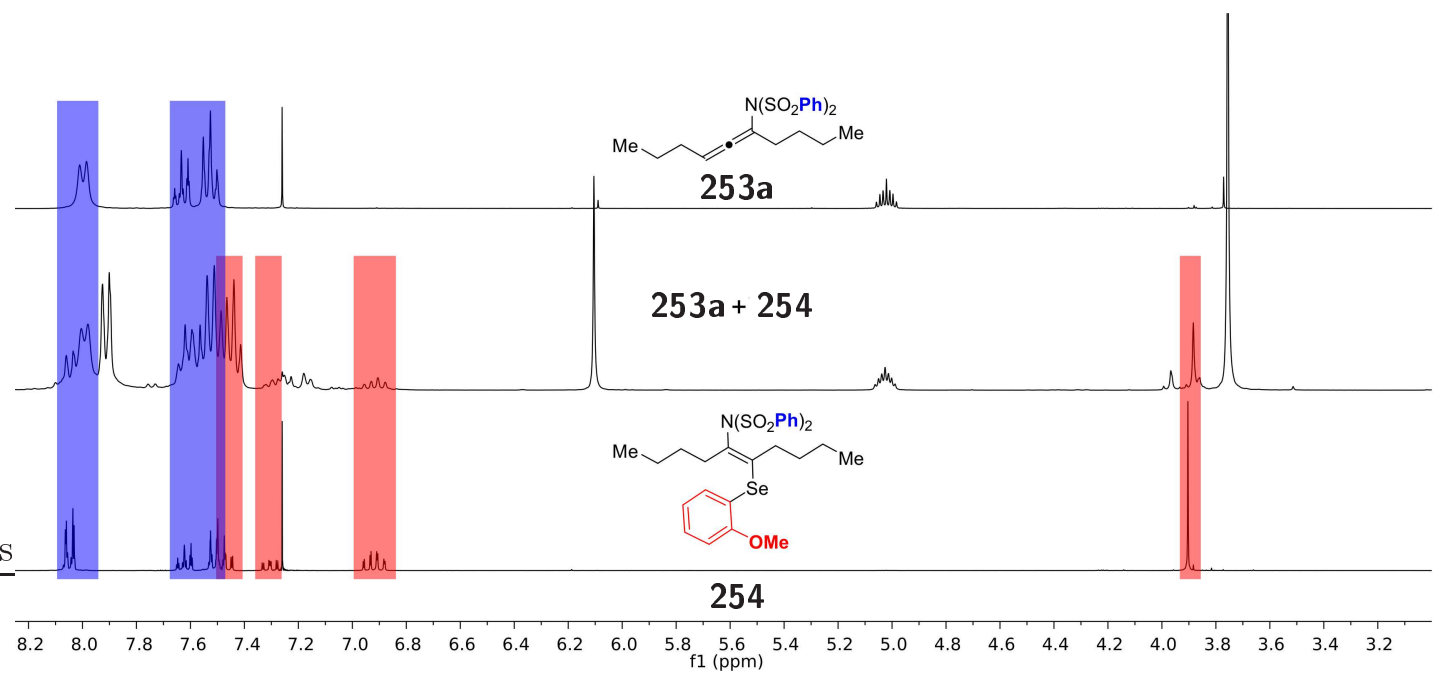

Figure 3.11: Extract from ${ }^{1} \mathrm{H}$ NMR spectra of allene 253a (top), the crude reaction mixture (middle) and selenofunctionalized compound 254 (bottom).

As the maximum amount of formed selenofunctionalized compound is twice the amount of applied catalyst, it appeared reasonable to decrease the catalyst loading. The stepwise decrease of the used amount of (2-anisyl) ${ }_{2} \mathrm{Se}_{2}$ from 10 to 5 and $2.5 \mathrm{~mol} \%$ increased the yield from $68 \%$ to 74 and $77 \%$ (Table 3.38, entries 1-3). Lowering the catalyst loading to $1 \mathrm{~mol} \%$ led to only $10 \%$ yield (Table 3.38, entry 4). 
Table 3.38: Optimization of catalyst loading in the allenylation.

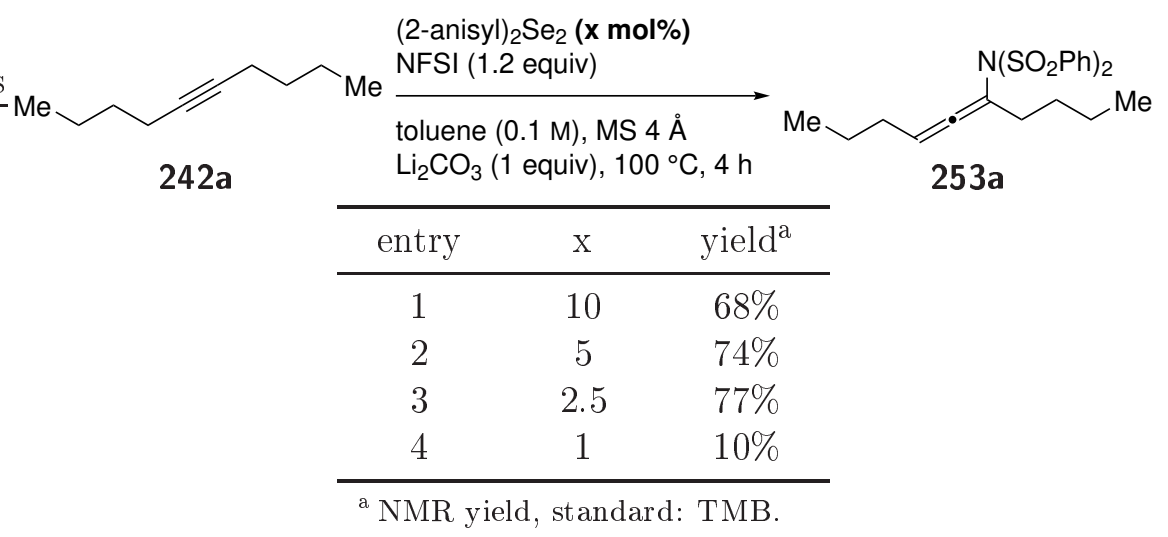

\subsubsection{Synthesis of alkynes and allenylamides}

With the optimized reaction conditions in hand, the synthesis of unsymmetrically and symmetrically substituted aliphatic alkynes was investigated. The first synthetic route was the nucleophilic substitution of dodecyl bromide with a lithiated terminal alkyne. Following a procedure by PÁPAI and REPO, the reaction was conducted using 3-methyl-1-butyne (255) or 1Boc-4-ethynylpiperidine (256), 1.0 equiv of $n$-butyl lithium and $3 \mathrm{~mol} \%$ of $\mathrm{CuCl} \cdot 2 \mathrm{LiCl}$ (Equation 3.35). Unfortunately, the reactions did not show complete conversion. Alkyne 257 could not be separated from equally unpolar starting materials and side products by column chromatography. Alkyne $\mathbf{2 5 8}$ was isolated in only 5\% yield.

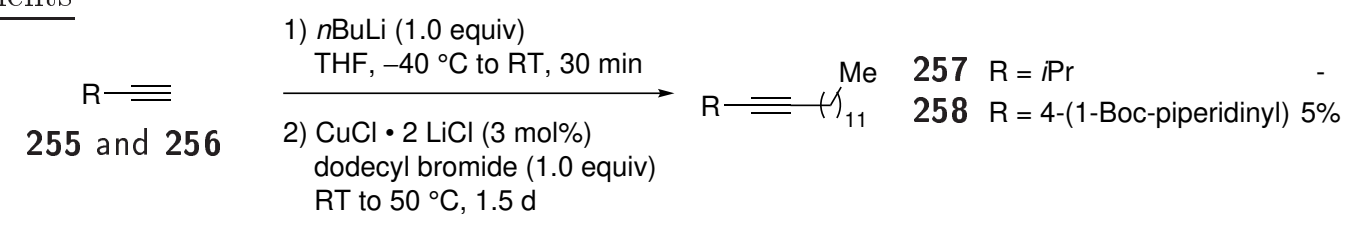

Instead of by a nucleophilic substitution, a terminal alkyne and a bromide could also be connected by a cross coupling reaction, e.g. a SonogAshiRA reaction. ${ }^{\text {[198] }}$ Therefore, the reaction of 1octyne (259) and dodecyl bromide (260) was conducted using conditions by Fu and colleagues with $2.5 \mathrm{~mol} \%$ of $[(\pi \text {-allyl }) \mathrm{PdCl}]_{2}, 5 \mathrm{~mol} \%$ of $\mathrm{NHC}$ ligand $262,7.5 \mathrm{~mol} \%$ of $\mathrm{CuI}$ and 1.4 equiv of $\mathrm{Cs}_{2} \mathrm{CO}_{3}$ as the base [Equation 3.36).

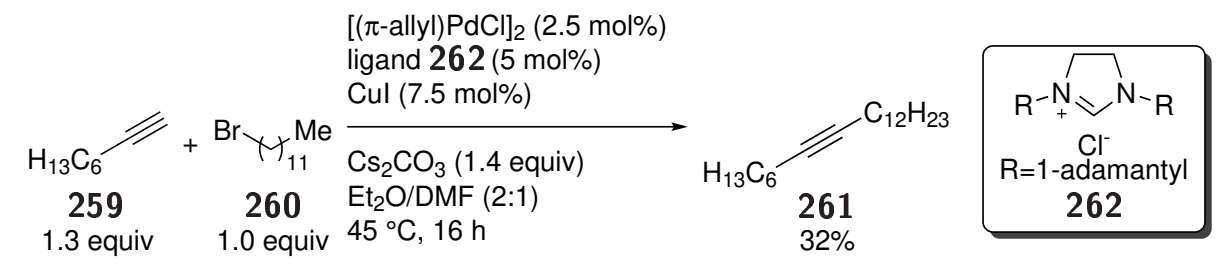


As the performance of a simple alkyne in the allenylation reaction had already been examined during the optimization, the substrate syntheses targeted molecules with different functional groups. In order to obtain alkynes with acid-derived side chains using the previously described cross coupling reaction, acids $\mathbf{2 6 3}$ and $\mathbf{2 6 5}$ were transferred into the respective methoxy esters $\mathbf{2 6 4}$ and 266 in 79 and $78 \%$ yield, respectively. The following SonOgASHIRA reaction was attempted with both the unprotected and the protected acid derivatives (Equation 3.37). ${ }^{[199]}$ In the reaction of the free acids, the side reaction of bromide $\mathbf{2 6 5}$ to $\gamma$-butyrolactone was predominant. In case of the protected acid derivatives, the signals of the starting materials disappeared, but the absence of new signals between 2 and $2.5 \mathrm{ppm}$ which matched the singlet at $3.68 \mathrm{ppm}$ of the methoxy groups led to the conclusion that acid derivatives were not suitable substrates for this reaction.

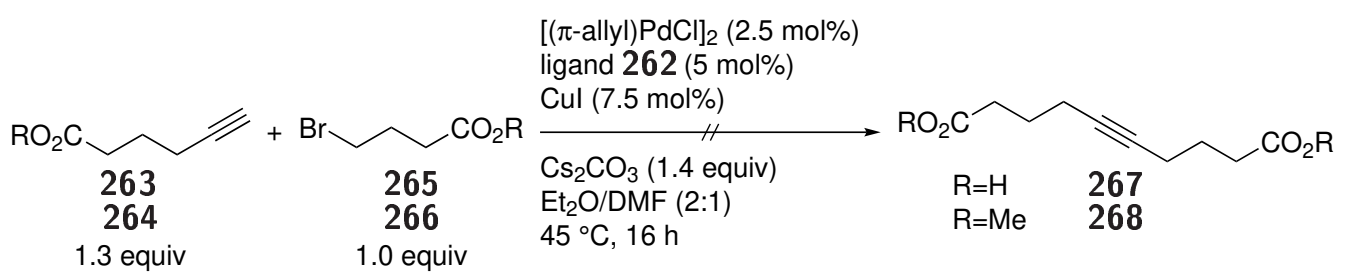

As an alternative synthetic route towards the formation of symmetric alkynes, their direct formation via alkyne metathesis was examined. For this purpose, 3-pentyne-1-ol (269) was transformed into TBS ether $\mathbf{2 4 2 \mathbf { b }}$ and pivalic acid ester 242d2. The homodimerization of 3-pentyne-1-ol (269) and TBS ether 242b was attempted with $\mathrm{Mo}(\mathrm{CO})_{6}(5 \mathrm{~mol} \%)$ and 2-fluorophenol (1.0 equiv), using a procedure by GRELA et al. (Equation 3.38, conditions A).201] Unfortunately, no reaction occurred and only the starting material was observed. Therefore, an alternative procedure by the group of TAMM was examined, which needed a substrate with a different protecting group to be compatible with the catalyst. The homodimerization of pivalic acid ester 242c was attempted using $1 \mathrm{~mol} \%$ of molybdenum catalyst $273^{3}$ (Equation 3.38, conditions B). ${ }^{[202,203]}$ Unfortunately, again only the starting material was observed.

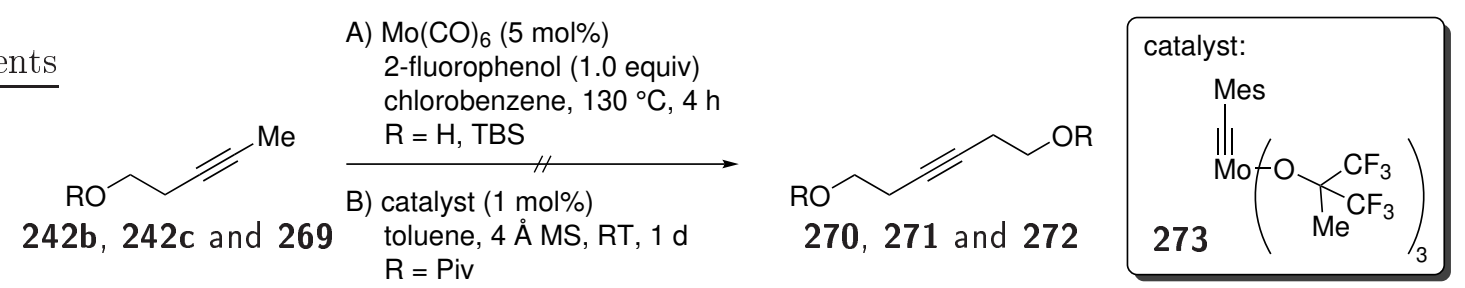

For the synthesis of an alkyne via COREY-FUCHS reaction, ketone 275 was synthesized by a palladium-catalyzed carbonylation of styrene (274). $\underline{204]}$ The reaction with 3.0 equiv formic acid,

${ }^{2}$ Pivalic acid ester 242c was synthesized by F. Krätzschmar. 200$]$

${ }^{3}$ Catalyst 273 was provided by Ò. Àrias, TU Braunschweig. 
1.5 equiv acetic anhydride, $5 \mathrm{~mol} \% \mathrm{Pd}(\mathrm{TFA})_{2}$ as the catalyst and $10 \mathrm{~mol} \%$ 1,3-bis(diphenylphosphino)propane (dppp) as the ligand afforded ketone $\mathbf{2 7 5}$ in $41 \%$ yield (Scheme 3.15). Consequently, the formation of dibromide $\mathbf{2 7 6}$ was attempted with a procedure by MAK. 205$]$ Unfortunately, the use of carbon tetrabromide (2.0 equiv) and triphenylphosphine ( 4.0 equiv) at room temperature or $40{ }^{\circ} \mathrm{C}$ did not lead to the formation of dibromoalkene 276 (Scheme 3.15). A slight upfield shift of the NMR signals of the methylene groups and the observation of a new unpolar species in the TLC hinted at the possible product formation, but only $64 \%$ of the starting material was isolated. In mass spectrometric analysis, the mass and isotopic pattern of the desired product could not be observed.

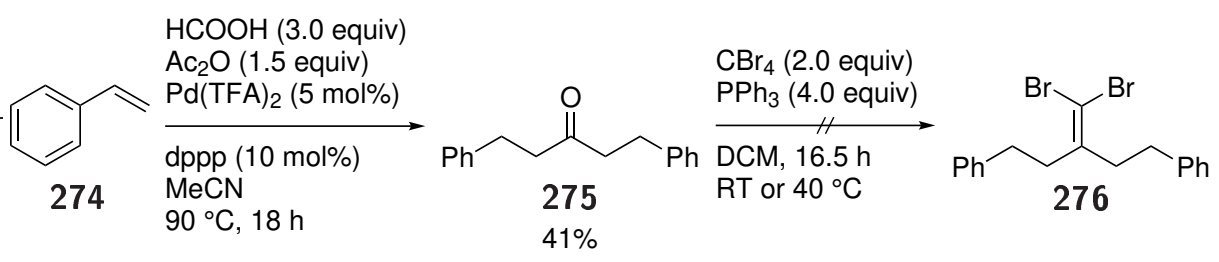

Scheme 3.15: Synthesis of ketone 275 and attempted CoREY-Fuchs reacion.

As the Seyferth-Gilbert homologation also uses carbonyl compounds as starting material, the reaction of ketone $\mathbf{2 7 5}$ with the OHIRA-BESTMAnN reagent $\mathbf{2 7 8}, 1.6$ equiv) and $\mathrm{K}_{2} \mathrm{CO}_{3}$ (2.5 equiv) was examined Equation 3.39. ${ }^{\text {.206] }}$ The analysis of the reaction mixture by ${ }^{1} \mathrm{H}$ and ${ }^{13} \mathrm{C}$ NMR only showed the starting material. In ${ }^{31} \mathrm{P}$ NMR, the shift of the signal of phosphonate 278 from $14.2 \mathrm{ppm}$ to $22.3 \mathrm{ppm}$ indicated its possible transformation into the reactive anionic intermediate.

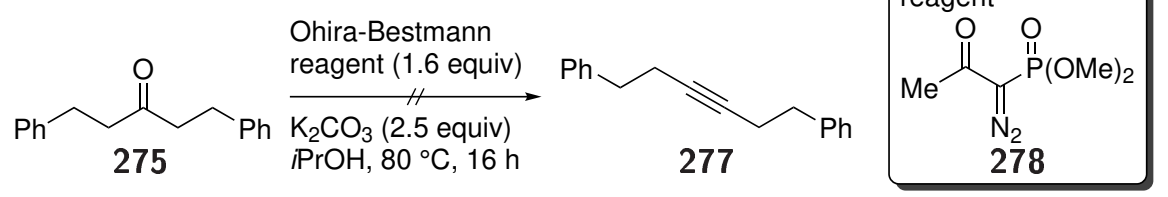

Due to the transformation of a carbonyl group into a triple bond not being successful, the introduction of the triple bond as a whole was considered. The groups of BROADBENT and ZHOU used acetylene bismagnesium bromide for the synthesis of symmetric alkynes. 207,208] Based on their work, the synthesis of propargylic diol $\mathbf{2 8 0}$ was conducted by first forming the bis-GRIGNARD reagent from ethinyl magnesium bromide and ethyl magnesium bromide and then adding phenyl acetaldehyde (2.2 equiv) Equation 3.40. Compound $\mathbf{2 8 0}$ was obtained in $35 \%$ yield.

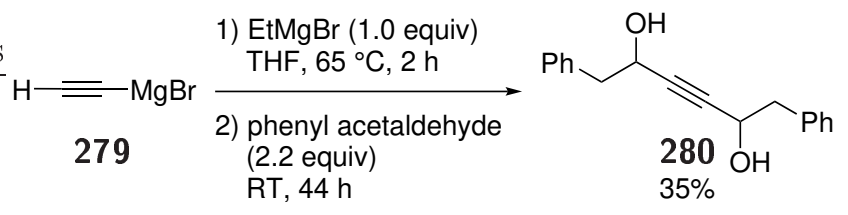


In order to reduce diol $\mathbf{2 8 0}$, the triple bond was protected as dicobalt complex $\mathbf{2 8 1}$ using 1.0 equiv $\mathrm{Co}_{2}(\mathrm{CO})_{8} \cdot \stackrel{[209]}{2}$ The reduction was conducted with 4.2 equiv of borane dimethylsulfide complex and the final deprotection of complex 282 with 3.8 equiv $\mathrm{Fe}\left(\mathrm{NO}_{3}\right)_{2}$ afforded alkyne 242d in $54 \%$ yield [Scheme 3.16). [209]
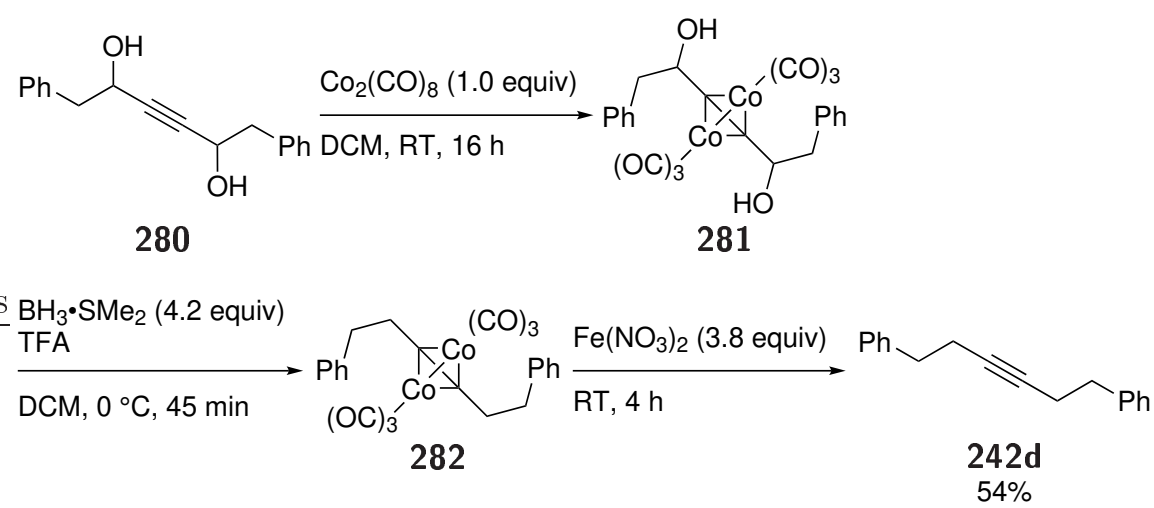

Scheme 3.16: Protection of alkyne $\mathbf{2 8 0}$ as dicobalt complex 281 and subsequent reduction and deprotection. [209]

The allenylation of the obtained alkynes 242 was conducted under optimized conditions with $2.5 \mathrm{~mol} \%$ (2-anisyl) ${ }_{2} \mathrm{Se}_{2}, 1.2$ equiv NFSI, 1.0 equiv $\mathrm{Li}_{2} \mathrm{CO}_{3}$ and molecular sieves in toluene at $100{ }^{\circ} \mathrm{C}$ (Table 3.39). Allenes $\mathbf{2 5 3}$ were obtained in moderate to good yields. Alkynes 242a and 242d, that contained simple alkyl or aryl moieties, gave similarly high yields of 60 and $58 \%$, respectively. With TBS ether $\mathbf{2 4 2 b}$ and pivalic acid ester 242c, two products, the internal allene and the terminal allene, were formed in combined yields of 32 and $28 \%$, respectively. The internal allenes have a signal for the vinylic proton at 5.18 or $5.12 \mathrm{ppm}$, respectively, which is similar to the shift of $5.02 \mathrm{ppm}$ of the respective signal in allene 253a. The signal of the allylic methylene group splits into two doublets of doublets at approx. $4.2 \mathrm{ppm}$ due to the axial chirality of the molecule. In comparison, in the spectrum of the terminal allene, two triplets at 4.7 and 3.7$4.2 \mathrm{ppm}$ were observed for the vinylic protons and the carbinol protons (Figure 3.12). The ratio of the two products was 1.5:1 (TBS ether) or 2.7:1 (pivalic acid ester) in favor of the internal allene.

As the allenylation uses an endogenous nucleophile originating from NFSI, the question came up if the use of $[\mathrm{TMPyF}]\left[\mathrm{BF}_{4}\right](\mathbf{6 5})$ as a bulky oxidant would allow for the use of external nucleophiles, like it was conducted by ZHAO et al. in their pyridination reaction. ${ }^{69]}$ The following reactions of 5-decyne (242a) with 1.2 equiv of $[\mathrm{TMPyF}]\left[\mathrm{BF}_{4}\right](\mathbf{6 5})$ as the oxidant and 1.0 equiv of either $\mathrm{HN}\left(\mathrm{SO}_{2} \mathrm{Ph}\right)_{2}$ or $\mathrm{KN}\left(\mathrm{SO}_{2} \mathrm{Ph}\right)_{2}$ as the nucleophile did not yield the desired product, but in both cases, traces of the selenofunctionalized product $\mathbf{2 5 4}$ were observed (Equation 3.41). 
Table 3.39: Synthesis of allenylamides 253. ${ }^{\mathrm{a}}$
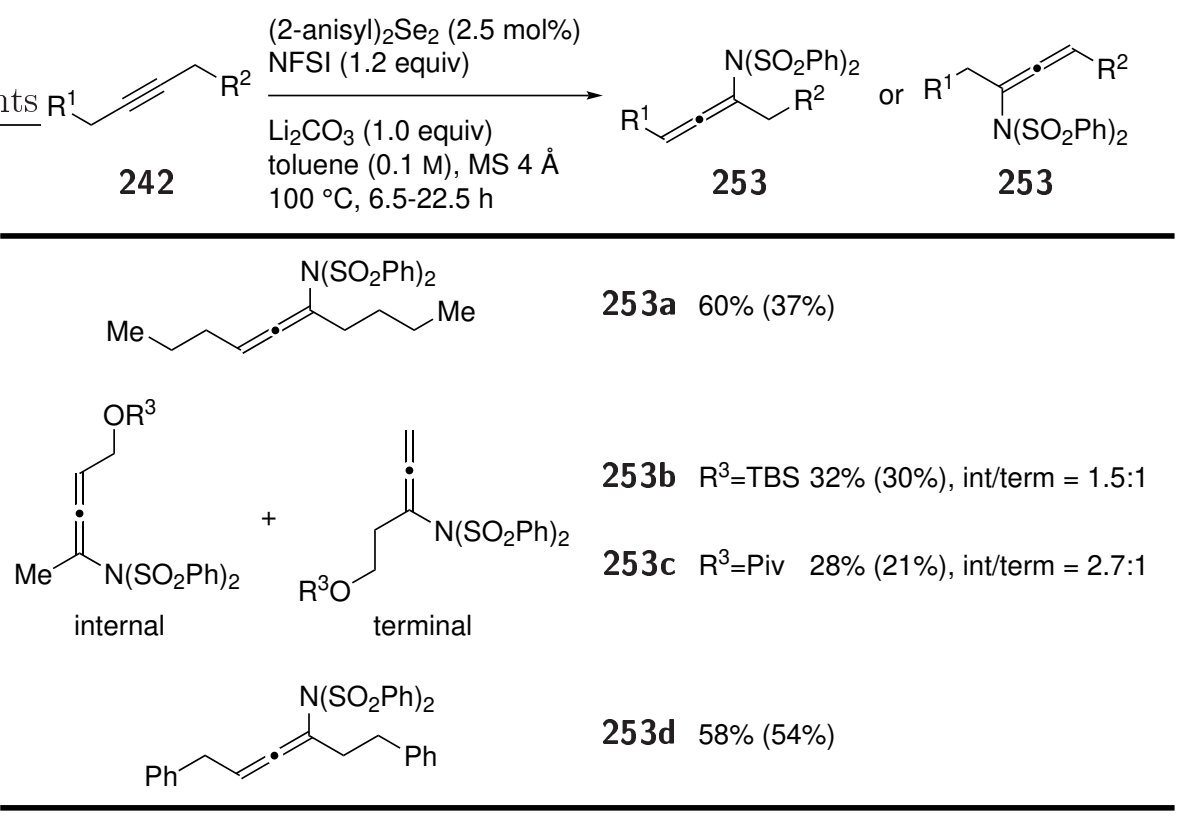

${ }^{\text {a }}$ NMR yield, standard: TMB, isolated yield in brackets.

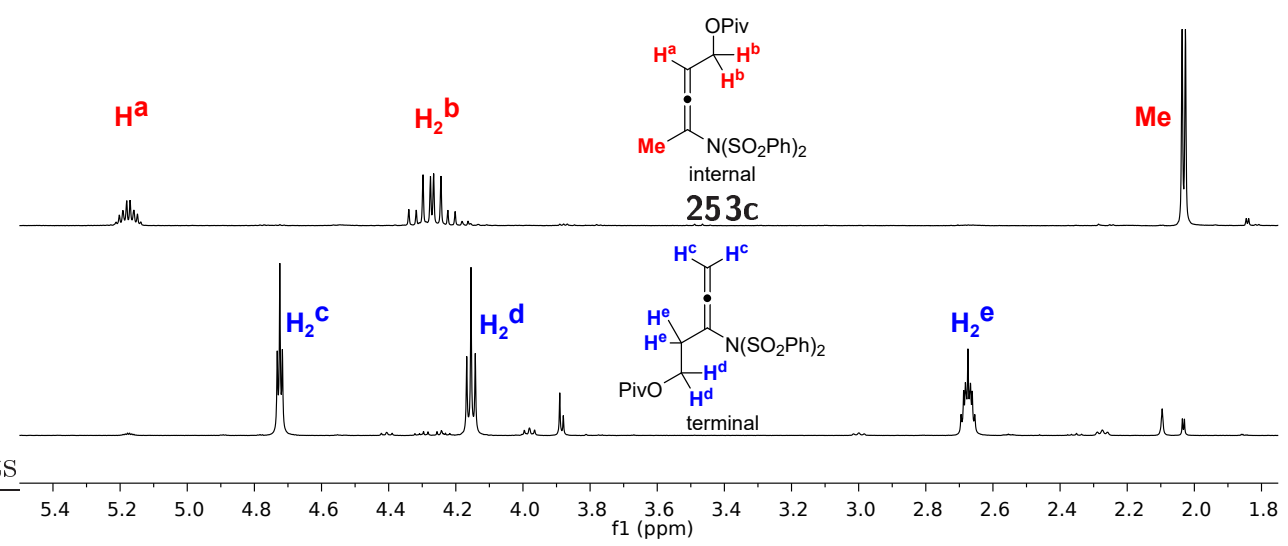

Figure 3.12: Comparison of relevant regions of the ${ }^{1} \mathrm{H}$ NMR spectra of the internal and terminal allene 253c.

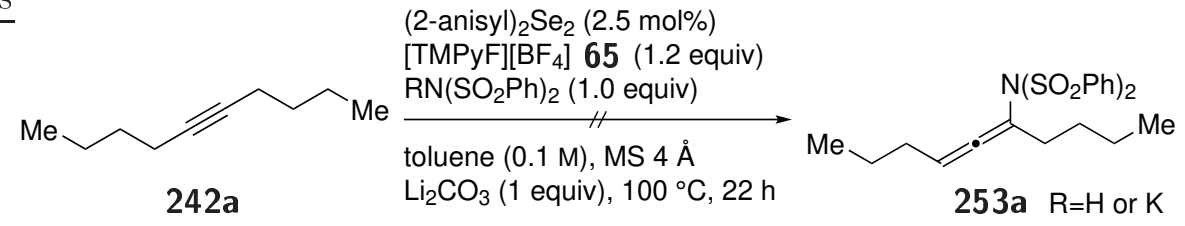

\subsubsection{Mechanistic investigations}

As was previously explained (cf. Subsection 1.1.1), selenium can act as a $\pi$-acid to activate the alkyne, but it could also activate the nucleophile by a LEWIS basic interaction. In order to find 
out if the reaction relied on a LEWIS basic activation, the reaction was carried out using different LEWIS bases instead of (2-anisyl) $)_{2} \mathrm{Se}_{2}$. When $5 \mathrm{~mol} \%$ of $\mathrm{Se}=\mathrm{PPh}_{3}$ were used, product 253a was formed in $10 \%$ yield (Table 3.40, entry 1). With $10 \mathrm{~mol} \%$ of DMF, no product formation was observed (Table 3.40, entry 2). From these results, it was concluded that LEWIS base activation played only a small role in the catalytic allenylation. To examine if the addition of a LEwIS base to the reaction under otherwise unchanged conditions would lead to an increased yield, $5 \mathrm{~mol} \%$ of $\mathrm{Se}=\mathrm{PPh}_{3}$ were added to the reaction along with $2.5 \mathrm{~mol} \%$ of (2-anisyl) ${ }_{2} \mathrm{Se}_{2}$ (Table 3.40, entry 3). The formation of allene 253a was observed in 56\%, which was a small decrease compared to the optimized reaction conditions.

Table 3.40: Allenylation of 5-decyne 242a using LEwIS base.

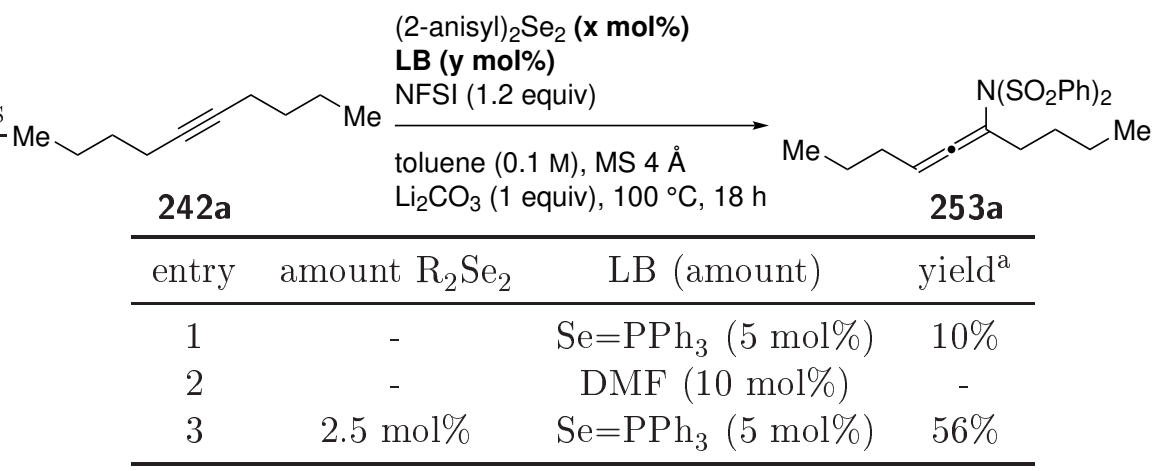

${ }^{a}$ NMR yield, standard: TMB, isolated yield in brackets.

As the allenylation of alkynes $\mathbf{2 4 2 b}$ and $\mathbf{2 4 2 c}$ resulted in moderate yields, it was attempted to increase the yield by adding $5 \mathrm{~mol} \% \mathrm{Se}=\mathrm{PPh}_{3}$. Indeed, allenes $\mathbf{2 5 3 \mathbf { b }}$ and $\mathbf{2 5 3 \mathbf { c }}$ were obtained in improved yields of 49 and 44\% (Table 3.41). Again, the formation of the internal allenes was preferred with ratios of 1.9:1 (TBS ether) or 2.3:1 (pivalic acid ester).

Table 3.41: Amidoallenylation of alkynes 242b and 242c using additional Lewis base. ${ }^{\mathrm{a}}$

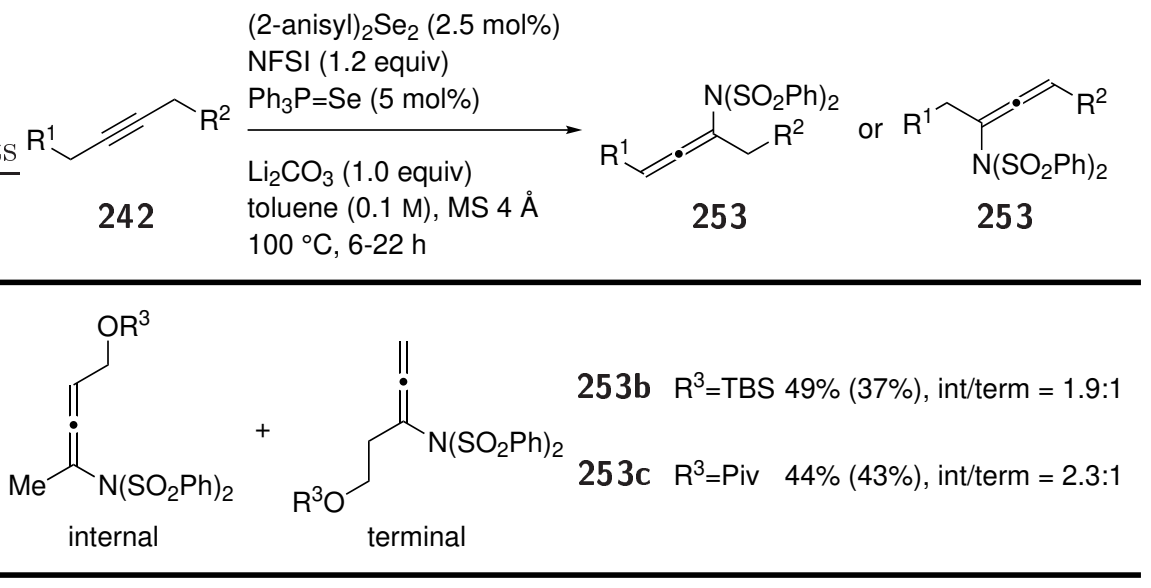

${ }^{\text {a }}$ NMR yield, standard: TMB, isolated yield in brackets. 
During the optimization of the reaction conditions, the formation of the selenofunctionalized compound $\mathbf{2 5 4}$ was observed in many cases, which is consistent with the isolation of such a species by REICH and BACK. $\stackrel{[190-193]}{-19}$ As BACK was able to transfer the selenium adduct into the respective allene by oxidizing the selenium moiety (cf. Scheme 3.13), it was assumed that compound $\mathbf{2 5 4}$ was an intermediate in the selenium-catalyzed allenylation and its oxidation would lead to the elimination of the selenium species and formation of allenes 253. In order to verify this assumption by further experiments, compound $\mathbf{2 5 4}$ was synthesized from 5-decyne (242a) with 0.5 equiv of (2-anisyl) ${ }_{2} \mathrm{Se}_{2}$ and 1.0 equiv of NFSI in $91 \%$ Equation 3.42.

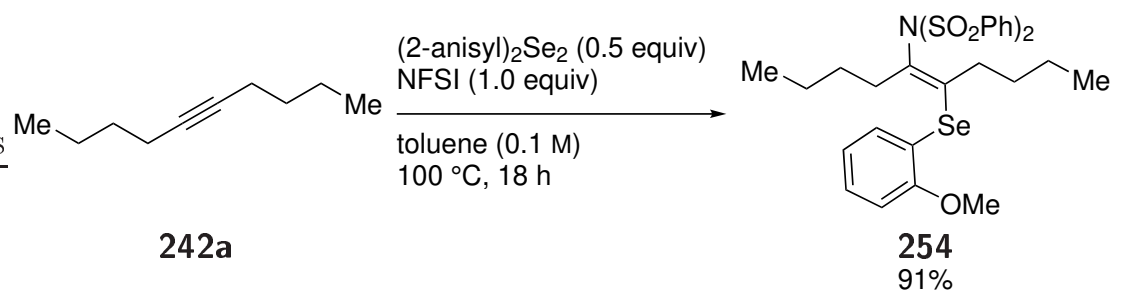

In the first experiments, selenofunctionalized product $\mathbf{2 5 4}$ was subjected to the optimized reaction conditions, but the diselenide catalyst was left out. The formation of product 253a was only observed in traces, leading to the conclusion that the oxidation of the selenium moiety by NFSI was not enough to initiate its elimination (Table 3.42, entries 1 and 2). During the mechanistic investigations of the photo-aerobic selenium- $\pi$-acid catalyzed lactonization, the formation of trimeric selenonium ions from diphenyl diselenide was observed.144] It was proposed that these trimers transfer a phenylselenium moiety onto the substrate. The hypothetical transfer of such a moiety onto selenofunctionalized product $\mathbf{2 5 4}$ could facilitate the elimination of the selenium moiety. Therefore the question arose, if the addition of diphenyl diselenide to the intermediate would lead to the formation of product 253a. Consequently, the reaction was repeated and 0.5 equiv $\mathrm{Ph}_{2} \mathrm{Se}_{2}$ were added (Table 3.42, entry 3). Unfortunately, no product formation was observed.

Table 3.42: Experiments on the formation of allene 253a from compound 254.

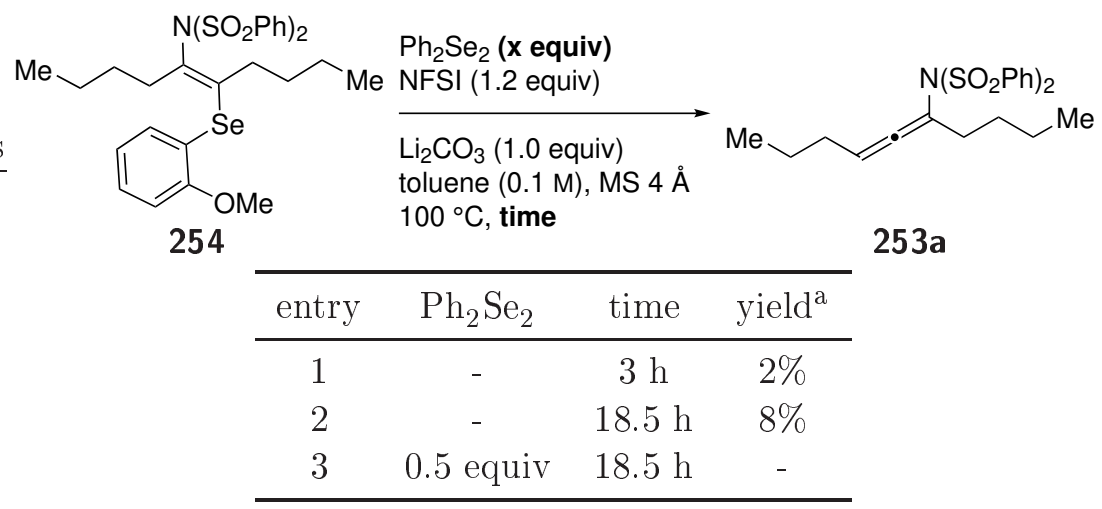

${ }^{\mathrm{a}}$ NMR yield, standard: TMB. 
As the product formation from compound $\mathbf{2 5 4}$ could not be initiated with an excess of NFSI or the addition of diphenyl diselenide, it came to mind that the transfer of the oxidized selenium moiety to an unreacted alkyne molecule could lead to product formation and propagate the reaction. Such a mechanism is remotely reminiscent of the transfer of chalcogenium groups between olefins which was reported by Denmark (cf. Subsection 1.2.4) and would explain the observation of leftover selenofunctionalized intermediate after full conversion of the alkyne starting material. 109,110$]$ To examine if the investigated reaction followed this mechanism, selenofunctionalized compound $\mathbf{2 5 4}$ was reacted with 1.0 equiv 2,9-dimethyl-5-decyne and 0.95 equiv NFSI to avoid the decomposition of the alkyne by an excess of oxidant (Equation 3.43). Indeed, the formation of allenes 253a and 253e in 33 and $18 \%$ yield, the formation of the selenofunctionalization product 284 from the added alkyne in $22 \%$ yield and the formation of (2-anisyl) ${ }_{2} \mathrm{Se}_{2}$ in $9 \%$ were observed.

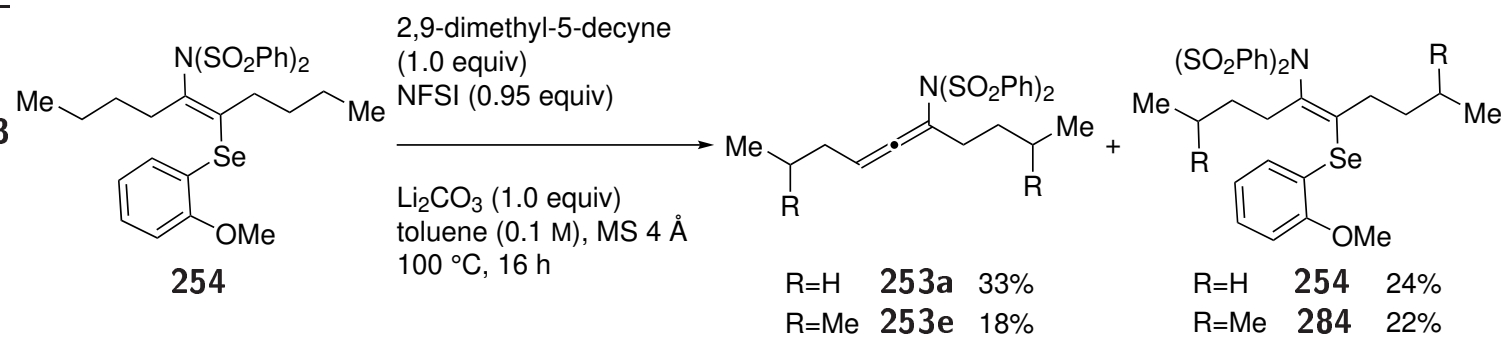

Based on these findings, a mechanism for the reaction was proposed (Scheme 3.17). In the initial phase, the diselenide catalyst is oxidized by NFSI and selenirenium ion $\mathbf{2 8 5}$ is formed from $\mathrm{ArSe}^{+}$and alkyne 242. Opening of the ion by $\mathrm{N}\left(\mathrm{SO}_{2} \mathrm{Ph}\right)_{2}{ }^{-}$and subsequent oxidation by another entity of NFSI leads to the formation of oxidized selenofunctionalized intermediate 287. The elimination of the selenium moiety is facilitated by the coordination of alkyne $\mathbf{2 4 2}$ (intermediate 288) and product $\mathbf{2 5 3}$ is released with simultaneous reformation of selenirenium ion $\mathbf{2 8 5}$ from the selenium moiety and the coordinated alkyne. 


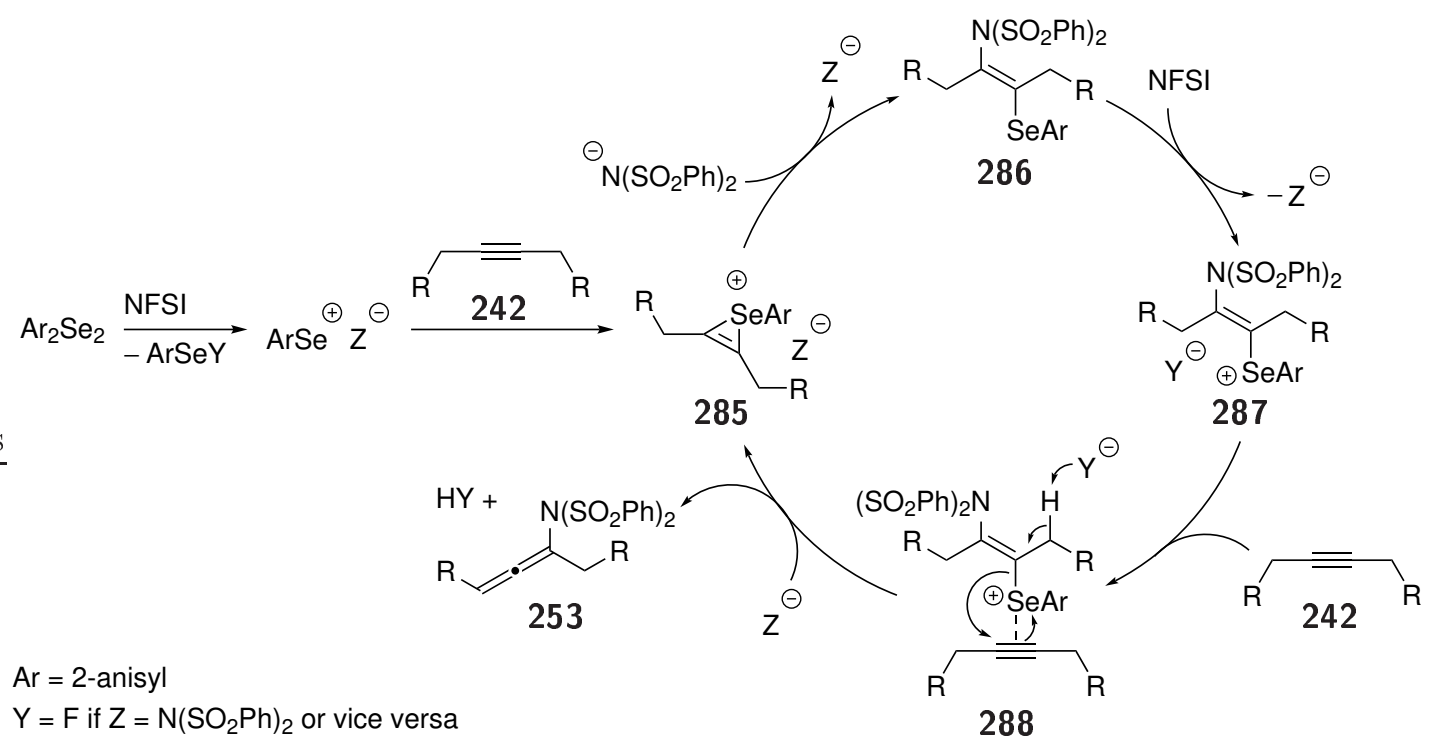

Scheme 3.17: Proposed catalytic cycle for the selenium- $\pi$-acid catalyzed synthesis of allenylamides. 


\subsection{Synthesis and application of chiral diselenide catalysts}

As was already explained (cf. Subsection 1.2.4, Section 2), the synthesis of sterically demanding diselenides was to be examined and binaphthyl derivatives appeared as suitable structural motifs. The first of two designs of chiral binaphthyl-derived diselenides, compound 121, is based on the chiral phosphoric acids 290 used by AKIYAma, TeradA and List. $\stackrel{[120-123]}{1}$ An important aspect in the design of phosphoric acids $\mathbf{2 9 0}$ was the attachment of substituted aryl moieties ortho to the oxygen atoms, as using an unsubstituted phenyl ring led to low selectivity, but substitution with bulky 2,4,6-triisopropylphenyl moieties resulted in high selectivity. ${ }^{[123]}$ Therefore, diselenide 121 should also feature this kind of substitution (Figure 3.13, left side). The second design of chiral binaphthyl diselenides is based on a work by TOMODA and IwAOKA, who used diselenide 291 in an asymmetric ring-opening reaction and achieved $e e$ 's up to 50\%. $\frac{[210]}{2}$ In order to improve the selectivity, envisioned catalyst $\mathbf{1 2 2}$ contains additional aryl moieties in proximity to selenium (Figure 3.13, right side).

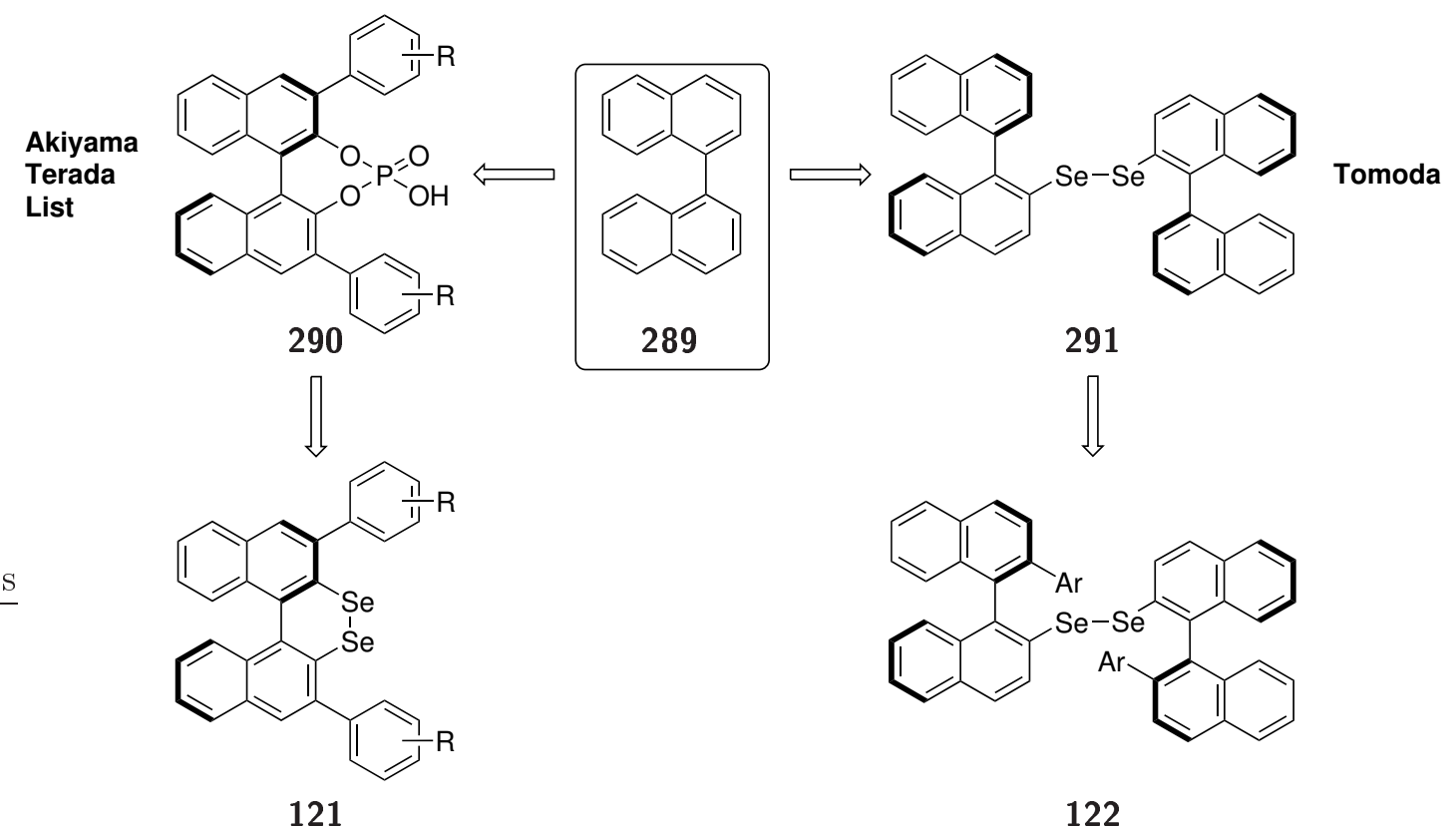

Figure 3.13: Binaphthyl (289) and derived chiral catalysts.

\subsubsection{Examination of selenation methods}

As the selenation step is crucial in the synthesis of diselenide catalysts, preliminary investigations on different methods for the introduction of the diselenide bridge in binaphthyl derivatives were carried out. A common method for diselenation is the metalation of an aryl halide and subsequent selenation with selenium powder.114,211] The reaction of racemic binaphthyl dibromide 292 with tert-butyl lithium (4.4 equiv) at $-78{ }^{\circ} \mathrm{C}$, subsequent addition of selenium powder (2.1 equiv) and heating at $60{ }^{\circ} \mathrm{C}$ afforded the diselenide 293 in $12 \%$ yield Equation 3.44. 


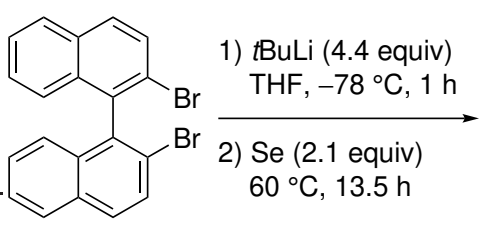

292

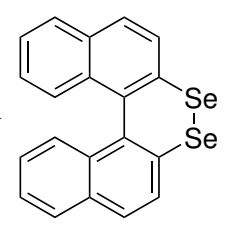

293

As an alternative method, the reduction of selenocyanates was examined. Selenocyanates are easily reduced or hydrolized to diselenides in reactions with hydrides, hydroxides or alkoxides. $.212-214]$ As they can be obtained in a SANDMEYER-type reaction from amines, the preparation of binaphthyl diselenide from binaphthyl diamine would only take two steps. The first attempt of the synthesis of $(R)$-2,2'-diselenocyanato-1,1'-binaphthalene (295) followed the synthesis of an aryl selenocyanate by NAKAmURA and coworkers. ${ }^{[215]}$ The diazonium compound was prepared from $(R)$-BINAM (294) by addition of $6 \mathrm{~N} \mathrm{HCl}$ (12 equiv) and $\mathrm{NaNO}_{2}$ (3.0 equiv) at $0{ }^{\circ} \mathrm{C}$. The selenocyanate source (KSeCN, 2.2 equiv) was added as an aqueous solution (Table 3.43, entry 1). The formation of the product was not observed, instead two side products were isolated. ${ }^{1} \mathrm{H}$ NMR analysis of the side products showed only signals in the aromatic region. In both cases, the integrals indicated symmetrically substituted products. The amount of signals in the ${ }^{13} \mathrm{C}$ NMR spectrum supported this assumption. Furthermore, as in both cases only 10 signals were observed, functionalization with a carbon-containing unit can be excluded. In both products, one proton was shifted to low field (8.62 ppm and $9.37 \mathrm{ppm}$, respectively) compared to the signals in $(R)$-BINAM (294), which are between 7 and 8 ppm, so an electron-withdrawing substituent seems probable. $[216]$ Since TOMODA had reported an indicative signal at $100.7 \mathrm{ppm}$ for the SeCN moiety, which was not observed in either of the products, and the correct mass was not found in mass spectrometric analysis, it was concluded that the desired product had not been formed. $[210]$ As a consequence, the conditions for the diazonium salt formation as well as the conditions for the addition of the nucleophile were changed. The diazonium salt was formed with 7.0 equiv tert-butyl nitrite and 8.0 equiv boron trifluoride diethyl etherate at $-30{ }^{\circ} \mathrm{C}$ and $-5{ }^{\circ} \mathrm{C}$. It was isolated by filtration and added to a solution of 2.2 equiv KSeCN in acetonitrile at $0{ }^{\circ} \mathrm{C}$ (Table 3.43, entry 2). The new conditions led to the formation of the product in $5 \%$ yield. The analytical data matched those reported by TOMODA et al.210]

In order to increase the yield, several variations for the addition of the nucleophile were tested. The diazonium salt was prepared with tert-butyl nitrite as described above, but the filtration of the diazonium salt was avoided in order not to lose too much of the compound in the filter. First, a one-pot synthesis was attempted. The diazonium salt was kept in THF and KSeCN was added in THF. As the solubility in THF of both the salt and KSeCN were low, the desired product was not formed (Table 3.43, entry 3). In the next reaction, the solvent was removed by a syringe and $\mathrm{KSeCN}$ was added in acetonitrile. The product was obtained in $14 \%$ yield (Table 3.43, entry 4). In order to increase the nucleophilicity of the selenocyanate, 2.2 equiv 18-crown- 6 were added to 
the solution of KSeCN in acetonitrile, which was then added to the diazonium salt. The previous results could not be improved and the yield in this reaction was $12 \%$ (Table 3.43, entry 5). As the yield was still quite low, no further attempts to synthesize the desired product were made.

Table 3.43: Tested conditions for the synthesis of $(R)-2,2^{\prime}$-diselenocyanato- $1,1^{\prime}$-binaphthalene (295).

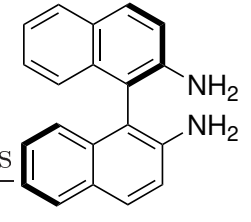

294

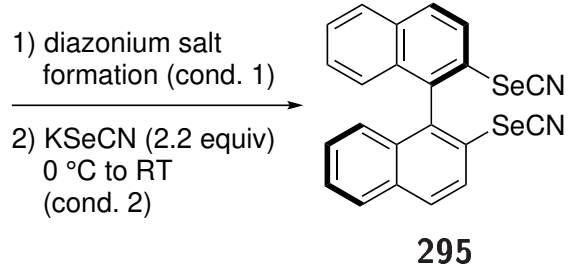

295

\begin{tabular}{cccc}
\hline entry & conditions 1 & conditions 2 & yield \\
\hline 1 & $\mathrm{NaNO}_{2}, 6 \mathrm{~N} \mathrm{HCl}, 0{ }^{\circ} \mathrm{C}$ & $\mathrm{H}_{2} \mathrm{O}$ & - \\
2 & $t \mathrm{BuNO}_{2}, \mathrm{BF}_{3} \cdot \mathrm{OEt}_{2}$ & $\mathrm{MeCN}$ & $5 \%$ \\
3 & $t \mathrm{BuNO}_{2}, \mathrm{BF}_{3} \cdot \mathrm{OEt}_{2}$ & one-pot; THF & - \\
4 & $t \mathrm{BuNO}_{2}, \mathrm{BF}_{3} \cdot \mathrm{OEt}_{2}$ & remove solvent by syringe; MeCN & $14 \%$ \\
5 & $t \mathrm{BuNO}_{2}, \mathrm{BF}_{3} \cdot \mathrm{OEt}_{2}$ & remove solvent by syringe; 18-crown-6 in MeCN & $12 \%$ \\
\hline
\end{tabular}

Another possible way for the selenation could be the synthesis of an aryl alkyl selenide, followed by cleavage of the alkyl residue. The attempted synthesis of the selenide was carried out using a triflate and dibenzyl diselenide. For this purpose, phenyl trifluoromethanesulfonate (297) was prepared from phenol (296) in 57\% yield, using pyridine (2.0 equiv) and trifluoromethanesulfonic anhydride (1.2 equiv) following a procedure by HuffmAN and colleagues (Equation 3.45). [217]

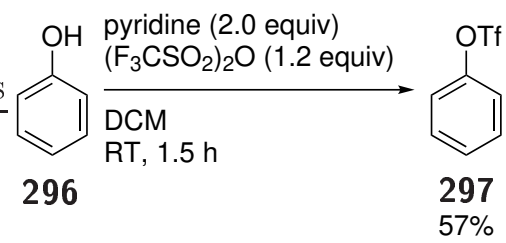

In order to synthesize phenyl benzyl selenide (300), the preparation of benzylselenol (299) was attempted using two different methods. $\frac{[218,219]}{2 n}$ the first method, 1.5 equiv sodium borohydride were added to a solution of dibenzyl diselenide (298) in $n$-butanol and stirred for $4 \mathrm{~h}$ at room temperature. In the second method, 2.0 equiv sodium borohydride were added to a solution of dibenzyl diselenide (298) in a mixture of THF and ethanol (3:1) and stirred for $1 \mathrm{~h}$ at room temperature. The decoloration of the formerly yellow solutions indicated the reduction of the diselenide to the selenolate. To both solutions, $\mathrm{Pd}\left(\mathrm{PPh}_{3}\right)_{4}(10 \mathrm{~mol} \%)$ and phenyl triflate (297, 2.0 equiv) were added and heated at $65{ }^{\circ} \mathrm{C}$ for $18 \mathrm{~h}$ (Equation 3.46). 220.221$]$ In both cases, the desired product could not be isolated, but dibenzyl diselenide was reisolated. A possible reason for this is the recombination reaction of benzylselenol to dibenzyl diselenide being faster than the reaction with phenyl triflate. 


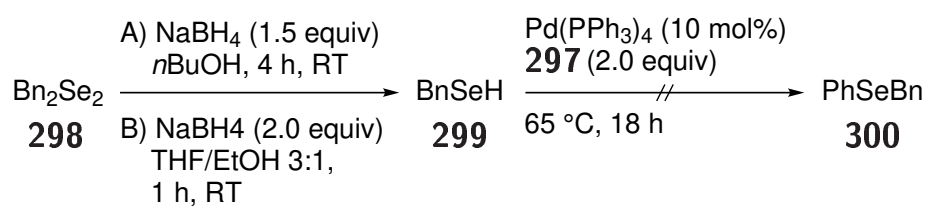

The Newman-KWART rearrangement is a way of obtaining thiophenols from phenols. This is accomplished by a rearrangement of thiocarbamates that can be either thermally induced [222,223] or palladium-catalyzed. [224] In 2013, PitTelkow and coworkers presented a variation of this reaction that allowed to make selenophenols from phenols by using a thermally induced rearrangement similar to the NEWMAN-KwART rearrangement. ${ }^{[225]}$ They used $O$-aryl selenocarbamates that undergo a thermally induced rearrangement to $S e$-aryl selenocarbamates. In order to apply this protocol to the synthesis of binaphthyl diselenides, binaphthyl di $(O$-aryl selenocarbamate) could be used for the rearrangement, and the carbamate moieties cleaved to obtain the diselenol, which could in turn be oxidized to the diselenide. The synthesis of $O$-aryl selenocarbamate 302 was performed according to PitTelkow et al. 225$]$ Racemic BINOL (301) was heated with 2.0 equiv of $N$-(dichloromethylene)- $N$-methylmethanaminium chloride (304) for $1 \mathrm{~h}$ and the solution was added to a freshly prepared NaHSe solution (Equation 3.47). Unfortunately, the desired disubstituted product was not formed and instead, two monosubstituted products were isolated in traces. The shifts of the ${ }^{1} \mathrm{H}$ NMR signals in the aromatic region differed only slightly, but the two singlets of the $\mathrm{NMe}_{2}$ moiety were observed at 3.30 and 2.59 ppm for product $\mathbf{3 0 2}$ and at 2.74 and $2.43 \mathrm{ppm}$ for product 303. By comparison with literature and mass spectrometric analysis, the compounds were identified as the mono-selenocarbamate (compound 302) and the mono-carbamate (compound 303).

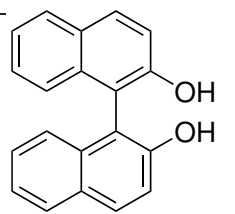

301

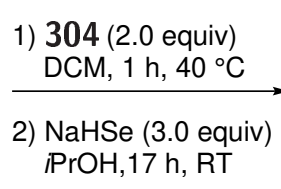

iPrOH,17 h, RT

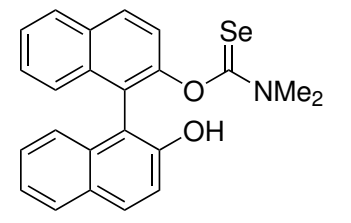

302

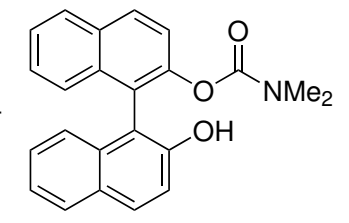

303

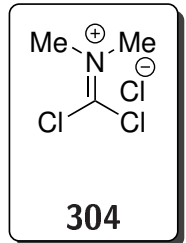

As for the synthesis of thiocarbamates, $N, N$-dimethylthiocarbamoyl chloride is used, the use of

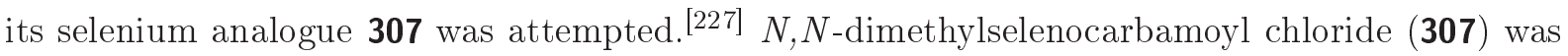
prepared from dichloromethylene-dimethyliminium chloride (306), 1.0 equiv lithium aluminum hydride and 1.0 equiv selenium according to a synthesis of IMAKUBO (Equation 3.48). [228]

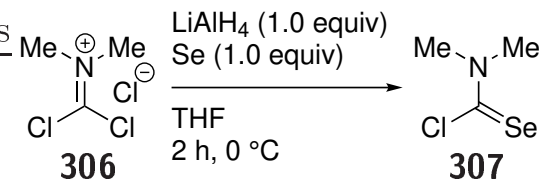


The prepared reagent was then used without further purification in a reaction with the freshly prepared sodium alcoholate of racemic BINOL (301) Equation 3.49). Unfortunately, the desired product was not formed and BINOL (301) was completely reisolated.

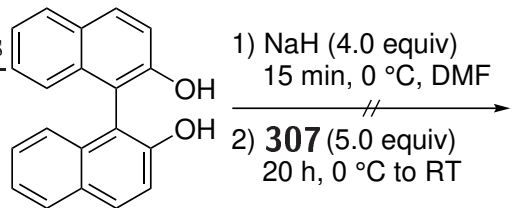

301<smiles>CNC(=O)Oc1ccc2ccccc2c1-c1c(OC([Se])=[Se])ccc2ccccc12</smiles>

308

\subsubsection{Synthesis of di(binaphthyl) diselenides}

The synthesis of di(binaphthyl)diselenides 122 began with the formation of binaphthyl dihalides 309, 310 and 311 from $(R)$-BINAM (294) via SANDMEYER-type reactions. 229-232] The formation of the diazonium salt was achieved with tert-butyl nitrite or sodium nitrite and the corresponding copper(I) or potassium salts as halogen source (Table 3.44). For the bromination and iodination, $48 \% \mathrm{HBr}$ and PTSA were used as additives. The best yield of $58 \%$ was obtained in the iodination reaction, the chlorination had a similar yield $(56 \%)$ and the bromination provided the product in $50 \%$ yield.

Table 3.44: Halogenation of ( $R$ )-BINAM (294) in SANDMEYER-type reactions.

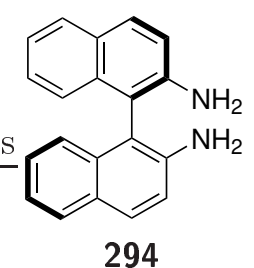

294

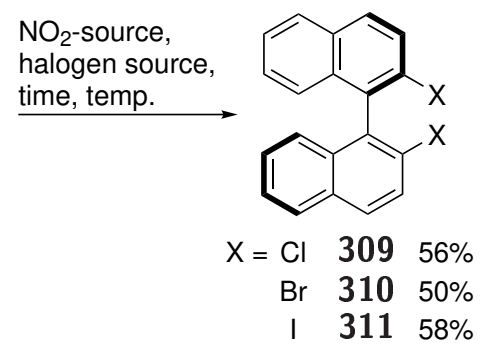

$\mathrm{NO}_{2}$-source, halogen source, time, temp.

$\mathrm{X}=\mathrm{Cl} \quad 309 \quad 56 \%$

$\mathrm{Br} 31050 \%$

I $31158 \%$

\begin{tabular}{cccccc}
\hline product & $\begin{array}{l}\mathrm{NO}_{2} \text {-source } \\
\text { (equiv) }\end{array}$ & $\begin{array}{l}\text { halogen source } \\
\text { (equiv) }\end{array}$ & $\begin{array}{l}\text { additive } \\
\text { (equiv) }\end{array}$ & time, temp. & yield \\
\hline $\mathbf{3 0 9}$ & $t \mathrm{BuNO}_{2}(4.2)$ & $\mathrm{CuCl}_{2}(2.6$ equiv) & - & $65{ }^{\circ} \mathrm{C}, 16 \mathrm{~h}$ & $56 \%$ \\
$\mathbf{3 1 0}$ & $\mathrm{NaNO}_{2}(3.0)$ & $\mathrm{CuBr}(2.5$ equiv) & $\mathrm{HBr}$ & $0{ }^{\circ} \mathrm{C}, 30 \mathrm{~min} ; \mathrm{RT}, 16.5 \mathrm{~h}$ & $50 \%$ \\
$\mathbf{3 1 1}$ & $\mathrm{NaNO}_{2}(4.0)$ & $\mathrm{KI}(5.0$ equiv) & $\mathrm{PTSA}(6.0)$ & $10{ }^{\circ} \mathrm{C}, 10 \mathrm{~min} ; \mathrm{RT}, 2 \mathrm{~h}$ & $58 \%$ \\
\hline
\end{tabular}

As the yields in the halogenation reactions were moderate, a method by HAYASHI and SHIRAKAWA for the formation of dibromide $\mathbf{3 1 0}$ from the respective bis(triflate) $\mathbf{3 1 2}$ was examined. [233] Binaphthyl bis(trifluoromethanesulfonate) (312) was prepared from BINOL (301) in $94 \%$ yield using 2.4 equiv trifluoromethanesulfonic anhydride (Equation 3.50). 217 


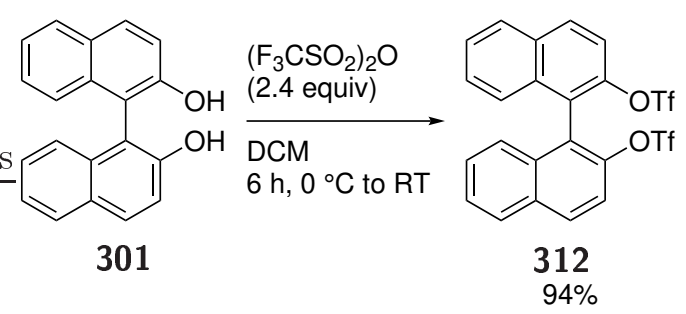

The reaction of triflate $\mathbf{3 1 2}$ with $\left[\mathrm{Cp} * \mathrm{Ru}(\mathrm{MeCN})_{3}\right] \mathrm{OTf}(5 \mathrm{~mol} \%)$ and lithium bromide (1.5 equiv) in DMI did not afford the desired product Equation 3.51]. 233] Instead, the entire starting material was reisolated.

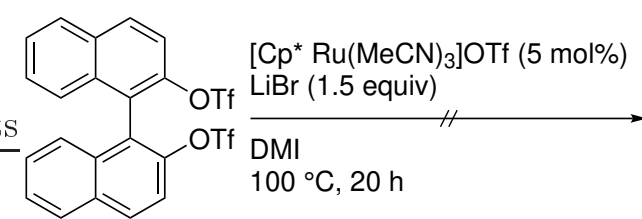

312

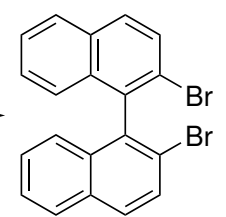

310

The monoarylation of binaphthyl dihalogenides 309, $\mathbf{3 1 0}$ and $\mathbf{3 1 1}$ was first examined by conducting a SUZUKI coupling with phenyl boronic acid. The couplings of dibromide $\mathbf{3 1 0}$ and diiodide 311 were conducted with $10 \mathrm{~mol} \% \mathrm{Pd}\left(\mathrm{PPh}_{3}\right)_{4}, 1.1$ equiv boronic acid and 4.0 equiv potassium carbonate as the base Equation 3.52]. 180$]$ Unfortunately, only a mixture of products was observed, which could not be separated by column chromatography. In the mass spectra, evidence of the desired products $\mathbf{3 1 3}$ and $\mathbf{3 1 4}$ was found, as well as a hydrogenated product $\mathbf{3 1 5}$ with a phenyl ring in 2-position and hydrogen instead of halogen in 2 -position. The diarylated product $\mathbf{3 1 6}$ was also present in the mixture. Due to the similarity of the products with only aromatic hydrogen atoms, the ratio of the products could not be determined in NMR spectroscopy. In the reaction with diiodide $\mathbf{3 1 1}, \mathbf{7 \%}$ of the starting material were reisolated.

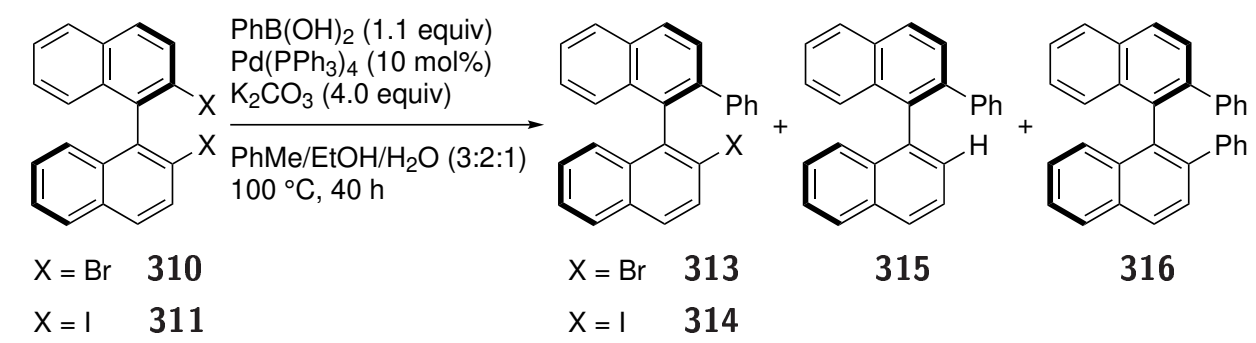

The conditions used for the dibromide and diiodide were applied in a SuzukI reaction of dichloride $\mathbf{3 0 9}$, but no conversion was observed and the starting material was recovered completely. Therefore, conditions used by CHATANI and TOBISU were applied instead and the reaction of dichloride 309 with palladium acetate ( $1 \mathrm{~mol} \%$ ), SPhos (2 mol\%), phenyl boronic acid (1.5 equiv) and potassium phosphate (2.0 equiv) showed conversion [Equation 3.53). 234$]$ A similar mixture of the desired, the hydrogenated and the diarylated product, like in the reactions of the dibro- 
mide and diiodide, was obtained, but could not be separated. $39 \%$ of the starting material were reisolated.<smiles>Clc1ccc2ccccc2c1-c1c(Cl)ccc2ccccc12</smiles>

309

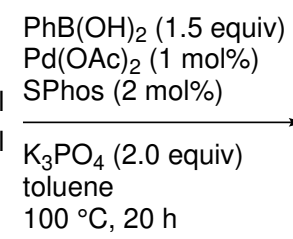

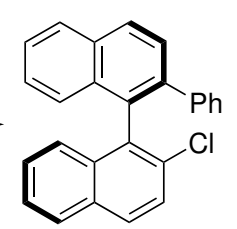

317<smiles>c1ccc(-c2cccc3cccc(-c4cccc5ccccc45)c23)cc1</smiles>

315<smiles>c1ccc(-c2cccc3cccc(-c4ccccc4)c23)cc1</smiles>

316

An alternative for this synthesis is the monoacetylation of BINAM, followed by a SANDMEYERtype transformation of the unprotected amino group into a halogen. In a cross-coupling reaction, this halogen atom could be substituted by an aryl residue. After deprotection and another SANDMEYER-type halogenation, the precursor for the selenation would be obtained (Scheme 3.18).<smiles>Nc1ccc2ccccc2c1-c1c(N)ccc2ccccc12</smiles>

294<smiles>Nc1ccc2ccccc2c1-c1c(N)ccc2ccccc12</smiles>

318<smiles>[X]c1ccc2ccccc2c1-c1c([Y])ccc2ccccc12</smiles>

319

Scheme 3.18: Possible synthesis of monoarylated binaphthyl halogenide $\mathbf{3 1 9}$.

The monoacetylation reaction was attempted with a procedure by SHI and colleagues using

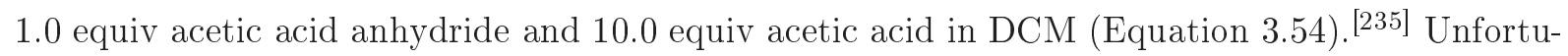
nately, all material was converted to the diacetylated product, which can be seen the ${ }^{1} \mathrm{H}$ NMR spectrum, where the symmetric substitution is indicated by a single set of aromatic protons between 7.0 and $8.5 \mathrm{ppm}$ and two matching singlets at $6.91(\mathrm{NH})$ and $1.83 \mathrm{ppm}$ (methyl at acetyl moiety) (Figure 3.14). This assumption was confirmed by a comparison with spectra available for the monoacetylated product. ${ }^{[236]}$

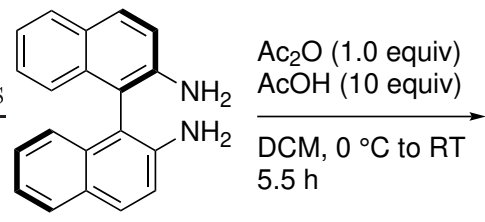

294

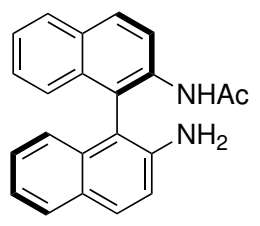

320

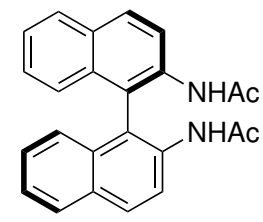

321

\subsubsection{Synthesis of arylated binaphthyl diselenides}

As the attempts in the synthesis of di(binapthyl) diselenides did not afford the desired products, the work was focussed on the synthesis of binaphthyl diselenides. In order to synthesize sterically demanding catalysts, the arylation of binaphthyl derivatives in 3 - and 3 '-position was 


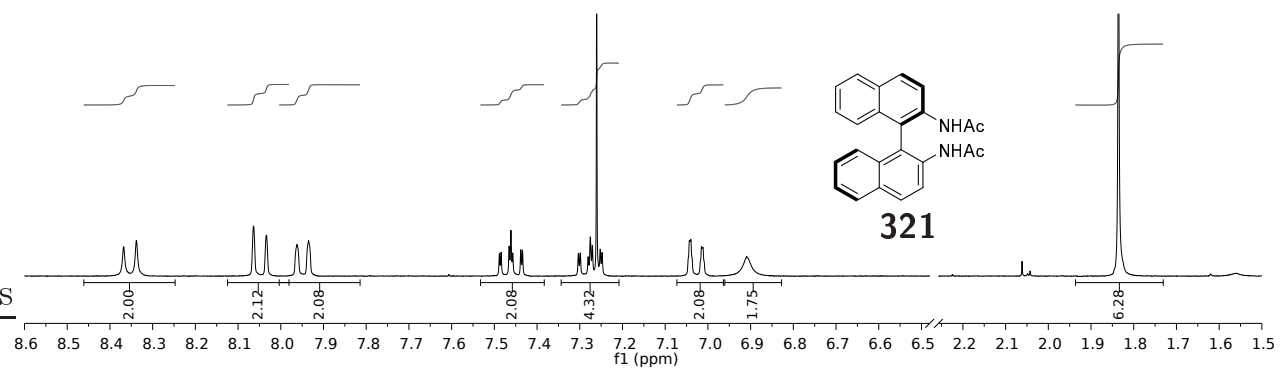

Figure 3.14: ${ }^{1} \mathrm{H}$ NMR spectrum of the product of the attempted monoacetylation.

attempted. One way of achieving this is the protection of the amino functions of BINAM (294) with acetyl moieties, followed by arylation via Daugulis-ZAitsev coupling. The acetylation of the amino groups was conducted with DMAP ( 0.1 equiv) and acetic acid anhydride as the solvent (12.0 equiv) Equation 3.55. [237] The desired product $\mathbf{3 2 1}$ was obtained in a good yield of $74 \%$, but side product 322 was isolated in $24 \%$ yield. This side product contains one additional acetyl moiety at one of the nitrogen atoms, which was determined from the three singlets at $2.28,1.96$ and $1.80 \mathrm{ppm}$ with a respective integral of $3(3 \times \mathrm{Ac})$ and the signals in the range between 6.8 and $8.5 \mathrm{ppm}$ with a combined integral of 13 (aromatic system and $\mathrm{NH}$ ). As in the previously attempted monoacetylation Equation 3.54, the diacetylated product was obtained in quantitative yield without a side product, that approach was preferred for future syntheses of diacetylated BINAM (321).

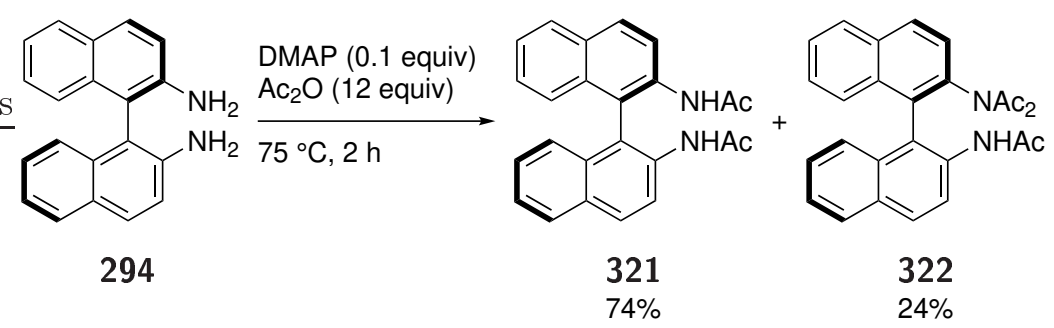

The arylation of the protected binaphthyl diamine $\mathbf{3 2 1}$ was carried out as described by STAHL et al. with a DAugulis-ZAitsev coupling. $\stackrel{\text { [237] }}{ }$ Bisamide 321 was heated to $72{ }^{\circ} \mathrm{C}$ with palladium acetate ( 0.15 equiv), silver acetate (2.3 equiv) and iodobenzene ( 5.0 equiv) in trifluoroacetic acid Equation 3.56). The reaction afforded a mixture of products that could not be separated. The formation of the desired product could be determined by mass spectrometry, but the isolation of the product was not successful.

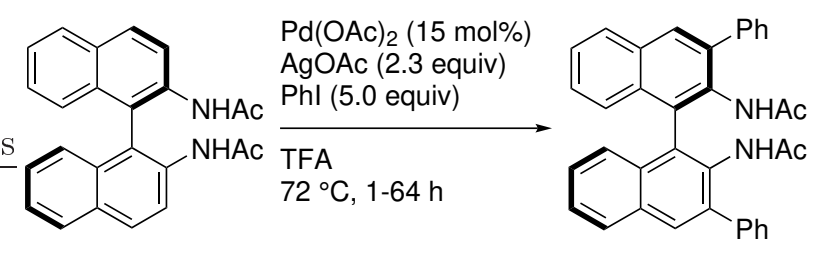


As an alternative route to $3,3^{\prime}$-arylated binaphthyl derivatives, a strategy based on works of WIDHALM ${ }^{[238]}$ and MATTSON ${ }^{[239]}$ was carried out. The synthesis started from $(R)-2,2^{\prime}$-dibromo- $1,1^{\prime}$ binaphthalene (310) with a disilylation in 3 and $3^{\prime}$ position. The reaction with LiTMP (5.0 equiv) and TMSCl (15 equiv) afforded the disilylated product $\mathbf{3 2 4}$ in $69 \%$ yield (Equation 3.57 ). The successful reaction was indicated by the appearance of the signals of the trimethylsilyl groups at $0.53 \mathrm{ppm}$ and the disappearance of the signals of two protons in the aromatic region. Additionally, the analytical data matched the reported data by Widhalm et al. ${ }^{\text {[238] }}$ As a side product, the monosilylated product was isolated in $2 \%$.

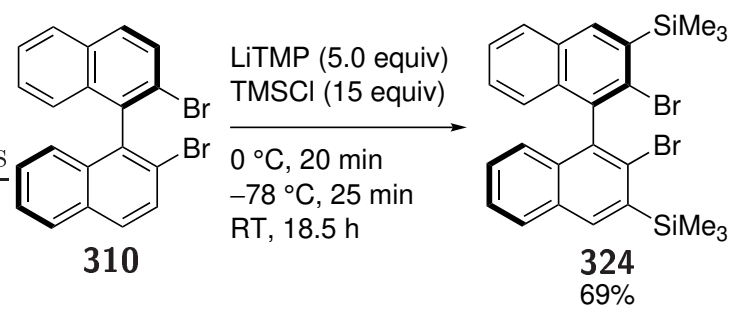

In order to facilitate a selective cross-coupling reaction, the trimethylsilyl moieties were replaced by iodides in the reaction with iodine monochloride (3.0 equiv). Dibromodiiodobinaphthalene 325 was obtained in $89 \%$ yield (Equation 3.58). In the ${ }^{1} \mathrm{H}$ NMR spectrum, the protons ortho to the iodides were shifted to lower field (singlet at $8.61 \mathrm{ppm}$ instead of $8.09 \mathrm{ppm}$ in the starting material) and the signals of the trimethylsilyl groups disappeared. The data was in agreement with the one reported by Widhalm et al. [238]

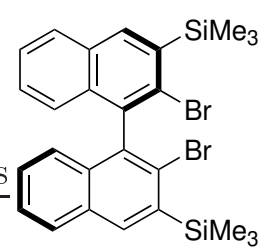

324

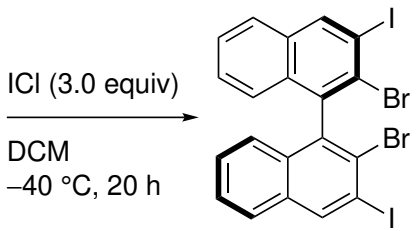

325

The Suzuki coupling of compound $\mathbf{3 2 5}$ with 2.1 equiv phenyl boronic acid to biarylated dibromide 326 was achieved in $49 \%$ yield with $5 \mathrm{~mol} \% \mathrm{Pd}\left(\mathrm{PPh}_{3}\right)_{4}$ as the catalyst and 2.2 equiv of sodium carbonate as the base (Equation 3.59). 238$]$ The reaction was chemoselective and only the iodine atoms were replaced by phenyl moieties. The formation of the product was confirmed by mass spectrometry and comparison of NMR data with literature data.[238] $7 \%$ of the starting material was reisolated, as well as $22 \%$ of the monoarylated product. The reaction with 2,4,6triisopropylphenylboronic acid under the same conditions did not afford the desired product $\mathbf{3 2 7}$, but $62 \%$ of the starting material $\mathbf{3 2 5}$ were reisolated. 


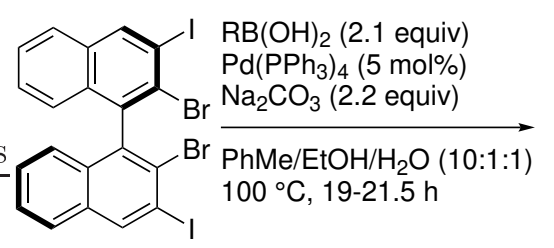

325

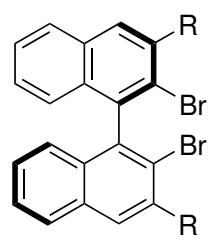

$32649 \% \mathrm{R}=\mathrm{Ph}$

$327-\mathrm{R}=2,4,6$-triisopropylphenyl

To obtain a diselenide, the diarylated binaphthyl derivative $\mathbf{3 2 6}$ was then treated with tert-butyl lithium (4.4 equiv) and selenium powder (2.1 equiv). Diselenide $\mathbf{3 2 8}$ was obtained in $21 \%$ yield Equation 3.60, which was confirmed by mass spectrometry with the help of its isotope pattern.<smiles></smiles>

326

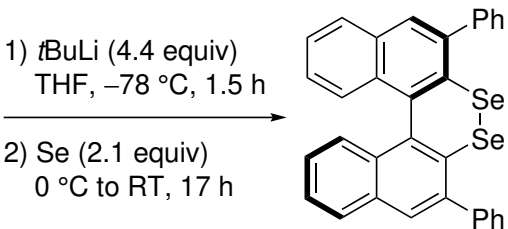

328

To see if trimethylsilyl groups were also useful as steric bulk, compound $\mathbf{3 2 4}$ was also selenated in order to examine its potential as a catalyst. The procedure corresponded to the one used for the arylated binaphthyl derivative $\mathbf{3 2 6}$ and the silylated diselenide $\mathbf{3 2 9}$ was obtained in $36 \%$ yield Equation 3.61. Its formation was also confirmed via mass spectrometry.

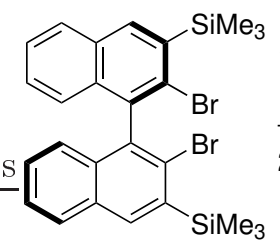

324

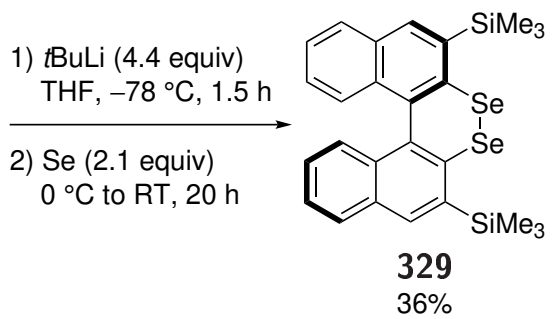

\subsubsection{Application of diselenide catalysts}

With the two binaphthyldiselenide catalysts $\mathbf{3 2 8}$ and $\mathbf{3 2 9}$ in hand, the amination of pentenoic acid ester $\mathbf{3 3 0}$ under the previously established conditions by BREDER et al. was performed (Table 3.45). W3] With catalyst 328, product 331 was obtained in 5\% yield after $114 \mathrm{~h}$. However, the enantiomeric excess was 53\%. This finding is in agreement with results by BREDER and MARUOKA, who also found that a rigid catalyst structure leads to stereoinduction. 119,240$]$ The reaction with catalyst $\mathbf{3 2 9}$ did not afford the desired product. 
Table 3.45: Amination of pentenoic acid ester 330 with chiral catalysts 328 and 329 .

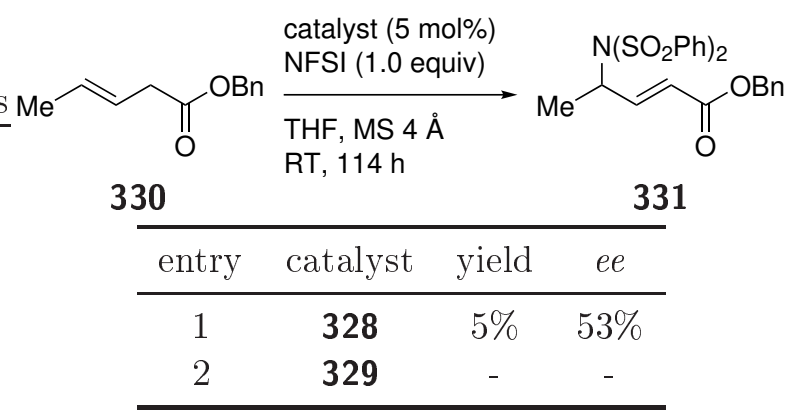




\section{Conclusion and Outlook}

Based on the recently disclosed work on photo-aerobic selenium- $\pi$-acid catalyzed acyloxylation of simple alkenes by BREDER et al., ${ }^{[11]}$ this methodology was successfully expanded to the use of alcohols as exogenous nucleophiles. ${ }^{[134]}$ Both the intramolecular and intermolecular reaction proceeded in good yields with (2-anisyl) ${ }_{2} \mathrm{Se}_{2}$ as the diselenide catalyst and $p$-MeO-TPT (95) as the photosensitizer (Scheme 4.1). In the formation of tetrahydrofurans $\mathbf{1 2 7}$ and tetrahydropyrans $\mathbf{1 4 4}$, various functional groups were tolerated. The functionalization of electron-poor alkenes $\mathbf{1 4 8}$ was most efficient with electron-poor alcohols. However, due to the weak nucleophilicity of the used alcohols, the intermolecular reaction needed higher catalyst loadings and longer reaction times which in some cases led to the decomposition of the starting material.

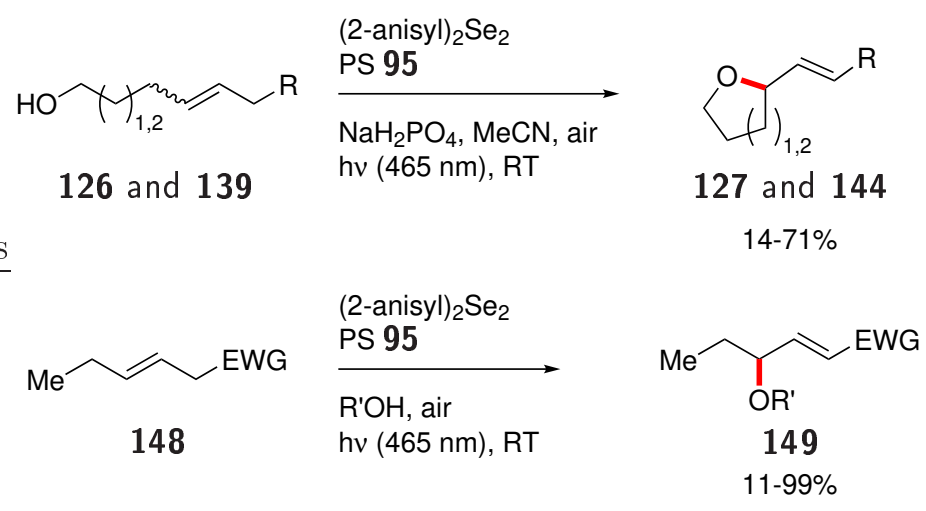

Scheme 4.1: Intra- and intermolecular etherification of alkenes 126, 139 and 148. 134$]$

Following these results, the scope of other photo-aerobic selenium- $\pi$-acid catalyzed transformations were examined after preliminary studies by C. Depken and R. Rieger. $\underline{[137,142,158]}$ In the formation of 5-membered and 6-membered ring lactones $\mathbf{7 7}$ and $\mathbf{1 7 0}$ from unsaturated acids $\mathbf{3 4}$ and 137, substrates containing carbonyl moieties, alcohols, protected amines, substituted aryls, sterically demanding branched groups and additional double bonds were tolerated and the products were afforded in good to excellent yields. 144$]$ Moreover, the 5-exo-cyclization of 4-alkenoic acid as well as the 7-exo-cyclization of a 6 -alkenoic acid showed the regioselectivity of the reaction. In the investigation of the phosphatation of alkenes, phosphates $\mathbf{1 7 3}$ were obtained in moderate to good yields, but many substrates turned out to be problematic. 159$]$ Nevertheless, these reactions demonstrate the versatility and further potential of photo-aerobic selenium- $\pi$-acid catalysis for the functionalization of alkenes with exogenous nucleophiles.

Aside from exogenous oxygen nucleophiles, the use of exogenous nitrogen nucleophiles was examined. Unfortunately, most of the used nitrogen compounds turned out to be unsuitable for the reaction. Only with saccharin, a moderate reactivity was observed, but two constitutional isomers were formed. Therefore, it was suspected that the use of intramolecular nucleophiles would lead to better results. The formation of indole 193 in 17\% yield confirmed this assumption and initiated the investigation of the formation of tetrahydroisoquinolines. Under the conditions of 
photo-aerobic selenium- $\pi$-acid catalysis, the desired product was obtained in up to $16 \%$ yield after full conversion in several optimization experiments. As this decomposition was attributed to the photocatalyst, the reaction was attempted using NFSI as the oxidant, which led to improved results. 185$]$ Hence, the scope of this reaction concerning the substitution of the aromatic core was examined and tetrahydroisoquinolines 209 were obtained in moderate to good yields.

As in previous examples for selenium- $\pi$-acid catalysis, olefins served as substrates, another topic of this work was the use of alkynes. Following preliminary experiments, 127$]$ the conditions for the selenium-catalyzed transformation of alkynes $\mathbf{2 4 2}$ into imidoallenes $\mathbf{2 5 3}$ using (2-anisyl) ${ }_{2} \mathrm{Se}_{2}$ as the catalyst and NFSI as the oxidant and nucleophile were optimized (Equation 4.1). Subsequently, different syntheses of alkynes were examined and allenes $\mathbf{2 5 3}$ were obtained in good yields. In order to understand the reaction mechanism, experiments with the selenofunctionalized reaction intermediate were conducted, which led to the conclusion that the transfer of the selenium moiety to another alkyne molecule plays an important role for the propagation of the reaction.

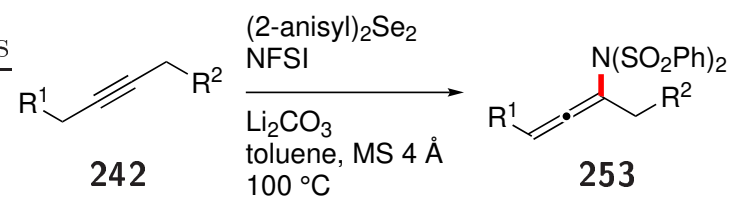

During the last part of this work, the synthesis of a binaphthyl-derived diselenide catalyst was realized and it was applied in the imidation of pentenoic acid ester $\mathbf{3 3 0}$. The product was obtained with an ee of $53 \%$, which suggested that the rigid character of the catalyst was beneficial for stereoinduction. In contrast, the yield of the reaction was very low, presumably due to the reactive center being blocked by the large phenyl moieties. Therefore, future catalyst designs should feature rigidity with simultaneous accessibility of the catalytic center.

Based on the results of this thesis, the expansion of photo-aerobic selenium- $\pi$-acid catalysis to further classes of nucleophiles, like halogens or cyanides, should be the focus of future investigations. For the application of nitrogen nucleophiles, further investigation into the oxidation potentials of the nucleophiles and formed intermediates, e.g. by CV measurements, could help to find suitable catalyst combinations and prevent decomposition of the starting materials. This methodology was already successfully applied in the investigations on the mechanism of the photo-aerobic selenium- $\pi$-acid catalyzed lactonization. $\frac{[126,127]}{12}$ Furthermore, the functionalization of alkynes provides many possibilities for further studies. Especially additional mechanistic investigations in the presented allenylation could help to develop strategies for the application of air as the terminal oxidant in cognate functionalizations with different nucleophiles. 


\section{Experimental Section}

\subsection{General Methods}

\subsubsection{Preparative Methods}

If not indicated otherwise, all reactions were performed in heat-dried glassware under an argon atmosphere. Dry solvents were distilled from a suitable drying agent and stored over molecular sieves under argon or taken from an MBraun SPS (THF, toluene, DCM, $\mathrm{Et}_{2} \mathrm{O}$ and DMF). Commercially available substances were used directly without further purification. For reactions at low temperatures, common freezing mixtures (liquid nitrogen/acetone, liquid nitrogen/isopropanol) or a cryostate TC100E-F from Huber or CORIO CD-601F from Julabo were used. For reactions with a syringe pump, an LA-100 from Landgraf Laborsysteme HLL was used. Irradiation experiments were performed at $\lambda=465 \mathrm{~nm}$ using commercially available blue LED strips (2835 super bright SMD-LEDs, 100 diodes/m, 400 lm/m, 24 V, 12 W).

\subsubsection{Chromatographic Methods}

Thin Layer Chromatography (TLC): Analytical thin layer chromatography was performed on TLC plates Silica gel $60 \mathrm{~F}_{254}$ from Merck. Reported are the $\mathrm{R}_{\mathrm{f}}$ values (substance level/solvent front level). Visualization was performed by fluorescence quenching at $254 \mathrm{~nm}$ and staining with solutions of potassium permanganate, ninhydrin or $p$-anisaldehyde.

Column chromatography: Column chromatographic separations were performed under increased pressure on silica gel 60 (0.063-0.200 mm, 70-230 mesh ASTM) from Merck.

\subsubsection{Instrumental Analysis}

IR spectra: IR spectra were measured on a Bruker FT-IR Alpha ATR or a Jasco FT/IR4600LE spectrometer.

NMR spectra: NMR spectra were recorded at $300 \mathrm{MHz}\left({ }^{1} \mathrm{H}\right)$ and $76 \mathrm{MHz}\left({ }^{13} \mathrm{C}\right)$ with Varian Unity-300, AMX 300, and Bruker Avance 300; at $400 \mathrm{MHz}\left({ }^{1} \mathrm{H}\right), 101 \mathrm{MHz}\left({ }^{13} \mathrm{C}\right), 162 \mathrm{MHz}$ $\left({ }^{31} \mathrm{P}\right), 376 \mathrm{MHz}\left({ }^{19} \mathrm{~F}\right)$ and $76 \mathrm{MHz}\left({ }^{77} \mathrm{Se}\right)$ with Varian Inova 400 and $500 \mathrm{MHz}\left({ }^{1} \mathrm{H}\right), 126 \mathrm{MHz}$ $\left({ }^{13} \mathrm{C}\right), 203 \mathrm{MHz}\left({ }^{31} \mathrm{P}\right)$ and $95 \mathrm{MHz}\left({ }^{77} \mathrm{Se}\right)$ with Varian Inova 500. Chemical shifts are reported as $\delta$-values in ppm relative to the residual peak of the deuterated solvent or its carbon atom. For characterization of the multiplicities, the following abbreviations are used: s (singlet), d (doublet), t (triplet), q (quartet), quint (quintet), sext (sextet), hept (heptet), m (multiplet), dd (doublet of doublet), td (triplet of doublet), and analogues. The coupling constants $J$ are reported in Hertz $(\mathrm{Hz})$.

Mass spectra: ESI and ESI-HRMS spectra were measured on a micrOTOF and a maXis mass spectrometer (both Bruker Daltonik). EI and EI-HRMS spectra were measured on a Joel AccuTOF. 
Melting points: Melting points were measured on a Büchi 540 apparatus or a Krüss M5000. The values were not corrected.

High Performance Liquid Chromatography: HPLC analyses were performed on an Agilent 1260 Infinity. The signals were detected on a diode array detector (DAD). The enantiomers were separated on a Chiralpak IA or Chiralcel OD column from Daicel. As solvent, a mixture of isopropanol and $n$-hexane was used.

\subsection{Synthesis of diaryl diselenides and photosensitizer 95}

\section{2,4,6-Tris(4-methoxyphenyl)pyrylium tetrafluoroborate (95) ${ }^{[182]}$}

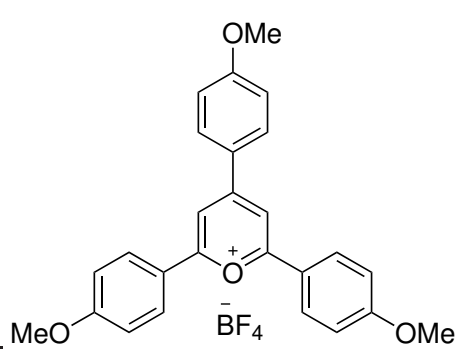

95

To a solution of $4^{\prime}$-methoxyacetophenone $(15.0 \mathrm{~g}, 100 \mathrm{mmol}$, 2.0 equiv) and freshly distilled 4-methoxybenzaldehyde $(6.08 \mathrm{~mL}$, $6.81 \mathrm{~g}, 50.0 \mathrm{mmol}, 1.0$ equiv) in dry toluene $(5 \mathrm{~mL})$ under an argon atmosphere, $\mathrm{BF}_{3} \cdot \mathrm{Et}_{2} \mathrm{O}(14.8 \mathrm{~mL}, 17.0 \mathrm{~g}, 120 \mathrm{mmol}, 2.4$ equiv) was slowly added and the mixture was stirred at $100{ }^{\circ} \mathrm{C}$ for $2 \mathrm{~h}$. The formed $\mathrm{Et}_{2} \mathrm{O}$ was removed under reduced pressure and the residue was dissolved in acetone. $\mathrm{Et}_{2} \mathrm{O}$ was added and the formed precipitate was filtered off and recrystallized from acetone. The title compound was obtained as a red solid (5.53 g, $11.4 \mathrm{mmol}, 23 \%)$.

IR (ATR): $\tilde{\nu}\left[\mathrm{cm}^{-1}\right]=2941,2841,1585,1482,1457,1434,1258,1235,1174,1016,829,562,518$; ${ }^{1} \mathbf{H}$ NMR $\left(300 \mathrm{MHz}\right.$, DMSO- $\left.d_{6}\right): \delta[\mathrm{ppm}]=8.54(\mathrm{~s}, 2 \mathrm{H}), 8.50-8.34(\mathrm{~m}, 2 \mathrm{H}), 8.34-8.18(\mathrm{~m}$, $4 \mathrm{H}), 7.28-7.00(\mathrm{~m}, 6 \mathrm{H}), 3.94(\mathrm{~s}, 3 \mathrm{H}), 3.91(\mathrm{~s}, 6 \mathrm{H}) ;{ }^{\mathbf{1 3}} \mathbf{C}\left\{{ }^{\mathbf{1}} \mathbf{H}\right\}$ NMR (126 MHz, DMSO- $\left.d_{6}\right)$ : $\delta[\mathrm{ppm}]=167.5,165.2,164.4,161.5,132.3,130.4,124.2,121.1,115.2,115.2,110.3,56.0,55.9$;

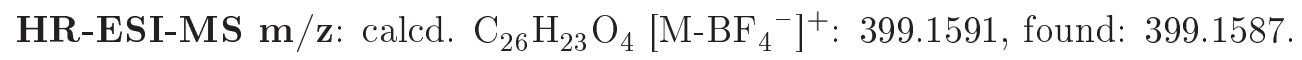

\section{1,2-Bis(2-methoxyphenyl)diselane (129)}<smiles>COc1ccccc1COc1ccccc1OC</smiles>

$332 \mathrm{a}$

2-Bromoanisole (3.78 g, $20.2 \mathrm{mmol}, 1.0$ equiv) was dissolved in THF (70 mL) and the solution was cooled to $-78{ }^{\circ} \mathrm{C}$. Tert-butyl lithium $(1.7 \mathrm{M}$ in pentane, $25 \mathrm{~mL}, 42.5 \mathrm{mmol}, 2.1$ equiv) was added dropwise over $15 \mathrm{~min}$, the solution was stirred for $15 \mathrm{~min}$ at $-78{ }^{\circ} \mathrm{C}$ and for $45 \mathrm{~min}$ at $0{ }^{\circ} \mathrm{C}$. Selenium powder ( $1.76 \mathrm{~g}, 22.3 \mathrm{mmol}, 1.1$ equiv) was added in one portion and the suspension was stirred for $15 \mathrm{~min}$ at $0{ }^{\circ} \mathrm{C}$ and for $18 \mathrm{~h}$ at room temperature. The reaction was quenched by the addition of $1 \mathrm{M}$ aq. $\mathrm{HCl}(14 \mathrm{~mL})$, the solution was diluted with $\mathrm{H}_{2} \mathrm{O}(70 \mathrm{~mL})$, the aq. phase was extracted with $\mathrm{Et}_{2} \mathrm{O}(3 \times 70 \mathrm{~mL})$, the combined org. phases were washed with sat. aq. $\mathrm{NaCl}$ solution $(70 \mathrm{~mL})$ and dried over $\mathrm{Na}_{2} \mathrm{SO}_{4}$. After evaporation of the solvent, the residue was dissolved in $\mathrm{Et}_{2} \mathrm{O}(35 \mathrm{~mL}), \mathrm{NaOH}$ (2 pellets) was added and stirred for $2 \mathrm{~h}$ at room temperature under air. Removal of the solvent in vacuum and purification by column 
chromatography (50:1 n-pentane:EtOAc) afforded the product as an orange oil. Recrystallization (9:1 hexane:DCM) afforded the product as yellow crystals (1.89 g, $5.09 \mathrm{mmol}, 50 \%)$.

$\mathbf{R}_{\mathbf{f}}=0.20$ (n-pentane:Et $\left.{ }_{2} \mathrm{O}, 40: 1\right) ; \mathbf{m} . \mathbf{p .}=80{ }^{\circ} \mathrm{C} ; \mathbf{I R}(\mathrm{ATR}): \tilde{\nu}\left[\mathrm{cm}^{-1}\right]=3057,3001,2962,2939$, 2835, 1573, 1465, 1430, 1303, 1266, 1234, 1182, 1159, 1122, 1051, 1016, 786, 747, 711, 653, 569, 539, 486, 433; ${ }^{\mathbf{1}} \mathbf{H}$ NMR (400 MHz, $\left.\mathrm{CDCl}_{3}\right): \delta[\mathrm{ppm}]=7.56(\mathrm{ddd}, J=7.8,1.6,0.3 \mathrm{~Hz}, 2 \mathrm{H})$, 7.21 (ddd, $J=8.1,7.4,1.6 \mathrm{~Hz}, 2 \mathrm{H}), 6.88$ (ddd, $J=7.4,1.2 \mathrm{~Hz}, 2 \mathrm{H}), 6.82(\mathrm{dd}, J=8.1,1.2 \mathrm{~Hz}$, $2 \mathrm{H}), 3.91(\mathrm{~s}, 6 \mathrm{H}) ;{ }^{13} \mathbf{C}\left\{{ }^{\mathbf{1}} \mathbf{H}\right\} \mathbf{N M R}\left(100 \mathrm{MHz}, \mathrm{CDCl}_{3}\right): \delta[\mathrm{ppm}]=157.0,130.8,128.3,122.0$, 118.9, 110.3, 56.1; ${ }^{77}$ Se NMR $\left(76 \mathrm{MHz}, \mathrm{CDCl}_{3}\right): \delta[\mathrm{ppm}]=332.8$; HR-ESI-MS m/z: calcd. $\mathrm{C}_{14} \mathrm{H}_{14} \mathrm{O}_{2} \mathrm{Se}_{2} \mathrm{Na}[\mathrm{M}+\mathrm{Na}]^{+}:$396.9219, found: 396.9212 .

\section{1,2-Bis(4-(trifluoromethyl)phenyl)diselane (130)}

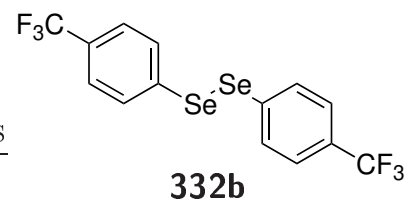

4-Bromobenzotrifluoride (4.56 g, $20.2 \mathrm{mmol}, 1.0$ equiv) was dissolved in THF $(70 \mathrm{~mL})$ and the solution was cooled to $-78{ }^{\circ} \mathrm{C}$. Tertbutyl lithium ( $1.7 \mathrm{M}$ in pentane, $25 \mathrm{~mL}, 42.5 \mathrm{mmol}, 2.1$ equiv) was added dropwise over $15 \mathrm{~min}$, the solution was stirred for $15 \mathrm{~min}$ at $-78{ }^{\circ} \mathrm{C}$ and for $45 \mathrm{~min}$ at $0{ }^{\circ} \mathrm{C}$. Selenium powder $(1.76 \mathrm{~g}, 22.3 \mathrm{mmol}$, 1.1 equiv) was added in one portion and the suspension was stirred for 15 min at $0{ }^{\circ} \mathrm{C}$ and for $18 \mathrm{~h}$ at room temperature. The reaction was quenched by the addition of $1 \mathrm{M}$ aq. $\mathrm{HCl}(14 \mathrm{~mL})$, the solution was diluted with $\mathrm{H}_{2} \mathrm{O}(70 \mathrm{~mL})$, the aq. phase was extracted with $\mathrm{Et}_{2} \mathrm{O}(3 \times 70 \mathrm{~mL})$, the combined org. phases were washed with sat. aq. $\mathrm{NaCl}$ solution $(70 \mathrm{~mL})$ and dried over $\mathrm{Na}_{2} \mathrm{SO}_{4}$. After evaporation of the solvent, the residue was dissolved in $\mathrm{EtOH}(35 \mathrm{~mL}), \mathrm{NaOH}$ (2 pellets) was added and stirred for $2 \mathrm{~h}$ at room temperature under air. Removal of the solvent in vacuo and purification by column chromatography ( $n$-pentane) afforded the product as an orange oil. Recrystallization (9:1 hexane:DCM) afforded the product as a yellow solid (1.98 g, $4.41 \mathrm{mmol}$, $44 \%$ ).

$\mathbf{R}_{\mathbf{f}}=0.34$ (n-pentane); m.p. $=58^{\circ} \mathrm{C} ; \mathbf{I R}(\mathrm{ATR}): \tilde{\nu}\left[\mathrm{cm}^{-1}\right]=1597,1495,1398,1321,1162,1101$, 1068, 1008, 948, 841, 820, 772, 719, 684, 587, 489, 426, 407; ${ }^{\mathbf{1}} \mathbf{H}$ NMR (400 MHz, $\left.\mathrm{CDCl}_{3}\right)$ : $\delta[\mathrm{ppm}]=7.77-7.66(\mathrm{~m}, 4 \mathrm{H}), 7.57-7.47(\mathrm{~m}, 4 \mathrm{H}) ;{ }^{\mathbf{1 3}} \mathbf{C}\left\{{ }^{\mathbf{1}} \mathbf{H}\right\}$ NMR $\left(100 \mathrm{MHz}, \mathrm{CDCl}_{3}\right): \delta$ $[\mathrm{ppm}]=135.1-134.7(\mathrm{~m}), 130.9,130.1\left(\mathrm{q},{ }^{2} J=32.7 \mathrm{~Hz}\right), 126.3\left(\mathrm{q},{ }^{3} J=3.8 \mathrm{~Hz}\right), 124.0$ (q, $\left.{ }^{1} J=272.2 \mathrm{~Hz}\right) ;{ }^{77} \mathbf{S e ~ N M R}\left(76 \mathrm{MHz}, \mathrm{CDCl}_{3}\right): \delta[\mathrm{ppm}]=453.1 ;{ }^{\mathbf{1 9}} \mathbf{F} \mathbf{N M R}\left(376 \mathrm{MHz}, \mathrm{CDCl}_{3}\right)$ : $\delta[\mathrm{ppm}]=-62.7$; HR-EI-MS m/z: calcd. $\mathrm{C}_{14} \mathrm{H}_{8} \mathrm{~F}_{6} \mathrm{Se}_{2}[\mathrm{M}]^{+}:$449.8863, found: 449.8847 . 


\subsection{Intramolecular etherification via photo-aerobic selenium- $\pi$-acid catalysis}

\subsubsection{Synthesis of unsaturated alcohols 126 and 139}

\section{General procedure A: Wittig reaction}

Under an atmosphere of argon, the carboxyalkyl triphenyl phosphonium bromide (2.0 equiv) is dissolved in anhydrous THF $(0.6 \mathrm{M})$. The suspension is cooled to $0{ }^{\circ} \mathrm{C}$ and $\mathrm{KO} t \mathrm{Bu}$ (powder or $1 \mathrm{M}$ solution in THF; 4.0 equiv) is added dropwise. After $30 \mathrm{~min}$ of stirring at room temperature, a solution of the aldehyde (1.0 equiv) in anhydrous THF $(2 \mathrm{M})$ is added dropwise at $0{ }^{\circ} \mathrm{C}$. The reaction is stirred at room temperature and after the aldehyde is consumed, the mixture is quenched with $1 \mathrm{M}$ aq. $\mathrm{HCl}$ solution or sat. aq. $\mathrm{NH}_{4} \mathrm{Cl}$, extracted with $\mathrm{Et}_{2} \mathrm{O}(3 \times)$ and washed with $\mathrm{H}_{2} \mathrm{O}(2 \times)$. The combined organic layers are washed with brine, dried over anhydrous $\mathrm{Na}_{2} \mathrm{SO}_{4}$, filtered and the solvent is removed under reduced pressure. The residue is purified on silica gel to yield the title compound.

\section{General procedure B; reduction with $\mathrm{LiAlH}_{4}$}

A solution of acid $\mathbf{3 4}$ or 137 (1.0 equiv) in anhydrous THF $(7 \mathrm{~mL})$ is added dropwise with stirring to a suspension of $\mathrm{LiAlH}_{4}(2.4 \mathrm{M}$ in THF; 1.5 equiv $)$ in anhydrous THF $(3 \mathrm{~mL})$ at $0{ }^{\circ} \mathrm{C}$. After stirring at RT, the reaction mixture is cooled to $0{ }^{\circ} \mathrm{C}$, diluted with $\mathrm{Et}_{2} \mathrm{O}(15 \mathrm{~mL})$, quenched with sodium sulfate decahydrate, and filtered. The filter cake is washed with a sat. aq. solution of potassium sodium tartrate (Rochelle salt) and extracted with $\mathrm{Et}_{2} \mathrm{O}(5 \times 10 \mathrm{~mL})$. The combined org. layers are washed with brine, dried over anhydrous $\mathrm{Na}_{2} \mathrm{SO}_{4}$, filtered and the solvent is removed under reduced pressure. The product is used without further purification.

\section{General procedure C: cross metathesis reaction}

Under an atmosphere of argon, 4-penten-1-ol (3 equiv) and the respective alkene (1.0 equiv) are dissolved in degassed, dry DCM (0.5 M). 2nd generation GruBBs catalyst (0.05 equiv) is added and the reaction mixture is stirred at $40{ }^{\circ} \mathrm{C}$. The solvent is removed under reduced pressure and the residue is purified on silica gel to yield the title compound.

Hept-6-ynal (333a)

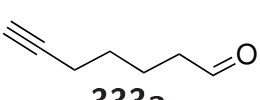

A solution of Dess-MARTin periodinane $(5.09 \mathrm{~g}, 12.0 \mathrm{mmol}, 2.0$ equiv) in 333a dry DCM $(27 \mathrm{~mL})$ was cooled to $0{ }^{\circ} \mathrm{C}$, 6-heptyn-1-ol $(673 \mathrm{mg}, 6.00 \mathrm{mmol}$, 1.0 equiv) in DCM $(3 \mathrm{~mL})$ was added and the mixture was stirred at RT for 5 h. DCM $(5 \mathrm{~mL})$ was added, the mixture was washed with sat. aq. $\mathrm{Na}_{2} \mathrm{~S}_{2} \mathrm{O}_{3}$ sol. $(30 \mathrm{~mL})$, $1 \mathrm{M} \mathrm{NaOH}(30 \mathrm{~mL})$, and sat. aq. $\mathrm{NaCl}(30 \mathrm{~mL})$. The aq. phase was extracted with DCM $(90 \mathrm{~mL})$, the combined organic layers were washed with brine, dried over anhydrous $\mathrm{Na}_{2} \mathrm{SO}_{4}$, filtered and the solvent was removed under reduced pressure. The residue was purified on silica 
gel (n-pentane: $\left.\mathrm{Et}_{2} \mathrm{O}, 9: 1\right)$ to yield the title compound as a colorless oil (337 $\mathrm{mg}, 3.06 \mathrm{mmol}$, $51 \%)$.

$\mathbf{R}_{\mathbf{f}}=0.29$ (n-pentane:Et $\left.{ }_{2} \mathrm{O}, 9: 1\right) ; \mathbf{I R}(\mathrm{ATR}): \tilde{\nu}\left[\mathrm{cm}^{-1}\right]=3296,2942,2869,2116,1706,1414,1289$, 1234, 1146, 1038, 934; ${ }^{\mathbf{1}} \mathbf{H}$ NMR $\left(400 \mathrm{MHz}, \mathrm{CDCl}_{3}\right): \delta[\mathrm{ppm}]=9.77$ (t, $\left.J=1.7 \mathrm{~Hz}, 1 \mathrm{H}\right), 2.46$ $(\mathrm{td}, J=7.3,1.7 \mathrm{~Hz}, 2 \mathrm{H}), 2.22(\mathrm{td}, J=7.0,2.7 \mathrm{~Hz}, 2 \mathrm{H}), 1.95(\mathrm{t}, J=2.7 \mathrm{~Hz}, 1 \mathrm{H}), 1.83-1.66$ $(\mathrm{m}, 2 \mathrm{H}), 1.65-1.42(\mathrm{~m}, 2 \mathrm{H}) ;{ }^{\mathbf{1 3}} \mathbf{C}\left\{{ }^{\mathbf{1}} \mathbf{H}\right\} \mathbf{N M R}\left(101 \mathrm{MHz}, \mathrm{CDCl}_{3}\right): \delta[\mathrm{ppm}]=202.3,83.9,68.9$, $43.4,27.9,21.2,18.3$.

\section{3-(4-Bromophenyl)propanal (333b)}

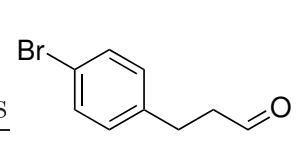

333b

A solution of Dess-MarTin periodinane (5.09 g, $12.0 \mathrm{mmol}, 2.0$ equiv) in dry DCM $(27 \mathrm{~mL})$ was cooled to $0{ }^{\circ} \mathrm{C}$, 3-(4-bromophenyl)propanol $(1.29 \mathrm{~g}$, $6.00 \mathrm{mmol}, 1.0$ equiv) in DCM ( $3 \mathrm{~mL})$ was added and the mixture was stirred at RT for $3 \mathrm{~h}$. DCM ( $5 \mathrm{~mL}$ ) was added, the mixture was washed with sat. aq. $\mathrm{Na}_{2} \mathrm{~S}_{2} \mathrm{O}_{3}$ sol. $(30 \mathrm{~mL}), 1 \mathrm{~m} \mathrm{NaOH}(30 \mathrm{~mL})$, and sat. aq. $\mathrm{NaCl}(30 \mathrm{~mL})$. The aq. phase was extracted with DCM $(90 \mathrm{~mL})$, the combined organic layers were washed with brine, dried over anhydrous $\mathrm{Na}_{2} \mathrm{SO}_{4}$, filtered and the solvent was removed under reduced pressure. The residue was purified on silica gel ( $n$-pentane:EtOAc, 10:1) to yield the title compound as a colorless oil (614 mg, $2.88 \mathrm{mmol}, 48 \%)$.

$\mathbf{R}_{\mathbf{f}}=0.38$ (n-pentane:EtOAc, 10:1); IR (ATR): $\tilde{\nu}\left[\mathrm{cm}^{-1}\right]=2928,2823,2725,2359,2341,1721$, 1488, 1444, 1405, 1389, 1358, 1201, 1106, 1071, 1011, 861, 801, 713; ${ }^{\mathbf{1}} \mathbf{H}$ NMR $(400 \mathrm{MHz}$, $\left.\mathrm{CDCl}_{3}\right): \delta[\mathrm{ppm}]=9.80(\mathrm{t}, J=1.3 \mathrm{~Hz}, 1 \mathrm{H}), 7.55-7.34(\mathrm{~m}, 2 \mathrm{H}), 7.14-6.86(\mathrm{~m}, 2 \mathrm{H}), 3.01-2.85$ $(\mathrm{m}, 2 \mathrm{H}), 2.85-2.63(\mathrm{~m}, 2 \mathrm{H}) ;{ }^{\mathbf{1 3}} \mathbf{C}\left\{{ }^{\mathbf{1}} \mathbf{H}\right\} \mathbf{N M R}\left(101 \mathrm{MHz}, \mathrm{CDCl}_{3}\right): \delta[\mathrm{ppm}]=201.1,139.5,131.8$, 130.2, 120.2, 45.2, 27.6; HR-EI-MS m/z: calcd. $\mathrm{C}_{9} \mathrm{H}_{9} \mathrm{BrO}[\mathrm{M}]^{+}:$211.9837, found: 211.9839 .

\section{(Z)-12-Hydroxy-8,12-dimethyltridec-5-enoic acid (137a)}

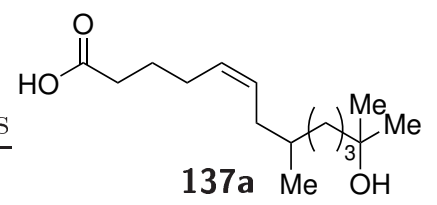

Following general procedure A (4-carboxybutyl)triphenylphosphonium bromide ( $3.1 \mathrm{~g}, 7.0 \mathrm{mmol}, 2.0$ equiv) in THF $(11 \mathrm{~mL})$, potassium tert-butoxide $(1.57 \mathrm{~g}, 14.0 \mathrm{mmol}, 4.0$ equiv), 7hydroxycitronellal (0.60 g, $3.5 \mathrm{mmol}, 1.0$ equiv) in THF (1 mL); reaction time $17 \mathrm{~h}$; eluting with $\mathrm{Et}_{2} \mathrm{O}$; colorless oil; $709 \mathrm{mg}, 2.77 \mathrm{mmol}$, $79 \%$.

$\mathbf{R}_{\mathbf{f}}=0.20\left(\mathrm{Et}_{2} \mathrm{O}\right) ; \mathbf{I R}(\mathrm{ATR}): \tilde{\nu}\left[\mathrm{cm}^{-1}\right]=2935,2869,1707,1459,1377,1198,1158,934,906,763$, 699, 495; ${ }^{1} \mathbf{H}$ NMR $\left(300 \mathrm{MHz}, \mathrm{CDCl}_{3}\right): \delta[\mathrm{ppm}]=6.40$ (s, $\left.2 \mathrm{H}\right), 5.59-5.17$ (m, $\left.2 \mathrm{H}\right), 2.34$ (t, $J=7.3 \mathrm{~Hz}, 2 \mathrm{H}), 2.19-1.97(\mathrm{~m}, 3 \mathrm{H}), 1.81(\mathrm{~m}, 1 \mathrm{H}), 1.68$ (quint, $J=7.1 \mathrm{~Hz}, 2 \mathrm{H}$ ), 1.57-1.24 $(\mathrm{m}, 6 \mathrm{H}), 1.21(\mathrm{~s}, 6 \mathrm{H}), 1.14(\mathrm{~m}, 1 \mathrm{H}), 0.87(\mathrm{~d}, J=6.7 \mathrm{~Hz}, 3 \mathrm{H}) ;{ }^{\mathbf{1 3}} \mathbf{C}\left\{{ }^{1} \mathbf{H}\right\}$ NMR $(101 \mathrm{MHz}$, $\left.\mathrm{CDCl}_{3}\right): \delta[\mathrm{ppm}]=178.9,129.8,129.3,71.6,44.1,37.2,34.3,33.6,33.5,29.3,29.2,26.7,24.8$, 21.8, 19.9; HR-ESI-MS m/z: calcd. $\mathrm{C}_{15} \mathrm{H}_{28} \mathrm{NaO}_{3}[\mathrm{M}+\mathrm{Na}]^{+}:$279.1931, found: 279.1929. 


\section{$(S, Z)-8,12-D i m e t h y l t r i d e c a-5,11-d i e n o i c ~ a c i d ~(137 b)$}

Following general procedure $\mathbf{A}$ (4-carboxybutyl)triphenylphos-<smiles>CC(C)=CCC(C)CC=CCCCC(=O)O</smilesphonium bromide (1.8 g, $4.0 \mathrm{mmol}, 2.0$ equiv) in THF $(7 \mathrm{~mL})$, potassium tert-butoxide $(0.90 \mathrm{~g}, 8.0 \mathrm{mmol}, 4.0$ equiv), (S)-citronellal (0.31 g, $2.0 \mathrm{mmol}, 1.0$ equiv) in THF (1 mL); reaction time $2 \mathrm{~h}$; eluting with $n$-pentane/ $\mathrm{Et}_{2} \mathrm{O}, 1: 1$; yellow oil; $377 \mathrm{mg}, 1.58 \mathrm{mmol}, 79 \%$.

$\mathbf{R}_{\mathbf{f}}=0.36$ (n-pentane:Et $\left.{ }_{2} \mathrm{O}, 1: 1\right)$; IR (ATR): $\tilde{\nu}\left[\mathrm{cm}^{-1}\right]=2954,2913,2870,1707,1437,1377$, 1267, 1240, 1205, 929, 692, 490; ${ }^{\mathbf{1}} \mathbf{H} \mathbf{N M R}\left(300 \mathrm{MHz}, \mathrm{CDCl}_{3}\right): \delta[\mathrm{ppm}]=5.51-5.29(\mathrm{~m}, 2 \mathrm{H})$, 5.10 (tdq, $J=7.1,2.9,1.4 \mathrm{~Hz}, 1 \mathrm{H}), 2.36$ (t, $J=7.5 \mathrm{~Hz}, 2 \mathrm{H}), 2.17-2.01$ (m, $2 \mathrm{H}), 1.99$ (tdd, $J=8.0,4.3,1.7 \mathrm{~Hz}, 2 \mathrm{H}), 1.96-1.78(\mathrm{~m}, 2 \mathrm{H}), 1.79-1.62(\mathrm{~m}, 5 \mathrm{H}), 1.61(\mathrm{~d}, J=1.4 \mathrm{~Hz}, 3 \mathrm{H}), 1.47$ $(\mathrm{m}, 1 \mathrm{H}), 1.35(\mathrm{~m}, 1 \mathrm{H}), 1.15(\mathrm{~m}, 1 \mathrm{H}), 0.87(\mathrm{~d}, J=6.6 \mathrm{~Hz}, 2 \mathrm{H}) ;{ }^{13} \mathbf{C}\left\{{ }^{1} \mathbf{H}\right\}$ NMR $(125 \mathrm{MHz}$, $\left.\mathrm{CDCl}_{3}\right): \delta[\mathrm{ppm}]=179.7,131.2,129.9,129.1,124.9,37.0,34.7,33.6,33.2,26.8,25.9,25.9,24.8$, 19.7, 17.9; HR-ESI-MS m/z: calcd. $\mathrm{C}_{15} \mathrm{H}_{25} \mathrm{O}_{2}[\mathrm{M}-\mathrm{H}]^{-}: 237.1860$, found: 237.1862 .

\section{(Z)-8-Phenyloct-5-enoic acid (137c)}

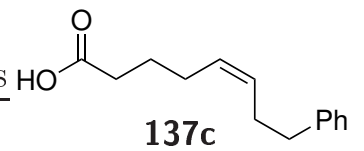

Following general procedure $\mathbf{A}$ : (4-carboxybutyl)triphenylphosphonium bromide (3.1 g, $7.0 \mathrm{mmol}, 2.0$ equiv) in THF (11 mL), potassium tert-butoxide (1.57 g, $14.0 \mathrm{mmol}, 4.0$ equiv), 3-phenylpropionaldehyde (0.47 g, $3.5 \mathrm{mmol}, 1.0$ equiv) in THF $(1 \mathrm{~mL})$; reaction time $2 \mathrm{~h}$; eluting with $n$-pentane/ $\mathrm{Et}_{2} \mathrm{O}, 4: 1$ to $0: 1$; colorless oil; $629 \mathrm{mg}, 2.88 \mathrm{mmol}, 82 \%$.

$\mathbf{R}_{\mathbf{f}}=0.13$ (n-pentane:Et $\left.{ }_{2} \mathrm{O}, 4: 1\right)$; IR (ATR): $\tilde{\nu}\left[\mathrm{cm}^{-1}\right]=3062,3026,2929,2857,1703,1603$, 1496, 1453, 1411, 1239, 1090, 1030, 931, 749, 725, 697, 584, 484; ${ }^{\mathbf{1}} \mathbf{H} \mathbf{~ N M R}\left(400 \mathrm{MHz}, \mathrm{CDCl}_{3}\right)$ : $\delta[\mathrm{ppm}]=7.33-7.25(\mathrm{~m}, 2 \mathrm{H}), 7.22-7.14(\mathrm{~m}, 2 \mathrm{H}), 5.46(\mathrm{~m}, 1 \mathrm{H}), 5.35(\mathrm{~m}, 1 \mathrm{H}), 2.72-2.59(\mathrm{~m}$, $2 \mathrm{H}), 2.39-2.32$ (m, $2 \mathrm{H}), 2.34-2.24$ (m, $2 \mathrm{H}), 2.09-1.98$ (m, $2 \mathrm{H}), 1.63$ (quint, $J=7.5 \mathrm{~Hz}, 2 \mathrm{H}$ ); ${ }^{13} \mathbf{C}\left\{{ }^{1} \mathbf{H}\right\}$ NMR $\left(101 \mathrm{MHz}, \mathrm{CDCl}_{3}\right): \delta[\mathrm{ppm}]=179.7,142.1,130.2,129.2,128.6,128.4,126.0$, 36.0, 33.4, 29.3, 26.6, 24.6; HR-ESI-MS m/z: calcd. $\mathrm{C}_{14} \mathrm{H}_{18} \mathrm{O}_{2} \mathrm{Na}[\mathrm{M}+\mathrm{Na}]^{+}:$241.1199, found: 241.1206 .

\section{(Z)-6-Cyclohexylhex-5-enoic acid (137d)}

Following general procedure $\mathbf{A}$ (4-carboxybutyl)triphenylphosphonium bro-<smiles>O=C(Br)/C=C\CCCC(=O)O</smilesmide (1.8 g, $4.0 \mathrm{mmol}, 2.0$ equiv) in THF $(7 \mathrm{~mL})$, potassium tert-butoxide (0.90 g, $8.0 \mathrm{mmol}, 4.0$ equiv), cyclohexanecarboxaldehyde (0.22 g, $2.0 \mathrm{mmol}$, 1.0 equiv) in THF $(1 \mathrm{~mL})$; reaction time $2 \mathrm{~h}$; eluting with $n$-pentane $/ \mathrm{Et}_{2} \mathrm{O}$, 1:1; colorless oil; $290 \mathrm{mg}, 1.48 \mathrm{mmol}, 74 \%$. 
$\mathbf{R}_{\mathbf{f}}=0.36$ (n-pentane:Et $\left.{ }_{2} \mathrm{O}, 1: 1\right) ; \mathbf{I R}(\mathrm{ATR}): \tilde{\nu}\left[\mathrm{cm}^{-1}\right]=2999,2922,2849,1704,1447,1411,1289$, 1241, 1203, 1164, 930, 889, 732, 487; ${ }^{\mathbf{1}} \mathbf{H}$ NMR $\left(500 \mathrm{MHz}, \mathrm{CDCl}_{3}\right): \delta[\mathrm{ppm}]=5.30-5.16(\mathrm{~m}$, $2 \mathrm{H}), 2.37$ (t, $J=7.5 \mathrm{~Hz}, 2 \mathrm{H}), 2.22(\mathrm{~m}, 1 \mathrm{H}), 2.16-2.07(\mathrm{~m}, 2 \mathrm{H}), 1.76-1.65(\mathrm{~m}, 5 \mathrm{H}), 1.63-1.54$ $(\mathrm{m}, 2 \mathrm{H}), 1.39-1.12(\mathrm{~m}, 3 \mathrm{H}), 1.11-0.99(\mathrm{~m}, 2 \mathrm{H}) ;{ }^{\mathbf{1 3}} \mathbf{C}\left\{{ }^{\mathbf{1}} \mathbf{H}\right\}$ NMR $\left(125 \mathrm{MHz}, \mathrm{CDCl}_{3}\right): \delta$ $[\mathrm{ppm}]=180.2$, 137.5, 126.4, 36.5, 33.6, 33.5, 26.8, 26.2, 26.1, 24.9; HR-ESI-MS m/z: calcd. $\mathrm{C}_{12} \mathrm{H}_{19} \mathrm{O}_{2}[\mathrm{M}-\mathrm{H}]^{-}:$195.1391, found: 195.1394 .

\section{(Z)-8-Methylnon-5-enoic acid (137e)}

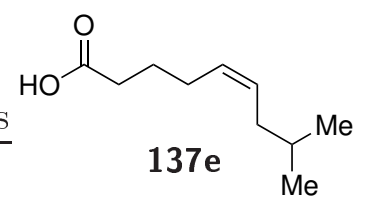

Following general procedure $\mathbf{A}$. (4-carboxybutyl)triphenylphosphonium bromide (1.8 g, $4.0 \mathrm{mmol}, 2.0$ equiv) in THF $(7 \mathrm{~mL})$, potassium tert-butoxide ( $0.90 \mathrm{~g}, 8.0 \mathrm{mmol}, 4.0$ equiv), isovaleraldehyde $(0.17 \mathrm{~g}$, $2.0 \mathrm{mmol}, 1.0$ equiv) in THF $(1 \mathrm{~mL})$; reaction time $2 \mathrm{~h}$; eluting with $n$-pentane/Et ${ }_{2} \mathrm{O}, 3: 1$ to $1: 1$; colorless oil; $204 \mathrm{mg}, 1.20 \mathrm{mmol}, 60 \%$.

$\mathbf{R}_{\mathbf{f}}=0.5$ (n-pentane:Et $\left.{ }_{2} \mathrm{O}, 1: 1\right)$; IR (ATR): $\tilde{\nu}\left[\mathrm{cm}^{-1}\right]=3008,2954,2870,1706,1461,1412$, 1384, 1366, 1314, 1241, 1167, 1122, 928, 826, 703, 481; ${ }^{\mathbf{1}} \mathbf{H} \mathbf{N M R}\left(300 \mathrm{MHz}_{\mathrm{C}} \mathrm{CDCl}_{3}\right): \delta$ $[\mathrm{ppm}]=5.54-5.24(\mathrm{~m}, 2 \mathrm{H}), 2.37(\mathrm{t}, J=7.5 \mathrm{~Hz}, 2 \mathrm{H}), 2.17-2.01(\mathrm{~m}, 2 \mathrm{H}), 1.95-1.87(\mathrm{~m}$, $2 \mathrm{H}), 1.77-1.53(\mathrm{~m}, 3 \mathrm{H}), 0.89(\mathrm{~d}, J=6.6 \mathrm{~Hz}, 6 \mathrm{H}) ;{ }^{13} \mathbf{C}\left\{{ }^{1} \mathbf{H}\right\} \mathbf{N M R}\left(125 \mathrm{MHz}, \mathrm{CDCl}_{3}\right)$ : $\delta[\mathrm{ppm}]=179.9,130.1,128.9,36.6,33.7,28.9,26.8,24.8,22.6 ;$ HR-ESI-MS m/z: calcd. $\mathrm{C}_{10} \mathrm{H}_{17} \mathrm{O}_{2}[\mathrm{M}-\mathrm{H}]^{-}:$169.1234, found: 169.1234 .

\section{(Z)-10-Chlorodec-5-enoic acid (137f)}

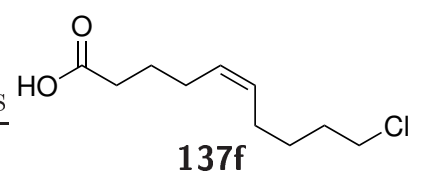

Following general procedure A (4-carboxybutyl)triphenylphosphonium bromide (3.6 g, $8.0 \mathrm{mmol}, 2.0$ equiv) in THF $(10 \mathrm{~mL})$, potassium tert-butoxide (1.80 g, $16.0 \mathrm{mmol}, 4.0$ equiv), 5chloropentanal ( $0.48 \mathrm{~g}, 4.0 \mathrm{mmol}, 1.0$ equiv) in THF $(1 \mathrm{~mL})$; reaction time $3 \mathrm{~h}$; eluting with $n$-pentane $/ \mathrm{Et}_{2} \mathrm{O}, 5: 1$ to 0:1; colorless oil; $491 \mathrm{mg}, 2.40 \mathrm{mmol}, 60 \%$.

$\mathbf{R}_{\mathbf{f}}=0.07$ (n-pentane:Et $\left.{ }_{2} \mathrm{O}, 5: 1\right)$; IR (ATR): $\tilde{\nu}\left[\mathrm{cm}^{-1}\right]=3007,2934,2863,1704,1412,1239$, 1204, 917, 716, 651, 485; ${ }^{1} \mathbf{H}$ NMR $\left(400 \mathrm{MHz}, \mathrm{CDCl}_{3}\right): \delta[\mathrm{ppm}]=5.56-5.23(\mathrm{~m}, 2 \mathrm{H}), 3.53(\mathrm{t}$, $J=6.7 \mathrm{~Hz}, 2 \mathrm{H}), 2.36(\mathrm{t}, J=7.5 \mathrm{~Hz}, 2 \mathrm{H}), 2.22-1.91(\mathrm{~m}, 4 \mathrm{H}), 1.91-1.59(\mathrm{~m}, 4 \mathrm{H}), 1.58-1.35$ $(\mathrm{m}, 2 \mathrm{H}) ;{ }^{13} \mathbf{C}\left\{{ }^{\mathbf{1}} \mathbf{H}\right\} \mathbf{N M R}\left(101 \mathrm{MHz}, \mathrm{CDCl}_{3}\right): \delta[\mathrm{ppm}]=179.8,130.5,129.1,45.1,33.5,32.3$, 27.0, 26.6, 26.6, 24.7; HR-ESI-MS m/z: calcd. $\mathrm{C}_{10} \mathrm{H}_{17} \mathrm{O}_{2} \mathrm{ClNa}[\mathrm{M}+\mathrm{Na}]^{+}:$227.0809, found: 227.0814 . 


\section{(Z)-7-(1-(tert-butoxycarbonyl)piperidin-4-yl)hept-5-enoic acid (137g)}

Following general procedure $\mathbf{A}$ (4-carboxybutyl)triphenylphos-<smiles>O=C(O)CC/C=C\CC1CCN(C(=O)[18O])CC1</smilesphonium bromide (1.8 g, $4.0 \mathrm{mmol}, 2.0$ equiv) in THF $(7 \mathrm{~mL})$, potassium tert-butoxide ( $0.90 \mathrm{~g}, 8.0 \mathrm{mmol}, 4.0$ equiv), $\mathrm{N}$-Boc4-piperidineacetaldehyde $(0.47 \mathrm{~g}, 2.0 \mathrm{mmol}, 1.0$ equiv $)$ in THF $(1 \mathrm{~mL})$; reaction time $2 \mathrm{~h}$; eluting with $n$-pentane/ $\mathrm{Et}_{2} \mathrm{O}, 3: 1$; yellow oil; $257 \mathrm{mg}, 0.83 \mathrm{mmol}$, $42 \%$.

$\mathbf{R}_{\mathbf{f}}=0.30$ (n-pentane:Et $\left.{ }_{2} \mathrm{O}, 1: 1\right)$; IR (ATR): $\tilde{\nu}\left[\mathrm{cm}^{-1}\right]=2922,2851,1736,1696,1422,1366$, 1278, 1243, 1160, 1127, 1084, 966, 947, 909, 863, 769, 733; ${ }^{\mathbf{1}} \mathbf{H}$ NMR $\left(400 \mathrm{MHz}, \mathrm{CDCl}_{3}\right): \delta$ $[\mathrm{ppm}]=5.48-5.33(\mathrm{~m}, 2 \mathrm{H}), 4.13-3.98(\mathrm{~m}, 2 \mathrm{H}), 2.66(\mathrm{t}, J=12.8 \mathrm{~Hz}, 2 \mathrm{H}), 2.35(\mathrm{t}, J=7.5 \mathrm{~Hz}$, $2 \mathrm{H}), 2.13-2.03(\mathrm{~m}, 2 \mathrm{H}), 1.97(\mathrm{dd}, J=6.7,5.6 \mathrm{~Hz}, 2 \mathrm{H}), 1.76-1.56(\mathrm{~m}, 3 \mathrm{H}), 1.45(\mathrm{~s}, 9 \mathrm{H})$, $1.09(\mathrm{qd}, J=12.6,4.3 \mathrm{~Hz}, 2 \mathrm{H}) ;{ }^{\mathbf{1 3}} \mathbf{C}\left\{{ }^{\mathbf{1}} \mathbf{H}\right\} \mathbf{N M R}\left(100 \mathrm{MHz}, \mathrm{CDCl}_{3}\right): \delta[\mathrm{ppm}]=178.8,154.9$, $129.7,128.5,79.3,36.5,34.0,33.3,32.0,28.5,26.5,24.6$; HR-ESI-MS m/z: calcd. $\mathrm{C}_{17} \mathrm{H}_{28} \mathrm{NO}_{4}$ $[\mathrm{M}-\mathrm{H}]^{-}:$310.2024, found: 310.2023 .

\section{(Z)-Hexadeca-5,15-dienoic acid (137h)}

Following general procedure A (4-carboxybutyl)triphenyl-<smiles>C=CCC=CCCCC(=O)O</smilesphosphonium bromide (3.55 g, $8.00 \mathrm{mmol}, 2.00$ equiv), potassium tert-butoxide (1 $\mathrm{M}$ in THF; $16 \mathrm{~mL}, 16.0 \mathrm{mmol}, 4.00$ equiv), 10undecenal (673 mg, $4.00 \mathrm{mmol}, 1.00$ equiv), THF (6 mL); reaction time $17 \mathrm{~h}$; eluting with $n$-pentane:Et ${ }_{2} \mathrm{O}, 3: 1$; yield: $733 \mathrm{mg}, 2.90 \mathrm{mmol}, 73 \%$, colorless oil.

$\mathbf{R}_{\mathbf{f}}=0.25$ (n-pentane:Et $\left.{ }_{2} \mathrm{O}, 3: 1\right)$; IR (ATR): $\tilde{\nu}\left[\mathrm{cm}^{-1}\right]=3005,2924,2853,2359,2341,1707,1640$, 1413, 1240, 1205, 1170, 992, 908, 721, 687; ${ }^{\mathbf{1}} \mathbf{H} \mathbf{~ N M R}\left(300 \mathrm{MHz}, \mathrm{CDCl}_{3}\right): \delta[\mathrm{ppm}]=5.81$ (ddt, $J=16.9,10.2,6.7 \mathrm{~Hz}, 1 \mathrm{H}), 5.59-5.17$ (m, $2 \mathrm{H}), 4.99$ (ddt, $J=17.1,2.3,1.6 \mathrm{~Hz}, 1 \mathrm{H}), 4.93$ (ddt, $J=10.2,2.3,1.2 \mathrm{~Hz}, 1 \mathrm{H}), 2.36(\mathrm{td}, J=7.6,4.3 \mathrm{~Hz}, 2 \mathrm{H}), 2.15-1.86(\mathrm{~m}, 6 \mathrm{H}), 1.85-1.58(\mathrm{~m}$, $2 \mathrm{H}), 1.49-1.10(\mathrm{~m}, 12 \mathrm{H}) ;{ }^{\mathbf{1 3}} \mathbf{C}\left\{{ }^{\mathbf{1}} \mathbf{H}\right\} \mathbf{N M R}\left(126 \mathrm{MHz}, \mathrm{CDCl}_{3}\right): \delta[\mathrm{ppm}]=179.6,139.3,131.4$, 128.2, 114.2, 34.0, 33.6, 29.9, 29.7, 29.7, 29.5, 29.4, 29.2, 27.5, 26.7, 24.8; HR-ESI-MS m/z: calcd. $\mathrm{C}_{16} \mathrm{H}_{29} \mathrm{O}_{2}[\mathrm{M}+\mathrm{H}]^{+}:$253.2162, found: 253.2154 .

\section{(Z)-Dodec-5-en-11-ynoic acid (137i)}

Following general procedure $\mathbf{A}$. (4-carboxybutyl)triphenyl-<smiles>C#CCNC=CCCCC(=O)O</smilesphosphonium bromide (2.66 g, $6.00 \mathrm{mmol}, 2.00$ equiv), potassium tert-butoxide (1 M in THF; $12 \mathrm{~mL}, 12.0 \mathrm{mmol}, 4.00$ equiv), hept-6ynal (300 mg, $3.00 \mathrm{mmol}, 1.00$ equiv), THF ( $5 \mathrm{~mL}$ ); reaction time $17 \mathrm{~h}$; eluting with $n$-pentane: $\mathrm{Et}_{2} \mathrm{O}, 3: 1$; yield: $317 \mathrm{mg}, 1.63 \mathrm{mmol}, 54 \%$, yellow oil. Contains allene (10\%). 
$\mathbf{R}_{\mathbf{f}}=0.14$ (n-pentane:Et $\left.{ }_{2} \mathrm{O}, 3: 1\right)$; IR (ATR): $\tilde{\nu}\left[\mathrm{cm}^{-1}\right]=3303,3006,2935,2860,1705,1457,1432$, 1412, 1267, 1239, 1206, 1098, 1040, 932; ${ }^{\mathbf{1}} \mathbf{H}$ NMR $\left(400 \mathrm{MHz}, \mathrm{CDCl}_{3}\right): \delta[\mathrm{ppm}]=9.25(\mathrm{~s}, 1 \mathrm{H})$, $5.47-5.29(\mathrm{~m}, 2 \mathrm{H}), 2.37$ (t, $J=7.4 \mathrm{~Hz}, 2 \mathrm{H}), 2.19(\mathrm{td}, J=6.9,2.7 \mathrm{~Hz}, 2 \mathrm{H}), 2.15-2.00(\mathrm{~m}, 4 \mathrm{H})$, $1.94(\mathrm{t}, J=2.7 \mathrm{~Hz}, 1 \mathrm{H}), 1.70$ (quint, $J=7.4 \mathrm{~Hz}, 2 \mathrm{H}), 1.60-1.36(\mathrm{~m}, 4 \mathrm{H}) ;{ }^{13} \mathbf{C}\left\{{ }^{1} \mathbf{H}\right\} \mathbf{N M R}$ $\left(101 \mathrm{MHz}, \mathrm{CDCl}_{3}\right): \delta[\mathrm{ppm}]=179.8,130.8,128.8,84.7,68.4,33.6,28.8,28.2,26.8,26.6,24.7$, 18.4; HR-ESI-MS m/z: calcd. $\mathrm{C}_{12} \mathrm{H}_{18} \mathrm{O}_{2} \mathrm{Na}[\mathrm{M}+\mathrm{Na}]^{+}: 217.1199$, found: 217.1198 .

\section{(Z)-8-(4-Bromophenyl)oct-5-enoic acid (137j)}

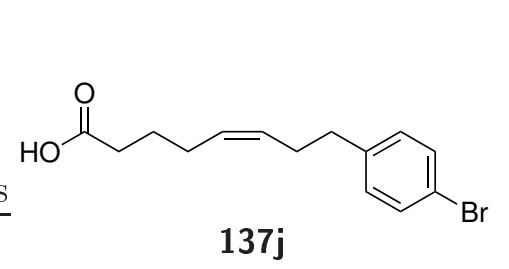

Following general procedure $\quad$ A carboxybutyl)triphenylphosphonium bromide (3.55 g, $8.00 \mathrm{mmol}, 2.00$ equiv), potassium tert-butoxide (1 M in THF; $16 \mathrm{~mL}, 16.0 \mathrm{mmol}, 4.00$ equiv), 3-(4-bromophenyl)propanal (864 mg, $4.00 \mathrm{mmol}, 1.00$ equiv), THF (6 mL); reaction time $17 \mathrm{~h}$; eluting with $n$-pentane:Et ${ }_{2} \mathrm{O}, 4: 1$; yield: $976 \mathrm{mg}, 3.28 \mathrm{mmol}, 81 \%$, yellow oil.

$\mathbf{R}_{\mathbf{f}}=0.06$ (n-pentane:Et $\left.{ }_{2} \mathrm{O}, 4: 1\right)$; IR (ATR): $\tilde{\nu}\left[\mathrm{cm}^{-1}\right]=3006,2928,2858,1899,1703,1487$, 1405, 1239, 1202, 1103, 1072, 1011, 934, 829, 813, 773, 714; ${ }^{\mathbf{1}} \mathbf{H}$ NMR (300 MHz, $\left.\mathrm{CDCl}_{3}\right): \delta$ $[\mathrm{ppm}]=7.45-7.30(\mathrm{~m}, 2 \mathrm{H}), 7.12-6.94(\mathrm{~m}, 2 \mathrm{H}), 5.52-5.25(\mathrm{~m}, 2 \mathrm{H}), 2.61(\mathrm{dd}, J=8.5,6.7 \mathrm{~Hz}$, $2 \mathrm{H}), 2.39-2.22(\mathrm{~m}, 4 \mathrm{H}), 2.08-1.90(\mathrm{~m}, 2 \mathrm{H}), 1.73-1.51(\mathrm{~m}, 2 \mathrm{H}) ;{ }^{\mathbf{1 3}} \mathbf{C}\left\{{ }^{1} \mathbf{H}\right\}$ NMR $(126 \mathrm{MHz}$, $\left.\mathrm{CDCl}_{3}\right): \delta[\mathrm{ppm}]=179.6,140.9,131.4,130.3,129.6,129.4,119.6,35.5,33.5,29.2,26.6,24.6$; HR-ESI-MS m/z: calcd. $\mathrm{C}_{14} \mathrm{H}_{17} \mathrm{O}_{2} \mathrm{BrNa}[\mathrm{M}+\mathrm{Na}]^{+}$: 319.0304, found: 319.0309 .

\section{Methyl (Z)-8-(4-bromophenyl)oct-5-enoate (334)}

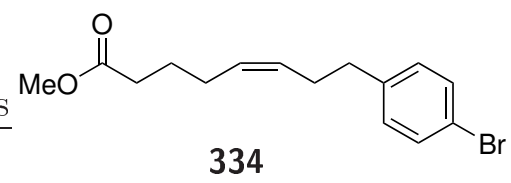

Acid 137j (446 mg, $1.5 \mathrm{mmol}, 1.0$ equiv) and conc. $\mathrm{HCl}$ $(0.1 \mathrm{~mL})$ were dissolved in $\mathrm{MeOH}(67 \mathrm{~mL})$ and stirred at $\mathrm{RT}$ for $15.5 \mathrm{~h}$. Washing of the mixture with $\mathrm{H}_{2} \mathrm{O}(70 \mathrm{~mL})$, sat. aq. $\mathrm{NaCl}(70 \mathrm{~mL})$ and extracting of the comb. aq. phases with EtOAc $(150 \mathrm{~mL})$ afforded the product as a colorless oil (275 mg, $884 \mu \mathrm{mol}, 59 \%)$.

$\mathbf{R}_{\mathbf{f}}=0.21$ (n-pentane:Et $\left.{ }_{2} \mathrm{O}, 2: 1\right)$; IR (ATR): $\tilde{\nu}\left[\mathrm{cm}^{-1}\right]=3006,2948,2859,1736,1488,1435$, 1404, 1366, 1313, 1242, 1197, 1170, 1072, 1011, 814, 773, 715; ${ }^{\mathbf{1}} \mathbf{H} \mathbf{N M R}\left(300 \mathrm{MHz}, \mathrm{CDCl}_{3}\right)$ : $\delta[\mathrm{ppm}]=7.51-7.32(\mathrm{~m}, 2 \mathrm{H}), 7.15-6.85(\mathrm{~m}, 2 \mathrm{H}), 5.58-5.18(\mathrm{~m}, 2 \mathrm{H}), 3.66(\mathrm{~s}, 3 \mathrm{H}), 2.61(\mathrm{t}$, $J=7.6 \mathrm{~Hz}, 2 \mathrm{H}), 2.47-2.14(\mathrm{~m}, 4 \mathrm{H}), 2.00(\mathrm{td}, J=7.5,6.2 \mathrm{~Hz}, 2 \mathrm{H}), 1.75-1.48(\mathrm{~m}, 2 \mathrm{H})$; ${ }^{13} \mathbf{C}\left\{{ }^{1} \mathbf{H}\right\} \mathbf{N M R}\left(126 \mathrm{MHz}, \mathrm{CDCl}_{3}\right): \delta[\mathrm{ppm}]=174.0,140.9,131.4,130.3,129.6,129.4,119.6$, 51.7, 35.5, 33.6, 29.1, 26.8, 24.9; HR-ESI-MS m/z: calcd. $\mathrm{C}_{15} \mathrm{H}_{20} \mathrm{BrO}_{2}[\mathrm{M}+\mathrm{H}]^{+}: 311.0641$, found: 311.0643 . 


\section{2-(But-3-en-1-yl)isoindoline-1,3-dione (335)}

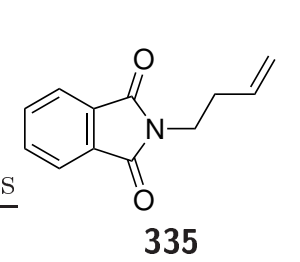

4-Bromo-1-butene (743 mg, $5.50 \mathrm{mmol}, 1.1$ equiv), phthalimide (736 mg, $5.01 \mathrm{mmol}, 1.0$ equiv) and $\mathrm{Cs}_{2} \mathrm{CO}_{3}(1.79 \mathrm{~g}, 5.49 \mathrm{mmol}, 1.1$ equiv) were dissolved in DMF $(3.5 \mathrm{~mL})$ and the solution was stirred at $70{ }^{\circ} \mathrm{C}$ for $4 \mathrm{~h}$. The mixture was poured in $\mathrm{H}_{2} \mathrm{O}$ and filtered. The resulting precipitate was washed with water and dried. The desired compound was obtained as a white solid (740 mg, $3.68 \mathrm{mmol}, 74 \%$ ).

$\mathbf{R}_{\mathbf{f}}=0.48$ (n-pentane:Et $\left.{ }_{2} \mathrm{O}, 2: 1\right)$; m.p. $=50{ }^{\circ} \mathrm{C}$; IR (ATR) $: \tilde{\nu}\left[\mathrm{cm}^{-1}\right]=2942,1769,1694,1641$, 1612, 1450, 1396, 1361, 1332, 1188, 1055, 1015, 983, 867, 798, 721, 712, 651, 611, 530; ${ }^{\mathbf{1}} \mathbf{H}$ NMR $\left(300 \mathrm{MHz}, \mathrm{CDCl}_{3}\right): \delta[\mathrm{ppm}]=7.96-7.77(\mathrm{~m}, 2 \mathrm{H}), 7.76-7.63(\mathrm{~m}, 2 \mathrm{H}), 5.79$ (ddt, $J=17.1,10.1$, $6.9 \mathrm{~Hz}, 1 \mathrm{H}), 5.1-4.97(\mathrm{~m}, 2 \mathrm{H}), 3.77$ (t, $J=7.1 \mathrm{~Hz}, 2 \mathrm{H}), 2.45$ (qt, $J=7.0,1.3 \mathrm{~Hz}, 2 \mathrm{H}$ ); ${ }^{13} \mathbf{C}\left\{{ }^{1} \mathbf{H}\right\}$ NMR $\left(126 \mathrm{MHz}, \mathrm{CDCl}_{3}\right): \delta[\mathrm{ppm}]=168.3,134.5,133.9,132.2,123.3,117.6,37.5$, 33.0; HR-ESI-MS m/z: calcd. $\mathrm{C}_{12} \mathrm{H}_{11} \mathrm{NO}_{2} \mathrm{Na}[\mathrm{M}+\mathrm{Na}]^{+}$: 224.0682, found: 224.0684 .

\section{2-(7-Hydroxyhept-3-en-1-yl)isoindoline-1,3-dione (126g)}

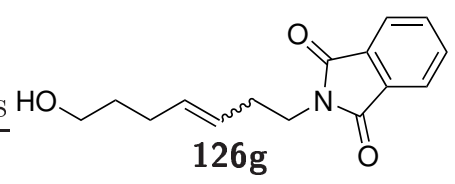

Following general procedure C: 4-pentene-1-ol (904 mg, $10.5 \mathrm{mmol}$, 3.0 equiv), 2-(but-3-en-1-yl)isoindoline-1,3-dione (335) (704 mg, $3.50 \mathrm{mmol}, 1.0$ equiv), DCM ( $7 \mathrm{~mL})$, 2nd gen. GrubBs catalyst (149 mg, 175 umol, 0.05 equiv); reaction time $20 \mathrm{~h}$; eluting with $n$-pentane/Et ${ }_{2} \mathrm{O}, 2: 1$; colorless liquid; $408 \mathrm{mg}, 1.57 \mathrm{mmol}, 45 \%$.

$\mathbf{R}_{\mathbf{f}}=0.25$ (n-pentane:EtOAc, 2:1); IR (ATR): $\tilde{\nu}\left[\mathrm{cm}^{-1}\right]=2935,2864,1771,1700,1614,1467$, 1436, 1393, 1359, 1187, 1130, 1057, 1015, 971, 870, 794, 718, 624, 530; ${ }^{\mathbf{1}} \mathbf{H}$ NMR $(300 \mathrm{MHz}$, $\left.\mathrm{CDCl}_{3}\right): \delta[\mathrm{ppm}]=7.85-7.81(\mathrm{~m}, 2 \mathrm{H}), 7.72-7.68(\mathrm{~m}, 2 \mathrm{H}), 5.48-5.37(\mathrm{~m}, 2 \mathrm{H}), 3.73(\mathrm{t}, J=7.0 \mathrm{~Hz}$, $2 \mathrm{H}), 3.53(\mathrm{t}, J=6.5 \mathrm{~Hz}, 2 \mathrm{H}), 2.43-2.31(\mathrm{~m}, 2 \mathrm{H}), 2.15-1.94(\mathrm{~m}, 3 \mathrm{H}), 1.66-1.40(\mathrm{~m}, 2 \mathrm{H})$; ${ }^{13} \mathbf{C}\left\{{ }^{1} \mathbf{H}\right\}$ NMR $\left(125 \mathrm{MHz}, \mathrm{CDCl}_{3}\right): \delta[\mathrm{ppm}]=$ major isomer: 168.4, 133.9, 132.8, 132.2, 126.7, 123.2, 62.3, 38.0, 32.3, 31.9, 28.9; HR-ESI-MS m/z: calcd. $\mathrm{C}_{15} \mathrm{H}_{18} \mathrm{NO}_{3}[\mathrm{M}+\mathrm{H}]^{+}: 260.1281$, found: 260.1284 .

\section{7-(Oxiran-2-yl)hept-4-en-1-ol (126i)}

Following general procedure C] 4-pentene-1-ol (904 mg, $10.5 \mathrm{mmol}$,

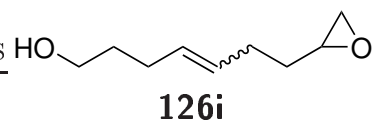

3.0 equiv), 1,2-epoxy-5-hexene ( $343 \mathrm{mg}, 3.50 \mathrm{mmol}, 1.0$ equiv), DCM

(7 mL), 2nd gen. GrubBs catalyst (149 mg, $175 \mu \mathrm{mol}, 0.05$ equiv); reaction time $22.5 \mathrm{~h}$; eluting with $n$-pentane/EtOAc, $5: 1$ to 0:1; brown liquid; $254 \mathrm{mg}, 1.63 \mathrm{mmol}$, $47 \%$.

$\mathbf{R}_{\mathbf{f}}=0.19$ (n-pentane:EtOAc, 5:1); IR (ATR): $\tilde{\nu}\left[\mathrm{cm}^{-1}\right]=3407,2928,2859,1723,1441,1410$, 1260, 1050, 970, 915, 833, 514, 439, 419, 402; ${ }^{\mathbf{1}} \mathbf{H} \mathbf{~ N M R}\left(300 \mathrm{MHz}, \mathrm{CDCl}_{3}\right): \delta[\mathrm{ppm}]=5.76-5.14$ 
$(\mathrm{m}, 2 \mathrm{H}), 3.64(\mathrm{t}, J=6.4 \mathrm{~Hz}, 2 \mathrm{H}), 2.92$ (dddd, $J=10.1,5.2,2.8,1.2 \mathrm{~Hz}, 1 \mathrm{H}), 2.75$ (dd, $J=5.0,4.0 \mathrm{~Hz}, 1 \mathrm{H}), 2.47(\mathrm{dt}, J=5.1,2.9 \mathrm{~Hz}, 1 \mathrm{H}), 2.40-1.85(\mathrm{~m}, 4 \mathrm{H}), 1.74-1.42(\mathrm{~m}, 5 \mathrm{H})$; ${ }^{13} \mathbf{C}\left\{{ }^{1} \mathbf{H}\right\}$ NMR $\left(126 \mathrm{MHz}, \mathrm{CDCl}_{3}\right): \delta[\mathrm{ppm}]=133.4,130.5,129.8,126.7,62.6,62.2,52.4,52.1$, $47.3,47.3,36.2,32.5,32.1,29.3,29.1,26.0$; HR-ESI-MS m/z: calcd. $\mathrm{C}_{9} \mathrm{H}_{16} \mathrm{O}_{2} \mathrm{Na}[\mathrm{M}+\mathrm{Na}]^{+}$: 179.1043, found: 179.1041 .

\section{4-Hydroxytetradec-10-enal (126I)}

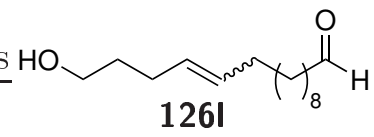

Following general procedure $\mathbf{C}$ 4-pentene-1-ol (904 mg, $10.5 \mathrm{mmol}$, 3.0 equiv), 10-undecenal (589 mg, $3.50 \mathrm{mmol}, 1.0$ equiv), DCM ( $7 \mathrm{~mL})$, 2nd gen. GRuBBs catalyst (149 mg, 175 umol, 0.05 equiv); reaction time $22 \mathrm{~h}$; eluting with $n$-pentane/EtOAc, 9:1; brown solid; $178 \mathrm{mg}$, 786 umol, $22 \%$.

$\mathbf{R}_{\mathbf{f}}=0.09$ (n-pentane:EtOAc, 9:1); m.p. $=46.2{ }^{\circ} \mathrm{C} ; \mathbf{I R}(\mathrm{ATR}): \tilde{\nu}\left[\mathrm{cm}^{-1}\right]=3419,2923,2853,2717$, 1723, 1461, 1409, 1391, 1051, 967, 723, 517; ${ }^{\mathbf{1}} \mathbf{H}$ NMR $\left(400 \mathrm{MHz}, \mathrm{CDCl}_{3}\right): \delta[\mathrm{ppm}]=9.76(\mathrm{t}$, $J=1.9 \mathrm{~Hz}, 1 \mathrm{H}), 5.63-5.24(\mathrm{~m}, 2 \mathrm{H}), 3.64(\mathrm{td}, J=6.6,3.7 \mathrm{~Hz}, 2 \mathrm{H}), 2.41(\mathrm{td}, J=7.3,1.9 \mathrm{~Hz}$, $2 \mathrm{H}), 2.35-1.89$ (m, $4 \mathrm{H}), 1.69-1.52(\mathrm{~m}, 4 \mathrm{H}), 1.49-1.19(\mathrm{~m}, 11 \mathrm{H}) ;{ }^{\mathbf{1 3}} \mathbf{C}\left\{{ }^{\mathbf{1}} \mathbf{H}\right\}$ NMR $(101 \mathrm{MHz}$, $\left.\mathrm{CDCl}_{3}\right): \delta[\mathrm{ppm}]=203.1,131.3,130.9,130.1,129.6,63.1,62.7,44.1,32.7,32.6,29.6,29.4,29.4$, 29.3, 29.3, 29.2, 29.1, 29.0, 22.2, 22.2; HR-ESI-MS m/z: calcd. $\mathrm{C}_{14} \mathrm{H}_{27} \mathrm{O}_{2}[\mathrm{M}+\mathrm{H}]^{+}: 227.2006$, found: 227.2015 .

\section{Oct-4-ene-1,8-diol (126m)}<smiles>OCCCC=CCCCO</smiles>

$126 \mathrm{~m}$

4-Pentene-1-ol (5.00 g, $58.1 \mathrm{mmol}, 1.0$ equiv) was dissolved in degassed

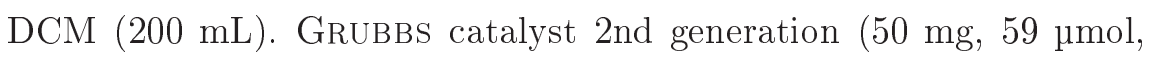
$0.1 \mathrm{~mol} \%$ ) was added and the reaction was stirred for $22 \mathrm{~h}$ at room temperature. After $3 \mathrm{~h}$, another portion of GRUBBs catalyst 2nd generation ( $50 \mathrm{mg}, 59$ umol, $0.1 \mathrm{~mol} \%$ ) was added, after 3 more hours, another portion (50 mg, 59 umol, $0.1 \mathrm{~mol} \%$ ) was added. After 3 more hours, GruBBs catalyst 2nd generation (100 mg, 118 umol, $0.2 \mathrm{~mol} \%$ ) in DCM $(6 \mathrm{~mL})$ was added via syringe pump over $6 \mathrm{~h}$. The solvent was removed in vacuum and the product afforded via column chromatography (n-pentane/EtOAc, 3:1 to 0:1) as a brown liquid (1.88 g, $13.0 \mathrm{mmol}, 45 \%)$.

$\mathbf{R}_{\mathbf{f}}=0.07$ (n-pentane:EtOAc, 3:1); IR (ATR): $\tilde{\nu}\left[\mathrm{cm}^{-1}\right]=3316,2931,2865,1440,1375,1351$, 1051, 966, 915, 631; ${ }^{\mathbf{1}} \mathbf{H}$ NMR (300 MHz, $\left.\mathrm{CDCl}_{3}\right): \delta[\mathrm{ppm}]=5.75-5.20$ (m, $\left.2 \mathrm{H}\right), 3.72-3.46$ $(\mathrm{m}, 4 \mathrm{H}), 2.86-1.89(\mathrm{~m}, 6 \mathrm{H}), 1.74-1.27(\mathrm{~m}, 4 \mathrm{H}) ;{ }^{13} \mathbf{C}\left\{{ }^{\mathbf{1}} \mathbf{H}\right\}$ NMR $\left(125 \mathrm{MHz}, \mathrm{CDCl}_{3}\right): \delta$ $[\mathrm{ppm}]=130.2,62.4,32.5,29.0$; HR-ESI-MS m/z: calcd. $\mathrm{C}_{8} \mathrm{H}_{16} \mathrm{O}_{2} \mathrm{Na}[\mathrm{M}+\mathrm{Na}]^{+}: 167.1043$, found: 167.1043 . 


\section{Ethyl (8-hydroxyoct-4-en-1-yl) carbonate (126n)}

Oct-4-ene-1,8-diol 126m (216 mg, $1.50 \mathrm{mmol}, 1.0$ equiv) and<smiles>CCOC(=O)OCCC/C=C/CCCO</smiles>

DMAP (4.6 mg, 38 umol, 0.025 equiv) were dissolved in dry DCM $(2 \mathrm{~mL})$ and cooled to $0{ }^{\circ} \mathrm{C}$. Pyridine $(237 \mathrm{mg}, 3.0 \mathrm{mmol}$, 2.0 equiv) was added dropwise, then ethyl chloroformate $(163 \mathrm{mg}$,

$1.50 \mathrm{mmol}, 1.5$ equiv) was added dropwise and the reaction was allowed to warm up to room temperature. The solution was stirred for $23 \mathrm{~h}$, subsequently washed with $\mathrm{H}_{2} \mathrm{O}(5 \times 2 \mathrm{~mL})$ and the comb. aq. phases were extracted with DCM $(10 \mathrm{~mL})$. The comb. org. phases were dried over $\mathrm{Na}_{2} \mathrm{SO}_{4}$, the solvent was removed in vacuum and the product was afforded via column chromatography ( $n$-pentane/EtOAc, 4:1) as a colorless oil (113 mg, $520 \mu \mathrm{mol}, 35 \%$ ).

$\mathbf{R}_{\mathbf{f}}=0.21$ (n-pentane:EtOAc, 4:1); IR (ATR): $\tilde{\nu}\left[\mathrm{cm}^{-1}\right]=2983,2934,2874,1742,1467,1448$, 1403, 1386, 1368, 1251, 1054, 1010, 969, 863, 791; ${ }^{\mathbf{1}} \mathbf{H} \mathbf{N M R}\left(300 \mathrm{MHz}, \mathrm{CDCl}_{3}\right): \delta$ $[\mathrm{ppm}]=5.69-5.29(\mathrm{~m}, 2 \mathrm{H}), 4.18(\mathrm{q}, J=7.1 \mathrm{~Hz}, 2 \mathrm{H}), 4.14-4.07(\mathrm{~m}, 2 \mathrm{H}), 3.64(\mathrm{t}, J=6.5 \mathrm{~Hz}$, $2 \mathrm{H}), 2.23-1.98(\mathrm{~m}, 4 \mathrm{H}), 1.83-1.67(\mathrm{~m}, 2 \mathrm{H}), 1.67-1.56(\mathrm{~m}, 2 \mathrm{H}), 1.51(\mathrm{~m}, 1 \mathrm{H}), 1.31(\mathrm{t}, J=7.1 \mathrm{~Hz}$, $3 \mathrm{H}) ;{ }^{13} \mathbf{C}\left\{{ }^{1} \mathbf{H}\right\}$ NMR $\left(126 \mathrm{MHz}, \mathrm{CDCl}_{3}\right): \delta[\mathrm{ppm}]=155.3,130.8,129.4,67.4,64.0,62.6,32.6$, 29.0, 28.8, 28.7, 14.5; HR-ESI-MS m/z: calcd. $\mathrm{C}_{11} \mathrm{H}_{20} \mathrm{O}_{4} \mathrm{Na}[\mathrm{M}+\mathrm{Na}]^{+}:$239.1254, found: 239.1256.

\section{8-Hydroxyoct-4-en-1-yl pivalate (126o)}<smiles>O=C(Br)CCC/C=C/CCCO</smiles>

temperature, subsequently washed with aq. sat. $\mathrm{Na}_{2} \mathrm{CO}_{3}$ solution $(5 \mathrm{~mL})$ and aq. $1 \mathrm{M} \mathrm{HCl}$ $(5 \mathrm{~mL})$ and the comb. aq. phases were extracted with DCM $(10 \mathrm{~mL})$. The comb. org. phases were dried over $\mathrm{Na}_{2} \mathrm{SO}_{4}$, the solvent was removed in vacuo and the product was afforded via column chromatography ( $n$-pentane/EtOAc, 4:1) as a colorless oil (113 mg, 520 umol, 35\%).

$\mathbf{R}_{\mathbf{f}}=0.29$ (n-pentane:EtOAc, 4:1); IR (ATR): $\tilde{\nu}\left[\mathrm{cm}^{-1}\right]=2958,2934,2872,1727,1480,1460$, 1398, 1366, 1284, 1153, 1055, 1036, 968, 892, 772; ${ }^{\mathbf{1}} \mathbf{H}$ NMR $\left(300 \mathrm{MHz}, \mathrm{CDCl}_{3}\right): \delta[\mathrm{ppm}]=5.83-$ $5.22(\mathrm{~m}, 2 \mathrm{H}), 4.04(\mathrm{t}, J=6.5 \mathrm{~Hz}, 2 \mathrm{H}), 3.64(\mathrm{t}, J=6.5 \mathrm{~Hz}, 2 \mathrm{H}), 2.20-1.90(\mathrm{~m}, 4 \mathrm{H}), 1.87-1.50$ $(\mathrm{m}, 4 \mathrm{H}), 1.41(\mathrm{~s}, 1 \mathrm{H}), 1.20(\mathrm{~d}, J=1.1 \mathrm{~Hz}, 9 \mathrm{H}) ;{ }^{\mathbf{1 3}} \mathbf{C}\left\{{ }^{\mathbf{1}} \mathbf{H}\right\} \mathbf{N M R}\left(126 \mathrm{MHz}, \mathrm{CDCl}_{3}\right): \delta$ $[\mathrm{ppm}]=178.6,130.6,129.6,63.9,62.6,39.0,32.6,29.1,28.7,27.4$; HR-ESI-MS m/z: calcd. $\mathrm{C}_{13} \mathrm{H}_{24} \mathrm{O}_{3} \mathrm{Na}[\mathrm{M}+\mathrm{Na}]^{+}: 251.1618$, found: 251.1624 . 


\section{8-((Triisopropylsilyl)oxy)oct-4-en-1-ol (126p)}

Oct-4-ene-1,8-diol 126m (216 mg, $1.50 \mathrm{mmol}, 1.0$ equiv) was dis-

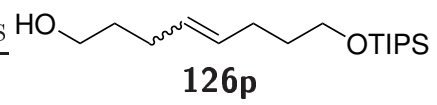

solved in dry DCM $(1.3 \mathrm{~mL})$, imidazole $(123 \mathrm{mg}, 1.80 \mathrm{mmol}$, 1.2 equiv) and TIPSCl (318 mg, $1.65 \mathrm{mmol}, 1.1$ equiv) were added and the solution stirred at room temperature for $17 \mathrm{~h}$. Subsequently, it was washed with aq. sat. $\mathrm{NaCl}(2 \times 1 \mathrm{~mL})$ and the aq. phase was extracted with DCM $(2 \times 3 \mathrm{~mL})$. The comb. org. phases were dried over $\mathrm{Na}_{2} \mathrm{SO}_{4}$, the solvent was removed in vacuo and the product was afforded via column chromatography ( $n$-pentane/EtOAc, 4:1) as a colorless oil (167 mg, 556 mol, 37\%).

$\mathbf{R}_{\mathbf{f}}=0.43$ (n-pentane:EtOAc, 4:1); IR (ATR): $\tilde{\nu}\left[\mathrm{cm}^{-1}\right]=2940,2865,2047,1968,1463,1383$, 1246, 1104, 1064, 1013, 995, 967, 919, 882, 788, 724, 679, 658, 507, 460, 419, 395; ${ }^{\mathbf{1}} \mathbf{H}$ NMR $\left(400 \mathrm{MHz}, \mathrm{CDCl}_{3}\right): \delta[\mathrm{ppm}]=5.77-5.20(\mathrm{~m}, 2 \mathrm{H}), 3.81-3.57(\mathrm{~m}, 4 \mathrm{H}), 2.43-1.92(\mathrm{~m}, 4 \mathrm{H})$, 1.69-1.49 (m, $4 \mathrm{H}), 1.25(\mathrm{~m}, 1 \mathrm{H}), 1.18-0.92(\mathrm{~m}, 21 \mathrm{H}) ;{ }^{\mathbf{1 3}} \mathbf{C}\left\{{ }^{\mathbf{1}} \mathbf{H}\right\}$ NMR (101 MHz, $\left.\mathrm{CDCl}_{3}\right)$ : $\delta[\mathrm{ppm}]=130.8,129.9,62.9,62.7,33.0,32.6,29.1,29.0,18.2,12.2 ;$ HR-ESI-MS m/z: calcd. $\mathrm{C}_{17} \mathrm{H}_{37} \mathrm{O}_{2} \mathrm{Si}[\mathrm{M}+\mathrm{H}]^{+}:$301.2557, found: 301.2554 .

\section{$(E / Z)-8,12-$ Dimethyltridec-5-ene-1,12-diol (139a)}

Following general procedure B: acid 137a (710 mg, $2.77 \mathrm{mmol}$,

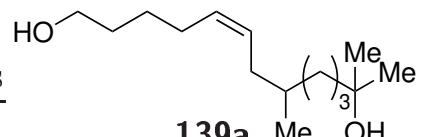
1.0 equiv), $\mathrm{LiAlH}_{4}(2.4 \mathrm{M}$ in THF; $1.73 \mathrm{~mL}, 4.16 \mathrm{mmol}, 1.5$ equiv), THF (9 mL); reaction time $4.5 \mathrm{~h}$; yield: $404 \mathrm{mg}, 1.89 \mathrm{mmol}, 68 \%$; mixture of $E / Z$ isomers, colorless liquid.

$\mathbf{R}_{\mathbf{f}}=0.21$ (n-pentane:Et $\left.{ }_{2} \mathrm{O}, 1: 1\right)$; IR (ATR): $\tilde{\nu}\left[\mathrm{cm}^{-1}\right]=3327,2933,2866,1459,1377,1198$, 1160, 1065, 937, 907, 688; ${ }^{\mathbf{1}} \mathbf{H}$ NMR $\left(300 \mathrm{MHz}, \mathrm{CDCl}_{3}\right): \delta[\mathrm{ppm}]=5.61-5.20(\mathrm{~m}, 2 \mathrm{H}), 3.63$ $(\mathrm{td}, J=6.5,0.6 \mathrm{~Hz}, 2 \mathrm{H}), 2.03(\mathrm{dtd}, J=13.5,6.4,5.4,4.0 \mathrm{~Hz}, 3 \mathrm{H}), 1.86(\mathrm{~m}, 1 \mathrm{H}), 1.67(\mathrm{~d}$, $J=0.8 \mathrm{~Hz}, 2 \mathrm{H}), 1.63-1.24(\mathrm{~m}, 11 \mathrm{H}), 1.20(\mathrm{~d}, J=0.5 \mathrm{~Hz}, 6 \mathrm{H}), 0.87(\mathrm{dd}, J=6.6,0.5 \mathrm{~Hz}$, $3 \mathrm{H}) ;{ }^{13} \mathbf{C}\left\{{ }^{1} \mathbf{H}\right\} \mathbf{N M R}\left(126 \mathrm{MHz}, \mathrm{CDCl}_{3}\right): \delta[\mathrm{ppm}]=$ major isomer: 130.3, 128.8, 71.2, 63.0, 44.4, 37.3, 34.6, 33.6, 32.6, 29.5, 29.4, 27.2, 26.0, 22.0, 19.9; minor isomer: 131.3, 129.3, 63.0, 40.2, 37.2, 33.3, 32.5, 32.4, 25.9, 22.0, 19.8; HR-ESI-MS m/z: calcd. $\mathrm{C}_{15} \mathrm{H}_{30} \mathrm{NaO}_{2}[\mathrm{M}+\mathrm{Na}]^{+}$: 265.2138, found: 265.2139 .

\section{(E/Z)-8,12-Dimethyltrideca-5,11-dien-1-ol (139b)}

Following general procedure B acid 137b $(663 \mathrm{mg}, 2.78 \mathrm{mmol}$,

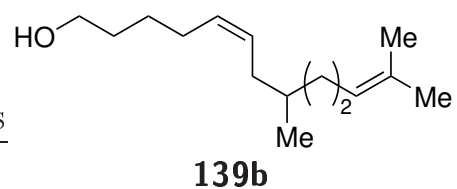

$139 b$ 1.0 equiv), $\mathrm{LiAlH}_{4}$ (2.4 M in THF; $1.74 \mathrm{~mL}, 4.17 \mathrm{mmol}, 1.5$ equiv), THF (9 mL); reaction time $17.5 \mathrm{~h}$; yield: $471 \mathrm{mg}, 2.11 \mathrm{mmol}, 76 \%$; mixture of $E / Z$ isomers, colorless liquid.

$\mathbf{R}_{\mathbf{f}}=0.21$ (n-pentane:Et $\left.{ }_{2} \mathrm{O}, 5: 1\right)$; IR (ATR): $\tilde{\nu}\left[\mathrm{cm}^{-1}\right]=3333,2924,2858,2042,2016,1966,1653$, 1454, 1376, 1341, 1063, 985, 824, 699; ${ }^{\mathbf{1}} \mathbf{H}$ NMR $\left(300 \mathrm{MHz}, \mathrm{CDCl}_{3}\right): \delta[\mathrm{ppm}]=5.56-5.23(\mathrm{~m}$, 
$2 \mathrm{H}), 5.10$ (tdt, $J=7.1,2.9,1.5 \mathrm{~Hz}, 1 \mathrm{H}), 3.65$ (t, $J=6.5 \mathrm{~Hz}, 2 \mathrm{H}), 2.15-1.80(\mathrm{~m}, 6 \mathrm{H}), 1.68$ $(\mathrm{q}, J=1.3 \mathrm{~Hz}, 3 \mathrm{H}), 1.64-1.52(\mathrm{~m}, 5 \mathrm{H}), 1.52-1.28(\mathrm{~m}, 4 \mathrm{H}), 1.25(\mathrm{~s}, 1 \mathrm{H}), 1.15(\mathrm{~m}, 1 \mathrm{H}), 0.88$ $(\mathrm{d}, J=6.5 \mathrm{~Hz}, 3 \mathrm{H}) ;{ }^{\mathbf{1 3}} \mathbf{C}\left\{{ }^{1} \mathbf{H}\right\} \mathbf{N M R}\left(101 \mathrm{MHz}, \mathrm{CDCl}_{3}\right): \delta[\mathrm{ppm}]=131.2,130.2,128.9,125.0$, 63.1, 37.0, 34.7, 33.3, 32.7, 27.3, 26.1, 25.9, 25.9, 19.8, 17.9; HR-ESI-MS m/z: calcd. $\mathrm{C}_{15} \mathrm{H}_{29} \mathrm{O}$ $[\mathrm{M}+\mathrm{H}]^{+}:$225.2213, found: 225.2211 .

\section{(Z)-8-Phenyloct-5-en-1-ol (139c)}

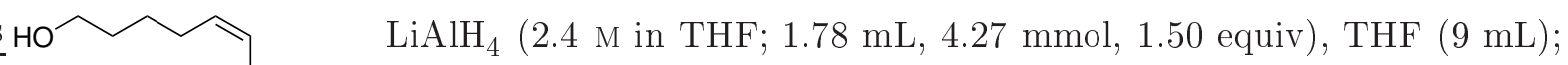
139c Ph reaction time 4 h; yield: $293 \mathrm{mg}, 1.43 \mathrm{mmol}, 51 \%$, colorless liquid.

$\mathbf{R}_{\mathbf{f}}=0.11$ (n-pentane:Et $\left.{ }_{2} \mathrm{O}, 9: 1\right)$; IR (ATR): $\tilde{\nu}\left[\mathrm{cm}^{-1}\right]=3332,3026,3005,2930,2857,1495,1453$, $1059,1031,746,722,696,583,489 ;{ }^{1} \mathbf{H}$ NMR $\left(300 \mathrm{MHz}, \mathrm{CDCl}_{3}\right): \delta[\mathrm{ppm}]=7.32-7.20(\mathrm{~m}, 2 \mathrm{H})$, $7.17(\mathrm{~d}, J=7.0 \mathrm{~Hz}, 3 \mathrm{H}), 5.62-5.01(\mathrm{~m}, 2 \mathrm{H}), 3.59(\mathrm{t}, J=6.5 \mathrm{~Hz}, 1 \mathrm{H}), 2.64(\mathrm{dd}, J=8.7,6.7 \mathrm{~Hz}$, $2 \mathrm{H}), 2.48-2.14(\mathrm{~m}, 2 \mathrm{H}), 2.07-1.83(\mathrm{~m}, 2 \mathrm{H}), 1.63-1.38(\mathrm{~m}, 2 \mathrm{H}), 1.32$ (dddd, $J=14.7,11.0$, 6.2, $2.2 \mathrm{~Hz}, 3 \mathrm{H}) ;{ }^{13} \mathbf{C}\left\{{ }^{1} \mathbf{H}\right\} \mathbf{N M R}\left(126 \mathrm{MHz}, \mathrm{CDCl}_{3}\right): \delta[\mathrm{ppm}]=142.2,130.3,129.2,128.6$, 128.4, 125.9, 63.0, 36.1, 32.5, 29.4, 27.0, 25.8; HR-ESI-MS m/z: calcd. $\mathrm{C}_{14} \mathrm{H}_{20} \mathrm{ONa}[\mathrm{M}+\mathrm{Na}]^{+}$: 227.1406, found: 227.1408 .

\section{(Z)-6-Cyclohexylhex-5-en-1-ol (139d)}

Following general procedure B] acid 137d (530 mg, $2.70 \mathrm{mmol}, 1.0$ equiv), 139d Cy $\mathrm{LiAlH}_{4}(2.4 \mathrm{M}$ in THF; $1.7 \mathrm{~mL}, 4.0 \mathrm{mmol}, 1.5$ equiv), THF (9 mL); reaction
time $17.5 \mathrm{~h}$; yield: $451 \mathrm{mg}, 2.47 \mathrm{mmol}, 91 \%$, colorless liquid.

$\mathbf{R}_{\mathbf{f}}=0.15$ (n-pentane:Et $\left.{ }_{2} \mathrm{O}, 7: 1\right) ; \mathbf{I R}(\mathrm{ATR}): \tilde{\nu}\left[\mathrm{cm}^{-1}\right]=3328,2999,2921,2849,1447,1059,993$, 889, 729; ${ }^{\mathbf{1}} \mathbf{H}$ NMR $\left(400 \mathrm{MHz}, \mathrm{CDCl}_{3}\right): \delta[\mathrm{ppm}]=5.53-4.73(\mathrm{~m}, 2 \mathrm{H}), 3.65(\mathrm{t}, J=6.6 \mathrm{~Hz}, 2 \mathrm{H})$, $2.23(\mathrm{tdq}, J=11.1,7.8,3.8 \mathrm{~Hz}, 1 \mathrm{H}), 2.14-2.01(\mathrm{~m}, 2 \mathrm{H}), 1.82-1.64(\mathrm{~m}, 2 \mathrm{H}), 1.64-1.49(\mathrm{~m}$, $5 \mathrm{H}), 1.49-1.37$ (m, $2 \mathrm{H}), 1.35-1.12(\mathrm{~m}, 4 \mathrm{H}), 1.10-0.97(\mathrm{~m}, 2 \mathrm{H}) ;{ }^{\mathbf{1 3}} \mathbf{C}\left\{{ }^{\mathbf{1}} \mathbf{H}\right\}$ NMR $(101 \mathrm{MHz}$, $\left.\mathrm{CDCl}_{3}\right): \delta[\mathrm{ppm}]=136.6,127.6,63.1,36.5,33.5,32.5,27.3,26.2,26.1 ;$ GC-MS (EI) $\mathbf{m} / \mathbf{z}$ : calcd. $\mathrm{C}_{12} \mathrm{H}_{22} \mathrm{O}[\mathrm{M}]^{+}:$: 182.2 , found: 182.3 .

\section{(Z)-8-Methylnon-5-en-1-ol (139e)}

Following general procedure B acid 137e (500 mg, $2.94 \mathrm{mmol}, 1.0$ equiv),

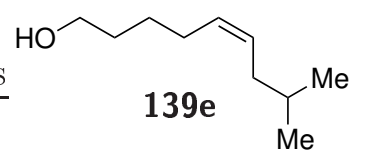
$\mathrm{LiAlH}_{4}(2.4 \mathrm{M}$ in THF; $1.8 \mathrm{~mL}, 4.4 \mathrm{mmol}, 1.5$ equiv), THF $(9 \mathrm{~mL})$; reaction time $4 \mathrm{~h}$; yield: $334 \mathrm{mg}, 2.14 \mathrm{mmol}, 71 \%$, colorless liquid.

$\mathbf{R}_{\mathbf{f}}=0.22$ (n-pentane:Et $\left.{ }_{2} \mathrm{O}, 9: 1\right) ; \mathbf{I R}$ (ATR): $\tilde{\nu}\left[\mathrm{cm}^{-1}\right]=3318,3006$, 2953, 2931, 2868, 1464, 1383, 1366, 1336, 1064, 989, 968, 937, 828, 707, 580; ${ }^{\mathbf{H}} \mathbf{H} \mathbf{~ N M R}(300 \mathrm{MHz}$, $\left.\mathrm{CDCl}_{3}\right): \delta[\mathrm{ppm}]=5.53-5.04(\mathrm{~m}, 2 \mathrm{H}), 3.61(\mathrm{t}, J=6.5 \mathrm{~Hz}, 2 \mathrm{H}), 2.04(\mathrm{dddd}, J=7.9,6.5,4.9$, 
$0.6 \mathrm{~Hz}, 2 \mathrm{H}), 1.95-1.76(\mathrm{~m}, 3 \mathrm{H}), 1.67-1.48(\mathrm{~m}, 3 \mathrm{H}), 1.48-1.22(\mathrm{~m}, 2 \mathrm{H}), 0.87(\mathrm{~d}, J=6.6 \mathrm{~Hz}$, $6 \mathrm{H}) ;{ }^{13} \mathbf{C}\left\{{ }^{\mathbf{1}} \mathbf{H}\right\}$ NMR $\left(126 \mathrm{MHz}, \mathrm{CDCl}_{3}\right): \delta[\mathrm{ppm}]=130.1,129.0,62.9,36.6,32.6,28.8,27.2$, 26.0, 22.6; GC-MS EI m/z: calcd. $\mathrm{C}_{10} \mathrm{H}_{20} \mathrm{O}[\mathrm{M}]^{+}$: 156.2, found: 156.2 .

\section{(E/Z)-10-Chlorodec-5-en-1-ol (139f)}

Following general procedure B acid 137f (477 mg, $2.34 \mathrm{mmol}$, HO 1.0 equiv), $\mathrm{LiAlH}_{4}(2.4 \mathrm{M}$ in THF; $1.46 \mathrm{~mL}, 3.51 \mathrm{mmol}, 1.5$ equiv), 139f $\sim \mathrm{Cl}$ THF $(8 \mathrm{~mL})$; reaction time $17.5 \mathrm{~h}$; yield: $161 \mathrm{mg}, 842 \mu \mathrm{mol}, 36 \%$; mixture of $E / Z$ isomers, colorless liquid.

$\mathbf{R}_{\mathbf{f}}=0.29$ (n-pentane:Et $\left.{ }_{2} \mathrm{O}, 3: 2\right) ; \mathbf{I R}(\mathrm{ATR}): \tilde{\nu}\left[\mathrm{cm}^{-1}\right]=3342,3004,2933,2859,1455,1363$, 1311, 1058, 715, 651; ${ }^{\mathbf{1}} \mathbf{H}$ NMR $\left(300 \mathrm{MHz}_{\mathrm{CDCl}}\right): \delta[\mathrm{ppm}]=5.57 .-5.19(\mathrm{~m}, 2 \mathrm{H}), 3.65$ (t, $J=6.5 \mathrm{~Hz}, 2 \mathrm{H}), 3.54(\mathrm{t}, J=6.7 \mathrm{~Hz}, 2 \mathrm{H}), 2.26-1.94(\mathrm{~m}, 4 \mathrm{H}), 1.94-1.69(\mathrm{~m}, 2 \mathrm{H}), 1.69-1.34$ $(\mathrm{m}, 6 \mathrm{H}) ;{ }^{\mathbf{1 3}} \mathbf{C}\left\{{ }^{\mathbf{1}} \mathbf{H}\right\} \mathbf{N M R}\left(101 \mathrm{MHz}, \mathrm{CDCl}_{3}\right): \delta[\mathrm{ppm}]=$ major isomer: 130.2, 129.4, 63.1, 45.2, 32.6, 32.4, 27.2, 27.1, 26.6, 26.0; minor isomer: 130.7, 130.0, 32.5, 32.3, 31.9, 27.8, 25.9; GC-MS (EI) $\mathbf{m} /$ z: calcd. $\mathrm{C}_{10} \mathrm{H}_{17} \mathrm{Cl}\left[\mathrm{M}-\mathrm{H}_{2} \mathrm{O}\right]^{+}$: 172.1 , found: 172.2 .

\section{tert-Butyl (Z)-4-(7-hydroxyhept-2-en-1-yl)piperidine-1-carboxylate (139g)}

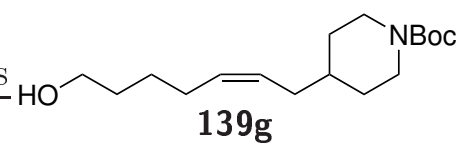

Following general procedure B acid $\mathbf{1 3 7 g}(829 \mathrm{mg}, 2.66 \mathrm{mmol}$, 1.0 equiv), $\mathrm{LiAlH}_{4}$ (2.4 M in THF; $1.7 \mathrm{~mL}, 4.0 \mathrm{mmol}, 1.5$ equiv), THF (9 mL); reaction time $4.5 \mathrm{~h}$; yield: $480 \mathrm{mg}, 1.61 \mathrm{mmol}, 61 \%$, colorless liquid.

IR (ATR): $\tilde{\nu}\left[\mathrm{cm}^{-1}\right]=3447,2927,2854,1693,1422,1365,1277,1243,1161,970,864,767 ;{ }^{\mathbf{1}} \mathbf{H}$ NMR $\left(500 \mathrm{MHz}, \mathrm{CDCl}_{3}\right): \delta[\mathrm{ppm}]=5.64-5.21(\mathrm{~m}, 2 \mathrm{H}), 4.06(\mathrm{~d}, J=13.1 \mathrm{~Hz}, 2, \mathrm{H}), 3.64(\mathrm{td}$, $J=6.5,1.5 \mathrm{~Hz}, 2 \mathrm{H}), 2.66(\mathrm{t}, J=12.8 \mathrm{~Hz}, 2 \mathrm{H}), 2.25(\mathrm{~s}, 1 \mathrm{H}), 2.05$ (q, $J=6.8 \mathrm{~Hz}, 2 \mathrm{H}), 1.97$ (t, $J=6.6 \mathrm{~Hz}, 2 \mathrm{H}), 1.83-1.45(\mathrm{~m}, 5 \mathrm{H}), 1.51-1.33(\mathrm{~m}, 11 \mathrm{H}), 1.19-0.97(\mathrm{~m}, 2 \mathrm{H}) ;{ }^{\mathbf{1 3}} \mathbf{C}\left\{{ }^{1} \mathbf{H}\right\}$ NMR $\left(125 \mathrm{MHz}, \mathrm{CDCl}_{3}\right): \delta[\mathrm{ppm}]=154.9,130.9,127.7,79.3,63.0,56.2,46.6,36.8,34.3,32.6,28.7$, 27.3, 26.0; HR-ESI-MS m/z: calcd. $\mathrm{C}_{17} \mathrm{H}_{31} \mathrm{NO}_{3} \mathrm{Na}[\mathrm{M}+\mathrm{Na}]^{+}: 320.2196$, found: 320.2197.

\section{(Z)-Hexadeca-5,15-dien-1-ol (139h)}

Following general procedure B acid 137h $(719 \mathrm{mg}, 2.85 \mathrm{mmol}$,

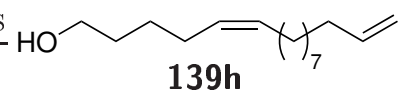
1.00 equiv), $\mathrm{LiAlH}_{4}$ (2.4 M in THF; $1.78 \mathrm{~mL}, 4.27 \mathrm{mmol}, 1.50$ equiv), THF (9 mL); reaction time $5.5 \mathrm{~h}$; yield: $578 \mathrm{mg}, 2.42 \mathrm{mmol}, 85 \%$, colorless liquid.

$\mathbf{R}_{\mathbf{f}}=0.36$ (n-pentane:Et $\left.{ }_{2} \mathrm{O}, 3: 1\right)$; IR (ATR): $\tilde{\nu}\left[\mathrm{cm}^{-1}\right]=3697,3322,3076,3004,2923,2853,2360$, 2341, 1726, 1640, 1460, 136, 1060, 991, 908, 721; ${ }^{\mathbf{1}} \mathbf{H} \mathbf{N M R}\left(400 \mathrm{MHz}, \mathrm{CDCl}_{3}\right): \delta[\mathrm{ppm}]=5.81$ (ddt, $J=16.9,10.2,6.7 \mathrm{~Hz}, 1 \mathrm{H}), 5.55-5.26(\mathrm{~m}, 2 \mathrm{H}), 4.99(\mathrm{ddt}, J=17.1,2.1,1.7 \mathrm{~Hz}, 1 \mathrm{H}), 4.93$ 
(ddt, $J=10.2,2.4,1.2 \mathrm{~Hz}, 1 \mathrm{H}), 3.65(\mathrm{t}, J=6.6 \mathrm{~Hz}, 2 \mathrm{H}), 2.26-1.91(\mathrm{~m}, 6 \mathrm{H}), 1.71-1.52(\mathrm{~m}$, $2 \mathrm{H}), 1.48-1.12(\mathrm{~m}, 15 \mathrm{H}) ;{ }^{13} \mathbf{C}\left\{{ }^{\mathbf{1}} \mathbf{H}\right\} \mathbf{N M R}\left(101 \mathrm{MHz}, \mathrm{CDCl}_{3}\right): \delta[\mathrm{ppm}]=139.4,130.5,129.4$, 114.2, 63.1, 34.0, 32.5, 29.9, 29.6, 29.6, 29.4, 29.3, 29.1, 27.4, 27.1, 26.0; HR-ESI-MS m/z: calcd. $\mathrm{C}_{16} \mathrm{H}_{31} \mathrm{O}[\mathrm{M}+\mathrm{H}]^{+}:$239.2369, found: 239.2368.

\section{(Z)-Dodec-5-en-11-yn-1-ol (139i)}

Following general procedure B: acid 137i $(310 \mathrm{mg}, 1.60 \mathrm{mmol}$, HO $ح 1.00$ equiv), $\mathrm{LiAlH}_{4}(2.4 \mathrm{M}$ in THF; $1.00 \mathrm{~mL}, 2.40 \mathrm{mmol}, 1.50$ equiv), $139 \mathrm{i}$ THF ( $8 \mathrm{~mL}$ ); reaction time $4 \mathrm{~h}$; yield: $262 \mathrm{mg}, 1.45 \mathrm{mmol}, 91 \%$, colorless liquid. Contains allene (10\%).

$\mathbf{R}_{\mathbf{f}}=0.22$ (n-pentane:Et $\left.{ }_{2} \mathrm{O}, 4: 1\right)$; IR (ATR): $\tilde{\nu}\left[\mathrm{cm}^{-1}\right]=3304,3005,2931,2858,2117,1955$, 1653, 1456, 1433, 1327, 1057, 972, 939, 843; ${ }^{\mathbf{1}} \mathbf{H}$ NMR $\left(300 \mathrm{MHz}, \mathrm{CDCl}_{3}\right): \delta[\mathrm{ppm}]=5.54-5.28$ $(\mathrm{m}, 2 \mathrm{H}), 3.65(\mathrm{t}, J=6.5 \mathrm{~Hz}, 2 \mathrm{H}), 2.27-2.14(\mathrm{~m}, 2 \mathrm{H}), 2.14-1.97(\mathrm{~m}, 4 \mathrm{H}), 1.94(\mathrm{t}, J=2.7 \mathrm{~Hz}$, $1 \mathrm{H}), 1.67-1.35(\mathrm{~m}, 9 \mathrm{H}) ;{ }^{13} \mathbf{C}\left\{{ }^{1} \mathbf{H}\right\} \mathbf{N M R}\left(126 \mathrm{MHz}, \mathrm{CDCl}_{3}\right): \delta[\mathrm{ppm}]=129.9,129.8,84.7,68.4$, 63.1, 32.6, 28.9, 28.3, 27.2, 26.9, 26.1, 18.5; HR-ESI-MS m/z: calcd. $\mathrm{C}_{12} \mathrm{H}_{20} \mathrm{ONa}[\mathrm{M}+\mathrm{Na}]^{+}$: 203.1406, found: 203.1401 .

\section{(Z)-8-(4-Bromophenyl)oct-5-en-1-ol (139j)}

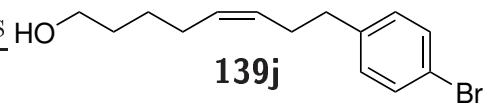

Following general procedure B: acid 137j $(650 \mathrm{mg}, 2.19 \mathrm{mmol}$, 1.00 equiv $), \mathrm{LiAlH}_{4}(2.4 \mathrm{M}$ in THF; $1.37 \mathrm{~mL}, 3.28 \mathrm{mmol}$, 1.50 equiv), THF (9 mL); reaction time $4 \mathrm{~h}$; yield: $303 \mathrm{mg}$, $1.07 \mathrm{mmol}, 49 \%$ (1:0.4 inseparable mixture with debrominated

product 139c), colorless liquid.

$\mathbf{R}_{\mathbf{f}}=0.11$ (n-pentane:Et $\left.{ }_{2} \mathrm{O}, 9: 1\right)$; IR (ATR): $\tilde{\nu}\left[\mathrm{cm}^{-1}\right]=3324,3005,2929,2857,1487,1453$, 1403, 1070, 1011, 813, 772, 698; ${ }^{\mathbf{1}} \mathbf{H}$ NMR $\left(400 \mathrm{MHz}, \mathrm{CDCl}_{3}\right): \delta[\mathrm{ppm}]=7.54-7.34(\mathrm{~m}, 2 \mathrm{H})$, 7.11-6.94 (m, $2 \mathrm{H}), 5.42-5.31(\mathrm{~m}, 2 \mathrm{H}), 3.61(\mathrm{t}, J=6.5 \mathrm{~Hz}, 2 \mathrm{H}), 2.61(\mathrm{t}, J=7.6 \mathrm{~Hz}, 2 \mathrm{H})$, 2.33 (dddd, $J=8.6,7.6,5.1,1.3 \mathrm{~Hz}, 2 \mathrm{H}), 2.03-1.90(\mathrm{~m}, 2 \mathrm{H}), 1.55-1.46(\mathrm{~m}, 2 \mathrm{H}), 1.39-1.29$ (m, $2 \mathrm{H}), 1.24(\mathrm{~s}, 1 \mathrm{H}) ;{ }^{13} \mathbf{C}\left\{{ }^{1} \mathbf{H}\right\} \mathbf{N M R}\left(126 \mathrm{MHz}, \mathrm{CDCl}_{3}\right): \delta[\mathrm{ppm}]=141.0,131.3,130.6,130.4$, 128.6, 119.6, 63.0, 35.5, 32.5, 29.2, 27.2, 25.9; HR-ESI-MS m/z: calcd. $\mathrm{C}_{14} \mathrm{H}_{20} \mathrm{OBr}[\mathrm{M}+\mathrm{H}]^{+}$: 283.0692, found: 283.0690 .

\subsubsection{Synthesis of tetrahydrofurans and tetrahydropyrans 127 and 144}

\section{General procedure D: intramolecular etherification}

To a solution of alcohol 126 or 139 (1.00 mmol, 1.0 equiv) in MeCN (0.2 M) are added (2anisyl) ${ }_{2} \mathrm{Se}_{2}$ (18.6 mg, 50.0 umol, 0.05 equiv), p-MeO-TPT (95) (14.6 mg, 30.0 umol, 0.03 equiv) 
and $\mathrm{NaH}_{2} \mathrm{PO}_{4}$ (96 mg, $0.8 \mathrm{mmol}, 0.8$ equiv). The mixture is stirred vigorously at room temperature under ambient air and irradiation at $\lambda=465 \mathrm{~nm}$ until complete conversion (monitored by TLC/NMR). Removal of the solvent in vacuo, followed by column chromatography afford products 127 or 144.

\section{(E)-2-(Hex-1-en-1-yl)tetrahydrofuran (127a)}

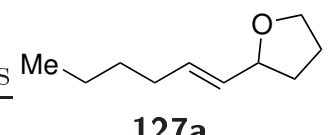

Following general procedure D: alcohol 126a; reaction time: $7 \mathrm{~h}$; eluting with $n$-pentane: $\mathrm{Et}_{2} \mathrm{O}$ (9:1); yield: $49 \mathrm{mg}, 0.32 \mathrm{mmol}, 32 \%$; colorless liquid; ${ }^{1} \mathrm{H}$ NMR yield using 1,3,5-trimethoxybenzene as the internal standard: $65 \%$.

$\mathbf{R}_{\mathbf{f}}=0.31$ (n-pentane:Et $\left.{ }_{2} \mathrm{O}, 9: 1\right) ; \mathbf{I R}(\mathrm{ATR}): \tilde{\nu}\left[\mathrm{cm}^{-1}\right]=2957,2926,2857,1459,1377,1153,1054$, 966, 731; ${ }^{1} \mathbf{H}$ NMR $\left(400 \mathrm{MHz}, \mathrm{CDCl}_{3}\right): \delta[\mathrm{ppm}]=5.67(\mathrm{dtd}, J=15.2,6.7,0.9 \mathrm{~Hz}, 1 \mathrm{H}), 5.44$ (ddt, $J=15.3,7.2,1.4 \mathrm{~Hz}, 1 \mathrm{H}), 4.22(\mathrm{q}, J=7.1 \mathrm{~Hz}, 1 \mathrm{H}), 3.89(\mathrm{ddd}, J=8.3,7.3,6.3 \mathrm{~Hz}, 1 \mathrm{H})$, $3.75(\mathrm{td}, J=7.9,6.1 \mathrm{~Hz}, 1 \mathrm{H}), 2.08-1.98(\mathrm{~m}, 3 \mathrm{H}), 1.97-1.79(\mathrm{~m}, 2 \mathrm{H}), 1.65-1.51(\mathrm{~m}, 2 \mathrm{H}), 1.33$ (tddd, $J=10.0,8.7,7.0,4.3 \mathrm{~Hz}, 3 \mathrm{H}), 0.88(\mathrm{t}, J=7.1 \mathrm{~Hz}, 3 \mathrm{H}) ;{ }^{\mathbf{1 3}} \mathbf{C}\left\{{ }^{\mathbf{1}} \mathbf{H}\right\} \mathbf{N M R}(101 \mathrm{MHz}$, $\left.\mathrm{CDCl}_{3}\right): \delta[\mathrm{ppm}]=133.0,130.7,80.2,68.0,32.4,32.0,31.4,26.1,22.4,14.1 ;$ HR-ESI-MS m/z: calcd. $\mathrm{C}_{10} \mathrm{H}_{19} \mathrm{O}[\mathrm{M}+\mathrm{H}]^{+}$: 155.1430 , found: 155.1433 . The analytical data was in agreement with literature. 125$]$

\section{(E)-2-(3-(Tetrahydrofuran-2-yl)allyl)isoindoline-1,3-dione (127g)}

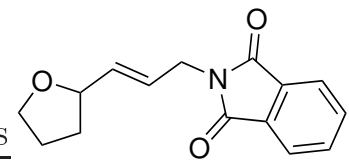

Following general procedure D alcohol 126g; reaction time: $7.5 \mathrm{~h}$; eluting with $n$-pentane:EtOAc (4:1); yield: $85 \mathrm{mg}, 0.33 \mathrm{mmol}, 37 \%$; yellow liquid; ${ }^{1} \mathrm{H}$ NMR yield using 1,1,2,2-tetrachloroethane as the internal standard: $45 \%$.

$127 \mathrm{~g}$

$\mathbf{R}_{\mathbf{f}}=0.19$ (n-pentane:EtOAc, 4:1); IR (ATR): $\tilde{\nu}\left[\mathrm{cm}^{-1}\right]=2972,2868,1770,1705,1613,1467$, 1428, 1391, 1188, 1112, 1087, 1050, 939, 854, 794, 718, 614, 529, 444; ${ }^{\mathbf{1}} \mathbf{H}$ NMR $(300 \mathrm{MHz}$, $\left.\mathrm{CDCl}_{3}\right): \delta[\mathrm{ppm}]=7.87-7.81(\mathrm{~m}, 2 \mathrm{H}), 7.76-7.66(\mathrm{~m}, 2 \mathrm{H}), 5.84-5.62(\mathrm{~m}, 2 \mathrm{H}), 4.39-4.13(\mathrm{~m}, 3 \mathrm{H})$, 3.87 (ddd, $J=8.3,7.1,6.4 \mathrm{~Hz}, 1 \mathrm{H}), 3.80-3.69(\mathrm{~m}, 1 \mathrm{H}), 2.08-1.96(\mathrm{~m}, 1 \mathrm{H}), 1.95-1.78(\mathrm{~m}$, $2 \mathrm{H}), 1.63-1.51(\mathrm{~m}, 1 \mathrm{H}) ;{ }^{13} \mathbf{C}\left\{{ }^{1} \mathbf{H}\right\} \mathbf{N M R}\left(125 \mathrm{MHz}, \mathrm{CDCl}_{3}\right): \delta[\mathrm{ppm}]=167.9,135.1,134.0$, 132.2, 124.2, 123.3, 78.7, 68.2, 39.2, 32.2, 25.9; HR-ESI-MS m/z: calcd. $\mathrm{C}_{15} \mathrm{H}_{16} \mathrm{NO}_{3}[\mathrm{M}+\mathrm{H}]^{+}$: 258.1125, found: 258.1124 .

\section{(E)-10-(Tetrahydrofuran-2-yl)dec-9-enal (127I)}

Following general procedure D alcohol 126l (170 mg, 751 umol, 1.0 equiv),<smiles>O=CCC=CC1CCCO1</smiles>

(2-anisyl) $\left.)_{2}\right)_{\mathrm{Se}_{2}}$ (14 mg, $0.038 \mathrm{mmol}, 0.05$ equiv), photosensitizer 95 (11 mg, $0.023 \mathrm{mmol}, 0.03$ equiv) and $\mathrm{NaH}_{2} \mathrm{PO}_{4}$ (72 $\mathrm{mg}, 0.60 \mathrm{mmol}, 0.8$ equiv); reaction 
time: $16.5 \mathrm{~h}$; eluting with $n$-pentane:EtOAc (20:1); yield: $12 \mathrm{mg}, 0.55 \mathrm{mmol}, 7 \%$; colorless liquid; ${ }^{1} \mathrm{H}$ NMR yield using 1,3,5-trimethoxybenzene as the internal standard: $24 \%$.

$\mathbf{R}_{\mathbf{f}}=0.21$ (n-pentane:EtOAc, 20:1); IR (ATR): $\tilde{\nu}\left[\mathrm{cm}^{-1}\right]=2926,2854,2716,2168,2015,1723$, 1461, 1410, 1371, 1179, 1051, 967, 920, 869, 726, 520, 401; ${ }^{\mathbf{1}} \mathbf{H}$ NMR (300 MHz, $\left.\mathrm{CDCl}_{3}\right): \delta$ $[\mathrm{ppm}]=9.75(\mathrm{t}, J=1.9 \mathrm{~Hz}, 1 \mathrm{H}), 5.65(\mathrm{dtd}, J=15.3,6.6,0.9 \mathrm{~Hz}, 1 \mathrm{H}), 5.44(\mathrm{ddt}, J=15.3$, $7.1,1.4 \mathrm{~Hz}, 1 \mathrm{H}), 4.21(\mathrm{q}, J=7.1 \mathrm{~Hz}, 1 \mathrm{H}), 3.89(\mathrm{~m}, 1 \mathrm{H}), 3.75(\mathrm{td}, J=7.8,6.1 \mathrm{~Hz}, 1 \mathrm{H}), 2.41$ $(\mathrm{td}, J=7.4,1.9 \mathrm{~Hz}, 2 \mathrm{H}), 2.11-1.77(\mathrm{~m}, 6 \mathrm{H}), 1.69-1.48(\mathrm{~m}, 2 \mathrm{H}), 1.45-1.16(\mathrm{~m}, 8 \mathrm{H}) ;{ }^{13} \mathbf{C}\left\{{ }^{\mathbf{1}} \mathbf{H}\right\}$ NMR $\left(126 \mathrm{MHz}, \mathrm{CDCl}_{3}\right): \delta[\mathrm{ppm}]=202.8,132.7,130.8,80.1,68.0,44.1,32.5,32.4,29.4,29.3$, 29.2, 29.1, 26.2, 22.3; HR-ESI-MS m/z: calcd. $\mathrm{C}_{14} \mathrm{H}_{25} \mathrm{O}_{2}[\mathrm{M}+\mathrm{H}]^{+}:$225.1849, found: 225.1847 .

\section{(E)-4-(Tetrahydrofuran-2-yl)but-3-en-1-ol (127m)}

Following general procedure D. alcohol $\mathbf{1 2 6 m}$; reaction time: $7 \mathrm{~h}$; eluting

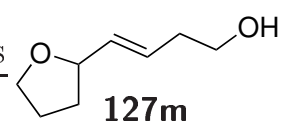

with $n$-pentane $/ \mathrm{Et}_{2} \mathrm{O}$ (9:1); yield: $49 \mathrm{mg}, 0.32 \mathrm{mmol}, 32 \%$; colorless liquid; ${ }^{1} \mathrm{H}$ NMR yield using 1,3,5-trimethoxybenzene as the internal standard: $65 \%$.

$\mathbf{R}_{\mathbf{f}}=0.31$ ( $n$-pentane:Et $\left.{ }_{2} \mathrm{O}, 9: 1\right)$; IR (ATR): $\tilde{\nu}\left[\mathrm{cm}^{-1}\right]=2957,2926,2857,1459,1377,1153,1054$, 966, 731; ${ }^{1} \mathbf{H}$ NMR $\left(400 \mathrm{MHz}, \mathrm{CDCl}_{3}\right): \delta[\mathrm{ppm}]=5.67(\mathrm{dtd}, J=15.2,6.7,0.9 \mathrm{~Hz}, 1 \mathrm{H}), 5.44$ (ddt, $J=15.3,7.2,1.4 \mathrm{~Hz}, 1 \mathrm{H}), 4.22(\mathrm{q}, J=7.1 \mathrm{~Hz}, 1 \mathrm{H}), 3.89(\mathrm{ddd}, J=8.3,7.3,6.3 \mathrm{~Hz}, 1 \mathrm{H})$, $3.75(\mathrm{td}, J=7.9,6.1 \mathrm{~Hz}, 1 \mathrm{H}), 2.08-1.98(\mathrm{~m}, 3 \mathrm{H}), 1.97-1.79(\mathrm{~m}, 2 \mathrm{H}), 1.65-1.51$ (m, $2 \mathrm{H}), 1.33$ (tddd, $J=10.0,8.7,7.0,4.3 \mathrm{~Hz}, 3 \mathrm{H}), 0.88(\mathrm{t}, J=7.1 \mathrm{~Hz}, 3 \mathrm{H}) ;{ }^{\mathbf{1 3}} \mathbf{C}\left\{{ }^{1} \mathbf{H}\right\}$ NMR $(101 \mathrm{MHz}$, $\left.\mathrm{CDCl}_{3}\right): \delta[\mathrm{ppm}]=133.0,130.7,80.2,68.0,32.4,32.0,31.4,26.1,22.4,14.1$; HR-ESI-MS m/z: calcd. $\mathrm{C}_{10} \mathrm{H}_{19} \mathrm{O}[\mathrm{M}+\mathrm{H}]^{+}$: 155.1430 , found: 155.1433 .

\section{(E)-Ethyl (4-(tetrahydrofuran-2-yl)but-3-en-1-yl) carbonate (127n)}

Following general procedure D alcohol 126n (113 mg, 524 umol,

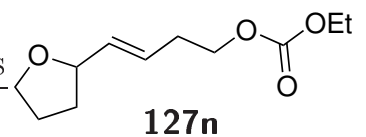
1.0 equiv), (2-anisyl) ${ }_{2} \mathrm{Se}_{2}$ (10 $\mathrm{mg}, 0.026 \mathrm{mmol}, 0.05$ equiv), photosensitizer 95 (7.6 mg, $0.016 \mathrm{mmol}, 0.03$ equiv) and $\mathrm{NaH}_{2} \mathrm{PO}_{4}(50 \mathrm{mg}$, $0.42 \mathrm{mmol}, 0.8$ equiv); reaction time: $7 \mathrm{~h}$; eluting with $n$-pentane $/$ EtOAc

(7:1); yield: $31 \mathrm{mg}, 0.14 \mathrm{mmol}, 28 \%$; colorless liquid; ${ }^{1} \mathrm{H}$ NMR yield using 1,1,2,2tetrachloroethane as the internal standard: $47 \%$.

$\mathbf{R}_{\mathbf{f}}=0.23$ (n-pentane:EtOAc, 7:1); IR (ATR): $\tilde{\nu}\left[\mathrm{cm}^{-1}\right]=2978,2868,1741,1464,1384,1366$, 1247, 1091, 1051, 1007, 968, 873, 791; ${ }^{\mathbf{1}} \mathbf{H}$ NMR $\left(300 \mathrm{MHz}, \mathrm{CDCl}_{3}\right): \delta[\mathrm{ppm}]=5.64(\mathrm{~m}, 1 \mathrm{H})$, $5.58(\mathrm{~m}, 1 \mathrm{H}), 4.22(\mathrm{~m}, 1 \mathrm{H}), 4.20-4.11(\mathrm{~m}, 4 \mathrm{H}), 3.88(\mathrm{~m}, 1 \mathrm{H}), 3.76(\mathrm{~m}, 1 \mathrm{H}), 2.51-2.30(\mathrm{~m}$, $2 \mathrm{H}), 2.14-1.76(\mathrm{~m}, 3 \mathrm{H}), 1.59(\mathrm{~m}, 1 \mathrm{H}), 1.30(\mathrm{t}, J=7.1 \mathrm{~Hz}, 3 \mathrm{H}) ;{ }^{13} \mathbf{C}\left\{{ }^{1} \mathbf{H}\right\}$ NMR $(125 \mathrm{MHz}$, $\left.\mathrm{CDCl}_{3}\right): \delta[\mathrm{ppm}]=155.1,134.1,126.6,79.6,68.1,67.1,64.0,32.3,31.8,26.1,14.5$; HR-ESI-MS m/z: calcd. $\mathrm{C}_{11} \mathrm{H}_{19} \mathrm{O}_{4}[\mathrm{M}+\mathrm{H}]^{+}:$215.1278, found: 215.1281 . 


\section{(E)-4-(Tetrahydrofuran-2-yl)but-3-en-1-yl pivalate (127o)}

Following general procedure D alcohol $\mathbf{1 2 6 0}$ (134 mg, 586 umol, 1.0 equiv),

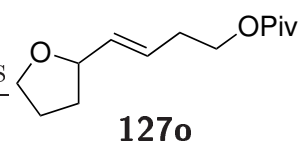

(2-anisyl) ${ }_{2} \mathrm{Se}_{2}$ (11 mg, $0.029 \mathrm{mmol}, 0.05$ equiv), photosensitizer 95 ( $8.5 \mathrm{mg}$, 0.018 mmol, 0.03 equiv) and $\mathrm{NaH}_{2} \mathrm{PO}_{4}(58 \mathrm{mg}, 0.47 \mathrm{mmol}, 0.8$ equiv); reaction time: $11 \mathrm{~h}$; eluting with $n$-pentane/EtOAc (7:1); yield: $33 \mathrm{mg}$,

$0.15 \mathrm{mmol}, 25 \%$; colorless liquid; ${ }^{1} \mathrm{H}$ NMR yield using 1,1,2,2-tetrachloroethane as the internal standard: $44 \%$.

$\mathbf{R}_{\mathbf{f}}=0.29$ (n-pentane:EtOAc, 7:1); IR (ATR): $\tilde{\nu}\left[\mathrm{cm}^{-1}\right]=2970,2872,1726,1480,1460,1398$, 1366, 1283, 1150, 1052, 967, 869, 770; ${ }^{\mathbf{1}} \mathbf{H} \mathbf{N M R}\left(300 \mathrm{MHz}, \mathrm{CDCl}_{3}\right): \delta[\mathrm{ppm}]=5.64(\mathrm{~m}, 1 \mathrm{H})$, $5.55(\mathrm{~m}, 1 \mathrm{H}), 4.24(\mathrm{~m}, 1 \mathrm{H}), 4.09(\mathrm{t}, J=6.7 \mathrm{~Hz}, 2 \mathrm{H}), 3.88$ (ddd, $J=8.0,7.1,6.3 \mathrm{~Hz}, 1 \mathrm{H})$, $3.76(\mathrm{~m}, 1 \mathrm{H}), 2.59-2.16(\mathrm{~m}, 2 \mathrm{H}), 2.01(\mathrm{~m}, 1 \mathrm{H}), 1.90(\mathrm{~m}, 2 \mathrm{H}), 1.58(\mathrm{~m}, 1 \mathrm{H}), 1.18(\mathrm{~s}, 9 \mathrm{H})$; ${ }^{13} \mathbf{C}\left\{{ }^{1} \mathbf{H}\right\}$ NMR $\left(125 \mathrm{MHz}, \mathrm{CDCl}_{3}\right): \delta[\mathrm{ppm}]=178.4,133.7,127.3,79.6,68.1,63.5,38.9,32.4$, 31.9, 27.4, 26.1; HR-ESI-MS m/z: calcd. $\mathrm{C}_{13} \mathrm{H}_{23} \mathrm{O}_{3}[\mathrm{M}+\mathrm{H}]^{+}:$227.1642, found: 227.1643.

\section{(E)-Triisopropyl((4-(tetrahydrofuran-2-yl)but-3-en-1-yl)oxy)silane (127p)}

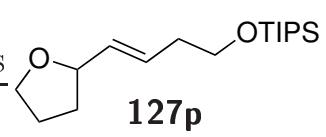

Following general procedure D alcohol 126p (163 mg, 541 umol, 1.0 equiv), (2-anisyl) $)_{2} \mathrm{Se}_{2}$ (10 mg, $0.027 \mathrm{mmol}, 0.05$ equiv), photosensitizer 95 (7.9 mg, $0.016 \mathrm{mmol}, 0.03$ equiv) and $\mathrm{NaH}_{2} \mathrm{PO}_{4}$ (52 mg, $0.43 \mathrm{mmol}, 0.8$ equiv); reaction time: $25 \mathrm{~h}$; eluting with $n$-pentane/EtOAc (75:1); yield: $37 \mathrm{mg}$, $0.13 \mathrm{mmol}, 23 \%$; colorless liquid; ${ }^{1} \mathrm{H}$ NMR yield using 1,1,2,2-tetrachloroethane as the internal standard: $29 \%$.

$\mathbf{R}_{\mathbf{f}}=0.33$ (n-pentane:EtOAc, 75:1); IR (ATR): $\tilde{\nu}\left[\mathrm{cm}^{-1}\right]=2942,2892,2865,1463,1382,1101$, 1055, 1013, 995, 966, 919, 881, 783, 737, 679, 657, 445; ${ }^{\mathbf{1}} \mathbf{H} \mathbf{N M R}\left(500 \mathrm{MHz}, \mathrm{CDCl}_{3}\right): \delta$ $[\mathrm{ppm}]=5.70(\mathrm{dtd}, J=15.4,6.8,0.9 \mathrm{~Hz}, 1 \mathrm{H}), 5.52(\mathrm{ddt}, J=15.3,7.0,1.3 \mathrm{~Hz}, 1 \mathrm{H}), 4.23(\mathrm{~m}$, $1 \mathrm{H}), 3.88(\mathrm{~m}, 1 \mathrm{H}), 3.81-3.63(\mathrm{~m}, 3 \mathrm{H}), 2.35-2.23(\mathrm{~m}, 2 \mathrm{H}), 2.09-1.76(\mathrm{~m}, 3 \mathrm{H}), 1.58(\mathrm{~m}, 1 \mathrm{H})$, 1.18-0.88 (m, $21 \mathrm{H}) ;{ }^{13} \mathbf{C}\left\{{ }^{\mathbf{1}} \mathbf{H}\right\}$ NMR $\left(125 \mathrm{MHz}, \mathrm{CDCl}_{3}\right): \delta[\mathrm{ppm}]=132.7,129.0,80.0,68.1$, 63.3, 36.3, 32.4, 26.2, 18.3, 12.3; HR-ESI-MS m/z: calcd. $\mathrm{C}_{17} \mathrm{H}_{35} \mathrm{O}_{2} \mathrm{Si}[\mathrm{M}+\mathrm{H}]^{+}:$299.2401, found: 299.2402 .

\section{(E)-2,6-Dimethyl-8-(tetrahydro-2H-pyran-2-yl)oct-7-en-2-ol (144a)}

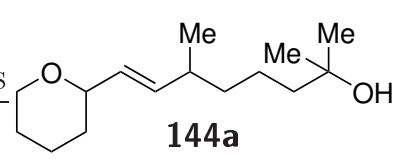

$\mathbf{R}_{\mathbf{f}}=0.19$ (n-pentane:Et $\left.{ }_{2} \mathrm{O}, 1: 1\right)$; IR (ATR): $\tilde{\nu}\left[\mathrm{cm}^{-1}\right]=3437,2934,2845,1463,1374,1203$, $1175,1083,1049,1033,968,938,895,754 ;{ }^{\mathbf{1}} \mathbf{H} \mathbf{~ N M R}\left(400 \mathrm{MHz}, \mathrm{CDCl}_{3}\right): \delta[\mathrm{ppm}]=5.53$ (ddd, 
$J=15.7,8.4,7.4,1.0 \mathrm{~Hz}, 1 \mathrm{H}), 5.42$ (dddd, $J=15.6,6.2,2.3,0.9 \mathrm{~Hz}, 1 \mathrm{H}), 4.00$ (ddt, $J=11.6$, 4.0, $1.6 \mathrm{~Hz}, 1 \mathrm{H}), 3.74(\mathrm{~m}, 1 \mathrm{H}), 3.47(\mathrm{~m}, 1 \mathrm{H}), 2.12(\mathrm{~m}, 1 \mathrm{H}), 1.82(\mathrm{~m}, 1 \mathrm{H}), 1.72-1.22(\mathrm{~m}$, $12 \mathrm{H}), 1.19(\mathrm{~s}, 6 \mathrm{H}), 0.98(\mathrm{dd}, J=6.7,3.4 \mathrm{~Hz}, 3 \mathrm{H}) ;{ }^{\mathbf{1 3}} \mathbf{C}\left\{{ }^{\mathbf{1}} \mathbf{H}\right\}$ NMR $\left(101 \mathrm{MHz}, \mathrm{CDCl}_{3}\right): \delta$ $[\mathrm{ppm}]=137.4,137.3,129.9,129.7,78.5,78.3,71.2,71.1,68.5,44.10,44.08,37.4,37.3,36.52$, $36.46,32.6,32.5,29.41,29.38,29.36,26.03,26.01,23.6,22.1,22.0,20.6,20.4$; HR-ESI-MS m/z: calcd. $\mathrm{C}_{15} \mathrm{H}_{29} \mathrm{O}_{2}[\mathrm{M}+\mathrm{H}]^{+}:$241.2162, found: 241.2160 .

\section{(E)-2-(3,7-Dimethylocta-1,6-dien-1-yl)tetrahydro-2 $H$-pyran (144b)}

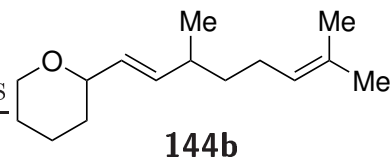

$144 b$

Following general procedure D alcohol $\mathbf{1 3 9 b}$; reaction time: $10 \mathrm{~h}$; eluting with $n$-pentane $/ \mathrm{Et}_{2} \mathrm{O}$ (5:1); yield: $43 \mathrm{mg}, 0.19 \mathrm{mmol}, 19 \%$; colorless liquid; mixture of diastereomers; ${ }^{1} \mathrm{H}$ NMR yield using $1,3,5-$ trimethoxybenzene as the internal standard: $25 \%$.

$\mathbf{R}_{\mathbf{f}}=0.71$ (n-pentane:Et $\left.{ }_{2} \mathrm{O}, 5: 1\right) ; \mathbf{I R}(\mathrm{ATR}): \tilde{\nu}\left[\mathrm{cm}^{-1}\right]=2929,2848,1452,1375,1266,1204,1175$, 1085, 1051, 1035, 968, 897, 847, 543, 442, 419; ${ }^{\mathbf{1}} \mathbf{H}$ NMR $\left(400 \mathrm{MHz}, \mathrm{CDCl}_{3}\right): \delta[\mathrm{ppm}]=5.54$ (dddd, $J=15.5,12.7,7.3,1.0 \mathrm{~Hz}, 1 \mathrm{H}$ ), 5.42 (dddd, $J=15.5,5.7,4.5,0.9 \mathrm{~Hz}, 1 \mathrm{H}$ ), 5.08 (dddd, $J=7.2,5.8,2.7,1.3 \mathrm{~Hz}, 1 \mathrm{H}$ ), 4.00 (ddt, $J=11.5,3.5,1.6 \mathrm{~Hz}, 1 \mathrm{H}$ ), 3.74 (ddt, $J=10.8,5.6$, $1.4 \mathrm{~Hz}, 1 \mathrm{H}), 3.47$ (tdd, $J=11.5,2.6,1.0 \mathrm{~Hz}, 1 \mathrm{H}), 2.10(\mathrm{dq}, J=13.6,6.7 \mathrm{~Hz}, 1 \mathrm{H}), 1.94$ (quint, $J=7.1 \mathrm{~Hz}, 2 \mathrm{H}), 1.83(\mathrm{~m}, 1 \mathrm{H}), 1.67(\mathrm{t}, J=1.2 \mathrm{~Hz}, 3 \mathrm{H}), 1.65-1.44(\mathrm{~m}, 6 \mathrm{H}), 1.45-1.16(\mathrm{~m}, 4 \mathrm{H})$, $0.98(\mathrm{dd}, J=6.7,2.7 \mathrm{~Hz}, 3 \mathrm{H}) ;{ }^{\mathbf{1 3}} \mathbf{C}\left\{{ }^{1} \mathbf{H}\right\} \mathbf{N M R}\left(101 \mathrm{MHz}, \mathrm{CDCl}_{3}\right): \delta[\mathrm{ppm}]=137.6,137.2$, $131.4,130.0,129.8,124.8,124.8,78.6,78.4,68.5,68.5,37.1,37.1,36.2,36.0,32.6,32.5,26.1$, 26.0, 25.9, 25.9, 25.9, 23.6, 23.6, 20.6, 20.4, 17.9; HR-ESI-MS m/z: calcd. $\mathrm{C}_{15} \mathrm{H}_{27} \mathrm{O}[\mathrm{M}+\mathrm{H}]^{+}$: 223.2056, found: 223.2058 .

\section{(E)-2-(3-Phenylprop-1-en-1-yl)tetrahydro-2 $H$-pyran (144c)}

Following general procedure D alcohol 139c; reaction time: $7 \mathrm{~h}$; eluting with

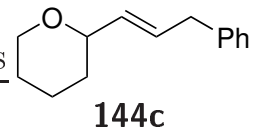
n-pentane/DCM (1:9); yield: $90 \mathrm{mg}, 0.44 \mathrm{mmol}$, 44\%; yellow liquid; ${ }^{1} \mathrm{H}$ NMR yield using 1,3,5-trimethoxybenzene as the internal standard: $54 \%$.

$\mathbf{R}_{\mathbf{f}}=0.43$ (n-pentane:DCM, 1:9); IR (ATR): $\tilde{\nu}\left[\mathrm{cm}^{-1}\right]=2933,2841,1495,1453,1438,1204$, 1175, 1083, 1048, 1033, 967, 894, 745, 697, 580, 543, 497, 456; ${ }^{\mathbf{1}} \mathbf{H}$ NMR $\left(300 \mathrm{MHz}, \mathrm{CDCl}_{3}\right): \delta$ $[\mathrm{ppm}]=7.38-7.24(\mathrm{~m}, 2 \mathrm{H}), 7.24-7.03(\mathrm{~m}, 3 \mathrm{H}), 5.83(\mathrm{dtd}, J=15.4,6.7,1.2 \mathrm{~Hz}, 1 \mathrm{H}), 5.54$ (ddt, $J=15.4,6.1,1.5 \mathrm{~Hz}, 1 \mathrm{H}), 4.01(\mathrm{~m}, 1 \mathrm{H}), 3.79(\mathrm{~m}, 1 \mathrm{H}), 3.47(\mathrm{~m}, 1 \mathrm{H}), 3.41-3.24(\mathrm{~m}, 2 \mathrm{H})$, $1.84(\mathrm{~m}, 1 \mathrm{H}), 1.74-1.30(\mathrm{~m}, 5 \mathrm{H}) ;{ }^{\mathbf{1 3}} \mathbf{C}\left\{{ }^{\mathbf{1}} \mathbf{H}\right\} \mathbf{N M R}\left(126 \mathrm{MHz}, \mathrm{CDCl}_{3}\right): \delta[\mathrm{ppm}]=140.2,132.8$, 130.2, 128.7, 128.4, 126.1, 78.1, 68.5, 39.0, 32.4, 26.1, 23.6; HR-ESI-MS m/z: calcd. $\mathrm{C}_{14} \mathrm{H}_{19} \mathrm{O}$ $[\mathrm{M}+\mathrm{H}]^{+}:$203.1430, found: 203.1434 . 


\section{2-(Cyclohexylidenemethyl)tetrahydro-2 $H$-pyran (144d)}

Following general procedure $\mathrm{D}$, alcohol 139d; reaction time: $17 \mathrm{~h}$; eluting with<smiles>C(=C1CCCCC1)C1CCCCO1</smiles>
n-pentane/DCM (1:9); yield: $62 \mathrm{mg}, 0.35 \mathrm{mmol}, 35 \%$; colorless liquid; ${ }^{1} \mathrm{H}$ NMR yield using 1,3,5-trimethoxybenzene as the internal standard: $53 \%$.

144d

$\mathbf{R}_{\mathbf{f}}=0.43$ (n-pentane:DCM, 1:9); IR (ATR): $\tilde{\nu}\left[\mathrm{cm}^{-1}\right]=2926,2851,1446,1204$, 1174, 1084, 1051, 1033, 992, 901, 846; ${ }^{\mathbf{1}} \mathbf{H}$ NMR (300 MHz, $\left.\mathrm{CDCl}_{3}\right): \delta[\mathrm{ppm}]=5.11$ (dquint, $J=8.0,1.2 \mathrm{~Hz}, 1 \mathrm{H}), 4.29-3.87(\mathrm{~m}, 2 \mathrm{H}), 3.47(\mathrm{td}, J=11.4,2.5 \mathrm{~Hz}, 1 \mathrm{H}), 2.35-1.94(\mathrm{~m}, 5 \mathrm{H})$, $1.83(\mathrm{~m}, 1 \mathrm{H}), 1.69-1.26(\mathrm{~m}, 10 \mathrm{H}) ;{ }^{\mathbf{1 3}} \mathbf{C}\left\{{ }^{\mathbf{1}} \mathbf{H}\right\} \mathbf{N M R}\left(126 \mathrm{MHz}, \mathrm{CDCl}_{3}\right): \delta[\mathrm{ppm}]=142.7,123.3$, $74.3,68.4,37.1,32.9,29.7,28.6,28.1,26.9,26.1,23.8$; HR-ESI-MS m/z: calcd. $\mathrm{C}_{12} \mathrm{H}_{21} \mathrm{O}$ $[\mathrm{M}+\mathrm{H}]^{+}:$181.1587, found: 181.1588 .

\section{(E)-2-(3-Methylbut-1-en-1-yl)tetrahydro-2 $H$-pyran (144e)}

Following general procedure D alcohol 139e; reaction time: $7 \mathrm{~h}$; eluting with<smiles>CC(C)(C)C=CC1CCCOC1</smiles>
n-pentane/Et ${ }_{2} \mathrm{O}$ (9:1); yield: $58 \mathrm{mg}, 0.38 \mathrm{mmol}, 38 \%$; colorless liquid; ${ }^{1} \mathrm{H}$ NMR yield using 1,3,5-trimethoxybenzene as the internal standard: $52 \%$.

$\mathbf{R}_{\mathbf{f}}=0.43$ (n-pentane:Et $\left.{ }_{2} \mathrm{O}, 9: 1\right) ; \mathbf{I R}(\mathrm{ATR}): \tilde{\nu}\left[\mathrm{cm}^{-1}\right]=2955,2934,2841,1464$, 1362, 1263, 1204, 1176, 1085, 1051, 1035, 968, 896, 847; ${ }^{\mathbf{1}} \mathbf{H} \mathbf{N M R}\left(300 \mathrm{MHz}, \mathrm{CDCl}_{3}\right): \delta$ $[\mathrm{ppm}]=5.64(\mathrm{ddd}, J=15.6,6.4,1.1 \mathrm{~Hz}, 1 \mathrm{H}), 5.41(\mathrm{ddd}, J=15.6,6.2,1.4 \mathrm{~Hz}, 1 \mathrm{H}), 4.01(\mathrm{~m}$, $1 \mathrm{H}), 3.73$ (dddd, $J=10.8,6.2,2.1,1.1 \mathrm{~Hz}, 1 \mathrm{H}), 3.47(\mathrm{~m}, 1 \mathrm{H}), 2.26(\mathrm{~m}, 1 \mathrm{H}), 1.83(\mathrm{~m}, 1 \mathrm{H})$, $1.70-1.29(\mathrm{~m}, 5 \mathrm{H}), 0.98(\mathrm{dd}, J=6.7,0.9 \mathrm{~Hz}, 6 \mathrm{H}) ;{ }^{\mathbf{1 3}} \mathbf{C}\left\{{ }^{\mathbf{1}} \mathbf{H}\right\} \mathbf{N M R}\left(126 \mathrm{MHz}, \mathrm{CDCl}_{3}\right): \delta$ $[\mathrm{ppm}]=138.8,128.4,78.5,68.5,32.5,30.9,26.1,23.7,22.5 ;$ HR-ESI-MS m/z: calcd. $\mathrm{C}_{10} \mathrm{H}_{19} \mathrm{O}$ $[\mathrm{M}+\mathrm{H}]^{+}:$155.1430, found: 155.1436 .

\section{(E)-2-(5-Chloropent-1-en-1-yl)tetrahydro-2 $\mathrm{H}$-pyran (144f)}

Following general procedure D alcohol $\mathbf{1 3 9 f}(156 \mathrm{mg}, 818$ umol), 1.0 equiv),<smiles>ClCCC/C=C/C1CCCCO1</smiles>

(2-aisyl) ${ }_{2} \mathrm{Se}_{2}$ (15 mg, $0.041 \mathrm{mmol}, 0.05$ equiv), photosensitizer 95 (12 mg, $0.025 \mathrm{mmol}, 0.03$ equiv) and $\mathrm{NaH}_{2} \mathrm{PO}_{4}$ (79 mg, $0.65 \mathrm{mmol}, 0.8$ equiv); reaction time: $7 \mathrm{~h}$; eluting with $n$-pentane/DCM (1:9); yield: $88 \mathrm{mg}$, $0.47 \mathrm{mmol}, 47 \%$; yellow liquid; ${ }^{1} \mathrm{H}$ NMR yield using 1,3,5-trimethoxybenzene as the internal standard: $62 \%$.

$\mathbf{R}_{\mathbf{f}}=0.44$ (n-pentane:DCM, 1:9); IR (ATR): $\tilde{\nu}\left[\mathrm{cm}^{-1}\right]=2934,2844,1440,1265,1204,1083$, 1049, 1034, 968, 895, 726, 653; ${ }^{\mathbf{1}} \mathbf{H}$ NMR $\left(300 \mathrm{MHz}, \mathrm{CDCl}_{3}\right): \delta[\mathrm{ppm}]=5.64(\mathrm{dtd}, J=15.5$, 6.3, $0.8 \mathrm{~Hz}, 1 \mathrm{H}), 5.52(\mathrm{ddt}, J=15.5,5.8,1.0 \mathrm{~Hz}, 1 \mathrm{H}), 4.00(\mathrm{~m}, 1 \mathrm{H}), 3.75(\mathrm{~m}, 1 \mathrm{H}), 3.53(\mathrm{t}$, $J=6.6 \mathrm{~Hz}, 2 \mathrm{H}), 2.18$ (dddd, $J=8.0,7.0,6.0,0.8 \mathrm{~Hz}, 2 \mathrm{H}), 2.03-1.74(\mathrm{~m}, 3 \mathrm{H}), 1.73-1.23(\mathrm{~m}$, $6 \mathrm{H}) ;{ }^{13} \mathbf{C}\left\{{ }^{1} \mathbf{H}\right\}$ NMR $\left(126 \mathrm{MHz}, \mathrm{CDCl}_{3}\right): \delta[\mathrm{ppm}]=132.8,129.4,78.1,68.5,44.6,32.4,32.1$, 29.6, 26.1, 23.6; HR-ESI-MS m/z: calcd. $\mathrm{C}_{10} \mathrm{H}_{17} \mathrm{ClONa}[\mathrm{M}+\mathrm{Na}]^{+}:$211.0860, found: 211.0865 . 


\section{(E)-2-(Undeca-1,10-dien-1-yl)tetrahydro-2 $H$-pyran (144h)}

Following general procedure $\mathbf{D}$ alcohol $\mathbf{1 3 9 h}$; reaction time: $17 \mathrm{~h}$; eluting<smiles>C=CCCC=CC1CCCCO1</smiles>
with $n$-pentane:Et ${ }_{2} \mathrm{O}$ (20:1); yield: $86 \mathrm{mg}, 0.37 \mathrm{mmol}, 37 \%$; colorless liquid; ${ }^{1} \mathrm{H}$ NMR yield using 1,3,5-trimethoxybenzene as the internal standard: $55 \%$.

$\mathbf{R}_{\mathbf{f}}=0.23$ (n-pentane:Et $\left.{ }_{2} \mathrm{O}, 20: 1\right) ; \mathbf{I R}(\mathrm{ATR}): \tilde{\nu}\left[\mathrm{cm}^{-1}\right]=2924,2852,1727$, $1640,1463,1439,1371,1263,1203,1175,1085,1035,966,907,861,843,810,723 ;{ }^{1} \mathbf{H}$ NMR $\left(300 \mathrm{MHz}, \mathrm{CDCl}_{3}\right): \delta[\mathrm{ppm}]=5.80(\mathrm{ddt}, J=16.9,10.2,6.7 \mathrm{~Hz}, 1 \mathrm{H}), 5.66(\mathrm{dtd}, J=15.5,6.6$, $1.1 \mathrm{~Hz}, 1 \mathrm{H}$ ), 5.45 (ddt, $J=15.5,6.2,1.4 \mathrm{~Hz}, 1 \mathrm{H}), 5.04-4.88(\mathrm{~m}, 2 \mathrm{H}), 4.00$ (ddt, $J=11.6$, $4.1,1.8 \mathrm{~Hz}, 1 \mathrm{H}), 3.73(\mathrm{ddd}, J=9.2,4.5,1.9 \mathrm{~Hz}, 1 \mathrm{H}), 3.47(\mathrm{~m}, 1 \mathrm{H}), 2.02$ (qdd, $J=6.9$, 5.4, $1.2 \mathrm{~Hz}, 4 \mathrm{H}), 1.84(\mathrm{~m}, 1 \mathrm{H}), 1.70-1.19(\mathrm{~m}, 15 \mathrm{H}) ;{ }^{\mathbf{1}} \mathbf{C}\left\{{ }^{\mathbf{1}} \mathbf{H}\right\} \mathbf{N M R}\left(126 \mathrm{MHz}, \mathrm{CDCl}_{3}\right): \delta$ $[\mathrm{ppm}]=139.3,131.9,131.3,114.2,78.4,68.5,34.0,32.5,32.4,29.5,29.4,29.31,29.28,29.1,26.1$, 23.7; HR-ESI-MS m/z: calcd. $\mathrm{C}_{16} \mathrm{H}_{28} \mathrm{ONa}[\mathrm{M}+\mathrm{Na}]^{+}: 259.2032$, found: 259.2034 .

\section{(E)-2-(Hept-1-en-6-yn-1-yl)tetrahydro-2 H-pyran (144i)}

Following general procedure D alcohol 139i; reaction time: $14.5 \mathrm{~h}$; eluting<smiles>C#CCCC=CC1CCCCO1</smiles>
with $n$-pentane: $\mathrm{Et}_{2} \mathrm{O}$ (30:1); yield: $54 \mathrm{mg}, 0.31 \mathrm{mmol}, 31 \%$; colorless liquid; ${ }^{1} \mathrm{H}$ NMR yield using 1,1,2,2-tetrachloroethane as the internal standard: $37 \%$.

$\mathbf{R}_{\mathbf{f}}=0.11$ (n-pentane:Et $\left.{ }_{2} \mathrm{O}, 30: 1\right)$; IR (ATR): $\tilde{\nu}\left[\mathrm{cm}^{-1}\right]=3304,2933,2843,1439,1344,1263$, 1203, 1175, 1083, 1049, 1034, 967, 896, 842, 810; ${ }^{\mathbf{1}} \mathbf{H}$ NMR $\left(300 \mathrm{MHz}, \mathrm{CDCl}_{3}\right): \delta[\mathrm{ppm}]=5.64$ $(\mathrm{dtd}, J=15.5,6.5,0.9 \mathrm{~Hz}, 1 \mathrm{H}), 5.50(\mathrm{ddt}, J=15.5,6.0,1.2 \mathrm{~Hz}, 1 \mathrm{H}), 4.00(\mathrm{~m}, 1 \mathrm{H}), 3.74$ $(\mathrm{m}, 1 \mathrm{H}), 3.47(\mathrm{~m}, 1 \mathrm{H}), 2.37-2.04(\mathrm{~m}, 4 \mathrm{H}), 1.93(\mathrm{t}, J=2.6 \mathrm{~Hz}, 1 \mathrm{H}), 1.83(\mathrm{~m}, 1 \mathrm{H}), 1.68-1.23$ $(\mathrm{m}, 7 \mathrm{H}) ;{ }^{13} \mathbf{C}\left\{{ }^{1} \mathbf{H}\right\} \mathbf{N M R}\left(126 \mathrm{MHz}, \mathrm{CDCl}_{3}\right): \delta[\mathrm{ppm}]=132.3,130.3,84.4,78.2,68.52,68.49$, 32.4, 31.4, 28.1, 26.1, 23.6, 18.1; HR-ESI-MS m/z: calcd. $\mathrm{C}_{12} \mathrm{H}_{19} \mathrm{O}[\mathrm{M}+\mathrm{H}]^{+}:$: 179.1430, found: 179.1429 .

\subsection{Intermolecular etherification via photo-aerobic selenium- $\pi$-acid catalysis}

\subsubsection{Synthesis of alkenes 148}

\section{Benzyl (E)-hex-3-enoate (148a)}

A solution of $(E)$-hex-3-enoic acid ( $3.00 \mathrm{~g}, 26.3 \mathrm{mmol}, 1.00$ equiv), benzyl<smiles>CC/C=C/CC(=O)O[Ga]CCC</smiles>

148a alcohol (5.68 g, $52.6 \mathrm{mmol}, 2.00$ equiv) and $p$-toluenesulfonic acid (350 mg, $1.31 \mathrm{mmol}, 0.05$ equiv) in toluene $(25 \mathrm{~mL})$ was stirred for $5 \mathrm{~h}$ at $150{ }^{\circ} \mathrm{C}$. After cooling down to room temperature, the solution was diluted with EtOAc $(50 \mathrm{~mL})$ and washed with sat. aq. $\mathrm{NaHCO}_{3}$ sol. $(3 \times 50 \mathrm{~mL})$ and sat. aq. $\mathrm{NaCl}$ sol. $(50 \mathrm{~mL})$. The organic layer was dried over $\mathrm{Na}_{2} \mathrm{SO}_{4}$ and concentrated under reduced pressure. Column 
chromatography ( $n$-pentane/EtOAc, 20:1) afforded the product as a yellow oil (4.62 g, $22.7 \mathrm{mmol}$, $86 \%)$.

$\mathbf{R}_{\mathbf{f}}=0.65$ (n-pentane:EtOAc, 20:1); IR (ATR): $\tilde{\nu}\left[\mathrm{cm}^{-1}\right]=2963,1735,1498,1455,1377,1317$, 1234, 1152, 966, 734, 696, 580, 481, 465; ${ }^{\mathbf{1}} \mathbf{H}$ NMR $\left(300 \mathrm{MHz}, \mathrm{CDCl}_{3}\right): \delta[\mathrm{ppm}]=7.45-7.28$ $(\mathrm{m}, 5 \mathrm{H}), 5.78-5.39(\mathrm{~m}, 2 \mathrm{H}), 5.13(\mathrm{~s}, 2 \mathrm{H}), 3.12-2.88(\mathrm{~m}, 2 \mathrm{H}), 2.19-1.94(\mathrm{~m}, 2 \mathrm{H}), 0.99$ (t, $J=7.5 \mathrm{~Hz}, 3 \mathrm{H}) ;{ }^{13} \mathbf{C}\left\{{ }^{\mathbf{1}} \mathbf{H}\right\} \mathbf{N M R}\left(101 \mathrm{MHz}, \mathrm{CDCl}_{3}\right): \delta[\mathrm{ppm}]=172.2,136.6,136.1,128.7$, 128.3, 128.3, 120.6, 66.4 38.2, 25.6, 13.6; HR-ESI-MS m/z: calcd. $\mathrm{C}_{13} \mathrm{H}_{16} \mathrm{O}_{2} \mathrm{Na}[\mathrm{M}+\mathrm{Na}]^{+}$: 227.1048, found: 227.1047 .

\section{(E)-3-(Hex-3-enoyl)oxazolidin-2-one (148b)}

To a solution of 2-oxazolidinone (435 $\mathrm{mg}, 5.00 \mathrm{mmol}, 1.0$ equiv) and<smiles>CC/C=C/CC(=O)N1CCOC1=O</smiles>

DMAP (79 mg, $0.65 \mathrm{mmol}, 0.13$ equiv) in DCM $(6.6 \mathrm{~mL}),(E)$-3-hexenoic acid (741 mg, $6.50 \mathrm{mmol}, 1.3$ equiv) was added and the solution was cooled to $0{ }^{\circ} \mathrm{C}$. DCC ( $1.35 \mathrm{~g}, 6.55 \mathrm{mmol}, 1.31$ equiv) was added, the solution was stirred for $10 \mathrm{~min}$ at $0{ }^{\circ} \mathrm{C}$ and $17.5 \mathrm{~h}$ at room temperature. The mixture was filtered, the precipitate was washed with DCM $(2 \times)$, the comb. org. phases were washed with sat. aq. $\mathrm{NaHCO}_{3}$ sol. $(2 \times)$ and dried over $\mathrm{Na}_{2} \mathrm{SO}_{4}$. Removal of the solvent in vacuo, followed by column chromatography ( $n$-pentane/EtOAc, 2:1) afforded the product as a yellow oil (292 $\mathrm{mg}, 1.59 \mathrm{mmol}$, $32 \%)$.

$\mathbf{R}_{\mathbf{f}}=0.36$ (n-pentane:EtOAc, 2:1); IR (ATR): $\tilde{\nu}\left[\mathrm{cm}^{-1}\right]=2965,2925,2876,2851,1711,1695$, 1479, 1385, 1362, 1328, 1290, 1218, 1189, 1104, 1035, 1017, 1005, 960, 757, 694; ${ }^{\mathbf{1}} \mathbf{H}$ NMR $\left(400 \mathrm{MHz}, \mathrm{CDCl}_{3}\right): \delta[\mathrm{ppm}]=5.65(\mathrm{~m}, 1 \mathrm{H}), 5.57(\mathrm{~m}, 1 \mathrm{H}), 4.41(\mathrm{t}, J=8.2 \mathrm{~Hz}, 2 \mathrm{H}), 4.04$ $3.99(\mathrm{~m}, 2 \mathrm{H}), 3.64(\mathrm{dq}, J=6.3,1.0 \mathrm{~Hz}, 2 \mathrm{H}), 2.06$ (qdq, $J=7.4,6.1,1.2 \mathrm{~Hz}, 2 \mathrm{H}$ ), 0.99 (t, $J=7.5 \mathrm{~Hz}, 3 \mathrm{H}) ;{ }^{13} \mathbf{C}\left\{{ }^{1} \mathbf{H}\right\}$ NMR $\left(101 \mathrm{MHz}, \mathrm{CDCl}_{3}\right): \delta[\mathrm{ppm}]=172.1,153.6,137.2,120.1$, 62.2, 42.7, 38.9, 25.7, 13.6; HR-ESI-MS m/z: calcd. $\mathrm{C}_{9} \mathrm{H}_{14} \mathrm{NO}_{3}[\mathrm{M}+\mathrm{H}]^{+}:$184.0968, found: 184.0971.

\section{Diethyl (E)-but-2-en-1-ylphosphonate (148c)}

Diethyl phosphite $(9.05 \mathrm{~g}, 66.0 \mathrm{mmol}, 1.10$ equiv) was dissolved in dry<smiles>CC=CCP(=O)(OCC)OCC</smiles>
THF $(200 \mathrm{~mL})$ and cooled to $-10{ }^{\circ} \mathrm{C} . n$-BuLi $(2.5 \mathrm{M}$ in hexane; $26.4 \mathrm{~mL}$, $66.0 \mathrm{mmol}, 1.1$ equiv) was added dropwise and the mixture was stirred for 5 min. A solution of $(E)$-crotyl bromide $(8.10 \mathrm{~g}, 60.0 \mathrm{mmol}, 1.00$ equiv) in dry THF $(24 \mathrm{~mL})$ was added dropwise, the solution was stirred for $15 \mathrm{~min}$ at $-10{ }^{\circ} \mathrm{C}$, allowed to warm up to $23{ }^{\circ} \mathrm{C}$ and stirred for $18 \mathrm{~h}$. The reaction was quenched by the addition of sat. aq. $\mathrm{NH}_{4} \mathrm{Cl}$-sol. $(25 \mathrm{~mL})$, the aq. phase was extracted with $\mathrm{Et}_{2} \mathrm{O}(3 \times 25 \mathrm{~mL})$, the comb. org. phases were washed with sat. aq. $\mathrm{NaCl}$ sol. $(3 \times 40 \mathrm{~mL})$ and dried over $\mathrm{Na}_{2} \mathrm{SO}_{4}$. Removal 
of the solvent in vacuo and vacuum distillation afforded the product as a colorless oil (7.06 g, $36.7 \mathrm{mmol}, 61 \%)$.

$\mathbf{R}_{\mathbf{f}}=0.32$ (n-pentane:EtOAc, 1:2); b.p. $=115{ }^{\circ} \mathrm{C}(19 \mathrm{mbar}) ; \mathbf{I R}(\mathrm{ATR}): \tilde{\nu}\left[\mathrm{cm}^{-1}\right]=2980,2935$, 2908, 1444, 1392, 1251, 1166, 1096, 1020, 956, 849, 831, 806, 776, 707, 683; ${ }^{\mathbf{1}} \mathbf{H}$ NMR $(500 \mathrm{MHz}$, $\left.\mathrm{CDCl}_{3}\right): \delta[\mathrm{ppm}]=5.61(\mathrm{dddt}, J=15.2,6.5,5.1,1.3 \mathrm{~Hz}, 1 \mathrm{H}), 5.41(\mathrm{~m}, 1 \mathrm{H}), 4.08$ (dqd, $J=7.8,7.1,3.9 \mathrm{~Hz}, 4 \mathrm{H}$ ), $2.52(\mathrm{ddt}, J=21.4,7.3,1.2 \mathrm{~Hz}, 2 \mathrm{H}$ ), 1.69 (dddd, $J=6.5,4.5$, 1.7, $1.2 \mathrm{~Hz}, 3 \mathrm{H}), 1.39-0.90(\mathrm{~m}, 6 \mathrm{H}) ;{ }^{\mathbf{1 3}} \mathbf{C}\left\{{ }^{\mathbf{1}} \mathbf{H}\right\} \mathbf{N M R}\left(126 \mathrm{MHz}, \mathrm{CDCl}_{3}\right): \delta[\mathrm{ppm}]=130.8(\mathrm{~d}$, $\left.J_{C P}=14.7 \mathrm{~Hz}\right), 119.7\left(\mathrm{~d}, J_{C P}=11.4 \mathrm{~Hz}\right), 61.9\left(\mathrm{~d}, J_{C P}=6.8 \mathrm{~Hz}\right), 30.6\left(\mathrm{~d}, J_{C P}=140.0 \mathrm{~Hz}\right)$, $18.2\left(\mathrm{~d}, J_{C P}=2.6 \mathrm{~Hz}\right), 16.6\left(\mathrm{~d}, J_{C P}=6.0 \mathrm{~Hz}\right) ;{ }^{\mathbf{3 1}} \mathbf{P}\left\{{ }^{1} \mathbf{H}\right\} \mathbf{N M R}\left(203 \mathrm{MHz}, \mathrm{CDCl}_{3}\right): \delta$ $[\mathrm{ppm}]=28.1$; HR-ESI-MS m/z: calcd. $\mathrm{C}_{8} \mathrm{H}_{18} \mathrm{O}_{3} \mathrm{P}[\mathrm{M}+\mathrm{H}]^{+}:$: 193.0988, found: 193.0992 .

\subsubsection{Synthesis of allylic ethers 149}

\section{General Procedure E: intermolecular etherification}

Alkene 148 (1.0 mmol), (2-anisyl) ${ }_{2} \mathrm{Se}_{2}(36 \mathrm{mg}, 0.10 \mathrm{mmol}, 0.1$ equiv) and $p$-MeO-TPT (95) (24 mg, $0.050 \mathrm{mmol}, 0.05$ equiv) are dissolved in the respective alcohol $(0.2 \mathrm{M} ; 5 \mathrm{~mL})$ and the solution is stirred vigorously at room temperature under an atmosphere of air and irradiation at $\lambda=465 \mathrm{~nm}$ until completion, which is determined by NMR or TLC. After removal of the solvent, the product is afforded via column chromatography.

\section{(E)-((Dec-6-en-5-yloxy)methyl)benzene (151)}

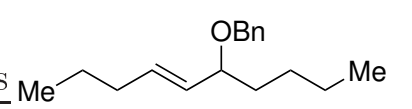

151

Isolated from a combination of three reaction mixtures, yield $12 \mathrm{mg}$, 48 umol, yellow oil.

$\mathbf{R}_{\mathbf{f}}=0.29$ ( $n$-pentane:EtOAc, 75:1); IR (ATR): $\tilde{\nu}\left[\mathrm{cm}^{-1}\right]=2958,2928$, 2861, 1362, 2340, 1722, 1456, 1263, 1090, 1067, 1028, 970, 801, 746, 734, 697, 672; ${ }^{1} \mathbf{H}$ NMR $\left(300 \mathrm{MHz}, \mathrm{CDCl}_{3}\right): \delta[\mathrm{ppm}]=7.45-7.27(\mathrm{~m}, 5 \mathrm{H}), 5.59(\mathrm{dt}, J=15.4$, $6.7 \mathrm{~Hz}, 1 \mathrm{H}), 5.33(\mathrm{ddt}, J=15.4,8.3,1.4 \mathrm{~Hz}, 1 \mathrm{H}), 4.58$ (d, $J=12.0 \mathrm{~Hz}, 1 \mathrm{H}), 4.33$ (d, $J=12.0 \mathrm{~Hz}, 1 \mathrm{H}), 3.66(\mathrm{dt}, J=8.2,6.5 \mathrm{~Hz}, 1 \mathrm{H}), 2.21-1.96(\mathrm{~m}, 2 \mathrm{H}), 1.81-1.07(\mathrm{~m}, 8 \mathrm{H})$, 1.02-0.71 (m, $6 \mathrm{H}) ;{ }^{\mathbf{1 3}} \mathbf{C}\left\{{ }^{\mathbf{1}} \mathbf{H}\right\} \mathbf{N M R}\left(75 \mathrm{MHz}, \mathrm{CDCl}_{3}\right): \delta[\mathrm{ppm}]=139.3,134.2,131.2,128.4$, 127.9, 127.4, 80.4, 69.7, 35.7, 34.5, 27.9, 22.8, 22.6, 14.2, 13.8. No HRMS could be obtained.

\section{Benzyl (E)-4-methoxyhex-2-enoate (149a)}

Following general procedure $\mathbf{E}$ alkene 148a (102 mg, $500 \mu \mathrm{mol}, 1.0$ equiv),<smiles>CCC(/C=C/C(=O)OCc1ccccc1)OC</smiles>
$\mathrm{MeOH}(2.5 \mathrm{~mL}),\left(2\right.$-anisyl) ${ }_{2} \mathrm{Se}_{2}(18 \mathrm{mg}, 50 \mu \mathrm{mol}, 0.1$ equiv) and $p$-MeOTPT 95 (12.0 mg, $0.025 \mathrm{mmol}, 0.05$ equiv); reaction time $70.5 \mathrm{~h}$; eluting with $n$-pentane:EtOAc (250:1); yield: $32 \mathrm{mg}, 0.14 \mathrm{mmol}, 27 \%$; colorless liquid; ${ }^{1} \mathrm{H}$ NMR yield using 1,1,2,2-tetrachloroethane as the internal standard: $32 \%$. 
$\mathbf{R}_{\mathbf{f}}=0.43$ (n-pentane:EtOAc, 10:1); IR (ATR): $\tilde{\nu}\left[\mathrm{cm}^{-1}\right]=2968,2933,2878,2824,1717,1657$, 1455, 1377, 1355, 1266, 1198, 1159, 1127, 1087, 981, 846, 737, 696; ${ }^{\mathbf{1}} \mathbf{H} \mathbf{N M R}\left(300 \mathrm{MHz}, \mathrm{CDCl}_{3}\right)$ : $\delta[\mathrm{ppm}]=7.47-7.30(\mathrm{~m}, 5 \mathrm{H}), 6.85(\mathrm{dd}, J=15.8,6.4 \mathrm{~Hz}, 1 \mathrm{H}), 6.04(\mathrm{dd}, J=15.8,1.3 \mathrm{~Hz}, 1 \mathrm{H})$, $5.20(\mathrm{~s}, 2 \mathrm{H}), 3.67(\mathrm{qd}, J=6.3,1.3 \mathrm{~Hz}, 1 \mathrm{H}), 3.31(\mathrm{~s}, 3 \mathrm{H}), 1.89-1.36(\mathrm{~m}, 2 \mathrm{H}), 0.92(\mathrm{t}, J=7.4 \mathrm{~Hz}$, $3 \mathrm{H}) ;{ }^{13} \mathbf{C}\left\{{ }^{1} \mathbf{H}\right\}$ NMR (126 MHz, $\left.\mathrm{CDCl}_{3}\right): \delta[\mathrm{ppm}]=166.2,148.9,136.0,128.7,128.4,128.4$, 121.9, 81.9, 66.4, 57.2, 27.7, 9.6; HR-ESI-MS m/z: calcd. $\mathrm{C}_{14} \mathrm{H}_{18} \mathrm{O}_{3} \mathrm{Na}[\mathrm{M}+\mathrm{Na}]^{+}: 257.1148$, found: 257.1146 .

\section{Benzyl (E)-4-ethoxyhex-2-enoate (149b)}

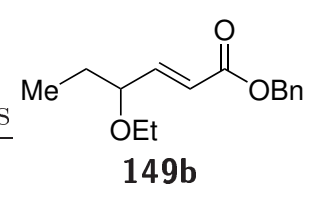

Following general procedure E alkene 148a (204 mg, $1.00 \mathrm{mmol}, 1.0$ equiv), EtOH $(5 \mathrm{~mL})$; reaction time $92 \mathrm{~h}$; eluting with $n$-pentane:EtOAc (50:1); yield: $48 \mathrm{mg}, 0.19 \mathrm{mmol}, 19 \%$; colorless liquid; ${ }^{1} \mathrm{H}$ NMR yield using 1,1,2,2tetrachloroethane as the internal standard: $31 \%$.

$\mathbf{R}_{\mathbf{f}}=0.31$ (n-pentane:EtOAc, 50:1); IR (ATR): $\tilde{\nu}\left[\mathrm{cm}^{-1}\right]=2972,2932,2875,1719,1658,1456$, 1377, 1338, 1268, 1164, 1126, 1092, 983, 748, 697; ${ }^{\mathbf{1}} \mathbf{H}$ NMR $\left(300 \mathrm{MHz}, \mathrm{CDCl}_{3}\right): \delta[\mathrm{ppm}]=7.47-$ $7.30(\mathrm{~m}, 5 \mathrm{H}), 6.87(\mathrm{dd}, J=15.8,6.2 \mathrm{~Hz}, 1 \mathrm{H}), 6.03(\mathrm{dd}, J=15.8,1.3 \mathrm{~Hz}, 1 \mathrm{H}), 5.19(\mathrm{~d}$, $J=0.5 \mathrm{~Hz}, 2 \mathrm{H}), 3.77(\mathrm{qd}, J=6.2,1.4 \mathrm{~Hz}, 1 \mathrm{H}), 3.53(\mathrm{dq}, J=9.3,7.0 \mathrm{~Hz}, 1 \mathrm{H}), 3.38$ (dqd, $J=9.2,7.0,0.9 \mathrm{~Hz}, 1 \mathrm{H}), 1.69-1.49(\mathrm{~m}, 2 \mathrm{H}), 1.20(\mathrm{t}, J=7.0 \mathrm{~Hz}, 3 \mathrm{H}), 1.08-0.79(\mathrm{~m}, 3 \mathrm{H})$; ${ }^{13} \mathbf{C}\left\{{ }^{1} \mathbf{H}\right\}$ NMR $\left(126 \mathrm{MHz}, \mathrm{CDCl}_{3}\right): \delta[\mathrm{ppm}]=166.2,149.5,136.1,128.6,128.32,128.30,121.3$, 80.1, 66.4, 64.9, 28.1, 15.6, 9.8; HR-ESI-MS m/z: calcd. $\mathrm{C}_{15} \mathrm{H}_{20} \mathrm{O}_{3} \mathrm{Na}[\mathrm{M}+\mathrm{Na}]^{+}: 271.1305$, found: 271.1303 .

\section{(E)-3-(4-Methoxyhex-2-enoyl)oxazolidin-2-one (149e)}

Following general procedure E alkene 148b (183 mg, $1.00 \mathrm{mmol}$,<smiles>COC(/C=C/C(=O)N1CCOC1=O)CO[Na]</smiles>
1.0 equiv), $\mathrm{MeOH}(5 \mathrm{~mL})$; reaction time $95 \mathrm{~h}$; eluting with $n$ pentane:EtOAc (3:1); yield: $22 \mathrm{mg}, 0.10 \mathrm{mmol}, 10 \%$; yellow liquid; ${ }^{1} \mathrm{H}$ NMR yield using 1,3,5-trimethoxybenzene as the internal standard: $11 \%$.

$\mathbf{R}_{\mathbf{f}}=0.14$ (n-pentane:EtOAc, 3:1); IR (ATR): $\tilde{\nu}\left[\mathrm{cm}^{-1}\right]=2965,2930,2881,2826,1769,1683$, 1640, 1476, 1462, 1440, 1385, 1362, 1335, 1194, 1105, 1088, 1033, 984, 972, 853, 759, 700; ${ }^{\mathbf{1}} \mathbf{H}$ $\operatorname{NMR}\left(300 \mathrm{MHz}, \mathrm{CDCl}_{3}\right): \delta[\mathrm{ppm}]=7.36(\mathrm{dd}, J=15.6,1.1 \mathrm{~Hz}, 1 \mathrm{H}), 6.96(\mathrm{dd}, J=15.6,6.8 \mathrm{~Hz}$, $1 \mathrm{H}), 4.55-4.27$ (m, $2 \mathrm{H}), 4.17-3.99(\mathrm{~m}, 2 \mathrm{H}), 3.75(\mathrm{~m}, 1 \mathrm{H}), 3.32(\mathrm{~s}, 3 \mathrm{H}), 1.79-1.49(\mathrm{~m}, 2 \mathrm{H}), 0.92$ $(\mathrm{t}, J=7.4 \mathrm{~Hz}, 3 \mathrm{H}) ;{ }^{\mathbf{1 3}} \mathbf{C}\left\{{ }^{\mathbf{1}} \mathbf{H}\right\} \mathbf{N M R}\left(126 \mathrm{MHz}, \mathrm{CDCl}_{3}\right): \delta[\mathrm{ppm}]=164.8,153.4,150.0,121.0$, 82.2, 62.2, 57.2, 42.9, 27.9, 9.7; HR-ESI-MS m/z: calcd. $\mathrm{C}_{10} \mathrm{H}_{15} \mathrm{NO}_{4} \mathrm{Na}[\mathrm{M}+\mathrm{Na}]^{+}: 236.0893$, found: 236.0897 . 


\section{Diethyl (E)-(3-methoxybut-1-en-1-yl)phosphonate (149f)}

Following general procedure $\mathbf{E}$ alkene 148c (192 mg, $1.00 \mathrm{mmol}, 1.0$ equiv),<smiles>CCOP(=O)(O)C=CC(C)OC</smiles>
$\mathrm{MeOH}(5 \mathrm{~mL})$; reaction time $8 \mathrm{~d}$; eluting with DCM:acetone $(20: 1)+1 \%$ AcOH; yield: $81 \mathrm{mg}, 0.36 \mathrm{mmol}, 36 \%$; yellow liquid; ${ }^{1} \mathrm{H}$ NMR yield using 1,3,5-trimethoxybenzene as the internal standard: $>99 \%$.

$\mathbf{R}_{\mathbf{f}}=0.07$ (DCM:acetone, 20:1 + 1\% AcOH); IR (ATR): $\tilde{\nu}\left[\mathrm{cm}^{-1}\right]=2980,2933,2905,2871,2826$, 1744, 1722, 1635, 1444, 1392, 1369, 1337, 1248, 1216, 1112, 1049, 1020, 956, 858, 826, 789, 746; ${ }^{1} \mathbf{H}$ NMR $\left(400 \mathrm{MHz}, \mathrm{CDCl}_{3}\right): \delta[\mathrm{ppm}]=6.66(\mathrm{ddd}, J=22.4,17.2,5.3 \mathrm{~Hz}, 1 \mathrm{H}), 5.85$ (ddd, $J=20.5,17.2,1.4 \mathrm{~Hz}, 1 \mathrm{H}$ ), 4.08 (dqt, $J=8.4,7.1,1.1 \mathrm{~Hz}, 4 \mathrm{H}$ ), 3.87 (qddd, $J=6.6,5.3,2.4$, $1.4 \mathrm{~Hz}, 1 \mathrm{H}), 3.31(\mathrm{~s}, 3 \mathrm{H}), 1.32(\mathrm{t}, J=7.1 \mathrm{~Hz}, 6 \mathrm{H}), 1.26(\mathrm{~d}, J=6.6 \mathrm{~Hz}, 3 \mathrm{H}){ }^{\mathbf{1 3}} \mathbf{C}\left\{{ }^{1} \mathbf{H}\right\} \mathbf{~ N M R}$ $\left(101 \mathrm{MHz}, \mathrm{CDCl}_{3}\right): \delta[\mathrm{ppm}]=153.5\left(\mathrm{~d}, J_{C P}=4.3 \mathrm{~Hz}\right), 116.9\left(\mathrm{~d}, J_{C P}=187.8 \mathrm{~Hz}\right), 77.4,61.9$ $\left(\mathrm{dd}, J_{C P}=5.6,2.1 \mathrm{~Hz}\right), 56.9,20.3\left(\mathrm{~d}, J_{C P}=1.9 \mathrm{~Hz}\right), 16.5\left(\mathrm{~d}, J_{C P}=6.2 \mathrm{~Hz}\right) ;{ }^{31} \mathbf{P}\left\{{ }^{1} \mathbf{H}\right\} \mathbf{N M R}$ $\left(162 \mathrm{MHz}, \mathrm{CDCl}_{3}\right): \delta[\mathrm{ppm}]=18.3$; HR-ESI-MS m/z: calcd. $\mathrm{C}_{9} \mathrm{H}_{20} \mathrm{O}_{4} \mathrm{P}[\mathrm{M}+\mathrm{H}]^{+}: 223.1094$, found: 223.1095 .

\section{Diethyl (E)-(3-ethoxybut-1-en-1-yl)phosphonate (149g)}<smiles>CCOP(=O)(OCC)OCC</smiles>

$\mathbf{R}_{\mathbf{f}}=0.10$ (DCM:acetone, 30:1 + 1\% AcOH); IR (ATR): $\tilde{\nu}\left[\mathrm{cm}^{-1}\right]=2978,2934,2904,2872,1721$, 1634, 1444, 1392, 1369, 1336, 1245, 1206, 1163, 1094, 1050, 1020, 957, 855, 828, 749; ${ }^{\mathbf{1}} \mathbf{H}$ NMR $\left(400 \mathrm{MHz}, \mathrm{CDCl}_{3}\right): \delta[\mathrm{ppm}]=6.68(\mathrm{ddd}, J=22.4,17.2,5.2 \mathrm{~Hz}, 1 \mathrm{H}), 5.85(\mathrm{ddd}, J=20.8$, 17.2, $1.4 \mathrm{~Hz}, 1 \mathrm{H}$ ), 4.16-4.02 (m, $4 \mathrm{H}$ ), 3.98 (dddd, $J=6.6,5.2,2.5,1.4 \mathrm{~Hz}, 1 \mathrm{H}$ ), 3.46 (dqd, $J=9.2,7.0,2.1 \mathrm{~Hz}, 2 \mathrm{H}), 1.32(\mathrm{tt}, J=7.1,0.3 \mathrm{~Hz}, 6 \mathrm{H}), 1.26(\mathrm{~d}, J=6.6 \mathrm{~Hz}, 3 \mathrm{H}), 1.20$ (t, $J=7.0 \mathrm{~Hz}, 3 \mathrm{H}) ;{ }^{13} \mathbf{C}\left\{{ }^{1} \mathbf{H}\right\} \mathbf{N M R}\left(101 \mathrm{MHz}, \mathrm{CDCl}_{3}\right): \delta[\mathrm{ppm}]=154.2\left(\mathrm{~d}, J_{C P}=4.4 \mathrm{~Hz}\right)$, $116.4\left(\mathrm{~d}, J_{C P}=188.1 \mathrm{~Hz}\right), 75.5\left(\mathrm{~d}, J_{C P}=21.9 \mathrm{~Hz}\right), 64.6,61.9\left(\mathrm{dd}, J_{C P}=5.6,2.5 \mathrm{~Hz}\right), 20.7$ $\left(\mathrm{d}, J_{C P}=2.1 \mathrm{~Hz}\right), 16.5\left(\mathrm{~d}, J_{C P}=6.4 \mathrm{~Hz}\right), 15.5 ;{ }^{31} \mathbf{P}\left\{{ }^{1} \mathbf{H}\right\} \quad \mathbf{N M R}\left(162 \mathrm{MHz}, \mathrm{CDCl}_{3}\right): \delta$ [ppm $]=18.5$; HR-ESI-MS m/z: calcd. $\mathrm{C}_{10} \mathrm{H}_{22} \mathrm{O}_{4} \mathrm{P}[\mathrm{M}+\mathrm{H}]^{+}:$237.1250, found: 237.1257.

\section{Diethyl (E)-(3-isopropoxybut-1-en-1-yl)phosphonate (149h)}<smiles>CCOC(=O)C=CC(=O)OCC</smiles>

Following general procedure E; alkene 148c (192 mg, $1.00 \mathrm{mmol}, 1.0$ equiv), isopropanol $(5 \mathrm{~mL})$; reaction time $12 \mathrm{~d}$; eluting with DCM:acetone $(30: 1)+$ 1\% AcOH; yield: $101 \mathrm{mg}, 402 \mu \mathrm{mol}, 40 \%$; yellow liquid; ${ }^{1} \mathrm{H}$ NMR yield using benzaldehyde as the internal standard: $43 \%$. 
$\mathbf{R}_{\mathbf{f}}=0.10$ (DCM:acetone, 30:1 $+1 \% \mathrm{AcOH}$ ); IR (ATR): $\tilde{\nu}\left[\mathrm{cm}^{-1}\right]=2975,2934,2909,2872$, 1722, 1633, 1445, 1369, 1247, 1121, 1020, 958, 851, 802, 751; ${ }^{\mathbf{1}} \mathbf{H}$ NMR $\left(400 \mathrm{MHz}, \mathrm{CDCl}_{3}\right): \delta$ $[\mathrm{ppm}]=6.70(\mathrm{ddd}, J=22.2,17.2,4.9 \mathrm{~Hz}, 1 \mathrm{H}), 5.86(\mathrm{ddd}, J=21.1,17.2,1.5 \mathrm{~Hz}, 1 \mathrm{H}), 4.14-4.01$ $(\mathrm{m}, 5 \mathrm{H}), 3.60$ (quint, $J=6.1 \mathrm{~Hz}, 1 \mathrm{H}), 1.32(\mathrm{td}, J=7.1,0.5 \mathrm{~Hz}, 6 \mathrm{H}), 1.23(\mathrm{~d}, J=6.6 \mathrm{~Hz}$, $3 \mathrm{H}), 1.14(\mathrm{dd}, J=6.1,3.2 \mathrm{~Hz}, 6 \mathrm{H}) ;{ }^{\mathbf{1 3}} \mathbf{C}\left\{{ }^{\mathbf{1}} \mathbf{H}\right\} \mathbf{N M R}\left(126 \mathrm{MHz}, \mathrm{CDCl}_{3}\right): \delta[\mathrm{ppm}]=155.1(\mathrm{~d}$, $\left.J_{C P}=4.5 \mathrm{~Hz}\right), 115.8\left(\mathrm{~d}, J_{C P}=188.3 \mathrm{~Hz}\right), 72.8\left(\mathrm{~d}, J_{C P}=21.7 \mathrm{~Hz}\right), 70.1,62.0\left(\mathrm{dd}, J_{C P}=5.6\right.$, $3.0 \mathrm{~Hz}), 23.1,22.1,21.3\left(\mathrm{~d}, J_{C P}=2.1 \mathrm{~Hz}\right), 16.5\left(\mathrm{~d}, J_{C P}=6.4 \mathrm{~Hz}\right) ;{ }^{\mathbf{3}} \mathbf{P}\left\{{ }^{1} \mathbf{H}\right\} \mathbf{N M R}(162 \mathrm{MHz}$, $\left.\mathrm{CDCl}_{3}\right): \delta[\mathrm{ppm}]=18.8$; HR-ESI-MS m/z: calcd. $\mathrm{C}_{11} \mathrm{H}_{24} \mathrm{O}_{4} \mathrm{P}[\mathrm{M}+\mathrm{H}]^{+}:$251.1407, found: 251.1400 .

\section{Diethyl (E)-(3-((4-chlorobenzyl)oxy)but-1-en-1-yl)phosphonate (149k)}<smiles>CCOP(=O)(OC)OC(C)COc1ccc(Cl)cc1</smiles>

750,$668 ;{ }^{\mathbf{1}} \mathbf{H}$ NMR $\left(500 \mathrm{MHz}, \mathrm{CDCl}_{3}\right): \delta[\mathrm{ppm}]=7.34-7.28(\mathrm{~m}, 2 \mathrm{H}), 7.28-7.23(\mathrm{~m}, 2 \mathrm{H}), 6.70$ (ddd, $J=22.4,17.2,5.4 \mathrm{~Hz}, 1 \mathrm{H}), 5.90(\mathrm{ddd}, J=20.4,17.2,1.4 \mathrm{~Hz}, 1 \mathrm{H}), 4.51(\mathrm{~d}, J=12.0 \mathrm{~Hz}$, $1 \mathrm{H}), 4.41(\mathrm{~d}, J=12.0 \mathrm{~Hz}, 1 \mathrm{H}), 4.21-3.98(\mathrm{~m}, 5 \mathrm{H}), 1.35-1.29(\mathrm{~m}, 9 \mathrm{H}) ;{ }^{13} \mathbf{C}\left\{{ }^{1} \mathbf{H}\right\} \mathbf{N M R}$ $\left(126 \mathrm{MHz}, \mathrm{CDCl}_{3}\right): \delta[\mathrm{ppm}]=153.4\left(\mathrm{~d}, J_{C P}=4.5 \mathrm{~Hz}\right), 136.7,133.6,129.0,128.7,117.1(\mathrm{~d}$, $\left.J_{C P}=188.1 \mathrm{~Hz}\right), 75.2\left(\mathrm{~d}, J_{C P}=22.1 \mathrm{~Hz}\right), 70.2,62.02\left(\mathrm{~d}, J_{C P}=2.2 \mathrm{~Hz}\right), 61.98\left(\mathrm{~d}, J_{C P}=\right.$ $2.3 \mathrm{~Hz}), 20.6\left(\mathrm{~d}, J_{C P}=1.9 \mathrm{~Hz}\right), 16.5\left(\mathrm{~d}, J_{C P}=6.3 \mathrm{~Hz}\right) ;{ }^{31} \mathbf{P}\left\{{ }^{1} \mathbf{H}\right\} \mathbf{N M R}\left(162 \mathrm{MHz}, \mathrm{CDCl}_{3}\right)$ : $\delta[\mathrm{ppm}]=18.1$; HR-ESI-MS m/z: calcd. $\mathrm{C}_{15} \mathrm{H}_{23} \mathrm{O}_{4} \mathrm{PCll}[\mathrm{M}+\mathrm{H}]^{+}:$333.1017, found: 333.1019 .

\section{Diethyl (E)-(3-((4-(trifluoromethyl)benzyl)oxy)but-1-en-1-yl)phosphonate (149I)}

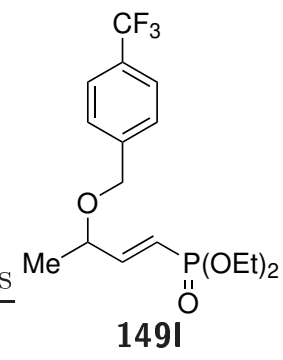

Following general procedure $\mathbf{E}$ alkene 148b (134 mg, 700 umol, 1.0 equiv), 4trifluoromethylbenzyl alcohol (3.5 mL), diphenyl diselenide (22 mg, 70 umol, 0.1 equiv) and $p$-MeO-TPT 95 (17 mg, 35 umol, 0.05 equiv); reaction time $73 \mathrm{~h}$; eluting with DCM:acetone $(30: 1)+1 \%$ AcOH; yield: $123 \mathrm{mg}$, $0.34 \mathrm{mmol}, 48 \%$; brown liquid; ${ }^{1} \mathrm{H}$ NMR yield using 1,3,5-trimethoxybenzene as the internal standard: $68 \%$.

$\mathbf{R}_{\mathbf{f}}=0.21$ (DCM:acetone, 30:1 $\left.+1 \% \mathrm{AcOH}\right) ; \mathbf{I R}(\mathrm{ATR}): \tilde{\nu}\left[\mathrm{cm}^{-1}\right]=2987$, 2935, 2871, 1623, 1444, 1424, 1392, 1369, 1325, 1248, 1161, 1121, 1066, 1051, 1017, 956, 823; ${ }^{1} \mathbf{H}$ NMR (400 MHz, $\left.\mathrm{CDCl}_{3}\right): \delta[\mathrm{ppm}]=7.70-7.53(\mathrm{~m}, 2 \mathrm{H}), 7.55-7.40(\mathrm{~m}, 2 \mathrm{H}), 6.72$ (ddd, $J=22.4,17.2,5.4 \mathrm{~Hz}, 1 \mathrm{H}), 5.92(\mathrm{ddd}, J=20.2,17.2,1.4 \mathrm{~Hz}, 1 \mathrm{H}), 4.61(\mathrm{~d}, J=12.5 \mathrm{~Hz}, 1 \mathrm{H})$, 
$4.51(\mathrm{~d}, J=12.5 \mathrm{~Hz}, 1 \mathrm{H}), 4.26-3.87(\mathrm{~m}, 5 \mathrm{H}), 1.37-1.30(\mathrm{~m}, 9 \mathrm{H}) ;{ }^{\mathbf{1 3}} \mathbf{C}\left\{{ }^{\mathbf{1}} \mathbf{H}\right\} \mathbf{N M R}(101 \mathrm{MHz}$, $\left.\mathrm{CDCl}_{3}\right): \delta[\mathrm{ppm}]=153.0\left(\mathrm{~d}, J_{C P}=4.5 \mathrm{~Hz}\right), 142.4,130.0\left(\mathrm{~d}, J_{C F}=32.3 \mathrm{~Hz}\right), 127.6,125.6(\mathrm{q}$, $\left.J_{C F}=271.8 \mathrm{~Hz}\right), 125.5\left(\mathrm{q}, J_{C F}=3.8 \mathrm{~Hz}\right), 117.4\left(\mathrm{~d}, J_{C P}=188.1 \mathrm{~Hz}\right), 75.6\left(\mathrm{~d}, J_{C P}=22.1 \mathrm{~Hz}\right)$, 70.2, 61.7-62.4 (m), $20.6\left(\mathrm{~d}, J_{C P}=1.9 \mathrm{~Hz}\right), 16.5\left(\mathrm{~d}, J_{C P}=6.2 \mathrm{~Hz}\right) ;{ }^{19} \mathbf{F}\left\{{ }^{\mathbf{1}} \mathbf{H}\right\} \mathbf{N M R}(376 \mathrm{MHz}$, $\left.\mathrm{CDCl}_{3}\right): \delta[\mathrm{ppm}]=-62.5 ;{ }^{\mathbf{3 1}} \mathbf{P}\left\{{ }^{\mathbf{1}} \mathbf{H}\right\} \mathbf{N M R}\left(162 \mathrm{MHz}, \mathrm{CDCl}_{3}\right): \delta[\mathrm{ppm}]=17.9$; HR-ESI-MS m/z: calcd. $\mathrm{C}_{16} \mathrm{H}_{23} \mathrm{~F}_{3} \mathrm{O}_{4} \mathrm{P}[\mathrm{M}+\mathrm{H}]^{+}$: 367.1281, found: 367.1283 .

\section{Diethyl (E)-(3-((perfluorophenyl)methoxy)but-1-en-1-yl)phosphonate (149m)}

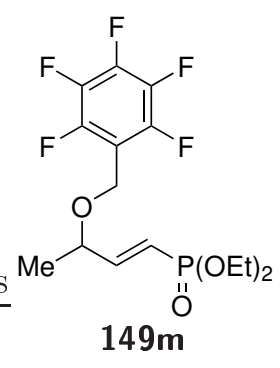

Following general procedure E: alkene 148c (192 mg, $1.00 \mathrm{mmol}, 1.0$ equiv), pentafluorobenzyl alcohol $(5 \mathrm{~mL})$; reaction time $1.5 \mathrm{~d}$; DCM:acetone $(30: 1)$ $+1 \% \mathrm{AcOH}$; yield: $148 \mathrm{mg}, 381 \mu \mathrm{mol}, 38 \%$; yellow liquid; ${ }^{1} \mathrm{H}$ NMR yield using benzaldehyde as the internal standard: $81 \%$.

$\mathbf{R}_{\mathbf{f}}=0.25$ (DCM:acetone, 30:1 $\left.+1 \% \mathrm{AcOH}\right) ; \mathbf{I R}(\mathrm{ATR}): \tilde{\nu}\left[\mathrm{cm}^{-1}\right]=2983$, 2936, 2909, 2875, 1750, 1722, 1656, 1636, 1522, 1504, 1393, 1305, 1248, 1123, 1046, 1022, 957, 936, 848, 821, 787, 753, 673; ${ }^{\mathbf{1}} \mathbf{H}$ NMR (400 MHz, $\left.\mathrm{CDCl}_{3}\right)$ : $\delta[\mathrm{ppm}]=6.67(\mathrm{ddd}, J=22.4,17.2,5.4 \mathrm{~Hz}, 1 \mathrm{H}), 5.90(\mathrm{ddd}, J=20.0,17.2,1.4 \mathrm{~Hz}, 1 \mathrm{H}), 4.60$ $(\mathrm{dt}, J=11.0,1.9 \mathrm{~Hz}, 1 \mathrm{H}), 4.52(\mathrm{dt}, J=11.2,1.9 \mathrm{~Hz}, 1 \mathrm{H}), 4.26-3.96(\mathrm{~m}, 5 \mathrm{H}), 1.33(\mathrm{td}, J=7.1$, $1.3 \mathrm{~Hz}, 6 \mathrm{H}), 1.29(\mathrm{~d}, J=6.5 \mathrm{~Hz}, 3 \mathrm{H}) ;{ }^{\mathbf{1 3}} \mathbf{C}\left\{{ }^{1} \mathbf{H}\right\} \mathbf{N M R}\left(126 \mathrm{MHz}, \mathrm{CDCl}_{3}\right): \delta[\mathrm{ppm}]=152.4$ $\left(\mathrm{d}, J_{C P}=4.8 \mathrm{~Hz}\right), 145.7\left(\mathrm{dddt}, J_{C F}=249.3,11.9,8.2,3.9 \mathrm{~Hz}\right), 141.6\left(\mathrm{dtt}, J_{C F}=255.0\right.$, 13.4, $5.3 \mathrm{~Hz}), 137.6(\mathrm{~m}), 117.6\left(\mathrm{~d}, J_{C P}=188.0 \mathrm{~Hz}\right), 111.3\left(\mathrm{td}, J_{C F}=18.0,3.8 \mathrm{~Hz}\right), 76.3(\mathrm{~d}$, $\left.J_{C P}=22.3 \mathrm{~Hz}\right), 62.1\left(\mathrm{dd}, J_{C P}=5.6,2.1 \mathrm{~Hz}\right), 58.0\left(\mathrm{~d}, J_{C P}=2.9 \mathrm{~Hz}\right), 20.7\left(\mathrm{~d}, J_{C P}=2.0 \mathrm{~Hz}\right)$, $16.5\left(\mathrm{~d}, J_{C P}=6.3 \mathrm{~Hz}\right) ;{ }^{19} \mathbf{F}\left\{{ }^{\mathbf{1}} \mathbf{H}\right\} \mathbf{N M R}\left(376 \mathrm{MHz}, \mathrm{CDCl}_{3}\right): \delta[\mathrm{ppm}]=-143.0(\mathrm{~m}),-153.5$ (m), $-161.8(\mathrm{~m}) ;{ }^{\mathbf{3 1}} \mathbf{P}\left\{{ }^{1} \mathbf{H}\right\}$ NMR $\left(162 \mathrm{MHz}, \mathrm{CDCl}_{3}\right): \delta[\mathrm{ppm}]=17.7 ; \mathbf{H R}-\mathbf{E S I}-\mathbf{M S} \mathbf{~ m} / \mathbf{z}$ : calcd. $\mathrm{C}_{15} \mathrm{H}_{19} \mathrm{~F}_{5} \mathrm{O}_{4} \mathrm{P}[\mathrm{M}+\mathrm{H}]^{+}$: 389.0936, found: 389.0933 .

\section{(E/Z)-4-((4-(Trifluoromethyl)benzyl)oxy)pent-2-enenitrile (149n)}

Following general procedure E 3-pentenenitrile ( $81 \mathrm{mg}, 1.0 \mathrm{mmol}, 1.0$ equiv),<smiles>CC(C=CC#N)OCc1ccc(C(F)(F)F)cc1</smiles>
4-trifluoromethylbenzyl alcohol $(5 \mathrm{~mL})$; reaction time $1 \mathrm{~d}$; eluting with $n$ pentane:EtOAc (30:1); yield: $63 \mathrm{mg}, 0.25 \mathrm{mmol}, 25 \%(E / Z=2: 1)$; yellow liquid; ${ }^{1} \mathrm{H}$ NMR yield using 1,1,2,2-tetrachloroethane as the internal standard: $54 \%(E / Z=2: 1)$.

$\mathbf{R}_{\mathbf{f}}=0.10$ (n-pentane:EtOAc, 30:1); IR (ATR): $\tilde{\nu}\left[\mathrm{cm}^{-1}\right]=E$ isomer: 2982, 2936, 2869, 2360, 2341, 2226, 1729, 1621, 1421, 1325, 1163, 1121, 1065, 1018, 965, 824; $Z$ isomer: 2984, 2934, 2872, 2360, 2341, 1326, 1163, 1122, 1066, 1019, 824; ${ }^{\mathbf{1}} \mathbf{H}$ NMR $\left(400 \mathrm{MHz}, \mathrm{CDCl}_{3}\right): \delta[\mathrm{ppm}](E$ isomer $)=7.74-7.55(\mathrm{~m}, 2 \mathrm{H}), 7.47-7.35(\mathrm{~m}, 2 \mathrm{H}), 6.70(\mathrm{dd}$, $J=16.3,5.2 \mathrm{~Hz}, 1 \mathrm{H}), 5.63(\mathrm{dd}, J=16.4,1.6 \mathrm{~Hz}, 1 \mathrm{H}), 4.59(\mathrm{~d}, J=12.4 \mathrm{~Hz}, 1 \mathrm{H}), 4.54(\mathrm{~d}$, $J=12.5 \mathrm{~Hz}, 1 \mathrm{H}), 4.12$ (qdd, $J=6.6,5.2,1.6 \mathrm{~Hz}, 1 \mathrm{H}), 1.35(\mathrm{~d}, J=6.5 \mathrm{~Hz}, 3 \mathrm{H}) ; \delta[\mathrm{ppm}]$ 
$(Z$ isomer $)=7.66-7.55(\mathrm{~m}, 2 \mathrm{H}), 7.49-7.44(\mathrm{~m}, 2 \mathrm{H}), 6.45(\mathrm{dd}, J=11.1,8.6 \mathrm{~Hz}, 1 \mathrm{H}), 5.48(\mathrm{dd}$, $J=11.1,0.9 \mathrm{~Hz}, 1 \mathrm{H}), 4.59(\mathrm{~d}, J=12.2 \mathrm{~Hz}, 1 \mathrm{H}), 4.52(\mathrm{~d}, J=12.0 \mathrm{~Hz}, 1 \mathrm{H}), 4.50(\mathrm{~m}, 1 \mathrm{H})$, $1.39(\mathrm{~d}, J=6.4 \mathrm{~Hz}, 3 \mathrm{H}) ;{ }^{\mathbf{1 3}} \mathbf{C}\left\{{ }^{\mathbf{1}} \mathbf{H}\right\} \mathbf{N M R}\left(101 \mathrm{MHz}, \mathrm{CDCl}_{3}\right): \delta[\mathrm{ppm}](E$ isomer $)=155.5$, $141.8,130.3\left(\mathrm{q}, J_{C F}=32.4 \mathrm{~Hz}\right), 127.6,125.6\left(\mathrm{q}, J_{C F}=3.8 \mathrm{~Hz}\right), 124.2\left(\mathrm{q}, J_{C F}=272.0 \mathrm{~Hz}\right)$, $117.0,100.0,74.4,70.3,20.3 ; \delta[\mathrm{ppm}](Z$ isomer $)=155.4,141.9,130.2\left(\mathrm{~d}, J_{C F}=32.4 \mathrm{~Hz}\right), 127.8$, $125.6\left(\mathrm{q}, J_{C F}=3.9 \mathrm{~Hz}\right), 125.3(\mathrm{~m}), 115.2,100.9,74.2,70.6,20.7 ;{ }^{\mathbf{1 9}} \mathbf{F}\left\{{ }^{\mathbf{1}} \mathbf{H}\right\} \mathbf{N M R}(376 \mathrm{MHz}$, $\left.\mathrm{CDCl}_{3}\right): \delta[\mathrm{ppm}]$ (both isomers) $=-62.6$; HR-ESI-MS $\mathbf{m} / \mathbf{z}$ : calcd. $\mathrm{C}_{13} \mathrm{H}_{13} \mathrm{~F}_{3} \mathrm{NO}[\mathrm{M}+\mathrm{H}]^{+}$: 256.0944, found: 256.0938 .

\section{(E/Z)-4-Phenethoxypent-2-enenitrile (149o)}

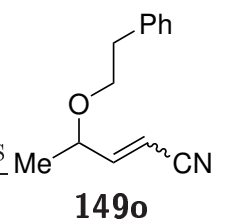

Following general procedure E 3-pentenenitrile ( $81 \mathrm{mg}, 1.0 \mathrm{mmol}, 1.0$ equiv), 2-phenyl ethanol (183 mg, $1.5 \mathrm{mmol}, 1.5$ equiv), MeCN $(2.5 \mathrm{~mL})$; reaction time $1 \mathrm{~d}$; eluting with $n$-pentane:EtOAc (20:1); yield: $29 \mathrm{mg}, 145 \mu \mathrm{mol}, 15 \%(E / Z=$ 6.5:1); yellow liquid; ${ }^{1} \mathrm{H}$ NMR yield using 1,3,5-trimethoxybenzene as the internal standard: $18 \%(E / Z=12: 1)$.

$\mathbf{R}_{\mathbf{f}}=0.22$ (n-pentane:EtOAc, 20:1); IR (ATR): $\tilde{\nu}\left[\mathrm{cm}^{-1}\right]=3087,3062,3028$, 2979, 2932, 2866, 2224, 1635, 1604, 1496, 1454, 1370, 1342, 1248, 1148, 1093, 1030, 963, 749, $699 ;{ }^{1} \mathbf{H}$ NMR $\left(500 \mathrm{MHz}, \mathrm{CDCl}_{3}\right): \delta[\mathrm{ppm}](E$ isomer $)=7.34-7.27(\mathrm{~m}, 2 \mathrm{H}), 7.25-7.19(\mathrm{~m}, 3 \mathrm{H})$, $6.60(\mathrm{dd}, J=16.3,4.8 \mathrm{~Hz}, 1 \mathrm{H}), 5.41(\mathrm{dd}, J=16.3,1.7 \mathrm{~Hz}, 1 \mathrm{H}), 3.98$ (qdd, $J=6.6,4.8$, $1.7 \mathrm{~Hz}, 1 \mathrm{H}), 3.65(\mathrm{t}, J=6.8 \mathrm{~Hz}, 1 \mathrm{H}), 3.61(\mathrm{t}, J=7.1 \mathrm{~Hz}, 1 \mathrm{H}), 2.88(\mathrm{t}, J=6.9 \mathrm{~Hz}, 2 \mathrm{H})$, $1.25(\mathrm{~d}, J=6.6 \mathrm{~Hz}, 3 \mathrm{H}) ; \delta[\mathrm{ppm}](Z$ isomer $)=7.36-7.27(\mathrm{~m}, 2 \mathrm{H}), 7.25-7.14(\mathrm{~m}, 3 \mathrm{H}), 6.35(\mathrm{dd}$, $J=11.2,8.6 \mathrm{~Hz}, 1 \mathrm{H}), 5.40(\mathrm{~m}, 1 \mathrm{H}), 4.39(\mathrm{dqd}, J=8.6,6.4,1.0 \mathrm{~Hz}, 1 \mathrm{H}), 3.67(\mathrm{t}, J=6.8 \mathrm{~Hz}$, $1 \mathrm{H}), 3.59(\mathrm{t}, J=7.1 \mathrm{~Hz}, 1 \mathrm{H}), 2.88(\mathrm{t}, J=6.9 \mathrm{~Hz}, 2 \mathrm{H}), 1.31(\mathrm{~d}, J=6.4 \mathrm{~Hz}, 3 \mathrm{H}) ;{ }^{13} \mathbf{C}\left\{{ }^{1} \mathbf{H}\right\}$ NMR $\left(126 \mathrm{MHz}, \mathrm{CDCl}_{3}\right): \delta[\mathrm{ppm}](E$ isomer $)=156.0,138.7,129.0,128.5,126.6,117.3,99.3$, $74.7,70.4,36.6,20.2 ; \delta[\mathrm{ppm}](Z$ isomer $)=156.2,138.7,129.0,128.5,126.6,117.3,100.2,74.2$, 70.4, 36.5, 20.6; HR-ESI-MS m/z: calcd. $\mathrm{C}_{13} \mathrm{H}_{16} \mathrm{NO}[\mathrm{M}+\mathrm{H}]^{+}: 202.1226$, found: 202.1226 .

\section{(E/Z)-4-((4-Chlorobenzyl)oxy)pent-2-enenitrile (149p)}

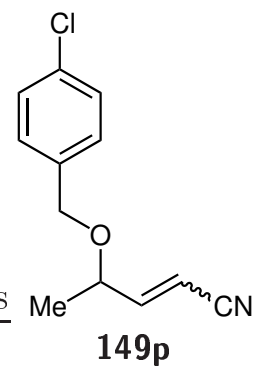

Following general procedure E 3-pentenenitrile ( $81 \mathrm{mg}, 1.0 \mathrm{mmol}, 1.0$ equiv), 4-chlorobenzyl alcohol (214 mg, $1.5 \mathrm{mmol}, 1.5$ equiv), MeCN (2.5 mL); reaction time $1 \mathrm{~d}$; eluting with $n$-pentane:EtOAc (15:1); yield: $6.9 \mathrm{mg}, 31 \mu \mathrm{mol}, 31 \%$ $(E / Z=3.5: 1)$; yellow liquid; ${ }^{1} \mathrm{H}$ NMR yield using $1,3,5$-trimethoxybenzene as the internal standard: $18 \%(E / Z=4.3: 1)$.

$\mathbf{R}_{\mathbf{f}}=0.19$ (n-pentane:EtOAc, 15:1); IR (ATR): $\tilde{\nu}\left[\mathrm{cm}^{-1}\right]=2979,2931,2868$, 2225, 1599, 1493, 1468, 1443, 1410, 1371, 1345, 1254, 1205, 1148, 1087, 1015, $965,809,761 ;{ }^{1} \mathbf{H}$ NMR $\left(300 \mathrm{MHz}, \mathrm{CDCl}_{3}\right): \delta[\mathrm{ppm}]=\delta[\mathrm{ppm}](E$ isomer $)=7.41-7.16(\mathrm{~m}, 4 \mathrm{H})$, $6.68(\mathrm{dd}, J=16.3,5.2 \mathrm{~Hz}, 1 \mathrm{H}), 5.61(\mathrm{dd}, J=16.3,1.6 \mathrm{~Hz}, 1 \mathrm{H}), 4.50(\mathrm{~d}, J=11.9 \mathrm{~Hz}, 1 \mathrm{H})$, 
$4.44(\mathrm{~d}, J=11.9 \mathrm{~Hz}, 1 \mathrm{H}), 4.09(\mathrm{qdd}, J=6.6,5.2,1.6 \mathrm{~Hz}, 1 \mathrm{H}), 1.32(\mathrm{~d}, J=6.6 \mathrm{~Hz}, 3 \mathrm{H}) ; \delta$ $[\mathrm{ppm}](Z$ isomer $)=7.41-7.16(\mathrm{~m}, 4 \mathrm{H}), 6.44(\mathrm{ddd}, J=11.2,8.7,0.5 \mathrm{~Hz}, 1 \mathrm{H}), 5.46(\mathrm{dd}, J=11.1$, $0.9 \mathrm{~Hz}, 1 \mathrm{H}), 4.55-4.40(\mathrm{~m}, 1 \mathrm{H}), 4.50(\mathrm{~d}, J=11.9 \mathrm{~Hz}, 1 \mathrm{H}), 4.43(\mathrm{~d}, J=11.9 \mathrm{~Hz}, 1 \mathrm{H}), 1.36$ $(\mathrm{d}, J=6.4 \mathrm{~Hz}, 3 \mathrm{H}) ;{ }^{\mathbf{1 3}} \mathbf{C}\left\{{ }^{\mathbf{1}} \mathbf{H}\right\} \mathbf{~ N M R}\left(126 \mathrm{MHz}, \mathrm{CDCl}_{3}\right): \delta[\mathrm{ppm}](E$ isomer $)=155.6,136.1$, $133.8,128.9,128.8,117.0,99.9,74.1,70.4,20.4 ; \delta[\mathrm{ppm}](Z$ isomer $)=155.5,136.3,133.7,129.2$, 128.7, 117.0, 100.6, 73.9, 70.7, 20.8; HR-ESI-MS m/z: calcd. $\mathrm{C}_{12} \mathrm{H}_{13} \mathrm{ClNO}[\mathrm{M}+\mathrm{H}]^{+}: 222.0680$, found: 222.0682 .

\subsection{Synthesis and lactonization of unsaturated acids via photo-aerobic selenium- $\pi$-acid catalysis}

\subsubsection{Synthesis of unsaturated carboxylic acids 34,137 and 167}

\section{General Procedure F: Knoevenagel reaction}

Malonic acid (2.2 equiv) is dissolved in DMSO (0.6 M), a solution of piperidine 0.02 equiv) and acetic acid (0.02 equiv) in DMSO (0.04 $\mathrm{M}$ for piperidine) is added dropwise. The solution is heated to $65{ }^{\circ} \mathrm{C}$, the respective aldehyde (1.0 equiv) is added dropwise and the solution is stirred at $65{ }^{\circ} \mathrm{C}$. The reaction is quenched by the addition of water, extracted with $\mathrm{Et}_{2} \mathrm{O}(3 \times)$, washed with water $(3 \times)$, extracted with sat. aq. $\mathrm{Na}_{2} \mathrm{CO}_{3}(3 \times)$, to the aq. phase, conc. $\mathrm{HCl}$ is added until $\mathrm{pH}=1$ and it is extracted with $\mathrm{Et}_{2} \mathrm{O}(2 \times)$. The org. phase is dried over $\mathrm{Na}_{2} \mathrm{SO}_{4}$. Removal of the solvent in vacuo, followed by column chromatography afford the product.

\section{(E)-5-Phenylpent-3-enoic acid (34b)}

Following general procedure F Malonic acid (2.56 g, $24.6 \mathrm{mmol}$,<smiles>O=C(O)C/C=C/Cc1ccccc1</smiles>

34b 2.2 equiv), piperidine (19.0 mg, 220 umol, 0.02 equiv), acetic acid (13.4 mg, 220 umol, 0.02 equiv) 3-phenylpropanal ( $1.50 \mathrm{~g}, 11.2 \mathrm{mmol}, 1.0$ equiv); reaction time $3 \mathrm{~h}$; eluting with $n$-pentane $/ \mathrm{Et}_{2} \mathrm{O}, 3: 1$; colorless liquid; yield: $550 \mathrm{mg}, 3.12 \mathrm{mmol}, 28 \%$.

$\mathbf{R}_{\mathbf{f}}=0.20$ (n-pentane:Et $\left.{ }_{2} \mathrm{O}, 3: 1\right)$; IR (ATR): $\tilde{\nu}\left[\mathrm{cm}^{-1}\right]=3027,2901,1704,1494,1417,1286$, 1220, 1156, 968, 932, 745, 698, 490; ${ }^{\mathbf{1}} \mathbf{H}$ NMR $\left(500 \mathrm{MHz}, \mathrm{CDCl}_{3}\right): \delta[\mathrm{ppm}]=10.5(\mathrm{~s}, 1 \mathrm{H})$, 7.39-7.15 (m, $5 \mathrm{H}), 5.90-5.54(\mathrm{~m}, 2 \mathrm{H}), 3.41(\mathrm{~d}, J=6.5 \mathrm{~Hz}, 2 \mathrm{H}), 3.14(\mathrm{dd}, J=6.7,1.0 \mathrm{~Hz}$, $2 \mathrm{H}) ;{ }^{13} \mathbf{C}\left\{{ }^{1} \mathbf{H}\right\} \mathbf{N M R}\left(125 \mathrm{MHz}, \mathrm{CDCl}_{3}\right): \delta[\mathrm{ppm}]=178.6,140.0,133.9,128.7,128.6,126.3$, 122.5, 39.0, 37.8; HR-ESI-MS m/z: calcd. $\mathrm{C}_{11} \mathrm{H}_{11} \mathrm{O}_{2}[\mathrm{M}-\mathrm{H}]^{-}:$: 175.0765 , found: 175.0766 . 


\section{(E)-5-(4-Methoxyphenyl)pent-3-enoic acid (34c)}<smiles>COc1ccc(C/C=C/CC(=O)O)cc1</smiles>

Following general procedure F: Malonic acid $(697 \mathrm{mg}, 6.70 \mathrm{mmol}$, 2.2 equiv), piperidine $(6.0 \mu \mathrm{L}, 5.2 \mathrm{mg}, 61$ umol, 0.02 equiv), acetic acid (3.5 $\mu \mathrm{L}, 3.7 \mathrm{mg}, 61$ umol, 0.02 equiv), 3-(4methoxyphenyl)propionaldehyde (500 mg, $3.05 \mathrm{mmol}, 1.0$ equiv); reaction time $10 \mathrm{~h}$; eluting with $n$-pentane $/ \mathrm{Et}_{2} \mathrm{O}, 5: 1$; white solid;

yield: $133 \mathrm{mg}, 0.645 \mathrm{mmol}, 43 \%$.

$\mathbf{R}_{\mathbf{f}}=0.07$ (n-pentane:Et $\left.{ }_{2} \mathrm{O}, 5: 1\right) ; \mathbf{m . p .} .=58{ }^{\circ} \mathrm{C}$; IR (ATR) $\tilde{\nu}\left[\mathrm{cm}^{-1}\right]=3033,3001,2953,2932$, 2906, 2836, 1704, 1610, 1584, 1510, 1464, 1418, 1299, 1176, 1108, 1034, 969, 937, 813, 557, 521; ${ }^{1} \mathbf{H}$ NMR $\left(500 \mathrm{MHz}, \mathrm{CDCl}_{3}\right): \delta[\mathrm{ppm}]=7.20-7.02(\mathrm{~m}, 2 \mathrm{H}), 6.91-6.65(\mathrm{~m}, 2 \mathrm{H}), 5.75(\mathrm{~m}, 1 \mathrm{H})$, $5.62(\mathrm{~m}, 1 \mathrm{H}), 3.80(\mathrm{~s}, 3 \mathrm{H}), 3.35(\mathrm{~d}, J=6.5 \mathrm{~Hz}, 3 \mathrm{H}), 3.13(\mathrm{dd}, J=6.7,1.1 \mathrm{~Hz}, 2 \mathrm{H}) ;{ }^{13} \mathbf{C}\left\{{ }^{1} \mathbf{H}\right\}$ NMR $\left(125 \mathrm{MHz}, \mathrm{CDCl}_{3}\right): \delta[\mathrm{ppm}]=178.4,158.0,134.3,132.0,129.5,122.0,114.0,55.4,38.2$, 37.8; HR-ESI-MS m/z: calcd. $\mathrm{C}_{12} \mathrm{H}_{14} \mathrm{O}_{3} \mathrm{Na}[\mathrm{M}+\mathrm{Na}]^{+}$: 229.0835, found: 229.0834 .

\section{Ethyl 2-acetyl-7-hydroxyheptanoate (159)}<smiles>CC(=O)OC(CCCCCO)C(C)=O</smiles>

$80{ }^{\circ} \mathrm{C}$. The solvent was removed in vacuo, the residue dissolved in EtOAc, filtered and concentrated in vacuo. Column chromatography (DCM/EtOAc, 2:1) afforded the product as a colorless liquid (1.31 g, $6.07 \mathrm{mmol}, 40 \%)$.

$\mathbf{R}_{\mathbf{f}}=0.29(\mathrm{DCM} / \mathrm{EtOAc}, 2: 1)$; IR (ATR): $\tilde{\nu}\left[\mathrm{cm}^{-1}\right]=3419,2935,2862,1735,1710,1463,1360$, 1242, 1196, 1149, 1053, 1025; ${ }^{\mathbf{1}} \mathbf{H}$ NMR $\left(500 \mathrm{MHz}, \mathrm{CDCl}_{3}\right): \delta[\mathrm{ppm}]=4.19(\mathrm{q}, J=7.1 \mathrm{~Hz}$, $2 \mathrm{H}), 3.62(\mathrm{t}, J=6.4 \mathrm{~Hz}, 2 \mathrm{H}), 3.39(\mathrm{t}, J=7.3 \mathrm{~Hz}, 1 \mathrm{H}), 2.21(\mathrm{~s}, 3 \mathrm{H}), 1.85$ (tdd, $J=8.2$, 6.5, $4.1 \mathrm{~Hz}, 2 \mathrm{H}), 1.65-1.49(\mathrm{~m}, 2 \mathrm{H}), 1.45-1.17$ (m, $7 \mathrm{H}) ;{ }^{\mathbf{1 3}} \mathbf{C}\left\{{ }^{\mathbf{1}} \mathbf{H}\right\}$ NMR $\left(75 \mathrm{MHz}, \mathrm{CDCl}_{3}\right)$ : $\delta[\mathrm{ppm}]=203.4,170.0,62.8,61.5,59.9,32.5,28.9,28.2,27.3,25.6,14.2 ;$ HR-ESI-MS m/z: calcd. $\mathrm{C}_{11} \mathrm{H}_{20} \mathrm{O}_{4} \mathrm{Na}[\mathrm{M}+\mathrm{Na}]^{+}: 239.1254$, found: 239.1259 .

\section{8-Hydroxyoctan-2-one (160)}

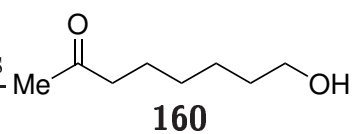

Ethyl 2-acetyl-7-hydroxyheptanoate (159) (1.30 g, $6.01 \mathrm{mmol}, 1.0$ equiv) was added to a solution of $\mathrm{NaOH}$ (601 mg, $15.0 \mathrm{mmol}, 2.5$ equiv) in $\mathrm{H}_{2} \mathrm{O}$ $(30 \mathrm{~mL})$. When everything was dissolved, a solution of $\mathrm{H}_{2} \mathrm{SO}_{4}(0.65 \mathrm{~mL}$, $12.0 \mathrm{mmol}, 2.0$ equiv) in $\mathrm{H}_{2} \mathrm{O}(60 \mathrm{~mL})$ was added and stirred at $50{ }^{\circ} \mathrm{C}$

for $1 \mathrm{~h}$. The solution was extracted with EtOAc $(2 \times 30 \mathrm{~mL})$, washed with sat. aq. $\mathrm{NaCl}$ solution $(15 \mathrm{~mL})$ and dried over $\mathrm{Na}_{2} \mathrm{SO}_{4}$. The product was obtained as a colorless oil $(814 \mathrm{mg}$, $5.65 \mathrm{mmol}, 94 \%)$. 
$\mathbf{R}_{\mathbf{f}}=0.21$ (DCM:EtOAc, 9:1); IR (ATR): $\tilde{\nu}\left[\mathrm{cm}^{-1}\right]=3399,2932,2859,1707,1410,1360,1165$, 1056, 597, 522; ${ }^{1} \mathbf{H}$ NMR $\left(300 \mathrm{MHz}, \mathrm{CDCl}_{3}\right): \delta[\mathrm{ppm}]=3.59$ (t, $\left.J=6.5 \mathrm{~Hz}, 2 \mathrm{H}\right), 2.53-2.30$ (m, $2 \mathrm{H}), 2.10(\mathrm{t}, J=0.4 \mathrm{~Hz}, 3 \mathrm{H}), 2.03(\mathrm{~s}, 1 \mathrm{H}), 1.73-1.44(\mathrm{~m}, 4 \mathrm{H}), 1.44-1.00(\mathrm{~m}, 4 \mathrm{H}) ;{ }^{13} \mathbf{C}\left\{{ }^{1} \mathbf{H}\right\}$ NMR $\left(75 \mathrm{MHz}, \mathrm{CDCl}_{3}\right): \delta[\mathrm{ppm}]=209.3,62.8,43.8,32.7,30.0,29.1,25.7,23.9$; HR-ESI-MS m/z: calcd. $\mathrm{C}_{8} \mathrm{H}_{16} \mathrm{O}_{2} \mathrm{Na}[\mathrm{M}+\mathrm{Na}]^{+}:$167.1043, found: 167.1045 .

\section{7-Oxooctanal (161)}

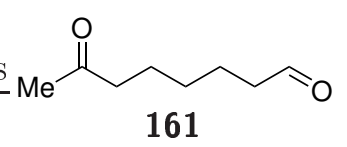

DMP (4.56 g, $10.7 \mathrm{mmol}, 2.0$ equiv) was dissolved in DCM $(22 \mathrm{~mL})$ and cooled to $0{ }^{\circ} \mathrm{C}$. 8-Hydroxyoctan-2-one (160) (775 mg, $5.37 \mathrm{mmol}$, 1.0 equiv) in DCM $(5 \mathrm{~mL})$ was added and the solution was stirred at $\mathrm{rt}$ for 3.5 h. DCM was added, the mixture washed with sat. aq. $\mathrm{Na}_{2} \mathrm{~S}_{2} \mathrm{O}_{3}$, $1 \mathrm{M}$ aq. $\mathrm{NaOH}$ and sat. aq. $\mathrm{NaCl}$. Extraction with $\mathrm{DCM}$, drying over $\mathrm{Na}_{2} \mathrm{SO}_{4}$, removal of the solvent in vacuo, followed by column chromatography (DCM/EtOAc, 9:1) afforded the product as a colorless liquid (210 $\mathrm{mg}, 1.48 \mathrm{mmol}, 28 \%)$.

$\mathbf{R}_{\mathbf{f}}=0.57$ (DCM:EtOAc, 9:1); ${ }^{\mathbf{1}} \mathbf{H}$ NMR $\left(300 \mathrm{MHz}, \mathrm{CDCl}_{3}\right): \delta[\mathrm{ppm}]=9.75(\mathrm{t}, J=1.7 \mathrm{~Hz}$, $1 \mathrm{H}), 2.43(\mathrm{td}, J=7.3,1.4 \mathrm{~Hz}, 4 \mathrm{H}), 2.12(\mathrm{t}, J=0.5 \mathrm{~Hz}, 3 \mathrm{H}), 1.74-1.48(\mathrm{~m}, 4 \mathrm{H}), 1.44-1.15$ $(\mathrm{m}, 2 \mathrm{H})$.

\section{(E)-10-Oxoundec-3-enoic acid (34e)}<smiles>CC(=O)CCCCCC=CCC(=O)O</smiles>

$34 \mathrm{e}$

DMAP (17 mg, $0.14 \mathrm{mmol}, 0.1$ equiv) was dissolved in DMF (3.5 mL), malonic acid (147 mg, $1.41 \mathrm{mmol}, 1.0$ equiv) and 161 (200 mg, $1.41 \mathrm{mmol}, 1.0$ equiv) were added and the solution was stirred at $\mathrm{rt}$ for $66 \mathrm{~h}$. $\mathrm{Et}_{2} \mathrm{O}$ was added, the org. phase was washed with $\mathrm{NH}_{4} \mathrm{Cl}, \mathrm{H}_{2} \mathrm{O}, \mathrm{NaHCO}_{3}$ and again water. The aq. phase was extracted with $\mathrm{Et}_{2} \mathrm{O}$ and dried over $\mathrm{Na}_{2} \mathrm{SO}_{4}$. Removal of the solvent in vacuo, followed by column chromatography ( $n$ pentane/ $\mathrm{Et}_{2} \mathrm{O}, 1: 1$ to $\mathrm{Et}_{2} \mathrm{O}$, pure) afforded the product as a colorless liquid (1:1.5 mixture of $\alpha$, $\beta$ and $\beta, \gamma$ unsaturated product; $61 \mathrm{mg}, 0.33 \mathrm{mmol}, 23 \%$ ).

$\mathbf{R}_{\mathbf{f}}=0.43\left(\mathrm{Et}_{2} \mathrm{O}\right) ; \mathbf{I R}(\mathrm{ATR}): \tilde{\nu}\left[\mathrm{cm}^{-1}\right]=2930,2859,1703,1654,1410,1361,1282,1222,1171$, 971; ${ }^{1} \mathbf{H}$ NMR $\left(300 \mathrm{MHz}, \mathrm{CDCl}_{3}\right): \delta[\mathrm{ppm}]=7.04(\mathrm{dt}, J=15.6,7.0 \mathrm{~Hz}, 1 \mathrm{H}), 5.80(\mathrm{dt}, J=$ 15.6, $1.5 \mathrm{~Hz}, 1 \mathrm{H}), 5.68-5.34(\mathrm{~m}, 2.9 \mathrm{H}), 3.09-2.98$ (m, $3 \mathrm{H}), 2.42(\mathrm{ddt}, J=9.6,4.5,2.2 \mathrm{~Hz}, 6 \mathrm{H})$, $2.34(\mathrm{t}, J=7.4 \mathrm{~Hz}, 1 \mathrm{H}), 2.28-2.15(\mathrm{~m}, 2 \mathrm{H}), 2.12(\mathrm{~d}, J=1.2 \mathrm{~Hz}, 8 \mathrm{H}), 2.10-1.97(\mathrm{~m}, 3 \mathrm{H})$, 1.74-1.17 (m, $20 \mathrm{H}) ;{ }^{13} \mathbf{C}\left\{{ }^{\mathbf{1}} \mathbf{H}\right\}$ NMR $\left(125 \mathrm{MHz}, \mathrm{CDCl}_{3}\right): \delta[\mathrm{ppm}]=209.3,209.1,179.4,177.8$, 171.6, 152.0, 134.7, 121.4, 120.8, 43.7, 43.7, 43.6, 37.9, 34.0, 32.3, 32.2, 30.1, 30.0, 28.8, 28.7, 28.7, 27.9, 24.6, 23.6, 23.5, 23.5; HR-ESI-MS m/z: calcd. $\mathrm{C}_{10} \mathrm{H}_{16} \mathrm{O}_{3} \mathrm{Na}[\mathrm{M}+\mathrm{Na}]^{+}: 207.0992$, found: 207.0994 . 


\section{$(E / Z)-13-0 x o t r i d e c-3-e n o i c$ acid (34f)}

To a degassed solution of 4-butenoic acid ( $0.26 \mathrm{~g}, 3.0 \mathrm{mmol}, 1.0$ equiv)<smiles>O=CC=CCC(=O)O</smiles>
and 10-undecenyl aldehyde (1.5 g, $9.0 \mathrm{mmol}, 3.0$ equiv) in DCM $(6.0 \mathrm{~mL})$, HoveYDA-GRuBBS catalyst 2nd gen. (94 mg, $0.15 \mathrm{mmol}, 0.05$ equiv) was added. The solution was heated to $40{ }^{\circ} \mathrm{C}$ for $13.5 \mathrm{~h}$. Removal of the solvent in vacuo, followed by column chromatography ( $n$-pentane/EtOAc, 3:1) afforded the product as a brown liquid (389 mg, $1.72 \mathrm{mmol}, 57 \%$ ).

$\mathbf{R}_{\mathbf{f}}=0.21$ (n-pentane:EtOAc, 3:1); IR (ATR): $\tilde{\nu}\left[\mathrm{cm}^{-1}\right]=2925,2854,1708,1463,1410,1290$, 1220, 1167, 968, 935; ${ }^{\mathbf{1}} \mathbf{H} \mathbf{N M R}\left(400 \mathrm{MHz}, \mathrm{CDCl}_{3}\right): \delta[\mathrm{ppm}]=9.76(\mathrm{t}, J=1.9 \mathrm{~Hz}, 1 \mathrm{H})$, $5.70-5.43(\mathrm{~m}, 2 \mathrm{H}), 3.23-2.96(\mathrm{~m}, 2 \mathrm{H}), 2.42(\mathrm{td}, J=7.4,1.9 \mathrm{~Hz}, 2 \mathrm{H}), 2.03(\mathrm{q}, J=6.6 \mathrm{~Hz}$, $2 \mathrm{H}), 1.62$ (quint, $J=7.2 \mathrm{~Hz}, 2 \mathrm{H}), 1.45-1.18(\mathrm{~m}, 11 \mathrm{H}) ;{ }^{\mathbf{1 3}} \mathbf{C}\left\{{ }^{\mathbf{1}} \mathbf{H}\right\} \mathbf{N M R}\left(100 \mathrm{MHz}, \mathrm{CDCl}_{3}\right): \delta$ $[\mathrm{ppm}]=203.2,178.2,135.6,120.9,44.0,37.9,32.5,29.4,29.3,29.3,29.2,29.1,22.2$; HR-ESI-MS m/z: calcd. $\mathrm{C}_{13} \mathrm{H}_{2103}[\mathrm{M}-\mathrm{H}]^{-}$: 225.1496, found: 225.1496 .

\section{(Z)-Hexadec-4-enoic acid (34g)}

Following general procedure $\mathbf{A}$ (3-carboxypropyl)triphenylphosphonium<smiles>CCCCCC(=O)O</smiles>
bromide (2.6 g, $6.0 \mathrm{mmol}, 2.0$ equiv) in THF $(9 \mathrm{~mL})$, potassium tertbutoxide (1.4 g, $12.0 \mathrm{mmol}, 4.0$ equiv), dodecanal $(0.55 \mathrm{~g}, 3.0 \mathrm{mmol}$, 1.0 equiv) in THF $(1 \mathrm{~mL})$; reaction time $2 \mathrm{~h}$; eluting with $n$-pentane $/ \mathrm{Et}_{2} \mathrm{O}$, $3: 1$; white solid; in vacuo, followed by column chromatography ( $n$-pentane/ $\operatorname{Et}_{2} \mathrm{O}, 3: 1$ ) afforded the product as a white solid (282 $\mathrm{mg}, 1.11 \mathrm{mmol}, 37 \%)$.

$\mathbf{R}_{\mathbf{f}}=0.50$ (n-pentane:Et $\left.{ }_{2} \mathrm{O}, 3: 1\right)$; IR (ATR): $\tilde{\nu}\left[\mathrm{cm}^{-1}\right]=2953,2915,2849,1710,1469,1432,1299$, 1215, 890, 718, 678; ${ }^{\mathbf{1}} \mathbf{H}$ NMR $\left(500 \mathrm{MHz}, \mathrm{CDCl}_{3}\right): \delta[\mathrm{ppm}]=11.48(\mathrm{~s}, 1 \mathrm{H}), 5.55-5.21(\mathrm{~m}, 2 \mathrm{H})$, 2.49-2.33 (m, $4 \mathrm{H}), 2.04$ (qd, $J=7.2,1.5 \mathrm{~Hz}, 2 \mathrm{H}), 1.44-1.16(\mathrm{~m}, 18 \mathrm{H}), 0.88(\mathrm{t}, J=6.9 \mathrm{~Hz}$, $3 \mathrm{H}) ;{ }^{13} \mathbf{C}\left\{{ }^{1} \mathbf{H}\right\}$ NMR $\left(125 \mathrm{MHz}, \mathrm{CDCl}_{3}\right): \delta[\mathrm{ppm}]=179.5,132.1,127.0,34.3,32.1,29.8,29.8$, 29.8, 29.8, 29.7, 29.5, 29.5, 27.4, 22.9, 22.7, 14.3; HR-ESI-MS m/z: calcd. $\mathrm{C}_{16} \mathrm{H}_{29} \mathrm{O}_{2}[\mathrm{M}-\mathrm{H}]^{-}$: 253.2173, found: 253.2173 .

\section{(Z)-7-Methyloct-5-enoic acid (137I)}<smiles>CC(C)/C=C\CCCC(=O)O</smiles>
1371

Following general procedure $\mathbf{A}$ (4-carboxybutyl)triphenylphosphonium bromide (1.8 g, $4.0 \mathrm{mmol}, 2.0$ equiv) in THF $(7 \mathrm{~mL})$, potassium tertbutoxide ( $0.90 \mathrm{~g}, 8.0 \mathrm{mmol}, 4.0$ equiv), isobutyraldehyde $(0.14 \mathrm{~g}, 2.0 \mathrm{mmol}$, 1.0 equiv) in THF $(1 \mathrm{~mL})$; reaction time $2 \mathrm{~h}$; eluting with $n$-pentane $/ \mathrm{Et}_{2} \mathrm{O}$, 2:1; colorless oil; $221 \mathrm{mg}, 1.41 \mathrm{mmol}, 71 \%$.

$\mathbf{R}_{\mathbf{f}}=0.50$ (n-pentane:Et $\left.{ }_{2} \mathrm{O}, 1: 1\right) ; \mathbf{I R}(\mathrm{ATR}): \tilde{\nu}\left[\mathrm{cm}^{-1}\right]=3001,2956,2869,1705,1459,1412,1361$, 1297, 1239, 1205, 1165, 1102, 927, 740, 486; ${ }^{\mathbf{1}} \mathbf{H}$ NMR $\left(600 \mathrm{MHz}, \mathrm{CDCl}_{3}\right): \delta[\mathrm{ppm}]=5.32-5.11$ 
(m, $2 \mathrm{H}$ ), 2.57 (dhept, $J=9.1,6.6 \mathrm{~Hz}, 1 \mathrm{H}$ ), 2.37 (t, $J=7.5 \mathrm{~Hz}, 2 \mathrm{H}$ ), 2.11 (dddd, $J=8.4,7.5$, $6.7,0.9 \mathrm{~Hz}, 2 \mathrm{H}), 1.70$ (quintd, $J=7.3,0.7 \mathrm{~Hz}, 2 \mathrm{H}), 0.94(\mathrm{~d}, J=6.6 \mathrm{~Hz}, 6 \mathrm{H}) ;{ }^{\mathbf{1}} \mathbf{C}\left\{{ }^{1} \mathbf{H}\right\} \mathbf{N M R}$ $\left(125 \mathrm{MHz}, \mathrm{CDCl}_{3}\right): \delta[\mathrm{ppm}]=179.9,138.9,125.8,33.6,26.8,26.7,25.0,23.4 ;$ HR-ESI-MS m/z: calcd. $\mathrm{C}_{9} \mathrm{H}_{15} \mathrm{O}_{2}[\mathrm{M}-\mathrm{H}]^{-}$: 155.1078 , found: 155.1083 .

\section{(Z)-8-(Naphthalen-1-yl)oct-5-enoic acid (137m)}

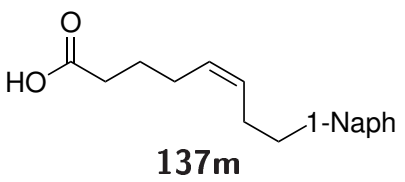

Following general procedure A (4-carboxybutyl)triphenylphosphonium bromide (1.8 g, $4.0 \mathrm{mmol}, 2.0$ equiv) in THF $(7 \mathrm{~mL})$, potassium tert-butoxide ( $0.90 \mathrm{~g}, 8.0 \mathrm{mmol}, 4.0$ equiv), 3-(naphthalen1-yl)propanal (0.37 g, $2.0 \mathrm{mmol}, 1.0$ equiv) in THF (1 mL); reaction time $2 \mathrm{~h}$; eluting with $n$-pentane/ $\mathrm{Et}_{2} \mathrm{O}, 4: 1$; yellow oil; $249 \mathrm{mg}$, 930 umol, $47 \%$.

$\mathbf{R}_{\mathbf{f}}=0.07$ (n-pentane:Et $\left.{ }_{2} \mathrm{O}, 4: 1\right)$; IR (ATR): $\tilde{\nu}\left[\mathrm{cm}^{-1}\right]=3043,3006,2921,2850,1703,1596,1510$, 1457, 1434, 1410, 1239, 1205, 937, 859, 796, 774, 732, 481, 423; ${ }^{\mathbf{1}} \mathbf{H} \mathbf{~ N M R}\left(400 \mathrm{MHz}, \mathrm{CDCl}_{3}\right): \delta$ $[\mathrm{ppm}]=8.05(\mathrm{~m}, 1 \mathrm{H}), 7.85(\mathrm{~m}, 1 \mathrm{H}), 7.72(\mathrm{~d}, J=8.2 \mathrm{~Hz}, 1 \mathrm{H}), 7.52(\mathrm{ddd}, J=8.5,6.7,1.7 \mathrm{~Hz}$, $2 \mathrm{H}), 7.48(\mathrm{~m}, 1 \mathrm{H}), 7.40(\mathrm{dd}, J=8.2,7.0 \mathrm{~Hz}, 1 \mathrm{H}), 7.33(\mathrm{~m}, 1 \mathrm{H}), 5.57(\mathrm{~m}, 1 \mathrm{H}), 5.38(\mathrm{~m}$, $1 \mathrm{H}), 3.16-3.10(\mathrm{~m}, 2 \mathrm{H}), 2.67-2.44(\mathrm{~m}, 2 \mathrm{H}), 2.22(\mathrm{t}, J=7.5 \mathrm{~Hz}, 2 \mathrm{H}), 2.04-1.90(\mathrm{~m}, 2 \mathrm{H}), 1.58$ (quint, $J=7.5 \mathrm{~Hz}, 2 \mathrm{H}) ;{ }^{13} \mathbf{C}\left\{{ }^{\mathbf{1}} \mathbf{H}\right\} \mathbf{N M R}\left(100 \mathrm{MHz}, \mathrm{CDCl}_{3}\right): \delta[\mathrm{ppm}]=179.8,138.0,134.0$, 132.0, 130.2, 129.3, 128.9, 126.8, 126.2, 125.9, 125.6, 125.5, 123.8, 33.4, 33.1, 28.6, 26.6, 24.6; HR-ESI-MS m/z: calcd. $\mathrm{C}_{18} \mathrm{H}_{19} \mathrm{O}_{2}[\mathrm{M}-\mathrm{H}]^{-}$: 267.1391, found: 267.1391.

\section{(Z)-8-(4-Cyanophenyl)oct-5-enoic acid (137q)}

Following general procedure [A] (4-carboxybutyl)triphenylphos-<smiles>N#Cc1ccc(CC/C=C\CCCC(=O)O)cc1</smilesphonium bromide (1.5 g, $3.4 \mathrm{mmol}, 2.0$ equiv) in THF $(6 \mathrm{~mL})$, potassium tert-butoxide ( $0.76 \mathrm{mg}, 6.8 \mathrm{mmol}, 4.0$ equiv), 3-(4cyanophenyl)propanal $(0.27 \mathrm{~g}, 1.7 \mathrm{mmol}, 1.0$ equiv) in THF $(1 \mathrm{~mL})$; reaction time $2 \mathrm{~h}$; eluting with $n$-pentane $/ \mathrm{Et}_{2} \mathrm{O}, 1: 1$; yellow oil; $210 \mathrm{mg}, 863$ umol, $51 \%$.

$\mathbf{R}_{\mathbf{f}}=0.21$ (n-pentane $\left./ \mathrm{Et}_{2} \mathrm{O}, 1: 1\right)$; IR (ATR): $\tilde{\nu}\left[\mathrm{cm}^{-1}\right]=3007,2932,2861,2227,1703,1607$, 1505, 1412, 1239, 1177, 936, 841, 823, 554; ${ }^{\mathbf{1}} \mathbf{H}$ NMR $\left(400 \mathrm{MHz}, \mathrm{CDCl}_{3}\right): \delta[\mathrm{ppm}]=7.66-7.52$ $(\mathrm{m}, 2 \mathrm{H}), 7.31-7.26(\mathrm{~m}, 2 \mathrm{H}), 5.50-5.29(\mathrm{~m}, 2 \mathrm{H}), 2.72(\mathrm{dd}, J=8.2,7.0 \mathrm{~Hz}, 2 \mathrm{H}), 2.39-2.32$ $(\mathrm{m}, 2 \mathrm{H}), 2.29(\mathrm{t}, J=7.4 \mathrm{~Hz}, 2 \mathrm{H}), 2.07-1.96(\mathrm{~m}, 2 \mathrm{H}), 1.65-1.56(\mathrm{~m}, 2 \mathrm{H}) ;{ }^{13} \mathbf{C}\left\{{ }^{1} \mathbf{H}\right\} \mathbf{N M R}$ $\left(100 \mathrm{MHz}, \mathrm{CDCl}_{3}\right): \delta[\mathrm{ppm}]=179.6,147.7,132.3,130.0,129.5,129.1,119.2,109.9,36.1,33.3$, 28.7, 26.5, 24.5; HR-ESI-MS m/z: calcd. $\mathrm{C}_{15} \mathrm{H}_{17} \mathrm{NO}_{2}[\mathrm{M}-\mathrm{H}]^{-}: 242.1187$, found: 242.1185 . 


\section{(Z)-8-(4-Methoxyphenyl)oct-5-enoic acid (137r)}

Following general procedure $\mathbf{A}$. (4-carboxybutyl)triphenylphos-<smiles>COc1ccc(CC/C=C\CCCC(=O)O)cc1</smiles>

$137 r$ phonium bromide ( $2.2 \mathrm{~g}, 5.0 \mathrm{mmol}, 2.0$ equiv) in THF $(6 \mathrm{~mL})$, potassium tert-butoxide (1.12 g, $10.0 \mathrm{mmol}, 4.0$ equiv), 3-(4methoxyphenyl)propanal ( $0.41 \mathrm{~g}, 2.5 \mathrm{mmol}, 1.0$ equiv) in THF $(1 \mathrm{~mL})$; reaction time $2 \mathrm{~h}$; eluting with $n$-pentane $/ \mathrm{Et}_{2} \mathrm{O}, 0: 1$; yellow oil; $468 \mathrm{mg}, 1.88 \mathrm{mmol}, 75 \%$.

$\mathbf{R}_{\mathbf{f}}=0.56\left(\mathrm{Et}_{2} \mathrm{O}\right) ; \mathbf{I R}(\mathrm{ATR}): \tilde{\nu}\left[\mathrm{cm}^{-1}\right]=3005,2932,2835,1704,1611,1584,1510,1440,1299$, 1241, 1176, 1109, 1036, 938, 823, 694, 516; ${ }^{\mathbf{1}} \mathbf{H} \mathbf{~ N M R}\left(400 \mathrm{MHz}, \mathrm{CDCl}_{3}\right): \delta[\mathrm{ppm}]=7.17-7.04$ $(\mathrm{m}, 2 \mathrm{H}), 6.92-6.77(\mathrm{~m}, 2 \mathrm{H}), 5.45(\mathrm{~m}, 1 \mathrm{H}), 5.35(\mathrm{~m}, 1 \mathrm{H}), 3.78(\mathrm{~s}, 3 \mathrm{H}), 2.60(\mathrm{dd}, J=8.5,6.8 \mathrm{~Hz}$, $2 \mathrm{H}$ ), 2.38-2.18 (m, $4 \mathrm{H}), 2.11-1.93(\mathrm{~m}, 2 \mathrm{H}), 1.63$ (quint, $J=7.5 \mathrm{~Hz}, 2 \mathrm{H}) ;{ }^{\mathbf{1 3}} \mathbf{C}\left\{{ }^{1} \mathbf{H}\right\} \mathbf{N M R}$ $\left(100 \mathrm{MHz}, \mathrm{CDCl}_{3}\right): \delta[\mathrm{ppm}]=179.6,157.9,134.2,130.3,129.5,129.1,113.8,55.4,35.1,33.4$, 29.6, 26.6, 24.6; HR-ESI-MS m/z: calcd. $\mathrm{C}_{15} \mathrm{H}_{20} \mathrm{O}_{3} \mathrm{Na}[\mathrm{M}+\mathrm{Na}]^{+}:$271.1305, found: 271.1308.

\section{6-Cyclopropylhex-5-enoic acid (137s)}

Following general procedure $\mathbf{A}$ (4-carboxybutyl)triphenylphosphonium bro-<smiles>O=C(O)CCC/C=C\C1CC1</smilesmide ( $1.8 \mathrm{~g}, 4.0 \mathrm{mmol}, 2.0$ equiv) in THF $(7 \mathrm{~mL})$, potassium tert-butoxide (0.90 g, 8.0 mmol, 4.0 equiv), cyclopropanecarboxaldehyde ( $0.14 \mathrm{~g}, 2.0 \mathrm{mmol}$, 1.0 equiv) in THF $(1 \mathrm{~mL})$; reaction time $2 \mathrm{~h}$; eluting with $n$-pentane $/ \mathrm{Et}_{2} \mathrm{O}$, 1:1; colorless oil; mixture of $Z / E 7: 1 ; 169 \mathrm{mg}, 1.09 \mathrm{mmol}, 55 \%$.

$\mathbf{R}_{\mathbf{f}}=0.28$ (n-pentane:Et $\left.{ }_{2} \mathrm{O}, 1: 1\right)$; IR (ATR): $\tilde{\nu}\left[\mathrm{cm}^{-1}\right]=3082,2005,2934,2868,1703,1412$, 1240, 1205, 1046, 1020, 933, 5, 810, 731, 601, 489; ${ }^{\mathbf{1}} \mathbf{H}$ NMR $\left(300 \mathrm{MHz}, \mathrm{CDCl}_{3}\right): \delta[\mathrm{ppm}](Z$ isomer $)=5.26(\mathrm{dtd}, J=10.7,7.4,0.9 \mathrm{~Hz}, 1 \mathrm{H}), 4.79(\mathrm{ddt}, J=11.1,9.9,1.5 \mathrm{~Hz}, 1 \mathrm{H}), 2.40$ (t, $J=7.5 \mathrm{~Hz}, 2 \mathrm{H}$ ), 2.23 (qd, $J=7.4,1.5 \mathrm{~Hz}, 2 \mathrm{H}$ ), 1.75 (quint, $J=7.7 \mathrm{~Hz}, 2 \mathrm{H}$ ), 1.51 (m, $1 \mathrm{H}), 0.78-0.68(\mathrm{~m}, 2 \mathrm{H}), 0.38-0.27(\mathrm{~m}, 2 \mathrm{H}) ; \delta[\mathrm{ppm}](E$ isomer $)=5.45(\mathrm{dt}, J=15.3,6.9 \mathrm{~Hz}$, $1 \mathrm{H}), 4.99$ (ddt, $J=15.2,8.5,1.4 \mathrm{~Hz}, 1 \mathrm{H}), 2.37-2.31(\mathrm{~m}, 2 \mathrm{H}), 2.12-1.97(\mathrm{~m}, 2 \mathrm{H}), 1.71$ (d, $J=7.5 \mathrm{~Hz}, 2 \mathrm{H}), 1.42-1.28(\mathrm{~m}, 1 \mathrm{H}), 0.69-0.59(\mathrm{~m}, 2 \mathrm{H}), 0.38-0.27(\mathrm{~m}, 2 \mathrm{H}) ;{ }^{13} \mathbf{C}\left\{{ }^{1} \mathbf{H}\right\} \mathbf{~ N M R}$ $\left(125 \mathrm{MHz}, \mathrm{CDCl}_{3}\right): \delta[\mathrm{ppm}]=180.3,135.3,135.2,126.6,33.6,33.6,31.9,27.0,24.9,24.7,13.7$, 9.8, 7.1, 6.6; HR-ESI-MS m/z: calcd. $\mathrm{C}_{9} \mathrm{H}_{14} \mathrm{O}_{2} \mathrm{Na}[\mathrm{M}+\mathrm{Na}]^{+}:$177.0886, found: 177.0878 .

\section{(Z)-8-(Benzyloxy)oct-5-enoic acid (137t)}

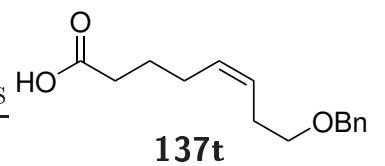

Following general procedure $\mathbf{A}$; (4-carboxybutyl)triphenylphosphonium bromide (1.8 g, $4.0 \mathrm{mmol}, 2.0$ equiv) in THF $(7 \mathrm{~mL})$, potassium tertbutoxide (0.90 g, $8.0 \mathrm{mmol}, 4.0$ equiv), 3-benzyloxypropionaldehyde

$(0.33 \mathrm{~g}, 2.0 \mathrm{mmol}, 1.0$ equiv) in THF $(1 \mathrm{~mL})$; reaction time $2 \mathrm{~h}$; eluting with $n$-pentane $/ \mathrm{Et}_{2} \mathrm{O}, 1: 1$; colorless oil; $60 \mathrm{mg}, 0.24 \mathrm{mmol}, 12 \%$. 
$\mathbf{R}_{\mathbf{f}}=0.36$ (n-pentane:Et $\left.{ }_{2} \mathrm{O}, 1: 1\right) ; \mathbf{I R}(\mathrm{ATR}): \tilde{\nu}\left[\mathrm{cm}^{-1}\right]=3087,3063,3030,3010,2931,2865,1705$, 1496, 1454, 1410, 1238, 1206, 1095, 1001, 904, 734, 697, 652, 595, 464; ${ }^{\mathbf{1}} \mathbf{H}$ NMR $(300 \mathrm{MHz}$, $\left.\mathrm{CDCl}_{3}\right): \delta[\mathrm{ppm}]=7.58-7.23(\mathrm{~m}, 10 \mathrm{H}), 6.62(\mathrm{dddd}, J=16.8,11.2,10.2,1.1 \mathrm{~Hz}, 1 \mathrm{H}), 6.06$ (dddd, $J=11.8,10.1,1.5,0.8 \mathrm{~Hz}, 1 \mathrm{H}), 5.52-5.36(\mathrm{~m}, 3 \mathrm{H}), 5.21$ (ddt, $J=17.0,1.6,0.8 \mathrm{~Hz}$, $1 \mathrm{H}), 5.12$ (dddd, $J=10.2,2.2,1.4,0.8 \mathrm{~Hz}, 2 \mathrm{H}), 4.69(\mathrm{~s}, 2 \mathrm{H}), 4.53(\mathrm{~s}, 2 \mathrm{H}), 3.49(\mathrm{t}, J=6.9 \mathrm{~Hz}$, $2 \mathrm{H}), 2.46-2.31$ (m, $8 \mathrm{H}), 2.26(\mathrm{qd}, J=7.5,1.6 \mathrm{~Hz}, 3 \mathrm{H}), 2.19-2.03(\mathrm{~m}, 3 \mathrm{H}), 1.82-1.60(\mathrm{~m}, 6 \mathrm{H})$; ${ }^{{ }^{13}} \mathbf{C}\left\{{ }^{1} \mathbf{H}\right\} \mathbf{N M R}\left(125 \mathrm{MHz}, \mathrm{CDCl}_{3}\right): \delta[\mathrm{ppm}]=179.4,179.3,140.7,138.4,137.0,133.6,132.0$, 131.1, 130.4, 130.4, 128.6, 128.4, 127.7, 127.7, 127.6, 127.1, 126.9, 117.6, 115.4, 73.0, 70.0, 65.4, 33.6, 33.5, 31.9, 28.1, 27.1, 26.7, 24.7, 24.5, 24.3; HR-ESI-MS m/z: calcd. $\mathrm{C}_{15} \mathrm{H}_{19} \mathrm{O}_{3}[\mathrm{M}-\mathrm{H}]^{-}$: 247.1340, found: 247.1342 .

\section{(Z)-Octadec-6-enoic acid (167)}

Following general procedure $\mathbf{A}$. (4-carboxybutyl)triphenylphos-

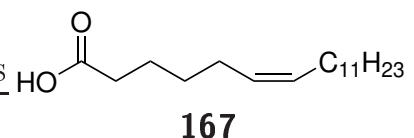
phonium bromide ( $2.3 \mathrm{~g}, 5.0 \mathrm{mmol}, 2.0$ equiv) in THF ( $6 \mathrm{~mL})$, potassium tert-butoxide (1.12 g, $10.0 \mathrm{mmol}, 4.0$ equiv), dodecanal ( $0.46 \mathrm{~g}$, $2.5 \mathrm{mmol}, 1.0$ equiv) in THF $(1 \mathrm{~mL})$; reaction time $2 \mathrm{~h}$; eluting with n-pentane/Et ${ }_{2} \mathrm{O}, 1: 1$; white solid; $582 \mathrm{mg}, 2.06 \mathrm{mmol}, 82 \%$.

$\mathbf{R}_{\mathbf{f}}=0.29$ (n-pentane $\left./ \mathrm{Et}_{2} \mathrm{O}, 1: 1\right) ; \mathbf{m} . \mathbf{p .}=35{ }^{\circ} \mathrm{C}$; IR (ATR): $\tilde{\nu}\left[\mathrm{cm}^{-1}\right]=2922,2853,1708,1461$, 1412, 1288, 1234, 934, 721; ${ }^{\mathbf{1}} \mathbf{H}$ NMR $\left(400 \mathrm{MHz}, \mathrm{CDCl}_{3}\right): \delta[\mathrm{ppm}]=5.45-5.26(\mathrm{~m}, 2 \mathrm{H})$, 2.42-2.28 (m, $2 \mathrm{H}), 2.20-1.90(\mathrm{~m}, 4 \mathrm{H}), 1.73-1.56(\mathrm{~m}, 2 \mathrm{H}), 1.49-1.35(\mathrm{~m}, 2 \mathrm{H}), 1.26$ ( $\mathrm{s}_{\mathrm{br}}$, $18 \mathrm{H}), 0.93-0.71(\mathrm{~m}, 3 \mathrm{H}) ;{ }^{\mathbf{1 3}} \mathbf{C}\left\{{ }^{\mathbf{1}} \mathbf{H}\right\} \mathbf{N M R}\left(100 \mathrm{MHz}, \mathrm{CDCl}_{3}\right): \delta[\mathrm{ppm}]=179.8,130.7,129.1$, 34.0, 32.1, 29.9, 29.8, 29.8, 29.7, 29.5, 29.5, 29.3, 27.4, 26.9, 24.4, 22.9, 14.3; HR-ESI-MS m/z: calcd. $\mathrm{C}_{18} \mathrm{H}_{33} \mathrm{O}_{2}[\mathrm{M}-\mathrm{H}]^{-}:$281.2486, found: 281.2487 .

\subsubsection{Synthesis of lactones 77,170 and 171}

\section{General procedure G: catalytic lactonization}

The respective acid 34 or 137 (1.0 mmol, 1.0 equiv), diphenyl diselenide (16 mg, 50 pmol, 0.05 equiv) and $p$-MeO-TPT (95) (24 mg, 50 umol, 0.05 equiv) are dissolved in acetonitrile $(10 \mathrm{~mL})$ and stirred vigorously under air and irradiation at $\lambda=465 \mathrm{~nm}$ at room temperature until complete conversion. The solvent is removed in vacuo and the product $\mathbf{7 7}$ or $\mathbf{1 7 0}$ afforded via column chromatography.

\section{5-(4-Methoxybenzyl)furan-2(5H)-one (77c)}

Following general procedure G; acid 34c, 23 h; yellow oil (106 mg,<smiles></smiles>

$77 \mathrm{c}$

$0.519 \mathrm{mmol}, 52 \%) ;$-pentane $/ \mathrm{Et}_{2} \mathrm{O}, 2: 1$.

$\mathbf{R}_{\mathbf{f}}=0.19$ (n-pentane:Et $\left.{ }_{2} \mathrm{O}, 1: 1\right) ; \mathbf{I R}(\mathrm{ATR}): \tilde{\nu}\left[\mathrm{cm}^{-1}\right]=2916,2837,1743$, 1612, 1584, 1511, 1464, 1337, 1300, 1244, 1178, 1159, 1097, 1071, 1025, 
981, 916, 902, 857, 843, 811, 782, 748, 695, 668, 615, 557, 532; ${ }^{\mathbf{1}} \mathbf{H}$ NMR (300 $\left.\mathrm{MHz}, \mathrm{CDCl}_{3}\right)$ : $\delta[\mathrm{ppm}]=7.39(\mathrm{dd}, J=5.7,1.5 \mathrm{~Hz}, 1 \mathrm{H}), 7.18-7.05(\mathrm{~m}, 2 \mathrm{H}), 6.91-6.75(\mathrm{~m}, 2 \mathrm{H}), 6.06$ (ddt, $J=5.7,2.0,0.4 \mathrm{~Hz}, 1 \mathrm{H}$ ), 5.19 (dddd, $J=7.0,6.2,2.0,1.5 \mathrm{~Hz}, 1 \mathrm{H}), 3.79$ (s, $3 \mathrm{H}), 3.10$ (dd, $J=14.0,6.2 \mathrm{~Hz}, 1 \mathrm{H}), 2.91(\mathrm{dd}, J=14.0,7.0 \mathrm{~Hz}, 1 \mathrm{H}) ;{ }^{13} \mathbf{C}\left\{{ }^{1} \mathbf{H}\right\} \mathbf{N M R}\left(125 \mathrm{MHz}, \mathrm{CDCl}_{3}\right)$ : $\delta[\mathrm{ppm}]=172.8,158.8,155.6,130.5,126.8,122.1,114.2,83.7,55.4,38.9 ;$ HR-ESI-MS m/z: calcd. $\mathrm{C}_{12} \mathrm{H}_{13} \mathrm{O}_{3}[\mathrm{M}+\mathrm{H}]^{+}:$205.0859, found: 205.0861 .

\section{5-(6-Oxoheptyl)furan-2(5H)-one (77d)}

Following general procedure $\mathbf{G}$ Acid 34e (35 mg, $0.19 \mathrm{mmol}, 1.0$ equiv), diphenyl<smiles>CC(=O)C(C)(C)C1C=CC(=O)O1</smiles>

$77 d$ diselenide (3.0 mg, 9.5 umol, 0.05 equiv) and $p$-MeO-TPT (4.6 mg, $9.5 \mu \mathrm{mol}$, 0.05 equiv), acetonitrile ( $2 \mathrm{~mL}) 19 \mathrm{~h}$; yellow oil (15 mg, $81 \mu \mathrm{mol}, 43 \%$ ); $n$ pentane $/ \mathrm{Et}_{2} \mathrm{O}, 0: 1$.

$\mathbf{R}_{\mathbf{f}}=0.21\left(\mathrm{Et}_{2} \mathrm{O}\right) ; \mathbf{I R}(\mathrm{ATR}): \tilde{\nu}\left[\mathrm{cm}^{-1}\right]=2930,2866,1745,1709,1358,1163,1102,1012,900$, $818 ;{ }^{1} \mathbf{H}$ NMR $\left(300 \mathrm{MHz}, \mathrm{CDCl}_{3}\right): \delta[\mathrm{ppm}]=7.44(\mathrm{dd}, J=5.7,1.5 \mathrm{~Hz}, 1 \mathrm{H}), 6.09(\mathrm{dd}, J=5.7$, $2.0 \mathrm{~Hz}, 1 \mathrm{H}), 5.03(\mathrm{ddt}, J=7.6,5.1,1.8 \mathrm{~Hz}, 1 \mathrm{H}), 2.44(\mathrm{t}, J=7.1 \mathrm{~Hz}, 2 \mathrm{H}), 2.12(\mathrm{~s}, 3 \mathrm{H})$, 1.86-1.36 (m, $6 \mathrm{H}) ;{ }^{13} \mathbf{C}\left\{{ }^{1} \mathbf{H}\right\}$ NMR $\left(75 \mathrm{MHz}, \mathrm{CDCl}_{3}\right): \delta[\mathrm{ppm}]=208.6,173.2,156.2,121.8$, 83.2, 43.3, 33.1, 30.1, 24.6, 23.3; HR-ESI-MS m/z: calcd. $\mathrm{C}_{10} \mathrm{H}_{15} \mathrm{O}_{3}[\mathrm{M}+\mathrm{H}]^{+}:$183.1016, found: 183.1019 .

\section{9-(5-Oxo-2,5-dihydrofuran-2-yl)nonanal (77e)}

Following general procedure $\mathbf{G}$ acid 34f, 48 h; yellow oil (78 mg, $0.35 \mathrm{mmol}, 35 \%$ );<smiles>O=CC1CCC(C=O)O1</smiles>
$n$-pentane $/ \mathrm{Et}_{2} \mathrm{O}, 3: 1$.

$77 \mathrm{e}$

$\mathbf{R}_{\mathbf{f}}=0.17$ (n-pentane:EtOAc, 3:1); IR (ATR): $\tilde{\nu}\left[\mathrm{cm}^{-1}\right]=2927,2855,1749,1720$, 1602, 1464, 1162, 1100, 1011, 898, 816, 706, 669, 511; ${ }^{\mathbf{1}} \mathbf{H}$ NMR $(400 \mathrm{MHz}$, $\left.\mathrm{CDCl}_{3}\right): \delta[\mathrm{ppm}]=9.76(\mathrm{t}, J=1.8 \mathrm{~Hz}, 1 \mathrm{H}), 7.44(\mathrm{dd}, J=5.7,1.5 \mathrm{~Hz}, 1 \mathrm{H}), 6.10(\mathrm{dd}, J=5.7$, $2.0 \mathrm{~Hz}, 1 \mathrm{H}), 5.03(\mathrm{ddt}, J=7.2,5.3,1.8 \mathrm{~Hz}, 1 \mathrm{H}), 2.42(\mathrm{td}, J=7.3,1.8 \mathrm{~Hz}, 2 \mathrm{H}), 1.84-1.17$ $(\mathrm{m}, 14 \mathrm{H}) ;{ }^{13} \mathbf{C}\left\{{ }^{1} \mathbf{H}\right\} \mathbf{N M R}\left(125 \mathrm{MHz}, \mathrm{CDCl}_{3}\right): \delta[\mathrm{ppm}]=202.7,173.1,156.2,121.7,83.5,44.1$, 33.4, 29.4, 29.4, 29.3, 29.3, 25.2, 22.2; HR-ESI-MS m/z: calcd. $\mathrm{C}_{13} \mathrm{H}_{21} \mathrm{O}_{3}[\mathrm{M}+\mathrm{H}]^{+}: 225.1485$, found: 225.1492 .

\section{(E)-5-(Dodec-1-en-1-yl)dihydrofuran-2(3H)-one (168)}

Following general procedure $\mathbf{G}$ acid 166; 16 h; white solid (161 mg, 637 umol,

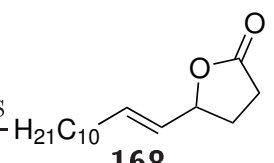

168 $64 \%) ; n$-pentane $/ \mathrm{Et}_{2} \mathrm{O} 3: 1$.

$\mathbf{R}_{\mathbf{f}}=0.43$ (n-pentane:Et $\left.{ }_{2} \mathrm{O}, 3: 1\right)$; IR (ATR): $\tilde{\nu}\left[\mathrm{cm}^{-1}\right]=2923,2853,1773$, $1459,1326,1173,968,914,756 ;{ }^{\mathbf{1}} \mathbf{H} \mathbf{N M R}\left(500 \mathrm{MHz}, \mathrm{CDCl}_{3}\right): \delta[\mathrm{ppm}]=5.80$

$(\mathrm{dtd}, J=15.3,6.6,1.0 \mathrm{~Hz}, 1 \mathrm{H}), 5.48(\mathrm{ddt}, J=15.3,7.1,1.5 \mathrm{~Hz}, 1 \mathrm{H}), 4.89(\mathrm{~m}, 1 \mathrm{H}), 2.54$ 
$(\mathrm{dd}, J=9.2,1.1 \mathrm{~Hz}, 1 \mathrm{H}), 2.52(\mathrm{dd}, J=9.2,2.2 \mathrm{~Hz}, 1 \mathrm{H}), 2.36(\mathrm{ddt}, J=13.0,7.5,6.4 \mathrm{~Hz}$, $1 \mathrm{H}), 2.18-1.88(\mathrm{~m}, 3 \mathrm{H}), 1.49-1.15(\mathrm{~m}, 16 \mathrm{H}), 0.91-0.84(\mathrm{~m}, 3 \mathrm{H}) ;{ }^{\mathbf{1 3}} \mathbf{C}\left\{{ }^{\mathbf{1}} \mathbf{H}\right\}$ NMR $(125 \mathrm{MHz}$, $\left.\mathrm{CDCl}_{3}\right): \delta[\mathrm{ppm}]=177.0,135.8,127.4,81.3,32.3,32.1,29.8,29.8,29.7,29.5,29.3,29.1,29.0$, 29.0, 22.9, 14.3; HR-ESI-MS m/z: calcd. $\mathrm{C}_{16} \mathrm{H}_{29} \mathrm{O}_{2}[\mathrm{M}+\mathrm{H}]^{+}:$: 253.2162, found: 253.2163.

\section{6-((S,E)-3,7-Dimethylocta-1,6-dien-1-yl)tetrahydro-2 $H$-pyran-2-one (170b)}<smiles>CC(C)=CCC(C)C=CC1CCCC(=O)O1</smiles>

$170 \mathrm{~b}$

Following general procedure $\mathbf{G}$; acid $\mathbf{1 3 7 b}$; $40 \mathrm{~h}$; orange oil (29 mg, $0.12 \mathrm{mmol}, 12 \%) ; n$-pentane/EtOAc 5:1.

$\mathbf{R}_{\mathbf{f}}=0.33$ ( $n$-pentane:EtOAc, 5:1); IR (ATR): $\tilde{\nu}\left[\mathrm{cm}^{-1}\right]=2959,2913$, 1872, 1733, 1441, 1376, 1341, 1233, 1161, 1034, 971, 928, 741, 465; ${ }^{1} \mathbf{H}$ NMR $\left(300 \mathrm{MHz}, \mathrm{CDCl}_{3}\right): \delta[\mathrm{ppm}]=5.62(\mathrm{dddd}, J=15.5,7.7,5.1,1.0 \mathrm{~Hz}, 1 \mathrm{H}), 5.43(\mathrm{~m}$, $1 \mathrm{H}$ ), 5.05 (tdquint, $J=7.2,2.9,1.4 \mathrm{~Hz}, 1 \mathrm{H}), 4.75(\mathrm{~m}, 1 \mathrm{H}), 2.74-2.27(\mathrm{~m}, 2 \mathrm{H}), 2.12$ (ddt, $J=13.7,9.7,6.8 \mathrm{~Hz}, 1 \mathrm{H}), 2.02-1.74(\mathrm{~m}, 6 \mathrm{H}), 1.65(\mathrm{~s}, 3 \mathrm{H}), 1.56(\mathrm{~m}, 3 \mathrm{H}), 1.39-1.19(\mathrm{~m}, 2 \mathrm{H})$, $0.96(\mathrm{dd}, J=6.7,2.0 \mathrm{~Hz}, 3 \mathrm{H}) ;{ }^{\mathbf{1 3}} \mathbf{C}\left\{{ }^{\mathbf{1}} \mathbf{H}\right\} \mathbf{N M R}\left(125 \mathrm{MHz}, \mathrm{CDCl}_{3}\right): \delta[\mathrm{ppm}]=171.4,140.0$, 131.5, 126.5, 124.4, 80.8, 36.9, 36.1, 29.7, 28.7, 25.9, 25.9, 20.4, 18.5, 17.9; HR-ESI-MS m/z: calcd. $\mathrm{C}_{15} \mathrm{H}_{25} \mathrm{O}_{2}[\mathrm{M}+\mathrm{H}]^{+}:$237.1849, found: 237.1853 .

\section{6-(Cyclohexylidenemethyl)tetrahydro-2 $\mathrm{H}$-pyran-2-one (170d)}<smiles>O=C1CCCC(C=C2CCCCC2)O1</smiles>

170d

Following general procedure $\mathbf{G}$ acid 137d; $16 \mathrm{~h}$; light yellow oil $(56 \mathrm{mg}$, $0.29 \mathrm{mmol}, 29 \%) ; n$-pentane/Et ${ }_{2} \mathrm{O}, 2: 1$.

$\mathbf{R}_{\mathbf{f}}=0.21$ (n-pentane:Et $\left.{ }_{2} \mathrm{O}, 1: 1\right)$; IR (ATR): $\tilde{\nu}\left[\mathrm{cm}^{-1}\right]=2926,2853,1727$, 1672, 1446, 1344, 1227, 1182, 1157, 1028, 987, 926, 831, 639, 469; ${ }^{\mathbf{1}} \mathbf{H}$ NMR (500 MHz, $\left.\mathrm{CDCl}_{3}\right)$ : $\delta[\mathrm{ppm}]=5.18(\mathrm{dt}, J=8.6,1.2 \mathrm{~Hz}, 1 \mathrm{H}), 5.07(\mathrm{ddd}, J=10.1,8.6,3.1 \mathrm{~Hz}, 1 \mathrm{H}), 2.58(\mathrm{~m}, 1 \mathrm{H})$, $2.46(\mathrm{~m}, 1 \mathrm{H}), 2.29-2.04(\mathrm{~m}, 4 \mathrm{H}), 1.99-1.78(\mathrm{~m}, 3 \mathrm{H}), 1.76-1.37(\mathrm{~m}, 7 \mathrm{H}) ;{ }^{13} \mathbf{C}\left\{{ }^{1} \mathbf{H}\right\} \mathbf{N M R}$ $\left(125 \mathrm{MHz}, \mathrm{CDCl}_{3}\right): \delta[\mathrm{ppm}]=171.8,145.9,120.0,37.1,29.7,29.6,29.1,28.5,27.9,26.7,18.8$; HR-ESI-MS m/z: calcd. $\mathrm{C}_{12} \mathrm{H}_{19} \mathrm{O}_{2}[\mathrm{M}+\mathrm{H}]^{+}:$195.1380, found: 195.1382 .

\section{(E)-6-(3-Methylbut-1-en-1-yl)tetrahydro-2H-pyran-2-one (170e)}<smiles>O=C1CC[Te]C(/C=C/[N+]([O-])[O-])O1</smiles>

Following general procedure $\mathbf{G}$ acid 137e; 14.5 h; yellow oil (116 mg, 689 umol, 69\%); n-pentane/Et ${ }_{2} \mathrm{O} 1: 1$.

$\mathbf{R}_{\mathbf{f}}=0.29$ (n-pentane:Et $\left.{ }_{2} \mathrm{O}, 1: 1\right) ; \mathbf{I R}(\mathrm{ATR}): \tilde{\nu}\left[\mathrm{cm}^{-1}\right]=2957,2870,1729$, 1464, 1339, 1231, 1160, 1032, 971, 927, 657, 570, 466; ${ }^{\mathbf{1}} \mathbf{H}$ NMR $(500 \mathrm{MHz}$, $\left.\mathrm{CDCl}_{3}\right): \delta[\mathrm{ppm}]=5.73(\mathrm{ddd}, J=15.5,6.6,1.2 \mathrm{~Hz}, 1 \mathrm{H}), 5.43(\mathrm{ddd}, J=15.5,6.6,1.4 \mathrm{~Hz}, 1 \mathrm{H})$, 4.75 (dddt, $J=10.2,6.7,3.5,0.8 \mathrm{~Hz}, 1 \mathrm{H}$ ), 2.57 (dddd, $J=17.7,6.8,5.4,1.3 \mathrm{~Hz}, 1 \mathrm{H}$ ), 2.46 (ddd, $J=17.7,8.3,6.8 \mathrm{~Hz}, 1 \mathrm{H}), 2.30(\mathrm{ddtd}, J=13.4,7.4,6.7,1.7 \mathrm{~Hz}, 1 \mathrm{H}), 1.99-1.78(\mathrm{~m}, 3 \mathrm{H}), 1.65$ $(\mathrm{m}, 1 \mathrm{H}), 0.99(\mathrm{dd}, J=6.8,1.4 \mathrm{~Hz}, 6 \mathrm{H}) ;{ }^{13} \mathbf{C}\left\{{ }^{1} \mathbf{H}\right\} \mathbf{N M R}\left(125 \mathrm{MHz}, \mathrm{CDCl}_{3}\right): \delta[\mathrm{ppm}]=171.6$, 
141.4, 125.2, 81.0, 30.8, 29.6, 28.6, 22.1, 18.4; HR-ESI-MS m/z: calcd. $\mathrm{C}_{10} \mathrm{H}_{16} \mathrm{O}_{2} \mathrm{Na}[\mathrm{M}+\mathrm{Na}]^{+}$: 191.1043, found: 191.1139 .

\section{tert-butyl(E)-4-(2-(6-Oxotetrahydro-2H-pyran-2-yl)vinyl)piperidine-1-carboxylate (170f)}

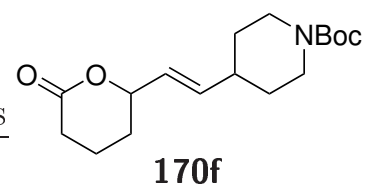

$170 f$

Following general procedure G Acid $\mathbf{1 3 7 g}(257 \mathrm{mg}, 0.83 \mathrm{mmol}$, 1.0 equiv), diphenyl diselenide ( $13 \mathrm{mg}, 42 \mu \mathrm{mol}, 0.05$ equiv) and $p$ MeO-TPT (20 mg, 42 umol, 0.05 equiv), acetonitrile ( $8.3 \mathrm{~mL}) ; 16 \mathrm{~h}$; light yellow oil (201 mg, $0.65 \mathrm{mmol}, 78 \%$ ) n-pentane/Et ${ }_{2} \mathrm{O} 1: 1$.

$\mathbf{R}_{\mathbf{f}}=0.10$ (n-pentane:Et $\left.{ }_{2} \mathrm{O}, 1: 1\right) ; \mathbf{I R}(\mathrm{ATR}): \tilde{\nu}\left[\mathrm{cm}^{-1}\right]=2974,2930$, 2851, 1733, 1684, 1421, 1365, 1274, 1231, 1160, 1092, 1033, 968, 929, 867, 753, 665, 461; ${ }^{\mathbf{1}} \mathbf{H}$ $\operatorname{NMR}\left(300 \mathrm{MHz}, \mathrm{CDCl}_{3}\right): \delta[\mathrm{ppm}]=5.70(\mathrm{ddd}, J=15.6,6.5,1.1 \mathrm{~Hz}, 1 \mathrm{H}), 5.47(\mathrm{ddd}, J=15.6$, $6.3,1.2 \mathrm{~Hz}, 1 \mathrm{H}), 4.74(\mathrm{ddt}, J=9.8,6.8,2.5 \mathrm{~Hz}, 1 \mathrm{H}$ ), 4.07 (d, $J=13.4 \mathrm{~Hz}, 2 \mathrm{H}), 2.70$ (t, $J=12.7 \mathrm{~Hz}, 2 \mathrm{H}), 2.63-2.35(\mathrm{~m}, 2 \mathrm{H}), 2.12(\mathrm{~m}, 1 \mathrm{H}), 2.01-1.74(\mathrm{~m}, 3 \mathrm{H}), 1.70-1.54(\mathrm{~m}, 3 \mathrm{H}), 1.43$ $(\mathrm{s}, 9 \mathrm{H}), 1.36-1.10(\mathrm{~m}, 2 \mathrm{H}) ;{ }^{13} \mathbf{C}\left\{{ }^{1} \mathbf{H}\right\} \mathbf{N M R}\left(75 \mathrm{MHz}, \mathrm{CDCl}_{3}\right): \delta[\mathrm{ppm}]=171.4,154.9,137.8$, 126.9, 80.6, 79.5, 43.7, 38.6, 31.5, 29.6, 28.6, 28.5, 18.4; HR-ESI-MS m/z: calcd. $\mathrm{C}_{17} \mathrm{H}_{27} \mathrm{NO}_{4}$ $[\mathrm{M}+\mathrm{Na}]^{+}: 332.1832$, found: 332.1835 .

\section{6-(2-Methylprop-1-en-1-yl)tetrahydro-2H-pyran-2-one (170g)}

Following general procedure G; acid 137l; 14.5 h; yellow oil (51 mg, $0.33 \mathrm{mmol}$,

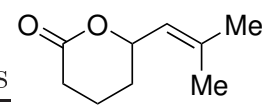

$33 \%) ; n$-pentane $/ \mathrm{Et}_{2} \mathrm{O}, 1: 1$.

170g $\quad \mathbf{R}_{\mathbf{f}}=0.29$ (n-pentane:Et $\left.{ }_{2} \mathrm{O}, 1: 1\right) ; \mathbf{I R}(\mathrm{ATR}): \tilde{\nu}\left[\mathrm{cm}^{-1}\right]=2934,2880,1725$, 1442, 1377, 1341, 1233, 1181, 1027, 925, 869, 820, 659, 571, 454; ${ }^{\mathbf{1}} \mathbf{H} \mathbf{N M R}\left(500 \mathrm{MHz}, \mathrm{CDCl}_{3}\right)$ : $\delta[\mathrm{ppm}]=5.23(\mathrm{ddt}, J=8.6,2.6,1.2 \mathrm{~Hz}, 1 \mathrm{H}), 5.01(\mathrm{ddd}, J=10.3,8.6,3.1 \mathrm{~Hz}, 1 \mathrm{H}), 2.58(\mathrm{~m}$, $1 \mathrm{H}), 2.45$ (ddd, $J=17.7,8.6,7.1 \mathrm{~Hz}, 1 \mathrm{H}), 2.04-1.77(\mathrm{~m}, 3 \mathrm{H}), 1.74(\mathrm{~d}, J=1.5 \mathrm{~Hz}, 3 \mathrm{H})$, $1.70(\mathrm{~d}, J=1.5 \mathrm{~Hz}, 3 \mathrm{H}), 1.62(\mathrm{~m}, 1 \mathrm{H}) ;{ }^{13} \mathbf{C}\left\{{ }^{1} \mathbf{H}\right\}$ NMR $\left(125 \mathrm{MHz}, \mathrm{CDCl}_{3}\right): \delta[\mathrm{ppm}]=171.8$, 138.5, 123.3, 77.6, 29.6, 28.6, 25.8, 18.7, 18.5; HR-EI-MS m/z: calcd. $\mathrm{C}_{9} \mathrm{H}_{14} \mathrm{O}_{2} \mathrm{Na}[\mathrm{M}+\mathrm{Na}]^{+}$: 177.0886, found: 177.0879 .

\section{(E)-6-(3-(Naphthalen-1-yl)prop-1-en-1-yl)tetrahydro-2 H-pyran-2-one (170h)}

Following general procedure G acid $\mathbf{1 3 7 m}$ (249 mg, $0.93 \mathrm{mmol}$,

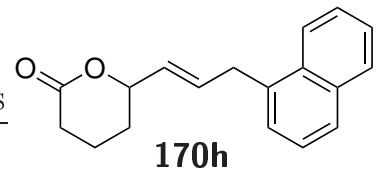

1.0 equiv), diphenyl diselenide ( $15 \mathrm{mg}, 47$ umol, 0.05 equiv) and $p$ MeO-TPT (23 mg, 47 pmol, 0.05 equiv), $(9.3 \mathrm{~mL}) ; 16 \mathrm{~h}$; yellow oil (141 mg, $530 \mu \mathrm{mol}, 57 \%$ ); n-pentane/Et ${ }_{2} \mathrm{O}$ 1:1.

$\mathbf{R}_{\mathbf{f}}=0.17$ (n-pentane:Et $\left.{ }_{2} \mathrm{O}, 1: 1\right) ; \mathbf{I R}(\mathrm{ATR}): \tilde{\nu}\left[\mathrm{cm}^{-1}\right]=3048,3011$, 2952, 2882, 1727, 1596, 1509, 1440, 1394, 1340, 1232, 1161, 1089, 1032, 969, 926, 777, 750, 665, 554, 466, 425, 407; ${ }^{1} \mathbf{H}$ NMR $\left(400 \mathrm{MHz}, \mathrm{CDCl}_{3}\right): \delta[\mathrm{ppm}]=7.98(\mathrm{~m}, 1 \mathrm{H}), 7.86(\mathrm{~m}, 1 \mathrm{H}), 7.75$ 
$(\mathrm{dt}, J=8.0,1.0 \mathrm{~Hz}, 1 \mathrm{H}), 7.56-7.45(\mathrm{~m}, 2 \mathrm{H}), 7.41(\mathrm{dd}, J=8.2,7.0 \mathrm{~Hz}, 1 \mathrm{H}), 7.33(\mathrm{~m}, 1 \mathrm{H}), 6.08$ $(\mathrm{dtd}, J=15.5,6.3,1.2 \mathrm{~Hz}, 1 \mathrm{H}), 5.55$ (ddt, $J=15.5,6.2,1.7 \mathrm{~Hz}, 1 \mathrm{H}$ ), 4.78 (dddd, $J=10.9$, 6.2, 2.8, $1.1 \mathrm{~Hz}, 1 \mathrm{H}), 3.85(\mathrm{dd}, J=6.4,1.6 \mathrm{~Hz}, 2 \mathrm{H}), 2.55(\mathrm{~m}, 1 \mathrm{H}), 2.44$ (ddd, $J=17.6$, 8.2, $6.8 \mathrm{~Hz}, 1 \mathrm{H}), 1.97-1.73(\mathrm{~m}, 4 \mathrm{H}), 1.61(\mathrm{~m}, 1 \mathrm{H}) ;{ }^{\mathbf{1 3}} \mathbf{C}\left\{{ }^{1} \mathbf{H}\right\}$ NMR $\left(100 \mathrm{MHz}, \mathrm{CDCl}_{3}\right): \delta$ $[\mathrm{ppm}]=171.3,135.6,134.0,132.4,132.0,129.8,128.9,127.3,126.6,126.1,125.7,124.0,80.4$, 35.7, 29.6, 28.4, 18.3; HR-EI-MS m/z: calcd. $\mathrm{C}_{18} \mathrm{H}_{18} \mathrm{O}_{2}[\mathrm{M}]^{+}:$: 266.1307, found: 266.1308.

\section{(E)-6-(3-(4-(Trifluoromethyl)phenyl)prop-1-en-1-yl)tetrahydro-2 $H$-pyran-2-one (170j)}

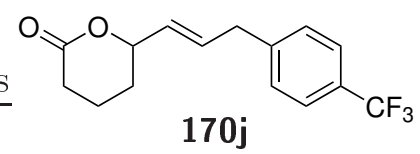

Following general procedure G acid 137o; 16 h; yellow oil (208 mg, 732 umol, $73 \%$ ); n-pentane/Et ${ }_{2} \mathrm{O}$ 2:1.

$\mathbf{R}_{\mathbf{f}}=0.21$ (n-pentane:Et $\left.{ }_{2} \mathrm{O}, 1: 1\right) ; \mathbf{I R}(\mathrm{ATR}): \tilde{\nu}\left[\mathrm{cm}^{-1}\right]=2954,1731$, $1618,1418,1322,1234,1159,1107,1065,1034,1018,971,927,848$, 822, 733, 594; ${ }^{\mathbf{1}} \mathbf{H}$ NMR $\left(300 \mathrm{MHz}, \mathrm{CDCl}_{3}\right): \delta[\mathrm{ppm}]=7.61-7.45(\mathrm{~m}, 2 \mathrm{H}), 7.33-7.23(\mathrm{~m}, 2 \mathrm{H})$, $5.92(\mathrm{dtd}, J=15.4,6.7,1.2 \mathrm{~Hz}, 1 \mathrm{H}), 5.57(\mathrm{ddt}, J=15.4,6.2,1.5 \mathrm{~Hz}, 1 \mathrm{H}), 4.80(\mathrm{~m}, 1 \mathrm{H})$, $3.44(\mathrm{~d}, J=6.7 \mathrm{~Hz}, 2 \mathrm{H}), 2.70-2.30(\mathrm{~m}, 2 \mathrm{H}), 2.07-1.74(\mathrm{~m}, 3 \mathrm{H}), 1.65(\mathrm{~m}, 1 \mathrm{H}) ;{ }^{13} \mathrm{C}\left\{{ }^{1} \mathbf{H}\right\}$ NMR $\left(125 \mathrm{MHz}, \mathrm{CDCl}_{3}\right): \delta[\mathrm{ppm}]=171.1,143.5(\mathrm{~d}, J=1.6 \mathrm{~Hz}), 131.5,130.2,129.0,128.7$ $(\mathrm{q}, J=32.4 \mathrm{~Hz}), 125.5$ (q, $J=3.9 \mathrm{~Hz}), 124.3$ (q, $J=271.3 \mathrm{~Hz}), 80.2$, 38.4, 29.7, 28.5, 18.4; ${ }^{19} \mathbf{F}$ NMR $\left(282 \mathrm{MHz}, \mathrm{CDCl}_{3}\right): \delta[\mathrm{ppm}]=-62.4$ (s); HR-ESI-MS m/z: calcd. $\mathrm{C}_{15} \mathrm{H}_{15} \mathrm{~F}_{3} \mathrm{O}_{2} \mathrm{Na}$ $[\mathrm{M}+\mathrm{Na}]^{+}$: 307.0916, found: 307.0918 .

(E)-4-(3-(6-Oxotetrahydro-2 $H$-pyran-2-yl)allyl)benzonitrile (170I)

Following general procedure G; acid 137q; 16 h; yellow oil $(152 \mathrm{mg}$,<smiles>N#Cc1ccc(C/C=C/C2CCCC(=O)O2)cc1</smiles>
629 umol, 81\%); n-pentane/Et ${ }_{2} \mathrm{O}$ 1:1.

$\mathbf{R}_{\mathbf{f}}=0.07$ (n-pentane:Et $\left.{ }_{2} \mathrm{O}, 1: 1\right)$; IR (ATR): $\tilde{\nu}\left[\mathrm{cm}^{-1}\right]=2952,2226$, $1726,1606,1504,1338,1232,1176,1088,1034,972,927,847,821$, $550 ;{ }^{1} \mathbf{H}$ NMR $\left(400 \mathrm{MHz}, \mathrm{CDCl}_{3}\right): \delta[\mathrm{ppm}]=7.70-7.54(\mathrm{~m}, 2 \mathrm{H}), 7.29-7.26(\mathrm{~m}, 2 \mathrm{H}), 5.90$ (dtd, $J=15.5,6.7,1.2 \mathrm{~Hz}, 1 \mathrm{H}), 5.58(\mathrm{ddt}, J=15.4,6.2,1.5 \mathrm{~Hz}, 1 \mathrm{H}), 4.80(\mathrm{~m}, 1 \mathrm{H}), 3.48-3.40$ $(\mathrm{m}, 2 \mathrm{H}), 2.58(\mathrm{~m}, 1 \mathrm{H}), 2.47$ (ddd, $J=17.7,8.4,6.8 \mathrm{~Hz}, 1 \mathrm{H}), 2.08-1.76(\mathrm{~m}, 3 \mathrm{H}), 1.64(\mathrm{~m}$, $1 \mathrm{H}) ;{ }^{13} \mathbf{C}\left\{{ }^{1} \mathbf{H}\right\} \mathbf{N M R}\left(100 \mathrm{MHz}, \mathrm{CDCl}_{3}\right): \delta[\mathrm{ppm}]=171.1,145.1,132.5,130.9,130.8,129.5$, 119.0, 110.5, 80.1, 38.6, 29.6, 28.4, 18.4; HR-ESI-MS m/z: calcd. $\mathrm{C}_{15} \mathrm{H}_{15} \mathrm{NO}_{2} \mathrm{Na}[\mathrm{M}+\mathrm{Na}]^{+}$: 264.0995, found: 264.0995 . 


\section{(E)-7-(Dodec-1-en-1-yl)oxepan-2-one (171)}

Following general procedure $\mathbf{G}$; acid $\mathbf{1 6 7} ; 21.5 \mathrm{~h}$; colorless oil (13 mg,<smiles>COC=CC1CCCCC(=O)O1</smiles>

171 46 umol, 5\%); n-pentane/Et ${ }_{2} \mathrm{O} 3: 1$.

$\mathbf{R}_{\mathbf{f}}=0.43$ (n-pentane:Et $\left.{ }_{2} \mathrm{O}, 3: 1\right) ; \mathbf{I R}(\mathrm{ATR}): \tilde{\nu}\left[\mathrm{cm}^{-1}\right]=2923,2853,1730$, 1457, 1347, 1328, 1277, 1251, 1226, 1175, 1039, 1009, 967, 847, 699, 564;

${ }^{1} \mathbf{H}$ NMR $\left(500 \mathrm{MHz}, \mathrm{CDCl}_{3}\right): \delta[\mathrm{ppm}]=5.74(\mathrm{dtd}, J=15.0,6.8,1.1 \mathrm{~Hz}$, $1 \mathrm{H}), 5.53(\mathrm{ddt}, J=15.4,6.8,1.5 \mathrm{~Hz}, 1 \mathrm{H}), 4.70(\mathrm{dd}, J=9.4,6.7 \mathrm{~Hz}, 1 \mathrm{H}), 2.73-2.57(\mathrm{~m}$, $2 \mathrm{H}), 2.06-1.88(\mathrm{~m}, 4 \mathrm{H}), 1.80-1.51(\mathrm{~m}, 3 \mathrm{H}), 1.41-1.18(\mathrm{~m}, 17 \mathrm{H}), 0.88(\mathrm{t}, J=6.9 \mathrm{~Hz}, 3 \mathrm{H})$; ${ }^{13} \mathbf{C}\left\{{ }^{1} \mathbf{H}\right\}$ NMR $\left(125 \mathrm{MHz}, \mathrm{CDCl}_{3}\right): \delta[\mathrm{ppm}]=175.4,133.5,128.8,81.1,35.6,35.2,32.36,32.1$, 29.86, 29.7, 29.6, 29.5, 29.3, 29.1, 28.3, 23.0, 22.8, 14.3; HR-ESI-MS m/z: calcd. $\mathrm{C}_{18} \mathrm{H}_{32} \mathrm{O}_{2} \mathrm{Na}$ $[\mathrm{M}+\mathrm{Na}]^{+}:$303.2295, found: 303.2297 .

\subsection{Synthesis and phosphatation of alkenes}

\subsubsection{Synthesis of alkenes 174}

Oct-4-ene-1,8-diyl bis(4-(trifluoromethyl)benzoate) (174a)<smiles>O=C(OCCCC=CCCCOC(=O)c1ccc(C(F)(F)F)cc1)c1ccc(C(F)(F)F)cc1</smiles>

Diol $\quad \mathbf{1 2 6} \mathbf{m} \quad(216 \quad \mathrm{mg}, \quad 1.50 \quad \mathrm{mmol}$, 1.0 equiv), 4-(trifluoromethyl)benzoylchloride (688 $\mathrm{mg}, 3.30 \mathrm{mmol}, 2.2$ equiv) and 4(dimethylamino)pyridine $(403 \mathrm{mg}, 3.30 \mathrm{mmol}$, 2.2 equiv) were dissolved in DCM $(5 \mathrm{~mL})$ and

stirred for $17 \mathrm{~h}$ at room temperature. The reaction was quenched by addition of conc. sat. $\mathrm{Na}_{2} \mathrm{CO}_{3}$ solution $(5 \mathrm{~mL})$, the org. phase was washed with sat. aq. $\mathrm{NaCl}$ solution $(5 \mathrm{~mL})$ and $1 \mathrm{M}$ aq. $\mathrm{HCl}$ solution $(5 \mathrm{~mL})$. The aq. phase was extracted with DCM $(10 \mathrm{~mL})$ and the org. phase was dried over $\mathrm{Na}_{2} \mathrm{SO}_{4}$. Removal of the solvent in vacuo, followed by column chromatography ( $n$-pentane/EtOAc, 10:1) afforded the product as a colorless liquid (653 mg, $1.34 \mathrm{mmol}, 89 \%$ ).

$\mathbf{R}_{\mathbf{f}}=0.57$ (n-pentane:EtOAc, 10:1); IR (ATR): $\tilde{\nu}\left[\mathrm{cm}^{-1}\right]=2957,2922,2873,2854,1721,1412$, 1323, 1270, 1163, 1097, 1065, 1017, 968, 862, 774, 703, 492; ${ }^{\mathbf{1}} \mathbf{H} \mathbf{~ N M R}\left(300 \mathrm{MHz}, \mathrm{CDCl}_{3}\right)$ : $\delta[\mathrm{ppm}]=8.36-8.00(\mathrm{~m}, 4 \mathrm{H}), 7.78-7.55(\mathrm{~m}, 4 \mathrm{H}), 5.72-5.32(\mathrm{~m}, 2 \mathrm{H}), 4.52-4.11(\mathrm{~m}, 4 \mathrm{H})$, 2.37-2.06 (m, $4 \mathrm{H}), 1.98-1.69(\mathrm{~m}, 4 \mathrm{H}) ;{ }^{\mathbf{1 3}} \mathbf{C}\left\{{ }^{\mathbf{1}} \mathbf{H}\right\}$ NMR $\left(125 \mathrm{MHz}, \mathrm{CDCl}_{3}\right): \delta[\mathrm{ppm}]=165.4$, 134.5 (q, $J=32.6 \mathrm{~Hz}), 133.7,130.0,129.5,125.47$ (q, $J=3.8 \mathrm{~Hz}), 123.73$ (q, $J=272.2 \mathrm{~Hz}$ ), 65.1, 29.1, 28.6; ${ }^{\mathbf{1 9}} \mathbf{F}$ NMR $\left(282 \mathrm{MHz}, \mathrm{CDCl}_{3}\right): \delta[\mathrm{ppm}]=-63.1$ (s); HR-ESI-MS m/z: calcd. $\mathrm{C}_{24} \mathrm{H}_{23} \mathrm{~F}_{6} \mathrm{O}_{4}[\mathrm{M}+\mathrm{H}]^{+}$: 489.1495, found: 489.1498. 


\section{Oct-4-ene-1,8-diyl bis(4-nitrobenzoate) (174b)}<smiles>O=C(OCCC/C=C/CCCOC(=O)c1ccc([N+](=O)[O-])cc1)c1ccc([N+](=O)[O-])cc1</smiles>

174b

Diol 126m (216 mg, $1.50 \mathrm{mmol}, 1.0$ equiv), 4-nitrobenzoylchloride $(612 \mathrm{mg}, 3.30 \mathrm{mmol}$, 2.2 equiv) and 4-(dimethylamino)pyridine (403 mg, $3.30 \mathrm{mmol}, 2.2$ equiv) were dissolved in DCM $(5 \mathrm{~mL})$ and stirred for $17 \mathrm{~h}$ at room temperature. The reaction was quenched by addition of conc. sat. $\mathrm{Na}_{2} \mathrm{CO}_{3}$ solution $(5 \mathrm{~mL})$, the org. phase was washed with sat. aq. $\mathrm{NaCl}$ solution $(5 \mathrm{~mL})$ and $1 \mathrm{M}$ aq. $\mathrm{HCl}$ solution $(5 \mathrm{~mL})$. The aq. phase was extracted with DCM $(10 \mathrm{~mL})$ and the org. phase was dried over $\mathrm{Na}_{2} \mathrm{SO}_{4}$. Removal of the solvent in vacuo, followed by column chromatography ( $n$-pentane/EtOAc, 10:1 to 5:1) afforded the product as a white solid (589 $\mathrm{mg}, 1.33 \mathrm{mmol}, 89 \%)$.

$\mathbf{R}_{\mathbf{f}}=0.14$ (n-pentane:EtOAc, 10:1); m.p. $=114.8{ }^{\circ} \mathrm{C} ; \mathbf{I R}(\mathrm{ATR}): \tilde{\nu}\left[\mathrm{cm}^{-1}\right]=3147,2955,2937$, 2854, 1713, 1599, 1522, 1462, 1346, 1321, 1270, 1167, 1123, 1102, 1010, 975, 911, 870, 857, 834, 787, 712, 505, 409; ${ }^{1} \mathbf{H}$ NMR (300 MHz, $\left.\mathrm{CDCl}_{3}\right): \delta[\mathrm{ppm}]=8.29-8.20(\mathrm{~m}, 4 \mathrm{H}), 8.20-8.11$ (m, $4 \mathrm{H}), 5.75-5.41(\mathrm{~m}, 2 \mathrm{H}), 4.33(\mathrm{td}, J=6.6,5.1 \mathrm{~Hz}, 4 \mathrm{H}), 2.28-2.00(\mathrm{~m}, 4 \mathrm{H}), 1.84(\mathrm{dq}, J=8.4$, $6.6 \mathrm{~Hz}, 4 \mathrm{H}) ;{ }^{13} \mathbf{C}\left\{{ }^{1} \mathbf{H}\right\} \mathbf{N M R}\left(125 \mathrm{MHz}, \mathrm{CDCl}_{3}\right): \delta[\mathrm{ppm}]=164.6,150.5,135.8,130.7,129.9$, 123.5, 65.4, 29.0, 28.5; HR-ESI-MS m/z: calcd. $\mathrm{C}_{22} \mathrm{H}_{22} \mathrm{~N}_{2} \mathrm{O}_{8} \mathrm{Na}[\mathrm{M}+\mathrm{Na}]^{+}$: 465.1268, found: 465.1279 .

\section{Oct-4-ene-1,8-diyl bis(4-bromobenzoate) (174c)}<smiles>O=C(OCCC/C=C/CCCOC(=O)c1ccc(Br)cc1)c1ccc(Br)cc1</smiles>

Diol 126m (216 mg, $1.50 \mathrm{mmol}, 1.0$ equiv), 4-bromobenzoylchloride (724 mg, $3.30 \mathrm{mmol}$, 2.2 equiv) and 4-(dimethylamino)pyridine (403 mg, $3.30 \mathrm{mmol}, 2.2$ equiv) were dissolved in DCM $(5 \mathrm{~mL})$ and stirred for $17 \mathrm{~h}$. The reaction was quenched by addition of conc. sat. $\mathrm{Na}_{2} \mathrm{CO}_{3}$ solution $(5 \mathrm{~mL})$, the org. phase was washed with sat. aq. $\mathrm{NaCl}$ solution $(5 \mathrm{~mL})$ and $1 \mathrm{M}$ aq. $\mathrm{HCl}$ solution $(5 \mathrm{~mL})$. The aq. phase was extracted with DCM $(10 \mathrm{~mL})$ and the org. phase was dried over $\mathrm{Na}_{2} \mathrm{SO}_{4}$. Removal of the solvent in vacuo, followed by column chromatography ( $n$-pentane/EtOAc, 10:1) afforded the product as a white solid (725 mg, $1.42 \mathrm{mmol}, 94 \%$ ).

$\mathbf{R}_{\mathbf{f}}=0.50$ (n-pentane:EtOAc, 10:1); m.p. $=55.7^{\circ} \mathrm{C} ; \mathbf{I R}(\mathrm{ATR}): \tilde{\nu}\left[\mathrm{cm}^{-1}\right]=2959,2935,2893$, 2847, 1714, 1589, 1472, 1396, 1267, 1173, 1102, 1066, 1008, 963, 848, 751, 708, 682, 469; ${ }^{\mathbf{1}} \mathbf{H}$ NMR $\left(300 \mathrm{MHz}, \mathrm{CDCl}_{3}\right): \delta[\mathrm{ppm}]=8.00-7.75(\mathrm{~m}, 4 \mathrm{H}), 7.70-7.39(\mathrm{~m}, 4 \mathrm{H}), 5.71-5.23(\mathrm{~m}$, $2 \mathrm{H}$ ), 4.40-4.08 (m, $4 \mathrm{H}$ ), 2.29-1.95 (m, $4 \mathrm{H}$ ), 1.82 (quint, $J=6.7 \mathrm{~Hz}, 4 \mathrm{H}$ ); ${ }^{\mathbf{1 3}} \mathbf{C}\left\{{ }^{\mathbf{1}} \mathbf{H}\right\} \mathbf{N M R}$ $\left(125 \mathrm{MHz}, \mathrm{CDCl}_{3}\right): \delta[\mathrm{ppm}]=165.9,131.7,131.1,130.0,129.4,128.0,64.8,29.2,28.7$; HR-ESIMS m/z: calcd. $\mathrm{C}_{22} \mathrm{H}_{23} \mathrm{Br}_{2} \mathrm{O}_{4}[\mathrm{M}+\mathrm{H}]^{+}$: 510.9939, found: 510.9945 . 


\section{Oct-4-ene-1,8-diyl bis(4-cyanobenzoate) (174d)}<smiles>N#Cc1ccc(C(=O)OCCC/C=C/CCCOC(=O)c2ccc([N+]#N)cc2)cc1</smiles>

Diol 126m (216 mg, $1.50 \mathrm{mmol}, 1.0$ equiv), 4-cyanobenzoylchloride (546 mg, $3.30 \mathrm{mmol}$, 2.2 equiv) and 4-(dimethylamino)pyridine (403 mg, $3.30 \mathrm{mmol}, 2.2$ equiv) were dissolved in $\mathrm{DCM}(5 \mathrm{~mL})$ and stirred for $17 \mathrm{~h}$ at room temperature. The reaction was quenched by addition of conc. sat. $\mathrm{Na}_{2} \mathrm{CO}_{3}$ solution $(5 \mathrm{~mL})$, the org. phase was washed with sat. aq. $\mathrm{NaCl}$ solution $(5 \mathrm{~mL})$ and $1 \mathrm{M}$ aq. $\mathrm{HCl}$ solution $(5 \mathrm{~mL})$. The aq. phase was extracted with DCM $(10 \mathrm{~mL})$ and the org. phase was dried over $\mathrm{Na}_{2} \mathrm{SO}_{4}$. Removal of the solvent in vacuo, followed by column chromatography ( $n$-pentane/EtOAc, 5:1) afforded the product as a white solid (488 $\mathrm{mg}, 1.20 \mathrm{mmol}, 81 \%$ ).

$\mathbf{R}_{\mathbf{f}}=0.25$ (n-pentane:EtOAc, 5:1); m.p. $=85.6{ }^{\circ} \mathrm{C} ; \mathbf{I R}(\mathrm{ATR}): \tilde{\nu}\left[\mathrm{cm}^{-1}\right]=2969,2951,2873,2847$, 2230, 1716, 1406, 1270, 1177, 1108, 1017, 957, 862, 766, 690, 546, 503; ${ }^{\mathbf{1}} \mathbf{H}$ NMR $(300 \mathrm{MHz}$, $\left.\mathrm{CDCl}_{3}\right): \quad \delta[\mathrm{ppm}]=8.35-7.99(\mathrm{~m}, 4 \mathrm{H}), 7.92-7.50(\mathrm{~m}, 4 \mathrm{H}), 5.69-5.09(\mathrm{~m}, 2 \mathrm{H}), 4.62-3.79$ $(\mathrm{m}, 4 \mathrm{H}), 2.33-1.97(\mathrm{~m}, 4 \mathrm{H}), 1.98-1.70(\mathrm{~m}, 4 \mathrm{H}) ;{ }^{\mathbf{1 3}} \mathbf{C}\left\{{ }^{\mathbf{1}} \mathbf{H}\right\}$ NMR $\left(125 \mathrm{MHz}, \mathrm{CDCl}_{3}\right)$ : $\delta$ $[\mathrm{ppm}]=164.9,134.3,132.3,130.1,130.0,118.0,116.5,65.3,29.1,28.6 ;$ HR-ESI-MS m/z: calcd. $\mathrm{C}_{24} \mathrm{H}_{22} \mathrm{~N}_{2} \mathrm{O}_{4} \mathrm{Na}[\mathrm{M}+\mathrm{Na}]^{+}$: 425.1472 , found: 425.1474 .

Oct-4-ene-1,8-diyl bis(4-methoxybenzoate) (174e)<smiles>COc1ccc(C(=O)OCCC/C=C/CCCOC(=O)c2ccc(OC)cc2)cc1</smiles>

Diol 126m (649 mg, $4.50 \mathrm{mmol}, 1.0$ equiv), 4-methoxybenzoylchloride $(1.7 \mathrm{~g}, 9.9 \mathrm{mmol}$, 2.2 equiv) and 4 -(dimethylamino)pyridine (1.21 g, $9.90 \mathrm{mmol}, 2.2$ equiv) were dissolved in DCM $(15 \mathrm{~mL})$ and stirred for $18 \mathrm{~h}$ at room temperature. The reaction was quenched by addition of conc. sat. $\mathrm{Na}_{2} \mathrm{CO}_{3}$ solution $(15 \mathrm{~mL})$, the org. phase was washed with sat. aq. $\mathrm{NaCl}$ solution $(15 \mathrm{~mL})$ and $1 \mathrm{M}$ aq. $\mathrm{HCl}$ solution $(15 \mathrm{~mL})$. The aq. phase was extracted with DCM $(30 \mathrm{~mL})$ and the org. phase was dried over $\mathrm{Na}_{2} \mathrm{SO}_{4}$. Removal of the solvent in vacuo, followed by column chromatography ( $n$-pentane/EtOAc, 3:1) afforded the product as a white solid (1.68 g, $4.08 \mathrm{mmol}, 91 \%)$.

$\mathbf{R}_{\mathbf{f}}=0.43$ (n-pentane:EtOAc, 3:1); m.p. $=57.8^{\circ} \mathrm{C} ; \mathbf{I R}(\mathrm{ATR}): \tilde{\nu}\left[\mathrm{cm}^{-1}\right]=3004,2958,2839,1700$, 1604, 1580, 1509, 1455, 1418, 1386, 1315, 1273, 1249, 1167, 1117, 1101, 1028, 971, 958, 850, 787, 770, 754, 695, 637, 613, 508; ${ }^{\mathbf{1}} \mathbf{H}$ NMR (400 MHz, $\left.\mathrm{CDCl}_{3}\right): \delta[\mathrm{ppm}]=8.11-7.85(\mathrm{~m}, 4 \mathrm{H})$, 6.99-6.66 (m, $4 \mathrm{H}), 5.62-5.40(\mathrm{~m}, 2 \mathrm{H}), 4.28(\mathrm{t}, J=6.6 \mathrm{~Hz}, 4 \mathrm{H}), 3.85(\mathrm{~d}, J=4.3 \mathrm{~Hz}, 6 \mathrm{H})$, 2.15 (tdd, $J=7.6,3.9,2.0 \mathrm{~Hz}, 4 \mathrm{H}), 1.88-1.71(\mathrm{~m}, 4 \mathrm{H}) ;{ }^{\mathbf{1 3}} \mathbf{C}\left\{{ }^{\mathbf{1}} \mathbf{H}\right\}$ NMR $\left(100 \mathrm{MHz}, \mathrm{CDCl}_{3}\right): \delta$ $[\mathrm{ppm}]=166.5,163.4,131.7,130.1,123.0,113.7,64.3,55.5,29.2,28.7 ;$ HR-ESI-MS m/z: calcd. $\mathrm{C}_{24} \mathrm{H}_{28} \mathrm{O}_{6} \mathrm{Na}[\mathrm{M}+\mathrm{Na}]^{+}:$435.1778, found: 435.1778 . 


\section{1,8-Bis(tert-butyldimethylsilyloxy)oct-4-ene (174f)}

Diol 126m (577 mg, $4.00 \mathrm{mmol}, 1.0$ equiv) was dissolved in DCM

TBSO

$174 f$
(3.5 mL), TBSCl (1.33 g, $8.80 \mathrm{mmol}, 2.2$ equiv) and imidazole (654 mg, $9.60 \mathrm{mmol}, 2.4$ equiv) were added sequentially and the solution was stirred at room temperature for $17 \mathrm{~h}$. The reaction

mixture was washed with sat. aq. $\mathrm{NaCl}$ solution $(2 \times 4 \mathrm{~mL})$, the aq. phase was extracted with DCM $(2 \times 8 \mathrm{~mL})$ and the comb. org. phase was dried over $\mathrm{Na}_{2} \mathrm{SO}_{4}$. Removal of the solvent in vacuo, followed by column chromatography ( $n$-pentane/EtOAc, 4:1) afforded the product as a colorless liquid (1.39 g, $3.72 \mathrm{mmol}, 93 \%)$.

$\mathbf{R}_{\mathbf{f}}=0.93$ (n-pentane:EtOAc, 4:1); IR (ATR): $\tilde{\nu}\left[\mathrm{cm}^{-1}\right]=2953,2929,2893,2857,1472,1387$, 1361, 1253, 1098, 1006, 966, 938, 832 772, 716, 662; ${ }^{\mathbf{1}} \mathbf{H}$ NMR (300 $\left.\mathrm{MHz}_{2} \mathrm{CDCl}_{3}\right): \delta$ $[\mathrm{ppm}]=5.67-5.15(\mathrm{~m}, 2 \mathrm{H}), 3.60(\mathrm{td}, J=6.6,1.6 \mathrm{~Hz}, 4 \mathrm{H}), 2.51-1.91(\mathrm{~m}, 4 \mathrm{H}), 1.73-1.44$ $(\mathrm{m}, 4 \mathrm{H}), 1.05-0.80(\mathrm{~m}, 18 \mathrm{H}), 0.14-(-0.02)(\mathrm{m}, 12 \mathrm{H}) ;{ }^{\mathbf{1 3}} \mathbf{C}\left\{{ }^{\mathbf{1}} \mathbf{H}\right\}$ NMR $\left(125 \mathrm{MHz}, \mathrm{CDCl}_{3}\right)$ : $\delta[\mathrm{ppm}]=130.2,62.8,32.9,29.0,26.2,18.6,-5.0 ;$ HR-ESI-MS $\mathbf{m} / \mathbf{z}$ : calcd. $\mathrm{C}_{14} \mathrm{H}_{45} \mathrm{O}_{2} \mathrm{Si}_{2}$ $[\mathrm{M}+\mathrm{H}]^{+}:$:373.2953, found: 373.2949 .

\section{Diethyl oct-4-ene-1,8-diyl bis(carbonate) (174g)}<smiles>CCOC(=O)OCCC/C=C/CCCOC(=O)OCC</smiles>

$174 \mathrm{~g}$

Diol 126m (577 mg, $4.00 \mathrm{mmol}, 1.0$ equiv) and DMAP (12 mg, $0.10 \mathrm{mmol}, 0.025$ equiv) were dissolved in DCM $(6.0 \mathrm{~mL})$ and cooled to $0{ }^{\circ} \mathrm{C}$. Pyridine $(633 \mathrm{mg}, 8.00 \mathrm{mmol}$, 2.0 equiv) and ethylchloroformate (434 mg, $4.00 \mathrm{mmol}$, 1.0 equiv) were added dropwise sequentially. The solution was allowed to warm to room temperature and stirred for $13 \mathrm{~h}$. Pyridine (633 mg, $8.00 \mathrm{mmol}, 2.0$ equiv), ethylchloroformate (434 mg, $4.00 \mathrm{mmol}, 1.0$ equiv) and DMAP (12 mg, $0.10 \mathrm{mmol}, 0.025$ equiv) were added and stirred for $24 \mathrm{~h}$. After stirring at $40{ }^{\circ} \mathrm{C}$ for $19 \mathrm{~h}$, the reaction was stopped, the solution was washed with $\mathrm{H}_{2} \mathrm{O}(3 \times 6 \mathrm{~mL})$. Drying of the org. phase over $\mathrm{Na}_{2} \mathrm{SO}_{4}$, removal of the solvent in vacuo, followed by column chromatography ( $n$-pentane/EtOAc, $2: 1$ ) afforded the product as a yellow liquid (881 mg, $3.05 \mathrm{mmol}, 76 \%$ ).

$\mathbf{R}_{\mathbf{f}}=0.86$ (n-pentane:EtOAc, 2:1); IR (ATR): $\tilde{\nu}\left[\mathrm{cm}^{-1}\right]=2983,2962,2939,2912,1739,1467$, 1402, 1367, 1244, 1091, 1007, 971, 864, 790; ${ }^{\mathbf{1}} \mathbf{H}$ NMR (400 MHz, $\left.\mathrm{CDCl}_{3}\right): \delta[\mathrm{ppm}]=5.67-5.29$ $(\mathrm{m}, 2 \mathrm{H}), 4.18(\mathrm{qd}, J=7.1,0.9 \mathrm{~Hz}, 4 \mathrm{H}), 4.12(\mathrm{td}, J=6.7,1.4 \mathrm{~Hz}, 4 \mathrm{H}), 2.45-2.00(\mathrm{~m}, 4 \mathrm{H}), 1.72$ $(\mathrm{dq}, J=8.3,6.6 \mathrm{~Hz}, 4 \mathrm{H}), 1.30(\mathrm{t}, J=7.1 \mathrm{~Hz}, 6 \mathrm{H}) ;{ }^{\mathbf{1 3}} \mathbf{C}\left\{{ }^{\mathbf{1}} \mathbf{H}\right\} \mathbf{N M R}\left(100 \mathrm{MHz}, \mathrm{CDCl}_{3}\right): \delta$ $[\mathrm{ppm}]=155.4$, 130.0, 67.4, 64.0, 28.7, 28.6, 14.4; HR-ESI-MS m/z: calcd. $\mathrm{C}_{14} \mathrm{H}_{25} \mathrm{O}_{6}[\mathrm{M}+\mathrm{H}]^{+}$: 289.1646, found: 289.1641 . 


\section{(E)-Hex-4-en-1-yl pivalate (174h)}

DMAP (202 mg, $1.65 \mathrm{mmol}, 1.1$ equiv) and 4-hexene-1-ol (150 mg, Me OPiv 1.50 mmol, 1.0 equiv) were dissolved in DCM (5 mL) and pivaloyl chlo174h $\quad$ ride (199 mg, $1.65 \mathrm{mmol}, 1.1$ equiv) was added dropwise. The solution was stirred at room temperature for $5 \mathrm{~h}$, washed with sat. aq. $\mathrm{Na}_{2} \mathrm{CO}_{3}$, sat. aq. $\mathrm{NaCl}$ and $1 \mathrm{M}$ aq. $\mathrm{HCl}$ solution. The comb. aq. phases were extracted with DCM and the comb. org. phases were dried over $\mathrm{Na}_{2} \mathrm{SO}_{4}$. Removal of the solvent in vacuo, followed by column chromatography (n-pentane/EtOAc, 20:1) afforded the product as a colorless liquid (207 mg, $1.12 \mathrm{mmol}, 75 \%$ ).

$\mathbf{R}_{\mathbf{f}}=0.71$ (n-pentane:EtOAc, 20:1); IR (ATR): $\tilde{\nu}\left[\mathrm{cm}^{-1}\right]=3015,2970,2872,1728,1480,1459$, $1398,1366,1283,1150,1150,1034,771,699 ;{ }^{1} \mathbf{H}$ NMR $\left(300 \mathrm{MHz}, \mathrm{CDCl}_{3}\right): \delta[\mathrm{ppm}]=5.56-5.43$ $(\mathrm{m}, 1 \mathrm{H}), 5.43-5.29(\mathrm{~m}, 1 \mathrm{H}), 4.05(\mathrm{t}, J=6.5 \mathrm{~Hz}, 2 \mathrm{H}), 2.32-1.97(\mathrm{~m}, 2 \mathrm{H}), 1.78-1.63(\mathrm{~m}, 2 \mathrm{H})$, 1.60 (ddt, $J=6.6,1.7,0.8 \mathrm{~Hz}, 3 \mathrm{H}), 1.20(\mathrm{~d}, J=0.4 \mathrm{~Hz}, 9 \mathrm{H}) ;{ }^{\mathbf{1 3}} \mathbf{C}\left\{{ }^{\mathbf{1}} \mathbf{H}\right\} \mathbf{N M R}(75 \mathrm{MHz}$, $\left.\mathrm{CDCl}_{3}\right): \delta[\mathrm{ppm}]=178.7,129.3,125.0,63.9,38.9,28.6,27.4,27.4,23.3,12.8$; HR-ESI-MS m/z: calcd. $\mathrm{C}_{11} \mathrm{H}_{20} \mathrm{O}_{2} \mathrm{Na}[\mathrm{M}+\mathrm{Na}]^{+}:$207.1356, found: 207.1363 .

\section{(E)-3,7-Dimethylocta-2,6-dien-1-yl pivalate (174i)}

DMAP (605 mg, $4.95 \mathrm{mmol}, 1.1$ equiv) and geraniol (694 mg,

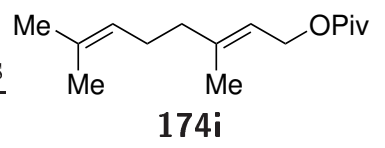

$4.50 \mathrm{mmol}, 1.0$ equiv) were dissolved in DCM $(15 \mathrm{~mL})$ and pivaloyl chloride (597 mg, $4.95 \mathrm{mmol}, 1.1$ equiv) was added dropwise. The solution was stirred at room temperature for $17 \mathrm{~h}$, washed with sat. aq. $\mathrm{Na}_{2} \mathrm{CO}_{3}$, sat. aq. $\mathrm{NaCl}$ and $1 \mathrm{M}$ aq. $\mathrm{HCl}$ solution. The comb. aq. phases were extracted with DCM and the comb. org. phases were dried over $\mathrm{Na}_{2} \mathrm{SO}_{4}$. Removal of the solvent in vacuo, followed by column chromatography ( $n$-pentane/EtOAc, 50:1) afforded the product as a colorless liquid (861 mg, $3.61 \mathrm{mmol}, 80 \%$ ).

$\mathbf{R}_{\mathbf{f}}=0.50$ (n-pentane:EtOAc, 50:1); IR (ATR): $\tilde{\nu}\left[\mathrm{cm}^{-1}\right]=2969,2931,2873,1727,1480,1455$, 1377, 1281, 1146, 1032, 951, 860, 830, 771; ${ }^{\mathbf{1}} \mathbf{H}$ NMR $\left(300 \mathrm{MHz}, \mathrm{CDCl}_{3}\right): \delta[\mathrm{ppm}]=5.32(\mathrm{tq}$, $J=6.9,1.3 \mathrm{~Hz}, 1 \mathrm{H}), 5.08(\mathrm{dddd}, J=8.2,6.8,2.9,1.4 \mathrm{~Hz}, 1 \mathrm{H}), 4.57(\mathrm{dq}, J=6.9,0.7 \mathrm{~Hz}, 2 \mathrm{H})$, 2.16-1.86 (m, $4 \mathrm{H}), 1.80-1.64$ (m, $6 \mathrm{H}), 1.60$ (d, $J=0.5 \mathrm{~Hz}, 3 \mathrm{H}), 1.20(\mathrm{~s}, 9 \mathrm{H}) ;{ }^{\mathbf{1 3}} \mathbf{C}\left\{{ }^{\mathbf{1}} \mathbf{H}\right\} \mathbf{~ N M R}$ $\left(125 \mathrm{MHz}, \mathrm{CDCl}_{3}\right): \delta[\mathrm{ppm}]=178.6,141.7,131.8,123.9,118.9,61.5,39.7,39.0,27.5,26.5,25.9$, 17.9, 16.7; HR-ESI-MS m/z: calcd. $\mathrm{C}_{15} \mathrm{H}_{26} \mathrm{O}_{2} \mathrm{Na}[\mathrm{M}+\mathrm{Na}]^{+}:$261.1825, found: 261.1838.

\section{(Z)-Dec-4-enal (174j)}

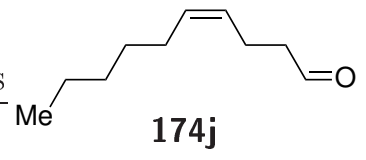

Dess-Martin-periodinane (4.24 g, $10.0 \mathrm{mmol}, 2.0$ equiv) was dissolved in DCM $(20 \mathrm{~mL})$ and cooled to $0{ }^{\circ} \mathrm{C}$. A solution of $(Z)$-4-decen-1-ol (782 mg, $5.00 \mathrm{mmol}, 1.0$ equiv) in DCM $(5 \mathrm{~mL})$ was added dropwise and the mixture was stirred at room temperature for $5 \mathrm{~h}$. After diluting the solution with DCM $(10 \mathrm{~mL})$, it was washed with sat. aq. $\mathrm{Na}_{2} \mathrm{~S}_{2} \mathrm{O}_{3}, 1 \mathrm{M}$ aq. $\mathrm{NaOH}$ and sat. 
aq. $\mathrm{NaCl}$ solution. The comb. aq. phases were extracted with DCM and the comb. org. phases were dried over $\mathrm{Na}_{2} \mathrm{SO}_{4}$. Removal of the solvent in vacuo, followed by column chromatography ( $n$-pentane/ $\left.\mathrm{Et}_{2} \mathrm{O}, 20: 1\right)$ afforded the product as a colorless liquid (581 $\mathrm{mg}, 3.77 \mathrm{mmol}, 75 \%$ ).

$\mathbf{R}_{\mathbf{f}}=0.36$ (n-pentane:Et $\left.{ }_{2} \mathrm{O}, 20: 1\right)$; IR (ATR): $\tilde{\nu}\left[\mathrm{cm}^{-1}\right]=3009,2956,2925,2856,2717,1725$, 1459, 1409, 1389, 1055, 857, 757, 725, 575, 503; ${ }^{\mathbf{1}} \mathbf{H}$ NMR $\left(400 \mathrm{MHz}, \mathrm{CDCl}_{3}\right): \delta[\mathrm{ppm}]=9.77$ $(\mathrm{t}, J=1.7 \mathrm{~Hz}, 1 \mathrm{H}), 5.43(\mathrm{dtt}, J=10.9,7.1,1.4 \mathrm{~Hz}, 1 \mathrm{H}), 5.32(\mathrm{~m}, 1 \mathrm{H}), 2.78-2.42(\mathrm{~m}, 2 \mathrm{H})$, 2.42-2.13 (m, $2 \mathrm{H}), 2.14-1.90(\mathrm{~m}, 2 \mathrm{H}), 1.46-1.06(\mathrm{~m}, 6 \mathrm{H}), 0.96-0.70(\mathrm{~m}, 3 \mathrm{H}) ;{ }^{13} \mathbf{C}\left\{{ }^{\mathbf{1}} \mathbf{H}\right\}$ NMR $\left(100 \mathrm{MHz}, \mathrm{CDCl}_{3}\right): \delta[\mathrm{ppm}]=202.4,131.9,127.2,44.0,31.6,29.4,27.3,22.7,20.2,14.2$; HR-ESI-MS m/z: calcd. $\mathrm{C}_{10} \mathrm{H}_{18} \mathrm{O}[\mathrm{M}-\mathrm{H}]^{+}:$153.1285, found: 153.1274 .

\section{Methyl ( $Z)$-tetracos-15-enoate (174k)}

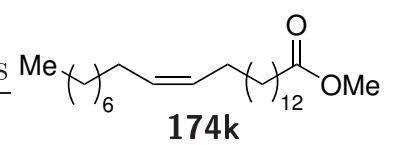

(Z)-15-Tetracosenoic acid (1.29 g, $3.52 \mathrm{mmol}, 1.0$ equiv) and PTSA (33 mg, $0.18 \mathrm{mmol}, 0.05$ equiv) were dissolved in $\mathrm{MeOH}(35 \mathrm{~mL})$ and stirred at $65{ }^{\circ} \mathrm{C}$ for $4 \mathrm{~h}$. The solvent was removed in vacuo, the residue was dissolved in EtOAc and washed with $\mathrm{H}_{2} \mathrm{O}$, sat. aq. $\mathrm{NaHCO}_{3}$ solution and again $\mathrm{H}_{2} \mathrm{O}$. Reextraction of the aq. phase with EtOAc, drying over $\mathrm{Na}_{2} \mathrm{SO}_{4}$ and removal of the solvent in vacuo afforded the product as a yellow liquid (1.26 g, $3.32 \mathrm{mmol}, 94 \%)$.

$\mathbf{R}_{\mathbf{f}}=0.86$ (DCM:EtOAc, 75:1); IR (ATR): $\tilde{\nu}\left[\mathrm{cm}^{-1}\right]=2922,2853,1743,1463,1436,1363,1195$, 1169,$721 ;{ }^{1} \mathbf{H} \mathbf{N M R}\left(400 \mathrm{MHz}, \mathrm{CDCl}_{3}\right): \delta[\mathrm{ppm}]=5.35(\mathrm{ddd}, J=5.6,4.4,1.1 \mathrm{~Hz}, 2 \mathrm{H}), 3.66$ $(\mathrm{s}, 3 \mathrm{H}), 2.30$ (t, $J=7.6 \mathrm{~Hz}, 2 \mathrm{H}), 2.18-1.87$ (m, $4 \mathrm{H}), 1.61$ (quint, $J=7.3 \mathrm{~Hz}, 2 \mathrm{H}$ ), 1.44-1.02 $(\mathrm{m}, 32 \mathrm{H}), 0.92-0.83(\mathrm{~m}, 3 \mathrm{H}) ;{ }^{\mathbf{1 3}} \mathbf{C}\left\{{ }^{\mathbf{1}} \mathbf{H}\right\}$ NMR $\left(100 \mathrm{MHz}, \mathrm{CDCl}_{3}\right): \delta[\mathrm{ppm}]=174.8,130.1$, 51.6, 34.3, 32.1, 30.0, 29.8, 29.8, 29.8, 29.7, 29.7, 29.6, 29.5, 29.4, 29.3, 27.4, 25.1, 22.8, 14.3; HR-ESI-MS m/z: calcd. $\mathrm{C}_{25} \mathrm{H}_{49} \mathrm{O}_{2}[\mathrm{M}+\mathrm{H}]^{+}:$: 381.3727, found: 381.3737 .

\subsubsection{Synthesis of phosphates 173}

\section{(E)-5-((Dibutoxyphosphoryl)oxy)oct-3-ene-1,8-diyl diethyl bis(carbonate) (173g)}<smiles>CCOC(=O)OCC/C=C/C(CCCOC(=O)OCC)O[P+](=O)OCC</smiles><smiles>CCOC(=O)OC/C=C/C(CCCCOC(=O)OCC)OP(=O)(O)OCC</smiles>

Alkene 174g (865 mg, $3.00 \mathrm{mmol}, 3.0$ equiv), dibutyl phosphate (210 mg, $1.00 \mathrm{mmol}, 1.0$ equiv), $\mathrm{Na}_{2} \mathrm{HPO}_{4}$ 6.6 (114 mg, 800 umol, 0.8 equiv), p-MeO-TPT (95) (49 mg, $0.10 \mathrm{mmol}, 0.1$ equiv), diphenyl diselenide (31 mg, $0.10 \mathrm{mmol}, 0.1$ equiv) and $4 \AA$ molecular sieves (30 mg) were dissolved in DCE $(10 \mathrm{~mL})$ and stirred under oxygen and irradiation with blue light at room temperature for $7.5 \mathrm{~h}$. The solvent was removed, the residue was dissolved in a mixture of $\mathrm{Et}_{2} \mathrm{O}$ and $\mathrm{H}_{2} \mathrm{O}$

$(1: 1)$ and the aq. phase was extracted with $\mathrm{Et}_{2} \mathrm{O}(3 \times)$. The comb. org. phases were washed 
with $\mathrm{H}_{2} \mathrm{O}$ and dried over $\mathrm{Na}_{2} \mathrm{SO}_{4}$. Removal of the solvent in vacuo, followed by column chromatography (DCM $\rightarrow n$-pentane/EtOAc, 5:1) afforded the product as a yellow liquid as a mixture of products with different double bond positions (3-alkene:2-alkene, 6.6:1; $96.6 \mathrm{mg}, 195 \mu \mathrm{mol}$, 20\%). ${ }^{31} \mathrm{P}$ NMR yield $42 \%$ (6:1 major/minor) with triphenylphosphine (117 mg, 447 pmol) as a standard.

$\mathbf{R}_{\mathbf{f}}=0.14$ (n-pentane:EtOAc, 5:1); IR (ATR): $\tilde{\nu}\left[\mathrm{cm}^{-1}\right]=2961,2936,2875,1741,1466,1403$, 1384, 1367, 1249, 976, 910, 868, 791, 736, 542; $\left.{ }^{\mathbf{1}} \mathbf{H} \mathbf{~ N M R ~ ( 5 0 0 ~ M H z , ~} \mathrm{CDCl}_{3}\right): \delta[\mathrm{ppm}]=$ major product: $5.74(\mathrm{dt}, J=15.4,6.8 \mathrm{~Hz}, 1 \mathrm{H}$ ), 5.57 (ddt, $J=15.5,7.4,1.4 \mathrm{~Hz}, 1 \mathrm{H}$ ), 4.73 (quint, $J=6.6 \mathrm{~Hz}, 1 \mathrm{H}), 4.18(\mathrm{q}, J=7.2 \mathrm{~Hz}, 4 \mathrm{H}), 4.15-4.11(\mathrm{~m}, 4 \mathrm{H}), 4.04-3.94(\mathrm{~m}, 4 \mathrm{H}), 2.42$ (qd, $J=6.7,1.3 \mathrm{~Hz}, 2 \mathrm{H}), 1.84-1.67(\mathrm{~m}, 4 \mathrm{H}), 1.63$ (sext, $J=6.8 \mathrm{~Hz}, 4 \mathrm{H}), 1.44-1.34$ (m, $4 \mathrm{H}), 1.30$ $(\mathrm{t}, J=7.1 \mathrm{~Hz}, 6 \mathrm{H}), 1.02-0.86(\mathrm{~m}, 6 \mathrm{H})$; minor product: $5.87(\mathrm{dt}, J=15.6,5.5 \mathrm{~Hz}, 1 \mathrm{H}), 5.79$ $(\mathrm{m}, 1 \mathrm{H}), 4.83(\mathrm{~m}, 1 \mathrm{H}), 4.61(\mathrm{~d}, J=5.5 \mathrm{~Hz}, 2 \mathrm{H}), 4.18(\mathrm{q}, J=7.2 \mathrm{~Hz}, 4 \mathrm{H}), 4.15-4.11(\mathrm{~m}, 2 \mathrm{H})$, 4.04-3.94 (m, $4 \mathrm{H}), 1.90-1.83$ (m, $2 \mathrm{H}), 1.84-1.67$ (m, $4 \mathrm{H}), 1.63$ (sext, $J=6.8 \mathrm{~Hz}, 4 \mathrm{H}), 1.44-1.33$ $(\mathrm{m}, 4 \mathrm{H}), 1.30(\mathrm{t}, J=7.1 \mathrm{~Hz}, 6 \mathrm{H}), 1.02-0.86(\mathrm{~m}, 6 \mathrm{H}) ;{ }^{\mathbf{1 3}} \mathbf{C}\left\{{ }^{\mathbf{1}} \mathbf{H}\right\} \mathbf{N M R}\left(126 \mathrm{MHz}, \mathrm{CDCl}_{3}\right)$ : $\delta[\mathrm{ppm}]=$ major product: 155.3, 155.2, $131.7\left(\mathrm{~d}, J_{C P}=3.3 \mathrm{~Hz}\right), 129.3,78.7\left(\mathrm{~d}, J_{C P}=5.7 \mathrm{~Hz}\right)$, 67.5, 67.4, 66.6, 64.0, 32.4, $32.4\left(\mathrm{~d}, J_{C P}=2.3 \mathrm{~Hz}\right), 31.7,24.4,18.8,14.4$, 13.7; minor product: 155.3, 155.2, $132.8\left(\mathrm{~d}, J_{C P}=3.6 \mathrm{~Hz}\right), 126.8,77.7\left(\mathrm{~d}, J_{C P}=5.6 \mathrm{~Hz}\right), 67.4,67.3,67.0,64.1,64.0$, 32.4, $29.1\left(\mathrm{~d}, J_{C P}=5.5 \mathrm{~Hz}\right), 24.3,24.2,18.8,14.4,13.7 ;{ }^{\mathbf{3 1}} \mathbf{P}\left\{{ }^{\mathbf{1}} \mathbf{H}\right\} \mathbf{N M R}\left(203 \mathrm{MHz}, \mathrm{CDCl}_{3}\right): \delta$ $[\mathrm{ppm}]=$ major product: -1.46 ; minor product: -1.24 ; HR-ESI-MS m/z: calcd. $\mathrm{C}_{22} \mathrm{H}_{42} \mathrm{O}_{10} \mathrm{P}$ $[\mathrm{M}+\mathrm{H}]^{+}:$497.2510, found: 497.2509 .

\section{4-((Dibutoxyphosphoryl)oxy)hex-5-en-1-yl pivalate (173i)}<smiles>C=CC(CCCO[Na])OP(O)OCC</smiles><smiles>CCCOCCOP(=O)(O)OC(C)C=CCCO</smiles>

Alkene 174i (553 mg, $3.00 \mathrm{mmol}, 3.0$ equiv), dibutyl phosphate 3.4 (210 mg, $1.00 \mathrm{mmol}, 1.0$ equiv), $\mathrm{Na}_{2} \mathrm{HPO}_{4}(114 \mathrm{mg}, 800 \mu \mathrm{mol}$, 0.8 equiv), $p$-MeO-TPT (95) (49 $\mathrm{mg}, 0.10 \mathrm{mmol}, 0.1$ equiv), to diphenyl diselenide ( $31 \mathrm{mg}, 0.10 \mathrm{mmol}, 0.1$ equiv) and $4 \AA$ molecular sieves $(30 \mathrm{mg})$ were dissolved in DCE $(10 \mathrm{~mL})$ and stirred under oxygen and irradiation with blue light at room temperature for $6.5 \mathrm{~h}$. After $3 \mathrm{~h}$, more $p$-MeO-TPT $(25 \mathrm{mg}, 0.05 \mathrm{mmol}$,

0.05 equiv) was added. The solvent was removed, the residue was dissolved in a mixture of $\mathrm{Et}_{2} \mathrm{O}$ and $\mathrm{H}_{2} \mathrm{O}(1: 1)$ and the aq. phase was extracted with $\mathrm{Et}_{2} \mathrm{O}(3 \times)$. The comb. org. phases were washed with $\mathrm{H}_{2} \mathrm{O}$ and dried over $\mathrm{Na}_{2} \mathrm{SO}_{4}$. Removal of the solvent in vacuo, followed by column chromatography (DCM $\rightarrow n$-pentane/EtOAc, 25:1 to 10:1) afforded the product as a yellow liquid (mixture of the terminal alkene and the internal alkene 3.4:1; $156 \mathrm{mg}, 397 \mathrm{mmol}, 40 \%$ ). ${ }^{1} \mathrm{H}$ NMR yield $74 \%$ (1:1 internal/terminal) with 1,3,5-trimethoxybenzene $(69.6 \mathrm{mg}, 414 \mathrm{\mu mol})$ as a standard.

$\mathbf{R}_{\mathbf{f}}=0.13$ (n-pentane:EtOAc, 5:1); IR (ATR): $\tilde{\nu}\left[\mathrm{cm}^{-1}\right]=2962,2936,2874,1725,1480,1460$, 1398, 1365, 1283, 1152, 989, 771, 755, 553; $\left.{ }^{\mathbf{1}} \mathbf{H} \mathbf{~ N M R ~ ( 5 0 0 ~ M H z , ~} \mathrm{CDCl}_{3}\right): \delta[\mathrm{ppm}]=$ major 
product: $5.82(\mathrm{ddd}, J=17.2,10.4,6.9 \mathrm{~Hz}, 1 \mathrm{H}), 5.32(\mathrm{dt}, J=17.2,1.2 \mathrm{~Hz}, 1 \mathrm{H}), 5.22$ (dt, $J=10.4,1.1 \mathrm{~Hz}, 1 \mathrm{H}), 4.77(\mathrm{~m}, 1 \mathrm{H}), 4.11-4.04(\mathrm{~m}, 2 \mathrm{H}), 4.04-3.96(\mathrm{~m}, 4 \mathrm{H}), 2.01-1.69(\mathrm{~m}$, $4 \mathrm{H}), 1.66-1.58(\mathrm{~m}, 4 \mathrm{H}), 1.46-1.34(\mathrm{~m}, 4 \mathrm{H}), 1.18(\mathrm{~s}, 9 \mathrm{H}), 0.98-0.88(\mathrm{~m}, 6 \mathrm{H})$; minor product: $5.70(\mathrm{dt}, J=15.5,6.5 \mathrm{~Hz}, 1 \mathrm{H}), 5.60(\mathrm{ddt}, J=15.5,6.6,1.2 \mathrm{~Hz}, 1 \mathrm{H}), 4.88$ (sext, $J=6.5 \mathrm{~Hz}$, $1 \mathrm{H}), 4.11-4.04(\mathrm{~m}, 2 \mathrm{H}), 4.04-3.96(\mathrm{~m}, 4 \mathrm{H}), 1.66-1.58(\mathrm{~m}, 4 \mathrm{H}), 1.46-1.34(\mathrm{~m}, 7 \mathrm{H}), 1.18$ (s, $9 \mathrm{H}), 0.98-0.88(\mathrm{~m}, 6 \mathrm{H}) ;{ }^{\mathbf{1 3}} \mathbf{C}\left\{{ }^{\mathbf{1}} \mathbf{H}\right\} \mathbf{~ N M R}\left(126 \mathrm{MHz}, \mathrm{CDCl}_{3}\right): \delta[\mathrm{ppm}]=$ major product: 178.6 , $136.7\left(\mathrm{~d}, J_{C P}=3.6 \mathrm{~Hz}\right), 117.6,79.2\left(\mathrm{~d}, J_{C P}=5.8 \mathrm{~Hz}\right), 67.5\left(\mathrm{~d}, J_{C P}=1.8 \mathrm{~Hz}\right), 67.5(\mathrm{~d}$, $\left.J_{C P}=1.6 \mathrm{~Hz}\right), 64.0,38.9,32.5,32.4\left(\mathrm{~d}, J_{C P}=2.7 \mathrm{~Hz}\right), 32.4\left(\mathrm{~d}, J_{C P}=2.2 \mathrm{~Hz}\right), 27.3,24.2$, $18.8\left(\mathrm{~d}, J_{C P}=0.7 \mathrm{~Hz}\right), 13.7$; minor product: 178.6, $133.0\left(\mathrm{~d}, J_{C P}=5.3 \mathrm{~Hz}\right), 128.3,75.5(\mathrm{~d}$, $\left.J_{C P}=5.5 \mathrm{~Hz}\right), 67.5\left(\mathrm{~d}, J_{C P}=1.8 \mathrm{~Hz}\right), 67.5\left(\mathrm{~d}, J_{C P}=1.6 \mathrm{~Hz}\right), 63.2,38.9,32.4\left(\mathrm{~d}, J_{C P}=\right.$ $2.7 \mathrm{~Hz}), 32.4\left(\mathrm{~d}, J_{C P}=2.2 \mathrm{~Hz}\right), 31.6,27.3,22.4\left(\mathrm{~d}, J_{C P}=4.8 \mathrm{~Hz}\right), 18.8\left(\mathrm{~d}, J_{C P}=0.7 \mathrm{~Hz}\right)$, 13.7; ${ }^{31} \mathbf{P}\left\{{ }^{1} \mathbf{H}\right\}$ NMR $\left(203 \mathrm{MHz}, \mathrm{CDCl}_{3}\right): \delta$ ppm]= major product: -1.42 ; minor product: -1.53; HR-ESI-MS m/z: calcd. $\mathrm{C}_{19} \mathrm{H}_{38} \mathrm{O}_{6} \mathrm{P}[\mathrm{M}+\mathrm{H}]^{+}$: 393.2401, found: 393.2400 .

\subsection{Light-driven intermolecular amination}

\section{(Z)-2-(Cyclooct-2-en-1-yl)benzo[d]isothiazol-3(2H)-one 1,1-dioxide (185a) and $(Z)-3-($ Cyclooct-2-en-1-yloxy)benzo[ $d$ ] isothiazole 1,1-dioxide (185b)}

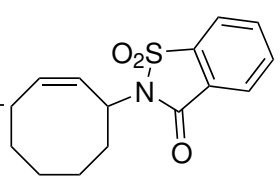

$185 a$

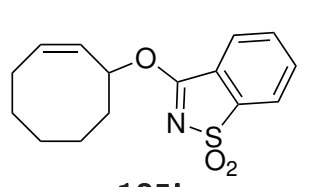

185b

Cyclooctene (55.0 mg, 500 pmol, 1.0 equiv), saccharin (275 mg, $1.50 \mathrm{mmol}, 3.0$ equiv), $\mathrm{CaF}_{2}$ (98.0 mg, $1.25 \mathrm{mmol}, 2.5$ equiv), diphenyl diselenide (16 mg, 50 umol, 0.1 equiv) and p-MeO-TPT (12 mg, $25 \mathrm{\mu mol}$, 0.05 equiv) were dissolved in acetonitrile- $d_{3}(5 \mathrm{~mL})$ and stirred under air and irradiation with blue light at room temperature for $16 \mathrm{~h}$. The solvent was removed in vacuo and the products afforded via column chromatography ( $n$-pentane/EtOAc, 7:1; 185a: white solid, $32 \mathrm{mg}, 0.11 \mathrm{mmol}, 22 \%$; 185b: white solid, $29 \mathrm{mg}, 0.10 \mathrm{mmol}, 20 \%)$.

185a: $\mathbf{R}_{\mathbf{f}}=0.54$ ( $n$-pentane:EtOAc, 5:1); IR (ATR): $\tilde{\nu}\left[\mathrm{cm}^{-1}\right]=2928,2856,1726,1461,1333$, 1292, 1252, 1179, 1126, 1061, 1049, 785, 750, 719, 676, 589, 575, 537, 508, 385; ${ }^{\mathbf{1}} \mathbf{H}$ NMR $\left(400 \mathrm{MHz}, \mathrm{CDCl}_{3}\right): \delta[\mathrm{ppm}]=8.04(\mathrm{~m}, 1 \mathrm{H}), 7.98-7.65(\mathrm{~m}, 3 \mathrm{H}), 6.04$ (ddd, $J=10.6,8.4$, $1.4 \mathrm{~Hz}, 1 \mathrm{H}$ ), 5.84 (dddd, $J=10.5,9.0,7.4,1.4 \mathrm{~Hz}, 1 \mathrm{H}$ ), 5.21 (dddd, $J=12.7,8.4,4.2,1.4 \mathrm{~Hz}$, $1 \mathrm{H}), 2.52(\mathrm{~m}, 1 \mathrm{H}), 2.37$ (dddd, $J=20.5,7.4,5.7,3.9 \mathrm{~Hz}, 2 \mathrm{H}$ ), 2.19 (dddd, $J=13.5,7.0$, $4.7,3.6 \mathrm{~Hz}, 1 \mathrm{H}), 2.02(\mathrm{ddt}, J=13.3,8.8,4.4 \mathrm{~Hz}, 1 \mathrm{H}), 1.84-1.32(\mathrm{~m}, 5 \mathrm{H}) ;{ }^{13} \mathbf{C}\left\{{ }^{1} \mathbf{H}\right\} \mathbf{N M R}$ $\left(125 \mathrm{MHz}, \mathrm{CDCl}_{3}\right): \delta[\mathrm{ppm}]=158.6,137.7,135.3,134.6,134.1,131.7,126.8,124.9,120.6,52.5$, 33.6, 29.1, 26.1, 26.0, 24.8.; HR-ESI-MS m/z: calcd. $\mathrm{C}_{15} \mathrm{H}_{17} \mathrm{NO}_{3} \mathrm{SNa}[\mathrm{M}+\mathrm{Na}]^{+}: 314.0821$, found: 314.0818 .

185b: $\mathbf{R}_{\mathbf{f}}=0.50(n$-pentane:EtOAc, $5: 1) ; \mathbf{m . p .}=150{ }^{\circ} \mathrm{C} ; \mathbf{I R}(\mathrm{ATR}): \tilde{\nu}\left[\mathrm{cm}^{-1}\right]=2928,2856,1728$, 1611, 1548, 1455, 1390, 1327, 1257, 1167, 1053, 1007, 920, 887, 868, 847, 785, 769, 750, 714, 701, 673, 651, 612, 575, 537, 453, 397; $\left.{ }^{\mathbf{1}} \mathbf{H} \mathbf{~ N M R ~ ( 6 0 0 ~ M H z , ~} \mathrm{CDCl}_{3}\right): \delta[\mathrm{ppm}]=7.87(\mathrm{~m}, 1 \mathrm{H})$, $7.78-7.67(\mathrm{~m}, 3 \mathrm{H}), 6.05(\mathrm{~m}, 1 \mathrm{H}), 5.79$ (dddd, $J=10.9,9.0,7.3,1.6 \mathrm{~Hz}, 1 \mathrm{H}), 5.64$ (ddd, 
$J=10.9,7.0,1.3 \mathrm{~Hz}, 1 \mathrm{H}), 2.33-2.10(\mathrm{~m}, 3 \mathrm{H}), 1.77-1.52(\mathrm{~m}, 7 \mathrm{H}), 1.43(\mathrm{~m}, 1 \mathrm{H}) ;{ }^{13} \mathbf{C}\left\{{ }^{\mathbf{1}} \mathbf{H}\right\}$ NMR $\left(125 \mathrm{MHz}, \mathrm{CDCl}_{3}\right): \delta[\mathrm{ppm}]=168.4,143.5,134.0,133.4,131.6,128.4,127.5,123.4,121.8$, 81.2, 34.8, 28.8, 26.7, 25.9, 23.4; HR-ESI-MS m/z: calcd. $\mathrm{C}_{15} \mathrm{H}_{17} \mathrm{NO}_{3} \mathrm{SNa}[\mathrm{M}+\mathrm{Na}]^{+}: 314.0821$, found: 314.0820 .

\section{4-Methyl- $N$-phenylbenzenesulfonamide (188)[163]}

Aniline (373 mg, $4.00 \mathrm{mmol}, 1.0$ equiv) and triethyl amine (607 mg, $6.00 \mathrm{mmol}$,

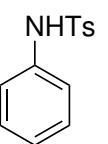

188

1.5 equiv) were dissolved in DCM $(11 \mathrm{~mL})$ and cooled to $0{ }^{\circ} \mathrm{C}$. Tosyl chloride $(915 \mathrm{mg}$, $4.80 \mathrm{mmol}, 1.2$ equiv) was added portionwise and the solution stirred for $1 \mathrm{~h}$ at room temperature. The reaction was quenched by addition of $1 \mathrm{M}$ aq. $\mathrm{HCl}$ solution $(10 \mathrm{~mL})$, the aq. phase was extracted with DCM $(2 \times 10 \mathrm{~mL})$, the comb. org. phases were dried over $\mathrm{Na}_{2} \mathrm{SO}_{4}$ and the solvent was removed under vacuum. Column chromatography ( $n$-pentane/EtOAc, 5:1) afforded the product as a white solid (989 mg, $4.00 \mathrm{mmol},>99 \%$ ).

$\mathbf{R}_{\mathbf{f}}=0.26$ (n-pentane:EtOAc, 5:1); m.p. $=96{ }^{\circ} \mathrm{C} ; \mathbf{I R}(\mathrm{ATR}): \tilde{\nu}\left[\mathrm{cm}^{-1}\right]=3248,1597,1482,1415$, 1337, 1319, 1289, 1154, 1122, 1089, 911, 823, 810, 755, 694, 659, 630, 562, 539, 506, 483; ${ }^{\mathbf{1}} \mathbf{H}$ $\operatorname{NMR}\left(300 \mathrm{MHz}, \mathrm{CDCl}_{3}\right): \delta[\mathrm{ppm}]=7.68(\mathrm{~d}, J=8.3 \mathrm{~Hz}, 2 \mathrm{H}), 7.24-7.14(\mathrm{~m}, 4 \mathrm{H}), 7.11-6.99(\mathrm{~m}$, $3 \mathrm{H}), 2.37(\mathrm{~s}, 3 \mathrm{H}) ;{ }^{\mathbf{1 3}} \mathbf{C}\left\{{ }^{\mathbf{1}} \mathbf{H}\right\} \mathbf{N M R}\left(100 \mathrm{MHz}, \mathrm{CDCl}_{3}\right): \delta[\mathrm{ppm}]=143.9,136.6,136.1,129.7$, 129.3, 127.3, 125.3, 121.6, 21.7; HR-ESI-MS m/z: calcd. $\mathrm{C}_{13} \mathrm{H}_{14} \mathrm{NO}_{2} \mathrm{~S}[\mathrm{M}+\mathrm{H}]^{+}: 248.0740$, found: 248.0740 . The data were in agreement with literature. $\underline{[163]}$

\section{4-Methyl- $N$-(tosyloxy)benzenesulfonamide (190)}

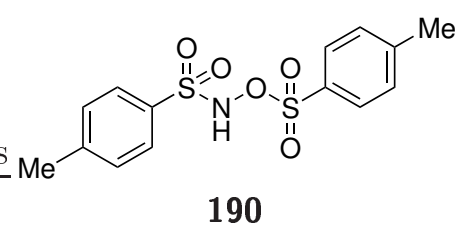

Hydroxylamine hydrochloride (347 mg, $5.00 \mathrm{mmol}$, 1.0 equiv) was dissolved in pyridine $(5 \mathrm{~mL})$ and cooled to $0{ }^{\circ} \mathrm{C}$. DMAP (61 mg, $0.50 \mathrm{mmol}, 0.1$ equiv) was added and tosyl chloride $(2.00 \mathrm{~g}$, $10.5 \mathrm{mmol}, 2.1$ equiv) was added portionwise. The reaction mixture was stirred at room temperature for $5 \mathrm{~h}$, EtOAc $(5 \mathrm{~mL})$ and

aq. $1 \mathrm{M} \mathrm{HCl}$ solution $(5 \mathrm{~mL})$ were added. The org. phase was washed with aq. $1 \mathrm{M} \mathrm{HCl}$ solution $(4 \times 7 \mathrm{~mL})$, the aq. phase was extracted with EtOAc $(2 \times 15 \mathrm{~mL})$ and the org. phase was dried over $\mathrm{Na}_{2} \mathrm{SO}_{4}$. Removal of the solvent in vacuo, followed by column chromatography (DCM/EtOAc, 50:1) afforded the product as a white solid (304 mg, $890 \mu \mathrm{mol}, 18 \%$ ).

$\mathbf{R}_{\mathbf{f}}=0.21$ (DCM:EtOAc, 50:1); m.p. $=155{ }^{\circ} \mathrm{C} ; \mathbf{I R}(\mathrm{ATR}): \tilde{\nu}\left[\mathrm{cm}^{-1}\right]=3194,1597,1374,1294$, 1267, 1194, 1169, 1121, 1088, 1019, 975, 910, 812, 776, 732, 681, 661, 574, 530, 469; ${ }^{\mathbf{1}} \mathbf{H}$ NMR $\left(300 \mathrm{MHz}, \mathrm{CDCl}_{3}\right): \delta[\mathrm{ppm}]=7.88-7.78(\mathrm{~m}, 2 \mathrm{H}), 7.76-7.67(\mathrm{~m}, 2 \mathrm{H}), 7.54(\mathrm{~s}, 1 \mathrm{H}), 7.44-7.28$ $(\mathrm{m}, 4 \mathrm{H}), 2.46(\mathrm{~d}, J=5.1 \mathrm{~Hz}, 6 \mathrm{H}) ;{ }^{\mathbf{1 3}} \mathbf{C}\left\{{ }^{\mathbf{1}} \mathbf{H}\right\} \mathbf{N M R}\left(100 \mathrm{MHz}, \mathrm{CDCl}_{3}\right): \delta[\mathrm{ppm}]=146.5$, 146.1, 132.3, 130.1, 129.9, 129.9, 129.0, 22.0, 21.9; HR-ESI-MS m/z: calcd. $\mathrm{C}_{14} \mathrm{H}_{15} \mathrm{NO}_{5} \mathrm{~S}_{2} \mathrm{Na}$ $[\mathrm{M}+\mathrm{Na}]^{+}: 364.0284$, found: 364.0295 . The data were in agreement with literature. $[164]$ 


\section{(Z)-N-(Cyclooct-2-en-1-yl)- N-(phenylsulfonyl)benzenesulfonamide (336)}

Cyclooctene (55.0 mg, 500 umol, 1.0 equiv), dibenzensulfonimide (446 mg,

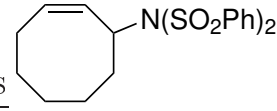

336

$1.50 \mathrm{mmol}, 3.0$ equiv), $\mathrm{CaF}_{2}$ (98.0 mg, $1.25 \mathrm{mmol}, 2.5$ equiv), diphenyl diselenide (16 mg, 50 umol, 0.1 equiv) and p-MeO-TPT (95) (12 mg, 25 umol, 0.05 equiv) were dissolved in acetonitrile- $d_{3}(5 \mathrm{~mL})$ and stirred under air and irradiation with blue light at room temperature for $16 \mathrm{~h}$. The solvent was removed in vacuo and the product afforded via column chromatography as a white solid (7.5 mg, 18 umol, 4\%).

$\mathbf{R}_{\mathbf{f}}=0.43$ (n-pentane:EtOAc, 7:1); m.p. $=124{ }^{\circ} \mathrm{C}$; IR (ATR) $: \tilde{\nu}\left[\mathrm{cm}^{-1}\right]=2922,2855,1711,1446$, 1377, 1349, 1289, 1157, 1081, 1021, 1001, 981, 898, 847, 833, 797, 756, 743, 720, 684, 614, 583, $548,475,388 ;{ }^{1} \mathbf{H}$ NMR $\left(300 \mathrm{MHz}, \mathrm{CDCl}_{3}\right): \delta[\mathrm{ppm}]=8.31-7.91(\mathrm{~m}, 4 \mathrm{H}), 7.79-7.40(\mathrm{~m}, 6 \mathrm{H})$, $5.98(\mathrm{dd}, J=10.6,8.5 \mathrm{~Hz}, 1 \mathrm{H}), 5.62(\mathrm{dt}, J=10.2,8.8 \mathrm{~Hz}, 1 \mathrm{H}), 5.11$ (ddd, $J=12.1,8.5$, $3.9 \mathrm{~Hz}, 1 \mathrm{H}), 2.38(\mathrm{td}, J=12.3,6.0 \mathrm{~Hz}, 1 \mathrm{H}), 2.24-1.98$ (m, $2 \mathrm{H}), 1.84-1.02$ (m, $7 \mathrm{H})$; HRESI-MS m/z: calcd. $\mathrm{C}_{20} \mathrm{H}_{23} \mathrm{NO}_{4} \mathrm{~S}_{2} \mathrm{Na}[\mathrm{M}+\mathrm{Na}]^{+}:$428.0961, found: 428.0952. The data were in agreement with literature. $\underline{\underline{241}]}$

\section{Sodium bis(phenylsulfonyl)amide (337)}

Dibenzene sulfonimide (500 mg, $1.7 \mathrm{mmol}, 1.0$ equiv) and $\mathrm{NaOH}$ (67 mg,

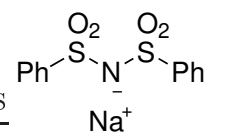
1.68 mmol, 1.0 equiv) were dissolved in a mixture of acetone and $\mathrm{H}_{2} \mathrm{O}$ (1:1; $2 \mathrm{~mL}$ ) and stirred for $16 \mathrm{~h}$ at room temperature. Removal of the solvent in vacuo 337 afforded the product as a white solid (526 $\mathrm{mg}, 1.65 \mathrm{mmol}, 98 \%$ ),

IR (ATR): $\tilde{\nu}\left[\mathrm{cm}^{-1}\right]=3061,1447,1366,1269,1159,1134,1082,998,874,791,751,721,686$, $625,575,553 ;{ }^{1} \mathbf{H}$ NMR $\left(300 \mathrm{MHz}, \mathrm{D}_{2} \mathrm{O}\right): \delta[\mathrm{ppm}]=7.64-7.54(\mathrm{~m}, 4 \mathrm{H}), 7.54-7.42(\mathrm{~m}, 2 \mathrm{H})$, 7.43-7.29 (m, $4 \mathrm{H})$; HR-ESI-MS m/z: calcd. $\mathrm{C}_{12} \mathrm{H}_{10} \mathrm{NO}_{4} \mathrm{Na}_{2} \mathrm{~S}_{2}[\mathrm{M}+\mathrm{Na}]^{+}:$341.9841, found: 341.9843. The data were in agreement with literature.165]

\section{2-Methyl-1-tosyl-1 $H$-indole (193)}

4-Methyl- $N$-(2-(prop-1-en-1-yl)phenyl) benzenesulfonamide (72 mg, $0.25 \mathrm{mmol}$,

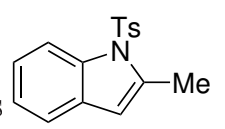

193 1.0 equiv), diphenyl diselenide ( $7.8 \mathrm{mg}, 25 \mathrm{\mu mol}, 0.1$ equiv) and $p$-MeO-TPT (95) (12 mg, 25 umol, 0.1 equiv) were dissolved in MeCN (2.5 mL) and stirred under air and irradiation with blue light at room temperature for $16 \mathrm{~h}$. Removal of the solvent in vacuo, followed by column chromatography ( $n$-pentane/EtOAc, 20:1) afforded the product as a white solid (12 mg, $43 \mu \mathrm{mol}, 17 \%)$.

$\mathbf{R}_{\mathbf{f}}=0.43$ (n-pentane:EtOAc, 10:1); ${ }^{1} \mathbf{H}$ NMR $\left(300 \mathrm{MHz}, \mathrm{CDCl}_{3}\right): \delta[\mathrm{ppm}]=8.18(\mathrm{~m}, 1 \mathrm{H})$, $7.82-7.58(\mathrm{~m}, 3 \mathrm{H}), 7.40(\mathrm{dd}, J=7.7,1.5 \mathrm{~Hz}, 1 \mathrm{H}), 7.25-7.00(\mathrm{~m}, 7 \mathrm{H}), 6.34(\mathrm{~m}, 1 \mathrm{H}), 2.60(\mathrm{~d}$, $J=1.1 \mathrm{~Hz}, 3 \mathrm{H}), 2.34(\mathrm{~s}, 3 \mathrm{H})$. The analytical data was in agreement with literature. $\underline{65]}$ 


\subsection{Synthesis of tetrahydroisoquinolines}

\section{General procedure $H$; Suzuki reaction $[180]$}

Under an atmosphere of argon, the aryl halide (1.0 equiv), the respective boronic acid (1.1 equiv) and potassium carbonate (4.0 equiv) are added to a mixture of toluene, ethanol and water (0.08 $\mathrm{M}, 5: 2: 1)$ and the suspension is degassed with argon for $30 \mathrm{~min}$. Tetrakis[triphenylphosphine]palladium(0) (0.1 equiv) is added and the mixture is heated to $100{ }^{\circ} \mathrm{C}$. After completion of the reaction, detected by NMR and TLC, the mixture is allowed to cool down to room temperature, EtOAc is added and the org. phase is washed with $\mathrm{H}_{2} \mathrm{O}$ and sat. aq. $\mathrm{NaCl}$ solution. The aq. phase is extracted with EtOAc, the combined org. phases are dried over $\mathrm{Na}_{2} \mathrm{SO}_{4}$ and the solvent is removed under reduced pressure. The residue is purified on silica gel to yield the title compound.

\section{General procedure [ nitrile reduction}

A solution of lithium aluminum hydride $\left(2.5\right.$ equiv) in diethyl ether $(0.35 \mathrm{M})$ is cooled to $0{ }^{\circ} \mathrm{C}$ and a solution of the respective nitrile (1.0 equiv) in diethyl ether $(0.4 \mathrm{M})$ is added dropwise. The solution is allowed to warm to room temperature and stirred until completion is detected via NMR and TLC. The reaction is quenched by addition of sodium sulfate decahydrate, filtered over celite and the residue is washed with EtOAc. Removal of the solvent affords the product, which is used without further purification.

\section{General procedure Џ: tosyl protection of amines $[181]$}

The amine (1.0 equiv) and $\mathrm{NEt}_{3}$ (1.1 equiv) are dissolved in DCM (0.5 M for the amine). Tosyl chloride (1.05 equiv) in DCM $(1.05 \mathrm{M})$ is added and the solution is stirred at room temperature until completion is detected via NMR and TLC. The reaction is quenched by the addition of $\mathrm{NH}_{4} \mathrm{Cl}$, the phases are separated and the org. phase is washed with sat. aq. $\mathrm{NaCl}$ solution. The combined aq. phases are reextracted with DCM, the combined org. phases were dried over $\mathrm{Na}_{2} \mathrm{SO}_{4}$ and the solvent is removed under reduced pressure. The residue is purified on silica gel to yield the title compound.

\subsubsection{Synthesis of ortho-vinyl homobenzylamides 207}

\section{(E)-2-(2-(Pent-1-en-1-yl)phenyl)acetonitrile (205a)}

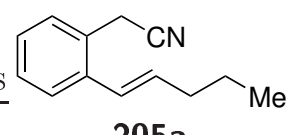

205a

Following general procedure $\mathbf{H}$ 2-bromophenylacetonitrile (1.96 g, $10.0 \mathrm{mmol}, 1.0$ equiv), 1-pentenylboronic acid (1.25 g, $11.0 \mathrm{mmol}, 1.1$ equiv), potassium carbonate $(5.53 \mathrm{~g}, 40.0 \mathrm{mmol}, 4.0$ equiv), toluene $(77.5 \mathrm{~mL})$, ethanol $(31 \mathrm{~mL})$, water $(15.5 \mathrm{~mL})$, tetrakis[triphenylphosphine]palladium(0) (1.2 g, $1.0 \mathrm{mmol}, 0.1$ equiv); reaction time $14.5 \mathrm{~h}$; eluting with $n$-pentane/EtOAc, 20:1; yellow oil; $1.78 \mathrm{~g}, 9.58 \mathrm{mmol}, 96 \%$. 
$\mathbf{R}_{\mathbf{f}}=0.29$ (n-pentane:EtOAc, 20:1); ${ }^{\mathbf{1}} \mathbf{H}$ NMR $\left(300 \mathrm{MHz}, \mathrm{CDCl}_{3}\right): \delta[\mathrm{ppm}]=7.45(\mathrm{dd}, J=7.6$, $1.7 \mathrm{~Hz}, 1 \mathrm{H}), 7.39-7.17(\mathrm{~m}, 3 \mathrm{H}), 6.48(\mathrm{dt}, J=15.6,1.6 \mathrm{~Hz}, 1 \mathrm{H}), 6.14(\mathrm{dt}, J=15.5,6.9 \mathrm{~Hz}, 1 \mathrm{H})$, $3.74(\mathrm{~s}, 2 \mathrm{H}), 2.24$ (qd, $J=7.2,1.5 \mathrm{~Hz}, 2 \mathrm{H}), 1.53$ (sext, $J=7.3 \mathrm{~Hz}, 2 \mathrm{H}), 0.98$ (t, $J=7.4 \mathrm{~Hz}$, $3 \mathrm{H})$. The analytical data are in agreement with literature. 185$]$

\section{(E)-2-(4,5-Dimethoxy-2-(pent-1-en-1-yl)phenyl)acetonitrile (205b)}

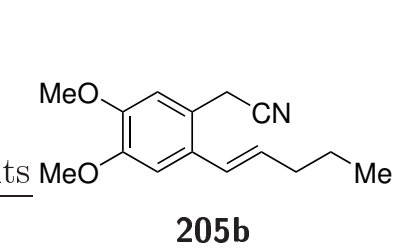

Following general procedure [H] 2-(2-bromo-4,5dimethoxyphenyl)acetonitrile (250 mg, $976 \mu \mathrm{mol}, 1.0$ equiv), 1pentenylboronic acid (122 mg, $1.07 \mathrm{mmol}, 1.1$ equiv), potassium carbonate (540 mg, $3.90 \mathrm{mmol}, 4.0$ equiv), toluene $(7.5 \mathrm{~mL})$, ethanol $(3.0 \mathrm{~mL})$, water $(1.5 \mathrm{~mL})$, tetrakis[triphenylphosphine]palladium(0) (113 mg, 97.8 pmol, 0.1 equiv); reaction time $16.5 \mathrm{~h}$; eluting with $n$-pentane/EtOAc, 5:1; yellow oil; $202 \mathrm{mg}$, $823 \mu \mathrm{mol}, 84 \%$.

$\mathbf{R}_{\mathbf{f}}=0.29$ (n-pentane:EtOAc, 5:1); IR (ATR): $\tilde{\nu}\left[\mathrm{cm}^{-1}\right]=3001,2958,2932,2870,2834,2249$, 1607, 1514, 1463, 1270, 1202, 1181, 1097, 1000, 962, 861, 751; ${ }^{\mathbf{1}} \mathbf{H} \mathbf{N M R}\left(500 \mathrm{MHz}, \mathrm{CDCl}_{3}\right): \delta$ $[\mathrm{ppm}]=6.93(\mathrm{~s}, 1 \mathrm{H}), 6.83(\mathrm{~s}, 1 \mathrm{H}), 6.40(\mathrm{dt}, J=15.5,1.5 \mathrm{~Hz}, 1 \mathrm{H}), 6.04(\mathrm{dt}, J=15.5,6.9 \mathrm{~Hz}$, $1 \mathrm{H}), 3.90(\mathrm{~s}, 1 \mathrm{H}), 3.90(\mathrm{~s}, 1 \mathrm{H}), 3.69(\mathrm{~s}, 2 \mathrm{H}), 2.27-2.16(\mathrm{~m}, 2 \mathrm{H}), 1.62-1.42(\mathrm{~m}, 2 \mathrm{H}), 0.97$ (t, $J=7.4 \mathrm{~Hz}, 3 \mathrm{H}) ;{ }^{\mathbf{1 3}} \mathbf{C}\left\{{ }^{\mathbf{1}} \mathbf{H}\right\} \mathbf{N M R}\left(126 \mathrm{MHz}, \mathrm{CDCl}_{3}\right): \delta[\mathrm{ppm}]=149.1,148.6,133.9,129.8$, 125.8, 118.9, 118.2, 111.9, 109.8, 56.2, 56.1, 35.5, 22.7, 21.4, 13.9; HR-ESI-MS m/z: calcd. $\mathrm{C}_{14} \mathrm{H}_{19} \mathrm{NO}_{2} \mathrm{Na}[\mathrm{M}+\mathrm{Na}]^{+}:$268.1308, found: 268.1310 .

\section{(E)-2-(4-Fluoro-2-(pent-1-en-1-yl)phenyl)acetonitrile (205c)}

Following general procedure [H] 2-(2-bromo-4-fluorophenyl)acetonitrile

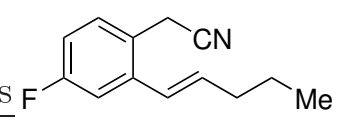

205c

(300 mg, $1.40 \mathrm{mmol}, 1.0$ equiv), 1-pentenylboronic acid (176 mg, $1.54 \mathrm{mmol}, 1.1$ equiv), potassium carbonate $(775 \mathrm{mg}, 5.61 \mathrm{mmol}$, 4.0 equiv), toluene $(10 \mathrm{~mL})$, ethanol $(4.0 \mathrm{~mL})$, water $(2 \mathrm{~mL})$, tetrakis[triphenylphosphine]palladium(0) (162 mg, 140 umol, 0.1 equiv); reaction time 16.5 h; eluting with $n$-pentane/EtOAc, 30:1; yellow oil; $235 \mathrm{mg}, 1.16 \mathrm{mmol}, 83 \%$.

$\mathbf{R}_{\mathbf{f}}=0.29$ (n-pentane:EtOAc, 20:1); IR (ATR): $\tilde{\nu}\left[\mathrm{cm}^{-1}\right]=2961,2931,2872,2250,1612,1584$, 1491, 1423, 1273, 1242, 1171, 1159, 962, 872, 806, 407; ${ }^{\mathbf{1}} \mathbf{H} \mathbf{N M R}\left(500 \mathrm{MHz}, \mathrm{CDCl}_{3}\right): \delta$ $[\mathrm{ppm}]=7.31(\mathrm{dd}, J=8.5,5.6 \mathrm{~Hz}, 1 \mathrm{H}), 7.15(\mathrm{dd}, J=9.9,2.7 \mathrm{~Hz}, 1 \mathrm{H}), 6.94(\mathrm{td}, J=8.2,2.7 \mathrm{~Hz}$, $1 \mathrm{H}), 6.43(\mathrm{dq}, J=15.5,1.5 \mathrm{~Hz}, 1 \mathrm{H}), 6.17(\mathrm{dt}, J=15.4,6.9 \mathrm{~Hz}, 1 \mathrm{H}), 3.69(\mathrm{~s}, 2 \mathrm{H}), 2.24$ (qd, $J=7.1,1.5 \mathrm{~Hz}, 2 \mathrm{H}), 1.52$ (sext, $J=7.4 \mathrm{~Hz}, 2 \mathrm{H}), 0.97(\mathrm{t}, J=7.4 \mathrm{~Hz}, 3 \mathrm{H}) ;{ }^{13} \mathbf{C}\left\{{ }^{\mathbf{1}} \mathbf{H}\right\} \mathbf{N M R}$ $\left(126 \mathrm{MHz}, \mathrm{CDCl}_{3}\right): \delta[\mathrm{ppm}]=162.9\left(\mathrm{~d},{ }^{1} J_{\mathrm{CF}}=246.7 \mathrm{~Hz}\right), 139.4\left(\mathrm{~d},{ }^{3} J_{\mathrm{CF}}=8.0 \mathrm{~Hz}\right), 136.9$, $130.6\left(\mathrm{~d},{ }^{3} J_{\mathrm{CF}}=8.7 \mathrm{~Hz}\right), 125.2\left(\mathrm{~d},{ }^{4} J_{\mathrm{CF}}=2.2 \mathrm{~Hz}\right), 122.5\left(\mathrm{~d},{ }^{4} J_{\mathrm{CF}}=3.0 \mathrm{~Hz}\right), 117.7,114.4(\mathrm{~d}$, $\left.{ }^{2} J_{\mathrm{CF}}=21.9 \mathrm{~Hz}\right), 113.6\left(\mathrm{~d},{ }^{2} J_{\mathrm{CF}}=22.4 \mathrm{~Hz}\right), 35.4,22.4,21.4,13.9 ;$ HR-ESI-MS m/z: calcd. $\mathrm{C}_{13} \mathrm{H}_{15} \mathrm{FN}[\mathrm{M}+\mathrm{H}]^{+}:$204.1183, found: 204.1175 . 


\section{(E)-2-(5-Fluoro-2-(pent-1-en-1-yl)phenyl)acetonitrile (205d)}

Following general procedure [H] 2-(2-bromo-5-fluorophenyl)acetonitrile<smiles>CCC/C=C/c1ccc(F)cc1CC#N</smiles>

205d (300 mg, $1.40 \mathrm{mmol}, 1.0$ equiv), 1-pentenylboronic acid (176 mg, $1.54 \mathrm{mmol}, 1.1$ equiv), potassium carbonate $(775 \mathrm{mg}, 5.61 \mathrm{mmol}$, 4.0 equiv), toluene $(10 \mathrm{~mL})$, ethanol $(4.0 \mathrm{~mL})$, water $(2 \mathrm{~mL})$, tetrakis[triphenylphosphine]palladium(0) (162 mg, 140 umol, 0.1 equiv); reaction time $16.5 \mathrm{~h}$; eluting with n-pentane/EtOAc, 30:1; yellow oil; $242 \mathrm{mg}, 1.19 \mathrm{mmol}, 85 \%$.

$\mathbf{R}_{\mathbf{f}}=0.26$ (n-pentane:EtOAc, 30:1); IR (ATR): $\tilde{\nu}\left[\mathrm{cm}^{-1}\right]=2960,2931,2873,2251,1610,1586$, 1492, 1463, 1426, 1250, 1186, 1150, 965, 861, 832, 801, 731, 449; ${ }^{\mathbf{1}} \mathbf{H}$ NMR (400 MHz, $\left.\mathrm{CDCl}_{3}\right)$ : $\delta[\mathrm{ppm}]=7.40(\mathrm{dd}, J=8.6,5.7 \mathrm{~Hz}, 1 \mathrm{H}), 7.11(\mathrm{dd}, J=9.2,2.7 \mathrm{~Hz}, 1 \mathrm{H}), 7.00(\operatorname{dddd}, J=8.7$, $8.1,2.7,0.5 \mathrm{~Hz}, 1 \mathrm{H}), 6.39(\mathrm{dd}, J=15.5,0.7 \mathrm{~Hz}, 1 \mathrm{H}), 6.07$ (dt, $J=15.5,6.9 \mathrm{~Hz}, 1 \mathrm{H}), 3.72$ $(\mathrm{s}, 2 \mathrm{H}), 2.22$ (qd, $J=7.1,1.5 \mathrm{~Hz}, 2 \mathrm{H}), 1.51$ (sext, $J=7.4 \mathrm{~Hz}, 2 \mathrm{H}$ ), 0.97 (t, $J=7.4 \mathrm{~Hz}$, $3 \mathrm{H}) ;{ }^{13} \mathbf{C}\left\{{ }^{1} \mathbf{H}\right\} \mathbf{N M R}\left(125 \mathrm{MHz}, \mathrm{CDCl}_{3}\right): \delta[\mathrm{ppm}]=162.0\left(\mathrm{~d},{ }^{1} J_{\mathrm{CF}}=247.5 \mathrm{~Hz}\right), 135.8(\mathrm{~d}$, $\left.{ }^{4} J_{\mathrm{CF}}=1.8 \mathrm{~Hz}\right), 133.46\left(\mathrm{~d},{ }^{4} J_{\mathrm{CF}}=3.5 \mathrm{~Hz}\right), 128.8\left(\mathrm{~d},{ }^{3} J_{\mathrm{CF}}=8.0 \mathrm{~Hz}\right), 128.6\left(\mathrm{~d},{ }^{3} J_{\mathrm{CF}}=7.6 \mathrm{~Hz}\right)$, $125.04,117.25,115.8\left(\mathrm{~d},{ }^{2} J_{\mathrm{CF}}=23.1 \mathrm{~Hz}\right), 115.6\left(\mathrm{~d},{ }^{2} J_{\mathrm{CF}}=21.0 \mathrm{~Hz}\right), 35.44,22.53,21.95(\mathrm{~d}$, $\left.{ }^{4} J_{\mathrm{CF}}=1.7 \mathrm{~Hz}\right), 13.83$; HR-ESI-MS m/z: calcd. $\mathrm{C}_{13} \mathrm{H}_{14} \mathrm{FNNa}[\mathrm{M}+\mathrm{Na}]^{+}: 226.1002$, found: 226.1006.

\section{(E)-2-(2-(Pent-1-en-1-yl)-4-(trifluoromethyl)phenyl)acetonitrile (205e)}

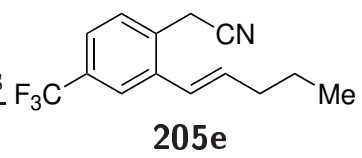

(1.75 mL), tetrakis[triphenylphosphine]palladium(0) (131 mg, 114 umol, 0.1 equiv); reaction time 16.5 h; eluting with $n$-pentane/EtOAc, 30:1; light yellow oil; $240 \mathrm{mg}, 946 \mu \mathrm{mol}, 83 \%$.

$\mathbf{R}_{\mathbf{f}}=0.14$ (n-pentane:EtOAc, 30:1); IR (ATR): $\tilde{\nu}\left[\mathrm{cm}^{-1}\right]=2962,2933,2874,2253,1650,1617$, 1424, 1330, 1273, 1218, 1163, 1121, 1075, 965, 824, 747, 660, 454; ${ }^{\mathbf{1}} \mathbf{H}$ NMR (400 MHz, $\left.\mathrm{CDCl}_{3}\right)$ : $\delta[\mathrm{ppm}]=7.68(\mathrm{~s}, 1 \mathrm{H}), 7.50(\mathrm{~d}, J=1.3 \mathrm{~Hz}, 2 \mathrm{H}), 6.47(\mathrm{dtd}, J=15.5,1.5,0.6 \mathrm{~Hz}, 1 \mathrm{H})$, $6.23(\mathrm{dt}, J=15.5,6.9 \mathrm{~Hz}, 1 \mathrm{H}), 3.78(\mathrm{~s}, 2 \mathrm{H}), 2.36-2.09(\mathrm{~m}, 2 \mathrm{H}), 1.54$ (sext, $J=7.3 \mathrm{~Hz}$, $2 \mathrm{H}), 0.98(\mathrm{t}, J=7.4 \mathrm{~Hz}, 3 \mathrm{H}) ;{ }^{\mathbf{1 3}} \mathbf{C}\left\{{ }^{\mathbf{1}} \mathbf{H}\right\} \mathbf{N M R}\left(100 \mathrm{MHz}, \mathrm{CDCl}_{3}\right): 138.1,137.7,131.1$ (q, $\left.{ }^{2} J_{\mathrm{CF}}=32.6 \mathrm{~Hz}\right), 130.5,129.3,124.9,124.2\left(\mathrm{q},{ }^{3} J_{\mathrm{CF}}=3.7 \mathrm{~Hz}\right), 124.0\left(\mathrm{q},{ }^{1} J_{\mathrm{CF}}=272.4 \mathrm{~Hz}\right)$, $123.8\left(\mathrm{q},{ }^{3} J_{\mathrm{CF}}=3.8 \mathrm{~Hz}\right), 117.0,35.5,22.4,22.0,13.8 ; \mathbf{H R}-\mathbf{E S I}-\mathbf{M S} \mathbf{m} / \mathbf{z}$ : calcd. $\mathrm{C}_{14} \mathrm{H}_{14} \mathrm{~F}_{3} \mathrm{NNa}$ $[\mathrm{M}+\mathrm{Na}]^{+}: 276.0971$, found: 276.0964 . 


\section{(E)-2-(2-(Pent-1-en-1-yl)-5-(trifluoromethyl)phenyl)acetonitrile (205f)}

Following general procedure $\mathbf{H}$; 2-(2-bromo-5-trifluoromethylphe-<smiles>CCCC/C=C/c1ccc(C(F)(F)F)cc1CN</smiles>

$205 f$ nyl)acetonitrile (300 mg, $1.14 \mathrm{mmol}, 1.0$ equiv), 1-pentenylboronic acid (142 mg, $1.25 \mathrm{mmol}, 1.1$ equiv), potassium carbonate $(628 \mathrm{mg}$, $4.54 \mathrm{mmol}$, 4.0 equiv), toluene $(8.75 \mathrm{~mL})$, ethanol $(3.5 .0 \mathrm{~mL})$, water $(1.75 \mathrm{~mL})$, tetrakis[triphenylphosphine]palladium(0) (131 mg, 114 mol, 0.1 equiv); reaction time $16.5 \mathrm{~h}$; eluting with $n$-pentane/EtOAc, 30:1; light yellow oil; $255 \mathrm{mg}, 1.01 \mathrm{mmol}, 88 \%$.

$\mathbf{R}_{\mathbf{f}}=0.29$ (n-pentane:EtOAc, 20:1); IR (ATR): $\tilde{\nu}\left[\mathrm{cm}^{-1}\right]=2962,2933,2875,2251,1650,1620$, 1422, 1330, 1285, 1264, 1161, 1119, 1098, 1081, 966, 882, 843; ${ }^{\mathbf{1}} \mathbf{H} \mathbf{N M R}\left(400 \mathrm{MHz}, \mathrm{CDCl}_{3}\right): \delta$ $[\mathrm{ppm}]=7.61(\mathrm{~s}, 1 \mathrm{H}), 7.56(\mathrm{~s}, 2 \mathrm{H}), 6.49(\mathrm{dt}, J=15.5,1.5 \mathrm{~Hz}, 1 \mathrm{H}), 6.25(\mathrm{dt}, J=15.5,6.9 \mathrm{~Hz}$, $1 \mathrm{H}), 3.78$ (s, $2 \mathrm{H}), 2.27$ (qd, $J=7.3,1.5 \mathrm{~Hz}, 2 \mathrm{H}), 1.61-1.45(\mathrm{~m}, 2 \mathrm{H}), 0.98(\mathrm{t}, J=7.4 \mathrm{~Hz}, 3 \mathrm{H})$; ${ }^{13} \mathbf{C}\left\{{ }^{1} \mathbf{H}\right\}$ NMR $\left(125 \mathrm{MHz}, \mathrm{CDCl}_{3}\right): \delta[\mathrm{ppm}]=140.9,138.3,129.7\left(\mathrm{q},{ }^{2} J_{\mathrm{CF}}=32.8 \mathrm{~Hz}\right), 127.5$, $127.4,125.9\left(\mathrm{q},{ }^{3} J_{\mathrm{CF}}=3.9 \mathrm{~Hz}\right), 125.6\left(\mathrm{q},{ }^{3} J_{\mathrm{CF}}=3.7 \mathrm{~Hz}\right), 124.9,123.9\left(\mathrm{q},{ }^{1} J_{\mathrm{CF}}=272.1 \mathrm{~Hz}\right)$, 117.0, 35.6, 22.4, 22.0, 13.9; HR-ESI-MS m/z: calcd. $\mathrm{C}_{14} \mathrm{H}_{14} \mathrm{~F}_{3} \mathrm{NNa}[\mathrm{M}+\mathrm{Na}]^{+}: 276.0971$, found: 276.0975 .

\section{(E)-4-Methyl-N-(2-(pent-1-en-1-yl)phenethyl)benzenesulfonamide (207a)}

Following general procedure I lithium aluminum hydride $(1.06 \mathrm{~g}, 28.0 \mathrm{mmol}$,

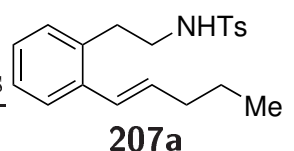

2.5 equiv) in diethyl ether ( $78 \mathrm{~mL})$, nitrile 205a ( $2.08 \mathrm{~g}, 11.2 \mathrm{mmol}, 1.0$ equiv) in diethyl ether $(27 \mathrm{~mL})$; reaction time $17 \mathrm{~h}$; yellow oil. Following general procedure J amine (1.42 g, $7.50 \mathrm{mmol}, 1.0$ equiv), $\mathrm{NEt}_{3}(835 \mathrm{mg}, 8.25 \mathrm{mmol}$, 1.1 equiv), DCM (16 mL), tosyl chloride (1.50 g, $7.88 \mathrm{mmol}, 1.05$ equiv) in DCM ( $8 \mathrm{~mL})$; reaction time $17 \mathrm{~h}$; eluting with $n$-pentane/EtOAc, 7:1; yellow oil; $1.86 \mathrm{~g}, 5.41 \mathrm{mmol}, 72 \%$.

$\mathbf{R}_{\mathbf{f}}=0.21$ (n-pentane:EtOAc, 7:1); ${ }^{\mathbf{1}} \mathbf{H}$ NMR $\left(300 \mathrm{MHz}, \mathrm{CDCl}_{3}\right): \delta[\mathrm{ppm}]=7.73-7.61(\mathrm{~m}, 2 \mathrm{H})$, $7.38(\mathrm{dd}, J=7.5,1.6 \mathrm{~Hz}, 1 \mathrm{H}), 7.31-7.23(\mathrm{~m}, 2 \mathrm{H}), 7.23-7.08(\mathrm{~m}, 2 \mathrm{H}), 7.01$ (dd, $J=7.4,1.7 \mathrm{~Hz}$, $1 \mathrm{H}), 6.47(\mathrm{~d}, J=15.4 \mathrm{~Hz}, 1 \mathrm{H}), 6.02(\mathrm{dt}, J=15.5,6.9 \mathrm{~Hz}, 1 \mathrm{H}), 4.35(\mathrm{t}, J=6.2 \mathrm{~Hz}, 1 \mathrm{H}), 3.16$ $(\mathrm{q}, J=6.9 \mathrm{~Hz}, 2 \mathrm{H}), 2.85(\mathrm{t}, J=7.1 \mathrm{~Hz}, 2 \mathrm{H}), 2.42(\mathrm{~s}, 3 \mathrm{H}), 2.16(\mathrm{qd}, J=7.1,1.5 \mathrm{~Hz}, 2 \mathrm{H})$, 1.46 (sext, $J=7.4 \mathrm{~Hz}, 2 \mathrm{H}$ ), $0.94(\mathrm{t}, J=7.3 \mathrm{~Hz}, 3 \mathrm{H}$ ). The analytical data are in agreement with literature. $\underline{\underline{[185]}}$

\section{(E)-N-(4,5-Dimethoxy-2-(pent-1-en-1-yl)phenethyl)-4-methylbenzenesulfonamide (207b)}<smiles>COc1cc(/C=C/CC[NH3+])c(CCN[NH3+])cc1OC</smiles>

207b

Following general procedure [ lithium aluminum hydride $(70 \mathrm{mg}$, $1.8 \mathrm{mmol}, 2.5$ equiv) in diethyl ether $(5 \mathrm{~mL})$, nitrile 205b (180 mg, 734 umol, 1.0 equiv) in diethyl ether $(1.7 \mathrm{~mL})$; reaction time $6 \mathrm{~h}$; colorless oil. Following general procedure J amine $(150 \mathrm{mg}, 602 \mu \mathrm{mol}$, 1.0 equiv), $\mathrm{NEt}_{3}$ (67 mg, $0.66 \mathrm{mmol}, 1.1$ equiv), DCM (1.2 mL), tosyl chloride (120 mg, 632 umol, 
1.05 equiv) in DCM $(0.6 \mathrm{~mL})$; reaction time $20 \mathrm{~h}$; eluting with $n$-pentane/EtOAc, $5: 1$; brown liquid; $68 \mathrm{mg}, 0.17 \mathrm{mmol}, 23 \%$ from nitrile.

$\mathbf{R}_{\mathbf{f}}=0.07$ (n-pentane:EtOAc, 10:1); IR (ATR): $\tilde{\nu}\left[\mathrm{cm}^{-1}\right]=3282,2957,2932,2870,2834,1603$, 1509, 1463, 1327, 1266, 1203, 1183, 1156, 1093, 1041, 1002, 964, 862, 814, 814, 752, 733, 707, $661,549,501,471 ;{ }^{\mathbf{1}} \mathbf{H} \mathbf{N M R}\left(500 \mathrm{MHz}, \mathrm{CDCl}_{3}\right): \delta[\mathrm{ppm}]=7.66(\mathrm{~d}, J=8.3 \mathrm{~Hz}, 2 \mathrm{H}), 7.26(\mathrm{~d}$, $J=7.9 \mathrm{~Hz}, 2 \mathrm{H}), 6.89(\mathrm{~s}, 1 \mathrm{H}), 6.50(\mathrm{~s}, 1 \mathrm{H}), 6.41(\mathrm{dt}, J=15.5,1.5 \mathrm{~Hz}, 1 \mathrm{H}), 5.94(\mathrm{dt}, J=15.5$, $7.0 \mathrm{~Hz}, 1 \mathrm{H}), 4.39(\mathrm{t}, J=6.2 \mathrm{~Hz}, 1 \mathrm{H}), 3.88(\mathrm{~s}, 3 \mathrm{H}), 3.82(\mathrm{~s}, 3 \mathrm{H}), 3.13(\mathrm{q}, J=6.9 \mathrm{~Hz}, 2 \mathrm{H}), 2.80$ (t, $J=7.1 \mathrm{~Hz}, 2 \mathrm{H}), 2.41$ (s, $3 \mathrm{H}), 2.15$ (qd, $J=7.2,1.5 \mathrm{~Hz}, 2 \mathrm{H}), 1.46$ (sext, $J=7.4 \mathrm{~Hz}, 2 \mathrm{H}$ ), $0.93(\mathrm{t}, J=7.4 \mathrm{~Hz}, 4 \mathrm{H}) ;{ }^{\mathbf{1 3}} \mathbf{C}\left\{{ }^{\mathbf{1}} \mathbf{H}\right\} \mathbf{N M R}\left(126 \mathrm{MHz}, \mathrm{CDCl}_{3}\right): \delta[\mathrm{ppm}]=148.3,148.1,143.5$, 137.0, 132.0, 129.8, 129.6, 127.2, 126.9, 126.6, 113.0, 109.3, 56.1, 56.0, 43.9, 35.5, 33.0, 22.8, 21.6, 13.9; HR-ESI-MS m/z: calcd. $\mathrm{C}_{22} \mathrm{H}_{29} \mathrm{NO}_{4} \mathrm{SNa}[\mathrm{M}+\mathrm{Na}]^{+}: 426.1710$, found: 426.1710 .

\section{(E)-N-(4-Fluoro-2-(pent-1-en-1-yl)phenethyl)-4-methylbenzenesulfonamide (207c)}

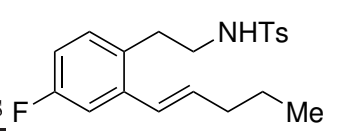

207c

Following general procedure I] lithium aluminum hydride $(107 \mathrm{mg}$, $2.83 \mathrm{mmol}, 2.5$ equiv) in diethyl ether $(8 \mathrm{~mL})$, nitrile 205c (230 mg, $1.13 \mathrm{mmol}, 1.0$ equiv) in diethyl ether $(2.7 \mathrm{~mL})$; reaction time $5 \mathrm{~h}$; yellow oil. Following general procedure J amine (200 mg, 965 umol, 1.0 equiv), $\mathrm{NEt}_{3}$ (108 mg, $1.06 \mathrm{mmol}, 1.1$ equiv), DCM (2 mL), tosyl chloride (193 mg,1.01 mmol, 1.05 equiv) in DCM (1 mL); reaction time $18 \mathrm{~h}$; eluting with $n$-pentane/EtOAc, 10:1; yellow oil; $31 \mathrm{mg}, 86 \mu \mathrm{mol}, 8 \%$ from nitrile.

$\mathbf{R}_{\mathbf{f}}=0.13$ (n-pentane:EtOAc, 10:1); IR (ATR): $\tilde{\nu}\left[\mathrm{cm}^{-1}\right]=3281,2959,2929,2872,1609,1582$, 1490, 1456, 1420, 1323, 1269, 1154, 1092, 1072, 964, 870, 812, 755, 735, 706, 659, 549, 489, $467 ;{ }^{1} \mathbf{H}$ NMR $\left(500 \mathrm{MHz}, \mathrm{CDCl}_{3}\right): \delta[\mathrm{ppm}]=7.83-7.59(\mathrm{~m}, 2 \mathrm{H}), 7.43-7.18(\mathrm{~m}, 2 \mathrm{H}), 7.06(\mathrm{dd}$, $J=10.3,2.8 \mathrm{~Hz}, 1 \mathrm{H}), 6.96(\mathrm{dd}, J=8.4,5.9 \mathrm{~Hz}, 1 \mathrm{H}), 6.79(\mathrm{td}, J=8.3,2.7 \mathrm{~Hz}, 1 \mathrm{H}), 6.42$ $(\mathrm{dq}, J=15.5,1.6 \mathrm{~Hz}, 1 \mathrm{H}), 6.04(\mathrm{dt}, J=15.5,7.0 \mathrm{~Hz}, 1 \mathrm{H}), 4.66(\mathrm{t}, J=6.2 \mathrm{~Hz}, 1 \mathrm{H}), 3.11$ $(\mathrm{q}, J=6.9 \mathrm{~Hz}, 2 \mathrm{H}), 2.80(\mathrm{t}, J=7.3 \mathrm{~Hz}, 2 \mathrm{H}), 2.41(\mathrm{~s}, 3 \mathrm{H}), 2.16(\mathrm{qd}, J=7.1,1.5 \mathrm{~Hz}, 2 \mathrm{H})$, 1.46 (sext, $J=\mathrm{Hz}, 2 \mathrm{H}), 0.93(\mathrm{t}, J=7.4 \mathrm{~Hz}, 3 \mathrm{H}) ;{ }^{13} \mathbf{C}\left\{{ }^{1} \mathbf{H}\right\} \mathbf{N M R}\left(126 \mathrm{MHz}, \mathrm{CDCl}_{3}\right): \delta$ $[\mathrm{ppm}]=162.1\left(\mathrm{~d},{ }^{1} J_{\mathrm{CF}}=244.4 \mathrm{~Hz}\right), 143.5,139.1\left(\mathrm{~d},{ }^{3} J_{\mathrm{CF}}=7.6 \mathrm{~Hz}\right), 137.0,135.0,131.6(\mathrm{~d}$, $\left.{ }^{3} J_{\mathrm{CF}}=8.2 \mathrm{~Hz}\right), 130.3\left(\mathrm{~d},{ }^{4} J_{\mathrm{CF}}=3.0 \mathrm{~Hz}\right), 129.8,127.1,126.1\left(\mathrm{~d},{ }^{4} J_{\mathrm{CF}}=2.3 \mathrm{~Hz}\right), 113.9(\mathrm{~d}$, $\left.{ }^{2} J_{\mathrm{CF}}=21.3 \mathrm{~Hz}\right), 112.8\left(\mathrm{~d},{ }^{3} J_{\mathrm{CF}}=21.8 \mathrm{~Hz}\right), 43.6,35.3,33.0,22.5,21.6,13.9$; HR-ESI-MS m/z: calcd. $\mathrm{C}_{20} \mathrm{H}_{24} \mathrm{NO}_{2} \mathrm{SFNa}[\mathrm{M}+\mathrm{Na}]^{+}$: 384.1404 , found: 384.1412 .

\section{(E)-N-(5-Fluoro-2-(pent-1-en-1-yl)phenethyl)-4-methylbenzenesulfonamide (207d)}

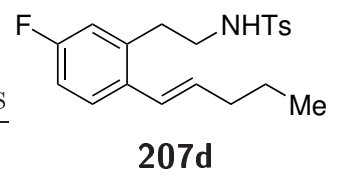

Following general procedure [1] lithium aluminum hydride $(107 \mathrm{mg}$, $2.83 \mathrm{mmol}, 2.5$ equiv) in diethyl ether $(8 \mathrm{~mL})$, nitrile 205d $(230 \mathrm{mg}$, $1.13 \mathrm{mmol}, 1.0$ equiv) in diethyl ether $(2.7 \mathrm{~mL})$; reaction time $23 \mathrm{~h}$; yellow oil. Following general procedure J amine (200 mg, 966 umol, 1.0 equiv), 
$\mathrm{NEt}_{3}$ (108 mg, $1.06 \mathrm{mmol}, 1.1$ equiv), DCM (2 mL), tosyl chloride (193 mg,1.01 mmol, 1.05 equiv) in DCM (1 mL); reaction time $17.5 \mathrm{~h}$; eluting with $n$-pentane/DCM, 5:1; light yellow oil; $65 \mathrm{mg}$, $0.18 \mathrm{mmol}, 16 \%$ from nitrile.

$\mathbf{R}_{\mathbf{f}}=0.07$ (n-pentane:EtOAc, 10:1); IR (ATR): $\tilde{\nu}\left[\mathrm{cm}^{-1}\right]=3277,2958,2929,2871,1600,1584$, 1491, 1324, 1248, 1153, 1091, 965, 871, 813, 706, 660, 548, 494; ${ }^{\mathbf{1}} \mathbf{H} \mathbf{N M R}\left(500 \mathrm{MHz}, \mathrm{CDCl}_{3}\right): \delta$ $[\mathrm{ppm}]=7.71-7.65(\mathrm{~m}, 2 \mathrm{H}), 7.33(\mathrm{dd}, J=8.6,5.8 \mathrm{~Hz}, 1 \mathrm{H}), 7.31-7.25(\mathrm{~m}, 2 \mathrm{H}), 6.86(\mathrm{td}, J=8.4$, $2.7 \mathrm{~Hz}, 1 \mathrm{H}), 6.68(\mathrm{dd}, J=9.4,2.7 \mathrm{~Hz}, 1 \mathrm{H}), 6.41(\mathrm{dt}, J=15.5,1.6 \mathrm{~Hz}, 1 \mathrm{H}), 5.96(\mathrm{dt}, J=15.5$, $6.9 \mathrm{~Hz}, 1 \mathrm{H}), 4.43(\mathrm{t}, J=6.3 \mathrm{~Hz}, 1 \mathrm{H}), 3.16(\mathrm{td}, J=7.3,6.3 \mathrm{~Hz}, 2 \mathrm{H}), 2.81(\mathrm{t}, J=7.2 \mathrm{~Hz}, 2 \mathrm{H})$, $2.42(\mathrm{~s}, 3 \mathrm{H}), 2.16$ (qd, $J=7.1,1.5 \mathrm{~Hz}, 2 \mathrm{H}), 1.46$ (sext, $J=7.4 \mathrm{~Hz}, 2 \mathrm{H}), 0.94$ (t, $J=7.4 \mathrm{~Hz}$, $3 \mathrm{H}) ;{ }^{1 \mathbf{3}} \mathbf{C}\left\{{ }^{1} \mathbf{H}\right\} \mathbf{N M R}\left(125 \mathrm{MHz}, \mathrm{CDCl}_{3}\right): \delta[\mathrm{ppm}]=161.9\left(\mathrm{~d},{ }^{1} J_{\mathrm{CF}}=246.4 \mathrm{~Hz}\right), 143.6,137.0$, $136.6\left(\mathrm{~d},{ }^{3} J_{\mathrm{CF}}=7.0 \mathrm{~Hz}\right), 133.9\left(\mathrm{~d},{ }^{4} J_{\mathrm{CF}}=1.7 \mathrm{~Hz}\right), 133.5\left(\mathrm{~d},{ }^{4} J_{\mathrm{CF}}=3.2 \mathrm{~Hz}\right), 129.9,128.2(\mathrm{~d}$, $\left.{ }^{3} J_{\mathrm{CF}}=7.9 \mathrm{~Hz}\right), 127.2,126.0,116.4\left(\mathrm{~d},{ }^{2} J_{\mathrm{CF}}=21.1 \mathrm{~Hz}\right), 114.2\left(\mathrm{~d},{ }^{2} J_{\mathrm{CF}}=21.0 \mathrm{~Hz}\right), 43.3,35.4$, $33.6\left(\mathrm{~d},{ }^{4} J_{\mathrm{CF}}=1.5 \mathrm{~Hz}\right), 22.7,21.7,13.9$; HR-ESI-MS m/z: calcd. $\mathrm{C}_{20} \mathrm{H}_{25} \mathrm{NO}_{2} \mathrm{SF}[\mathrm{M}+\mathrm{H}]^{+}$: 362.1585 , found: 362.1581 .

\section{(E)-4-Methyl-N-(2-(pent-1-en-1-yl)-4-(trifluoromethyl)phenethyl)benzenesulfonamide} (207e)

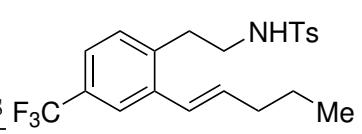

207e

Following general procedure [? lithium aluminum hydride $(81 \mathrm{mg}$, $2.1 \mathrm{mmol}, 2.5$ equiv) in diethyl ether $(6 \mathrm{~mL})$, nitrile 205e $(215 \mathrm{mg}$, $849 \mathrm{mmol}, 1.0$ equiv) in diethyl ether $(2 \mathrm{~mL})$; reaction time $23 \mathrm{~h}$; red liquid. Following general procedure J amine (170 mg, 661 umol, 1.0 equiv), $\mathrm{NEt}_{3}$ (74 mg, $0.73 \mathrm{mmol}, 1.1$ equiv), DCM (1.3 mL), tosyl chloride (132 mg, 695 umol, 1.05 equiv) in DCM $(0.6 \mathrm{~mL})$; reaction time $65 \mathrm{~h}$; eluting with $n$-pentane/EtOAc, 5:1; yellow oil; $50.0 \mathrm{mg}$, 121 umol, $14 \%$ from nitrile.

$\mathbf{R}_{\mathbf{f}}=0.19$ (n-pentane:EtOAc, 5:1); IR (ATR): $\tilde{\nu}\left[\mathrm{cm}^{-1}\right]=3302,2959,2932,2873,1650,1616$, 1598, 1578, 1422, 1325, 1275, 1156, 1118, 1092, 1076, 966, 925, 903, 830, 815, 662, 550; ${ }^{\mathbf{1}} \mathbf{H}$ NMR $\left(500 \mathrm{MHz}, \mathrm{CDCl}_{3}\right): \delta[\mathrm{ppm}]=7.79-7.62(\mathrm{~m}, 2 \mathrm{H}), 7.59(\mathrm{~d}, J=1.9 \mathrm{~Hz}, 1 \mathrm{H}), 7.35(\mathrm{dd}$, $J=8.0,1.9 \mathrm{~Hz}, 1 \mathrm{H}), 7.30-7.21(\mathrm{~m}, 2 \mathrm{H}), 7.13(\mathrm{~d}, J=7.9 \mathrm{~Hz}, 1 \mathrm{H}), 6.48(\mathrm{dt}, J=15.6,1.6 \mathrm{~Hz}$, $1 \mathrm{H}), 6.10(\mathrm{dt}, J=15.5,7.0 \mathrm{~Hz}, 1 \mathrm{H}), 4.50(\mathrm{t}, J=6.3 \mathrm{~Hz}, 1 \mathrm{H}), 3.16(\mathrm{q}, J=6.9 \mathrm{~Hz}, 2 \mathrm{H})$, $2.90(\mathrm{t}, J=7.2 \mathrm{~Hz}, 2 \mathrm{H}), 2.41(\mathrm{~s}, 3 \mathrm{H}), 2.19(\mathrm{qd}, J=7.1,1.5 \mathrm{~Hz}, 2 \mathrm{H}), 1.49(\mathrm{q}, J=7.4 \mathrm{~Hz}$, $2 \mathrm{H}), 0.95(\mathrm{t}, J=7.4 \mathrm{~Hz}, 3 \mathrm{H}) ;{ }^{\mathbf{1 3}} \mathbf{C}\left\{{ }^{\mathbf{1}} \mathbf{H}\right\} \mathbf{N M R}\left(126 \mathrm{MHz}, \mathrm{CDCl}_{3}\right): \delta[\mathrm{ppm}]=143.6,138.4$ $\left(\mathrm{d},{ }^{4} J_{\mathrm{CF}}=1.6 \mathrm{~Hz}\right), 138.0,136.9,135.8,130.5,129.8,129.6\left(\mathrm{q},{ }^{2} J_{\mathrm{CF}}=32.4 \mathrm{~Hz}\right), 127.2,125.9$, $124.3\left(\mathrm{q},{ }^{1} J_{\mathrm{CF}}=272.1 \mathrm{~Hz}\right), 123.6\left(\mathrm{~d},{ }^{3} J_{\mathrm{CF}}=3.8 \mathrm{~Hz}\right), 123.3\left(\mathrm{~d},{ }^{3} J_{\mathrm{CF}}=3.8 \mathrm{~Hz}\right), 43.2,35.5$, 33.7, 22.5, 21.6, 13.9; HR-ESI-MS m/z: calcd. $\mathrm{C}_{21} \mathrm{H}_{24} \mathrm{NO}_{2} \mathrm{SF}_{3} \mathrm{Na}[\mathrm{M}+\mathrm{Na}]^{+}:$434.1372, found: 434.1369. 


\section{(E)-4-Methyl-N-(2-(pent-1-en-1-yl)-5-(trifluoromethyl)phenethyl)benzenesulfonamide} (207f)

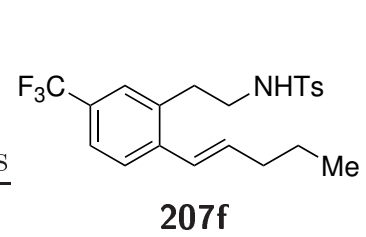

Following general procedure [ lithium aluminum hydride $(90.0 \mathrm{mg}$, $2.37 \mathrm{mmol}, 2.5$ equiv) in diethyl ether $(6.6 \mathrm{~mL})$, nitrile $\mathbf{2 0 5 f}(240 \mathrm{mg}$, 948 umol, 1.0 equiv) in diethyl ether $(2.3 \mathrm{~mL})$; reaction time $41 \mathrm{~h}$; yellow oil. Following general procedure J: amine (195 mg, 758 mol, 1.0 equiv), $\mathrm{NEt}_{3}$ ( $84 \mathrm{mg}, 0.83 \mathrm{mmol}, 1.1$ equiv), DCM (1.5 mL), tosyl chloride (152 mg, 796 mol, 1.05 equiv) in DCM $(0.75 \mathrm{~mL})$; reaction time $22.5 \mathrm{~h}$; eluting with $n$-pentane/DCM, 3:1; light yellow oil; $21 \mathrm{mg}, 51 \mu \mathrm{mol}, 5 \%$ from nitrile.

$\mathbf{R}_{\mathbf{f}}=0.13$ (n-pentane:EtOAc, 10:1); IR (ATR): $\tilde{\nu}\left[\mathrm{cm}^{-1}\right]=3282,2960,2930,2873,1648,1616$, 1599, 1459, 1419, 1327, 1286, 1155, 1118, 1083, 968, 896, 841, 813, 746, 735, 706, 664, 549, 500; ${ }^{1} \mathbf{H}$ NMR $\left(400 \mathrm{MHz}, \mathrm{CDCl}_{3}\right): \delta[\mathrm{ppm}]=7.79-7.58(\mathrm{~m}, 2 \mathrm{H}), 7.47(\mathrm{~d}, J=8.2 \mathrm{~Hz}, 1 \mathrm{H}), 7.40$ $(\mathrm{dd}, J=8.2,2.0 \mathrm{~Hz}, 1 \mathrm{H}), 7.30-7.23(\mathrm{~m}, 2 \mathrm{H}), 7.20(\mathrm{~d}, J=1.9 \mathrm{~Hz}, 1 \mathrm{H}), 6.52(\mathrm{dt}, J=15.7$, $1.6 \mathrm{~Hz}, 1 \mathrm{H}), 6.14(\mathrm{dt}, J=15.5,7.0 \mathrm{~Hz}, 1 \mathrm{H}), 4.64(\mathrm{t}, J=6.3 \mathrm{~Hz}, 1 \mathrm{H}), 3.18(\mathrm{td}, J=7.3$, $6.2 \mathrm{~Hz}, 2 \mathrm{H}), 2.88(\mathrm{dd}, J=8.1,6.6 \mathrm{~Hz}, 2 \mathrm{H}), 2.42(\mathrm{~s}, 3 \mathrm{H}), 2.21(\mathrm{qd}, J=7.1,1.5 \mathrm{~Hz}, 2 \mathrm{H})$, 1.50 (sext, $J=7.4 \mathrm{~Hz}, 2 \mathrm{H}), 0.96(\mathrm{t}, J=7.4 \mathrm{~Hz}, 3 \mathrm{H}) ;{ }^{\mathbf{1 3}} \mathbf{C}\left\{{ }^{\mathbf{1}} \mathbf{H}\right\} \mathbf{N M R}\left(101 \mathrm{MHz}, \mathrm{CDCl}_{3}\right)$ : $\delta[\mathrm{ppm}]=143.7,140.9,137.1,136.4,135.3,129.9,129.0\left(\mathrm{q},{ }^{2} J_{\mathrm{CF}}=32.4 \mathrm{~Hz}\right), 127.1,126.8,126.7$ $\left(\mathrm{q},{ }^{3} J_{\mathrm{CF}}=3.8 \mathrm{~Hz}\right), 126.0,124.2\left(\mathrm{q},{ }^{1} J_{\mathrm{CF}}=271.9 \mathrm{~Hz}\right), 124.0\left(\mathrm{q},{ }^{3} J_{\mathrm{CF}}=3.8 \mathrm{~Hz}\right), 43.4,35.5$, 33.7, 22.5, 21.6, 13.9; HR-ESI-MS m/z: calcd. $\mathrm{C}_{21} \mathrm{H}_{24} \mathrm{NO}_{2} \mathrm{SF}_{3} \mathrm{Na}[\mathrm{M}+\mathrm{Na}]^{+}$: 434.1372 , found: 434.1375 .

\section{1-Bromo-4-methoxy-2-(2-nitroethyl)benzene (212)}

Copper(I)bromide (31 mg, 0.21 mmol, 0.2 equiv), $\quad N-((2 Z, 4 E)-4$<smiles>COc1ccc(Br)c(CCN=O)c1</smiles>

212

((2,6-Dimethylphenyl)imino)pent-2-en-2-yl)-2,6-dimethylaniline $(66 \mathrm{mg}$, $0.21 \mathrm{mmol}, 0.2$ equiv) and sodium tert-butoxide (124 mg, $1.29 \mathrm{mmol}$, 1.2 equiv) were dissolved in 1,4-dioxane $(6 \mathrm{~mL})$. Nitromethane $(430 \mu \mathrm{L}$, $491 \mathrm{mg}, 8.04 \mathrm{mmol}, 7.5$ equiv) and 1-bromo-2-(bromomethyl)-4-methoxybenzene (300 mg, $1.07 \mathrm{mmol}, 1.0$ equiv) were added and the solution was heated to $60{ }^{\circ} \mathrm{C}$ for $15 \mathrm{~h}$. Sat. aq. $\mathrm{NH}_{4} \mathrm{Cl}(10 \mathrm{~mL})$ was added, the aq. phase was extracted with $\mathrm{Et}_{2} \mathrm{O}(10 \mathrm{~mL})$, the combined org. phases were washed with sat. aq. $\mathrm{NaCl}(20 \mathrm{~mL})$ and dried over $\mathrm{Na}_{2} \mathrm{SO}_{4}$. Removal of the solvent in vacuum and purification by column chromatography (12:1 n-pentane:EtOAc) afforded the product as a brown oil (192 mg, $740 \mu \mathrm{mol}, 69 \%)$.

$\mathbf{R}_{\mathbf{f}}=0.25$ (n-pentane:EtOAc, $\left.12: 1\right) ;{ }^{\mathbf{1}} \mathbf{H}$ NMR $\left(300 \mathrm{MHz}, \mathrm{CDCl}_{3}\right): \delta[\mathrm{ppm}]=7.45(\mathrm{~d}, J=$ $8.8 \mathrm{~Hz}, 1 \mathrm{H}), 6.80(\mathrm{~d}, J=3.0 \mathrm{~Hz}, 1 \mathrm{H}), 6.72(\mathrm{dd}, J=8.8,3.0 \mathrm{~Hz}, 1 \mathrm{H}), 4.67-4.60(\mathrm{~m}, 2 \mathrm{H}), 3.78$ (s, $3 \mathrm{H}), 3.41$ (t, $J=7.3 \mathrm{~Hz}, 2 \mathrm{H})$. 


\section{5-Bromo-6-(2-nitroethyl)benzo[d][1,3]dioxole (213)}

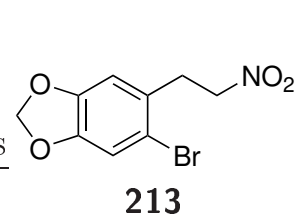

Copper(I)bromide (98 mg, 0.68 mmol, 0.2 equiv), $\quad N-((2 Z, 4 E)-4-$ ((2,6-Dimethylphenyl)imino)pent-2-en-2-yl)-2,6-dimethylaniline (209 mg, 680 umol, 0.2 equiv) and sodium tert-butoxide $(392 \mathrm{mg}, 4.08 \mathrm{mmol}$, 1.2 equiv) were dissolved in 1,4-dioxane $(20 \mathrm{~mL})$. Nitromethane $(1.37 \mathrm{~mL}$, $1.56 \mathrm{~g}, 25.5 \mathrm{mmol}, 7.5$ equiv) and 5-bromo-6-(bromomethyl) benzo[d][1,3]dioxole (1.0 g, $3.4 \mathrm{mmol}$, 1.0 equiv) were added and the solution was heated to $60{ }^{\circ} \mathrm{C}$ for $19 \mathrm{~h}$. Sat. aq. $\mathrm{NH}_{4} \mathrm{Cl}(20 \mathrm{~mL})$ was added, the aq. phase was extracted with $\mathrm{Et}_{2} \mathrm{O}(15 \mathrm{~mL})$, the combined org. phases were washed with water $(20 \mathrm{~mL})$ and dried over $\mathrm{Na}_{2} \mathrm{SO}_{4}$. Removal of the solvent in vacuo, purification by column chromatography (30:1 n-pentane:EtOAc) and recrystallization from $n$-hexane/MeOH afforded the product as a white solid (330 $\mathrm{mg}, 1.20 \mathrm{mmol}, 35 \%)$.

$\mathbf{R}_{\mathbf{f}}=0.14$ (n-pentane:EtOAc, 30:1); m.p. $=58-60{ }^{\circ} \mathrm{C} ; \mathbf{I R}(\mathrm{ATR}): \tilde{\nu}\left[\mathrm{cm}^{-1}\right]=3111,3093,3055$, 3008, 2975, 2913, 1542, 1501, 1476, 1421, 1408, 1377, 1359, 1331, 1232, 1162, 1118, 1032, 990, $922,877,855,826,744,654,592,536,500,428 ;{ }^{1} \mathbf{H} \mathbf{~ N M R}\left(500 \mathrm{MHz}, \mathrm{CDCl}_{3}\right): \delta[\mathrm{ppm}]=7.01(\mathrm{~s}$, $1 \mathrm{H}), 6.73(\mathrm{~s}, 1 \mathrm{H}), 5.97(\mathrm{~s}, 2 \mathrm{H}), 4.59(\mathrm{t}, J=7.2 \mathrm{~Hz}, 2 \mathrm{H}), 3.35(\mathrm{t}, J=7.2 \mathrm{~Hz}, 2 \mathrm{H}) ;{ }^{13} \mathrm{C}\left\{{ }^{1} \mathbf{H}\right\}$ NMR $\left(126 \mathrm{MHz}, \mathrm{CDCl}_{3}\right): \delta[\mathrm{ppm}]=148.2,147.8,127.9,114.8,113.2,110.8,102.1,74.7,33.9$; HR-ESI-MS m/z: calcd. $\mathrm{C}_{9} \mathrm{H}_{7} \mathrm{BrNO}_{4}[\mathrm{M}-\mathrm{H}]^{-}:$271.9564, found: 271.9564 .

\section{N-(2-Bromo-5-methoxybenzyl)-4-methylbenzenesulfonamide (215)}

Nitroethyl compound 212 (700 mg, $2.69 \mathrm{mmol}, 1.0$ equiv) and zinc (3.5 g,<smiles>COc1ccc(Br)c(CCNS)c1</smiles>

215 $54 \mathrm{mmol}, 20$ equiv) were added to a mixture of methanol $(27 \mathrm{~mL})$, conc. acetic acid $(9 \mathrm{~mL})$ and $2 \mathrm{M}$ aq. $\mathrm{HCl}(18 \mathrm{~mL})$ and the mixture was stirred at room temperature for $6.5 \mathrm{~h}$. The mixture was filtered over celite, methanol was removed in vacuo and the $\mathrm{pH}$ value of the aq. solution was adjusted to 10 with sat. aq. $\mathrm{Na}_{2} \mathrm{CO}_{3}$ solution. The aq. phase was extracted with DCM $(4 \times 25 \mathrm{~mL})$ and the combined org. phases were dried over $\mathrm{Na}_{2} \mathrm{SO}_{4}$. Removal of the solvent afforded the amine, which was used without further purification.

The amine (200 mg, 869 umol, 1.0 equiv) was dissolved in DCM (2 mL) and triethylamine (97 mg, $0.96 \mathrm{mmol}, 1.1$ equiv) was added. $p$-Toluenesulfonyl chloride (174 mg, $912 \mu \mathrm{mol}, 1.05$ equiv) in DCM $(1 \mathrm{~mL})$ was added dropwise and the mixture was stirred at room temperature for $19.5 \mathrm{~h}$. The reaction was quenched by the addition of $\mathrm{NH}_{4} \mathrm{Cl}(3 \mathrm{~mL})$ and the phases were separated. The aq. phase was extracted with DCM $(3 \mathrm{~mL})$ and the combined organic phases were washed with sat. aq. $\mathrm{NaCl}$ solution $(6 \mathrm{~mL})$ and dried over $\mathrm{Na}_{2} \mathrm{SO}_{4}$. Purification by column chromatography (5:1 $n$-pentane/EtOAc) afforded the product as a yellow oil (226 mg, $588 \mu \mathrm{mol}, 67 \%)$.

$\mathbf{R}_{\mathbf{f}}=0.36$ (n-pentane:EtOAc, 5:1); IR (ATR): $\tilde{\nu}\left[\mathrm{cm}^{-1}\right]=3278,2936,2837,1596,1572,1473$, 1416, 1321, 1241, 1153, 1092, 1058, 1010, 811, 660, 548; ${ }^{\mathbf{1}} \mathbf{H} \mathbf{N M R}\left(400 \mathrm{MHz}, \mathrm{CDCl}_{3}\right)$ : $\delta[\mathrm{ppm}]=7.76-7.64(\mathrm{~m}, 2 \mathrm{H}), 7.36(\mathrm{~d}, J=8.7 \mathrm{~Hz}, 1 \mathrm{H}), 7.30-7.26(\mathrm{~m}, 2 \mathrm{H}), 6.69(\mathrm{~d}, J=3.0 \mathrm{~Hz}$, 
$1 \mathrm{H}), 6.64(\mathrm{dd}, J=8.7,3.1 \mathrm{~Hz}, 1 \mathrm{H}), 4.50(\mathrm{t}, J=6.3 \mathrm{~Hz}, 1 \mathrm{H}), 3.76(\mathrm{~s}, 3 \mathrm{H}), 3.30-3.14(\mathrm{~m}, 2 \mathrm{H})$, $2.87(\mathrm{t}, J=7.0 \mathrm{~Hz}, 2 \mathrm{H}), 2.42(\mathrm{~s}, 3 \mathrm{H}) ;{ }^{\mathbf{1 3}} \mathbf{C}\left\{{ }^{\mathbf{1}} \mathbf{H}\right\} \mathbf{N M R}\left(101 \mathrm{MHz}, \mathrm{CDCl}_{3}\right): \delta[\mathrm{ppm}]=159.2$, 143.6, 138.2, 137.1, 133.7, 129.9, 127.2, 116.8, 114.8, 114.4, 55.6, 42.7, 36.7, 21.7; HR-ESI-MS m/z: calcd. $\mathrm{C}_{16} \mathrm{H}_{17} \mathrm{NO}_{3} \mathrm{BrS}[\mathrm{M}-\mathrm{H}]^{+}:$382.0118, found: 382.0110 .

\section{N-(2-(6-Bromobenzo[d][1,3]dioxol-5-yl)ethyl)-4-methylbenzenesulfonamide (216)}

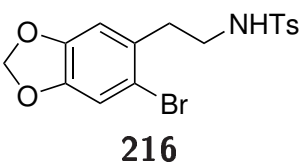

216

Nitroethyl compound 213 (330 mg, $1.20 \mathrm{mmol}, 1.0$ equiv) and zinc (1.57 g, $24.1 \mathrm{mmol}, 20$ equiv) were added to a mixture of methanol $(12 \mathrm{~mL})$, conc. acetic acid $(4 \mathrm{~mL})$ and $2 \mathrm{M}$ aq. $\mathrm{HCl}(8 \mathrm{~mL})$ and the mixture was stirred at room temperature for $20 \mathrm{~h}$. The mixture was filtered over celite, methanol

was removed in vacuo and the $\mathrm{pH}$ value of the aq. solution was adjusted to 10 with sat. aq. $\mathrm{Na}_{2} \mathrm{CO}_{3}$ solution. The aq. phase was extracted with DCM $(4 \times 10 \mathrm{~mL})$ and the combined org. phases were dried over $\mathrm{Na}_{2} \mathrm{SO}_{4}$. Removal of the solvent afforded the amine, which was used without further purification.

The amine (275 mg, $1.13 \mathrm{mmol}, 1.0$ equiv) was dissolved in DCM (2.3 mL) and triethylamine (125 mg, $1.24 \mathrm{mmol}, 1.1$ equiv) was added. $p$-Toluenesulfonyl chloride (236 mg, $1.24 \mathrm{mmol}$, 1.05 equiv) in DCM $(1.2 \mathrm{~mL})$ was added dropwise and the mixture was stirred at room temperature for $20 \mathrm{~h}$. The reaction was quenched by the addition of $\mathrm{NH}_{4} \mathrm{Cl}(3 \mathrm{~mL})$ and the phases were separated. The aq. phase was extracted with DCM $(3 \mathrm{~mL})$ and the combined organic phases were washed with sat. aq. $\mathrm{NaCl}$ solution $(6 \mathrm{~mL})$ and dried over $\mathrm{Na}_{2} \mathrm{SO}_{4}$. Purification by column chromatography (4:1 n-pentane/EtOAc) afforded the product as a yellow solid (321 mg, $805 \mu \mathrm{mol}, 71 \%)$.

$\mathbf{R}_{\mathbf{f}}=0.14$ (n-pentane:EtOAc, 4:1); m.p. $=103{ }^{\circ} \mathrm{C}$; IR (ATR): $\tilde{\nu}\left[\mathrm{cm}^{-1}\right]=3301,3287,3276,2924$, 2904, 2881, 1598, 1504, 1478, 1410, 1324, 1243, 1158, 1116, 1093, 1041, 932, 814, 662, 551, 407; ${ }^{1} \mathbf{H}$ NMR $\left(300 \mathrm{MHz}, \mathrm{CDCl}_{3}\right): \delta[\mathrm{ppm}]=7.79-7.66(\mathrm{~m}, 2 \mathrm{H}), 7.28(\mathrm{dd}, J=8.0,0.7 \mathrm{~Hz}, 1 \mathrm{H})$, $6.93(\mathrm{~s}, 1 \mathrm{H}), 6.61(\mathrm{~s}, 1 \mathrm{H}), 5.95(\mathrm{~s}, 2 \mathrm{H}), 4.46(\mathrm{t}, J=6.1 \mathrm{~Hz}, 1 \mathrm{H}), 3.19(\mathrm{q}, J=6.8 \mathrm{~Hz}, 2 \mathrm{H})$, $2.81(\mathrm{t}, J=7.0 \mathrm{~Hz}, 2 \mathrm{H}), 2.42(\mathrm{~s}, 3 \mathrm{H}) ;{ }^{\mathbf{1 3}} \mathbf{C}\left\{{ }^{\mathbf{1}} \mathbf{H}\right\} \mathbf{N M R}\left(126 \mathrm{MHz}, \mathrm{CDCl}_{3}\right): \delta[\mathrm{ppm}]=147.5$, 147.4, 143.5, 137.0, 130.1, 129.7, 127.2, 114.6, 113.0, 110.7, 101.9, 43.0, 36.4, 21.7; HR-ESI-MS m/z: calcd. $\mathrm{C}_{16} \mathrm{H}_{16} \mathrm{BrNO}_{4} \mathrm{~S}[\mathrm{M}+\mathrm{H}]^{+}$: 398.0056 , found: 398.0046 .

\section{(E)-N-(5-Methoxy-2-(pent-1-en-1-yl)phenethyl)-4-methylbenzenesulfonamide (207g)}

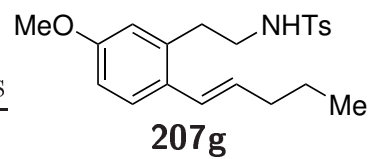

Following general procedure $\mathbf{H}$; toluenesulfonic acid amide $\mathbf{2 1 5}$ (200 mg, 520 umol, 1.0 equiv), 1-pentenylboronic acid (65 mg, $0.57 \mathrm{mmol}$, 1.1 equiv), potassium carbonate (288 $\mathrm{mg}, 2.08 \mathrm{mmol}, 4.0$ equiv), toluene $(3.75 \mathrm{~mL})$, ethanol $(1.5 \mathrm{~mL})$, water $(0.75 \mathrm{~mL})$, tetrakis[tri$n$-pentane/EtOAc, 6:1; yellow oil; $129 \mathrm{mg}, 346 \mu \mathrm{mol}, 67 \%$. 
$\mathbf{R}_{\mathbf{f}}=0.5$ (n-pentane:EtOAc, 2:1); IR (ATR): $\tilde{\nu}\left[\mathrm{cm}^{-1}\right]=3279,2957,2929,2871,2836,1606$, 1494, 1463, 1324, 1254, 1155, 1092, 966, 872, 813, 661, 549, 501; ${ }^{\mathbf{1}} \mathbf{H}$ NMR (300 MHz, $\left.\mathrm{CDCl}_{3}\right)$ : $\delta[\mathrm{ppm}]=7.73-7.60(\mathrm{~m}, 2 \mathrm{H}), 7.36-7.21(\mathrm{~m}, 3 \mathrm{H}), 6.72(\mathrm{dd}, J=8.6 \mathrm{~Hz}, J=2.7 \mathrm{~Hz}, 1 \mathrm{H}), 6.56$ $(\mathrm{d}, J=2.7 \mathrm{~Hz}, 1 \mathrm{H}), 6.39(\mathrm{~m}, 1 \mathrm{H}), 5.91(\mathrm{dt}, J=15.5,7.0 \mathrm{~Hz}, 1 \mathrm{H}), 4.69(\mathrm{t}, J=6.1 \mathrm{~Hz}, 1 \mathrm{H})$, $3.76(\mathrm{~s}, 3 \mathrm{H}), 3.15$ (ddd, $J=7.3,6.7,5.9 \mathrm{~Hz}, 2 \mathrm{H}), 2.81$ (t, $J=7.2 \mathrm{~Hz}, 2 \mathrm{H}), 2.41(\mathrm{~s}, 3 \mathrm{H})$, 2.27-2.05 (m, $2 \mathrm{H}), 1.45$ (sext, $J=7.3 \mathrm{~Hz}, 2 \mathrm{H}), 0.93(\mathrm{t}, J=7.3 \mathrm{~Hz}, 3 \mathrm{H}) ;{ }^{\mathbf{1 3}} \mathbf{C}\left\{{ }^{1} \mathbf{H}\right\} \mathbf{N M R}$ $\left(151 \mathrm{MHz}, \mathrm{CDCl}_{3}\right): \delta[\mathrm{ppm}]=158.9,143.4,136.0,132.1,130.1,129.8,127.8,127.2,126.6,126.6$, 115.3, 113.0, 55.4, 43.6, 35.4, 33.8, 22.8, 21.5, 13.8; HR-ESI-MS m/z: calcd. $\mathrm{C}_{21} \mathrm{H}_{28} \mathrm{NO}_{3} \mathrm{~S}$ $[\mathrm{M}+\mathrm{H}]^{+}:$:374.1784, found: 374.1781 .

\section{(E)-4-Methyl-N-(2-(6-(pent-1-en-1-yl)benzo[d][1,3]dioxol-5-yl)ethyl)benzenesulfonamide} (207h)

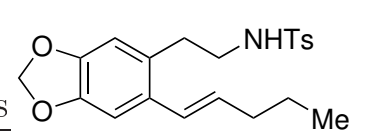

$207 \mathrm{~h}$

Following general procedure $\mathbf{H}$; toluenesulfonic acid amide $\mathbf{2 1 6}(310 \mathrm{mg}$, 778 umol, 1.0 equiv), 1-pentenylboronic acid (98 mg, $0.86 \mathrm{mmol}$, 1.1 equiv), potassium carbonate (430 $\mathrm{mg}, 3.11 \mathrm{mmol}, 4.0$ equiv), toluene $(6.25 \mathrm{~mL})$, ethanol $(2.5 \mathrm{~mL})$, water $(1.25 \mathrm{~mL})$, tetrakis[triphenylphosphine]palladium(0) (90 mg, 78 umol, 0.1 equiv); reaction time $25 \mathrm{~h}$; eluting with $n$-pentane/EtOAc, 8:1; yellow solid; $143 \mathrm{mg}, 368$ umol, $47 \%$.

$\mathbf{R}_{\mathbf{f}}=0.29$ (n-pentane:EtOAc, 4:1); m.p. $=84^{\circ} \mathrm{C}$; IR $($ ATR $): \tilde{\nu}\left[\mathrm{cm}^{-1}\right]=3256,2955,2926,2872$, 1596, 1503, 1479, 1421, 1373, 1316, 1255, 1159, 1092, 1073, 1039, 963, 939, 879, 856, 814, 665, $571,551,495,469,400 ;{ }^{1} \mathbf{H} \mathbf{N M R}\left(500 \mathrm{MHz}, \mathrm{CDCl}_{3}\right): \delta[\mathrm{ppm}]=7.68(\mathrm{~d}, J=8.3 \mathrm{~Hz}, 2 \mathrm{H})$, 7.31-7.22 (m, $2 \mathrm{H}), 6.87$ (s, $1 \mathrm{H}), 6.47(\mathrm{~s}, 1 \mathrm{H}), 6.38$ (dt, $J=15.4,1.5 \mathrm{~Hz}, 1 \mathrm{H}), 6.05-5.73$ (m, $3 \mathrm{H}), 4.41(\mathrm{t}, J=6.2 \mathrm{~Hz}, 1 \mathrm{H}), 3.10(\mathrm{td}, J=7.1,6.1 \mathrm{~Hz}, 2 \mathrm{H}), 2.75(\mathrm{t}, J=7.1 \mathrm{~Hz}, 2 \mathrm{H}), 2.42$ (s, $3 \mathrm{H}), 2.13$ (qd, $J=7.1,1.5 \mathrm{~Hz}, 2 \mathrm{H}), 1.45$ (sext, $J=7.4 \mathrm{~Hz}, 2 \mathrm{H}), 0.92$ (t, $J=7.4 \mathrm{~Hz}, 3 \mathrm{H}$ ); ${ }^{13} \mathbf{C}\left\{{ }^{1} \mathbf{H}\right\}$ NMR $\left(126 \mathrm{MHz}, \mathrm{CDCl}_{3}\right): \delta[\mathrm{ppm}]=147.0,146.8,143.5,137.0,132.3,130.9,129.8$, 128.1, 127.2, 126.6, 109.9, 106.3, 101.1, 43.8, 35.4, 33.4, 22.8, 21.7, 13.9; HR-ESI-MS m/z: calcd. $\mathrm{C}_{21} \mathrm{H}_{26} \mathrm{NO}_{4} \mathrm{~S}[\mathrm{M}+\mathrm{H}]^{+}: 388.1577$, found: 388.1570 .

\section{2-(2-Bromo-5-iodophenyl)acetonitrile (221)}

2-Bromo-5-iodobenzyl alcohol (1.0 g, $3.2 \mathrm{mmol}, 1.0$ equiv) was dissolved in DCM<smiles>N#CCc1cc(I)ccc1Br</smiles>
$\left(10 \mathrm{~mL}\right.$ ), $\mathrm{NEt}_{3}$ (485 mg, $4.79 \mathrm{mmol}, 1.5$ equiv) was added and the solution was cooled to $0{ }^{\circ} \mathrm{C}$. A solution of methanesulfonyl chloride (403 mg, $3.52 \mathrm{mmol}$, 2211.1 equiv) in DCM $(6 \mathrm{~mL})$ was added and the mixture was stirred at room temperature for $3 \mathrm{~h}$. Sat. aq. $\mathrm{NaHCO}_{3}$ solution $(15 \mathrm{~mL})$ was added, the aq. phase was extracted with DCM $(3 \times 15 \mathrm{~mL})$, the combined org. phases were washed with $\mathrm{H}_{2} \mathrm{O}(30 \mathrm{~mL})$ and sat. aq. $\mathrm{NaCl}$ solution $(30 \mathrm{~mL})$ and dried over $\mathrm{Na}_{2} \mathrm{SO}_{4}$. After removal of the solvent in vacuo, the crude product was used without further purification. It was dissolved in DMF (6 mL) and added to a solution of $\mathrm{NaCN}$ (235 mg, $4.79 \mathrm{mmol}, 1.5$ equiv) in DMF (4 mL). The solution 
was stirred at room temperature for $18.5 \mathrm{~h}, \mathrm{H}_{2} \mathrm{O}(10 \mathrm{~mL})$ was added, the aq. phase was extracted with $\mathrm{Et}_{2} \mathrm{O}(3 \times 10 \mathrm{~mL})$ and the combined org. phases were dried over $\mathrm{Na}_{2} \mathrm{SO}_{4}$. Removal of the solvent in vacuo and purification by column chromatography (5:1 n-pentane:DCM) afforded the product as a white solid (829 $\mathrm{mg}, 2.57 \mathrm{mmol}, 80 \%$ ).

$\mathbf{R}_{\mathbf{f}}=0.13$ (n-pentane:DCM, 5:1); m.p. $=82{ }^{\circ} \mathrm{C}$; IR (ATR) $\tilde{\nu}\left[\mathrm{cm}^{-1}\right]=2963,2929,2909,2243$, 1458, 1397, 1377, 1259, 1078, 1018, 878, 794, 683, 426; ${ }^{\mathbf{1}} \mathbf{H} \mathbf{~ N M R}\left(300 \mathrm{MHz}, \mathrm{CDCl}_{3}\right): \delta$ $[\mathrm{ppm}]=7.84(\mathrm{~d}, J=2.0 \mathrm{~Hz}, 1 \mathrm{H}), 7.54(\mathrm{dd}, J=8.5 \mathrm{~Hz}, J=2.1 \mathrm{~Hz}, 1 \mathrm{H}), 7.32(\mathrm{~d}, J=8.4 \mathrm{~Hz}$, $1 \mathrm{H}), 3.79(\mathrm{~s}, 2 \mathrm{H}) ;{ }^{13} \mathbf{C}\left\{{ }^{1} \mathbf{H}\right\} \mathbf{N M R}\left(75 \mathrm{MHz}, \mathrm{CDCl}_{3}\right): \delta[\mathrm{ppm}]=139.1,138.5,134.7,132.2$, 123.5, 116.4, 93.0, 24.6; HR-ESI-MS $\mathbf{m} / \mathbf{z}$ : calcd. $\mathrm{C}_{8} \mathrm{H}_{4} \mathrm{BrIN}[\mathrm{M}-\mathrm{H}]^{-}$: 319.8577 , found: 319.8562

\section{1-Bromo-2-(bromomethyl)-4-iodobenzene (224)}

To a solution of 2-bromo-5-iodobenzyl alcohol (300 mg, 957 umol, 1.0 equiv) in<smiles>BrCc1cc(I)ccc1Br</smiles>

224

THF $(7.0 \mathrm{~mL}), \mathrm{PBr}_{3}\left(112 \mathrm{mg}, 414 \mu \mathrm{mol}, 0.43\right.$ equiv) was added dropwise at $0{ }^{\circ} \mathrm{C}$. The solution was stirred at $0{ }^{\circ} \mathrm{C}$ for $2.5 \mathrm{~h}$ and at room temperature for $14.5 \mathrm{~h}$. Removal of the solvent in vacuo and purification by column chromatography (5:1 $n$-pentane:DCM) afforded the product as a white solid (226 mg, $601 \mu \mathrm{mol}, 63 \%$ ).

$\mathbf{R}_{\mathbf{f}}=0.64\left(n\right.$-pentane:DCM, 5:1); m.p. $=110-113{ }^{\circ} \mathrm{C} ; \mathbf{I R}(\mathrm{ATR}): \tilde{\nu}\left[\mathrm{cm}^{-1}\right]=3068,3045,1901$, 1886, 1762, 1630, 1547, 1460, 1431, 1373, 1273, 1213, 1074, 1024, 886, 872, 814, 718, 677, 615, 566, 501, 444, 430; ${ }^{1} \mathbf{H} \mathbf{N M R}\left(500 \mathrm{MHz}, \mathrm{CDCl}_{3}\right): \delta[\mathrm{ppm}]=7.77(\mathrm{~d}, J=2.2 \mathrm{~Hz}, 1 \mathrm{H}), 7.47$ $(\mathrm{dd}, J=8.4 \mathrm{~Hz}, J=2.2 \mathrm{~Hz}, 1 \mathrm{H}), 7.29(\mathrm{~d}, J=8.4 \mathrm{~Hz}, 1 \mathrm{H}), 4.50(\mathrm{~s}, 2 \mathrm{H}) ;{ }^{13} \mathbf{C}\left\{{ }^{1} \mathbf{H}\right\} \mathbf{N M R}$ $\left(125 \mathrm{MHz} \mathrm{CDCl}_{3}\right): \delta[\mathrm{ppm}]=139.9,139.2,139.0,134.9,124.3,92.6,32.2 ; \mathbf{H R}-\mathbf{E I}-\mathbf{M S} \mathbf{m} / \mathbf{z}$ : calcd. $\mathrm{C}_{7} \mathrm{H}_{5} \mathrm{Br}_{2} \mathrm{I}[\mathrm{M}]^{+}: 373.7803$, found: 373.7810 .

\section{1-Bromo-4-iodo-2-(2-nitroethyl)benzene (223)}

Copper(I)bromide (76 mg, $0.53 \mathrm{mmol}, 0.2$ equiv), $N$-((2Z,4E)-4-((2,6-<smiles>O=[N+]([O-])Cc1cc(I)ccc1Br</smiles>

223 dimethylphenyl)imino)pent-2-en-2-yl)-2,6-dimethylaniline (163 mg, $532 \mu \mathrm{mol}$, 0.2 equiv) and sodium tert-butoxide ( $307 \mathrm{mg}, 3.19 \mathrm{mmol}, 1.2$ equiv) were dissolved in 1,4-dioxane $(16 \mathrm{~mL})$. Nitromethane $(1.10 \mathrm{~mL}, 1.22 \mathrm{~g}, 20.0 \mathrm{mmol}$, 7.5 equiv) and 1-bromo-2-(bromomethyl)-4-iodobenzene (1.0 g, $2.7 \mathrm{mmol}, 1.0$ equiv) were added and the solution was heated to $60{ }^{\circ} \mathrm{C}$ for $18.5 \mathrm{~h}$. Sat. aq. $\mathrm{NH}_{4} \mathrm{Cl}(16 \mathrm{~mL})$ was added, the aq. phase was extracted with $\mathrm{Et}_{2} \mathrm{O}(16 \mathrm{~mL})$, the combined org. phases were washed with water $(16 \mathrm{~mL})$ and dried over $\mathrm{Na}_{2} \mathrm{SO}_{4}$. Removal of the solvent in vacuo, purification by column chromatography (5:1 n-pentane:DCM) and afforded the product as a light yellow solid (279 $\mathrm{mg}$, $0.784 \mathrm{mmol}, 30 \%)$.

$\mathbf{R}_{\mathbf{f}}=0.11$ (n-pentane:DCM, 5:1); m.p. $=65{ }^{\circ} \mathrm{C} ; \mathbf{I R}(\mathrm{ATR}): \tilde{\nu}\left[\mathrm{cm}^{-1}\right]=1548,1535,1456,1432$, 1374, 1323, 1274, 1215, 1173, 1131, 1080, 1041, 1022, 973, 950, 888, 862, 807, 717, 605, 526, 480, 
$454 ;{ }^{1} \mathbf{H}$ NMR $\left(400 \mathrm{MHz}, \mathrm{CDCl}_{3}\right): \delta[\mathrm{ppm}]=7.59(\mathrm{~d}, J=2.2 \mathrm{~Hz}, 1 \mathrm{H}), 7.47(\mathrm{dd}, J=8.4 \mathrm{~Hz}$, $J=2.2 \mathrm{~Hz}, 1 \mathrm{H}), 7.29(\mathrm{~d}, J=8.4 \mathrm{~Hz}, 1 \mathrm{H}), 4.63(\mathrm{t}, J=7.3 \mathrm{~Hz}, 2 \mathrm{H}), 3.39(\mathrm{t}, J=7.3 \mathrm{~Hz}$, $2 \mathrm{H}) ;{ }^{13} \mathbf{C}\left\{{ }^{1} \mathbf{H}\right\}$ NMR (101 MHz, $\left.\mathrm{CDCl}_{3}\right): \delta[\mathrm{ppm}]=139.9,138.5,137.4,134.9,124.3,93.0$, 74.0, 33.5; HR-ESI-MS m/z: calcd. $\mathrm{C}_{8} \mathrm{H}_{7} \mathrm{BrINO}_{2}[\mathrm{M}]^{+}: 354.8705$, found: 354.8710 .

\section{2-(4-Bromo-4'-(trifluoromethyl)-[1,1'-biphenyl]-3-yl)acetonitrile (229)}

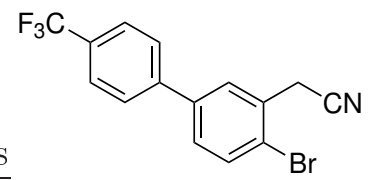

229

Following general procedure [H] 2-(2-bromo-5-iodophenyl)acetonitrile (300 mg, 932 umol, 1.0 equiv), 4-(trifluoromethyl)phenylboronic acid

(195 mg, $1.03 \mathrm{mmol}, 1.1$ equiv), potassium carbonate (515 mg, $3.73 \mathrm{mmol}, 4.0$ equiv), toluene $(7.5 \mathrm{~mL})$, ethanol $(3.0 \mathrm{~mL})$, water (1.5 mL), tetrakis[triphenylphosphine]palladium(0) (108 mg, $93.2 \mu \mathrm{mol}$, 0.1 equiv); reaction time $65 \mathrm{~h}$; eluting with $n$-pentane/EtOAc, 75:1; white solid; $113 \mathrm{mg}$, $332 \mu \mathrm{mol}, 36 \%$.

$\mathbf{R}_{\mathbf{f}}=0.07$ ( $n$-pentane:EtOAc, 75:1); m.p. $=105{ }^{\circ} \mathrm{C}$; IR (ATR): $\tilde{\nu}\left[\mathrm{cm}^{-1}\right]=2934,2910,2248$, 1616, 1472, 1398, 1388, 1322, 1164, 1103, 1072, 1014, 956, 928, 849, 817, 745, 715, 669, 607, $518,442,410 ;{ }^{1} \mathbf{H}$ NMR $\left(300 \mathrm{MHz}, \mathrm{CDCl}_{3}\right): \delta[\mathrm{ppm}]=7.80-7.60(\mathrm{~m}, 6 \mathrm{H}), 7.44(\mathrm{dd}, J=8.3$, $2.3 \mathrm{~Hz}, 1 \mathrm{H}), 3.92(\mathrm{~s}, 2 \mathrm{H}) ;{ }^{\mathbf{1 3}} \mathbf{C}\left\{{ }^{\mathbf{1}} \mathbf{H}\right\} \mathbf{N M R}\left(126 \mathrm{MHz}, \mathrm{CDCl}_{3}\right): \delta[\mathrm{ppm}]=142.7,140.1,133.7$, $132.3,130.7,130.3\left(\mathrm{q},{ }^{2} J_{\mathrm{CF}}=32.7 \mathrm{~Hz}\right), 128.6,128.4,127.4,126.1\left(\mathrm{q},{ }^{3} J_{\mathrm{CF}}=3.7 \mathrm{~Hz}\right), 124.1$ $\left(\mathrm{q},{ }^{1} J_{\mathrm{CF}}=271.6\right), 123.6,116.8,25.2 ;{ }^{\mathbf{1 9}} \mathbf{F}\left\{{ }^{\mathbf{1}} \mathbf{H}\right\} \mathbf{N M R}\left(282 \mathrm{MHz}, \mathrm{CDCl}_{3}\right): \delta[\mathrm{ppm}]=-62.6$; HR-EI-MS m/z: calcd. $\mathrm{C}_{15} \mathrm{H}_{9} \mathrm{BrF}_{3} \mathrm{~N}[\mathrm{M}]^{+}: 338.9870$, found: 338.9866 .

\section{(E)-2-(4-(Pent-1-en-1-yl)-4'-(trifluoromethyl)-[1,1'-biphenyl]-3-yl)acetonitrile (232)}

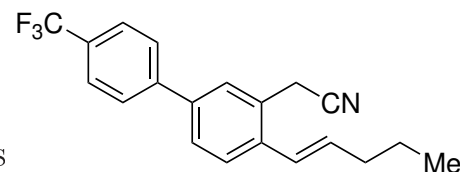

232

Following general procedure $\mathbf{H}$ 2-(4-bromo-4'-(trifluoromethyl)[1,1'-biphenyl]-3-yl)acetonitrile (100 mg, 294 umol, 1.0 equiv), 1-pentenylboronic acid (61 mg, $0.32 \mathrm{mmol}, 1.1$ equiv), potassium carbonate $(163 \mathrm{mg}, 1.18 \mathrm{mmol}, 4.0$ equiv), toluene $(2.5 \mathrm{~mL})$, ethanol $(1.0 \mathrm{~mL})$, water $(0.5 \mathrm{~mL})$, tetrakis[triphenylphosphine]palladium(0) (34 mg, 29 umol, 0.1 equiv); reaction time $16.5 \mathrm{~h}$; eluting with $n$ pentane/DCM, 5:1; white solid; $87 \mathrm{mg}, 0.26 \mathrm{mmol}, 89 \%$.

$\mathbf{R}_{\mathbf{f}}=0.09$ (n-pentane:DCM, 5:1); m.p. $=75{ }^{\circ} \mathrm{C}$; IR (ATR): $\tilde{\nu}\left[\mathrm{cm}^{-1}\right]=2960,2931,2866,2838$, 2246, 1614, 1493, 1427, 1395, 1319, 1170, 1128, 1112, 1070, 1015, 968, 893, 836, 812, 741, 707, $601 ;{ }^{1} \mathbf{H}$ NMR $\left(500 \mathrm{MHz}, \mathrm{CDCl}_{3}\right): \delta[\mathrm{ppm}]=7.70(\mathrm{~s}, 4 \mathrm{H}), 7.60(\mathrm{~d}, J=1.3 \mathrm{~Hz}, 1 \mathrm{H}), 7.56(\mathrm{~d}$, $J=8.0 \mathrm{~Hz}, 1 \mathrm{H}), 7.53(\mathrm{dd}, J=8.1,1.8 \mathrm{~Hz}, 1 \mathrm{H}), 6.50(\mathrm{dt}, J=15.4,1.5 \mathrm{~Hz}, 1 \mathrm{H}), 6.23(\mathrm{dt}$, $J=15.5,7.0 \mathrm{~Hz}, 1 \mathrm{H}), 3.82(\mathrm{~s}, 2 \mathrm{H}), 2.27$ (qd, $J=7.1,1.5 \mathrm{~Hz}, 2 \mathrm{H}), 1.55$ (sext, $J=7.4 \mathrm{~Hz}, 2 \mathrm{H}$ ), $0.99(\mathrm{t}, J=7.4 \mathrm{~Hz}, 3 \mathrm{H}) ;{ }^{13} \mathbf{C}\left\{{ }^{1} \mathbf{H}\right\} \mathbf{N M R}\left(126 \mathrm{MHz}, \mathrm{CDCl}_{3}\right): \delta[\mathrm{ppm}]=143.6,139.0,137.1$, $136.4,129.8\left(\mathrm{q},{ }^{2} J_{\mathrm{CF}}=32.4 \mathrm{~Hz}\right), 127.7,127.6,127.5,127.4,127.3,126.0\left(\mathrm{q},{ }^{3} J_{\mathrm{CF}}=3.8 \mathrm{~Hz}\right)$, 
125.3, $124.4\left(\mathrm{q},{ }^{1} J_{\mathrm{CF}}=271.9 \mathrm{~Hz}\right), 117.7,35.6,22.5,22.1,13.9 ;$ HR-ESI-MS m/z: calcd. $\mathrm{C}_{20} \mathrm{H}_{18} \mathrm{NF}_{3} \mathrm{Na}[\mathrm{M}+\mathrm{Na}]^{+}$: 352.1284 , found: 352.1273 .

\subsubsection{Synthesis of tetrahydroisoquinolines 209}

\section{General procedure $\mathrm{K}$. Synthesis of tetrahydroisoquinolines}

Tosyl amide 207 (1.0 equiv), NFSI (1.05 equiv), diphenyl diselenide (10 M in toluene, 0.05 equiv) and activated molecular sieves $4 \AA$ (spatula tip) are dissolved in toluene $(0.1 \mathrm{M})$ and stirred at room temperature until completion, detected by NMR and TLC. The solvent is removed in vacuo and the residue purified on silica gel to yield the title compound.

\section{(E)-1-(But-1-en-1-yl)-2-tosyl-1,2,3,4-tetrahydroisoquinoline (208)}

Tosyl amide 207a (86 mg, $0.25 \mathrm{mmol}, 1.0$ equiv), diphenyl diselenide (7.8 mg,

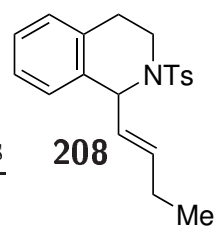
25 umol, 0.1 equiv) and $p$-MeO-TPT (95) (6.0 mg, $12.5 \mu \mathrm{mol}, 0.05$ equiv) were dissolved in MeCN $(2.5 \mathrm{~mL})$ and stirred under air and irradiation with blue light at room temperature for $4 \mathrm{~h}$. Removal of the solvent in vacuo, followed by column chromatography ( $n$-pentane/EtOAc, $7: 1$ ) afforded the product as a yellow oil (12 mg, 34 mol, 13\%).

$\mathbf{R}_{\mathbf{f}}=0.43$ (n-pentane:EtOAc, 7:1); IR (ATR): $\tilde{\nu}\left[\mathrm{cm}^{-1}\right]=2961,2928,2872,1598,1493,1453$, 1334, 1275, 1158, 1091, 1015, 964, 923, 813, 762, 713, 663, 569, 545; ${ }^{\mathbf{H}} \mathbf{H} \mathbf{N M R}\left(600 \mathrm{MHz}, \mathrm{C}_{6} \mathrm{D}_{6}\right)$ : $\delta[\mathrm{ppm}]=7.78-7.65(\mathrm{~m}, 2 \mathrm{H}), 6.96-6.76(\mathrm{~m}, 3 \mathrm{H}), 6.72-6.62(\mathrm{~m}, 3 \mathrm{H}), 5.75(\mathrm{~d}, J=3.6 \mathrm{~Hz}, 1 \mathrm{H})$, $5.47-5.45(\mathrm{~m}, 2 \mathrm{H}), 3.85$ (dddd, $J=13.4,6.3,2.4,1.1 \mathrm{~Hz}, 1 \mathrm{H}), 3.13$ (ddd, $J=13.4,11.6$, $4.3 \mathrm{~Hz}, 1 \mathrm{H}), 2.57$ (ddd, $J=17.3,11.6,6.3 \mathrm{~Hz}, 1 \mathrm{H}), 2.14$ (ddd, $J=16.4,4.3,2.3 \mathrm{~Hz}, 1 \mathrm{H}$ ), $1.82(\mathrm{~s}, 3 \mathrm{H}), 1.80-1.63(\mathrm{~m}, 1 \mathrm{H}), 0.72(\mathrm{t}, J=7.4 \mathrm{~Hz}, 3 \mathrm{H}) ;{ }^{\mathbf{1 3}} \mathbf{C}\left\{{ }^{\mathbf{1}} \mathbf{H}\right\}$ NMR $\left(125 \mathrm{MHz}, \mathrm{C}_{6} \mathrm{D}_{6}\right)$ : $\delta[\mathrm{ppm}]=142.4,139.5,135.6,135.4,133.9,129.4,129.2,129.1,128.4,127.7,126.9,126.2,58.3$, 39.6, 28.2, 25.6, 21.2, 13.4; HR-ESI-MS m/z: calcd. $\mathrm{C}_{20} \mathrm{H}_{24} \mathrm{NO}_{2} \mathrm{~S}[\mathrm{M}+\mathrm{H}]^{+}$: 342.1522 , found: 342.1528 .

\section{(Z)-1-Butylidene-7-fluoro-2-tosyl-1,2,3,4-tetrahydroisoquinoline (209c)}

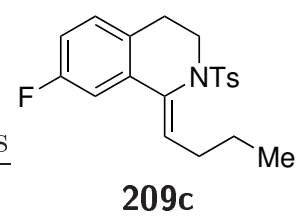

$\mathbf{R}_{\mathbf{f}}=0.29$ (n-pentane:EtOAc, 10:1); IR (ATR): $\tilde{\nu}\left[\mathrm{cm}^{-1}\right]=2958,2929,2871,1613,1597,1583$, 1491, 1455, 1429, 1346, 1268, 1184, 1159, 1133, 1090, 1063, 1020, 934, 889, 866, 811, 777, 722, 708, $685,657,573,544,502,487,470 ;{ }^{\mathbf{1}} \mathbf{H} \mathbf{N M R}\left(600 \mathrm{MHz}, \mathrm{CDCl}_{3}\right): \delta[\mathrm{ppm}]=7.52(\mathrm{~d}, J=8.2 \mathrm{~Hz}$, 
$2 \mathrm{H}), 7.10(\mathrm{dd}, J=10.1,2.6 \mathrm{~Hz}, 1 \mathrm{H}), 7.00(\mathrm{~d}, J=8.1 \mathrm{~Hz}, 2 \mathrm{H}), 6.71(\mathrm{td}, J=8.3,2.6 \mathrm{~Hz}, 1 \mathrm{H})$, $6.66(\mathrm{dd}, J=8.5,5.8 \mathrm{~Hz}, 1 \mathrm{H}), 6.21(\mathrm{t}, J=7.3 \mathrm{~Hz}, 1 \mathrm{H}), 3.80(\mathrm{~s}, 2 \mathrm{H}), 2.55-2.40(\mathrm{~m}, 4 \mathrm{H})$, 2.27 (s, $3 \mathrm{H}), 1.49$ (q, $J=7.4 \mathrm{~Hz}, 2 \mathrm{H}), 0.96$ (t, $J=7.3 \mathrm{~Hz}, 3 \mathrm{H}) ;{ }^{\mathbf{1 3}} \mathbf{C}\left\{{ }^{1} \mathbf{H}\right\}$ NMR $(126 \mathrm{MHz}$, $\left.\mathrm{CDCl}_{3}\right): \delta[\mathrm{ppm}]=161.2\left(\mathrm{~d},{ }^{1} J_{\mathrm{CF}}=243.6 \mathrm{~Hz}\right), 143.4,137.3,134.7\left(\mathrm{~d},{ }^{3} J_{\mathrm{CF}}=7.1 \mathrm{~Hz}\right), 132.6$, $132.5\left(\mathrm{~d},{ }^{4} J_{\mathrm{CF}}=3.0 \mathrm{~Hz}\right), 130.2\left(\mathrm{~d},{ }^{3} J_{\mathrm{CF}}=8.0 \mathrm{~Hz}\right), 129.0,128.0\left(\mathrm{~d},{ }^{4} J_{\mathrm{CF}}=2.8 \mathrm{~Hz}\right), 127.6,114.4$ $\left(\mathrm{d},{ }^{2} J_{\mathrm{CF}}=21.7 \mathrm{~Hz}\right), 110.4\left(\mathrm{~d},{ }^{2} J_{\mathrm{CF}}=22.5 \mathrm{~Hz}\right), 46.0,31.2,25.3,22.5,21.6,14.2$; HR-ESI-MS m/z: calcd. $\mathrm{C}_{20} \mathrm{H}_{23} \mathrm{FNO}_{2} \mathrm{~S}[\mathrm{M}+\mathrm{H}]^{+}: 350.1428$, found: 360.1428 .

\section{(Z)-1-Butylidene-6-fluoro-2-tosyl-1,2,3,4-tetrahydroisoquinoline (209d)}

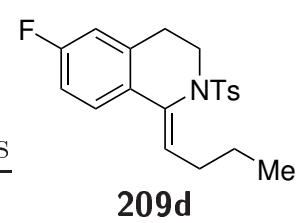

Following general procedure $\mathbf{K}$ tosyl amide 207d $(50 \mathrm{mg}, 0.14 \mathrm{mmol}$, 1.0 equiv), NFSI (46 mg, $0.15 \mathrm{mmol}, 1.05$ equiv), diphenyl diselenide (10 M in toluene, $0.2 \mathrm{~mL}, 2.0 \mathrm{mg}, 6.9 \mathrm{\mu mol}, 0.05$ equiv), activated molecular sieves $4 \AA$ (spatula tip), toluene $(1.4 \mathrm{~mL})$; reaction time $16 \mathrm{~h}$; eluting with $n$ pentane/EtOAc, 10:1; yellow oil; $10 \mathrm{mg}, 28$ umol, $21 \%$.

$\mathbf{R}_{\mathbf{f}}=0.43$ (n-pentane:EtOAc, 10:1); IR (ATR): $\tilde{\nu}\left[\mathrm{cm}^{-1}\right]=2958,2929,2871,1611,1597,1492$, 1454, 1345, 1305, 1247, 1227, 1160, 1107, 1089, 1063, 1019, 967, 888, 863, 813, 708, 688, 663, 636, 608, 587, 567, 547, 507; ${ }^{\mathbf{1}} \mathbf{H}$ NMR $\left(400 \mathrm{MHz}, \mathrm{CDCl}_{3}\right): \delta[\mathrm{ppm}]=7.55-7.46(\mathrm{~m}, 2 \mathrm{H}), 7.38$ $(\mathrm{dd}, J=8.7,5.6 \mathrm{~Hz}, 1 \mathrm{H}), 7.04-6.96(\mathrm{~m}, 2 \mathrm{H}), 6.80(\mathrm{td}, J=8.6,2.7 \mathrm{~Hz}, 1 \mathrm{H}), 6.41(\mathrm{dd}, J=9.3$, $2.6 \mathrm{~Hz}, 1 \mathrm{H}), 6.15(\mathrm{t}, J=7.3 \mathrm{~Hz}, 1 \mathrm{H}), 3.82(\mathrm{~s}, 2 \mathrm{H}), 2.47$ (t, $J=6.9 \mathrm{~Hz}, 4 \mathrm{H}), 2.29(\mathrm{~s}, 3 \mathrm{H})$, $1.49(\mathrm{q}, J=7.4 \mathrm{~Hz}, 2 \mathrm{H}), 0.96(\mathrm{t}, J=7.4 \mathrm{~Hz}, 3 \mathrm{H}) ;{ }^{\mathbf{1 3}} \mathbf{C}\left\{{ }^{\mathbf{1}} \mathbf{H}\right\} \mathbf{N M R}\left(101 \mathrm{MHz}, \mathrm{CDCl}_{3}\right): \delta$ $[\mathrm{ppm}]=161.9\left(\mathrm{~d},{ }^{1} J_{\mathrm{CF}}=247.1 \mathrm{~Hz}\right), 143.5,137.5,134.7\left(\mathrm{~d},{ }^{3} J_{\mathrm{CF}}=7.7 \mathrm{~Hz}\right), 132.5,131.4(\mathrm{~d}$, $\left.{ }^{4} J_{\mathrm{CF}}=1.7 \mathrm{~Hz}\right), 129.5\left(\mathrm{~d},{ }^{4} J_{\mathrm{CF}}=3.1 \mathrm{~Hz}\right), 129.1,127.7,126.0\left(\mathrm{~d},{ }^{3} J_{\mathrm{CF}}=8.2 \mathrm{~Hz}\right), 114.9(\mathrm{~d}$, $\left.{ }^{2} J_{\mathrm{CF}}=21.1 \mathrm{~Hz}\right), 113.8\left(\mathrm{~d},{ }^{2} J_{\mathrm{CF}}=21.9 \mathrm{~Hz}\right), 45.6,31.1,25.2,22.5,21.6,14.1$; HR-ESI-MS $\mathbf{m} / \mathbf{z}$ : calcd. $\mathrm{C}_{20} \mathrm{H}_{23} \mathrm{FNO}_{2} \mathrm{~S}[\mathrm{M}+\mathrm{H}]^{+}: 360.1428$, found: 360.1426 .

\section{(Z)-1-Butylidene-2-tosyl-7-(trifluoromethyl)-1,2,3,4-tetrahydroisoquinoline (209e)}

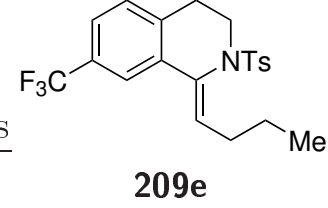

$\mathbf{R}_{\mathbf{f}}=0.19$ (n-pentane:EtOAc, 10:1); IR (ATR): $\tilde{\nu}\left[\mathrm{cm}^{-1}\right]=2959,2931,2874,1722,1692,1652$, 1620, 1597, 1496, 1457, 1430, 1331, 1310, 1285, 1241, 1159, 1119, 1080, 893, 813, 678; ${ }^{\mathbf{1}} \mathbf{H}$ NMR $\left(400 \mathrm{MHz}, \mathrm{CDCl}_{3}\right): \delta[\mathrm{ppm}]=7.59(\mathrm{~d}, J=1.8 \mathrm{~Hz}, 1 \mathrm{H}), 7.51(\mathrm{~d}, J=8.3 \mathrm{~Hz}, 2 \mathrm{H}), 7.23(\mathrm{~m}$, $1 \mathrm{H}), 7.09-6.91(\mathrm{~m}, 2 \mathrm{H}), 6.84(\mathrm{~d}, J=8.0 \mathrm{~Hz}, 1 \mathrm{H}), 6.29(\mathrm{t}, J=7.3 \mathrm{~Hz}, 1 \mathrm{H}), 3.86(\mathrm{~s}, 2 \mathrm{H}), 2.59$ $(\mathrm{t}, J=6.7 \mathrm{~Hz}, 2 \mathrm{H}), 2.51(\mathrm{q}, J=7.3 \mathrm{~Hz}, 2 \mathrm{H}), 2.25(\mathrm{~s}, 3 \mathrm{H}), 1.51$ (q, $J=7.4 \mathrm{~Hz}, 2 \mathrm{H}), 0.97$ $(\mathrm{t}, J=7.4 \mathrm{~Hz}, 3 \mathrm{H}) ;{ }^{13} \mathbf{C}\left\{{ }^{1} \mathbf{H}\right\} \mathbf{N M R}\left(101 \mathrm{MHz}, \mathrm{CDCl}_{3}\right): \delta[\mathrm{ppm}]=143.7,137.0,136.5,134.1$, 
133.4, 132.4, 129.3, 129.1, $128.9\left(\mathrm{q},{ }^{2} J_{\mathrm{CF}}=32.4 \mathrm{~Hz}\right), 127.8,124.2\left(\mathrm{q},{ }^{1} J_{\mathrm{CF}}=272.1 \mathrm{~Hz}\right), 123.5$ $\left(\mathrm{q},{ }^{3} J_{\mathrm{CF}}=3.7 \mathrm{~Hz}\right), 121.3\left(\mathrm{q},{ }^{3} J_{\mathrm{CF}}=4.0 \mathrm{~Hz}\right), 45.8,31.1,26.0,22.4,21.4,14.1$; HR-ESI-MS m/z: calcd. $\mathrm{C}_{21} \mathrm{H}_{23} \mathrm{NO}_{2} \mathrm{SF}_{3}[\mathrm{M}+\mathrm{H}]^{+}:$410.1396, found: 410.1395 .

\section{(Z)-1-Butylidene-2-tosyl-6-(trifluoromethyl)-1,2,3,4-tetrahydroisoquinoline (209f)}

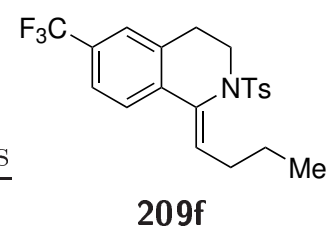

Following general procedure $\mathbf{K}$ tosyl amide $\mathbf{2 0 7 f}$ (20 mg, 49 umol, 1.0 equiv), NFSI (16 mg, 51 mol, 1.05 equiv), diphenyl diselenide $(10 \mathrm{M}$ in toluene, $76 \mu \mathrm{L}, 0.76 \mathrm{mg}, 2.4 \mu \mathrm{mol}, 0.05$ equiv), activated molecular sieves $4 \AA$ (spatula tip), toluene $(0.5 \mathrm{~mL})$; reaction time $16 \mathrm{~h}$; eluting with $n$-pentane/EtOAc, 15:1; yellow oil; $4.7 \mathrm{mg}, 12 \mu \mathrm{mol}, 23 \%$.

$\mathbf{R}_{\mathbf{f}}=0.36$ (n-pentane:EtOAc, 15:1); ${ }^{\mathbf{1}} \mathbf{H}$ NMR $\left(300 \mathrm{MHz}, \mathrm{CDCl}_{3}\right): \delta[\mathrm{ppm}]=7.64(\mathrm{~d}, J=$ $8.0 \mathrm{~Hz}, 1 \mathrm{H}), 7.60-7.42(\mathrm{~m}, 2 \mathrm{H}), 7.32(\mathrm{~d}, J=8.2 \mathrm{~Hz}, 1 \mathrm{H}), 7.17$ (t, $J=8.3 \mathrm{~Hz}, 1 \mathrm{H}), 7.07-6.85$ $(\mathrm{m}, 2 \mathrm{H}), 6.34(\mathrm{t}, J=7.3 \mathrm{~Hz}, 1 \mathrm{H}), 3.87(\mathrm{~s}, 2 \mathrm{H}), 2.54(\mathrm{dt}, J=15.1,7.1 \mathrm{~Hz}, 4 \mathrm{H}), 2.25(\mathrm{~s}, 3 \mathrm{H})$, 1.79-1.39 (m, $2 \mathrm{H}), 0.97$ (t, $J=7.4 \mathrm{~Hz}, 3 \mathrm{H})$; HR-ESI-MS m/z: calcd. $\mathrm{C}_{21} \mathrm{H}_{22} \mathrm{NO}_{2} \mathrm{SF}_{3} \mathrm{Na}$ $[\mathrm{M}+\mathrm{Na}]^{+}:$432.1216, found: 432.1215 .

\section{(Z)-1-Butylidene-6-methoxy-2-tosyl-1,2,3,4-tetrahydroisoquinoline (209g)}

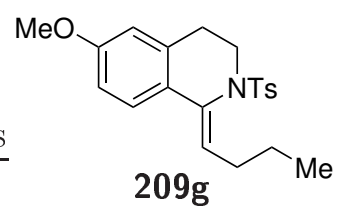

Following general procedure $\mathbf{K}$ tosyl amide $\mathbf{2 0 7 g}$ (50 $\mathrm{mg}, 0.13 \mathrm{mmol}$, 1.0 equiv), NFSI (44 mg, $0.14 \mathrm{mmol}, 1.05$ equiv), diphenyl diselenide (10 M in toluene, $0.2 \mathrm{~mL}, 2.0 \mathrm{mg}, 6.7$ umol, 0.05 equiv), activated molecular sieves $4 \AA$ (spatula tip), toluene $(1.4 \mathrm{~mL})$; reaction time $16 \mathrm{~h}$; eluting with $n$-pentane/EtOAc, 6:1; yellow oil; $23 \mathrm{mg}, 62 \mu \mathrm{mol}, 48 \%$.

$\mathbf{R}_{\mathbf{f}}=0.71$ (n-pentane:EtOAc, 6:1); ${ }^{\mathbf{1}} \mathbf{H}$ NMR $\left(300 \mathrm{MHz}, \mathrm{CDCl}_{3}\right): \delta[\mathrm{ppm}]=7.56-7.46(\mathrm{~m}, 2 \mathrm{H})$, $7.35(\mathrm{~d}, J=8.7 \mathrm{~Hz}, 1 \mathrm{H}), 7.06-6.87(\mathrm{~m}, 2 \mathrm{H}), 6.67(\mathrm{dd}, J=8.7,2.7 \mathrm{~Hz}, 1 \mathrm{H}), 6.21(\mathrm{~d}, J=2.7 \mathrm{~Hz}$, $1 \mathrm{H}), 6.10(\mathrm{t}, J=7.3 \mathrm{~Hz}, 1 \mathrm{H}), 3.90-3.73(\mathrm{~m}, 2 \mathrm{H}), 3.70(\mathrm{~d}, J=0.7 \mathrm{~Hz}, 3 \mathrm{H}), 2.45(\mathrm{q}, J=6.6 \mathrm{~Hz}$, $4 \mathrm{H}), 2.28$ (s, $3 \mathrm{H}), 1.48$ (q, $J=7.4 \mathrm{~Hz}, 2 \mathrm{H}), 0.95$ (t, $J=7.4 \mathrm{~Hz}, 3 \mathrm{H})$; HR-ESI-MS m/z: calcd. $\mathrm{C}_{21} \mathrm{H}_{26} \mathrm{NO}_{3} \mathrm{~S}[\mathrm{M}+\mathrm{H}]^{+}$: 372.1628 , found: 372.1625 .

\section{(Z)-5-Butylidene-6-tosyl-5,6,7,8-tetrahydro-[1,3]dioxolo[4,5-g]isoquinoline (209h)}

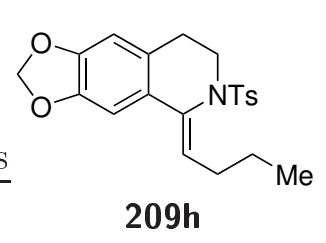

Following general procedure $[\mathbf{K}$ tosyl amide $\mathbf{2 0 7 h}(50 \mathrm{mg}, 0.13 \mathrm{mmol}$, 1.0 equiv), NFSI (43 mg, $0.14 \mathrm{mmol}, 1.05$ equiv), diphenyl diselenide $(10 \mathrm{M}$ in toluene, $0.20 \mathrm{~mL}, 2.0 \mathrm{mg}, 6.5 \mu \mathrm{mol}, 0.05$ equiv), activated molecular sieves $4 \AA$ (spatula tip), toluene $(0.8 \mathrm{~mL})$; reaction time $21 \mathrm{~h}$; eluting with $n$-pentane/EtOAc, 20:1; yellow oil; $28 \mathrm{mg}, 74$ umol, 57\%.

$\mathbf{R}_{\mathbf{f}}=0.50$ (n-pentane:EtOAc, 5:1); IR (ATR): $\tilde{\nu}\left[\mathrm{cm}^{-1}\right]=2956,2923,2872,1502,1483,1382$, 1346, 1222, 1159, 1091, 1039, 964, 939, 862, 841, 810, 785, 707, 682, 640, 603, 572, 552, 421; ${ }^{\mathbf{1}} \mathbf{H}$ 
NMR $\left(300 \mathrm{MHz}, \mathrm{CDCl}_{3}\right): \delta[\mathrm{ppm}]=7.64-7.38(\mathrm{~m}, 2 \mathrm{H}), 7.11-6.93(\mathrm{~m}, 2 \mathrm{H}), 6.88(\mathrm{~s}, 1 \mathrm{H}), 6.15$ $(\mathrm{s}, 1 \mathrm{H}), 6.04(\mathrm{t}, J=7.3 \mathrm{~Hz}, 1 \mathrm{H}), 5.87(\mathrm{~s}, 2 \mathrm{H}), 4.00-3.52(\mathrm{~m}, 2 \mathrm{H}), 2.46(\mathrm{q}, J=7.4 \mathrm{~Hz}, 2 \mathrm{H})$, 2.40-2.32 (m, $2 \mathrm{H}), 2.30$ (s, $3 \mathrm{H}), 1.47$ (sext, $J=7.3 \mathrm{~Hz}, 2 \mathrm{H}), 0.94(\mathrm{t}, J=7.3 \mathrm{~Hz}, 3 \mathrm{H})$.

\subsection{Synthesis of allenylamides}

\subsubsection{Synthesis of alkynes 242}

\section{tert-Butyl 4-(tetradec-1-yn-1-yl)piperidine-1-carboxylate (258)}

1-Boc-4-ethynylpiperidine (500 mg, $2.39 \mathrm{mmol}, 1.0$ equiv) was dissolved in

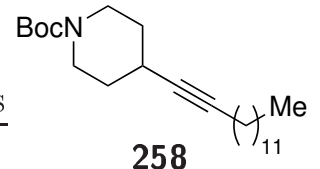
dry THF $(5 \mathrm{~mL})$ and cooled to $-40{ }^{\circ} \mathrm{C} . n$-BuLi $(2.5 \mathrm{M}$ in hexane; $0.96 \mathrm{~mL}$, $2.39 \mathrm{mmol}, 1.0$ equiv) was added dropwise and the solution was allowed to warm to room temperature. $\mathrm{CuCl}(7.0 \mathrm{mg}, 72 \mu \mathrm{mol}, 0.03$ equiv), $\mathrm{LiCl}$ (6.0 mg, $0.14 \mathrm{mmol}, 0.06$ equiv) and dodecyl bromide ( $595 \mathrm{mg}, 2.39 \mathrm{mmol}, 1.0$ equiv) were added and the mixture was stirred at RT for $22 \mathrm{~h}$ and at $50{ }^{\circ} \mathrm{C}$ for $13.5 \mathrm{~h}$. The reaction was quenched by addition of aq. $\mathrm{HCl}(1 \mathrm{M}, 3 \mathrm{~mL})$, the solvent was removed in vacuo, $n$-pentane $(5 \mathrm{~mL})$ was added and the org. phase washed with aq. $\mathrm{HCl}(1 \mathrm{M}, 5 \mathrm{~mL})$ and dried over $\mathrm{Na}_{2} \mathrm{SO}_{4}$. Removal of the solvent in vacuo and purification on silica gel ( $n$-pentane:EtOAc, 20:1) yielded the title compound as a yellow oil (49 $\mathrm{mg}, 0.13 \mathrm{mmol}, 5 \%)$.

$\mathbf{R}_{\mathbf{f}}=0.57$ (n-pentane:EtOAc, 20:1); IR (ATR): $\tilde{\nu}\left[\mathrm{cm}^{-1}\right]=2923,2853,1696,1465,1417,1364$, 1317, 1297, 1271, 1230, 1168, 1119, 1099, 1014, 933, 869, 810, 768, 722; ${ }^{\mathbf{1}} \mathbf{H}$ NMR $(500 \mathrm{MHz}$, $\left.\mathrm{CDCl}_{3}\right): \delta[\mathrm{ppm}]=3.66(\mathrm{ddd}, J=13.3,6.9,3.9 \mathrm{~Hz}, 2 \mathrm{H}), 3.18(\mathrm{ddd}, J=13.4,8.3,3.4 \mathrm{~Hz}$, $2 \mathrm{H}), 2.53$ (dddquint, $J=8.1,6.1,4.0,2.0 \mathrm{~Hz}, 1 \mathrm{H}), 2.14(\mathrm{td}, J=7.1,2.2 \mathrm{~Hz}, 2 \mathrm{H}), 1.73$ (ddt, $J=13.9,7.2,3.8 \mathrm{~Hz}, 2 \mathrm{H}), 1.58-1.41(\mathrm{~m}, 13 \mathrm{H}), 1.41-1.17(\mathrm{~m}, 18 \mathrm{H}), 1.00-0.69(\mathrm{~m}, 3 \mathrm{H})$; ${ }^{13} \mathbf{C}\left\{{ }^{1} \mathbf{H}\right\}$ NMR $\left(126 \mathrm{MHz}, \mathrm{CDCl}_{3}\right): \delta[\mathrm{ppm}]=154.9,82.3,82.0,79.5,42.4,32.1,32.1,29.9$, 29.9, 29.9, 29.8, 29.6, 29.4, 29.3, 29.1, 28.7, 27.3, 22.9, 18.9, 14.4; HR-ESI-MS m/z: calcd. $\mathrm{C}_{24} \mathrm{H}_{43} \mathrm{NO}_{2} \mathrm{Na}[\mathrm{M}+\mathrm{Na}]^{+}:$400.3186, found: 400.3186 .

\section{Icos-7-yne (261)}

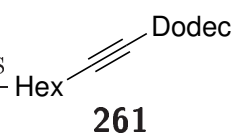

1,3-Bis(1-adamantyl)imidazolium chloride (19 mg, 50 umol, 0.05 equiv), CuI (14 mg, 75 umol, 0.075 equiv), allylpalladium(II) chloride dimer (9.0 mg, $25 \mathrm{\mu mol}, 0.025$ equiv) and $\mathrm{CsCO}_{3}(456 \mathrm{mg}, 1.40 \mathrm{mmol}, 1.4$ equiv) were dissolved in a mixture of dry $\mathrm{Et}_{2} \mathrm{O}$ and dry DMF $(2: 1 \mathrm{v} / \mathrm{v}, 2 \mathrm{~mL}), 1$-octyne $(143 \mathrm{mg}$, $1.30 \mathrm{mmol}, 1.3$ equiv) and dodecyl bromide (249 mg, $1.0 \mathrm{mmol}, 1.0$ equiv) were added and the mixture was stirred at $45{ }^{\circ} \mathrm{C}$ for $15 \mathrm{~h}$. Removal of the solvent in vacuo and purification on silica gel ( $n$-pentane) afforded the title compound as a yellow oil ( $88 \mathrm{mg}, 0.32 \mathrm{mmol}, 32 \%$ ).

$\mathbf{R}_{\mathbf{f}}=0.57$ (n-pentane); IR (ATR): $\tilde{\nu}\left[\mathrm{cm}^{-1}\right]=2921,2853,1465,1378,1332,722 ;{ }^{\mathbf{1}} \mathbf{H} \mathbf{~ N M R}$ $\left(400 \mathrm{MHz}, \mathrm{CDCl}_{3}\right): \delta[\mathrm{ppm}]=2.19-2.10(\mathrm{~m}, 4 \mathrm{H}), 1.56-1.20(\mathrm{~m}, 28 \mathrm{H}), 0.98-0.82(\mathrm{~m}, 6 \mathrm{H})$; 
${ }^{13} \mathbf{C}\left\{{ }^{1} \mathbf{H}\right\}$ NMR $\left(101 \mathrm{MHz}, \mathrm{CDCl}_{3}\right): \delta[\mathrm{ppm}]=80.40,80.39,32.1,31.6,29.8,29.8,29.8,29.7$, 29.5, 29.3, 29.3, 29.0, 28.7, 22.9, 22.7, 18.9, 14.3, 14.2; HR-EI-MS m/z: calcd. $\mathrm{C}_{20} \mathrm{H}_{38}[\mathrm{M}]^{+}$: 278.2973, found: 278.2970 .

\section{Methyl hex-5-ynoate (264)}

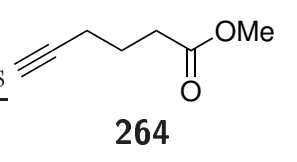

5-Hexynoic acid (561 mg, $5.0 \mathrm{mmol}, 1.0$ equiv), PTSA (43 mg, $0.25 \mathrm{mmol}$, 0.05 equiv), $\mathrm{MeOH}\left(0.41 \mathrm{~mL}, 320 \mathrm{mg}, 10 \mathrm{mmol}, 2.0\right.$ equiv) and $\mathrm{Na}_{2} \mathrm{SO}_{4}$ $(300 \mathrm{mg})$ were added to toluene $(5 \mathrm{~mL})$ and the mixture was stirred at $120{ }^{\circ} \mathrm{C}$ for $4 \mathrm{~h}$. The mixture was allowed to cool down, EtOAc $(5 \mathrm{~mL})$ was added, the org. phase was washed with sat. aq. $\mathrm{NaHCO}_{3}(3 \times 10 \mathrm{~mL})$ and sat. aq. $\mathrm{NaCl}(10 \mathrm{~mL})$. The comb. aq. phases were extracted with EtOAc $(30 \mathrm{~mL})$ and dried over $\mathrm{Na}_{2} \mathrm{SO}_{4}$. Removal of the solvent in vacuo afforded the product as a brown liquid (491 mg, $3.89 \mathrm{mmol}, 78 \%$ ).

IR (ATR): $\tilde{\nu}\left[\mathrm{cm}^{-1}\right]=3293,2952,2362,1732,1436,1369,1317,1215,1157,1058,1015,993$, 898, 867, 773; ${ }^{1} \mathbf{H}$ NMR (400 MHz, $\left.\mathrm{CDCl}_{3}\right): \delta[\mathrm{ppm}]=3.68(\mathrm{~s}, 3 \mathrm{H}), 2.46(\mathrm{t}, J=7.4 \mathrm{~Hz}$, $2 \mathrm{H}), 2.27(\mathrm{td}, J=6.9,2.6 \mathrm{~Hz}, 2 \mathrm{H}), 1.97(\mathrm{t}, J=2.7 \mathrm{~Hz}, 1 \mathrm{H}$ ), 1.85 (quintd, $J=7.2,0.5 \mathrm{~Hz}$, $2 \mathrm{H}) ;{ }^{13} \mathbf{C}\left\{{ }^{1} \mathbf{H}\right\}$ NMR $\left(101 \mathrm{MHz}, \mathrm{CDCl}_{3}\right): \delta[\mathrm{ppm}]=173.6,83.4,69.2,51.7,32.8,23.8,18.0$; HR-ESI-MS m/z: calcd. $\mathrm{C}_{7} \mathrm{H}_{11} \mathrm{O}_{2}[\mathrm{M}+\mathrm{H}]^{+}:$: 27.0754 , found: 127.0745 .

\section{Methyl 4-bromobutanoate (266)}

4-Bromobutyric acid ( $835 \mathrm{mg}, 5.0 \mathrm{mmol}, 1.0$ equiv), PTSA (43 mg, $0.25 \mathrm{mmol}$,<smiles>COC(=O)CCCBr</smiles>
0.05 equiv), $\mathrm{MeOH}\left(0.41 \mathrm{~mL}, 320 \mathrm{mg}, 10 \mathrm{mmol}, 2.0\right.$ equiv) and $\mathrm{Na}_{2} \mathrm{SO}_{4}$ $(300 \mathrm{mg})$ were added to toluene $(5 \mathrm{~mL})$ and the mixture was stirred at $120{ }^{\circ} \mathrm{C}$ for $2.5 \mathrm{~h}$. The mixture was allowed to cool down, EtOAc $(5 \mathrm{~mL})$ was added, the org. phase was washed with sat. aq. $\mathrm{NaHCO}_{3}(3 \times 10 \mathrm{~mL})$ and sat. aq. $\mathrm{NaCl}(10 \mathrm{~mL})$. The comb. aq. phases were extracted with EtOAc $(30 \mathrm{~mL})$ and dried over $\mathrm{Na}_{2} \mathrm{SO}_{4}$. Removal of the solvent in vacuo afforded the product as a brown liquid (718 $\mathrm{mg}, 3.96 \mathrm{mmol}, 79 \%)$.

IR (ATR): $\tilde{\nu}\left[\mathrm{cm}^{-1}\right]=2952,1732,1436,1366,1312,1251,1205,1171,1130,1061,1026,993$, 874, 779; ${ }^{1} \mathbf{H}$ NMR $\left(400 \mathrm{MHz}, \mathrm{CDCl}_{3}\right): \delta[\mathrm{ppm}]=3.69(\mathrm{~s}, 3 \mathrm{H}), 3.47(\mathrm{t}, J=6.4 \mathrm{~Hz}, 2 \mathrm{H}), 2.51$ $(\mathrm{t}, J=7.2 \mathrm{~Hz}, 2 \mathrm{H}), 2.26-1.98(\mathrm{~m}, 2 \mathrm{H}) ;{ }^{\mathbf{1 3}} \mathbf{C}\left\{{ }^{\mathbf{1}} \mathbf{H}\right\} \mathbf{N M R}\left(101 \mathrm{MHz}, \mathrm{CDCl}_{3}\right): \delta[\mathrm{ppm}]=173.1$, 51.9, 32.8, 32.4, 27.9; HR-EI-MS m/z: calcd. $\mathrm{C}_{5} \mathrm{H}_{9} \mathrm{BrO}_{2}[\mathrm{M}]^{+}:$179.9786, found: 179.9777 .

\section{tert-Butyldimethyl(pent-3-yn-1-yloxy)silane (242b)}

3-Pentyne-1-ol (2.52 g, $30.0 \mathrm{mmol}, 1.0$ equiv) was dissolved in dry DCM

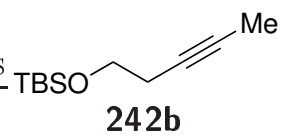

(25 mL), TBSCl (4.97 g, $33.0 \mathrm{mmol}, 1.1$ equiv) and imidazole (2.45 g, $36.0 \mathrm{mmol}, 1.2$ equiv) were added subsequently and the solution was stirred at RT for $18 \mathrm{~h}$. The reaction mixture was washed with sat. aq. $\mathrm{NaCl}$ $(2 \times 25 \mathrm{~mL})$, the comb. aq. phases were extracted with DCM $(2 \times 25 \mathrm{~mL})$ and the comb. org. 
phases were dried over $\mathrm{Na}_{2} \mathrm{SO}_{4}$. Removal of the solvent in vacuo and purification on silica gel ( $n$-pentane:EtOAc, 9:1) afforded the product as a colorless oil (5.72 g, $28.8 \mathrm{mmol}, 96 \%$ ).

$\mathbf{R}_{\mathbf{f}}=0.86$ ( $n$-pentane:EtOAc, 9:1); IR (ATR): $\tilde{\nu}\left[\mathrm{cm}^{-1}\right]=2954,2928,2857,1471,1387,1362$, 1335, 1254, 1145, 1098, 1058, 1006, 938, 913, 833, 773, 716, 661; ${ }^{\mathbf{1}} \mathbf{H} \mathbf{N M R}\left(500 \mathrm{MHz}, \mathrm{CDCl}_{3}\right)$ : $\delta[\mathrm{ppm}]=3.69(\mathrm{t}, J=7.3 \mathrm{~Hz}, 2 \mathrm{H}), 2.34(\mathrm{tq}, J=7.3,2.5 \mathrm{~Hz}, 2 \mathrm{H}), 1.77(\mathrm{t}, J=2.5 \mathrm{~Hz}, 3 \mathrm{H})$, $0.90(\mathrm{~s}, 9 \mathrm{H}), 0.07(\mathrm{~s}, 6 \mathrm{H}) ;{ }^{13} \mathbf{C}\left\{{ }^{\mathbf{1}} \mathbf{H}\right\} \mathbf{N M R}\left(126 \mathrm{MHz}, \mathrm{CDCl}_{3}\right): \delta[\mathrm{ppm}]=76.8,76.2,62.5$, 26.1, 23.4, 18.6, 3.7, -5.0; HR-ESI-MS m/z: calcd. $\mathrm{C}_{11} \mathrm{H}_{23} \mathrm{OSi}[\mathrm{M}+\mathrm{H}]^{+}$: 199.1513, found: 199.1514.

\section{1,5-Diphenylpentan-3-one (275)}

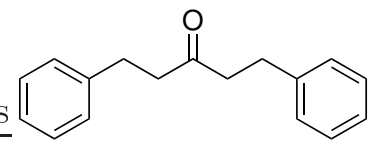

275

Styrene (1.04 g, $10.0 \mathrm{mmol}, 1.0$ equiv), freshly distilled formic acid ( $1.38 \mathrm{~g}, 30.0 \mathrm{mmol}, 3.0$ equiv) and freshly distilled acetic acid anhydride (1.53 g, $15.0 \mathrm{mmol}, 1.5$ equiv) were dissolved in dry MeCN (0.5 mL) and the solution was degassed (freeze-pump-thaw, $3 \times$ ). Palladium(II) trifluoroacetate (166 mg, 500 umol, 0.05 equiv) and 1,3-bis(diphenylphosphino)propane (412 mg, $1.0 \mathrm{mmol}, 0.1$ equiv) were added and the mixture was stirred at $90{ }^{\circ} \mathrm{C}$ for $18.5 \mathrm{~h}$. Removal of the solvent in vacuo and purification on silica gel ( $n$-pentane:EtOAc, 50:1) afforded the product as a yellow oil (492 mg, $2.06 \mathrm{mmol}, 41 \%)$. 204]

Alternative procedure: 1,5,-diphenylpenta-1,4-dien-3-one (2.34 g, $10.0 \mathrm{mmol}, 1.0$ equiv), bis(pinacolato)diboron (10.1 g, $40.0 \mathrm{mmol}, 4.0$ equiv), $\mathrm{CuBr}$ (143 mg, $1.00 \mathrm{mmol}, 0.1$ equiv) and $\mathrm{Cs}_{2} \mathrm{CO}_{3}$ (9.8 g, $30 \mathrm{mmol}, 3.0$ equiv) were dissolved in a mixture of THF (40 mL) and toluene $(200 \mathrm{~mL})$ and the mixture was stirred at $90{ }^{\circ} \mathrm{C}$ under air for $19.5 \mathrm{~h}$ before being allowed to cool to RT. Filtration over celite and purification on silica gel (n-pentane:EtOAc, 20:1) afforded the product as a yellow oil (237 mg, $1.0 \mathrm{mmol}, 10 \%) . \underline{242]}$

$\mathbf{R}_{\mathbf{f}}=0.29$ (n-pentane:EtOAc, 50:1); IR (ATR): $\tilde{\nu}\left[\mathrm{cm}^{-1}\right]=3083,3060,3026,2925,2896,2860$, 1711, 1603, 1495, 1452, 1405, 1368, 1179, 1143, 1092, 1076, 1030, 979, 909, 857, 746, 696; ${ }^{\mathbf{1}} \mathbf{H}$ NMR $\left(400 \mathrm{MHz}, \mathrm{CDCl}_{3}\right): \delta[\mathrm{ppm}]=7.35-7.24(\mathrm{~m}, 4 \mathrm{H}), 7.24-7.11(\mathrm{~m}, 6 \mathrm{H}), 2.89(\mathrm{t}, J=$ $7.6 \mathrm{~Hz}, 4 \mathrm{H}), 2.80-2.65(\mathrm{~m}, 4 \mathrm{H}) ;{ }^{\mathbf{1 3}} \mathbf{C}\left\{{ }^{\mathbf{1}} \mathbf{H}\right\} \mathbf{N M R}\left(101 \mathrm{MHz}, \mathrm{CDCl}_{3}\right): \delta[\mathrm{ppm}]=209.2,141.1$, 128.6, 128.4, 126.2, 44.6, 29.9; HR-ESI-MS m/z: calcd. $\mathrm{C}_{17} \mathrm{H}_{19} \mathrm{O}[\mathrm{M}+\mathrm{H}]^{+}:$239.1430, found: 239.1435 .

\section{1,6-Diphenylhex-3-yne-2,5-diol (280)}<smiles>OC(C#CC(O)c1ccccc1)Cc1ccccc1</smiles>

At RT, a solution of ethinylmagnesium bromide $(0.5 \mathrm{M}$ in THF; $3.3 \mathrm{~mL}$, $3.0 \mathrm{mmol}, 1.0$ equiv) was added dropwise to a solution of ethylmagnesium bromide (0.9 M in THF; $6.0 \mathrm{~mL}, 3.0 \mathrm{mmol}, 1.0$ equiv) and the mixture was stirred at $65{ }^{\circ} \mathrm{C}$ for $2 \mathrm{~h}$ before being allowed to cool down to RT. A solution of phenylacetaldehyde $(0.79 \mathrm{~g}, 6.6 \mathrm{mmol}, 2.2$ equiv) in dry THF (15 mL) 
was added dropwise and the solution was stirred at $\mathrm{RT}$ for $44 \mathrm{~h}$. The reaction was quenched by addition of sat. aq. $\mathrm{NH}_{4} \mathrm{Cl}(15 \mathrm{~mL})$, the aq. phase was extracted with $\mathrm{Et}_{2} \mathrm{O}(2 \times 15 \mathrm{~mL})$, the comb. org. phases were washed with sat. aq. $\mathrm{NaHCO}_{3}$ and dried over $\mathrm{Na}_{2} \mathrm{SO}_{4}$. Removal of the solvent in vacuo and purification on silica gel ( $n$-pentane:EtOAc, 4:1) afforded the product as a white solid (283 mg, $1.06 \mathrm{mmol}, 35 \%$ ).

$\mathbf{R}_{\mathbf{f}}=0.50$ (n-pentane:EtOAc, 4:1); m.p. $=80.1{ }^{\circ} \mathrm{C} ; \mathbf{I R}$ (ATR): $\tilde{\nu}\left[\mathrm{cm}^{-1}\right]=3312,3060,3028$, 2923, 1708, 1603, 1495, 1453, 1335, 1260, 1123, 1077, 1029, 909, 854, 826, 734, 696; ${ }^{\mathbf{1}} \mathbf{H}$ NMR $\left(400 \mathrm{MHz}, \mathrm{CDCl}_{3}\right): \delta[\mathrm{ppm}]=7.34-7.26(\mathrm{~m}, 6 \mathrm{H}), 7.24-7.17(\mathrm{~m}, 4 \mathrm{H}), 4.68-4.48(\mathrm{~m}, 2 \mathrm{H}), 2.97$ $(\mathrm{dd}, J=6.4,1.5 \mathrm{~Hz}, 4 \mathrm{H}), 1.91(\mathrm{~s}, 2 \mathrm{H}) ;{ }^{\mathbf{1 3}} \mathbf{C}\left\{{ }^{\mathbf{1}} \mathbf{H}\right\} \mathbf{N M R}\left(101 \mathrm{MHz}, \mathrm{CDCl}_{3}\right): \delta[\mathrm{ppm}]=136.5$, 136.5, 129.9, 128.6, 127.1, 86.3, 63.2, 63.2, 44.0, 44.0; HR-ESI-MS m/z: calcd. $\mathrm{C}_{18} \mathrm{H}_{18} \mathrm{O}_{2} \mathrm{Na}$ $[\mathrm{M}+\mathrm{Na}]^{+}:$289.1199, found: 289.1204 .

\section{1,6-Diphenylhex-3-yne (242d)}

Diol 280 (100 mg, 375 mol, 1.0 equiv) was dissolved in dry DCM (1.6 mL),

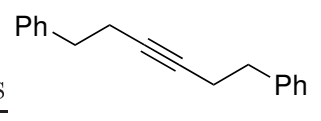

242d $\mathrm{Co}_{2}(\mathrm{CO})_{8}$ (129 mg, 375 umol, 1.0 equiv) was added portionwise and the mixture was stirred at RT for $16 \mathrm{~h}$. It was cooled to $0{ }^{\circ} \mathrm{C}, \mathrm{BH}_{3} \cdot \mathrm{SMe}_{2}$ (120 mg, $1.58 \mathrm{mmol}, 4.2$ equiv) was added dropwise, then TFA $(0.75 \mathrm{~mL})$ was added dropwise. The mixture was stirred at $0{ }^{\circ} \mathrm{C}$ for $30 \mathrm{~min}$, poured into ice water and stirred for $15 \mathrm{~min}$. The phases were separated, the org. phase was washed with $\mathrm{H}_{2} \mathrm{O}(2 \mathrm{~mL})$ and $\mathrm{Fe}\left(\mathrm{NO}_{3}\right) \cdot 9 \mathrm{H}_{2} \mathrm{O}$ (576 mg, $1.43 \mathrm{mmol}, 3.8$ equiv) was added portionwise while stirring the mixture vigorously. After $4 \mathrm{~h}$, the org. phase was decanted, the solvent removed under vacuum and the residue was purified on silica gel ( $n$-pentane:EtOAc, 50:1). The product was afforded as a yellow oil (47 mg, $0.20 \mathrm{mmol}, 54 \%$ ).

$\mathbf{R}_{\mathbf{f}}=0.15$ (n-pentane:EtOAc, 50:1); IR (ATR): $\tilde{\nu}\left[\mathrm{cm}^{-1}\right]=3085,3061,3026,2925,2858,1738$, 1603, 1495, 1453, 1430, 1341, 1219, 1154, 1076, 1030, 906, 745, 695; ${ }^{\mathbf{1}} \mathbf{H}$ NMR $(400 \mathrm{MHz}$, $\left.\mathrm{CDCl}_{3}\right): \delta[\mathrm{ppm}]=7.35-7.27(\mathrm{~m}, 4 \mathrm{H}), 7.25-7.18(\mathrm{~m}, 6 \mathrm{H}), 2.80(\mathrm{t}, J=7.5 \mathrm{~Hz}, 4 \mathrm{H}), 2.60-2.30$ $(\mathrm{m}, 4 \mathrm{H}) ;{ }^{13} \mathbf{C}\left\{{ }^{\mathbf{1}} \mathbf{H}\right\} \mathbf{N M R}\left(101 \mathrm{MHz}, \mathrm{CDCl}_{3}\right): \delta[\mathrm{ppm}]=141.1,128.6,128.4,126.3,80.4,35.6$, 21.1; EI-MS m/z: calcd. $\mathrm{C}_{18} \mathrm{H}_{18}[\mathrm{M}]^{+}$: 234.1, found: 234.1.

\subsubsection{Synthesis of imidoallenes 253}

\section{General Procedure ㄴ: allenylation}

Alkyne 242 (1.00 mmol, 1.0 equiv), $\mathrm{Li}_{2} \mathrm{CO}_{3}$ (74 mg, $1.0 \mathrm{mmol}, 1.0$ equiv), (2-anisyl) ${ }_{2} \mathrm{Se}_{2}(9.0 \mathrm{mg}$, 25 umol, 0.025 equiv) and MS powder $(4 \AA, 30 \mathrm{mg})$ are dissolved in dry toluene $(4 \mathrm{~mL})$ and heated to $100{ }^{\circ} \mathrm{C}$. NFSI $(378 \mathrm{mg}, 1.20 \mathrm{mmol}, 1.2$ equiv) is dissolved in dry toluene $(6 \mathrm{~mL})$ and added over the course of $3.5 \mathrm{~h}$ to the heated mixture using a syringe pump $(1.7 \mathrm{~mL} / \mathrm{h})$. The reaction is stirred at $100{ }^{\circ} \mathrm{C}$ until complete conversion of the alkyne is determined by NMR or TLC. After removal of the solvent, the product is afforded via column chromatography. 


\section{$N$-(Deca-5,6-dien-5-yl)- $N$-(phenylsulfonyl)benzenesulfonamide (253a)}

Following general procedure $\mathrm{L}$ 5-decyne 242a $(138 \mathrm{mg}, 1.00 \mathrm{mmol}$,

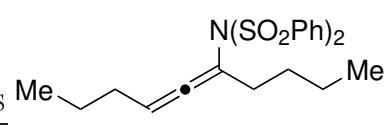

$253 a$

1.0 equiv); reaction time $28 \mathrm{~h}$; eluting with $n$-pentane:EtOAc, 10:1; yield: $161 \mathrm{mg}, 371 \mu \mathrm{mol}, 37 \%$, yellow oil; ${ }^{1} \mathrm{H}$ NMR yield using 1,1,2,2tetrachloroethane as the internal standard: $60 \%$.

$\mathbf{R}_{\mathbf{f}}=0.43$ (n-pentane:EtOAc, 10:1); IR (ATR): $\tilde{\nu}\left[\mathrm{cm}^{-1}\right]=3066,2957,2930,2871,1585,1478$, 1448, 1407, 1372, 1356, 1312, 1291, 1254, 1165, 1121, 1084, 1024, 1000, 937, 894, 782, 752, 718, $684 ;{ }^{1} \mathbf{H}$ NMR $\left(300 \mathrm{MHz}, \mathrm{CDCl}_{3}\right): \delta[\mathrm{ppm}]=8.00(\mathrm{~d}, J=7.7 \mathrm{~Hz}, 4 \mathrm{H}), 7.63(\mathrm{ddt}, J=8.3,6.6$, $1.3 \mathrm{~Hz}, 2 \mathrm{H}), 7.58-7.45(\mathrm{~m}, 4 \mathrm{H}), 5.02(\mathrm{tt}, J=7.4,3.9 \mathrm{~Hz}, 1 \mathrm{H}), 2.34-2.22(\mathrm{~m}, 2 \mathrm{H}), 1.86-1.74$ $(\mathrm{m}, 2 \mathrm{H}), 1.44-1.12(\mathrm{~m}, 6 \mathrm{H}), 0.95-0.85(\mathrm{~m}, 3 \mathrm{H}), 0.81(\mathrm{t}, J=7.3 \mathrm{~Hz}, 3 \mathrm{H}) ;{ }^{\mathbf{1 3}} \mathbf{C}\left\{{ }^{1} \mathbf{H}\right\}$ NMR $\left(126 \mathrm{MHz}, \mathrm{CDCl}_{3}\right): \delta[\mathrm{ppm}]=206.0,133.7,128.9,128.6,106.8,99.0,33.4,30.6,29.1,22.4,22.2$, 14.2, 13.8; HR-ESI-MS m/z: calcd. $\mathrm{C}_{22} \mathrm{H}_{28} \mathrm{NO}_{4} \mathrm{~S}_{2}[\mathrm{M}+\mathrm{H}]^{+}$: 434.1454, found: 434.1447 .

\section{$N$-(5-((tert-Butyldimethylsilyl)oxy)penta-2,3-dien-2-yl)- $N$-(phenylsulfonyl)benzenesulfon-} amide and

$N$-(5-((tert-butyldimethylsilyl)oxy)penta-1,2-dien-3-yl)- $N$-(phenylsulfonyl)benzenesulfonamide

(253b)

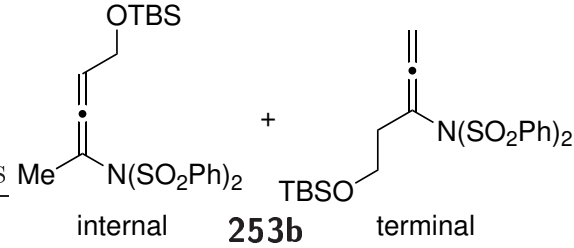

trimethoxybenzene as the internal standard: $31 \%$ (internal allene), 18\% (terminal allene). 1.00 mmol, 1.0 equiv), additional triphenylphosphineselenide (17 mg, 50 umol, 0.05 equiv); reaction time $5.5 \mathrm{~h}$; eluting with $n$-pentane: $\mathrm{Et}_{2} \mathrm{O}, 15: 1$; yield: $184 \mathrm{mg}$, 372 umol, $37 \%$ (mixture of internal and terminal allene 1.7:1), white solid; ${ }^{1} \mathrm{H}$ NMR yield using 1,3,5-

Following general procedure $\mathbf{L}$; alkyne 242b (198 mg,

Internal allene: $\mathbf{R}_{\mathbf{f}}=0.14$ ( $n$-pentane:Et $\left.{ }_{2} \mathrm{O}, 15: 1\right) ; \mathbf{m} . \mathbf{p .}=95.2{ }^{\circ} \mathrm{C} ; \mathbf{I R}(\mathrm{ATR}): \tilde{\nu}\left[\mathrm{cm}^{-1}\right]=2952$, 2927, 2887, 2854, 1471, 1447, 1400, 1363, 1360, 1309, 1294, 1255, 1167, 1151, 1085, 1051, 1002, 973, 902, 875, 835, 774, 754, 719, 687; ${ }^{\mathbf{1}} \mathbf{H}$ NMR $\left(300 \mathrm{MHz}, \mathrm{CDCl}_{3}\right): \delta[\mathrm{ppm}]=8.12-7.75(\mathrm{~m}$, $4 \mathrm{H}), 7.73-7.59(\mathrm{~m}, 2 \mathrm{H}), 7.59-7.45(\mathrm{~m}, 4 \mathrm{H}), 5.12(\mathrm{tq}, J=6.6,6.0,3.1 \mathrm{~Hz}, 1 \mathrm{H}), 3.98(\mathrm{dd}$, $J=12.9,6.7 \mathrm{~Hz}, 1 \mathrm{H}), 3.91(\mathrm{dd}, J=12.9,5.9 \mathrm{~Hz}, 1 \mathrm{H}), 2.02(\mathrm{~d}, J=3.0 \mathrm{~Hz}, 3 \mathrm{H}), 0.87(\mathrm{~s}$, $9 \mathrm{H}), 0.03(\mathrm{~s}, 3 \mathrm{H}), 0.02(\mathrm{~s}, 3 \mathrm{H}) \cdot ;{ }^{\mathbf{1 3}} \mathbf{C}\left\{{ }^{\mathbf{1}} \mathbf{H}\right\} \mathbf{N M R}\left(126 \mathrm{MHz}, \mathrm{CDCl}_{3}\right): \delta[\mathrm{ppm}]=205.6,139.6$, 133.8, 128.9, 128.6, 103.7, 97.3, 60.3, 26.0, 21.0, 18.5, -4.9, -5.0; HR-ESI-MS m/z: calcd. $\mathrm{C}_{23} \mathrm{H}_{32} \mathrm{NO}_{5} \mathrm{~S}_{2} \mathrm{Si}[\mathrm{M}+\mathrm{H}]^{+}:$494.1486, found: 494.1486 .

Terminal allene: $\mathbf{R}_{\mathbf{f}}=0.1$ ( $n$-pentane:Et $\left.{ }_{2} \mathrm{O}, 15: 1\right) ; \mathbf{m . p .}=94.3{ }^{\circ} \mathrm{C} ; \mathbf{I R}(\mathrm{ATR}): \tilde{\nu}\left[\mathrm{cm}^{-1}\right]=2952$, 2926, 2894, 2853, 1472, 1448, 1427, 1365, 1351, 1309, 1295, 1254, 1164, 1085, 1059, 1007, 902, $871,856,835,772,755,719,686 ;{ }^{\mathbf{1}} \mathbf{H}$ NMR $\left(400 \mathrm{MHz}, \mathrm{CDCl}_{3}\right): \delta[\mathrm{ppm}]=8.15-7.88(\mathrm{~m}, 4 \mathrm{H})$, 7.76-7.59 (m, 2 H), 7.59-7.42 (m, $4 \mathrm{H}), 4.69$ (t, $J=3.8 \mathrm{~Hz}, 2 \mathrm{H}), 3.70$ (t, $J=7.0 \mathrm{~Hz}, 2 \mathrm{H}), 2.54$ 
(tt, $J=7.2,3.8 \mathrm{~Hz}, 2 \mathrm{H}), 0.90(\mathrm{~s}, 9 \mathrm{H}), 0.05(\mathrm{~s}, 6 \mathrm{H}) ;{ }^{\mathbf{1 3}} \mathbf{C}\left\{{ }^{\mathbf{1}} \mathbf{H}\right\} \mathbf{N M R}\left(101 \mathrm{MHz}, \mathrm{CDCl}_{3}\right): \delta$ $[\mathrm{ppm}]=211.1,139.4,134.0,129.0,128.8,104.4,82.1,60.3,36.5,26.0,18.4,-5.2$; HR-ESI-MS m/z: calcd. $\mathrm{C}_{23} \mathrm{H}_{32} \mathrm{NO}_{5} \mathrm{~S}_{2} \mathrm{Si}[\mathrm{M}+\mathrm{H}]^{+}$: 494.1486, found: 494.1482 .

\section{4-( $N$-(Phenylsulfonyl)phenylsulfonamido)penta-2,3-dien-1-yl pivalate and} 3-(N-(phenylsulfonyl)phenylsulfonamido)penta-3,4-dien-1-yl pivalate (253c)

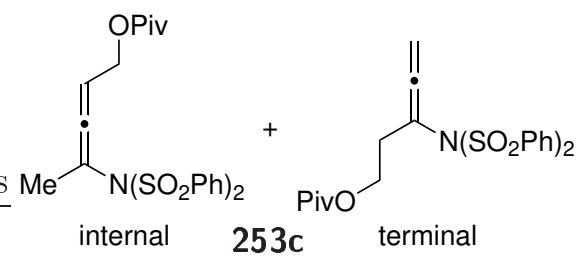

Following general procedure $\mathrm{L}$ alkyne 242c (168 mg, $1.00 \mathrm{mmol}, 1.0$ equiv), additional triphenylphosphineselenide (17 mg, 50 umol, 0.05 equiv); reaction time $22 \mathrm{~h}$;

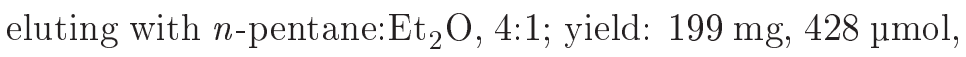
$43 \%$ (mixture of internal and terminal allene 2.3:1), yellow oil (internal), white solid (terminal); ${ }^{1} \mathrm{H}$ NMR yield using 1,3,5-trimethoxybenzene as the internal standard: $25 \%$ (internal allene), $11 \%$ (terminal allene).

Internal allene: $\mathbf{R}_{\mathbf{f}}=0.25$ (n-pentane:Et $\left.{ }_{2} \mathrm{O}, 4: 1\right)$; IR (ATR): $\tilde{\nu}\left[\mathrm{cm}^{-1}\right]=3067,2971,2932$, 2873, 1726, 1584, 1479, 1448, 1377, 1359, 1313, 1281, 1167, 1140, 1084, 1033, 1000, 970, 932, 891, 796, 752, 720, 685; ${ }^{\mathbf{1}} \mathbf{H}$ NMR $\left(300 \mathrm{MHz}, \mathrm{CDCl}_{3}\right): \delta[\mathrm{ppm}]=8.20-7.95(\mathrm{~m}, 4 \mathrm{H})$, 7.76-7.61 $(\mathrm{m}, 2 \mathrm{H}), 7.61-7.40(\mathrm{~m}, 4 \mathrm{H}), 5.18(\mathrm{tq}, J=6.6,3.0 \mathrm{~Hz}, 1 \mathrm{H}), 4.31(\mathrm{dd}, J=12.7,6.7 \mathrm{~Hz}, 1 \mathrm{H})$, $4.23(\mathrm{dd}, J=12.7,6.5 \mathrm{~Hz}, 1 \mathrm{H}), 2.03(\mathrm{~d}, J=3.0 \mathrm{~Hz}, 3 \mathrm{H}), 1.19(\mathrm{~s}, 9 \mathrm{H}) ;{ }^{\mathbf{1 3}} \mathbf{C}\left\{{ }^{\mathbf{1}} \mathbf{H}\right\} \mathbf{N M R}$ $\left(126 \mathrm{MHz}, \mathrm{CDCl}_{3}\right): \delta[\mathrm{ppm}]=207.5,178.0,139.5,133.9,129.0,128.5,104.6,92.6,60.5,38.9$, 27.3, 21.0; HR-ESI-MS m/z: calcd. $\mathrm{C}_{22} \mathrm{H}_{26} \mathrm{NO}_{6} \mathrm{~S}_{2}[\mathrm{M}+\mathrm{H}]^{+}:$464.1196, found: 464.1199 .

Terminal allene: $\mathbf{R}_{\mathbf{f}}=0.19$ (n-pentane:Et $\left.{ }_{2} \mathrm{O}, 4: 1\right) ; \mathbf{m} . \mathbf{p .}=79.8^{\circ} \mathrm{C} ; \mathbf{I R}(\mathrm{ATR}): \tilde{\nu}\left[\mathrm{cm}^{-1}\right]=3067$, 2971, 2934, 2872, 2359, 1725, 1479, 1449, 1376, 1285, 1167, 1085, 1036, 895, 754, 720, 686; ${ }^{\mathbf{1}} \mathbf{H}$ NMR $\left(500 \mathrm{MHz}, \mathrm{CDCl}_{3}\right): \delta[\mathrm{ppm}]=8.15-7.90(\mathrm{~m}, 4 \mathrm{H}), 7.72-7.60(\mathrm{~m}, 2 \mathrm{H}), 7.60-7.45(\mathrm{~m}, 4 \mathrm{H})$, $4.72(\mathrm{t}, J=3.8 \mathrm{~Hz}, 2 \mathrm{H}), 4.15(\mathrm{t}, J=6.4 \mathrm{~Hz}, 2 \mathrm{H}), 2.67$ (tt, $J=6.4,3.8 \mathrm{~Hz}, 2 \mathrm{H}), 1.21(\mathrm{~s}, 9 \mathrm{H})$; ${ }^{13} \mathbf{C}\left\{{ }^{1} \mathbf{H}\right\}$ NMR $\left(126 \mathrm{MHz}, \mathrm{CDCl}_{3}\right): \delta[\mathrm{ppm}]=210.7,178.5,139.2,134.1,129.1,128.8,104.0$, 82.8, 61.0, 38.8, 32.6, 27.3; HR-ESI-MS m/z: calcd. $\mathrm{C}_{22} \mathrm{H}_{26} \mathrm{NO}_{6} \mathrm{~S}_{2}[\mathrm{M}+\mathrm{H}]^{+}:$464.1196, found: 464.1201 .

\section{$N$-(1,6-Diphenylhexa-3,4-dien-3-yl)- $N$-(phenylsulfonyl)benzenesulfonamide (253d)}

Following general procedure $\mathbf{L}$ alkyne 242d (234 mg, $1.0 \mathrm{mmol}, 1.0$ equiv);

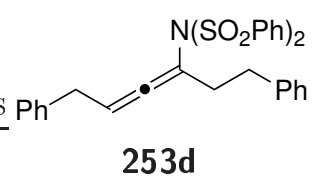
reaction time $6.5 \mathrm{~h}$; eluting with $n$-pentane:EtOAc, 50:1 to 10:1; yield: $288 \mathrm{mg}, 544 \mathrm{\mu mol}, 54 \%$, light yellow solid; ${ }^{1} \mathrm{H}$ NMR yield using 1,3,5trimethoxybenzene as the internal standard: $58 \%$.

$\mathbf{R}_{\mathbf{f}}=0.29$ (n-pentane:EtOAc, 10:1); m.p. $=99.5^{\circ} \mathrm{C} ; \mathbf{I R}(\mathrm{ATR}): \tilde{\nu}\left[\mathrm{cm}^{-1}\right]=3084,3059,3029$, 2921, 2851, 1602, 1582, 1495, 1476, 1447, 1406, 1372, 1357, 1312, 1184, 1164, 1120, 1084, 1025, 1000, 934, 895, 858, 753, 728, 684; ${ }^{1} \mathbf{H}$ NMR $\left(400 \mathrm{MHz}, \mathrm{CDCl}_{3}\right): \delta[\mathrm{ppm}]=8.07-7.83(\mathrm{~m}, 4 \mathrm{H})$, 7.65-7.51 (m, 2 H), 7.51-7.36 (m, $4 \mathrm{H}), 7.33-7.27$ (m, $2 \mathrm{H}), 7.26-7.17(\mathrm{~m}, 6 \mathrm{H})$, 7.02-6.92 (m, 
$2 \mathrm{H}), 5.12$ (tt, $J=7.3,3.6 \mathrm{~Hz}, 1 \mathrm{H}), 3.15-3.11(\mathrm{~m}, 2 \mathrm{H}), 2.78-2.71(\mathrm{~m}, 2 \mathrm{H}), 2.71-2.64(\mathrm{~m}, 2 \mathrm{H})$; ${ }^{13} \mathbf{C}\left\{{ }^{1} \mathbf{H}\right\}$ NMR $\left(101 \mathrm{MHz}, \mathrm{CDCl}_{3}\right): \delta[\mathrm{ppm}]=207.0,140.8,138.7,133.9,129.0,128.7,128.6$, 128.5, 128.4 126.6, 126.2, 106.6, 98.6, 34.7, 34.6, 32.9; HR-ESI-MS m/z: calcd. $\mathrm{C}_{30} \mathrm{H}_{28} \mathrm{NO}_{4} \mathrm{~S}_{2}$ $[\mathrm{M}+\mathrm{H}]^{+}:$530.1454, found: 530.1455 .

\section{Potassium bis(phenylsulfonyl)amide (338)}

Dibenzenesulfonimide (500 mg, $1.68 \mathrm{mmol}, 1.0$ equiv) and $\mathrm{KOH}$ (94 mg, $1.7 \mathrm{mmol}$, $\mathrm{Ph}^{-\mathrm{S}_{-}^{\mathrm{O}_{2}}} \underset{\mathrm{K}^{+}}{\mathrm{O}^{-}} \mathrm{S}_{-\mathrm{Ph}}$ 1.0 equiv) were dissolved in a mixture of acetone and $\mathrm{H}_{2} \mathrm{O}(1: 1 \mathrm{v} / \mathrm{v}, 2 \mathrm{~mL})$ and 338 stirred at RT for $16 \mathrm{~h}$. Removal of the solvent in vacuo afforded the product as a white solid.

IR (ATR): $\tilde{\nu}\left[\mathrm{cm}^{-1}\right]=2359,1737,1445,1265,1148,1088,746,718,683 ;{ }^{1} \mathbf{H}$ NMR $(400 \mathrm{MHz}$, $\left.\mathrm{D}_{2} \mathrm{O}\right): \delta[\mathrm{ppm}]=7.64-7.59(\mathrm{~m}, 4 \mathrm{H}), 7.56-7.51(\mathrm{~m}, 2 \mathrm{H}), 7.44-7.38(\mathrm{~m}, 4 \mathrm{H}) ;{ }^{13} \mathbf{C}\left\{{ }^{1} \mathbf{H}\right\} \mathbf{N M R}$ $\left(101 \mathrm{MHz}, \mathrm{D}_{2} \mathrm{O}\right): \delta[\mathrm{ppm}]=140.9,132.3,128.8,126.0$; HR-ESI-MS m/z: calcd. $\mathrm{C}_{12} \mathrm{H}_{10} \mathrm{NO}_{4} \mathrm{~S}_{2}$ $[\mathrm{M}-\mathrm{K}]^{-}: 296.0057$, found: 296.0057.

\section{Mechanistic investigations}

(E)-N-(6-((2-Methoxyphenyl)selanyl)dec-5-en-5-yl)-N-(phenylsulfonyl)benzenesulfonamide (254)

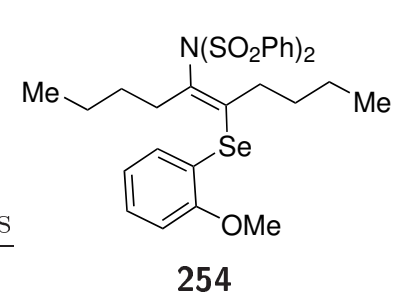

5-Decyne (553 mg, $4.00 \mathrm{mmol}, 1.0$ equiv), (2-anisyl) ${ }_{2} \mathrm{Se}_{2}$ (744 mg, $2.00 \mathrm{mmol}, 0.5$ equiv) and NFSI (1.26 g, $4.00 \mathrm{mmol}, 1.0$ equiv) were dissolved in dry toluene $(40 \mathrm{~mL})$ and stirred at $100{ }^{\circ} \mathrm{C}$ for $18 \mathrm{~h}$. Removal of the solvent in vacuo and purification on silica gel $(n$ pentane:EtOAc, 10:1) afforded the product as a yellow oil $(2.25 \mathrm{~g}$, $3.63 \mathrm{mmol}, 91 \%)$.

$\mathbf{R}_{\mathbf{f}}=0.29$ (n-pentane:EtOAc, 10:1); IR (ATR): $\tilde{\nu}\left[\mathrm{cm}^{-1}\right]=3064,2955,2930,2871,2261,2035$, 1902, 1725, 1579, 1474, 1447, 1432, 1373, 1356, 1312, 1290, 1272, 1244, 1166, 1123, 1083, 1058, 1023, 1000, 983, 930, 866, 792, 750, 718, 684; ${ }^{\mathbf{1}} \mathbf{H}$ NMR $\left(300 \mathrm{MHz}, \mathrm{CDCl}_{3}\right): \delta[\mathrm{ppm}]=8.10-8.00$ $(\mathrm{m}, 4 \mathrm{H}), 7.66-7.59$ (m, $2 \mathrm{H}), 7.54-7.42(\mathrm{~m}, 5 \mathrm{H}), 7.31$ (ddd, $J=8.2,7.4,1.7 \mathrm{~Hz}, 1 \mathrm{H}), 7.08-6.75$ $(\mathrm{m}, 2 \mathrm{H}), 3.90(\mathrm{~s}, 3 \mathrm{H}), 2.66-2.48(\mathrm{~m}, 2 \mathrm{H}), 1.68-1.49(\mathrm{~m}, 4 \mathrm{H}), 1.33-1.16(\mathrm{~m}, 4 \mathrm{H}), 0.91-0.72(\mathrm{~m}$, $5 \mathrm{H}), 0.61-0.49(\mathrm{~m}, 3 \mathrm{H}) ;{ }^{13} \mathbf{C}\left\{{ }^{\mathbf{1}} \mathbf{H}\right\} \mathbf{N M R}\left(126 \mathrm{MHz}, \mathrm{CDCl}_{3}\right): \delta[\mathrm{ppm}]=158.5,146.7,140.0$, 135.3, 133.8, 133.5, 129.4, 129.0, 128.9, 121.4, 118.2, 110.8, 56.1, 38.2, 34.1, 31.5, 31.3, 23.2, 22.7, 14.0, 13.6; ${ }^{77} \mathbf{S e ~ N M R ~}\left(76 \mathrm{MHz}, \mathrm{CDCl}_{3}\right): \delta[\mathrm{ppm}]=314.1$; HR-ESI-MS m/z: calcd. $\mathrm{C}_{29} \mathrm{H}_{35} \mathrm{NO}_{5} \mathrm{SeS}_{2} \mathrm{Na}[\mathrm{M}+\mathrm{Na}]^{+}:$644.1015, found: 644.1011 . 


\section{(E)-N-(6-((2-Methoxyphenyl)selanyl)-2,9-dimethyldec-5-en-5-yl)- $N$-(phenylsulfonyl)ben-} zenesulfonamide

(284)

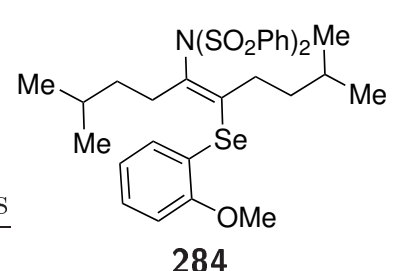

284

2,9-Dimethyl-5-decyne (83 mg, $0.5 \mathrm{mmol}, 1.0$ equiv), alkene $\mathbf{2 5 4}$ (311 mg, 500 umol, 1.0 equiv), $\mathrm{Li}_{2} \mathrm{CO}_{3}$ (37 mg, 0.5 mmol, 1.0 equiv) and MS powder ( $4 \AA, 30 \mathrm{mg})$ were dissolved in dry toluene $(6 \mathrm{~mL})$ and heated to $100{ }^{\circ} \mathrm{C}$. NFSI $(150 \mathrm{mg}, 475$ umol, 0.95 equiv) was dissolved in dry toluene $(4 \mathrm{~mL})$ and added over the course of $2.5 \mathrm{~h}$ to the heated mixture via syringe pump $(1.7 \mathrm{~mL} / \mathrm{h})$. The reaction was

stirred at $100{ }^{\circ} \mathrm{C}$ for $16 \mathrm{~h}$. Removal of the solvent in vacuo and purification on silica gel $(n$ pentane:EtOAc, 15:1) afforded the title compound as a yellow oil (70 $\mathrm{mg}, 0.11 \mathrm{mmol}, 22 \%$ ). Furthermore, diselenide 129 (8 mg, 22 umol, 9\%), starting material 254 (75 mg, 0.12 mmol, 24\%) and a mixture of allenes 253a and 253e $(1.8: 1 ; 114 \mathrm{mg}, 257$ umol) were isolated.

$\mathbf{R}_{\mathbf{f}}=0.29$ (n-pentane:EtOAc, 15:1); IR (ATR): $\tilde{\nu}\left[\mathrm{cm}^{-1}\right]=3065,2953,2929,2868,1580,1474$, 1448, 1433, 1374, 1291, 1272, 1245, 1166, 1124, 1084, 1058, 1023, 984, 880, 793, 749, 730, 686; ${ }^{\mathbf{1}} \mathbf{H}$ NMR $\left(500 \mathrm{MHz}, \mathrm{CDCl}_{3}\right): \delta[\mathrm{ppm}]=8.09-7.99(\mathrm{~m}, 4 \mathrm{H}), 7.67-7.57(\mathrm{~m}, 2 \mathrm{H}), 7.57-7.40(\mathrm{~m}, 5 \mathrm{H})$, 7.32 (ddd, $J=8.2,7.4,1.7 \mathrm{~Hz}, 1 \mathrm{H}), 7.02-6.83(\mathrm{~m}, 2 \mathrm{H}), 3.91(\mathrm{~s}, 3 \mathrm{H}), 2.68-2.51$ (m, $2 \mathrm{H}), 1.59$ $1.47(\mathrm{~m}, 3 \mathrm{H}), 1.47-1.37(\mathrm{~m}, 2 \mathrm{H}), 1.22(\mathrm{ddd}, J=12.1,9.7,6.4 \mathrm{~Hz}, 2 \mathrm{H}), 0.96$ (hept, $J=6.7 \mathrm{~Hz}$, $1 \mathrm{H}), 0.84(\mathrm{~d}, J=6.5 \mathrm{~Hz}, 6 \mathrm{H}), 0.46(\mathrm{~d}, J=6.6 \mathrm{~Hz}, 6 \mathrm{H}) ;{ }^{\mathbf{1 3}} \mathbf{C}\left\{{ }^{\mathbf{1}} \mathbf{H}\right\} \mathbf{N M R}\left(126 \mathrm{MHz}, \mathrm{CDCl}_{3}\right)$ : $\delta[\mathrm{ppm}]=159.0,147.4,140.0,136.4,133.9,132.5,130.0,129.1,128.9,121.3,117.7,110.9,56.1$, 38.0, 37.7, 36.6, 32.0, 28.8, 28.2, 22.5, 22.0; ${ }^{77}$ Se NMR (76 MHz, $\left.\mathrm{CDCl}_{3}\right): \delta[\mathrm{ppm}]=315.9$; HR-ESI-MS m/z: calcd. $\mathrm{C}_{31} \mathrm{H}_{43} \mathrm{~N}_{2} \mathrm{O}_{5} \mathrm{~S}_{2} \mathrm{Se}\left[\mathrm{M}+\mathrm{NH}_{4}\right]^{+}$: 667.1774 , found: 667.1772.

\subsection{Syntheses towards binaphthyl diselenides and catalysis}

Dinaphtho[2,1-c: $\left.1^{\prime}, 2^{\prime}-\mathrm{e}\right][1,2]$ diselenine (293)

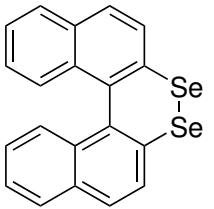

293 tert-Butyllithium (1.7 $\mathrm{M}$ in pentane; $0.63 \mathrm{~mL}, 1.1 \mathrm{mmol}, 4.4$ equiv) was cooled to $-78{ }^{\circ} \mathrm{C}$. 2,2'-dibromo-1,1'-binaphthalene (100 mg, 243 umol, 1.0 equiv) in THF $(0.4 \mathrm{~mL})$ was addred dropwise. The solution was stirred for $2 \mathrm{~h}$ while being allowed to warm to room temperature. Selenium powder (40 mg, $0.51 \mathrm{mmol}, 2.1$ equiv) was added and stirred at $60{ }^{\circ} \mathrm{C}$ for $13.5 \mathrm{~h}$. The reaction was quenched by the addition of $\mathrm{H}_{2} \mathrm{O}(4 \mathrm{~mL})$ and filtered over celite. The phases were separated, the aq. phase washed with EtOAc $(4 \mathrm{~mL})$, the combined org. phases were dried over $\mathrm{Na}_{2} \mathrm{SO}_{4}$ and the solvent removed in vacuo. Purification by column chromatography (40:1 PE:DCM) afforded the product as an orange solid (12 mg, 28 umol, 12\%).

$\mathbf{R}_{\mathbf{f}}=0.14$ (PE:DCM, 20:1); m.p. $=170-175{ }^{\circ} \mathrm{C}$; IR (ATR): $\tilde{\nu}\left[\mathrm{cm}^{-1}\right]=2920,2852,1498,1461$, 1255, 904, 806, 771, 727, 687, 669, 453, 407; ${ }^{\mathbf{1}} \mathbf{H} \mathbf{N M R}\left(600 \mathrm{MHz}, \mathrm{CDCl}_{3}\right): \delta[\mathrm{ppm}]=8.17(\mathrm{~d}$, 
$J=8.5 \mathrm{~Hz}, 2 \mathrm{H}), 7.96(\mathrm{dd}, J=8.6,0.8 \mathrm{~Hz}, 2 \mathrm{H}), 7.94(\mathrm{~d}, J=8.2 \mathrm{~Hz}, 2 \mathrm{H}), 7.50(\mathrm{ddd}, J=8.2$, $6.8,1.2 \mathrm{~Hz}, 2 \mathrm{H}), 7.30(\mathrm{ddd}, J=8.4,6.8,1.3 \mathrm{~Hz}, 2 \mathrm{H}), 6.99(\mathrm{dd}, J=8.5,1.1 \mathrm{~Hz}, 2 \mathrm{H}) ;{ }^{13} \mathrm{C}\left\{{ }^{1} \mathbf{H}\right\}$ NMR $\left(126 \mathrm{MHz}, \mathrm{CDCl}_{3}\right): \delta[\mathrm{ppm}]=146.0,134.2,133.4,133.1,130.4,129.7,128.3,127.7,127.6$, 127.3.; HR-EI-MS m/z: calcd. $\mathrm{C}_{20} \mathrm{H}_{12} \mathrm{Se}_{2}[\mathrm{M}]^{+}:$: 411.9, found: 411.9 .

\section{(R)-2,2'-diselenocyanato-1,1'-binaphthalene (295)}

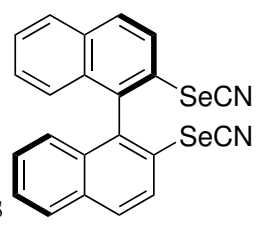

295

Boron trifluoride diethyl etherate (200 $\mathrm{mg}, 1.41 \mathrm{mmol}, 8.0$ equiv) was cooled to $-30{ }^{\circ} \mathrm{C}$. A solution of $(R)-2,2^{\prime}$-diamino- $1,1^{\prime}$-binaphthalene $(50 \mathrm{mg}$, $0.18 \mathrm{mmol}, 1.0$ equiv) in THF $(0.8 \mathrm{~mL})$ and a solution of tert-butyl nitrite (127 mg, $1.23 \mathrm{mmol}, 7.0$ equiv) in THF $(1.2 \mathrm{~mL})$ were added dropwise and the mixture was stirred at $-5{ }^{\circ} \mathrm{C}$ for $2.5 \mathrm{~h}$. Diethyl ether $(1.0 \mathrm{~mL})$ was added and stirred at $-5{ }^{\circ} \mathrm{C}$ for 15 min, then the supernatant was removed with a syringe. Potassium selenocyanate $(56 \mathrm{mg}, 0.39 \mathrm{mmol}, 2.2$ equiv) in acetonitrile $(1.8 \mathrm{~mL})$ was added dropwise at $0{ }^{\circ} \mathrm{C}$. The solution was stirred for $2 \mathrm{~h}$ and allowed to warm to room temperature. It was washed with water $(2 \mathrm{~mL})$ and sat. aq. $\mathrm{NaCl}$ solution $(2 \mathrm{~mL})$, dried over $\mathrm{Na}_{2} \mathrm{SO}_{4}$ and the solvent removed in vacuo. Purification by column chromatography (8:1 PE:EtOAc) afforded the product as a brown solid (12 mg, 27 mol, 15\%).

$\mathbf{R}_{\mathbf{f}}=0.36$ (PE:EtOAc, 5:1); m.p. $=146-150{ }^{\circ} \mathrm{C}$; IR (ATR) $\tilde{\nu}\left[\mathrm{cm}^{-1}\right]=3055,2923,2151,1577$, 1500, 1312, 811, 784, 772, 747, 689, 524, 460; ${ }^{\mathbf{1}} \mathbf{H} \mathbf{N M R}\left(300 \mathrm{MHz}, \mathrm{CDCl}_{3}\right): \delta[\mathrm{ppm}]=8.12$ $(\mathrm{d}, J=9.0 \mathrm{~Hz}, 2 \mathrm{H}), 8.06-7.89(\mathrm{~m}, 4 \mathrm{H}), 7.60$ (ddd, $J=8.2,6.9,1.2 \mathrm{~Hz}, 2 \mathrm{H}), 7.41$ (ddd, $J=8.3,6.9,1.3 \mathrm{~Hz}, 2 \mathrm{H}), 7.05(\mathrm{dd}, J=8.4,1.0 \mathrm{~Hz}, 2 \mathrm{H}) ;{ }^{\mathbf{1 3}} \mathbf{C}\left\{{ }^{\mathbf{1}} \mathbf{H}\right\} \mathbf{N M R}\left(126 \mathrm{MHz}, \mathrm{CDCl}_{3}\right)$ : $\delta[\mathrm{ppm}]=134.1,133.4,132.5,132.1,129.0,128.8,127.9,127.2,124.8,124.8,101.1 ; \mathbf{E I}-\mathbf{M S} \mathbf{m} / \mathbf{z}:$ calcd. $\mathrm{C}_{22} \mathrm{H}_{12} \mathrm{~N}_{2} \mathrm{Se}_{2}[\mathrm{M}]^{+}$: 463.9, found: 463.9. The analytical data was in agreement with literature. 210$]$

\section{Phenyl trifluoromethanesulfonate (297)}

Phenol (98 mg, $1.0 \mathrm{mmol}, 1.0$ equiv) was dissolved in DCM (3 mL). Pyridine

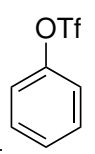

297 (168 mg, $2.13 \mathrm{mmol}, 2.0$ equiv) was added and the mixture cooled to $0{ }^{\circ} \mathrm{C}$. Trifluoromethanesulfonic anhydride (360 mg, $1.28 \mathrm{mmol}, 1.2$ equiv) was added dropwise, the solution was allowed to warm to room temperature and stirred for $1.5 \mathrm{~h} .1 \mathrm{M} \mathrm{HCl}$ solution $(3 \mathrm{~mL})$ was added and the phases separated. The org. phase was washed with sat. aq. $\mathrm{NaHCO}_{3}$ solution $(2 \times 3 \mathrm{~mL})$ and sat. aq. $\mathrm{NaCl}$ solution $(3 \mathrm{~mL})$ and dried over $\mathrm{Na}_{2} \mathrm{SO}_{4}$. Removal of the solvent in vacuo and purification by column chromatography (8:1 PE:EtOAc) afforded the product as a yellow liquid $(134 \mathrm{mg}$, 592 umol, $57 \%)$.

$\mathbf{R}_{\mathbf{f}}=0.57$ (PE:EtOAc, 8:1); ${ }^{1} \mathbf{H}$ NMR $\left(300 \mathrm{MHz}, \mathrm{CDCl}_{3}\right): \delta[\mathrm{ppm}]=7.51-7.36(\mathrm{~m}, 3 \mathrm{H}), 7.32-$ $7.24(\mathrm{~m}, 2 \mathrm{H}) ;{ }^{\mathbf{1 3}} \mathbf{C}\left\{{ }^{\mathbf{1}} \mathbf{H}\right\} \mathbf{N M R}\left(126 \mathrm{MHz}, \mathrm{CDCl}_{3}\right): \delta[\mathrm{ppm}]=149.8,130.4,128.5,121.5,118.9$ 
$\left(\mathrm{q},{ }^{1} J_{C F}=318.8 \mathrm{~Hz}\right) ;{ }^{\mathbf{1 9}} \mathbf{F}$ NMR $\left(282 \mathrm{MHz}, \mathrm{CDCl}_{3}\right): \delta[\mathrm{ppm}]=-72.9 ; \mathbf{H R}-\mathbf{E I}-\mathbf{M S} \mathbf{~ m} / \mathbf{z}$ : calcd. $\mathrm{C}_{7} \mathrm{H}_{5} \mathrm{~F}_{3} \mathrm{O}_{3} \mathrm{~S}[\mathrm{M}]^{+}:$225.9912, found: 225.9920 . The analytical data was in agreement with literature. .243$]$

\section{(R)-2,2'-dichloro-1, $1^{\prime}$-binaphthalene (309)}

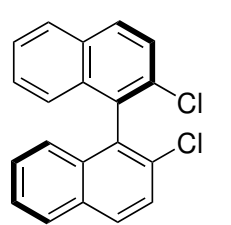

309

$\mathrm{CuCl}_{2}$ (123 mg, 914 umol, 2.6 equiv) and $t \mathrm{BuNO}_{2}(152 \mathrm{mg}, 1.48 \mathrm{mmol}$, 4.2 equiv) were dissolved in acetonitrile $(2 \mathrm{~mL})$. A suspension of $(R)-2,2^{\prime}-$ diamino-1,1'-binaphthalene (100 mg, $352 \mu \mathrm{mol}, 1.0$ equiv) in acetonitrile $(2 \mathrm{~mL})$ was added dropwise and the mixture was stirred at $65{ }^{\circ} \mathrm{C}$ for $16 \mathrm{~h}$. Aq. $1 \mathrm{~N} \mathrm{HCl}(4 \mathrm{~mL})$ was added and the phases were separated. The org. phase was washed with $\mathrm{H}_{2} \mathrm{O}(4 \mathrm{~mL})$ and sat. aq. $\mathrm{NaCl}$ solution $(4 \mathrm{~mL})$, the aq. phase was extracted with DCM $(8 \mathrm{~mL})$ and the combined org. phases were dried over $\mathrm{Na}_{2} \mathrm{SO}_{4}$. Removal of the solvent in vacuo and purification by column chromatography (30:1 PE:DCM) afforded the product as a yellow solid (63 mg, $0.19 \mathrm{mmol}, 56 \%$ ).

$\mathbf{R}_{\mathbf{f}}=0.28$ (PE:DCM, 30:1); m.p. $=134-138{ }^{\circ} \mathrm{C}$; IR (ATR): $\tilde{\nu}\left[\mathrm{cm}^{-1}\right]=3054,2923,1581,1502$, 1128, 1117, 857, 845, 808, 771, 741, 612, 555, 458, 412; ${ }^{\mathbf{1}} \mathbf{H}$ NMR (300 MHz, $\left.\mathrm{CDCl}_{3}\right): \delta$ $[\mathrm{ppm}]=7.95(\mathrm{dd}, J=8.4,3.5 \mathrm{~Hz}, 4 \mathrm{H}), 7.67(\mathrm{~d}, J=8.8 \mathrm{~Hz}, 2 \mathrm{H}), 7.49$ (ddd, $J=8.2,6.8,1.2 \mathrm{~Hz}$, $2 \mathrm{H}), 7.33$ (ddd, $J=8.3,6.9,1.3 \mathrm{~Hz}, 2 \mathrm{H}), 7.10(\mathrm{~d}, J=8.5 \mathrm{~Hz}, 2 \mathrm{H}) ;{ }^{\mathbf{1 3}} \mathbf{C}\left\{{ }^{\mathbf{1}} \mathbf{H}\right\}$ NMR $(126 \mathrm{MHz}$, $\left.\mathrm{CDCl}_{3}\right): \delta[\mathrm{ppm}]=133.3,133.1,132.4,132.2,129.9,128.4,127.5,127.4,126.3,125.7$; HR-EIMS m/z: calcd. $\mathrm{C}_{20} \mathrm{H}_{12} \mathrm{Cl}_{2}[\mathrm{M}]^{+}: 322.0316$, found: 322.0310 .

\section{(R)-2,2'-dibromo-1,1'-binaphthalene (310)}

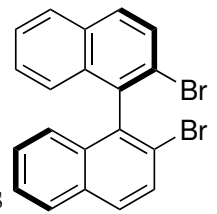

310

(R)-2,2'-diamino-1,1'-binaphthalene (100 $\mathrm{mg}, 0.352 \mathrm{mmol}, 1.0$ equiv) was dissolved in $\mathrm{HBr}(48 \%, 0.6 \mathrm{~mL})$ and cooled to $0{ }^{\circ} \mathrm{C} . \mathrm{NaNO}_{2}(73.0 \mathrm{mg}$, $1.06 \mathrm{mmol}, 3.0$ equiv) was dissolved in $\mathrm{H}_{2} \mathrm{O}(0.4 \mathrm{~mL})$ and added to the first solution. The mixture was stirred at $0{ }^{\circ} \mathrm{C}$ for $30 \mathrm{~min}$ and a solution of $\mathrm{CuBr}$ (126 mg, 878 umol, 2.5 equiv) in $\mathrm{HBr}(48 \%, 0.8 \mathrm{~mL})$ was added. Subsequently, the mixture was allowed to warm to room temperature and stirred for $16.5 \mathrm{~h}$. The reaction was quenched by the addition of ammonia solution $(2 \mathrm{~mL})$ and extracted with DCM. The organic phase was washed with sat. aq. $\mathrm{NaCl}$ solution, dried over $\mathrm{Na}_{2} \mathrm{SO}_{4}$ and the solvent removed in vacuo. Purification by column chromatography (20:1 PE:DCM) afforded the product as a yellow solid ( $73 \mathrm{mg}, 0.18 \mathrm{mmol}, 50 \%)$.

$\mathbf{R}_{\mathbf{f}}=0.31$ (n-pentane:DCM, 20:1); m.p. $=152-158{ }^{\circ} \mathrm{C} ; \mathbf{I R}(\mathrm{ATR}): \tilde{\nu}\left[\mathrm{cm}^{-1}\right]=3057,1579,1501$, 1110, 838, 808, 773, 747, 551, 453, 410; ${ }^{\mathbf{1}} \mathbf{H}$ NMR (300 MHz, $\left.\mathrm{CDCl}_{3}\right): \delta[\mathrm{ppm}]=7.94(\mathrm{~d}$, $J=8.3 \mathrm{~Hz}, 2 \mathrm{H}), 7.88(\mathrm{~d}, J=8.9 \mathrm{~Hz}, 2 \mathrm{H}), 7.82(\mathrm{~d}, J=8.9 \mathrm{~Hz}, 2 \mathrm{H}), 7.50$ (ddd, $J=8.3,6.8$, $1.3 \mathrm{~Hz}, 2 \mathrm{H}), 7.31(\mathrm{ddd}, J=8.4,6.8,1.3 \mathrm{~Hz}, 2 \mathrm{H}), 7.09(\mathrm{dd}, J=8.4,1.3 \mathrm{~Hz}, 2 \mathrm{H}) ;{ }^{13} \mathbf{C}\left\{{ }^{1} \mathbf{H}\right\}$ NMR $\left(126 \mathrm{MHz}, \mathrm{CDCl}_{3}\right): \delta[\mathrm{ppm}]=137.2,133.4,132.5,130.1,129.9,128.4,127.5,126.5$, 
126.0, 122.9; HR-EI-MS m/z: calc. $\mathrm{C}_{20} \mathrm{H}_{12} \mathrm{Br}_{2}[\mathrm{M}]^{+}:$409.9306, found: 409.9315; HPLC: 13.387 min, 15.369 min (Daicel Chiralcel OD; solvents: $n$-hexane/isopropanol, 99.5/0.5, v/v; flow rate: $0.6 \mathrm{~mL} / \mathrm{min})$. The analytical data was in agreement with literature. [244]

\section{(R)-2,2'-diiodo-1,1'-binaphthalene $(311)^{[232]}$}

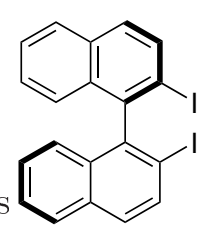

311

A suspension of $(R)$-2,2'-diamino-1,1'-binaphthalene (100 $\mathrm{mg}, 0.352 \mathrm{mmol}$, 1.0 equiv) and para-toluenesulfonic acid monohydrate (401 mg, $2.11 \mathrm{mmol}$, 6.0 Äq.) in $\mathrm{MeCN}(5 \mathrm{~mL})$ was cooled to $10{ }^{\circ} \mathrm{C}$. A solution of $\mathrm{KI}(292 \mathrm{mg}$, $1.76 \mathrm{mmol}, 5.0$ Äq.) and $\mathrm{NaNO}_{2}\left(97.3 \mathrm{mg}, 1.41 \mathrm{mmol}, 4.0\right.$ Äq.) in $\mathrm{H}_{2} \mathrm{O}$ $(0.5 \mathrm{~mL})$ was added dropwise and the solution stirred for $10 \mathrm{~min}$ at $10{ }^{\circ} \mathrm{C}$.

The solution was allowed to warm to room temperature and stirred for $2 \mathrm{~h}$. The reaction was quenched by the addition of $\mathrm{H}_{2} \mathrm{O}(10 \mathrm{~mL})$, sat. aq. $\mathrm{NaHCO}_{3}$ solution $(6 \mathrm{~mL}), 10 \% \mathrm{Na}_{2} \mathrm{~S}_{2} \mathrm{O}_{3}$ solution $(2.5 \mathrm{~mL})$. The aq. phase was extracted with DCM $(3 \times 10 \mathrm{~mL})$, the combined org. phases were dried over $\mathrm{Na}_{2} \mathrm{SO}_{4}$ and the solvent removed in vacuo. Purification by column chromatography (20:1 PE:DCM) afforded the product as a white solid (104 mg, $0.205 \mathrm{mmol}$, $58 \%)$.

$\mathbf{R}_{\mathbf{f}}=0.37(\mathrm{PE}: \mathrm{DCM}, 20: 1) ;{ }^{\mathbf{1}} \mathbf{H} \mathbf{N M R}\left(300 \mathrm{MHz}, \mathrm{CDCl}_{3}\right): \delta[\mathrm{ppm}]=8.06(\mathrm{~d}, J=8.7 \mathrm{~Hz}, 2 \mathrm{H})$, $7.93(\mathrm{~m}, 2 \mathrm{H}), 7.72(\mathrm{~d}, J=8.6 \mathrm{~Hz}, 2 \mathrm{H}), 7.51$ (ddd, $J=8.1,6.8,1.2 \mathrm{~Hz}, 2 \mathrm{H}), 7.29$ (ddd, $J=8.3$, $6.8,1.3 \mathrm{~Hz}, 2 \mathrm{H}), 7.07(\mathrm{~m}, 2 \mathrm{H})$. The analytical data was in agreement with literature. 232$]$

\section{[1,1'-binaphthalene]-2,2'-diyl bis(trifluoromethanesulfonate) (312)}

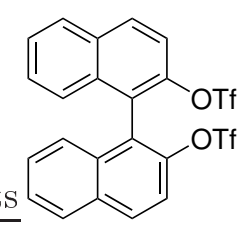

312

BINOL (200 mg, $0.699 \mathrm{mmol}, 1.0$ equiv) was dissolved in DCM (2 mL) and cooled to $0{ }^{\circ} \mathrm{C}$. Trifluoromethanesulfonic anhydride $(473 \mathrm{mg}, 1.68 \mathrm{mmol}$, 2.4 equiv) was added dropwise, the reaction was allowed to warm to room temperature and stirred for $6 \mathrm{~h}$. The reaction was quenched by the addition of aq. $1 \mathrm{M} \mathrm{HCl}$ solution $(2 \mathrm{~mL})$, the org. phase was washed with sat. aq. $\mathrm{NaHCO}_{3}$ solution $(2 \mathrm{~mL})$ and sat. aq. $\mathrm{NaCl}$ solution $(2 \mathrm{~mL})$ and the aq. phase was extracted with DCM $(6 \mathrm{~mL})$. The combined org. phases were dried over $\mathrm{Na}_{2} \mathrm{SO}_{4}$ and the solvent removed in vacuo. Purification by column chromatography (8:1 PE:EtOAc) afforded the product as a white solid (362 mg, $0.658 \mathrm{mmol}, 94 \%$ ).

$\mathbf{R}_{\mathbf{f}}=0.57$ (PE:EtOAc, 8:1); m.p. $=118-123{ }^{\circ} \mathrm{C}$; IR (ATR): $\tilde{\nu}\left[\mathrm{cm}^{-1}\right]=1417,1207,1173,1136$, $954,934,829,814,750,685,629,616,494 ;{ }^{\mathbf{1}} \mathbf{H} \mathbf{N M R}\left(300 \mathrm{MHz}, \mathrm{CDCl}_{3}\right): \delta[\mathrm{ppm}]=8.15$ $(\mathrm{dd}, J=9.2,0.8 \mathrm{~Hz}, 2 \mathrm{H}), 8.02(\mathrm{dd}, J=8.3,1.0 \mathrm{~Hz}, 2 \mathrm{H}), 7.68-7.54(\mathrm{~m}, 4 \mathrm{H}), 7.42$ (ddd, $J=8.3,6.8,1.2 \mathrm{~Hz}, 2 \mathrm{H}), 7.26(\mathrm{dt}, J=8.6,0.9 \mathrm{~Hz}, 2 \mathrm{H}) ;{ }^{\mathbf{1 3}} \mathbf{C}\left\{{ }^{\mathbf{1}} \mathbf{H}\right\}$ NMR $(126 \mathrm{MHz}$, $\left.\mathrm{CDCl}_{3}\right): \delta[\mathrm{ppm}]=145.5,133.3,132.5,132.1,128.5,128.1,127.5,126.9,123.6,119.5,118.3$ (q, $\left.J_{C F}=320.2 \mathrm{~Hz}\right) ;{ }^{\mathbf{1 9}} \mathbf{F}$ NMR $\left(282 \mathrm{MHz}, \mathrm{CDCl}_{3}\right): \delta[\mathrm{ppm}]=-74.6 ;$ HR-ESI-MS m/z: calcd. 
$\mathrm{C}_{22} \mathrm{H}_{13} \mathrm{~F}_{6} \mathrm{O}_{6} \mathrm{~S}_{2}[\mathrm{M}+\mathrm{H}]^{+}:$551.0052, found: 551.0058. The analytical data was in agreement with literature. 245$]$

\section{(R)-2,2'-Di[acetylamino]-1,1'-binaphthalene (321)}

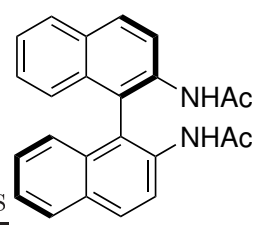

321

\section{Procedure A:}

(R)-2,2'-diamino-1,1'-binaphthalene (100 mg, $352 \mu \mathrm{mol}, 1.0$ equiv) and DMAP (4.0 mg, $35 \mu \mathrm{mol}, 1.0$ equiv) were dissolved in acetic anhydride (399 $\mathrm{\mu L}, 430 \mathrm{mg}, 4.22 \mathrm{mmol}, 12.0$ equiv) and stirred at $75^{\circ} \mathrm{C}$ for $2 \mathrm{~h}$. The solution was allowed to cool to room temperature, slowly added to $0.2 \mathrm{M}$ aq. HCl-solution $(3.5 \mathrm{~mL}$ ) and stirred for $20 \mathrm{~min}$. The phases were separated, the aq. phase was extracted with DCM $(2 \times 4 \mathrm{~mL})$, the organic phase was dried over $\mathrm{Na}_{2} \mathrm{SO}_{4}$ and the solvent removed in vacuo. Purification by column chromatography (1:1 PE:EtOAc) afforded the product as a yellow solid (96 mg, $0.26 \mathrm{mmol}, 74 \%$ ).

\section{Procedure B:}

(R)-2,2'-diamino-1,1'-binaphthalene (100 mg, $352 \mu \mathrm{mol}, 1.0$ equiv) and acetic acid $(211 \mathrm{mg}$, $3.52 \mathrm{mmol}, 10.0$ equiv) were dissolved in DCM $(3.5 \mathrm{~mL})$ and cooled to $0{ }^{\circ} \mathrm{C}$. Acetic acid anhydride (36 mg, $0.35 \mathrm{mmol}, 1.0$ equiv) was added dropwise, the cooling bath was removed and stirred at room temperature for $4.5 \mathrm{~h}$. Addition of $2.5 \mathrm{~N}$ aq. $\mathrm{NaOH}$ until $\mathrm{pH}=7$, extraction of the aq. phase with DCM $(2 \times 2 \mathrm{~mL})$, washing of the combined org. phases with sat. aq. $\mathrm{NaCl}$ solution $(5 \mathrm{~mL})$, drying over $\mathrm{Na}_{2} \mathrm{SO}_{4}$ and removal of the solvent in vacuo afforded the product as a yellow solid (114 mg, 349 umol, >99\%).

$\mathbf{R}_{\mathbf{f}}=0.07$ (PE:EtOAc, 1:1); m.p. $=94-96{ }^{\circ} \mathrm{C} ; \mathbf{I R}(\mathrm{ATR}): \tilde{\nu}\left[\mathrm{cm}^{-1}\right]=3400,3236,1686,1630,1598$, 1494, 1425, 1278, 820, 773, 752, 729, 700, 573, 498; ${ }^{\mathbf{1}} \mathbf{H} \mathbf{~ N M R}\left(300 \mathrm{MHz}, \mathrm{CDCl}_{3}\right): \delta[\mathrm{ppm}]=8.31$ $(\mathrm{d}, J=9.0 \mathrm{~Hz}, 2 \mathrm{H}), 8.04(\mathrm{dd}, J=8.8 \mathrm{~Hz}, 2 \mathrm{H}), 7.94(\mathrm{~d}, J=8.2 \mathrm{~Hz}, 2 \mathrm{H}), 7.45$ (ddd, $J=8.1,6.8$, $1.2 \mathrm{~Hz}, 2 \mathrm{H}), 7.26(\mathrm{ddd}, J=8.2,6.8,1.3 \mathrm{~Hz}, 2 \mathrm{H}), 7.07-6.89(\mathrm{~m}, 2 \mathrm{H}), 1.82(\mathrm{~s}, 6 \mathrm{H}) ;{ }^{13} \mathbf{C}\left\{{ }^{\mathbf{1}} \mathbf{H}\right\}$ NMR $\left(126 \mathrm{MHz}, \mathrm{CDCl}_{3}\right): \delta[\mathrm{ppm}]=169.5,135.0,132.6,131.6,130.0,128.5,127.4,125.8,125.4$, 122.6, 121.7, 24.3; HR-ESI-MS m/z: calcd. $\mathrm{C}_{24} \mathrm{H}_{21} \mathrm{~N}_{2} \mathrm{O}_{2}[\mathrm{M}+\mathrm{H}]^{+}:$369.1598, found: 369.1600 .

\section{2,2'-Dibromo-3,3'-bis(trimethylsilyl)-1,1'-binaphthyl (324)}

Tetramethylpiperidine ( $850 \mathrm{mg}, 6.02 \mathrm{mmmol}, 4.96$ equiv) was dissolved in<smiles>COc1cc2ccccc2c(-c2c(Br)c(C)cc3ccccc23)c1Br</smiles>

324

THF $(10 \mathrm{~mL})$ and cooled to $0{ }^{\circ} \mathrm{C}$. $n$-Butyl lithium $(2.5 \mathrm{M}$ in hexane, $2.0 \mathrm{~mL}$, $6.02 \mathrm{mmol}, 4.96$ equiv) was added dropwise, the solution was stirred at $0{ }^{\circ} \mathrm{C}$ for $20 \mathrm{~min}$ and cooled to $-78{ }^{\circ} \mathrm{C}$. TMSCl $(1.96 \mathrm{~g}, 18.1 \mathrm{mmol}, 14.9$ equiv) was added dropwise and the mixture was stirred for $25 \mathrm{~min}$ at $-78{ }^{\circ} \mathrm{C}$. A solution of binaphthyl dibromide $\mathbf{3 1 0}$ (500 mg, $1.21 \mathrm{mmol}, 1.0$ equiv) in THF

$(5.0 \mathrm{~mL})$ was cooled to $-78{ }^{\circ} \mathrm{C}$ and added to the reaction mixture which was then allowed to warm to room temperature and stirred for $18.5 \mathrm{~h}$. After addition of $1 \mathrm{M}$ aq. $\mathrm{HCl}(2.5 \mathrm{~mL})$, the aq. phase was extracted with DCM $(2 \times 10 \mathrm{~mL})$, the combined org. phases were washed with 
sat. aq. $\mathrm{NaCl}$ solution $(2 \times 10 \mathrm{~mL})$ and dried over $\mathrm{Na}_{2} \mathrm{SO}_{4}$. Removal of the solvent in vacuo and purification by column chromatography ( $n$-pentane) afforded the product as a white solid (465 mg, $0.84 \mathrm{mmol}, 69 \%$ ).

$\mathbf{R}_{\mathbf{f}}=0.33$ (PE); m.p. $=160-163{ }^{\circ} \mathrm{C}$; IR (ATR): $\tilde{\nu}\left[\mathrm{cm}^{-1}\right]=2952,1246,1116,972,901,878$, 835, 788, 753, 688, 627, 561; ${ }^{\mathbf{1}} \mathbf{H} \mathbf{~ N M R}\left(300 \mathrm{MHz}, \mathrm{CDCl}_{3}\right): \delta[\mathrm{ppm}]=8.09$ (s, $\left.2 \mathrm{H}\right), 7.92(\mathrm{~d}$, $J=8.0 \mathrm{~Hz}, 2 \mathrm{H}), 7.48(\mathrm{ddd}, J=8.1,6.8,1.2 \mathrm{~Hz}, 2 \mathrm{H}), 7.29(\mathrm{ddd}, J=8.3,6.8,1.3 \mathrm{~Hz}, 2 \mathrm{H}), 7.02$ $(\mathrm{d}, J=8.7 \mathrm{~Hz}, 2 \mathrm{H}), 0.53(\mathrm{~s}, 36 \mathrm{H}) ;{ }^{\mathbf{1 3}} \mathbf{C}\left\{{ }^{\mathbf{1}} \mathbf{H}\right\} \mathbf{N M R}\left(126 \mathrm{MHz}, \mathrm{CDCl}_{3}\right): \delta[\mathrm{ppm}]=140.0,137.9$, 137.0, 133.9, 131.9, 129.8, 128.5, 127.8, 126.3, 125.8, 0.3; HR-EI-MS m/z: calcd. $\mathrm{C}_{26} \mathrm{H}_{28} \mathrm{Br}_{2} \mathrm{Si}_{2}$ $[\mathrm{M}]^{+}$: 554.0096, found: 554.0100; HPLC: $6.123 \mathrm{~min}, 6.488 \mathrm{~min}$ (Daicel Chiralpak IA; solvents: $n$-hexane/isopropanol, $99.8 / 0.2, \mathrm{v} / \mathrm{v}$; flow rate: $0.6 \mathrm{~mL} / \mathrm{min})$. The analytical data was in agreement with literature. 238$]$

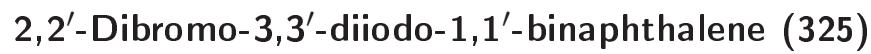

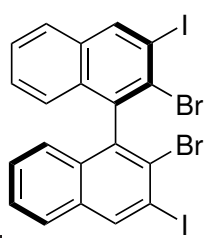

325

Dibromide 324 (230 mg, 413 pmol, 1.0 equiv) was dissolved in DCM (6 mL) and cooled to $-40{ }^{\circ} \mathrm{C}$. Iodine monochloride $(1 \mathrm{M}$ in DCM, $1.24 \mathrm{~mL}, 1.24 \mathrm{mmol}$, 3.0 equiv) was added dropwise and the mixture was stirred at $-40{ }^{\circ} \mathrm{C}$ for $20 \mathrm{~h}$. The reaction was quenched by the addition of $10 \%$ aq. $\mathrm{NaHSO}_{3}(4.3 \mathrm{~mL})$ and allowed to warm to room temperature. The mixture was washed with sat. aq. $\mathrm{NaCl}$ solution $(4 \mathrm{~mL})$, the aq. phase extracted with DCM $(4 \mathrm{~mL})$ and the combined org. phases were dried over $\mathrm{Na}_{2} \mathrm{SO}_{4}$. Removal of the solvent in vacuo and purification by column chromatography (PE) afforded the product as a white solid (242 mg, $364 \mu \mathrm{mol}, 89 \%$ ).

$\mathbf{R}_{\mathbf{f}}=0.57$ (PE:EtOAc, 4:1); m.p. $=190-194{ }^{\circ} \mathrm{C} ; \mathbf{I R}(\mathrm{ATR}): \tilde{\nu}\left[\mathrm{cm}^{-1}\right]=3057,2919,1546,1486$, 1366, 1297, 1112, 947, 891, 858, 780, 746, 732, 554; ${ }^{\mathbf{1}} \mathbf{H} \mathbf{N M R}\left(300 \mathrm{MHz}, \mathrm{CDCl}_{3}\right): \delta[\mathrm{ppm}]=8.61$ $(\mathrm{s}, 2 \mathrm{H}), 7.81(\mathrm{~d}, J=8.2 \mathrm{~Hz}, 2 \mathrm{H}), 7.51(\mathrm{ddd}, J=8.1,6.8,1.2 \mathrm{~Hz}, 2 \mathrm{H}), 7.32(\mathrm{ddd}, J=8.3$, 6.9, $1.3 \mathrm{~Hz}, 2 \mathrm{H}), 6.98(\mathrm{dd}, J=8.5,1.0 \mathrm{~Hz}, 2 \mathrm{H}) ;{ }^{13} \mathbf{C}\left\{{ }^{1} \mathbf{H}\right\} \operatorname{NMR}\left(125 \mathrm{MHz}, \mathrm{CDCl}_{3}\right): \delta$ $[\mathrm{ppm}]=140.2,139.7,133.8,132.1,128.6,128.2,127.4,127.3,126.0,99.1 ;$ HR-EI-MS m/z: calcd. $\mathrm{C}_{20} \mathrm{H}_{10} \mathrm{Br}_{2} \mathrm{I}_{2}[\mathrm{M}]^{+}:$:661.7239, found: 661.7249; HPLC: $16.513 \mathrm{~min}, 17.996 \mathrm{~min}$ (Daicel Chiralpak IA; solvents: $n$-hexane/isopropanol, $99.8 / 0.2, \mathrm{v} / \mathrm{v}$; flow rate: $0.6 \mathrm{~mL} / \mathrm{min})$. The analytical data was in agreement with literature. $\underline{[238]}$

\section{2,2'-Dibromo-3,3'-diphenyl-1,1'-binaphthalene (326)}

Phenylboronic acid (93 mg, $0.76 \mathrm{mmol}, 2.1$ equiv), $\mathrm{Na}_{2} \mathrm{CO}_{3}(84 \mathrm{mg}, 0.80 \mathrm{mmol}$,<smiles>Brc1cc2ccccc2c(-c2ccccc2)c1-c1c(Br)c(-c2ccccc2)cc2ccccc12</smiles>

326
2.2 equiv) and tetrakis[triphenylphosphine]palladium(0) (21 mg, 18 umol, 0.05 equiv) were dissolved in a mixture of ethanol $(0.3 \mathrm{~mL})$ and $\mathrm{H}_{2} \mathrm{O}$ $(0.45 \mathrm{~mL})$ and the solution was degassed (30 min). 2,2'-Dibromo-3,3'-diiodo1,1'-binaphthalene 325 (240 mg, $0.36 \mathrm{mmol}, 1.0$ equiv) was dissolved in toluene $(4.5 \mathrm{~mL})$ and added dropwise to the other solution. The reaction mixture was 
heated at $100{ }^{\circ} \mathrm{C}$ for $19 \mathrm{~h} . \mathrm{H}_{2} \mathrm{O}(4 \mathrm{~mL})$ was added, the phases were separated and the org. phase was washed with sat. aq. $\mathrm{NaCl}$ solution $(4 \mathrm{~mL})$. The combined aq. phases were extracted with DCM $(5 \mathrm{~mL})$ and the combined org. phases were dried over $\mathrm{Na}_{2} \mathrm{SO}_{4}$. Removal of the solvent in vacuo and purification by column chromatography (40:1 PE:DCM) afforded the product as a white solid (100 mg, $177 \mu \mathrm{mol}, 49 \%)$.

$\mathbf{R}_{\mathbf{f}}=0.07$ (PE:DCM, 40:1); m.p. $=205-208{ }^{\circ} \mathrm{C}$; IR (ATR): $\tilde{\nu}\left[\mathrm{cm}^{-1}\right]=3054,2922,2852,1494$, 1089, 1029, 891, 873, 778, 761, 746, 697, 584, 521, 464; ${ }^{\mathbf{1}} \mathbf{H} \mathbf{N M R}\left(300 \mathrm{MHz}, \mathrm{CDCl}_{3}\right): \delta$ $[\mathrm{ppm}]=7.97(\mathrm{~s}, 2 \mathrm{H}), 7.94(\mathrm{~d}, J=8.2 \mathrm{~Hz}, 2 \mathrm{H}), 7.63-7.57(\mathrm{~m}, 4 \mathrm{H}), 7.57-7.39(\mathrm{~m}, 8 \mathrm{H}), 7.35$ (ddd, $J=8.3,6.8,1.3 \mathrm{~Hz}, 2 \mathrm{H}), 7.18$ (d, $8.5 \mathrm{~Hz}, 2 \mathrm{H}) ;{ }^{\mathbf{1 3}} \mathbf{C}\left\{{ }^{\mathbf{1}} \mathbf{H}\right\} \mathbf{N M R}\left(126 \mathrm{MHz}, \mathrm{CDCl}_{3}\right)$ : $\delta[\mathrm{ppm}]=141.7,140.9,139.1,132.5,130.1,130.0,128.4,128.0,127.8,127.5,126.9,126.1,124.0 ;$ HR-EI-MS m/z: calcd. $\mathrm{C}_{32} \mathrm{H}_{20} \mathrm{Br}_{2}[\mathrm{M}]^{+}:$561.9932, found: 561.9934 . The analytical data was in agreement with literature. 2238$]$

\section{(R)-2,5-Diphenyldinaphtho[2,1-c:1', 2'-e][1,2]diselenine (328)}

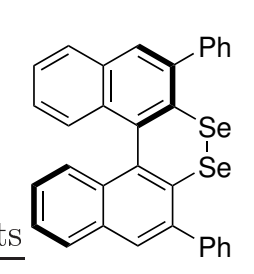

328

(R)-2,2'-Dibromo-3,3'-diphenyl-1,1'-binaphthalene 326 (33 mg, 58 umol, 1.0 equiv) was dissolved in THF $(0.1 \mathrm{~mL})$, cooled to $-78{ }^{\circ} \mathrm{C}$ and tert-butyl lithium ( $1.7 \mathrm{M}$ in pentane, $0.15 \mathrm{~mL}, 0.26 \mathrm{mmol}, 4.4$ equiv) was added dropwise. The solution was stirred for $1.5 \mathrm{~h}$ at $-78{ }^{\circ} \mathrm{C}$ and then allowed to warm to $0{ }^{\circ} \mathrm{C}$. Selenium powder (10 $\mathrm{mg}, 0.12 \mathrm{mmol}, 2.1$ equiv) was added and the solution was stirred for $17 \mathrm{~h}$ while allowed to warm to room temperature. The reaction was quenched by addition of $\mathrm{H}_{2} \mathrm{O}(1 \mathrm{~mL})$, the phases were separated, the aq. phase was extracted with EtOAc $(2 \mathrm{~mL})$ and the combined org. phases were dried over $\mathrm{Na}_{2} \mathrm{SO}_{4}$. The residue was dissolved in ethanol $(2 \mathrm{~mL})$, sodium hydroxide (1 pellet) was added and stirred for $30 \mathrm{~min}$. The solution was neutralized by addition of $1 \mathrm{M}$ aq. $\mathrm{HCl}$ solution. Removal of the solvent in vacuo and purification by column chromatography (40:1 PE:DCM) afforded the product as a yellow solid (8.0 mg, 13 umol, 21\%).

$\mathbf{R}_{\mathbf{f}}=0.07$ (PE:DCM, 20:1); m.p. $=215-220{ }^{\circ} \mathrm{C}$; IR (ATR): $\tilde{\nu}\left[\mathrm{cm}^{-1}\right]=2921,2852,1728,1579$, 1492, 1446, 1260, 1074, 1026, 890, 793, 748, 699, 582, 511; ${ }^{\mathbf{1}} \mathbf{H} \mathbf{N M R}\left(300 \mathrm{MHz}, \mathrm{CDCl}_{3}\right): \delta$ $[\mathrm{ppm}]=8.00-7.92(\mathrm{~m}, 4 \mathrm{H}), 7.60-7.39(\mathrm{~m}, 12 \mathrm{H}), 7.19(\mathrm{ddd}, J=8.3,6.8,1.3 \mathrm{~Hz}, 2 \mathrm{H}), 7.04$ $(\mathrm{d}, J=8.5 \mathrm{~Hz}, 2 \mathrm{H}) ;{ }^{\mathbf{1 3}} \mathbf{C}\left\{{ }^{1} \mathbf{H}\right\} \mathbf{N M R}\left(126 \mathrm{MHz}, \mathrm{CDCl}_{3}\right): \delta[\mathrm{ppm}]=142.4,142.0,141.6$, 139.9, 132.7, 132.4, 128.9, 128.9, 128.4, 128.3, 128.0, 127.4, 126.6, 126.3; EI-MS m/z: calcd. $\mathrm{C}_{32} \mathrm{H}_{20} \mathrm{Se}_{2}[\mathrm{M}]^{+}:$564.0, found: 563.9 . 


\section{(R)-2,5-Bis(trimethylsilyl)dinaphtho[2,1-c:1',2'-e][1,2]diselenine (329)}

(R)-2,2'-dibromo-3,3'-bis(trimethylsilyl)-1,1'-binaphthyl $324 \quad(55 \mathrm{mg}$,

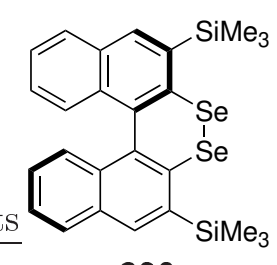

329

$99 \mu \mathrm{mol}, 1.0$ equiv) was dissolved in THF $(0.2 \mathrm{~mL})$ and cooled to $-78{ }^{\circ} \mathrm{C}$. tert-Butyl lithium (1.7 $\mathrm{M}$ in pentane, $0.26 \mathrm{~mL}, 0.43 \mathrm{mmol}, 4.4$ equiv) was added dropwise, the solution was stirred for $1.5 \mathrm{~h}$ at $-78{ }^{\circ} \mathrm{C}$ and was allowed to warm to $0{ }^{\circ} \mathrm{C}$. Selenium powder $(16 \mathrm{mg}, 0.21 \mathrm{mmol}, 2.1$ equiv) was added and the solution was stirred for $2 \mathrm{~h}$ at $0{ }^{\circ} \mathrm{C}$ and $17.5 \mathrm{~h}$ at room temperature.

The reaction was quenched by addition of $\mathrm{H}_{2} \mathrm{O}(1 \mathrm{~mL})$ and filtered over celite. The phases were separated, the aq. phase was extracted with EtOAc $(2 \mathrm{~mL})$ and the combined org. phases were dried over $\mathrm{Na}_{2} \mathrm{SO}_{4}$. Removal of the solvent in vacuo and purification by column chromatography (PE) afforded the product as an orange solid (20 mg, $36 \mu \mathrm{mol}, 36 \%)$.

$\mathbf{R}_{\mathbf{f}}=0.21(\mathrm{PE}) ; \mathbf{I R}(\mathrm{ATR}): \tilde{\nu}\left[\mathrm{cm}^{-1}\right]=2953,2924,1248,1163,1119,906,868,836,751,734 ;{ }^{\mathbf{1}} \mathbf{H}$ $\operatorname{NMR}\left(300 \mathrm{MHz}, \mathrm{CDCl}_{3}\right): \delta[\mathrm{ppm}]=7.94(\mathrm{~s}, 2 \mathrm{H}), 7.86(\mathrm{~d}, J=8.5 \mathrm{~Hz}, 2 \mathrm{H}), 7.37$ (ddd, $J=8.1$, 6.8, $1.2 \mathrm{~Hz}, 2 \mathrm{H}), 7.10$ (ddd, $J=8.3,6.8,1.4 \mathrm{~Hz}, 2 \mathrm{H}), 6.85$ (d, $J=8.4 \mathrm{~Hz}, 1 \mathrm{H}), 0.52(\mathrm{~s}, 18 \mathrm{H})$; ${ }^{13} \mathbf{C}\left\{{ }^{1} \mathbf{H}\right\}$ NMR $\left(126 \mathrm{MHz}, \mathrm{CDCl}_{3}\right): \delta[\mathrm{ppm}]=144.5,140.3,139.6,134.2,133.5,132.5,128.3$, 127.8, 126.8, 125.9, 0.4; EI-MS m/z: calcd. $\mathrm{C}_{26} \mathrm{H}_{28} \mathrm{Se}_{2} \mathrm{Si}_{2}[\mathrm{M}]^{+}$: 556.0, found: 556.0.

\section{(E)-Benzyl-4-N-di(phenylsulfonyl)imido pent-2-enoate (331)}

(E)-Benzyl pent-3-enoate (50 mg, $0.26 \mathrm{mmol}, 1.0$ equiv), NFSI ( $83 \mathrm{mg}$,<smiles>CC1=CC(=O)[O+]([O-])c2ccccc2O1</smiles>

331 $0.26 \mathrm{mmol}, 1.0$ equiv), diselenide 328 ( $7.5 \mathrm{mg}, 13$ mol, 0.05 equiv) and molecular sieves (4 A powder, spatula tip) were added to THF $(1.5 \mathrm{~mL})$ and the suspension was stirred at room temperature for $114 \mathrm{~h}$. Removal of the solvent in vacuo and purification by column chromatography (10:1 $\mathrm{PE}: \mathrm{Et}_{2} \mathrm{O}$ ) afforded the product as a colorless oil (4.5 $\mathrm{mg}, 9.3 \mathrm{mmol}, 4 \%$ ).

$\mathbf{R}_{\mathbf{f}}=0.11\left(\mathrm{PE}: \mathrm{Et}_{2} \mathrm{O}, 10: 1\right) ;{ }^{\mathbf{1}} \mathbf{H} \mathbf{N M R}\left(300 \mathrm{MHz}, \mathrm{CDCl}_{3}\right): \delta[\mathrm{ppm}]=8.04-7.99(\mathrm{~m}, 4 \mathrm{H}), 7.65-$ 7.58 (m, $2 \mathrm{H}), 7.54-7.49$ (m, $4 \mathrm{H}), 7.40-7.34(\mathrm{~m}, 5 \mathrm{H}), 7.00$ (dd, $J=15.9,5.6 \mathrm{~Hz}, 1 \mathrm{H}), 5.79$ (dd, $J=15.9,1.8 \mathrm{~Hz}, 1 \mathrm{H}), 5.17(\mathrm{~s}, 2 \mathrm{H}), 4.91(\mathrm{~m}, 1 \mathrm{H}), 1.54(\mathrm{~d}, J=7.0 \mathrm{~Hz}, 3 \mathrm{H})$. The analytical data was in agreement with literature. $\underline{63]}$ 


\section{References}

[1] P. A. Wender, M. P. Croatt, B. Witulski, Tetrahedron 2006, 62, 7505-7511.

[2] P. A. Wender, V. A. Verma, T. J. Paxton, T. H. Pillow, Acc. Chem. Res. 2008, 41, 40-49.

[3] P. A. Wender, B. L. Miller, Nature 2009, 460, 197-201.

[4] E. M. Beccalli, G. Broggini, M. Martinelli, S. Sottocornola, Chem. Rev. 2007, 107, 53185365.

[5] H. M. L. Davies, P. Ren, Q. Jin, Org. Lett. 2001, 3, 3587-3590.

[6] R. W. Kubiak, J. D. Mighion, S. M. Wilkerson-Hill, J. S. Alford, T. Yoshidomi, H. M. L. Davies, Org. Lett. 2016, 18, 3118-3121.

[7] R. I. McDonald, G. Liu, S. S. Stahl, Chem. Rev. 2011, 111, 2981-3019.

[8] G. Broggini, T. Borelli, S. Giofré, A. Mazza, Synthesis 2017, 49, 2803-2818.

[9] A. García-Cabeza, F. Moreno-Dorado, M. Ortega, F. Guerra, Synthesis 2016, 48, 23232342.

[10] L. Bayeh, P. Q. Le, U. K. Tambar, Nature 2017, 547, 196-200.

[11] T. Hori, K. B. Sharpless, J. Org. Chem. 1979, 44, 4204-4208.

[12] J. A. Tunge, S. R. Mellegaard, Org. Lett. 2004, 6, 1205-1207.

[13] S. Mann, L. Benhamou, T. Sheppard, Synthesis 2015, 47, 3079-3117.

[14] G. Yin, X. Mu, G. Liu, Acc. Chem. Res. 2016, 49, 2413-2423.

[15] M. Çelik, C. Alp, B. Coşkun, M. S. Gültekin, M. Balci, Tetrahedron Lett. 2006, 47, 36593663.

[16] A. De Mico, R. Margarita, L. Parlanti, G. Piancatelli, A. Vescovi, Tetrahedron 1997, 53, $16877-16882$.

[17] G. F. Koser, L. Rebrovic, R. H. Wettach, J. Org. Chem. 1981, 46, 4324-4326.

[18] N. Zefirov, V. Zhdankin, Y. Dan'kov, V. Sorokin, V. Semerikov, A. Koz'min, R. Caple, B. Berglund, Tetrahedron Lett. 1987, 28, 243-250.

[19] R. M. Moriarty, J. S. Khosrowshahi, Tetrahedron Lett. 1986, 27, 2809-2812.

[20] P. Magnus, J. Lacour, P. A. Evans, M. B. Roe, C. Hulme, J. Am. Chem. Soc. 1996, 118, $3406-3418$.

[21] P. Magnus, M. B. Roe, C. Hulme, J. Chem. Soc. Chem. Commun. 1995, 263.

[22] R. Chung, E. Yu, C. D. Incarvito, D. J. Austin, Org. Lett. 2004, 6, 3881-3884.

[23] S. Hara, J. Nakahigashi, K. Ishi-i, M. Sawaguchi, H. Sakai, T. Fukuhara, N. Yoneda, Synlett 1998, 1998, 495-496. 
[24] S. M. Banik, J. W. Medley, E. N. Jacobsen, J. Am. Chem. Soc. 2016, 138, 5000-5003.

[25] I. G. Molnár, R. Gilmour, J. Am. Chem. Soc. 2016, 138, 5004-5007.

[26] R. M. Romero, T. H. Wöste, K. Muñiz, Chem. Asian J. 2014, 9, 972-983.

[27] X. Li, P. Chen, G. Liu, Beilstein J. Org. Chem. 2018, 14, 1813-1825.

[28] J. A. Souto, D. Zian, K. Muñiz, J. Am. Chem. Soc. 2012, 134, 7242-7245.

[29] T. Fuchigami, T. Fujita, J. Org. Chem. 1994, 59, 7190-7192.

[30] M. Ochiai, Y. Takeuchi, T. Katayama, T. Sueda, K. Miyamoto, J. Am. Chem. Soc. 2005, 127, 12244-12245.

[31] T. Dohi, A. Maruyama, M. Yoshimura, K. Morimoto, H. Tohma, Y. Kita, Angew. Chemie Int. Ed. 2005, 44, 6193-6196.

[32] M. Fujita, Y. Yoshida, K. Miyata, A. Wakisaka, T. Sugimura, Angew. Chemie Int. Ed. 2010, 49, 7068-7071.

[33] M. Fujita, K. Mori, M. Shimogaki, T. Sugimura, Org. Lett. 2012, 14, 1294-1297.

[34] U. Farid, T. Wirth, Angew. Chemie Int. Ed. 2012, 51, 3462-3465.

[35] S. Haubenreisser, T. H. Wöste, C. Martínez, K. Ishihara, K. Muñiz, Angew. Chemie Int. Ed. 2016, 55, 413-417.

[36] A. Breder, S. Ortgies, Tetrahedron Lett. 2015, 56, 2843-2852.

[37] S. Ortgies, A. Breder, ACS Catal. 2017, 7, 5828-5840.

[38] H. Lecher, F. Holschneider, K. Köberle, W. Speer, P. Stöcklin, Eur. J. Inorg. Chem. 1925, $58,409-416$.

[39] N. Kharasch, H. L. Wehrmeister, H. Tigerman, J. Am. Chem. Soc. 1947, 69, 1612-1615.

[40] R. A. Turner, R. Connor, J. Am. Chem. Soc. 1947, 69, 1009-1012.

[41] W. Jenny, Helv. Chim. Acta 1953, 36, 1278-1282.

[42] G. Hölzle, W. Jenny, Helv. Chim. Acta 1958, 41, 593-603.

[43] N. Kharasch, C. M. Buess, J. Am. Chem. Soc. 1949, 71, 2724-2728.

[44] D. J. Cram, J. Am. Chem. Soc. 1949, 71, 3883-3889.

[45] D. G. Garratt, G. H. Schmid, Can. J. Chem. 1974, 52, 3599-3606.

[46] W. H. Mueller, P. E. Butler, J. Am. Chem. Soc. 1968, 90, 2075-2081.

[47] D. J. Pettitt, G. K. Helmkamp, J. Org. Chem. 1963, 28, 2932-2933.

[48] D. J. Pettitt, G. K. Helmkamp, J. Org. Chem. 1964, 29, 2702-2706.

[49] D. G. Garratt, G. H. Schmid, Can. J. Chem. 1974, 52, 1027-1028.

[50] G. H. Schmid, D. G. Garratt, Tetrahedron Lett. 1975, 16, 3991-3994. 
[51] P. Raynolds, S. Zonnebelt, S. Bakker, R. M. Kellogg, J. Am. Chem. Soc. 1974, 96, 31463154.

[52] C. A. Kingsbury, D. J. Cram, J. Am. Chem. Soc. 1960, 82, 1810-1819.

[53] D. N. Jones, D. Mundy, R. D. Whitehouse, J. Chem. Soc. D Chem. Commun. 1970, 86.

[54] S. E. Denmark, G. L. Beutner, Angew. Chemie Int. Ed. 2008, 47, 1560-1638.

[55] S. A. Snyder, D. S. Treitler, A. P. Brucks, J. Am. Chem. Soc. 2010, 132, 14303-14.

[56] S. A. Snyder, D. S. Treitler, A. P. Brucks, W. Sattler, J. Am. Chem. Soc. 2011, 133, $15898-15901$.

[57] S. R. Mellegaard, J. A. Tunge, J. Org. Chem. 2004, 69, 8979-8981.

[58] S. E. Denmark, M. T. Burk, Proc. Natl. Acad. Sci. 2010, 107, 20655-20660.

[59] C. K. Tan, Y.-Y. Yeung, Chem. Commun. 2013, 49, 7985.

[60] G. Ciancaleoni, ACS Omega 2018, 3, 16292-16300.

[61] A. Fürstner, P. W. Davies, Angew. Chemie Int. Ed. 2007, 46, 3410-3449.

[62] S. E. Denmark, M. G. Edwards, J. Org. Chem. 2006, 71, 7293-7306.

[63] J. Trenner, C. Depken, T. Weber, A. Breder, Angew. Chem. Int. Ed. 2013, 52, 8952-6.

[64] Z. Deng, J. Wei, L. Liao, H. Huang, X. Zhao, Org. Lett. 2015, 17, 1834-1837.

[65] S. Ortgies, A. Breder, Org. Lett. 2015, 17, 2748-2751.

[66] X. Zhang, R. Guo, X. Zhao, Org. Chem. Front. 2015, 2, 1334-1337.

[67] R. Guo, J. Huang, H. Huang, X. Zhao, Org. Lett. 2016, 18, 504-507.

[68] A. J. Cresswell, S. T.-C. Eey, S. E. Denmark, Nat. Chem. 2015, 7, 146-152.

[69] L. Liao, R. Guo, X. Zhao, Angew. Chemie Int. Ed. 2017, 56, 3201-3205.

[70] J. Szudkowska-Fratczak, B. Marciniec, G. Hreczycho, M. Kubicki, P. Pawluć, Org. Lett. 2015, 17, 2366-2369.

[71] B. L. Kohn, T. Rovis, Chem. Sci. 2014, 5, 2889-2892.

[72] N. Kirai, S. Iguchi, T. Ito, J. Takaya, N. Iwasawa, Bull. Chem. Soc. Jpn. 2013, 86, 784799.

[73] T. Jeffery, Tetrahedron Lett. 1992, 33, 1989-1992.

[74] M. Iwaoka, S. Tomoda, J. Chem. Soc. Chem. Commun. 1992, 1165.

[75] M. Tiecco, L. Testaferri, M. Tingoli, L. Bagnoli, C. Santi, J. Chem. Soc. Chem. Commun. 1993, 637-639.

[76] M. Tiecco, L. Testaferri, M. Tingoli, L. Bagnoli, C. Santi, Synlett 1993, 1993, 798-800.

[77] M. Tiecco, L. Testaferri, C. Santi, Eur. J. Org. Chem. 1999, 1999, 797-803. 
[78] M. Tiecco, L. Testaferri, M. Tingoli, F. Marini, Synlett 1994, 1994, 373-374.

[79] M. Tingoli, M. Tiecco, D. Chianelli, R. Balducci, A. Temperini, J. Org. Chem. 1991, 56, 6809-6813.

[80] M. Tingoli, M. Tiecco, L. Testaferri, R. Balducci, Synlett 1993, 1993, 211-212.

[81] M. Tingoli, M. Tiecco, L. Testaferri, A. Temperini, Synth. Commun. 1998, 28, 1769-1778.

[82] D. M. Browne, O. Niyomura, T. Wirth, Org. Lett. 2007, 9, 3169-3171.

[83] S. A. Shahzad, C. Venin, T. Wirth, Eur. J. Org. Chem. 2010, 2010, 3465-3472.

[84] F. V. Singh, T. Wirth, Org. Lett. 2011, 13, 6504-6507.

[85] S. Torii, K. Uneyama, K. Handa, Tetrahedron Lett. 1980, 21, 1863-1866.

[86] S. Torii, K. Uneyama, M. Ono, Tetrahedron Lett. 1980, 21, 2653-2654.

[87] S. Torii, K. Uneyama, M. Ono, Tetrahedron Lett. 1980, 21, 2741-2744.

[88] S. Torii, K. Uneyama, M. Ono, T. Bannou, J. Am. Chem. Soc. 1981, 103, 4606-4608.

[89] O. Niyomura, M. Cox, T. Wirth, Synlett 2006, 251-254.

[90] F. Krätzschmar, M. Kaßel, D. Delony, A. Breder, Chem. Eur. J. 2015, 21, 7030-7034.

[91] S. Ortgies, C. Depken, A. Breder, Org. Lett. 2016, 18, 2856-2859.

[92] G. Pandey, V. J. Rao, U. T. Bhalerao, J. Chem. Soc. Chem. Commun. 1989, 416.

[93] G. Pandey, B. B. V. Soma Sekhar, U. T. Bhalerao, J. Am. Chem. Soc. 1990, 112, 56505651.

[94] G. Pandey, B. B. V. Soma Sekhar, J. Org. Chem. 1992, 57, 4019-4023.

[95] G. Pandey, B. B. V. Soma Sekhar, J. Org. Chem. 1994, 59, 7367-7372.

[96] E. S. Conner, K. E. Crocker, R. G. Fernando, F. R. Fronczek, G. G. Stanley, J. R. Ragains, Org. Lett. 2013, 15, 5558-5561.

[97] A. Kunai, J. Harada, J. Izumi, H. Tachihara, K. Sasaki, Electrochim. Acta 1983, 28, $1361-1366$.

[98] A. J. Cresswell, S. T. Eey, S. E. Denmark, Angew. Chemie Int. Ed. 2015, 54, 1564215682.

[99] C. Nilewski, R. W. Geisser, E. M. Carreira, Nature 2009, 457, 573-6.

[100] D. K. Bedke, G. M. Shibuya, A. R. Pereira, W. H. Gerwick, C. D. Vanderwal, J. Am. Chem. Soc. 2010, 132, 2542-3.

[101] A. R. Pereira, T. Byrum, G. M. Shibuya, C. D. Vanderwal, W. H. Gerwick, J. Nat. Prod. 2010, 73, 279-83.

[102] G. M. Shibuya, J. S. Kanady, C. D. Vanderwal, J. Am. Chem. Soc. 2008, 130, 12514-8. 
[103] R. Guo, J. Huang, X. Zhao, ACS Catal. 2018, 8, 926-930.

[104] T. Wirth, Angew. Chem. 2000, 39, 3740-3749.

[105] T. I. Sølling, S. B. Wild, L. Radom, Chem. Eur. J. 1999, 5, 509-514.

[106] G. I. Borodkin, E. I. Chernyak, M. M. Shakirov, V. G. Shubin, Russ. J. Org. Chem. 1997, 33, 418-419.

[107] G. I. Borodkin, E. I. Chernyak, M. M. Shakirov, V. G. Shubin, Russ. J. Org. Chem. 1998, $34,1563-1568$.

[108] T. Wirth, G. Fragale, M. Spichty, J. Am. Chem. Soc. 1998, 120, 3376-3381.

[109] S. E. Denmark, W. R. Collins, M. D. Cullen, J. Am. Chem. Soc. 2009, 131, 3490-3492.

[110] S. E. Denmark, D. Kalyani, W. R. Collins, J. Am. Chem. Soc. 2010, 132, 15752-15765.

[111] A. J. Mukherjee, S. S. Zade, H. B. Singh, R. B. Sunoj, Chem. Rev. 2010, 110, 4357-416.

[112] Y. Nishibayashi, S. K. Srivastava, H. Takada, S.-i. Fukuzawa, S. Uemura, J. Chem. Soc. Chem. Commun. 1995, 2321.

[113] K.-I. Fujita, M. Iwaoka, S. Tomoda, Chem. Lett. 1994, 923-926.

[114] S.-I. Fukuzawa, K. Takahashi, H. Kato, H. Yamazaki, J. Org. Chem. 1997, 62, 7711-7716.

[115] M. Tiecco, L. Testaferri, C. Santi, F. Marini, L. Bagnoli, A. Temperini, Tetrahedron Lett. 1998, 39, 2809-2812.

[116] M. Tiecco, L. Testaferri, F. Marini, C. Santi, L. Bagnoli, A. Temperini, Tetrahedron: Asymmetry 1999, 10, 747-757.

[117] T. Wirth, S. Häuptli, M. Leuenberger, Tetrahedron: Asymmetry 1998, 9, 547-550.

[118] M. Tiecco, L. Testaferri, C. Santi, C. Tomassini, F. Marini, L. Bagnoli, A. Temperini, Chem. Eur. J. 2002, 8, 1118.

[119] Y. Kawamata, T. Hashimoto, K. Maruoka, J. Am. Chem. Soc. 2016, 138, 5206-5209.

[120] T. Akiyama, J. Itoh, K. Yokota, K. Fuchibe, Angew. Chemie Int. Ed. 2004, 43, 15661568.

[121] D. Uraguchi, M. Terada, J. Am. Chem. Soc. 2004, 126, 5356-5357.

[122] S. J. Connon, Angew. Chemie Int. Ed. 2006, 45, 3909-3912.

[123] S. Hoffmann, A. M. Seayad, B. List, Angew. Chemie Int. Ed. 2005, 44, 7424-7427.

[124] S. Leisering, I. Riaño, C. Depken, L. J. Gross, M. Weber, D. Lentz, R. Zimmer, C. B. W. Stark, A. Breder, M. Christmann, Org. Lett. 2017, 19, 1478-1481.

[125] D. R. Owen, R. J. Whitby, Synthesis 2005, 2005, 2061-2074.

[126] M. Wilken, S. Ortgies, A. Breder, I. Siewert, ACS Catal. 2018, 8, 10901-10912.

[127] S. Ortgies, PhD thesis, Georg-August-Universität Göttingen, 2018. 
[128] R. J. Crutchley, A. B. P. Lever, J. Am. Chem. Soc. 1980, 102, 7128-7129.

[129] N. A. Romero, D. A. Nicewicz, Chem. Rev. 2016, 116, 10075-10166.

[130] A. Joshi-Pangu, F. Lévesque, H. G. Roth, S. F. Oliver, L.-C. Campeau, D. Nicewicz, D. A. DiRocco, J. Org. Chem. 2016, 81, 7244-7249.

[131] G. O. Schenck, Naturwissenschaften 1948, 35, 28-29.

[132] M. Prein, W. Adam, Angew. Chem. 1996, 108, 519-538.

[133] M. Palomba, Georg-August-Universität Göttingen, 2017.

[134] K. Rode, M. Palomba, S. Ortgies, R. Rieger, A. Breder, Synthesis 2018, 50, 3875-3885.

[135] J. A. Marshall, J. J. Sabatini, Org. Lett. 2006, 8, 3557-3560.

[136] S. Ortgies, Georg-August-Universität Göttingen, 2017.

[137] R. Rieger, Master Thesis, Georg-August-Universität Göttingen, 2016.

[138] Structure Determination of Organic Compounds, Springer, Berlin, Heidelberg, 2009.

[139] G. R. Fulmer, A. J. M. Miller, N. H. Sherden, H. E. Gottlieb, A. Nudelman, B. M. Stoltz, J. E. Bercaw, K. I. Goldberg, Organometallics 2010, 29, 2176-2179.

[140] M. R. Binns, R. K. Haynes, A. G. Katsifis, P. A. Schober, S. C. Vonwiller, J. Am. Chem. Soc. 1988, 110, 5411-5423.

[141] T. Wada, T. Hata, Tetrahedron Lett. 1990, 31, 7461-7462.

[142] R. Rieger, Georg-August-Universität Göttingen, 2017.

[143] D. W. Knight, Contemp. Org. Synth. 1994, 1, 287.

[144] S. Ortgies, R. Rieger, K. Rode, K. Koszinowski, J. Kind, C. M. Thiele, J. Rehbein, A. Breder, ACS Catal. 2017, 7, 7578-7586.

[145] C. Milite, M. Viviano, M. Santoriello, F. Aricò, G. Sbardella, S. Castellano, RSC Adv. 2012, 2, 5229 .

[146] D. B. Dess, J. C. Martin, J. Org. Chem. 1983, 48, 4155-4156.

[147] B. List, A. Doehring, M. T. Hechavarria Fonseca, A. Job, R. Rios Torres, Tetrahedron 2006, 62, 476-482.

[148] L. Löffler, Georg-August-Universität Göttingen, 2016.

[149] S. Ortgies, Georg-August-Universität Göttingen, 2016.

[150] F. G. Bordwell, Acc. Chem. Res. 1988, 21, 456-463.

[151] T. Calogeropoulou, P. Angelou, A. Detsi, I. Fragiadaki, E. Scoulica, J. Med. Chem. 2008, 51, 897-908.

[152] H. Chen, Z. Huang, X. Hu, G. Tang, P. Xu, Y. Zhao, C.-H. Cheng, J. Org. Chem. 2011, $76,2338-2344$. 
[153] D. Parmar, E. Sugiono, S. Raja, M. Rueping, Chem. Rev. 2014, 114, 9047-9153.

[154] I. van der Veen, J. de Boer, Chemosphere 2012, 88, 1119-1153.

[155] Y. Ren, B. Cheng, L. Xu, A. Jiang, Y. Lu, J. Appl. Polym. Sci. 2010, 115, 1489-1494.

[156] L. A. Slotin, Synthesis 1977, 1977, 737-752.

[157] P. Lemmen, W. Richter, B. Werner, R. Karl, R. Stumpf, I. Ugi, Synthesis 1993, 1993, $1-10$.

[158] C. Depken, Georg-August Universität Göttingen, 2017.

[159] C. Depken, F. Krätzschmar, R. Rieger, K. Rode, A. Breder, Angew. Chemie Int. Ed. 2018, 57, 2459-2463.

[160] F. Krätzschmar, Georg-August-Universität Göttingen, 2017.

[161] W. Yan, Z. Li, Y. Kishi, J. Am. Chem. Soc. 2015, 137, 6219-6225.

[162] D. L. Haire, E. G. Janzen, V. J. Robinson, I. Hrvoic, Magn. Reson. Chem. 2004, 42, $835-843$.

[163] P. R. Sultane, T. B. Mete, R. G. Bhat, Org. Biomol. Chem. 2014, 12, 261-264.

[164] Ł. Albrecht, H. Jiang, G. Dickmeiss, B. Gschwend, S. G. Hansen, K. A. Jørgensen, J. Am. Chem. Soc. 2010, 132, 9188-9196.

[165] F. Buckingham, A. K. Kirjavainen, S. Forsback, A. Krzyczmonik, T. Keller, I. M. Newington, M. Glaser, S. K. Luthra, O. Solin, V. Gouverneur, Angew. Chemie Int. Ed. 2015, 54, 13366-13369.

[166] C. C. Pattillo, I. I. Strambeanu, P. Calleja, N. A. Vermeulen, T. Mizuno, M. C. White, J. Am. Chem. Soc. 2016, 138, 1265-1272.

[167] C. Defieber, M. A. Ariger, P. Moriel, E. M. Carreira, Angew. Chemie Int. Ed. 2007, 46, $3139-3143$.

[168] C. Puerto Galvis, V. Kouznetsov, Synthesis 2017, 49, 4535-4561.

[169] A. Gualandi, L. Mengozzi, E. Manoni, P. G. Cozzi, Catal. Letters 2015, 145, 398-419.

[170] W. Liu, S. Liu, R. Jin, H. Guo, J. Zhao, Org. Chem. Front. 2015, 2, 288-299.

[171] S. Kotha, D. Deodhar, P. Khedkar, Org. Biomol. Chem. 2014, 12, 9054-9091.

[172] L. F. Tietze, O. Burkhardt, Synthesis 1994, 1994, 1331-1336.

[173] L. F. Tietze, O. Burkhardt, M. Henrich, Liebigs Ann. 1997, 1997, 1407-1413.

[174] N. Kawai, R. Abe, M. Matsuda, J. Uenishi, J. Org. Chem. 2011, 76, 2102-2114.

[175] N. Kawai, R. Abe, J. Uenishi, Tetrahedron Lett. 2009, 50, 6580-6583.

[176] J. F. Teichert, M. Fañanás-Mastral, B. L. Feringa, Angew. Chemie Int. Ed. 2011, 50, $688-691$. 
[177] J. Eustache, P. Van de Weghe, D. L. Nouen, H. Uyehara, C. Kabuto, Y. Yamamoto, J. Org. Chem. 2005, 70, 4043-4053.

[178] N. Z. Burns, P. S. Baran, R. W. Hoffmann, Angew. Chemie Int. Ed. 2009, 48, 2854-2867.

[179] D. L. J. Clive, V. Farina, A. Singh, C. K. Wong, W. A. Kiel, S. M. Menchen, J. Org. Chem. 1980, 45, 2120-2126.

[180] M. Shen, B. E. Leslie, T. G. Driver, Angew. Chemie Int. Ed. 2008, 47, 5056-5059.

[181] C.-B. Yu, Y.-G. Zhou, Angew. Chemie Int. Ed. 2013, 52, 13365-13368.

[182] M. Martiny, E. Steckhan, T. Esch, Chem. Ber. 1993, 126, 1671-1682.

[183] T. Shono, Y. Matsumura, K. Tsubata, K. Uchida, T. Kanazawa, K. Tsuda, J. Org. Chem. 1984, 49, 3711-3716.

[184] D. S. Hamilton, D. A. Nicewicz, J. Am. Chem. Soc. 2012, 134, 18577-18580.

[185] C. Schlawis, Master Thesis, Georg-August-Universität Göttingen, 2015.

[186] P. G. Gildner, A. A. S. Gietter, D. Cui, D. A. Watson, J. Am. Chem. Soc. 2012, 134, 9942-9945.

[187] Y. Zhou, Y. Zhu, S. Yan, Y. Gong, Angew. Chemie Int. Ed. 2013, 52, 10265-10269.

[188] Y.-N. Zhang, X.-G. Zhong, Z.-P. Zheng, X.-D. Hu, J.-P. Zuo, L.-H. Hu, Bioorg. Med. Chem. 2007, 15, 988-996.

[189] N. Kharasch, S. J. Assony, J. Am. Chem. Soc. 1953, 75, 1081-1082.

[190] H. J. Reich, J. Org. Chem. 1974, 39, 428-429.

[191] T. G. Back, S. Collins, Tetrahedron Lett. 1981, 22, 5111-5114.

[192] T. G. Back, M. V. Krishna, K. R. Muralidharan, Tetrahedron Lett. 1987, 28, 1737-1740.

[193] T. G. Back, M. V. Krishna, K. R. Muralidharan, J. Org. Chem. 1989, 54, 4146-4153.

[194] T. G. Back, S. Collins, U. Gokhale, K. W. Law, J. Org. Chem. 1983, 48, 4776-4779.

[195] L. Liao, H. Zhang, X. Zhao, ACS Catal. 2018, 8, 6745-6750.

[196] R. Rieger, Georg-August-Universität Göttingen, 2018.

[197] K. Chernichenko, Á. Madarász, I. Pápai, M. Nieger, M. Leskelä, T. Repo, Nat. Chem. 2013, 5, 718-723.

[198] K. Sonogashira, Y. Tohda, N. Hagihara, Tetrahedron Lett. 1975, 16, 4467-4470.

[199] M. Eckhardt, G. C. Fu, J. Am. Chem. Soc. 2003, 125, 13642-13643.

[200] F. Krätzschmar, Georg-August-Universität Göttingen, 2018.

[201] V. Sashuk, J. Ignatowska, K. Grela, J. Org. Chem. 2004, 69, 7748-7751.

[202] B. Haberlag, M. Freytag, C. G. Daniliuc, P. G. Jones, M. Tamm, Angew. Chemie Int. Ed. 2012, 51, 13019-13022. 
[203] Ò. Àrias, H. Ehrhorn, J. Härdter, P. G. Jones, M. Tamm, Organometallics 2018, 37, $4784-4800$.

[204] W. Chang, J. Dai, J. Li, Y. Shi, W. Ren, Y. Shi, Org. Chem. Front. 2017, 4, 1074-1078.

[205] S. C. K. Hau, T. C. W. Mak, J. Am. Chem. Soc. 2014, 136, 902-905.

[206] S. E. Lazerwith, W. Lew, J. Zhang, P. Morganelli, Q. Liu, E. Canales, M. O. Clarke, E. Doerffler, D. Byun, M. Mertzman, H. Ye, L. Chong, L. Xu, T. Appleby, X. Chen, M. Fenaux, A. Hashash, S. A. Leavitt, E. Mabery, M. Matles, J. W. Mwangi, Y. Tian, Y.-J. Lee, J. Zhang, C. Zhu, B. P. Murray, W. J. Watkins, J. Med. Chem. 2014, 57, 1893-1901.

[207] H. Zhou, Q. Zhou, Q. Zhou, L. Ni, Q. Chen, RSC Adv. 2015, 5, 12161-12167.

[208] W. B. Sudweeks, H. S. Broadbent, J. Org. Chem. 1975, 40, 1131-1136.

[209] D. F. McComsey, A. B. Reitz, C. A. Maryanoff, B. E. Maryanoff, Synth. Commun. 1986, $16,1535-1549$.

[210] S. Tomoda, M. Iwaoka, J. Chem. Soc. Chem. Commun. 1988, 1283-4.

[211] T. G. Back, B. P. Dyck, M. Parvez, J. Org. Chem. 1995, 60, 703-710.

[212] Y. Lee, G. M. Morales, L. Yu, Angew. Chemie Int. Ed. 2005, 44, 4228-4231.

[213] M. Iwaoka, T. Katsuda, H. Komatsu, S. Tomoda, J. Org. Chem. 2005, 70, 321-327.

[214] M. Minozzi, D. Nanni, J. C. Walton, J. Org. Chem. 2004, 69, 2056-2069.

[215] T. Sato, I. Nakamura, M. Terada, Eur. J. Org. Chem. 2009, 5509-5512.

[216] X.-L. Li, J.-H. Huang, L.-M. Yang, Org. Lett. 2011, 13, 4950-4953.

[217] A. Thompson, G. Kabalka, M. Akula, J. Huffman, Synthesis 2005, 2005, 547-550.

[218] I. A. Cotgreave, R. Morgenstern, L. Engman, J. Ahokas, Chem. Biol. Interact. 1992, 84, $69-76$

[219] W. Chen, X. Yue, H. Zhang, W. Li, L. Zhang, Q. Xiao, C. Huang, J. Sheng, X. Song, Anal. Chem. 2017, 89, 12984-12991.

[220] I. P. Beletskaya, A. S. Sigeev, A. S. Peregudov, P. V. Petrovskii, Russ. J. Org. Chem. 2001, 37, 1463-1475.

[221] Y. Nishiyama, K. Tokunaga, N. Sonoda, Org. Lett. 1999, 1, 1725-1727.

[222] M. S. Newman, H. A. Karnes, J. Org. Chem. 1966, 31, 3980-3984.

[223] H. Kwart, E. R. Evans, J. Org. Chem. 1966, 31, 410-413.

[224] H.-J. Gais, A. Böhme, J. Org. Chem. 2002, 67, 1153-1161.

[225] A. Sørensen, B. Rasmussen, S. Agarwal, M. Schau-Magnussen, T. I. Sølling, M. Pittelkow, Angew. Chem. Int. Ed. 2013, 52, 12346-9. 
[226] S. M. Azad, S. M. W. Bennett, S. M. Brown, J. Green, E. Sinn, C. M. Topping, S. Woodward, J. Chem. Soc. Perkin Trans. 1 1997, 687-694.

[227] P. Garcia-Garcia, F. Lay, P. Garcia-Garcia, C. Rabalakos, B. List, Angew. Chem. Int. Ed. 2009, 48, 4363-4366.

[228] T. Imakubo, T. Shirahata, M. Kibune, Chem. Comm. 2004, 1590-1591.

[229] E. B. Prage, S. C. Pawelzik, L. S. Busenlehner, K. Kim, R. Morgenstern, P. J. Jakobsson, R. N. Armstrong, Biochemistry 2011, 50, 7684-7693.

[230] E. Elmalem, F. Biedermann, K. Johnson, R. H. Friend, W. T. S. Huck, J. Am. Chem. Soc. 2012, 134, 17769-17777.

[231] L. F. Tietze, T. Hungerland, A. Düfert, I. Objartel, D. Stalke, Chem. Eur. J. 2012, 18, $3286-3291$.

[232] S. Ortgies, Master Thesis, Georg-August-Universität Göttingen, 2013.

[233] Y. Imazaki, E. Shirakawa, R. Ueno, T. Hayashi, J. Am. Chem. Soc. 2012, 134, 1476014763

[234] M. Tobisu, K. Koh, T. Furukawa, N. Chatani, Angew. Chemie Int. Ed. 2012, 51, 1136311366.

[235] C.-J. Wang, M. Shi, J. Org. Chem. 2003, 68, 6229-6237.

[236] J. Wang, H. Li, X. Yu, L. Zu, W. Wang, Science 2005, 7, 2003-2006.

[237] C. C. Scarborough, R. I. McDonald, C. Hartmann, G. T. Sazama, A. Bergant, S. S. Stahl, J. Org. Chem. 2009, 74, 2613-5.

[238] M. Widhalm, C. Aichinger, K. Mereiter, Tetrahedron Lett. 2009, 50, 2425-2429.

[239] A. G. Schafer, J. M. Wieting, A. E. Mattson, Org. Lett. 2011, 13, 5228-31.

[240] F. Krätzschmar, S. Ortgies, R. Willing, A. Breder, Catalysts 2019, 9, 153.

[241] J. Trenner, Master Thesis, Georg-August-Universität Göttingen, 2013.

[242] W. Ding, Q. Song, Org. Chem. Front. 2016, 3, 14-18.

[243] S. Zhu, C. Wang, L. Chen, R. Liang, Y. Yu, H. Jiang, Org. Lett. 2011, 13, 1146-9.

[244] J. Clayden, P. M. Kubinski, F. Sammiceli, M. Helliwell, L. Diorazio, Tetrahedron 2004, 60, 4387-4397.

[245] P. C. Bulman Page, B. R. Buckley, M. M. Farah, A. John Blacker, Eur. J. Org. Chem. 2009, 3413-3426. 


\section{List of Abbreviations}

\begin{tabular}{|c|c|}
\hline Ac & acetyl \\
\hline Alk & alkyl \\
\hline approx. & approximately \\
\hline aq. & aqueous \\
\hline $\mathrm{Ar}$ & aryl \\
\hline ATP & adenosine triphosphate \\
\hline BINAM & $1,1^{\prime}$-binaphthalene- $2,2^{\prime}$-diamine \\
\hline BINOL & 1,1'-binaphthalene- $2,2^{\prime}$-diol \\
\hline $\mathrm{Bn}$ & benzyl \\
\hline Boc & tert-butyloxycarbonyl \\
\hline b.p. & boiling point \\
\hline bpz & $2,2^{\prime}$-bipyrazine \\
\hline calc. & calculated \\
\hline $\mathrm{Cbz}$ & carboxybenzyl, benzyloxycarbonyl \\
\hline $\mathrm{Ch}$ & chalcogen \\
\hline conc. & concentrated \\
\hline COSY & correlation spectroscopy \\
\hline $\mathrm{CV}$ & cyclic voltammetry \\
\hline $\mathrm{Cy}$ & cyclohexyl \\
\hline DAD & diode array detector \\
\hline DB & double bond \\
\hline DCC & $N, N^{\prime}$-dicyclohexylcarbodiimide \\
\hline DCE & 1,2-dichloroethane \\
\hline DCM & dichloromethane \\
\hline $\mathrm{DCN}$ & 1,4-dicyanonaphthalene \\
\hline$d e$ & diastereomeric excess \\
\hline DIBAL-H & diisobutylaluminum hydride \\
\hline DMAP & 4-(N,N-dimethylamino)pyridin \\
\hline DMB & 1,4-dimethoxybenzene \\
\hline DMF & $N, N$-dimethylformamide \\
\hline DMI & 1,3-dimethyl-2-imidazolidinone \\
\hline $\mathrm{DMP}$ & Dess-Martin periodinane \\
\hline DMSO & dimethylsulfoxide \\
\hline DMTA & $\begin{array}{l}\text { 10-(3,5-dimethoxyphenyl)-9-mesityl-1,3,6,8-tetramethoxyacridinium } \\
\text { fluoroborate } \mathbf{1 3 1}\end{array}$ \\
\hline DNA & deoxyribonucleic acid \\
\hline Dodec & dodecyl \\
\hline
\end{tabular}




\begin{tabular}{|c|c|}
\hline dppf & [1,1'-bis(diphenylphosphino)ferrocene] \\
\hline dppp & 1,3-bis(diphenylphosphino)propane \\
\hline $\mathrm{E}$ & electrophile \\
\hline$e e$ & enantiomeric excess \\
\hline EI & electron ionization \\
\hline$E_{o x}$ & oxidation potential \\
\hline equiv & equivalents \\
\hline$E_{\text {red }}$ & reduction potential \\
\hline ESI & electrospray ionization \\
\hline $\mathrm{Et}_{2} \mathrm{O}$ & diethyl ether \\
\hline Et & ethyl \\
\hline EtOAc & ethyl acetate \\
\hline $\mathrm{EtOH}$ & ethanol \\
\hline EWG & electron withdrawing group \\
\hline $\mathrm{GC}$ & gas chromatography \\
\hline gen. & generation \\
\hline Hal & halide \\
\hline Hex & hexyl \\
\hline HPLC & high-performance liquid chromatography \\
\hline HR-MS & high resolution mass spectrometry \\
\hline$i \mathrm{Bu}$ & isobutyl \\
\hline$i \operatorname{Pr}$ & isopropyl \\
\hline IR & infrared \\
\hline $\mathrm{LB}$ & LEWIS base \\
\hline LED & light-emitting diodes \\
\hline m.p. & melting point \\
\hline M & molar \\
\hline$m \mathrm{CPBA}$ & meta-chloroperbenzoic acid \\
\hline $\mathrm{MeCN}$ & acetonitrile \\
\hline $\mathrm{Me}$ & methyl \\
\hline $\mathrm{MeOH}$ & methanol \\
\hline Mes & mesityl \\
\hline $\mathrm{m}$ & mass \\
\hline MS & mass spectrometry or molecular sieves \\
\hline Ms & mesyl, methanesulfonyl \\
\hline $\mathrm{N}$ & normal \\
\hline Naph & naphthyl \\
\hline NBS & $N$-bromosuccinimid \\
\hline$n \mathrm{Bu}$ & $n$-butyl \\
\hline
\end{tabular}




\begin{tabular}{|c|c|}
\hline NCS & $N$-chlorosuccinimide $(\mathbf{2 2})$ \\
\hline NFSI & $N$-fluorobenzenesulfonimide \\
\hline NMR & nuclear magnetic resonance \\
\hline NOESY & nuclear overhauser enhancement spectroscopy \\
\hline NPSS & $N$-phenylselenosuccinimide $(\mathbf{2 6})$ \\
\hline $\mathrm{nr}$ & no reaction \\
\hline $\mathrm{Nu}$ & nucleophile \\
\hline ox & oxidant \\
\hline$p$-MeO-TPT & 2,4,6-tris(4-methoxyphenyl)pyrylium tetrafluoroborate $(\mathbf{9 5})$ \\
\hline $\mathrm{PE}$ & petroleum ether \\
\hline PG & protecting group \\
\hline $\mathrm{Ph}$ & phenyl \\
\hline PIDA & phenyliodine(III) diacetate, (diacetoxyiodo)benzene \\
\hline PIFA & phenyliodine(III) bis(trifluoroacetate), (bis(trifluoroacetoxy)iodo)benzene \\
\hline Pin & pinacolyl \\
\hline Piv & pivaloyl \\
\hline PMB & para-methoxybenzyl \\
\hline PS & photosensitizer \\
\hline PTSA & para-toluenesulfonic acid \\
\hline$[\mathrm{PyF}]$ & $N$-fluoropyridinium \\
\hline $\mathrm{rac}$ & racemic \\
\hline RT & room temperature, $23{ }^{\circ} \mathrm{C}$ \\
\hline sat. & saturated \\
\hline $\mathrm{SCE}$ & saturated calomel electrode \\
\hline SET & single electron transfer \\
\hline SPhos & 2-dicyclohexylphosphino-2', $6^{\prime}$-dimethoxybiphenyl \\
\hline SPS & solvent purification system \\
\hline TBS & tert-butyldimethylsilyl \\
\hline$t \mathrm{Bu}$ & tert-butyl \\
\hline TCE & 1,1,2,2-tetrachloroethane \\
\hline TEMPO & (2,2,6,6-tetramethylpiperidin-1-yl)oxyl \\
\hline temp. & temperature \\
\hline TFAA & trifluoroacetic acid anhydride \\
\hline TFA & trifluoroacetic acid \\
\hline Tf & triflate, trifluoromethanesulfonate \\
\hline THF & tetrahydrofurane \\
\hline TIPS & triisopropyl silyl \\
\hline TLC & thin layer chromatography \\
\hline TMB & 1,3,5-trimethoxybenzene \\
\hline
\end{tabular}




$\begin{array}{ll}\text { TMP } & 2,2,6,6 \text {-tetramethylpiperidine } \\ \text { [TMPyF] } & N \text {-fluoro-2,4,6-trimethylpyridinium } \\ \text { TMS } & \text { trimethylsilyl } \\ \text { Tol } & p \text {-tolyl } \\ \text { Ts } & \text { tosyl, } p \text {-toluenesulfonyl } \\ \text { z } & \text { charge }\end{array}$




\section{List of Figures}

1.1 First synthesized thiiranium and seleniranium ions. . . . . . . . . . . 3

1.2 Proposed orbital interactions in the $\pi$-acid activation of olefins by a) chalcogenium ions and b) transition metals. . . . . . . . . . . . . . . . 6

1.3 Strategies for the stabilization of enantioenriched seleniranium ions: a) LEWIS basic side chains, b) steric bulk, c) electron-withdrawing substituents at the selenium moiety. . . . . . . . . . . . . . . . . . . .

1.4 Chiral selenium catalysts synthesized by Tomoda, Fukuzawa, WirTh, and

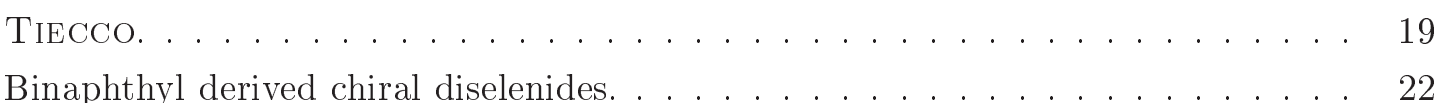
$126 \mathrm{~m}$.

3.2 Crude ${ }^{1} \mathrm{H}$ NMR spectra of assumed products $\mathbf{1 2 7 h}$ and $\mathbf{1 2 7 i}$.

3.3 Crude ${ }^{1} \mathrm{H}$ NMR spectrum of ethers $\mathbf{1 4 4} \mathbf{j}$ and $\mathbf{1 4 4 \mathbf { c }}$ compared with a clean spectrum of compound 144c. . . . . . . . . . . . . . . . 36

$3.4{ }^{1} \mathrm{H}$ NMR spectrum of assumed allyl alcohols 155. . . . . . . . . . . . . . . . 43

3.5 Knoevenagel reaction of aldehyde $\mathbf{1 6 1}$ (top), extract from the ${ }^{1} \mathrm{H}$ NMR spectrum of the mixture of $\mathbf{3 4 e}$ and $\mathbf{1 6 2}$ (bottom) . . . . . . . . . 46

3.6 NOESY spectrum of unsaturated acid 1371. . . . . . . . . . . . . . . . 48

3.7 Selected H,C-HMBC (blue) and P,H-HMBC (red) couplings of product mixture 173g.

3.8 Nucleophiles for the amination of olefins.

3.9 Spectroscopic data used for the assignment of the structures of compounds 185a and 185b.

3.10 Assignment of the ${ }^{1} \mathrm{H}$ NMR signals of tetrahydroisoquinoline 208.

3.11 Extract from ${ }^{1} \mathrm{H}$ NMR spectra of allene 253a (top), the crude reaction mixture (middle) and selenofunctionalized compound 254 (bottom).

3.12 Comparison of relevant regions of the ${ }^{1} \mathrm{H}$ NMR spectra of the internal and terminal allene $253 \mathrm{c}$.

\section{List of Schemes}

1.1 Iodine(III)-mediated oxidative vicinal functionalization of alkenes. . . . . . . . . .

1.2 Formation of thiiranium ion $\mathbf{1 1}$ via an ionic or a radical pathway. . . . . . . . . .

1.3 Allylic alcohol synthesis by SHARPLESS and LAUER.

1.4 Proposed mechanism of the catalytic chlorination by Hori and SHarpless. . . . 
1.5 LEWIS base activation of an electrophile and subsequent alkene functionalization.

1.6 Oxidative functionalization of alkene 37 via $\pi$-acid activation. . . . . . . . . . . .

1.7 Formation of selenolactone $\mathbf{4 4}$ and adducts $\mathbf{4 2}$ and $\mathbf{4 3}$ from seleniranium ion $\mathbf{4 1}$. .

1.8 Selenium- $\pi$-acid catalyzed allylic and vinylic imidation by BREDER et al. 63$]$. . .

1.9 Indole formation under different conditions by BREDER et al. and ZHAO et al. $.65,66]$

1.10 Subsequent oxygenation reactions by TIECCO et al. . . . . . . . . . . . . .

1.11 Formation of isocoumarins $\mathbf{7 9}$ and dihydropyranones $\mathbf{8 0}$ by WIRTH et al. . . . . .

1.12 Suggested mechanism for the formation of isobenzofuranones $\mathbf{8 8}$.

1.13 Envisioned catalytic cycle for the intermolecular esterification. . . . . . . . . . . .

1.14 Erosion of enantioenriched chalcogeniranium ion $\mathbf{1 0 8}$ via a) olefin-to-olefin transfer and b) nucleophilic attack on the chalcogenium. ${ }^{[37,110]}$

3.1 Palladium-mediated (top), selenium/NFSI-mediated (middle) and seleniumphotoredox-mediated (bottom) formation of $(+)$-Greek tobacco lactone (124). . .

3.2 Synthesis of 7 -oxooctanal $(\mathbf{1 6 1}) .145] \ldots \ldots \ldots \ldots \ldots$

3.3 Previous methods for the synthesis of tetrahydroisoquinolines (194). . . . . . . .

3.4 Synthesis of tetrahydroisoquinolines (194) via direct allylic $\mathrm{C}-\mathrm{H}$ amination. . . .

3.5 Synthesis of tosyl amide 207a. $.180,181]$

3.6 Reduction and following tosyl amide protection of nitro compounds 212 and 213. 181,187$]$

3.7 Retrosynthetic considerations for the synthesis of arylated ortho-vinyl homobenzylamides 219 .

3.8 Cyanation and attempted reduction and following tosyl amide protection of benzyl alcohol 221.

3.9 Bromination and following nitromethylation of benzyl alcohol 222.

3.10 Suzuki reaction and attempted tosylation of aryl bromide $\mathbf{2 2 9}$.

3.11 Addition of phenylselenyl trifluoroacetate to phenyl acetylene (234) and the following hydrolysis. . . . . . . . . . . . . . . . .

3.12 Addition of Se-phenyl $p$-tolueneselenosulfonate to alkynes 237 and the following reactions to alkynes 239 or allenes $241 \ldots \ldots \ldots \ldots \ldots$

3.13 Addition of $S e$-phenyl $p$-tolueneselenosulfonate to 5-decyne (242a) and the following elimination to allene $\mathbf{2 4 4}$

3.14 Proposed mechanism of the propargylic oxidation by ZHAO et al.

3.15 Synthesis of ketone $\mathbf{2 7 5}$ and attempted COREY-Fuchs reacion.

3.16 Protection of alkyne $\mathbf{2 8 0}$ as dicobalt complex $\mathbf{2 8 1}$ and subsequent reduction and deprotection. 209]

3.17 Proposed catalytic cycle for the selenium- $\pi$-acid catalyzed synthesis of allenylamides.

3.18 Possible synthesis of monoarylated binaphthyl halogenide $\mathbf{3 1 9}$.

4.1 Intra- and intermolecular etherification of alkenes 126, 139 and 148. 


\section{List of Tables}

3.1 Stern-Volmer constants for different diselenide/photosensitizer combinations, determined by Dr. S. Ortgies. 127$] \ldots \ldots \ldots \ldots \ldots$. . . . . . . . . . . 25

3.2 Comparison of photosensitizers in different solvents in the intramolecular etheri-

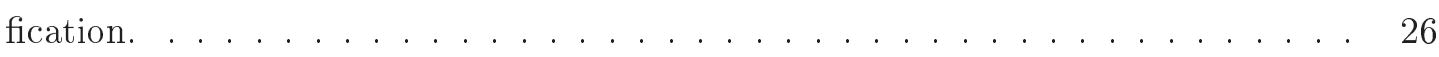

3.3 Comparison of photosensitizers in the intramolecular etherification. . . . . . . . 26

3.4 Optimization of catalyst loading and control experiments in the intramolecular

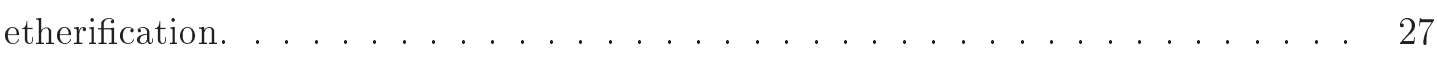

3.5 Optimization of bases used in the intramolecular etherification. . . . . . . . . 28

3.6 Wittig reaction of aldehydes $\mathbf{1 3 3}$ to carboxylic acids $\mathbf{1 3 6}$ and 137. Products 136 $(\mathrm{n}=0)$ with yields in gray synthesized by Dr. M. Palomba.133,134] . . . . . . . 29

3.7 Reduction of carboxylic acids $\mathbf{1 3 6}$ and $\mathbf{1 3 7}$ to alcohols $\mathbf{1 2 6}$ and 139. Products 126 $(\mathrm{n}=0)$ with yields in gray synthesized by Dr. M. Palomba.133,134] . . . . . . . 30

3.8 Synthesis of alcohols $\mathbf{1 2 6} \mathbf{g}, \mathbf{h}, \mathbf{i}, \mathbf{j}, \mathbf{k}, \mathbf{l}$. Grayed products were synthesized by Dr. M. Palomba. 133,134$]$

3.9 Synthesis of tetrahydrofurans $127 .{ }^{a}$ Grayed products were synthesized by Dr. M. Palomba. [133,134] .

3.10 Synthesis of tetrahydropyrans $1444^{\text {a }[134]}$

3.11 Synthesis of tetrahydrofurans 147. ${ }^{\mathrm{a}}$ All syntheses by Dr. S. Ortgies. 134,136$]$

3.12 Comparison of diselenides in the intermolecular etherification of 5-trans-decen.

3.13 Influence of base on the intermolecular etherification of 5-trans-decen.

3.14 Synthesis of allylic ethers $\mathbf{1 4 9} \mathbf{a}, \mathbf{b}, \mathbf{c}, \mathbf{d} .^{\text {a }}$ Grayed product $\mathbf{1 4 9 c}$ was synthesized by R. Rieger. 134,142$]$

3.15 Synthesis of allylic ethers $\mathbf{1 4 9 f}, \mathbf{g}, \mathbf{h}, \mathbf{i}, \mathbf{j}, \mathbf{k}, \mathbf{l}, \mathbf{m}$. ${ }^{a}[134]$

3.16 Synthesis of allylic ethers $149 \mathbf{n}, \mathbf{o}, \mathbf{p}, \mathbf{q} .{ }^{\text {a }}{ }^{[134]}$

3.17 Synthesis of carboxylic acids $\mathbf{3 4 b}, \mathbf{c}, \mathbf{d} . \underline{144]}$

3.18 WitTig reaction of aldehydes 133 to carboxylic acids 137, 166 and 167. Acids with yield in gray synthesized by L. Löffler and Dr. S. Ortgies..144,148,149] . . . . .

3.19 Synthesis of lactones 77. Grayed product 77b synthesized by R. Rieger.1137,144] . .

3.20 Assignment of ${ }^{1} \mathrm{H}$ NMR signals of lactone $\mathbf{1 6 8}$ and COSY couplings.

3.21 Lactonization of acids 137. Grayed products synthesized by L. Löffler, Dr. S. Ortgies and R. Rieger.[137,144,148,149]

3.22 Esterification of diol $\mathbf{1 2 6 m}$.

3.23 Phosphatation reactions of asymmetric alkenes $174 .^{\text {a }}$ Reactions for products in gray conducted by F. Krätzschmar and R. Rieger.[142,159,160] . . . . . . . . . .

3.24 Phosphatation reactions of asymmetric alkenes $174{ }^{a} \stackrel{\text { a } 159]}{ }$

3.25 Amination of cyclooctene (181) with different nucleophiles and bases. . . . . . . . .

3.26 Amination of cyclooctene (181) with saccharin and different bases. 
3.27 Attempted amination of cyclooctene (181) with different nucleophiles. . . . . . 61

3.28 Attempted functionalization of cyclooctene (181) with sulfamic acid. . . . . . . 62

3.29 Optimization of used solvents in the intramolecular amination. . . . . . . . . 66

3.30 Optimization of used bases in the intramolecular amination. . . . . . . . . 67

3.31 SuZuKI reaction of bromophenylacetonitriles 204 to nitriles 205. 180$] \ldots$. . . . . 68

3.32 Reduction of nitriles $\mathbf{2 0 5}$ to amines $\mathbf{2 0 6}$ and subsequent reaction to tosyl amides

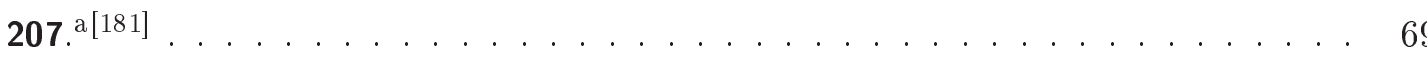

3.33 Cyclization of tosyl amides 207. ${ }^{\mathrm{a}}$

3.34 Experiments on the used catalyst in the allenylation. Grayed reaction by $\mathrm{R}$. Rieger.196] ........................... 77

3.35 Experiments on temperature and used solvents in the allenylation. Grayed reactions by R. Rieger.196] . . . . . . . . . . . . . . . . . . . . 77

3.36 Optimization of reagent amounts in the allenylation. Grayed reactions by $\mathrm{R}$. Rieger $[196] \ldots \ldots \ldots \ldots \ldots \ldots \ldots \ldots$

3.37 Optimization of bases used in the allenylation. Grayed reaction by R. Rieger. ${ }^{[196]}$

3.38 Optimization of catalyst loading in the allenylation. . . . . . . . . . . . . .

3.39 Synthesis of allenylamides $253{ }^{\mathrm{a}} \ldots \ldots \ldots \ldots \ldots \ldots \ldots \ldots \ldots \ldots \ldots \ldots$

3.40 Allenylation of 5-decyne 242a using LEWIS base. . . . . . . . . . . . . . . . 85

3.41 Amidoallenylation of alkynes $242 \mathbf{b}$ and 242c using additional LEwis base. ${ }^{\mathrm{a}}$. . . 85

3.42 Experiments on the formation of allene 253a from compound 254. . . . . . . . 86

3.43 Tested conditions for the synthesis of $(R)-2,2^{\prime}$-diselenocyanato-1,1'-binaphthalene (295).

3.44 Halogenation of $(R)$-BINAM (294) in SANDMEYER-type reactions. . . . . . . 93

3.45 Amination of pentenoic acid ester $\mathbf{3 3 0}$ with chiral catalysts 328 and $\mathbf{3 2 9}$. . . . . 99 



\section{Acknowledgements}

First of all, I would like to thank Prof. Dr. Alexander Breder for the opportunity to write my thesis in his group and his dedicated supervision. I am very grateful for your assistance in lab problems and motivating me, as well as the discussions about chemistry, scientific writing and everyday topics.

Furthermore, I would like to thank Prof. Dr. Lutz Ackermann and Prof. Dr. Daniel B. Werz for being my second and third supervisor and Prof. Dr. Ackermann for providing our group with generous material and infrastructural support.

I would also like to thank all further members of the examination board: Dr. H. Frauendorf, Prof. Dr. K. Koszinowski, Prof. Dr. I. Siewert and Prof. Dr. D. Stalke.

Thank you to Gabi Keil-Knepel for helping a lot with all kinds of administrative questions!

For measuring my spectra and your always friendly support with problems, I would like to thank the staff of the NMR department and the central analytics department.

For your always friendly help with lab-related and $\mathrm{PhD}$-hat-related constructions and repairing I would like to thank the staff of the electronics, mechanics and glassware workshops!

Many thanks for proofreading large parts of this thesis and giving helpful advice despite all your other obligations, Poorva, Stefan, Susi and Tobi!

A big thank you goes to my former and current lab colleagues, bachelor students and lab rotation students. I'm especially grateful to: Christian Depken for Rocket Beans, for being such a laidback/northern-German-style relaxed person, for bringing new music of different genres and for reminding me of old music; Felix "Harry" Krätzschmar for sharing your competence in lab techniques and devices as well as for entertainment and craziness (but also being down-to-earth when needed). Thank you for being the best writing buddy I could wish for, helping me by sharing the motivation/demotivation periods, discussing questions, papers and topics that confused or annoyed me and posing me occasional chemistry challenges; Stefan Ortgies for all your competence, for starting to train me in chemistry with the Kürti-Czakó-challenge, for leaving your Clayden in the lab when I was too lazy to bring mine, for answering questions about my thesis long after your time in the group, for being the nicest person in the world when in a good mood, for sharing some music moments and for being unexpectedly and enjoyably nerdy; Martina Palomba for Italian flair and contributing a lot to my project; Poorva Ramadas Narasimhamurthy for making me more active (for example with swimming dates), for becoming my scientific language tandem partner and for becoming a friend right from the start; and Rene Rieger for reliably making me laugh by his constant and generous contributions to the Phrasenschwein. All of you, I would like to thank for the good working atmosphere in the lab, the good teamwork, your help with problems, the discussions about chemistry and more, for being totally crazy and for making me 
smile again when my chemistry really brought me down. Last but not least the Phrasenschwein, our Laborhymnen-CDs, the vampire murder mystery dinner, the legendary Gloryhammer concert, the barbecues and several group trips added a lot to the fun I had.

(... und gut, dass wir uns darin einig waren: "Immer erst mal pöbeln!")

To my friends, I am grateful that you care about me. Thank you for advice, comfort and all the things we do together, like trips in Germany and trips abroad, trips to the lake, many cooking evenings, board games, video games, pen and paper, going out, movie nights, ...

Thank you to the Friday Lunch Group for sticking together since the first semester and bringing diversion and different topics into my workday.

Thank you to everyone I talked to about my writing process (sometimes when meeting randomly in the corridors of the institute) for listening, your motivating words and believing in me.

Thank you Daniel for happiness, dreams and support during the last tasks on the way to my $\mathrm{PhD}$.

Eventually, I would like to thank my parents and my brother for their unconditional and constant interest, caring and support during these years. I would not be where I am without you. 


\section{Spectra}

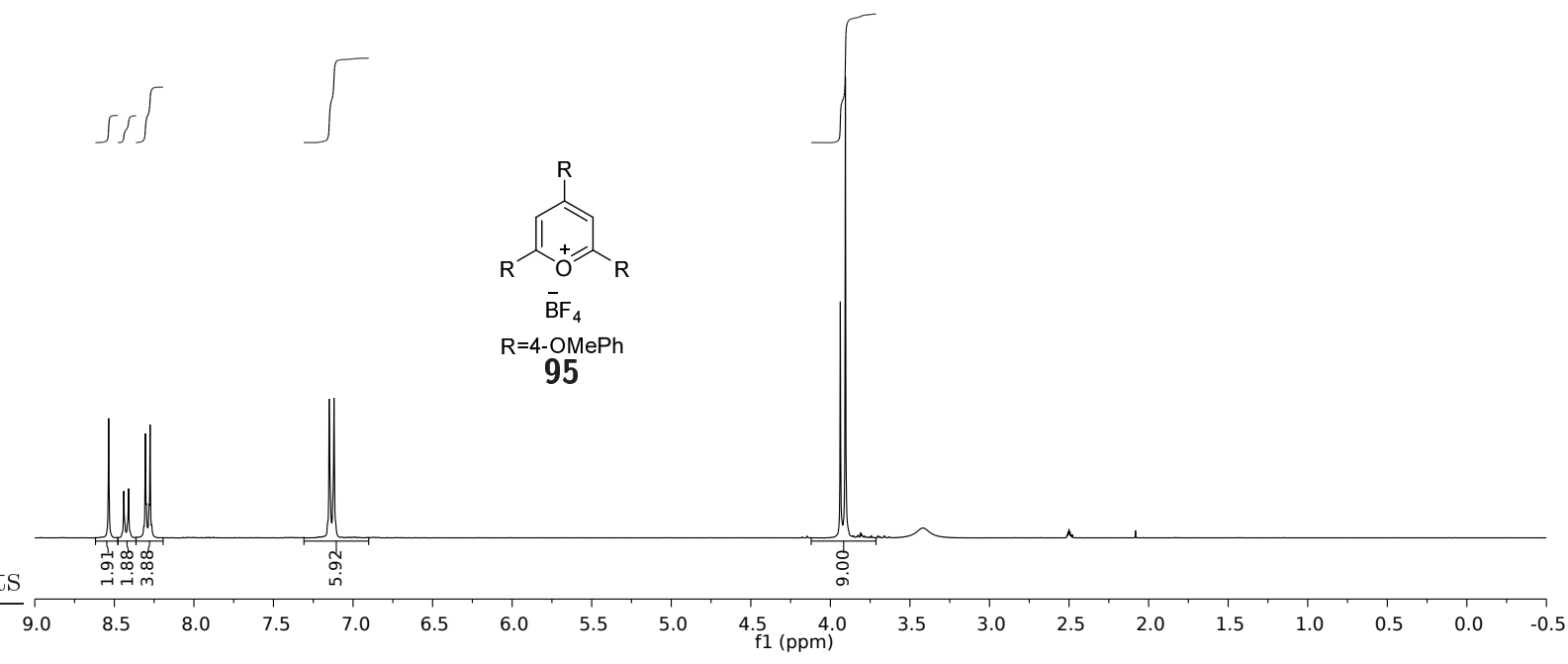

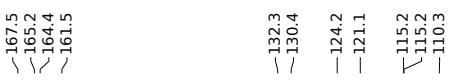

ทั่
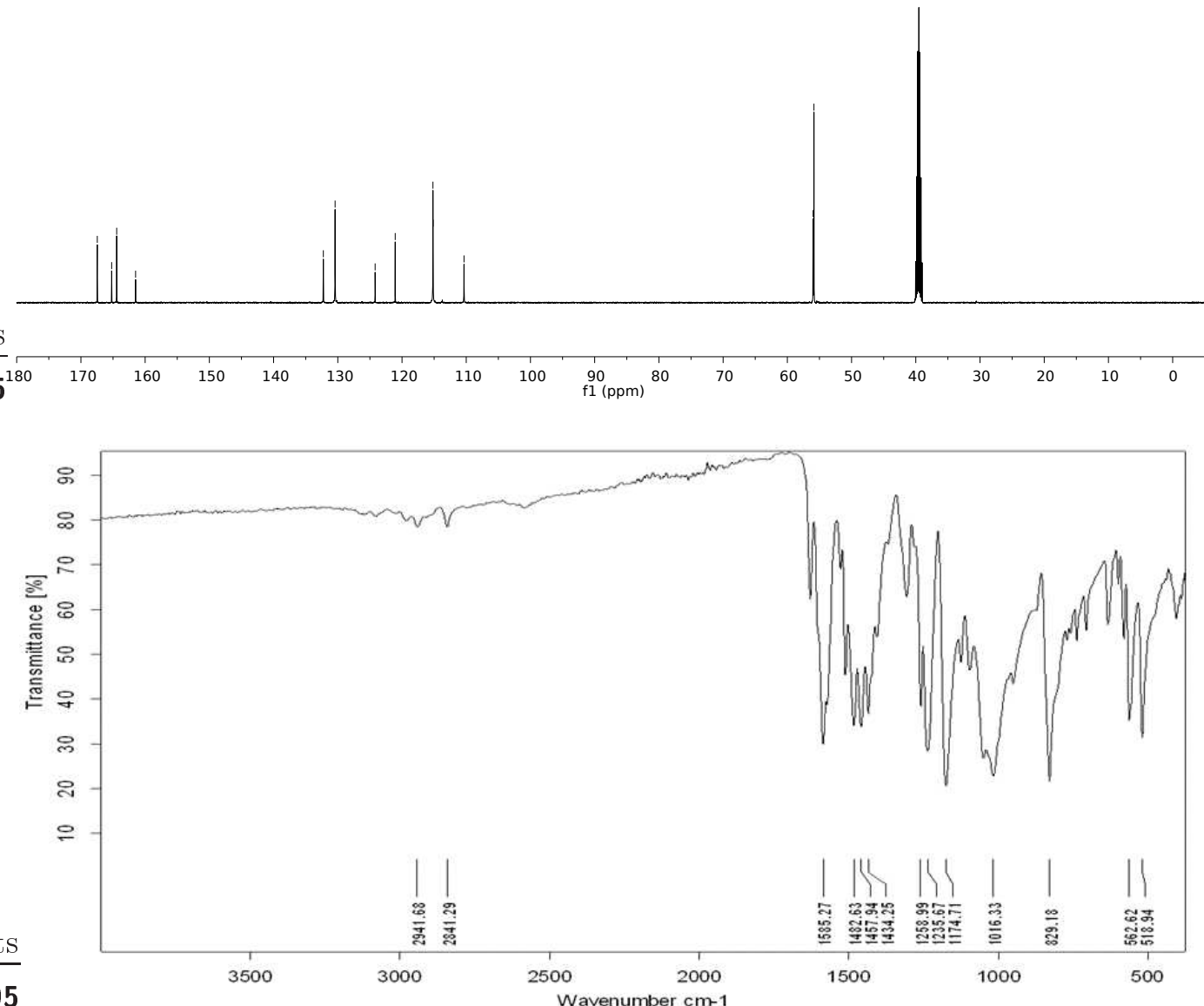


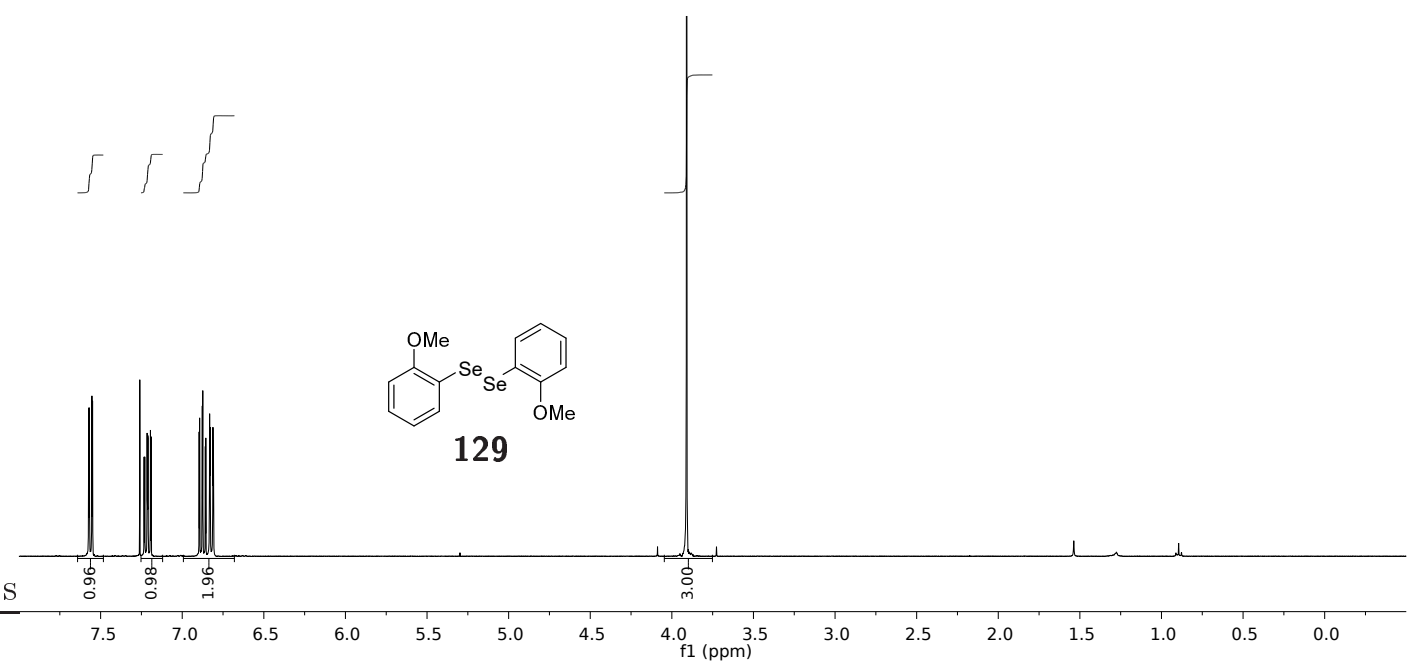

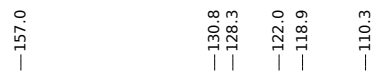

i̊n.
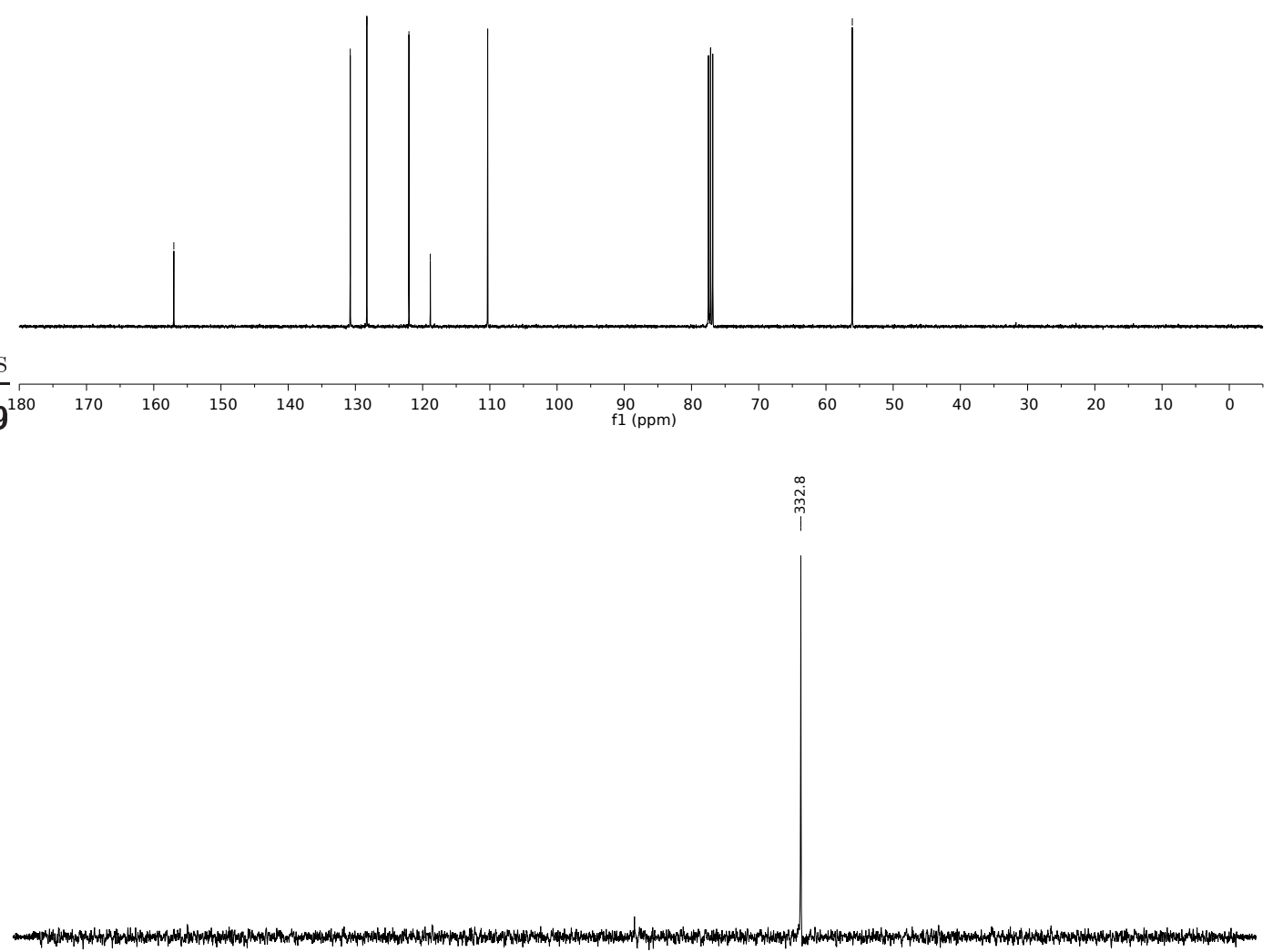

\begin{tabular}{llllllllll}
\hline 650 & 600 & 550 & 500 & 450 & 400 & 350 & 300 & 250 & 200
\end{tabular} 


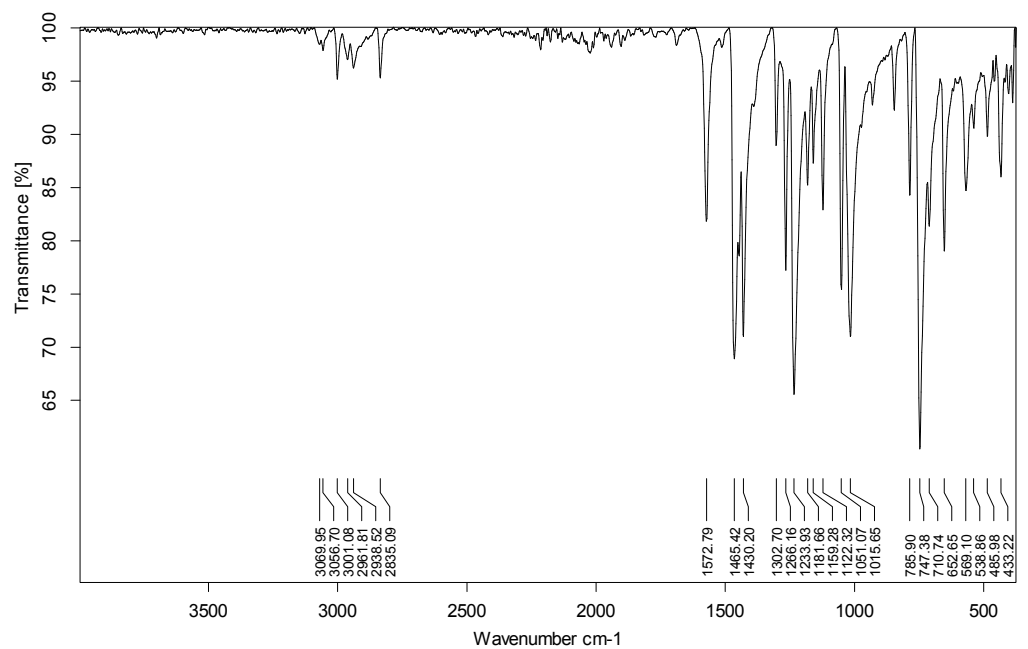


$\int$

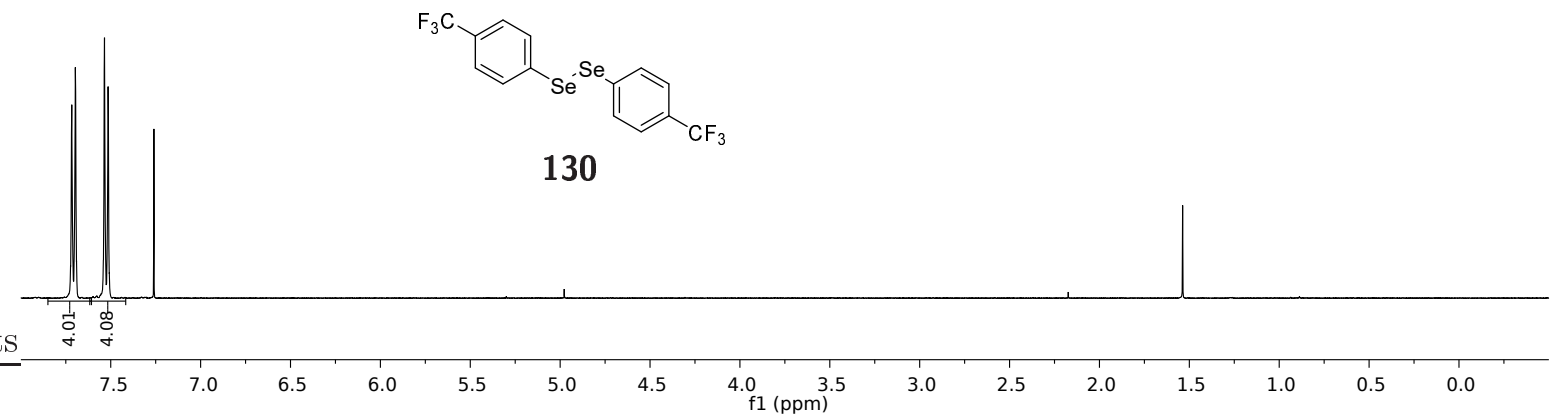

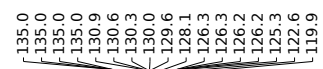

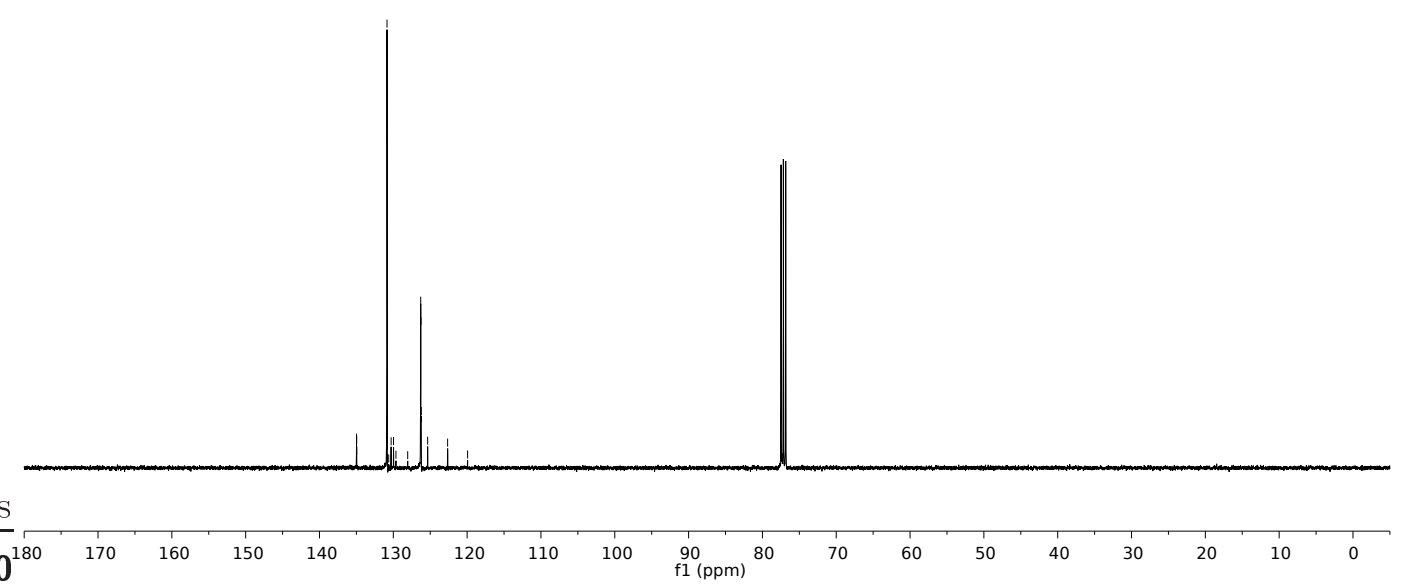

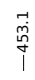

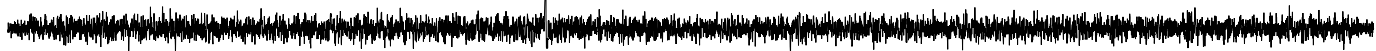

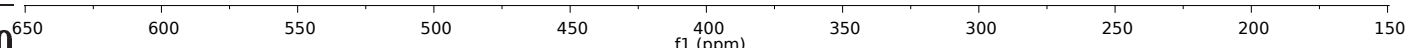




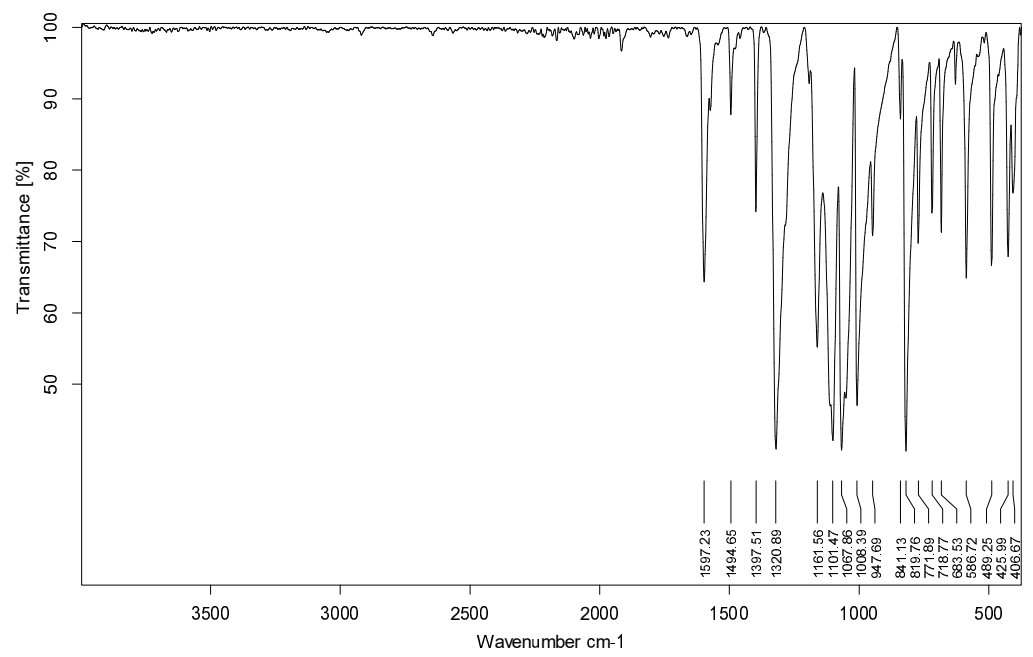




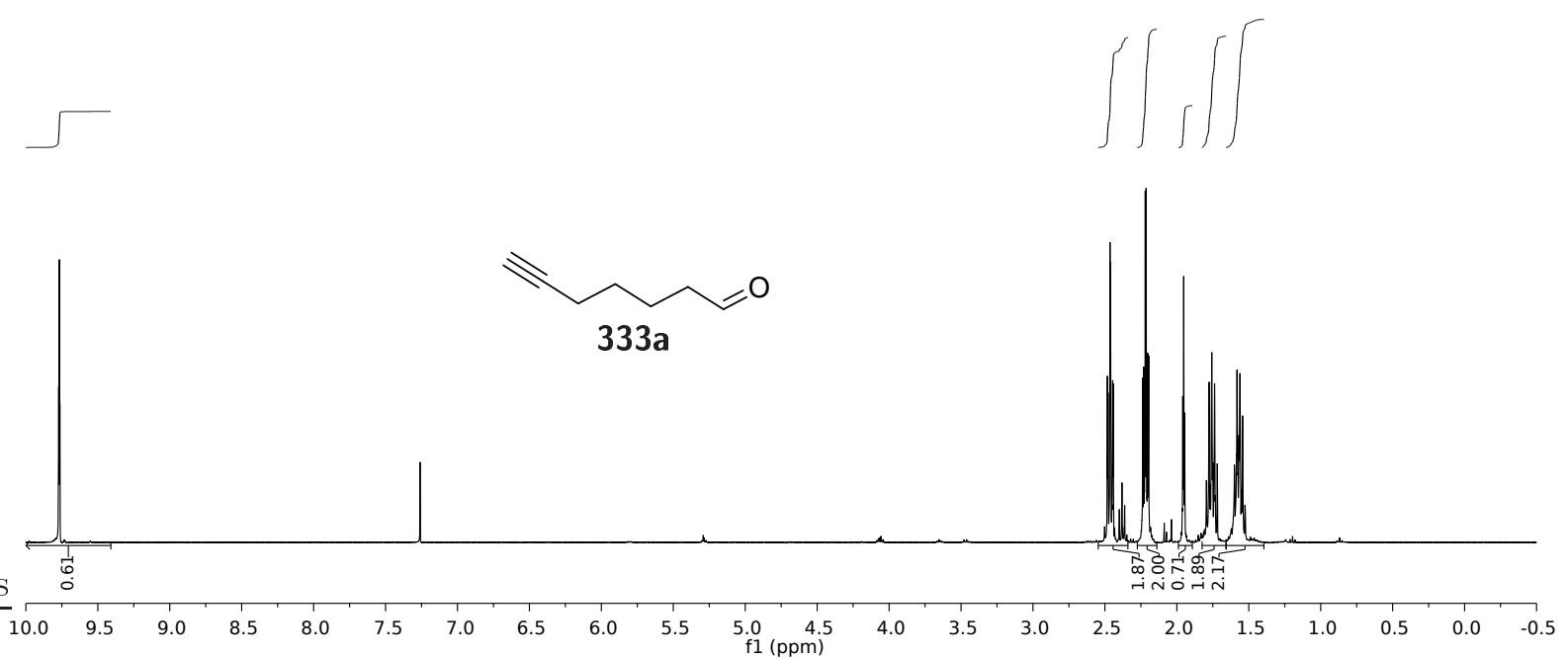

$\stackrel{m}{\stackrel{m}{i}}$

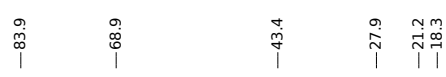
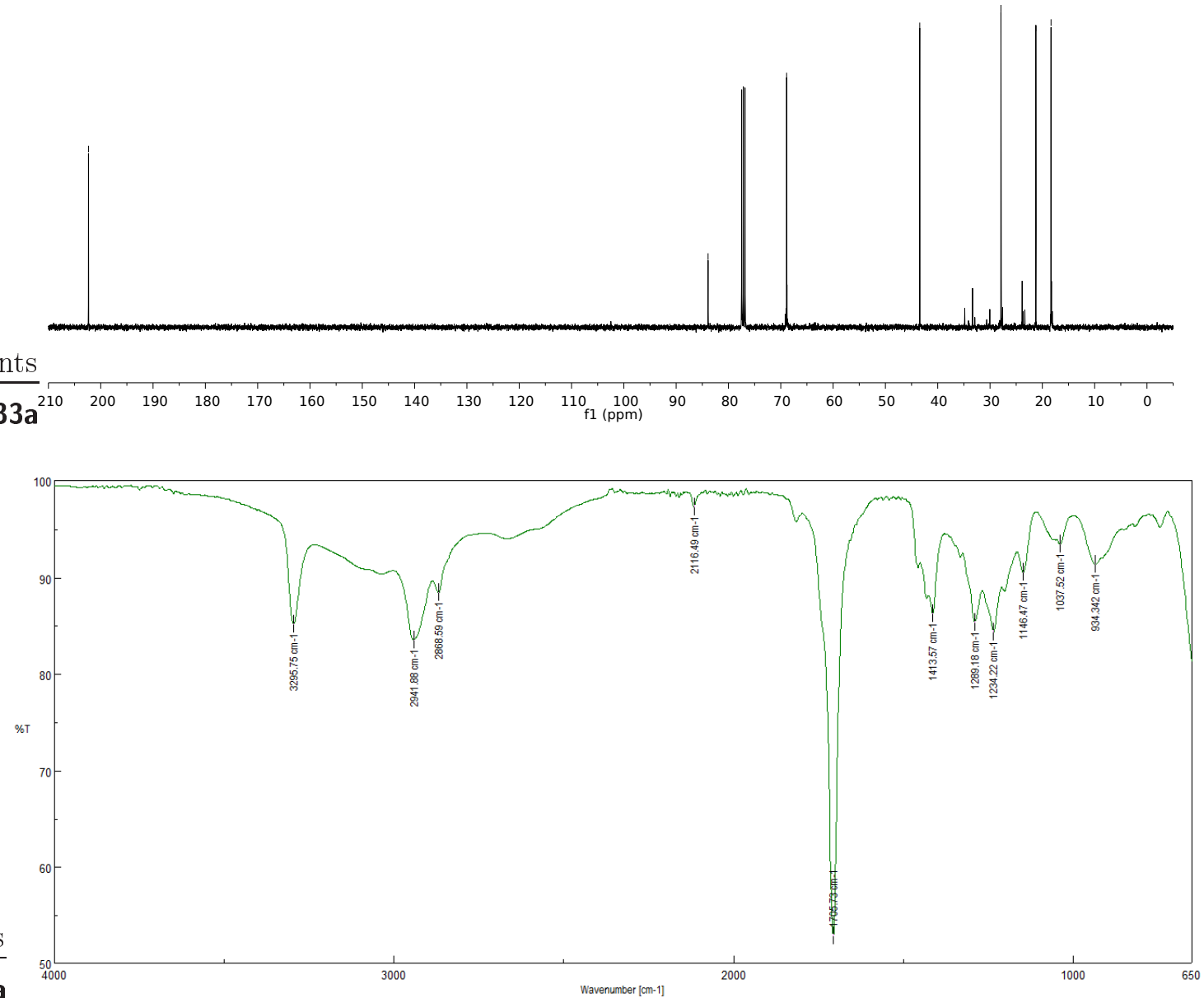

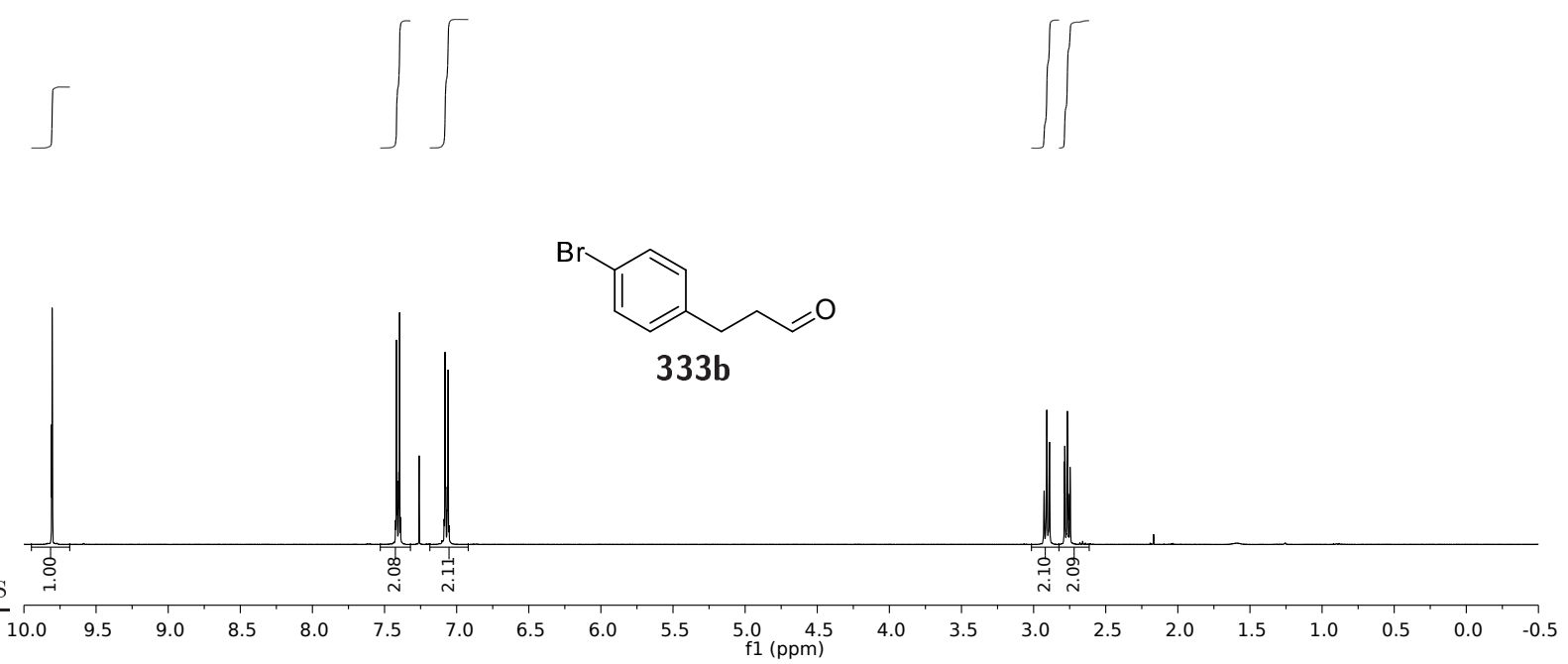

ঙํำ

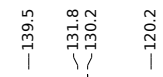

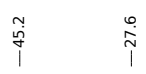
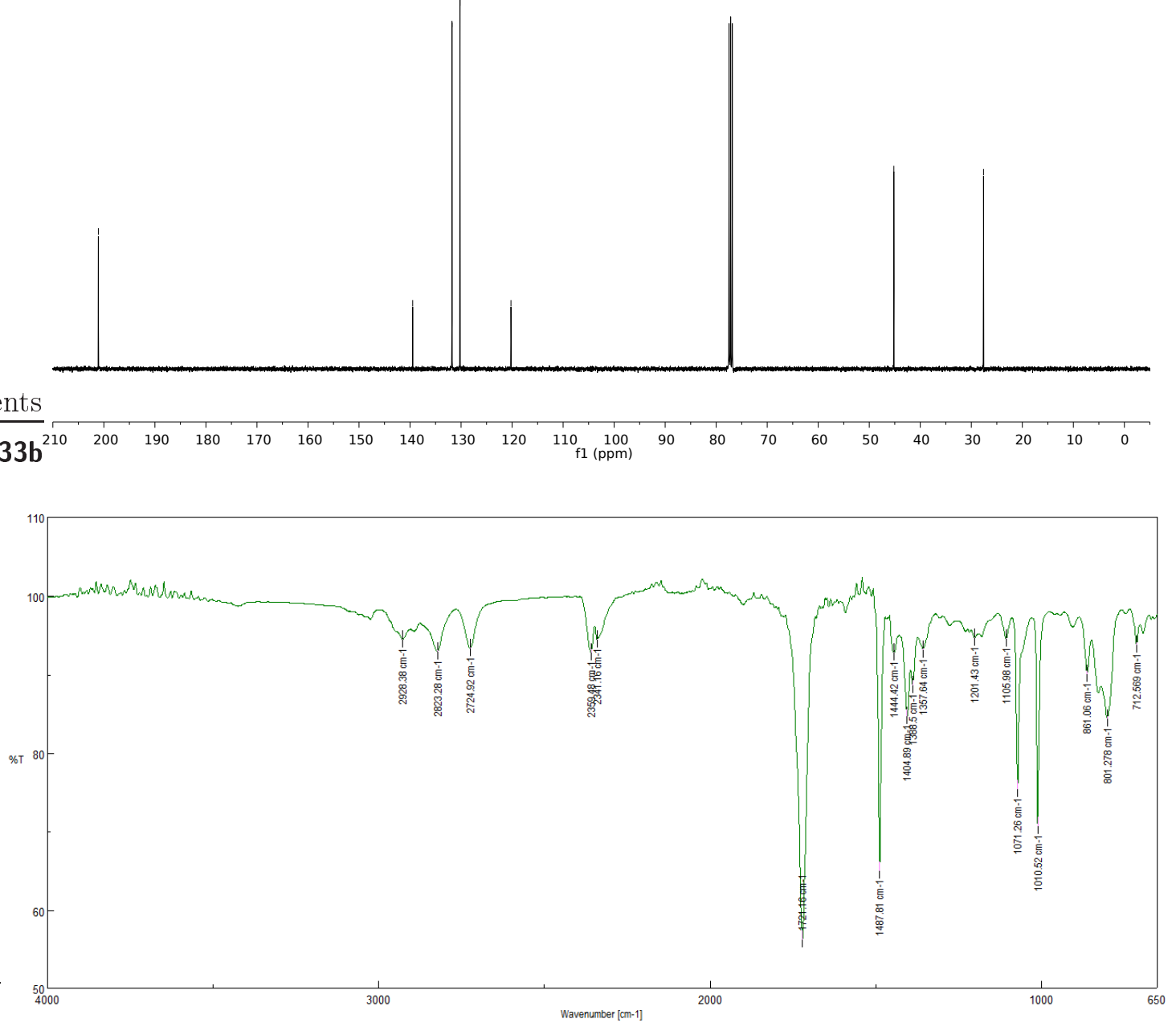

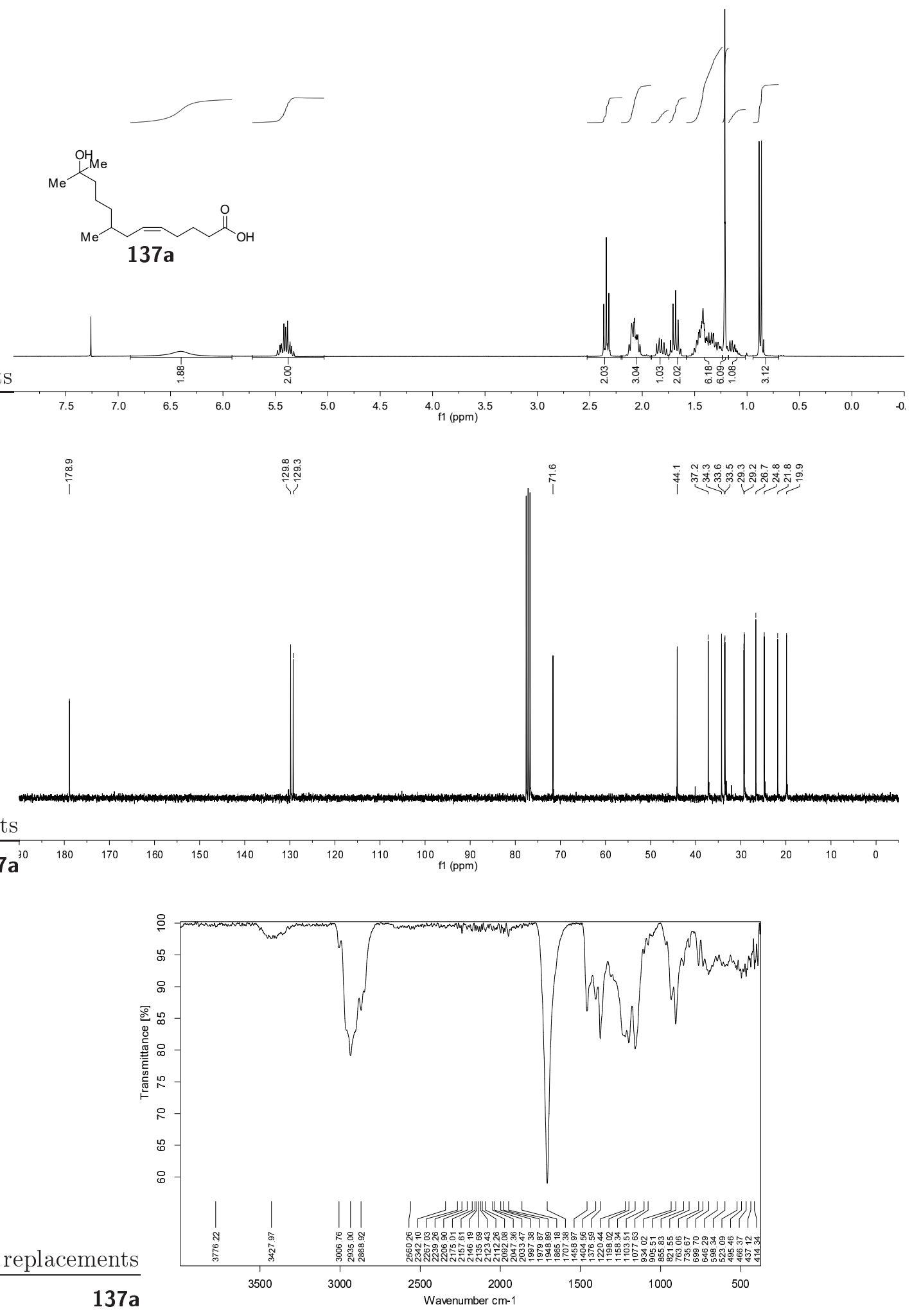

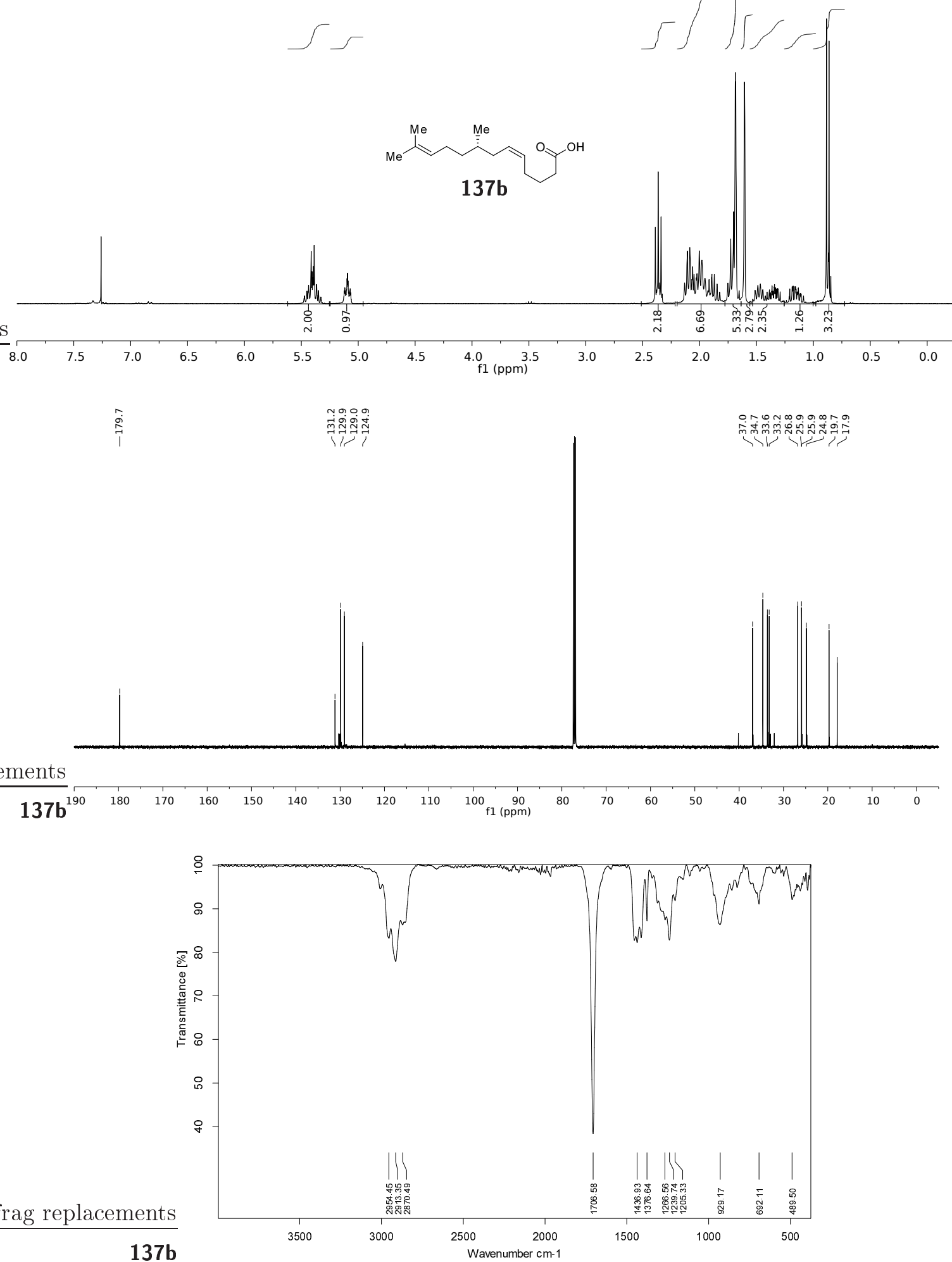

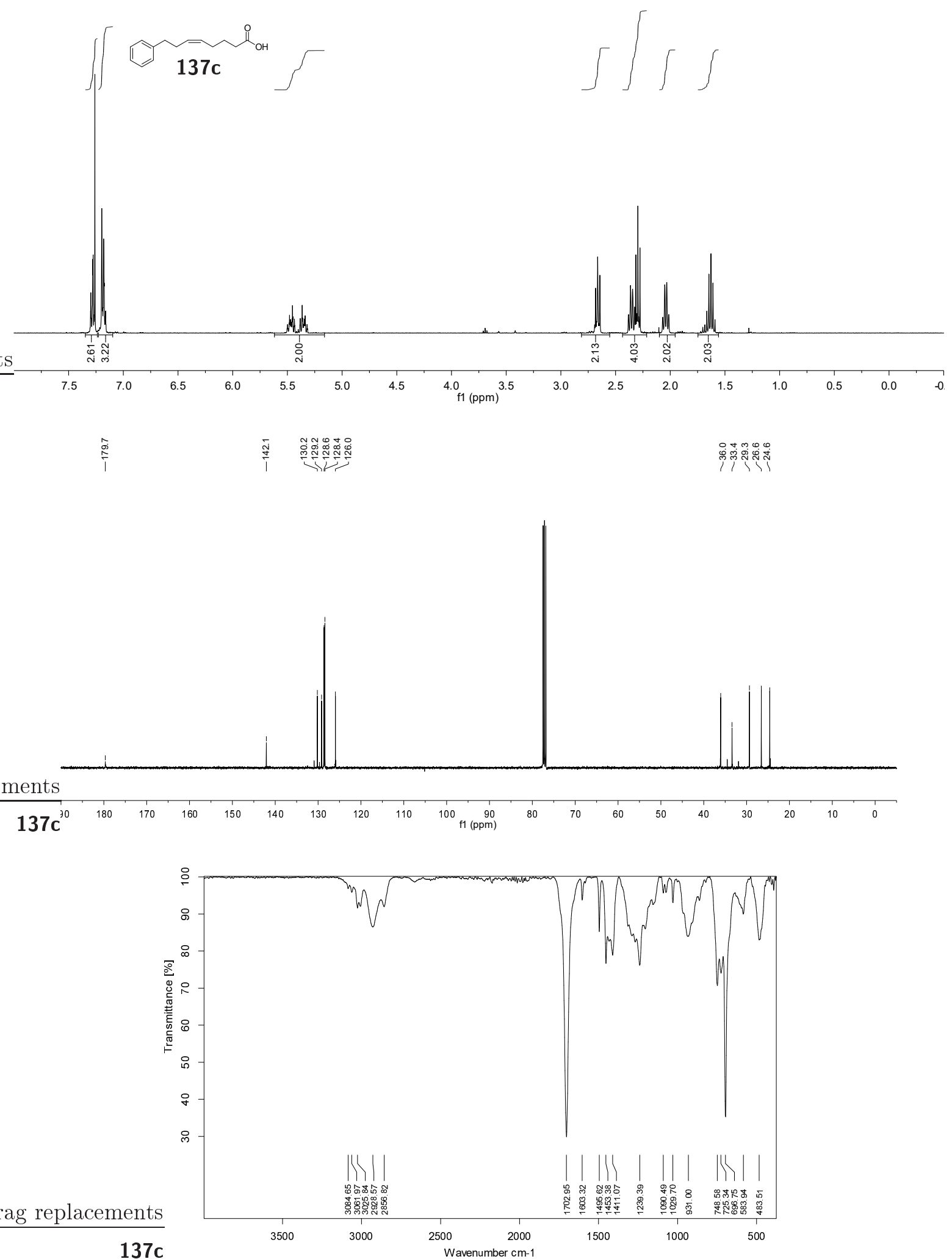

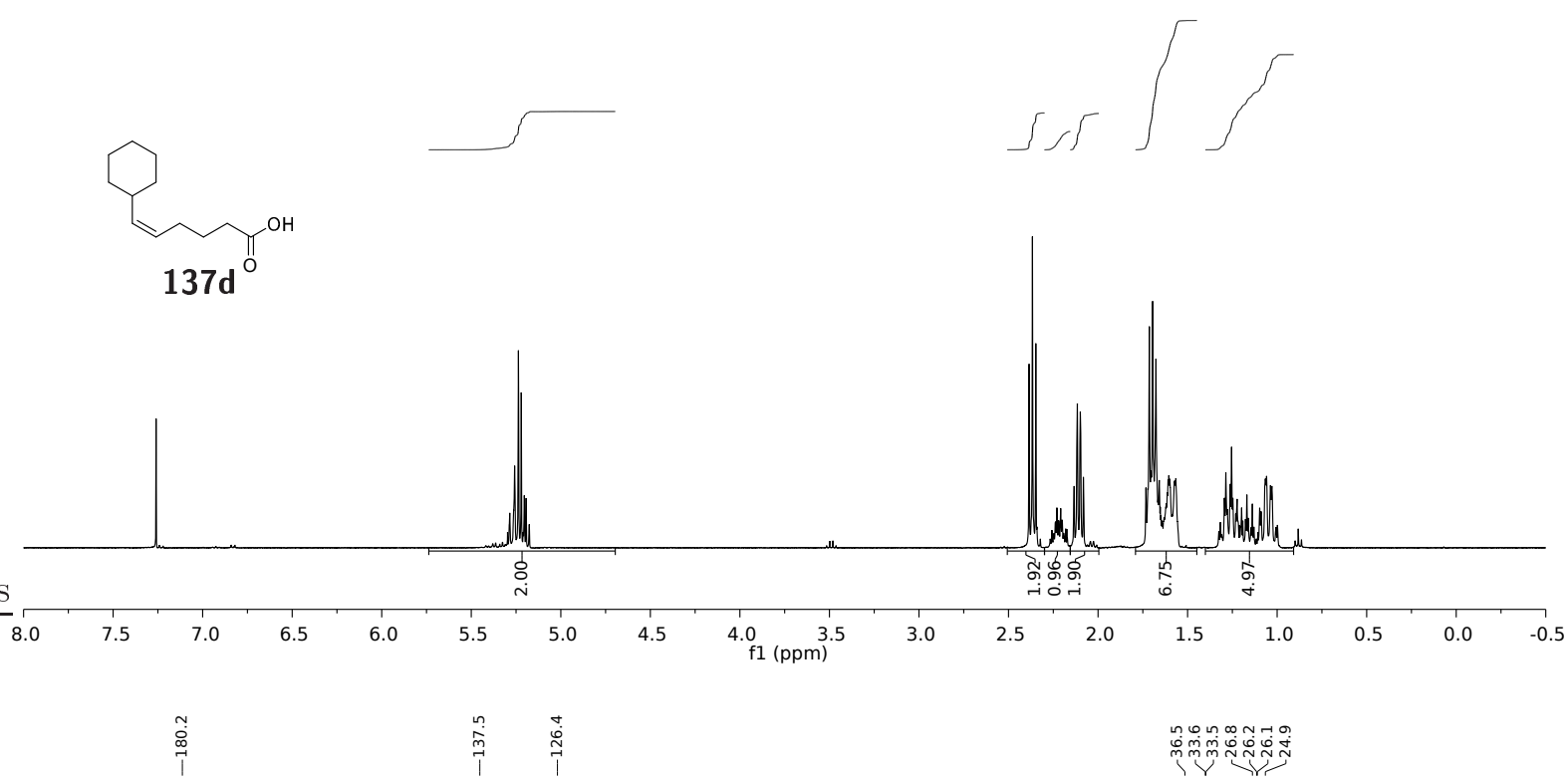

$\underbrace{n}$
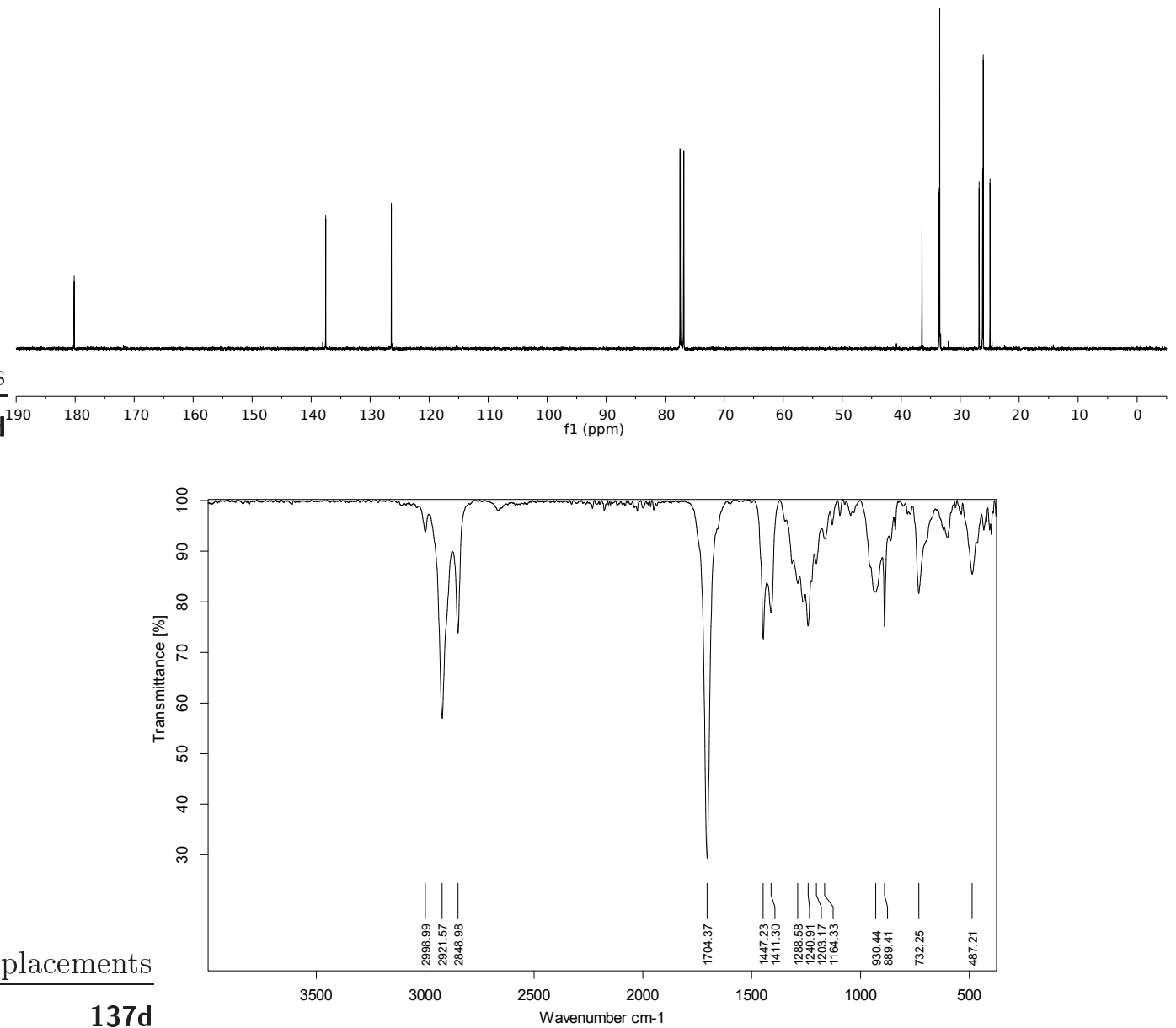


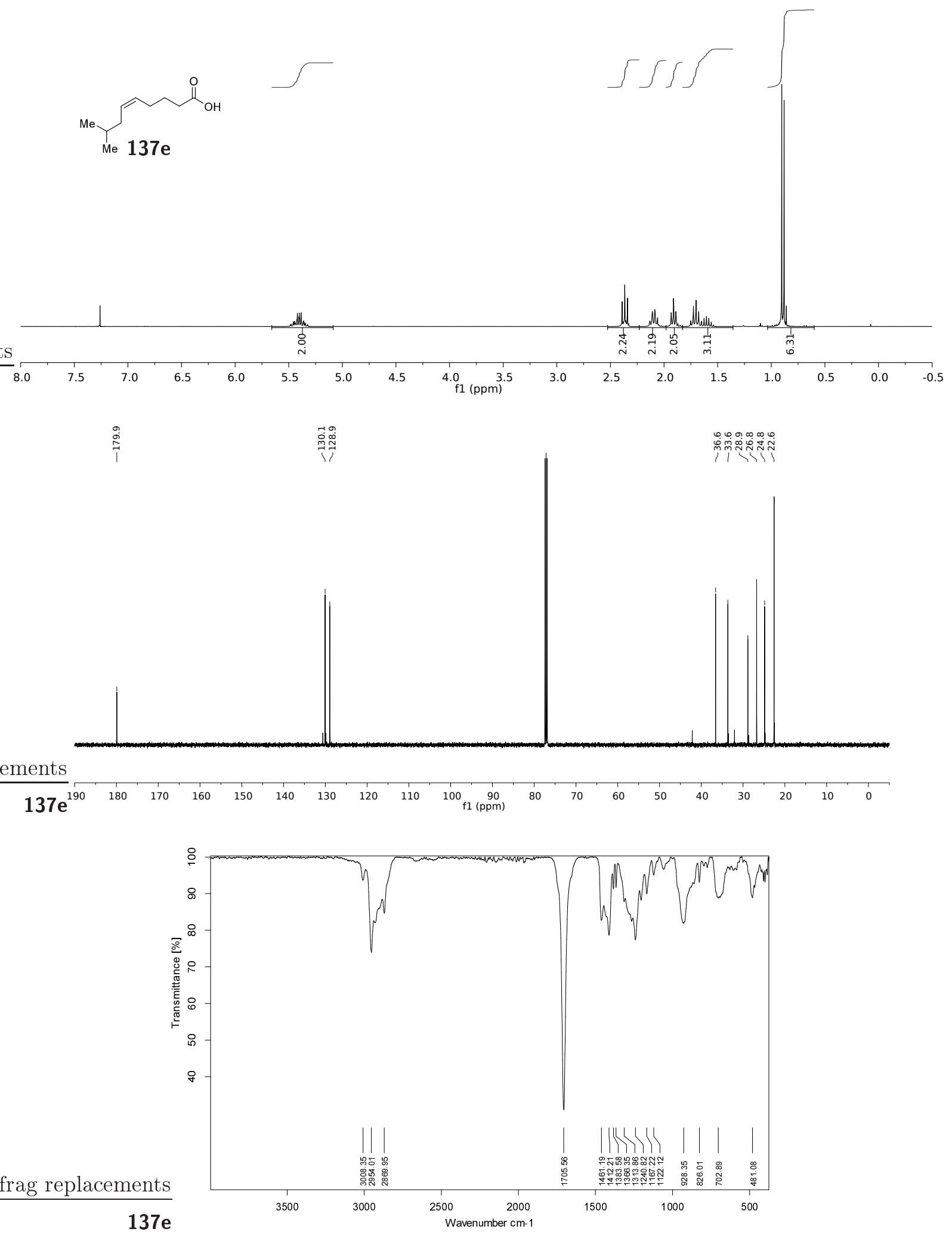




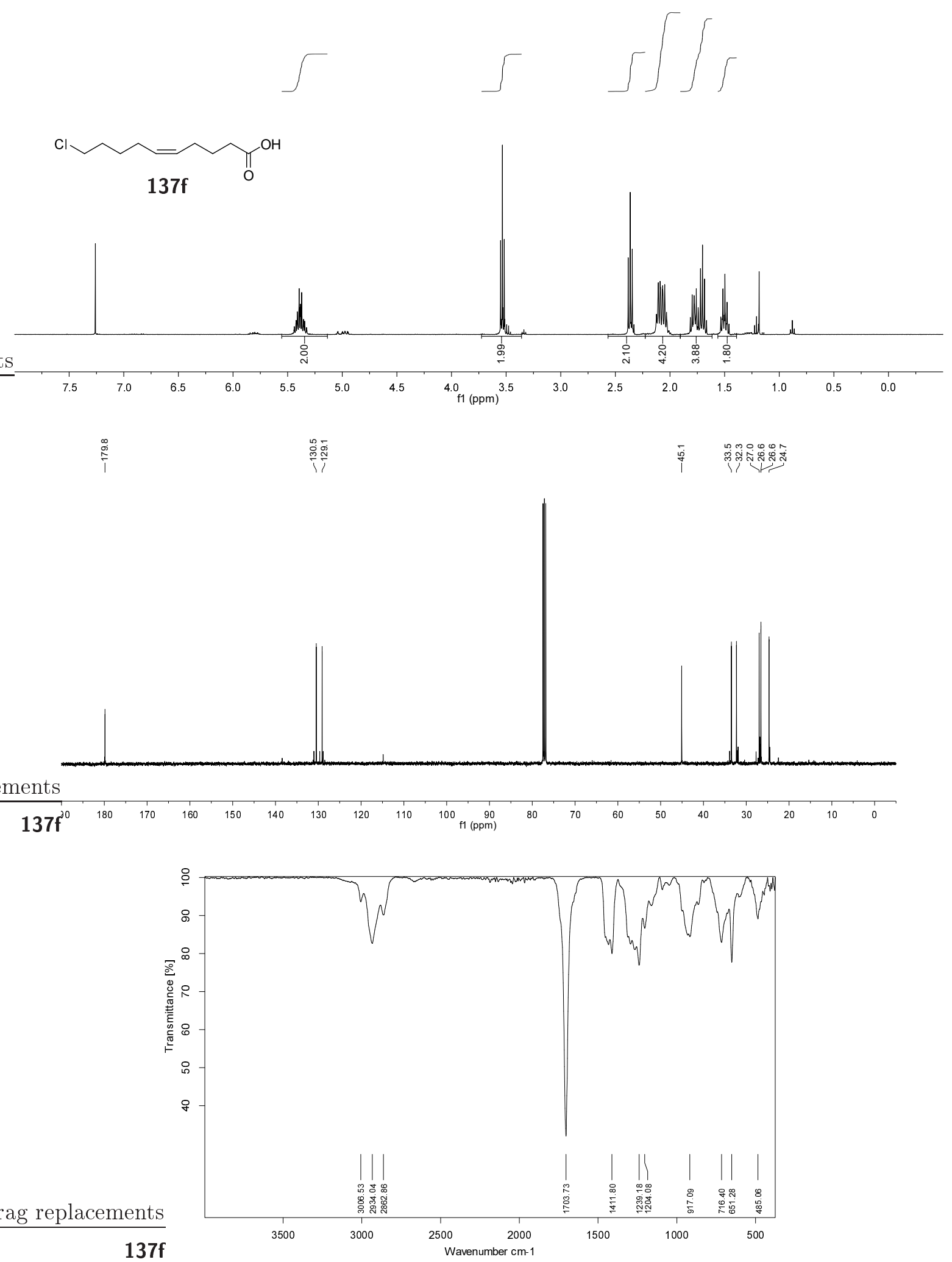



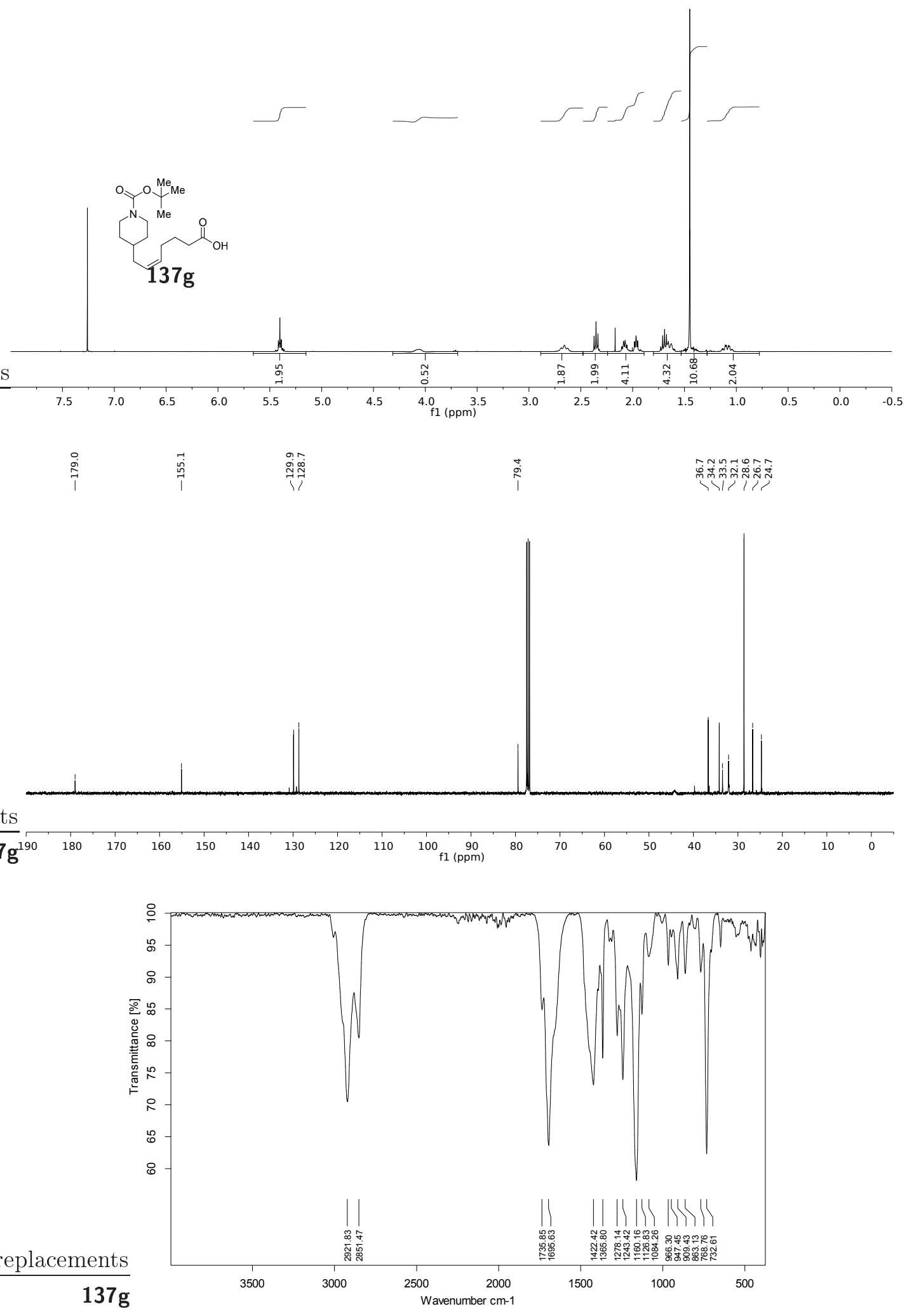


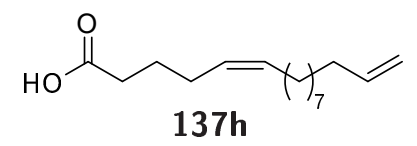

$137 \mathrm{~h}$
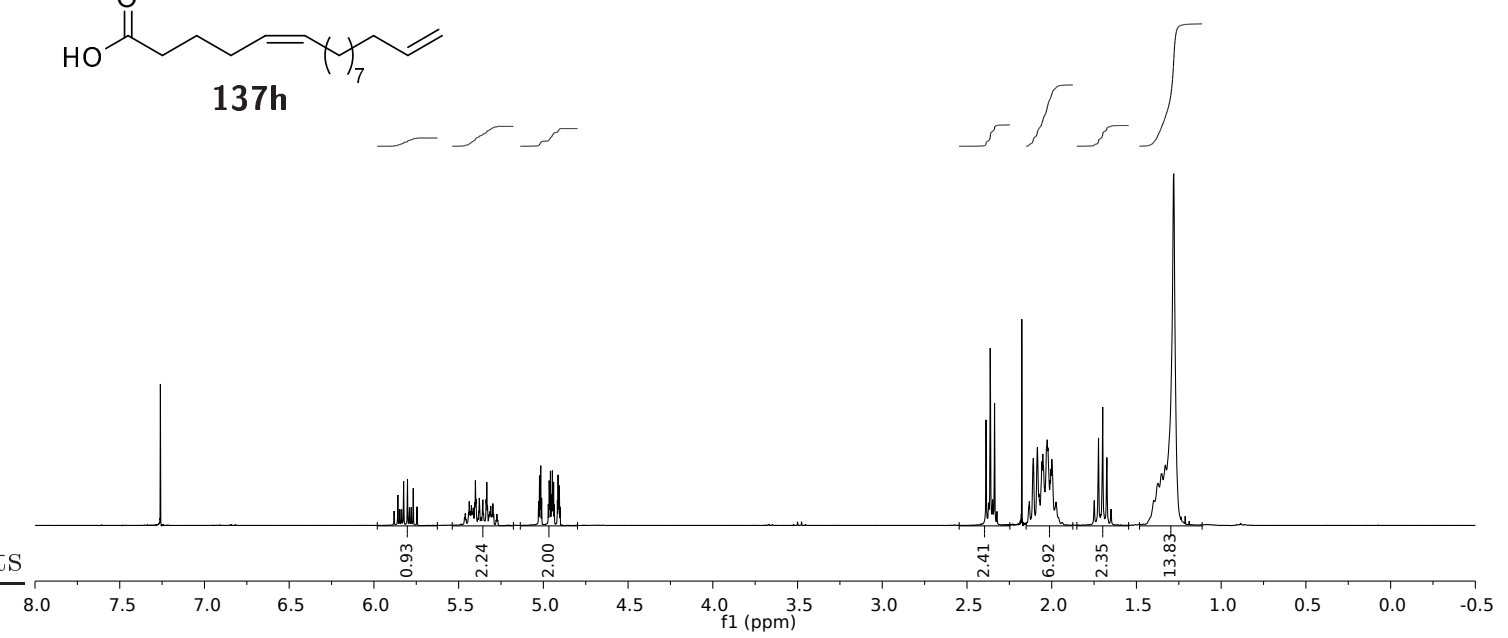

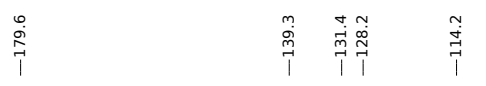

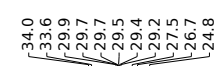
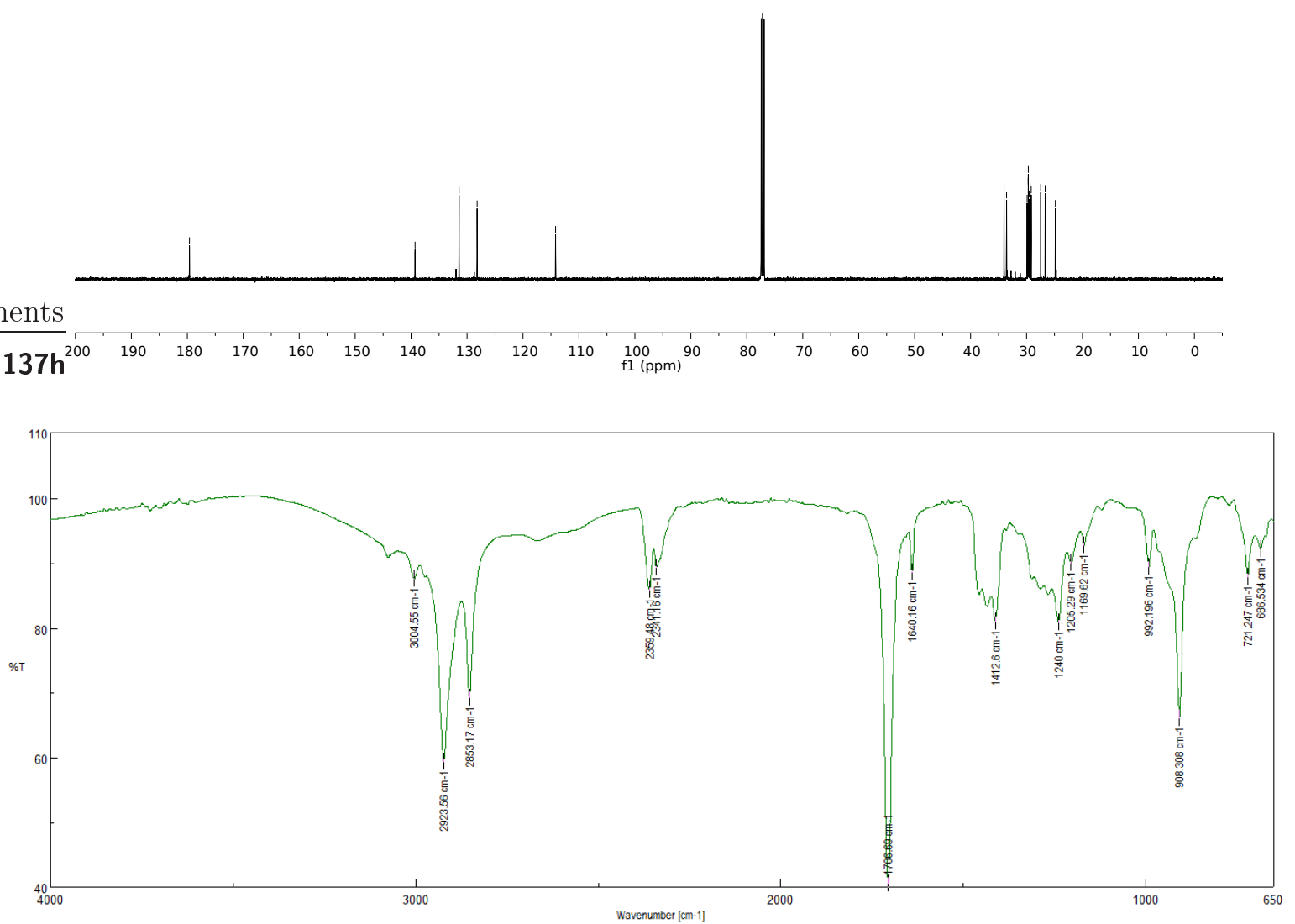

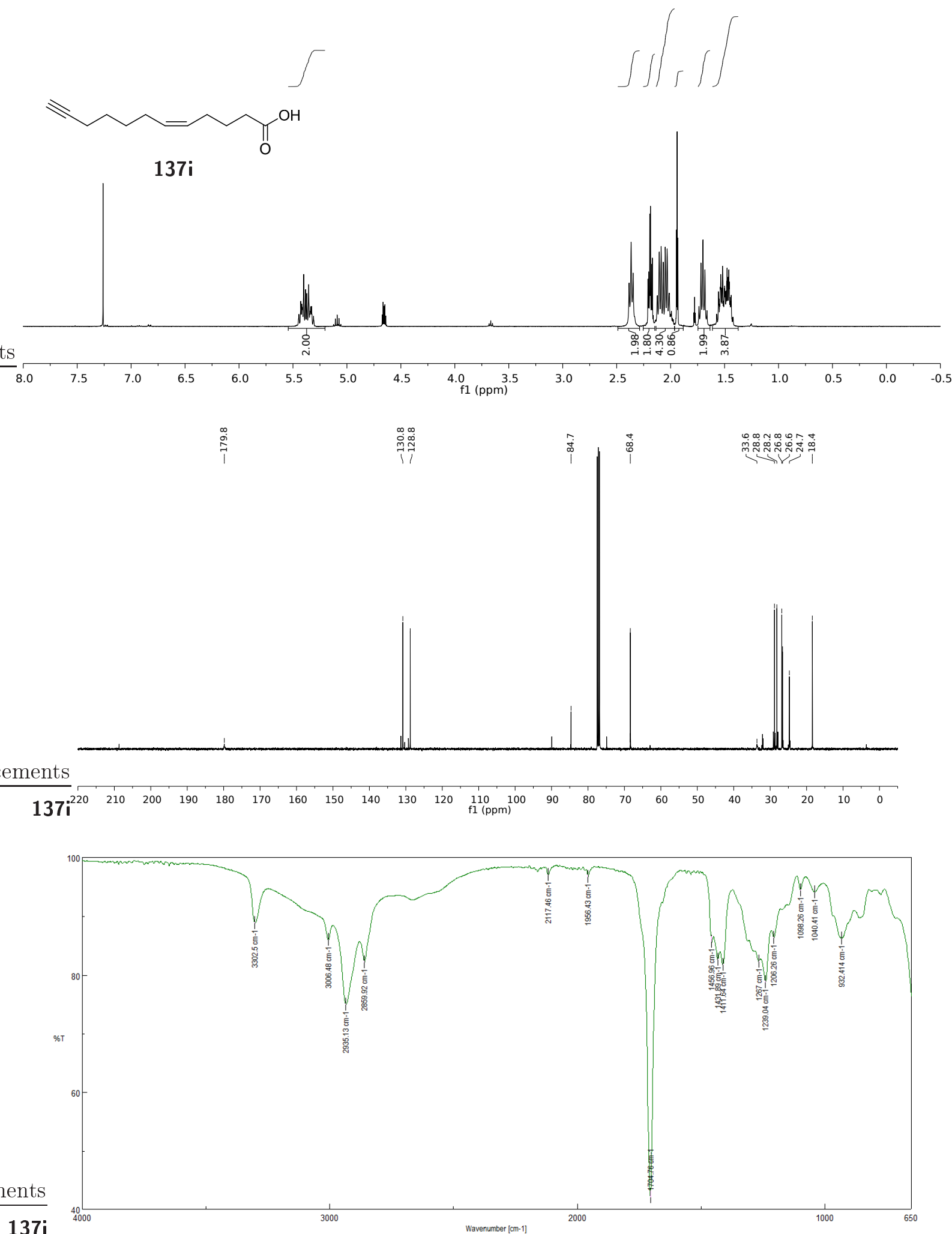

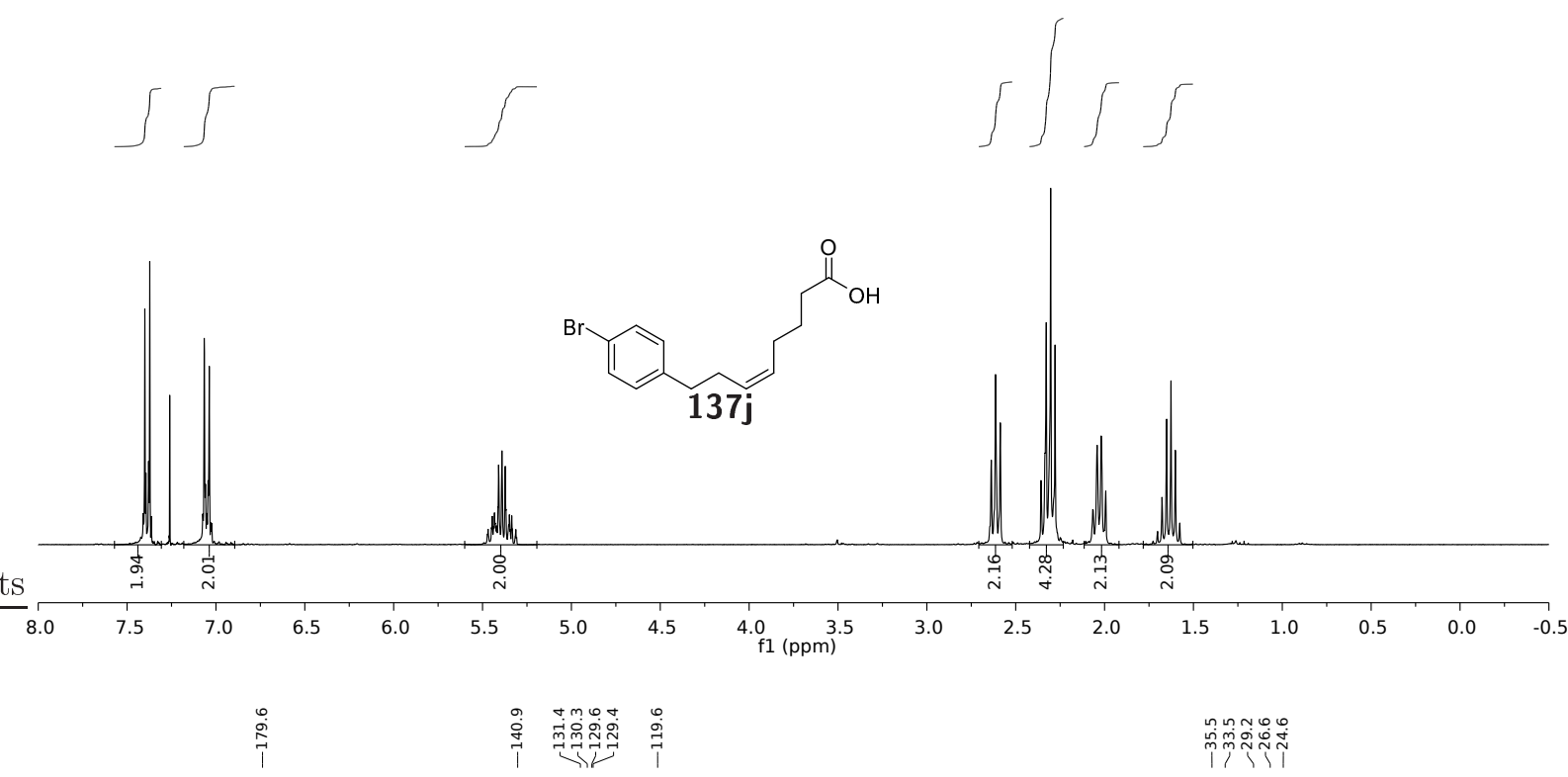

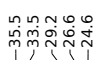
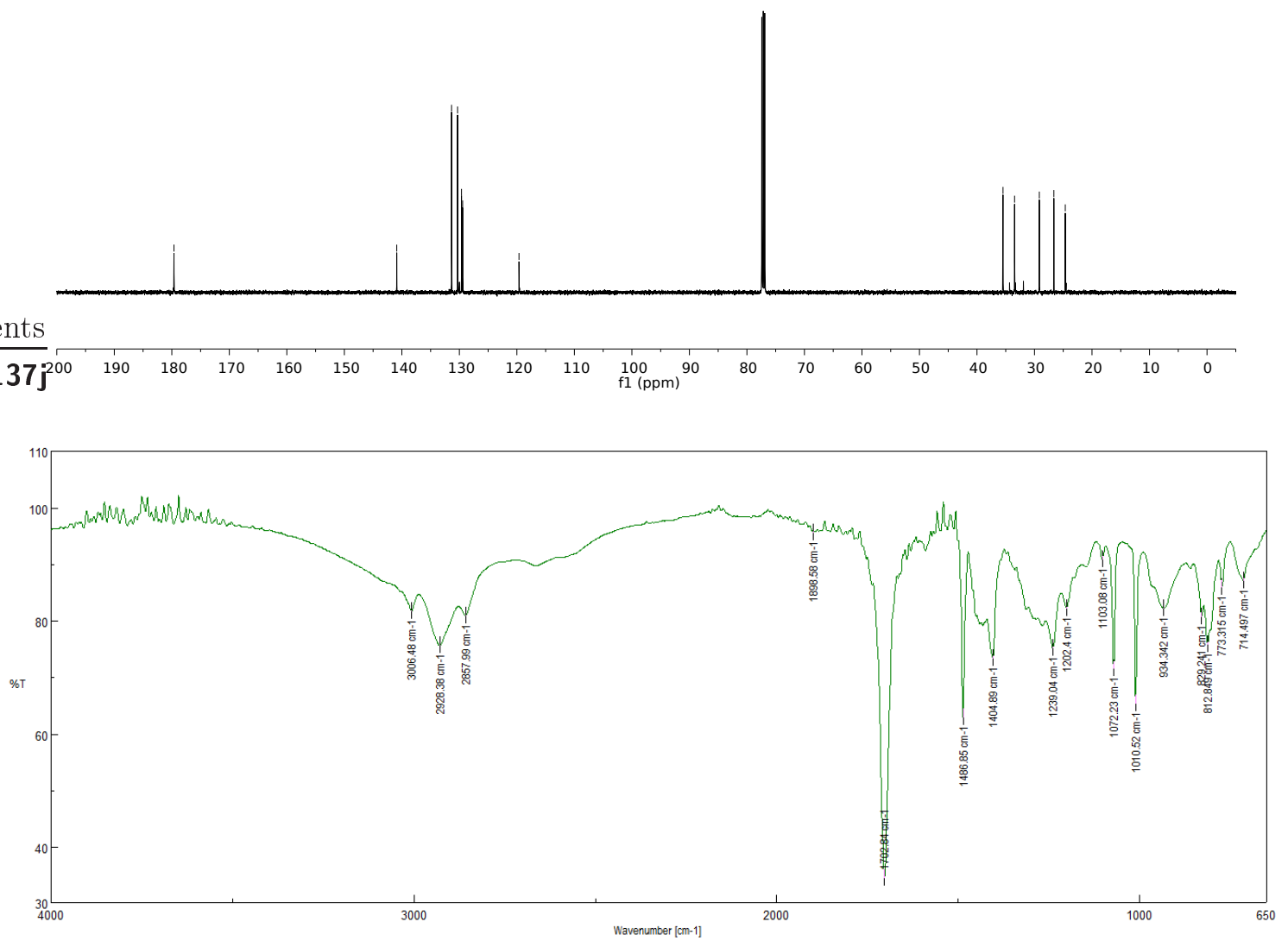


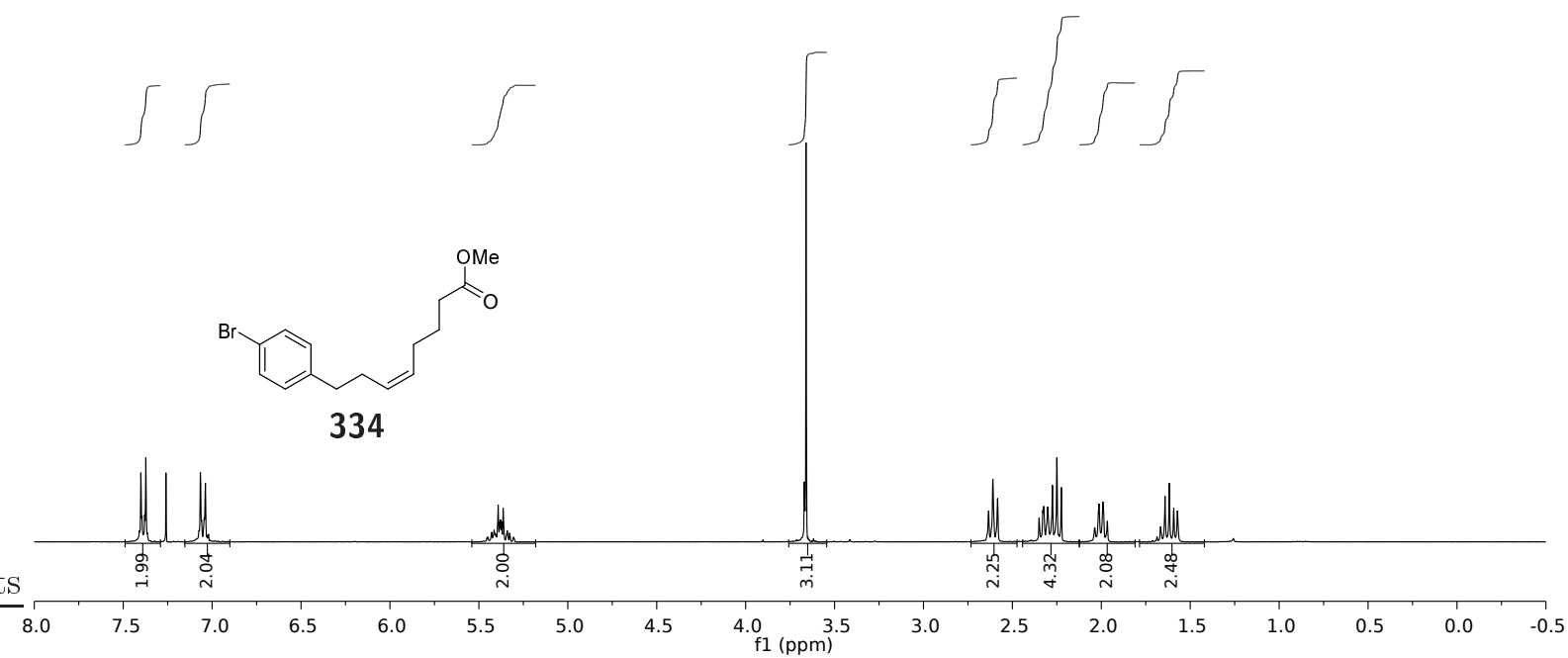

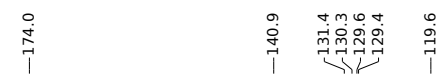

ํ.
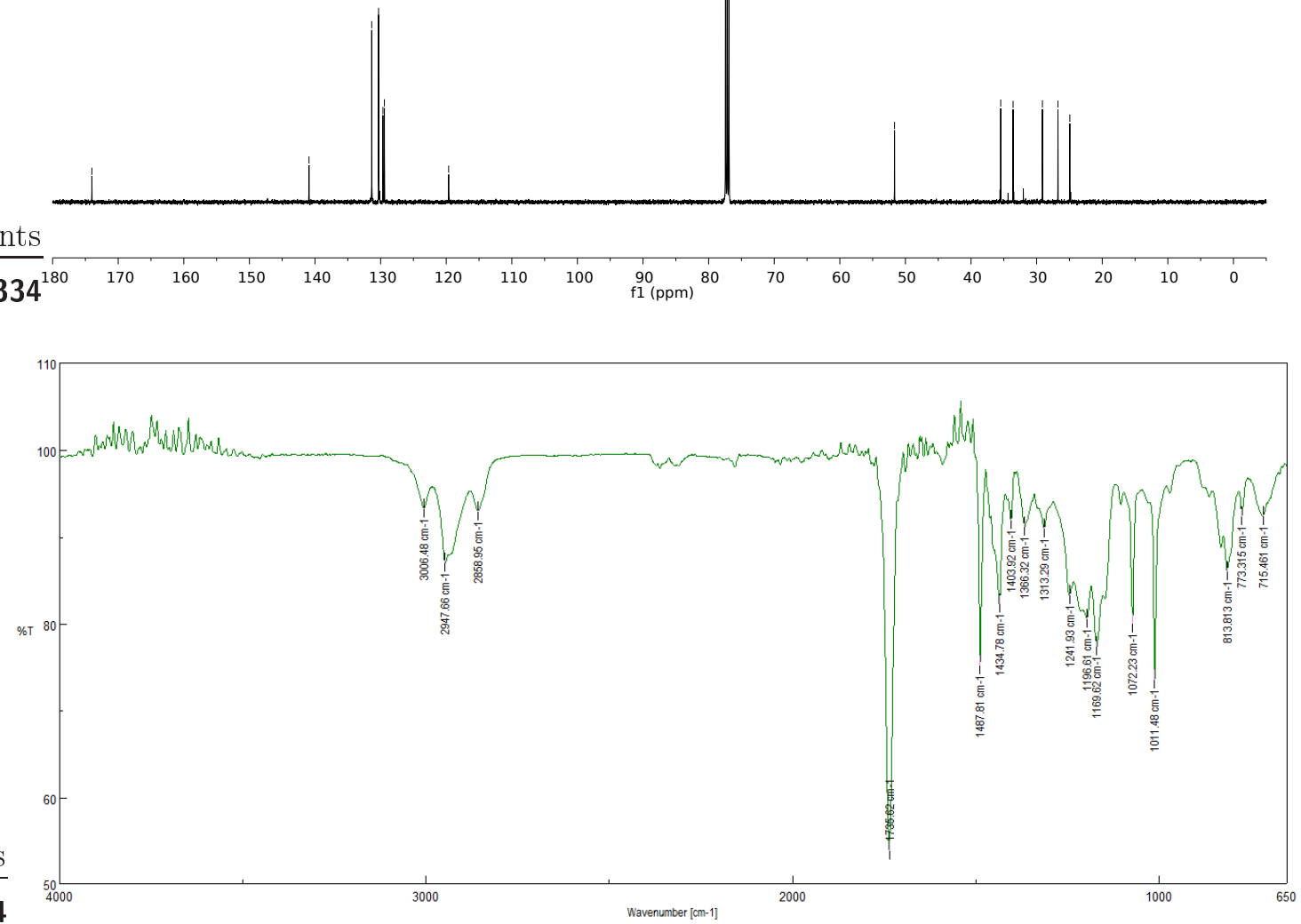

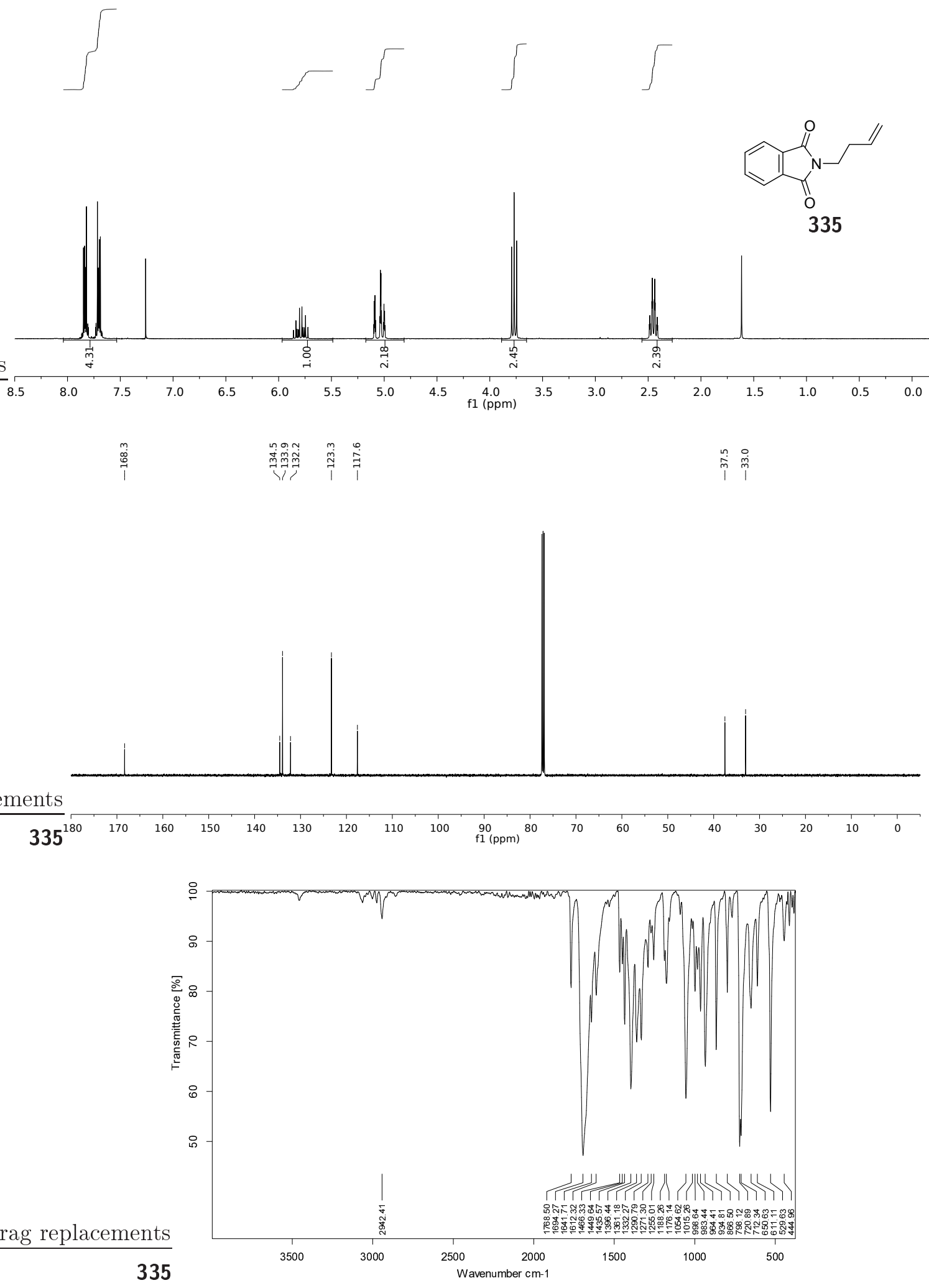


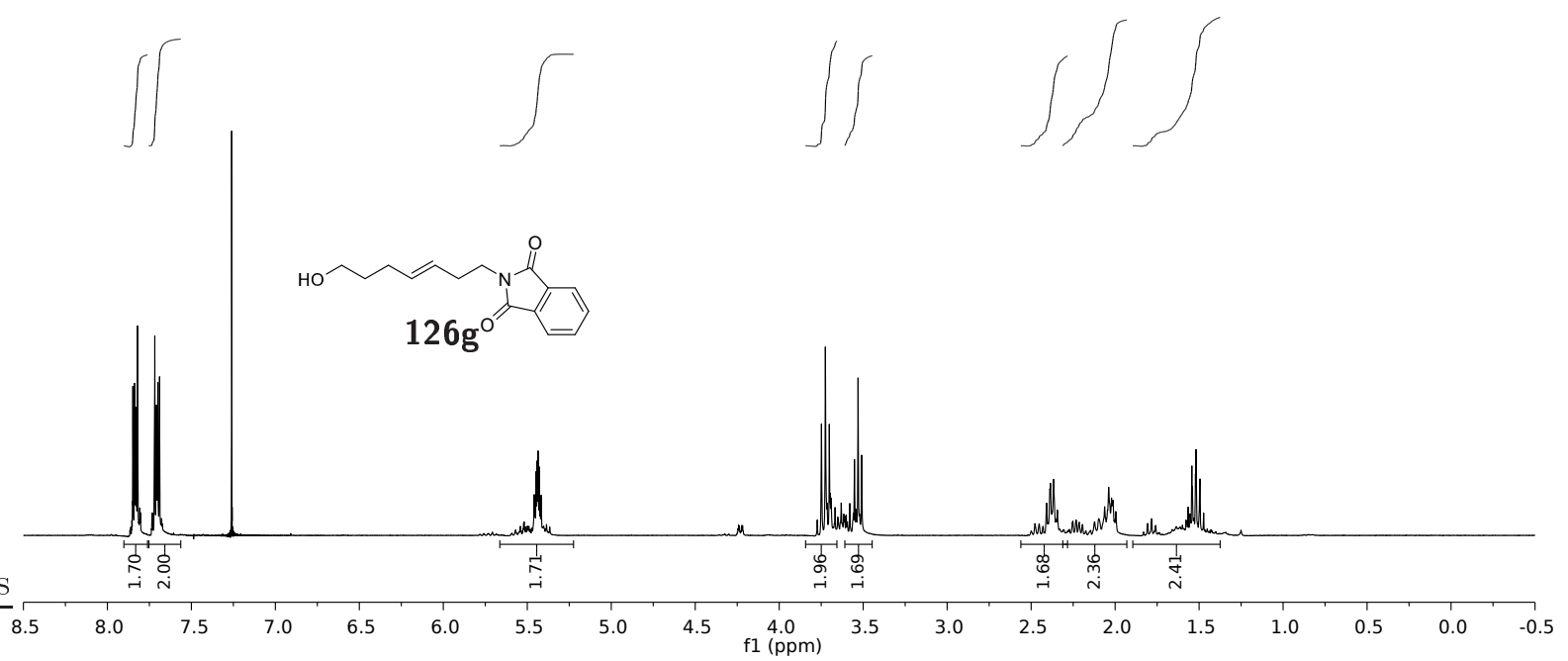

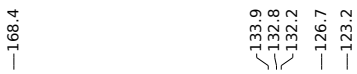
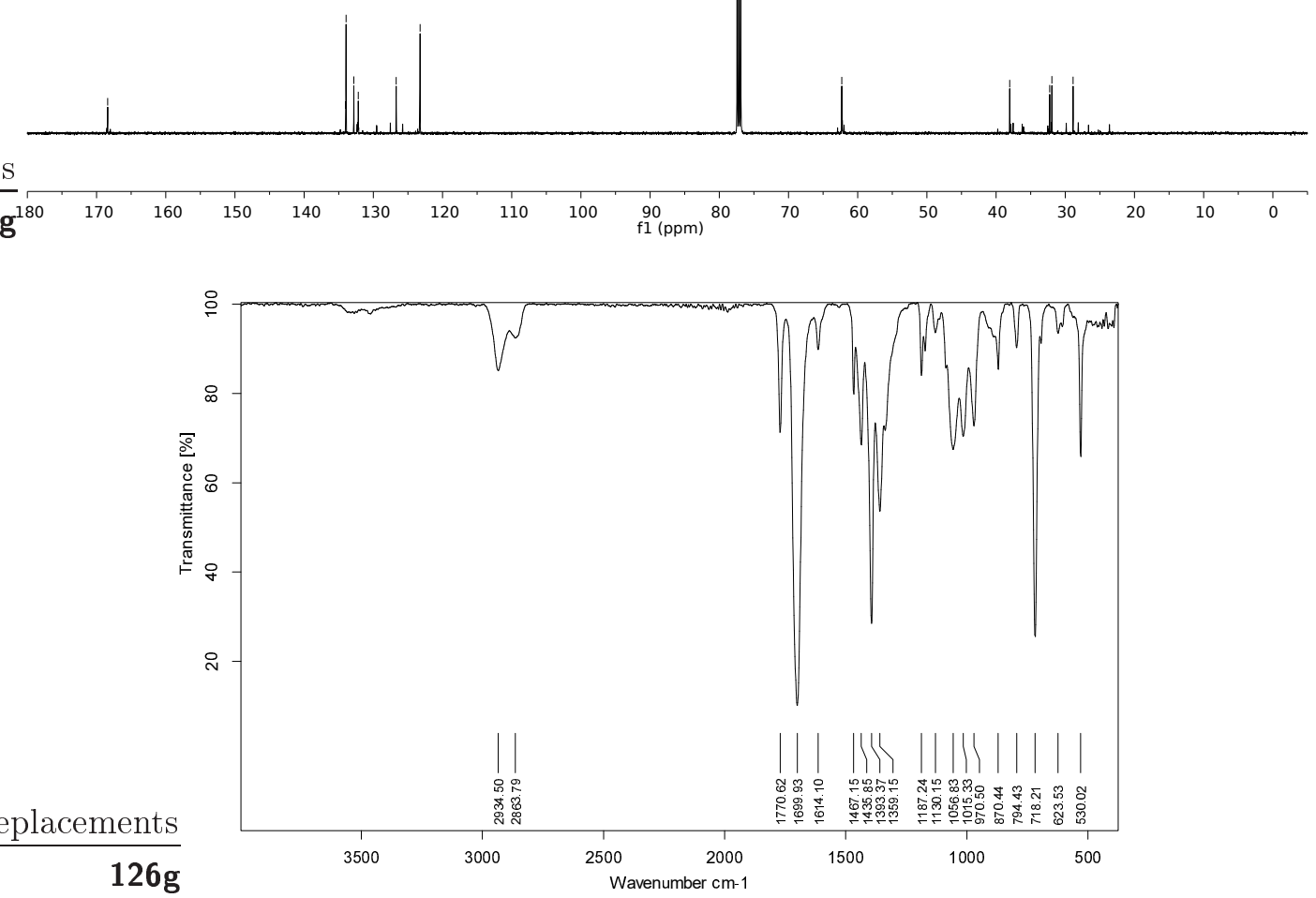


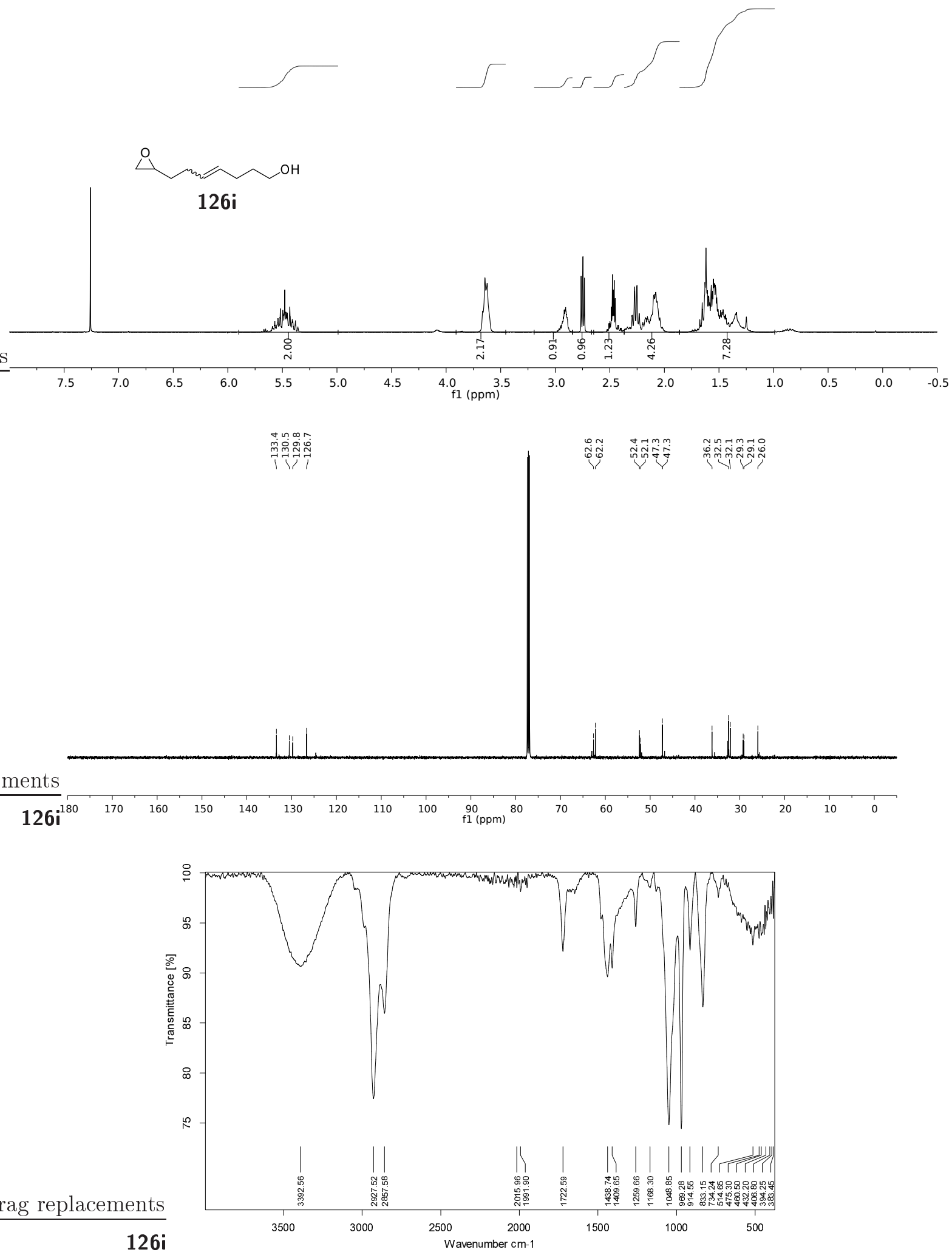



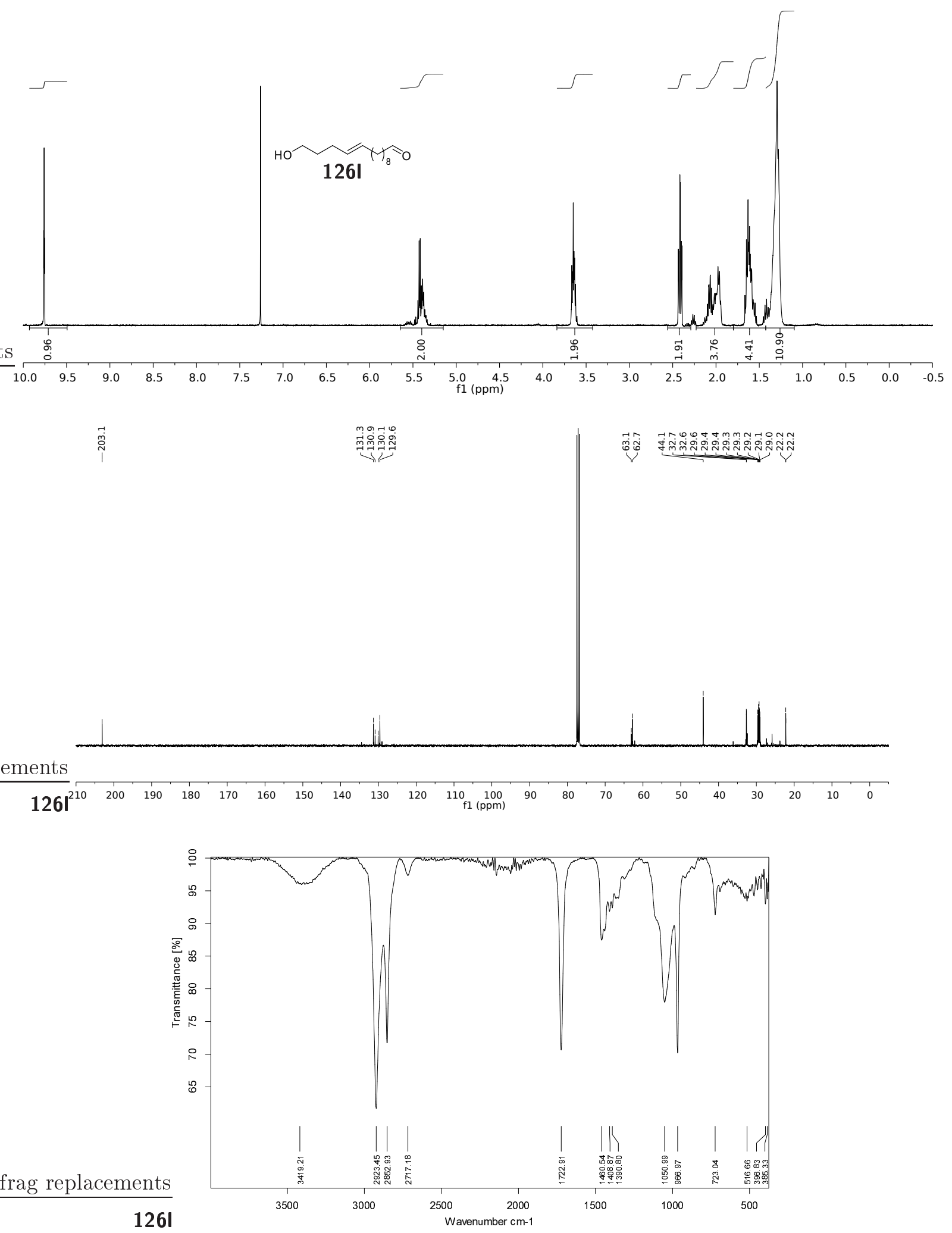

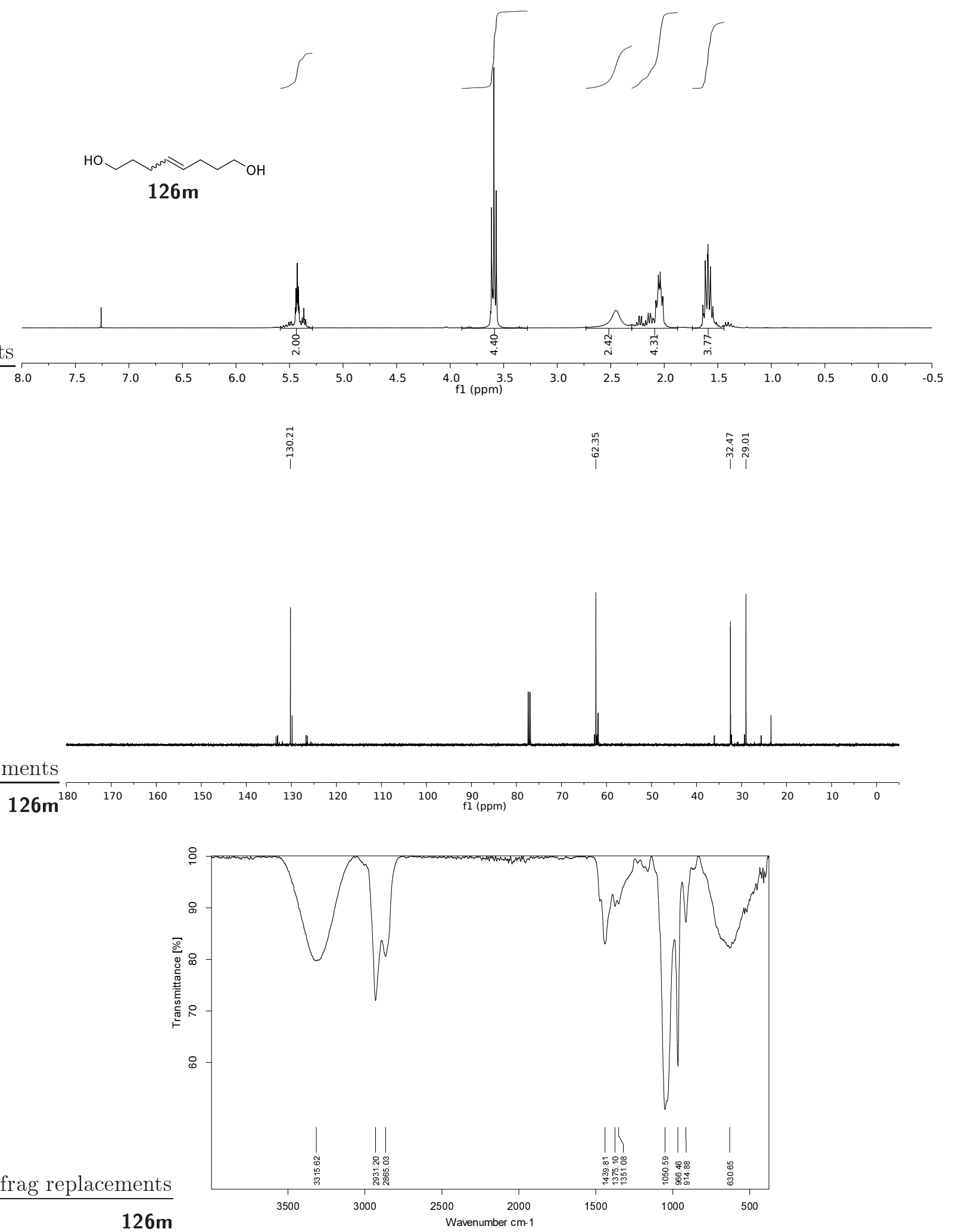


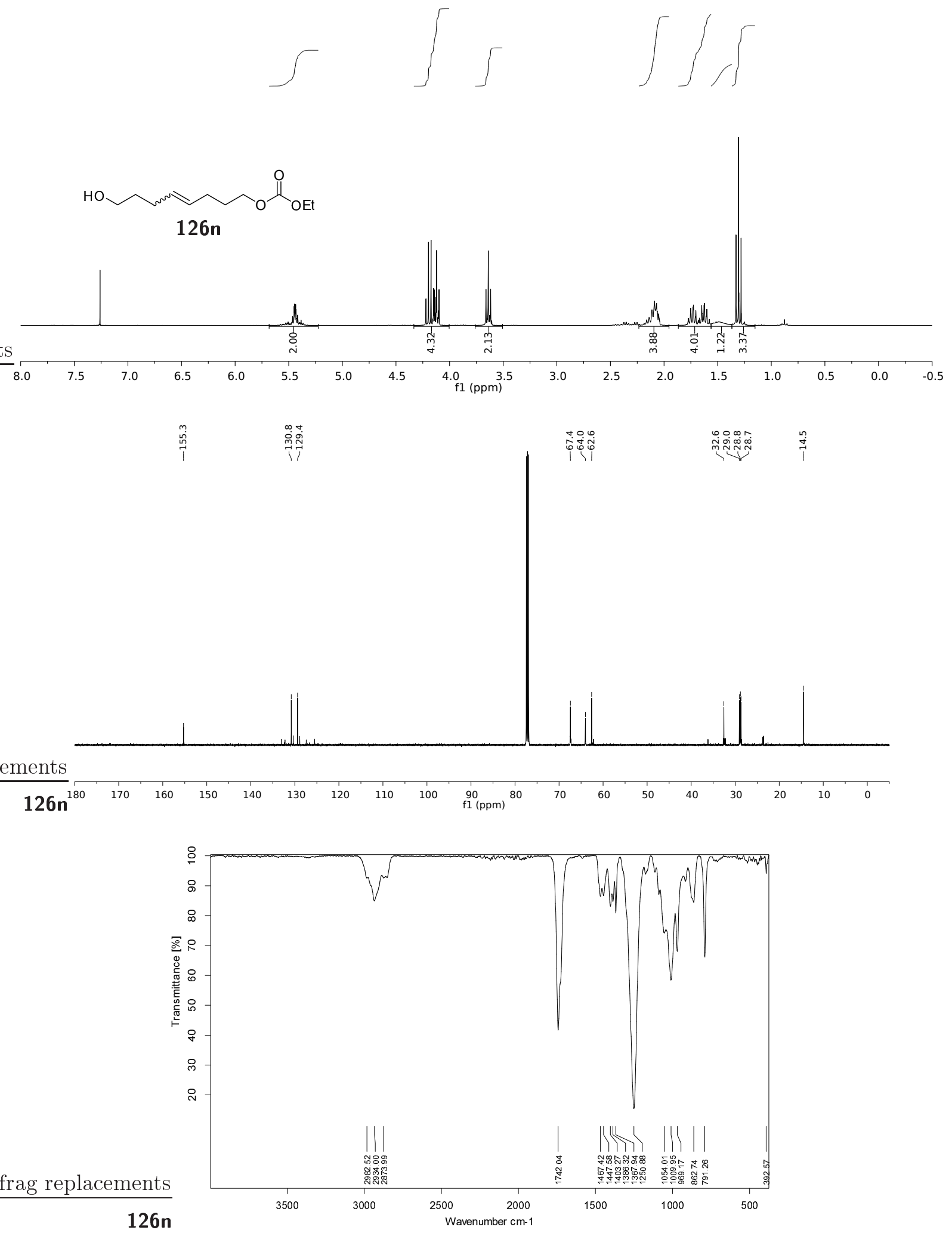



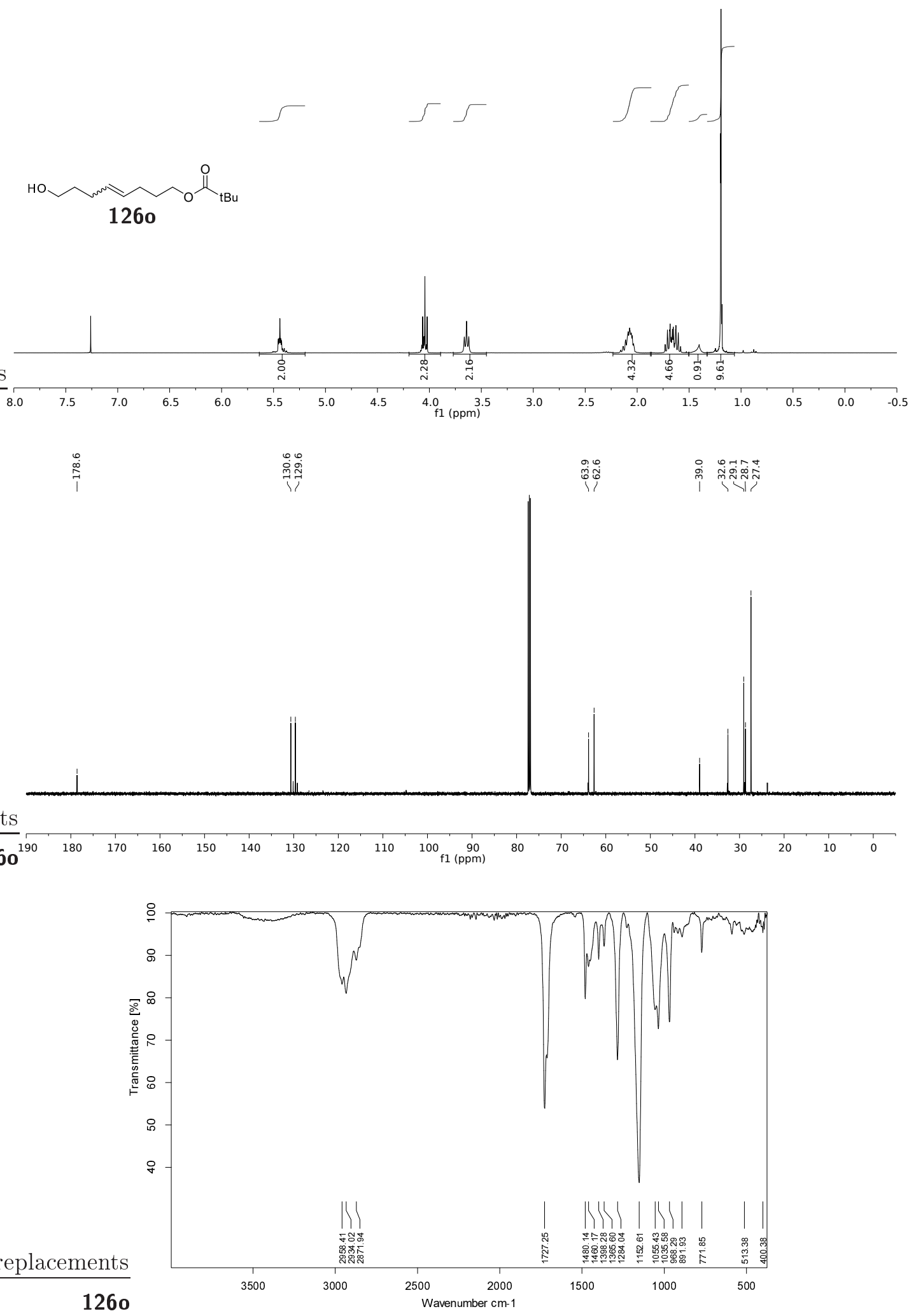

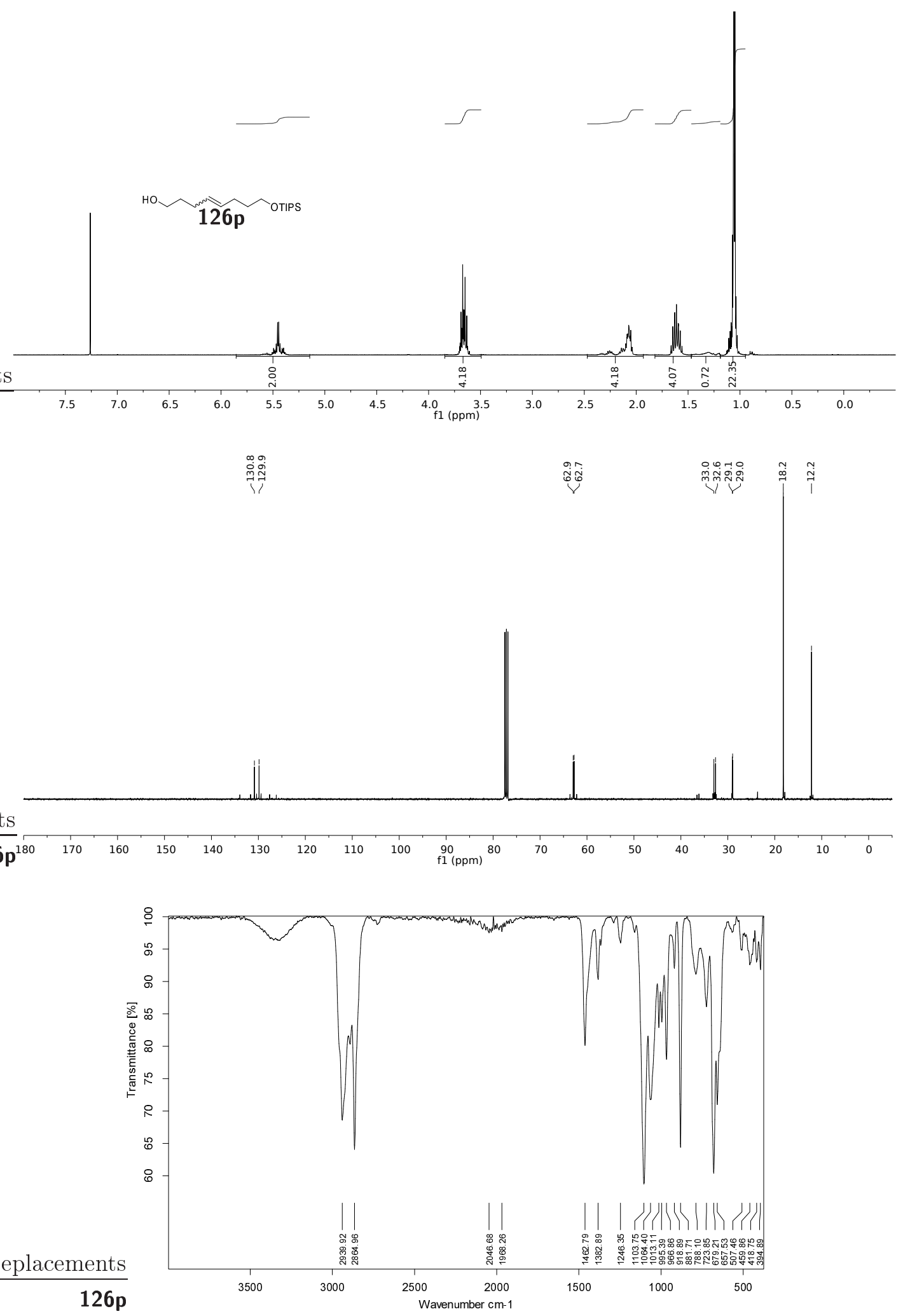

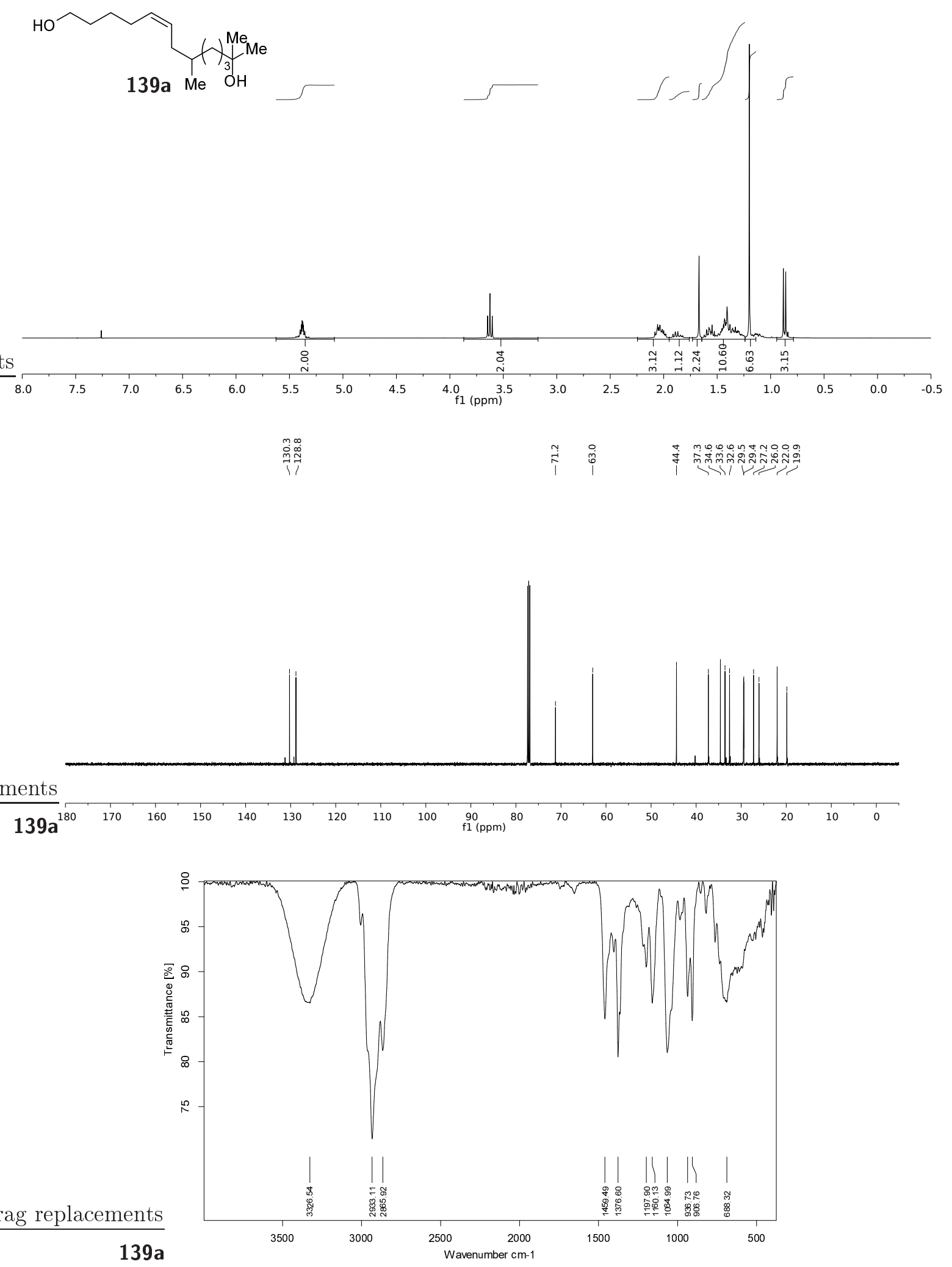

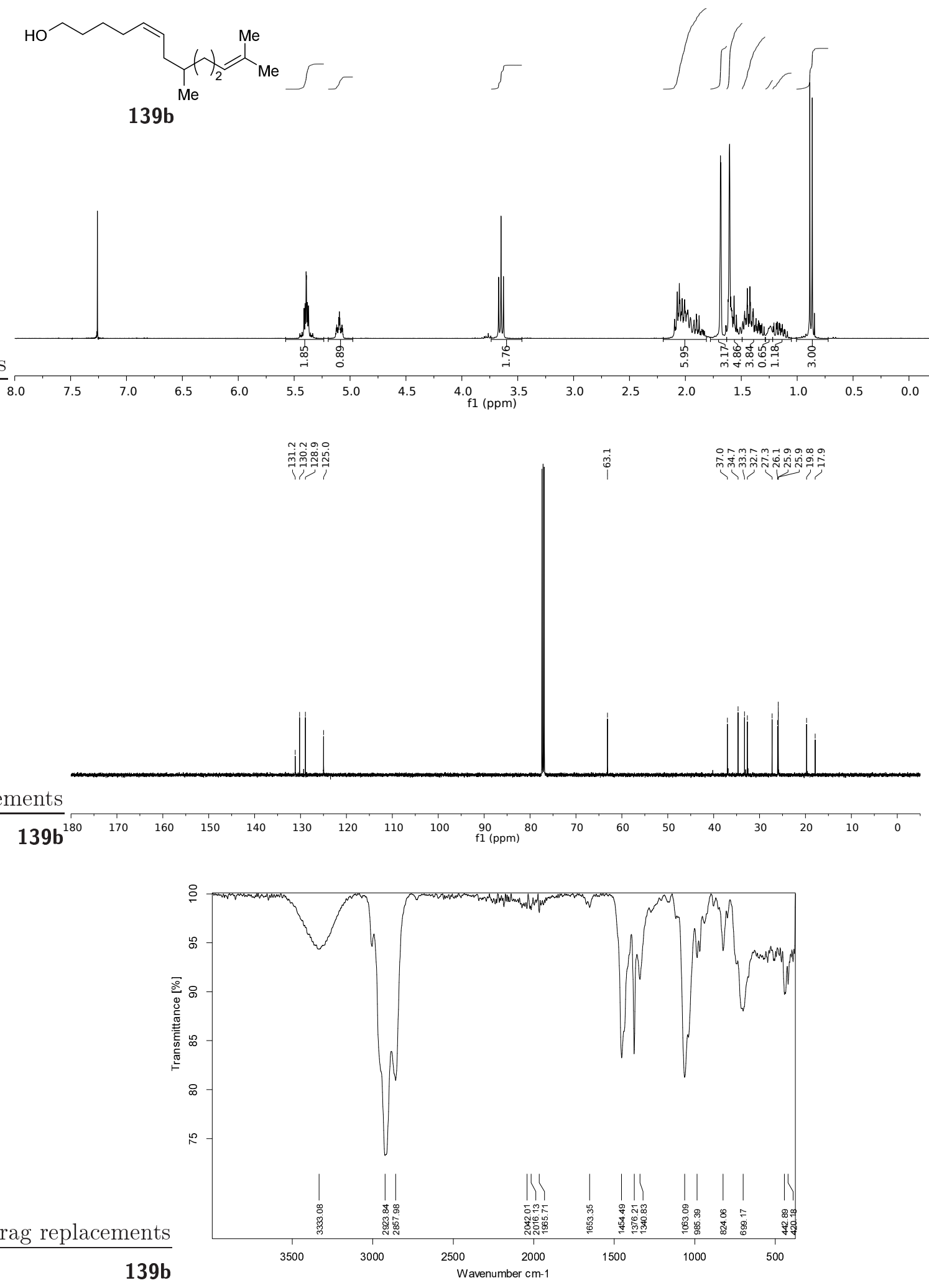


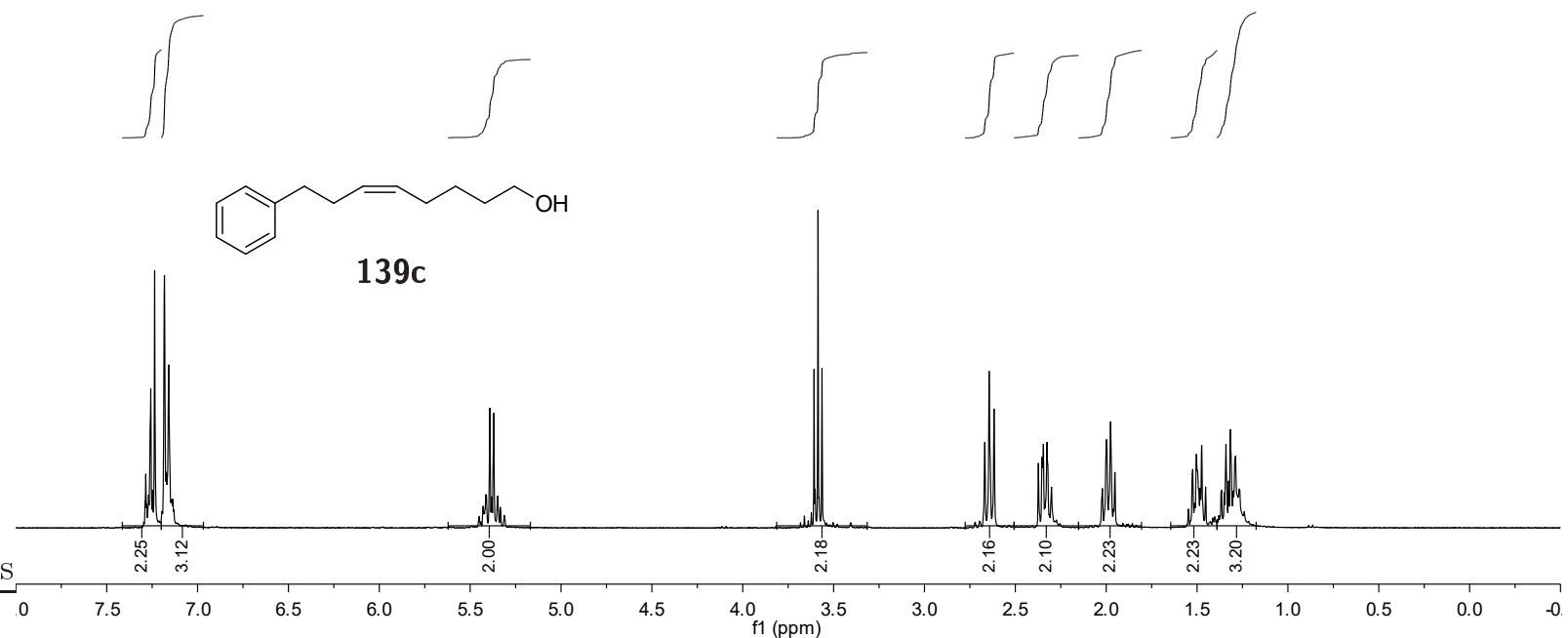

年
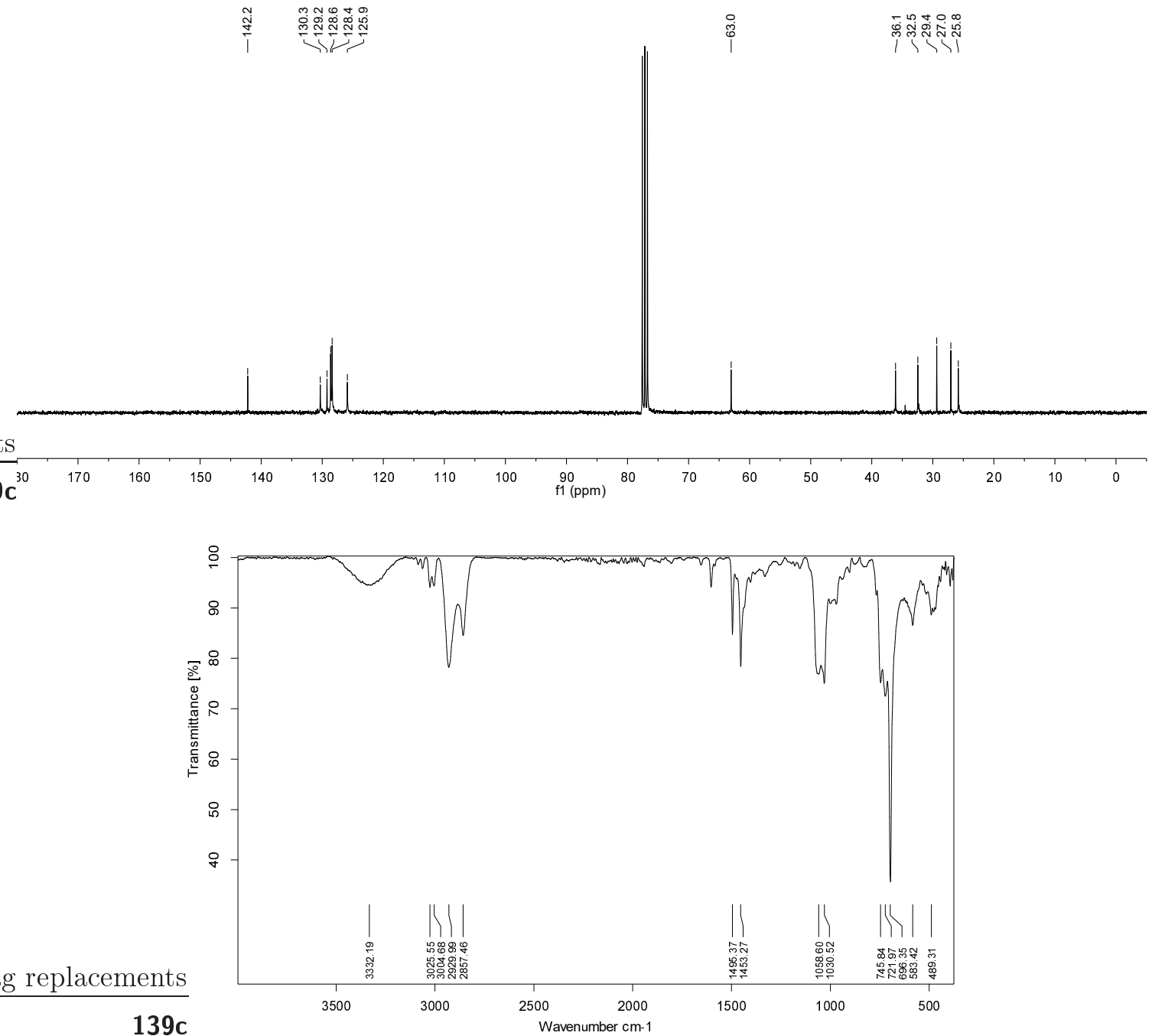


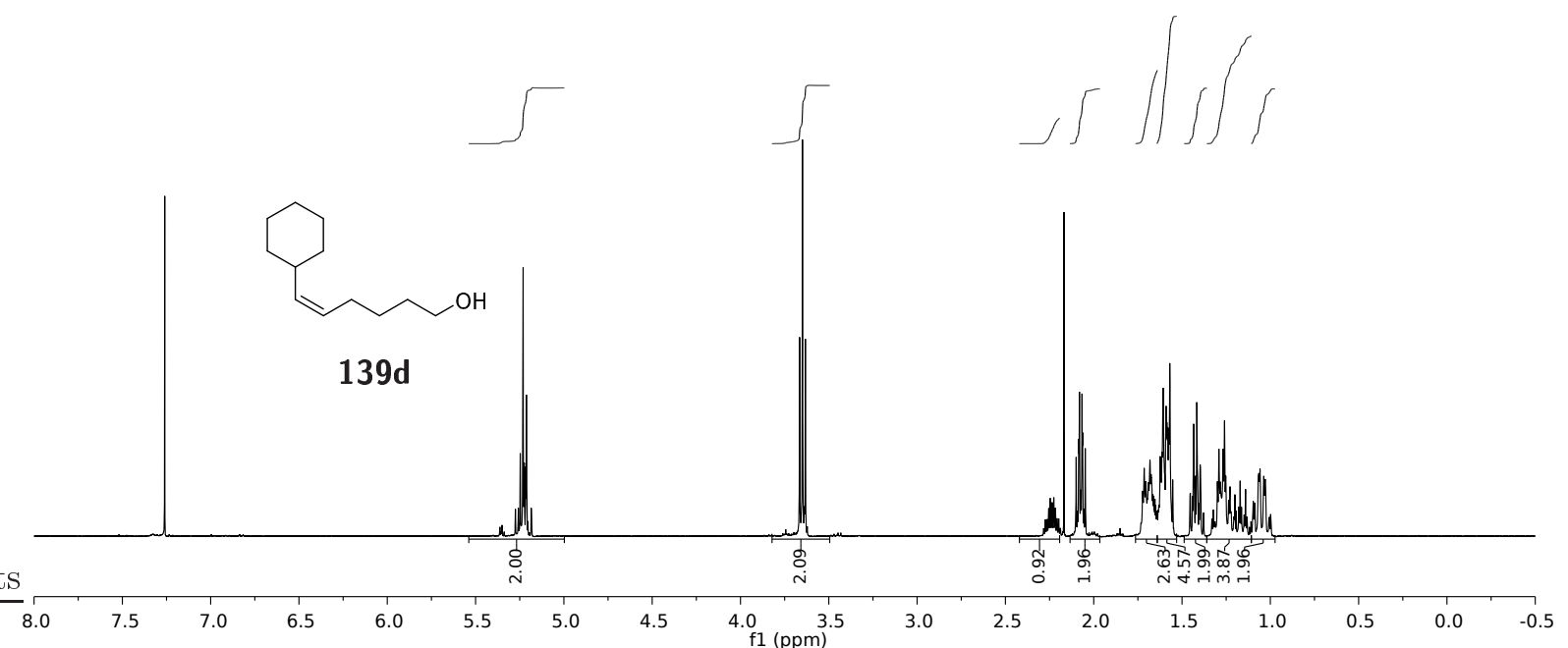

$\begin{array}{ll}0 \\ \stackrel{0}{0} & \stackrel{0}{7} \\ 1 & 1\end{array}$

)

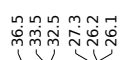
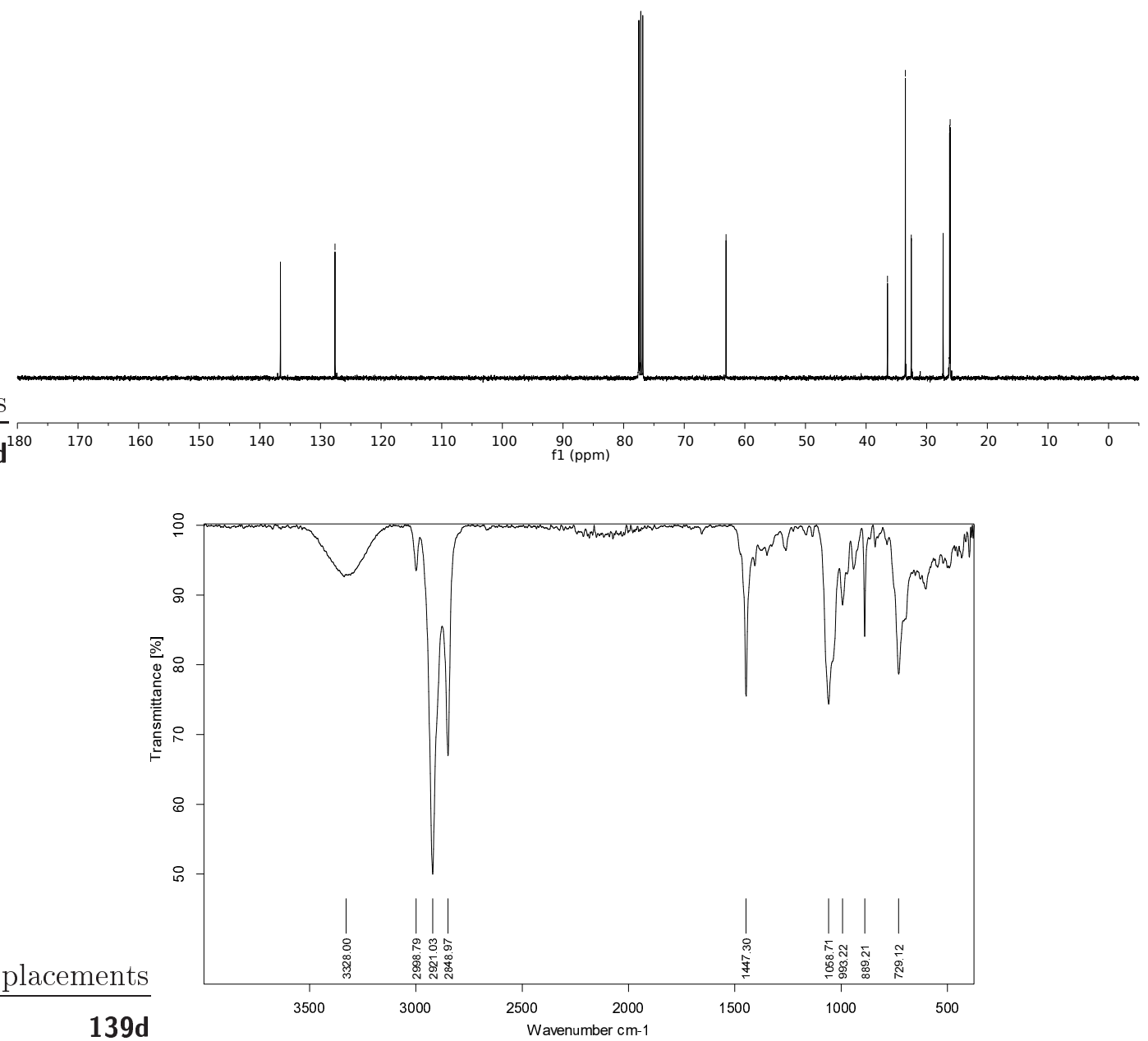


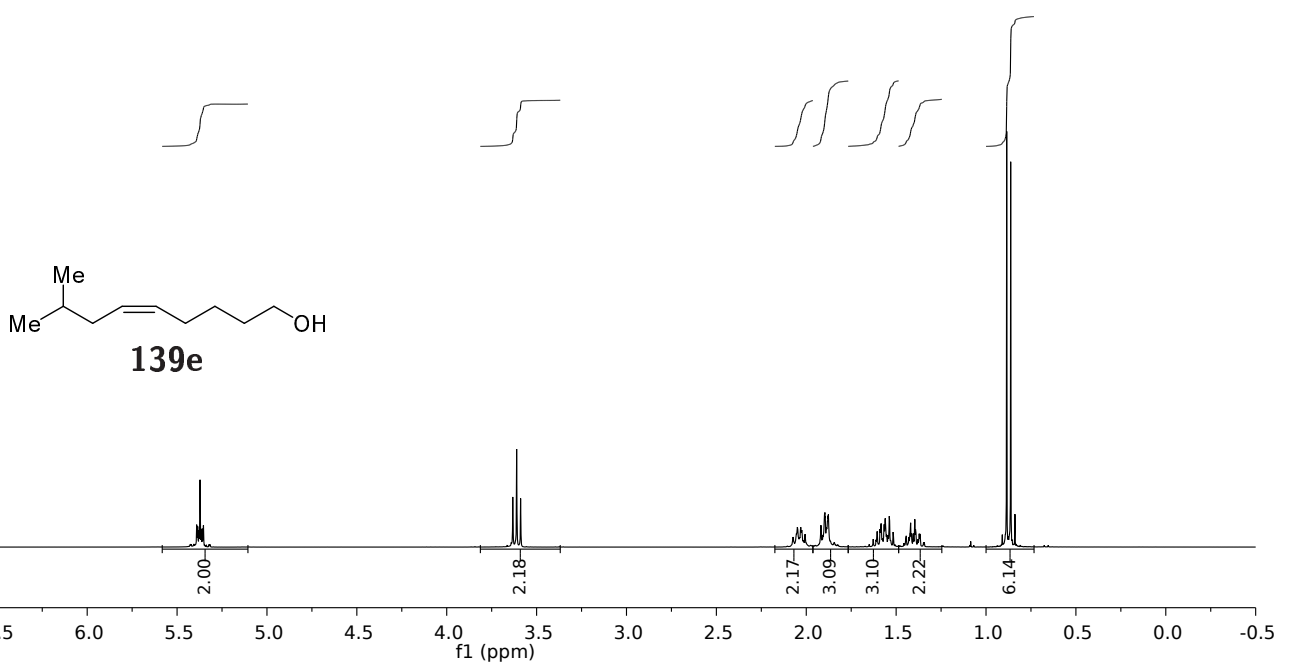

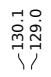
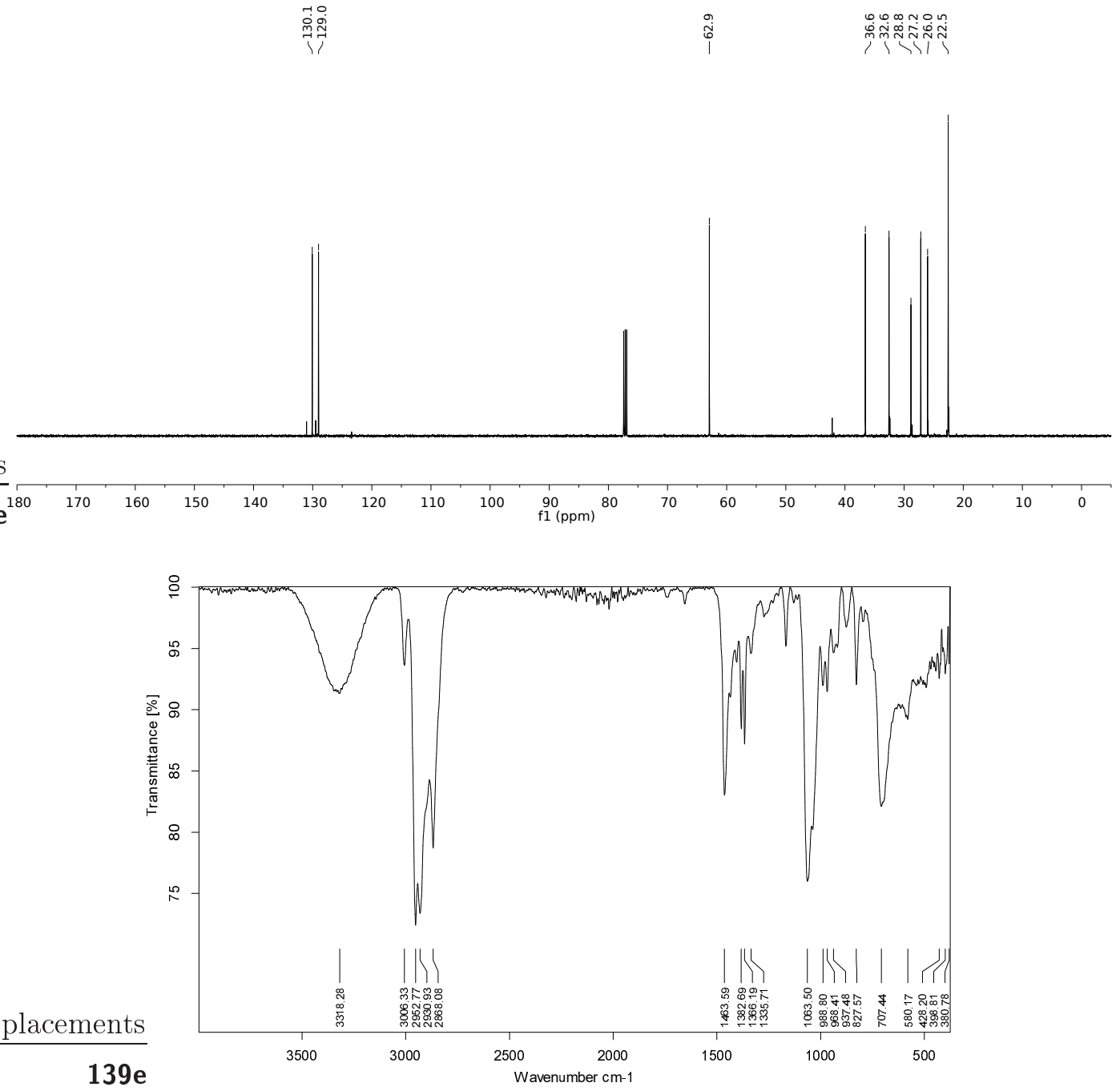

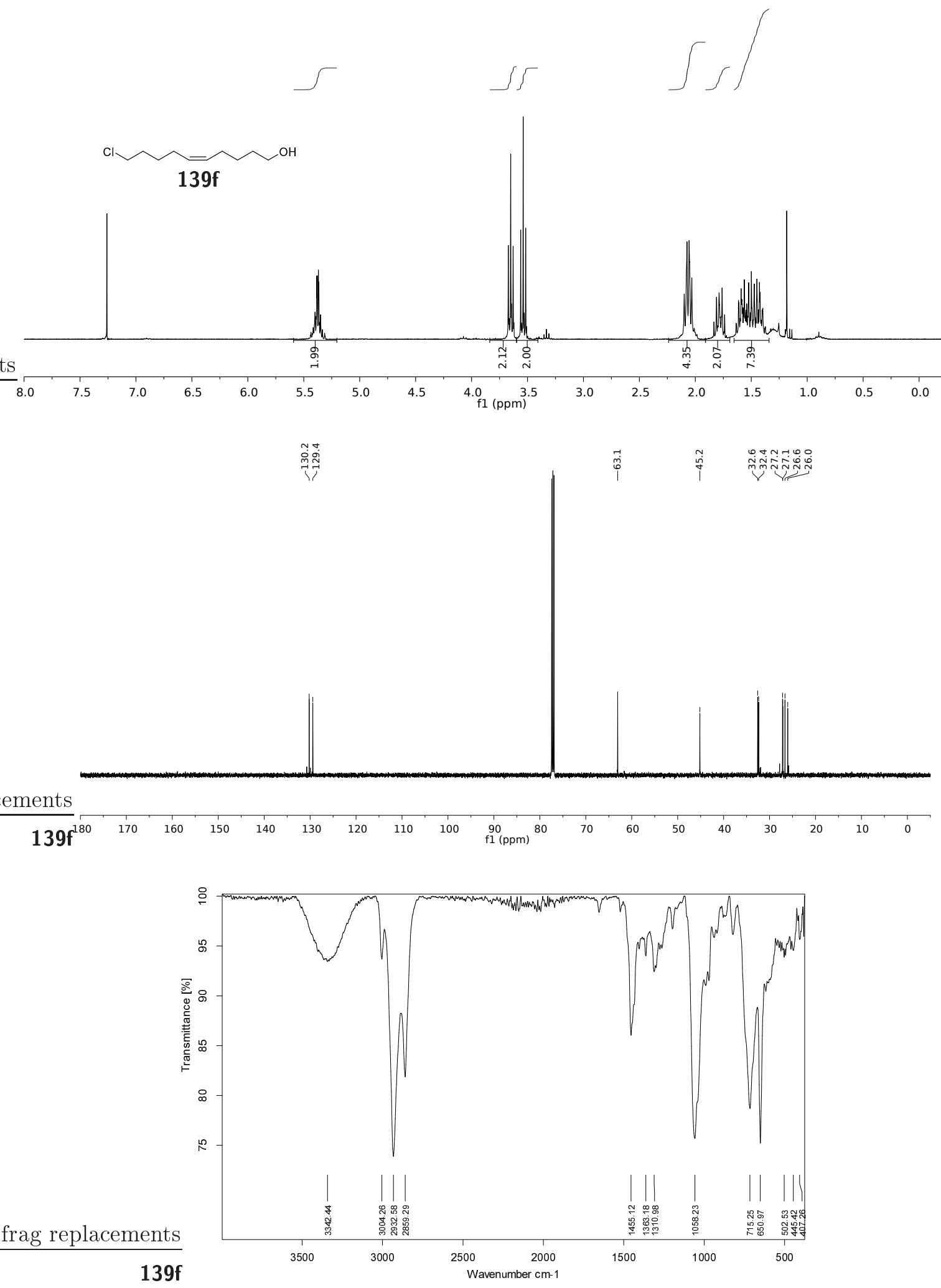

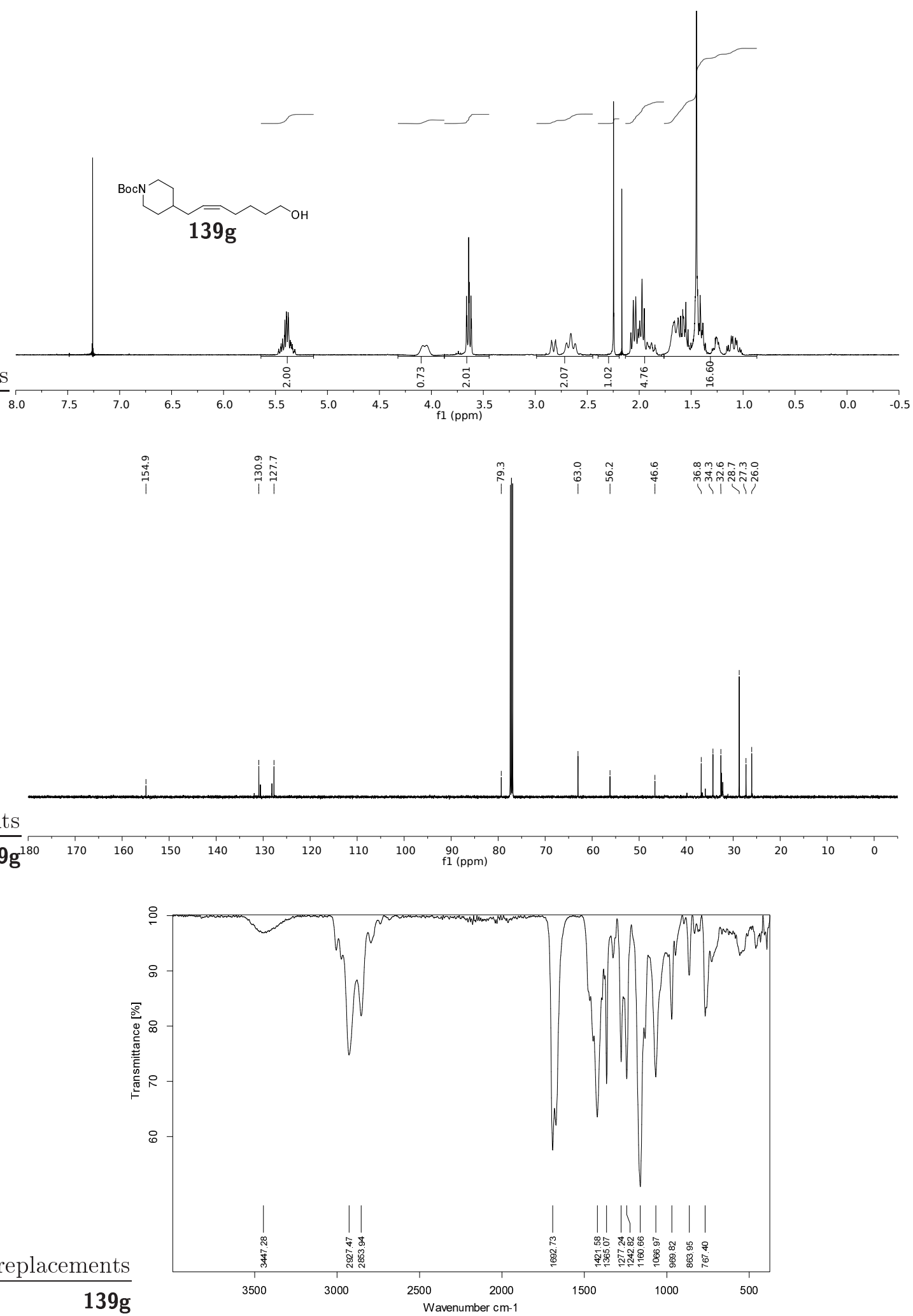

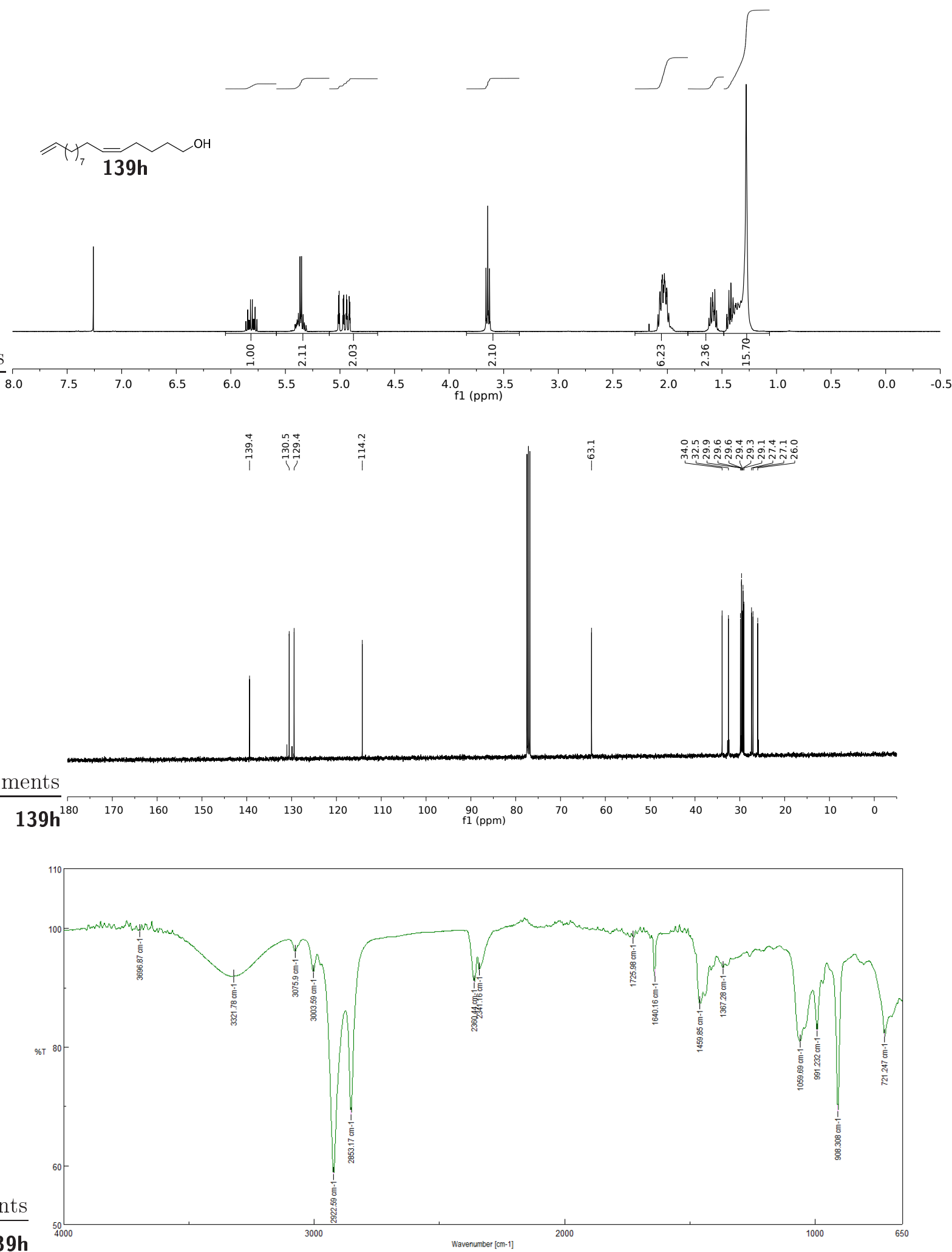

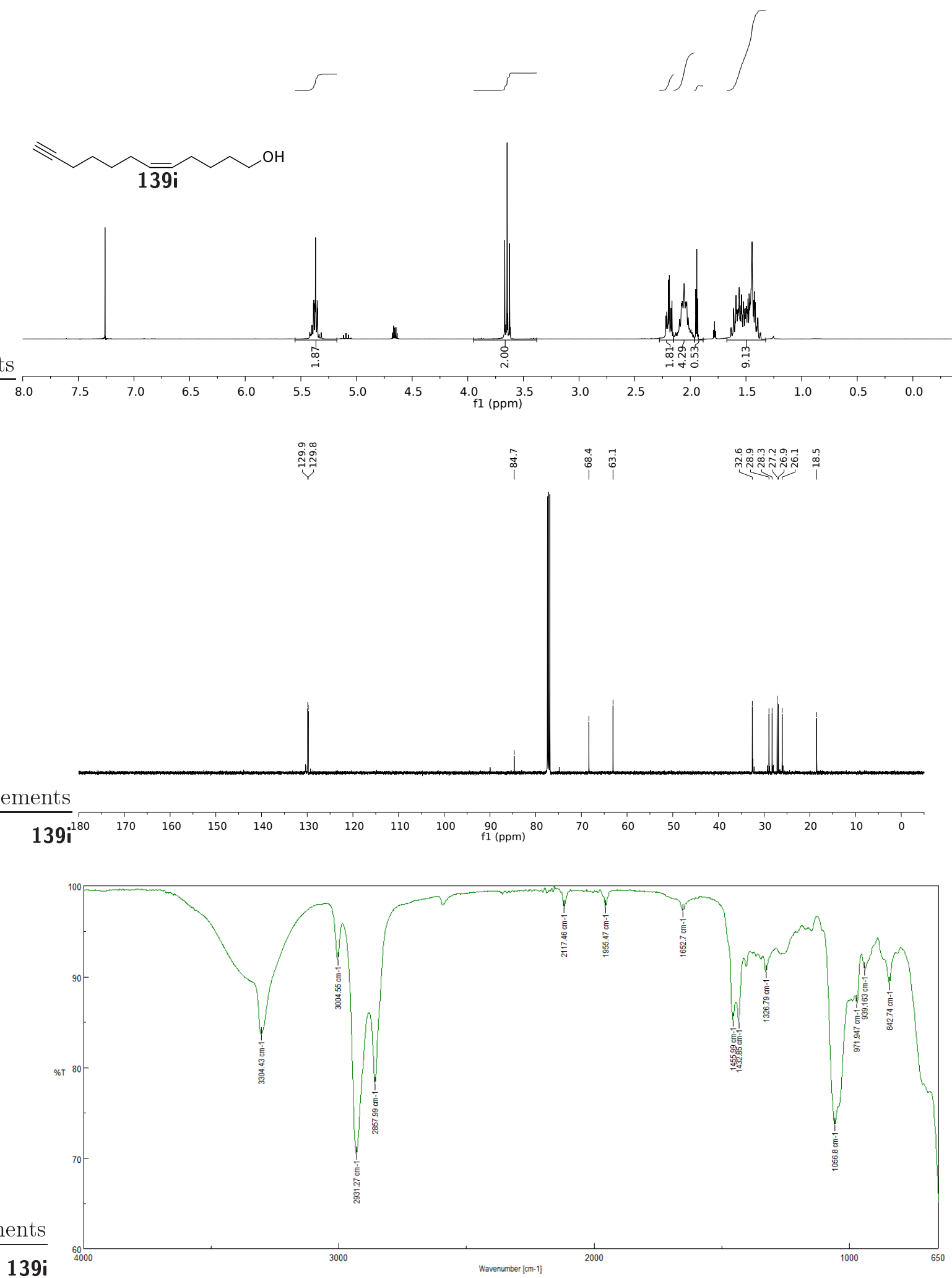

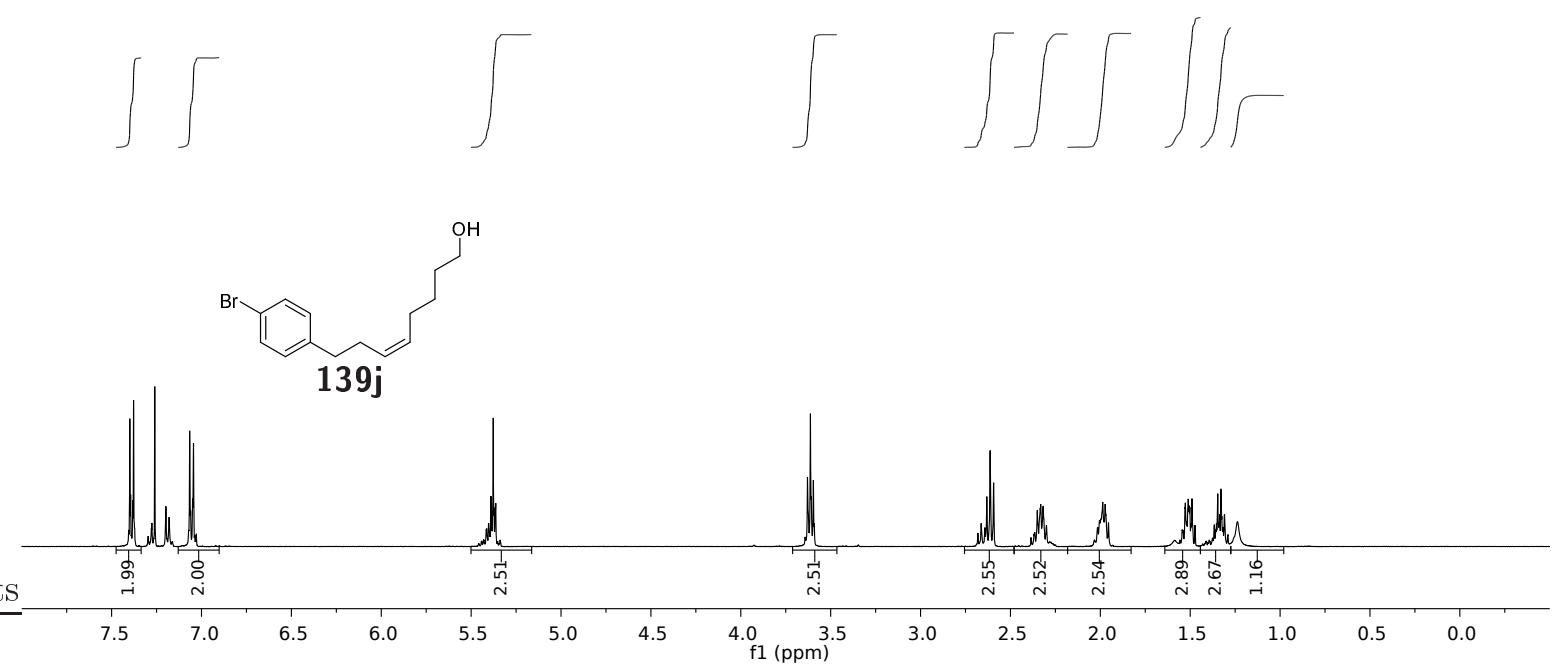

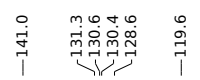

i

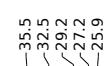
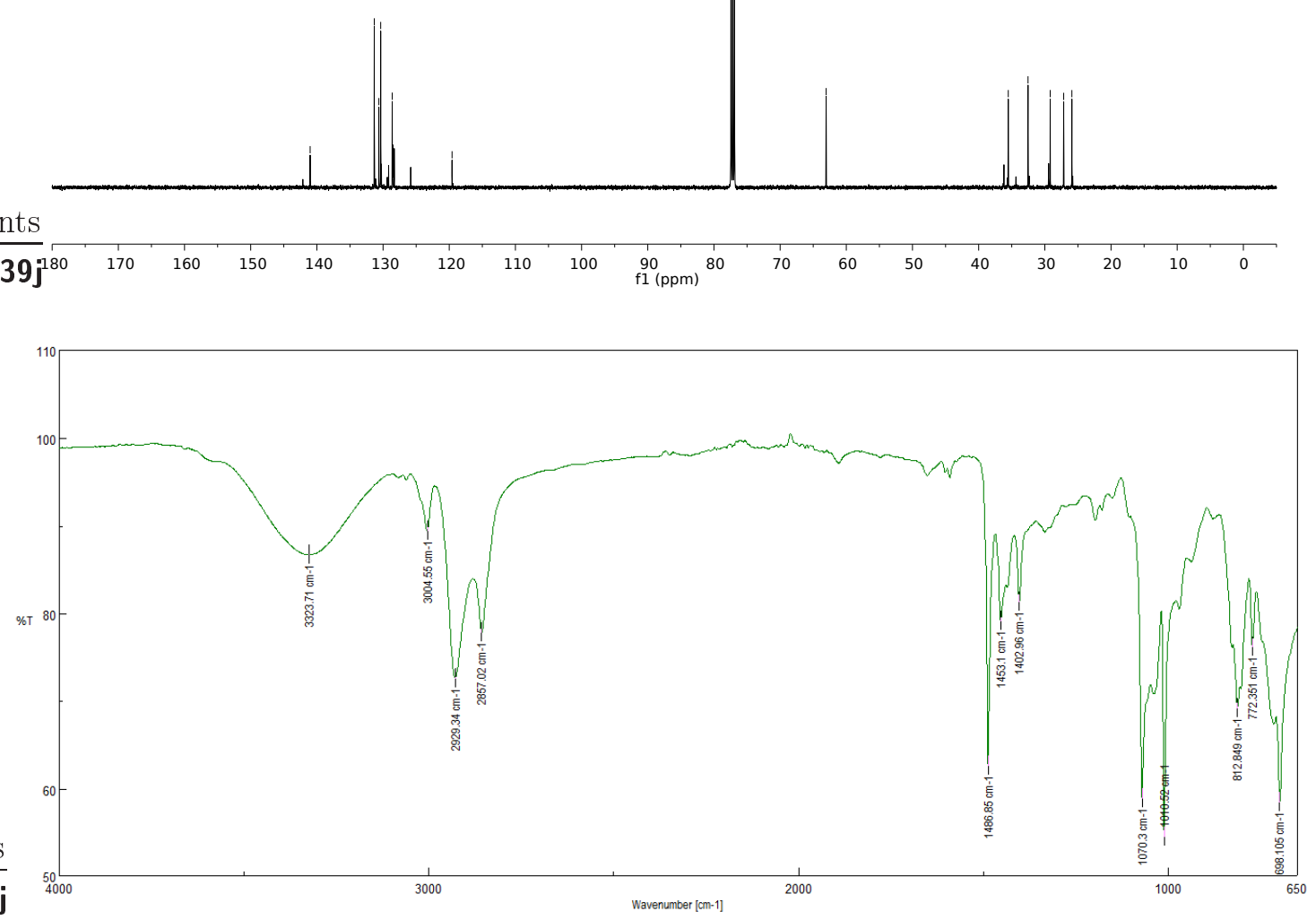


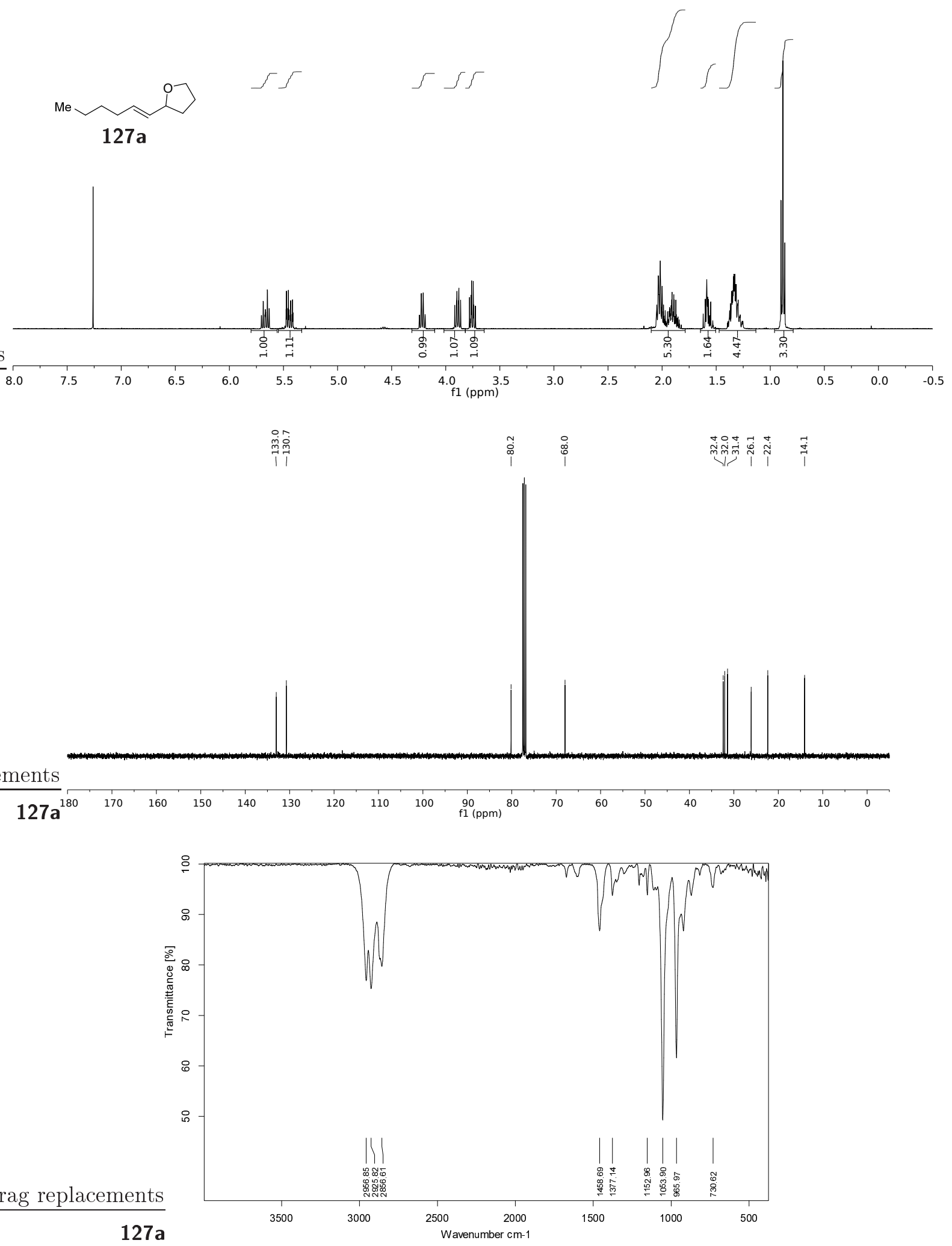



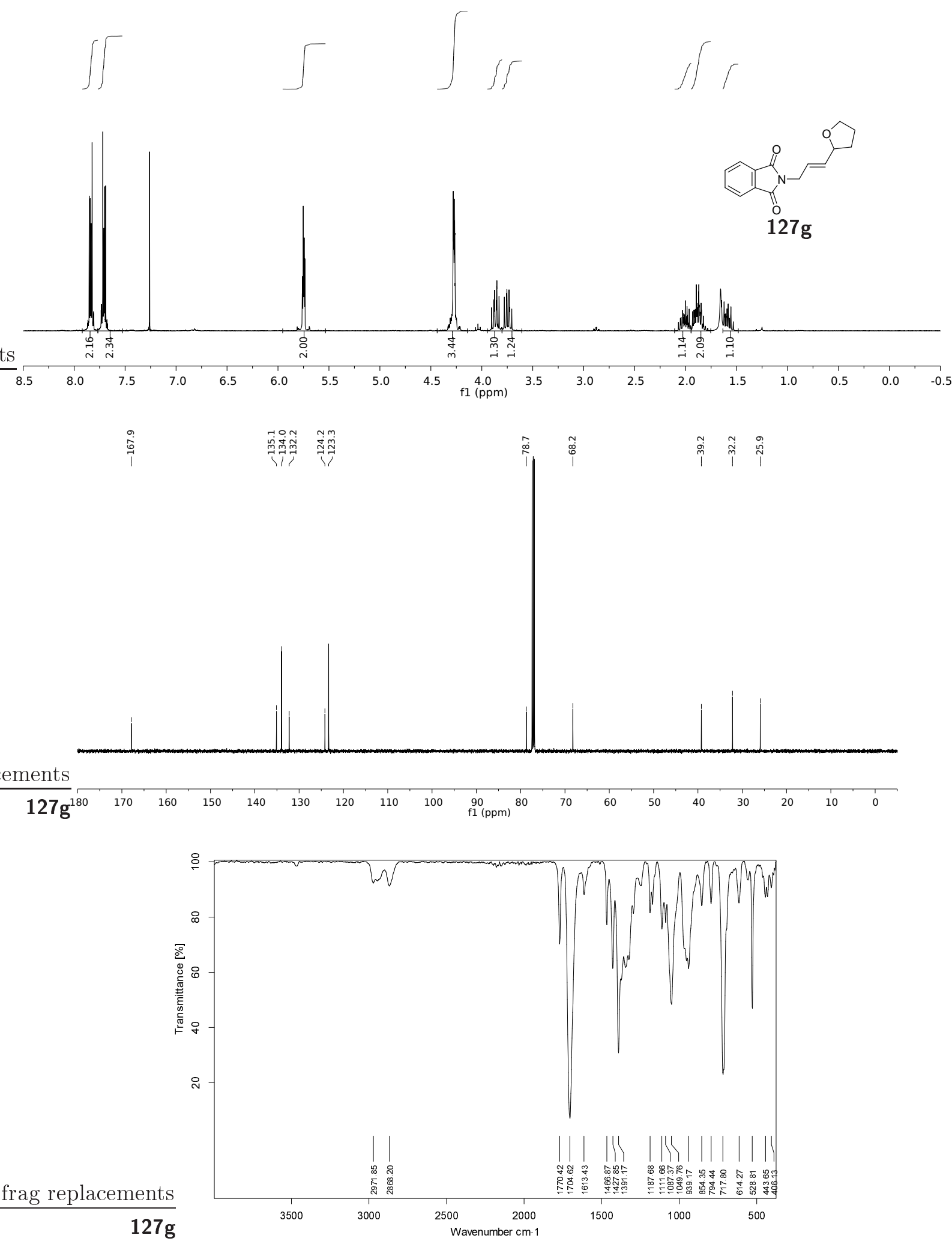


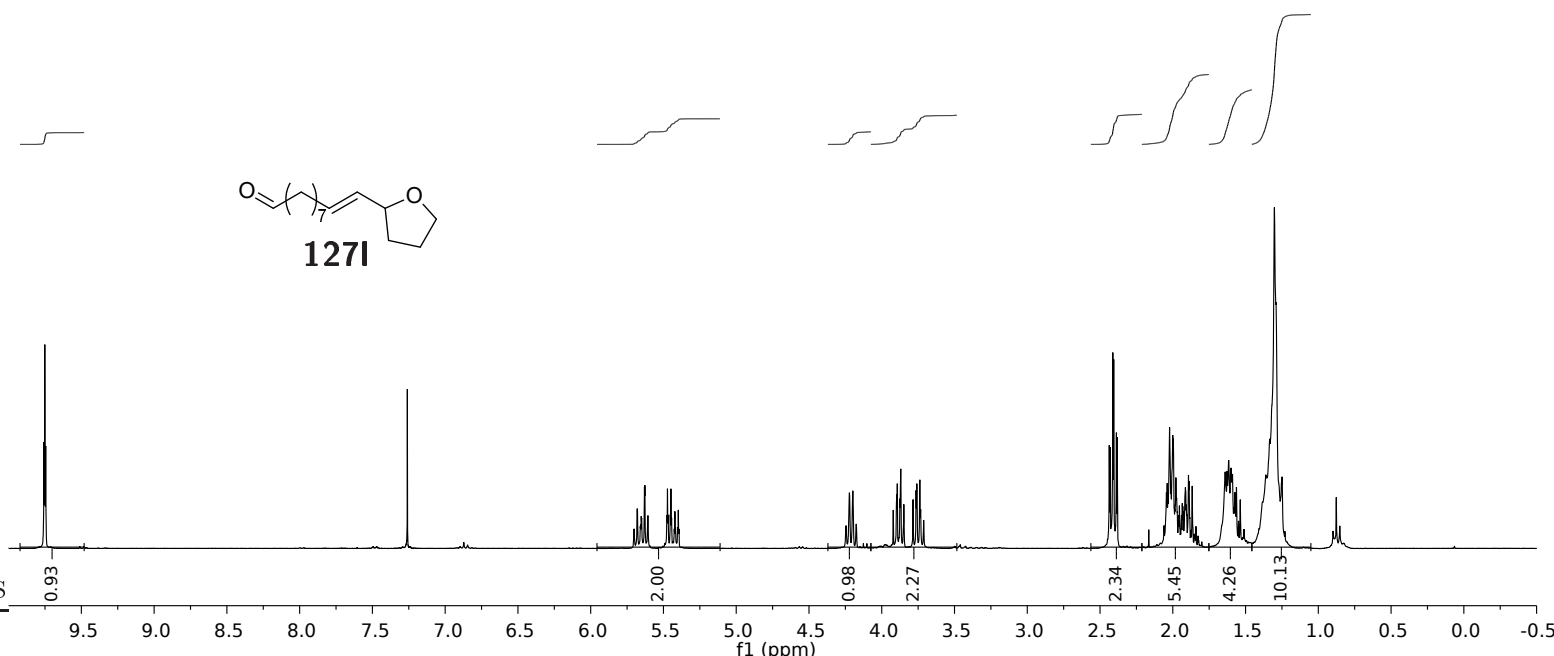

$\stackrel{\infty}{\stackrel{\sim}{N}}$

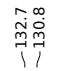

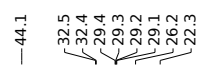
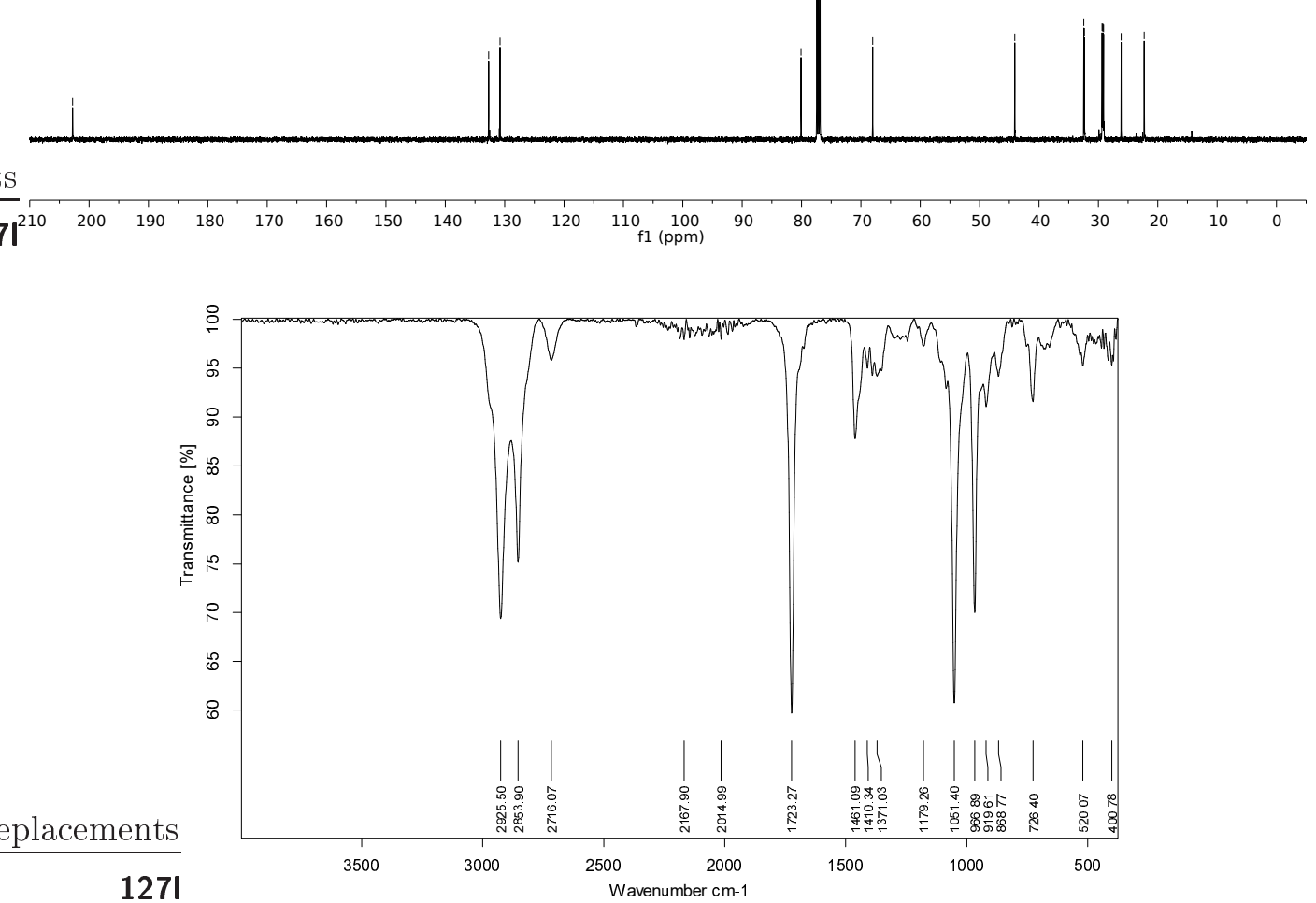

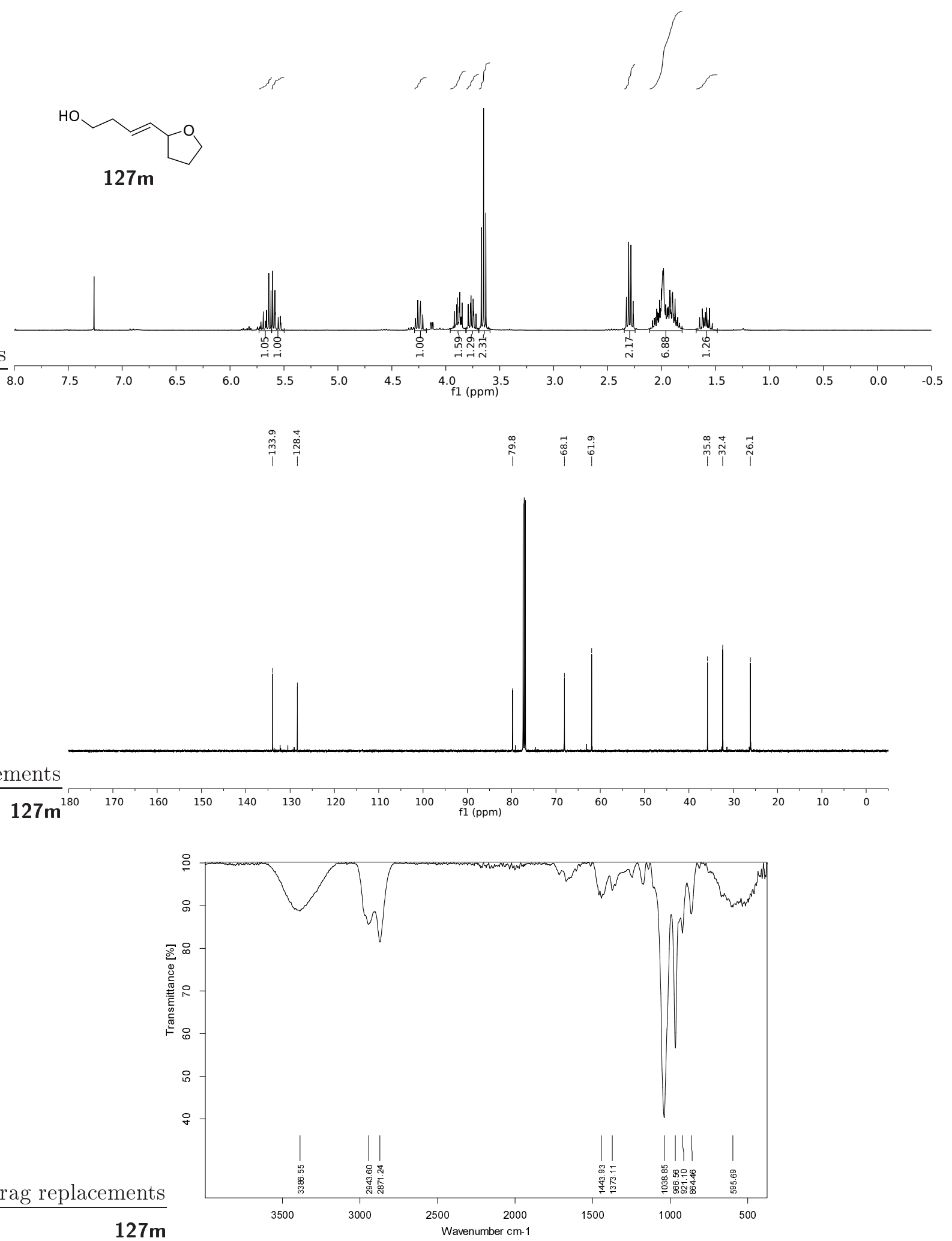


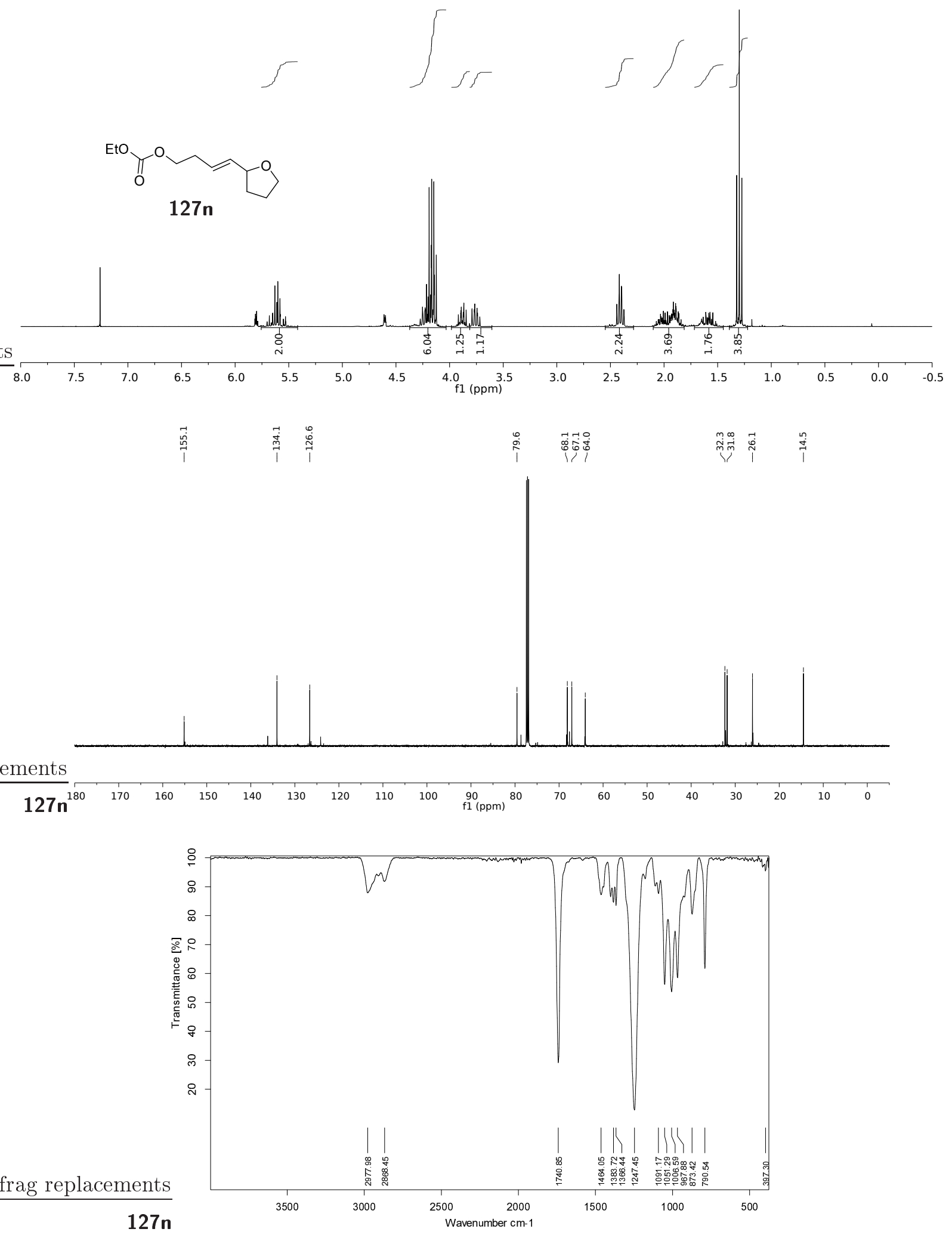



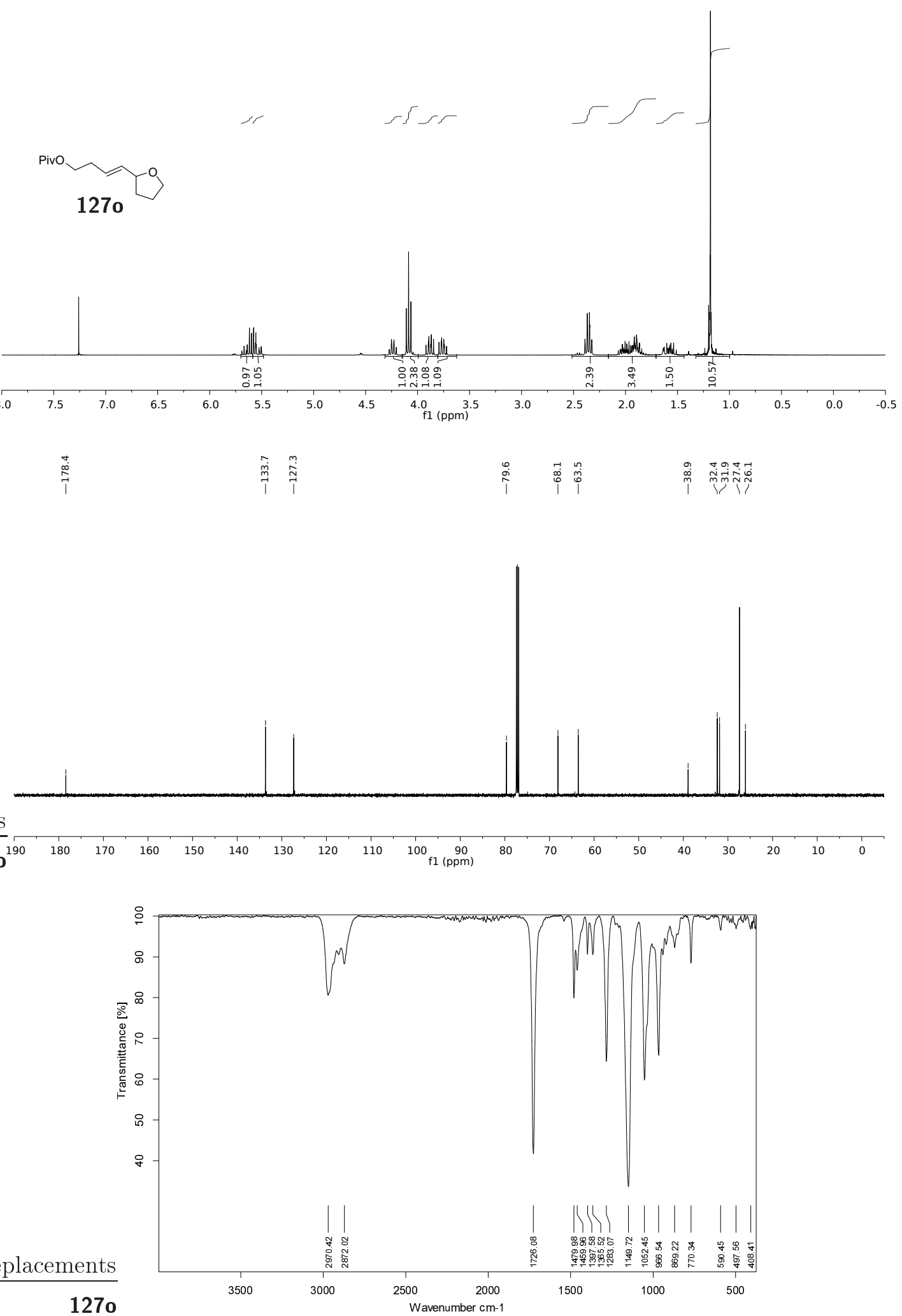

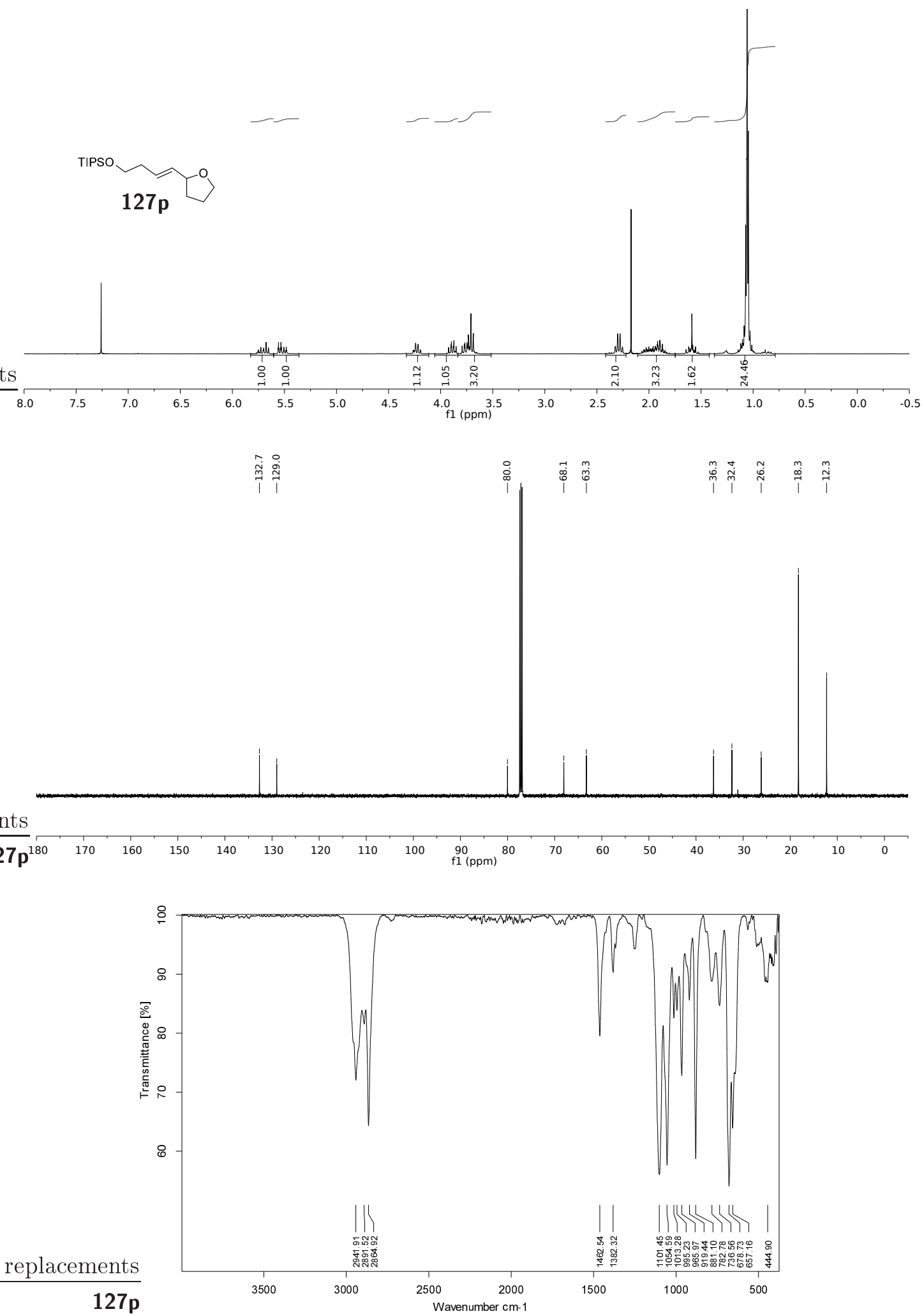


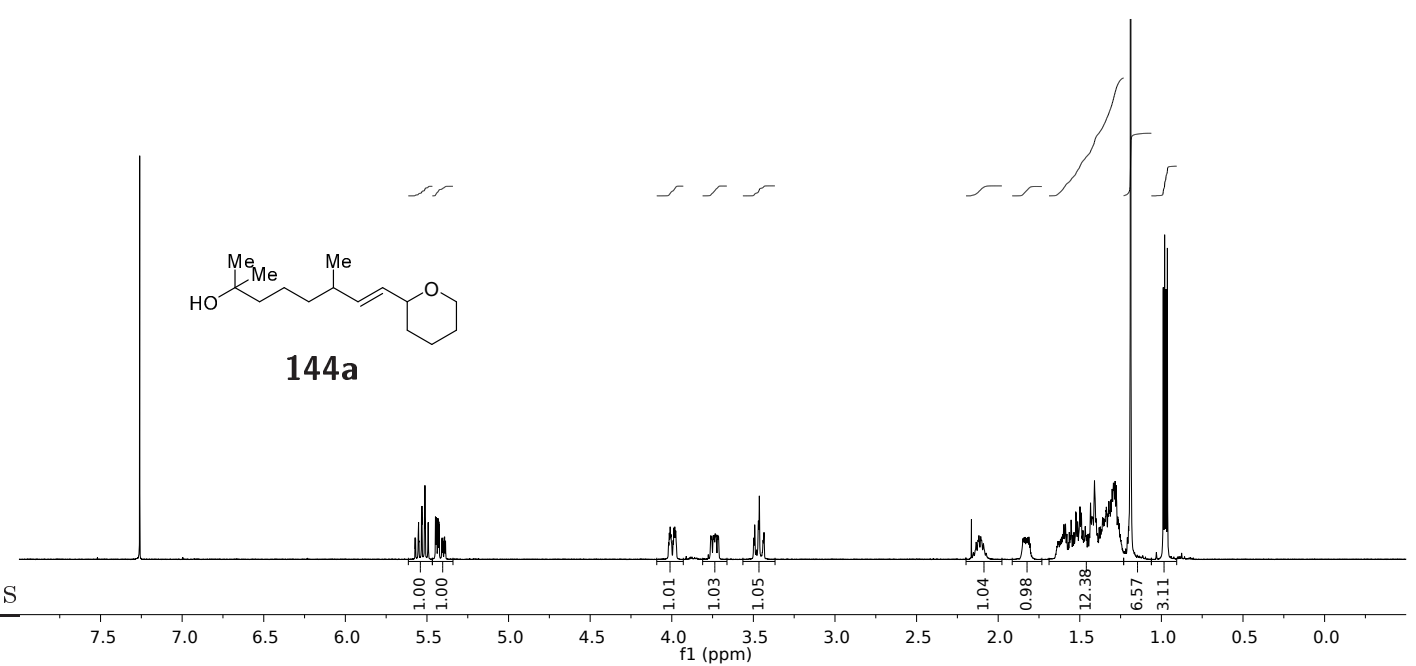

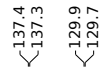

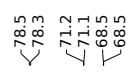

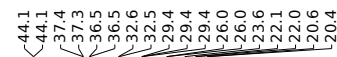
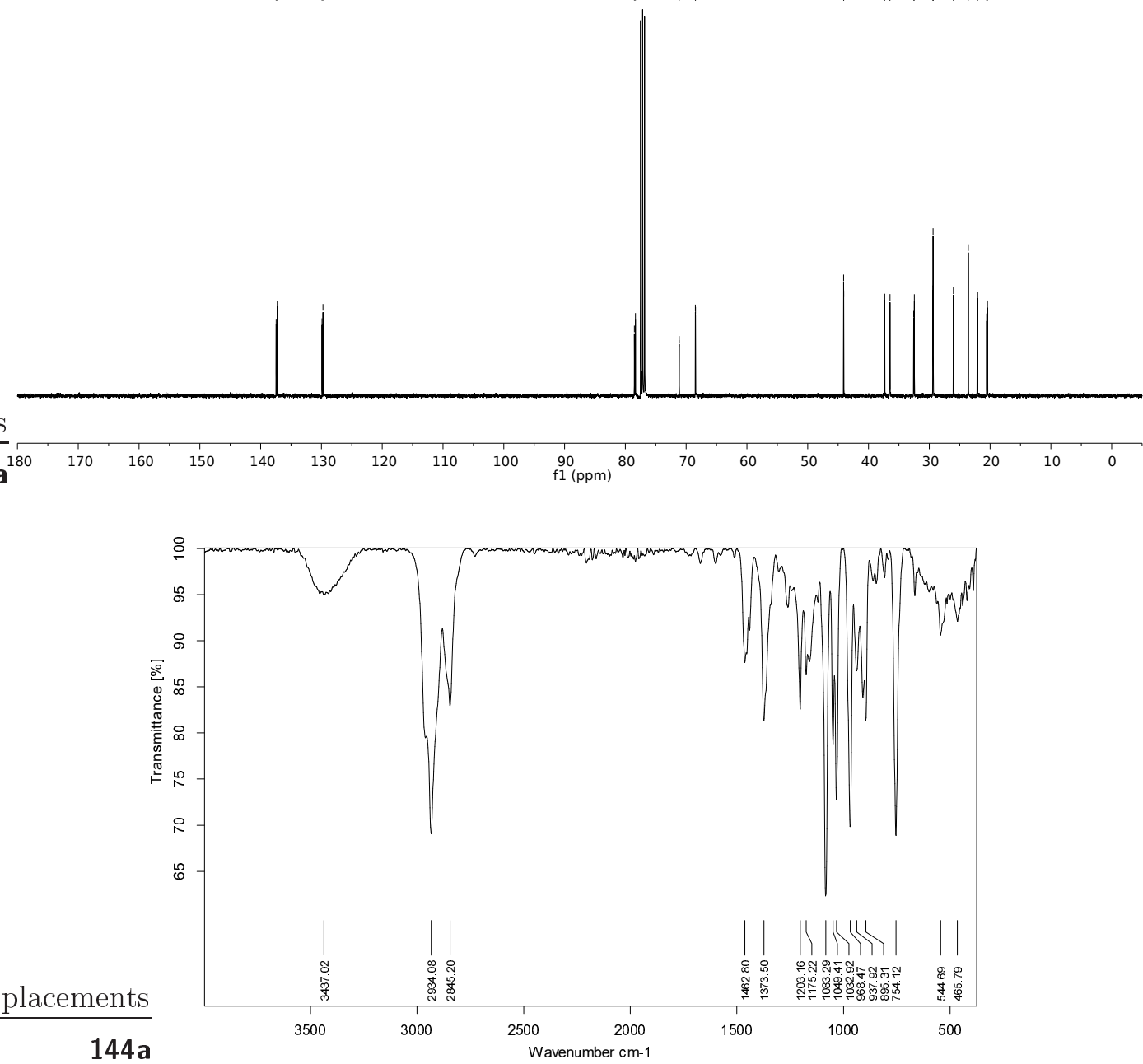


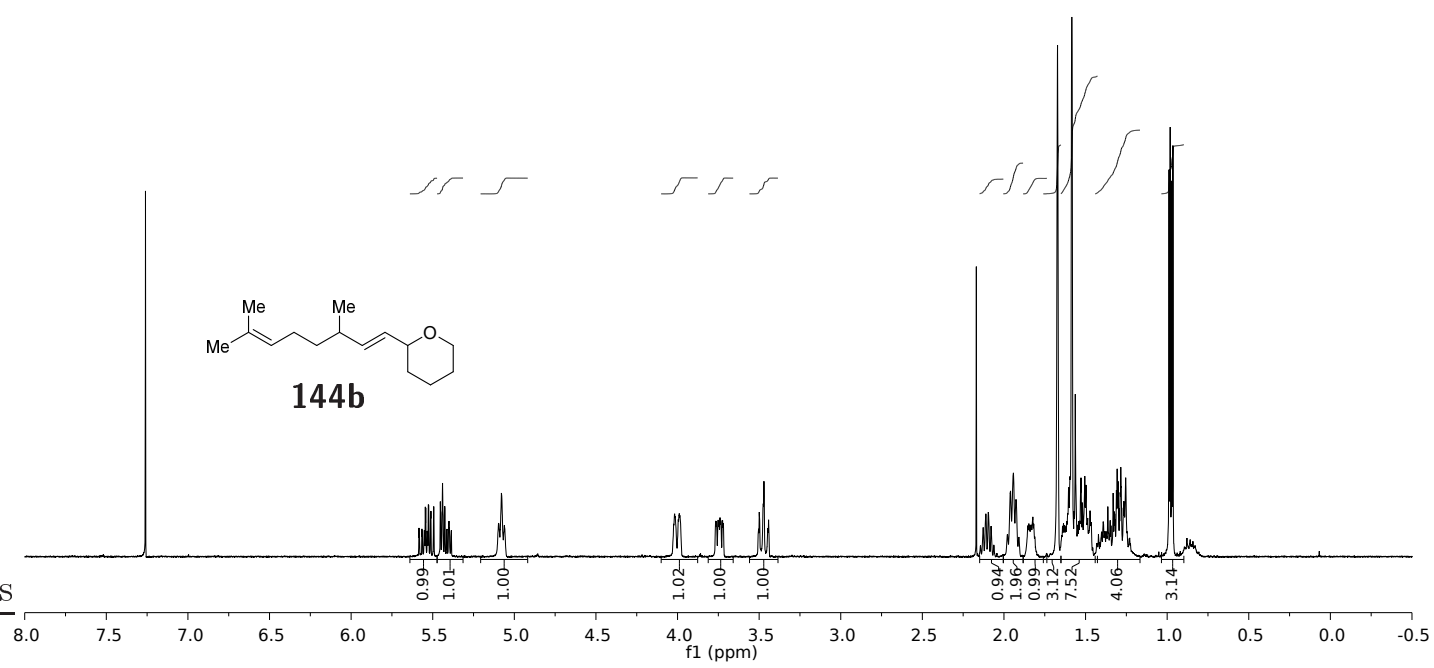

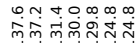
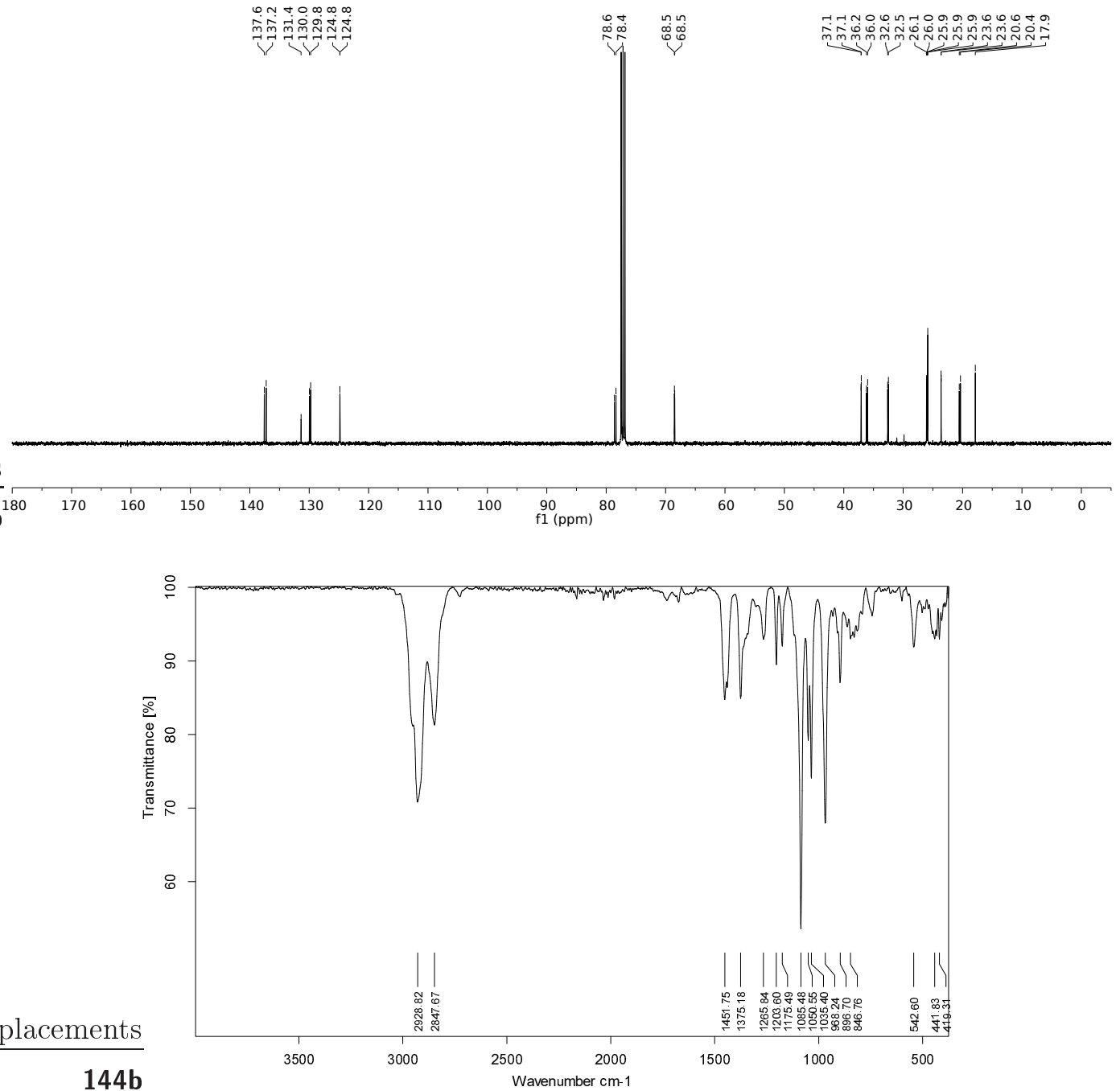

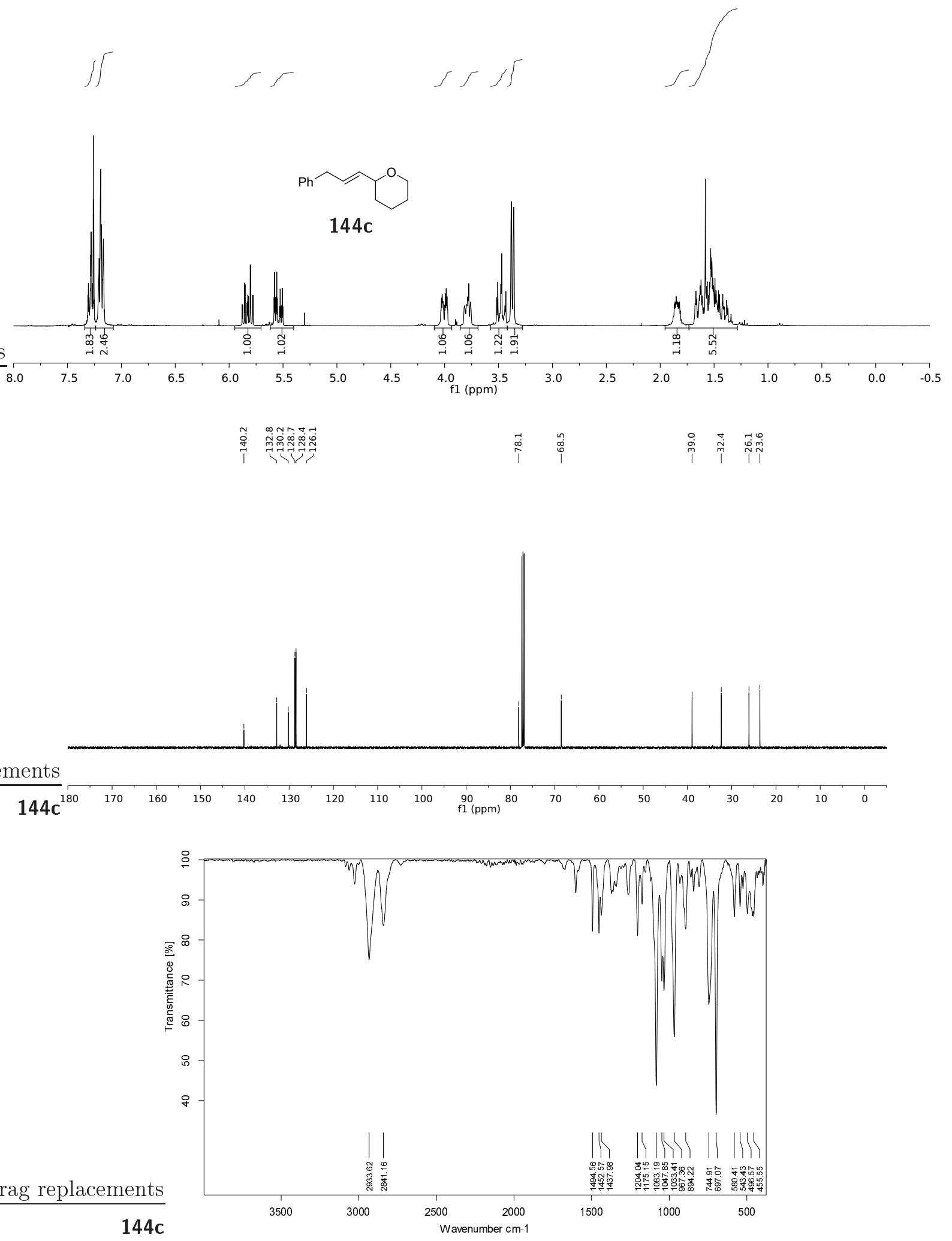

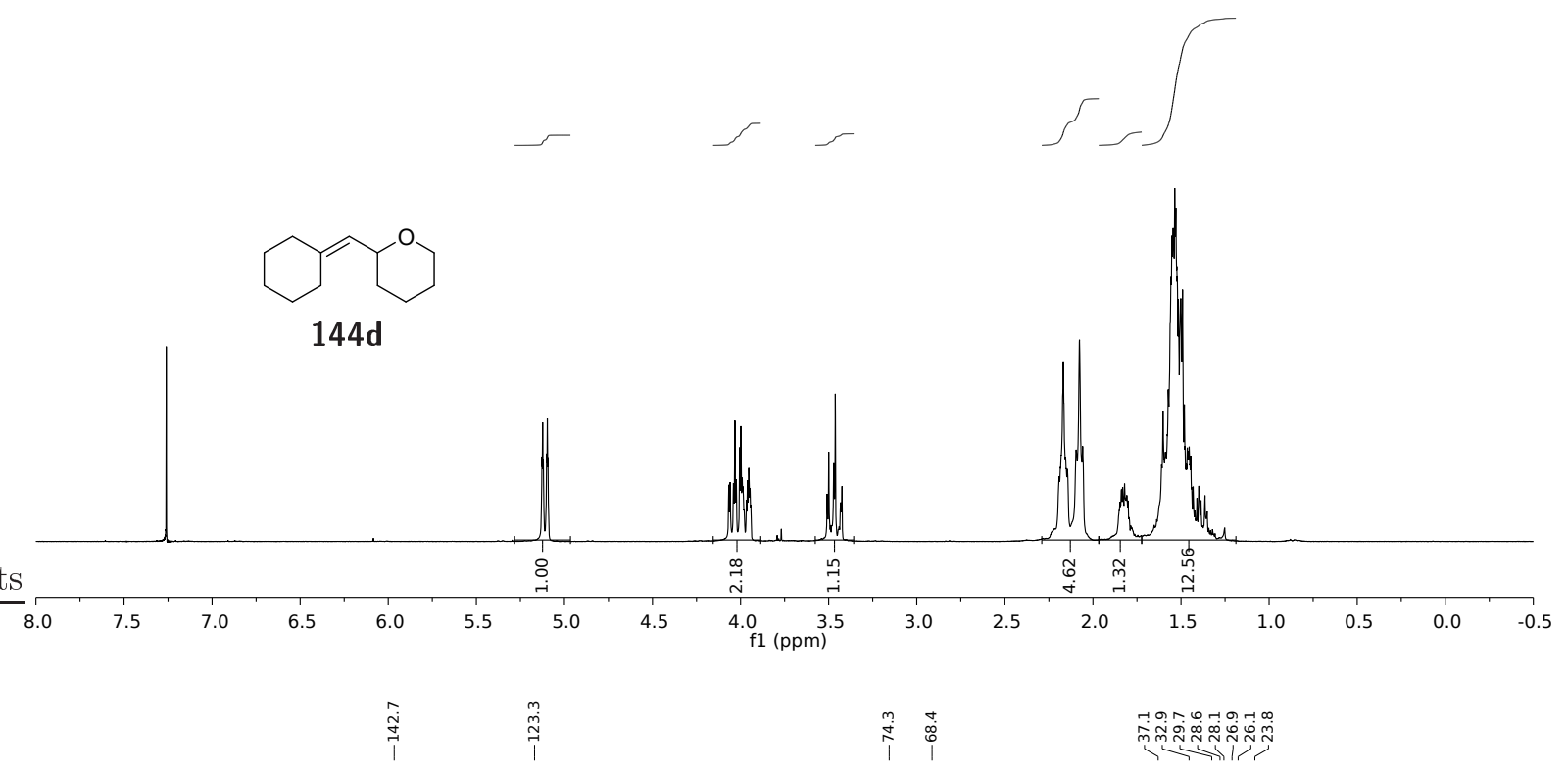

نู
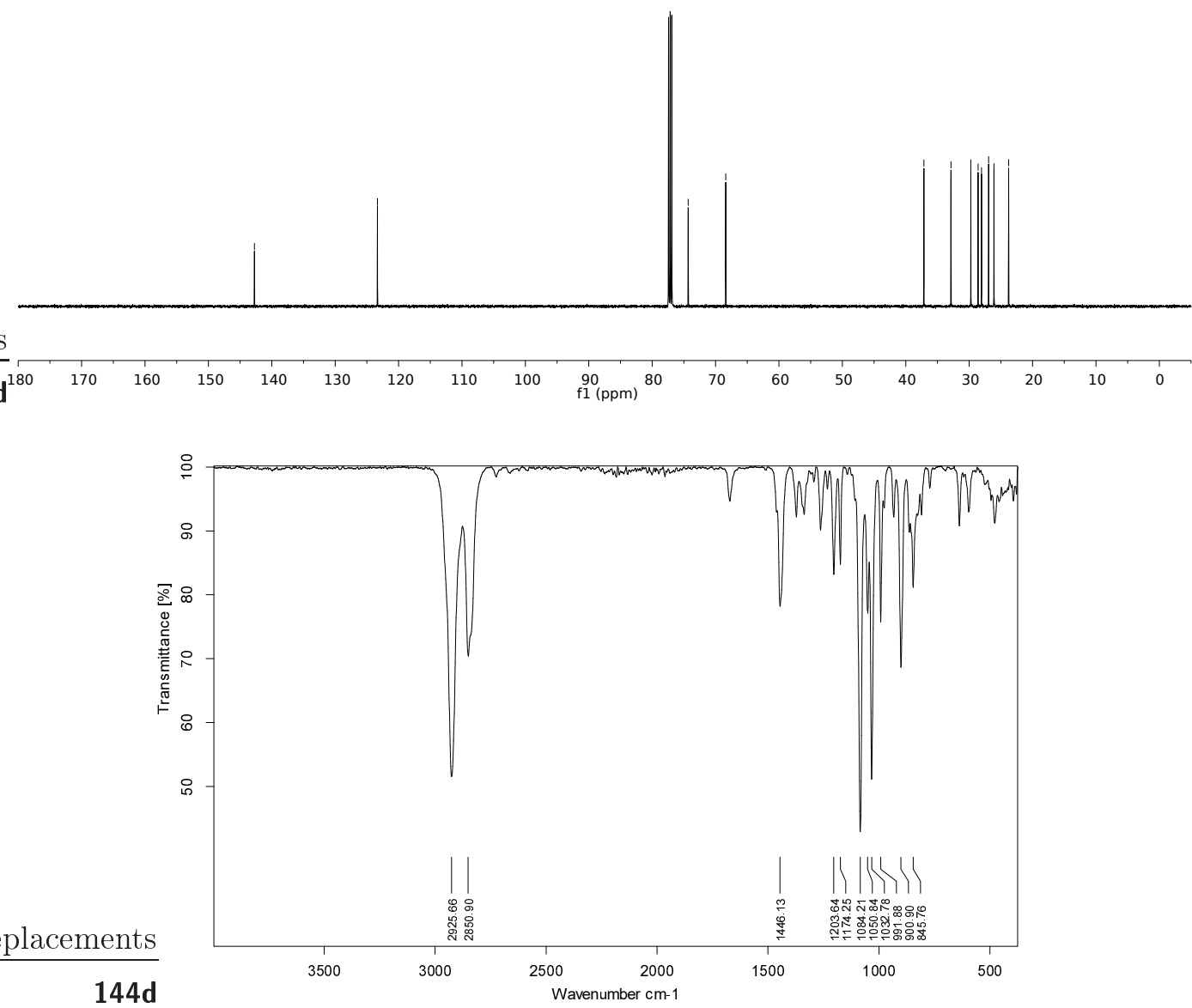


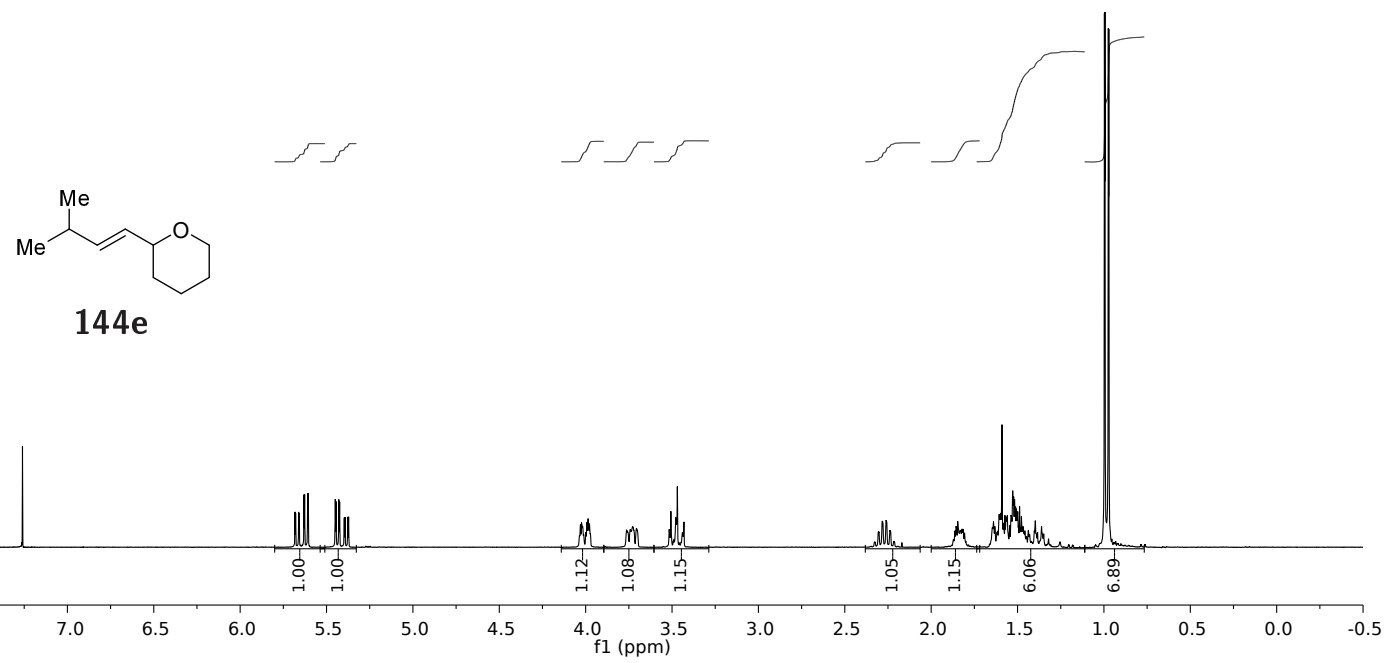

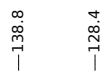

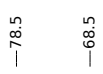

นึำกำ
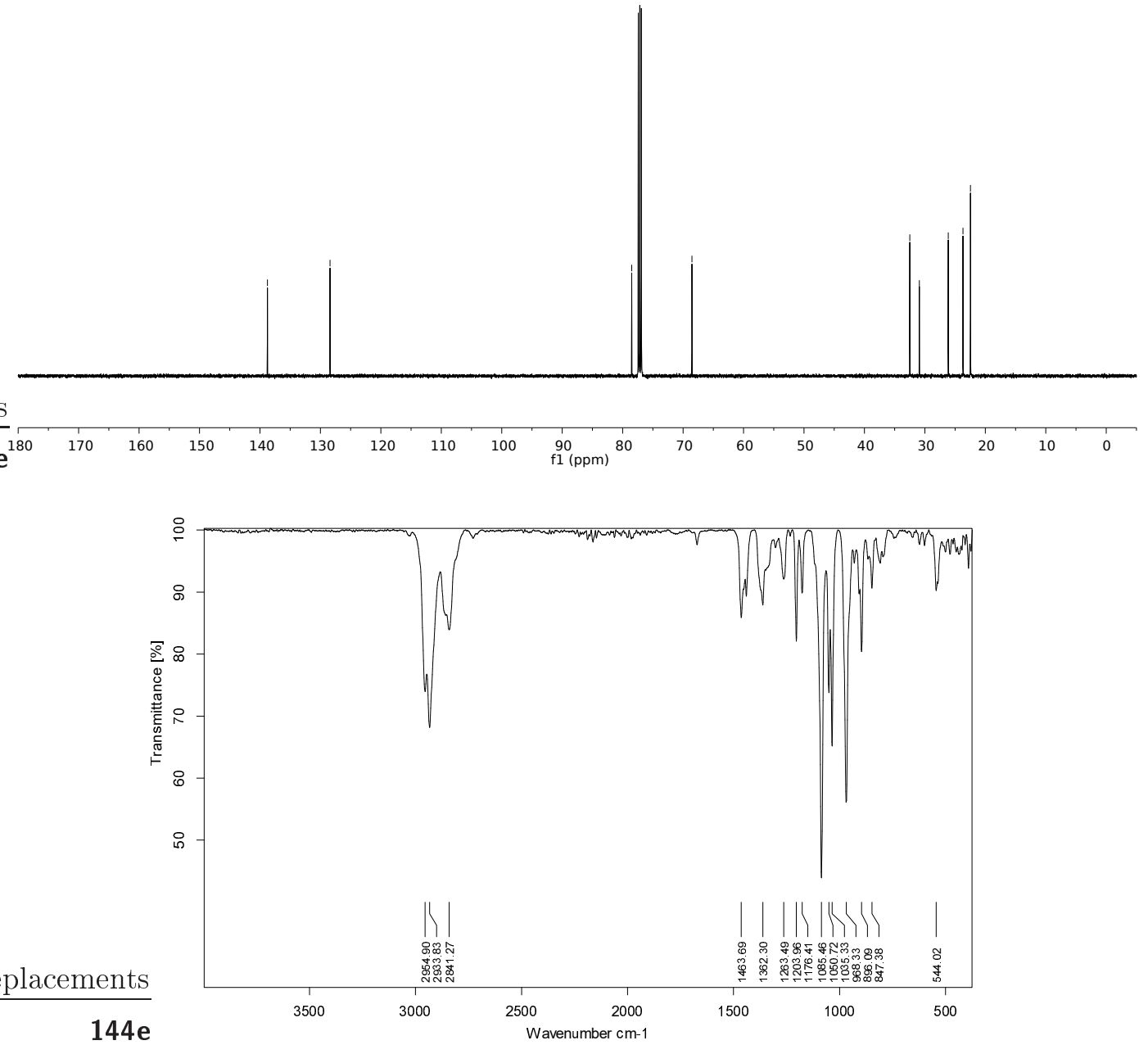

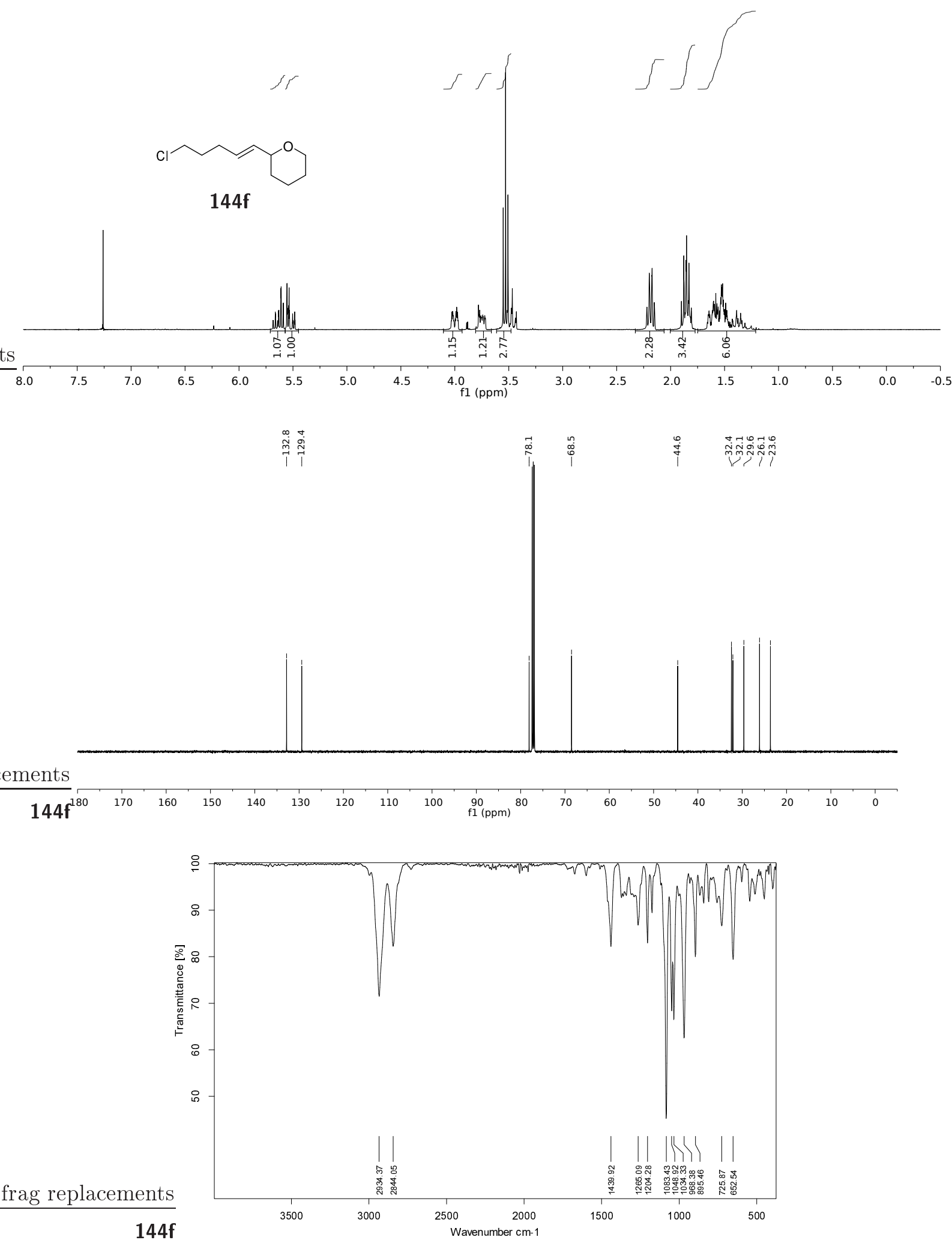

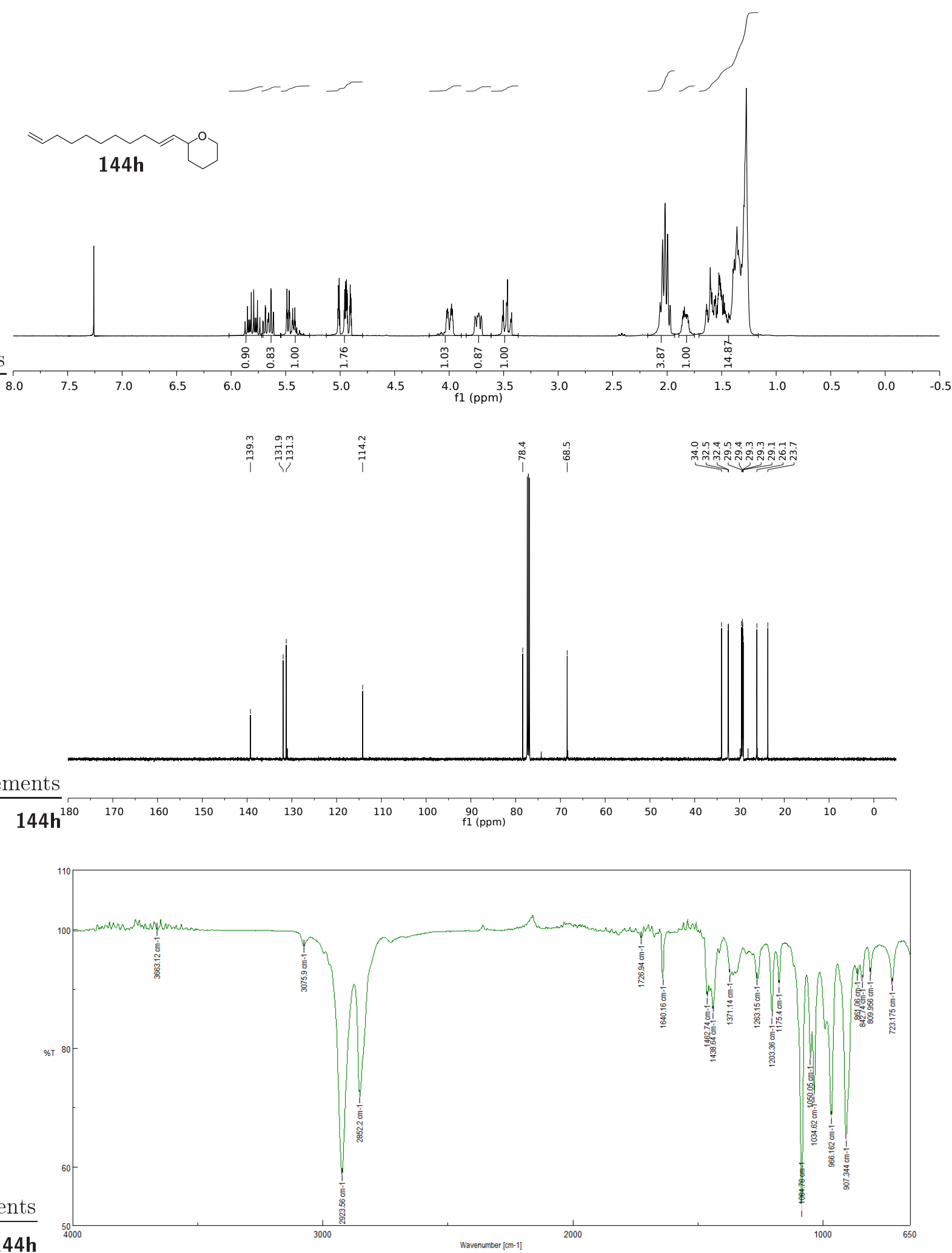


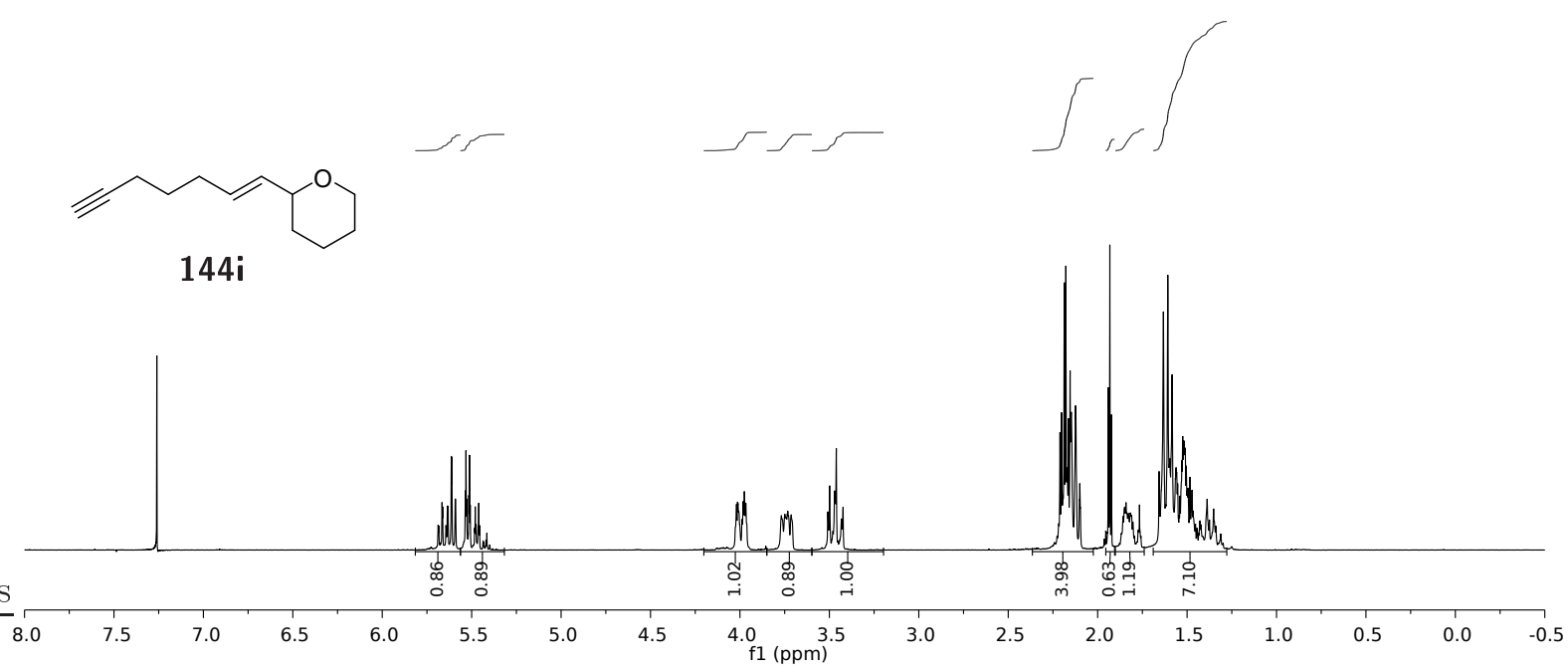

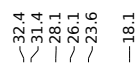
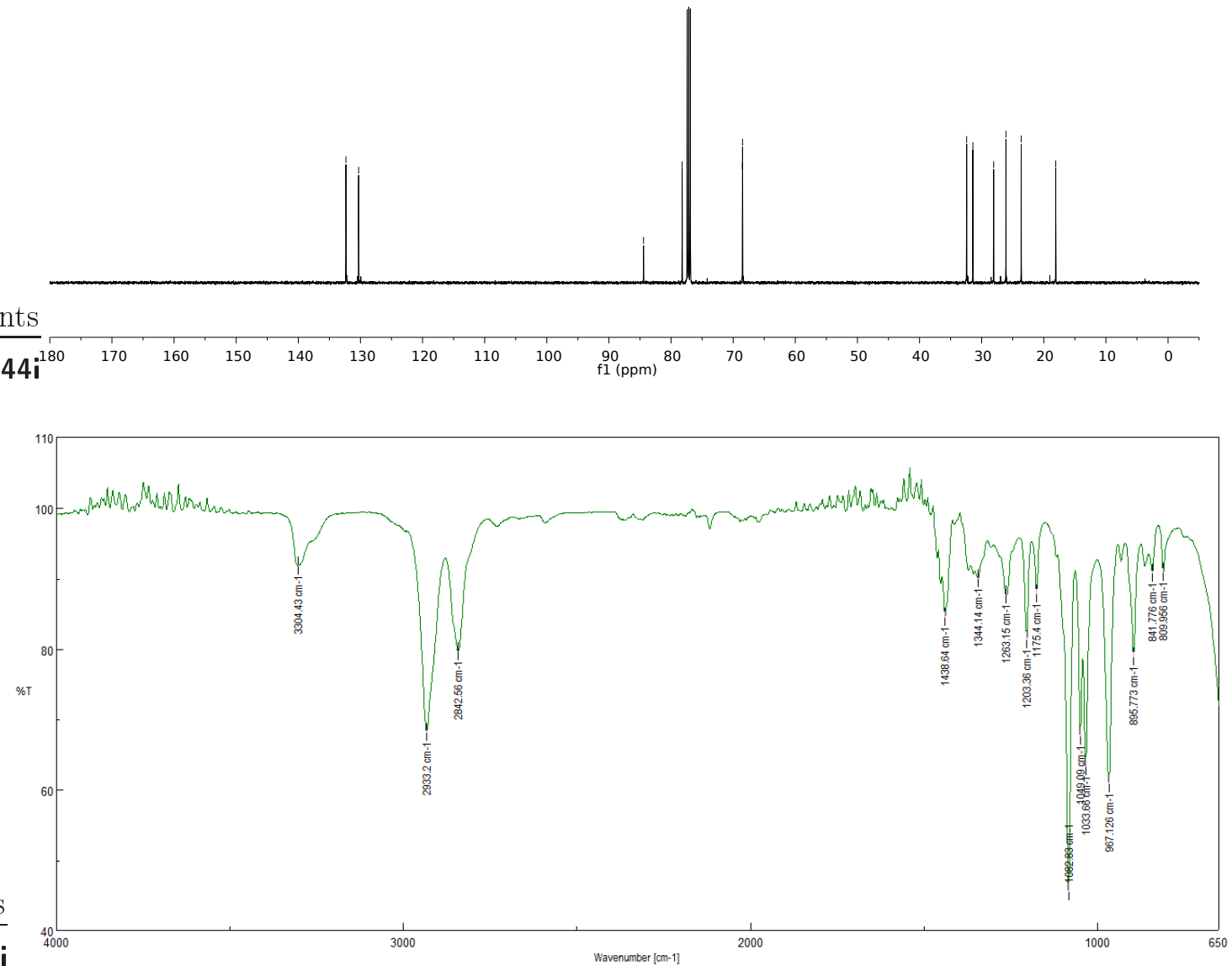


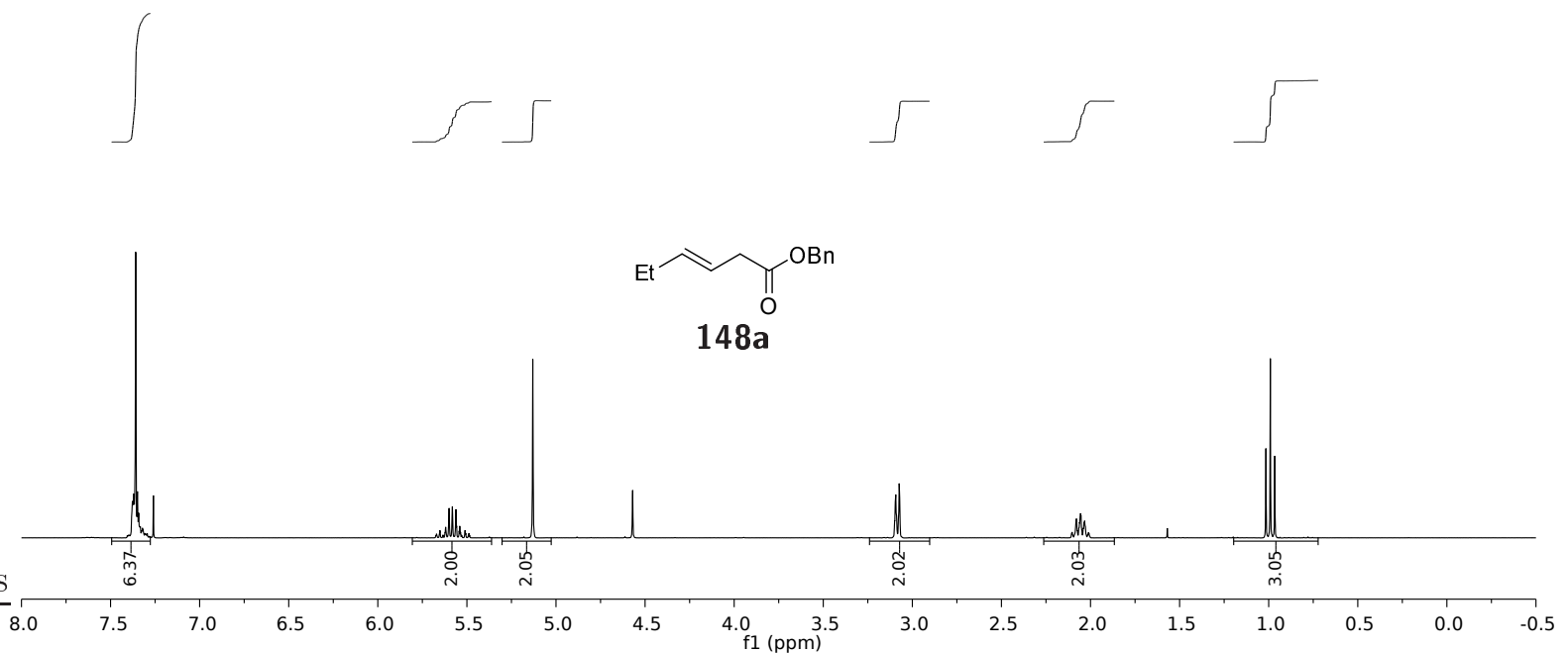

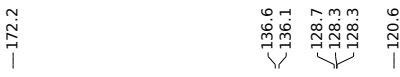

过
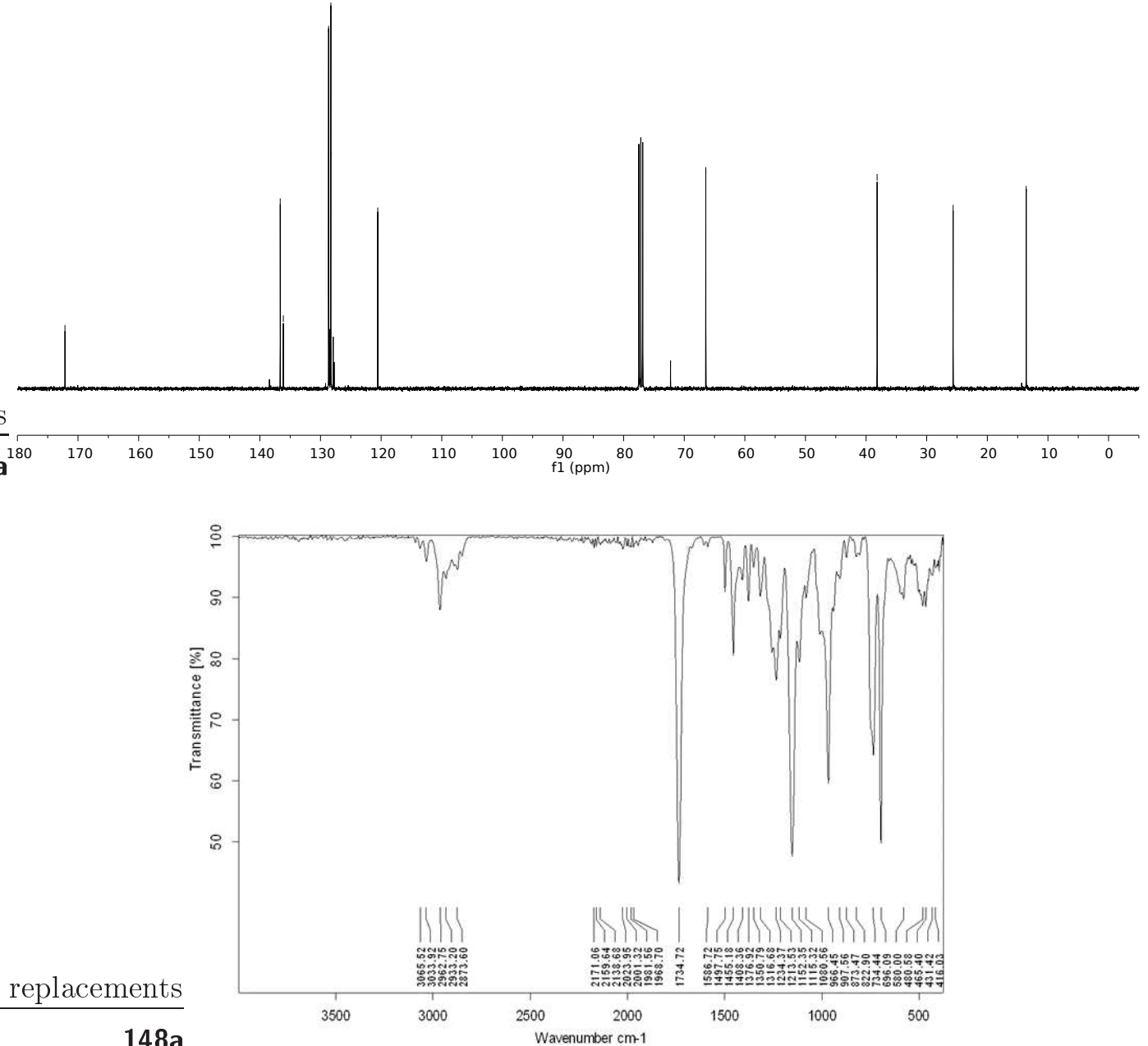

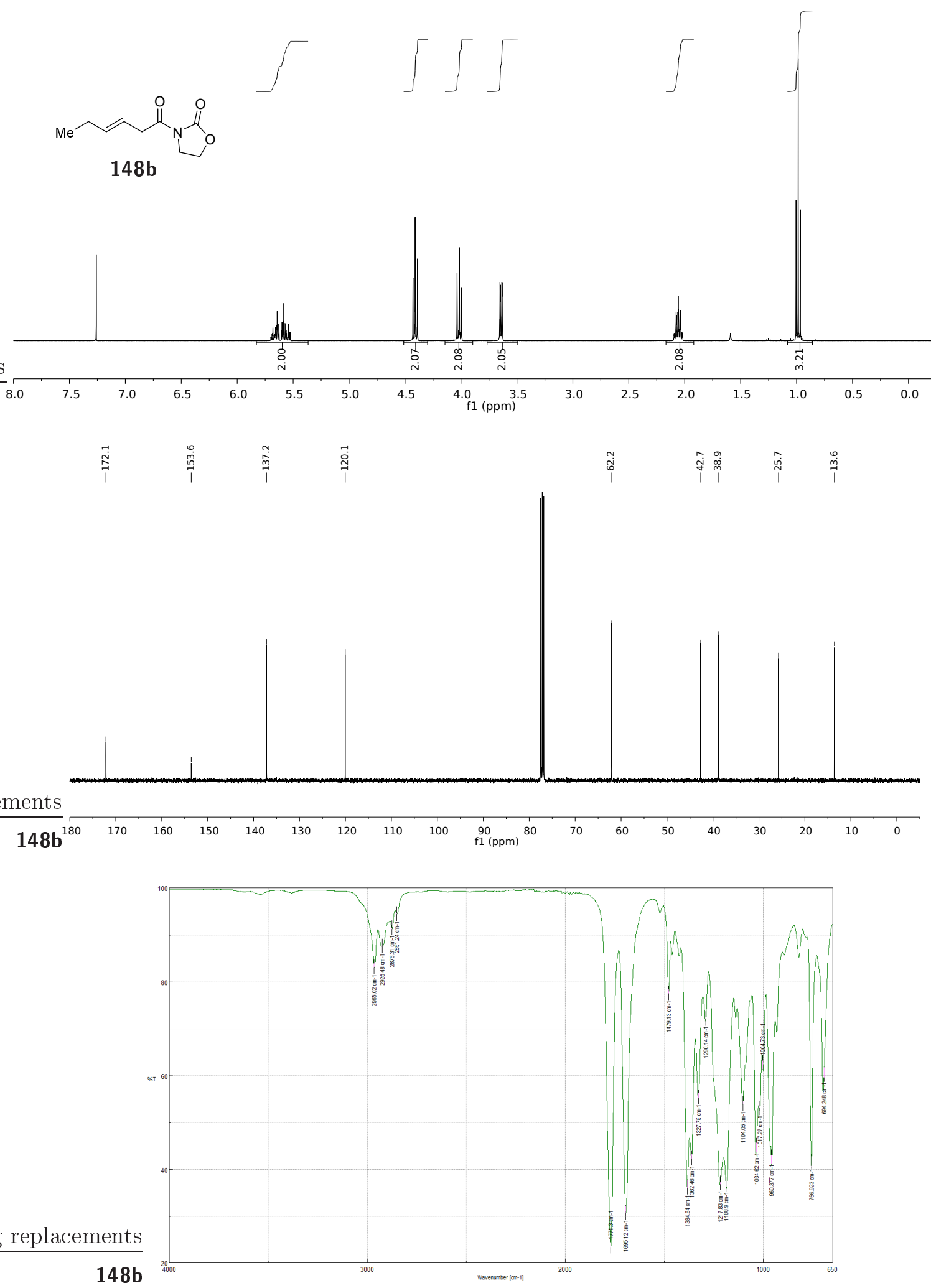


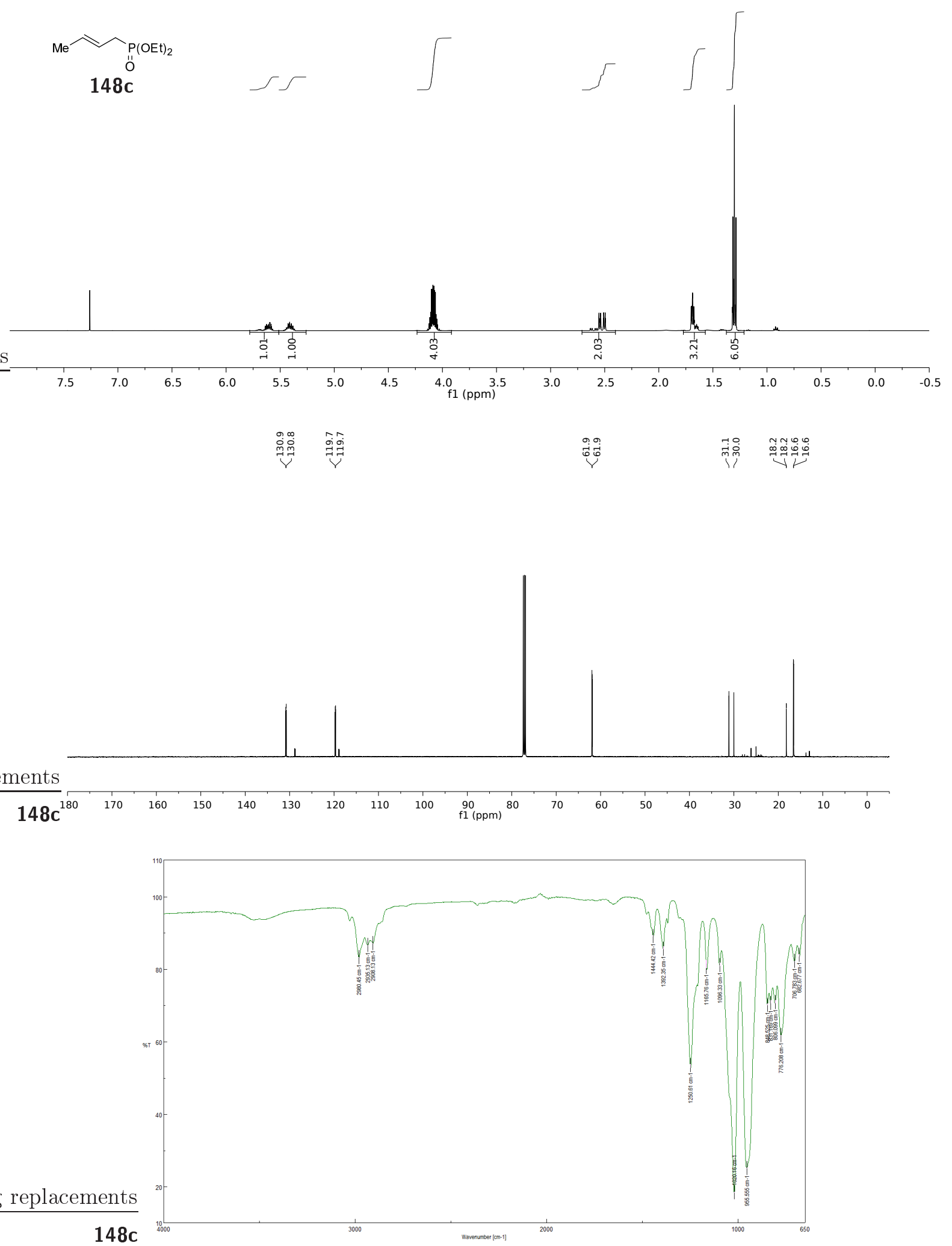



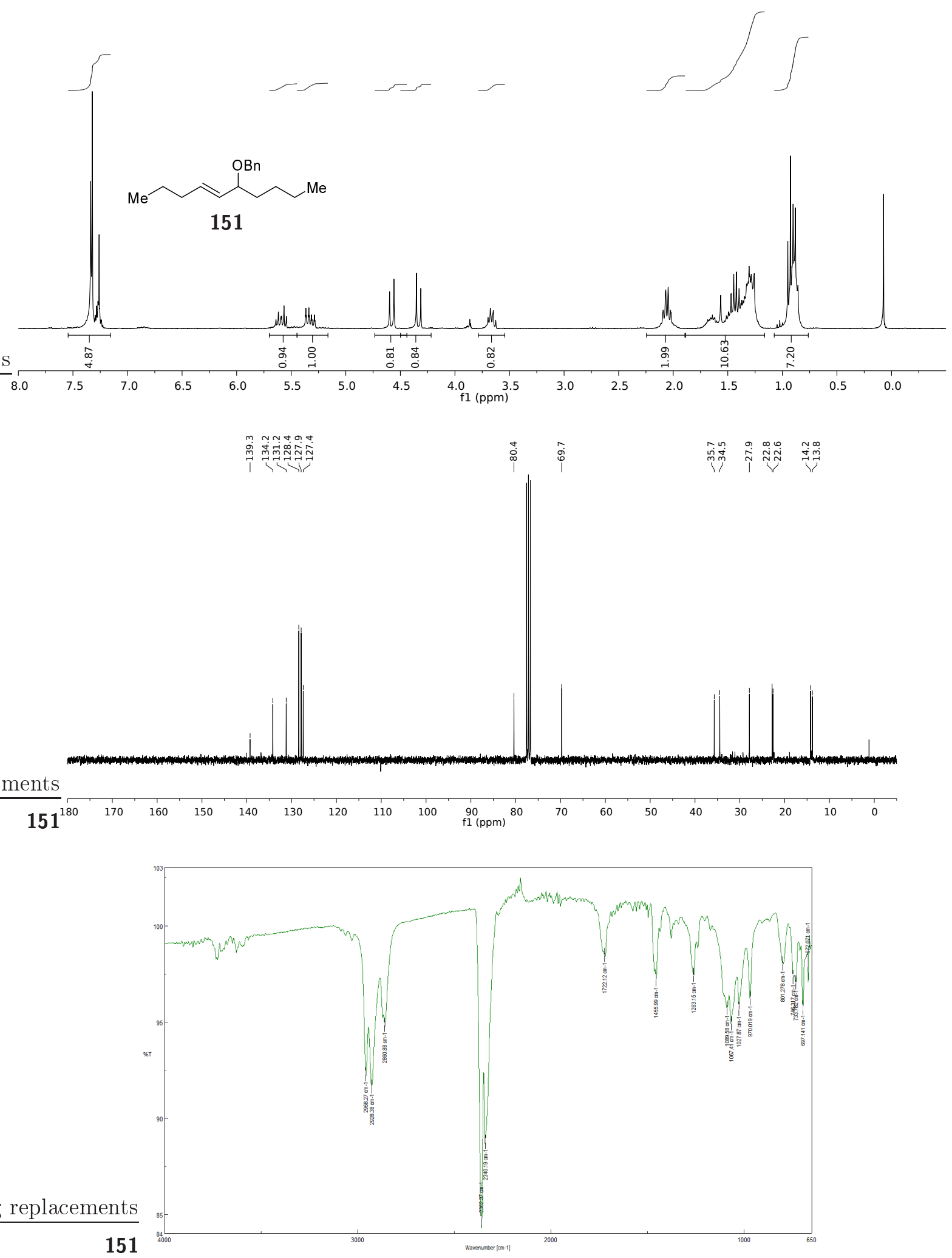


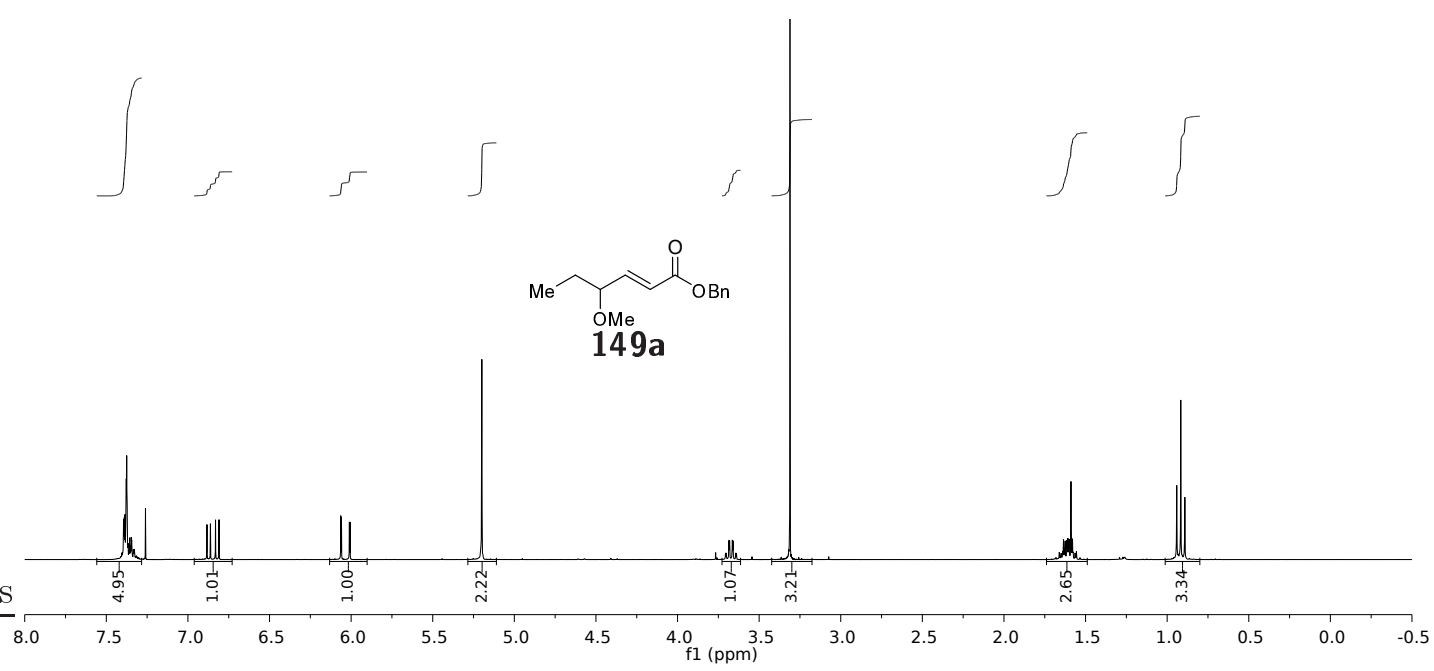

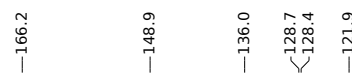
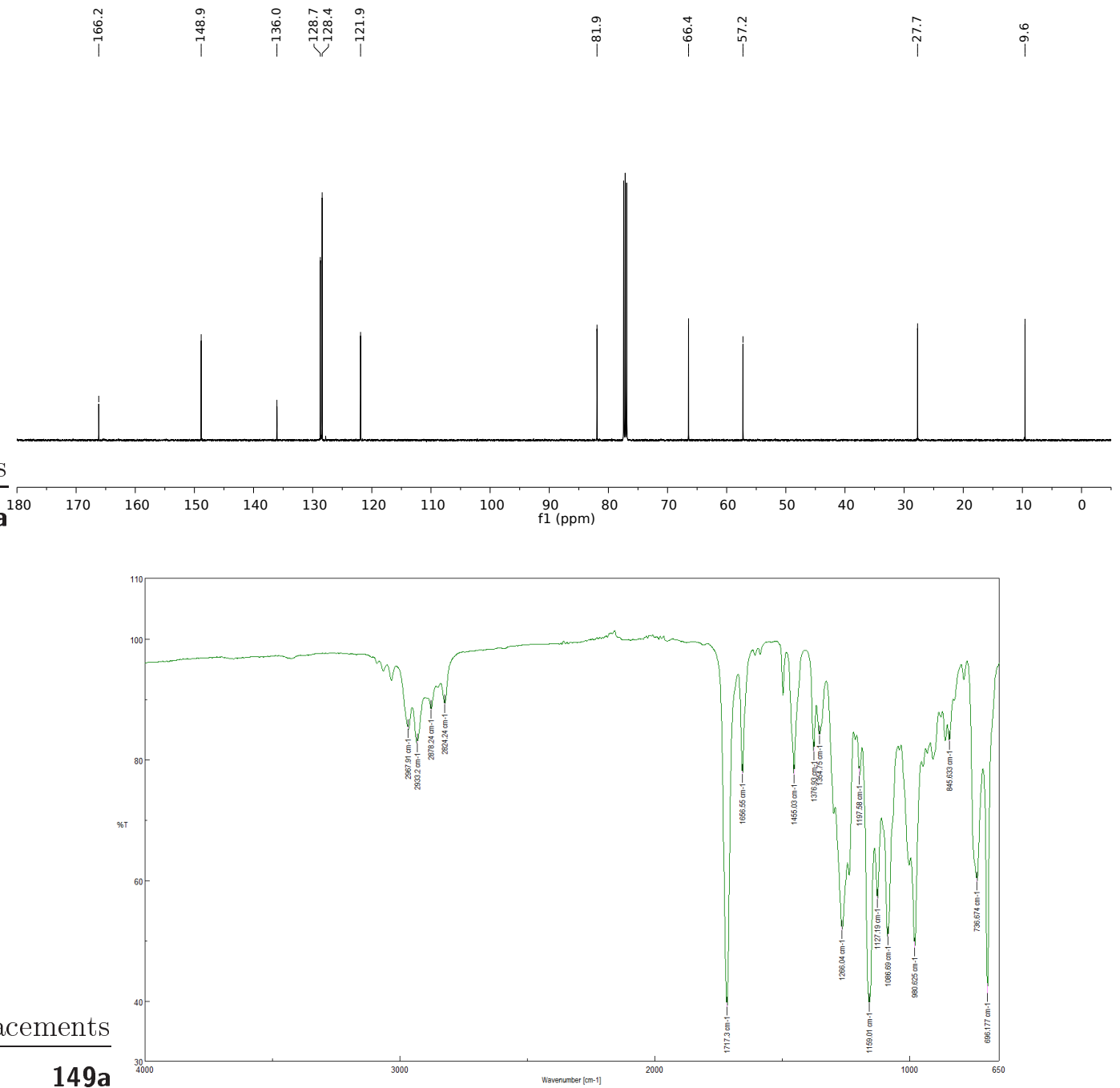

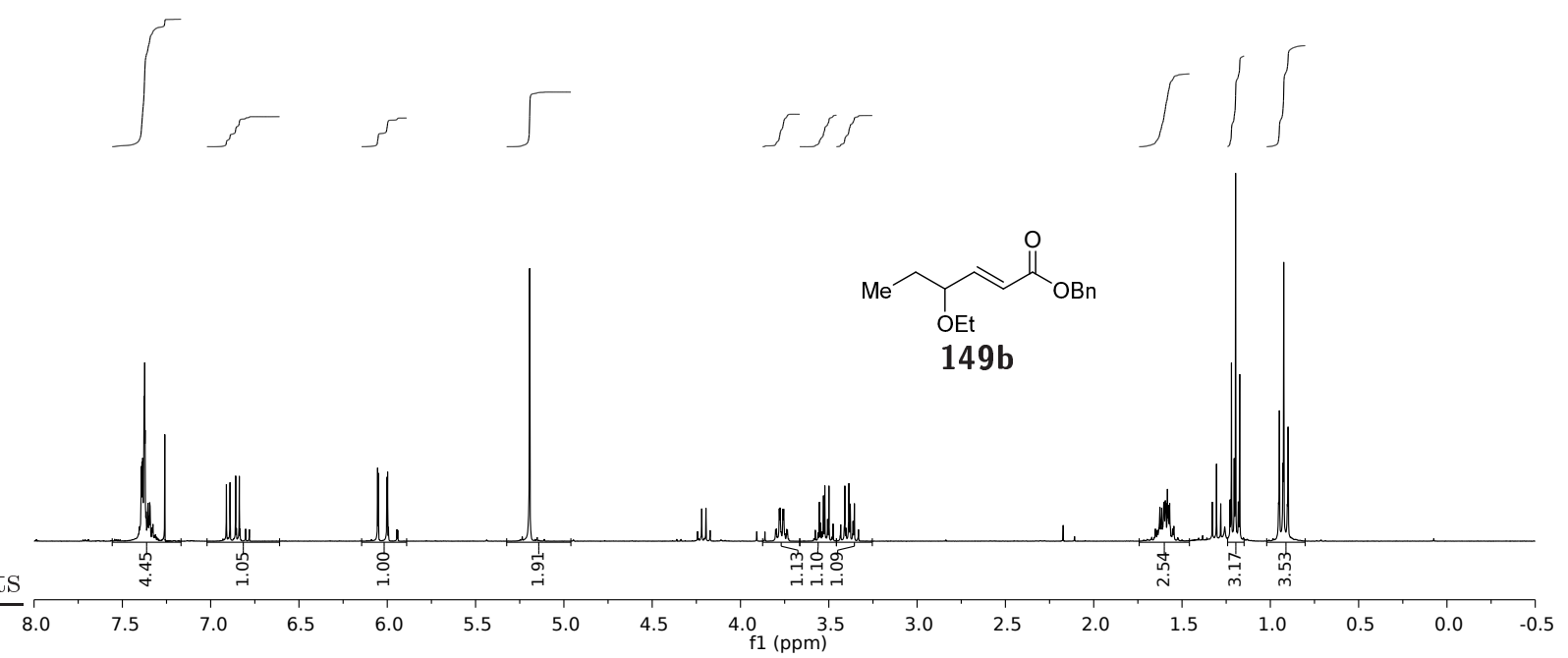

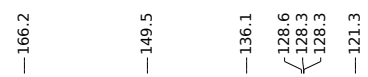

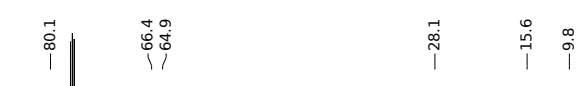
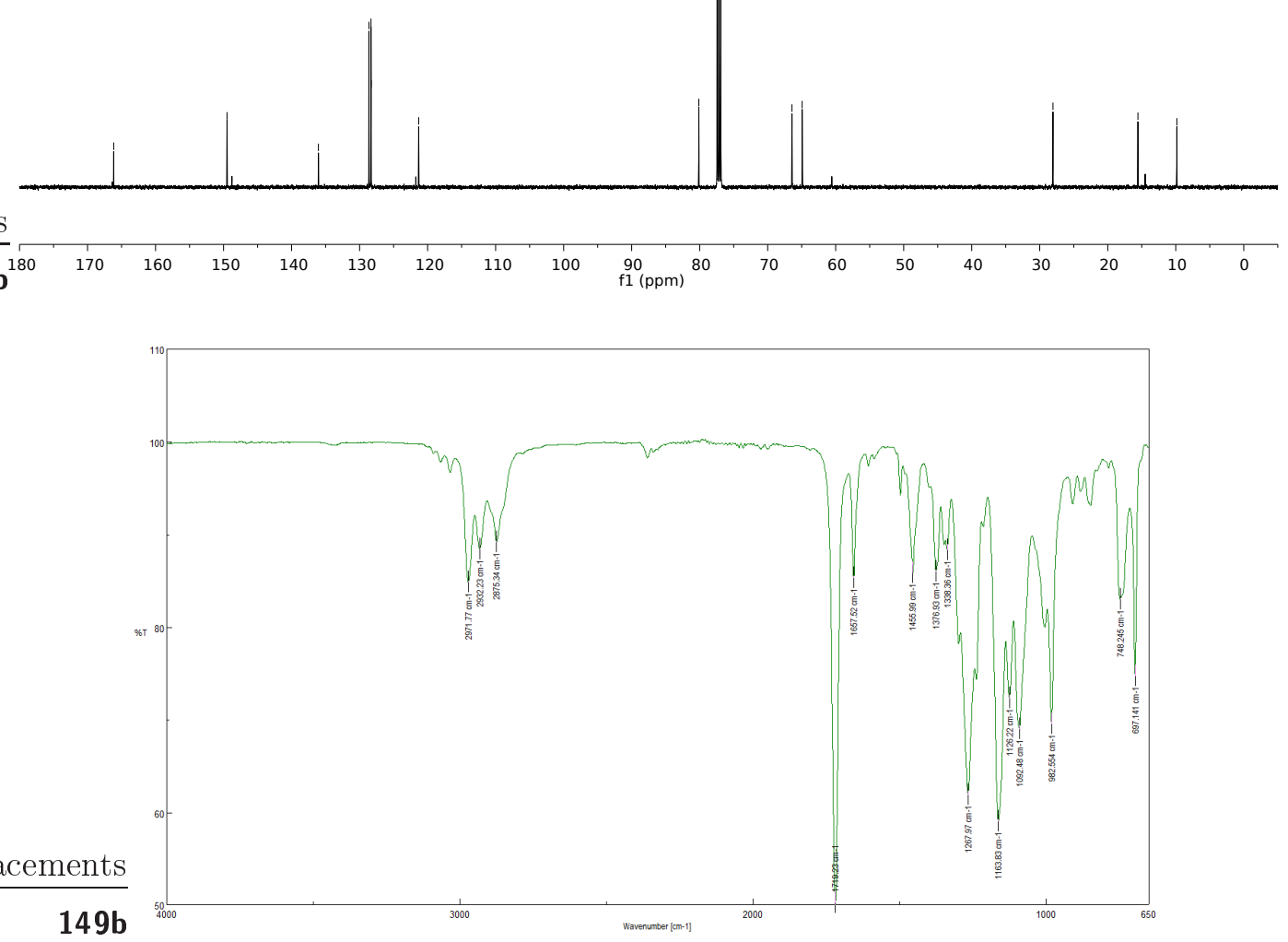


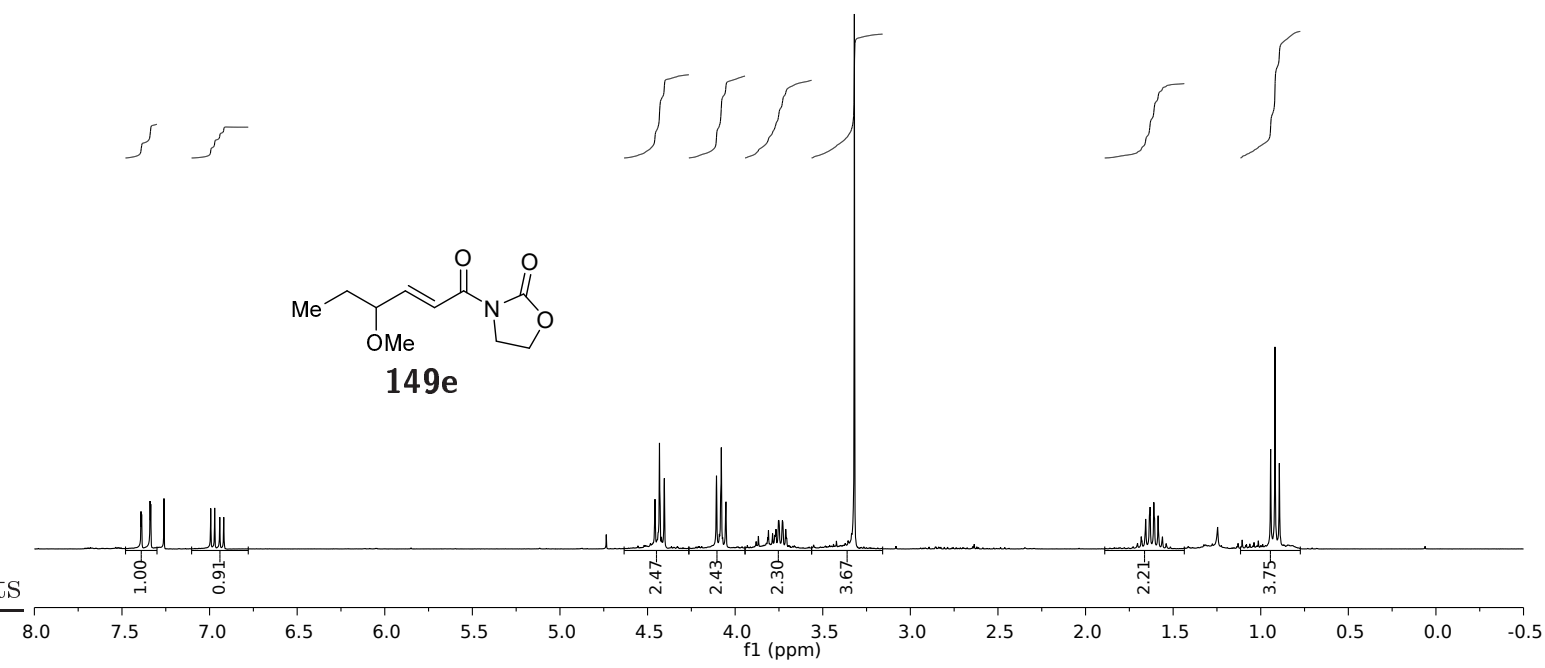

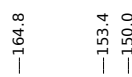

$\stackrel{\circ}{\stackrel{i}{I}}$

ָ艹
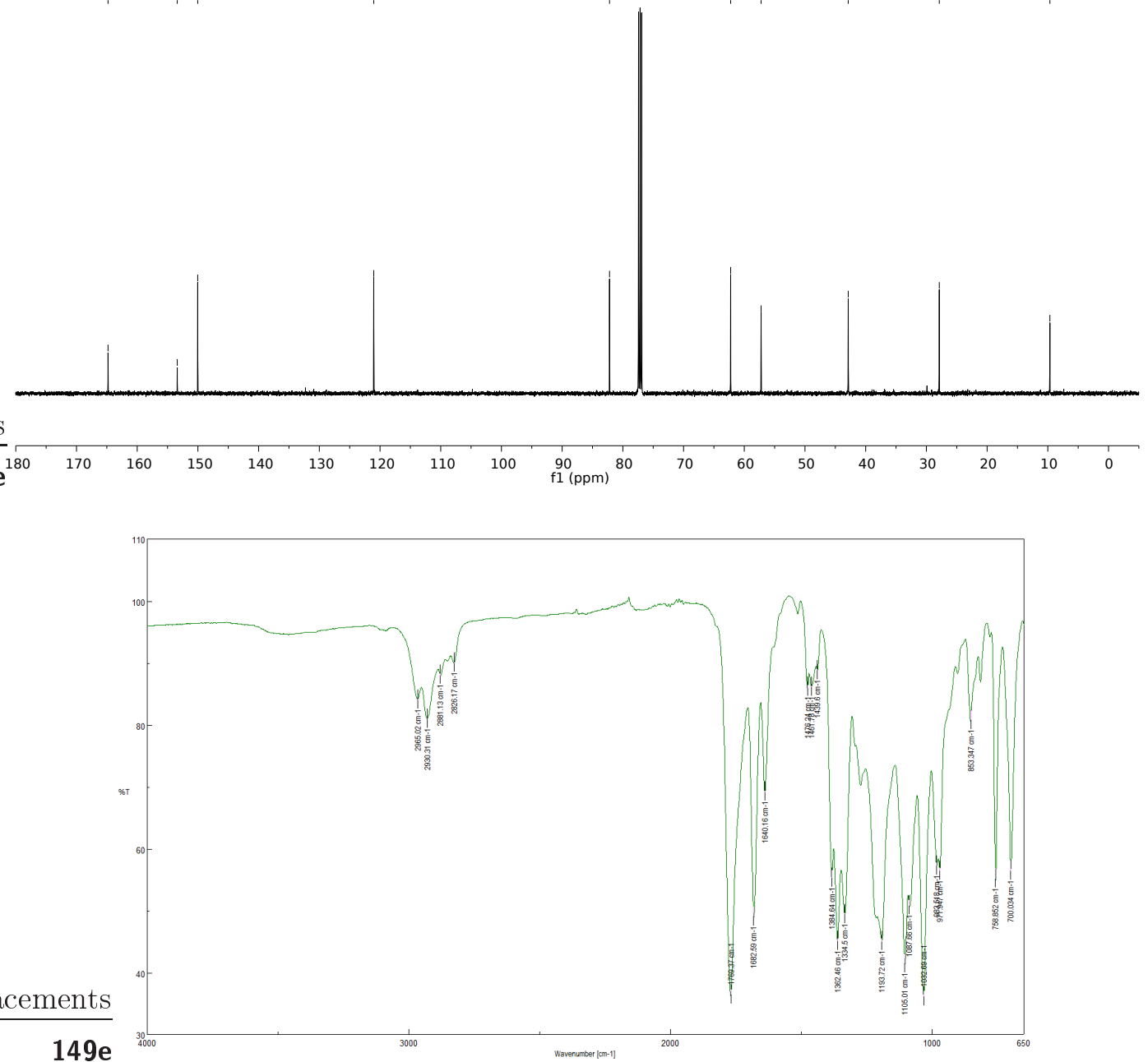


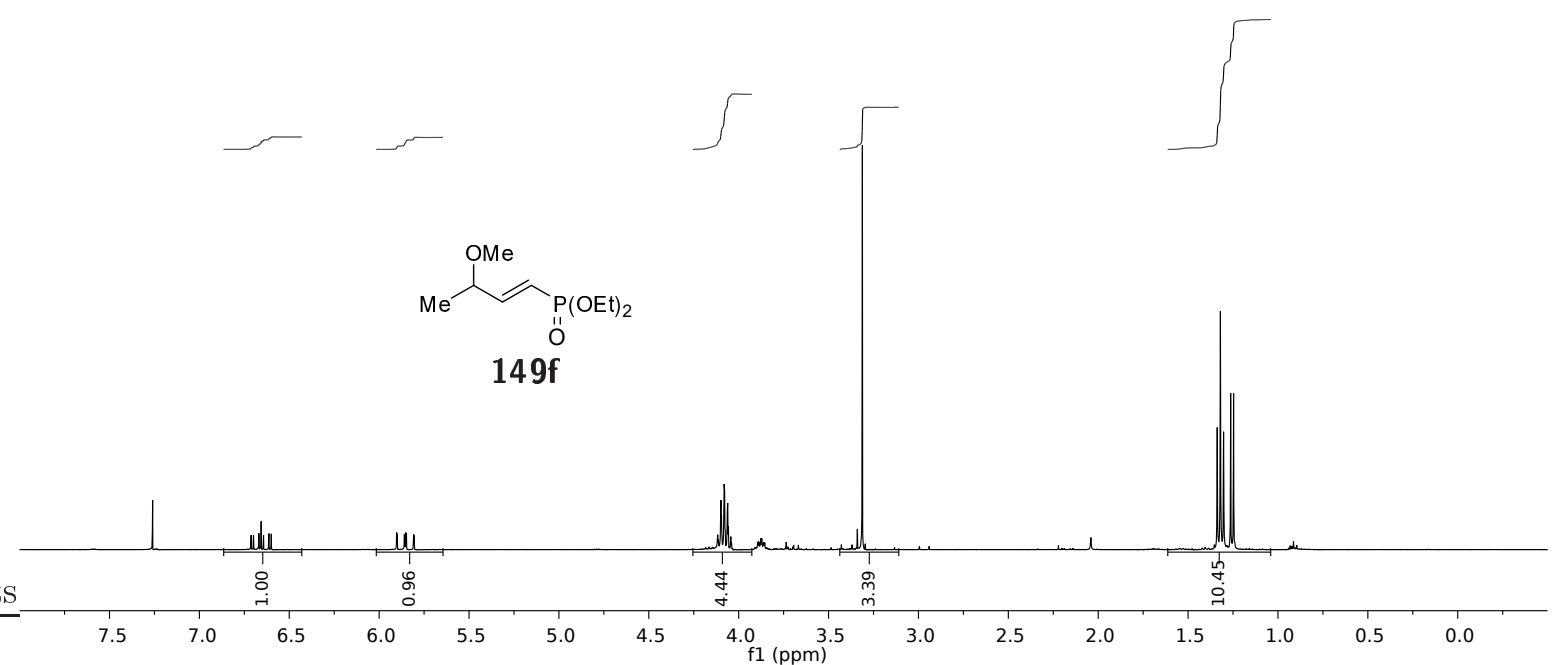

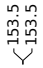

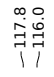

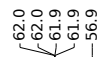

ํํำ
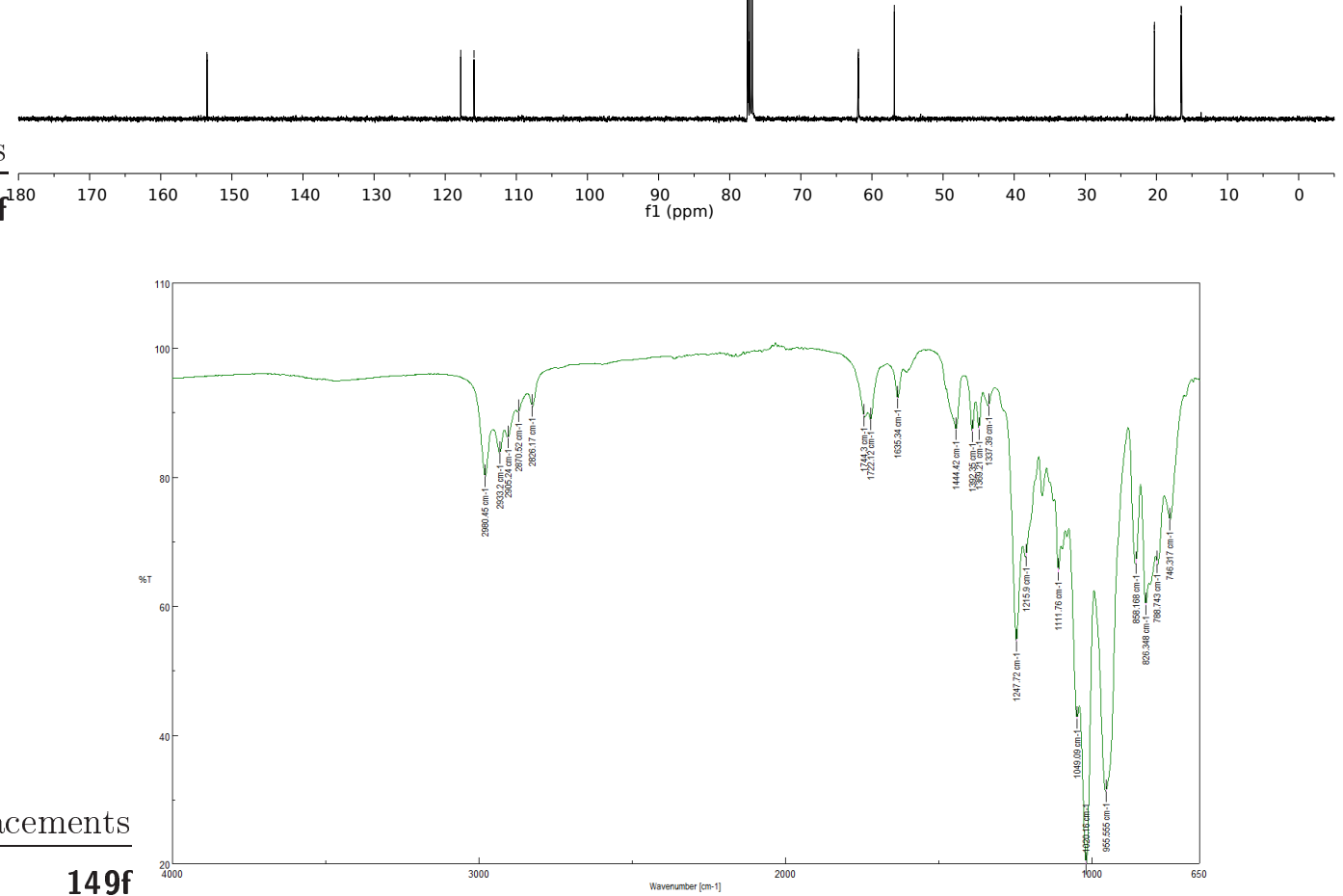

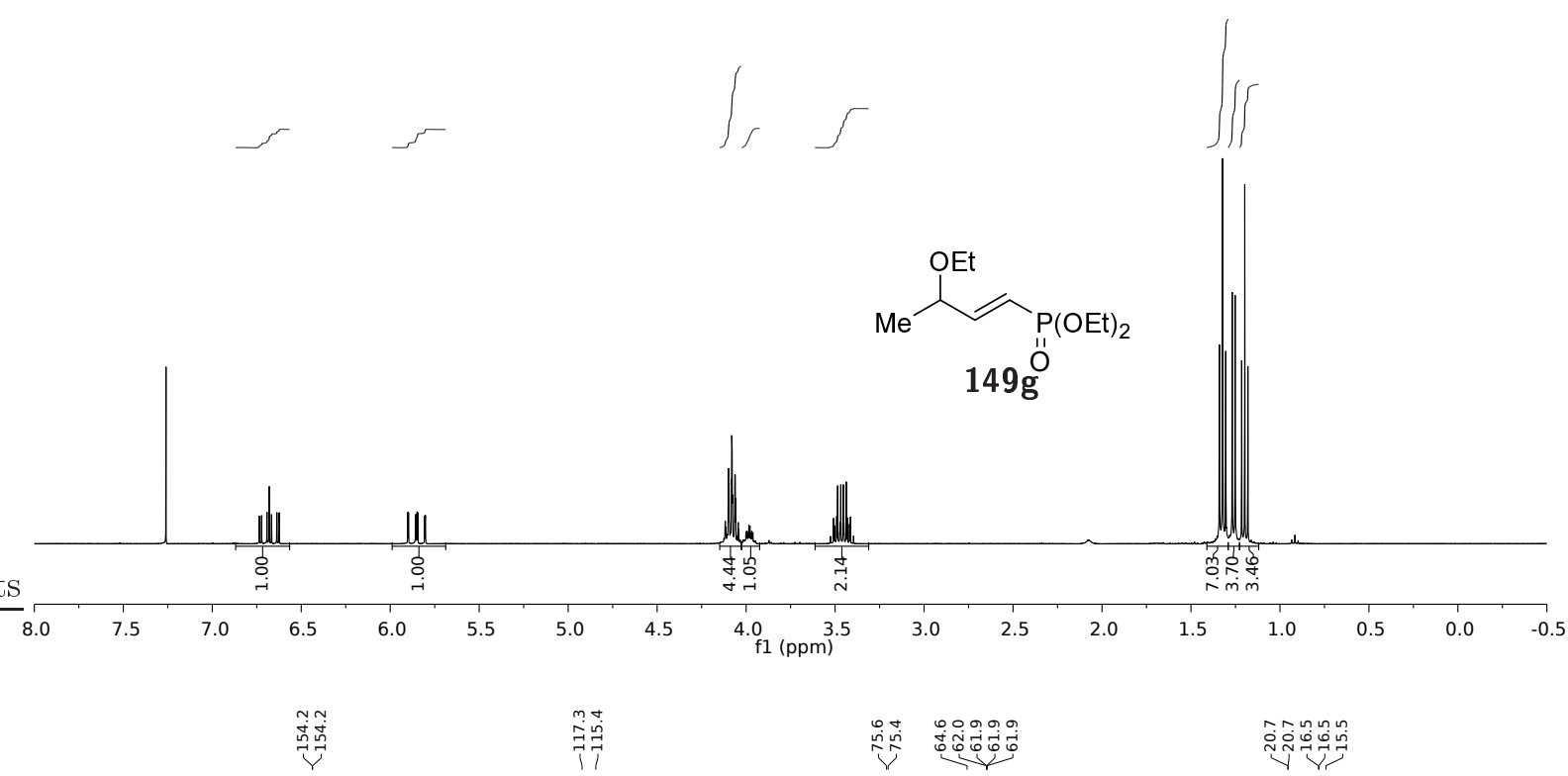

ํำ
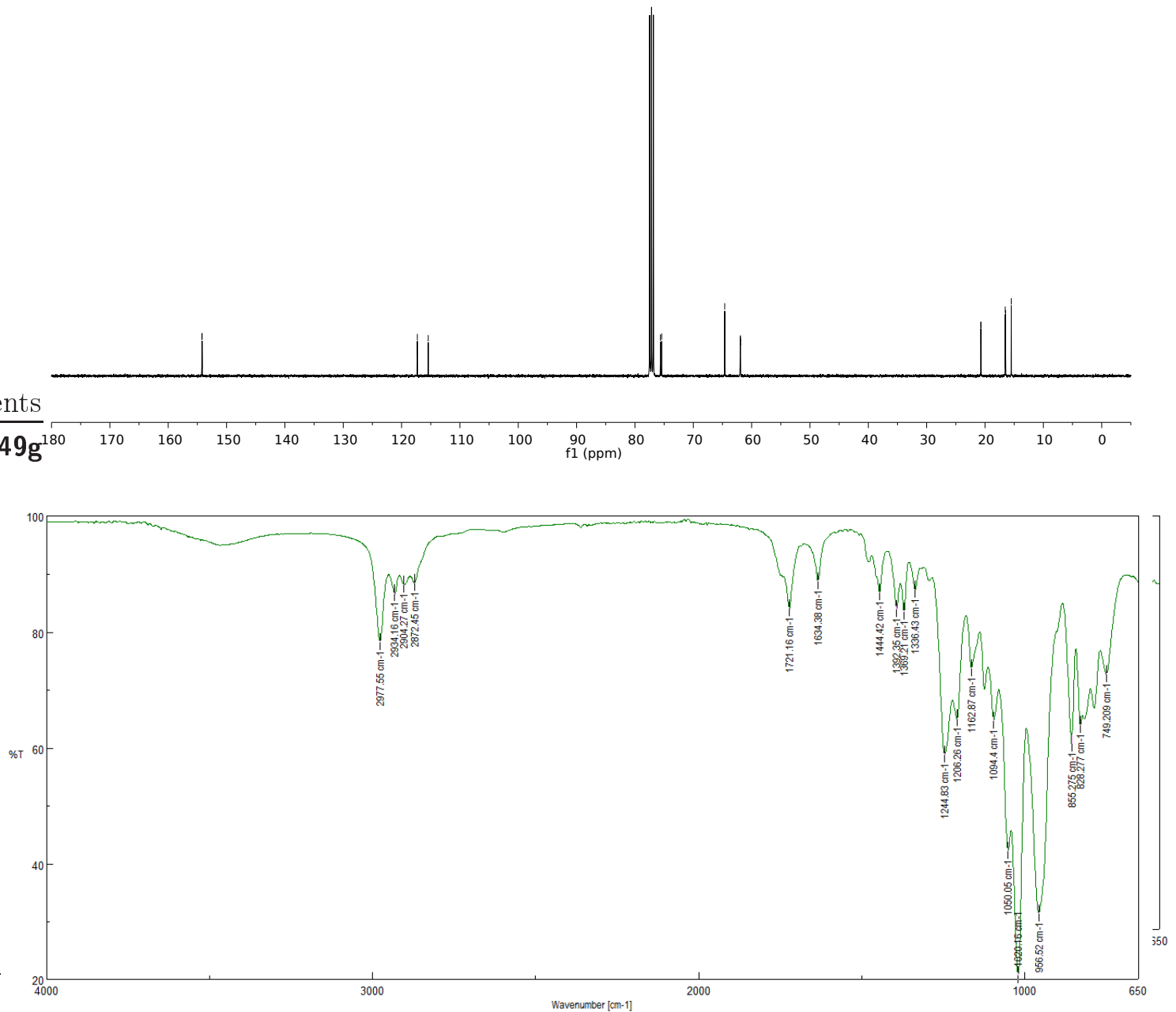

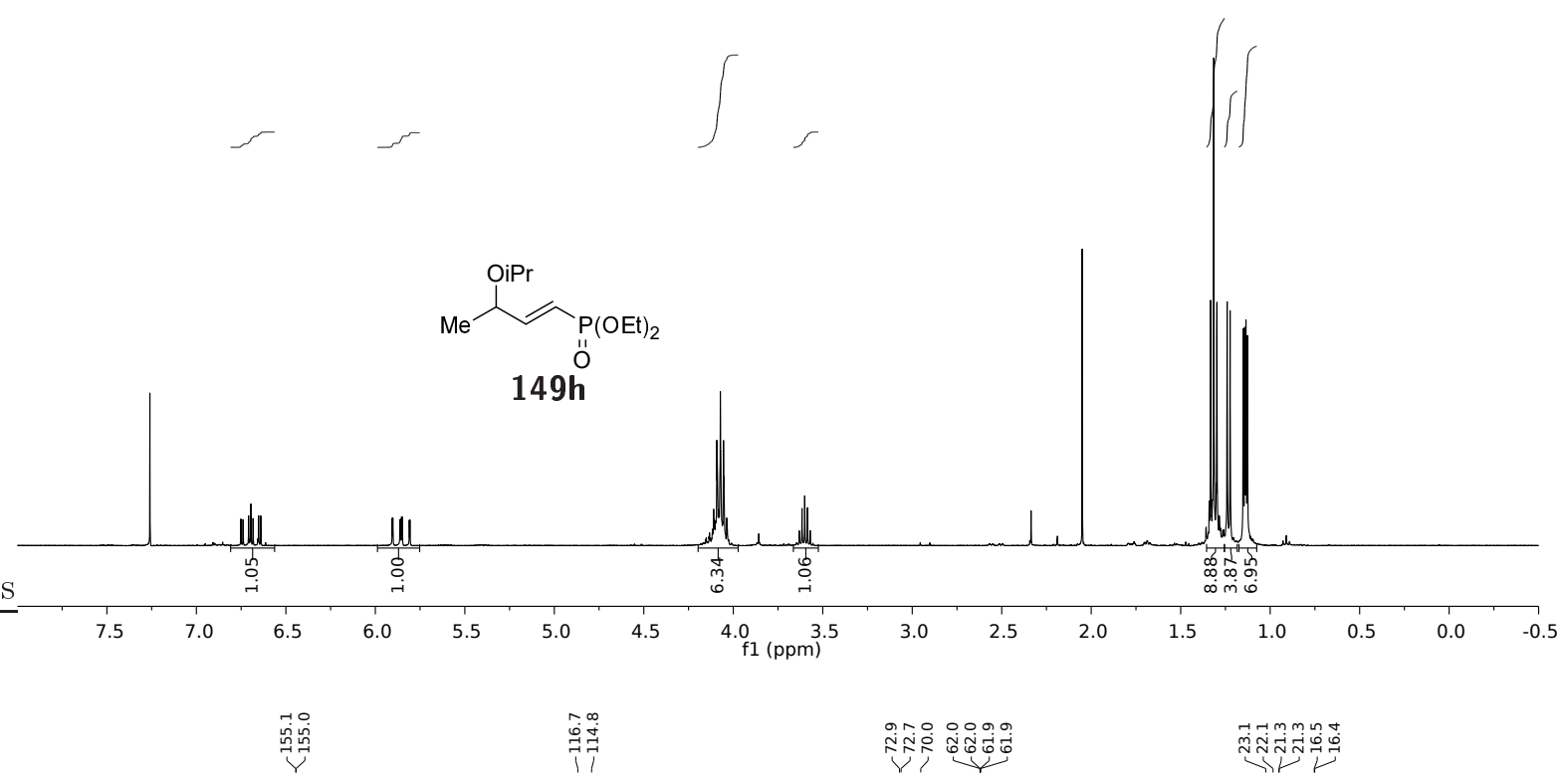

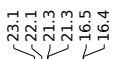
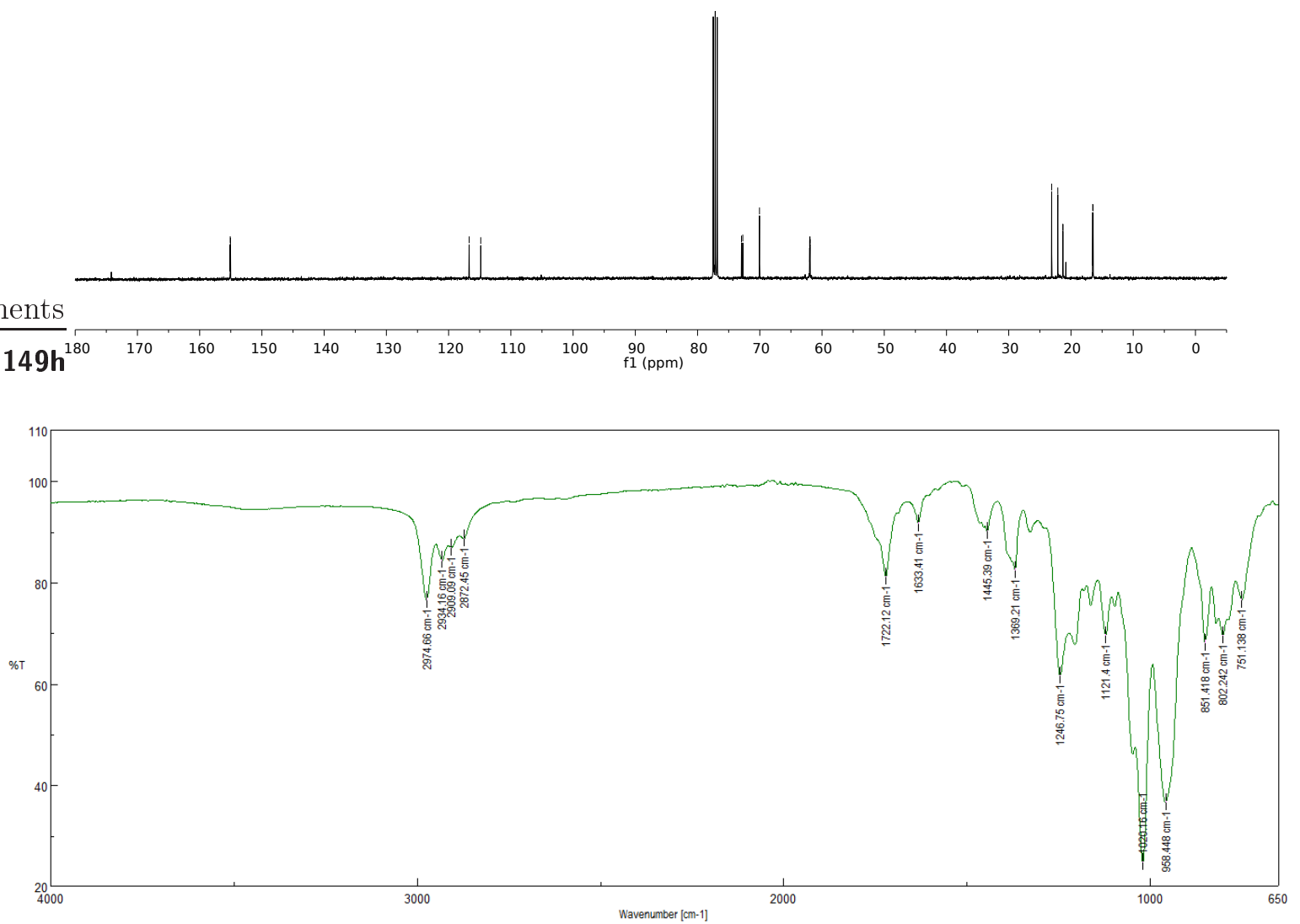


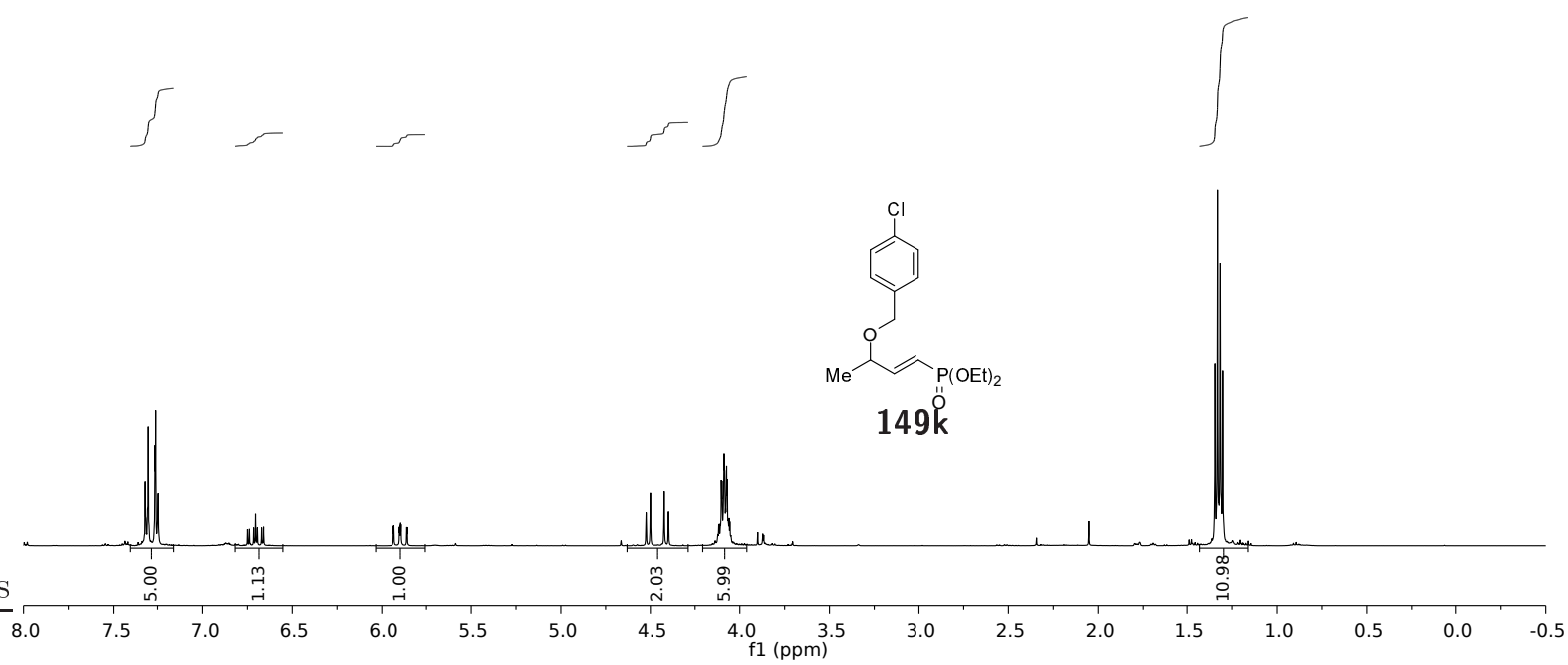

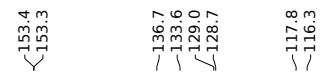

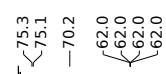

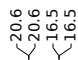
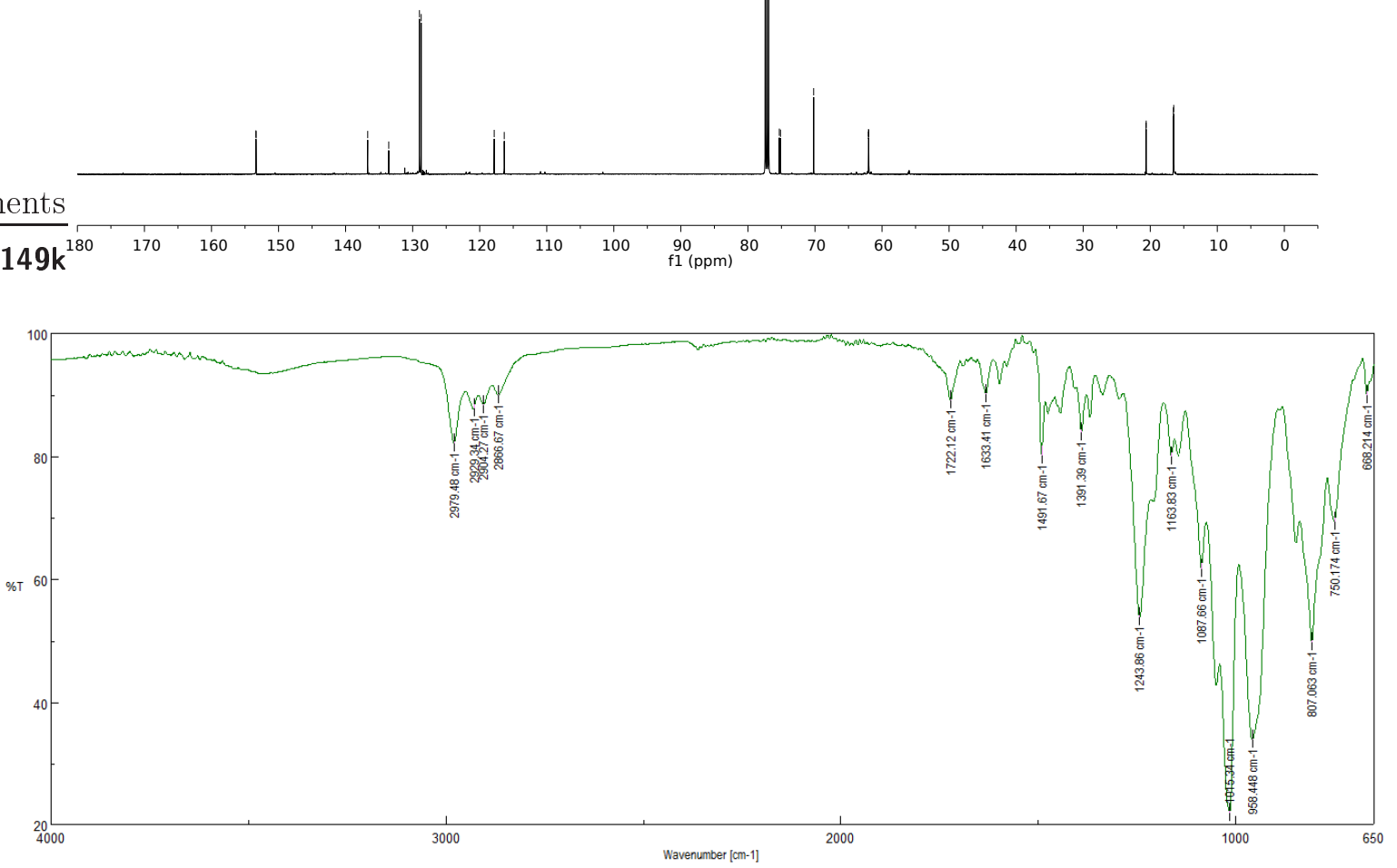


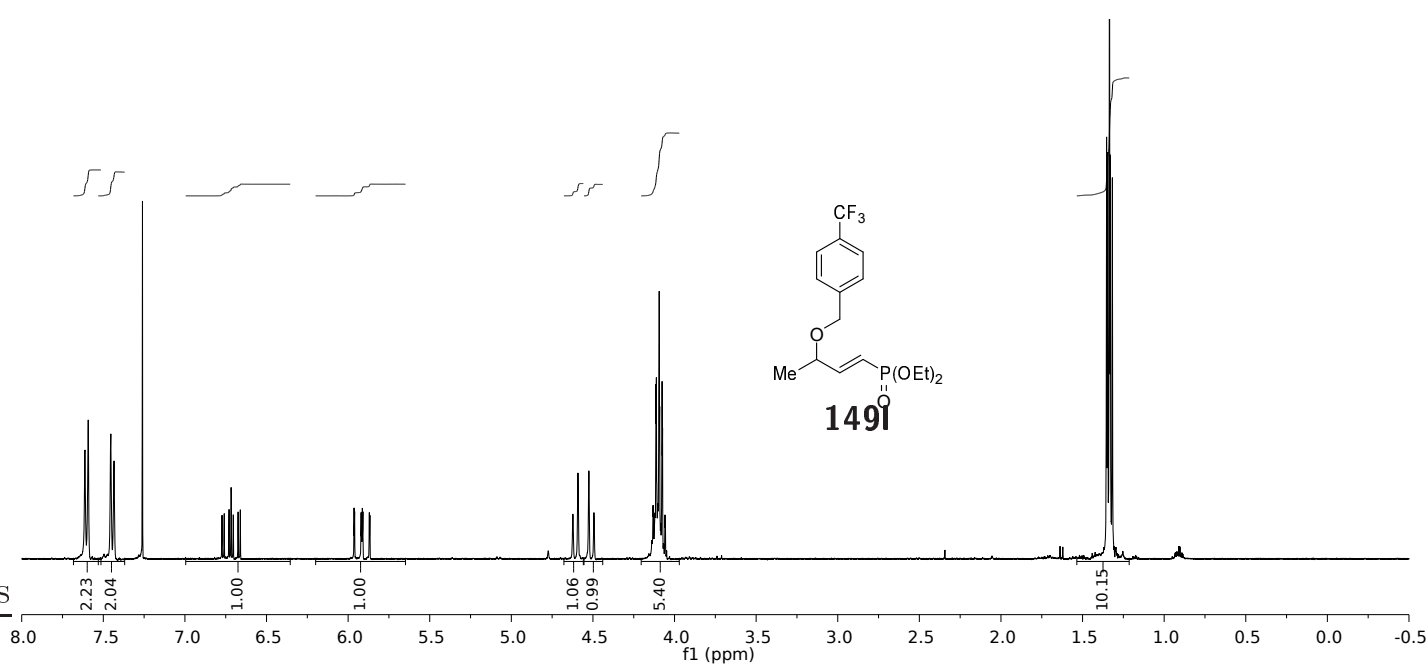

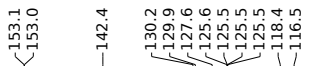

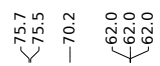

ขึ่
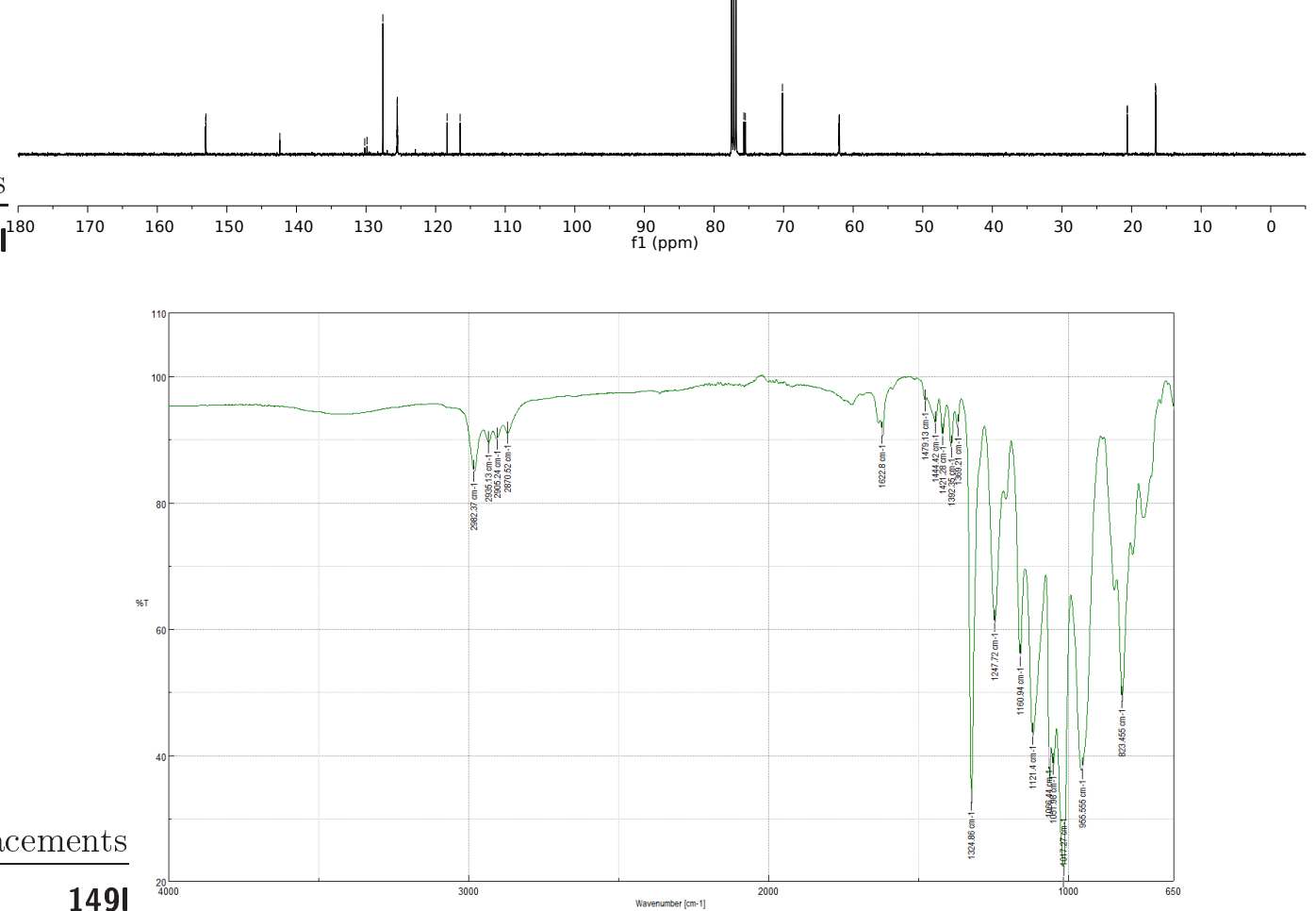


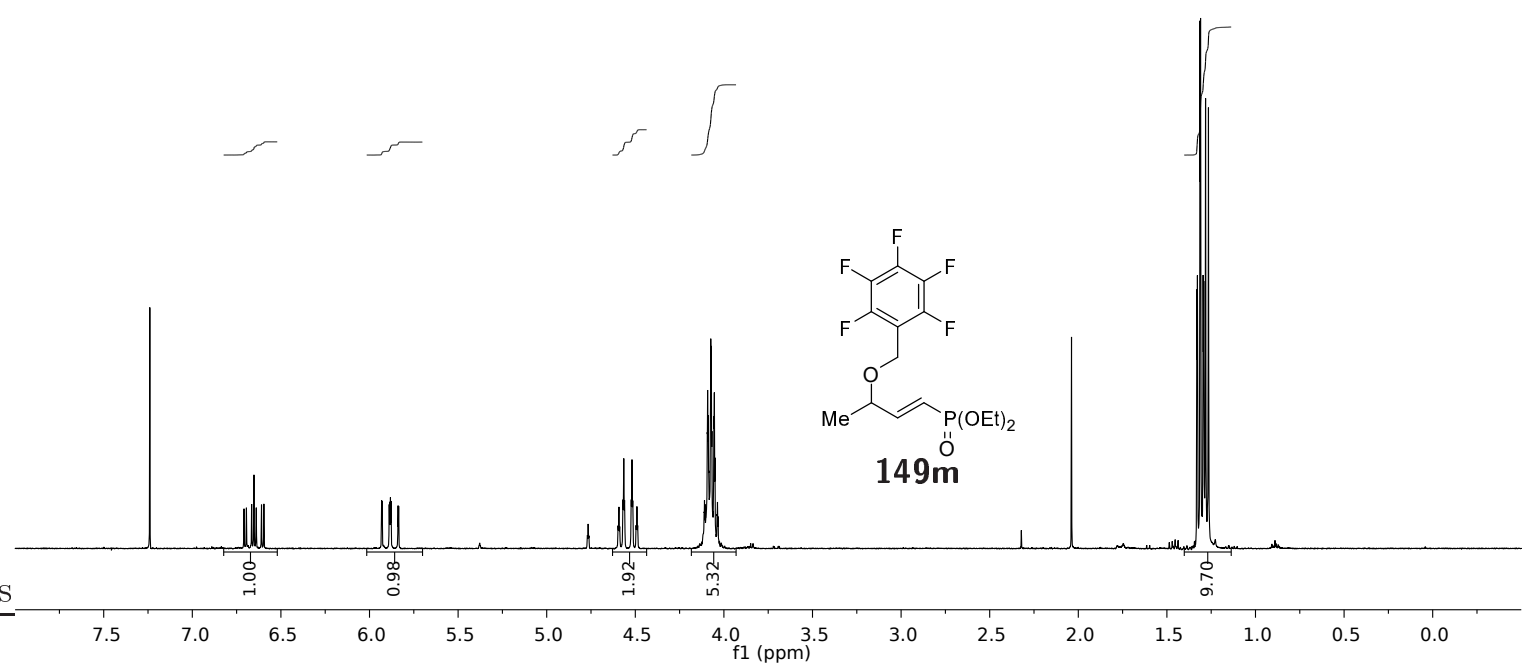

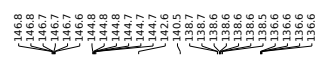

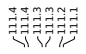
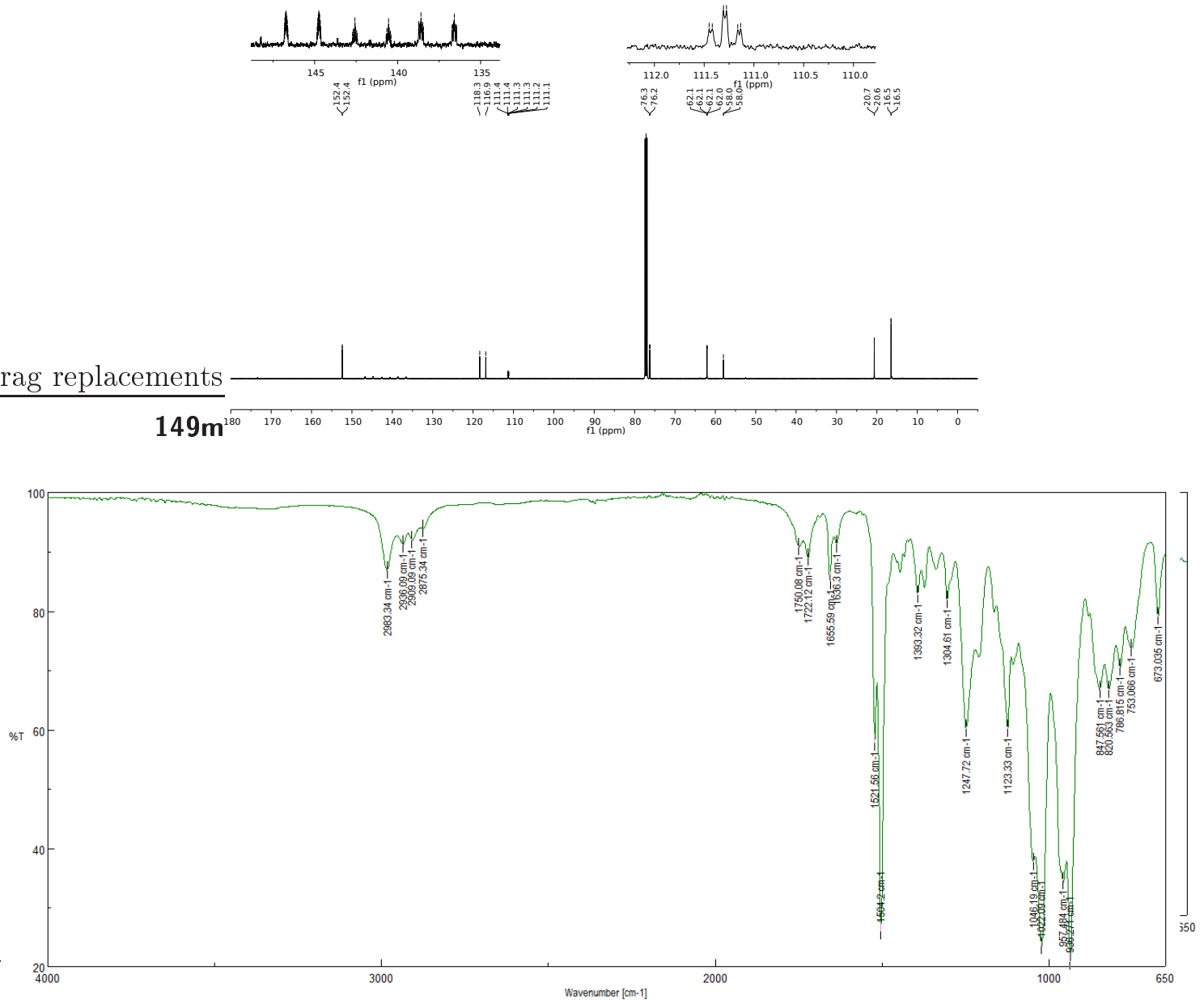


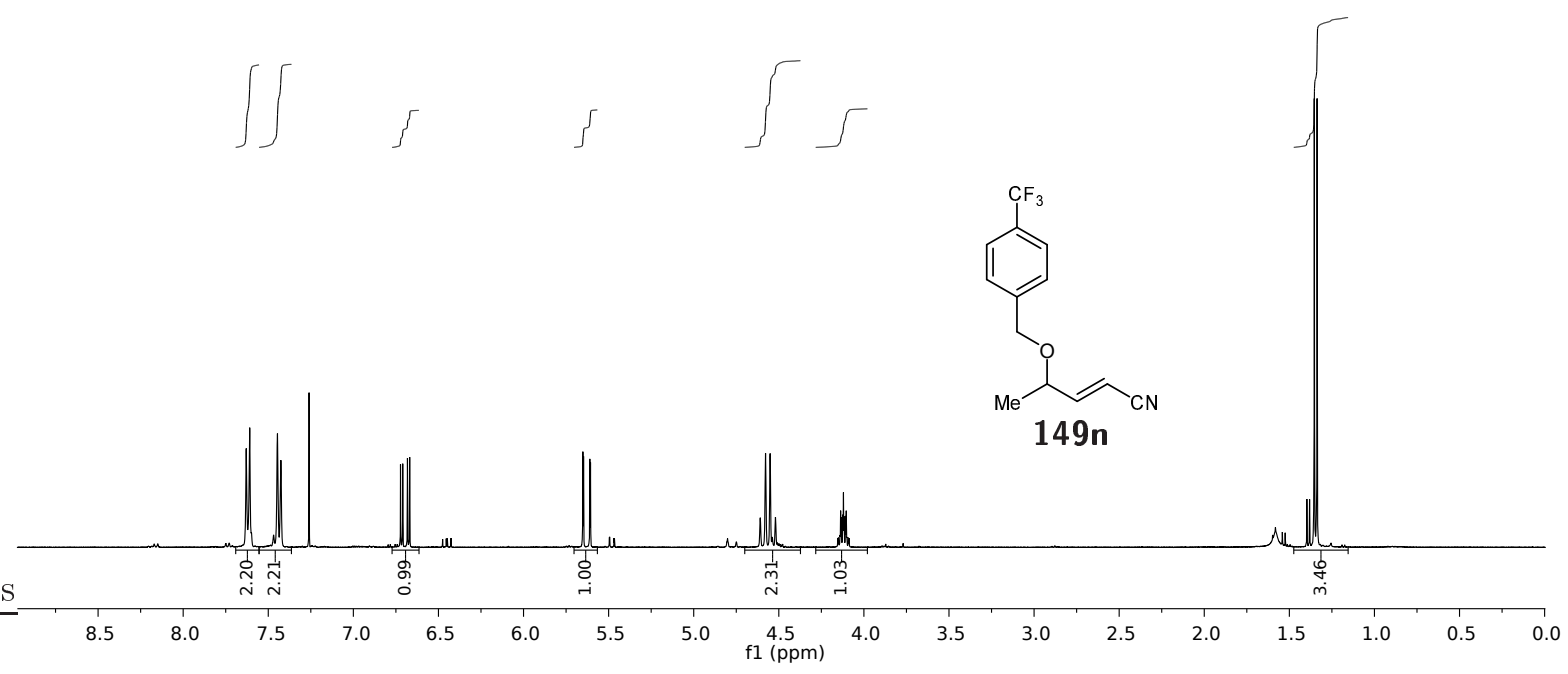

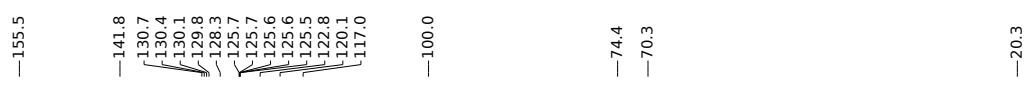
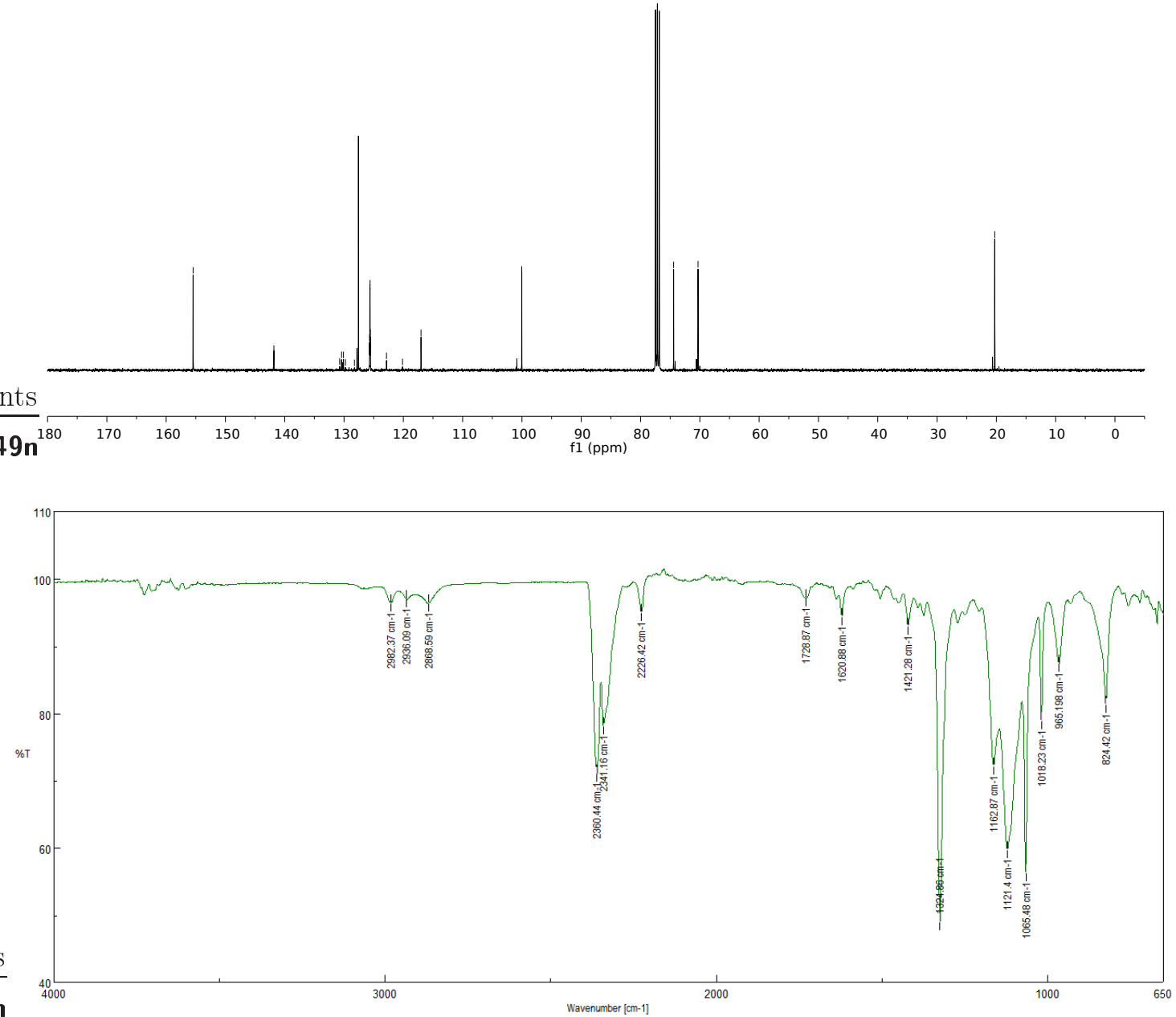


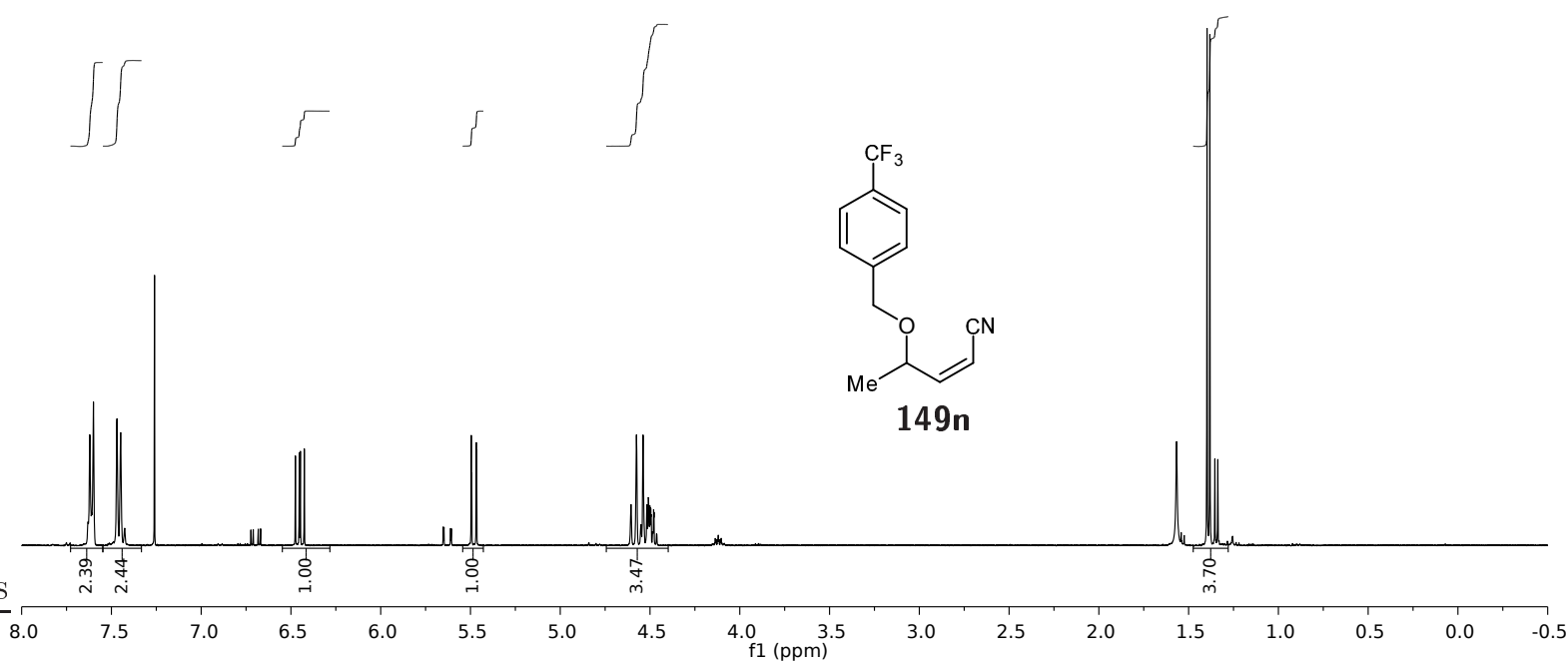

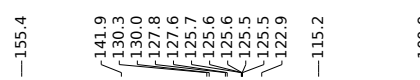

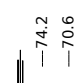

$\stackrel{i}{i}$
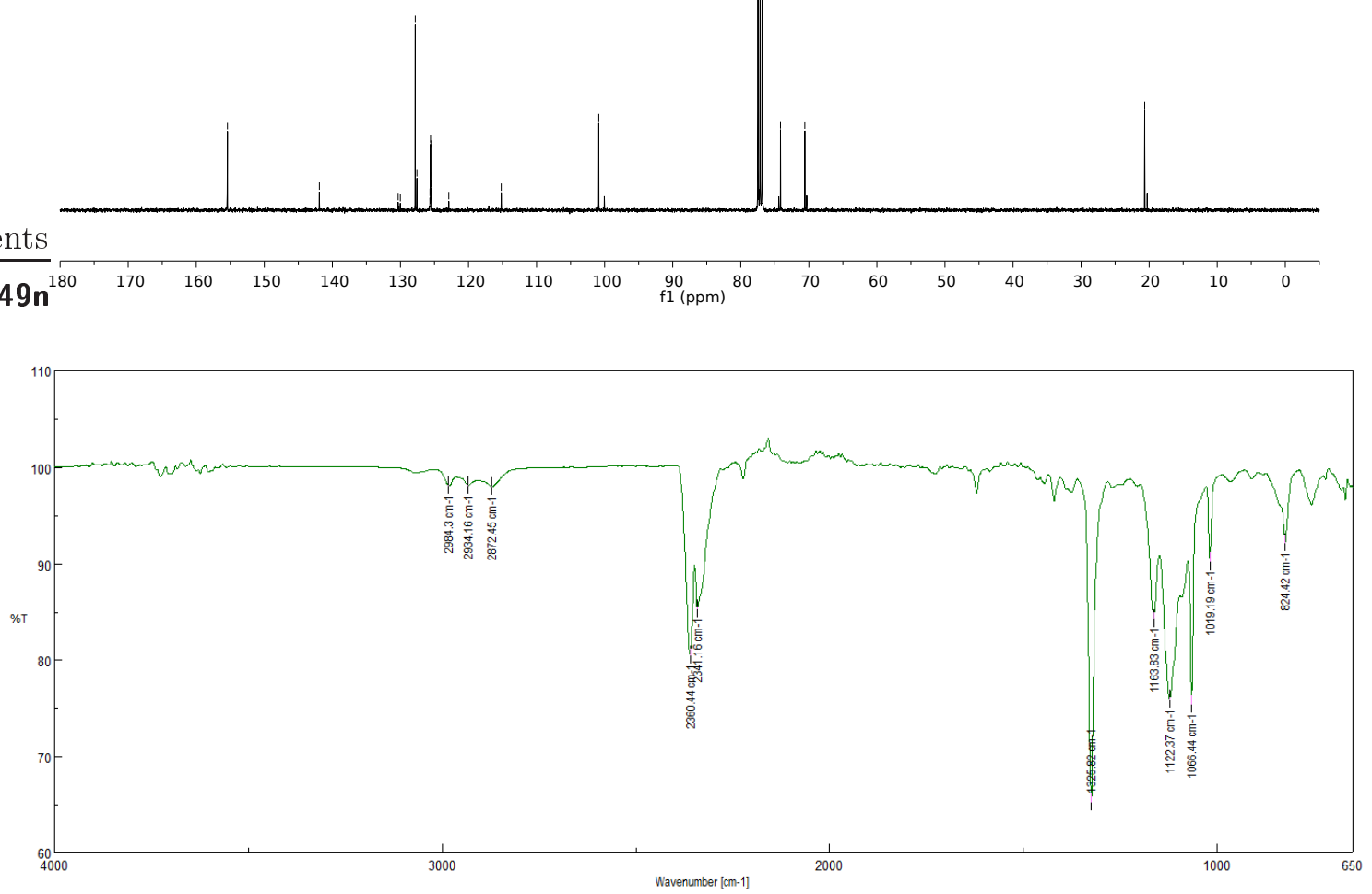


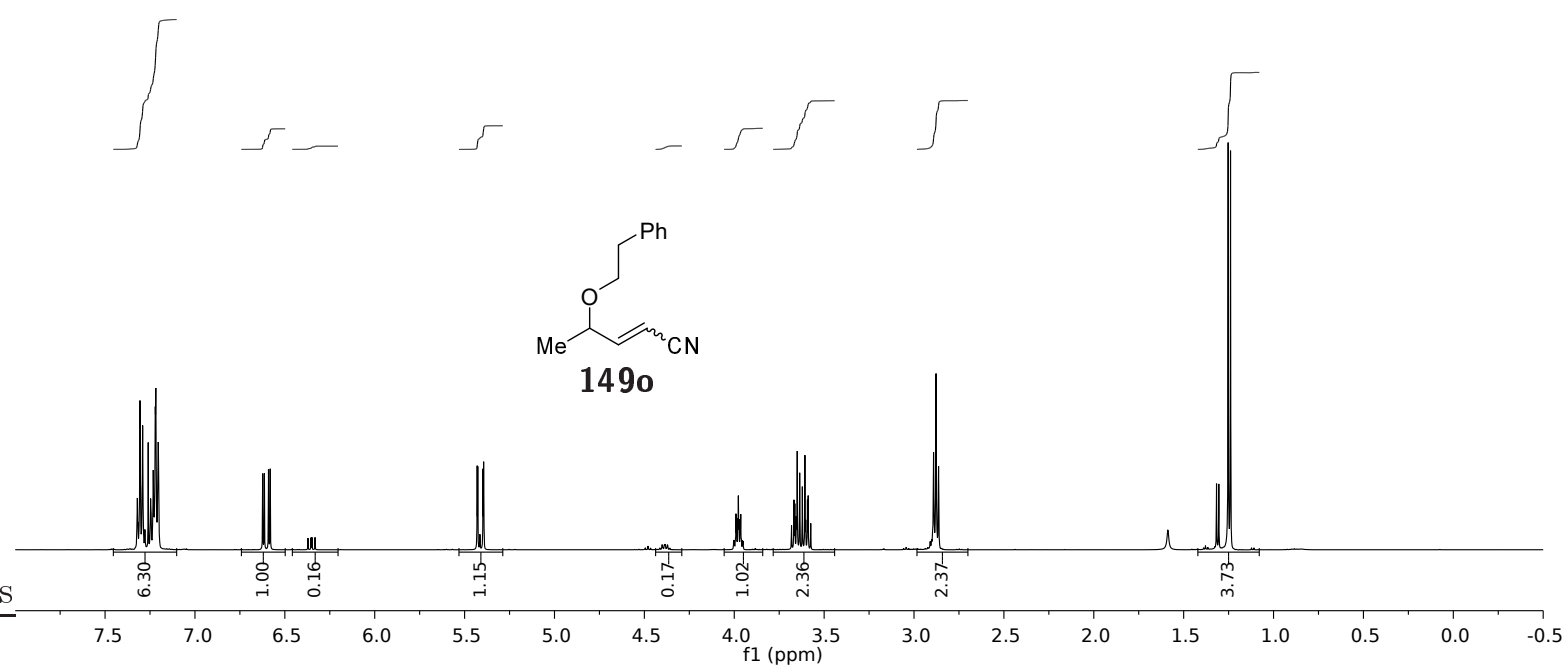

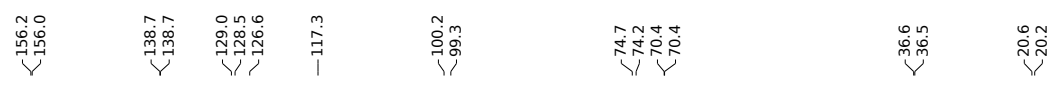
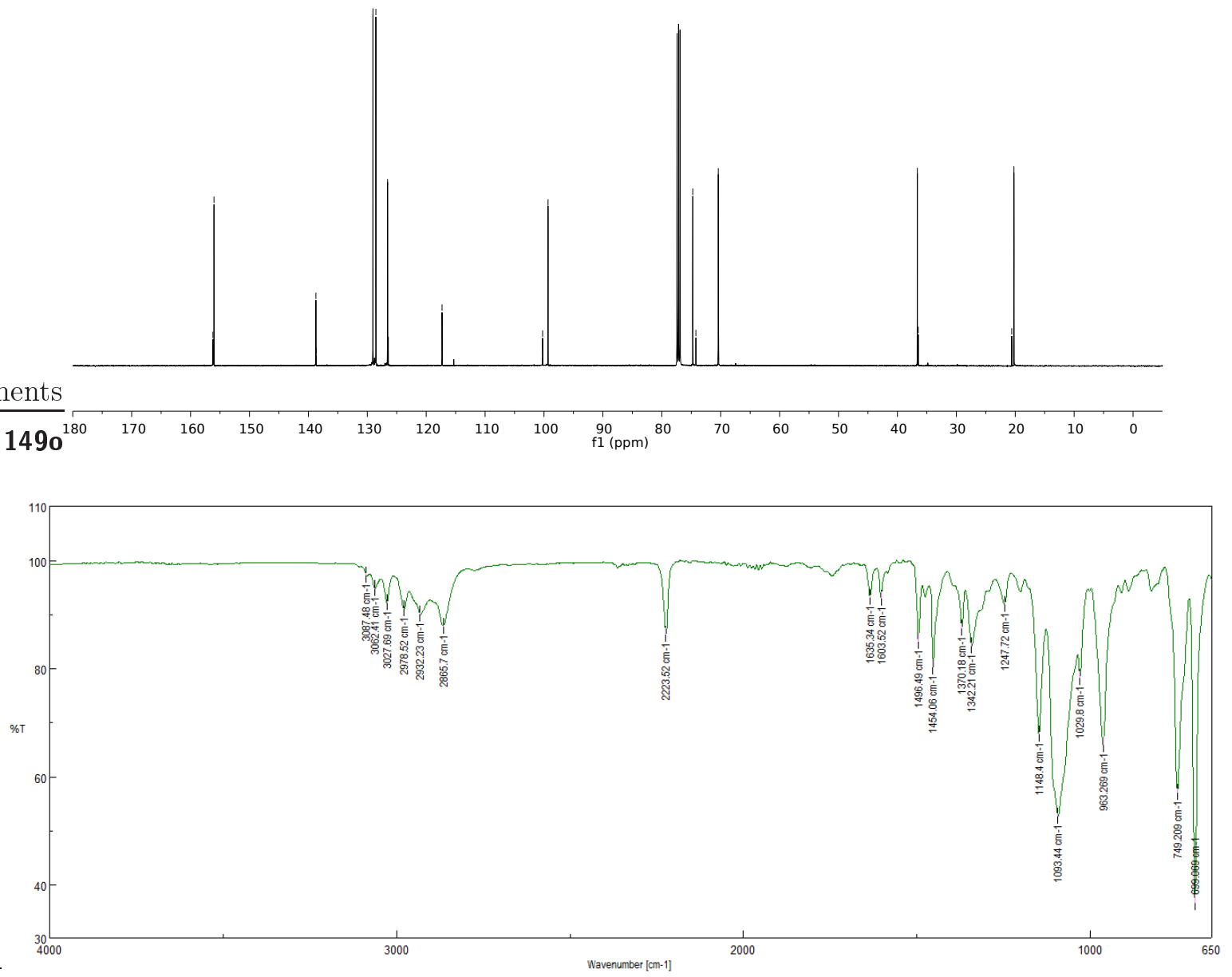


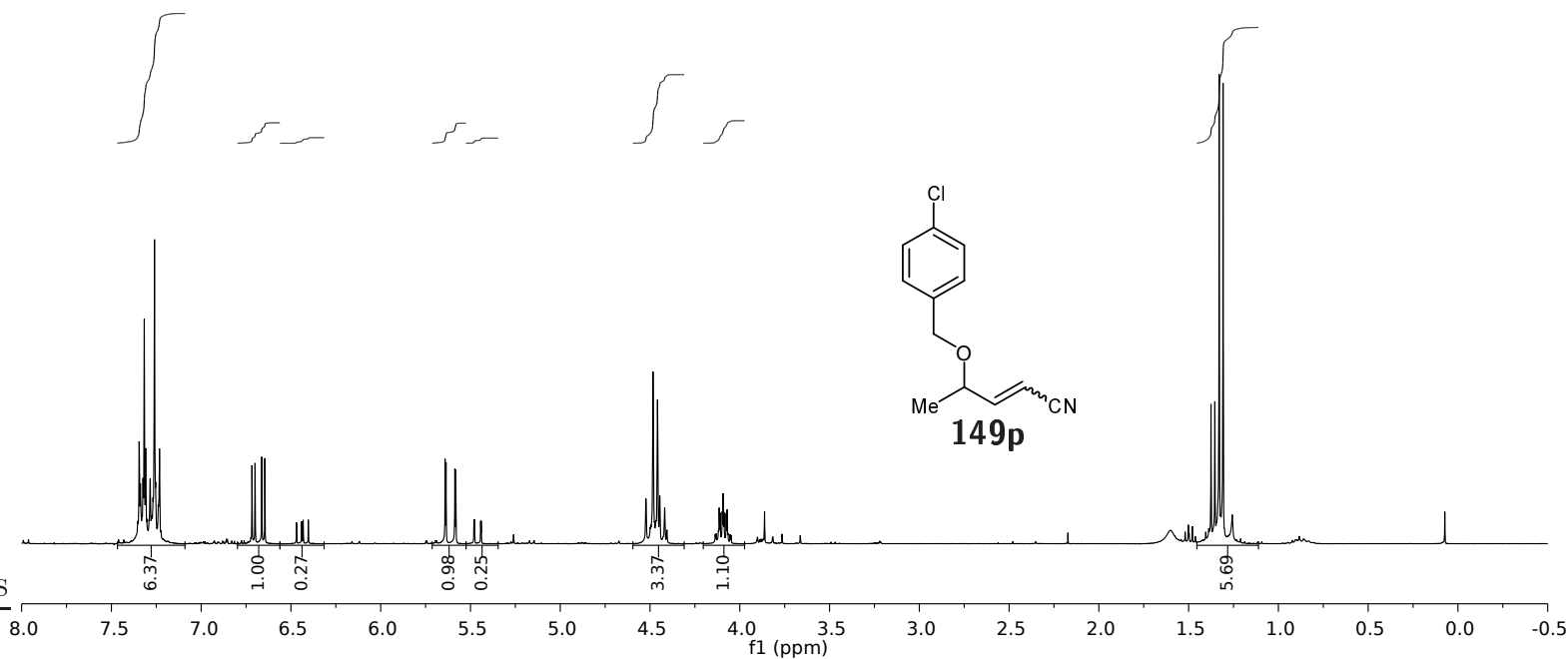

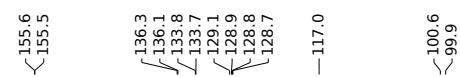

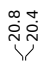
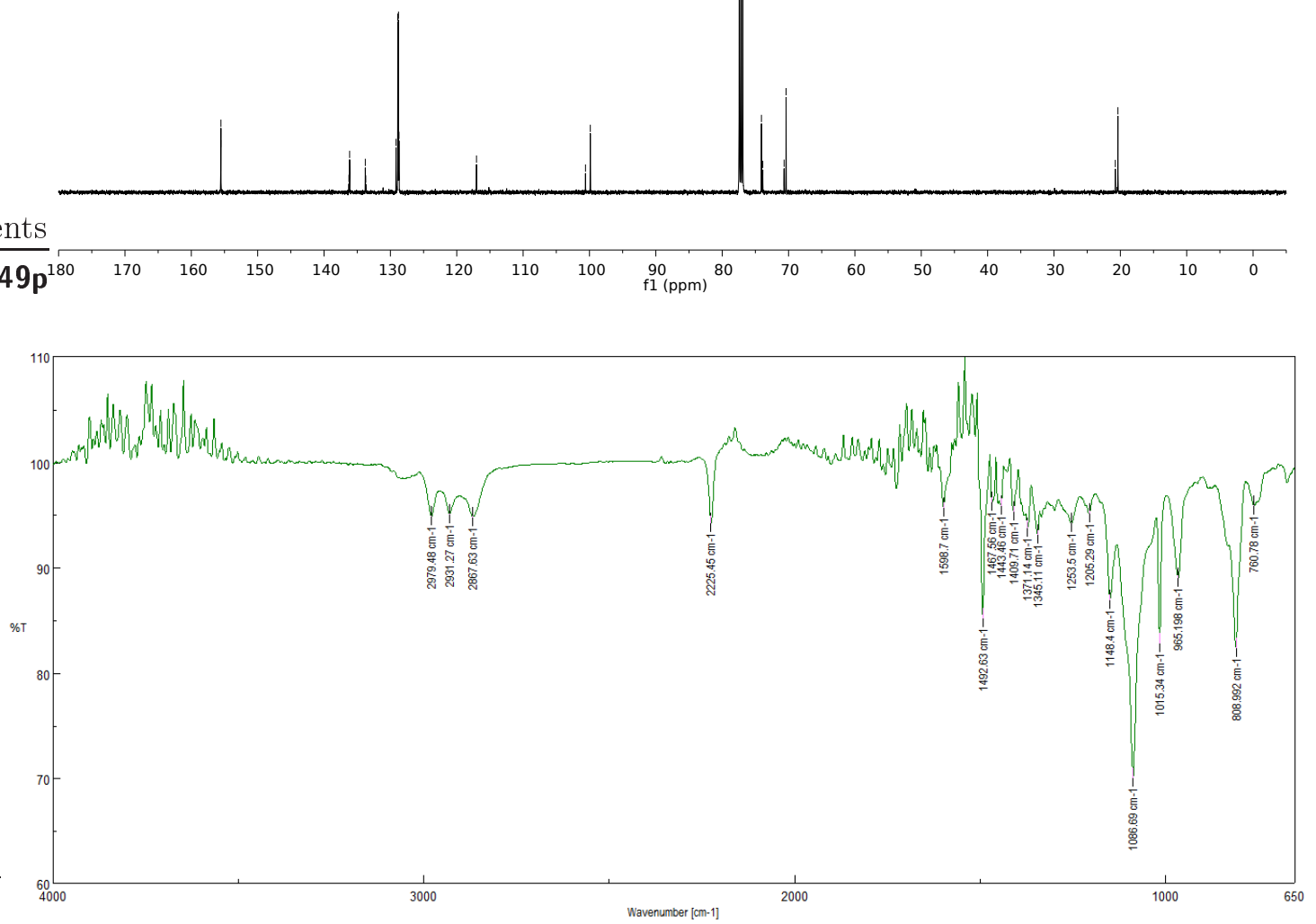


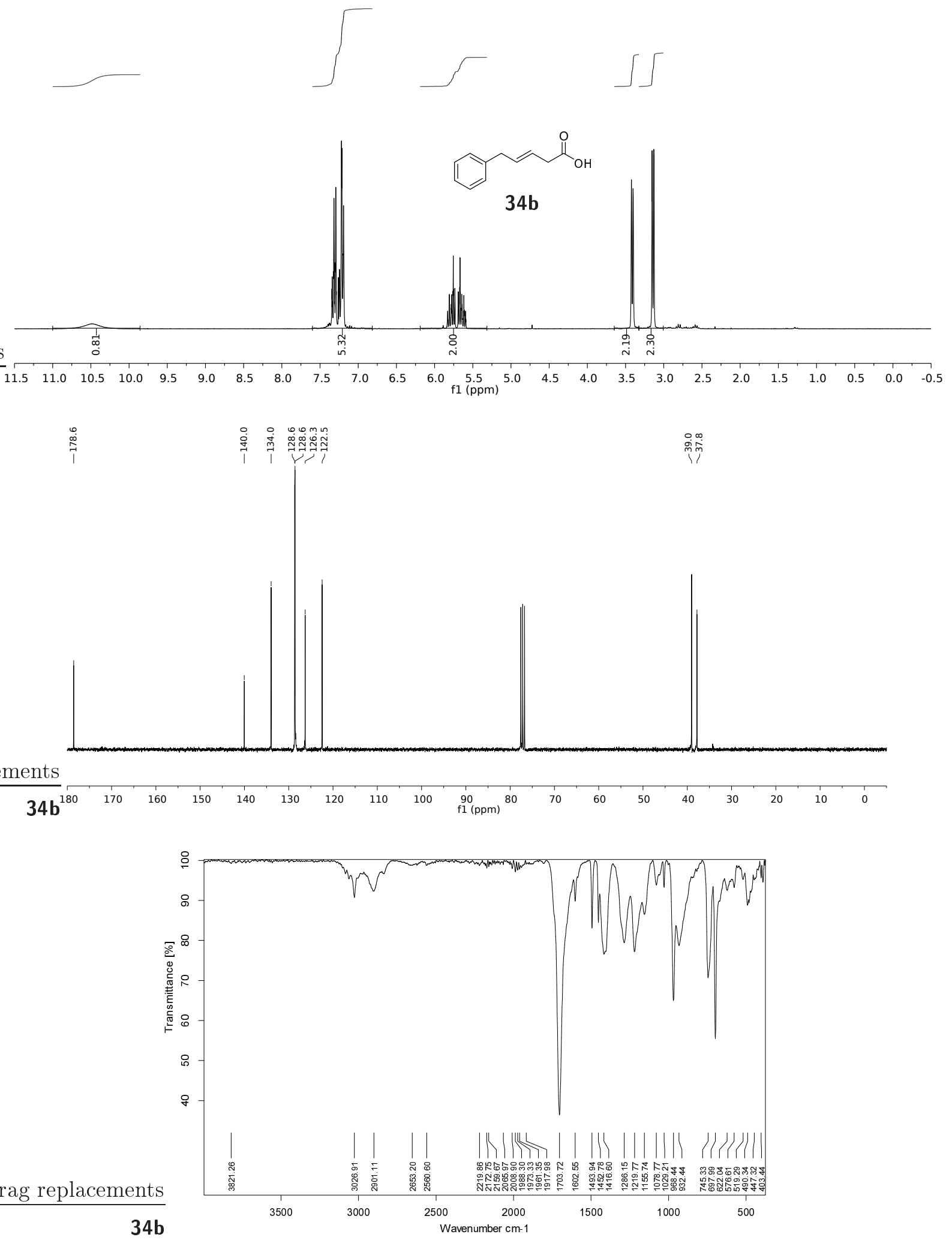




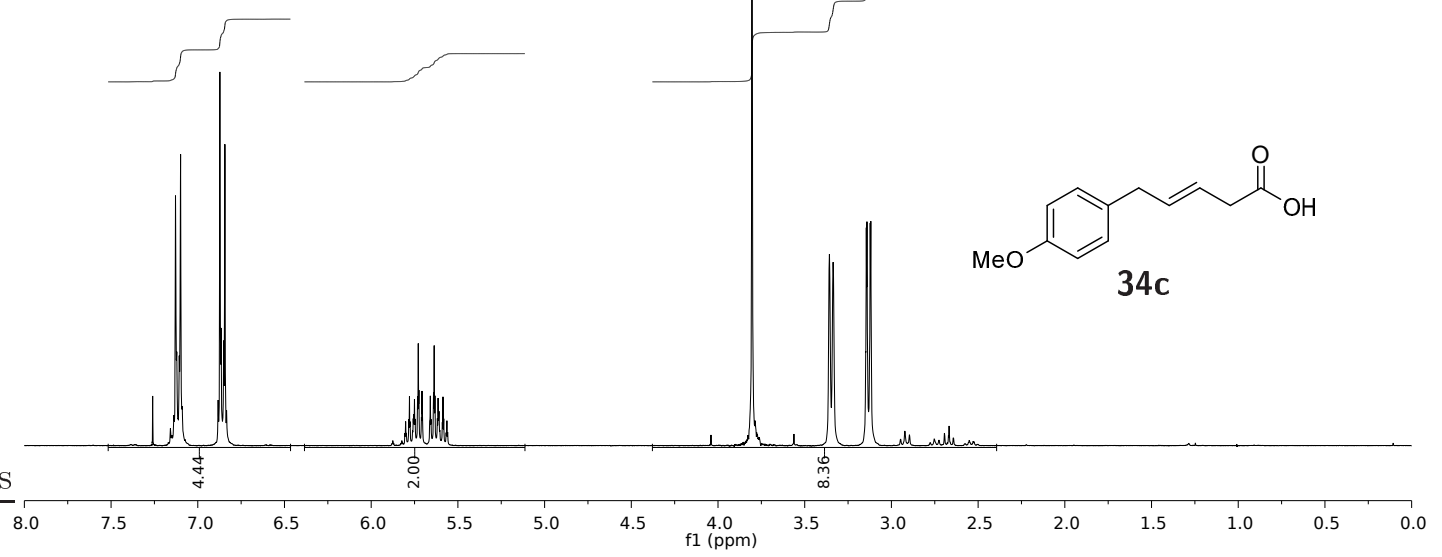

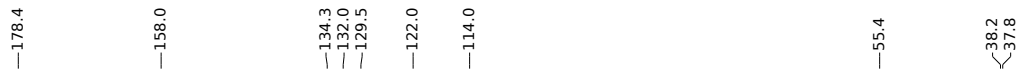
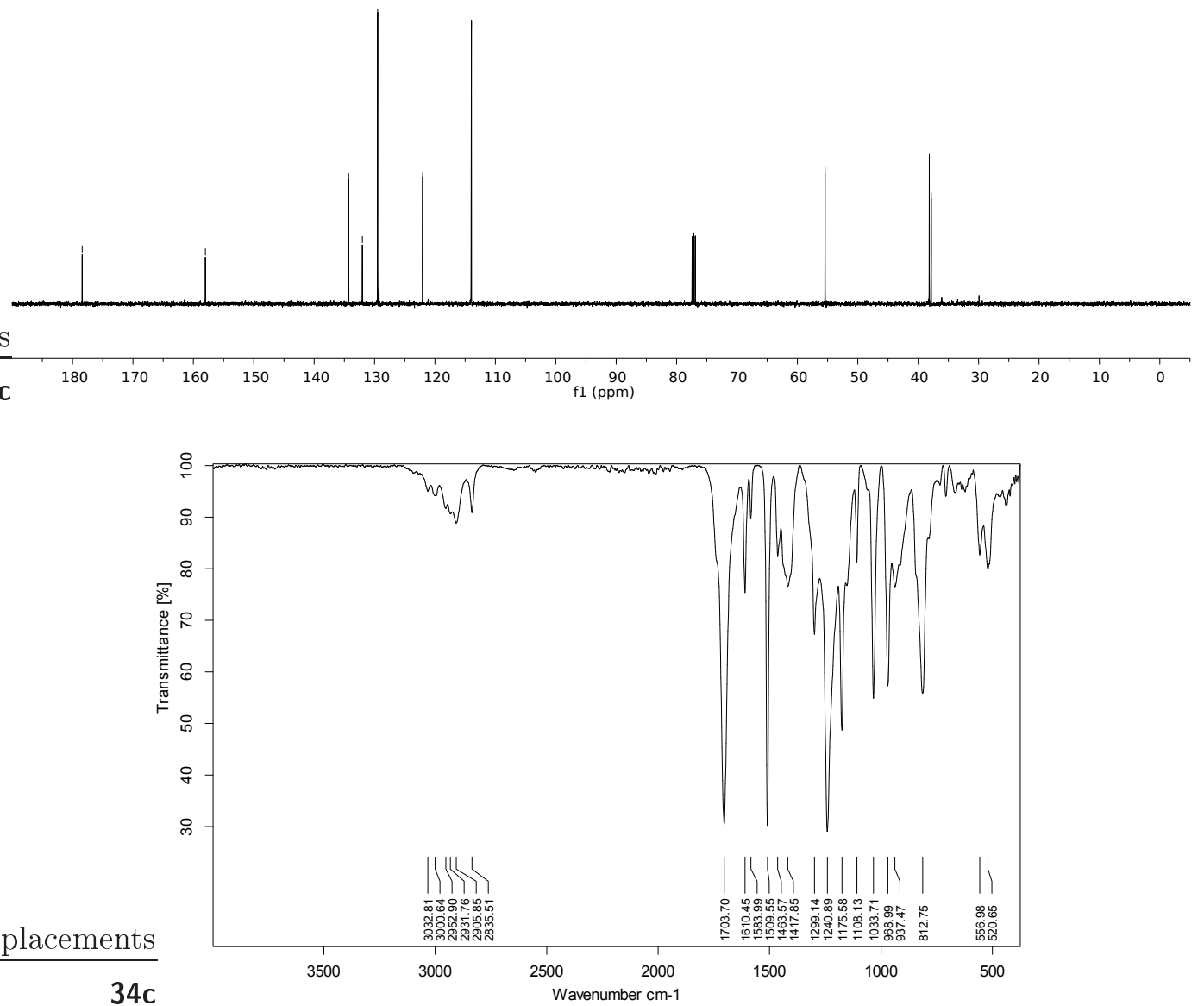


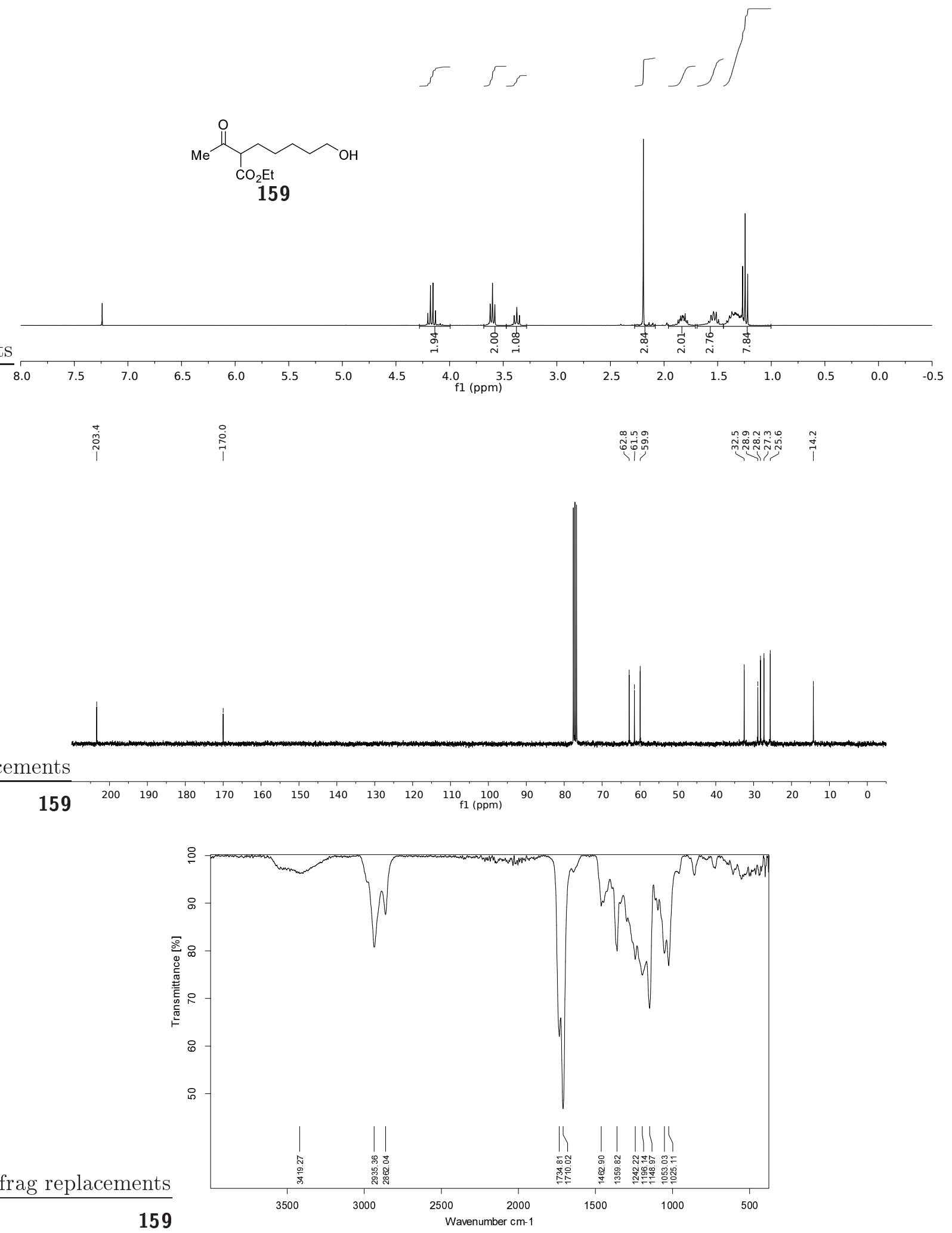




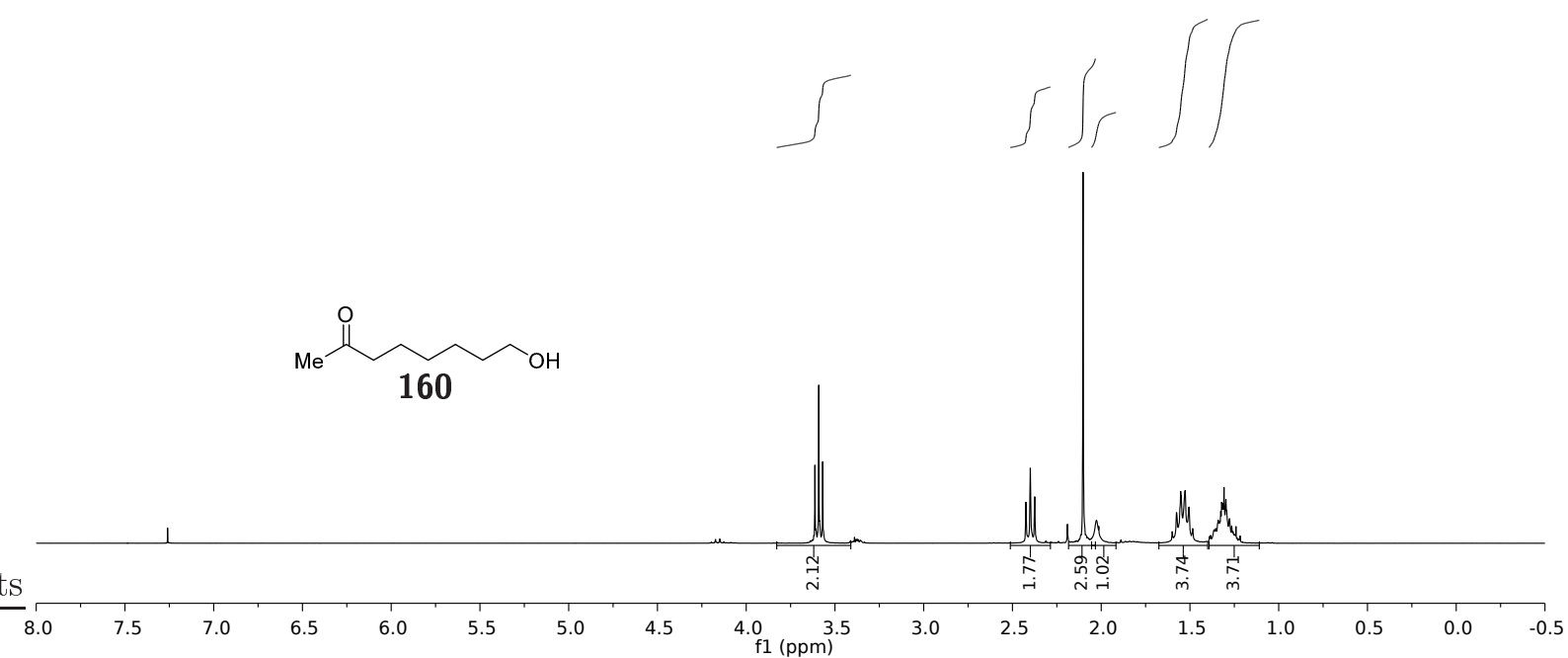

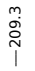

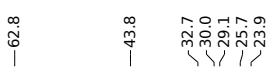
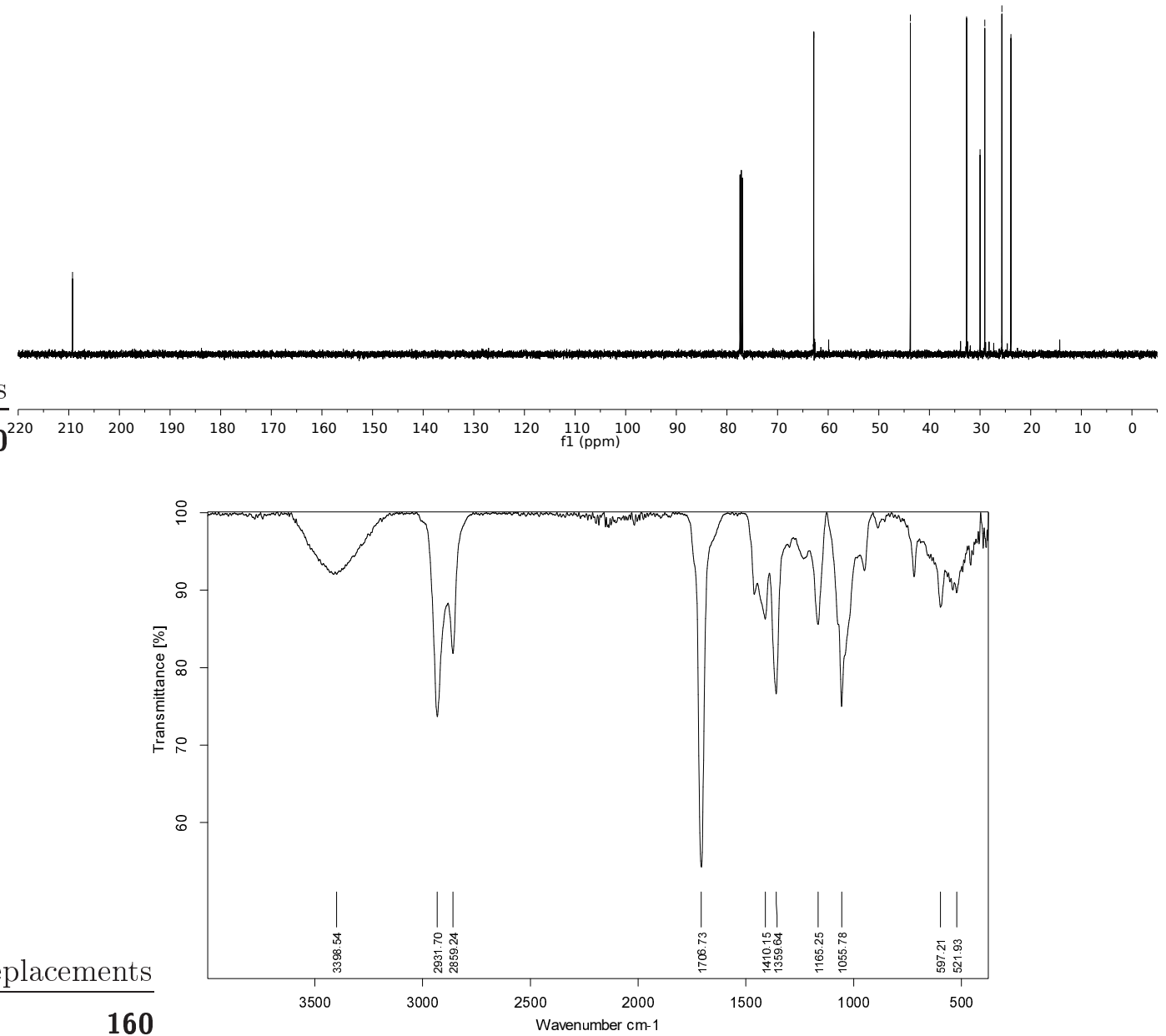


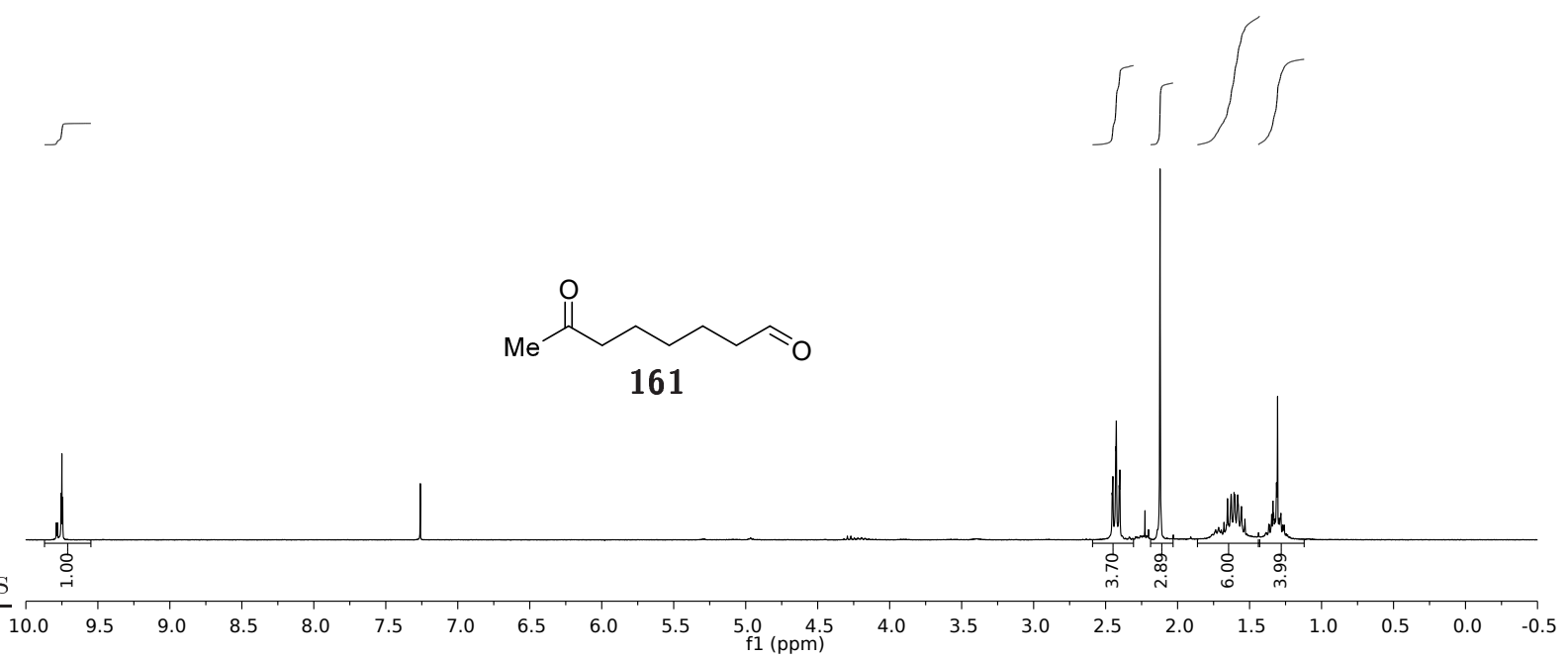




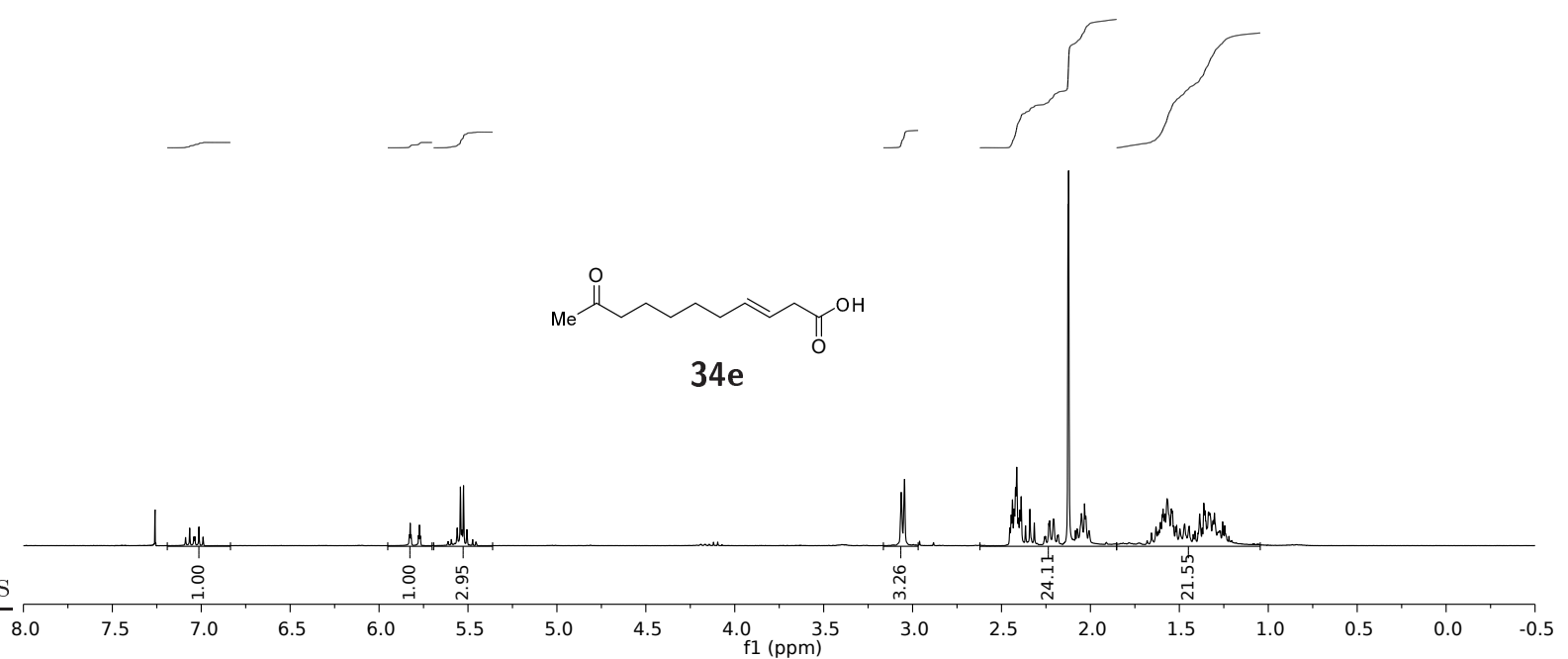

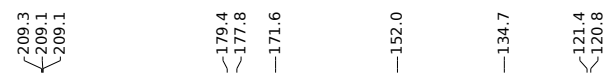

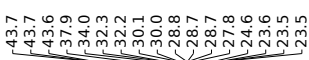

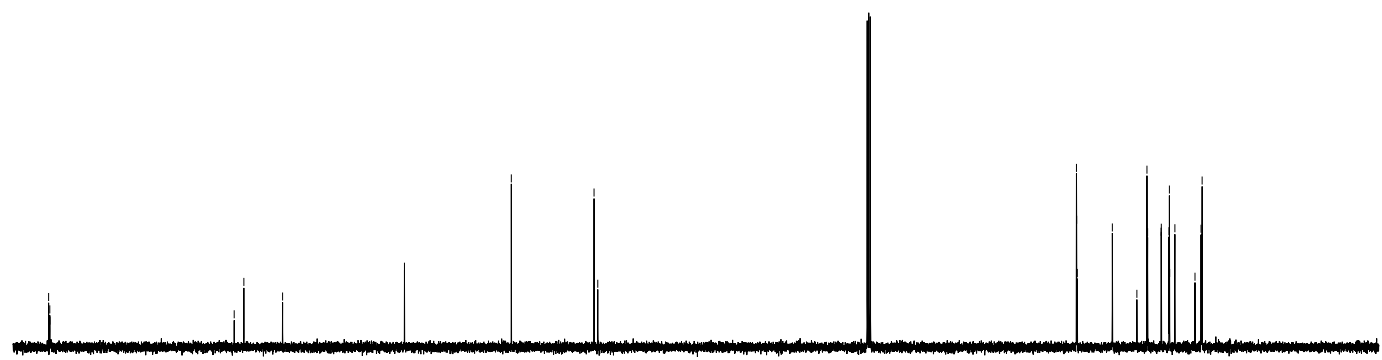

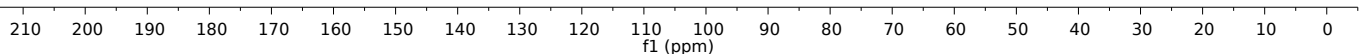

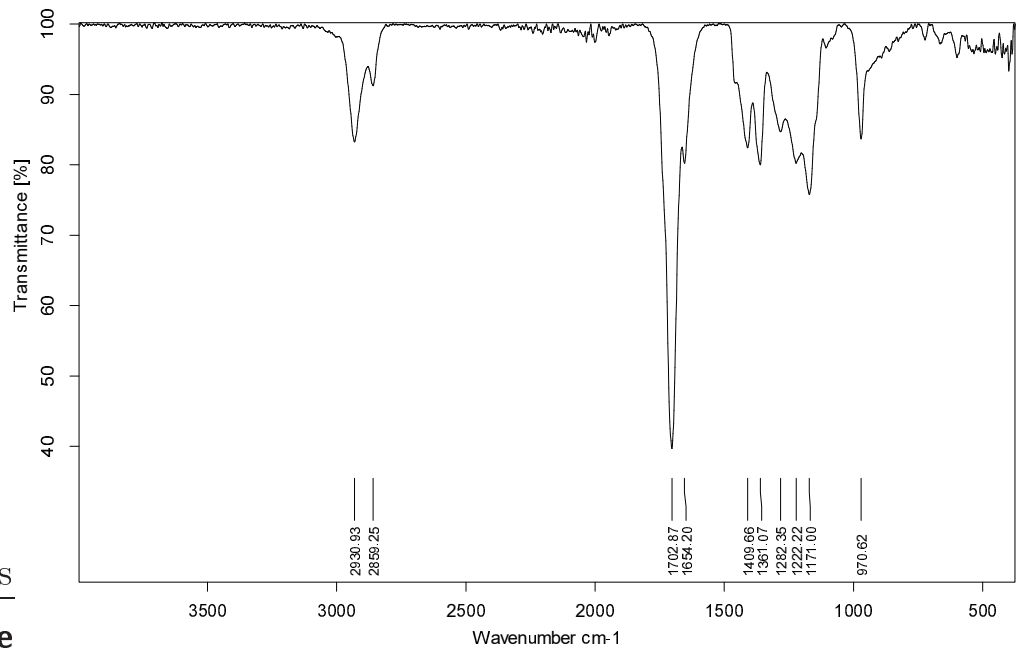




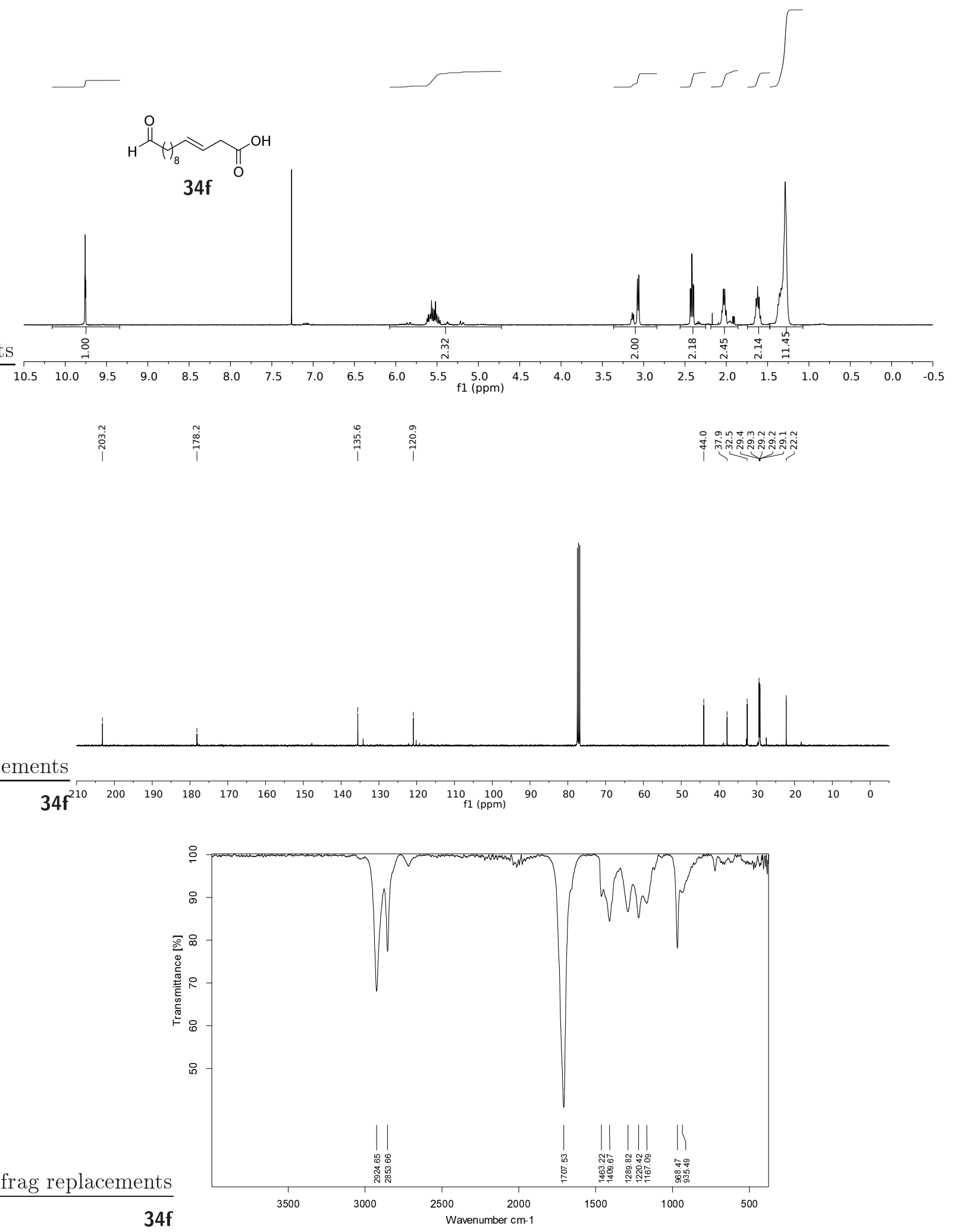



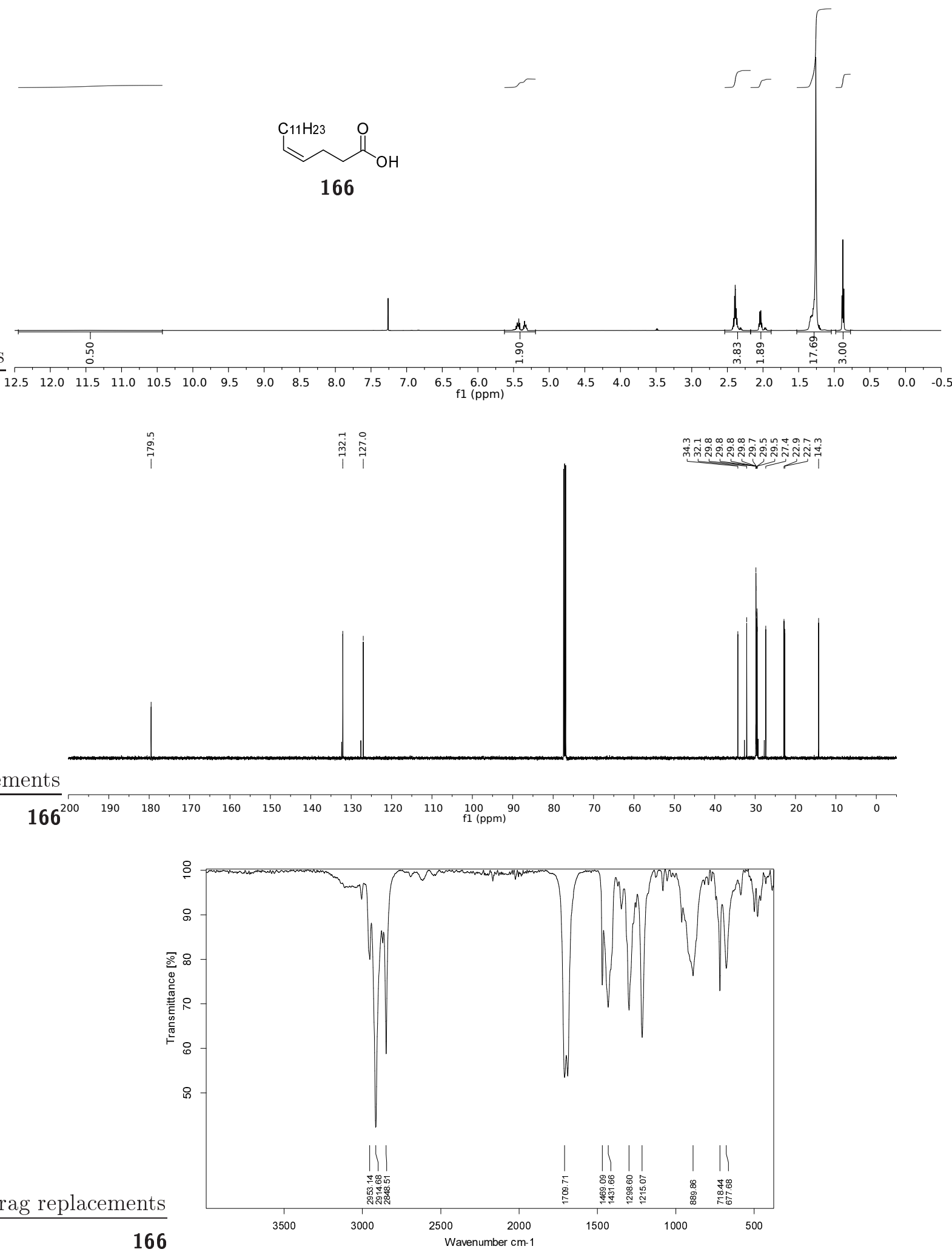

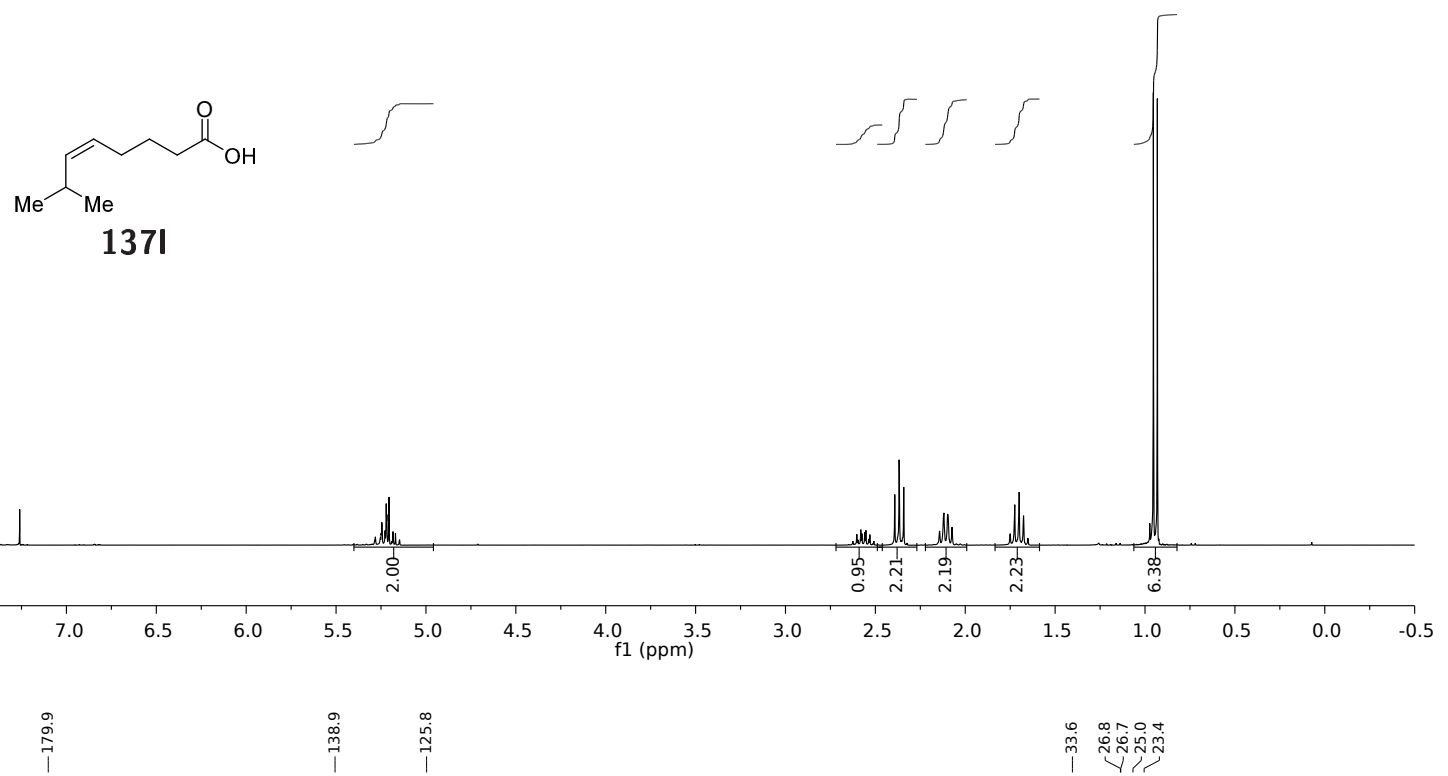

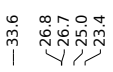
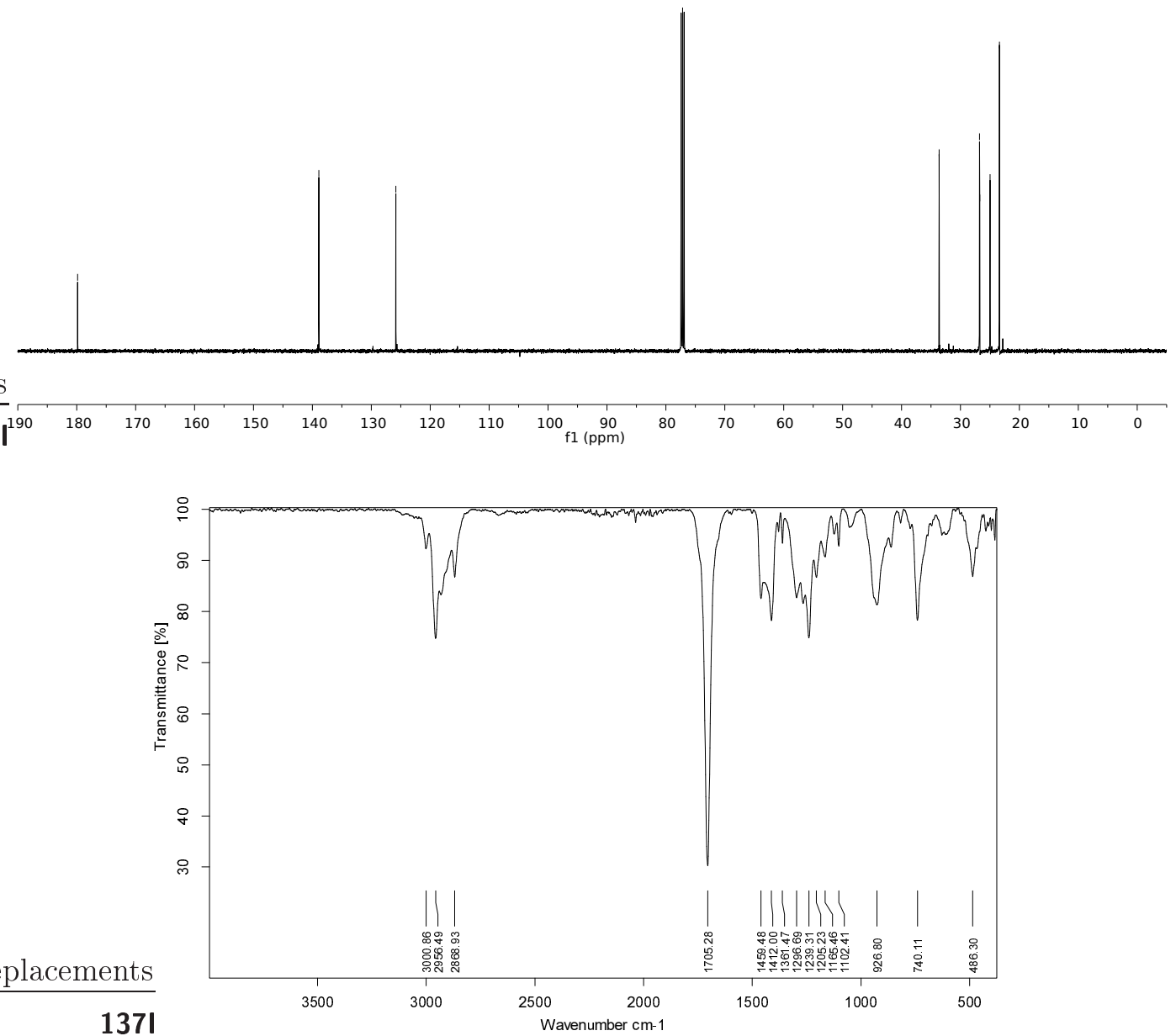

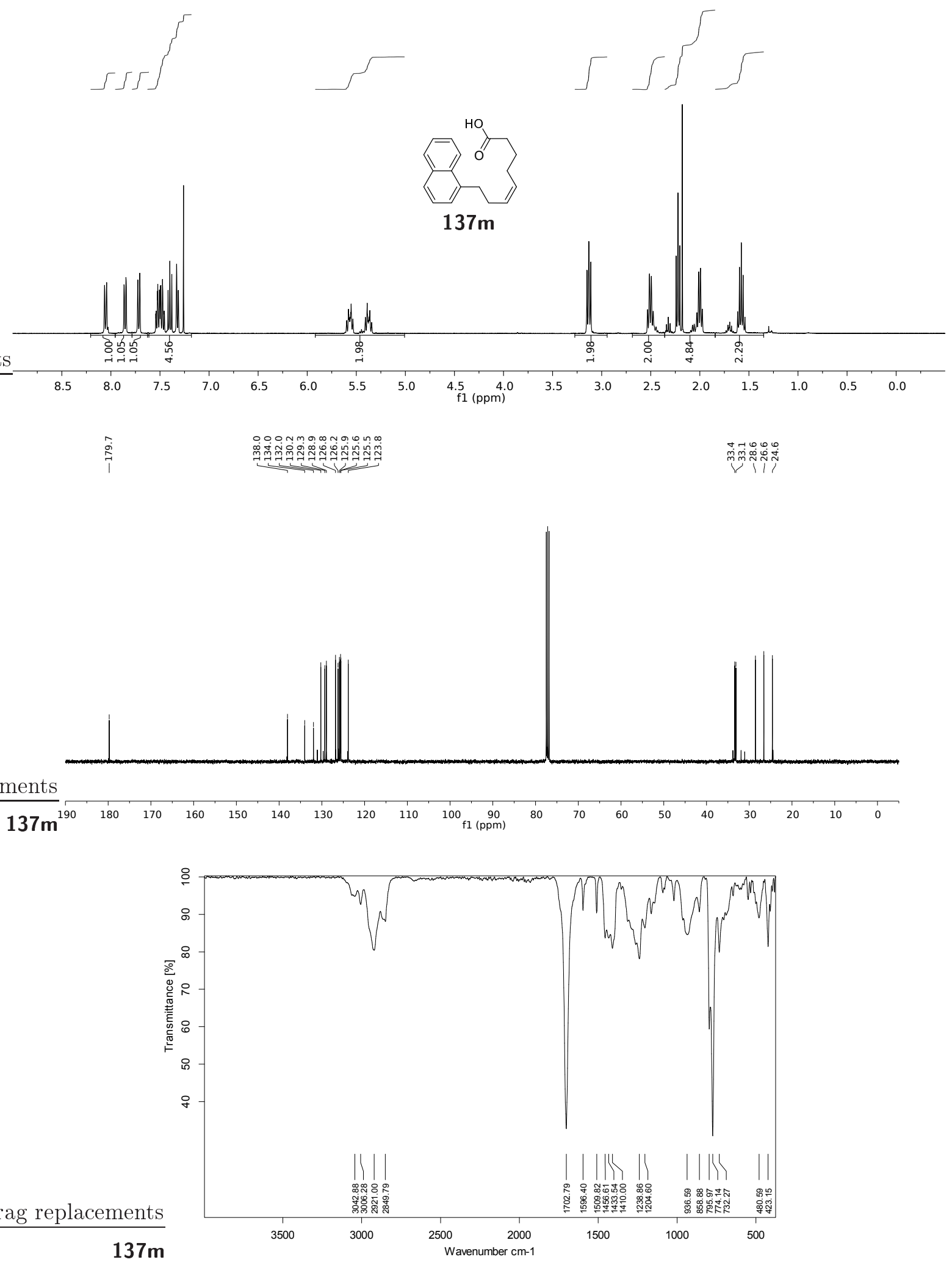


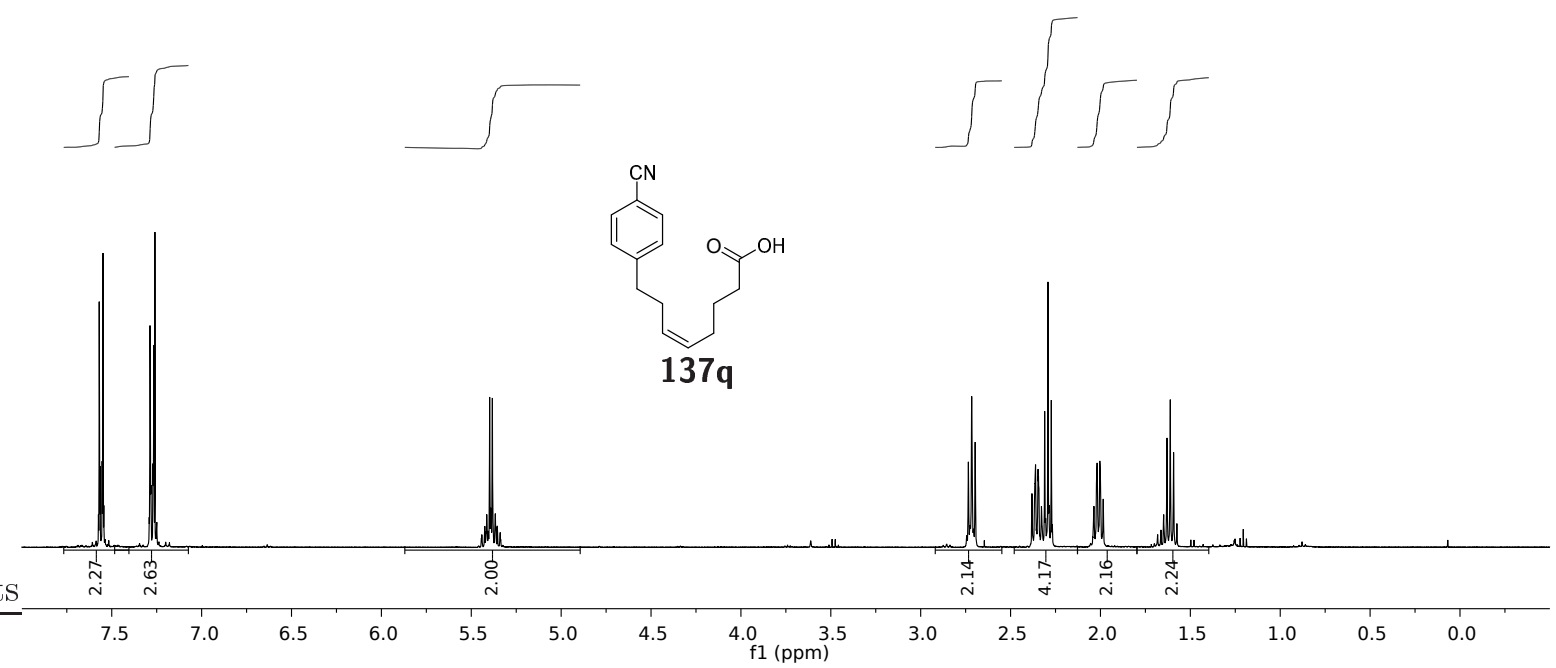

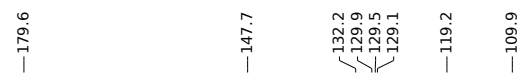

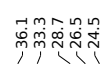
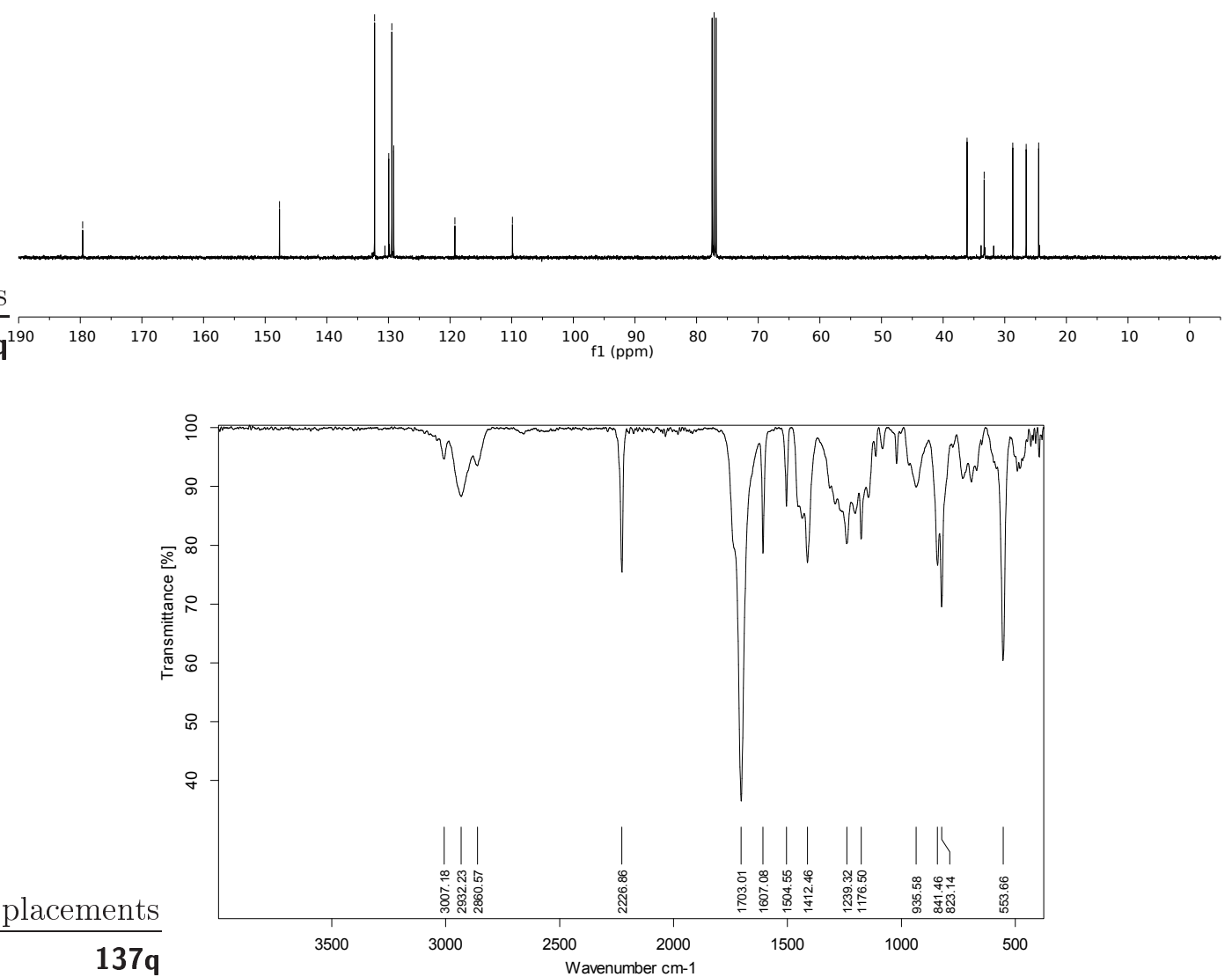

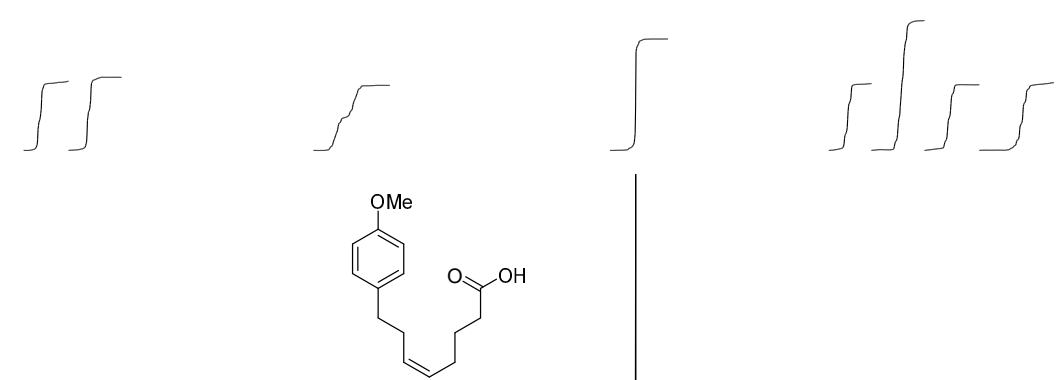

$137 r$

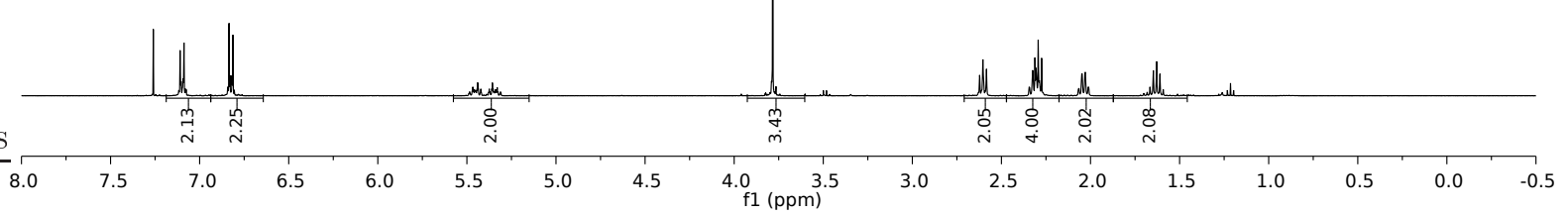

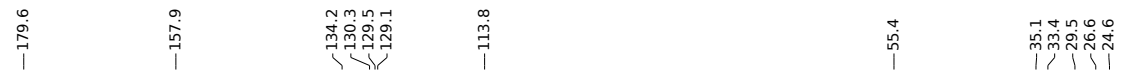
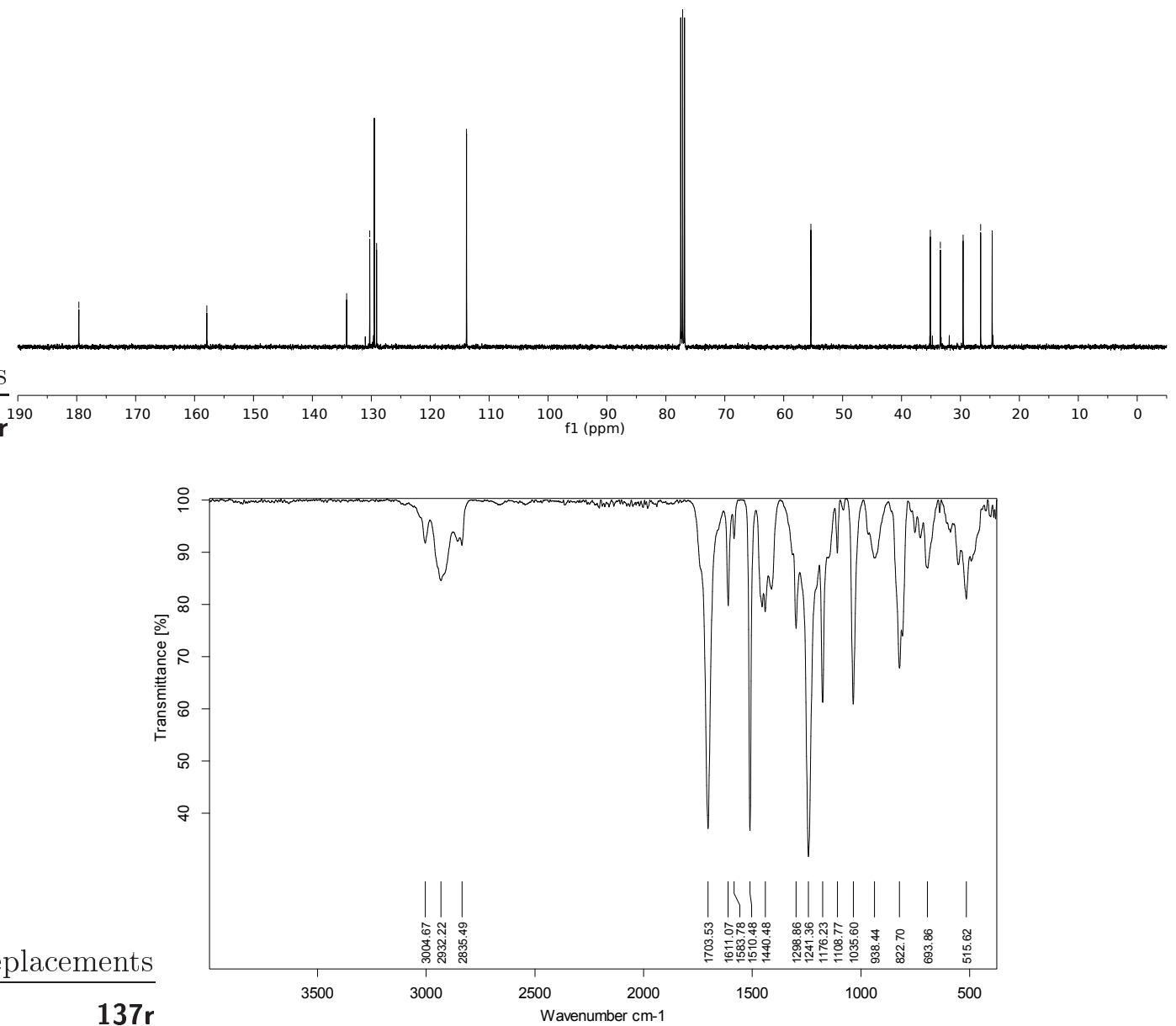

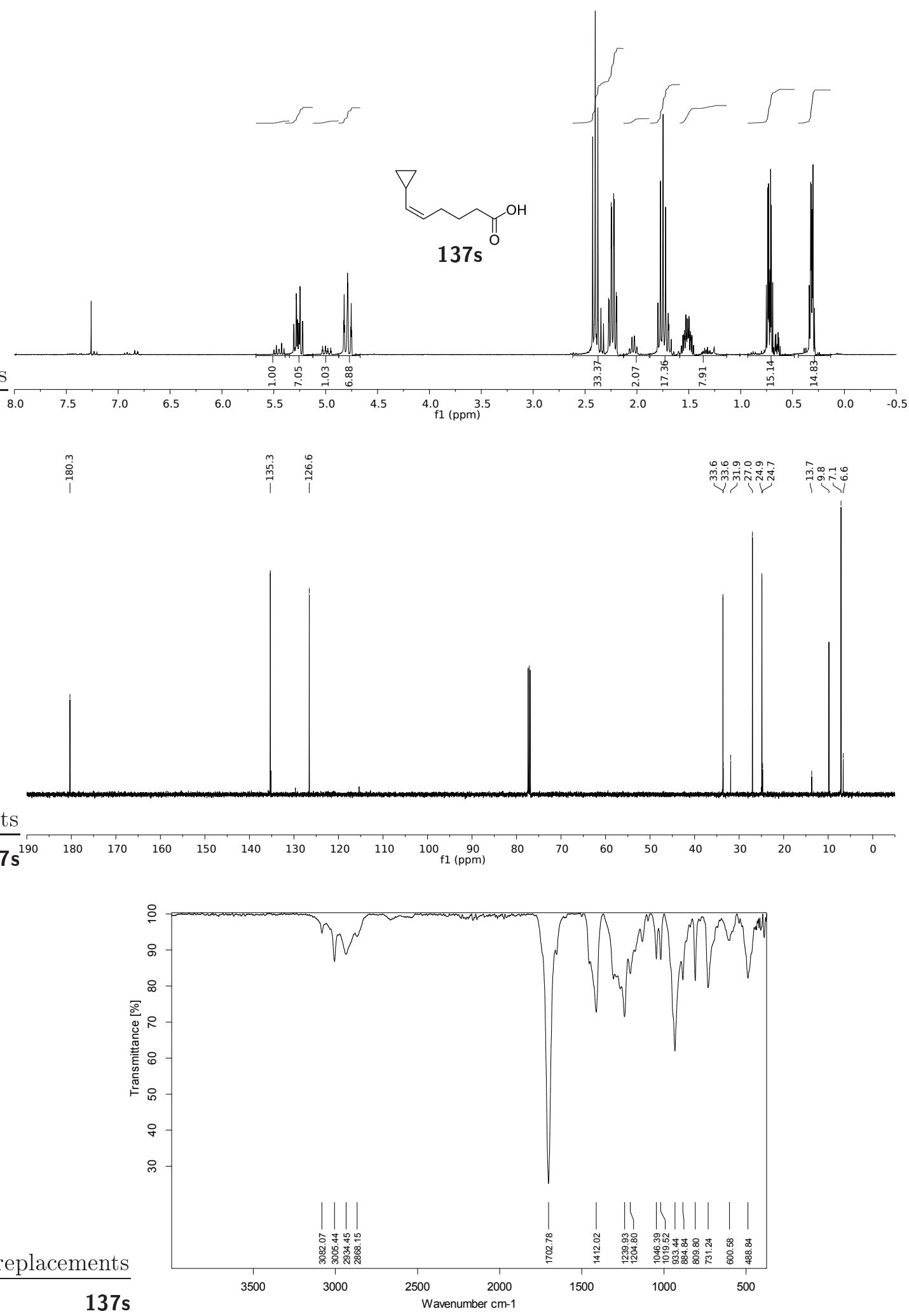


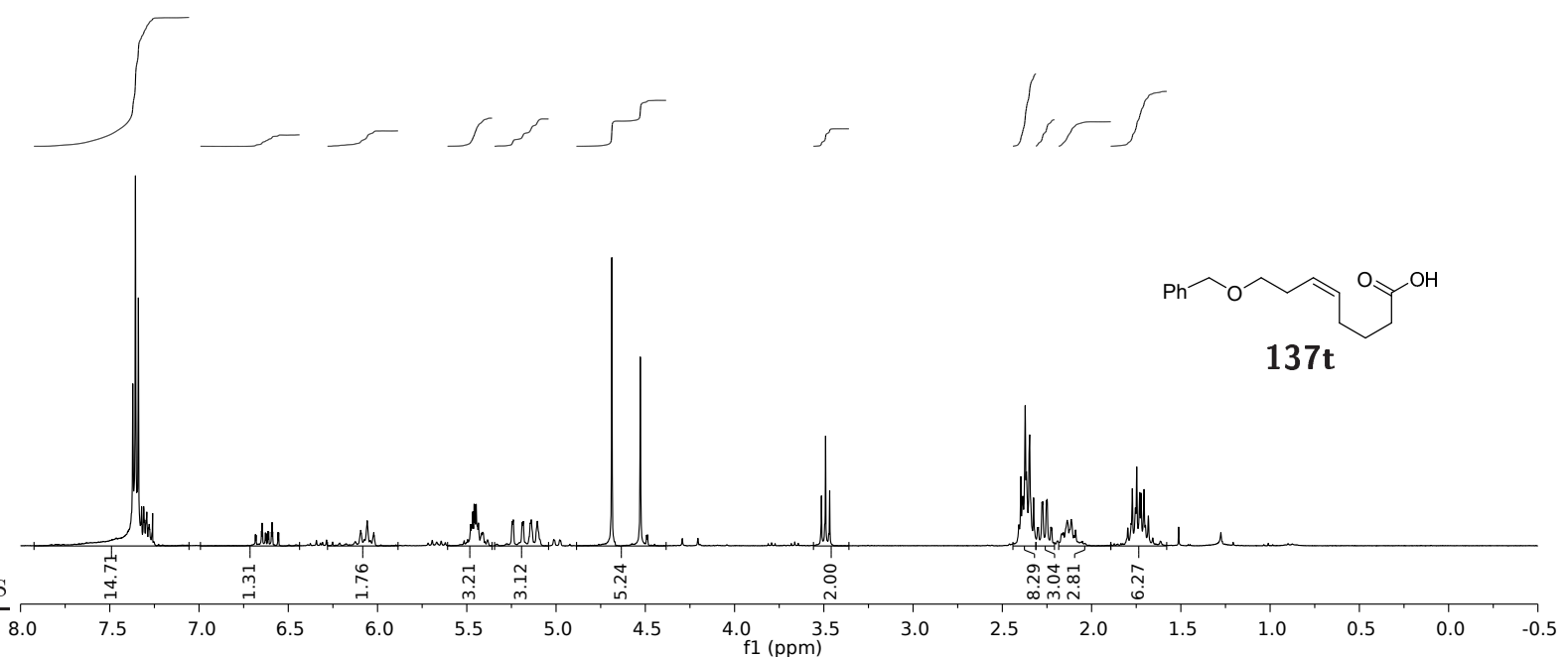

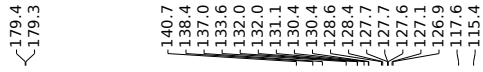

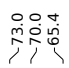

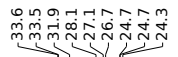
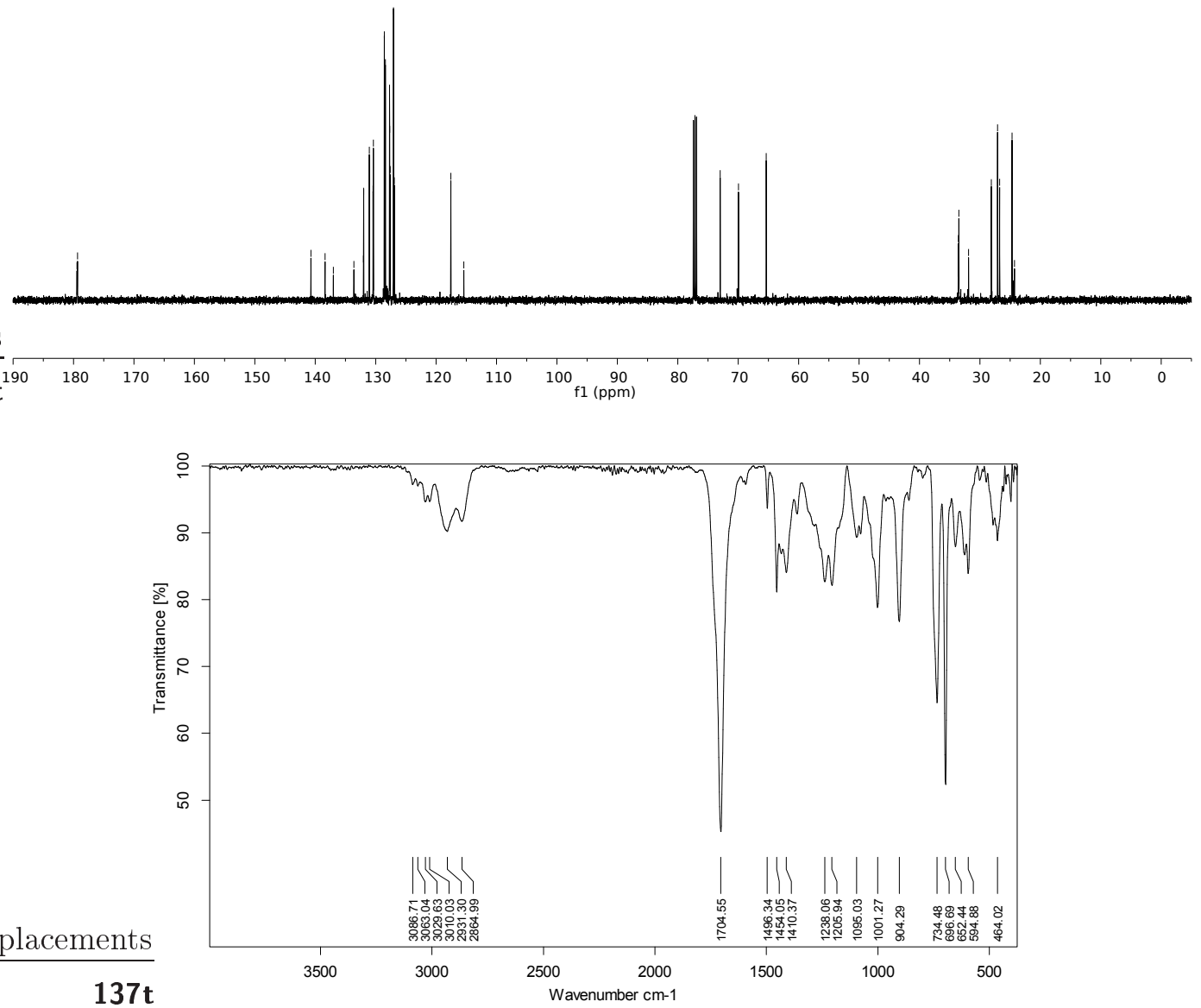

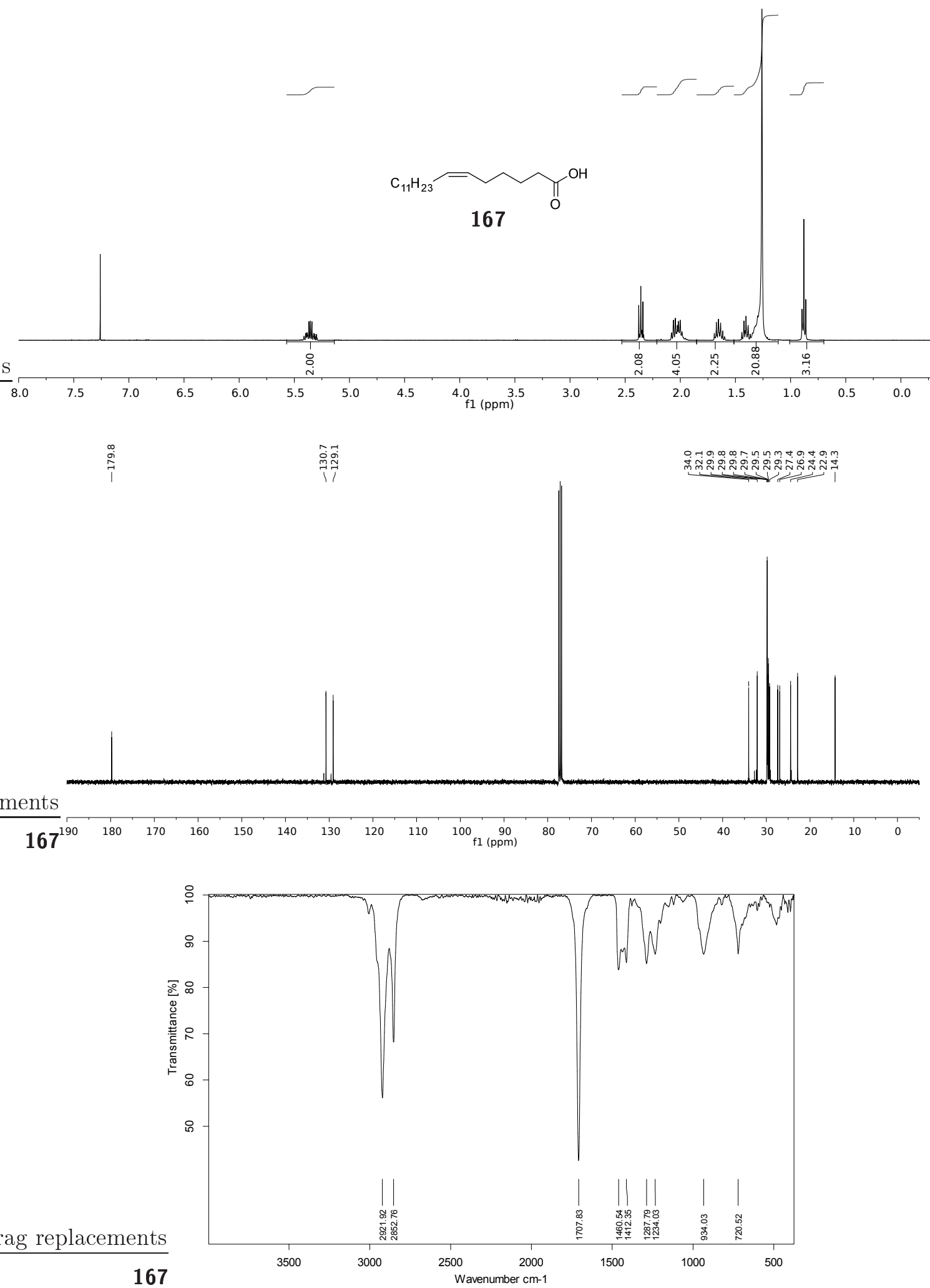


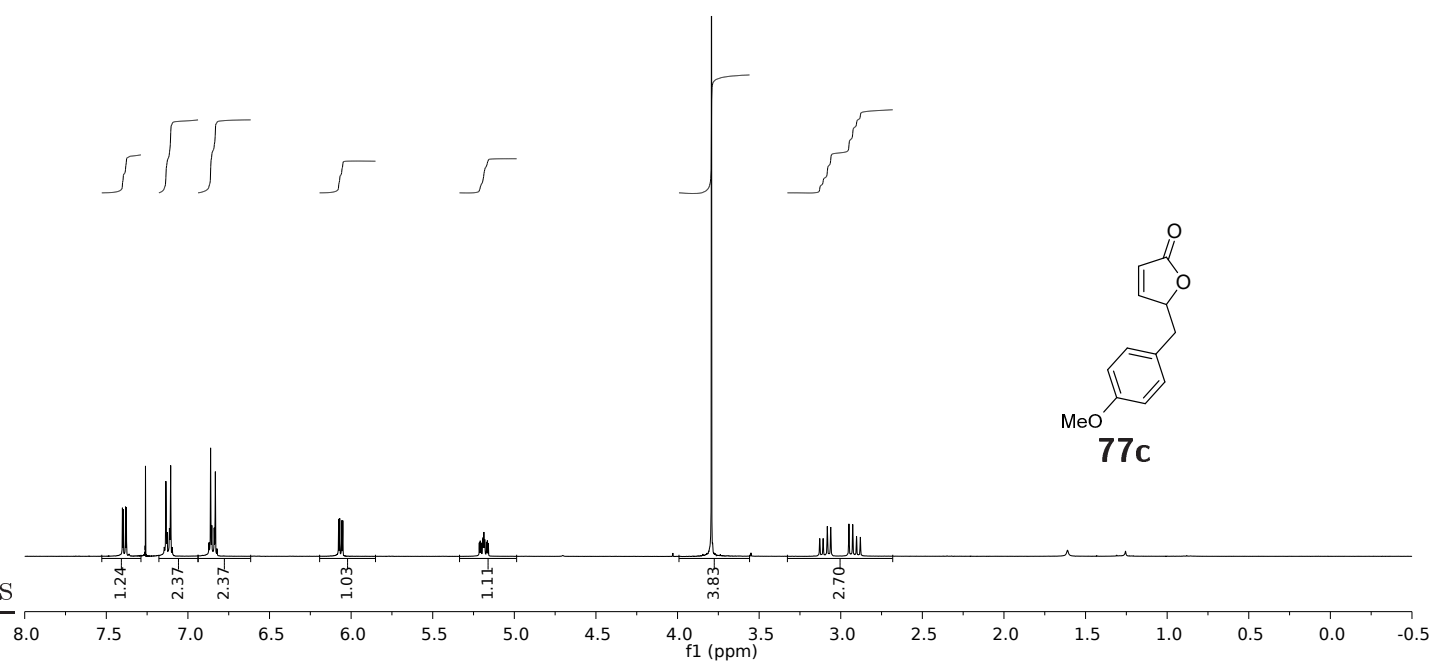

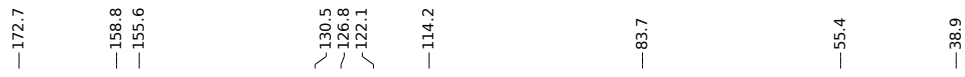
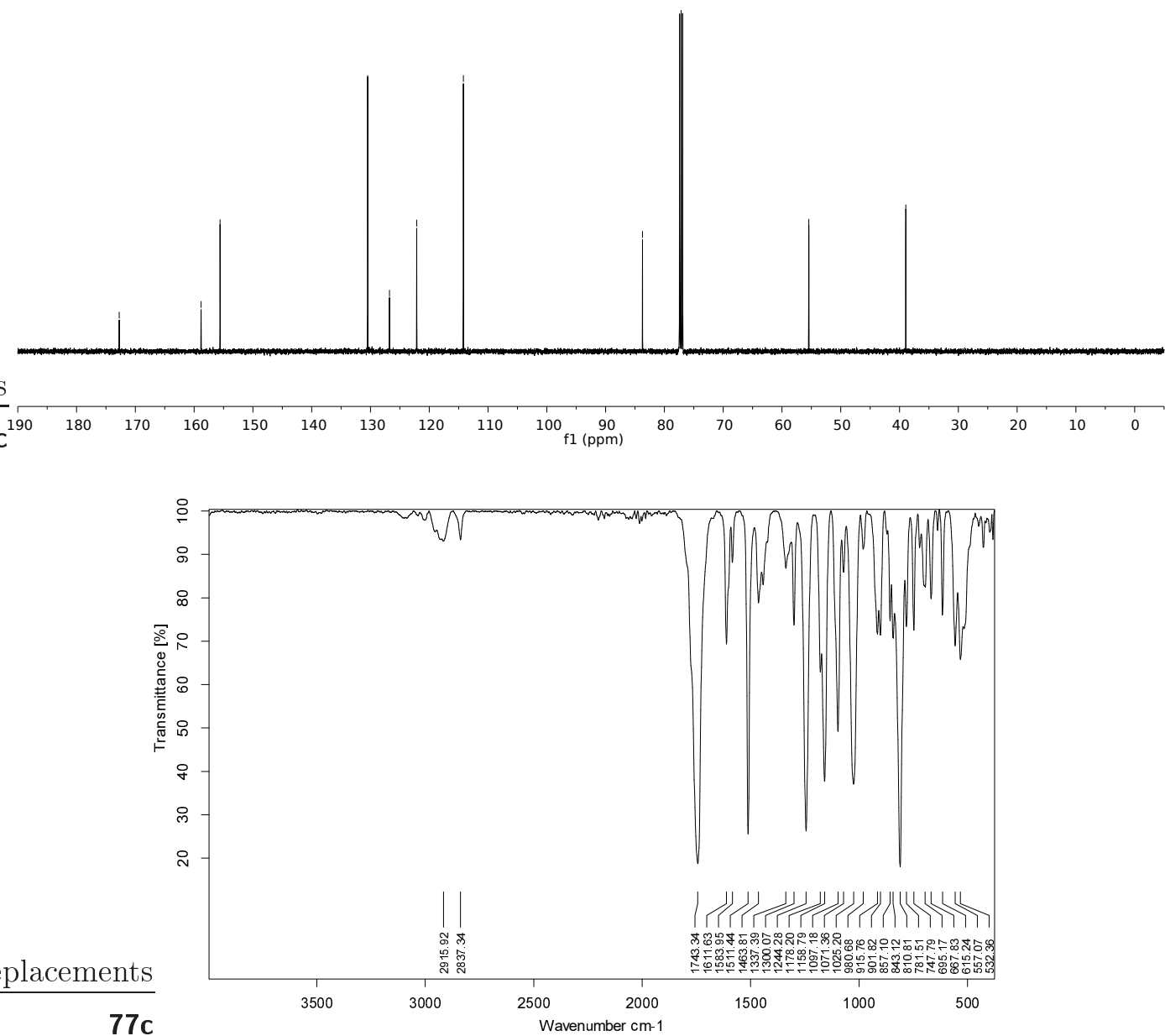


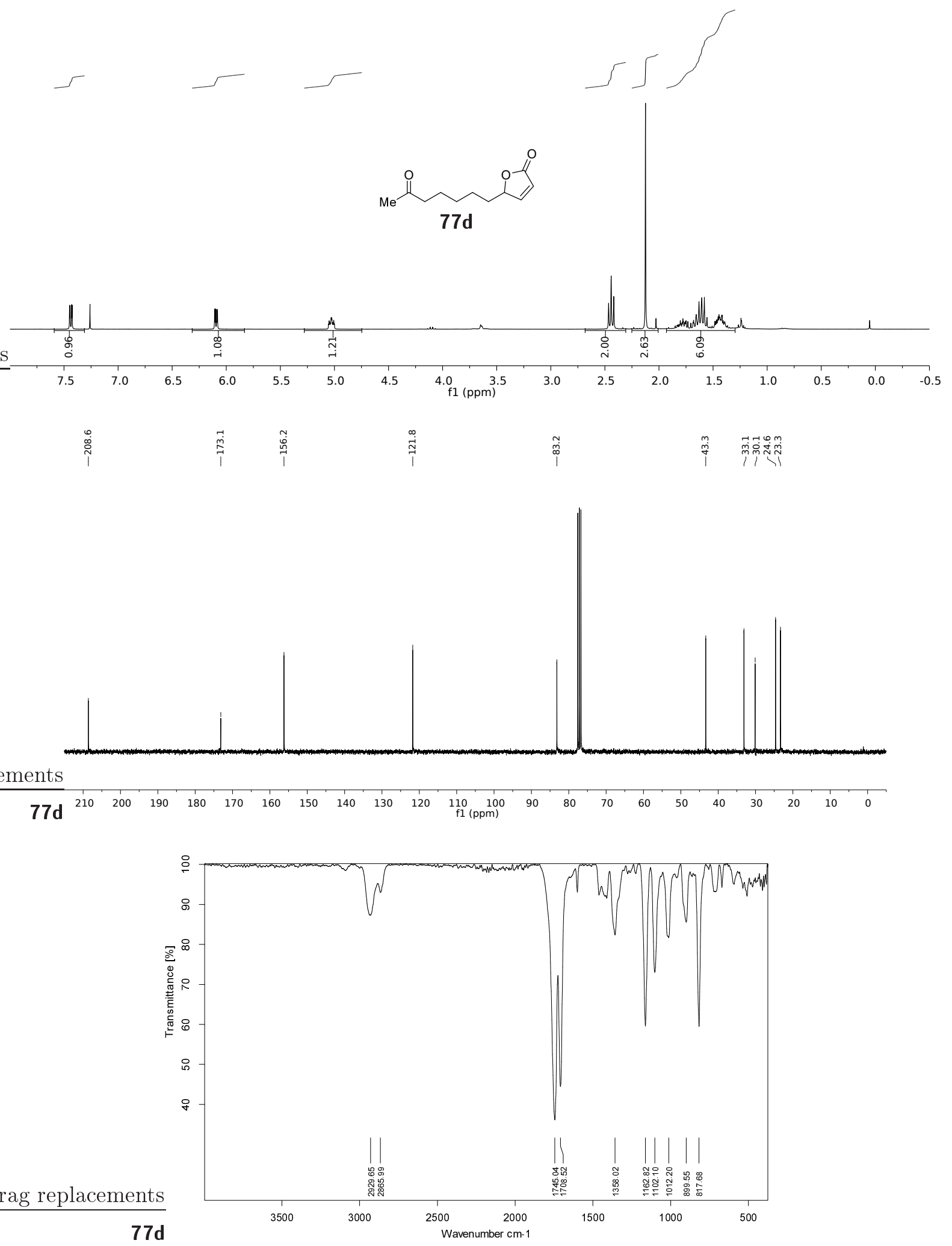




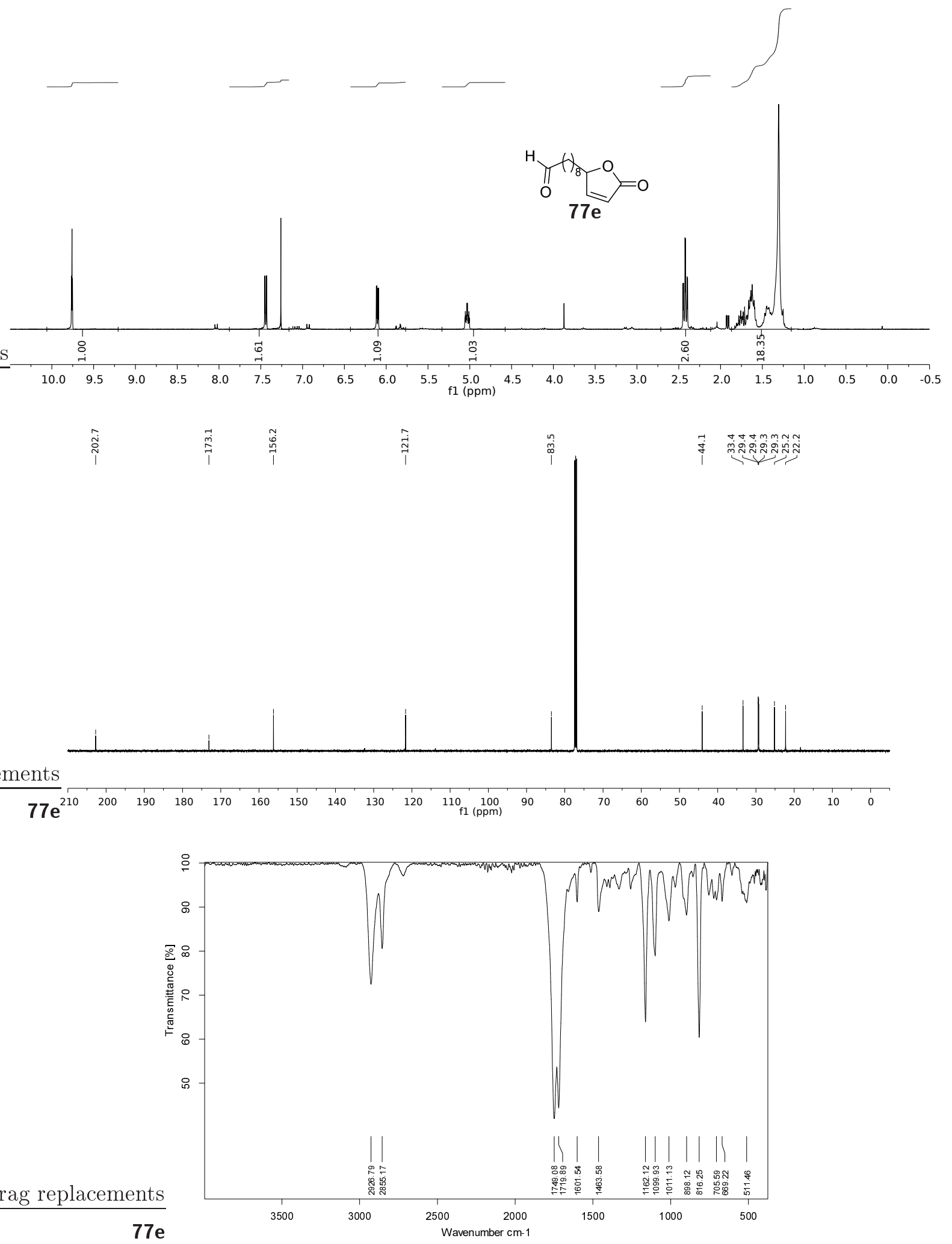




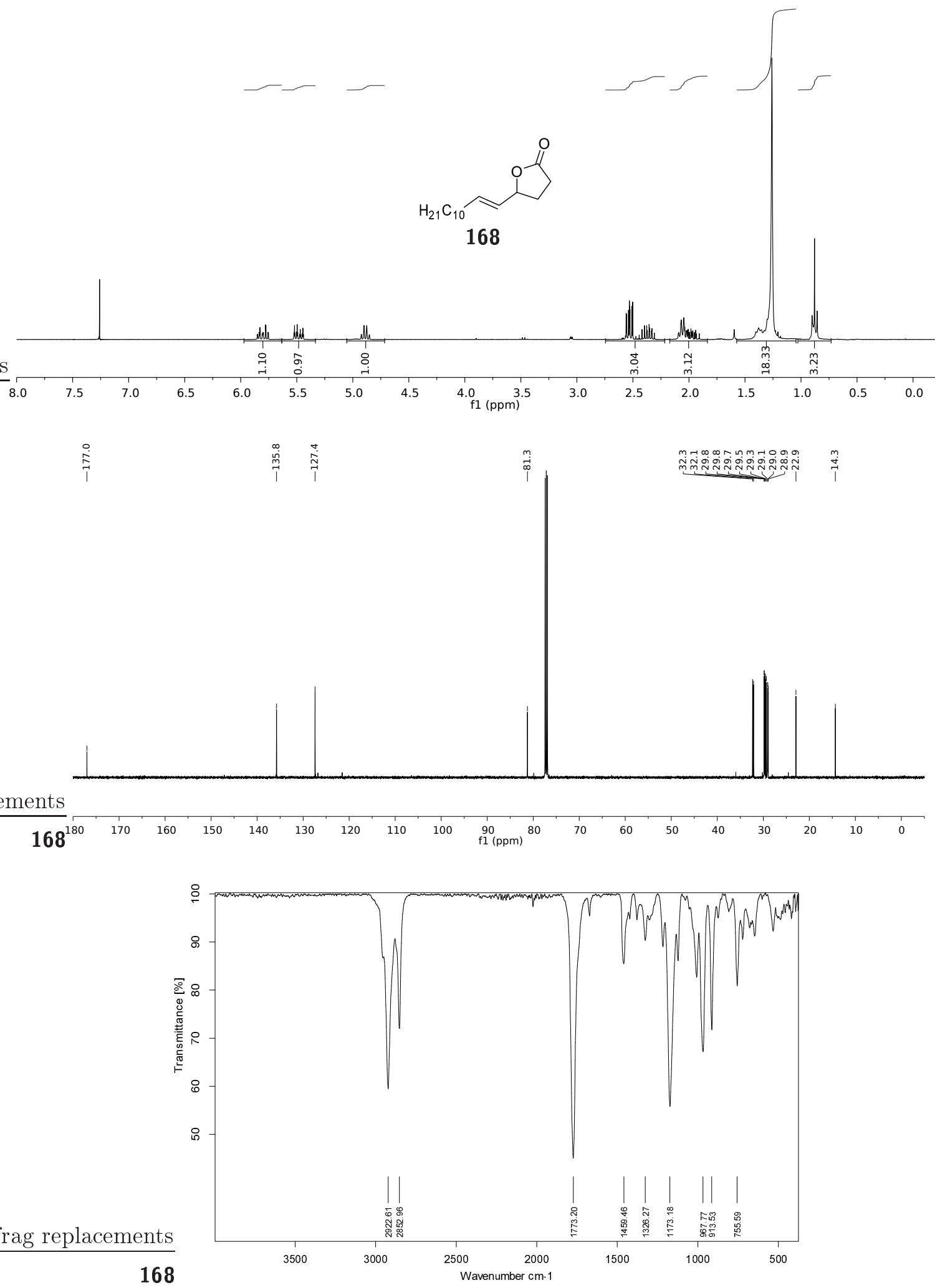



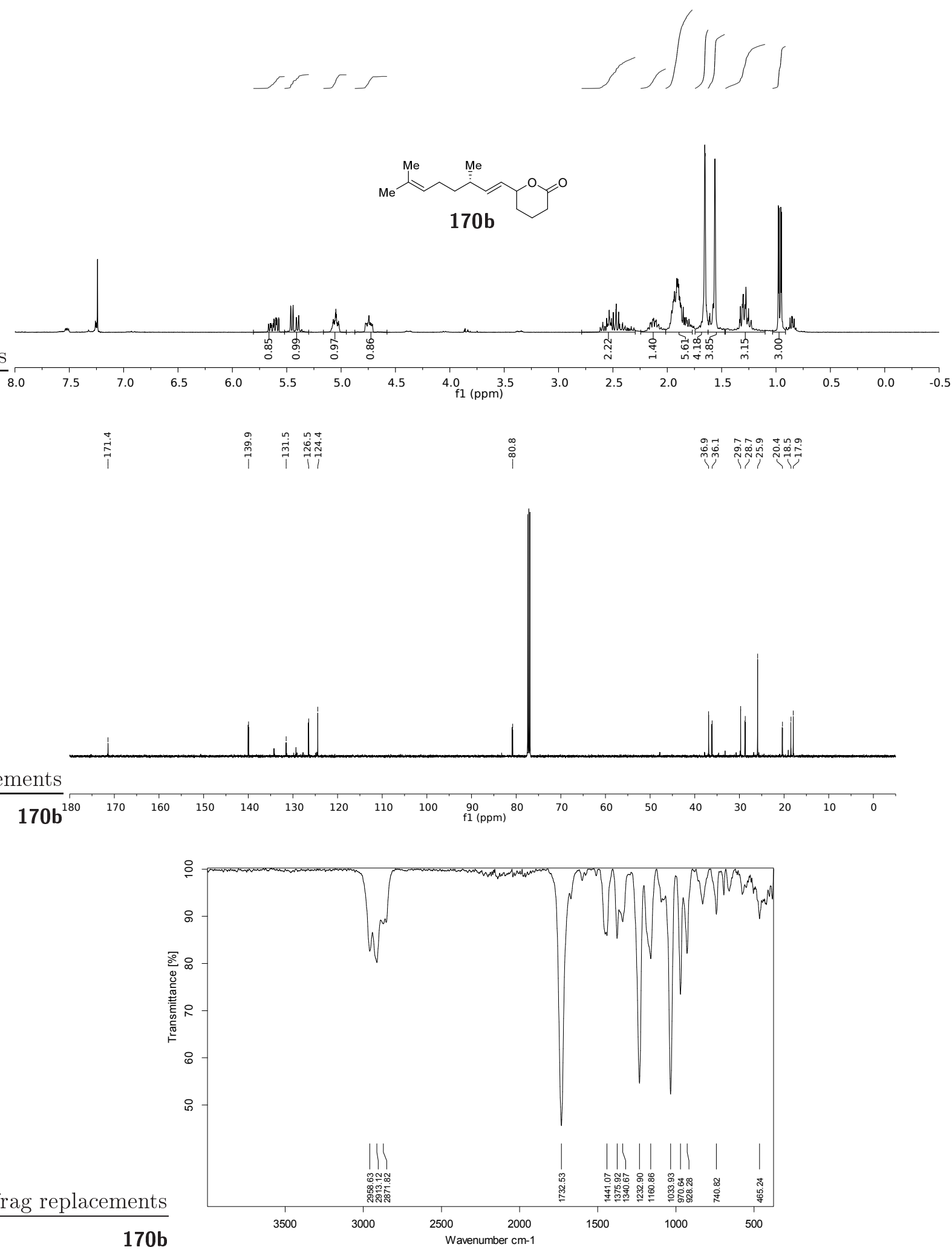

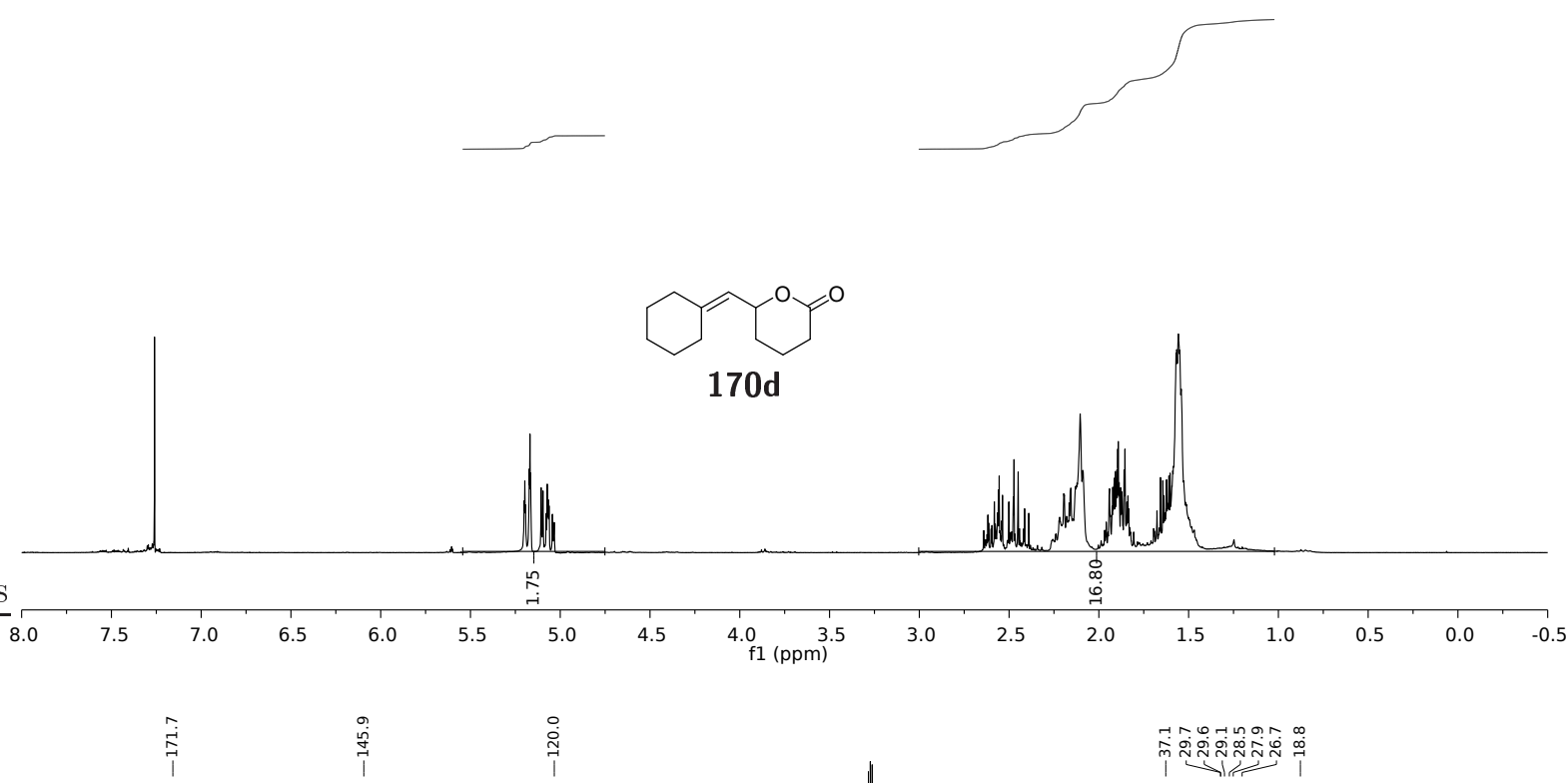

菅
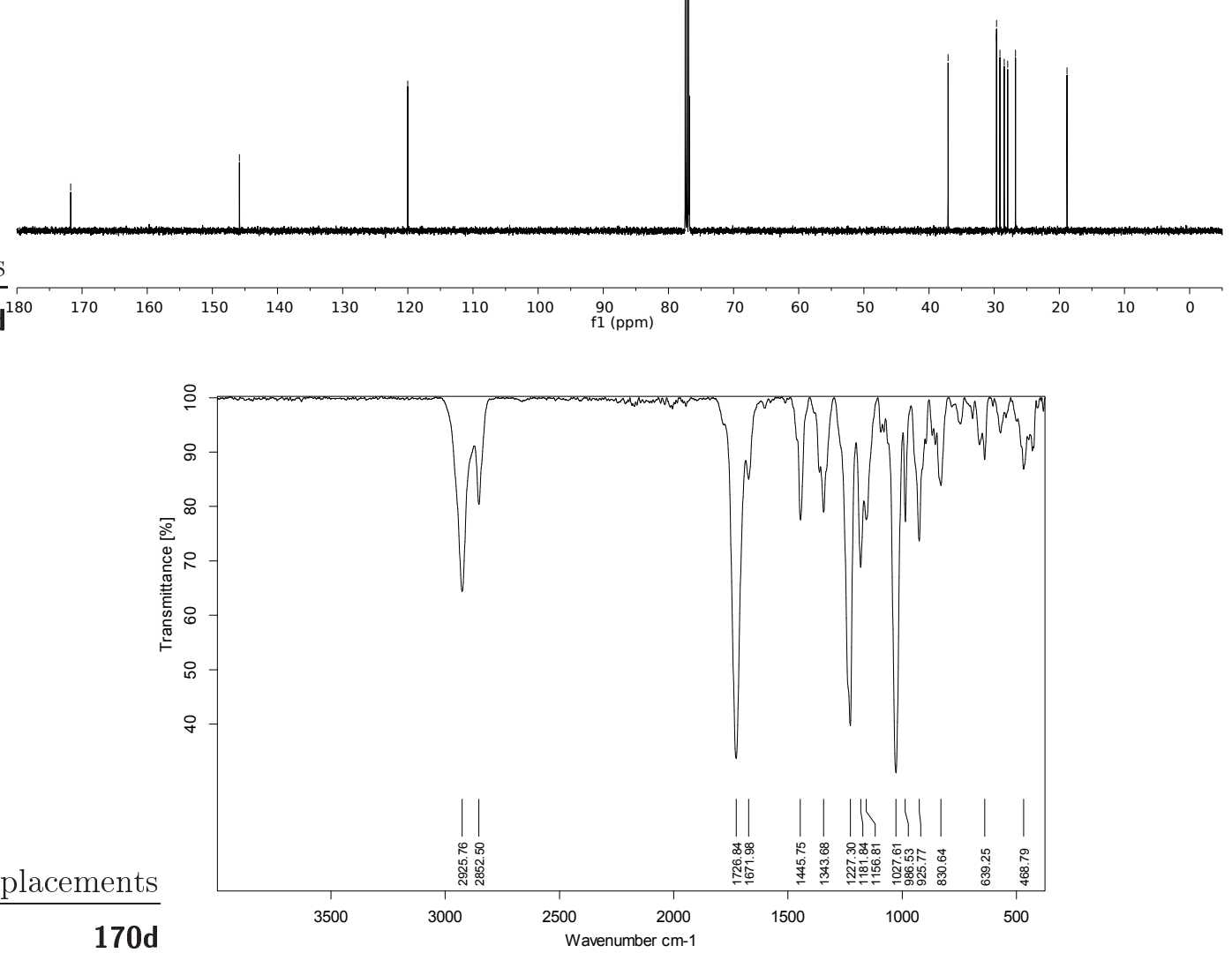


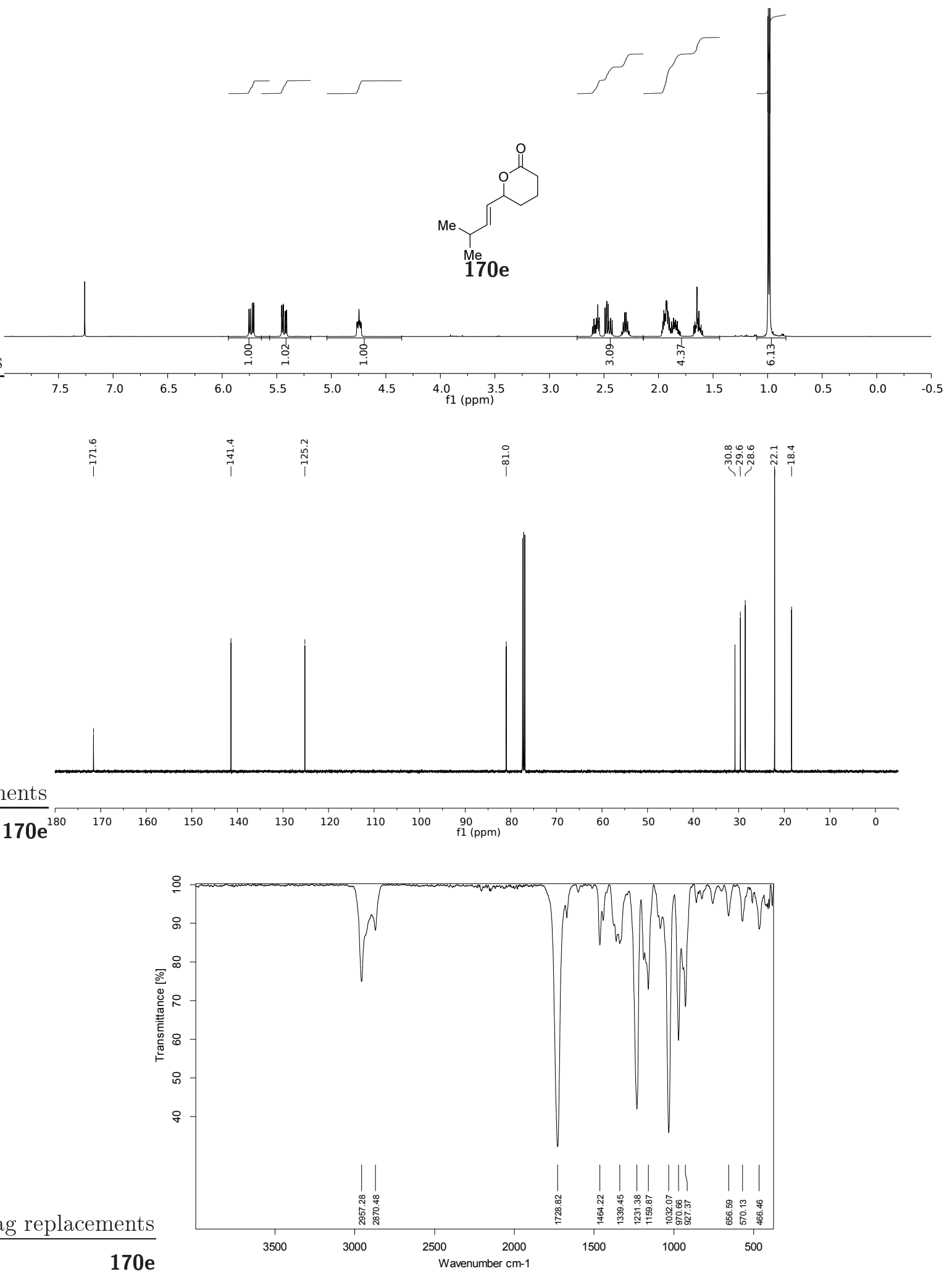



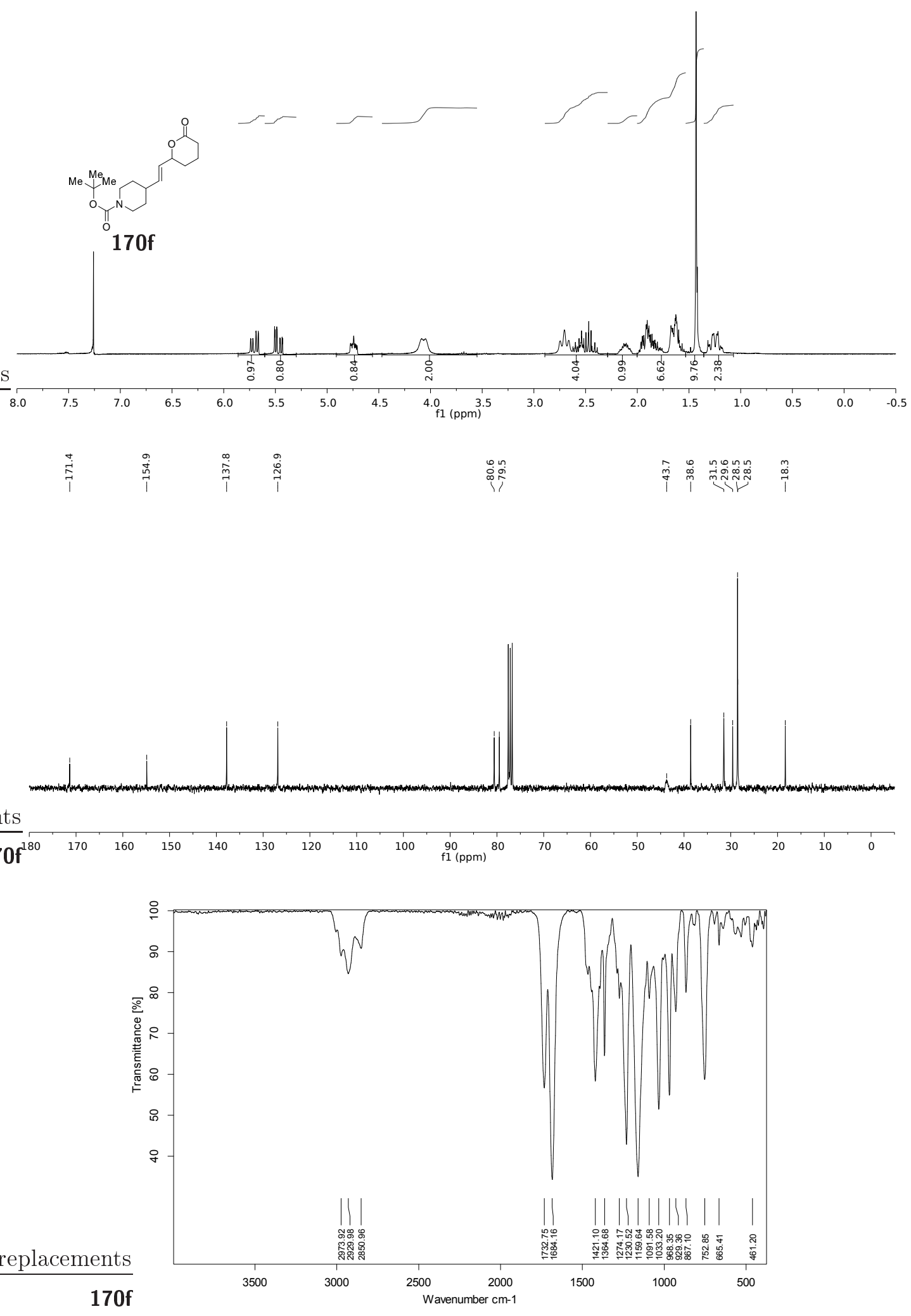


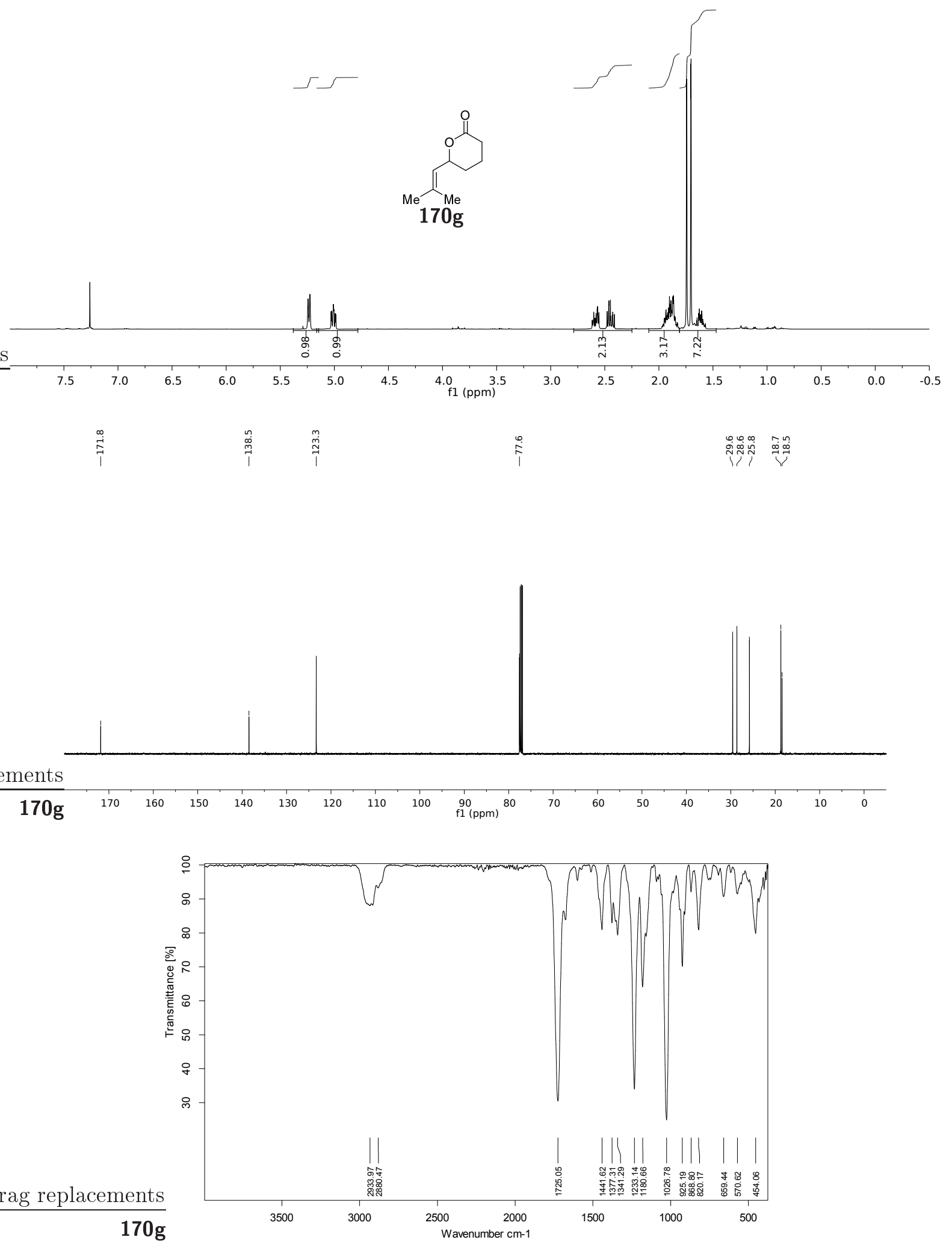




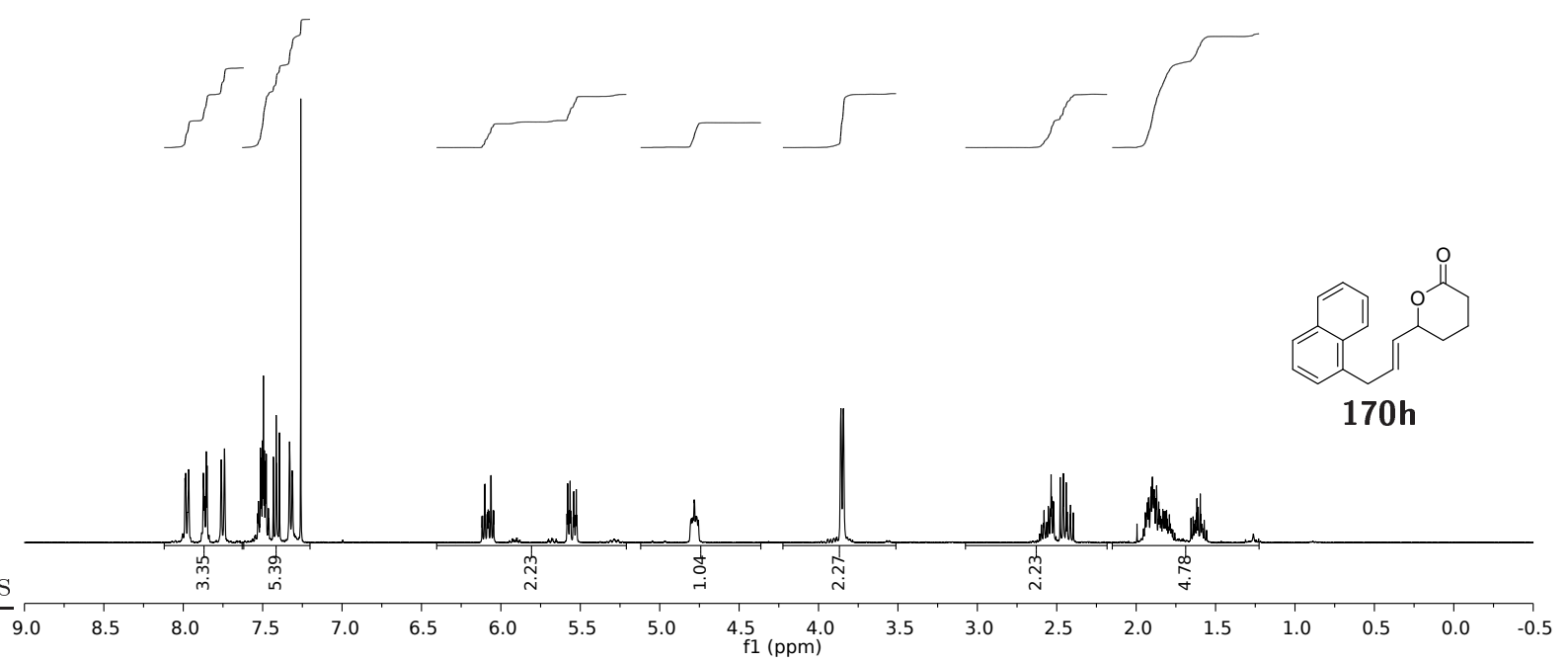

美
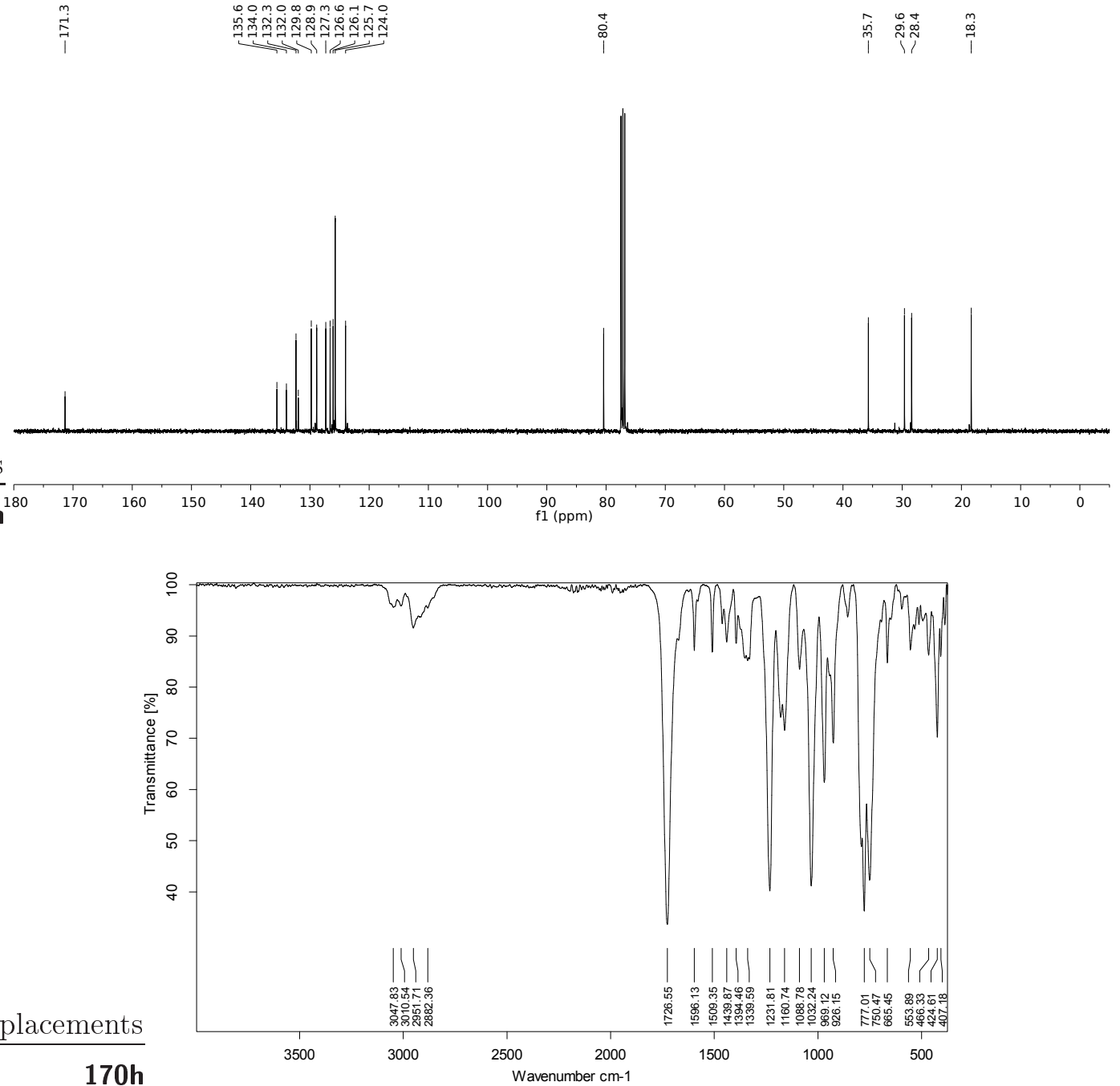

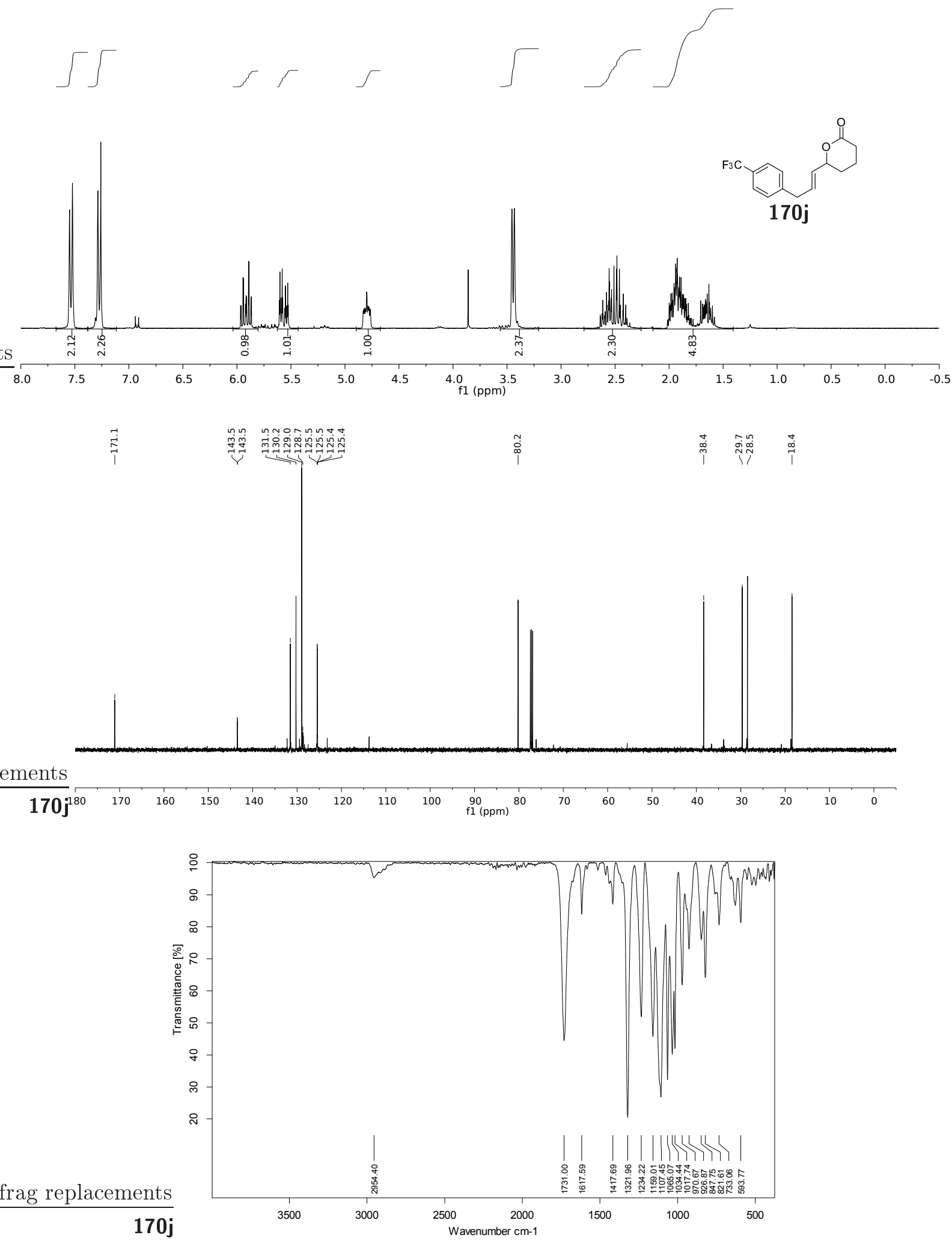

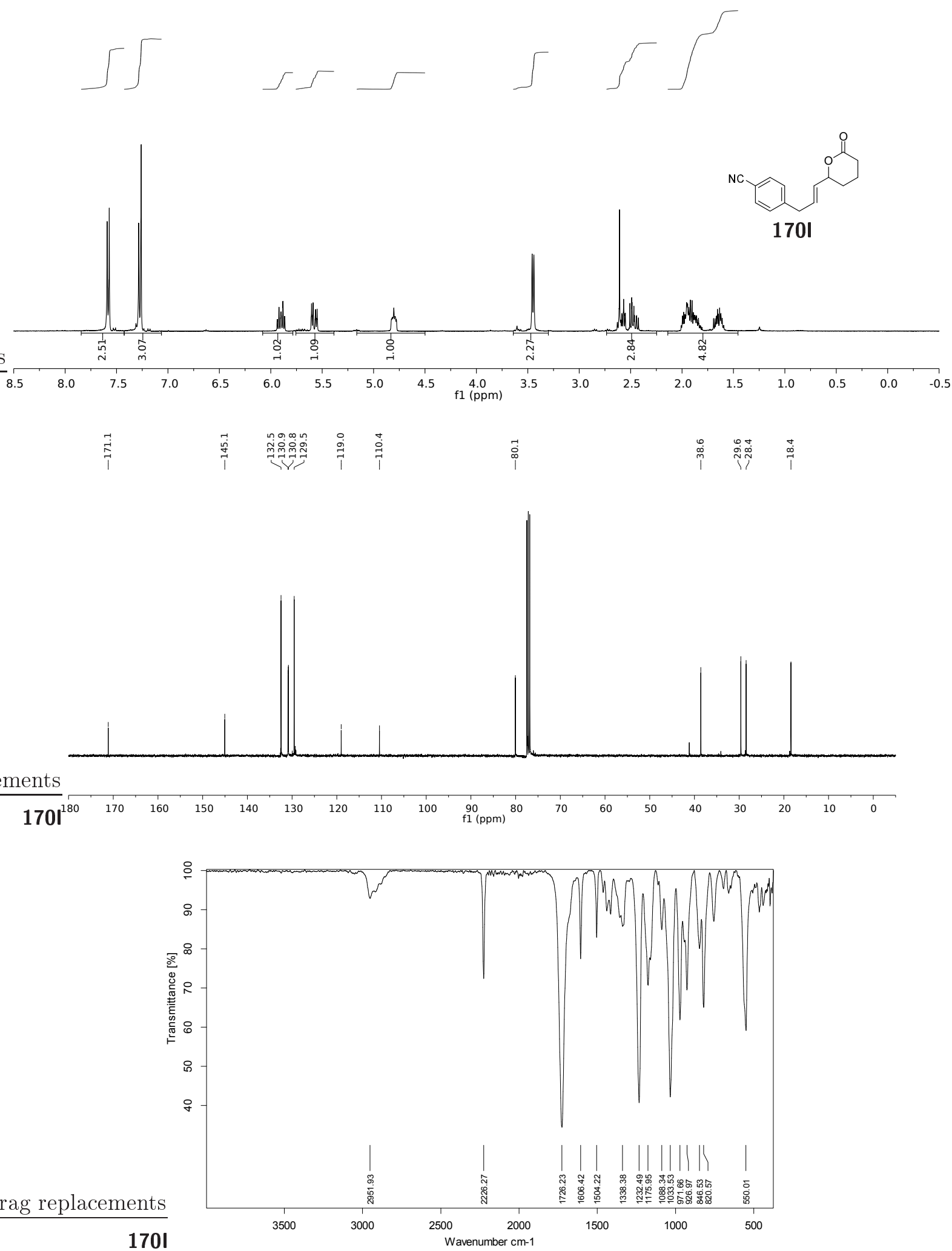

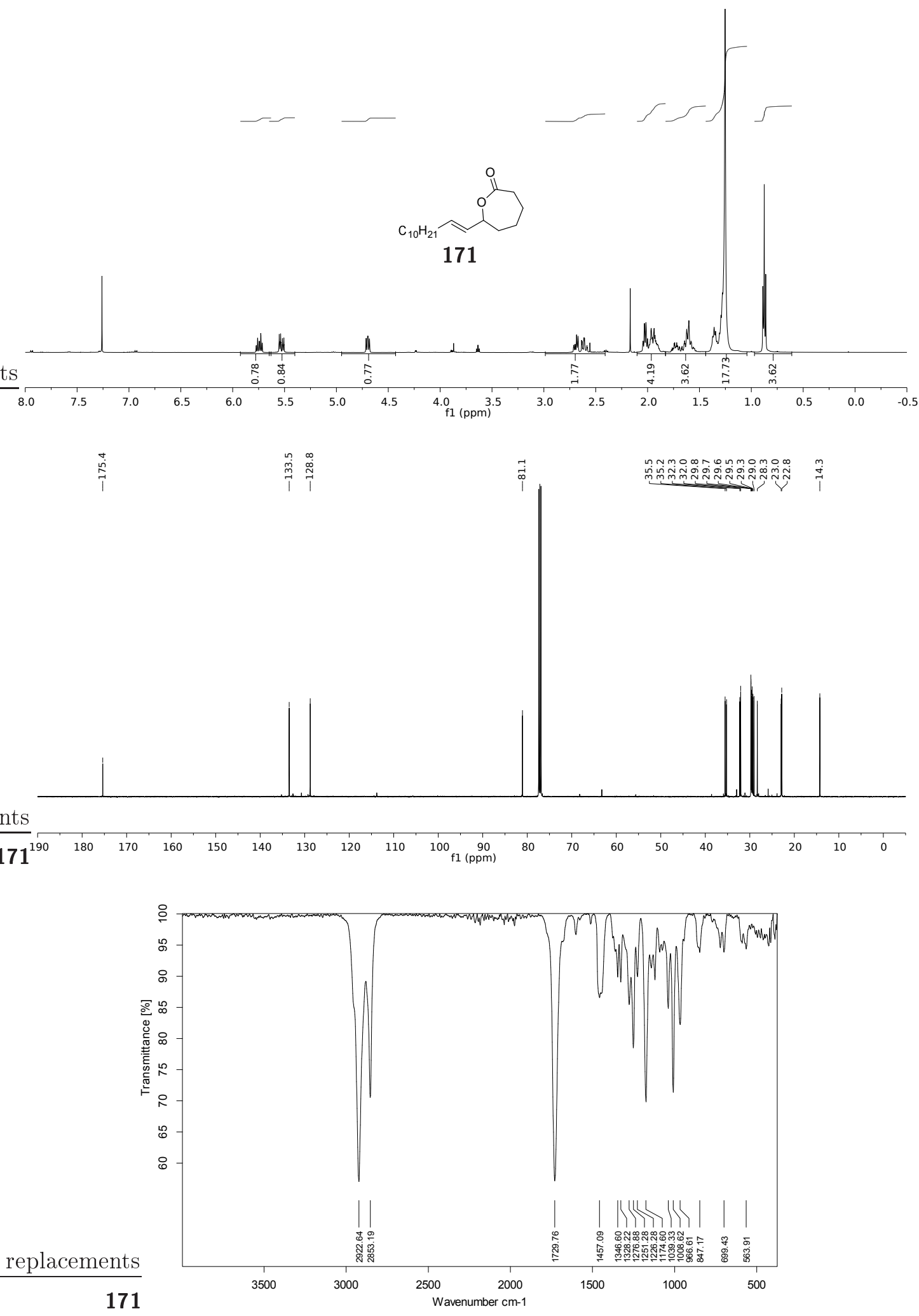

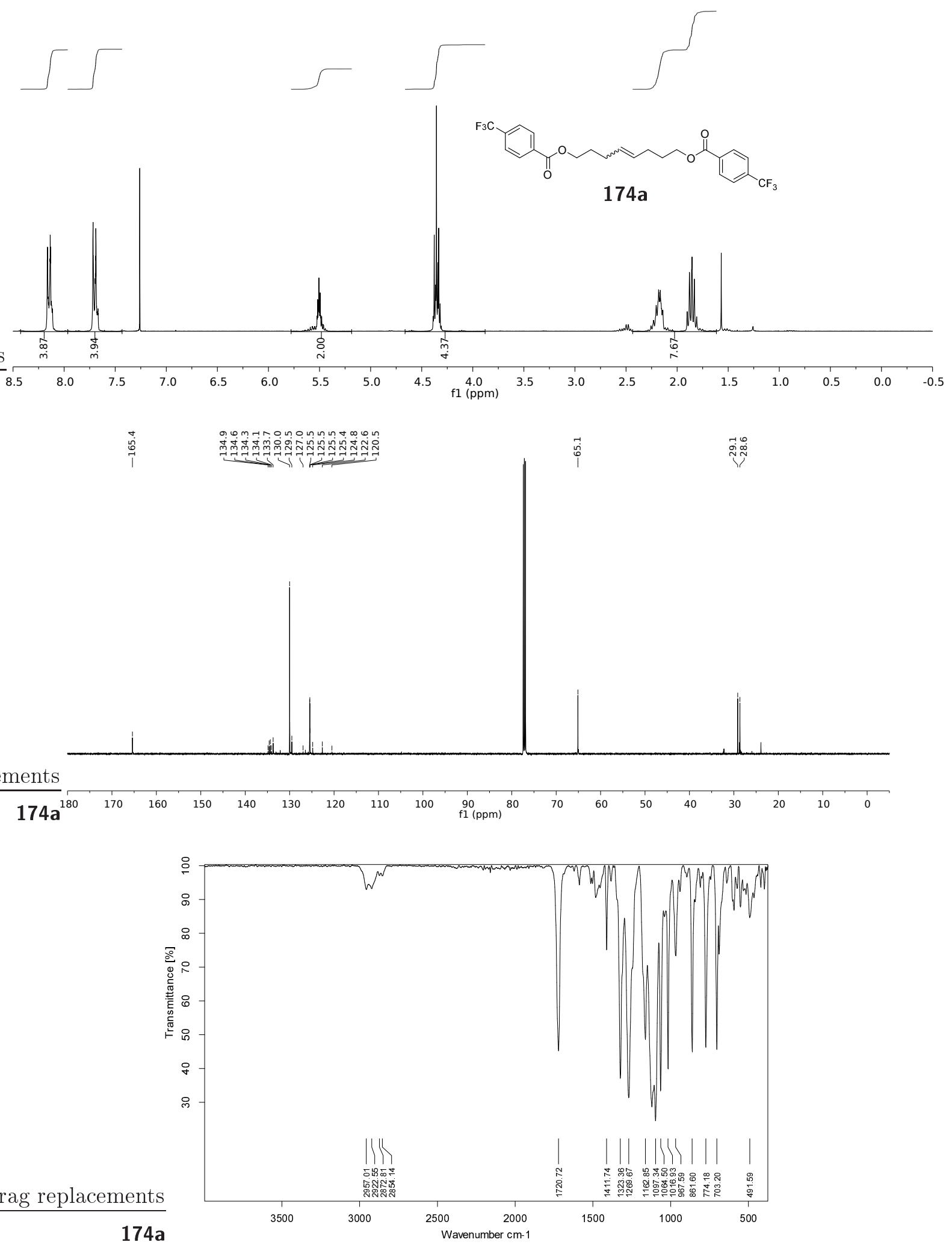


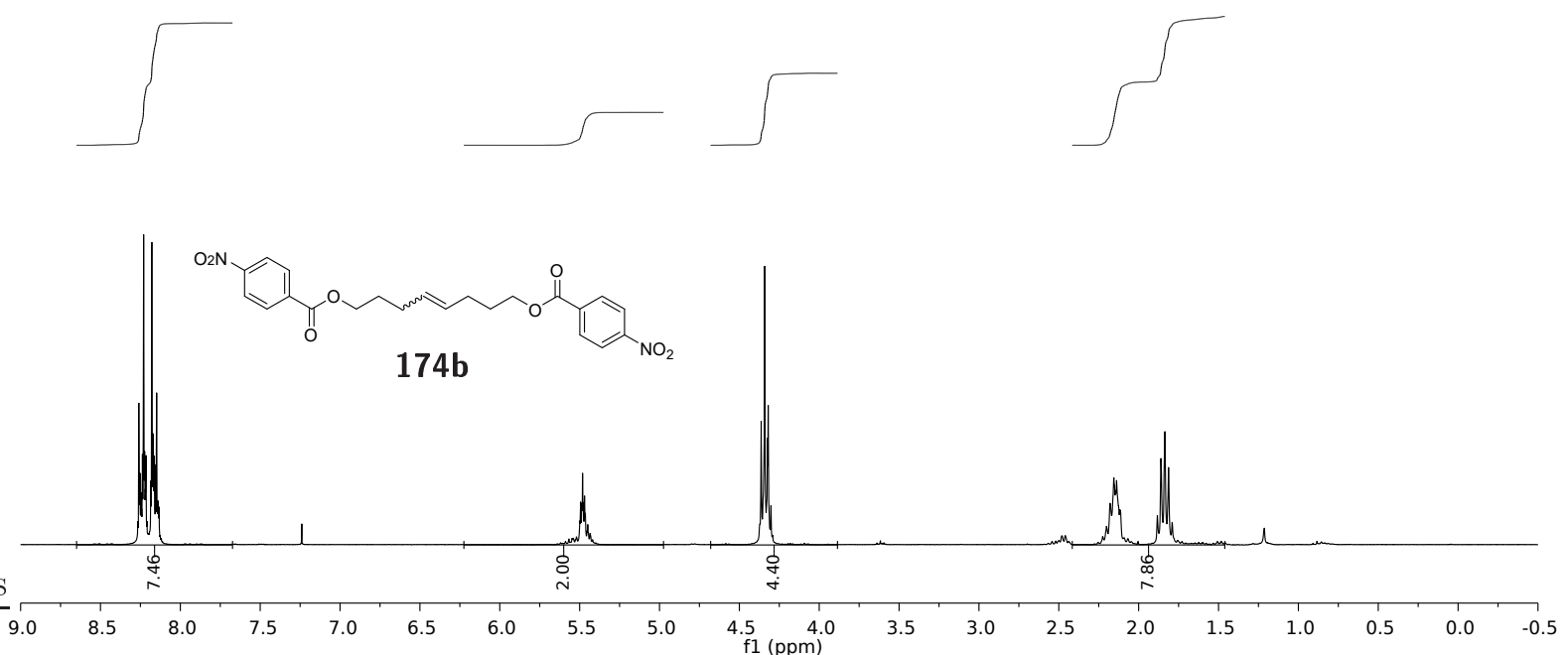

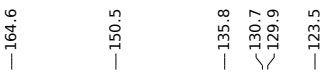

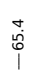

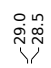
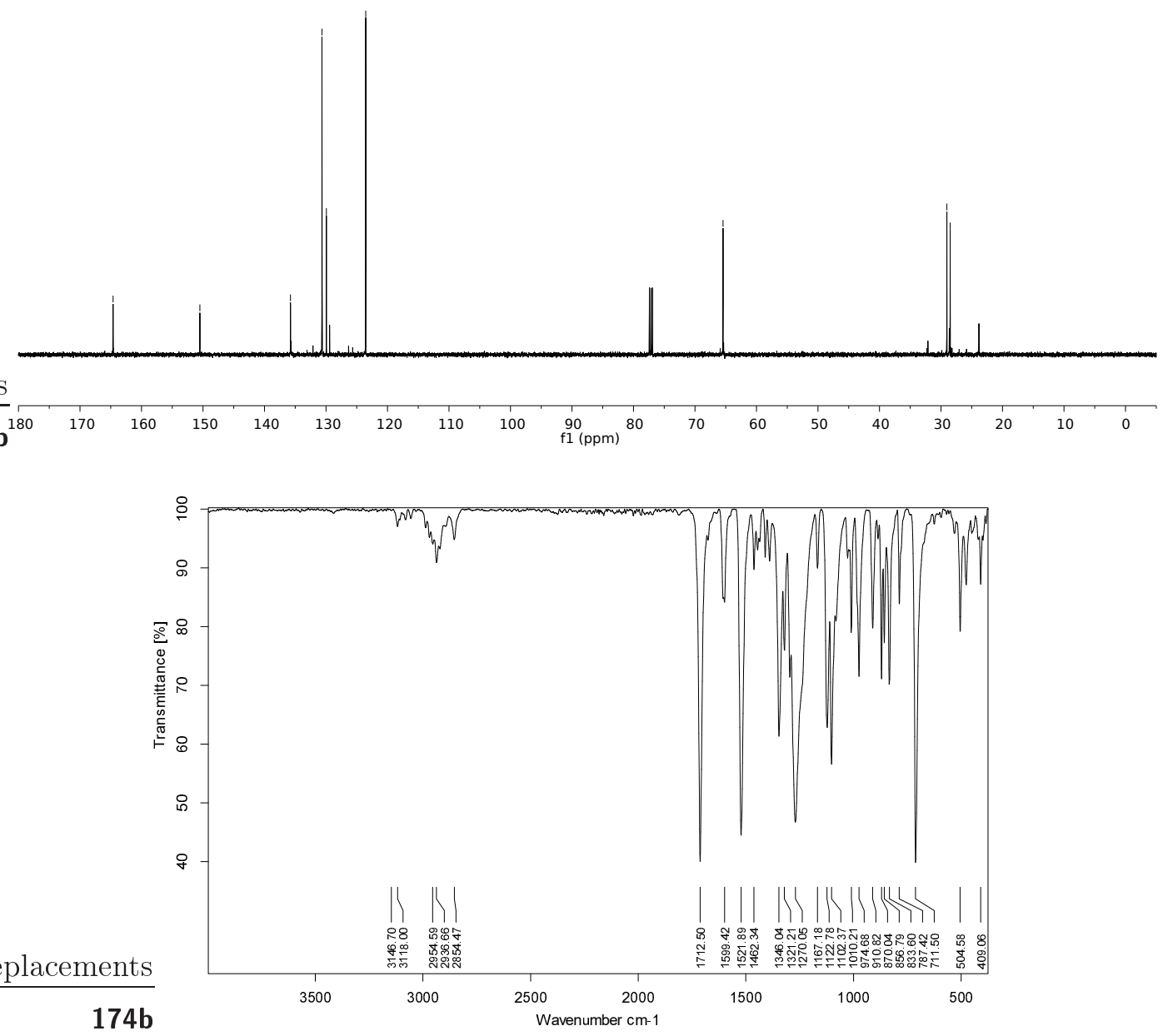

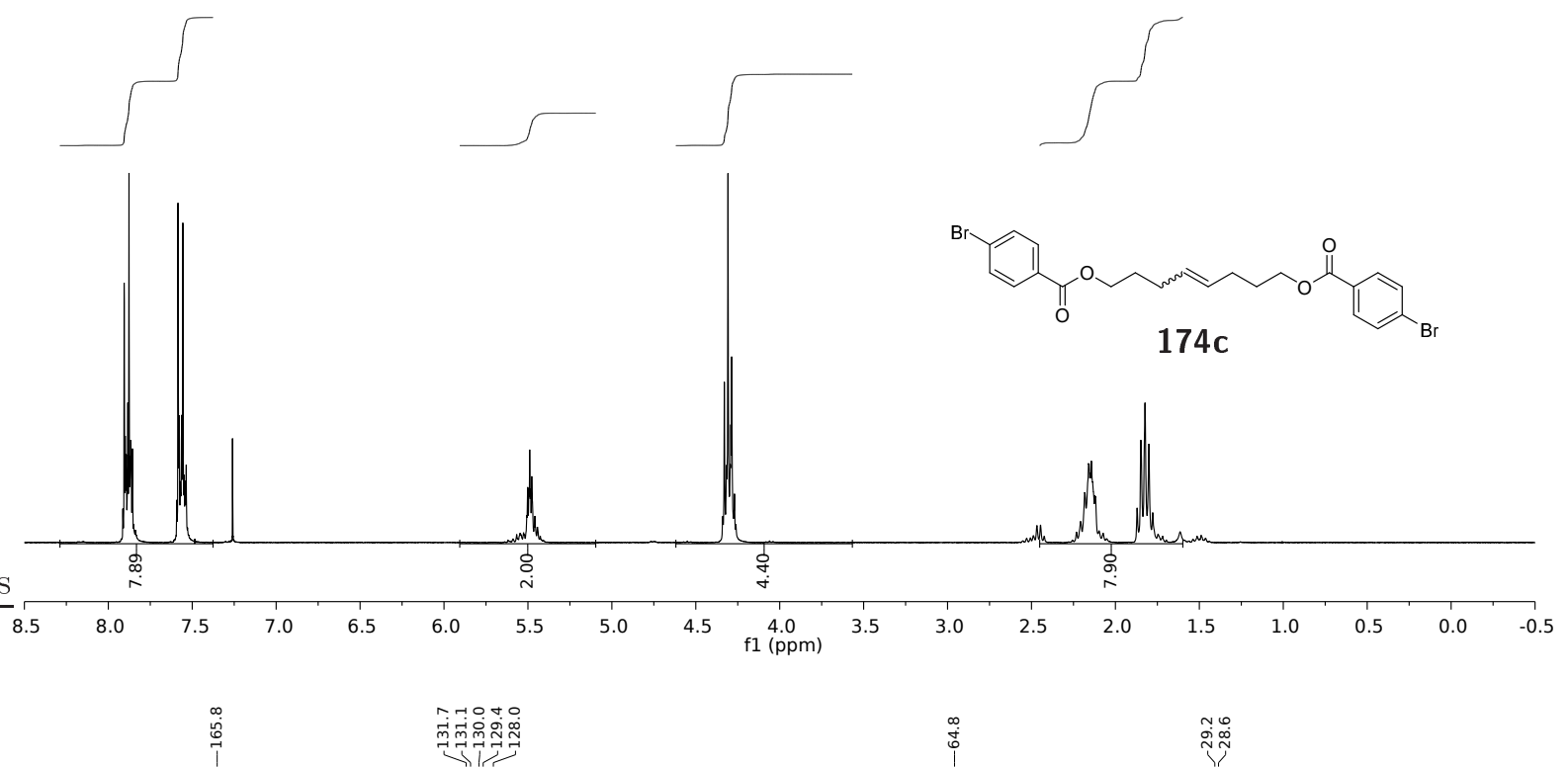

离

กู๐
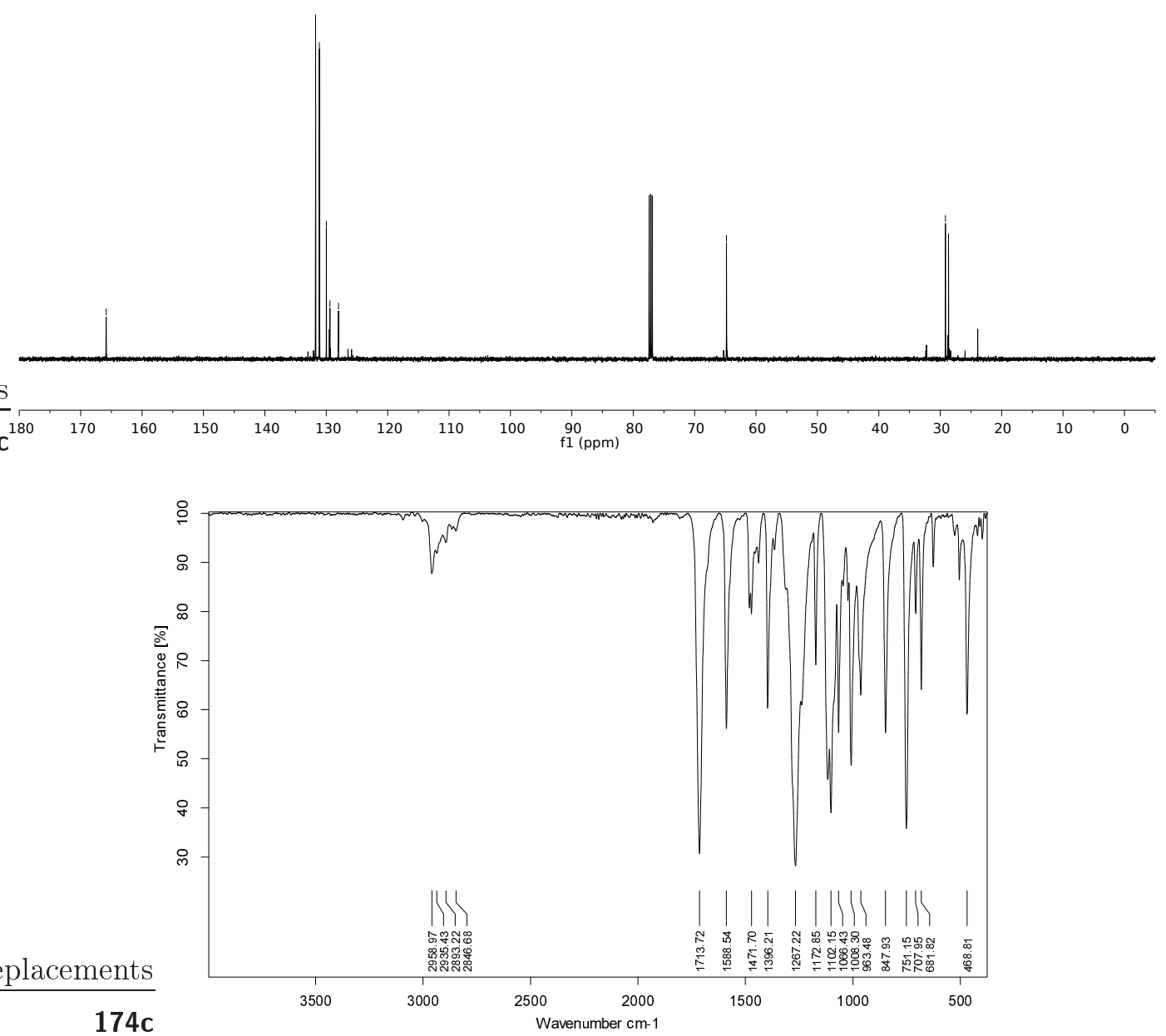

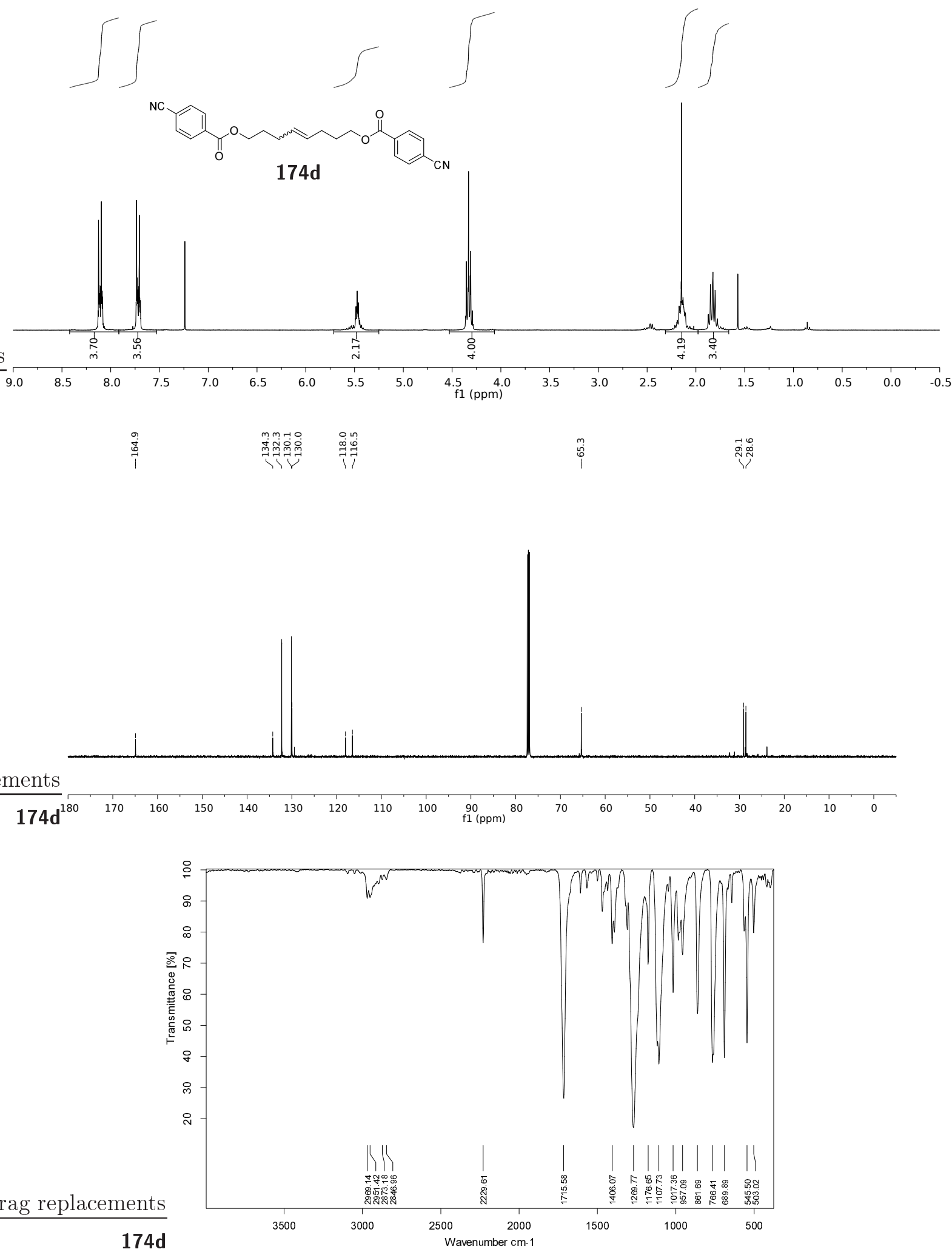

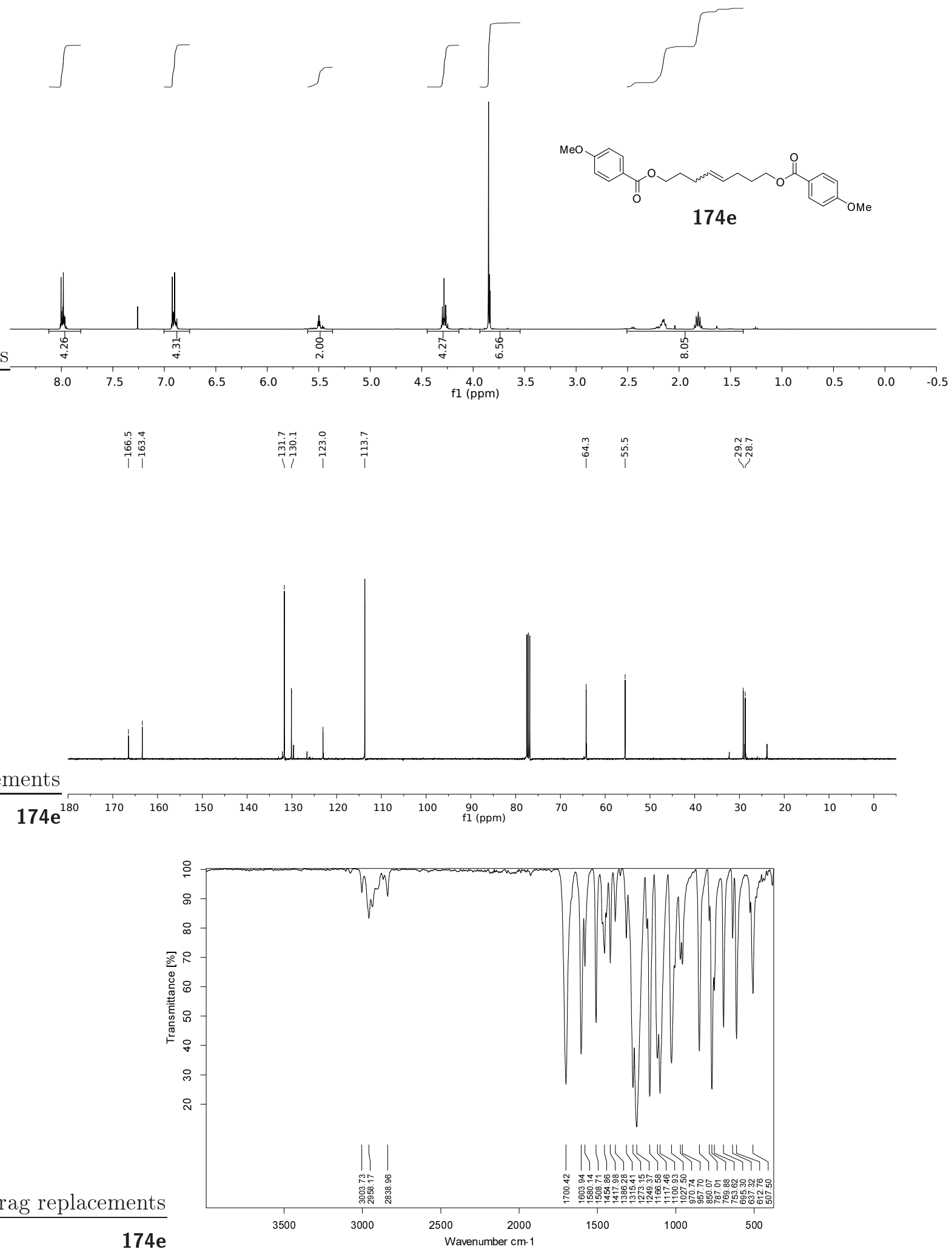


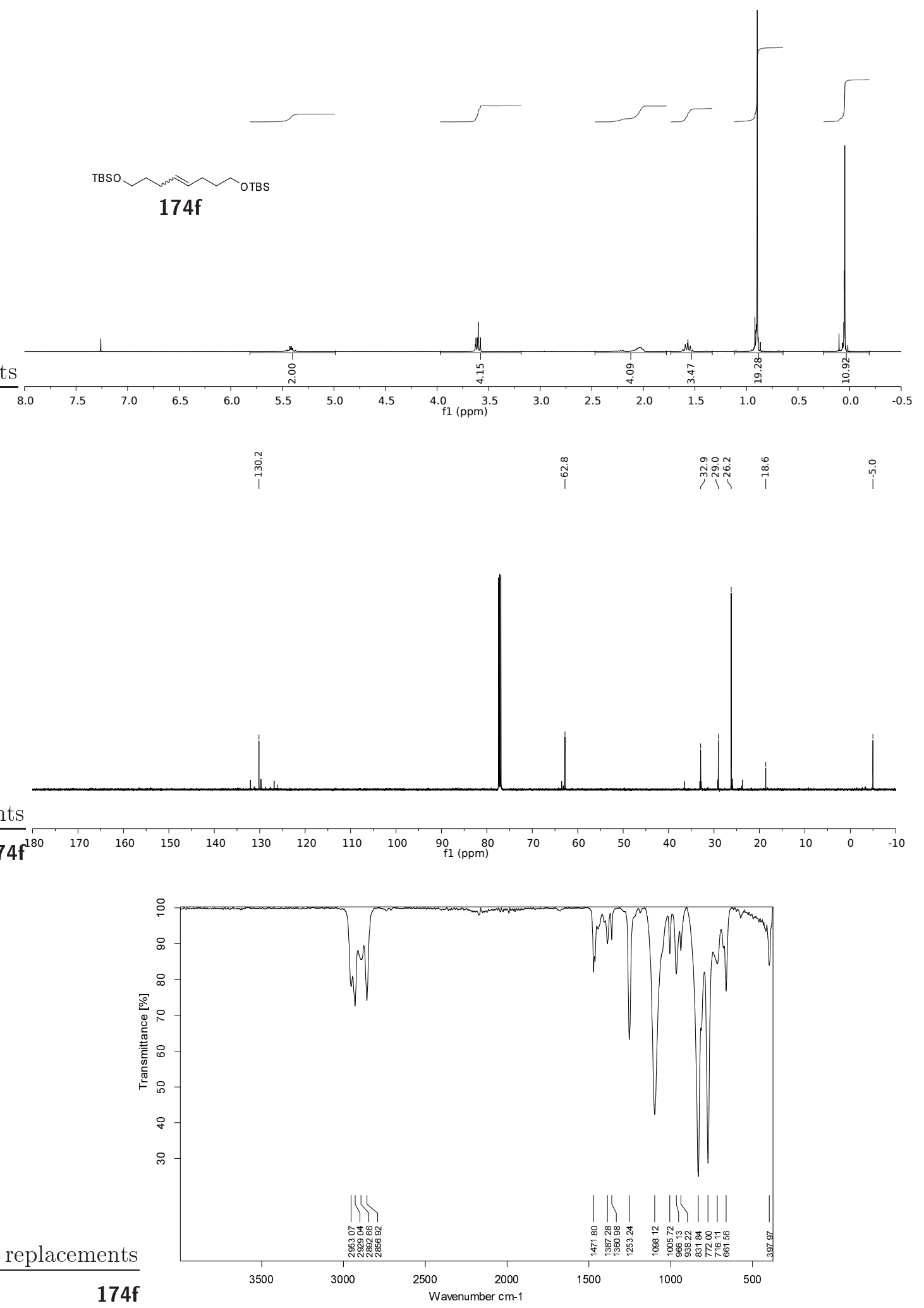



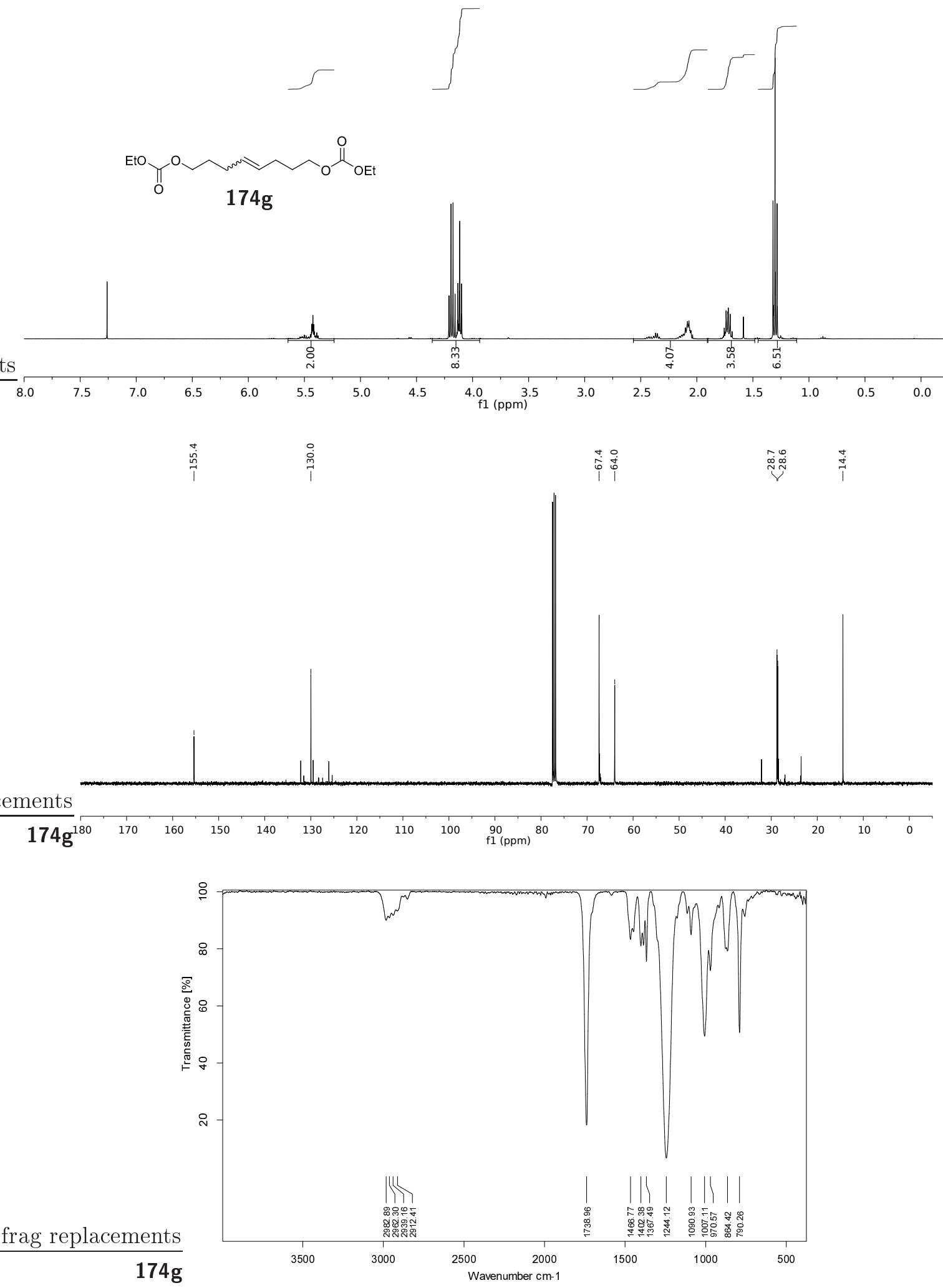

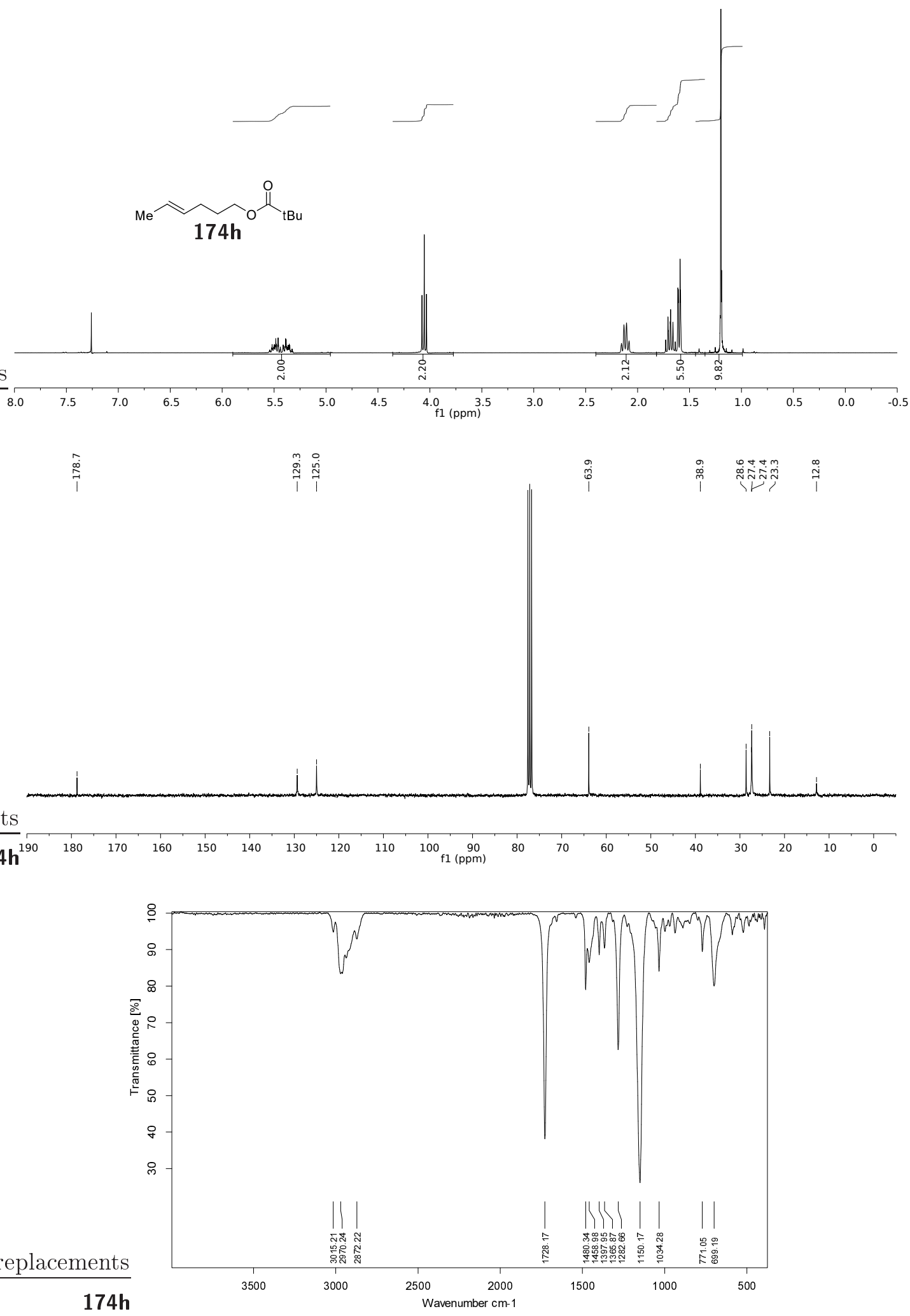

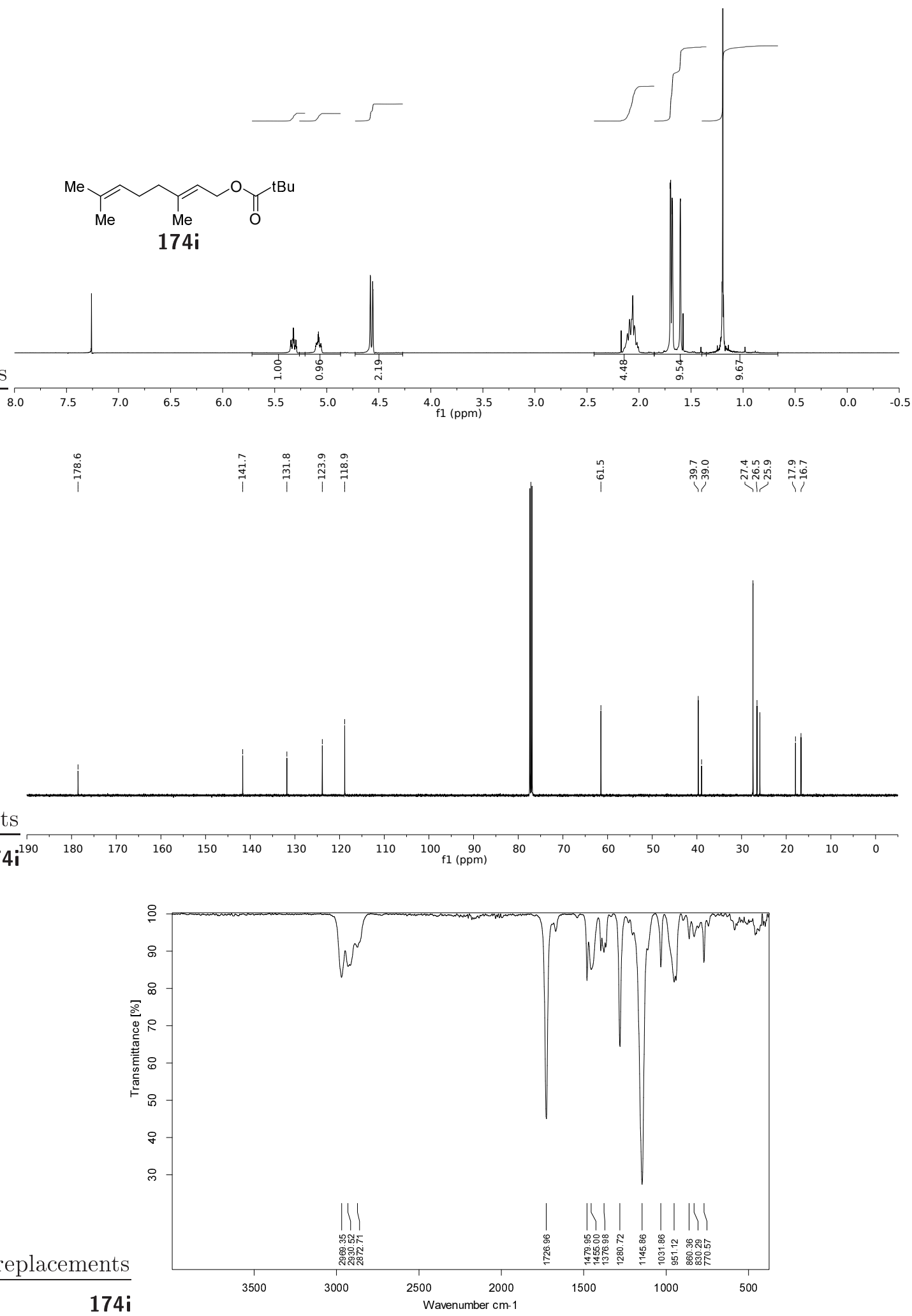

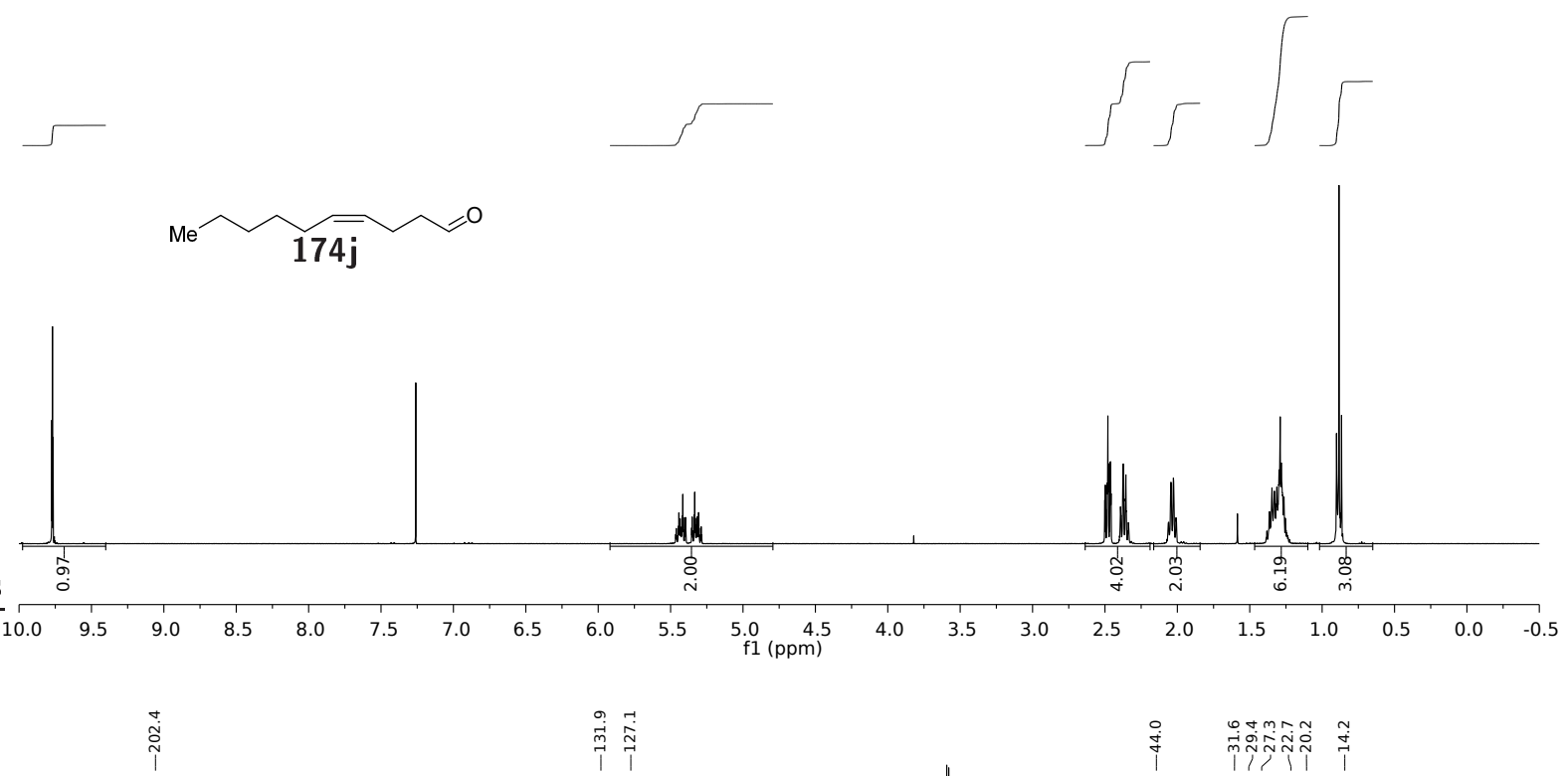

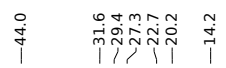

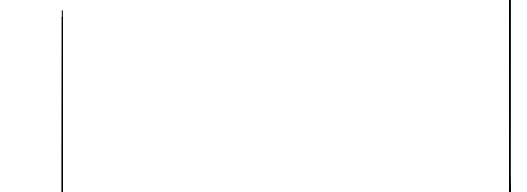

$\overrightarrow{\stackrel{1}{*}}$
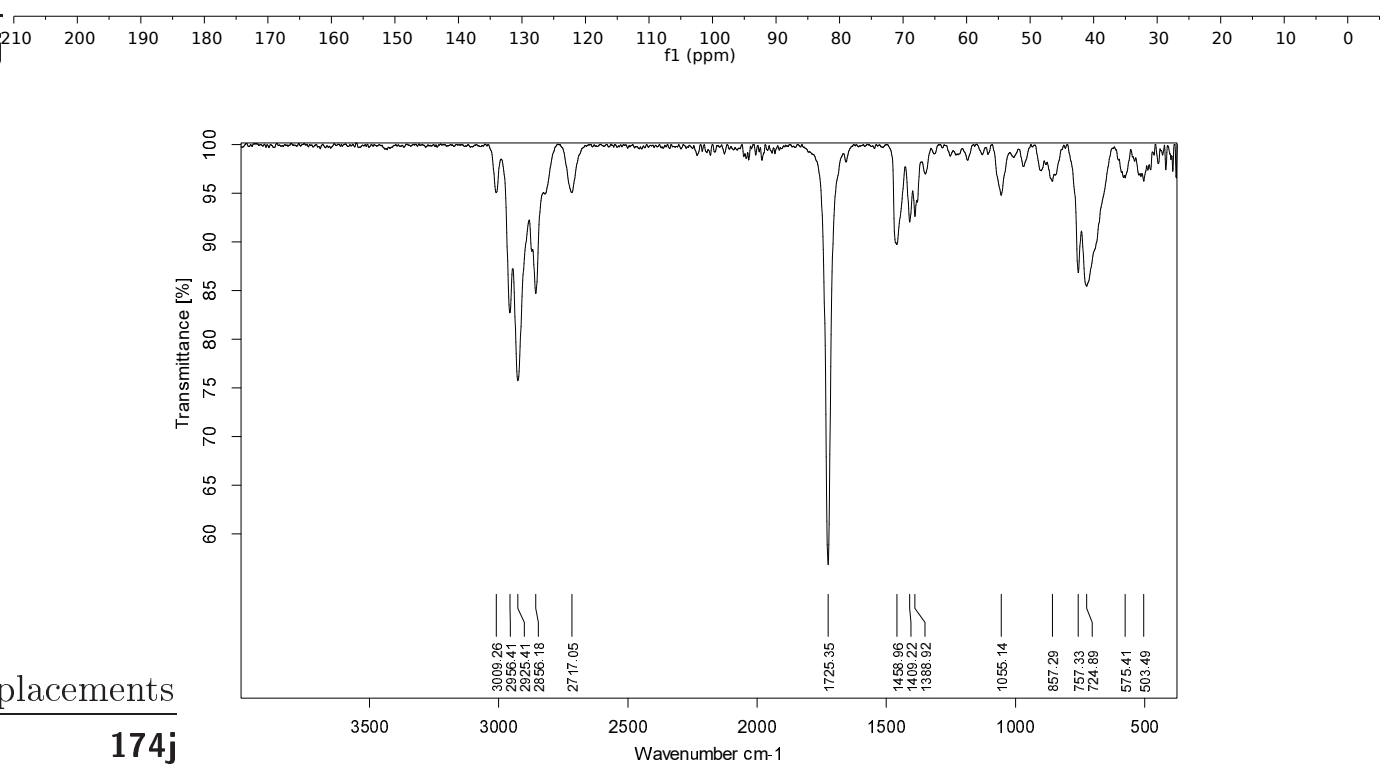

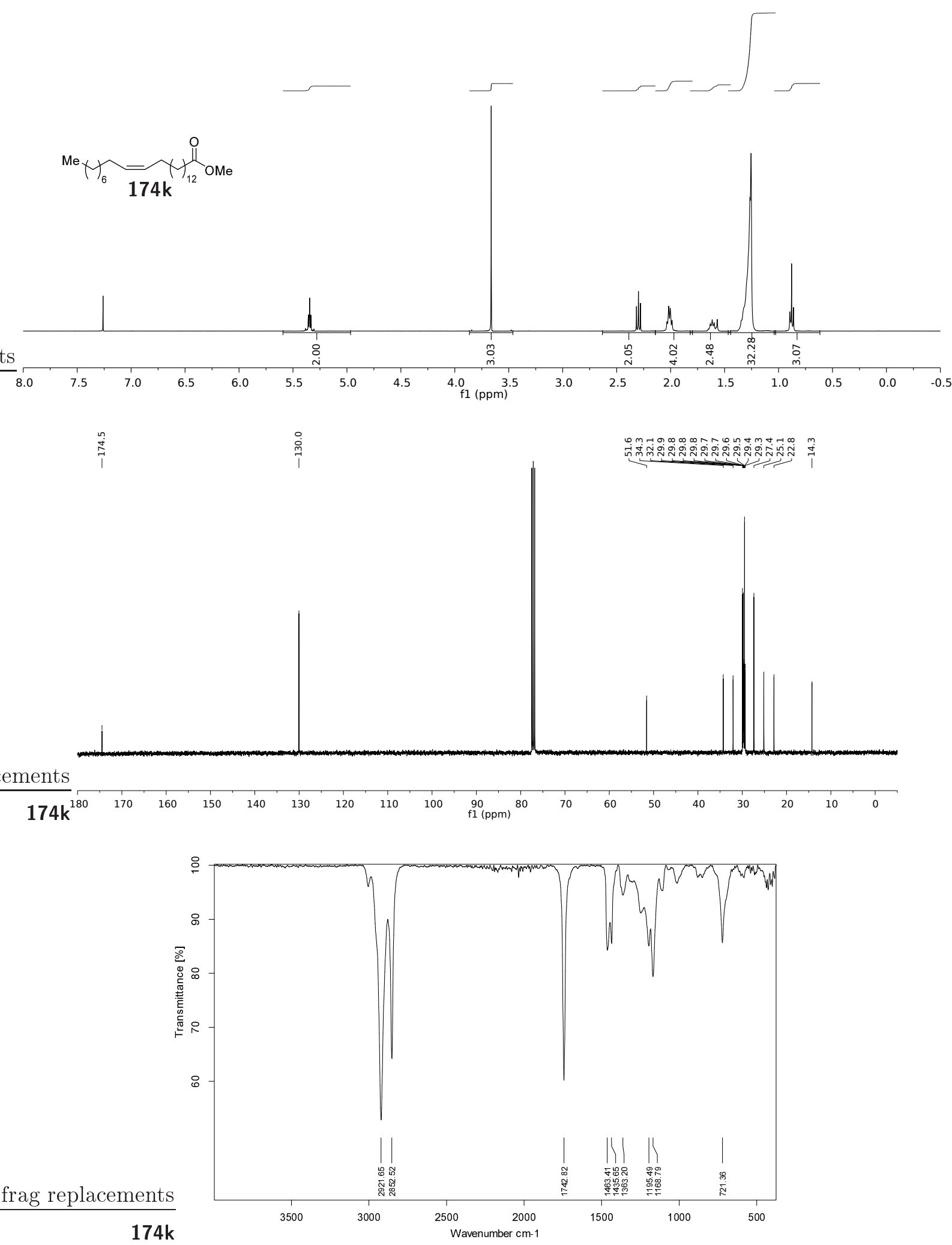

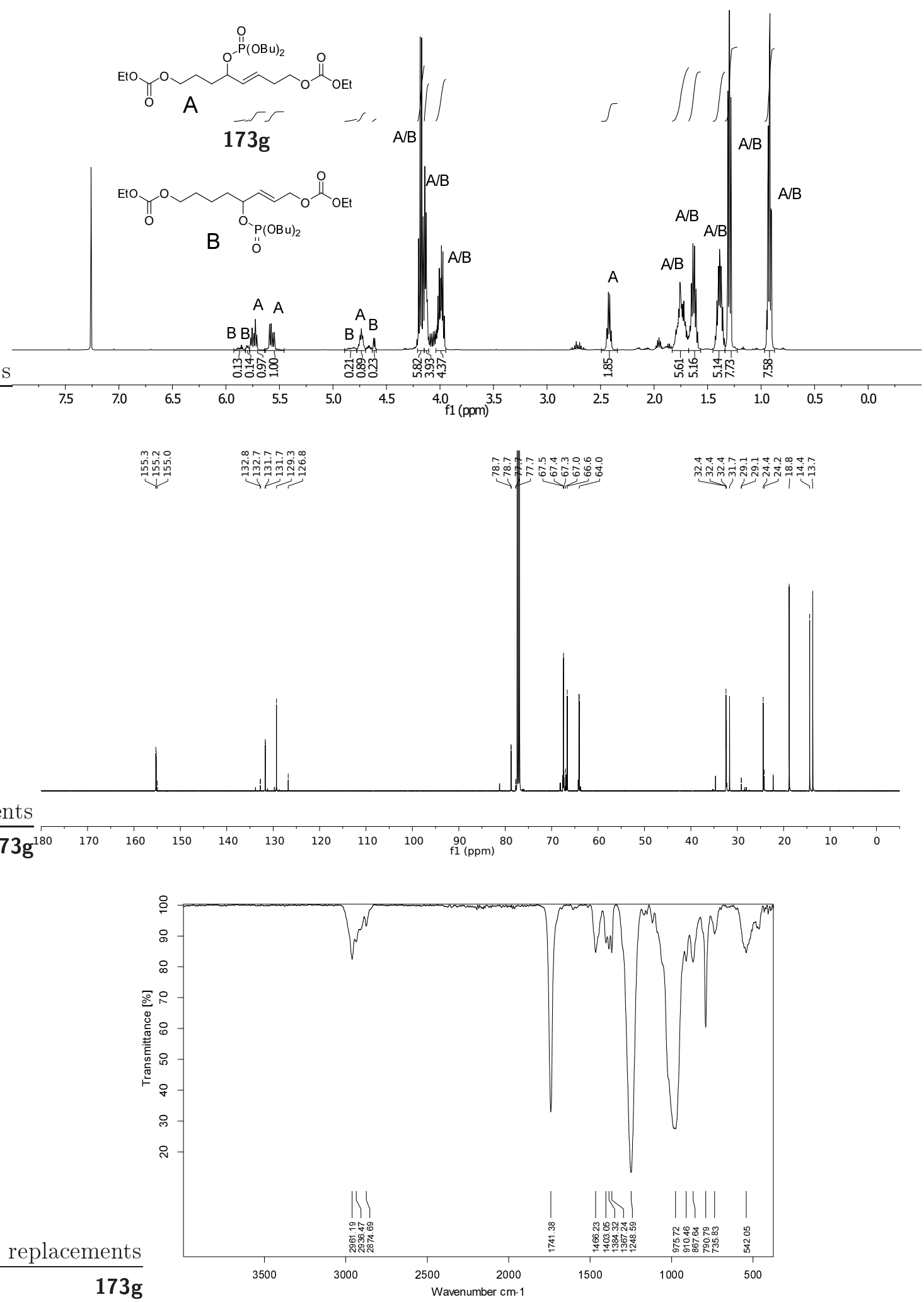

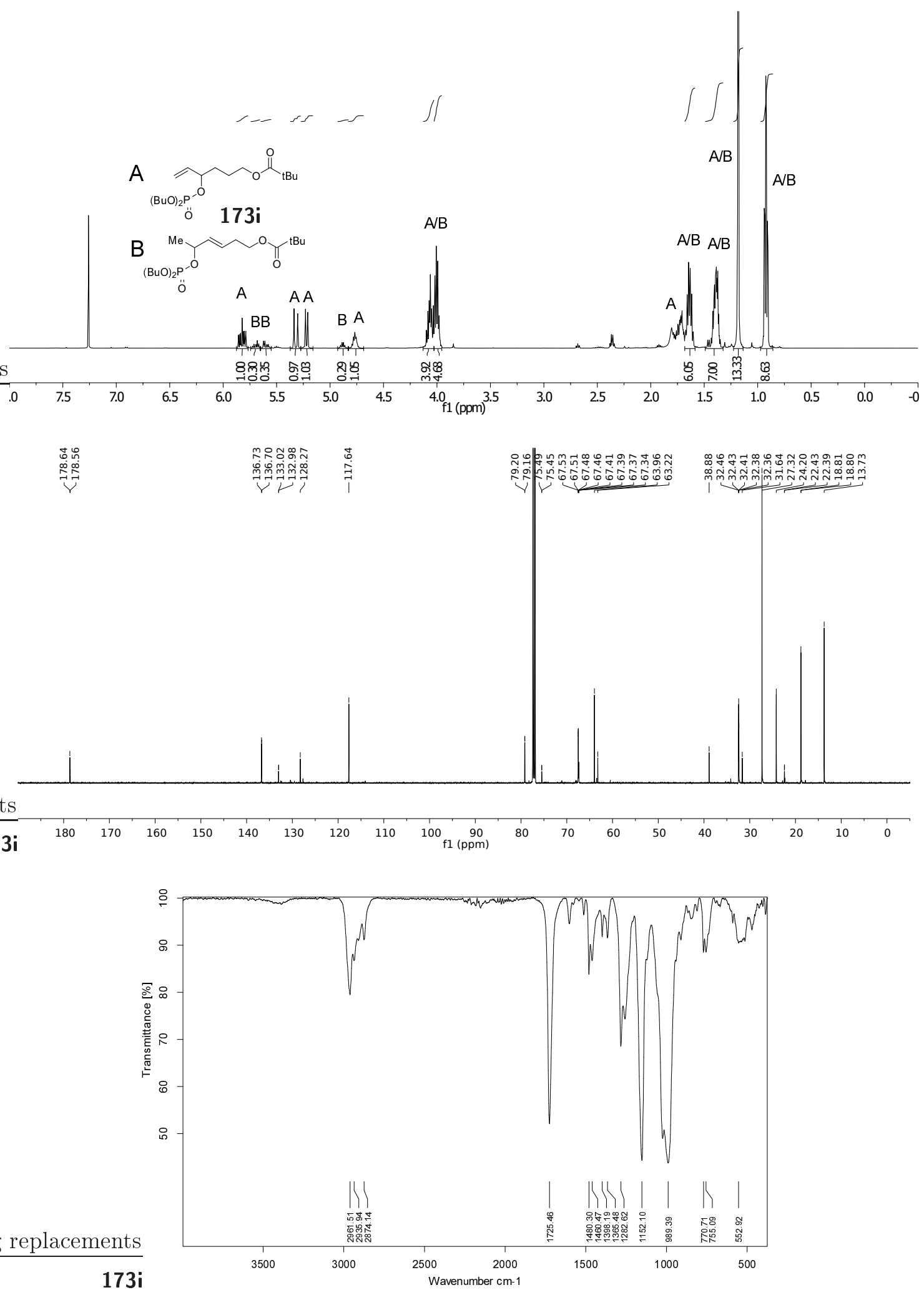


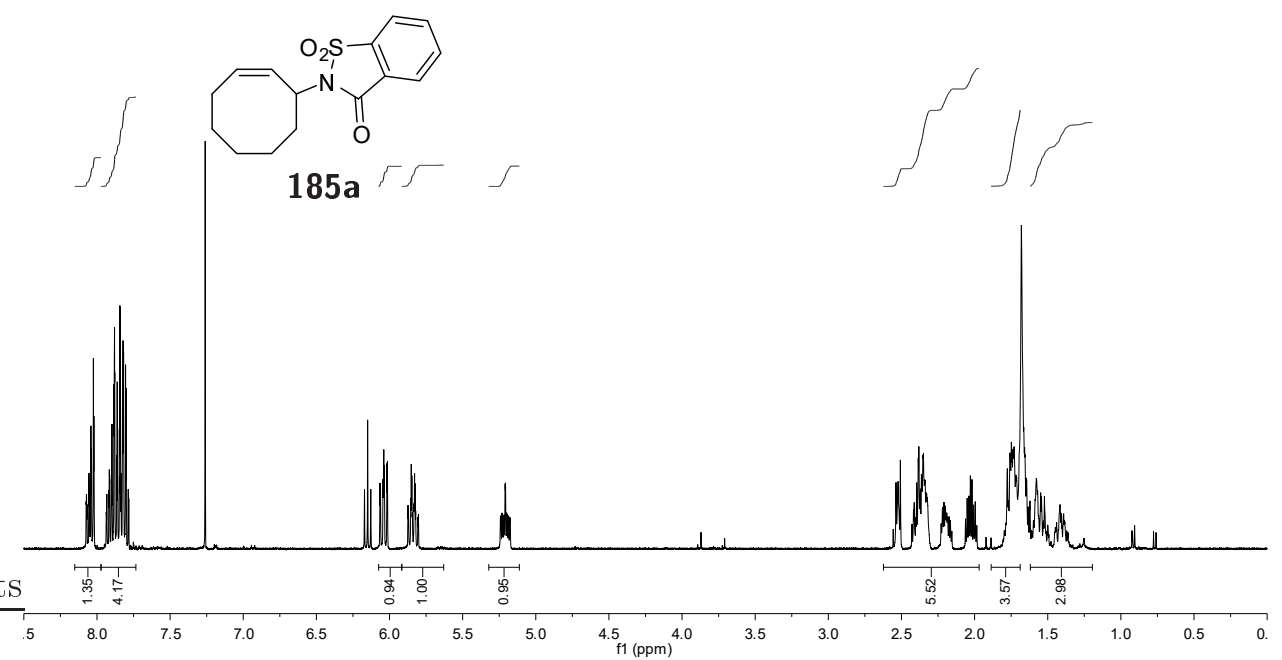

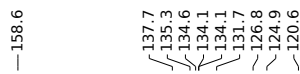

กับ
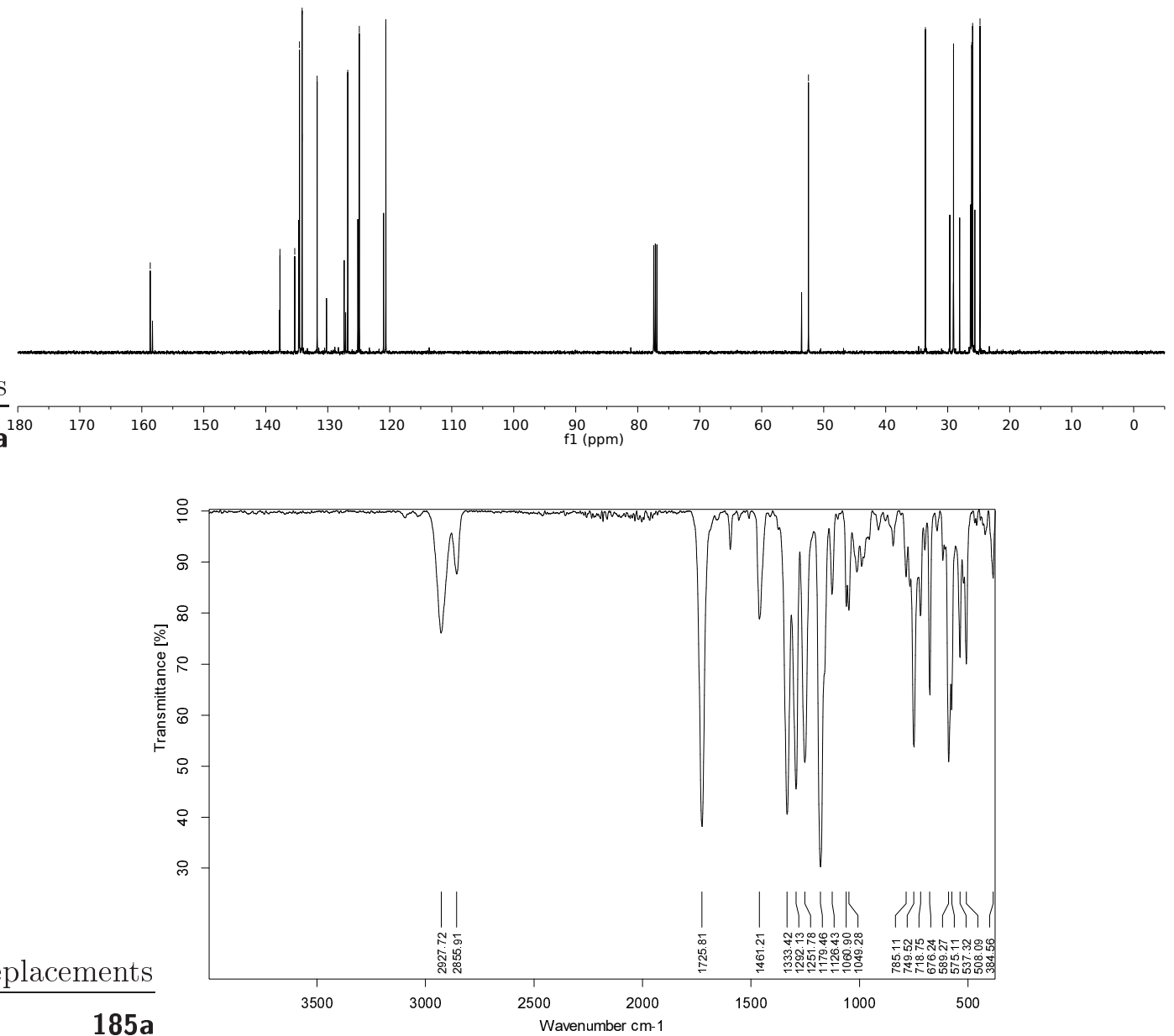

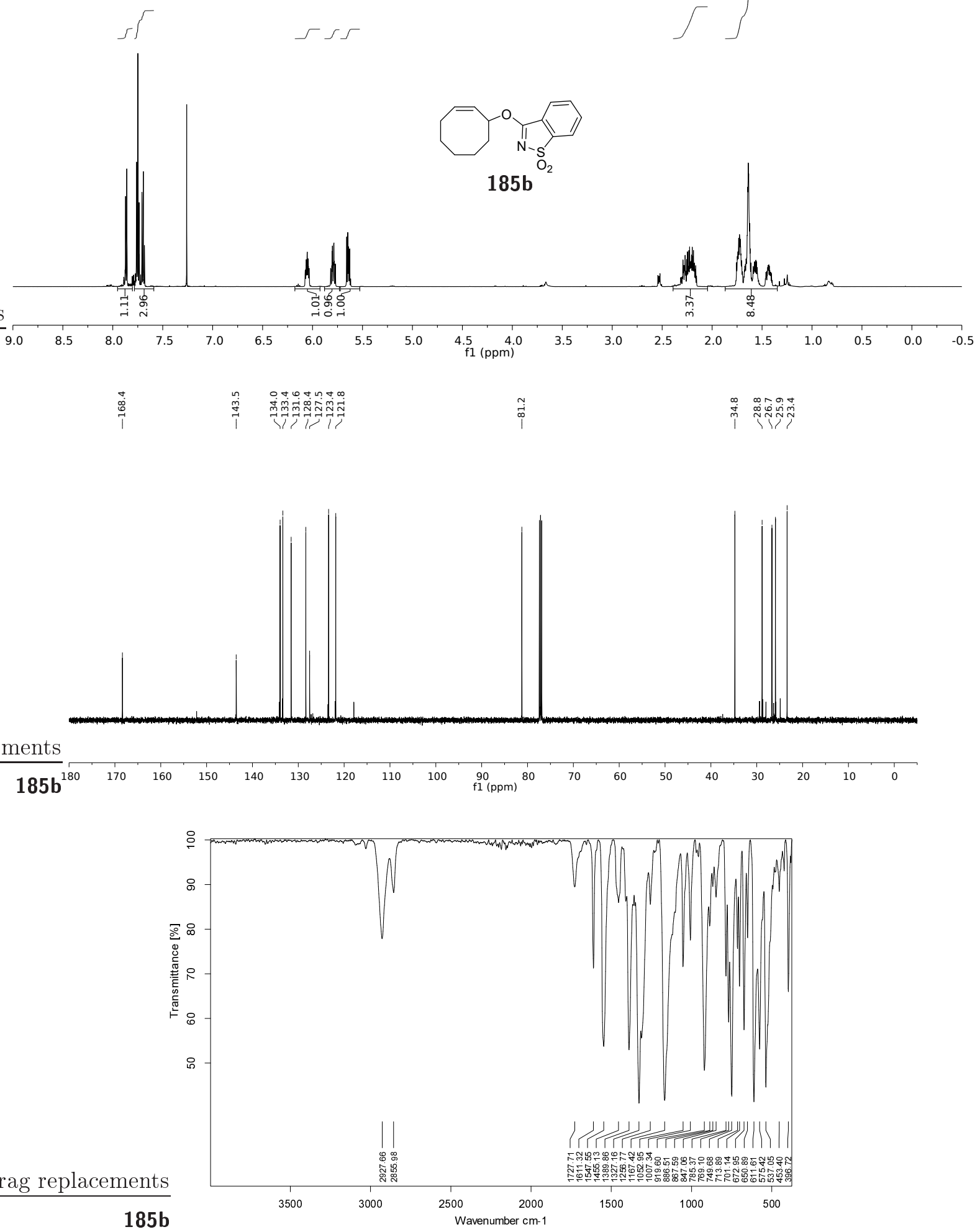
$-\sqrt{1}$
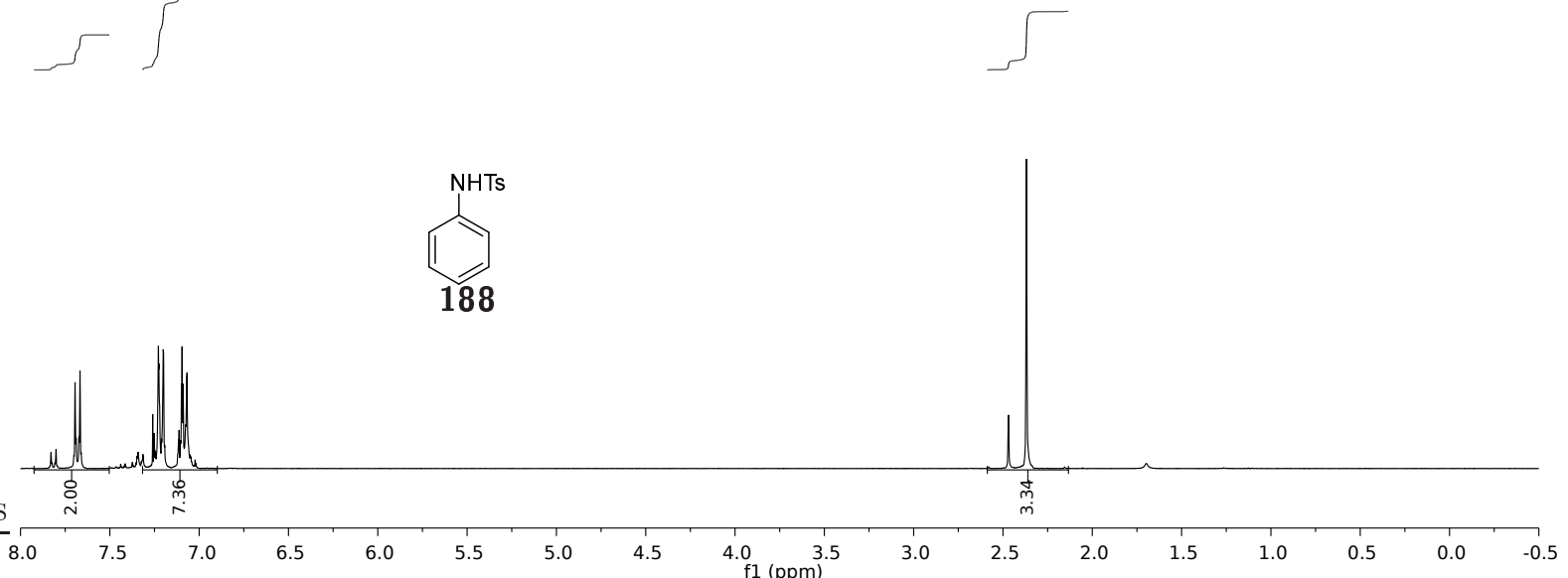

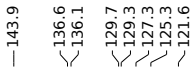
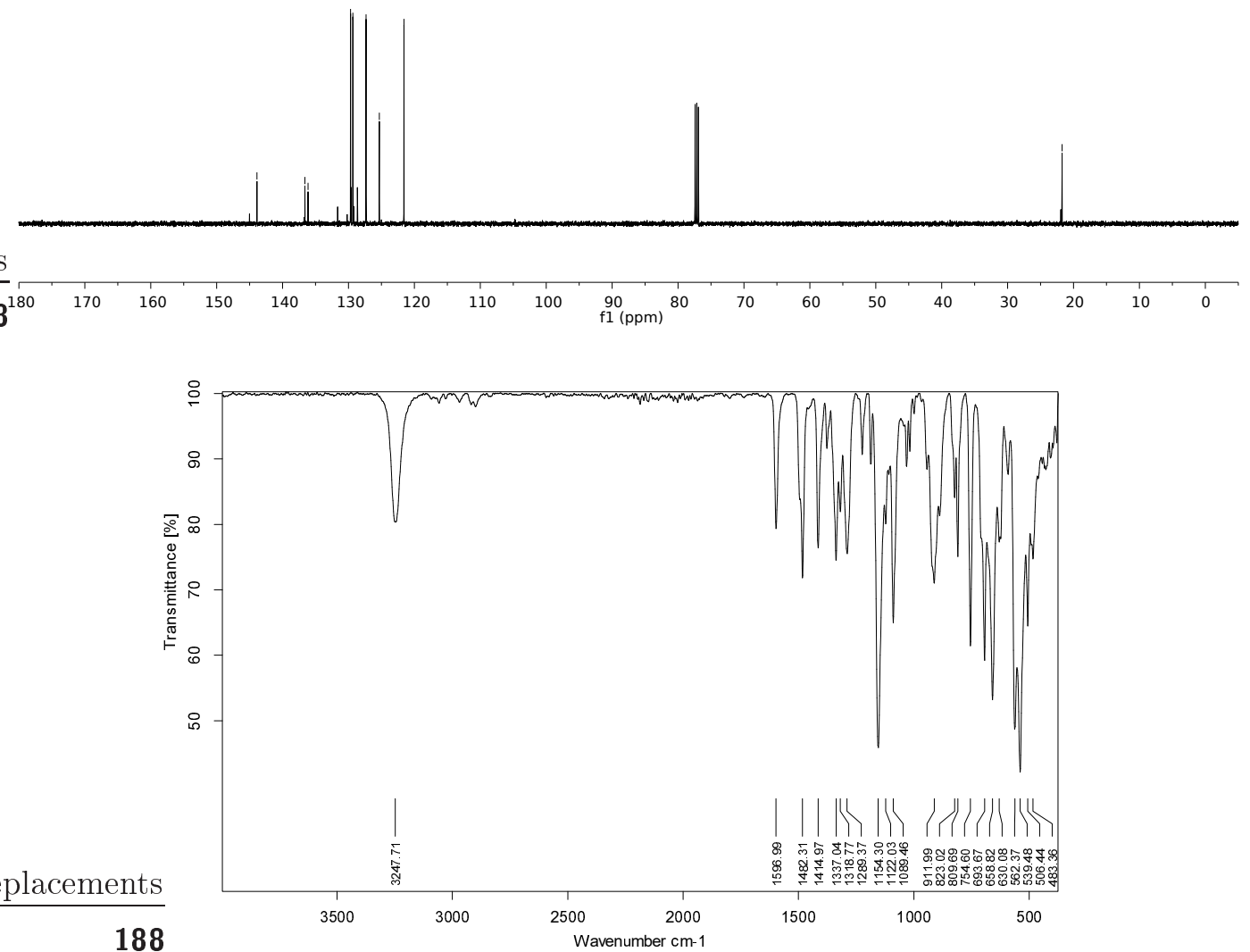


$$
\text { Ses }
$$
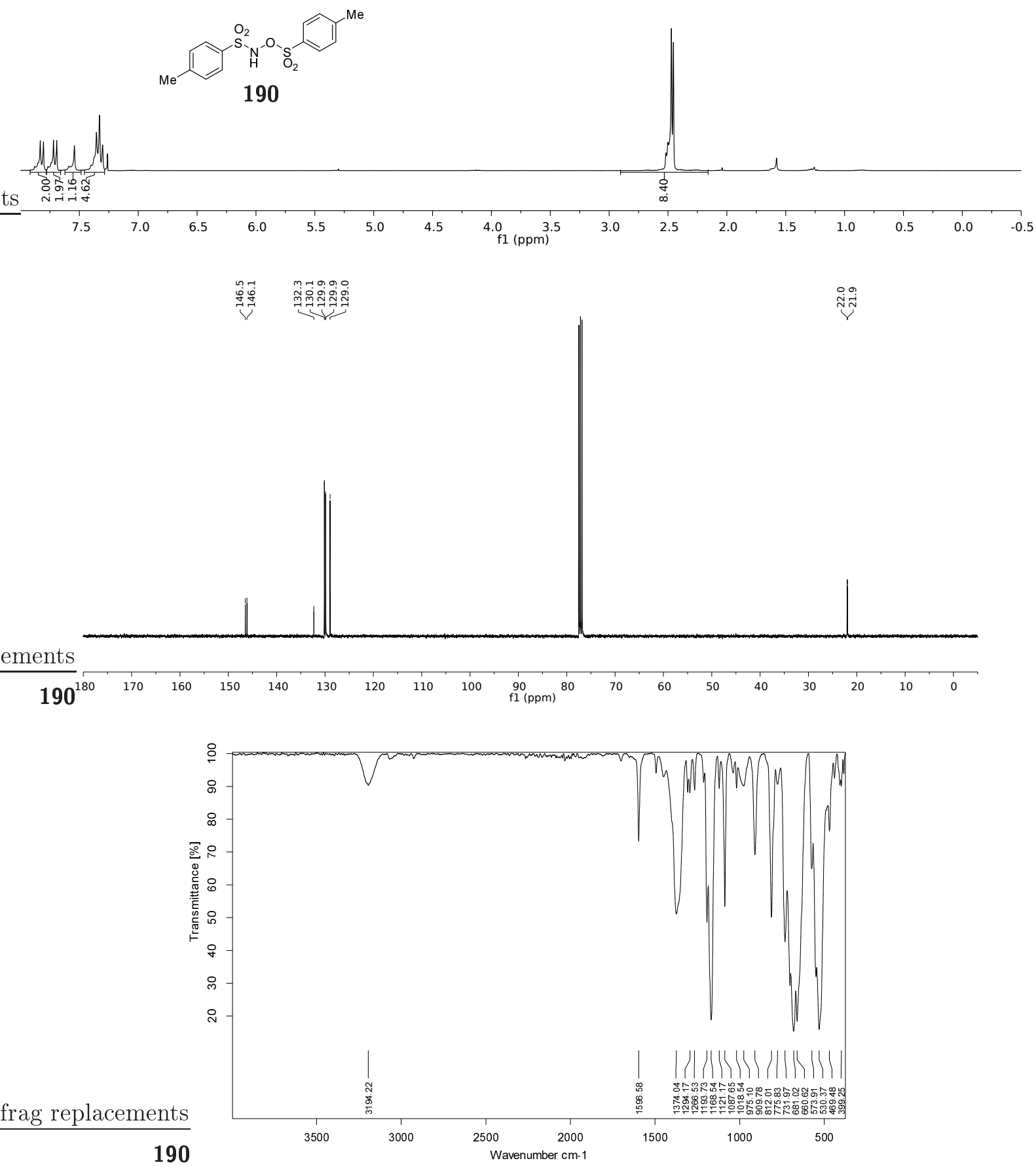

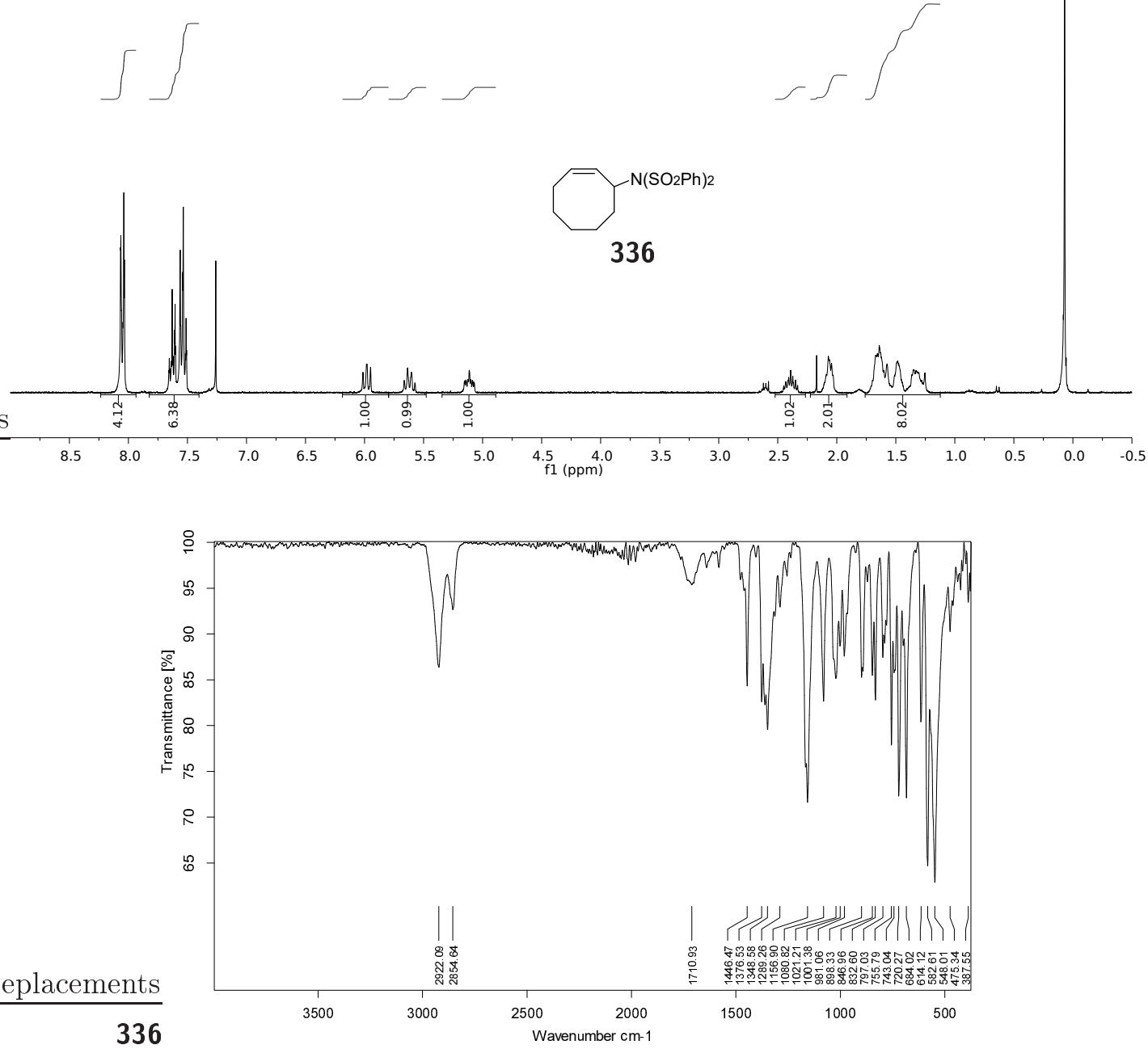

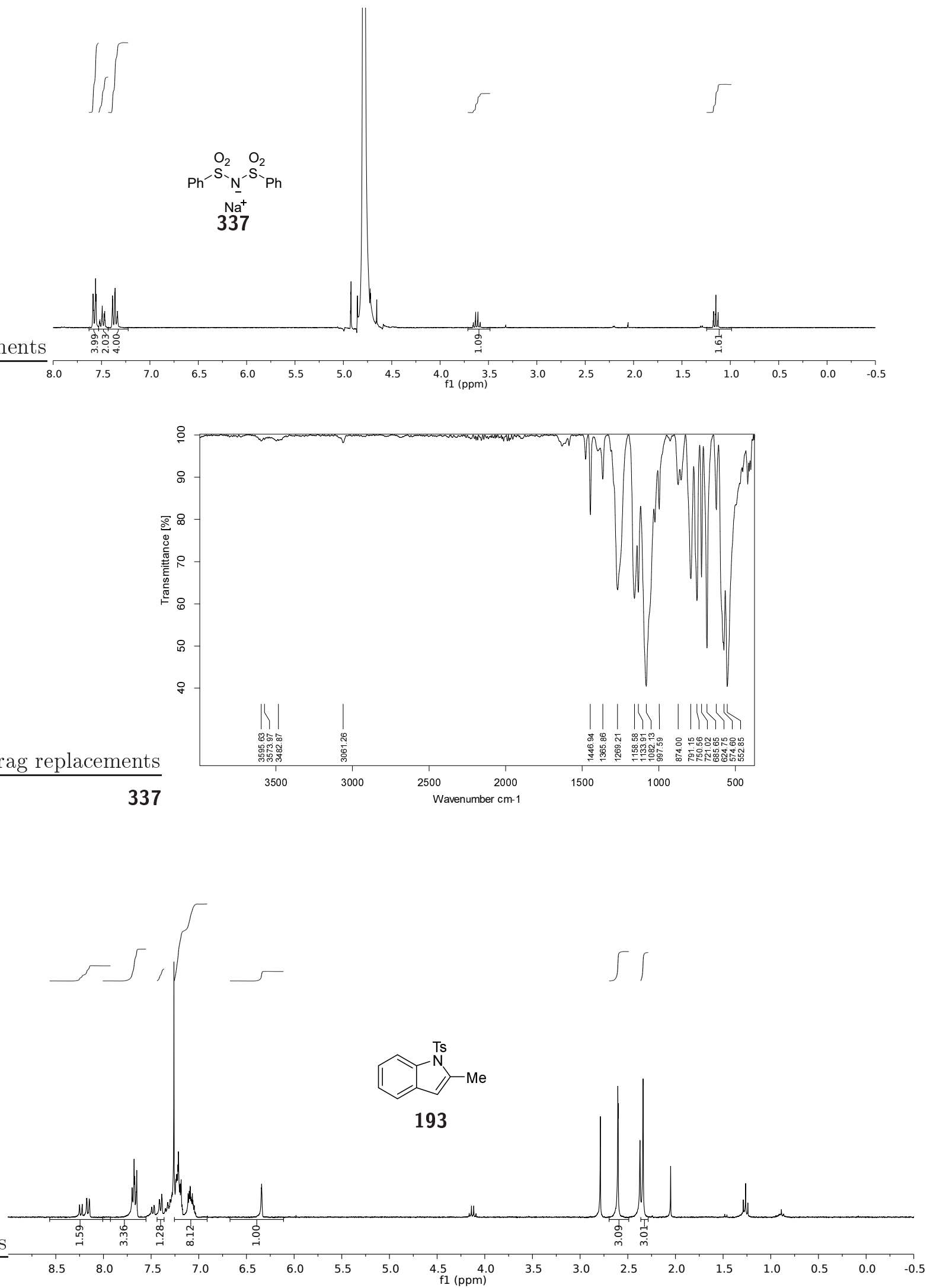


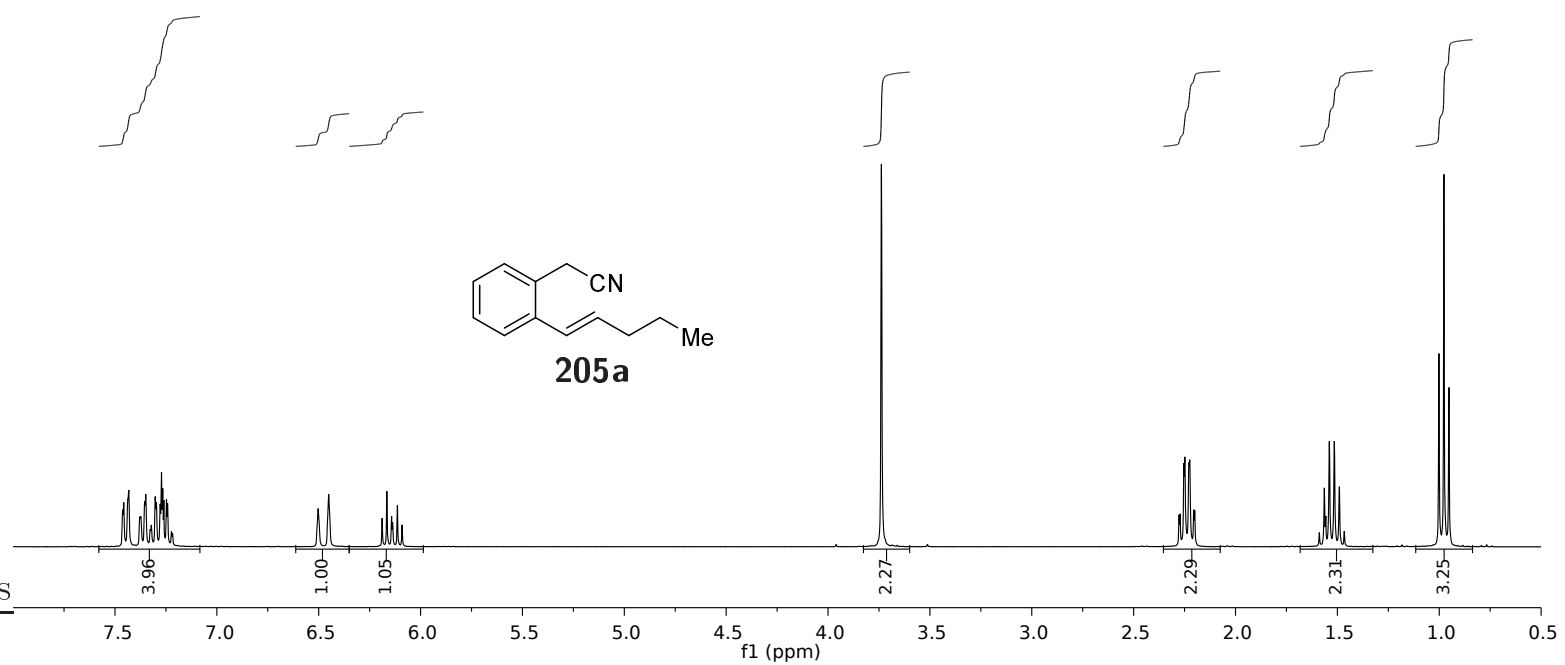




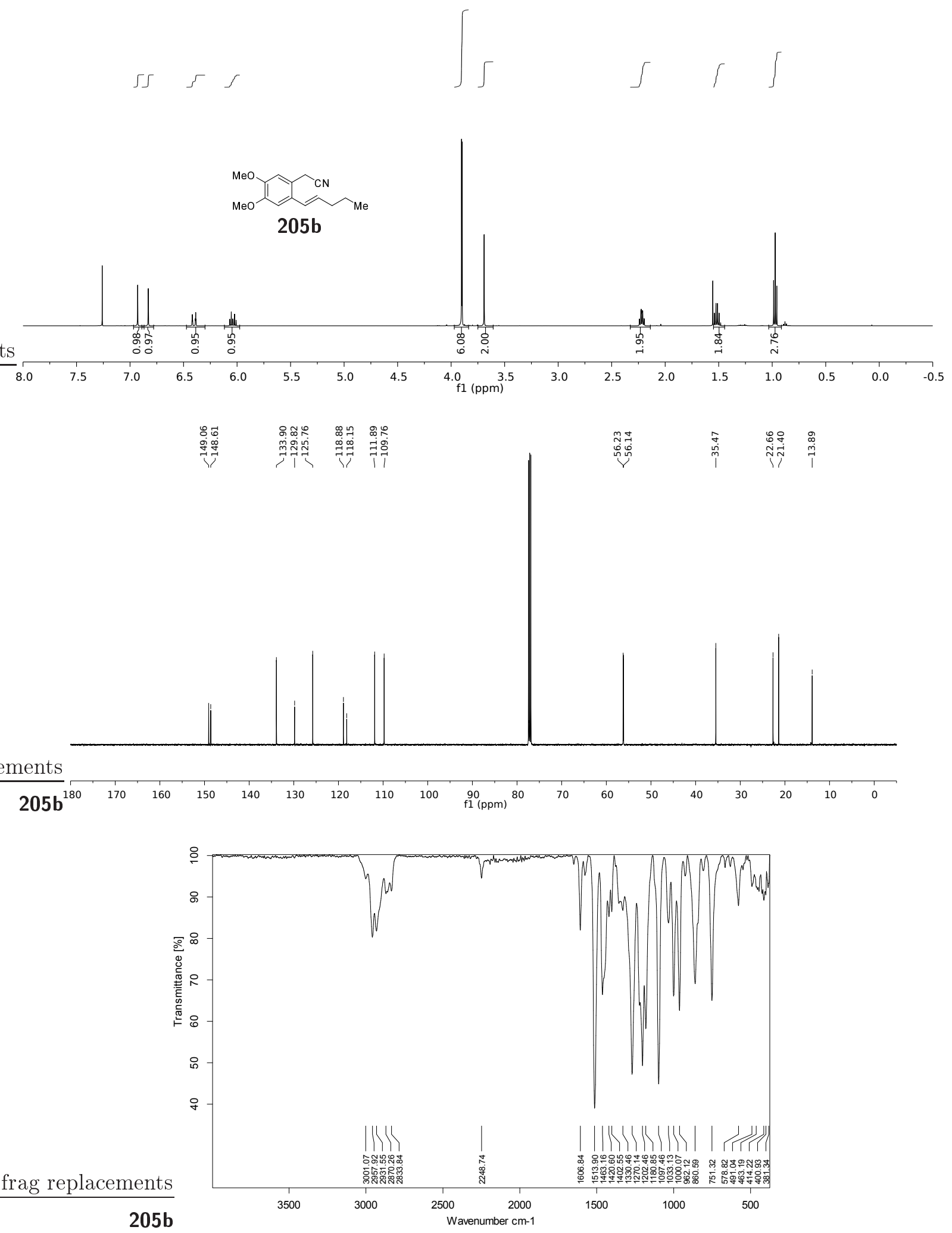



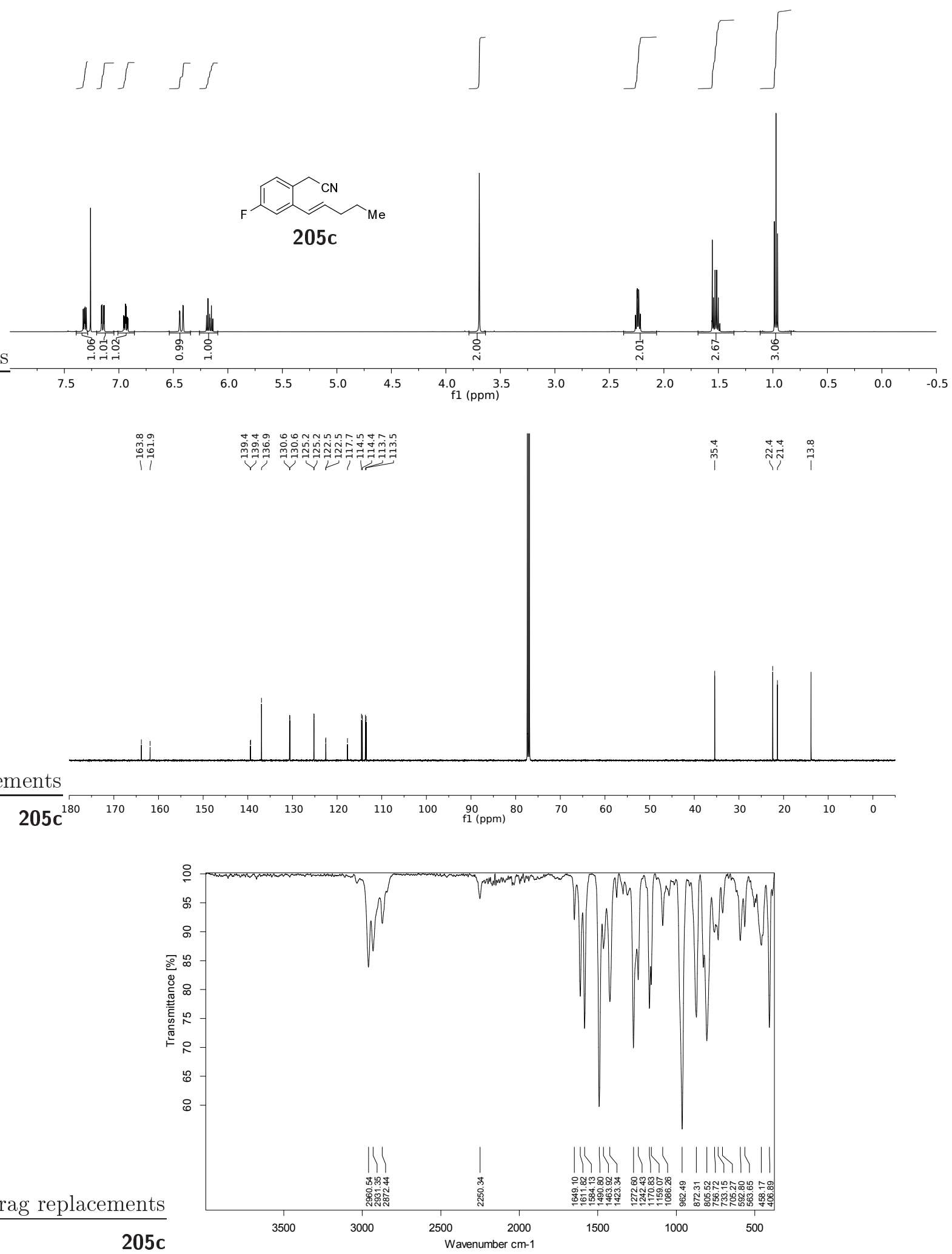


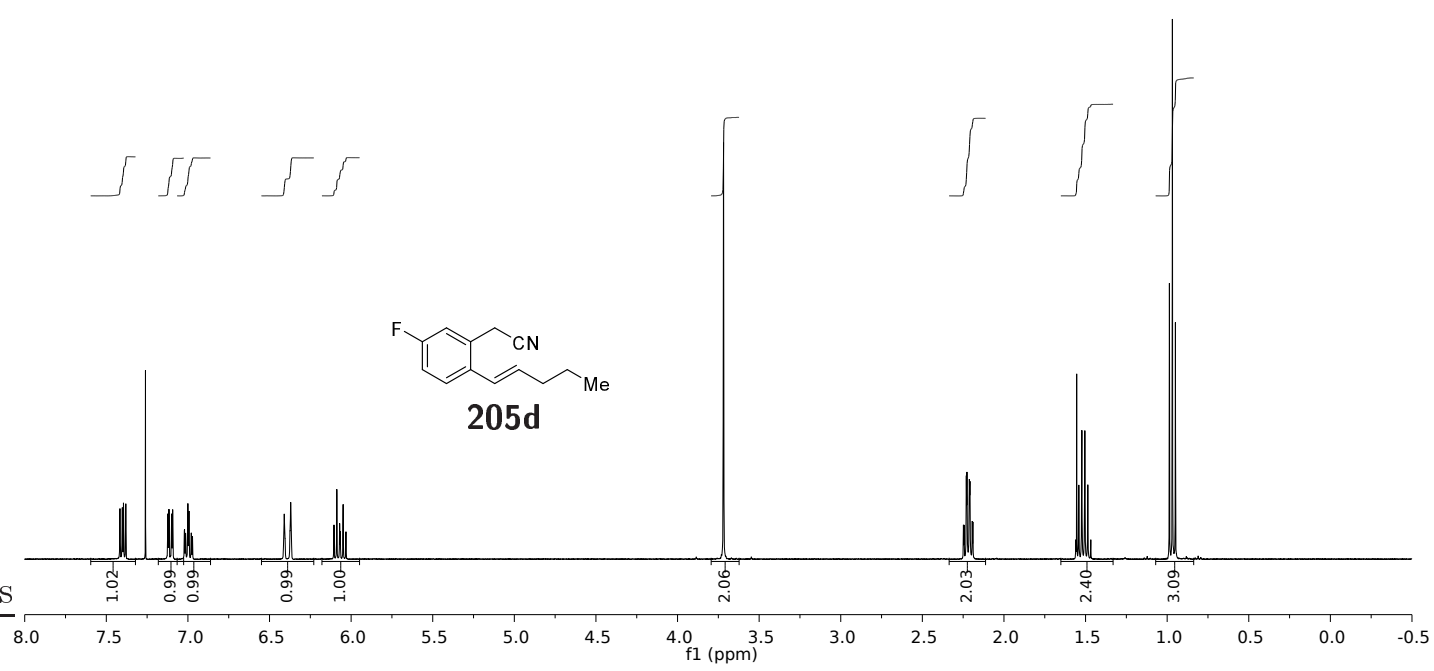

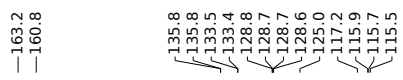

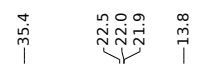
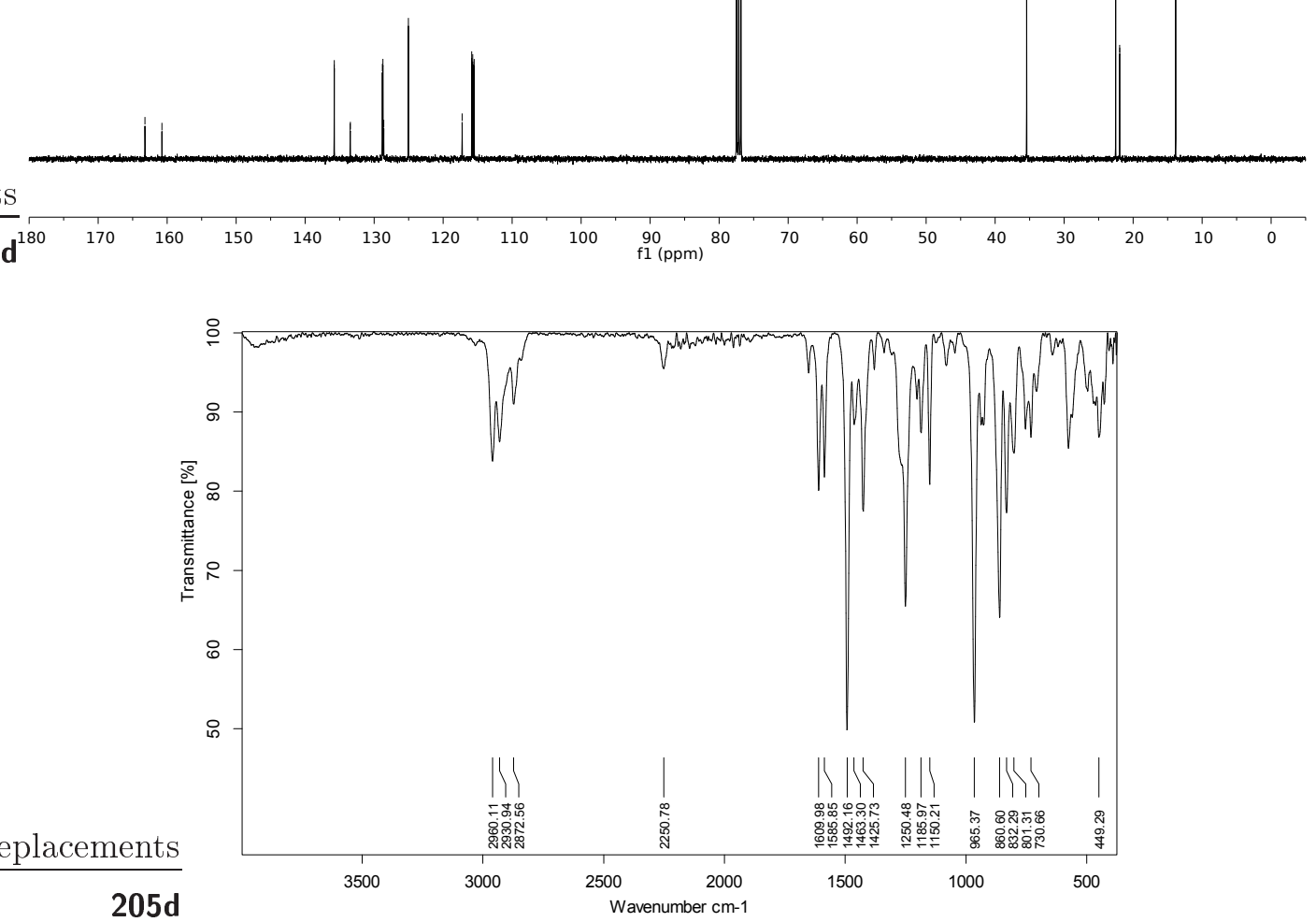

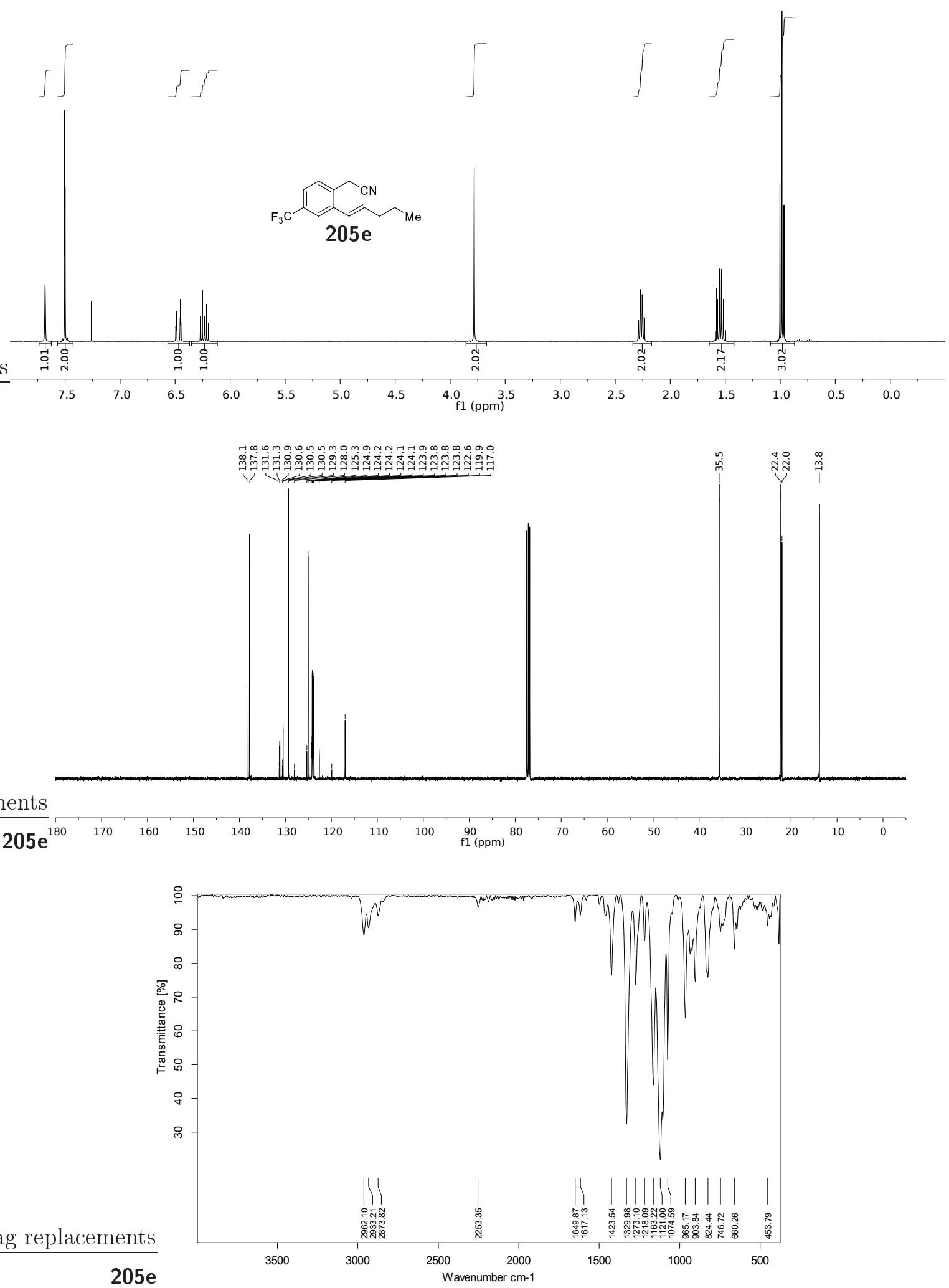

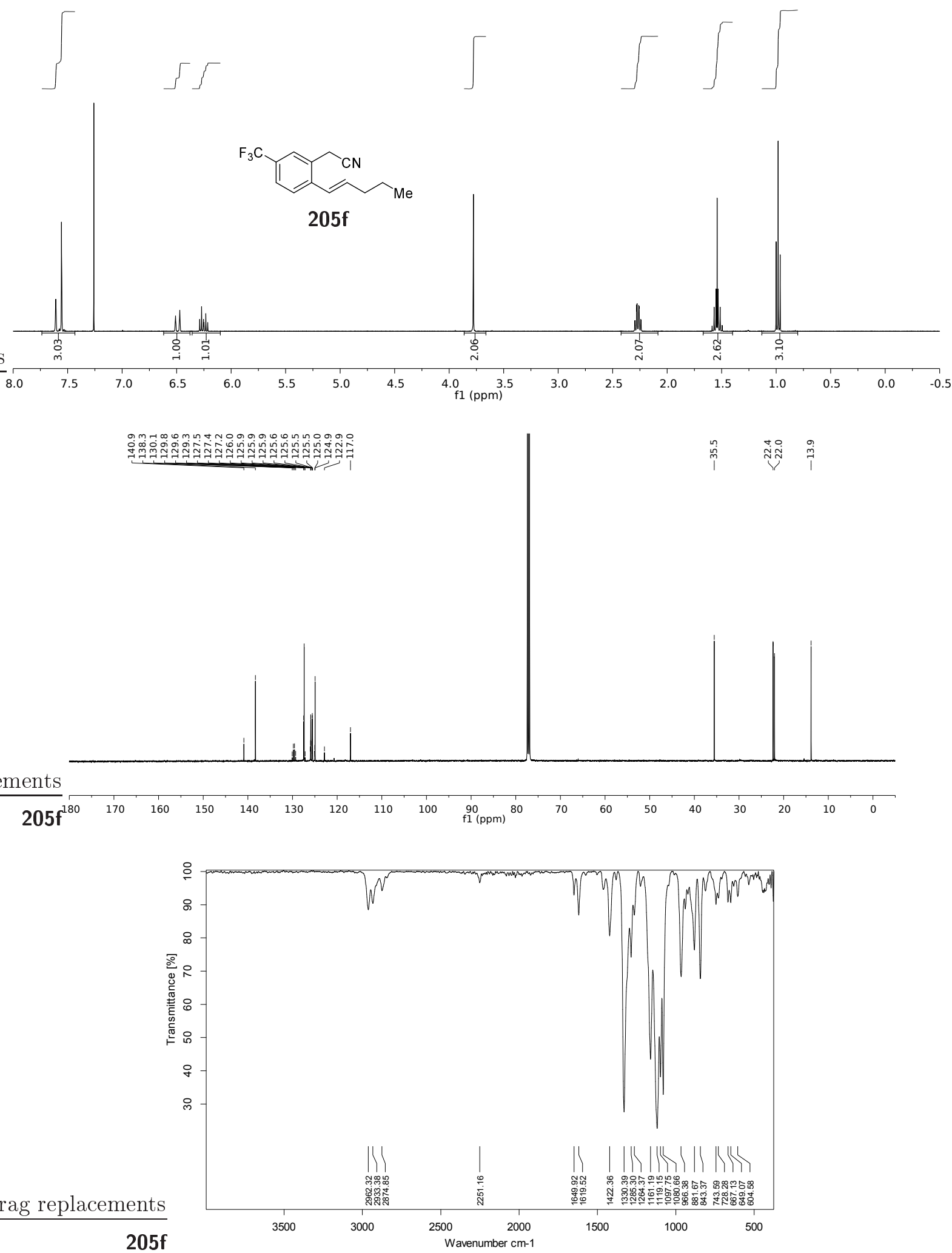


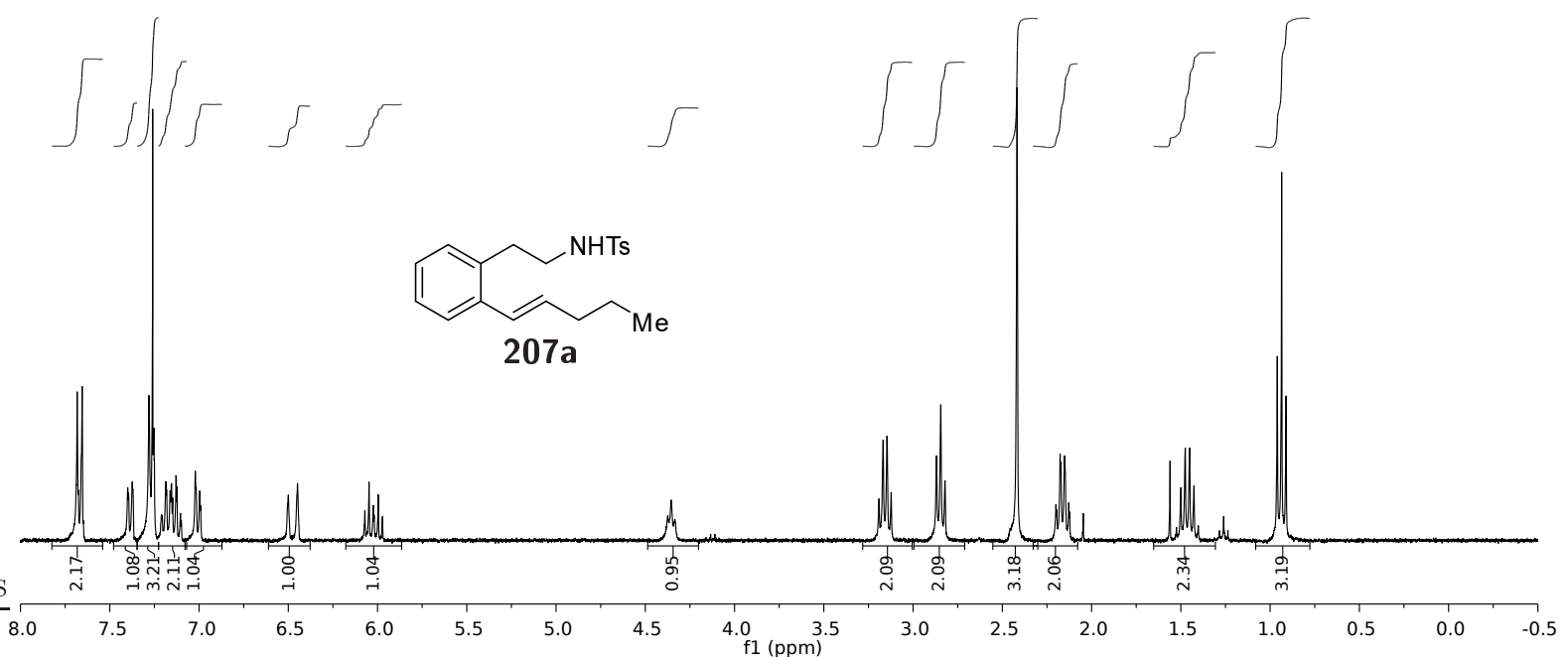




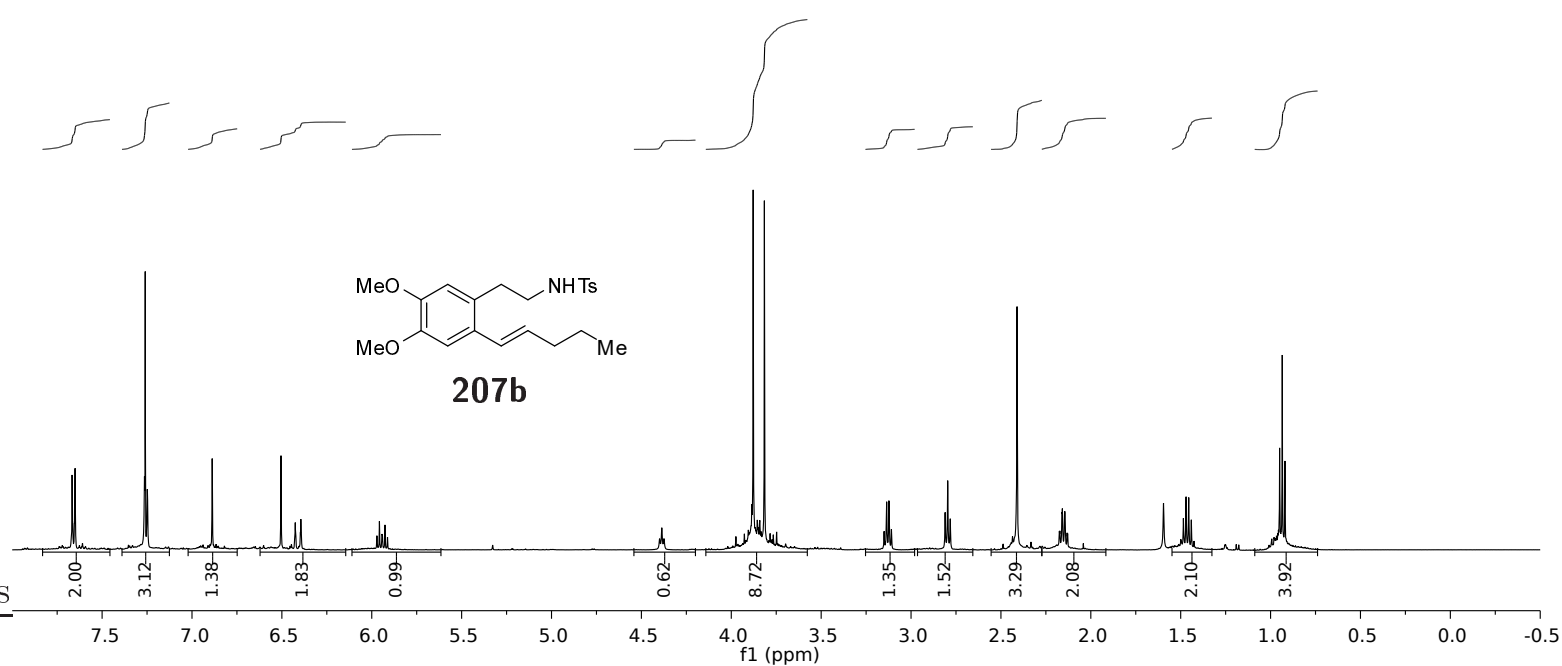

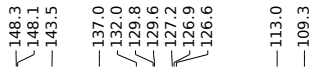

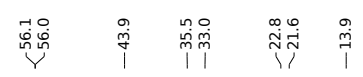
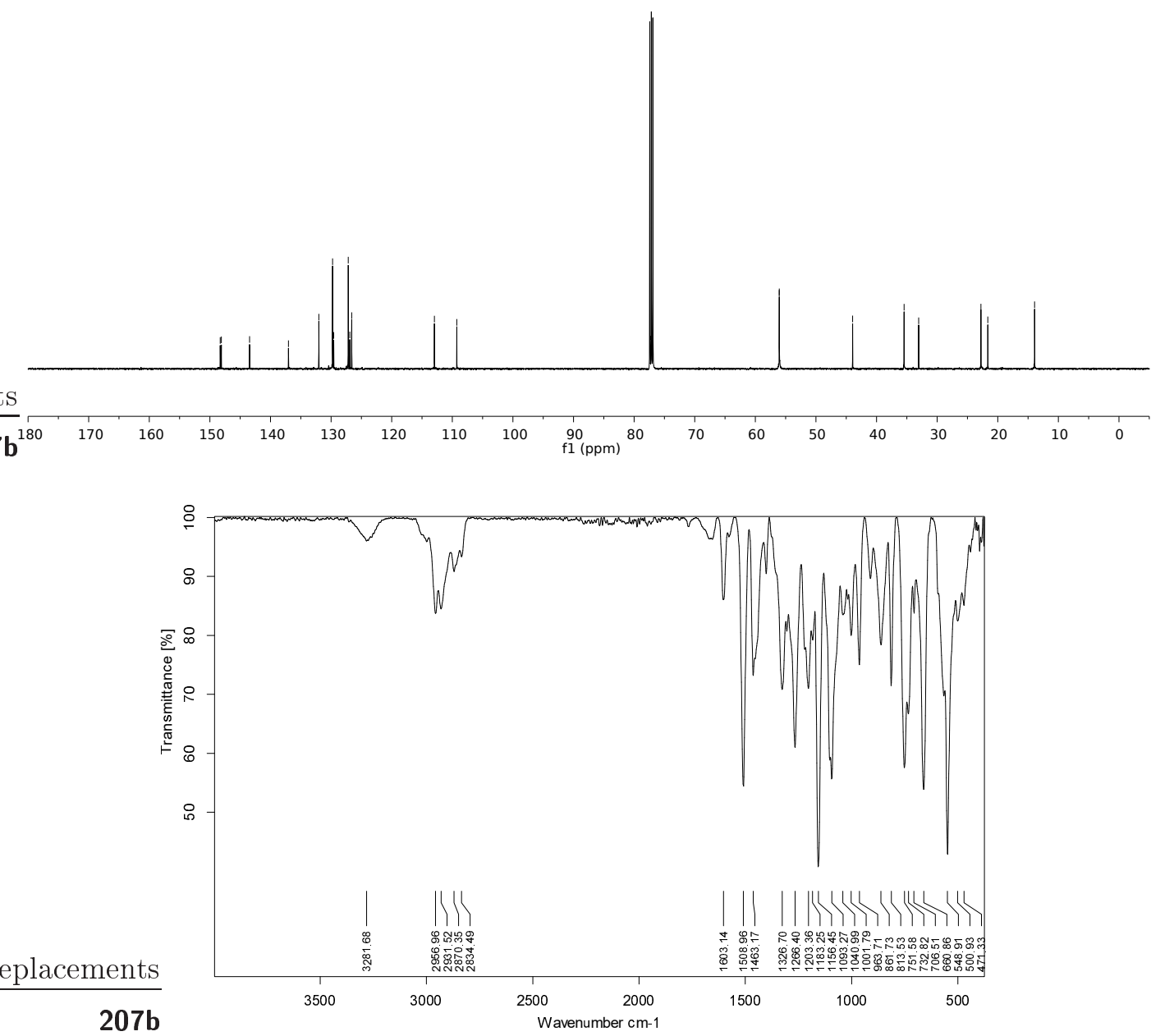

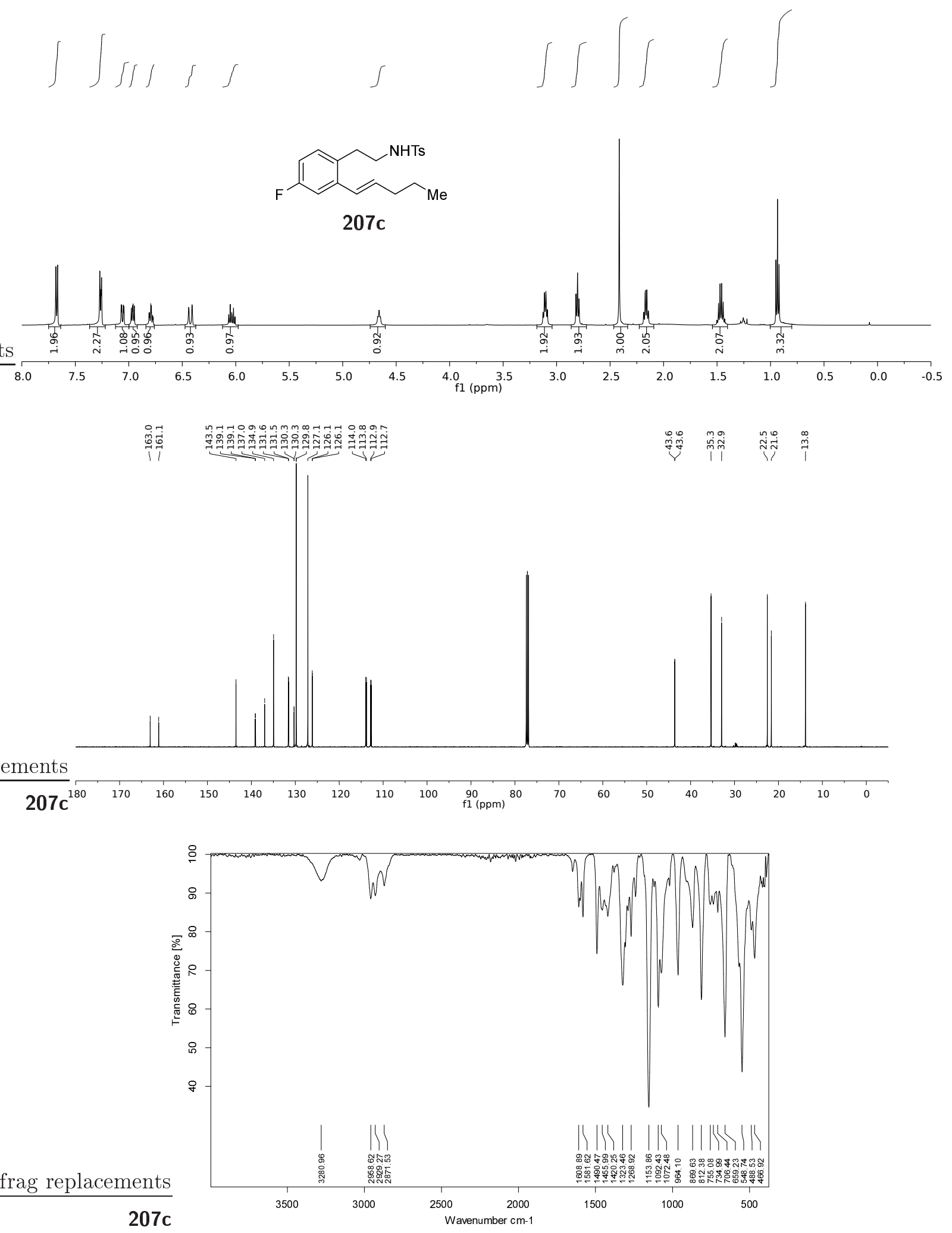


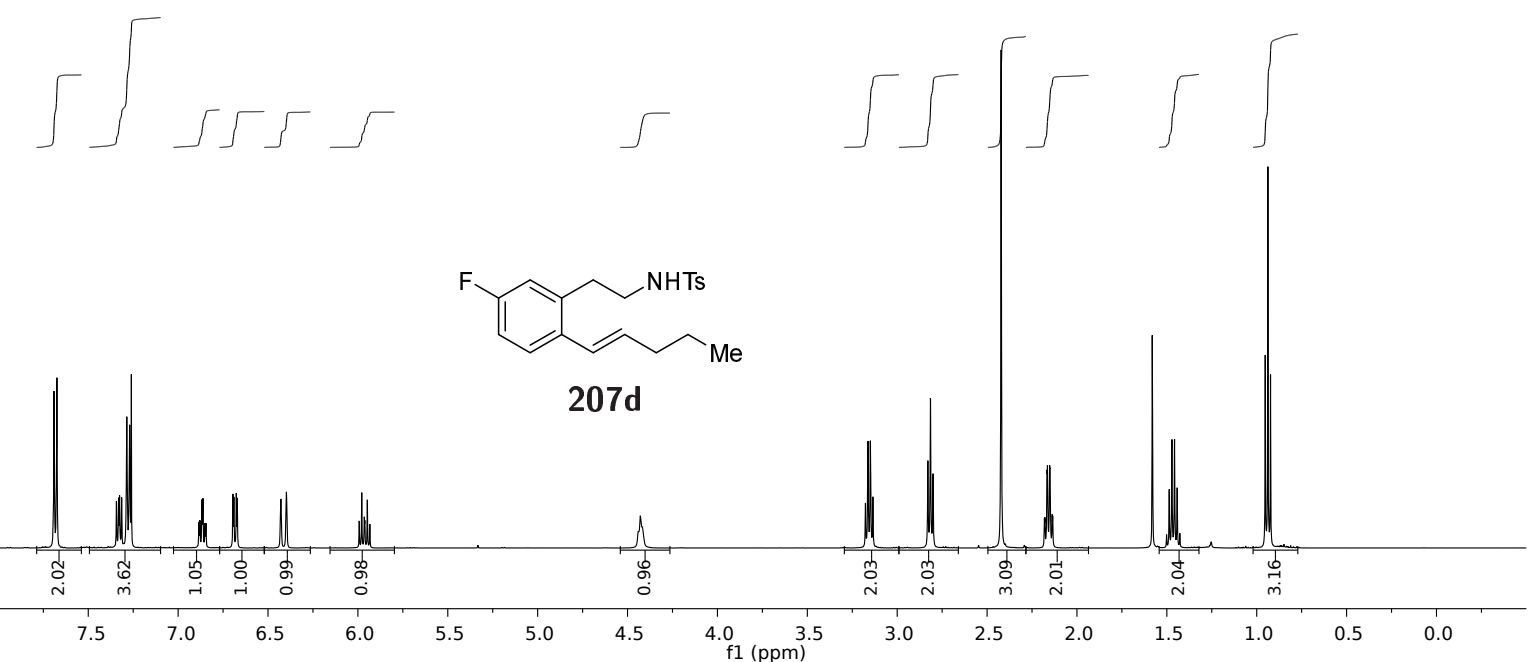

我

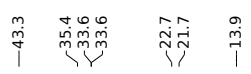
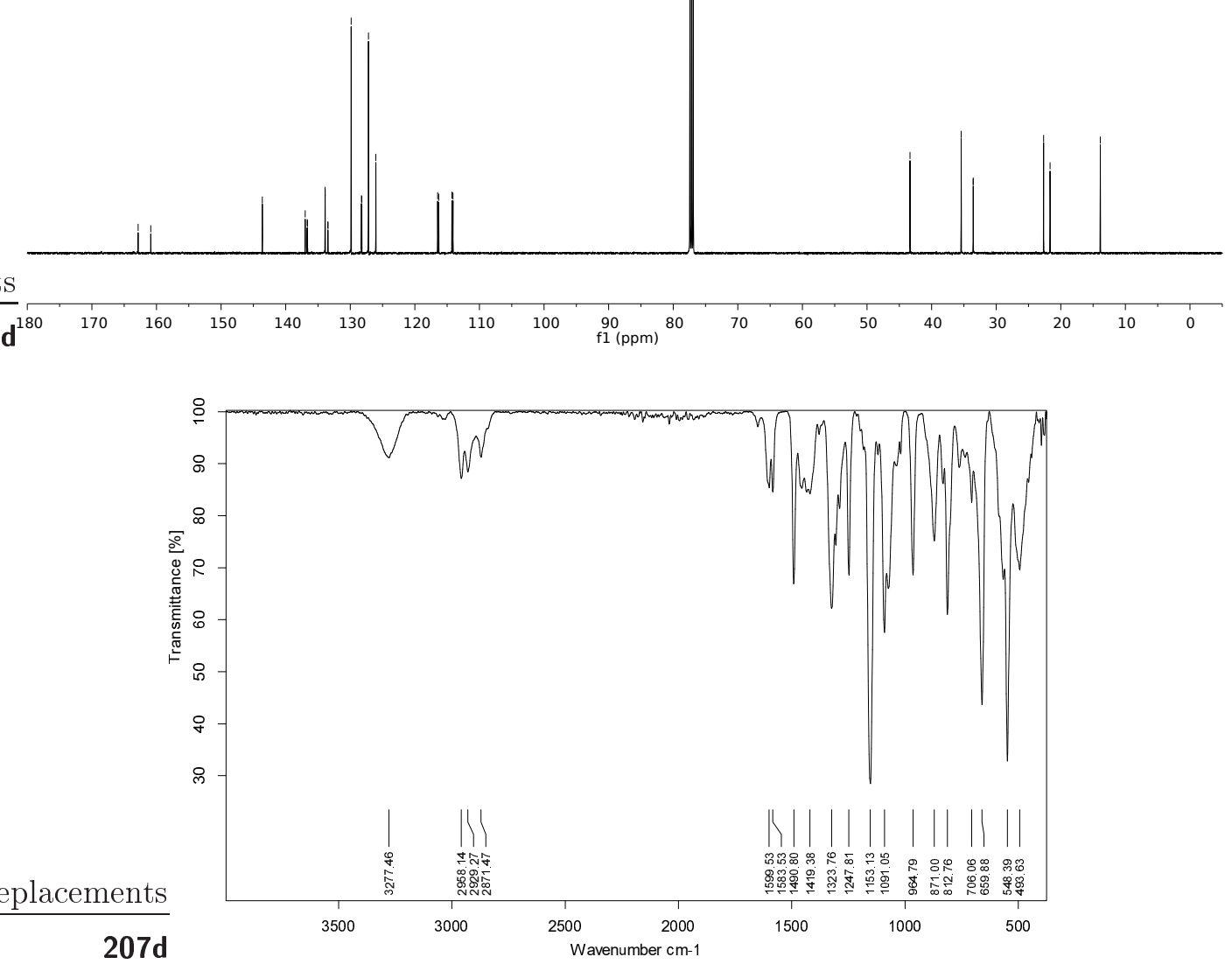

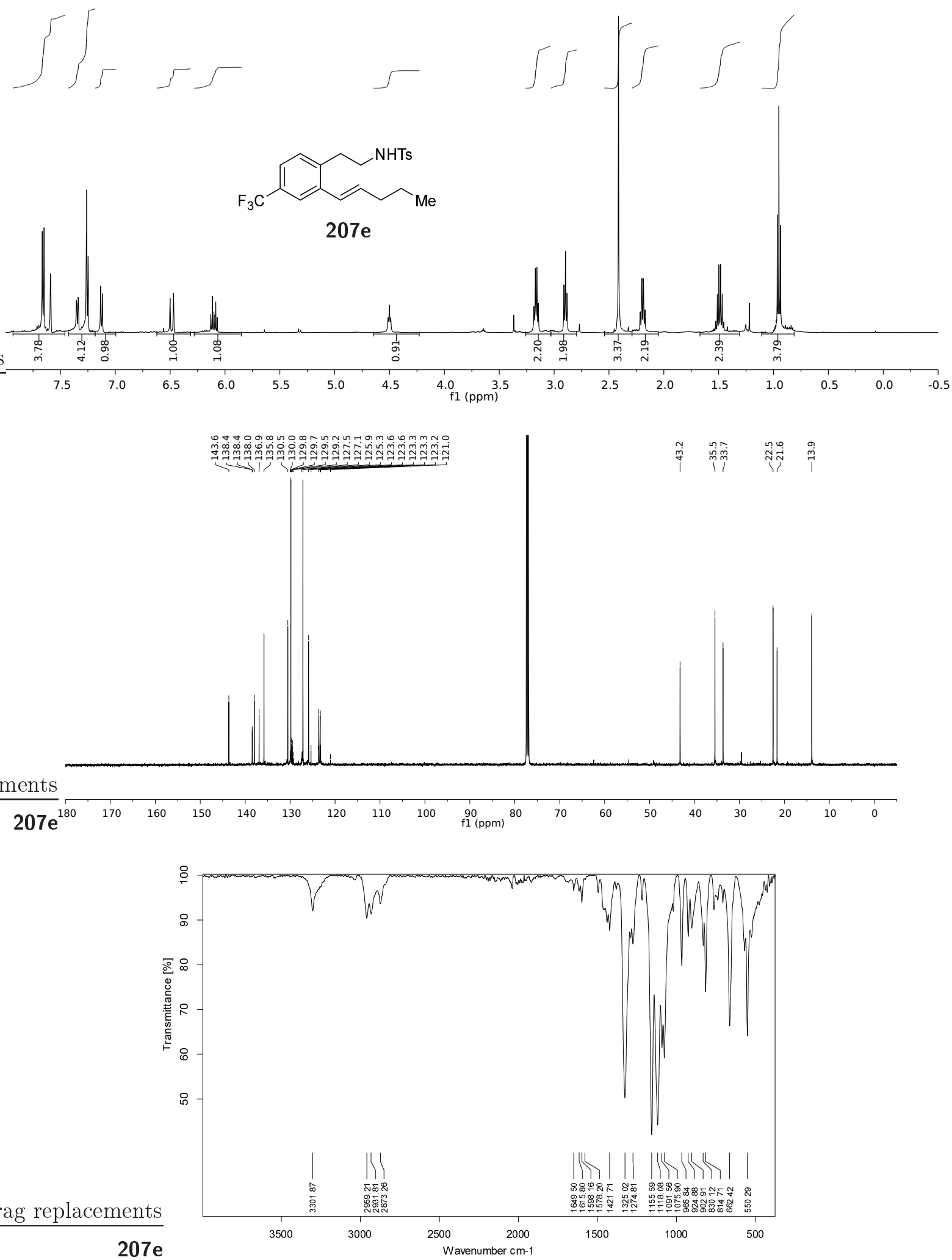


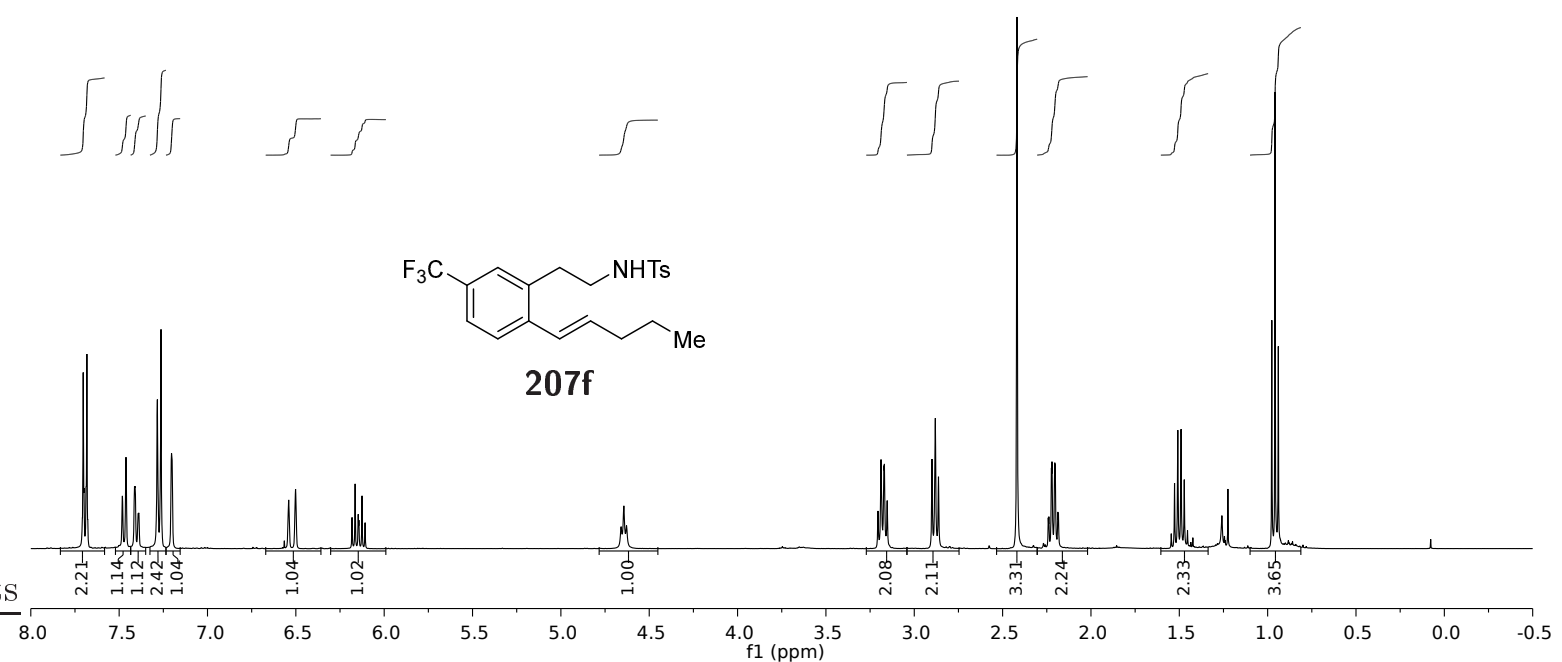

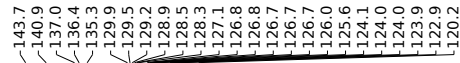

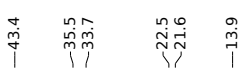
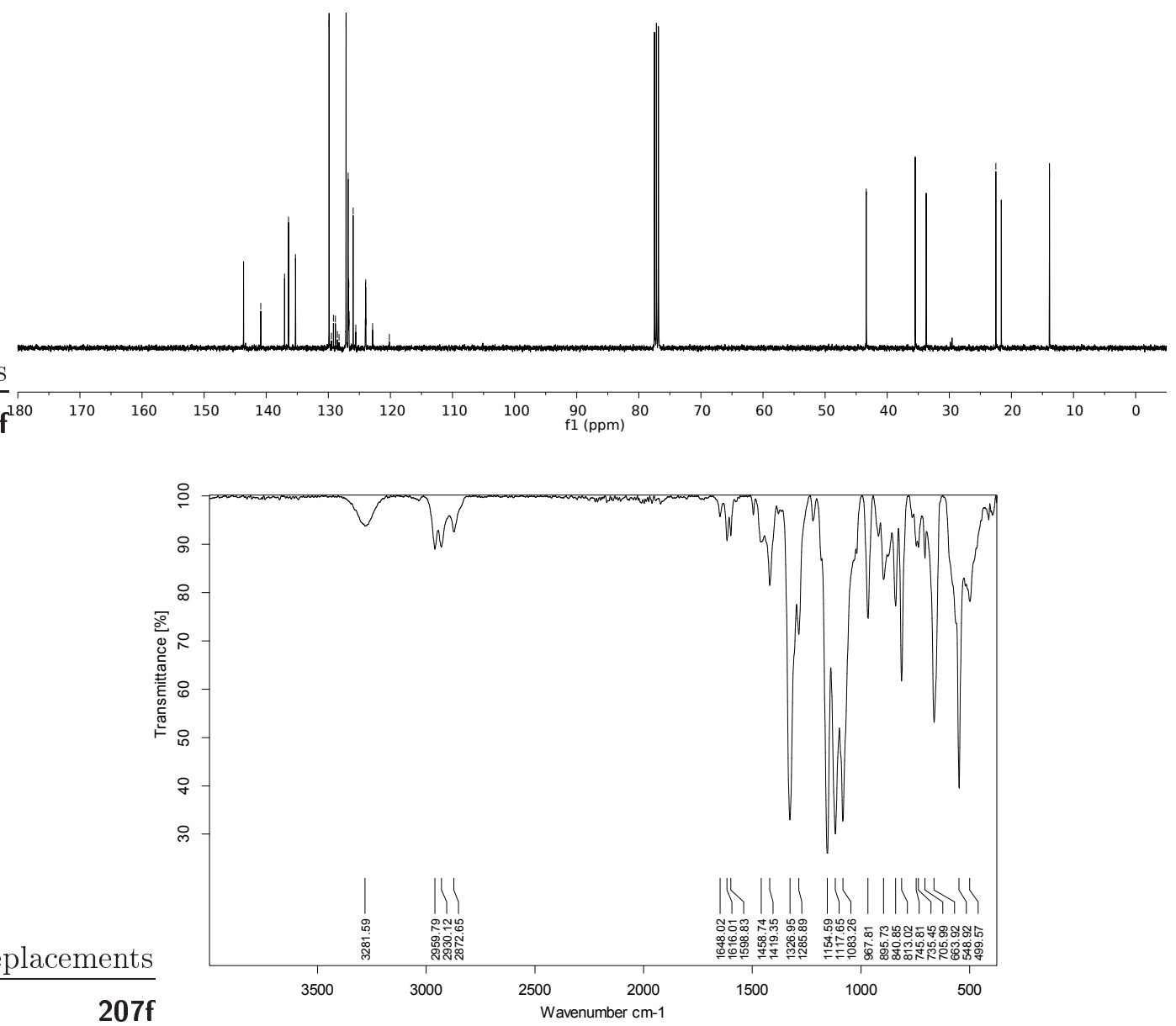


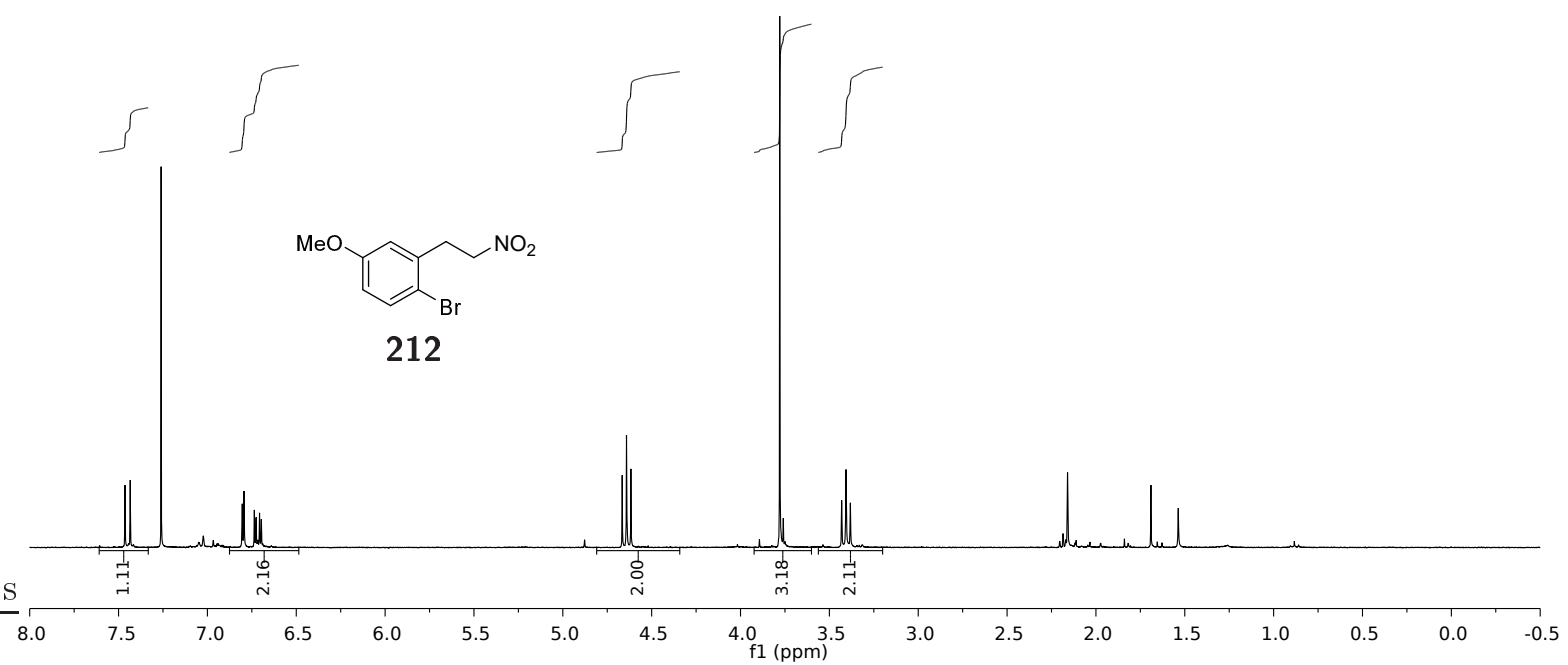



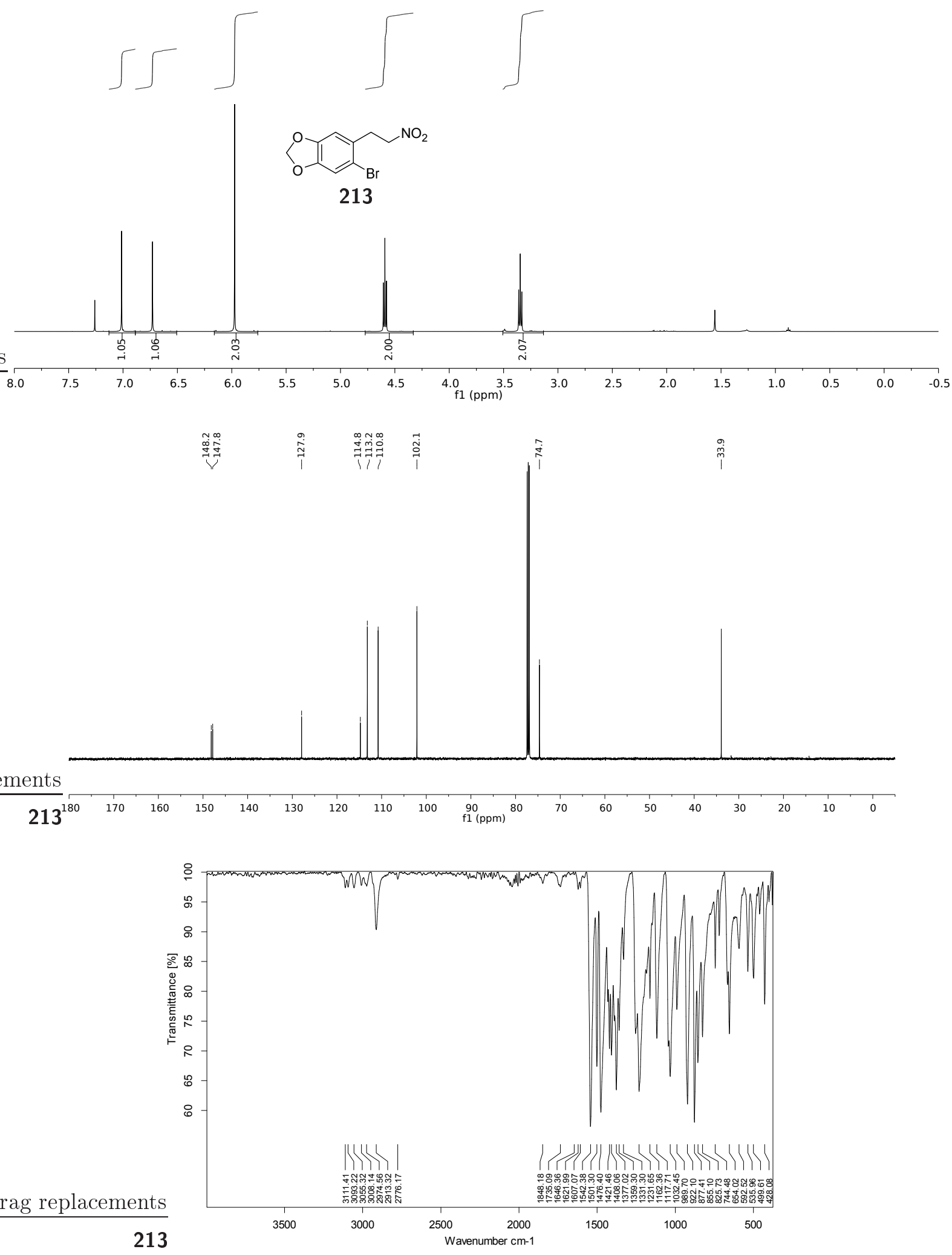


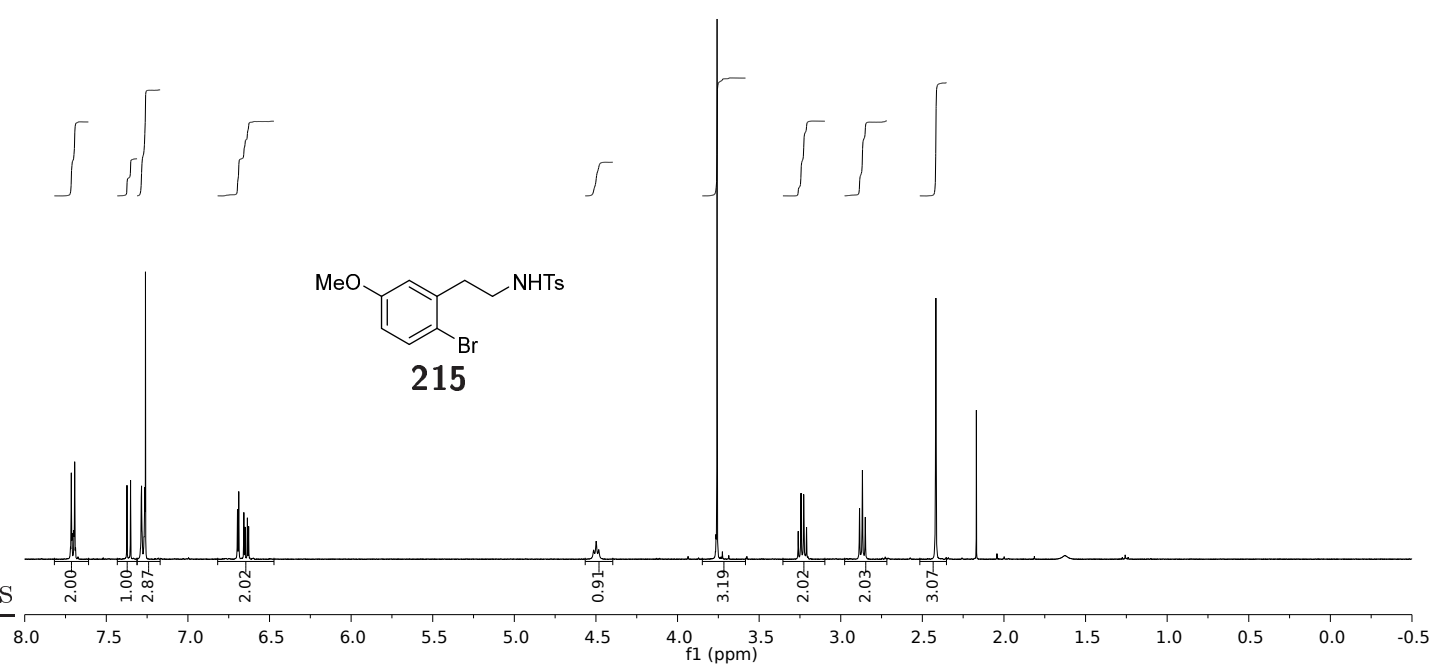

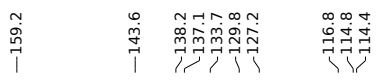

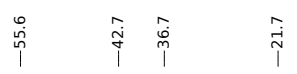
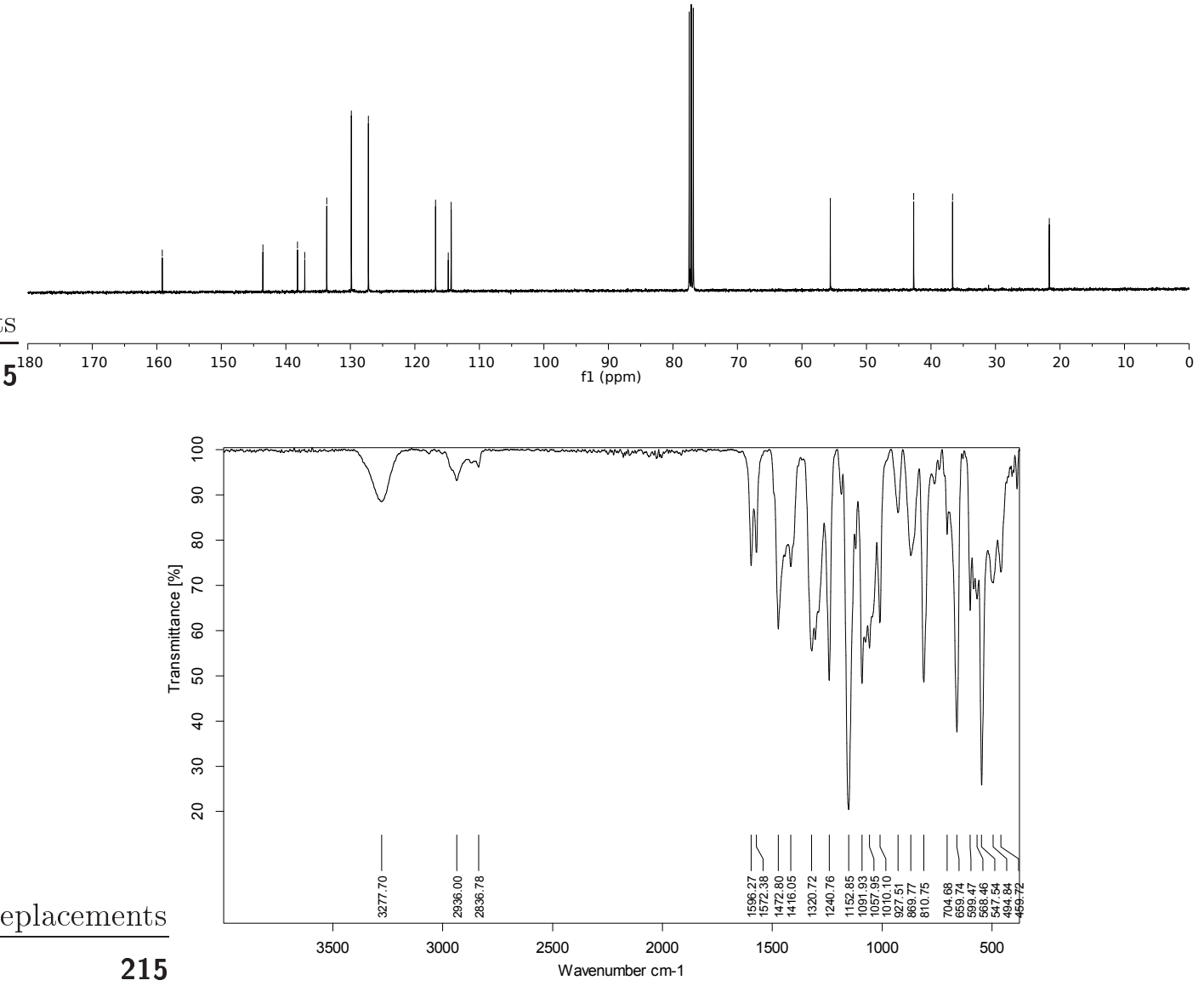


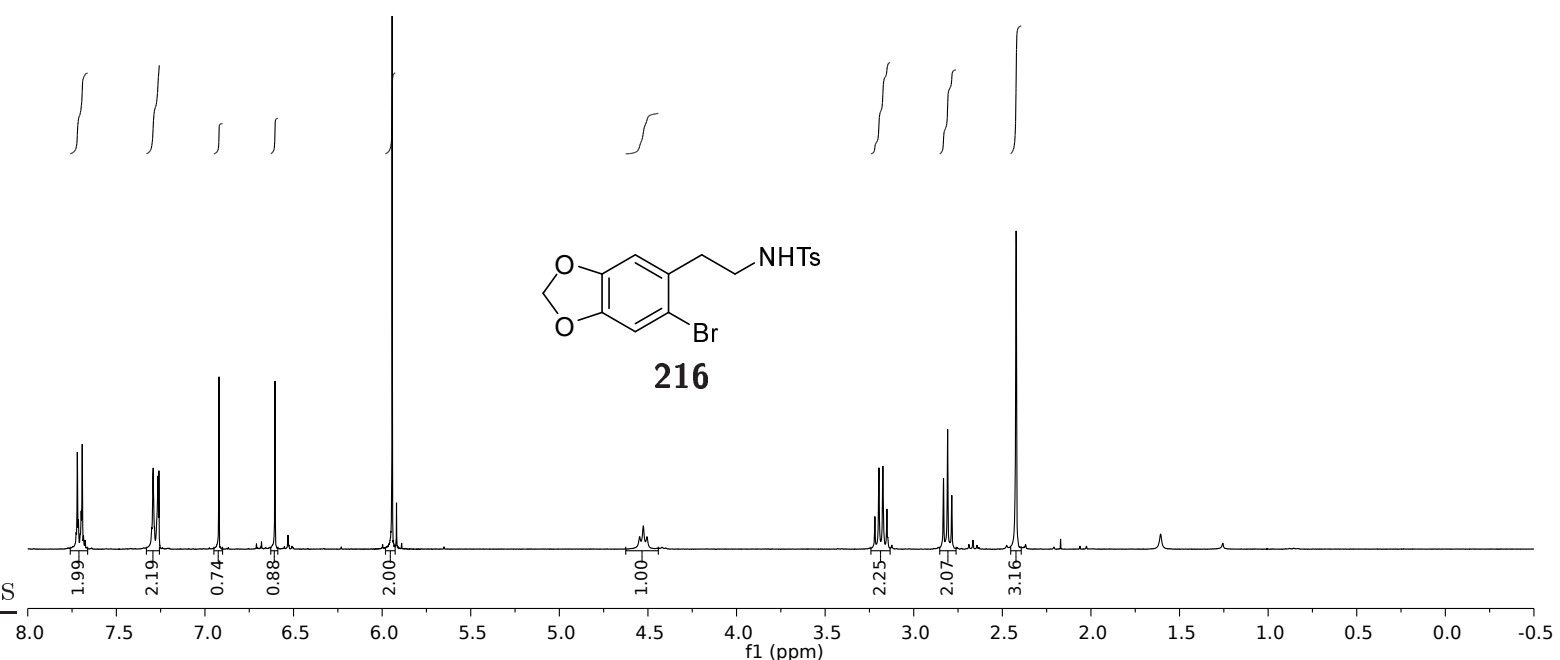

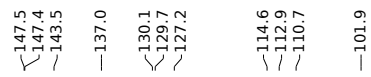

隽索市
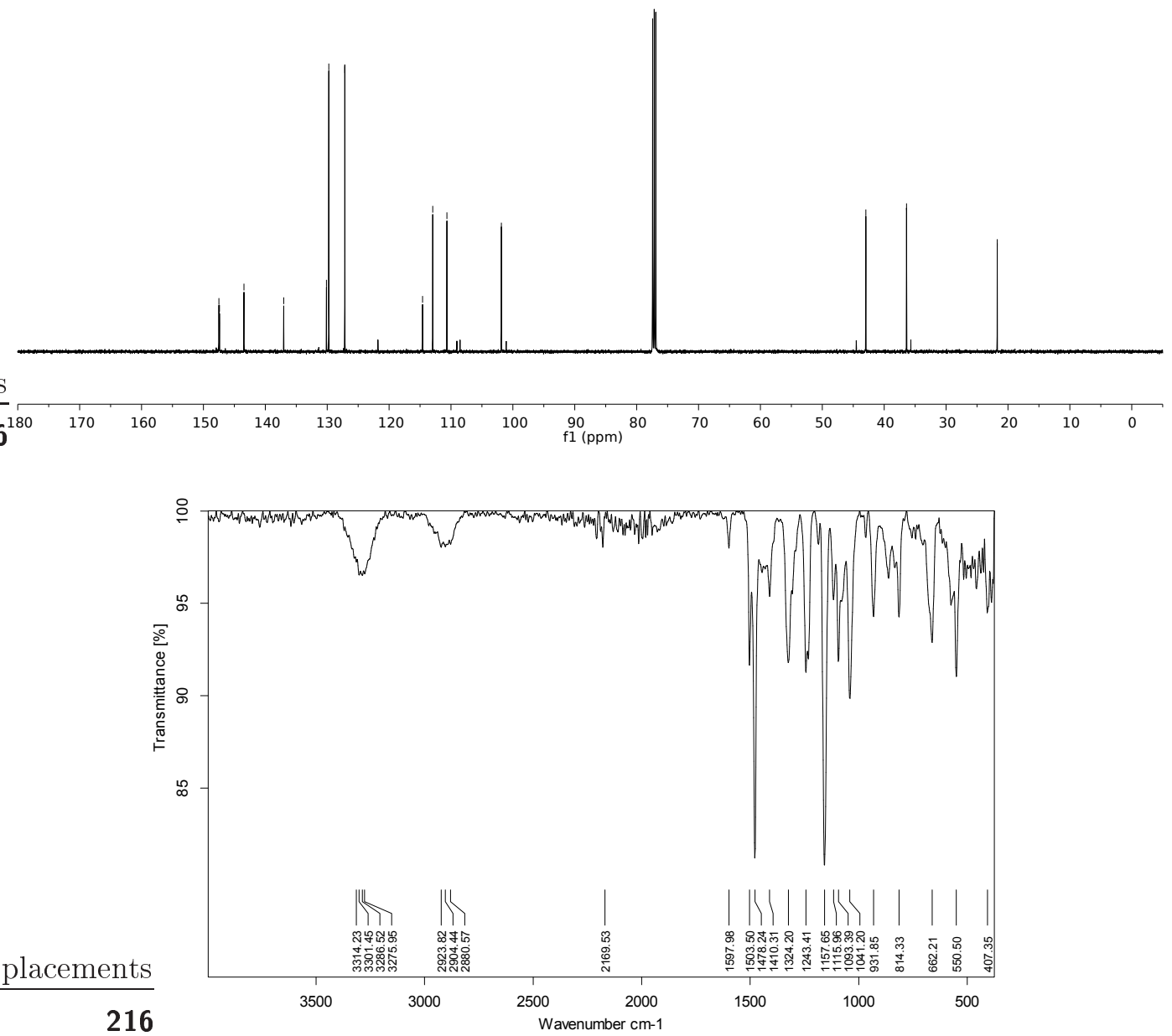

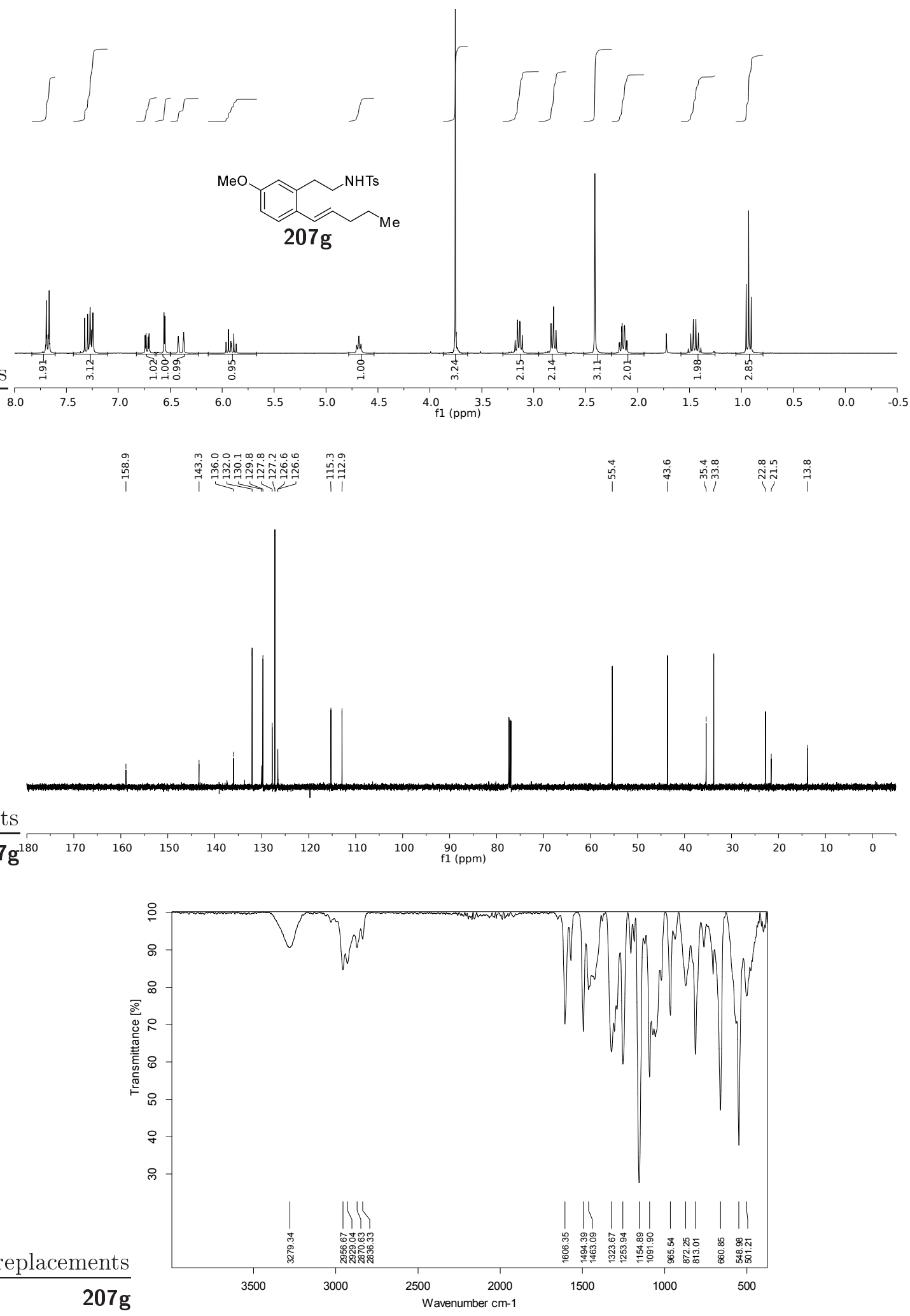


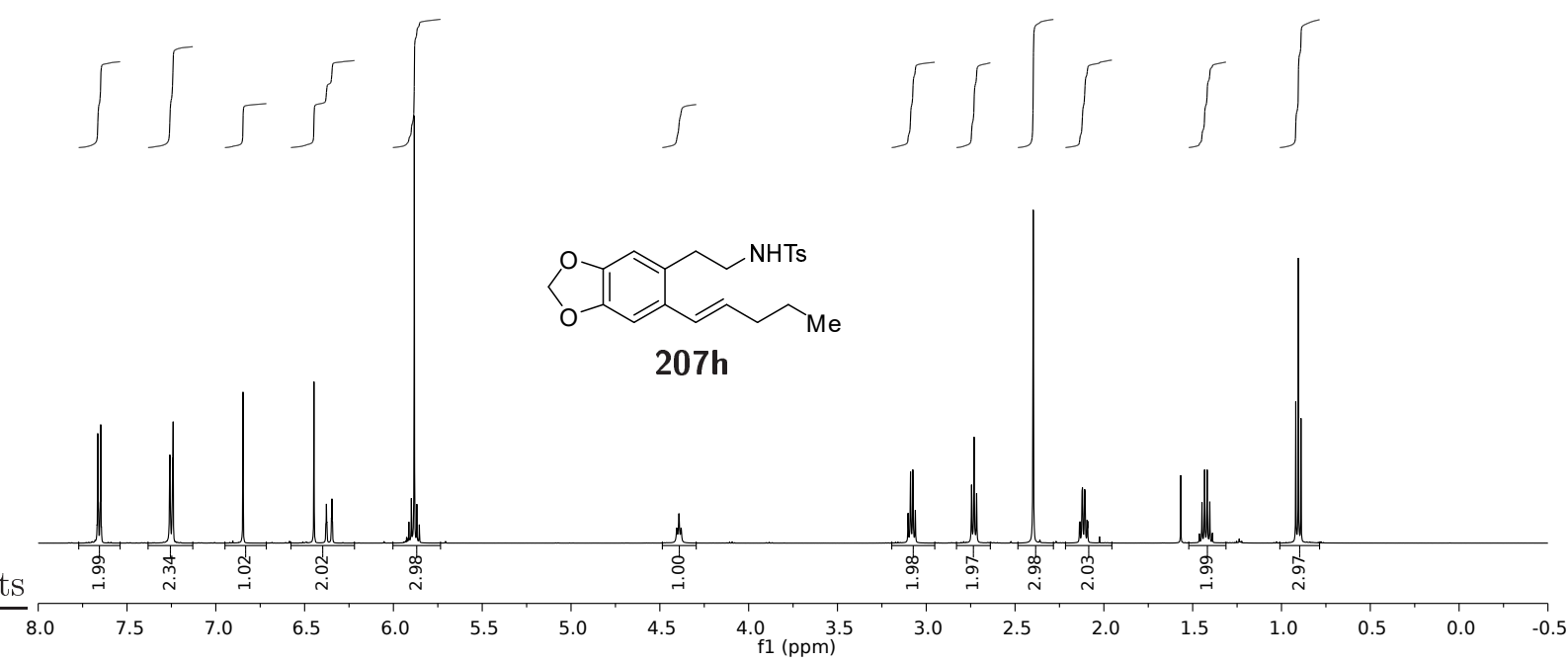

$\begin{array}{ll} & \\ 0 & 0\end{array}$

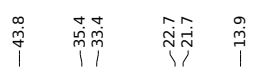
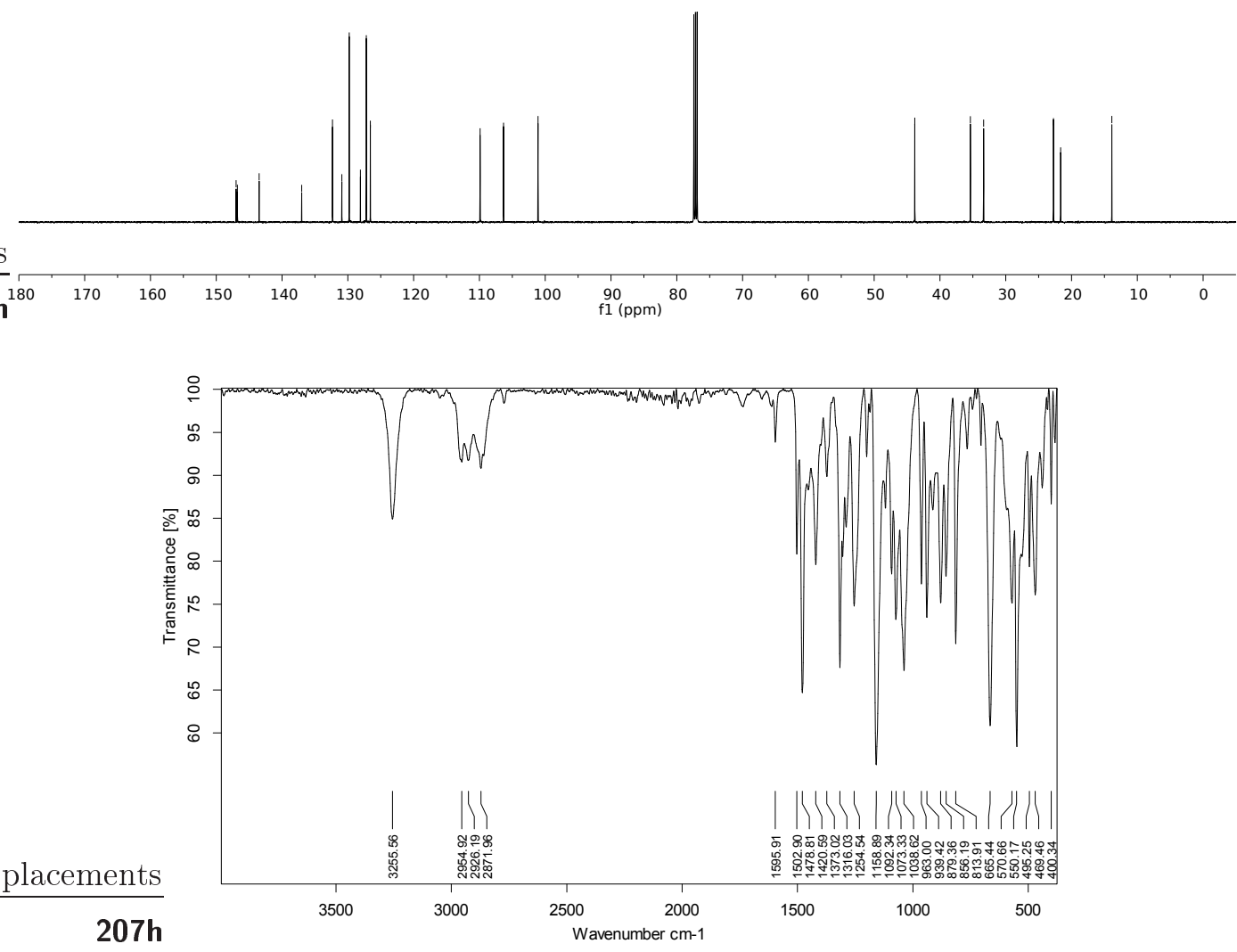


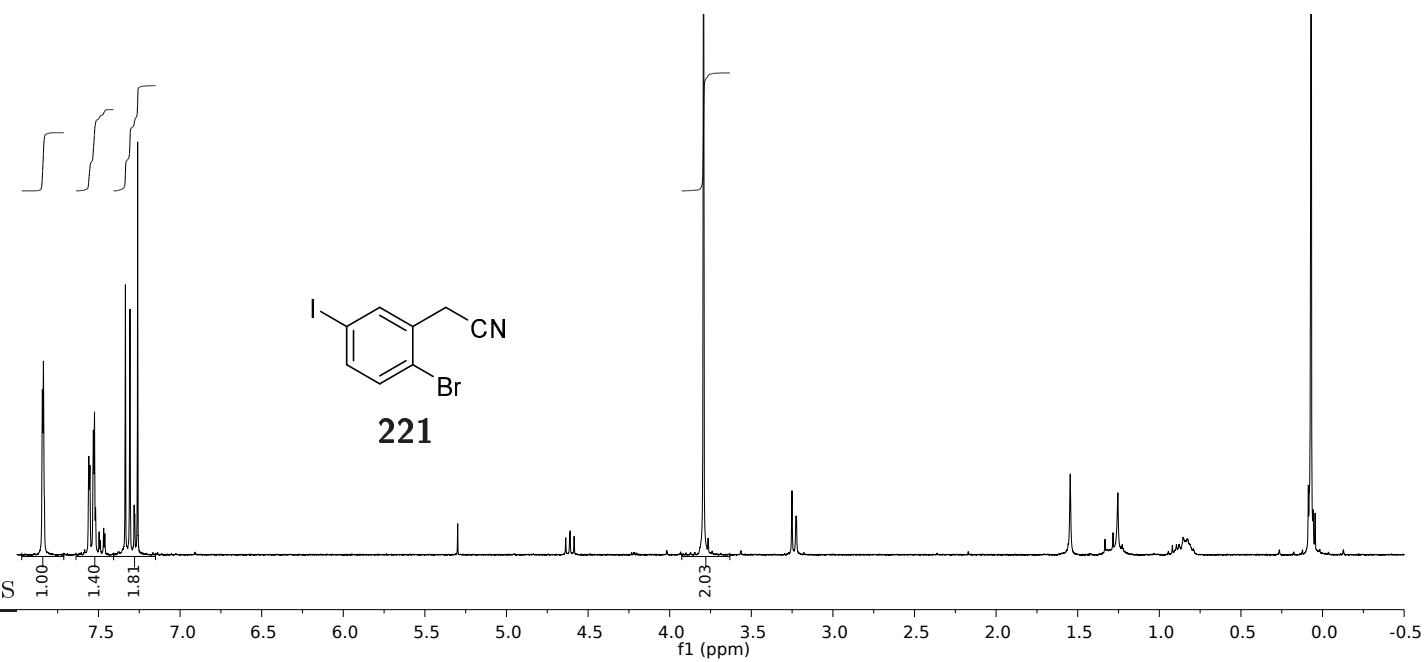

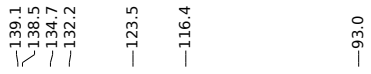

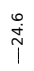
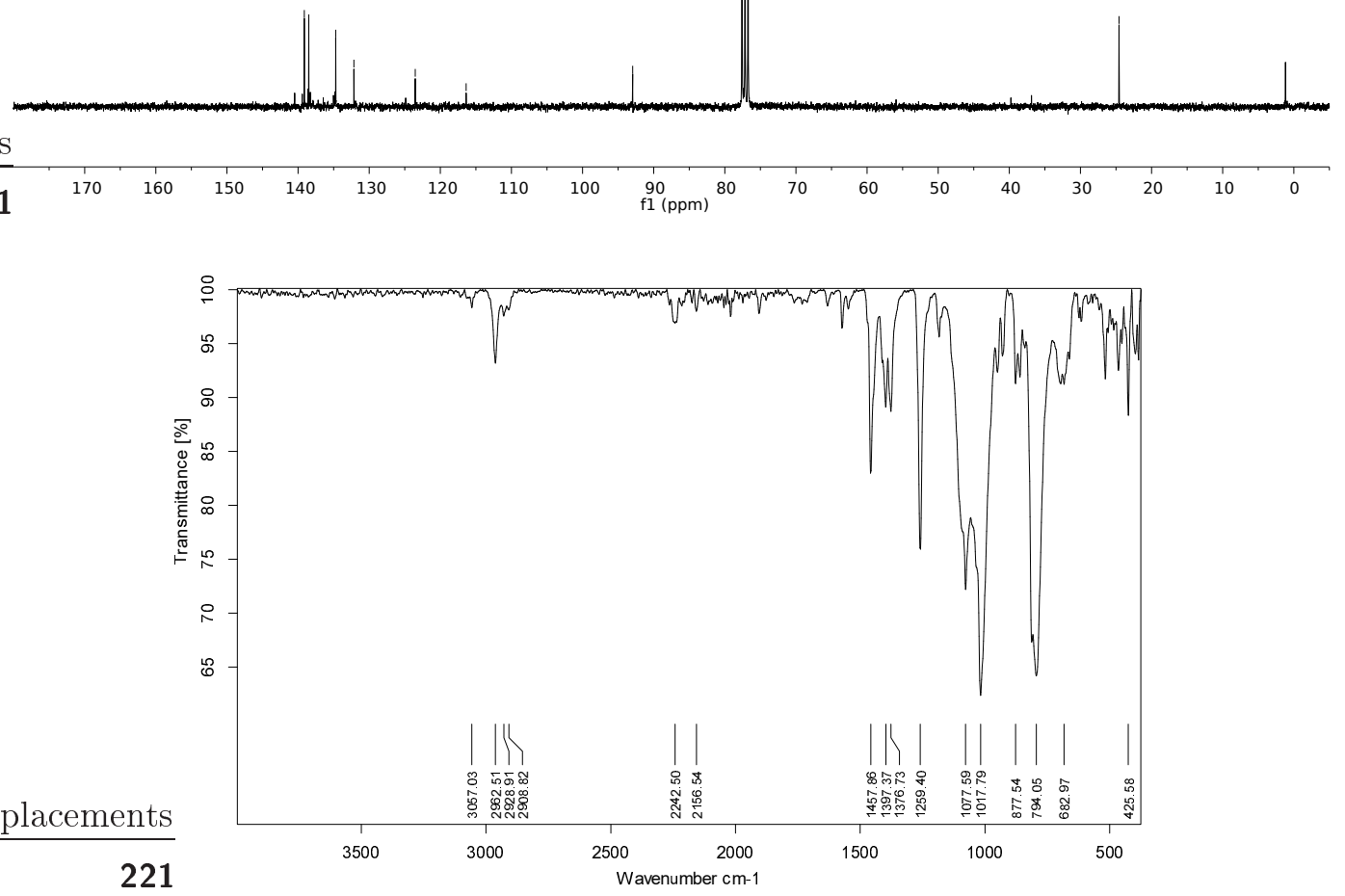

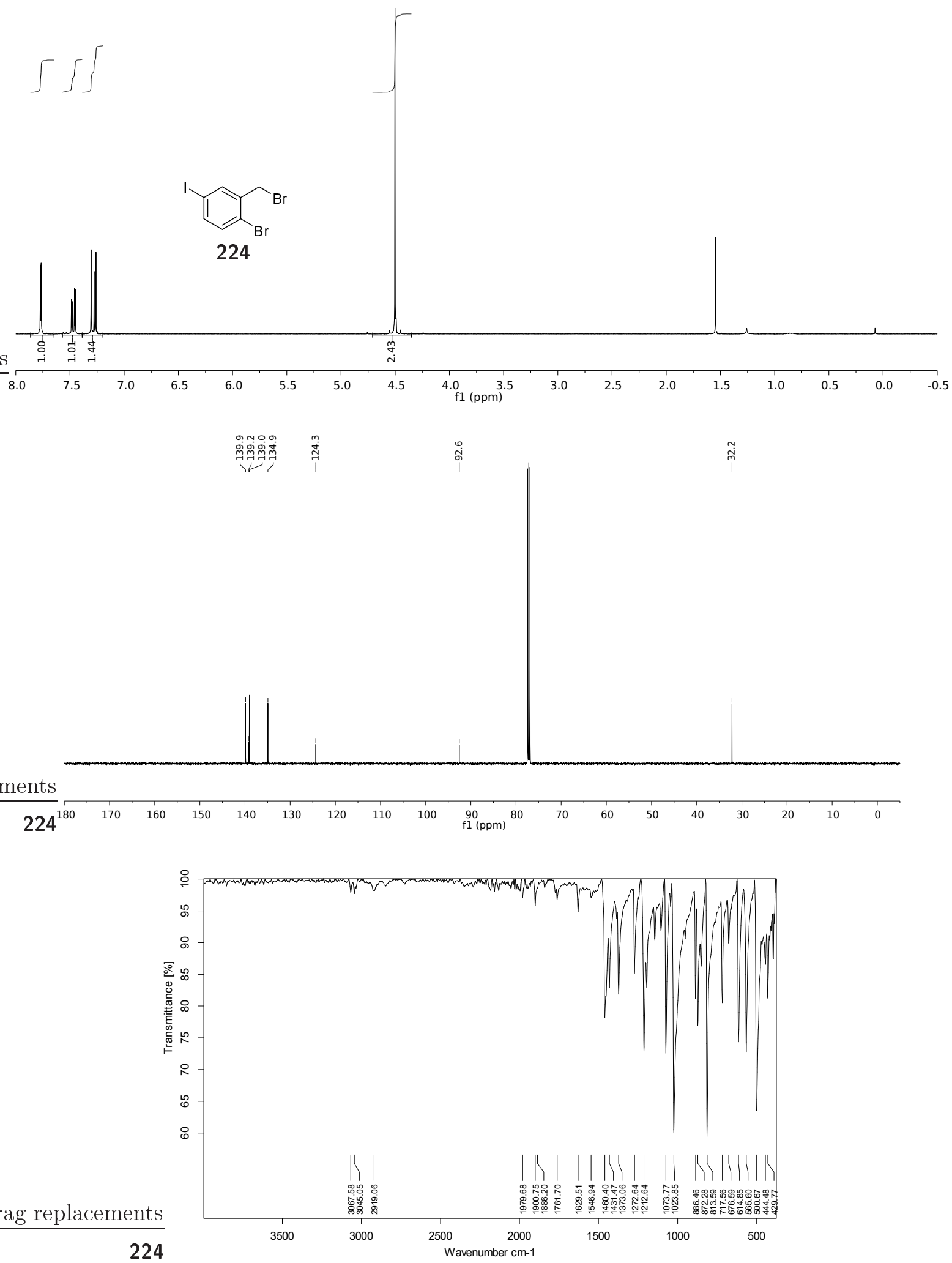

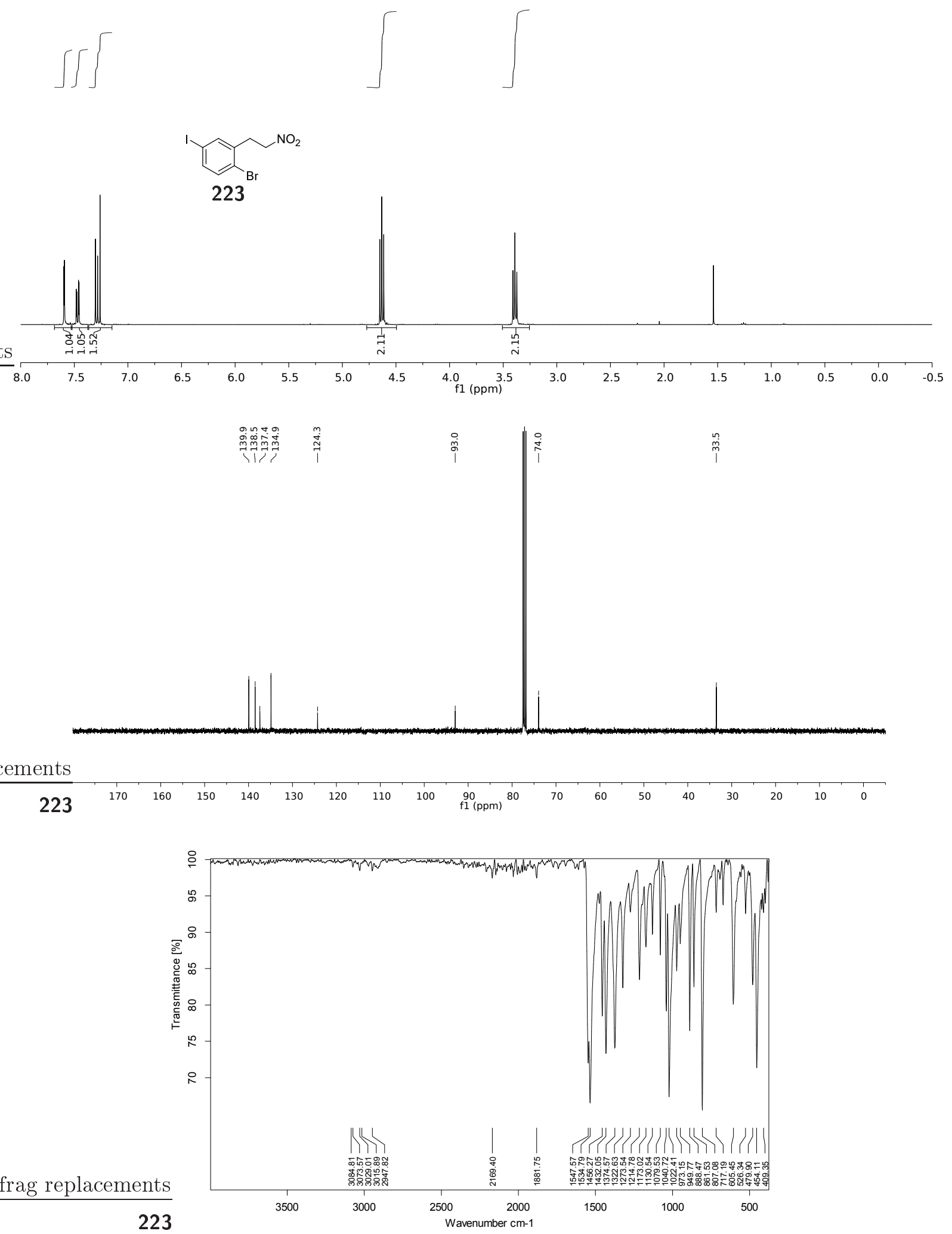
$\int_{5}$
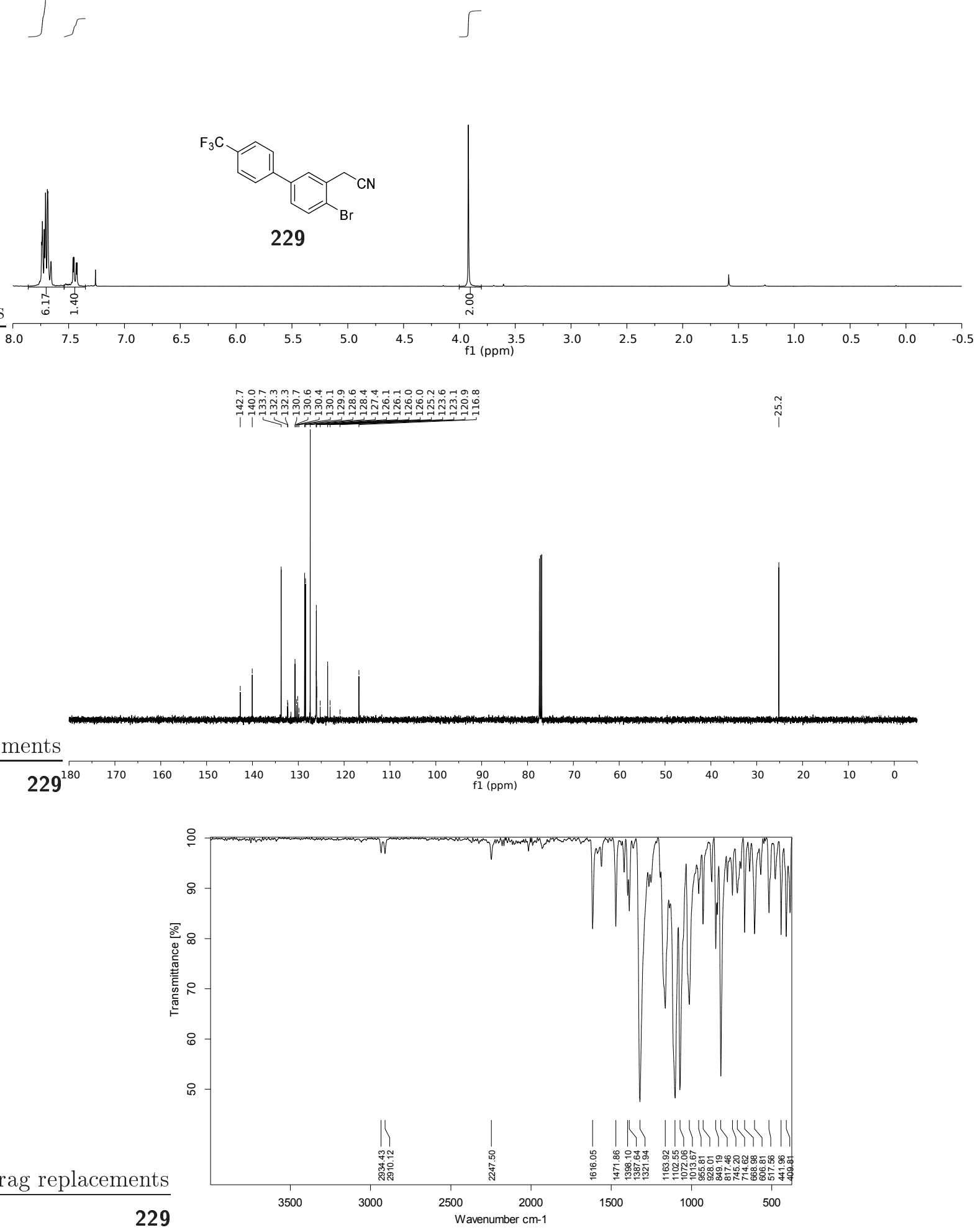

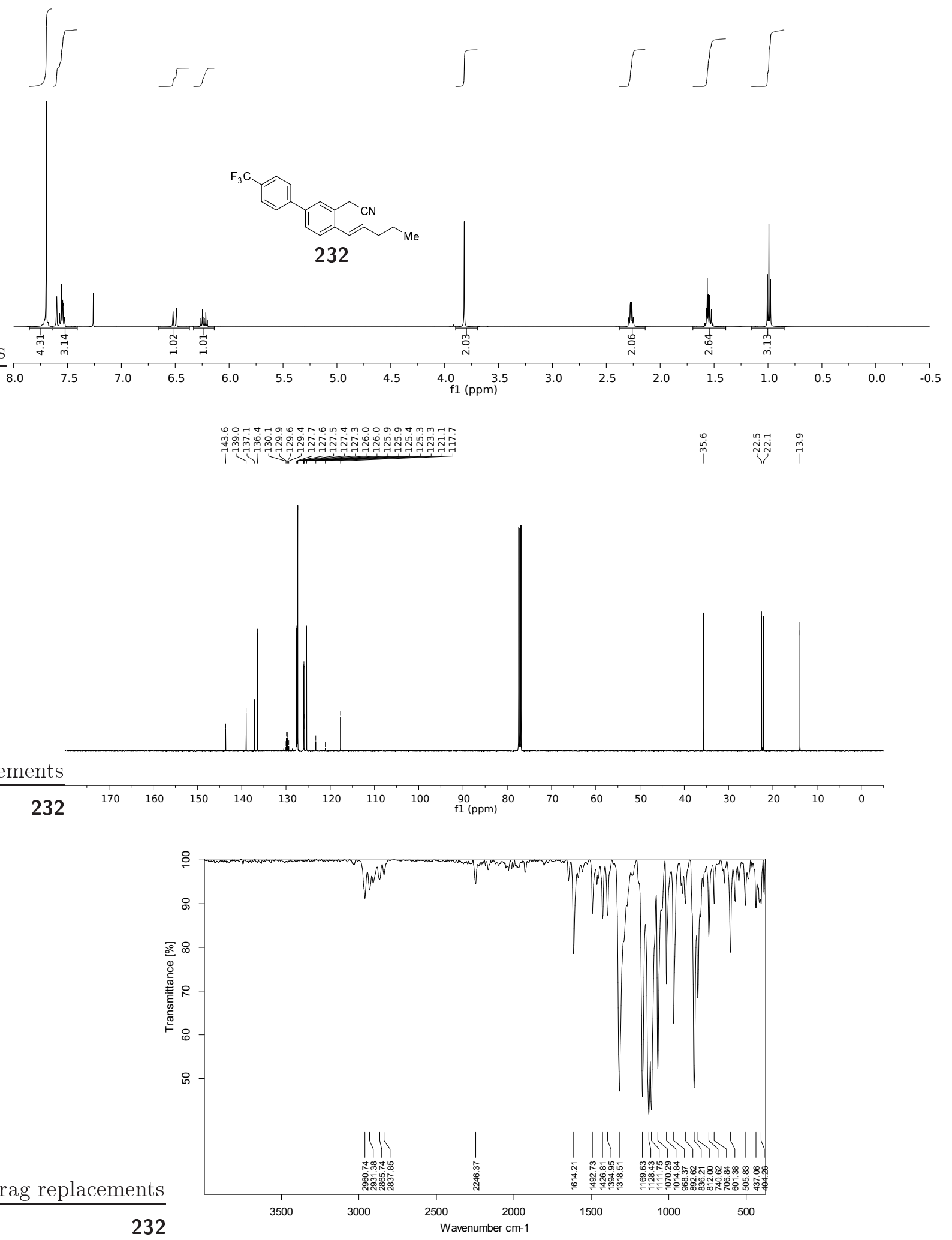

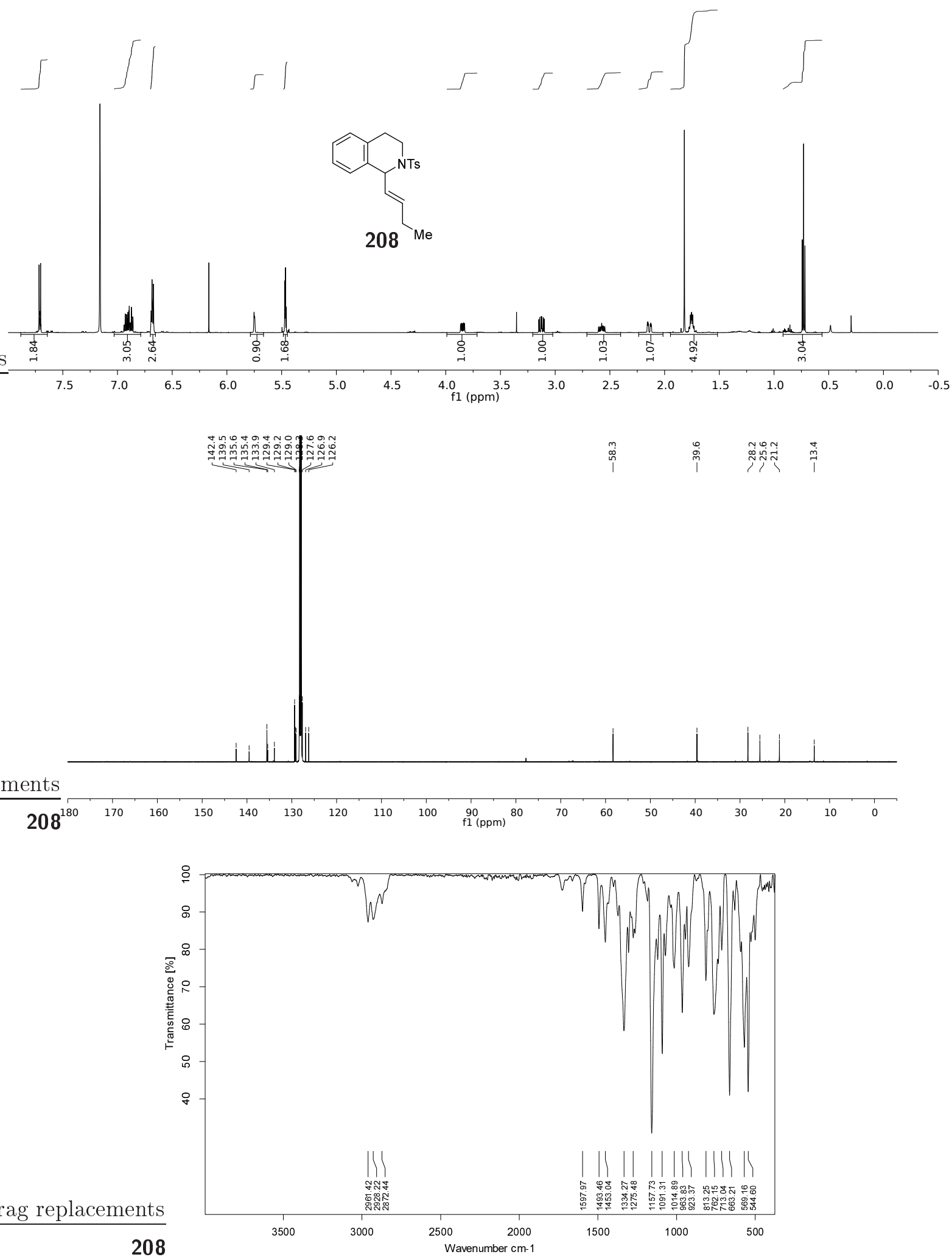

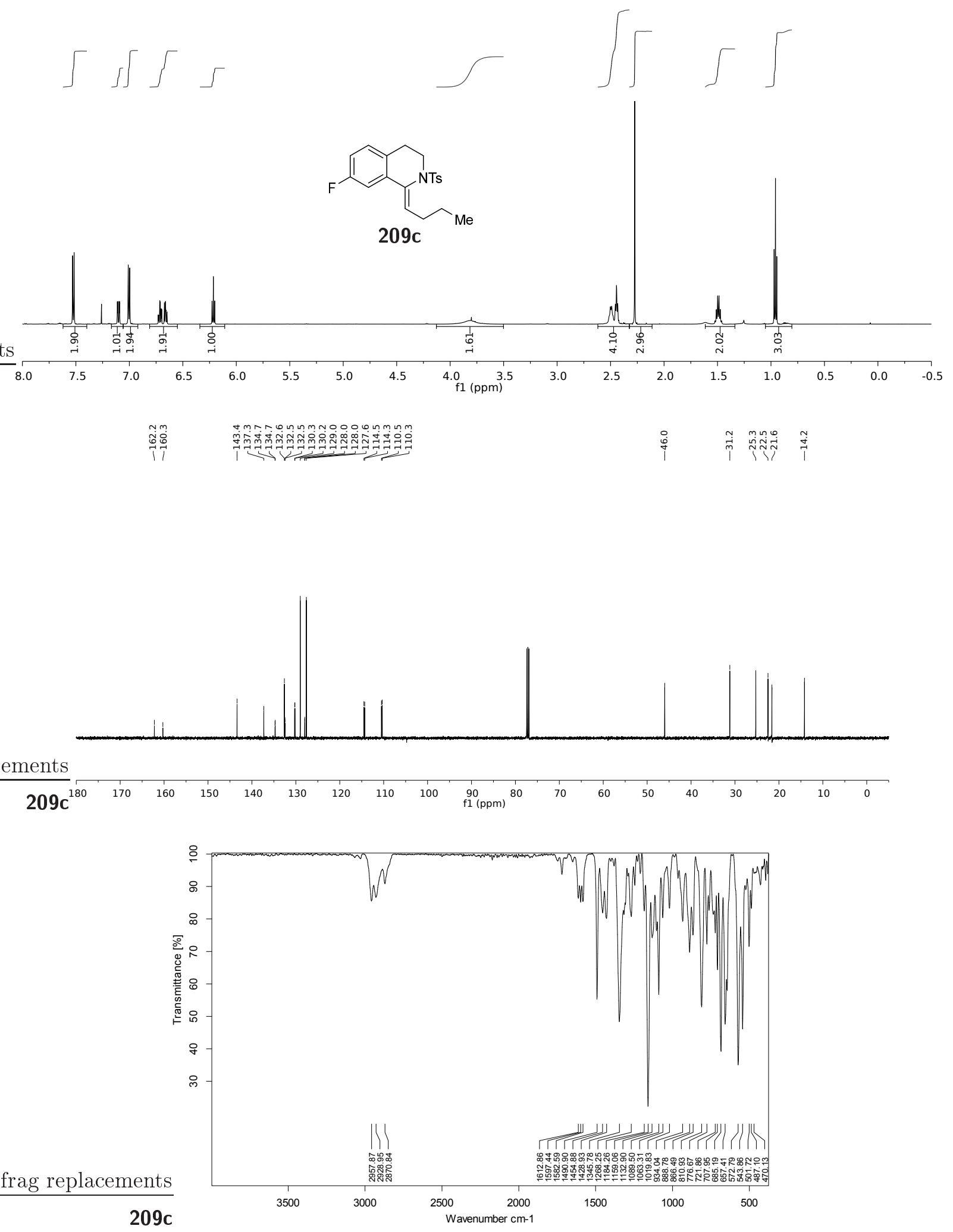

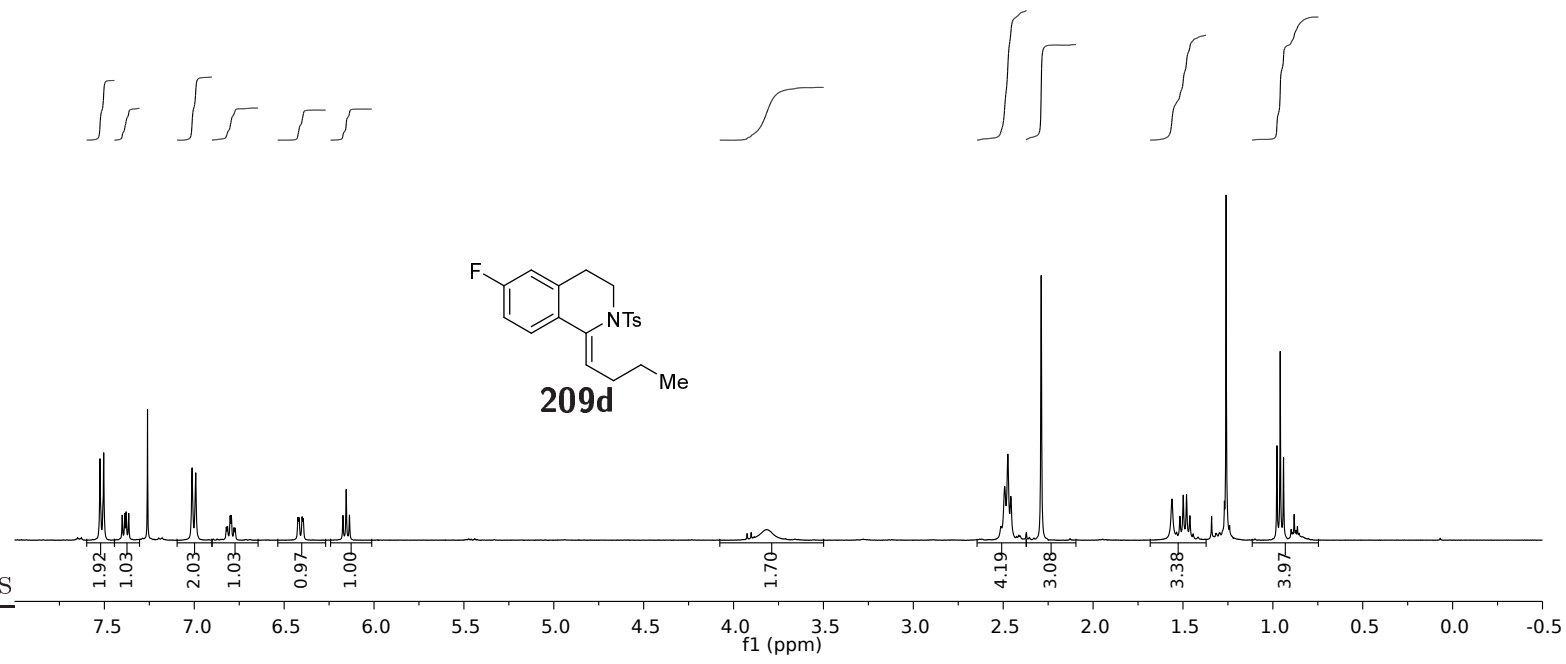

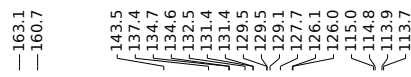

\section{$\stackrel{\substack{\infty \\ i}}{i}$}

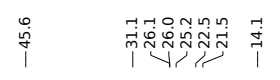
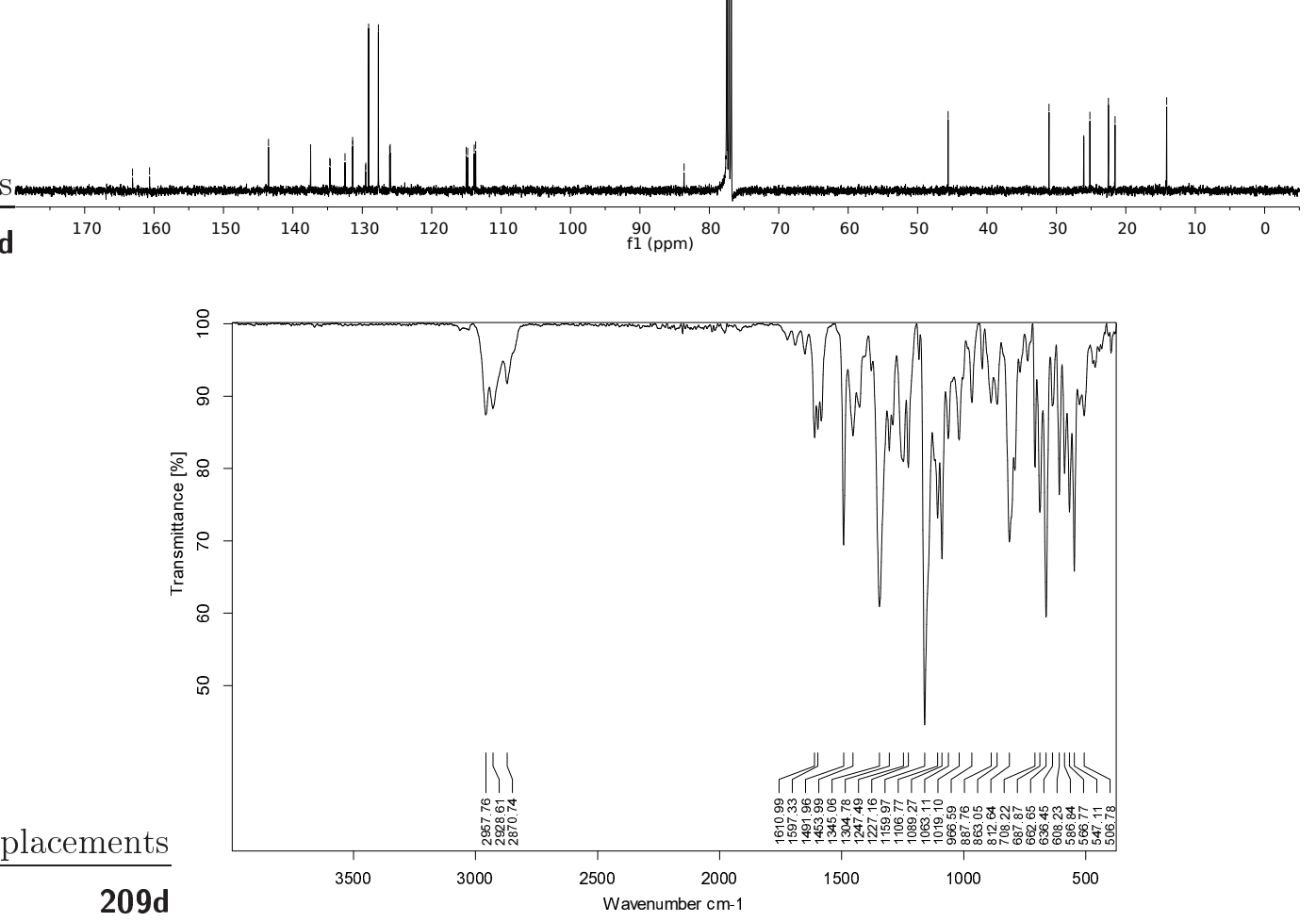

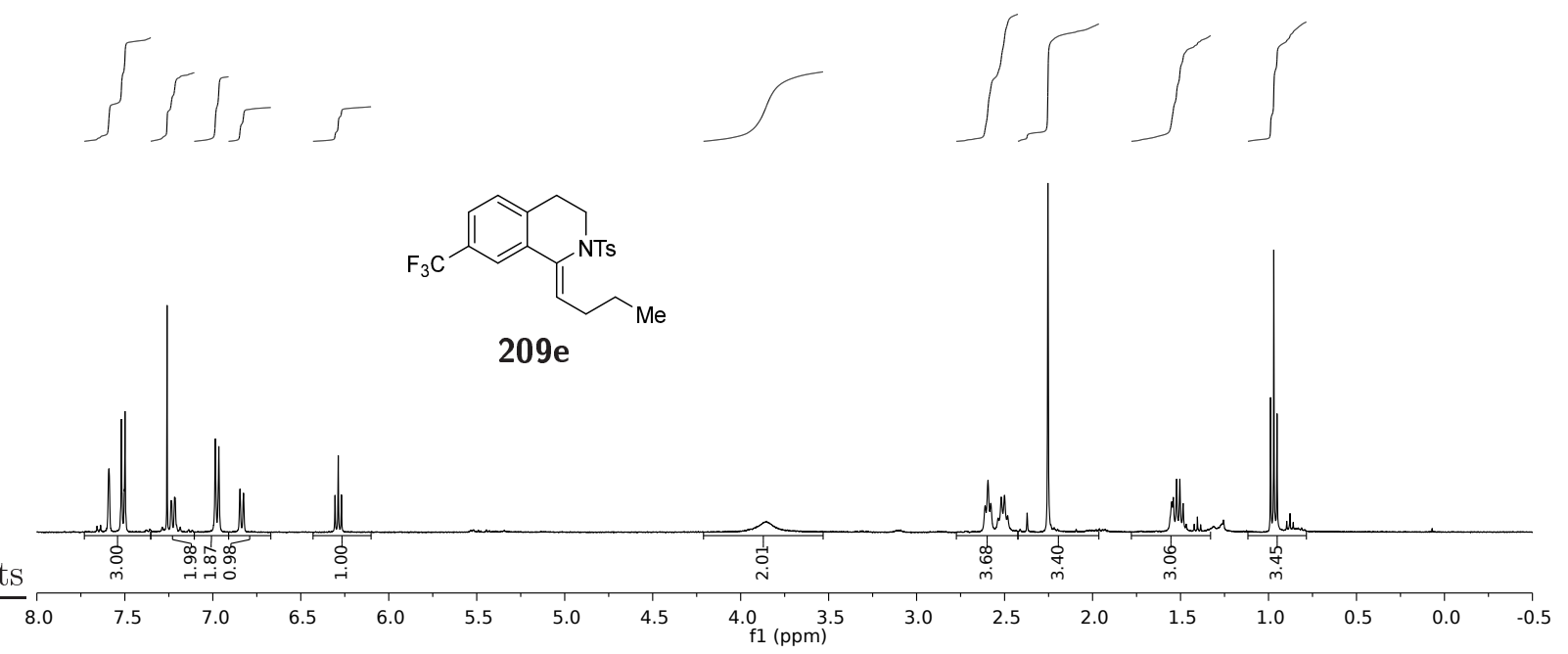

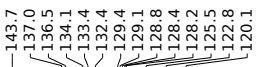

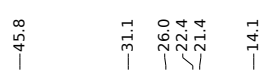
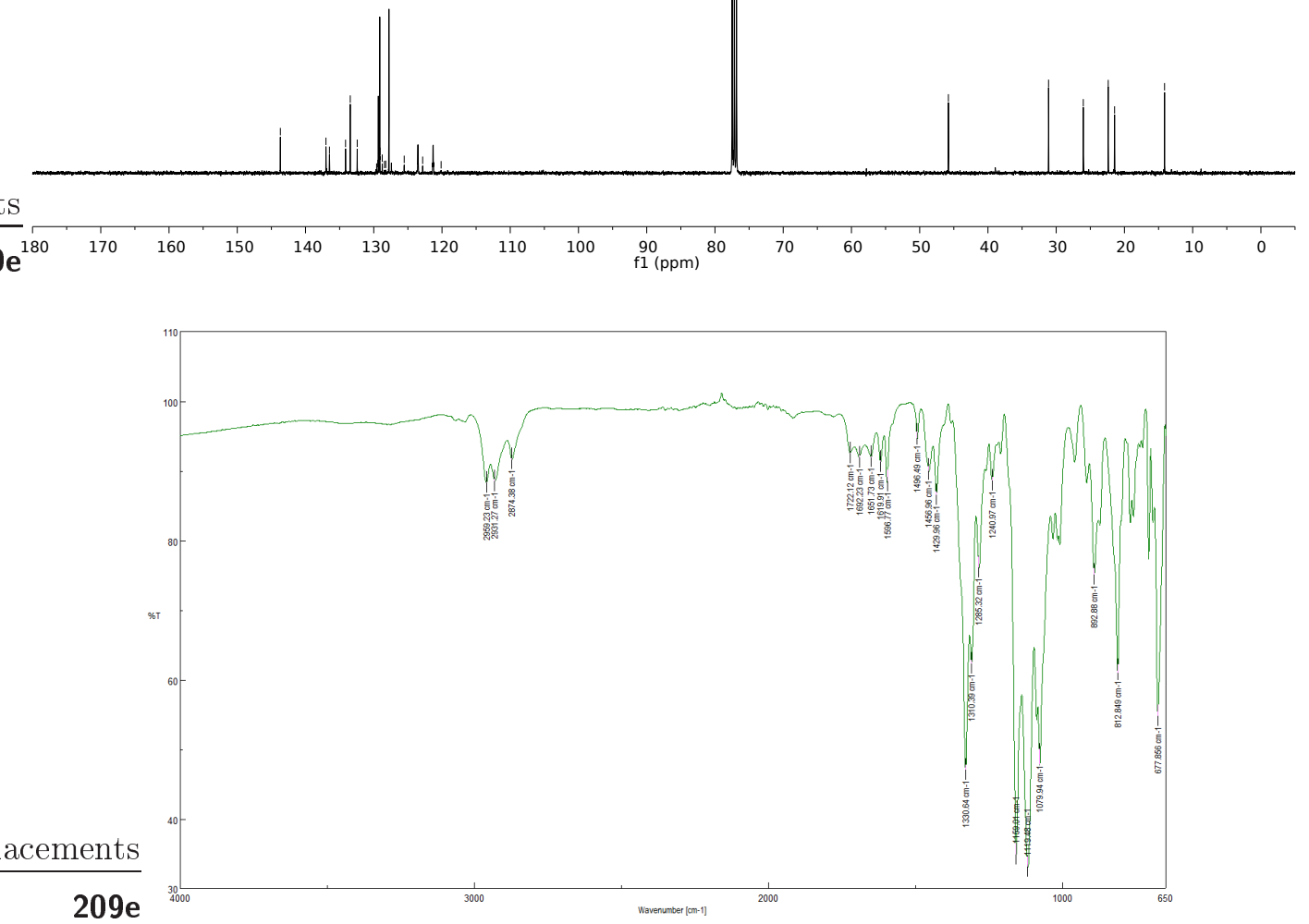

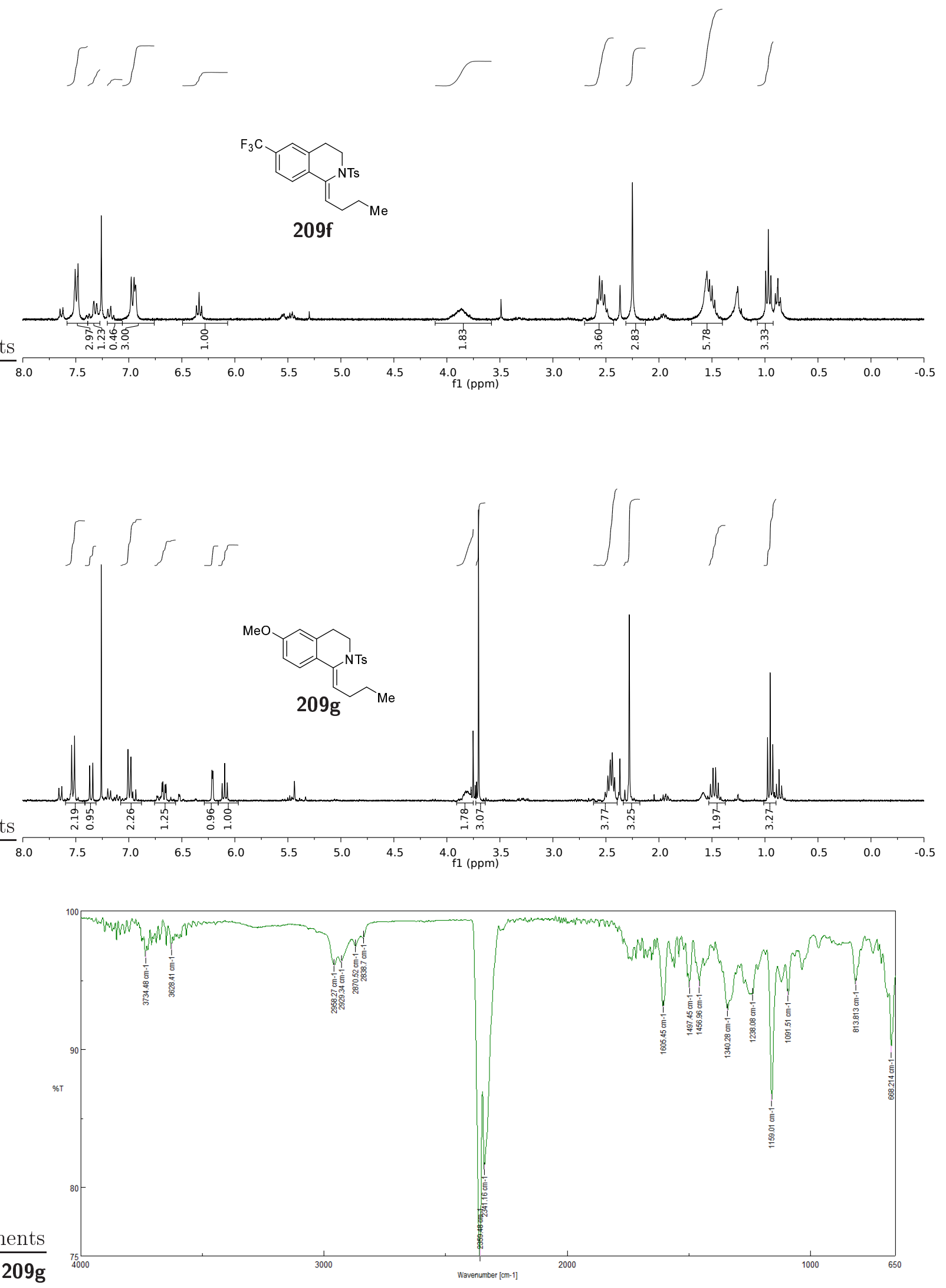

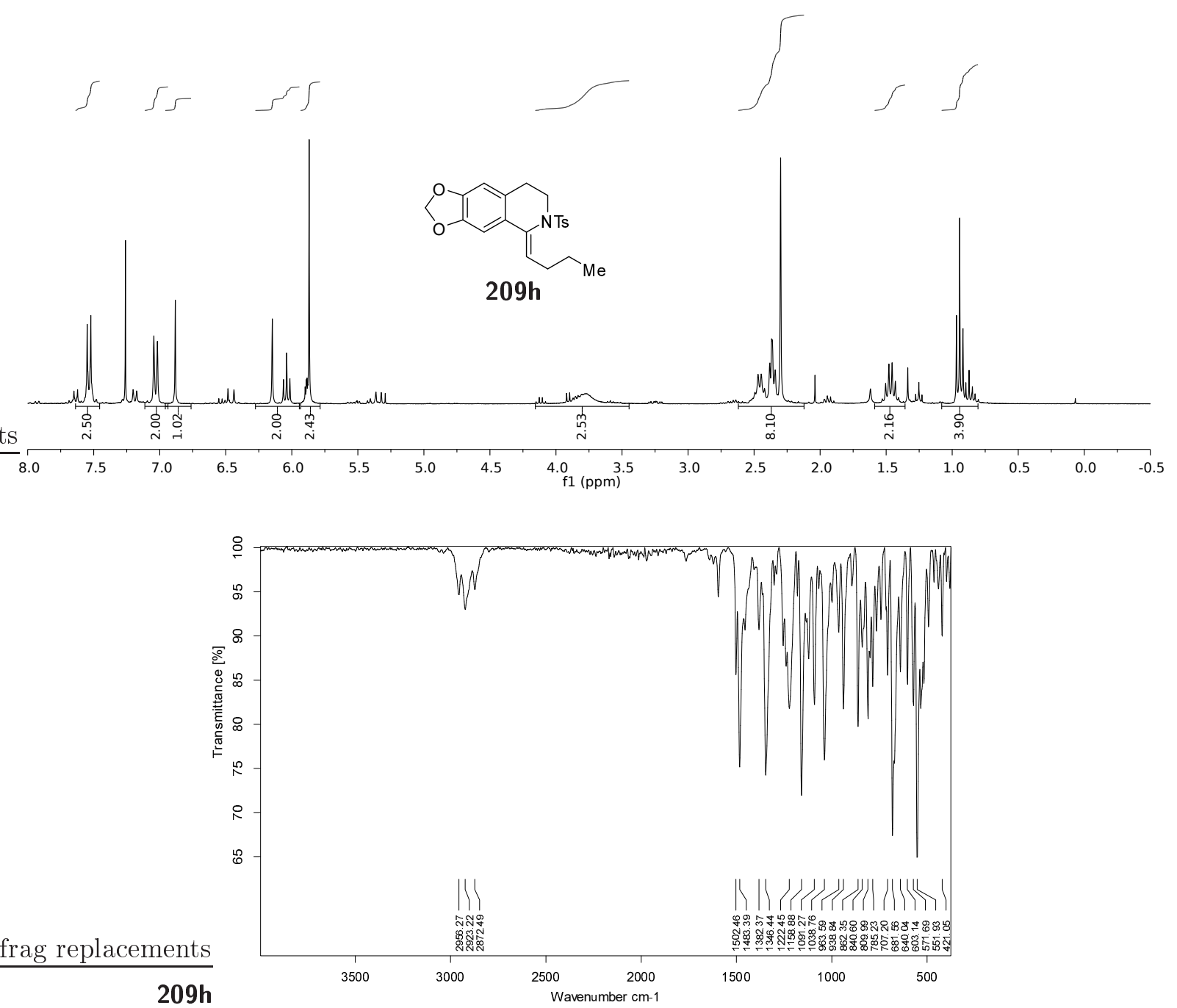

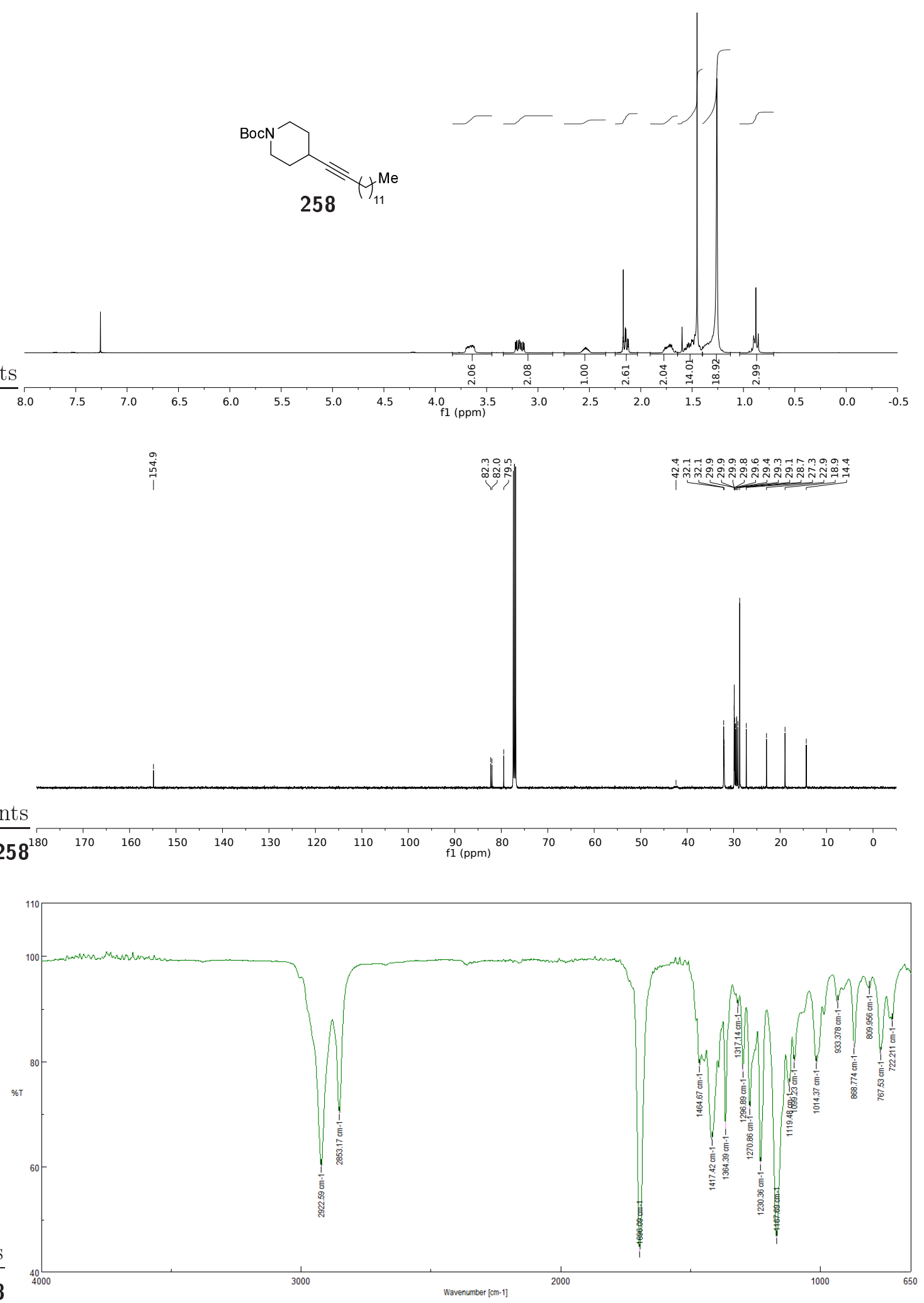

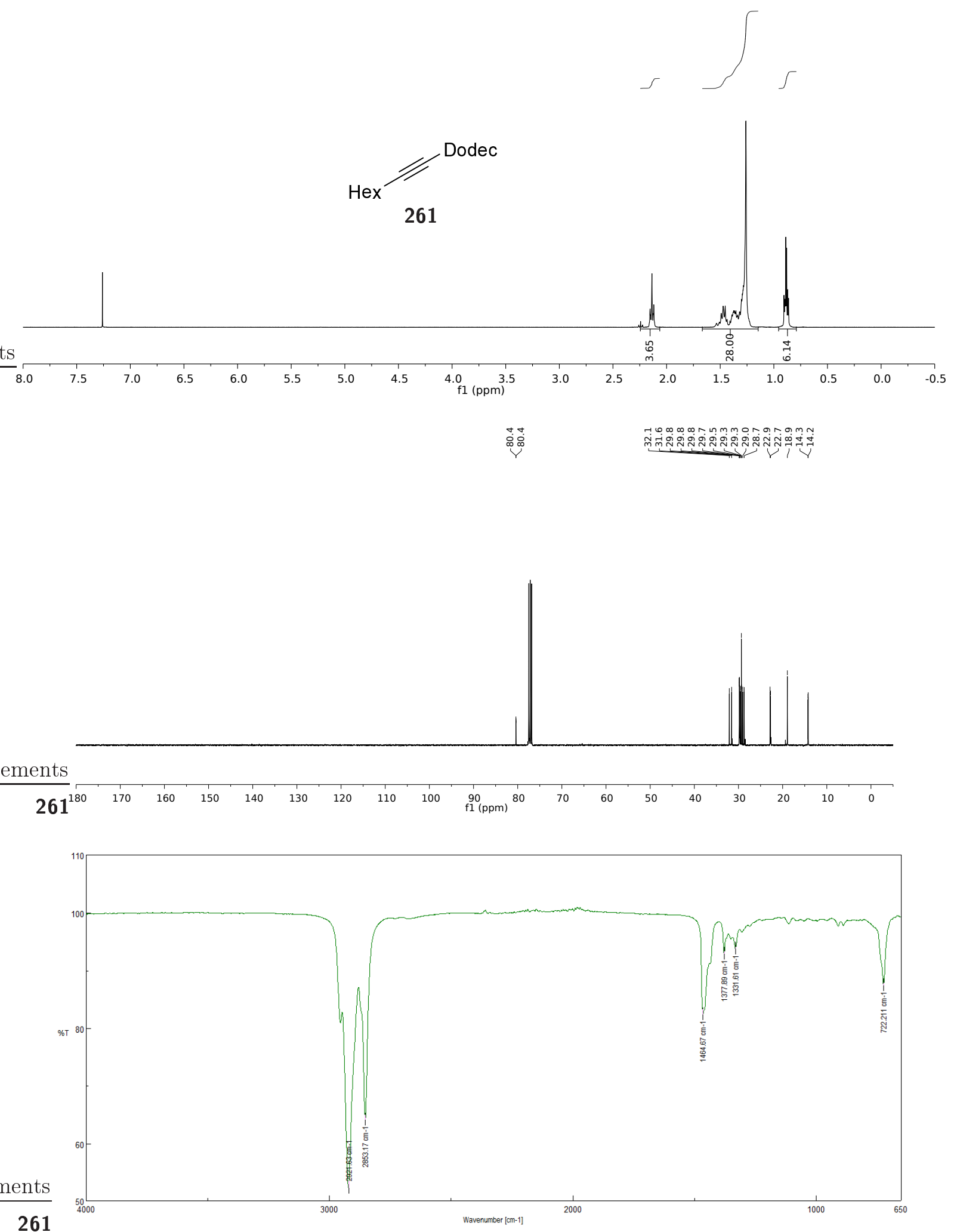

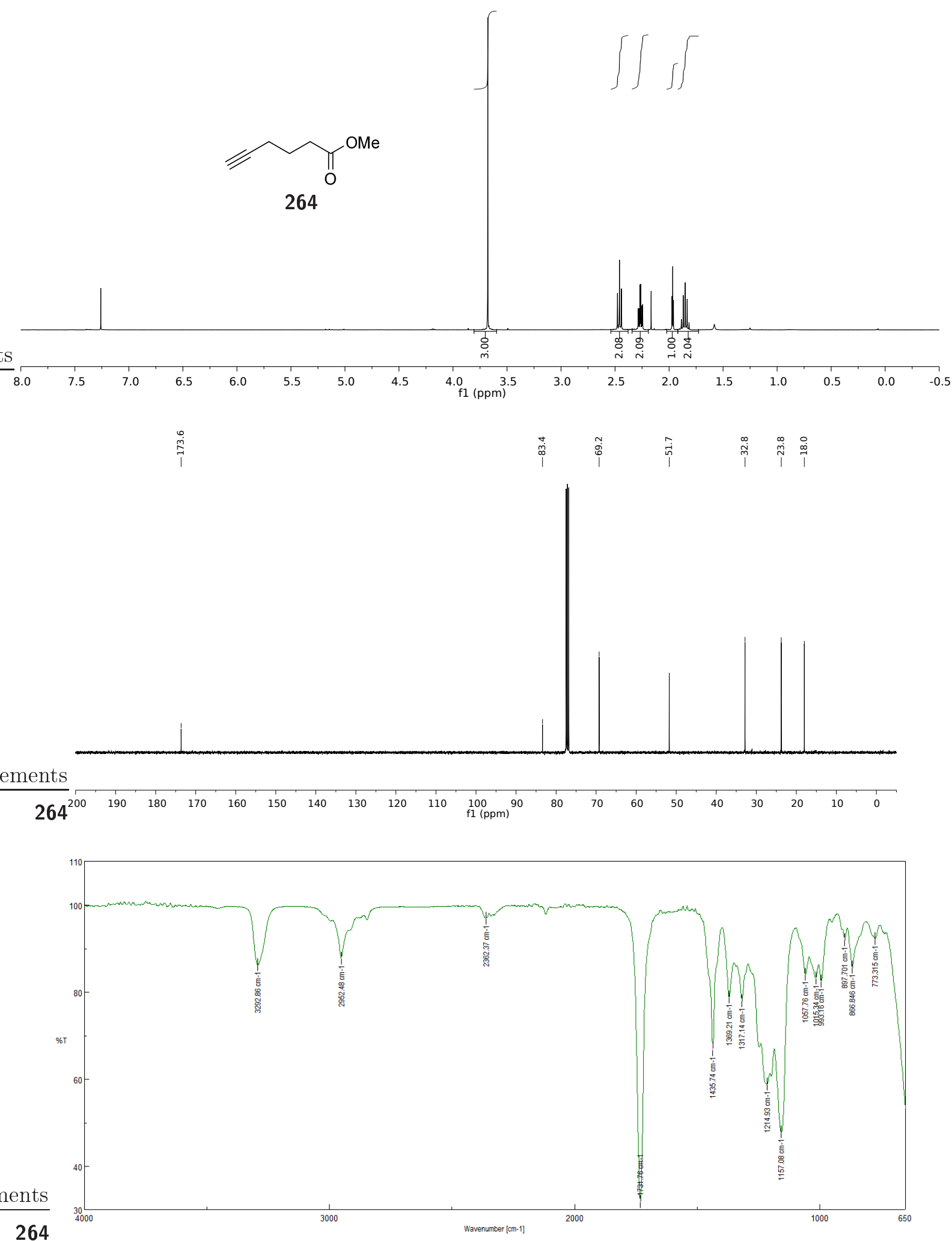


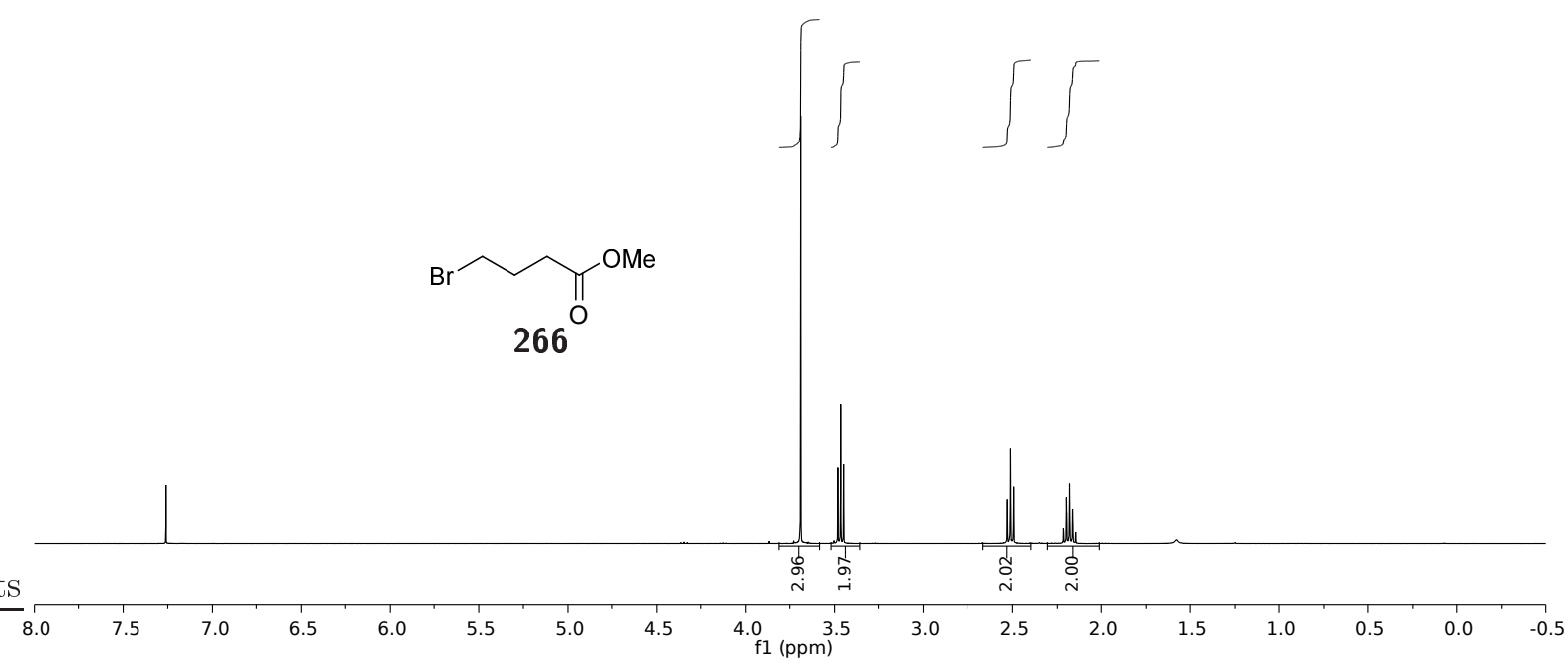

$\stackrel{\vec{m}}{\overrightarrow{1}}$

में
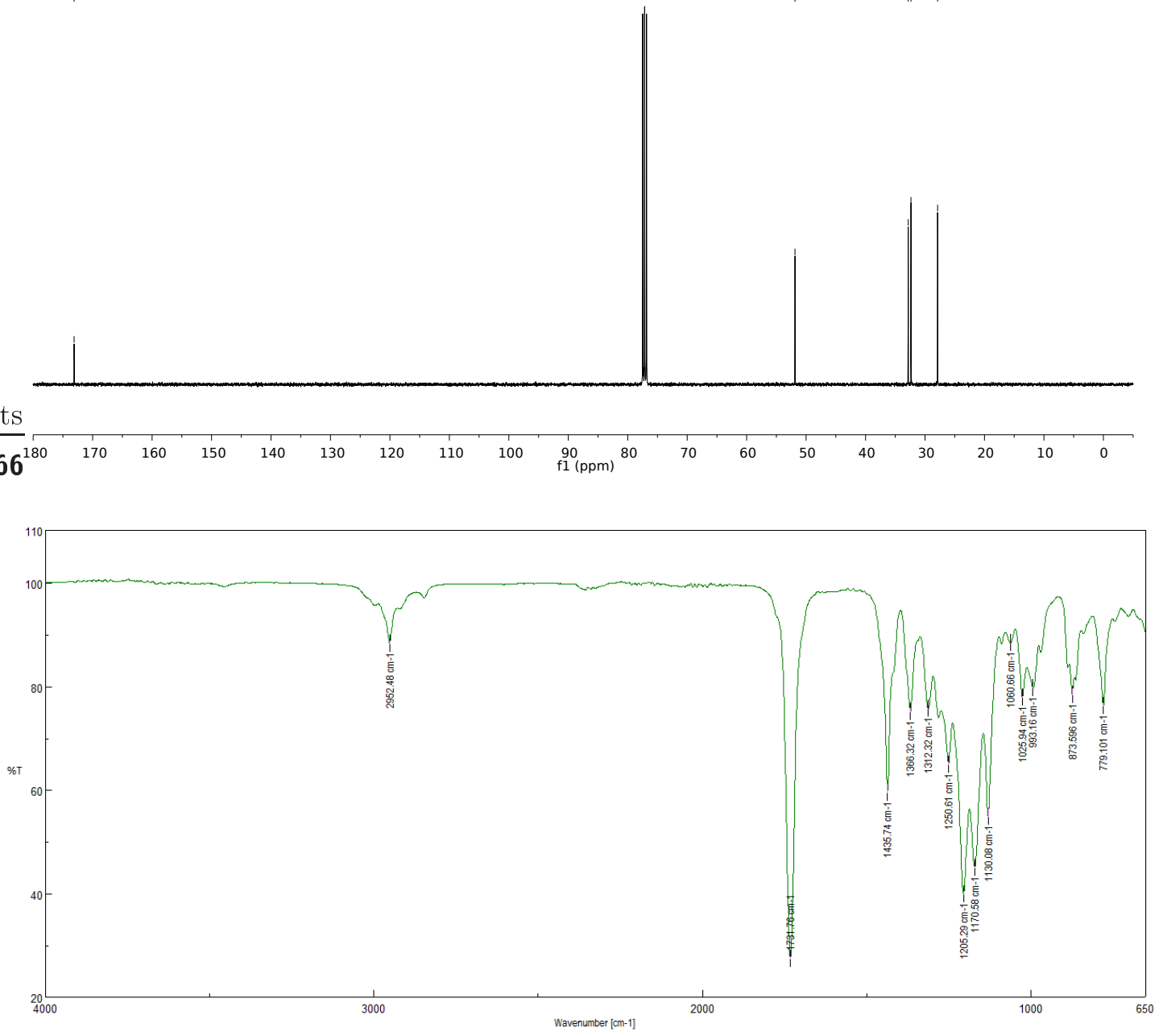

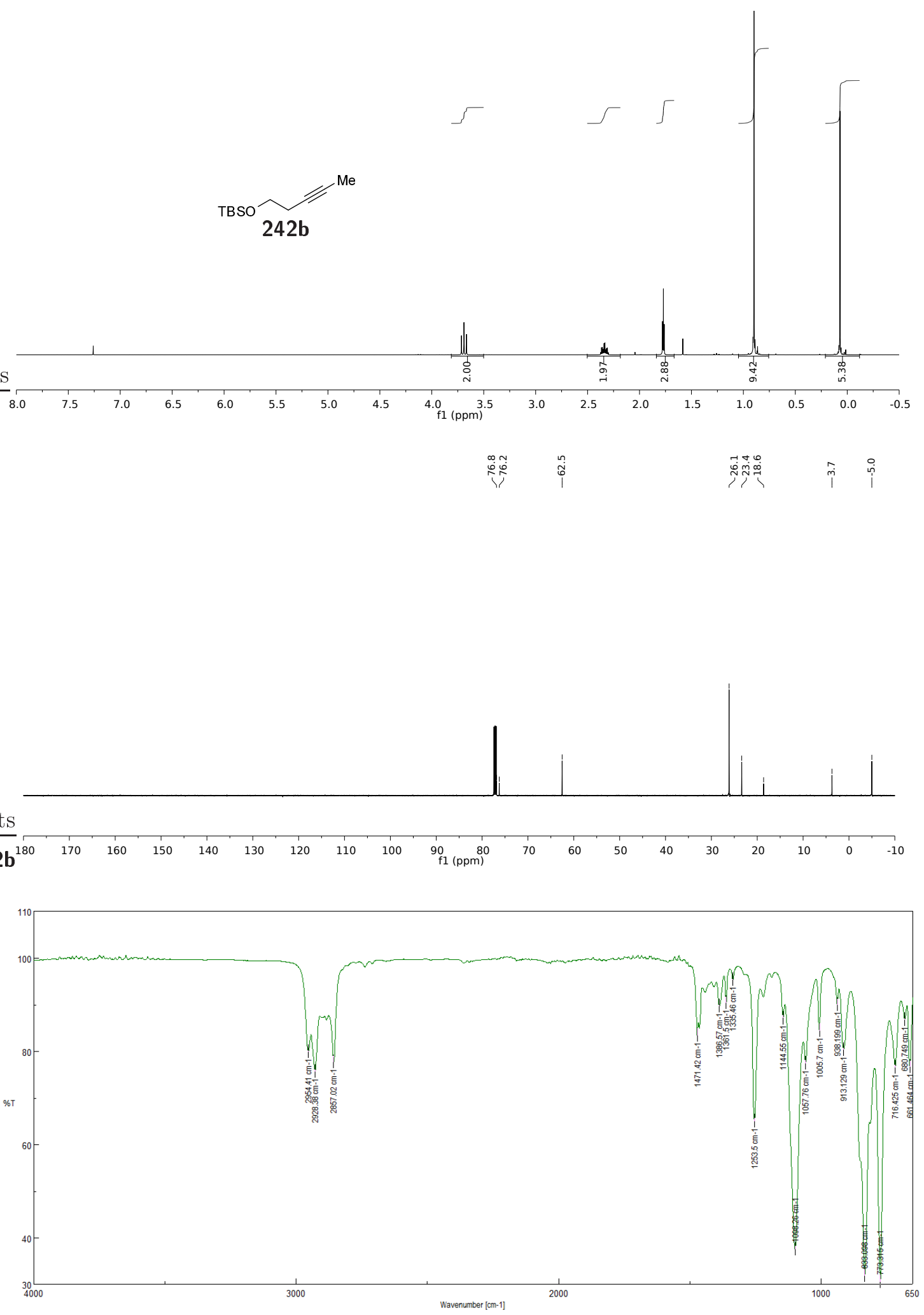

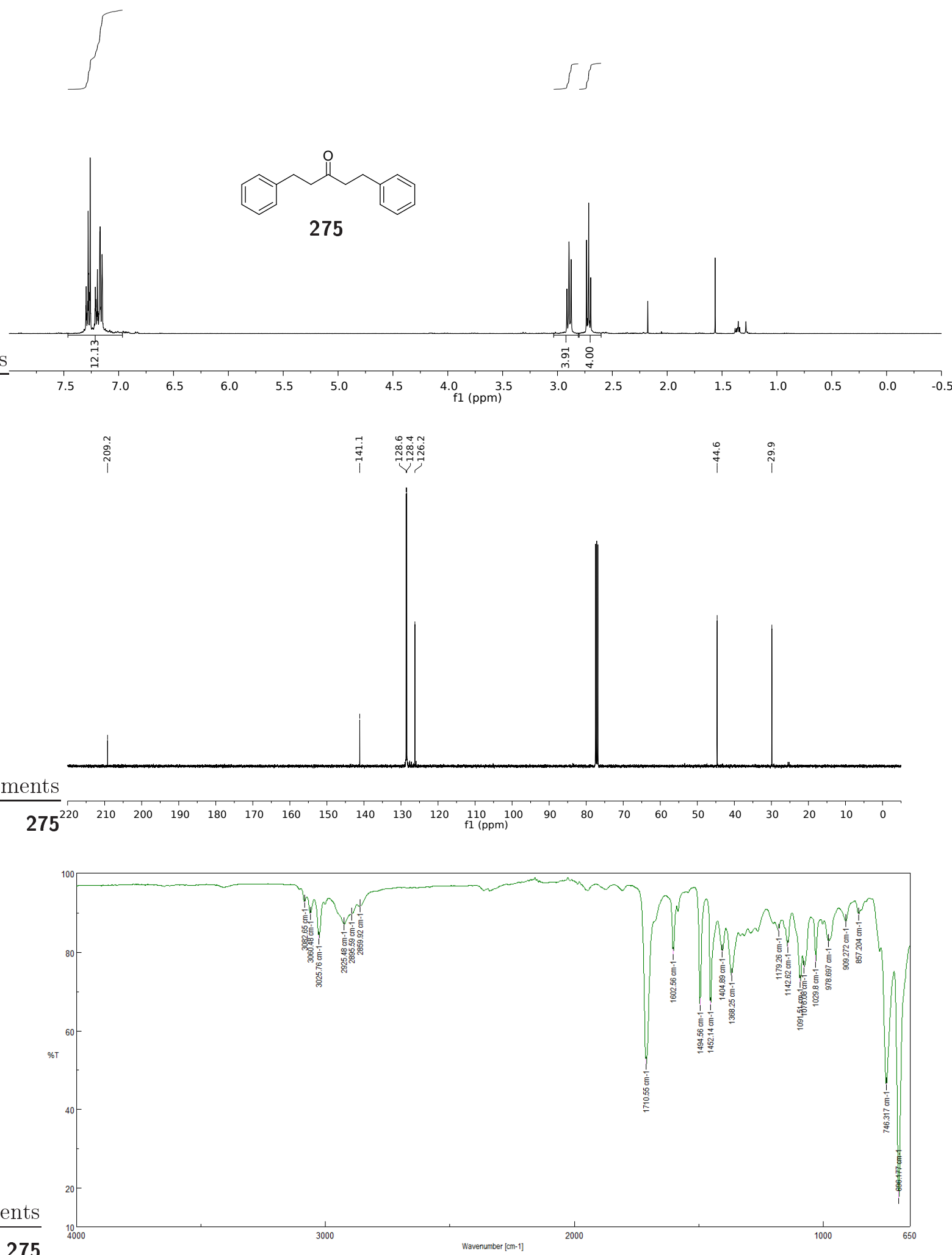

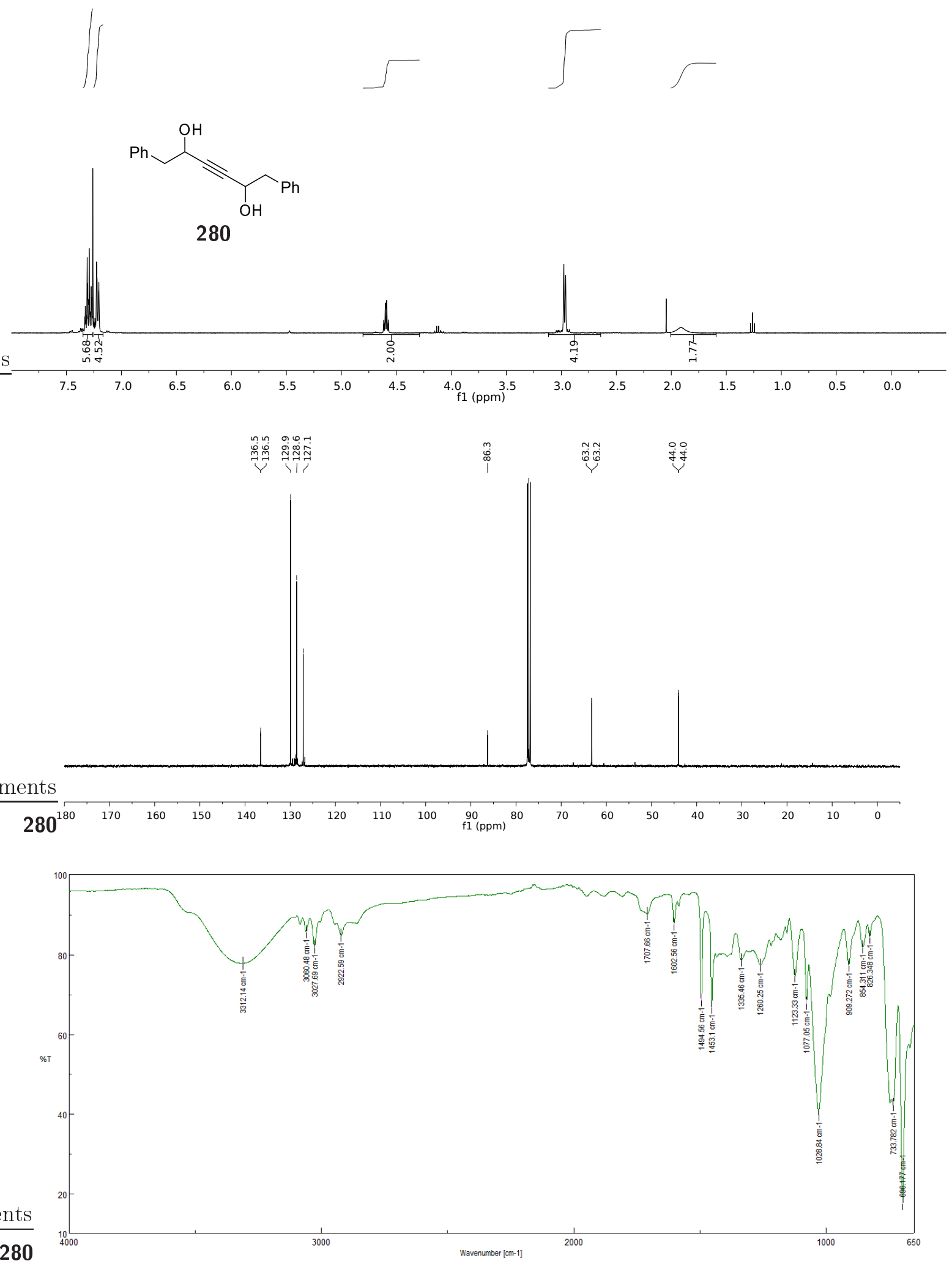

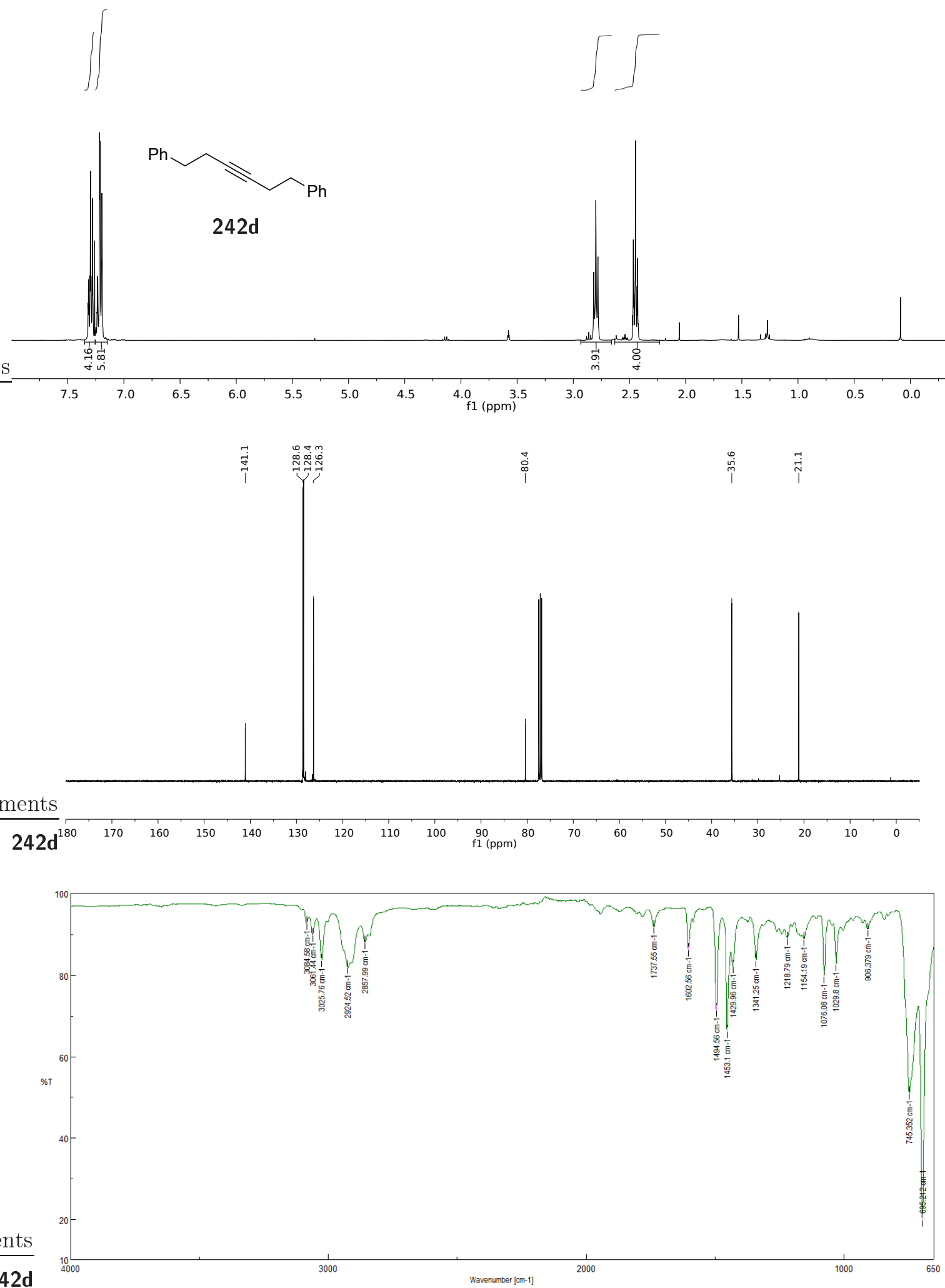


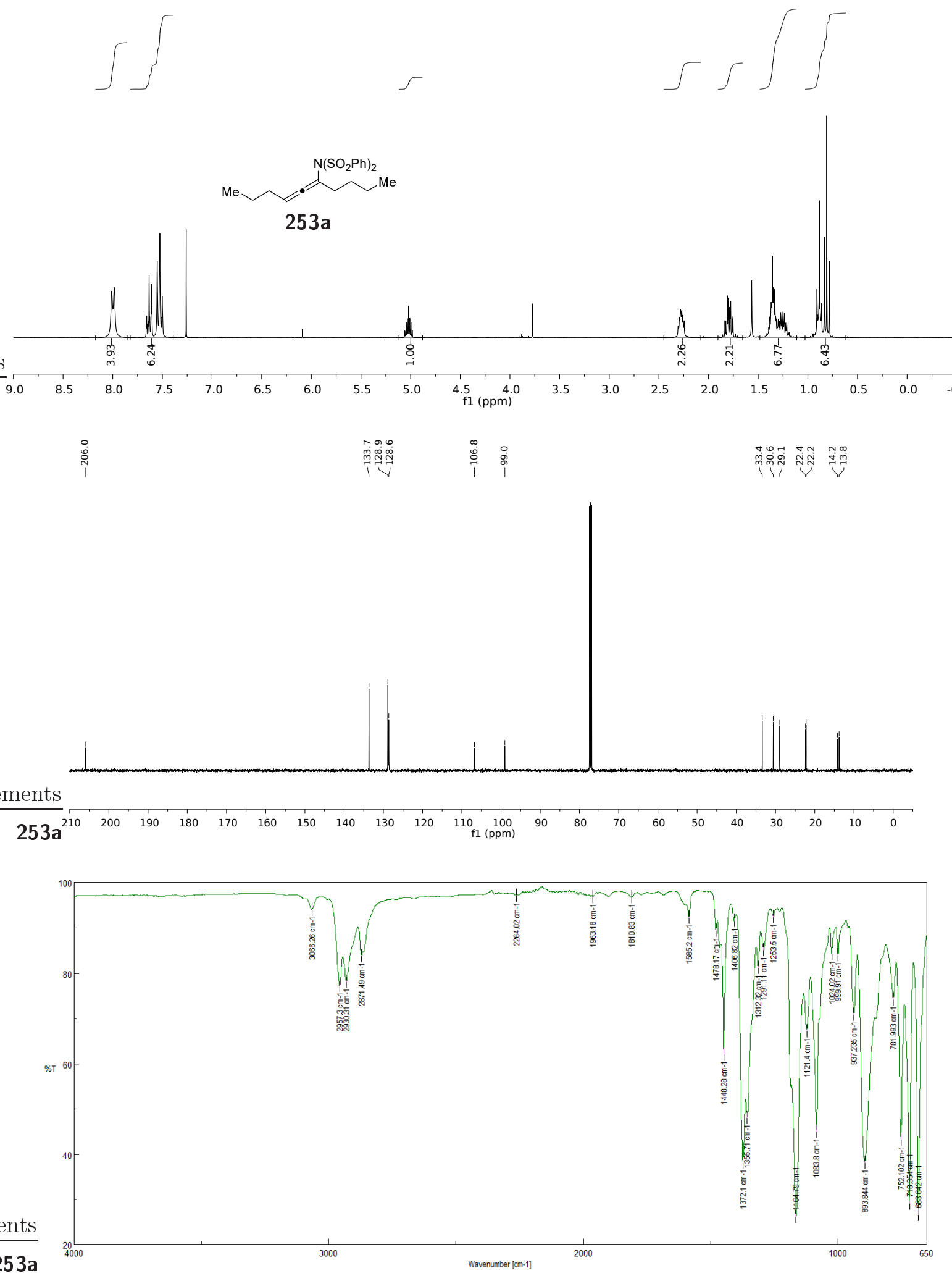



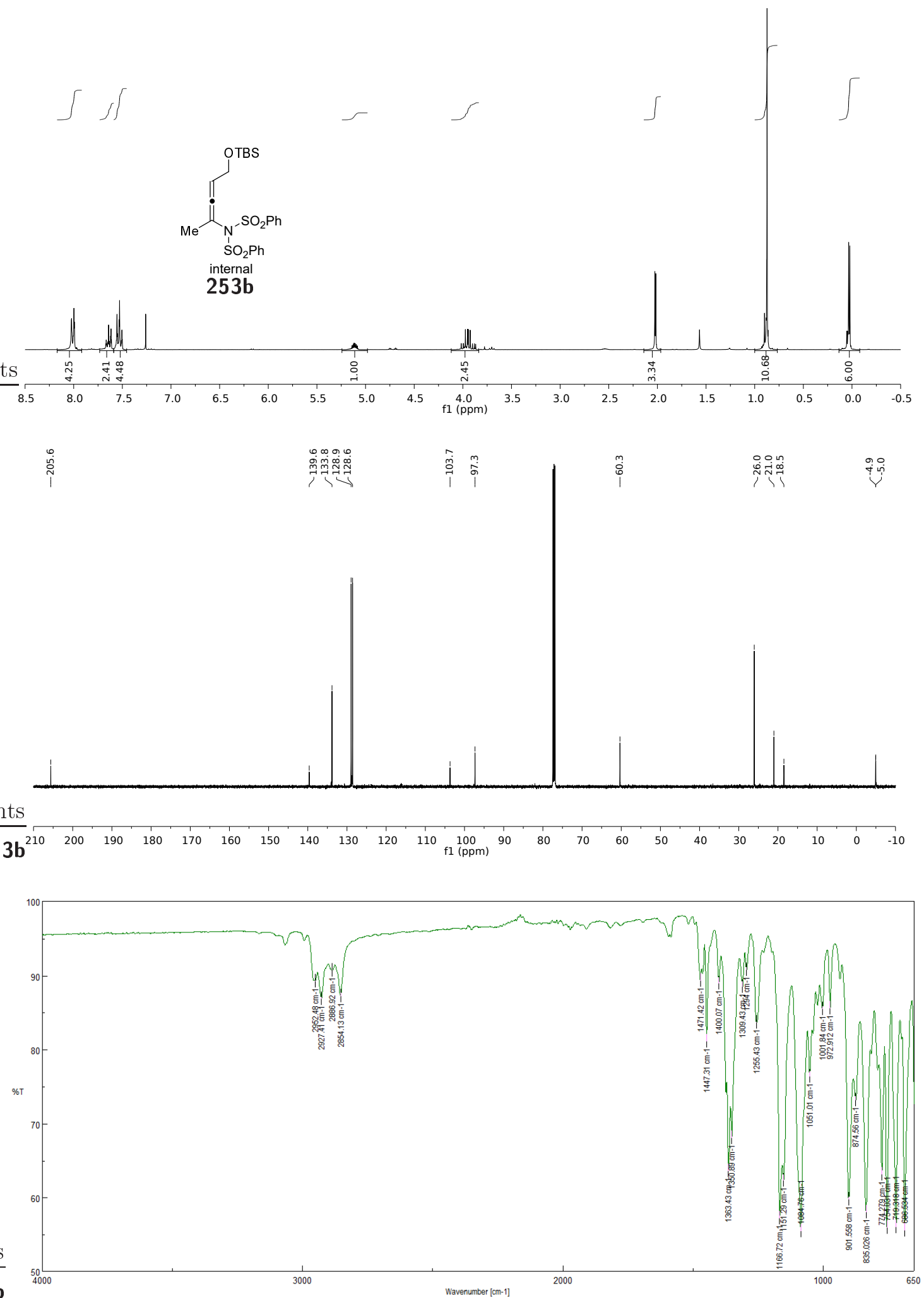

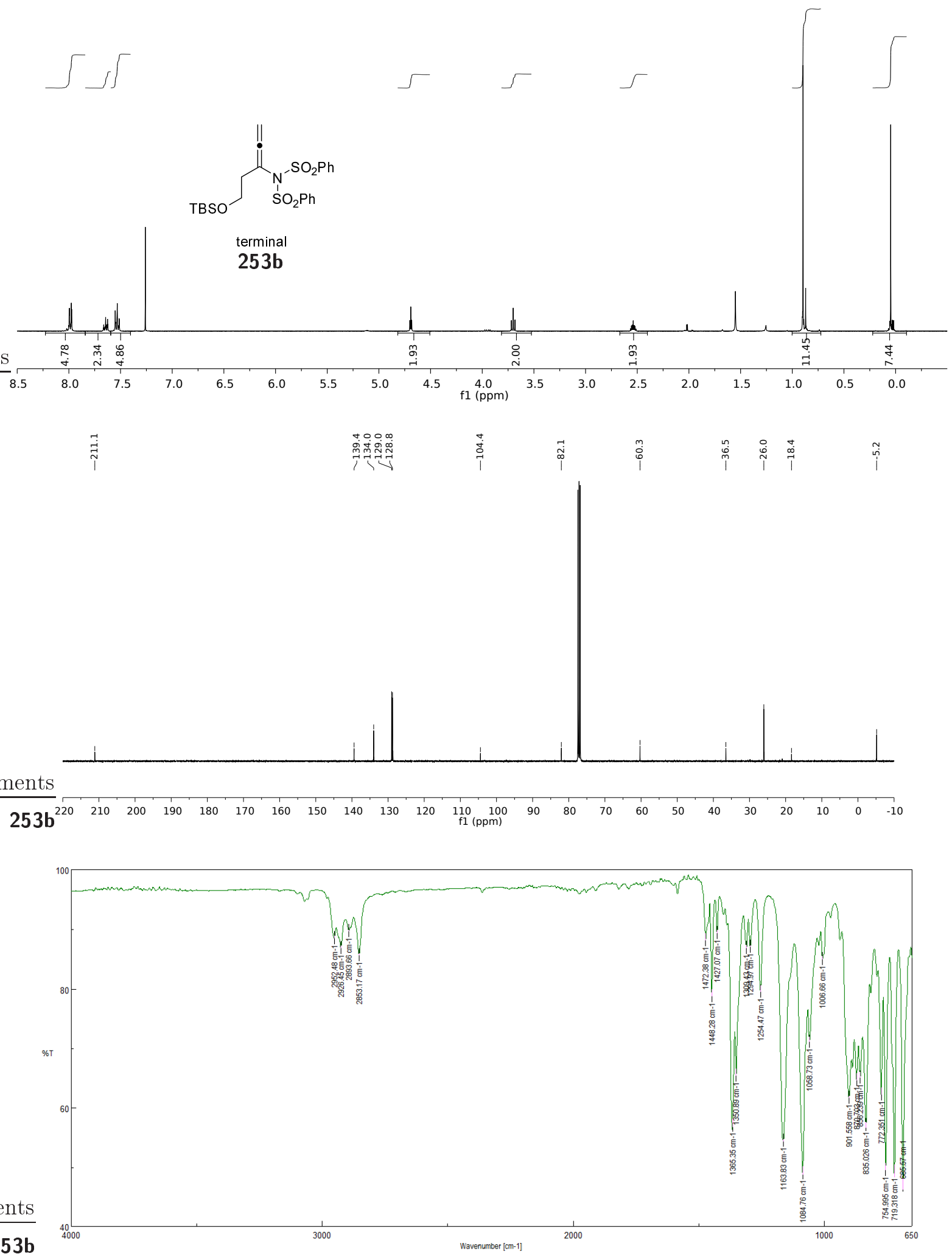


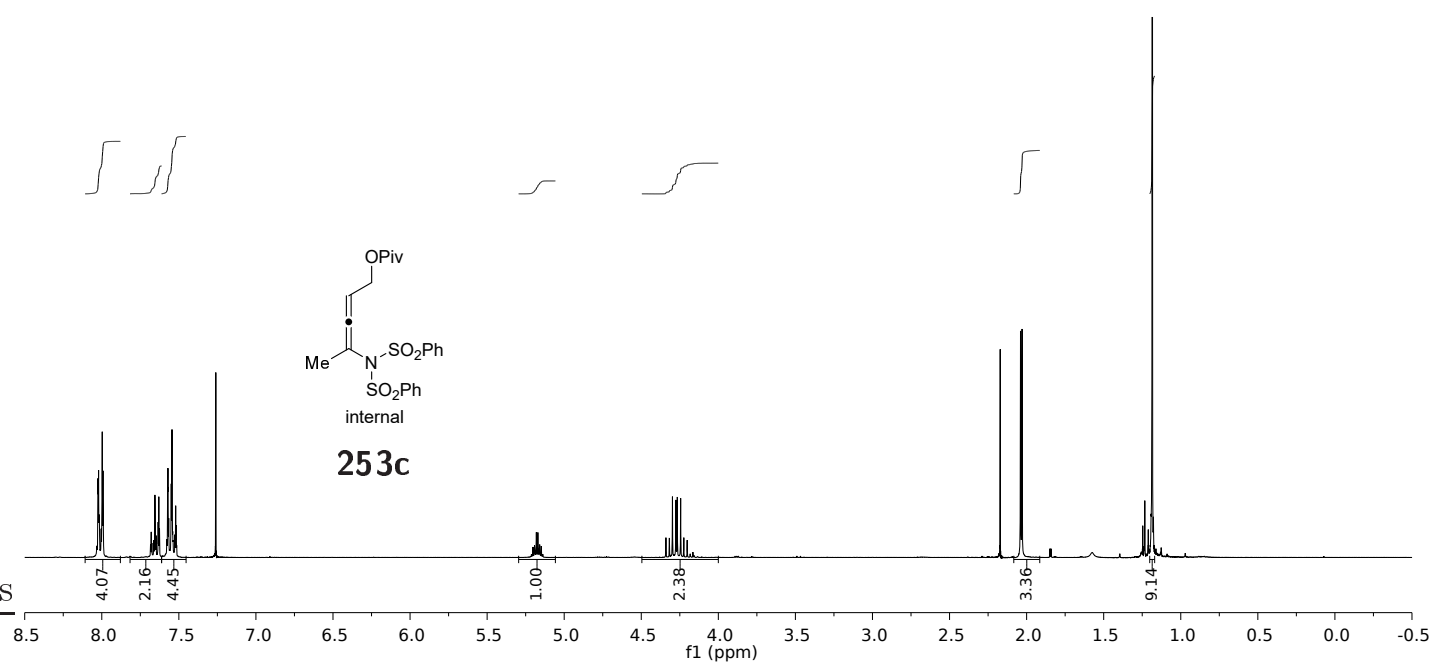

in

maำ

草

$\stackrel{\infty}{\infty} \stackrel{m}{\stackrel{m}{i}} \stackrel{0}{i}$
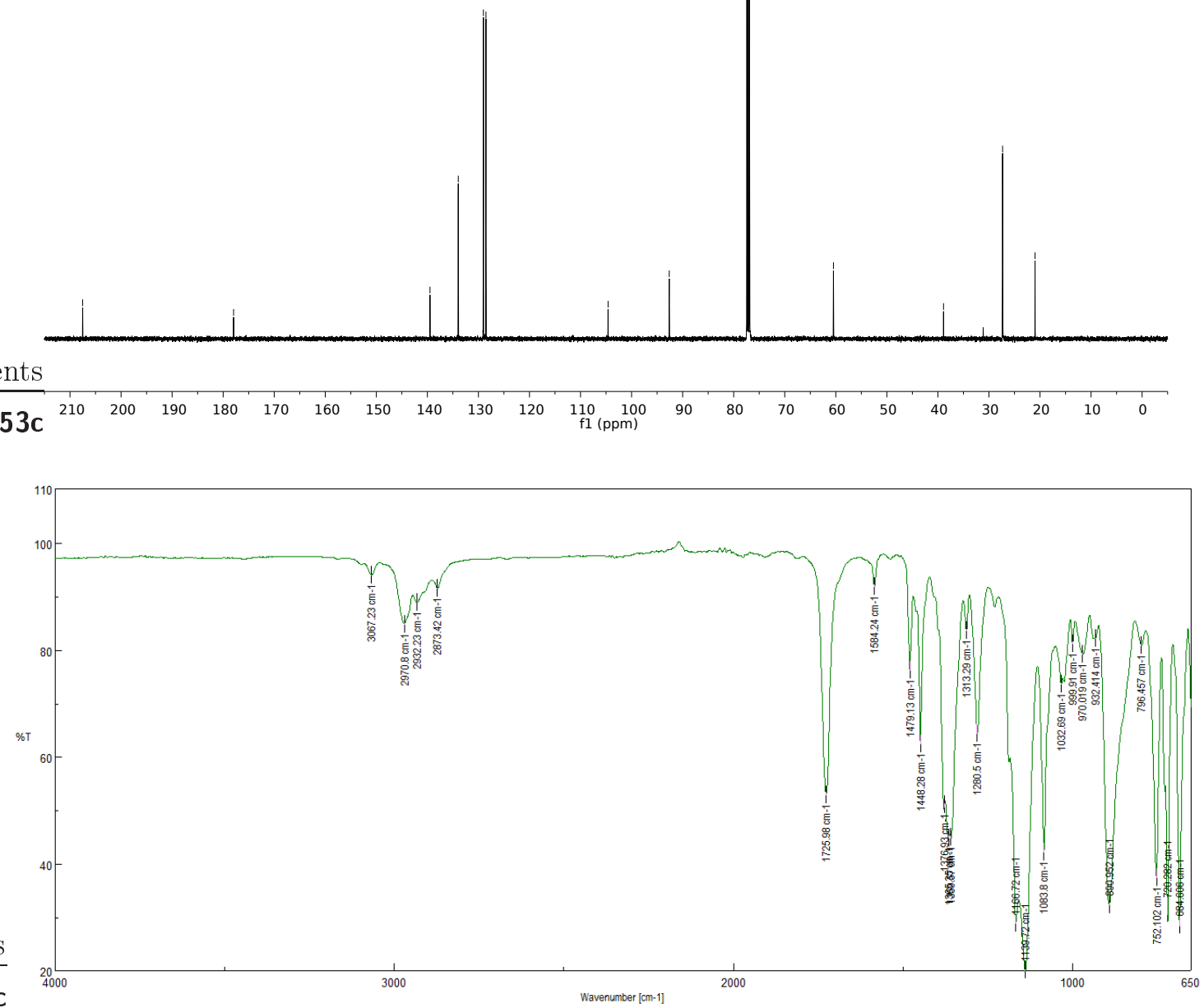


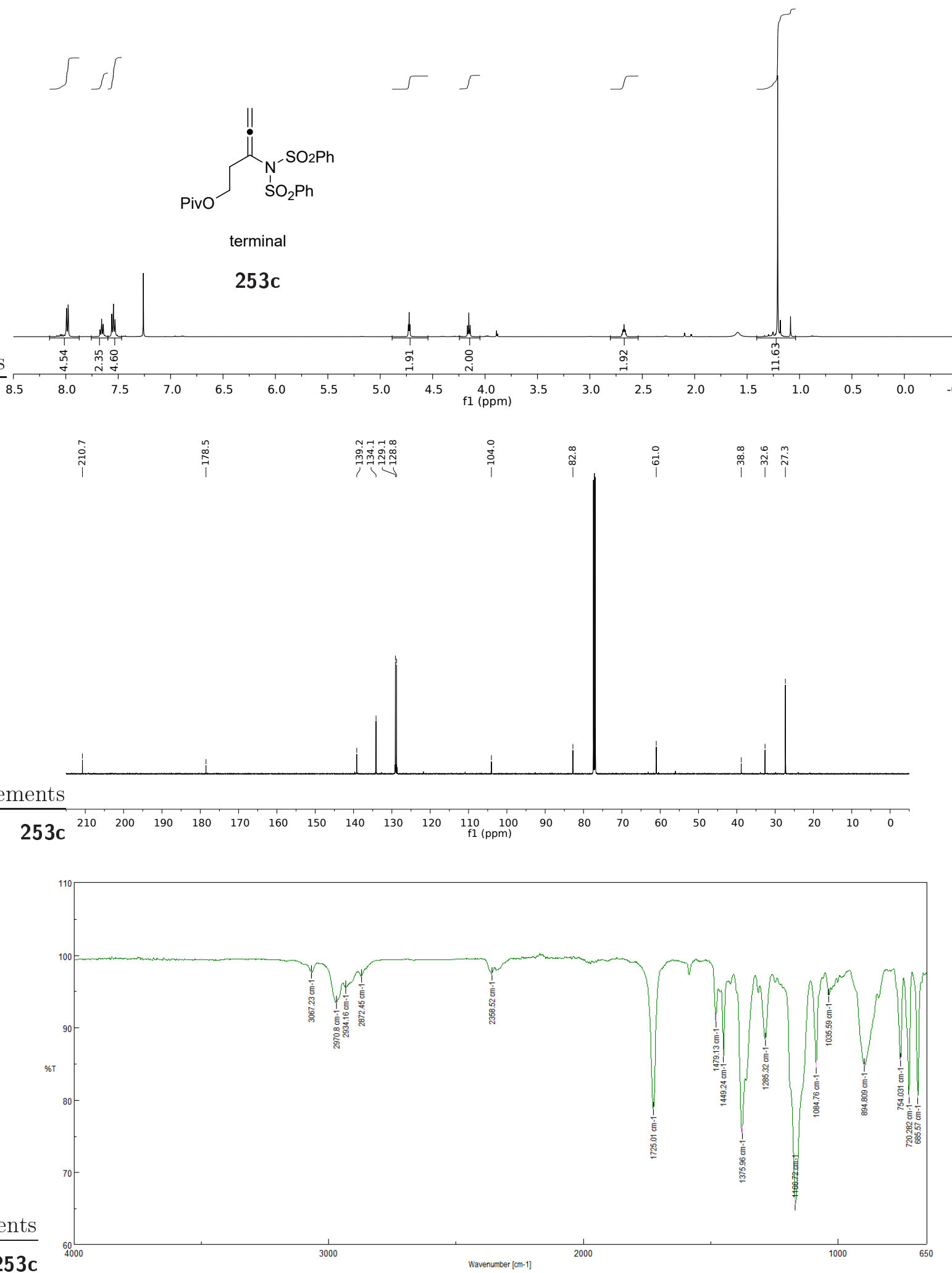



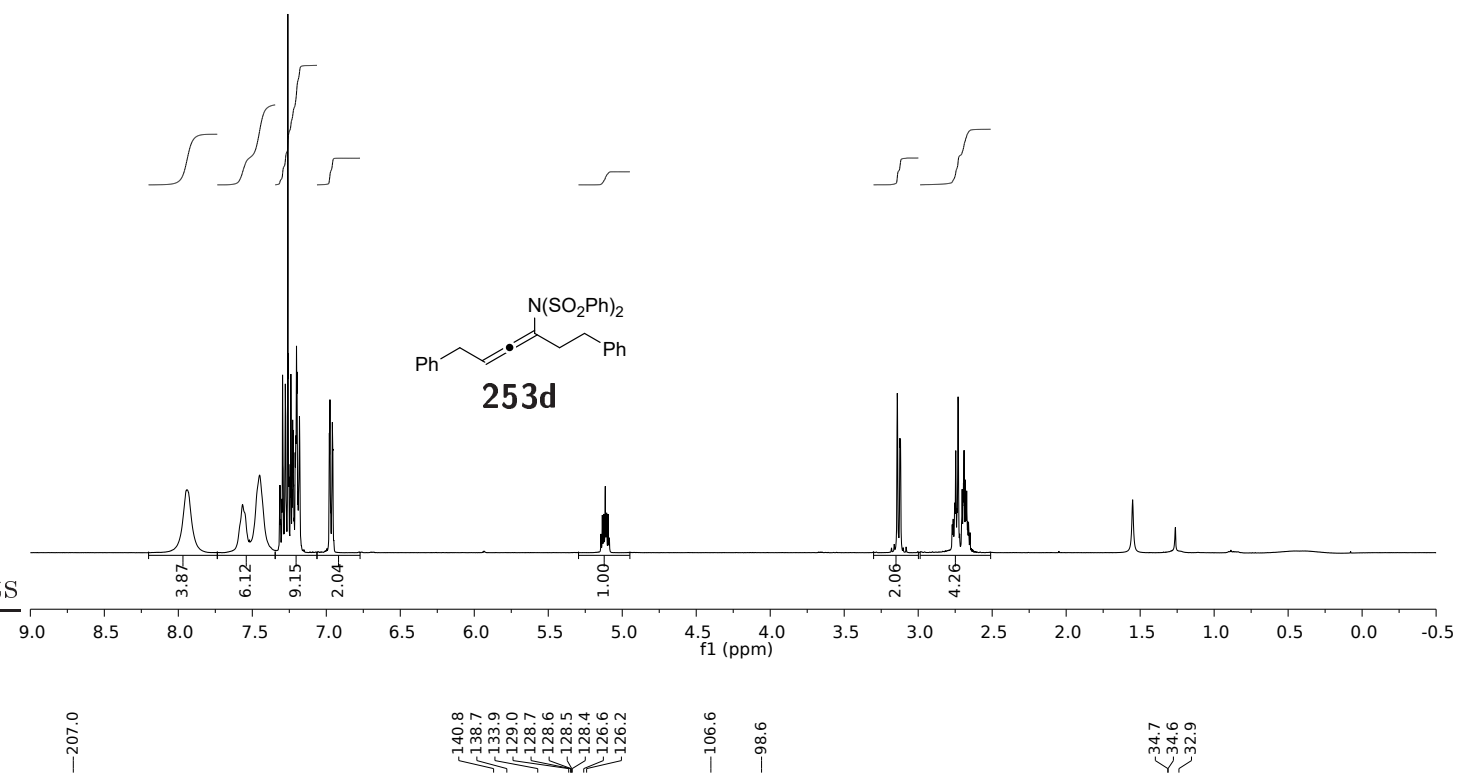

ํํำำ
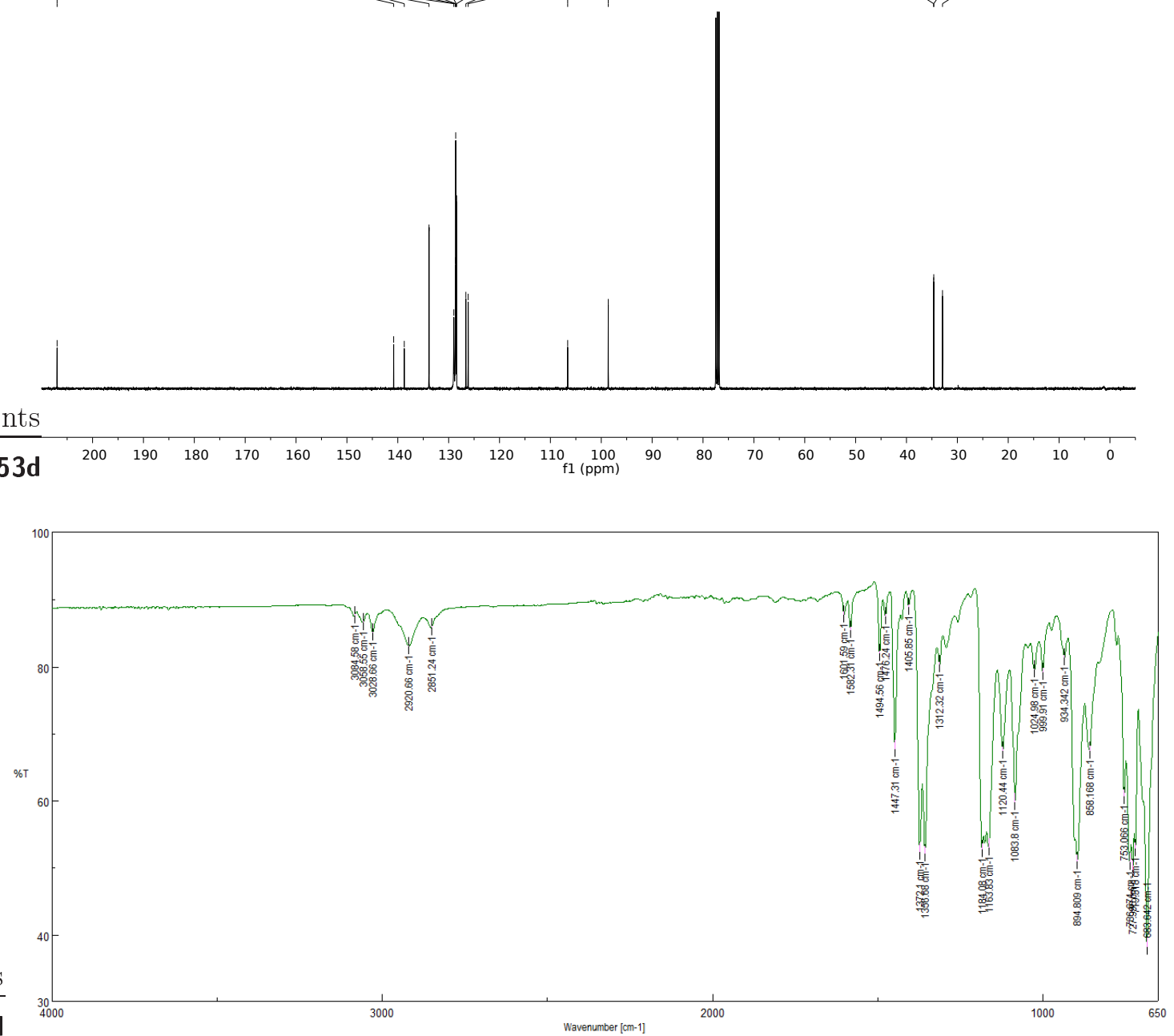


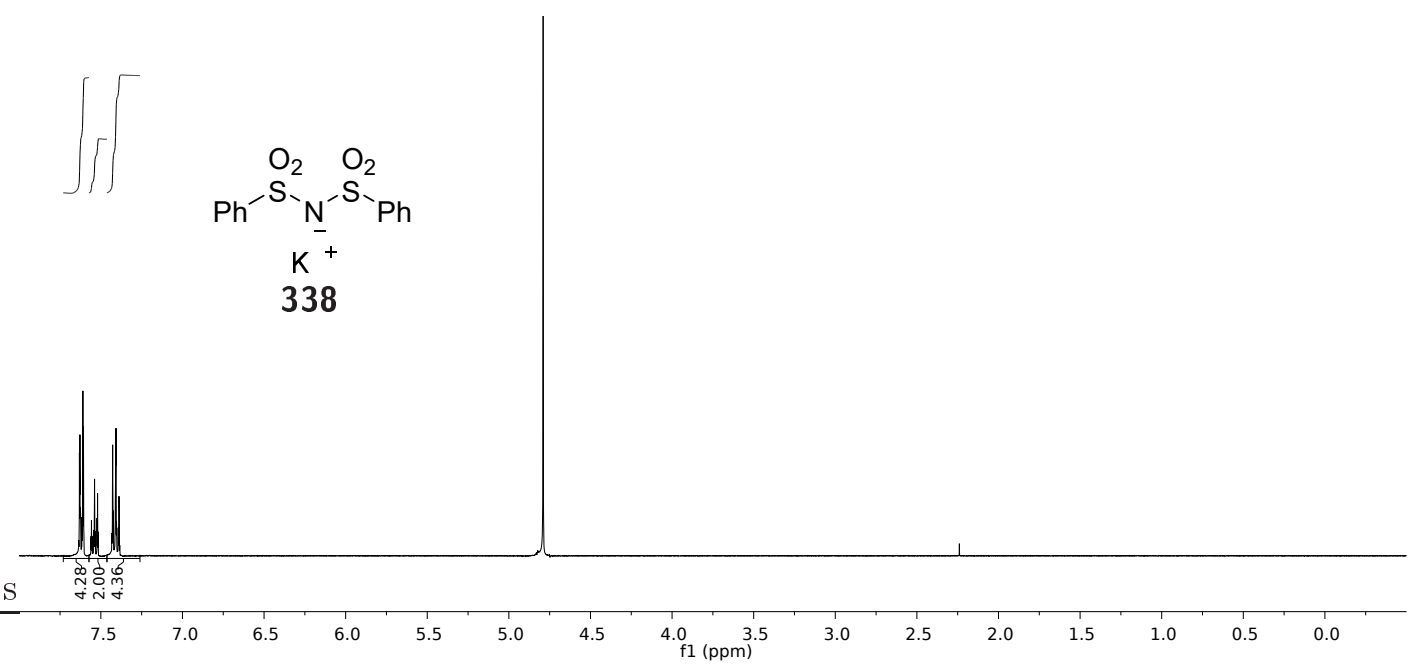

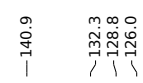
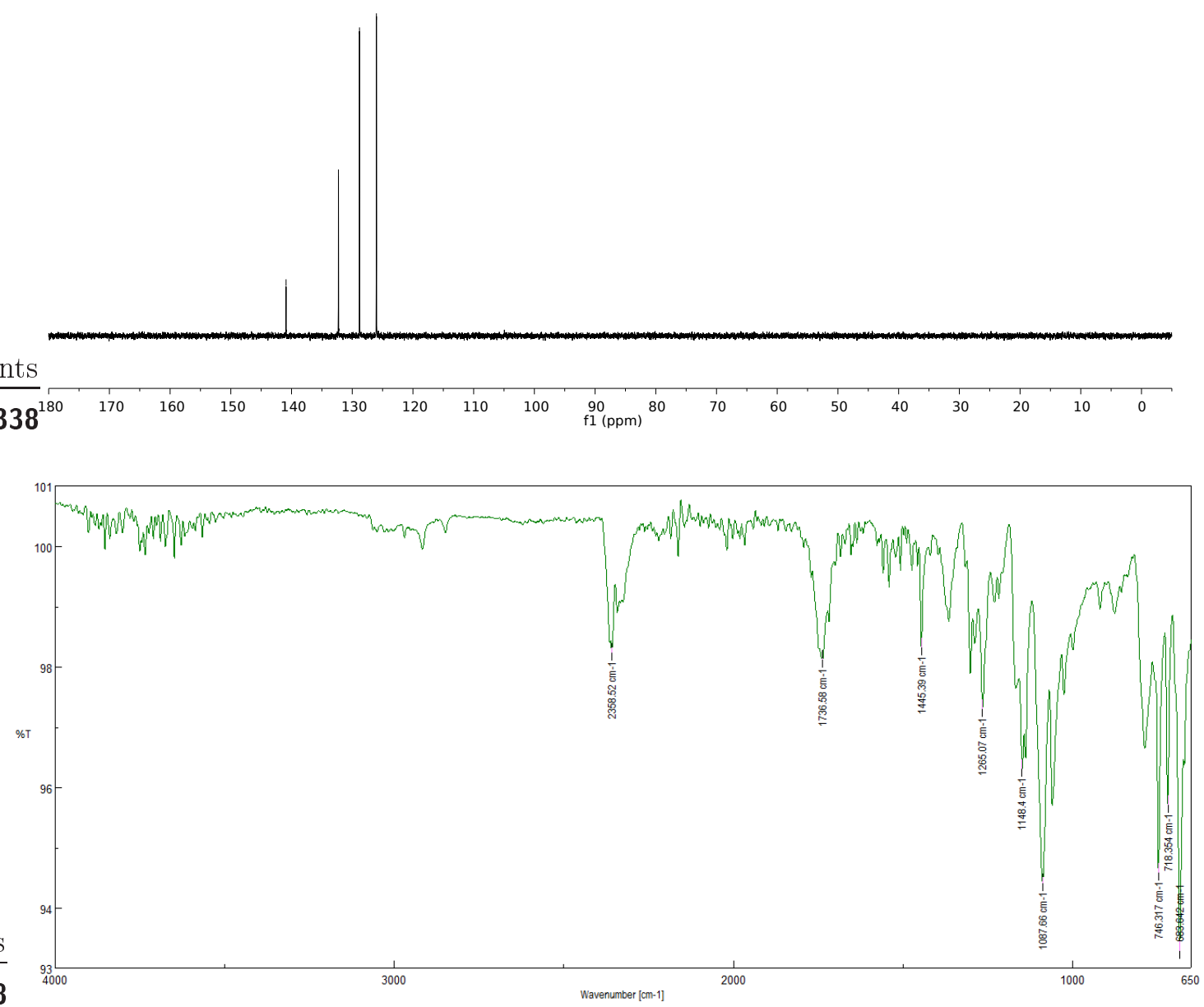


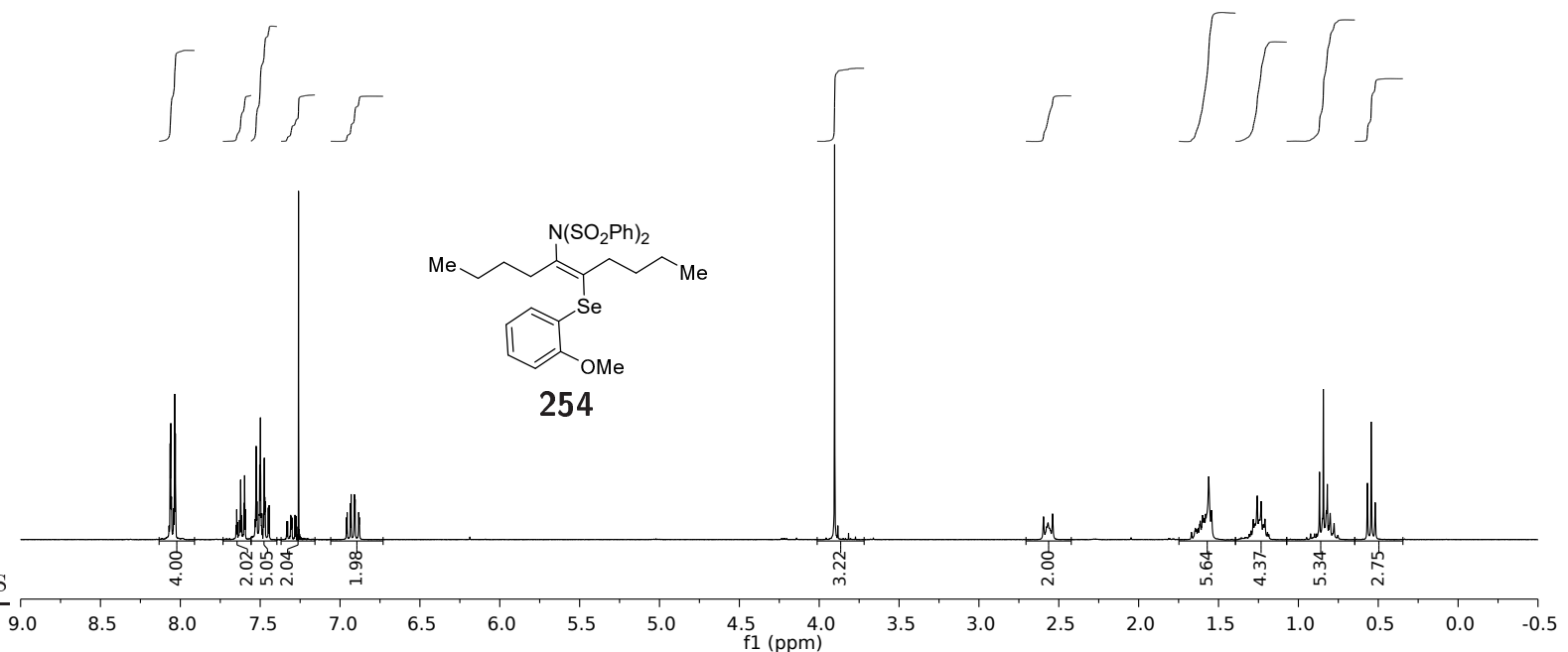

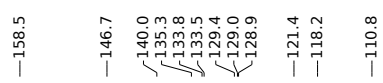

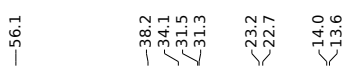
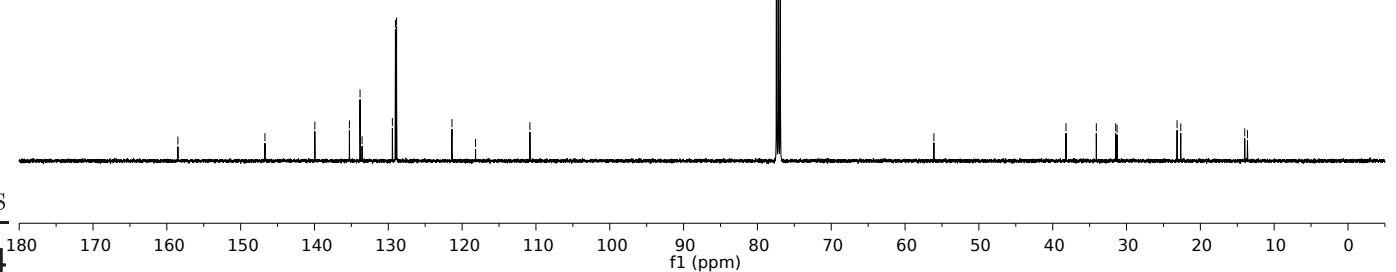

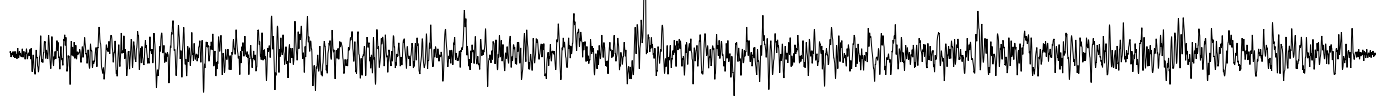




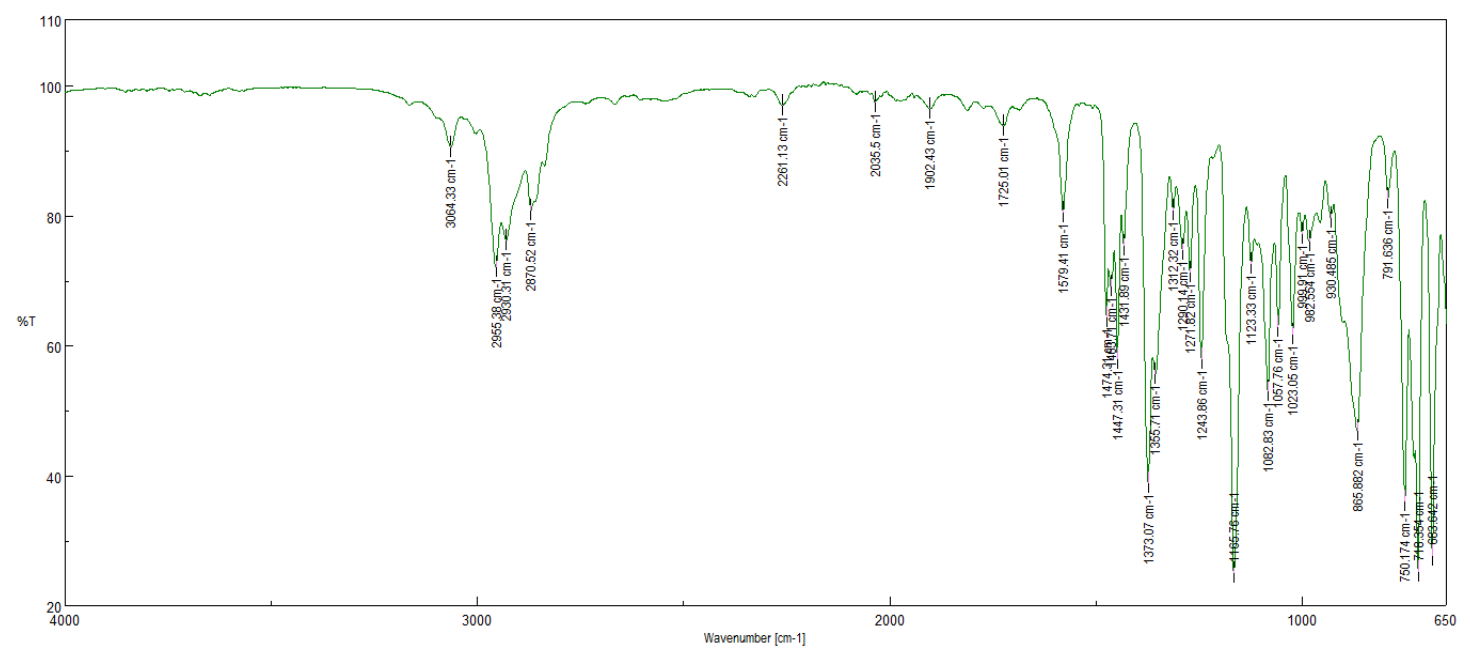




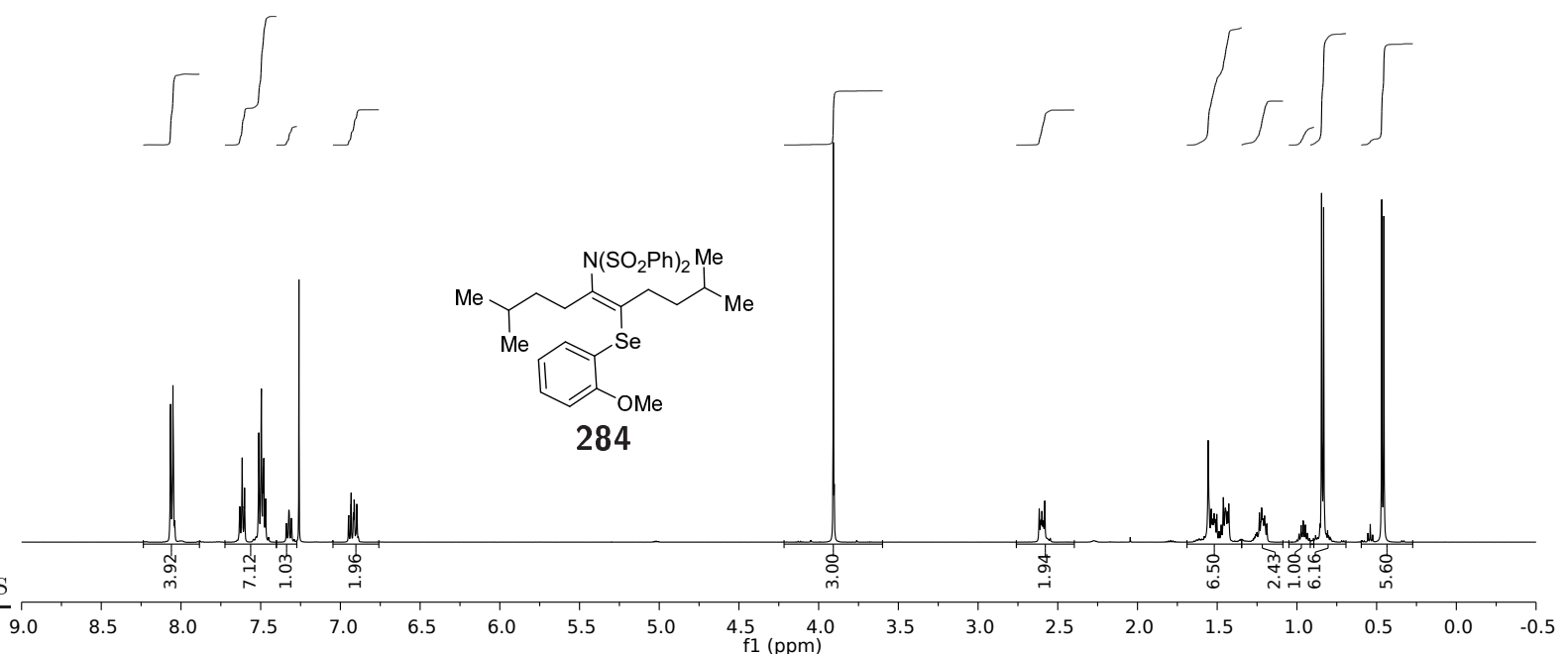

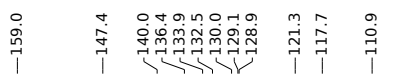

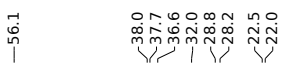

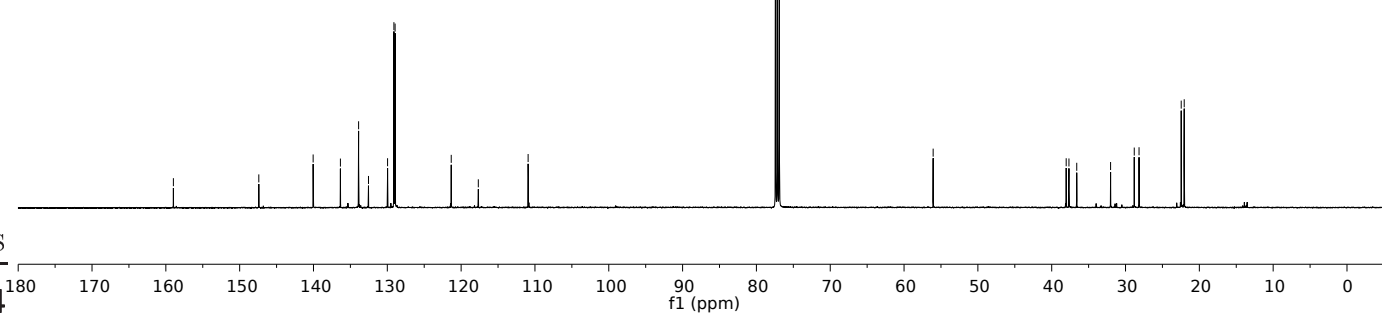




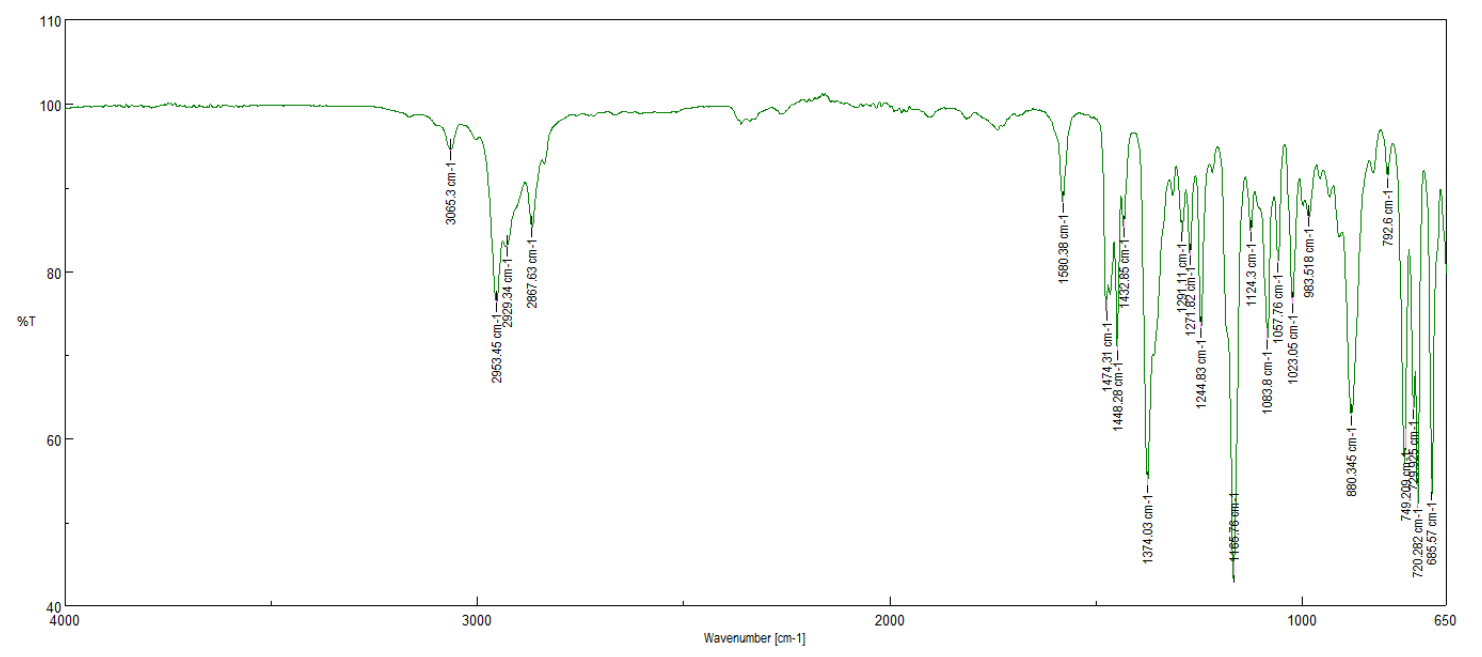




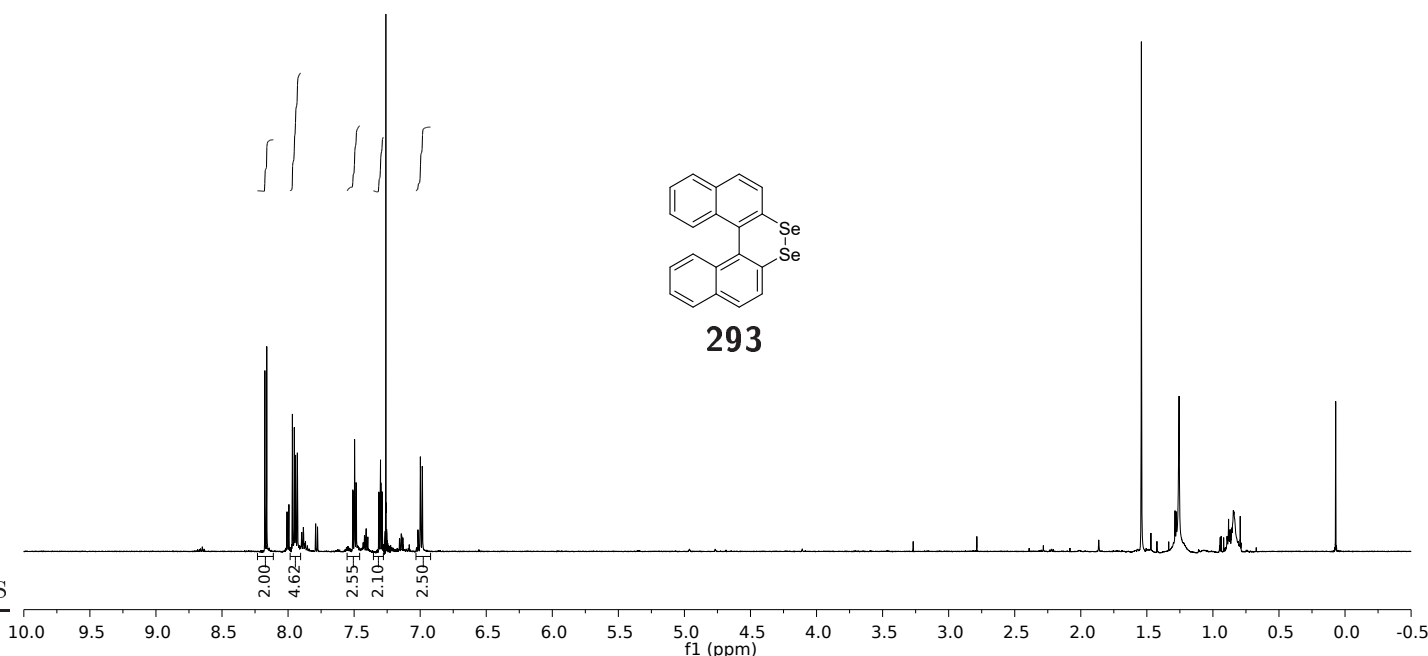

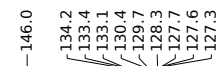
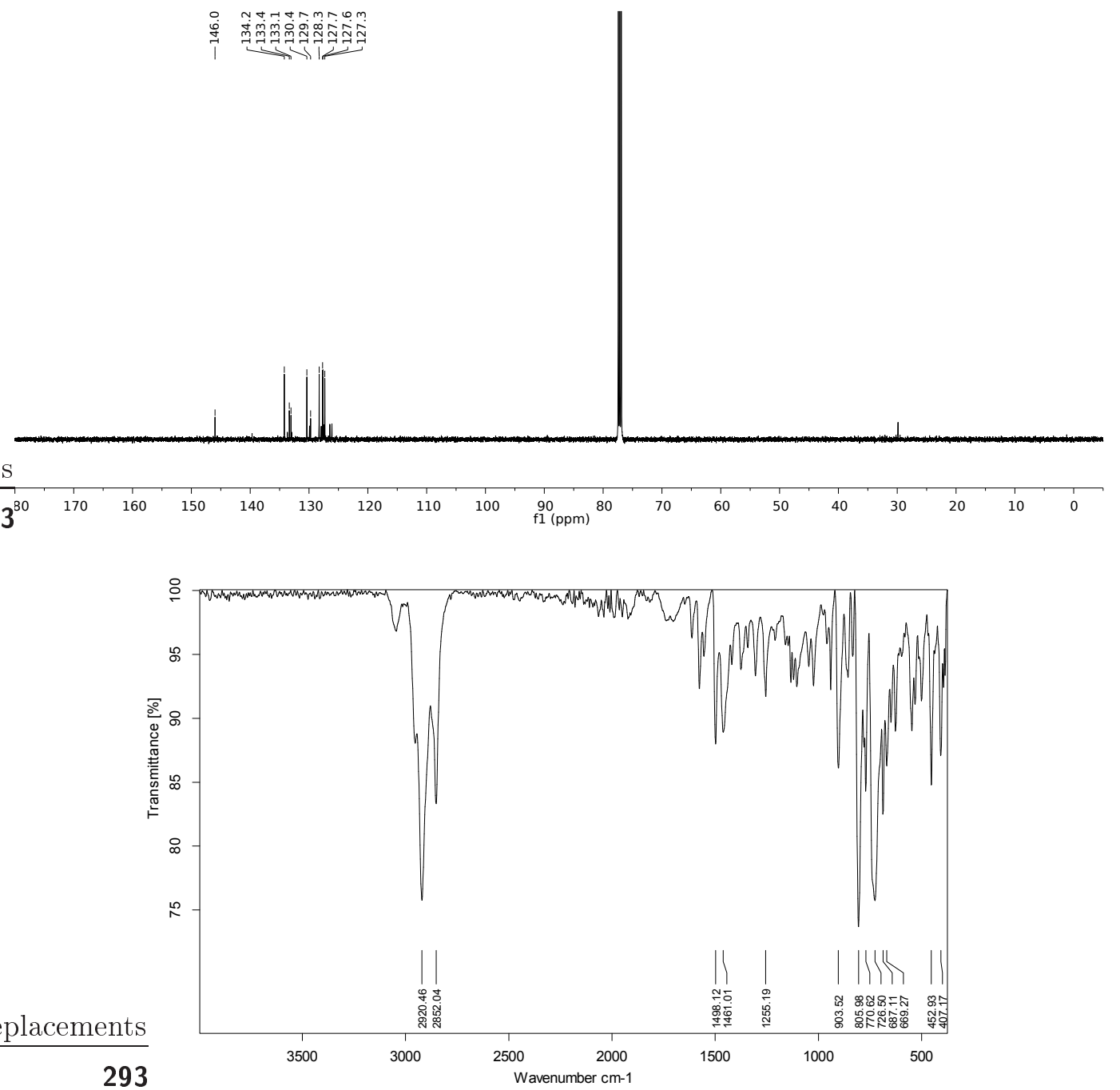


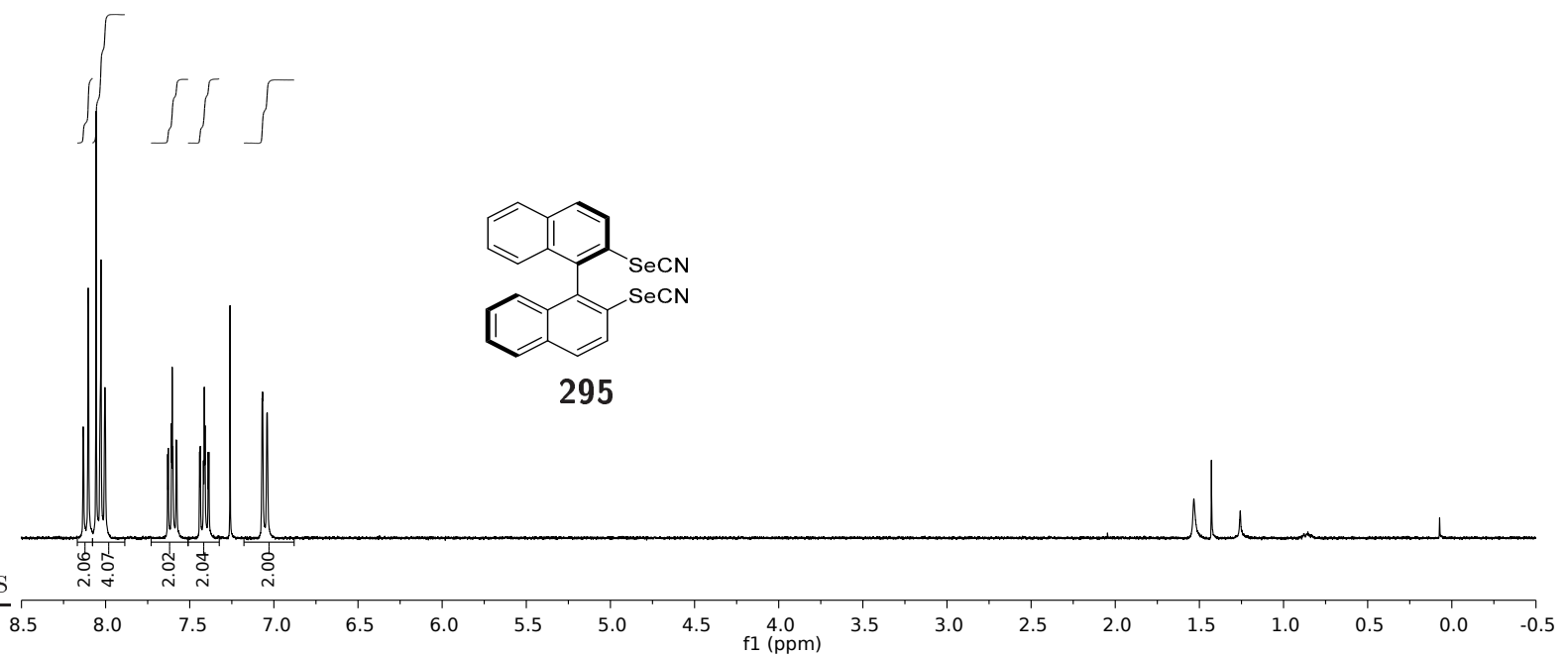

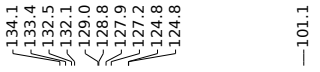
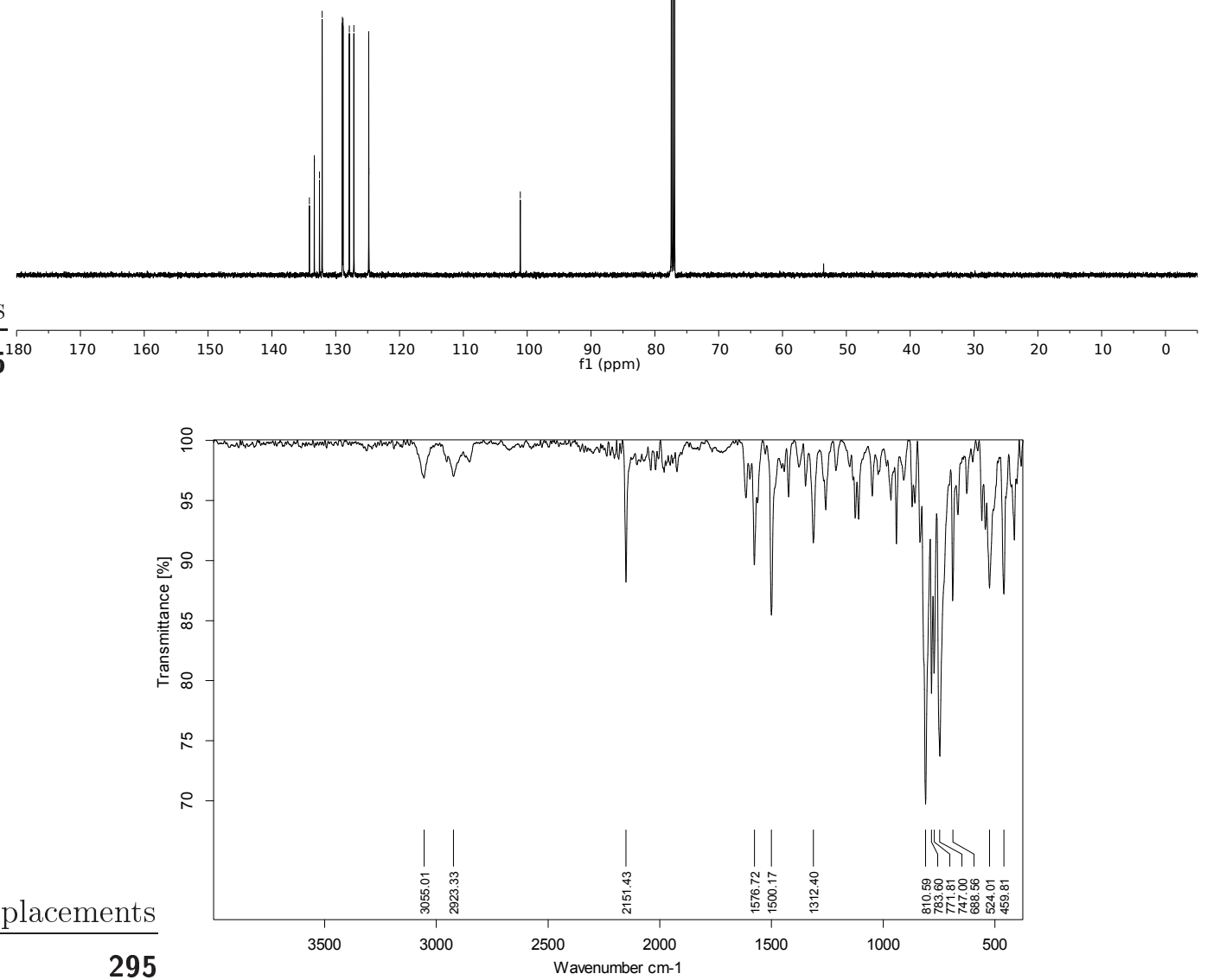

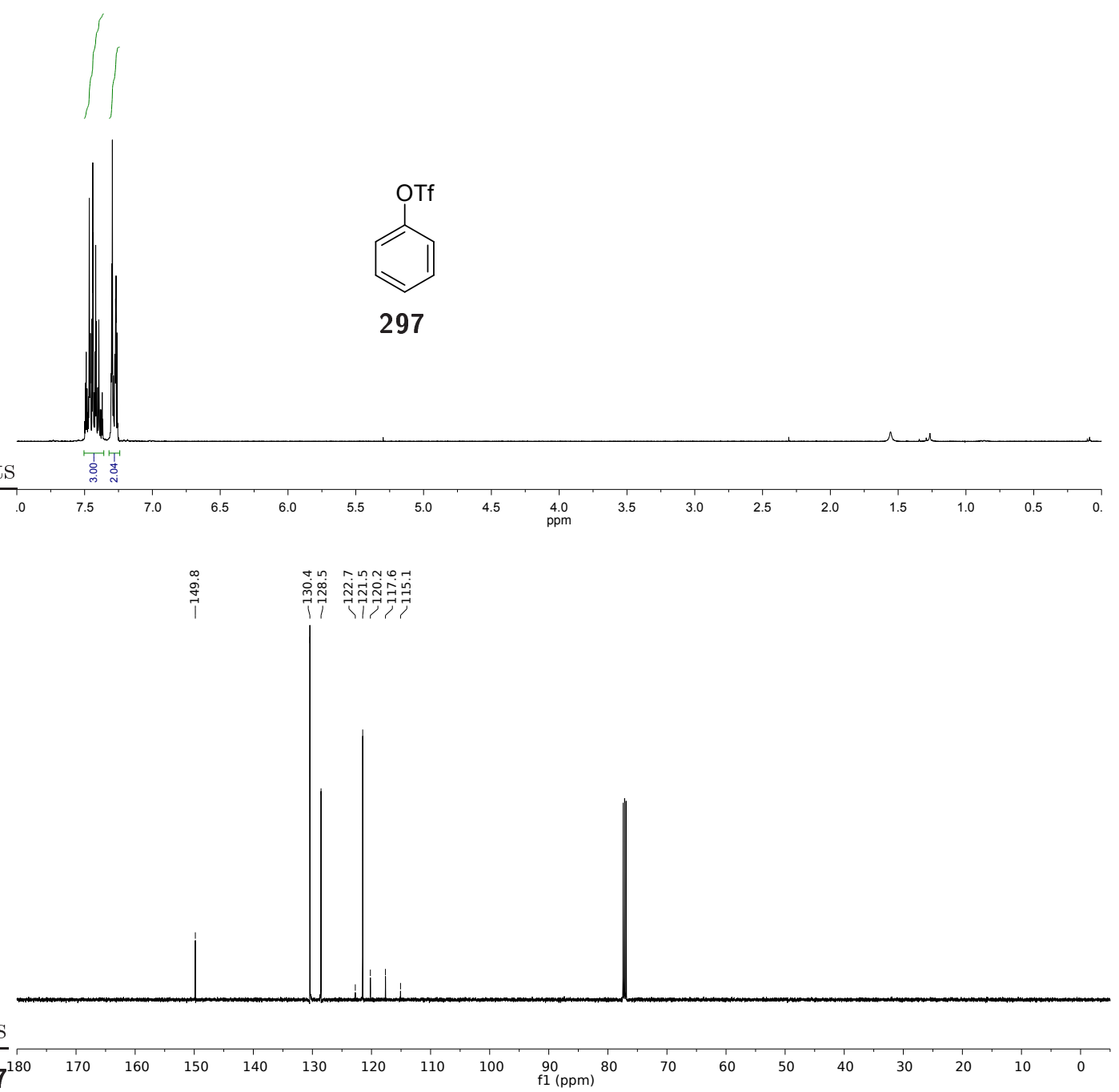


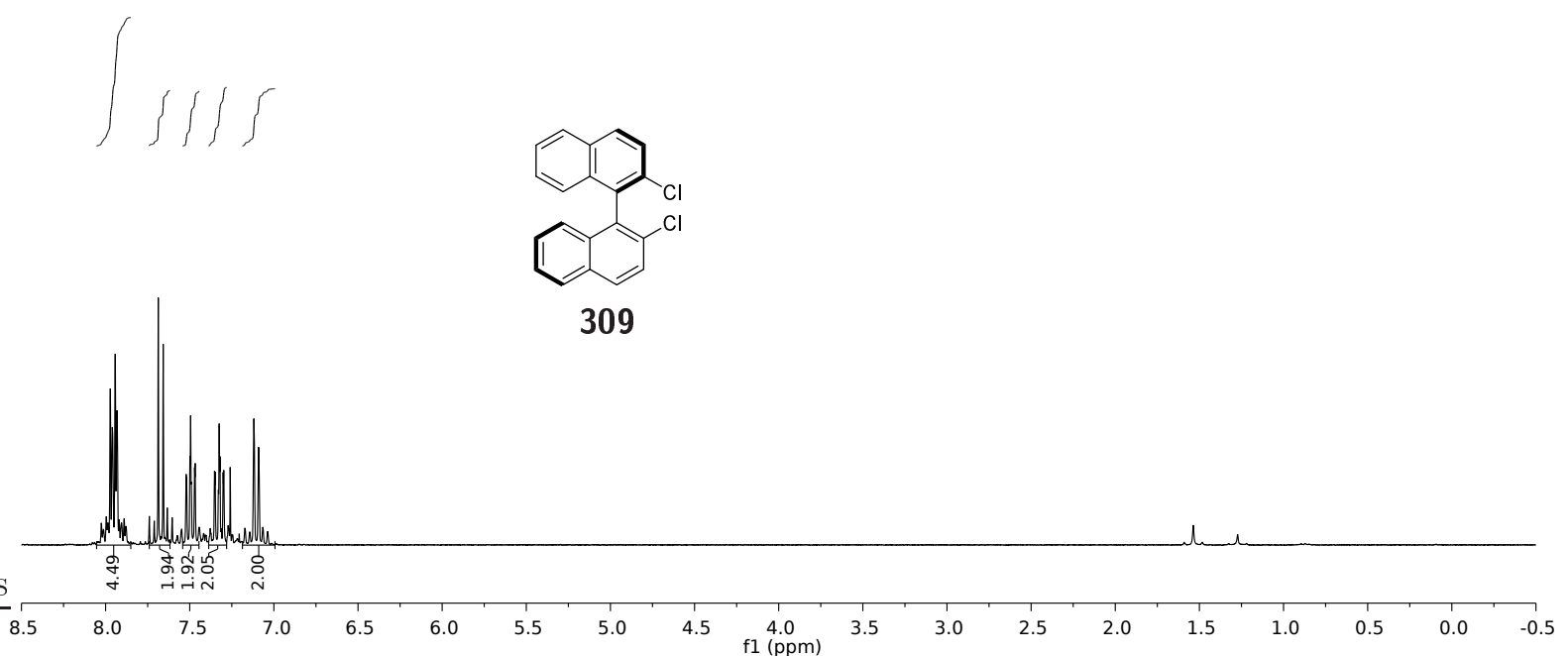

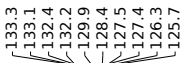
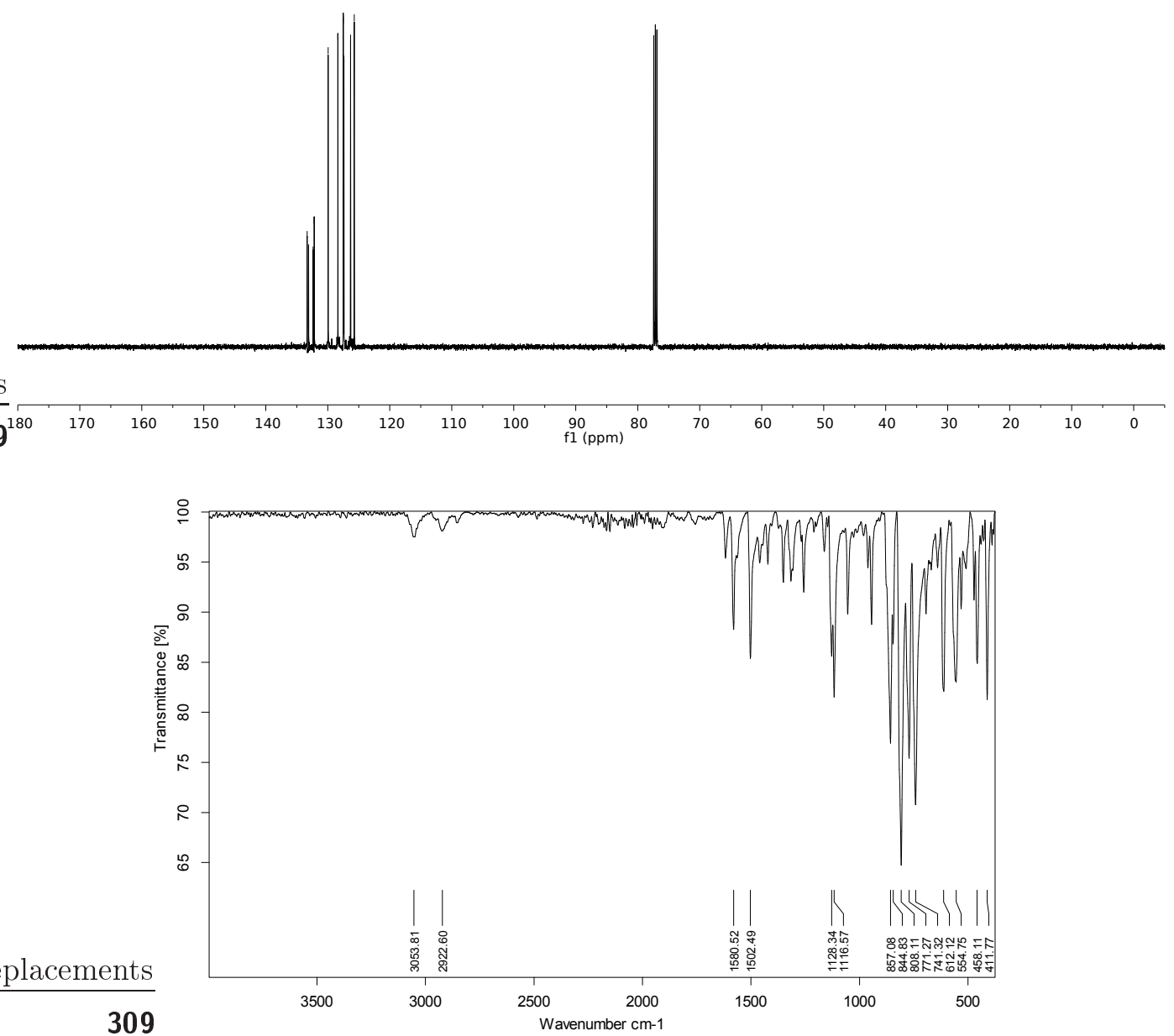

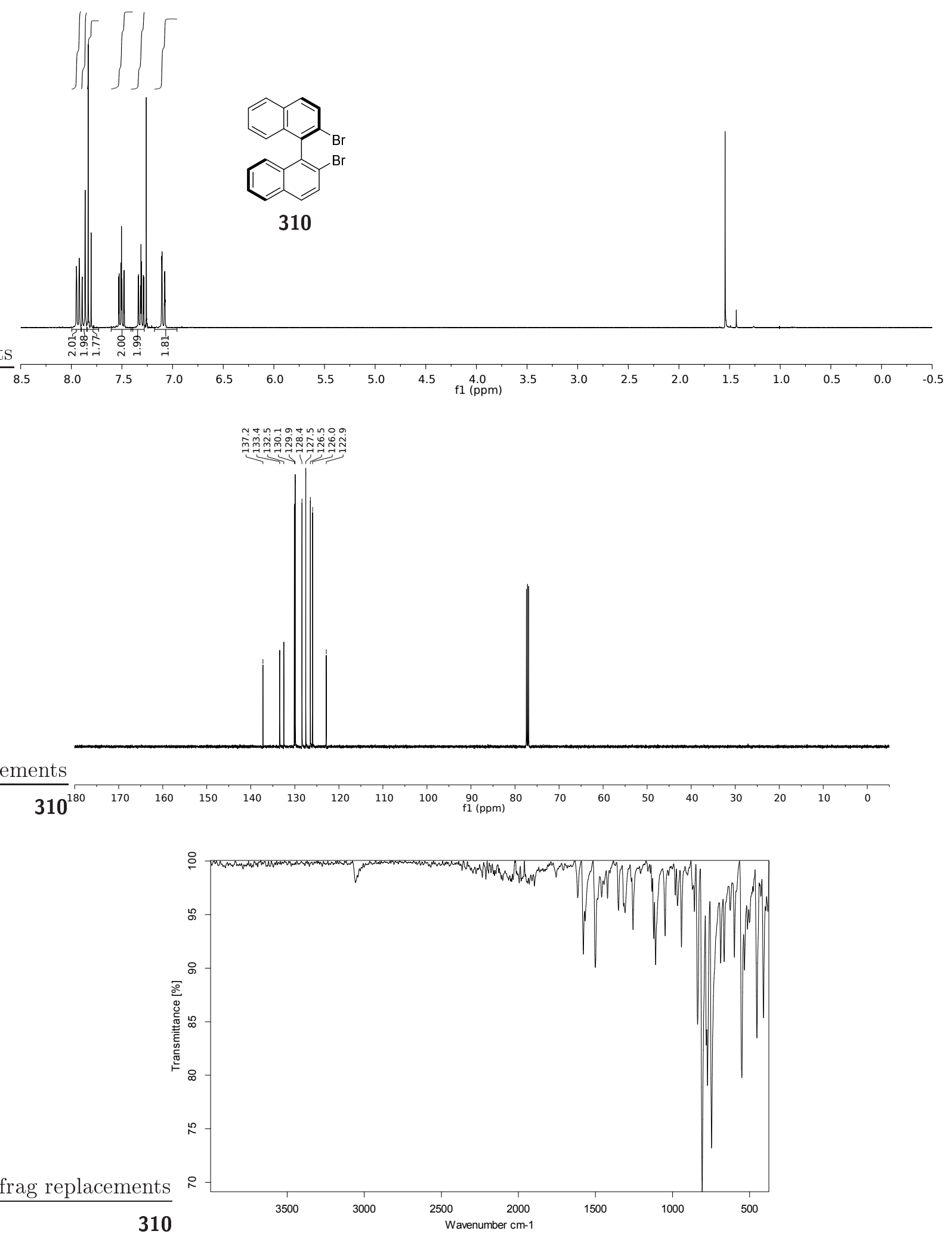
Column: Chiralcel OD

Eluent: $n$-hexane/ $i \mathrm{PrOH}(99.5 / 0.5)$

Flow rate: $0.6 \mathrm{~mL} / \mathrm{min}$

Detection: UV $250.4 \mathrm{~nm}$
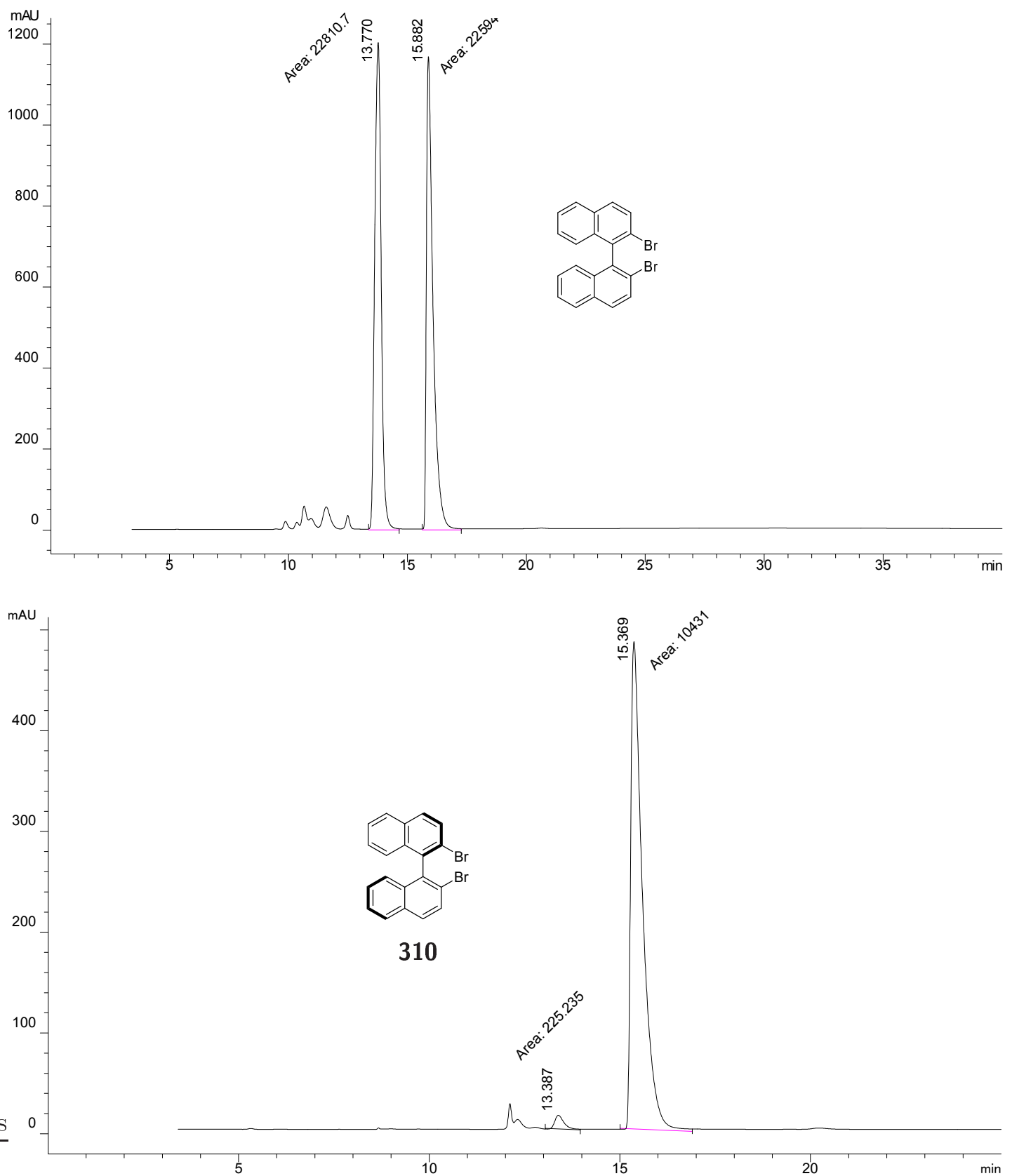


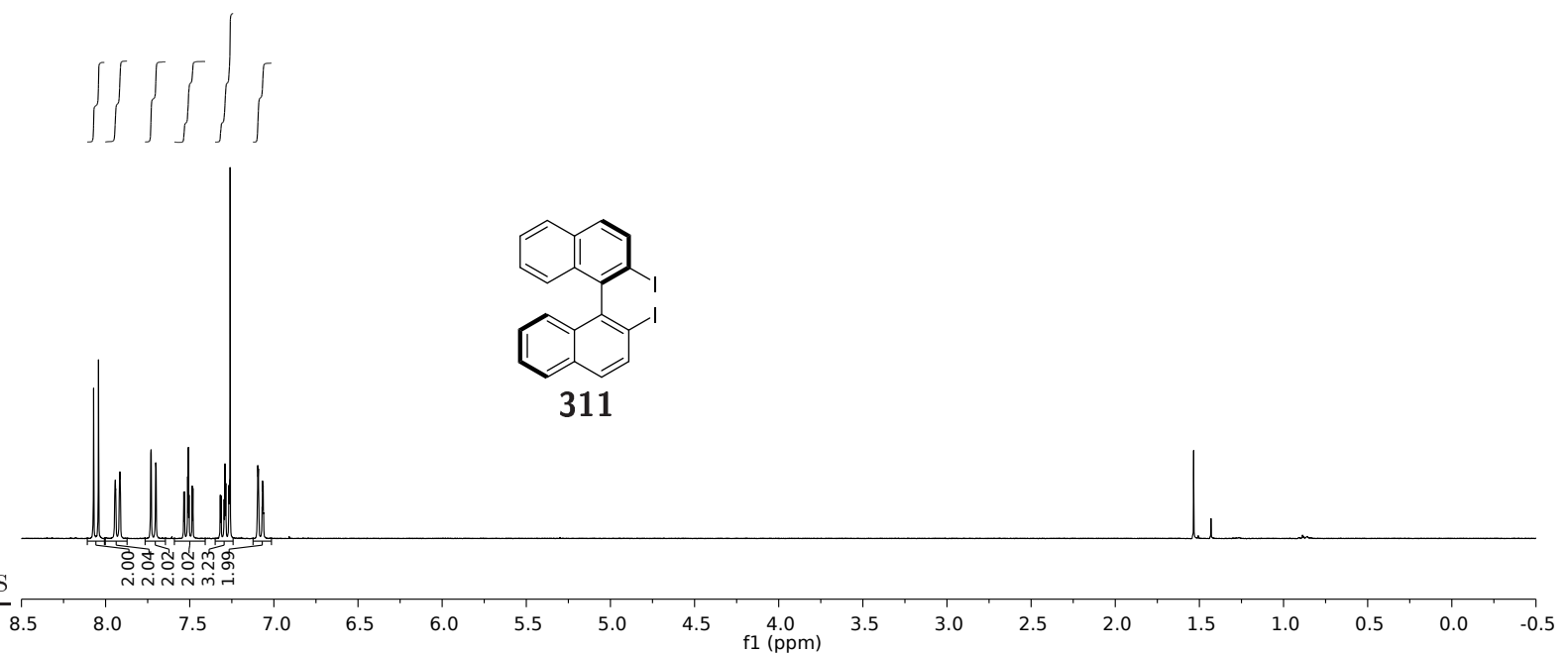




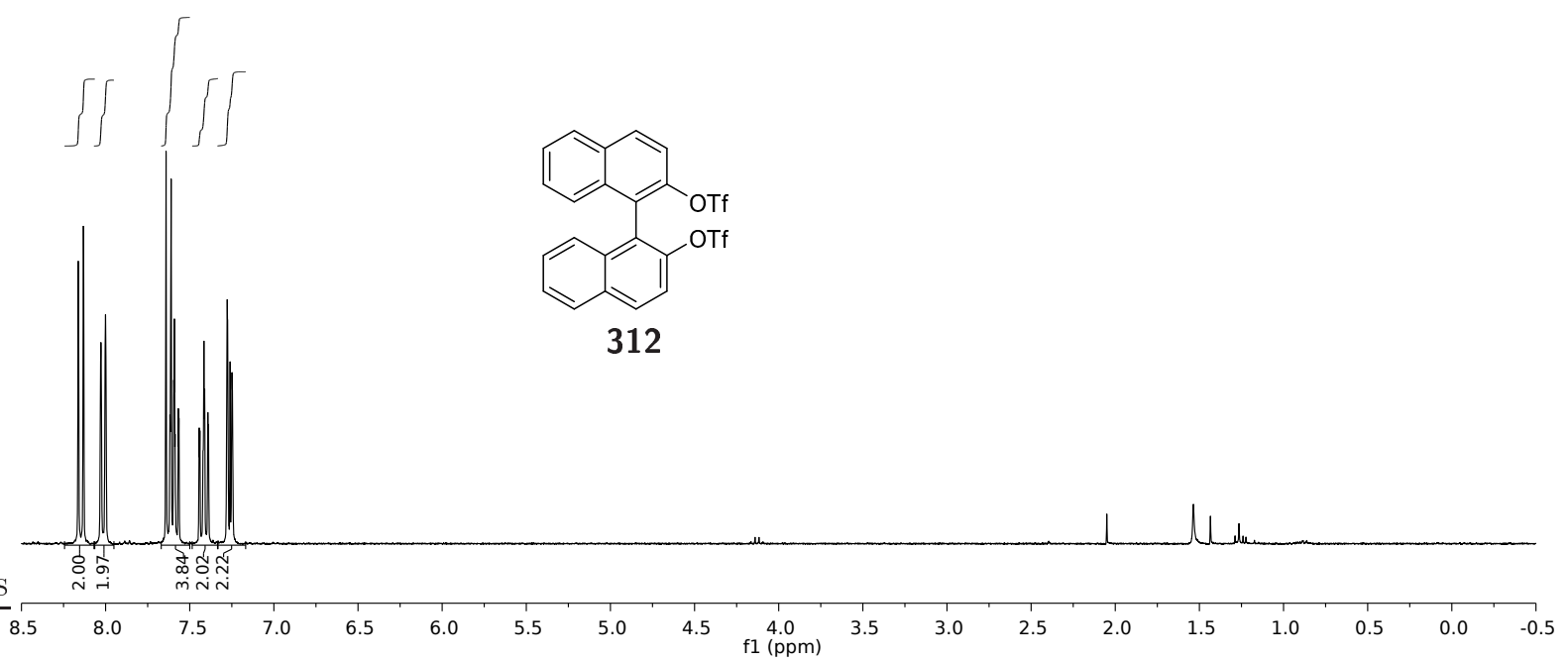

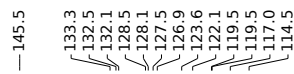
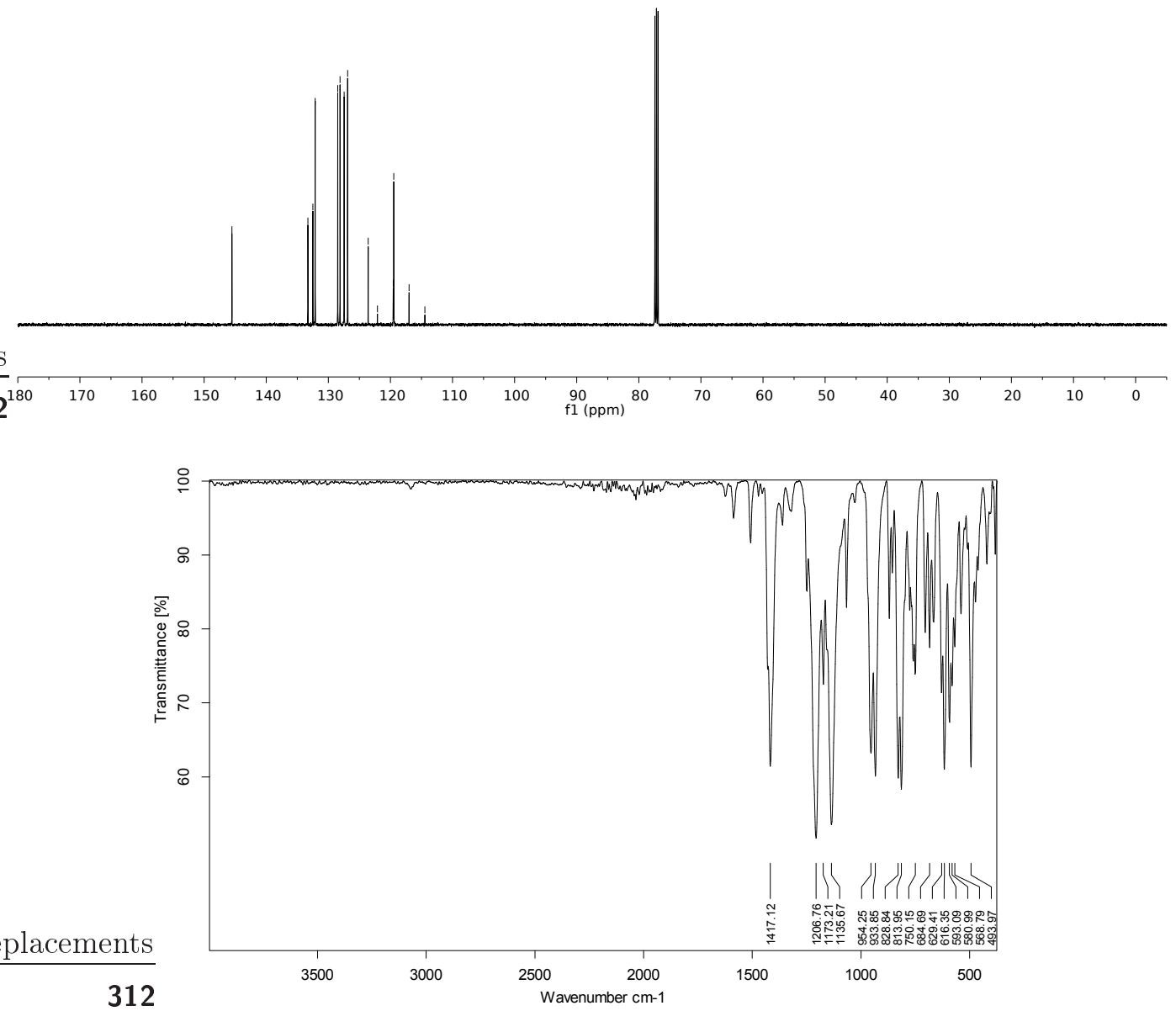
$\iiint \iint S$
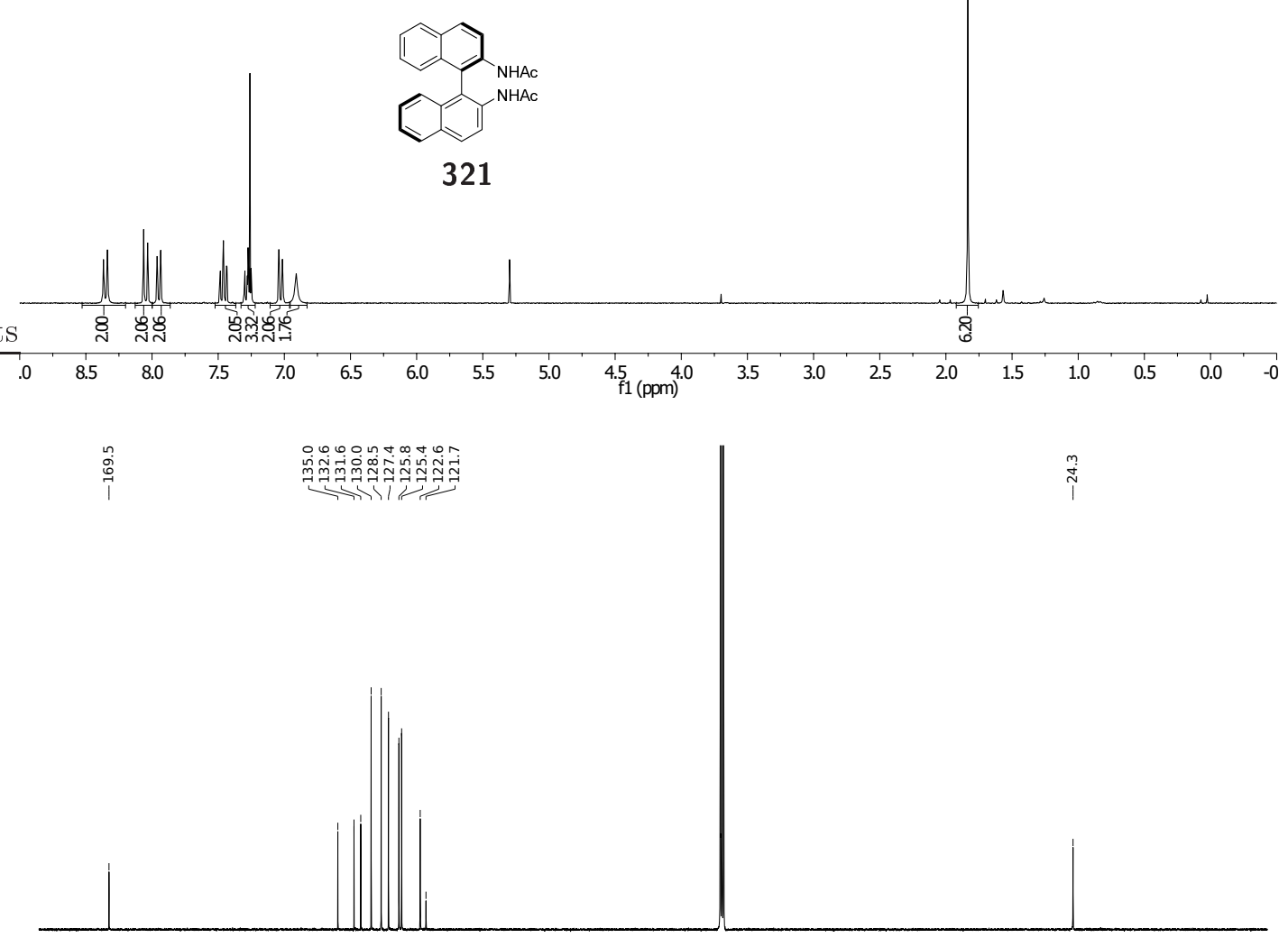

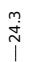
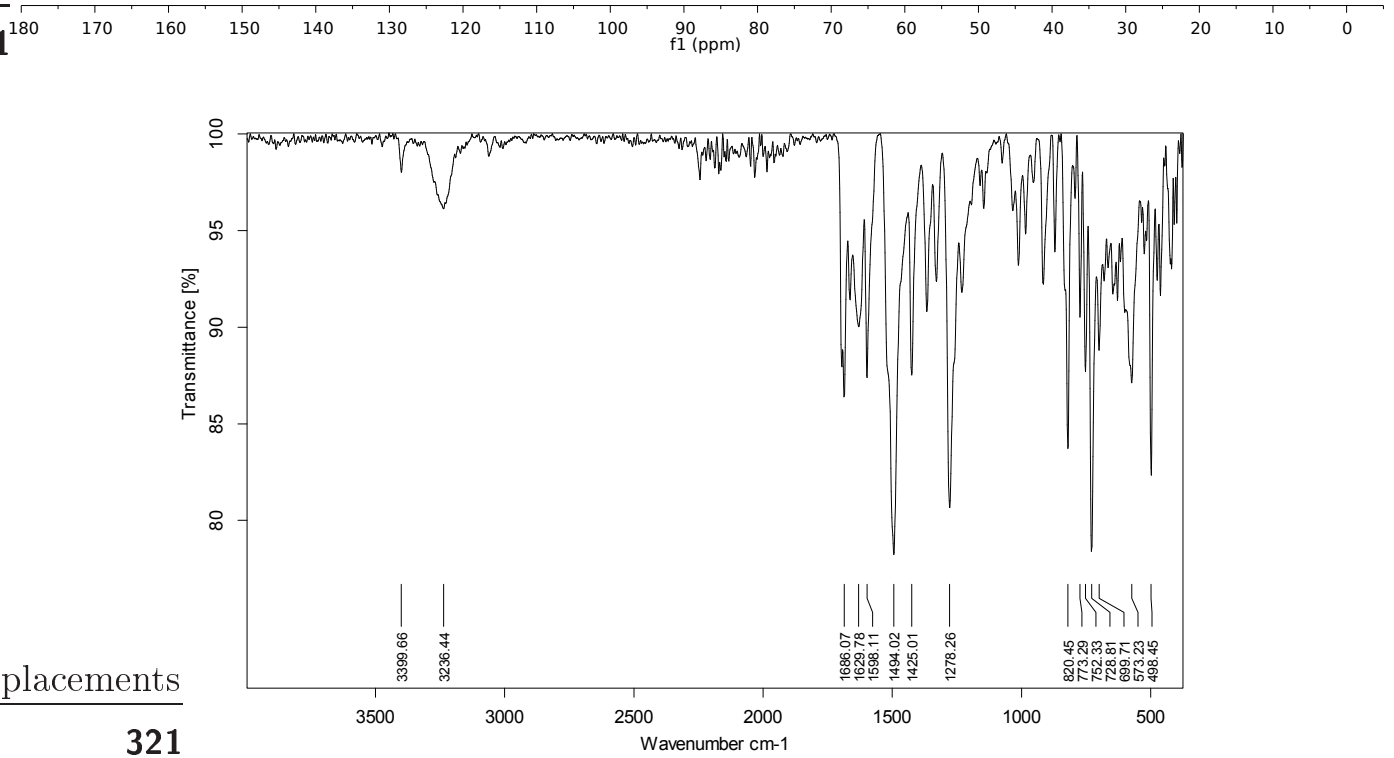
$22+2 \pi s$

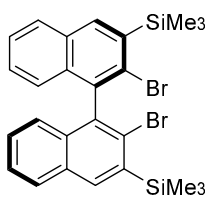

324

$\mid$

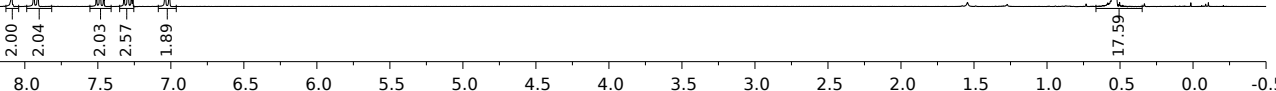

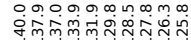

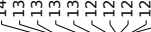

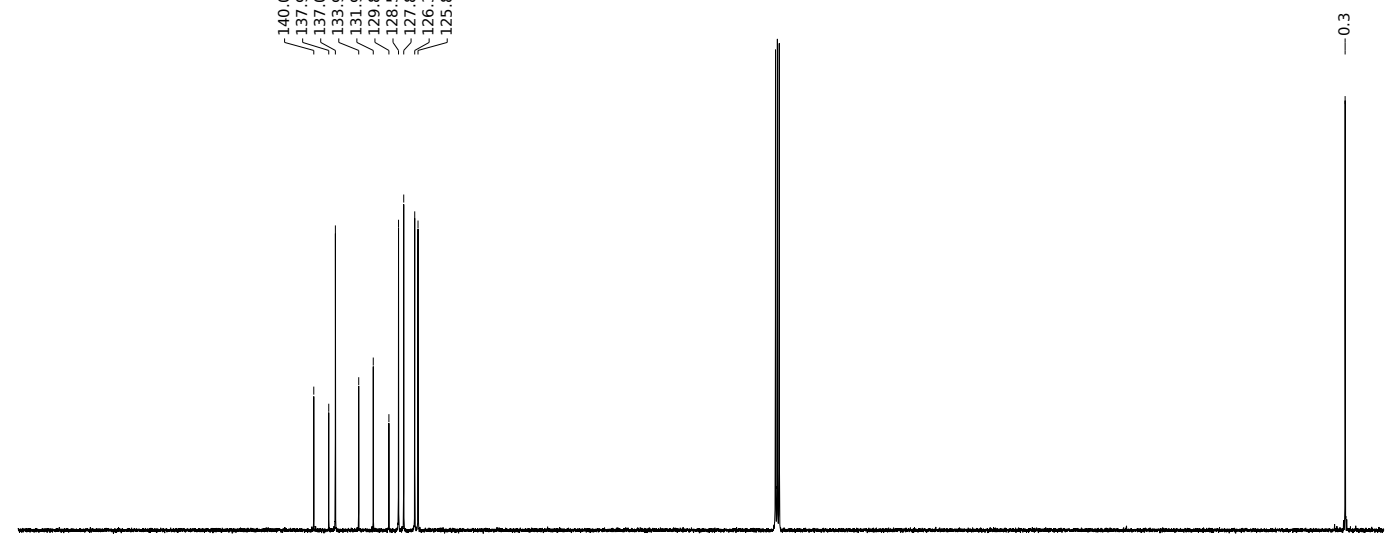

$\begin{array}{lllllllllllllllllll}180 & 170 & 160 & 150 & 140 & 130 & 120 & 110 & 100 & \begin{array}{c}90 \\ \mathrm{f} 1(\mathrm{ppm})\end{array} & 80 & 70 & 60 & 50 & 40 & 30 & 20 & 10 & 0\end{array}$

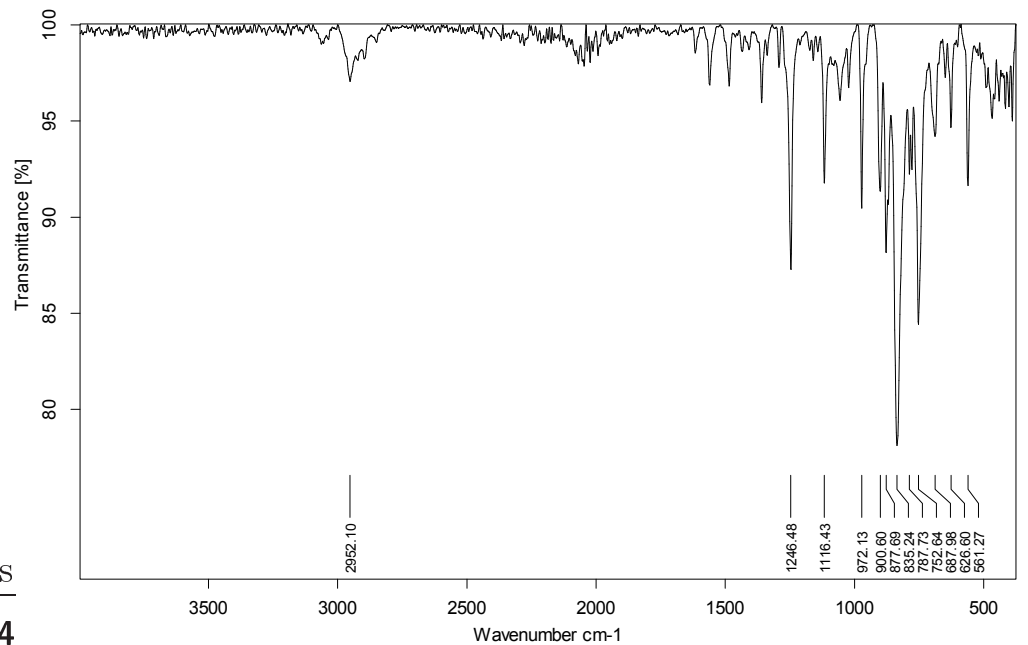


Column: Chiralpak IA

Eluent: $n$-hexane/ $i \mathrm{PrOH}(99.8 / 0.2)$

Flow rate: $0.6 \mathrm{~mL} / \mathrm{min}$

Detection: UV $250.4 \mathrm{~nm}$
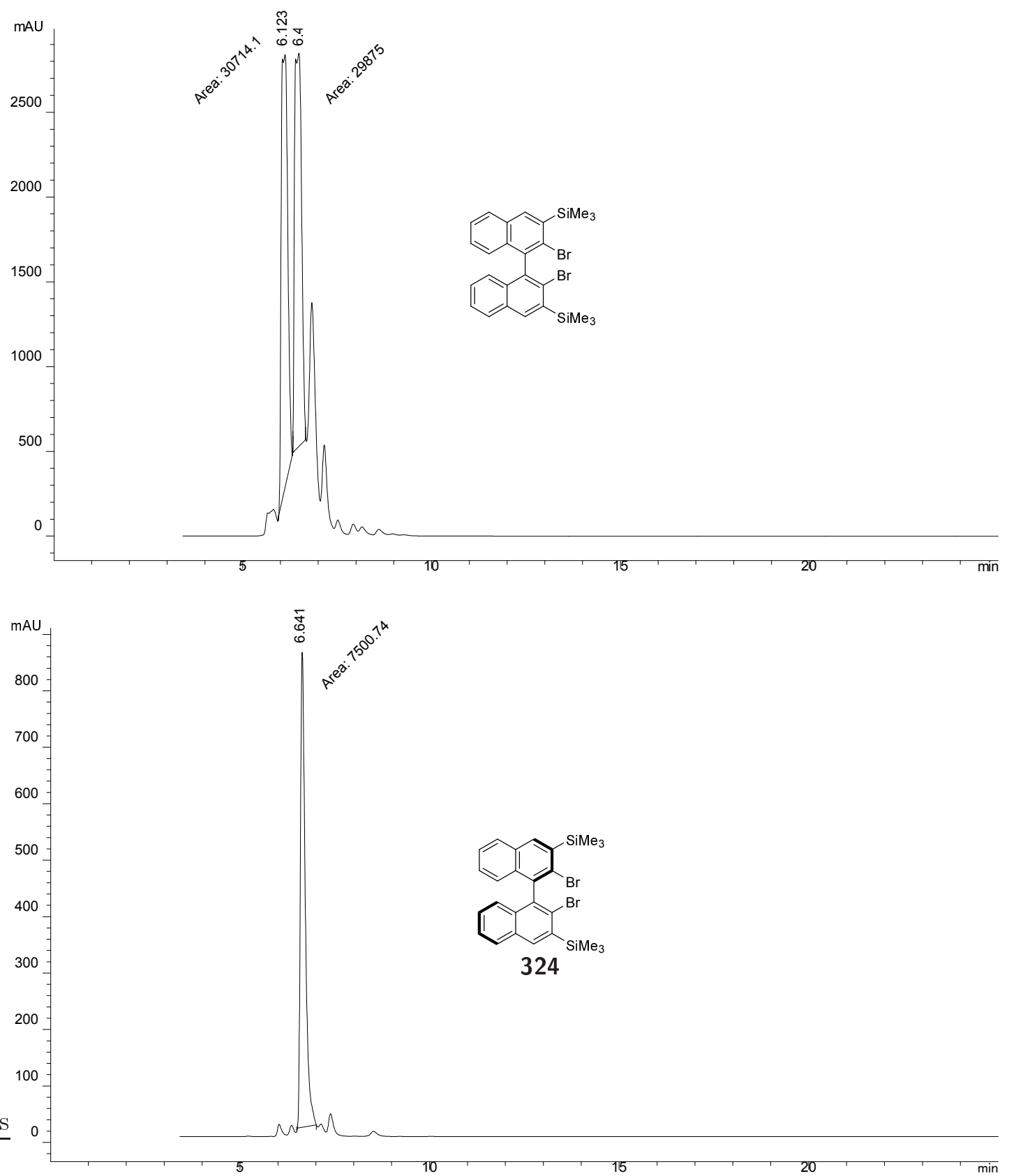


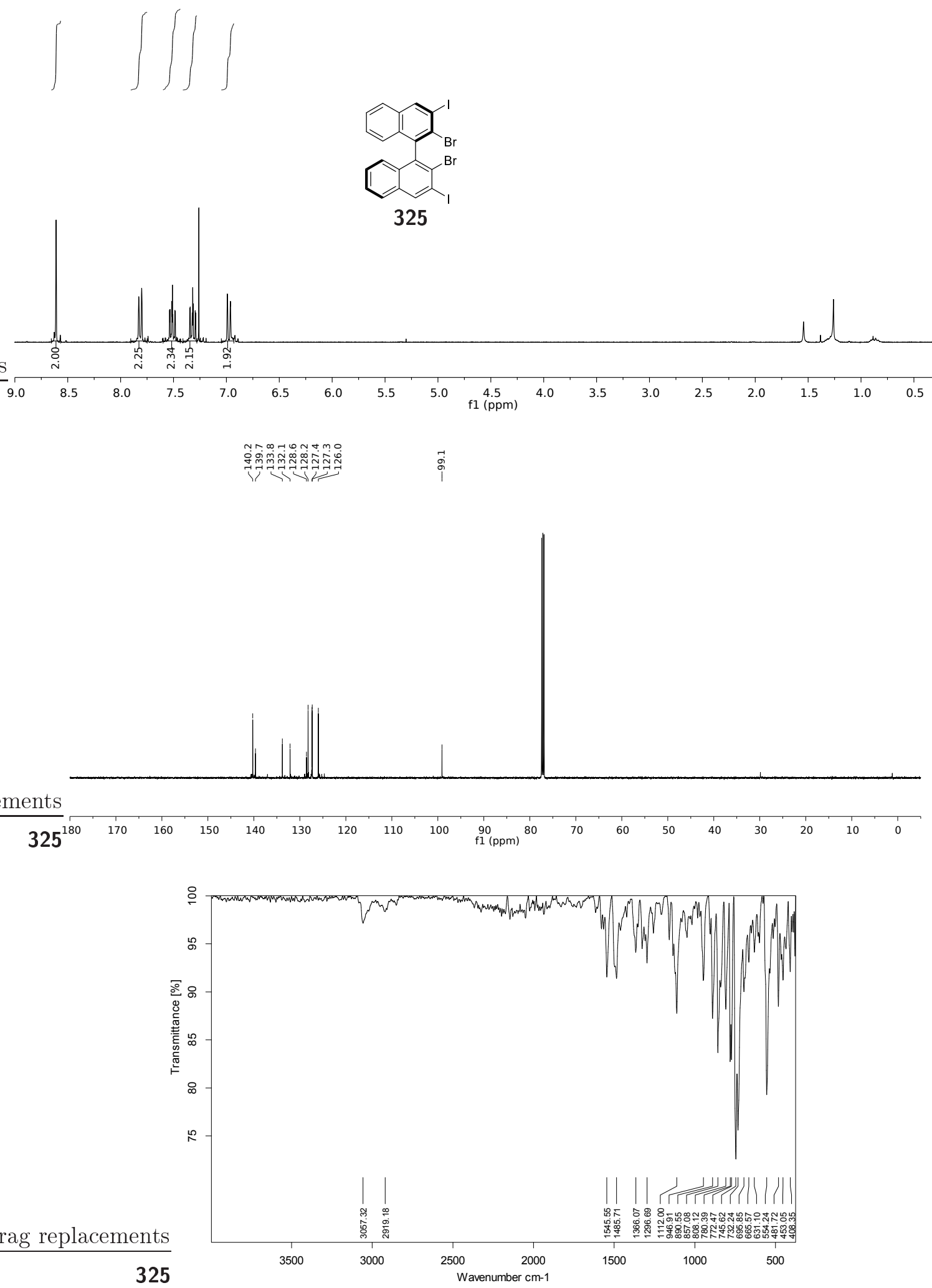


Column: Chiralpak IA

Eluent: $n$-hexane/ $i \mathrm{PrOH}(99.8 / 0.2)$

Flow rate: $0.6 \mathrm{~mL} / \mathrm{min}$

Detection: UV $250.4 \mathrm{~nm}$
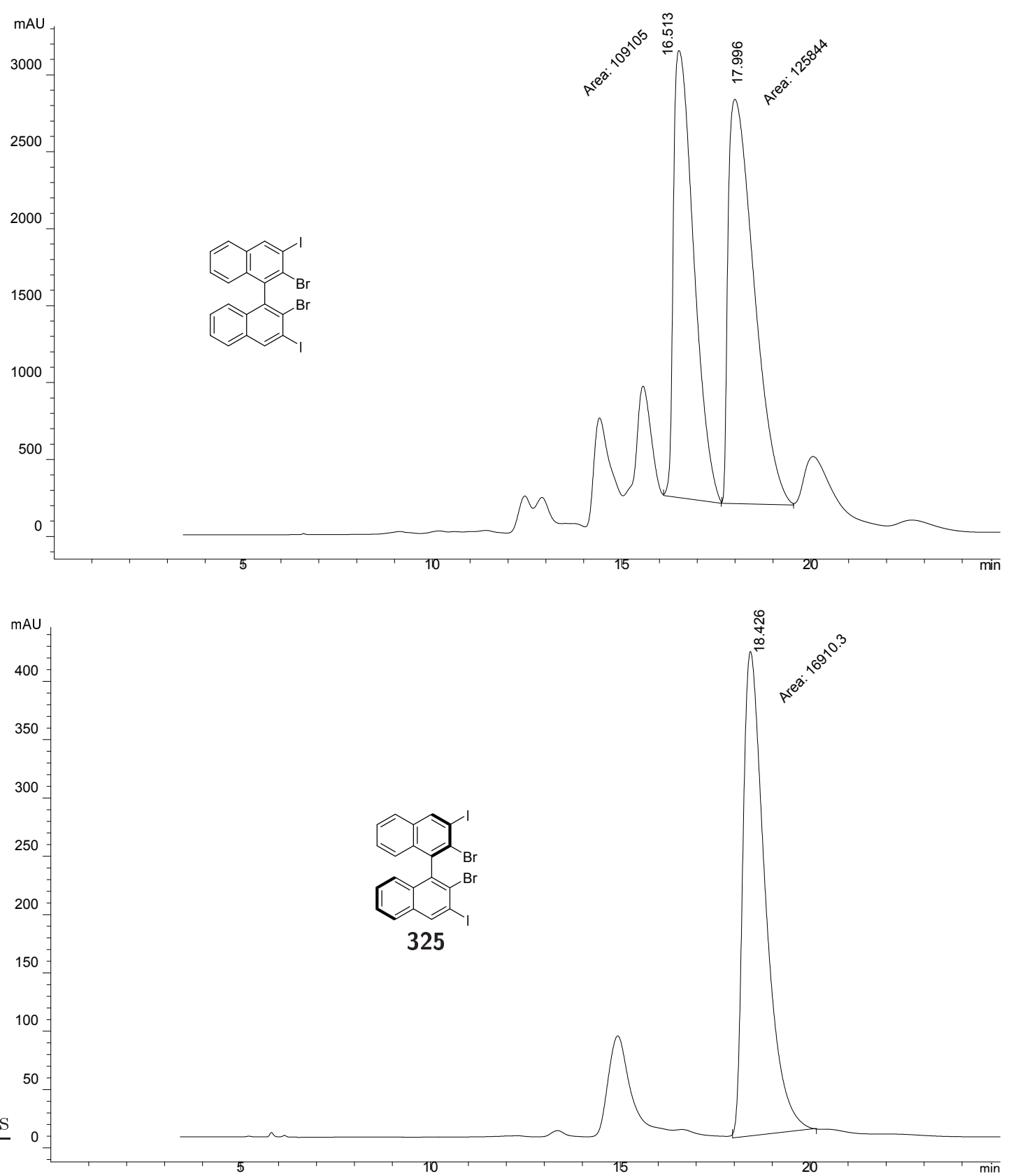


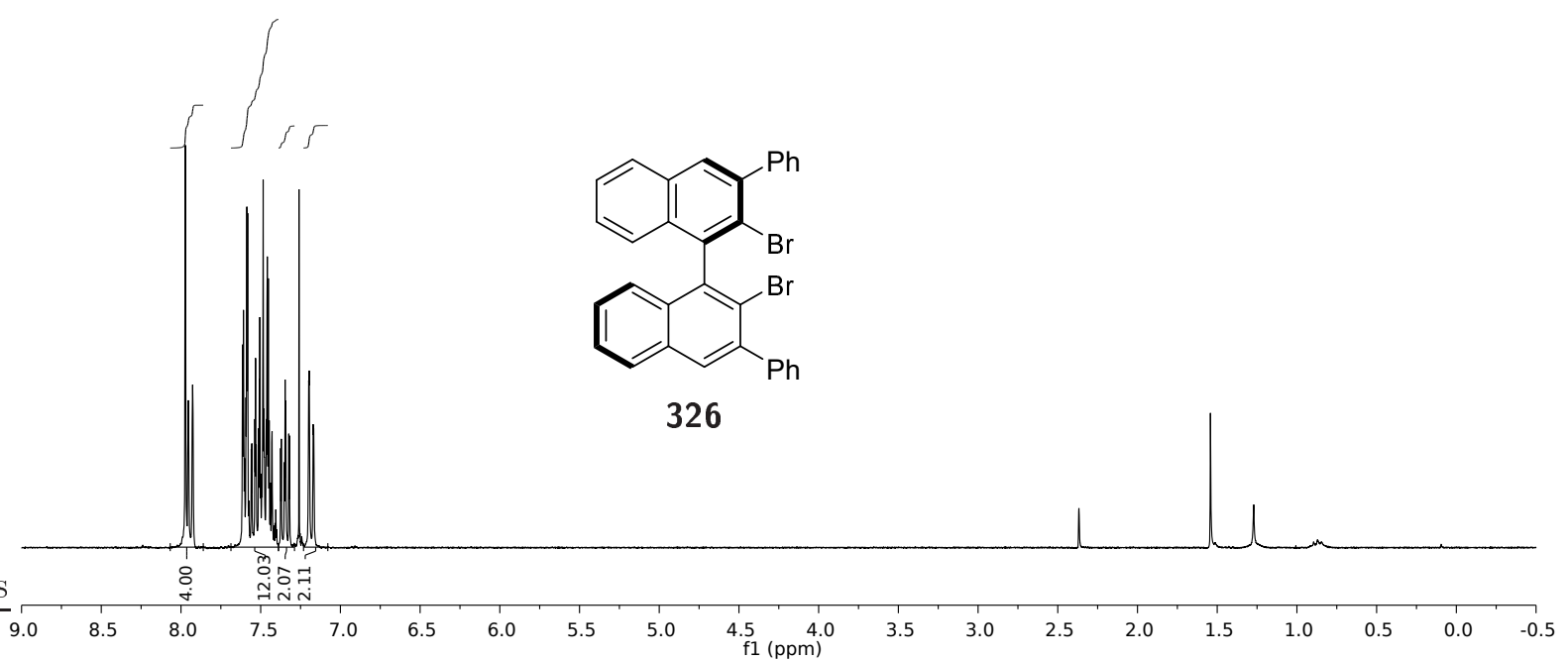

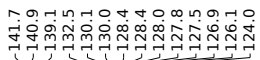
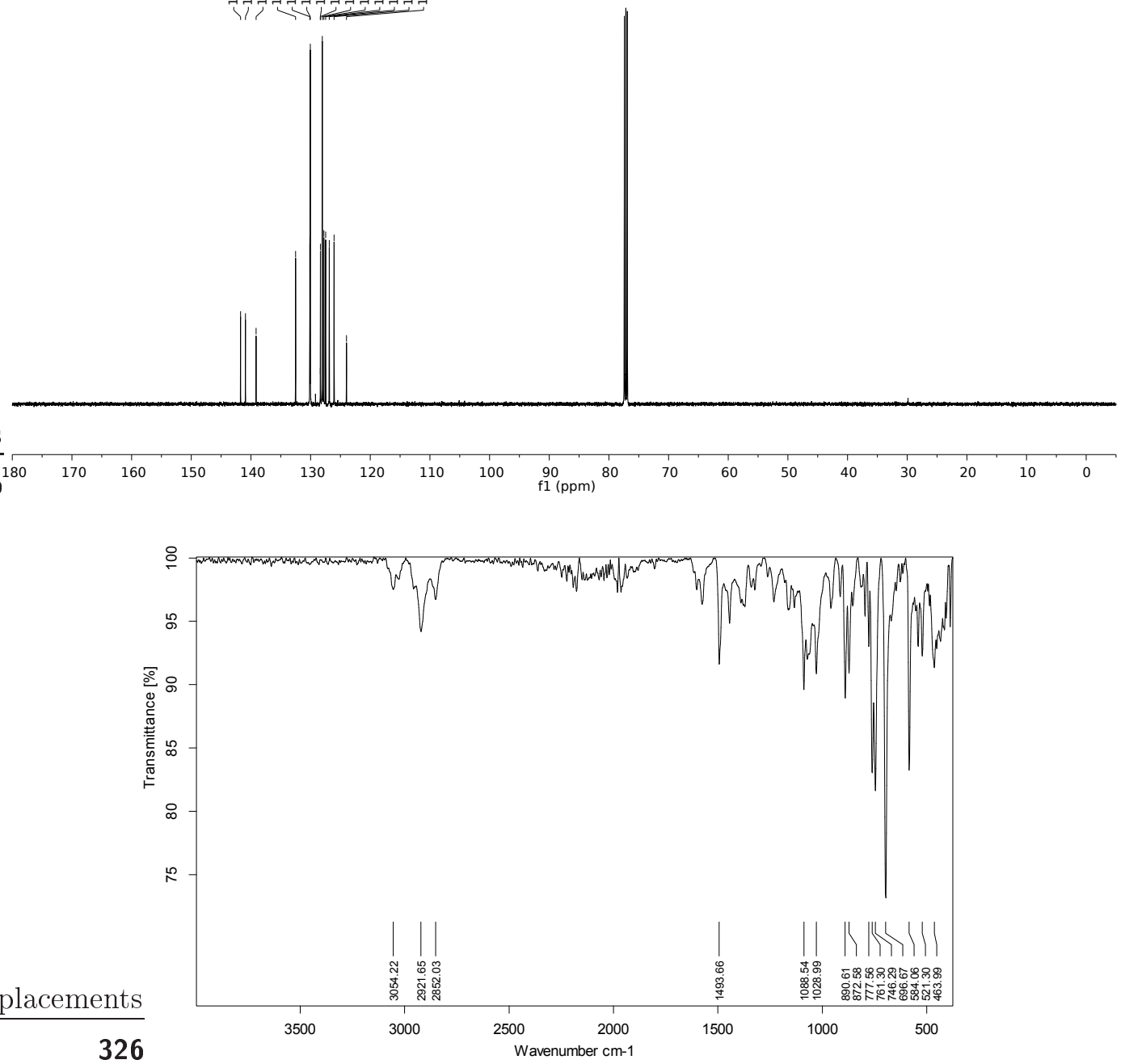


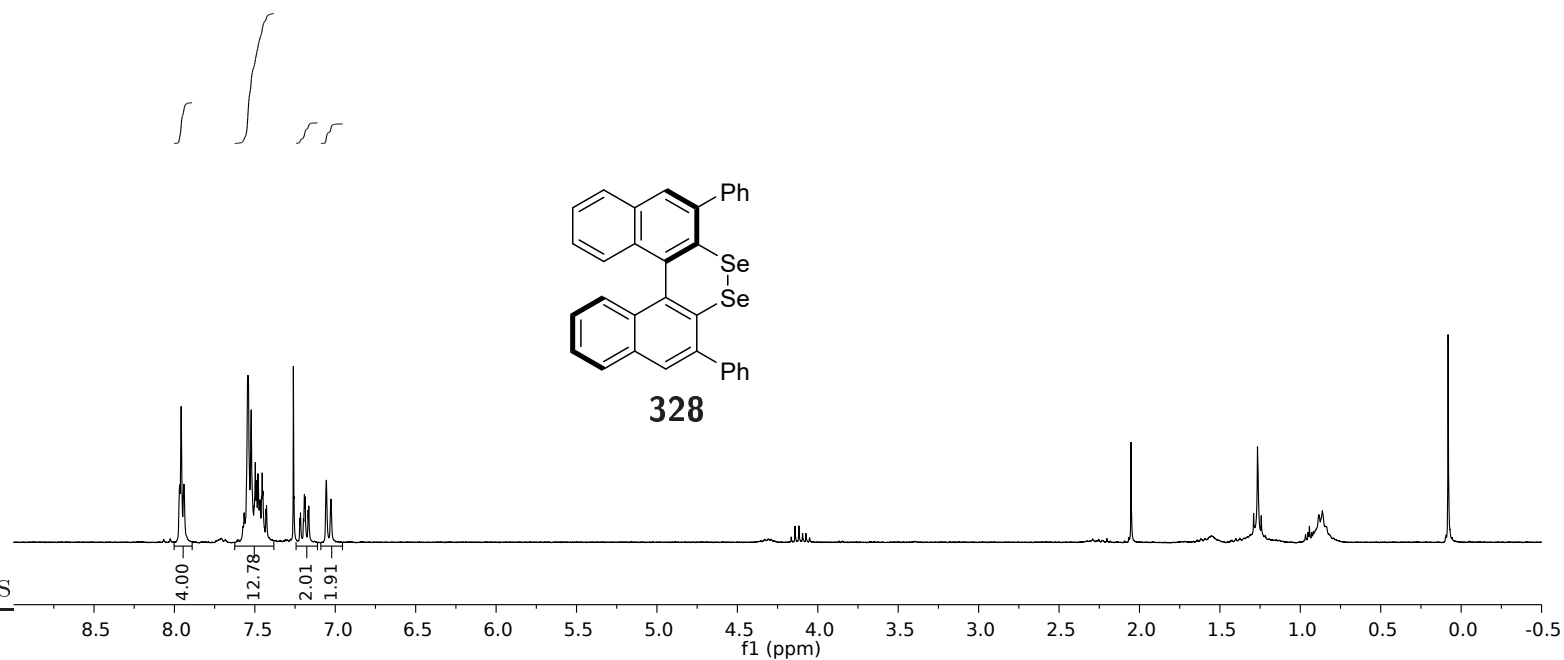

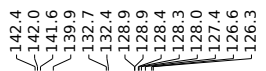
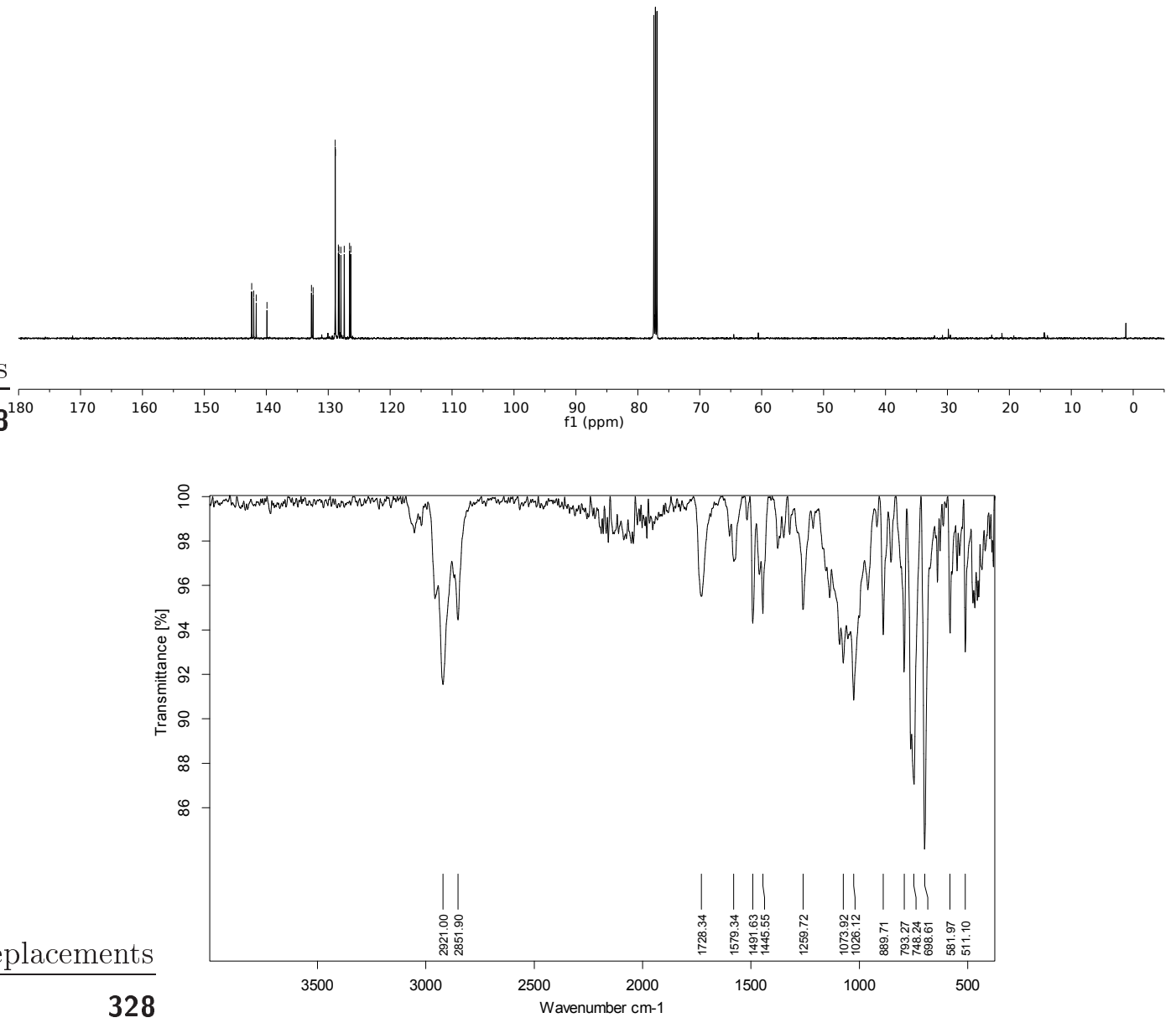


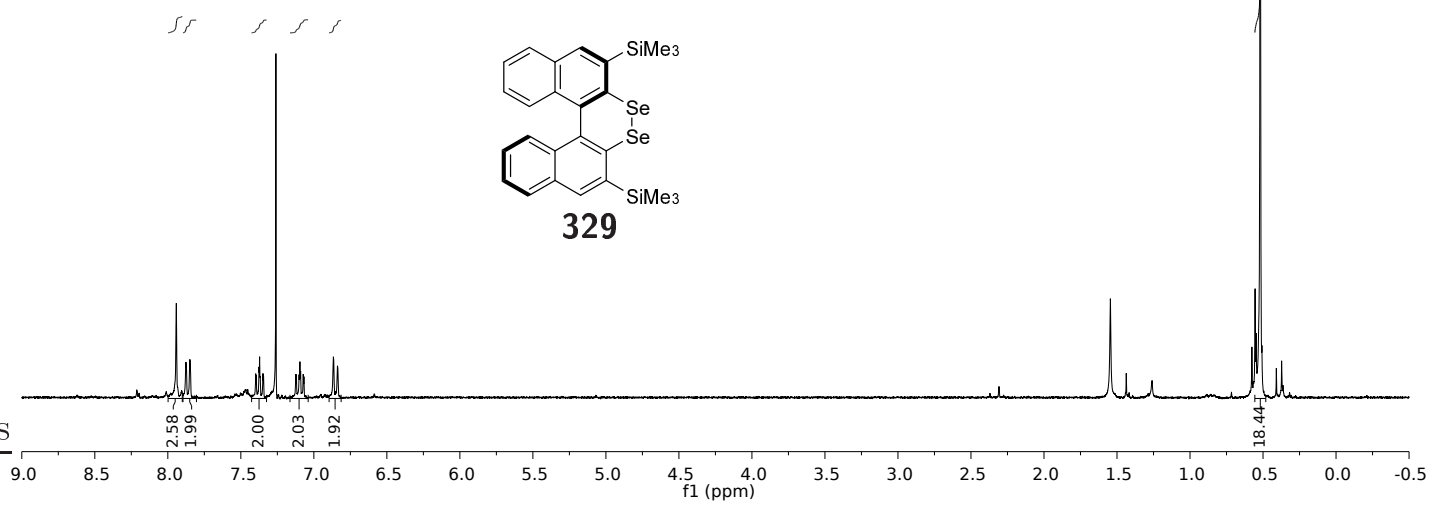

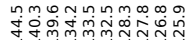

가국궈
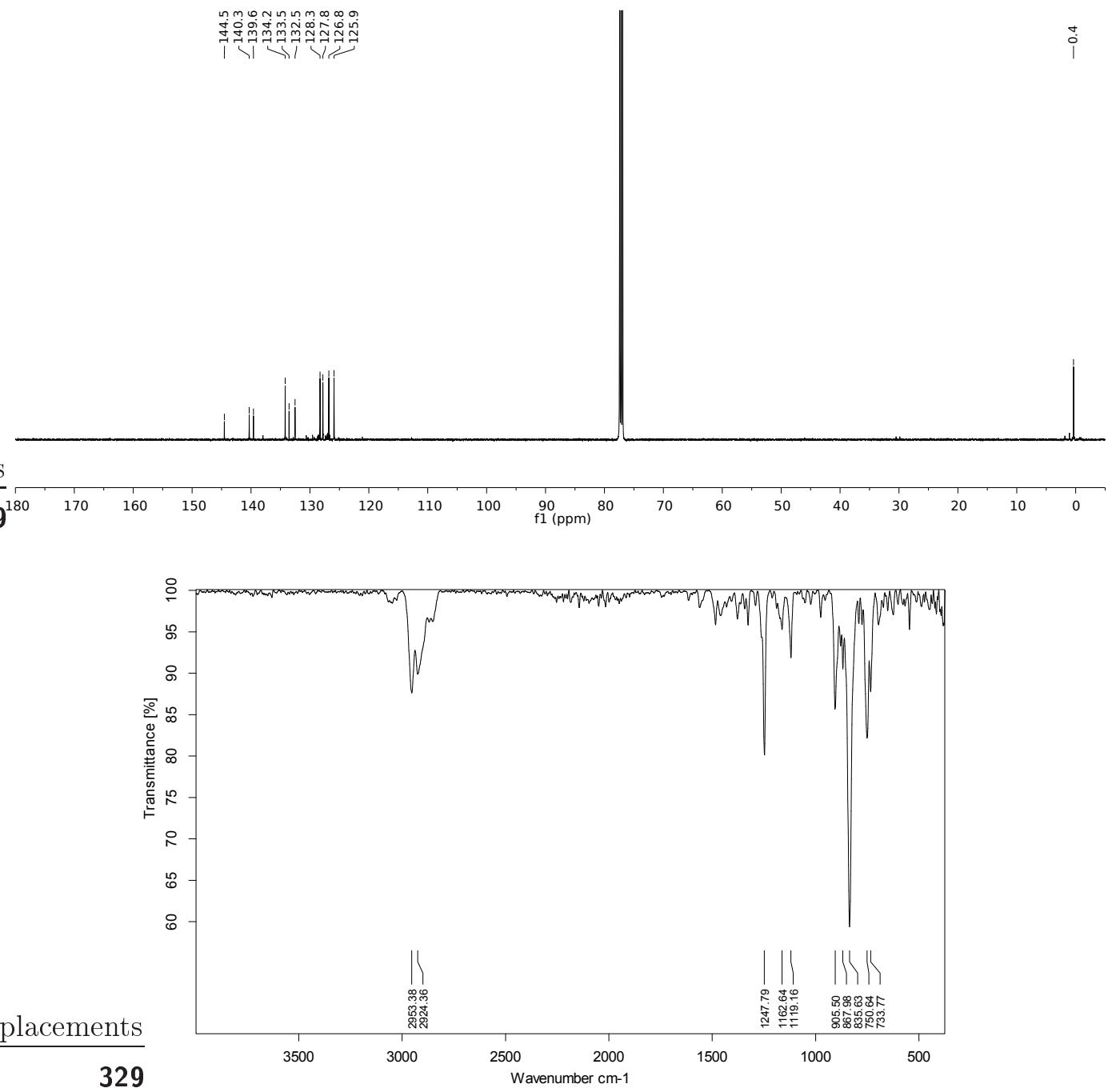


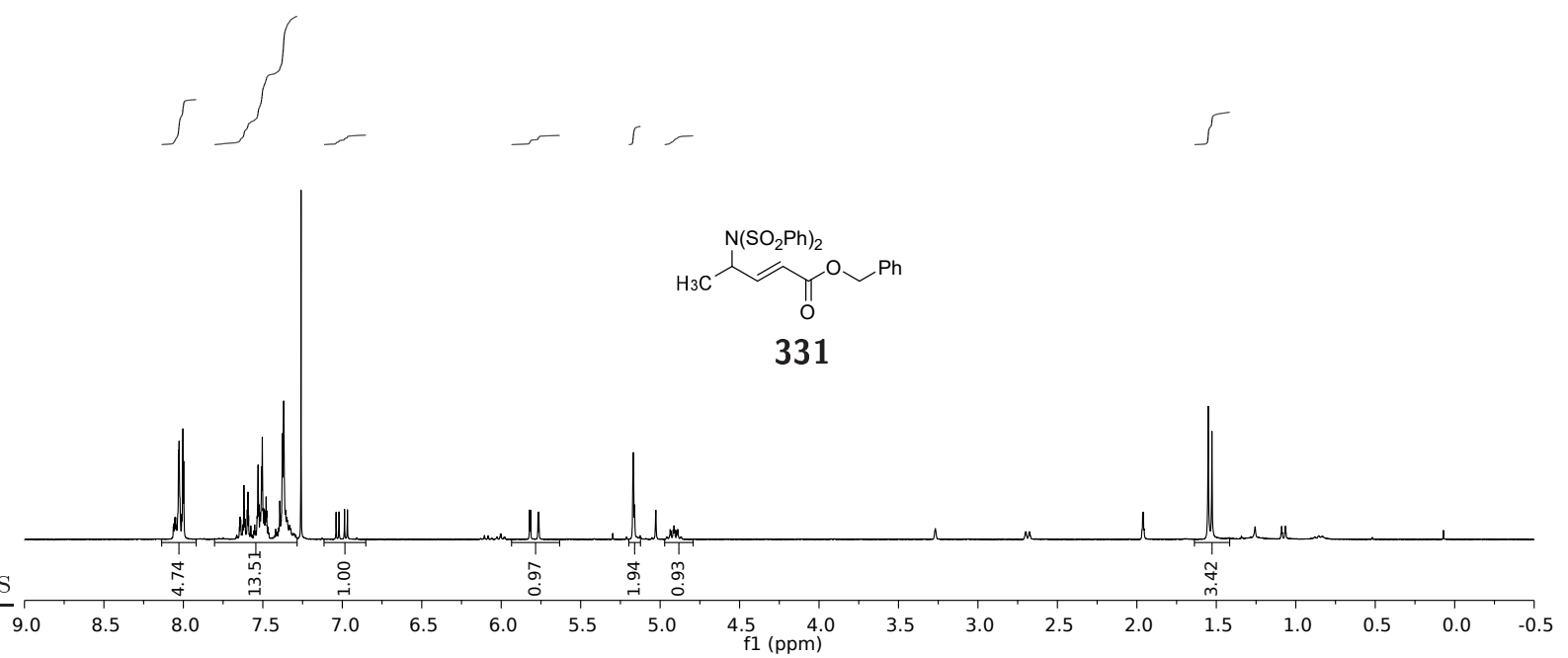

